\title{
WATER RESOURCES DATA-CALIFORNIA, WATER YEAR 1999 VOLUME 4-NORTHERN CENTRAL VALLEY BASINS AND THE GREAT BASIN FROM HONEY LAKE BASIN TO OREGON STATE LINE
}

\author{
By M.D. Webster, S.W. Anderson, G.L. Rockwell, J.R. Smithson, and M.F. Friebel
}

\section{INTRODUCTION}

The Water Resources Division of the U.S. Geological Survey, in cooperation with State and Federal agencies, obtains a large amount of data pertaining to the water resources of California each water year. These data, accumulated during many water years, constitute a valuable database for developing an improved understanding of the water resources of the State. To make these data readily available to interested parties outside the U.S. Geological Survey, the data are published annually in this report series entitled "Water Resources Data-California."

This volume of the report includes records on surface water in the State. Specifically, it contains: (1) discharge records for 176 streamflow-gaging stations and 1 partial-record station; (2) stage and content records for 45 lakes and reservoirs; (3) gageheight records for 1 station; (4) precipitation records for 3 stations; and (5) water-quality records for 14 streamflow-gaging stations and 7 water-quality partial-record stations. Records included for stream stages are only a small fraction of those obtained during the water year.

The series of annual reports for California began with the 1961 water year with a report that contained only data relating to the quantities of surface water. For the 1964 water year, a similar report was introduced that contained only data relating to water quality. Beginning with the 1975 water year, the report format changed to include data on quantities of surface water, quality of surface and ground water, and ground-water levels. From the 1985 through the 1993 water years, a separate volume for groundwater levels and quality was published for California.

Prior to introduction of this series and for several water years concurrent with it, water-resources data for California were published in U.S. Geological Survey Water-Supply Papers. Data on stream discharge and stage and on lake or reservoir contents and stage, through September 1960, were published annually under the title "Surface-Water Supply of the United States, Parts 10 and 11." For the 1961 through 1970 water years, the data were published in two 5-year reports. Data on chemical quality, temperature, and suspended sediment for the 1941 through 1970 water years were published annually under the title "Quality of Surface Waters of the United States," and water levels for the 1935 through 1974 water years were published under the title "Ground-Water Levels in the United States." These Water-Supply Papers may be consulted in public libraries of principal cities of the United States, or if not out of print, they may be purchased from U.S. Geological Survey, Information Services, Box 25286, Denver Federal Center, Denver, CO 80225-0046.

Publications similar to this report are published annually by the U.S. Geological Survey for all States. Each report has an identification number consisting of the two-letter State abbreviation, the last two digits of the water year, and the volume number. For example, this volume is identified as "U.S. Geological Survey Water-Data Report CA-99-4." For archiving and general distribution, the reports for 1971-74 water years also are identified as water-data reports. These water-data reports are for sale, in paper copy or on microfiche, by the National Technical Information Service, 5285 Port Royal Road, Springfield, VA 22161. For further ordering information, the Customer Inquiries telephone number is (703) 487-4650, between 8:30 a.m. and 5:30 p.m. Eastern Standard Time.

Additional information for ordering specific reports may be obtained from the District Office at the address given on the back of the title page or by telephone at (916) 278-3100.

\section{COOPERATION}

The U.S. Geological Survey and organizations of the State of California have had cooperative agreements for the systematic collection of records since 1903. Organizations that supplied data are acknowledged in station descriptions. Organizations that assisted in collecting data through cooperative agreement with the Survey are:

California Department of Water Resources, David N. Kennedy, Director.

Georgetown Divide Public Utility District, Marie E. Davis, General Manager.

Hidden Valley Lake Community Services District, Mel Aust, General Manager. 
Placer County, Edward McCarthy, Senior Civil Engineer.

Sacramento County Department of Public Works, Mark Rains, Associate Civil Engineer.

Yolo County Flood Control and Water Conservation District, James F. Eagan, General Manager.

Yuba County Water Agency, Donn Wilson, Engineer-Administrator.

Assistance in the form of funds or services was given by the Bureau of Reclamation, U.S. Department of Interior.

The following organizations aided in collecting records: Arbuckle Mountain Project; California Department of Water Resources; Energy Growth Partnership I; Five Bears Hydro, Inc.; Malacha Power Project, Inc.; Nelson Creek Power Co.; Nevada and Oroville-Wyandotte Irrigation Districts; Pacific Gas and Electric Co.; Placer and Yuba County Water Agencies; Sacramento Municipal Utility District; Shasta Hydroelectric; Sithe Energies, Inc.; Snow Mountain Hydroelectric; South Sutter Water District; STS Hydropower; and Synergics, Inc.

\section{SPECIAL NETWORKS AND PROGRAMS}

Hydrologic Benchmark Network is a network of 50 sites in small drainage basins around the country whose purpose is to provide consistent data on the hydrology, including water quality, and related factors in representative undeveloped watersheds nationwide, and to provide analyses on a continuing basis to compare and contrast conditions observed in basins more obviously affected by human activities.

National Stream-Quality Accounting Network (NASQAN) monitors the water quality of large rivers within four of the Nation's largest river basins - the Mississippi, the Columbia, the Colorado, and the Rio Grande. The network consists of 39 stations. Samples are collected with sufficient frequency that the flux of a wide range of constituents can be estimated. The objective of NASQAN is to characterize the water quality of these large rivers by measuring concentration and mass transport of a wide range of dissolved and suspended constituents, including nutrients, major ions, dissolved and sediment-bound heavy metals, common pesticides, and inorganic and organic forms of carbon. This information will be used (1) to describe the long-term trends and changes in concentration and transport of these constituents; (2) to test findings of the National Water-Quality Assessment Program (NAWQA); (3) to characterize processes unique to large-river systems such as storage and re-mobilization of sediments and associated contaminants; and (4) to refine existing estimates of off-continent transport of water, sediment, and chemicals for assessing human effects on the world's oceans and for determining global cycles of carbon, nutrients, and other chemicals.

The National Atmospheric Deposition Program/National Trends Network (NADP/NTN) provides continuous measurement and assessment of the chemical climate of precipitation throughout the United States. As the lead Federal agency, the USGS works together with over 100 organizations to accomplish the following objectives: (1) provide a long-term, spatial and temporal record of atmospheric deposition generated from a network of 191 precipitation chemistry monitoring sites; (2) provide the mechanism to evaluate the effectiveness of the significant reduction in $\mathrm{SO}_{2}$ emissions that began in 1995 as implementation of the Clean Air Act Amendments (CAAA) occurred; (3) provide the scientific basis and nationwide evaluation mechanism for implementation of the Phase II CAAA emission reductions for $\mathrm{SO}_{2}$ and $\mathrm{NO}_{\mathrm{x}}$ scheduled to begin in 2000.

Data from the network, as well as information about individual sites, are available through the world wide web at:

$$
\text { http://nadp.nrel.colostate.edu/NADP }
$$

The National Water-Quality Assessment (NAWQA) Program of the U.S. Geological Survey is a long-term program with goals to describe the status and trends of water-quality conditions for a large, representative part of the Nation's ground- and surface-water resources; provide an improved understanding of the primary natural and human factors affecting these observed conditions and trends; and provide information that supports development and evaluation of management, regulatory, and monitoring decisions by other agencies.

Assessment activities are being conducted in 53 study units (major watersheds and aquifer systems) that represent a wide range of environmental settings nationwide and that account for a large percentage of the Nation's water use. A wide array of chemical constituents will be measured in ground water, surface water, streambed sediments, and fish tissues. The coordinated application of comparative hydrologic studies at a wide range of spatial and temporal scales will provide information for decision making by water-resources managers and a foundation for aggregation and comparison of findings to address water-quality issues of regional and national interest.

Communication and coordination between USGS personnel and other local, State, and Federal interests are critical components of the NAWQA Program. Each study unit has a local liaison committee consisting of representatives from key Federal, State, and local water resources agencies, Indian nations, and universities in the study unit. Liaison committees typically meet semiannually to discuss their information needs, monitoring plans and progress, desired information products, and opportunities to collaborate efforts among the agencies.

Additional information about the NAWQA Program is available through the world wide web at:

http://wwwrvares.er.usgs.gov/nawqa/nawqa_home.html 


\section{EXPLANATION OF THE RECORDS}

The surface-water records published in this report are for the 1999 water year that began October 1, 1998, and ended September 30, 1999. A calendar of the water year is provided on the inside of the front cover. The records contain streamflow data, stage and contents data for lakes and reservoirs, and water-quality data for surface water. The following sections of the introductory text are presented to provide users with a more detailed explanation of how the hydrologic data published in this report were collected, analyzed, computed, and arranged for presentation.

\section{Station-Identification Numbers}

Each streamsite data station in this report is assigned a unique identification number. This number is unique in that it applies specifically to a given station and to no other. The number usually is assigned when a station is first established and is retained for that station indefinitely. The systems used by the U.S. Geological Survey to assign identification numbers for surface-water stations and for ground-water well sites differ, but both are based on geographic location. The "downstream-order" system is used for regular surface-water stations and the "latitude-longitude" system is used for surface-water stations in California where only miscellaneous measurements are made.

\section{Downstream-Order System}

Since October 1, 1950, the order of listing hydrologic-station records in Survey reports has been in a downstream direction along the main stream. All stations on a tributary entering upstream from a mainstream station are listed before that station. A station on a tributary that enters between two mainstream stations is listed between them. A similar order is followed in listing stations on first rank, second rank, and other ranks of tributaries. The rank of any tributary with respect to the stream to which it is immediately tributary is indicated by an indentation in the "List of Stations" in the front of this report. Each indentation represents one rank. This downstream order and system of indentation show which stations are on tributaries between any two stations and the rank of the tributary on which each station is situated.

The station-identification number is assigned according to downstream order. In assigning station numbers, no distinction is made between partial-record stations and other stations; therefore, the station number for a partial-record station indicates downstream-order position in a list made up of both types of stations. Gaps are left in the series of numbers to allow for new stations that may be established; hence, the numbers are not consecutive. The complete eight-digit number for each station such as 11396310, which appears just to the left of the station name, includes the two-digit part number "11" plus the six-digit downstreamorder number "396310." The part number designates the major river basin; for example, part "11" is the Pacific Slope Basins in California.

\section{Latitude-Longitude System}

The identification numbers for miscellaneous surface-water sites are assigned according to the grid system of latitude and longitude. The number consists of 15 digits. The first six digits denote the degrees, minutes, and seconds of latitude, the next seven digits denote degrees, minutes, and seconds of longitude, and the last two digits (assigned sequentially) identify the other sites within a 1-second grid. This site-identification number, once assigned, is a pure number and has no locational significance. In the rare instance where the initial determination of latitude and longitude are found to be in error, the station will retain its initial identification number; however, its true latitude and longitude will be listed in the LOCATION paragraph of the station description (fig. 1).

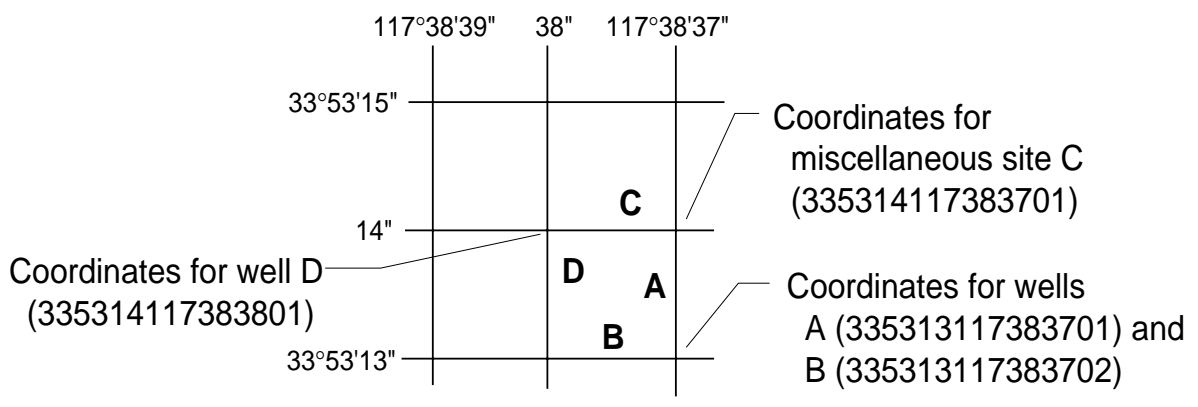

Figure 1. System for numbering miscellaneous sites (latitude and longitude). 


\section{Records of Stage and Water Discharge}

Records of stage and water discharge may be complete or partial. Complete records of discharge are those obtained using a continuous stage-recording device through which either instantaneous or mean daily discharges may be computed for any time, or any period of time, during the period of record. Complete records of lake and reservoir contents, similarly, are those for which stage or contents may be computed or estimated with reasonable accuracy for any time, or period of time. They may be obtained using a continuous stage-recording device, but need not be. Because daily mean discharges and end-of-day contents commonly are published for such stations, they are referred to as "daily stations."

By contrast, partial records are obtained through discrete measurements without using a continuous stage-recording device and pertain only to a few flow characteristics, or perhaps only one. The nature of the partial record is indicated by table titles such as "Crest-Stage Partial Records" or "Low-Flow Partial Records." Records of miscellaneous discharge measurements or of measurements from special studies, such as low-flow seepage studies, may be considered as partial records, but they are presented separately in this report. Location of all complete-record stations for which data are given in this report are shown, by county, in figures 2 through 23 .

\section{Data Collection and Computation}

The data obtained at a complete-record gaging station on a stream or canal consist of a continuous record of stage, individual measurements of discharge throughout a range of stages, and notations regarding factors that may affect the relation between stage and discharge. These data, together with supplemental information, such as weather records, are used to compute daily discharges. The data obtained at a complete-record gaging station on a lake or reservoir consist of a record of stage and of notations regarding factors that may affect the relation between stage and lake contents. These data are used with stage-area and stage-capacity curves or tables to compute water-surface areas and lake storage.

Continuous records of stage are obtained with digital recorders, data-collection platforms, or data loggers that sample stage values at selected time intervals. Measurements of discharge are made with current meters using methods adapted by the U.S. Geological Survey as a result of experience accumulated since 1880. These methods are described in standard textbooks, in U.S. Geological Survey Water-Supply Paper 2175, and in U.S. Geological Survey Techniques of Water-Resources Investigations (TWRI), Book 3, Chapters A1 through A19, and Book 8, Chapters A2 and B2. The methods are consistent with the American Society for Testing and Materials (ASTM) standards and generally follow the standards of the International Organization for Standards (ISO).

In computing discharge records, results of individual measurements are plotted against the corresponding stages, and stagedischarge relation curves are then constructed. From these curves, rating tables indicating the approximate discharge are prepared for any stage within the range of the measurements. If it is necessary to define extremes of discharge outside the range of currentmeter measurements, the curves are extended using (1) logarithmic plotting; (2) velocity-area studies; (3) results of indirect measurements of peak discharge, such as slope-area or contracted-opening measurements, and computations of flow-over-dam or weirs; or (4) step-backwater techniques.

Daily mean discharges are computed by applying the daily mean stages (gage heights) to the stage-discharge curves or tables. If the stage-discharge relation is subject to change because of frequent or continual change in the physical features that form the control, the daily mean discharge is determined by the shifting-control method, in which correction factors based on individual discharge measurements and notes of the personnel making the measurements are applied to the gage heights before the discharges are determined from the curves or tables. This shifting-control method also is used if the stage-discharge relation is changed temporarily because of aquatic growth or debris on the control. For some stations, formation of ice in the winter may so obscure the stage-discharge relations that daily mean discharges must be estimated from other information such as temperature and precipitation records, notes or observations, and records for other stations in the same or nearby basins for comparable periods.

At some stream-gaging stations, the stage-discharge relation is affected by backwater from reservoirs, tributary streams, or other sources. This necessitates the use of the slope method in which the slope or fall in a reach of the stream is a factor in computing discharge. The slope or fall is obtained by means of an auxiliary gage set at some distance from the base gage. At some stations the stage-discharge relation is affected by changing stage; at these stations the rate of change in stage is used as a factor in computing discharge.

At some gaging stations, acoustic-velocity meter (AVM) systems are used to compute discharge. The AVM system measures the stream's velocity at one or more paths in the cross section. Coefficients are developed to relate this path velocity to the mean velocity in the cross section. Because the AVM sensors are fixed in position, the adjustment coefficients generally vary with stage. Cross-sectional area curves are developed to relate stage, recorded as noted above, to cross-section area. Discharge is computed by multiplying path velocity by the appropriate stage-related coefficient and area.

In computing records of lake or reservoir contents, it is necessary to have available surveys, curves, or tables defining the relation of stage and contents. The application of stage to the stage-content curves or tables gives the contents from which daily, monthly, or yearly changes then are determined. If the stage-content relation changes because of deposition of sediment in a lake or reservoir, periodic resurveys may be necessary to redefine the relation. When this is done, the contents computed may become increasingly in error as time increases since the last survey. Discharges over lake or reservoir spillways are computed from stagedischarge relations in the same manner as other stream discharges are computed. 
For some gaging stations, there are periods when no gage-height record is obtained, or the recorded gage height is so faulty that it cannot be used to compute daily discharge or contents. This happens when the recorder stops or otherwise fails to operate properly, intakes are plugged, the float is frozen in the well, or for various other reasons. For such periods, the daily discharges are estimated from the recorded range in stage, previous or following record, discharge measurements, weather records, and comparison with other station records from the same or nearby basins. Likewise, daily contents may be estimated from operator's logs, previous or following records, inflow-outflow studies, and other information. Information explaining how estimated dailydischarge values are identified in station records is included in the next two sections, "Data Presentation" (REMARKS paragraph) and "Identifying Estimated Daily Discharge."

\section{Data Presentation}

Streamflow data in this report are presented in a new format that is considerably different from the format in data reports prior to the 1991 water year. The major changes are that statistical characteristics of discharge now appear in tabular summaries following the water-year data table and less information is provided in the text or station manuscript above the table. These changes represent the results of a pilot program to reformat the annual water-data report to meet current user needs and data preferences.

The records published for each continuous-record surface-water discharge station (gaging station) now consist of four parts, the manuscript or station description; the data table of daily mean values of discharge for the current water year with summary data; a tabular statistical summary of monthly mean flow data for a designated period, by water year; and a summary-statistics table that includes statistical data of annual, daily, and instantaneous flows as well as data pertaining to annual runoff, 7-day low-flow minimums, and flow duration.

\section{Station manuscript}

The manuscript provides, under various headings, descriptive information, such as station location; period of record; historical extremes outside the period of record; record accuracy; and other remarks pertinent to station operation and regulation. The following information, as appropriate, is provided with each continuous record of discharge or lake content. Comments follow to clarify information presented under the various headings of the station description.

LOCATION.- Information on locations is obtained from the most accurate maps available. The location of the gaging station is given with respect to the cultural and physical features in the vicinity and with respect to the reference place mentioned in the station name. River mileages, given for only a few stations, were determined by methods given in "River Mileage

Measurement," Bulletin 14, Revision of October 1968, prepared by the Water Resources Council, or were provided by the U.S. Army Corps of Engineers.

DRAINAGE AREA.-Drainage areas are measured using the most accurate maps available. Because the type of maps available varies from one drainage basin to another, the accuracy of drainage areas likewise varies. Drainage areas are updated as better maps become available.

PERIOD OF RECORD.- - This indicates the period for which there are published records for the station or for an equivalent station. An equivalent station is one that was in operation at a time when the present station was not, and whose location was such that records from it reasonably can be considered equivalent with records from the present station.

REVISED RECORDS.-Published records, because of new information, occasionally are incorrect, and revisions are printed in later reports. Listed under this heading are all the reports in which revisions have been published for the station and the water years to which the revisions apply. If a revision did not include daily, monthly, or annual figures of discharge, that fact is noted after the year dates as follows: "(M)" means that only the instantaneous maximum discharge was revised; "(m)" that only the instantaneous minimum was revised; and "(P)" that only peak discharges were revised. If the drainage area has been revised, the report is given in which the most recently revised figure was published.

GAGE.-The type of gage currently in use, the datum of the current gage referred to sea level (see glossary), and a condensed history of the types, locations, and datums of previous gages are given under this heading.

REMARKS.-All periods of estimated daily-discharge record will either be identified by date in this paragraph of the station description for water-discharge stations or flagged in the daily-discharge table. (See next section, "Identifying Estimated Daily Discharge.") If a REMARKS paragraph is used to identify estimated record, the paragraph will begin with this information presented as the first entry. The paragraph also is used to present information relative to the accuracy of the records, to special methods of computation, to conditions that affect natural flow at the station, and possibly to other pertinent items. For reservoir stations, information is given on the dam forming the reservoir, the capacity, outlet works and spillway, and purpose and use of the reservoir.

COOPERATION.- Records provided by a cooperating organization or obtained for the U.S. Geological Survey by a cooperating organization are identified.

EXTREMES FOR PERIOD OF RECORD.-Extremes may include maximum and minimum discharges or content. Unless otherwise qualified, the maximum discharge or content is the instantaneous maximum corresponding to the highest stage that occurred. The highest stage may have been obtained from a graphic or digital recorder, a crest-stage gage, or by direct observation of a nonrecording gage. If the maximum stage did not occur on the same day as the maximum discharge or content, it is given 
separately. Similarly, the minimum is the instantaneous minimum discharge, unless otherwise qualified, and was determined and is reported in the same manner as the maximum.

EXTREMES OUTSIDE PERIOD OF RECORD.-Included is information concerning major floods or unusually low flows that occurred outside the stated period of record. The information may or may not have been obtained by the U.S. Geological Survey.

EXTREMES FOR CURRENT YEAR.-Extremes given are similar to those for the period of record, except the peak discharge listing may include secondary peaks. For stations meeting certain criteria, all peak discharges and stages occurring during the water year that are greater than a selected base discharge are presented under this heading. The peaks greater than the base discharge, excluding the highest one, are referred to as secondary peaks. Peak discharges are not published for canals, ditches, drains, or streams for which the peaks are subject to substantial control by man. The time of occurrence for peaks is expressed in 24-hour local standard time. For example, 12:30 a.m. is 0030, and 1:30 p.m. is 1330.

REVISIONS.-If a critical error is discovered in published records, a revision is included in the first report published following discovery of the error.

Occasionally the records of a discontinued gaging station may need revision. Because for these stations there would be no current or, possible, future station manuscript published to document the revision in a "Revised Records" entry, users of data for these stations who obtained the record from previously published data reports may wish to contact the District Office to determine if the published records were revised after the station was discontinued. If the data were obtained by computer retrieval, the data would be current and there would be no need to check because any published revision of data is always accompanied by revision of the corresponding data in computer storage.

Manuscript information for lake or reservoir stations differs from that for stream-gaging stations in the nature of the "Remarks" and in the inclusion of a skeleton stage-capacity table when daily contents are given.

\section{Data table of daily mean values}

The daily table of discharge records for stream-gaging stations gives mean discharge for each day of the water year. In the monthly summary for the table, the line headed "TOTAL" gives the sum of the daily figures for each month; the line headed "MEAN" gives the average flow in cubic feet per second for the month; and the lines headed "MAX" and "MIN" give the maximum and minimum daily mean discharges, respectively, for each month. Discharge for the month also usually is expressed in cubic feet per second per square mile (line headed "CFSM"); or in inches (line headed "IN."); or in acre-feet (line headed "AC-FT"). Figures for cubic feet per second per square mile and runoff in inches or in acre-feet may be omitted if there is extensive regulation or diversion or if the drainage area includes large noncontributing areas. At some stations monthly and (or) yearly observed discharges are adjusted for reservoir storage or diversion, or diversion data or reservoir contents are given. These figures are identified by a symbol and corresponding footnote.

\section{Statistics of monthly mean data}

A tabular summary of the mean (line headed "MEAN"), maximum (line headed "MAX"), and minimum (line headed "MIN") of monthly mean flows for each month for a designated period is provided below the mean values table. The water years of the first occurrence of the maximum and minimum monthly flows are provided immediately below those figures. The designated period will be expressed as "FOR WATER YEARS ____ BY WATER YEAR (WY)," and will list the first and last water years of the range of years selected from the PERIOD OF RECORD paragraph in the station manuscript. It will consist of all of the station record within the specified water years, inclusive, including complete months of record for partial water years, if any, and may coincide with the period of record for the station. The water years for which the statistics are computed will be consecutive, unless a break in the station record is indicated in the manuscript.

\section{Summary statistics}

A table titled "SUMMARY STATISTICS" follows the statistics of monthly mean data tabulation for tables containing complex data for the current water year. This table consists of four columns, with the first column containing the line headings of the statistics being reported. The table provides a statistical summary of yearly, daily, and instantaneous flows, not only for the current water year but also for the previous calendar year and for a designated period, as appropriate. The designated period selected, "WATER YEARS ____, will consist of all of the station record within the specified water years, inclusive, including complete months of record for partial water years, if any, and may coincide with the period of record for the station. The water years for which the statistics are computed will be consecutive, unless a break in the station record is indicated in the manuscript. All of the calculations for the statistical characteristics designated ANNUAL (see line headings below), except for the "ANNUAL 7-DAY MINIMUM" statistic, are calculated for the designated period using complete water years. The other statistical characteristics may be calculated using partial water years.

The date or water year, as appropriate, of the first occurrence of each statistic reporting extreme values of discharge is provided adjacent to the statistic. Repeated occurrences may be noted in the REMARKS paragraph of the manuscript or in footnotes. Because the designated period may not be the same as the station period of record published in the manuscript, occasionally the dates of occurrence listed for the daily and instantaneous extremes in the designated-period column may not be within the selected water years listed in the heading. When this occurs, it will be noted in the REMARKS paragraph or in footnotes. 
Selected streamflow duration curve statistics and runoff data also are given. Runoff data may be omitted if there is extensive regulation or diversion of flow in the drainage basin.

The following summary statistics data, as appropriate, are provided with each continuous record of discharge. Comments follow to clarify information presented under the various line headings of the summary statistics table.

ANNUAL TOTAL.- The sum of the daily mean values of discharge for the year. At some stations the annual total discharge is adjusted for reservoir storage or diversion. The adjusted figures are identified by a symbol and corresponding footnotes.

ANNUAL MEAN.- The arithmetic mean of the individual daily mean discharges for the year noted or for the designated period. At some stations the yearly mean discharge is adjusted for reservoir storage or diversion. The adjusted figures are identified by a symbol and corresponding footnotes.

HIGHEST ANNUAL MEAN.-The maximum annual mean discharge occurring for the designated period.

LOWEST ANNUAL MEAN.-The minimum annual mean discharge occurring for the designated period.

HIGHEST DAILY MEAN.-The maximum daily mean discharge for the year or for the designated period.

LOWEST DAILY MEAN.-The minimum daily mean discharge for the year or for the designated period.

INSTANTANEOUS PEAK FLOW.- The maximum instantaneous discharge occurring for the water year or for the designated period. Note that secondary instantaneous peak discharges above a selected base discharge are stored in District computer files for stations meeting certain criteria. Those discharge values may be obtained by writing to the District Office. (See address on back of title page of this report.)

INSTANTANEOUS PEAK STAGE.-The maximum instantaneous stage occurring for the water year or for the designated period. If the dates of occurrence for the instantaneous peak flow and instantaneous peak stage differ, the REMARKS paragraph in the manuscript or a footnote may be used to provide further information.

INSTANTANEOUS LOW FLOW.-The minimum instantaneous discharge occurring for the water year or for the designated period.

ANNUAL RUNOFF.- Indicates the total quantity of water in runoff for a drainage area for the year. Data reports may use any of the following units of measurement in presenting annual runoff data:

Acre-foot (AC-FT) is the quantity of water required to cover 1 acre to a depth of 1 foot and is equivalent to 43,560 cubic feet, or about 326,000 gallons, or 1,233 cubic meters.

Cubic feet per second per square mile (CFSM) is the average number of cubic feet of water flowing per second from each square mile of area drained, assuming that the runoff is distributed uniformly in time and area.

Inches (IN.) indicates the depth to which the drainage area would be covered if all the runoff for a given period were distributed on it uniformly.

10 PERCENT EXCEEDS.- The discharge that is exceeded 10 percent of the time for the designated period.

50 PERCENT EXCEEDS.- - The discharge that is exceeded 50 percent of the time for the designated period.

90 PERCENT EXCEEDS. - The discharge that is exceeded 90 percent of the time for the designated period.

Data collected at partial-record stations follow the information for continuous-record sites. Data for partial-record discharge stations are presented in two tables. The first is a table of annual maximum stage and discharge at crest-stage stations, and the second is a table of discharge measurements at low-flow partial-record stations. The tables of partial-record stations are followed by a listing of discharge measurements made at sites other than continuous-record or partial-record stations. These measurements generally are made in times of drought or flood to give better areal coverage to those events. Those measurements and others collected for some special reason are called measurements at miscellaneous sites.

\section{Identifying Estimated Daily Discharge}

Estimated daily-discharge values published in the water-discharge tables of annual State data reports are identified either by flagging individual daily values with the letter symbol "e" and printing the table footnote, "e Estimated," or by listing the dates of the estimated record in the REMARKS paragraph of the station description.

\section{Accuracy of the Records}

The accuracy of streamflow records depends primarily on (1) the stability of the stage-discharge relation or, if the control is unstable, the frequency of discharge measurements, and (2) the accuracy of measurements of stage and discharge, and interpretation of records.

The accuracy attributed to the records is indicated under "REMARKS." "Excellent" means that about 95 percent of the daily discharges are within 5 percent of the true; "good," within 10 percent; and "fair," within 15 percent. Records that do not meet the criteria mentioned, are rated "poor." Different accuracies may be attributed to different parts of a given record.

Daily mean discharges in this report are given to the nearest hundredth of a cubic foot per second ( $\mathrm{ft}^{3} / \mathrm{s}$ ) for values less than $1 \mathrm{ft}^{3} / \mathrm{s}$, to the nearest tenth between 1.0 and $10 \mathrm{ft}^{3} / \mathrm{s}$, to whole numbers between 10 and $1,000 \mathrm{ft}^{3} / \mathrm{s}$, and to three significant figures 
for more than $1,000 \mathrm{ft}^{3} / \mathrm{s}$. The number of significant figures used is based solely on the magnitude of the discharge value. The same rounding rules apply to discharges listed for partial-record stations and miscellaneous sites.

Discharge at many stations, as indicated by the monthly mean, may not reflect natural runoff due to the effects of diversion, consumption, regulation by storage, increase or decrease in evaporation due to artificial causes, or to other factors. For such stations, figures of cubic feet per second per square mile and of runoff, in inches, are not published unless satisfactory adjustments can be made for diversions, for changes in contents of reservoirs, or for other changes incident to use and control. Evaporation from a reservoir is not included in the adjustments for changes in reservoir contents, unless it is so stated. Even at those stations where adjustments are made, large errors in computed runoff may occur if adjustments or losses are large in comparison with the measured discharge.

\section{Other Records Available}

The National Water Data Exchange (NAWDEX), U.S. Geological Survey, Reston, VA 20192, maintains an index of sites as well as an index of records of discharge collected by other agencies but not published by the U.S. Geological Survey. Information on records at specific sites can be obtained from that office upon request.

Information used in the preparation of the records in this publication, such as discharge measurement notes, gage-height records, temperature measurements, and rating tables are on file in the District Office. Also, most of the daily mean discharges are in computer-readable form and have been analyzed statistically. Information on the availability of the unpublished information or on the results of statistical analyses of the published records may be obtained from the District Office.

\section{Records of Surface-Water Quality}

Records of surface-water quality ordinarily are obtained at or near stream-gaging stations because interpretation of records of surface-water quality nearly always requires corresponding discharge data. Records of surface-water quality in this report may involve various types of data and measurement frequencies.

\section{Classification of Records}

Water-quality data for surface-water sites are grouped into one of three classifications. A continuing-record station is a site where data are collected on a regularly scheduled basis. Frequency may be one or more times daily, weekly, monthly, or quarterly. A partial-record station is a site where limited water-quality data are collected systematically over a period of years. Frequency of sampling is usually less than quarterly. A miscellaneous sampling site is a location other than a continuing or partial-record station, where random samples are collected to give better areal coverage to define water-quality conditions in the river basin.

A careful distinction needs to be made between "continuing records" as used in this report and "continuous recordings," which refers to a continuous graph or a series of discrete values punched at short intervals on a paper tape or stored electronically in a data logger. Some records of water quality, such as temperature and specific conductance, may be obtained through continuous recordings; however, because of costs, most data are obtained only monthly or less frequently. Locations of stations for which records on the quality of surface water appear in this report are shown in figures 2 through 23.

\section{Arrangement of Records}

Water-quality records collected at a surface-water daily record station are published immediately following that record, regardless of the frequency of sample collection. Station number and name are the same for both records. Where a surface-water daily record station is not available or where the water quality differs significantly from that at the nearby surface-water station, the continuing water-quality record is published with its own station number and name in the regular downstream order sequence. Water-quality data for partial-record stations and for miscellaneous sampling sites appear in separate tables following the table of discharge measurements at miscellaneous sites.

\section{Onsite Measurements and Sample Collection}

In obtaining water-quality data, a major concern is the assurance that the data obtained represent the in situ quality of the water. To assure this, certain measurements, such as water temperature, $\mathrm{pH}$, and dissolved oxygen, are made onsite when samples are taken. To assure that measurements made in the laboratory also represent the in situ water, carefully prescribed procedures are followed in collecting the samples, in treating the samples to prevent changes in quality pending analysis, and in shipping the samples to the laboratory. Procedures for onsite measurements and for collecting, treating, and shipping samples are given in "Techniques of Water-Resources Investigations," Book 1, Chapter D2; Book 3, Chapter C2; and Book 5, Chapters A1, A3, and A4. All these references are listed in the section "Publications on Techniques of Water-Resources Investigations." Also, detailed information on collecting, treating, and shipping samples may be obtained from the District Office. 
One sample can adequately define the water quality at a given time if the mixture of solutes throughout the stream cross section is homogeneous. However, the concentration of solutes at different locations in the cross section may vary widely with different rates of water discharge, depending on the source of material and the turbulence and mixing of the stream. Some streams must be sampled through several vertical sections to obtain a representative sample needed for an accurate mean concentration and for use in calculating load. All samples obtained for the National Stream-Quality Accounting Network (see definitions) are obtained from at least several verticals. Whether samples are obtained from the centroid of flow or from several verticals depends on flow conditions and other factors which must be evaluated by the collector.

Chemical-quality data published in this report are considered to be the most representative value available for the stations listed. The values reported represent water-quality conditions at the time of sampling as much as possible, consistent with available sampling techniques and methods of analysis. In the rare case where an apparent inconsistency exists between a reported $\mathrm{pH}$ value and the relative abundance of carbon dioxide species (carbonate and bicarbonate), the inconsistency is the result of a slight uptake of carbon dioxide from the air by the sample between measurement of $\mathrm{pH}$ in the field and determination of carbonate and bicarbonate in the laboratory.

For chemical-quality stations equipped with digital monitors, the records consist of daily maximum and minimum values for each constituent measured and are based on hourly punches beginning at 0100 hours and ending at 2400 hours for the day of record. More detailed records (hourly values) may be obtained from the District Office.

Historical and current (1999) dissolved trace-element concentrations are reported herein for water that was collected, processed, and analyzed by using either ultraclean or other than ultraclean techniques. If ultraclean techniques were used, then those concentrations are reported in nanograms per liter $(\mathrm{ng} / \mathrm{L})$. If other than ultraclean techniques were used, then those concentrations are reported in micrograms per liter $(\mu \mathrm{g} / \mathrm{L})$ and could reflect contamination introduced during some phase of the procedure.

\section{Water Temperature}

Water temperatures are measured at the water-quality stations. In addition, water temperatures are taken at time of discharge measurements for water-discharge stations. For stations where water temperatures are taken manually once or twice daily, the water temperatures are taken at about the same time each day. Large streams have a small diurnal temperature change; shallow streams may have a daily range of several degrees and may follow closely the changes in air temperature. Some streams may be affected by waste-heat discharges.

At stations where recording instruments are used, either mean temperatures or maximum and minimum temperatures for each day are published. Water temperatures measured at the time of water-discharge measurements are on file in the District Office.

\section{Sediment}

Suspended-sediment concentrations are determined from samples collected by using depth-integrating samplers. Samples usually are obtained at several verticals in the cross section, or a single sample may be obtained at a fixed point and a coefficient applied to determine the mean concentration in the cross section.

During periods of rapidly changing flow or rapidly changing concentration, samples may have been collected more frequently (twice daily or, in some instances, hourly). The published sediment discharges for days of rapidly changing flow or concentration were computed by the subdivided-day method (time-discharge weighted average). Therefore, for those days when the published sediment discharge value differs from the value computed as the product of discharge times mean concentration times 0.0027, the reader can assume that the sediment discharge for that day was computed by the subdivided-day method. For periods when no samples were collected, daily discharges of suspended sediment were estimated on the basis of water discharge, sediment concentrations measured immediately before and after the periods, and suspended-sediment loads for other periods of similar discharge. Methods used in the computation of sediment records are described in the TWRI Book 3, Chapters C1 and C3. These methods are consistent with the ASTM standards and generally follow ISO standards.

At other stations, suspended-sediment samples were collected periodically at many verticals in the stream cross section. Although data collected periodically may represent conditions only at the time of observation, such data are useful in establishing seasonal relations between quality and streamflow and in predicting long-term sediment-discharge characteristics of the stream.

In addition to the records of suspended-sediment discharge, records of the periodic measurements of the particle-size distribution of suspended sediment, bed material, and bed load are included for some stations.

Estimates of bed-load and total-sediment discharge are included for some stations. Computations of monthly bed-load discharges are based on the relation between instantaneous water discharge and corresponding bed-load discharge for the station. Values of bed-load discharge used in defining this relation are based on samples obtained by use of the Helley-Smith or BL 84 bedload samplers or by modified-Einstein or Meyer-Peter Muller computation procedures. Application of the bed-load-transport relation at a station was made on a daily basis or subdivided-day basis. The bed-load samplers are designed to collect time-weighted samples for the sediment moving within $0.25 \mathrm{ft}$ of the streambed. Sediment moving in this portion of the flow cannot be sampled with standard suspended-sediment samplers. Calibration of the bed-load samplers has not been completed, and a trap efficiency of 1.0 has been assumed applicable to these devices. Error sources in the theoretical methods, based on analysis of bed-material 
characteristics, channel geometry, and associated hydraulic factors, are also undefined. In consequence, figures of bed-load discharge must be used with caution. They are estimates, at best, and are subject to revision.

\section{Cross-Sectional Data}

Cross-sectional surveys of water temperature, $\mathrm{pH}$, specific conductance, dissolved oxygen, and suspended sediment are done at all NASQAN and Hydrologic Benchmark Stations during various seasons and surface-water discharges. Documentation of crosssection variation of water quality is essential in order to determine how many samples in a cross section are necessary to ensure a representative composite sample.

\section{Laboratory Measurements}

Sediment samples, biochemical-oxygen-demand (BOD) samples, indicator-bacteria samples, and daily specificconductance samples are analyzed locally. All other samples are analyzed in the U.S. Geological Survey's National Water-Quality Laboratory in Arvada, Colorado. Methods used to analyze sediment samples and to compute sediment records are described in the Techniques of Water-Resources Investigations, Book 5, Chapter C1. Methods used by the U.S. Geological Survey laboratories are given in TWRI Book 1, Chapter D2; Book 3, Chapter C2; and Book 5, Chapters A1, A3, A4, and A5. These methods are consistent with ASTM standards and generally follow ISO standards.

\section{Water Quality-Control Data}

Data generated from quality-control (QC) samples are a requisite for evaluating the quality of the sampling and processing techniques as well as data from the actual samples themselves. Without QC data, environmental-sample data cannot be interpreted adequately because the errors associated with the sample data are unknown. The various types of QC samples collected by this District are described in the following section. Procedures have been established for the storage of water quality-control data within the U. S. Geological Survey. These procedures allow for storage of all derived QC data and are identified so that they can be related to corresponding environmental samples.

\section{Blank Samples}

Blank samples are collected and analyzed to ensure the environmental samples have not been contaminated by the overall data-collection process. The blank solution used to develop specific types of blank samples is a solution that is free of the analytes of interest. Any measured value signal in a blank sample for an analyte (a specific component measured in a chemical analysis) that was absent in the blank solution is believed to be due to contamination. There are many types of blank samples possible, each designed to segregate a different part of the overall data-collection process. The types of blank samples collected in this District are:

Field blank-a blank solution that is subjected to all aspects of sample collection, field processing preservation, transportation, and laboratory handling as an environmental sample.

Trip blank-a blank solution that is put in the same type of bottle used for an environmental sample and kept with the set of sample bottles before and after sample collection.

Equipment blank-a blank solution that is processed through all equipment used for collecting and processing an environmental sample (similar to a field blank but normally done in the more controlled conditions of the office).

Sampler blank - a blank solution that is poured or pumped through the same field sampler used for collecting an environmental sample.

Filter blank - a blank solution that is filtered in the same manner and through the same filter apparatus used for an environmental sample.

Splitter blank-a blank solution that is mixed and separated using a field splitter in the same manner and through the same apparatus used for an environmental sample.

Preservation blank - a blank solution that is treated with the sampler preservatives used for an environmental sample.

\section{$\underline{\text { Reference Samples }}$}

Reference material is a solution or material prepared by a laboratory whose composition is certified for one or more properties so that it can be used to assess a measurement method. Samples of reference material are submitted for analysis to ensure that an analytical method is accurate for the known properties of the reference material. Generally, the selected reference material properties are similar to the environmental sample properties.

\section{Replicate Samples}

Replicate samples are a set of environmental samples collected in a manner such that the samples are thought to be essentially identical in composition. Replicate is the general case for which a duplicate is the special case consisting of two samples. Replicate samples are collected and analyzed to establish the amount of variability in the data contributed by some part of the collection and 
analytical process. There are many types of replicate samples possible, each of which may yield slightly different results in a dynamic hydrologic setting, such as a flowing stream. The types of replicate samples collected in this District are:

Sequential samples-a type of replicate sample in which the samples are collected one after the other, typically over a short time.

Split sample - a type of replicate sample in which a sample is split into subsamples contemporaneous in time and space.

\section{Spike Samples}

Spike samples are samples to which known quantities of a solution with one or more well-established analyte concentrations have been added. These samples are analyzed to determine the extent of matrix interference or degradation on the analyte concentration during sample processing and analysis.

\section{Data Presentation}

For continuing-record stations, information pertinent to the history of station operation is provided in descriptive headings preceding the tabular data. These descriptive headings give details regarding location, drainage area, period of record, type of data available, instrumentation, general remarks, cooperation, and extremes for parameters currently measured daily. Tables of chemical, physical, biological, radiochemical data, and other data obtained at a frequency less than daily are presented first. Tables of "daily values" of specific conductance, $\mathrm{pH}$, water temperature, dissolved oxygen, and suspended sediment follow in sequence.

In the descriptive headings, if the location is identical to that of the discharge gaging station, neither the LOCATION nor the DRAINAGE AREA statements are repeated. The following information, as appropriate, is provided with each continuous-record station. Comments that follow clarify information presented under the various headings of the station description.

LOCATION.-See Data Presentation under "Records of Stage and Water Discharge"; same comments apply.

DRAINAGE AREA.-See Data Presentation under "Records of Stage and Water Discharge"; same comments apply.

PERIOD OF RECORD.- - This indicates the periods for which there are published water-quality records for the station. The periods are shown separately for records of parameters measured daily or continuously and those measured less than daily. For those measured daily or continuously, periods of record are given for the individual parameters.

INSTRUMENTATION.- Information on instrumentation is given only if a water-quality monitor, temperature recorder, sediment-pumping sampler, or other sampling device is in operation at a station.

REMARKS.- - Remarks provide added information pertinent to the collection, analysis, or computation of the records.

COOPERATION.--Records provided by a cooperating organization or obtained for the U.S. Geological Survey by a cooperating organization are identified here.

EXTREMES.-Maximums and minimums are given only for parameters measured daily or more frequently. None are given for parameters measured weekly or less frequently because the true maximums or minimums may not have been sampled. Extremes, when given, are provided for both the period of record and for the current water year.

REVISIONS.- - If errors in water-quality records are discovered after publication, appropriate updates are made to the Water-Quality File in the U.S. Geological Survey's computerized data system, National Water Information System (NWIS), and subsequently by monthly transfer of update transactions to the U.S. Environmental Protection Agency's STORET system. Because the usual volume of updates makes it impractical to document individual changes in the State data-report series or elsewhere, potential users of U.S. Geological Survey water-quality data are encouraged to obtain all required data from the appropriate computer file to ensure the most recent updates.

The surface-water-quality records for partial-record stations and miscellaneous sampling sites are published in separate tables following the table of discharge measurements at miscellaneous sites. No descriptive statements are given for these records. Each station is published with its own station number and name in the regular downstream-order sequence.

\section{ACCESS TO USGS WATER DATA}

The U.S. Geological Survey provides near real-time stage and discharge data for many of the gaging stations equipped with the necessary telemetry and historic daily-mean and peak-flow discharge data for most current or discontinued gaging stations through the world wide web (WWW). These data may be accessed at

\section{http://water.usgs.gov}

Some water-quality and ground-water data also are available through the WWW. In addition, data can be provided in various machine-readable formats on magnetic tape or 3-1/2 inch floppy disk. Information about the availability of specific types of additional data or products, and user charges, can be obtained locally from each of the Water Resources Division District Offices. (See address on the back of the title page.) 


\section{DEFINITION OF TERMS}

Terms related to streamflow, water-quality, and other hydrologic data, as used in this report, are defined below. See also the table for converting English (inch-pound) units to International System (SI) Units on the inside of the back cover.

Acid neutralizing capacity (ANC) is the equivalent sum of all bases or base-producing materials, solutes plus particulates, in an aqueous system that can be titrated with acid to an equivalence point. This term designates titration of an "unfiltered" sample (formerly reported as alkalinity).

Acre-foot (AC-FT, acre-ft) is the quantity of water required to cover 1 acre to a depth of 1 foot and is equivalent to 43,560 cubic feet, 325,851 gallons, or 1,233 cubic meters.

Adenosine triphosphate (ATP) is an organic, phosphate-rich, compound important in the transfer of energy in organisms. Its central role in living cells makes it an excellent indicator of the presence of living material in water. A measurement of ATP therefore provides a sensitive and rapid estimate of biomass. ATP is reported in micrograms per liter. leaves.

Algae are mostly aquatic single-celled, colonial, or multicelled plants containing chlorophyll and lacking roots, stems, and

Algal growth potential (AGP) is the maximum algal dry weight biomass that can be produced in a natural water sample under standardized laboratory conditions. The growth potential is the algal biomass present at stationary phase and is expressed as milligrams dry weight of algae produced per liter of sample.

Alkalinity is the capacity of solutes in an aqueous system to neutralize acid. This term designates titration of a "filtered" sample.

Annual runoff is the total quantity of water in runoff for a drainage area for the year. Data reports may use any of the following units of measurement in presenting annual runoff data:

Acre-foot (AC-FT, acre-ft) is the quantity of water required to cover 1 acre to a depth of 1 foot and is equal to 43,560 cubic feet, 325,851 gallons, or 1,233 cubic meters.

Cubic foot per second per square mile $\left[\mathrm{CFSM},\left(\mathrm{ft}^{3} / \mathrm{s}\right) / \mathrm{mi}^{2}\right]$ is the average number of cubic feet of water flowing per second from each square mile of area drained, assuming the runoff is distributed uniformly in time and area.

Inch (IN., in.) as used in this report, refers to the depth to which the drainage area would be covered with water if all of the runoff for a given time period were uniformly distributed on it.

Aroclor is the registered trademark for a group of polychlorinated biphenyls that were manufactured by the Monsanto Company prior to 1976. Aroclors are assigned specific 4-digit reference numbers dependent upon molecular type and degree of substitution of the biphenyl ring hydrogen atoms by chlorine atoms. The first two digits of a numbered aroclor represent the molecular type and the last two digits represent the weight percent of the hydrogen substituted chlorine.

Aquifer is a geologic formation, group of formations, or part of a formation that contains sufficient saturated permeable material to yield significant quantities of water to wells and springs.

Artesian means confined and is used to describe a well in which the water level stands above the top of the aquifer tapped by a well. A flowing artesian well is one in which the water level is above the land surface.

Bacteria are microscopic unicellular organisms, typically spherical, rodlike, or spiral and threadlike in shape, often clumped into colonies. Some bacteria cause disease, while others perform an essential role in nature in the recycling of materials; for example, by decomposing organic matter into a form available for reuse by plants.

Total coliform bacteria are a particular group of bacteria that are used as indicators of possible sewage pollution. This group includes coliforms that inhabit the intestines of warm-blooded animals and those that inhabit soils. They are characterized as aerobic or facultative anaerobic, gram-negative, nonspore-forming, rod-shaped bacteria that ferment lactose with gas formation within 48 hours at $35^{\circ} \mathrm{C}$. In the laboratory, these bacteria are defined as all the organisms that produce colonies with a golden-green metallic sheen within 24 hours when incubated at $35^{\circ} \mathrm{C}$ plus or minus $1.0^{\circ} \mathrm{C}$ on $\mathrm{M}$-Endo medium (nutrient medium for bacterial growth). Their concentrations are expressed as number of colonies per $100 \mathrm{~mL}$ of sample.

Fecal coliform bacteria are bacteria that are present in the intestines or feces of warm-blooded animals. They are often used as indicators of the sanitary quality of the water. In the laboratory, they are defined as all the organisms that produce blue colonies within 24 hours when incubated at $44.5^{\circ} \mathrm{C}$ plus or minus $0.2^{\circ} \mathrm{C}$ on M-FC medium (nutrient medium for bacterial growth). Their concentrations are expressed as number of colonies per $100 \mathrm{~mL}$ of sample.

Fecal streptococcal bacteria are bacteria found in the intestines of warm-blooded animals. Their presence in water is considered to verify fecal pollution. They are characterized as gram-positive, cocci bacteria that are capable of growth in brain-heart infusion broth. In the laboratory, they are defined as all the organisms that produce red or pink colonies within 48 hours at $35^{\circ} \mathrm{C}$ plus or minus $1.0^{\circ} \mathrm{C}$ on $\mathrm{KF}$-streptococcus medium (nutrient medium for bacterial growth). Their concentrations are expressed as number of colonies per $100 \mathrm{~mL}$ of sample.

Enterococcus bacteria are commonly found in the feces of humans and other warm-blooded animals. Although some strains are ubiquitous and not related to fecal pollution, the presence of enterococci in water is an indication of fecal pollution and the possible presence of enteric pathogens. Enterococcus bacteria are those bacteria that produce pink to red colonies 
with black or reddish-brown precipitate after incubation at $41^{\circ} \mathrm{C}$ on $\mathrm{mE}$ agar and subsequent transfer to EIA medium. Enterococci include Streptococcus feacalis, Streptococcus feacium, Streptococcus avium, and their variants.

Escherichia coli (E. coli) are bacteria present in the intestine and feces of warm-blooded animals. E. coli are a member species of the fecal coliform group of indicator bacteria. In the laboratory, they are defined as those bacteria that produce yellow or yellow-brown colonies on a filter pad saturated with urea substrate broth after primary culturing for 22 to 24 hours at $44.5^{\circ} \mathrm{C}$ on mTEC medium. Their concentrations are expressed as number of colonies per $100 \mathrm{~mL}$ of sample.

Base flow is flow in a channel sustained by ground-water discharge in the absence of direct runoff.

Bed load is the sediment which moves along in essentially continuous contact with the streambed by rolling, sliding, and making brief excursions into the flow a few diameters above the bed.

Bed material is the sediment mixture of which a streambed, lake, pond, reservoir, or estuary bottom is composed.

Benthic organisms (invertebrates) are the group of animals inhabiting the bottom of an aquatic environment. They include a number of types of organisms, such as bacteria, fungi, insect larvae and nymphs, snails, clams, and crayfish. They are useful as indicators of water quality.

Biochemical oxygen demand (BOD) is a measure of the quantity of dissolved oxygen, in milligrams per liter, necessary for the decomposition of organic matter by microorganisms, such as bacteria.

Biomass is the amount of living matter present at any given time, expressed as the mass per unit area or volume of habitat.

Ash mass is the mass or amount of residue present after the residue from the dry mass determination has been ashed in a muffle furnace at a temperature of $500^{\circ} \mathrm{C}$ for 1 hour. The ash-mass values of zooplankton and phytoplankton are expressed in grams per cubic meter $\left(\mathrm{g} / \mathrm{m}^{3}\right)$, and periphyton and benthic organisms in grams per square meter $\left(\mathrm{g} / \mathrm{m}^{2}\right)$.

Dry mass refers to the mass of residue present after drying in an oven at $105^{\circ} \mathrm{C}$ for zooplankton and periphyton, until the mass remains unchanged. This mass represents the total organic matter, ash, and sediment in the sample. Dry-mass values are expressed in the same units as ash mass.

Organic mass or volatile mass of the living substance is the difference between the dry mass and ash mass and represents the actual mass of the living matter. Organic mass is expressed in the same units as for ash mass and dry mass.

Wet mass is the mass of living matter plus contained water.

Biomass pigment ratio is an indicator of the total proportion of periphyton which are autotrophic (plants). This is also called the Autotrophic Index.

Bottom material: See Bed material.

Cells/volume (cells per volume) refers to the number of plankton cells or natural units counted using a microscope and grid or counting cell. Results are generally reported as cells or units per milliliter.

Cells volume (biovolume) determination is one of several common methods used to estimate biomass of algae in aquatic systems. Cell numbers of algae are frequently used in aquatic surveys as an indicator of algal production. However, cell numbers alone cannot represent true biomass because of considerable cell-size variation among the algal species. Cell volume $\left(\mu \mathrm{m}^{3}\right)$ is determined by obtaining critical cell measurements on cell dimensions (for example, length, width, height, or radius) for 20 to 50 cells of each important species to obtain an average biovolume per cell. Cells are categorized according to the correspondence of their cellular shape to the nearest geometric solid or combinations of simple solids (for example, spheres, cones, or cylinders). Representative formulae used to compute biovolume are as follows:

$$
\text { sphere } 4 / 3 \pi r^{3} \quad \text { cone } 1 / 3 \pi r^{3} h \quad \text { cylinder } \pi r^{3} h \text {. }
$$

From cell volume, total algal biomass expressed as biovolume $\left(\mu \mathrm{m}^{3} / \mathrm{mL}\right)$ is thus determined by multiplying the number of cells of a given species by its average cell volume and then summing these volumes over all species.

Chemical oxygen demand (COD) is a measure of the chemically oxidizable material in the water and furnishes an approximation of the amount of organic and reducing material present. The determined value may correlate with BOD or with carbonaceous organic pollution from sewage or industrial wastes.

Chlorophyll refers to the green pigments of plants. Chlorophyll $a$ and $b$ are the two most common green pigments in plants.

Colloid is any substance with particles in such a fine state of subdivision dispersed in a medium (for example, water) that they do not settle out; but not in so fine a state of subdivision that they can be said to be truly dissolved.

Color unit is produced by 1 milligram per liter of platinum in the form of the chloroplatinate ion. Color is expressed in units of the platinum-cobalt scale.

Confined aquifer is a term used to describe an aquifer containing water between two relatively impermeable boundaries. The water level in a well tapping a confined aquifer stands above the top of the confined aquifer and can be higher or lower than the water table that may be present in the material above it. In some cases the water level can rise above the ground surface, yielding a flowing well.

Contents is the volume of water in a reservoir or lake. Unless otherwise indicated, volume is computed on the basis of a level pool and does not include bank storage. 
Continuous-record station is a site that meets either of the following conditions:

1. Stage or streamflow are recorded at some interval on a continuous basis. The recording interval is usually 15 minutes, but may be less or more frequent.

2. Water-quality, sediment, or other hydrologic measurements are recorded at least daily.

Control designates a feature in the channel downstream from a gaging station that physically influences the water-surface elevation and thereby determines the stage-discharge relation at the station. This feature may be a constriction of the channel, a bedrock outcrop, a gravel bar, an artificial structure, or a uniform cross section over a long reach of the channel.

Control structure as used in this report is a structure on a stream or canal that is used to regulate the flow or stage of the stream or to prevent the intrusion of saltwater.

Cubic foot per second (CFS, $\left.\mathrm{cfs} \mathrm{ft}^{3} / \mathrm{s}\right)$ is the rate of discharge representing a volume of 1 cubic foot passing a given point in 1 second. It is equivalent to approximately 7.48 gallons per second, 448.8 gallons per minute, or 0.02832 cubic meters per second.

Cubic foot per second per day (CFS-DAY, cfs-day, $\mathrm{cfs} / \mathrm{d}$, or $\left.\left[\left(\mathrm{ft}^{3} / \mathrm{s}\right) / \mathrm{d}\right]\right)$ is the volume of water represented by a flow of 1 cubic foot per second for 24 hours. It is equivalent to 86,400 cubic feet, 1.9835 acre-feet, 646,317 gallons, or 2,447 cubic meters.

Daily record is a summary of streamflow, sediment, or water-quality values computed from data collected with sufficient frequency to obtain reliable estimates of daily mean values.

Daily record station is a site for which daily records of streamflow, sediment, or water-quality values are computed.

Datum, as used in this report, is an elevation above mean sea level to which all gage height readings are referenced.

Diel is of or pertaining to a 24-hour period of time; a regular daily cycle.

Discharge, or flow, is the volume of water (or more broadly, volume of fluid including solid- and dissolved-phase material), that passes a given point in a given period of time.

Annual 7-day minimum is the lowest mean discharge for 7 consecutive days in a year. Note that most low-flow frequency analyses of annual 7-day minimum flows use a climatic year (April 1-March 31). The date shown in the summarystatistics table is the initial date of the 7-day period. (This value should not be confused with the

7-day 10-year low-flow statistic.)

Instantaneous discharge is the discharge at a particular instant of time.

Mean discharge (MEAN) is the arithmetic mean of individual daily mean discharges during a specific period.

Dissolved refers to that material in a representative water sample which passes through a 0.45 -micrometer membrane filter. This is a convenient operational definition used by Federal agencies that collect water data. Determinations of "dissolved" constituents are made on subsamples of the filtrate.

Dissolved oxygen (DO) content of water in equilibrium with air is a function of atmospheric pressure, temperature, and dissolved-solids concentration of the water. The ability of water to retain oxygen decreases with increasing temperature or dissolved solids, with small temperature changes having the more significant offset. Photosynthesis and respiration may cause diurnal variations in dissolved-oxygen concentration in water from some streams.

Dissolved-solids concentration of water is determined either analytically by the "residue-on-evaporation" method, or mathematically by totaling the concentrations of individual constituents reported in a comprehensive chemical analysis. During that analytical determination of dissolved solids, the bicarbonate (generally a major dissolved component of water) is converted to carbonate. Therefore, in the mathematical calculation of dissolved-solids concentration, the bicarbonate value, in milligrams per liter, is multiplied by 0.4926 to reflect the change. Alternatively, alkalinity concentration $\left(\mathrm{as} \mathrm{mg} / \mathrm{L} \mathrm{CaCO}_{3}\right)$ can be converted to carbonate concentration by multiplying by 0.60 .

Diversity index is a numerical expression of evenness of distribution of aquatic organisms. The formula for diversity index is:

$$
\bar{d}=-\sum_{i \approx 1}^{s} \frac{n_{i}}{n} \log _{2} \frac{n_{i}}{n},
$$

where $n_{i}$ is the number of individuals per taxon, $n$ is the total number of individuals, and $s$ is the total number of taxa in the sample of the community. Diversity index values range from zero, when all the organisms in the samples are the same, to some positive number, when some or all the organisms in the sample are different.

Drainage area of a site on a stream is that area, measured in a horizontal plane, that has a common outlet at the site for its surface runoff. Figures of drainage area given herein include all closed basins, or noncontributing areas, within the area unless otherwise specified.

Drainage basin is a part of the Earth's surface that is occupied by a drainage system with a common outlet for its surface runoff (see "Drainage area").

Dry weight refers to the weight of animal tissue after it has been dried in an oven at $65^{\circ} \mathrm{C}$ until a constant weight is achieved. Dry weight represents total organic and inorganic matter in the tissue. 
Extractable-organic halides (EOX) are organic compounds which contain halogen atoms such as chlorine. These organic compounds are semi-volatile and extractable by ethyl acetate from air-dried stream-bottom sediments. The ethyl-acetate extract is combusted, and the concentration is determined by microcoulometric determination of the halides formed. The concentration is reported as micrograms of chlorine per gram of the dry weight of the stream-bottom sediments.

Flow-duration percentiles are values on a scale of 100 that indicate the percentage of time for which a flow is not exceeded. For example, the 90th percentile of river flow is greater than or equal to 90 percent of all recorded flow rates.

Gage datum is the elevation of the zero point of the reference gage from which gage height is determined as compared to sea level (see "Datum"). This elevation is established by a system of levels from known benchmarks, by approximation from topographic maps, or by geographical positioning system.

Gage height (G.H.) is the water-surface elevation referenced to the gage datum. Gage height is often used interchangeably with the more general term "stage," although gage height is more appropriate when used with a reading on a gage.

Gaging station is a site on a stream, canal, lake, or reservoir where systematic observations of stage, discharge, or other hydrologic data are obtained. When used in connection with a discharge record, the term is applied only to those gaging stations where a continuous record of discharge is computed.

Gas chromatography/flame ionization detector (GC/FID) is a laboratory analytical method used as a screening technique for semivolatile organic compounds that are extractable from water in methylene chloride.

Ground-water level is the elevation of the water table or another potentiometric surface at a particular location.

Hardness of water is a physical-chemical characteristic that is commonly recognized by the increased quantity of soap required to produce lather. It is attributable to the presence of alkaline earths (principally calcium and magnesium) and is expressed as the equivalent concentration of calcium carbonate $\left(\mathrm{CaCO}_{3}\right)$.

High tide is the maximum height reached by each rising tide. The high-high and low-high tides are the higher and lower of the two high tides, respectively, of each tidal day. See NOAA web site: http://www.co-ops.nos.noaa.gov/tideglos.html

Hydrologic benchmark station is one that provides hydrologic data for a basin in which the hydrologic regimen will likely be governed solely by natural conditions. Data collected at a benchmark station may be used to separate effects of natural from human-induced changes in other basins that have been developed and in which the physiography, climate, and geology are similar to those in the undeveloped benchmark basin.

Hydrologic unit is a geographic area representing part or all of a surface drainage basin or distinct hydrologic feature as defined by the former Office of Water Data Coordination and delineated on the State Hydrologic Unit Maps by the U.S. Geological Survey. Each hydrologic unit is identified by an 8-digit number.

Land-surface datum (lsd) is a datum plane that is approximately at land surface at each ground-water observation well.

Light-attenuation coefficient, also known as the extinction coefficient, is a measure of water clarity. Light is attenuated according to the Lambert-Beer equation

$$
I=I_{o} e^{-\lambda L}
$$

where $I_{o}$ is the source light intensity, $I$ is the light intensity at length $L$ (in meters) from the source, $\lambda$ is the light-attenuation coefficient, and $e$ is the base of the natural logarithm. The light-attenuation coefficient is defined as

$$
\lambda=-\frac{1}{L} \log _{e} \frac{I}{I_{o}} .
$$

Lipid is any one of a family of compounds that are insoluble in water and that make up one of the principal components of living cells. Lipids include fats, oils, waxes, and steroids. Many environmental contaminants such as organochlorine pesticides are lipophilic.

Low tide is the minimum height reached by each falling tide. The high-low and low-low tides are the higher and lower of the two low tides, respectively, of each tidal day. See NOAA web site: http://www.co-ops.nos.noaa.gov/tideglos.html

Macrophytes are the macroscopic plants in the aquatic environment. The most common macrophytes are the rooted vascular plants that are usually arranged in zones in aquatic ecosystems and restricted in the area by the extent of illumination through the water and sediment deposition along the shoreline.

Mean high tide is the average of all high tides over a specified period.

Mean low tide is the average of all low tides over a specified period.

Mean water level is the average of all tides over a specified period.

Measuring point (MP) is an arbitrary permanent reference point from which the distance to water surface in a well is measured to obtain water level.

Membrane filter is a thin microporous material of specific pore size used to filter bacteria, algae, and other very small particles from water. 
Metamorphic stage refers to the stage of development that an organism exhibits during its transformation from an immature form to an adult form. This developmental process exists for most insects, and the degree of difference from the immature stage to the adult form varies from relatively slight to pronounced, with many intermediates. Examples of metamorphic stages of insects are egg-larva-adult or egg-nymph-adult.

Methylene blue active substances (MBAS) are apparent detergents. This determination depends on the formation of a blue color when methylene blue dye reacts with synthetic anionic detergent compounds.

Micrograms per gram (UG/G, $\mu \mathrm{g} / \mathrm{g}$ ) is a unit expressing the concentration of a chemical constituent as the mass (micrograms) of the element per unit mass (gram) of material analyzed.

Micrograms per kilogram $(\mathrm{UG} / \mathrm{KG}, \mu \mathrm{g} / \mathrm{kg})$ is a unit expressing the concentration of a chemical constituent as the mass (micrograms) of the constituent per unit mass (kilogram) of the material analyzed. One microgram per kilogram is equivalent to 1 part per billion.

Micrograms per liter (UG/L, $\mu \mathrm{g} / \mathrm{L})$ is a unit expressing the concentration of chemical constituents in water as mass (micrograms) of constituent per unit volume (liter) of water. One thousand micrograms per liter is equivalent to 1 milligram per liter.

Microsiemens per centimeter (US/CM, $\mu \mathrm{S} / \mathrm{cm}$ ) is a unit expressing the amount of electrical conductivity of a solution as measured between opposite faces of a centimeter cube of solution at a specified temperature. Siemens is the International System of Units nomenclature. It is synonymous with mhos and is the reciprocal of resistance in ohms.

Milligrams per liter (MG/L, mg/L) is a unit for expressing the concentration of chemical constituents in water as the mass (milligrams) of constituent per unit volume (liter) of water. Concentration of suspended sediment also is expressed in $\mathrm{mg} / \mathrm{L}$ and is based on the mass of dry sediment per liter of water-sediment mixture.

Miscellaneous site, or miscellaneous station, is a site where streamflow, sediment, and/or water-quality data are collected once, or more often on a random or discontinuous basis.

Most probable number (MPN) is an index of the number of coliform bacteria that, more probably than any other number, would give the results shown by the laboratory examination; it is not an actual enumeration. MPN is determined from the distribution of gas-positive cultures among multiple inoculated tubes.

Multiple-plate samplers are artificial substrates of known surface area used for obtaining benthic-invertebrate samples. They consist of a series of spaced, hardboard plates on an eyebolt.

Nanograms per liter (NG/L, ng/L) is a unit expressing the concentration of chemical constituents in solution as mass (nanograms) of solute per unit volume (liter) of water. One million nanograms per liter is equivalent to 1 milligram per liter.

National Geodetic Vertical Datum of 1929 (NGVD of 1929) is a geodetic datum derived from a general adjustment of the first order level nets of both the United States and Canada. It was formerly called "Sea Level Datum of 1929" or "mean sea level" in this series of reports. Although the datum was derived from the average sea level over a period of many years at 26 tide stations along the Atlantic, Gulf of Mexico, and Pacific Coasts, it does not necessarily represent local mean sea level at any particular place. See NOAA web site: http://www.ngs.noaa.gov/faq.shtml\#WhatVD29VD88

Nekton are the consumers in the aquatic environment and consist of large free-swimming organisms that are capable of sustained, directed mobility.

Nephelometric turbidity unit (NTU) is the measurement for reporting turbidity that is based on use of a standard suspension of Formazin. Turbidity measured in NTU uses nephelometric methods that depend on passing specific light of a specific wavelength through the sample.

Open or screened interval is the length of unscreened opening or of well screen through which water enters a well, in feet below land surface.

Organic carbon (OC) is a measure of organic matter present in aqueous solution, suspension, or bottom sediments. May be reported as dissolved organic carbon (DOC), suspended organic carbon (SOC), or total organic carbon (TOC).

Organism is any living entity.

Organism count/area refers to the number of organisms collected and enumerated in a sample and adjusted to the number per unit area of habitat, usually square meter $\left(\mathrm{m}^{2}\right)$, acre, or hectare. Periphyton, benthic organisms, and macrophytes are expressed in these terms.

Organism count/volume refers to the number of organisms collected and enumerated in a sample and adjusted to the number per sample volume, usually milliliter $(\mathrm{mL})$ or liter $(\mathrm{L})$. Numbers of planktonic organisms can be expressed in these terms.

Total organism count is the total number of organisms collected and enumerated in any particular sample.

Organochlorine compounds are any chemicals that contain carbon and chlorine. Organochlorine compounds that are important in investigations of water, sediment, and biological quality include certain pesticides and industrial compounds.

Parameter Code is a 5-digit number used in the U.S. Geological Survey's computerized data system, National Water Information System (NWIS), to uniquely identify a specific constituent or property.

Partial-record station is a site where discrete measurements of one or more hydrologic parameters are obtained over a period of time without continuous data being recorded or computed. A common example is a crest-stage gage partial-record station at which only peak stages and flows are recorded. 
Particle size is the diameter, in millimeters $(\mathrm{mm})$, of a particle determined by sieve or sedimentation methods. The sedimentation method utilizes the principle of Stokes Law to calculate sediment particle sizes. Sedimentation methods (pipet, bottom-withdrawal tube, visual-accumulation tube, Sedigraph) determine fall diameter of particles in either distilled water (chemically dispersed) or in native water (the river water at the time and point of sampling).

Particle-size classification used in this report agrees with the recommendations made by the American Geophysical Union Subcommittee on Sediment Terminology. The classification is as follows:

\begin{tabular}{|c|c|c|}
\hline Classification & Size (mm) & Method of analysis \\
\hline Clay & $0.00024-0.004$ & Sedimentation \\
\hline Silt . & $.004-.062$ & Sedimentation \\
\hline Sand .... & $.062-2.0$ & Sedimentation/sieve \\
\hline Gravel... . & $2.0-64.0$ & Sieve \\
\hline
\end{tabular}

The particle-size distributions given in this report are not necessarily representative of all particles in transport in the stream. Most of the organic material is removed, and the sample is subjected to mechanical and chemical dispersion before analysis in distilled water. Chemical dispersion is not used for native-water analysis.

Percent composition or percent of total is a unit for expressing the ratio of a particular part of a sample or population to the total sample or population, in terms of types, numbers, weight, or volume.

Periodic station is a site where stage, discharge, sediment, chemical, or other hydrologic measurements are made one or more times during a year, but at a frequency insufficient to develop a daily record.

Periphyton is the assemblage of microorganisms attached to and living upon submerged solid surfaces. While primarily consisting of algae, they also include bacteria, fungi, protozoa, rotifers, and other small organisms. Periphyton are useful indicators of water quality.

Pesticides are chemical compounds used to control undesirable organisms. Major categories of pesticides include insecticides, miticides, fungicides, herbicides, and rodenticides.

pH of water is the negative logarithm of the hydrogen-ion activity. Solutions with pH less than 7 are termed "acidic," and solutions with a $\mathrm{pH}$ greater than 7 are termed "basic." Solutions with a $\mathrm{pH}$ of 7 are neutral. The presence and concentration of many dissolved chemical constituents found in water are, in part, influenced by the hydrogen-ion activity of water. Biological processes including growth, distribution of organisms, and toxicity of the water to organisms are also influenced, in part, by the hydrogen-ion activity of water.

Picocurie (PC, pCi) is one trillionth $\left(1 \times 10^{-12}\right)$ of the amount of radioactivity represented by a curie $(\mathrm{Ci})$. A curie is the amount of radioactivity that yields $3.7 \times 10^{10}$ radioactive disintegrations per second. A picocurie yields $2.22 \mathrm{dpm}$ (disintegrations per minute).

Plankton is the community of suspended, floating, or weakly swimming organisms that live in the open water of lakes and rivers. Concentrations are expressed as a number of cells per milliliter (cells $/ \mathrm{mL}$ ) of sample.

Phytoplankton is the plant part of the plankton. They are usually microscopic, and their movement is subject to the water currents. Phytoplankton growth is dependent upon solar radiation and nutrient substances. Because they are able to incorporate as well as release materials to the surrounding water, the phytoplankton have a profound effect on the quality of the water. They are the primary food producers in the aquatic environment and are commonly known as algae.

Blue-green algae (Cyanophyta) are a group of phytoplankton organisms having a blue pigment, in addition to the green pigment called chlorophyll. Blue-green algae often cause nuisance conditions in water.

Diatoms are the unicellular or colonial algae having a siliceous shell. Their concentrations are expressed as number of cells per milliliter (cells $/ \mathrm{mL}$ ) of sample.

Euglenoids (Euglenophyta) are a group of algae that are usually free-swimming and rarely creeping. They have the ability to grow either photosynthetically in the light or heterotrophically in the dark.

Fire algae (Pyrrhophyta) are a group of algae that are free-swimming unicells characterized by a red pigment spot.

Green algae have chlorophyll pigments similar in color to those of higher green plants. Some forms produce algae mats or floating "moss" in lakes. Their concentrations are expressed as number of cells per milliliter (cells $/ \mathrm{mL})$ of sample.

Zooplankton is the animal part of the plankton. Zooplankton are capable of extensive movements within the water column and are often large enough to be seen with the unaided eye. Zooplankton are secondary consumers feeding upon bacteria, phytoplankton, and detritus. Because they are the grazers in the aquatic environment, the zooplankton are a vital part of the aquatic food web. The zooplankton community is dominated by small crustaceans and rotifers.

Polychlorinated biphenyls (PCB's) are industrial chemicals that are mixtures of chlorinated biphenyl compounds having various percentages of chlorine. They are similar in structure to organochlorine insecticides. 
Polychlorinated naphthalenes (PCN's) are industrial chemicals that are mixtures of chlorinated naphthalene compounds. They have properties and applications similar to polychlorinated biphenyls (PCB's) and have been identified in commercial PCB preparations.

Primary productivity is a measure of the rate at which new organic matter is formed and accumulated through photosynthetic and chemosynthetic activity of producer organisms (chiefly, green plants). The rate of primary production is estimated by measuring the amount of oxygen released (oxygen method) or the amount of carbon assimilated (carbon method) by the plants.

Primary productivity (carbon method) is expressed as milligrams of carbon per area per unit time $\left[\mathrm{mg} \mathrm{C} /\left(\mathrm{m}^{2} / \mathrm{time}\right)\right]$ for periphyton and macrophytes or per volume $\left[\mathrm{mg} \mathrm{C} /\left(\mathrm{m}^{3} / \mathrm{time}\right)\right]$ for phytoplankton. Carbon method defines the amount of carbon dioxide consumed as measured by radioactive carbon (carbon-14). The carbon-14 method is of greater sensitivity than the oxygen light and dark bottle method and is preferred for use in unenriched waters. Unit time may be either the hour or day, depending on the incubation period.

Primary productivity (oxygen method) is expressed as milligrams of oxygen per area per unit time $\left[\mathrm{mg} \mathrm{O} /\left(\mathrm{m}^{2} / \mathrm{time}\right)\right]$ for periphyton and macrophytes or per volume $\left[\mathrm{mg} \mathrm{O} /\left(\mathrm{m}^{3} /\right.\right.$ time $\left.)\right]$ for phytoplankton. Oxygen method defines production and respiration rates as estimated from changes in the measured dissolved-oxygen concentration. The oxygen light and dark bottle method is preferred if the rate of primary production is sufficient for accurate measurements to be made within 24 hours. Unit time may be either the hour or day, depending on the incubation period.

Radioisotopes are isotopic forms of an element that exhibit radioactivity. Isotopes are varieties of a chemical element that differ in atomic weight, but are very nearly alike in chemical properties. The difference arises because the atoms of the isotopic forms of an element differ in the number of neutrons in the nucleus; for example, ordinary chlorine is a mixture of isotopes having atomic weights of 35 and 37, and the natural mixture has an atomic weight of about 35.453. Many of the elements similarly exist as mixtures of isotopes, and a great many new isotopes have been produced in the operation of nuclear devices such as the cyclotron. There are 275 isotopes of the 81 stable elements, in addition to more than 800 radioactive isotopes.

Recoverable from bottom material is the amount of a given constituent that is in solution after a representative sample of bottom material has been digested by a method (usually using an acid or mixture of acids) that results in dissolution of readily soluble substances. Complete dissolution of all bottom material is not achieved by the digestion treatment and thus, the determination represents less than the total amount (that is, less than 95 percent) of the constituent in the sample. To achieve comparability of analytical data, equivalent digestion procedures would be required of all laboratories performing such analyses because different digestion procedures are likely to produce different analytical results.

Recurrence interval, also referred to as return period, is the average time, usually expressed in years, between occurrences of hydrologic events of a specified type (such as exceedances of a specified high flow or non-exceedance of a specified low flow). The terms "return period" and "recurrence interval" do not imply regular cyclic occurrence. The actual times between occurrences vary randomly, with most of the times being less than the average and a few being substantially greater than the average. For example, the 100-year flood is the flow rate that is exceeded by the annual maximum peak flow at intervals whose average length is 100 years (that is, once in 100 years, on average); almost two-thirds of all exceedances of the 100-year flood occur less than 100 years after the previous exceedance, half occur less than 70 years after the previous exceedance, and about one-eighth occur more than 200 years after the previous exceedance. Similarly, the 7-day 10 -year low flow $\left(7 \mathrm{Q}_{10}\right)$ is the flow rate below which the annual minimum 7-day-mean flow dips at intervals whose average length is 10 years (that is, once in 10 years, on average); almost two-thirds of the non-exceedances of the $7 \mathrm{Q}_{10}$ occur less than 10 years after the previous non-exceedance, half occur less than 7 years after, and about one-eighth occur more than 20 years after the previous non-exceedance. The recurrence interval for annual events is the reciprocal of the annual probability of occurrence. Thus, the 100-year flood has a 1-percent chance of being exceeded by the maximum peak flow in any year, and there is a 10-percent chance in any year that the annual minimum 7-day-mean flow will be less than the $7 Q_{10}$.

Replicate samples are a group of samples collected in a manner such that the samples are thought to be essentially identical in composition.

Return period is the average time interval between occurrences of a hydrological event of a given or greater magnitude, usually expressed in years. May also be called recurrence interval.

River mile is the distance of a point on a river measured in miles from the river's mouth along the low-water channel.

River mileage is the linear distance along the meandering path of a stream channel determined in accordance with Bulletin No. 14 (October 1968) of the Water Resources Council.

Runoff in inches (IN., in.) is the depth, in inches, to which the drainage area would be covered if all the runoff for a given time period were uniformly distributed on it.

Sea level refers to the National Geodetic Vertical Datum of 1929 (NGVD of 1929) - a geodetic datum derived from a general adjustment of the first-order level nets of both the United States and Canada, formerly called Sea Level Datum of 1929.

See: http://www.co-ops.nos.noaa.gov/glossary/gloss_n.html\#NGVD

Sediment is solid material that is transported by, suspended in, or deposited from water. It originates mostly from disintegrated rocks; it also includes chemical and biochemical precipitates and decomposed organic material, such as humus. The quantity, characteristics, and cause of the occurrence of sediment in streams are influenced by environmental factors. Some major factors are degree of slope, length of slope, soil characteristics, land usage, and quantity and intensity of precipitation. 
Bed load is the sediment that is transported in a stream by rolling, sliding, or skipping along or very close to the bed. In this report, bed load is considered to consist of particles in transit from the bed to an elevation equal to the top of the bedload sampler nozzle (usually within $0.25 \mathrm{ft}$ of the streambed).

Bed-load discharge (tons per day) is the quantity of sediment moving as bed load, reported as dry weight, that passes a cross section in a given time.

Suspended sediment is the sediment that is maintained in suspension by the upward components of turbulent currents or that exists in suspension as a colloid.

Suspended-sediment concentration is the velocity-weighted concentration of suspended sediment in the sampled zone (from the water surface to a point approximately $0.3 \mathrm{ft}$ above the bed) expressed as milligrams of dry sediment per liter of water-sediment mixture $(\mathrm{mg} / \mathrm{L})$. The entire sample is used for the analysis.

Mean concentration of suspended sediment is the time-weighted concentration of suspended sediment passing a stream section during a 24-hour day.

Suspended-sediment discharge (tons/day) is the quantity of sediment moving in suspension, reported as dry weight, that passes a cross section in a given time. It is calculated in units of tons per day as follows: concentration $(\mathrm{mg} / \mathrm{L}) \mathrm{x}$ discharge $\left(\mathrm{ft}^{3} / \mathrm{s}\right) \times 0.0027$.

Suspended-sediment load is a term that refers to material in suspension. The term needs to be qualified, such as "annual suspended-sediment load" or "sand-size suspended-sediment load," and so on. It is not synonymous with either suspendedsediment discharge or concentration.

Suspended total residue at $\mathbf{1 0 5}^{\circ} \mathrm{C}$ concentration is the concentration of suspended sediment in the sampled zone expressed as milligrams of dry sediment per liter of water-sediment mixture (mg/L). A small aliquot of the sample is used for the analysis.

Total sediment discharge (tons/day) is the sum of the suspended-sediment discharge and the bed-load discharge. It is the total quantity of sediment, reported as dry weight, that passes a cross section in a given time.

Total sediment load or total load is a term that refers to the total sediment (bed load plus suspended-sediment load) that is in transport. The term needs to be qualified, such as "annual suspended-sediment load" or "sand-size suspended-sediment load," and so on. It is not synonymous with total sediment discharge.

Seven-day 10-year low flow $\left(7 \mathrm{Q} 10,7 \mathrm{Q}_{10}\right)$ is the minimum flow averaged over 7 consecutive days that is expected to occur on average, once in any 10-year period. The 7Q10 has a 10-percent chance of occurring in any given year.

Sodium adsorption ratio (SAR) is the expression of relative activity of sodium ions in exchange reactions within soil and is an index of sodium or alkali hazard to the soil. Water ranges in respect to sodium hazard from those which can be used for irrigation on almost all soils to those which are generally unsatisfactory for irrigation.

Solute is any substance that is dissolved in water.

Specific conductance is a measure of the ability of a water to conduct an electrical current. It is expressed in microsiemens per centimeter at $25^{\circ} \mathrm{C}$. Specific conductance is related to the type and concentration of ions in solution and can be used for approximating dissolved-solids content of the water. Commonly, the concentration of dissolved solids (in milligrams per liter) is from 55 to 75 percent of the specific conductance (in microsiemens). This relation is not constant from stream to stream, and it may vary in the same source with changes in the composition of the water.

Stable isotope ratio (per MILL/MIL) is a unit expressing the ratio of the abundance of two radioactive isotopes. Isotope ratios are used in hydrologic studies to determine the age or source of specific waters, to evaluate mixing of different waters, as an aid in determining reaction rates, and other chemical or hydrologic processes.

Stage: See "Gage height."

Stage-discharge relation is the relation between the water-surface elevation, termed stage (gage height), and the volume of water flowing in a channel per unit time.

Streamflow is the discharge that occurs in a natural channel. Although the term "discharge" can be applied to the flow of a canal, the word "streamflow" uniquely describes the discharge in a surface stream course. The term "streamflow" is more general than "runoff" as streamflow may be applied to discharge whether or not it is affected by diversion or regulation.

Substrate is the physical surface upon which an organism lives.

Artificial substrate is a device which is purposely placed in a stream or lake for colonization of organisms. The artificial substrate simplifies the community structure by standardizing the substrate from which each sample is taken. Examples of artificial substrates are basket samplers (made of wire cages filled with clean streamside rocks) and multiplate samplers (made of hardboard) for benthic-organism collection and plexiglass strips for periphyton collection.

Natural substrate refers to any naturally occurring immersed or submersed solid surface, such as a rock or tree, upon which an organism lives.

Surface area of a lake or impoundment is that area encompassed by the boundary of the lake or impoundment as shown on U.S. Geological Survey topographic maps, or on other available maps or photographs. The computed surface areas reflect the water levels of the lakes or impoundments at the times when the information for the maps or photographs was obtained.

Surficial bed material is the top 0.1 to $0.2 \mathrm{ft}$ of the bed material that is sampled using U.S. Series Bed-Material Samplers. 
Suspended (as used in tables of chemical analyses) refers to the amount (concentration) of undissolved material in a watersediment mixture. It is associated with the material retained on a 0.45 -micrometer filter.

Suspended, recoverable is the amount of a given constituent that is in solution after the part of a representative suspended-sediment sample that is retained on a 0.45 -micrometer membrane filter has been digested by a method (usually using a dilute acid solution) that results in dissolution of only readily soluble substances. Complete dissolution of all the particulate matter is not achieved by the digestion treatment and thus, the determination represents something less than the "total" amount (that is, less than 95 percent) of the constituent present in the sample. To achieve comparability of analytical data, equivalent digestion procedures are required of all laboratories performing such analyses because different digestion procedures are likely to produce different analytical results.

Determinations of "suspended, recoverable" constituents are made either by analyzing portions of the material collected on the filter or, more commonly, by difference, based on determinations of (1) dissolved and (2) total recoverable concentrations of the constituent.

Suspended, total is the total amount of a given constituent in the part of a representative suspended-sediment sample that is retained on a 0.45 -micrometer membrane filter. This term is used only when the analytical procedure assures measurement of at least 95 percent of the constituent determined. Knowledge of the expected form of the constituent in the sample, as well as the analytical methodology used, is required to determine when the results should be reported as "suspended, total."

Determinations of "suspended, total" constituents are made either by analyzing portions of the material collected on the filter or, more commonly, by difference, based on determinations of (1) dissolved and (2) total concentrations of the constituent.

Synoptic Studies are short-term investigations of specific water-quality conditions during selected seasonal or hydrologic periods to provide improved spatial resolution for critical water-quality conditions. For the period and conditions sampled, they assess the spatial distribution of selected water-quality conditions in relation to causative factors, such as land use and contaminant sources.

Taxonomy is the division of biology concerned with the classification and naming of organisms. The classification of organisms is based upon a hierarchial scheme beginning with Kingdom and ending with Species at the base. The higher the classification level, the fewer features the organisms have in common. For example, the taxonomy of a particular mayfly, Hexagenia limbata is the following:

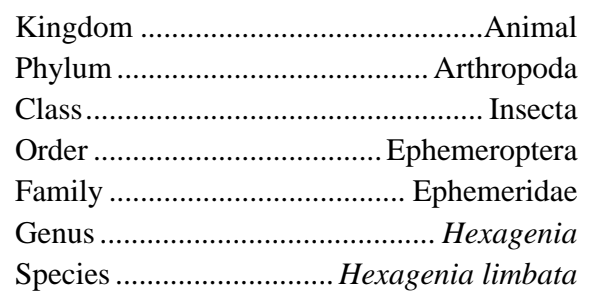

Thermograph is a thermometer that continuously and automatically records, on a chart, the water temperature of a stream. "Temperature recorder" is the term used to indicate the presence of a thermograph or a digital mechanism that records water temperature in a digital format on punched paper tape.

Time-weighted average is computed by multiplying the number of days in the sampling period by the concentrations of individual constituents for the corresponding period and dividing the sum of the products by the total number of days. A timeweighted average represents the composition of water that would be contained in a vessel or reservoir that had received equal quantities of water from the stream each day for the year.

Tons per acre-foot is the dry mass of dissolved solids in 1 acre-foot of water. It is computed by multiplying the concentration of the constituent, in milligrams per liter, by 0.00136 .

Tons per day (T/DAY, tons/d) is the rate representing a mass of 1 ton of a constituent in streamflow passing a cross section in 1 day. It is equivalent to 2,000 pounds per day, or 0.9072 metric tons per day.

Total is the total amount of a given constituent in a representative suspended-sediment sample, regardless of the constituent's physical or chemical form. This term is used only when the analytical procedure assures measurement of at least 95 percent of the constituent present in both the dissolved and suspended phases of the sample. Knowledge of the expected form of the constituent in the sample, as well as the analytical methodology used, is required to judge when the results should be reported as "total." (Note that the word "total" does double duty here, indicating both that the sample consists of a suspended-sediment mixture and that the analytical method determines all the constituent in the sample.)

Total discharge is the quantity of a given constituent, measured as dry mass or volume, that passes a stream cross section per unit of time. When referring to constituents other than water, this term needs to be qualified, such as "total sediment discharge," "total chloride discharge," and so on.

Total in bottom material is the total amount of a given constituent in a representative sample of bottom material. This term is used only when the analytical procedure assures measurement of at least 95 percent of the constituent determined. Knowledge of 
the expected form of the constituent in the sample, as well as the analytical methodology used, is required to judge when the results should be reported as "total in bottom material."

Total length (fish) is the straight-line distance from the anterior point of a fish specimen's snout, with the mouth closed, to the posterior end of the caudal (tail) fin, with the lobes of the caudal fin squeezed together.

Total load refers to all of a constituent in transport. When referring to sediment, it includes suspended load plus bed load.

Total, recoverable is the amount of a given constituent that is in solution after a representative suspended-sediment sample has been digested by a method (usually using a dilute acid solution) that results in dissolution of only readily soluble substances. Complete dissolution of all particulate matter is not achieved by the digestion treatment and thus, the determination represents something less than the "total" amount (that is, less than 95 percent) of the constituent present in the dissolved and suspended phases of the sample. To achieve comparability of analytical data, equivalent digestion procedures are required of all laboratories performing such analyses because different digestion procedures are likely to produce different analytical results.

Turbidity is a measurement of the collective optical properties of a water sample that cause light to be scattered and absorbed rather than transmitted in straight lines; the higher the intensity of scattered light, the higher the turbidity. Turbidity is expressed in nephelometric turbidity units (NTU) or Formazin turbidity units (FTU) depending on the method and equipment used.

Volatile organic compounds (VOC's) are organic compounds that can be isolated from the water phase of a sample by purging the water sample with inert gas, such as helium, and subsequently analyzed by gas chromatography. Many VOC's are manmade chemicals that are used and produced in the manufacture of paints, adhesives, petroleum products, pharmaceuticals, and refrigerants. They are often components of fuels, solvents, hydraulic fluids, paint thinners, and dry cleaning agents commonly used in urban settings. VOC contamination of drinking-water supplies is a human health concern because many are toxic and are known or suspected human carcinogens (U.S. Environmental Protection Agency, 1996).

Water level is the water-surface elevation or stage of the free surface of a body of water above or below any datum (see "Gage height"), or the surface of water standing in a well, usually indicative of the position of the water table or other potentiometric surface.

Water table is the surface of a ground-water body at which the water is at atmospheric pressure.

Water-table aquifer is an unconfined aquifer within which is found the water table.

Water year in U.S. Geological Survey reports dealing with surface-water supply is the 12-month period, October 1 through September 30. The water year is designated by the calendar year in which it ends and which includes 9 of the 12 months. Thus, the year ending September 30, 1999, is called the "1999 water year."

WDR is used as an abbreviation for "Water-Data Report" in the REVISED RECORDS paragraph to refer to State annual hydrologic-data reports. (WRD was used as an abbreviation for "Water-Resources Data" in reports published prior to 1976.)

Weighted average is used in this report to indicate discharge-weighted average. It is computed by multiplying the discharge for a sampling period by the concentrations of individual constituents for the corresponding period and dividing the sum of the products by the sum of the discharges. A discharge-weighted average approximates the composition of water that would be found in a reservoir containing all the water passing a given location during the water year after thorough mixing in the reservoir.

Well is an excavation (pit, hole, tunnel), generally cylindrical in form and often walled in, drilled, dug, driven, bored, or jetted into the ground to such a depth as to penetrate water-yielding geologic material and allow the water to flow or to be pumped to the surface.

Wet weight refers to the weight of animal tissue or other substance including its contained water.

WSP is used as an abbreviation for "Water-Supply Paper" in reference to previously published reports. 



\section{PUBLICATIONS ON TECHNIQUES OF WATER-RESOURCES INVESTIGATIONS}

The U.S. Geological Survey publishes a series of manuals describing procedures for planning and conducting specialized work in water-resources investigations. The material is grouped under major subject headings called books and is further divided into sections and chapters. For example, Section A of Book 3 (Applications of Hydraulics) pertains to surface water. The chapter, the unit of publication, is limited to a narrow field of subject matter. This format permits flexibility in revision and publication as the need arises.

The reports listed below are for sale by the U.S. Geological Survey, Branch of Information Services, Box 25286, Federal Center, Denver, Colorado 80225 (authorized agent of the Superintendent of Documents, Government Printing Office). Prepayment is required. Remittance should be sent by check or money order payable to the U.S. Geological Survey. Prices are not included because they are subject to change. Current prices can be obtained by writing to the above address. When ordering or inquiring about prices for any of these publications, please give the title, book number, chapter number, and "U.S. Geological Survey Techniques of Water-Resources Investigations."

\section{Book 1. Collection of Water Data by Direct Measurement}

\section{Section D. Water Quality}

1-D1. Water temperature—influential factors, field measurement, and data presentation, by H. H. Stevens, Jr., J.F. Ficke, and G. F. Smoot: USGS-TWRI Book 1, Chapter D1. 1975. 65 p.

1-D2. Guidelines for collection and field analysis of ground-water samples for selected unstable constituents, by W.W. Wood: USGS-TWRI Book 1, Chapter D2. 1976. 24 p.

Book 2. Collection of Environmental Data

\section{Section D. Surface Geophysical Methods}

2-D1. Application of surface geophysics to ground-water investigations, by A.A. R. Zohdy, G.P. Eaton, and D.R. Mabey: USGS-TWRI Book 2, Chapter D1. 1974. 116 p.

2-D2. Application of seismic-refraction techniques to hydrologic studies, by F.P. Haeni: USGS-TWRI Book 2, Chapter D2. $1988.86 \mathrm{p}$.

\section{Section E. Subsurface Geophysical Methods}

2-E1. Application of borehole geophysics to water-resources investigations, by W.S. Keys and L.M. MacCary: USGS-TWRI Book 2, Chapter E1. 1971. 126 p.

2-E2. Borehole geophysics applied to ground-water investigations, by W.S. Keys: USGS-TWRI Book 2, Chapter E2. 1990. $150 \mathrm{p}$.

\section{Section F. Drilling and Sampling Methods}

2-F1. Application of drilling, coring, and sampling techniques to test holes and wells, by Eugene Shuter and W.E. Teasdale: USGS-TWRI Book 2, Chapter F1. 1989. 97 p.

Book 3. Applications of Hydraulics

Section A. Surface-Water Techniques

3-A1. General field and office procedures for indirect discharge measurements, by M.A. Benson and Tate Dalrymple: USGS-TWRI Book 3, Chapter A1. 1967. 30 p.

3-A2. Measurement of peak discharge by the slope-area method, by Tate Dalrymple and M.A. Benson: USGS-TWRI Book 3, Chapter A2. 1967. 12 p.

3-A3. Measurement of peak discharge at culverts by indirect methods, by G.L. Bodhaine: USGS-TWRI Book 3, Chapter A3. 1968. $60 \mathrm{p}$.

3-A4. Measurement of peak discharge at width contractions by indirect methods, by H.F. Matthai: USGS-TWRI Book 3 , Chapter A4. 1967. 44 p.

3-A5. Measurement of peak discharge at dams by indirect methods, by Harry Hulsing: USGS-TWRI Book 3, Chapter A5. 1967. $29 \mathrm{p}$.

3-A6. General procedure for gaging streams, by R.W. Carter and Jacob Davidian: USGS-TWRI Book 3, Chapter A6. 1968. $13 \mathrm{p}$. 
3-A7. Stage measurement at gaging stations, by T.J. Buchanan and W.P. Somers: USGS-TWRI Book 3, Chapter A7. 1968. $28 \mathrm{p}$.

3-A8. Discharge measurements at gaging stations, by T.J. Buchanan and W.P. Somers: USGS-TWRI Book 3, Chapter A8. 1969. $65 \mathrm{p}$.

3-A9. Measurement of time of travel in streams by dye tracing, by F.A. Kilpatrick and J.F. Wilson, Jr.: USGS-TWRI Book 3, Chapter A9. 1989. 27 p.

3-A10. Discharge ratings at gaging stations, by E.J. Kennedy: USGS-TWRI Book 3, Chapter A10. 1984. 59 p.

3-A11. Measurement of discharge by the moving-boat method, by G.F. Smoot and C.E. Novak: USGS-TWRI Book 3, Chapter A11. 1969. 22 p.

3-A12. Fluorometric procedures for dye tracing, Revised, by J.F. Wilson, Jr., E.D. Cobb, and F.A. Kilpatrick: USGS-TWRI Book 3, Chapter A12. 1986. 41 p.

3-A13. Computation of continuous records of streamflow, by E.J. Kennedy: USGS-TWRI Book 3, Chapter A13. 1983. 53 p.

3-A14. Use of flumes in measuring discharge, by F.A. Kilpatrick and V.R. Schneider: USGS-TWRI Book 3, Chapter A14. 1983. $46 \mathrm{p}$.

3-A15. Computation of water-surface profiles in open channels, by Jacob Davidian: USGS-TWRI Book 3, Chapter A15. 1984. $48 \mathrm{p}$.

3-A16. Measurement of discharge using tracers, by F.A. Kilpatrick and E.D. Cobb: USGS-TWRI Book 3, Chapter A16. 1985. $52 \mathrm{p}$.

3-A17. Acoustic velocity meter systems, by Antonius Laenen: USGS-TWRI Book 3, Chapter A17. 1985. 38 p.

3-A18. Determination of stream reaeration coefficients by use of tracers, by F.A. Kilpatrick, R.E. Rathbun, Nobuhiro Yotsukura, G.W. Parker, and L.L. DeLong: USGS-TWRI Book 3, Chapter A18. 1989. 52 p.

3-A19. Levels at streamflow gaging stations, by E.J. Kennedy: USGS-TWRI Book 3, Chapter A19. 1990. 31 p.

3-A20. Simulation of soluable waste transport and buildup in surface waters using tracers, by F.A. Kilpatrick: USGS-TWRI Book 3, Chapter A20. 1993. 38 p.

3-A21 Stream-gaging cableways, by C. Russell Wagner: USGS-TWRI Book 3, Chapter A21. 1995. 56 p.

Section B. Ground-Water Techniques

3-B1. Aquifer-test design, observation, and data analysis, by R.W. Stallman: USGS-TWRI Book 3, Chapter B1. 1971. 26 p.

3-B2. Introduction to ground-water hydraulics, a programed text for self-instruction, by G.D. Bennett: USGS-TWRI Book 3, Chapter B2. 1976. 172 p.

3-B3. Type curves for selected problems of flow to wells in confined aquifers, by J.E. Reed: USGS-TWRI Book 3, Chapter B3. 1980. $106 \mathrm{p}$.

3-B4. Regression modeling of ground-water flow, by R.L. Cooley and R.L. Naff: USGS-TWRI Book 3, Chapter B4. 1990. $232 \mathrm{p}$.

3-B4. Supplement 1. Regression modeling of ground-water flow-Modifications to the computer code for nonlinear regression solution of steady-state ground-water flow problems, by R.L. Cooley: USGS-TWRI Book 3, Chapter B4. 1993. 8 p.

3-B5. Definition of boundary and initial conditions in the analysis of saturated ground-water flow systems-An introduction, by O.L. Franke, T.E. Reilly, and G.D. Bennett: USGS-TWRI Book 3, Chapter B5. 1987. 15 p.

3-B6. The principle of superposition and its application in ground-water hydraulics, by T.E. Reilly, O.L. Franke, and G.D. Bennett: USGS-TWRI Book 3, Chapter B6. 1987. 28 p.

3-B7. Analytical solutions for one-, two-, and three-dimensional solute transport in ground-water systems with uniform flow, by E.J. Wexler: USGS-TWRI Book 3, Chapter B7. 1992. 190 p.

Section C. Sedimentation and Erosion Techniques

3-C1. Fluvial sediment concepts, by H.P. Guy: USGS-TWRI Book 3, Chapter C1. 1970. 55 p.

3-C2. Field methods for measurement of fluvial sediment, by H.P. Guy and V.W. Norman: USGS-TWRI Book 3, Chapter C2. 1970.59 p.

3-C3. Computation of fluvial-sediment discharge, by George Porterfield: USGS-TWRI Book 3, Chapter C3. 1972. 66 p. 
Book 4. Hydrologic Analysis and Interpretation

Section A. Statistical Analysis

4-A1. Some statistical tools in hydrology, by H.C. Riggs: USGS-TWRI Book 4, Chapter A1. 1968. 39 p.

4-A2. Frequency curves, by H.C. Riggs: USGS-TWRI Book 4, Chapter A2. 1968. 15 p.

Section B. Surface Water

4-B1. Low-flow investigations, by H.C. Riggs: USGS-TWRI Book 4, Chapter B1. 1972.18 p.

4-B2. $\quad$ Storage analyses for water supply, by H.C. Riggs and C.H. Hardison: USGS-TWRI Book 4, Chapter B2. 1973. 20 p.

4-B3. Regional analyses of streamflow characteristics, by H.C. Riggs: USGS-TWRI Book 4, Chapter B3. 1973. 15 p.

Section D. Interrelated Phases of the Hydrologic Cycle

4-D1. Computation of rate and volume of stream depletion by wells, by C.T. Jenkins: USGS-TWRI Book 4, Chapter D1. 1970. $17 \mathrm{p}$.

\section{Book 5. Laboratory Analysis}

Section A. Water Analysis

5-A1. Methods for determination of inorganic substances in water and fluvial sediments, by M.J. Fishman and L.C. Friedman, editors: USGS-TWRI Book 5, Chapter A1. 1989. 545 p.

5-A2. Determination of minor elements in water by emission spectroscopy, by P.R. Barnett and E.C. Mallory, Jr.: USGS-TWRI Book 5, Chapter A2. 1971. 31 p.

5-A3. Methods for the determination of organic substances in water and fluvial sediments, edited by R.L. Wershaw, M.J. Fishman, R.R. Grabbe, and L.E. Lowe: USGS-TWRI Book 5, Chapter A3. 1987. 80 p.

5-A4. Methods for collection and analysis of aquatic biological and microbiological samples, by L.J. Britton and P.E. Greeson, editors: USGS-TWRI Book 5, Chapter A4. 1989. 363 p.

5-A5. Methods for determination of radioactive substances in water and fluvial sediments, by L.L. Thatcher, V.J. Janzer, and K.W. Edwards: USGS-TWRI Book 5, Chapter A5. 1977. 95 p.

5-A6. Quality assurance practices for the chemical and biological analyses of water and fluvial sediments, by L.C. Friedman and D.E. Erdmann: USGS-TWRI Book 5, Chapter A6. 1982. 181 p.

Section C. Sediment Analysis

5-C1. Laboratory theory and methods for sediment analysis, by H.P. Guy: USGS-TWRI Book 5, Chapter C1. 1969. 58 p.

Book 6. Modeling Techniques

Section A. Ground Water

6-A1. A modular three-dimensional finite-difference ground-water flow model, by M.G. McDonald and A.W. Harbaugh: USGS-TWRI Book 6, Chapter A1. 1988. 586 p.

6-A2. Documentation of a computer program to simulate aquifer-system compaction using the modular finite-difference ground-water flow model, by S.A. Leake and D.E. Prudic: USGS-TWRI Book 6, Chapter A2. 1991. 68 p.

6-A3. A modular finite-element model (MODFE) for areal and axisymmetric ground-water-flow problems, Part 1: Model Description and User's Manual, by L.J. Torak: USGS-TWRI Book 6, Chapter A3. 1993. 136 p.

6-A4. A modular finite-element model (MODFE) for areal and axisymmetric ground-water-flow problems, Part 2: Derivation of finite-element equations and comparisons with analytical solutions, by R.L. Cooley: USGS-TWRI Book 6, Chapter A4. 1992. 108 p.

6-A5. A modular finite-element model (MODFE) for areal and axisymmetric ground-water-flow problems, Part 3: Design philosophy and programming details, by L.J. Torak: USGS-TWRI Book 6, Chapter A5, 1993. 243 p.

6-A6. A coupled surface-water and ground-water flow model (MODBRANCH) for simulation of stream-aquifer interaction, by Eric D. Swain and Eliezer J. Wexler. 1996. 125 p. 
Book 7. Automated Data Processing and Computations

Section C. Computer Programs

7-C1. Finite difference model for aquifer simulation in two dimensions with results of numerical experiments, by P.C. Trescott, G.F. Pinder, and S.P. Larson: USGS-TWRI Book 7, Chapter C1. 1976. 116 p.

7-C2. Computer model of two-dimensional solute transport and dispersion in ground water, by L.F. Konikow and J.D. Bredehoeft: USGS-TWRI Book 7, Chapter C2. 1978. 90 p.

7-C3. A model for simulation of flow in singular and interconnected channels, by R.W. Schaffranek, R.A. Baltzer, and D.E. Goldberg: USGS-TWRI Book 7, Chapter C3. 1981. 110 p.

Book 8. Instrumentation

Section A. Instruments for Measurement of Water Level

8-A1. Methods of measuring water levels in deep wells, by M.S. Garber and F.C. Koopman: USGS-TWRI Book 8, Chapter A1. 1968. $23 \mathrm{p}$.

8-A2. Installation and service manual for U.S. Geological Survey manometers, by J.D. Craig: USGS-TWRI Book 8, Chapter A2. 1983.57 p.

Section B. Instruments for Measurement of Discharge

8-B2. Calibration and maintenance of vertical-axis type current meters, by G.F. Smoot and C.E. Novak: USGS-TWRI Book 8, Chapter B2. 1968. 15 p.

Book 9. Handbooks for Water-Resources Investigations

Section A. National Field Manual for the Collection of Water-Quality Data

9-A1. National Field Manual for the Collection of Water-Quality Data: Preparations for Water Sampling, by F.D. Wilde, D.B. Radtke, Jacob Gibs, and R.T. Iwatsubo: USGS-TWRI Book 9, Chapter A1. 1998. 47 p.

9-A2. National Field Manual for the Collection of Water-Quality Data: Selection of Equipment for Water Sampling, edited by F.D. Wilde, D.B. Radtke, Jacob Gibs, and R.T. Iwatsubo: USGS-TWRI Book 9, Chapter A2. 1998. 94 p.

9-A3. National Field Manual for the Collection of Water-Quality Data: Cleaning of Equipment for Water Sampling, edited by F.D. Wilde, D.B. Radtke, Jacob Gibs, and R.T. Iwatsubo: USGS-TWRI Book 9, Chapter A3. 1998. 75 p.

9-A5. National Field Manual for the Collection of Water-Quality Data: Processing of Water Samples, edited by F.D. Wilde, D.B. Radtke, Jacob Gibs, and R.T. Iwatsubo: USGS-TWRI Book 9, Chapter A5. 1999. 149 p.

9-A6. National Field Manual for the Collection of Water-Quality Data: Field Measurements, edited by F.D. Wilde and D.B. Radtke: USGS-TWRI Book 9, Chapter A6. 1998. Variously paginated.

9-A7. National Field Manual for the Collection of Water-Quality Data: Biological Indicators, by D.N. Myers and F.D. Wilde: USGS-TWRI Book 9, Chapter A7.1. 1997. 49 p.

9-A7. National Field Manual for the Collection of Water-Quality Data: Five-Day Biological Oxygen Demand, by G.C. Delzer and S.W. McKenzie: USGS-TWRI Book 9, Chapter A7.2. 1999. 28 p.

9-A8. National Field Manual for the Collection of Water-Quality Data: Bottom-Material Samples, by D.B. Radtke: USGS-TWRI Book 9, Chapter A8. 1998. 48 p.

9-A9. National Field Manual for the Collection of Water-Quality Data: Safety in Field Activities, by S.L. Lane and R.G. Fay: USGS-TWRI Book 9, Chapter A9. 1998. 60 p. 


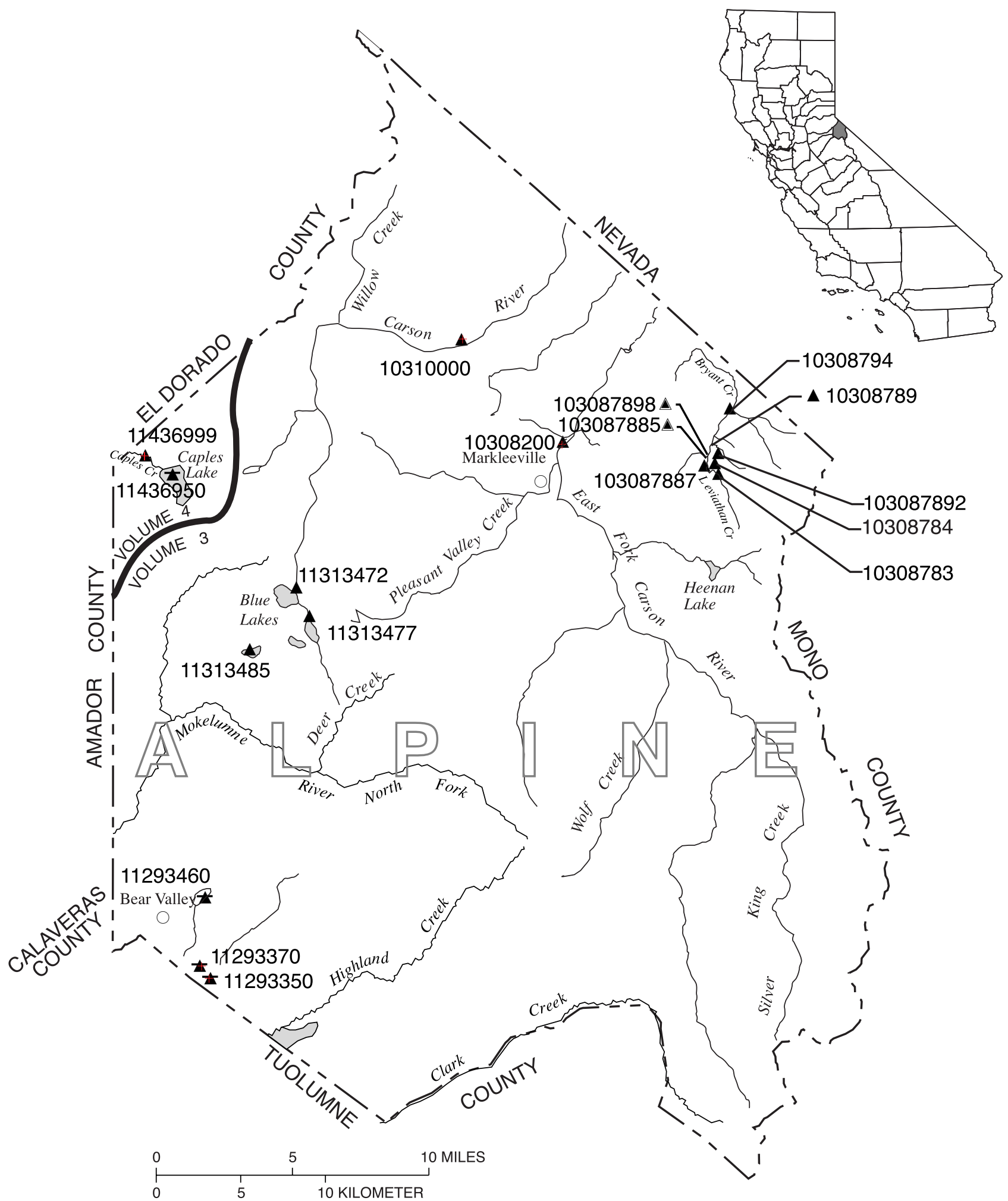

EXPLANATION

- GAGING STATION

$\triangle$ GAGING STATION (PARTIAL RECORD)

× RESERVOIR SITE AND CONTENTS

Figure 2. Location of discharge stations in Alpine County.

(NOTE: Records for stations 10308200 through 10310000 and 11293350 through 11313485 published in volume 3.) 


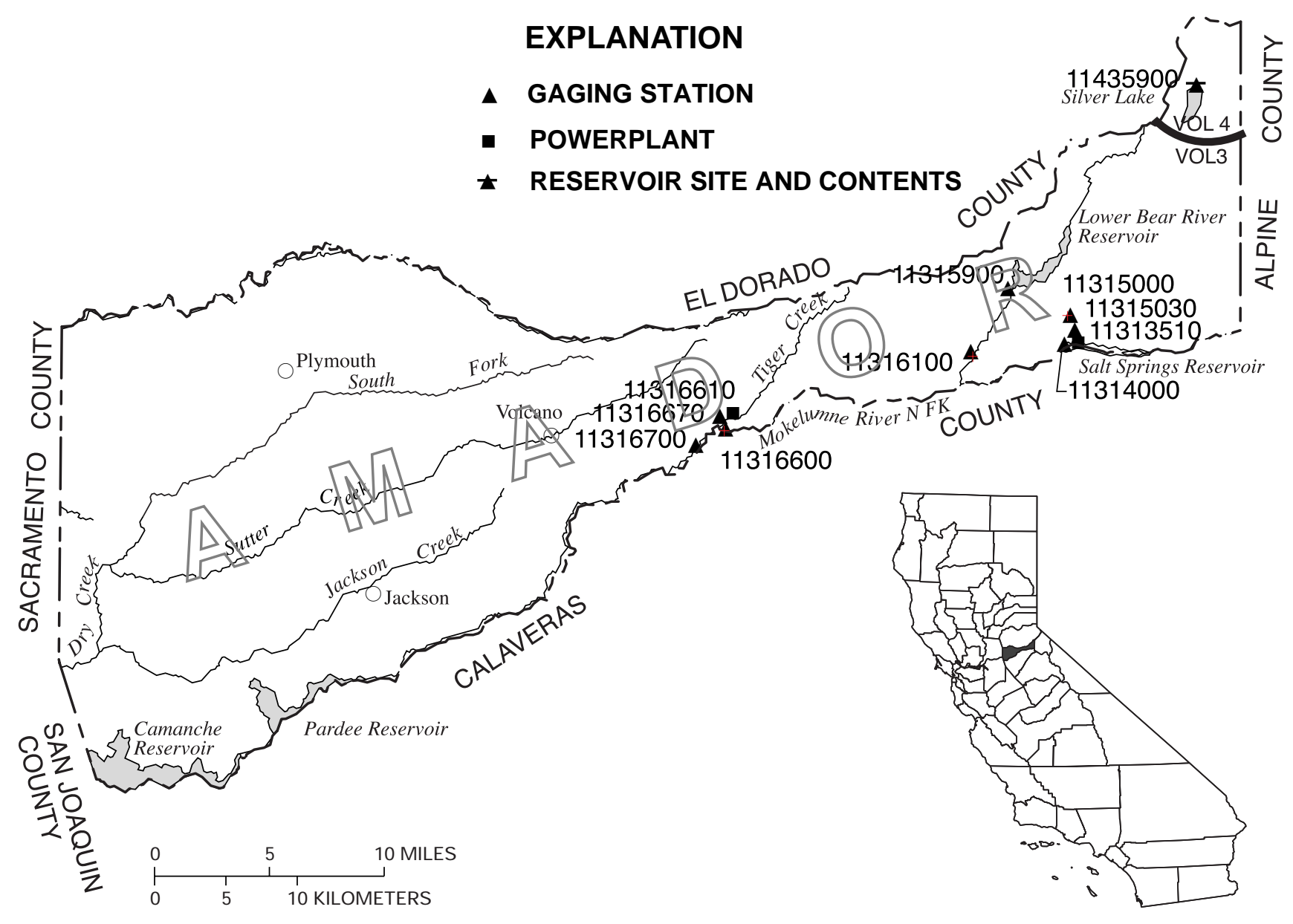

Figure 3. Location of discharge stations in Amador County.

(NOTE: Records for stations 11313510 through 11316700 published in volume 3.) 


\section{EXPLANATION}

\ GAGING STATION

GAGING AND WATER-QUALITY

(TEMPERATURE) STATION WITH

TELEMETRY

- POWERPLANT

× RESERVOIR SITE AND CONTENTS

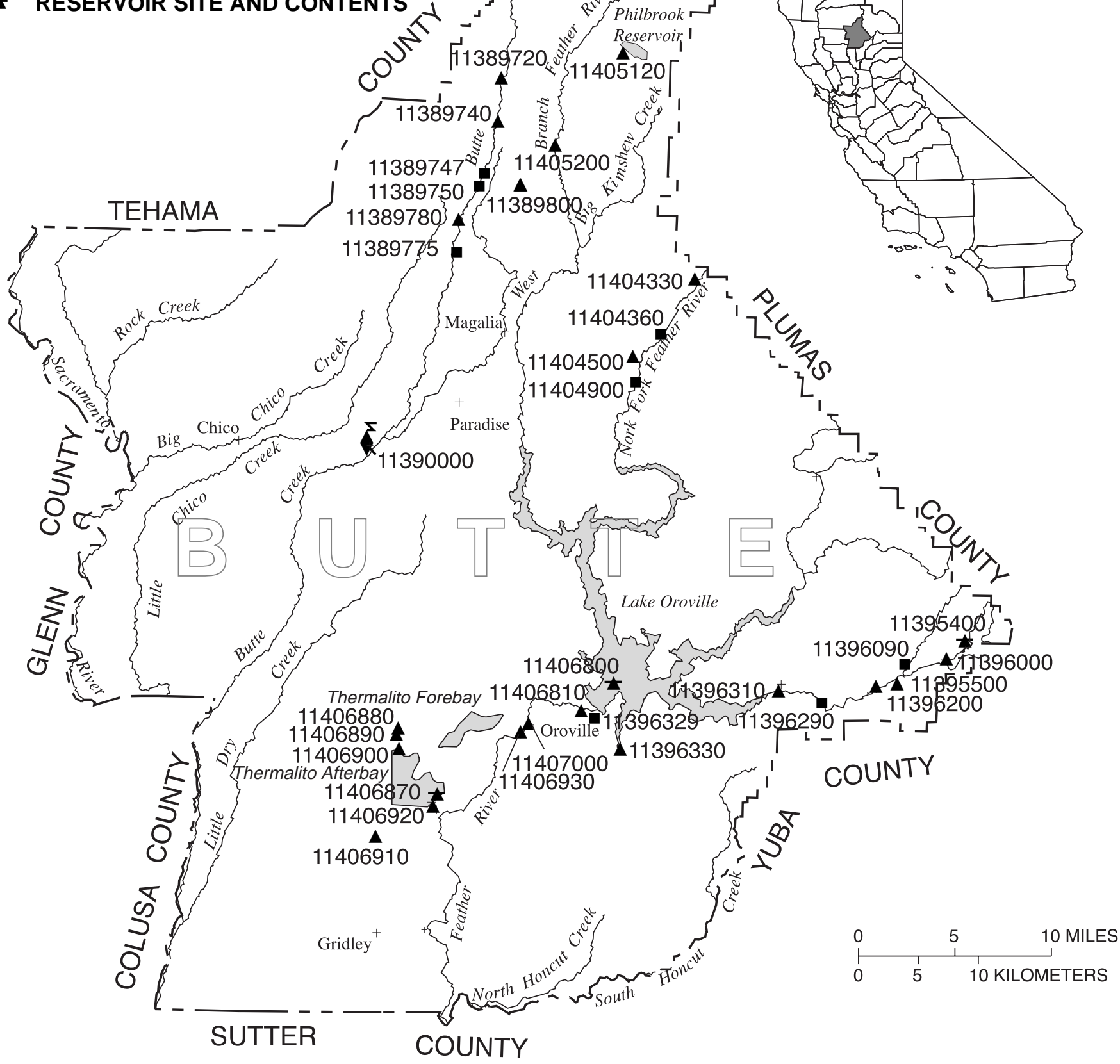

Figure 4. Location of discharge and water-quality stations in Butte County. 


\section{EXPLANATION}

$\Sigma$ GAGING STATION WITH TELEMETRY

GAGING AND WATER-QUALITY (TEMPERATURE)

STATION WITH DATA COLLECTION PLATFORM

\& GAGING AND WATER-QUALITY (SEDIMENT, CHEMICAL ) STATION WITH DATA COLLECTION PLATFORM

$\star \quad$ RESERVOIR SITE AND CONTENTS

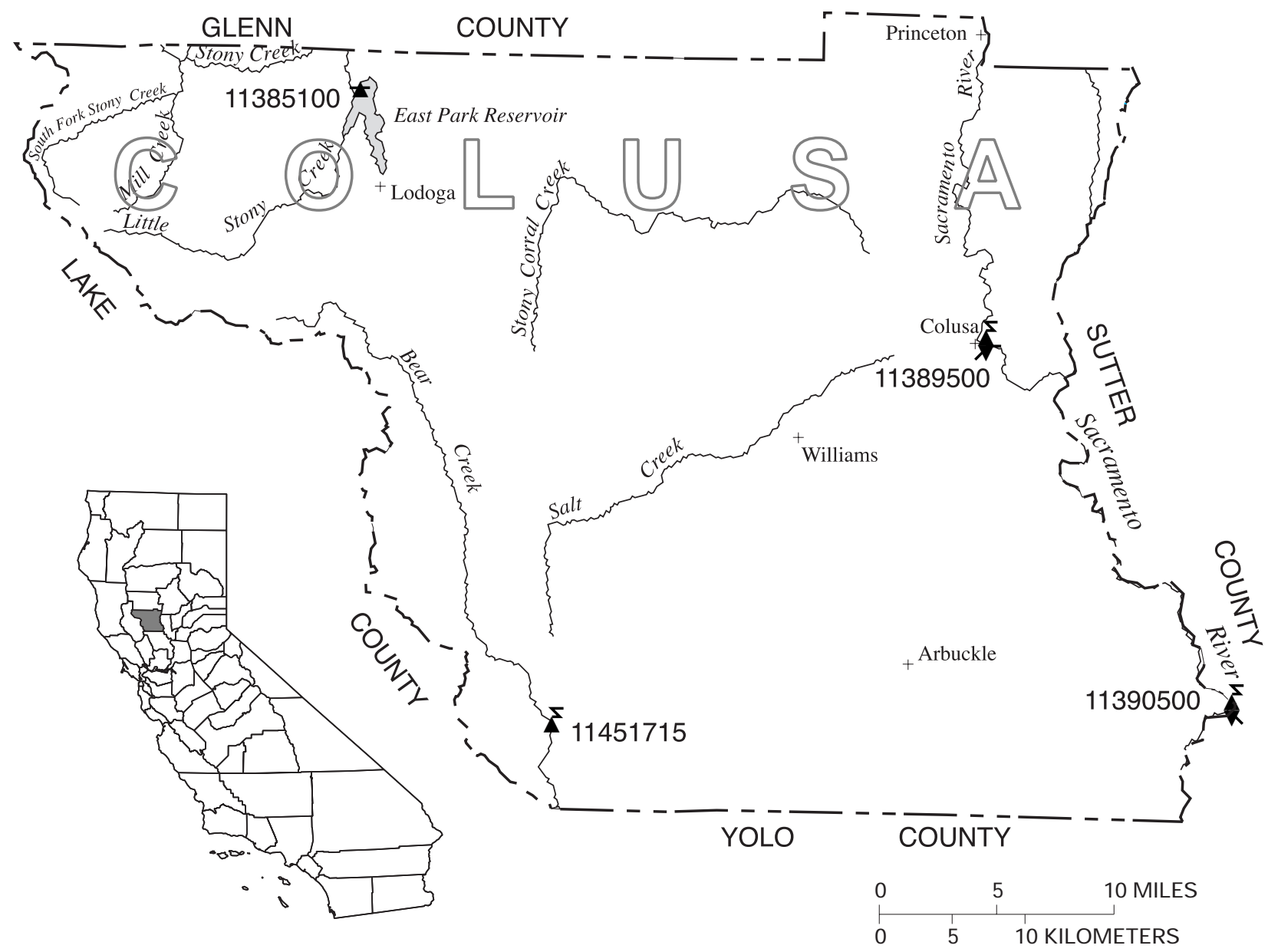

Figure 5. Location of discharge and water-quality stations in Colusa County. 

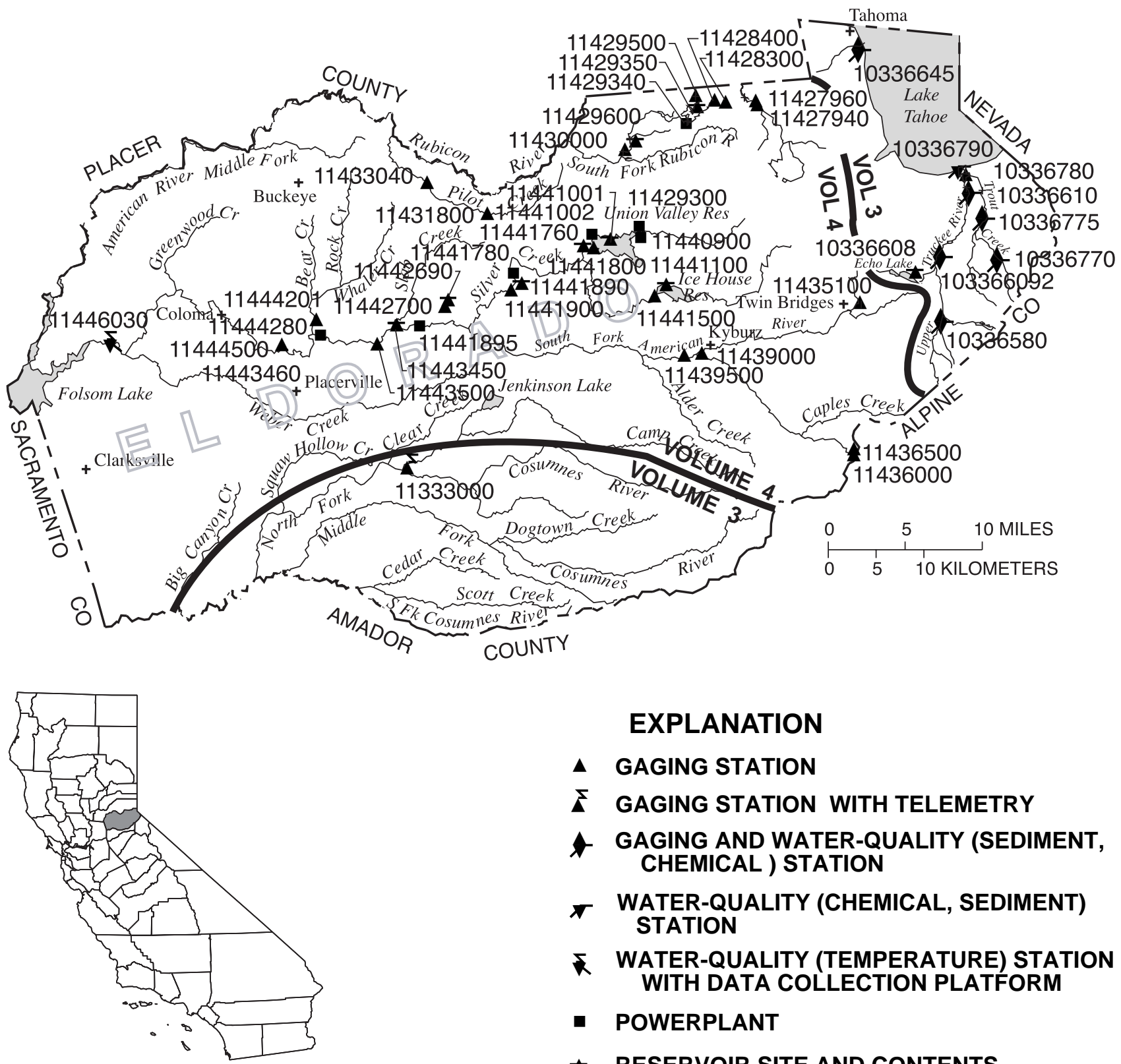

EXPLANATION

- GAGING STATION

$\Sigma$ GAGING STATION WITH TELEMETRY

f GAGING AND WATER-QUALITY (SEDIMENT, CHEMICAL ) STATION

$\pi$ WATER-QUALITY (CHEMICAL, SEDIMENT) STATION

\ WATER-QUALITY (TEMPERATURE) STATION WITH DATA COLLECTION PLATFORM

- POWERPLANT

$\star \quad$ RESERVOIR SITE AND CONTENTS

Figure 6. Location of discharge and water-quality stations in El Dorado County. (NOTE: Records for stations 10336580 through 10336790 and 11333000 published in volume 3.) 


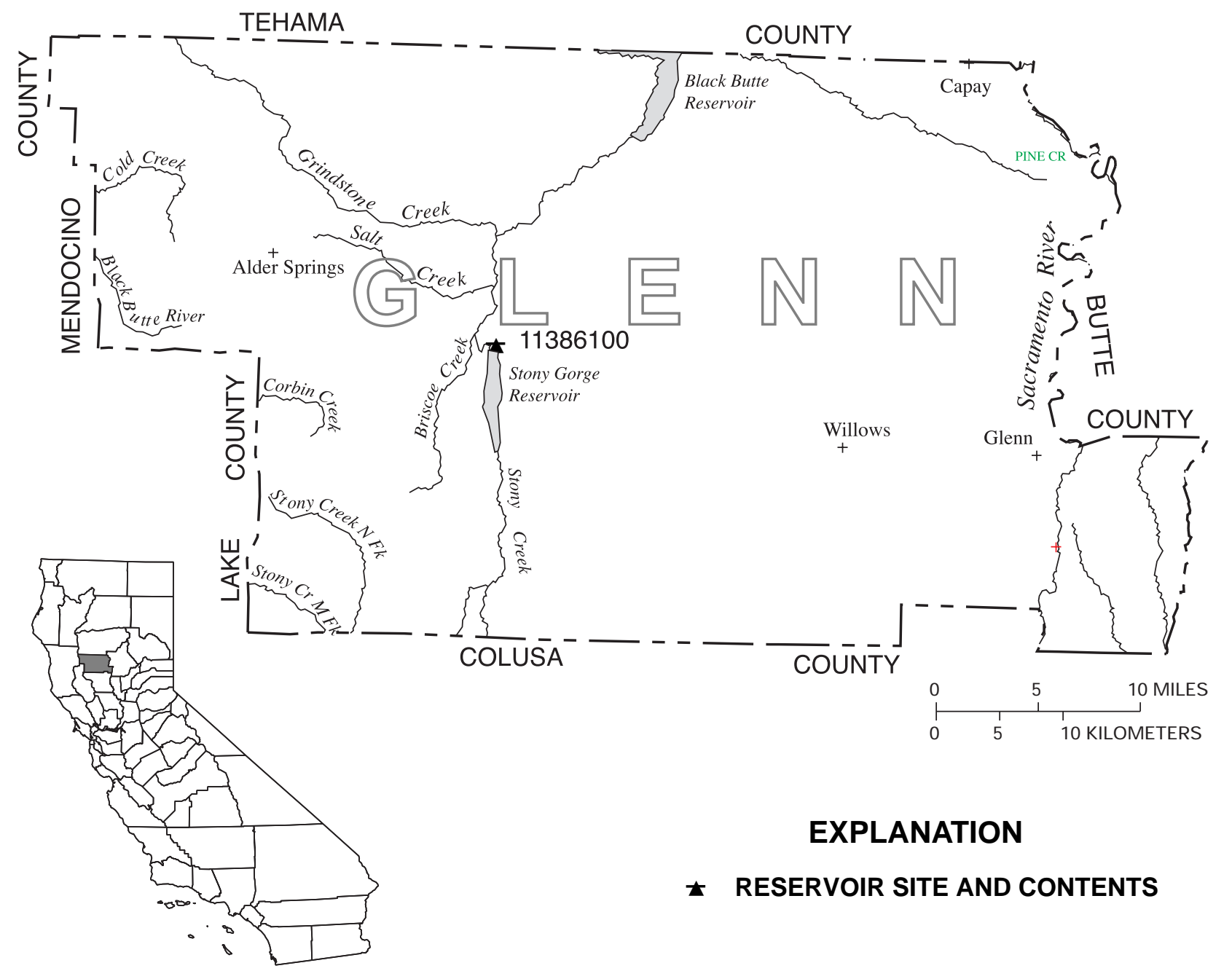

Figure 7. Location of discharge station in Glenn County. 


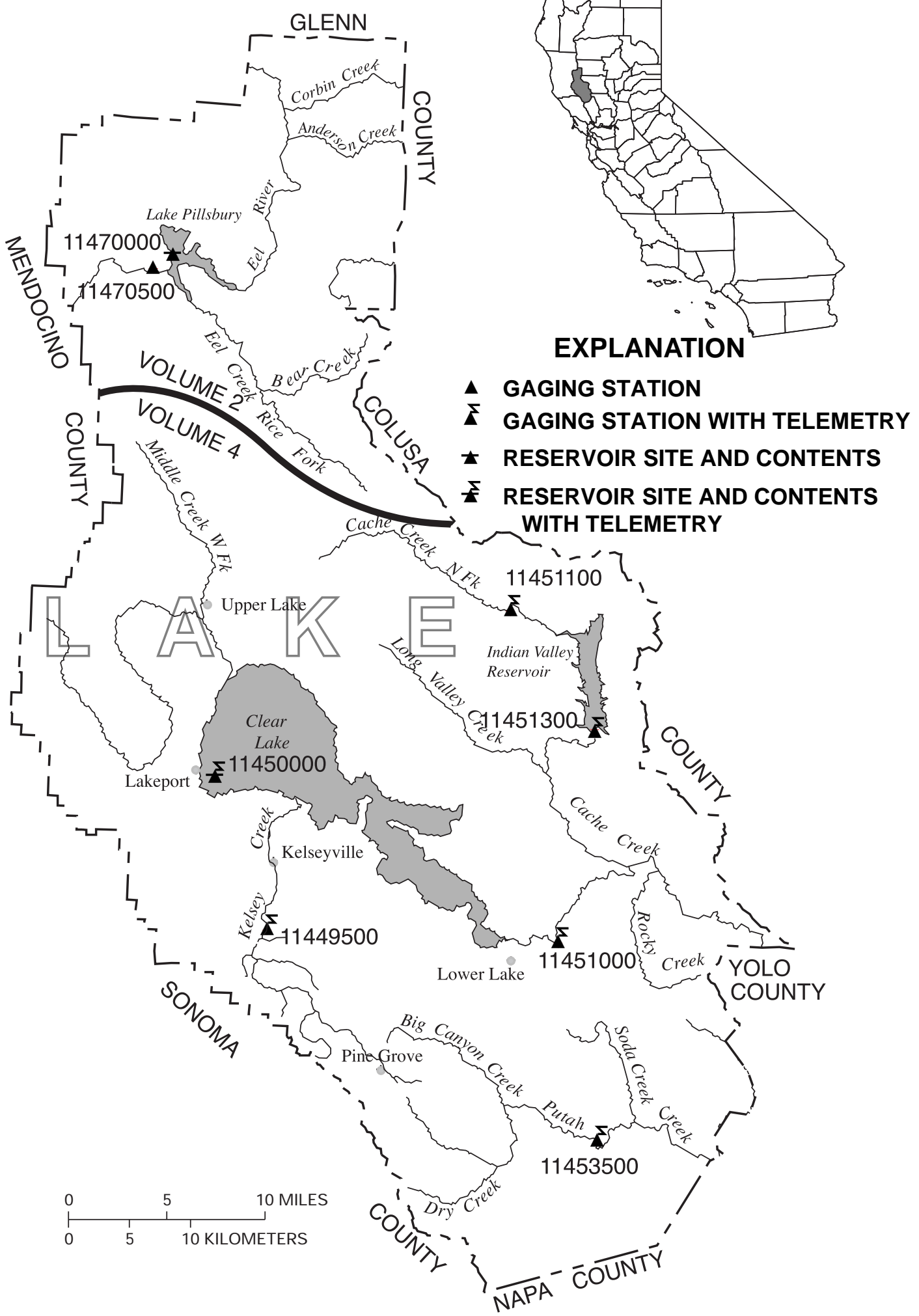

Figure 8. Location of discharge stations in Lake County.

(NOTE: Records for stations 11470000 and 11470500 published in volume 2.) 


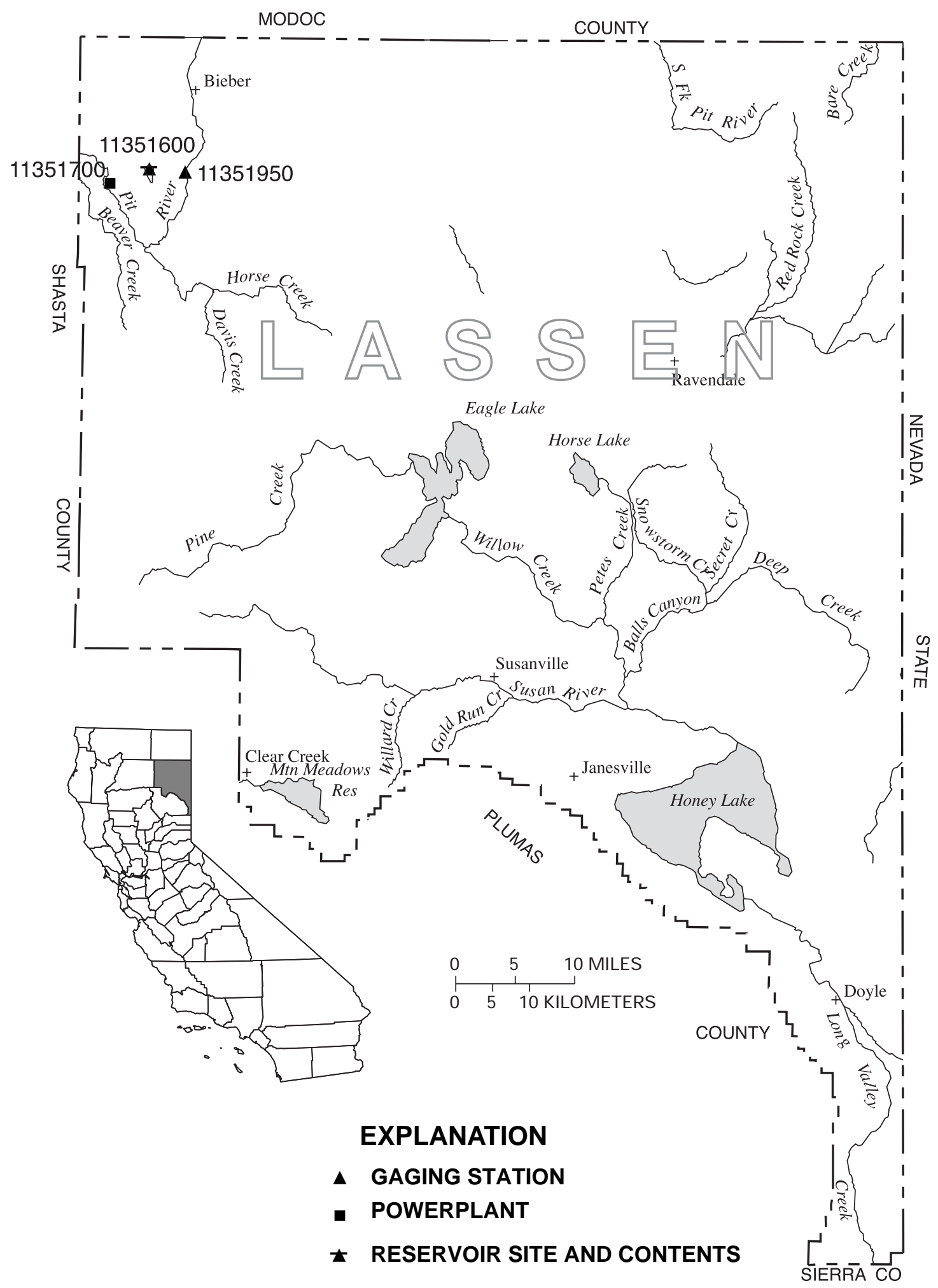

Figure 9. Location of discharge stations in Lassen County. 

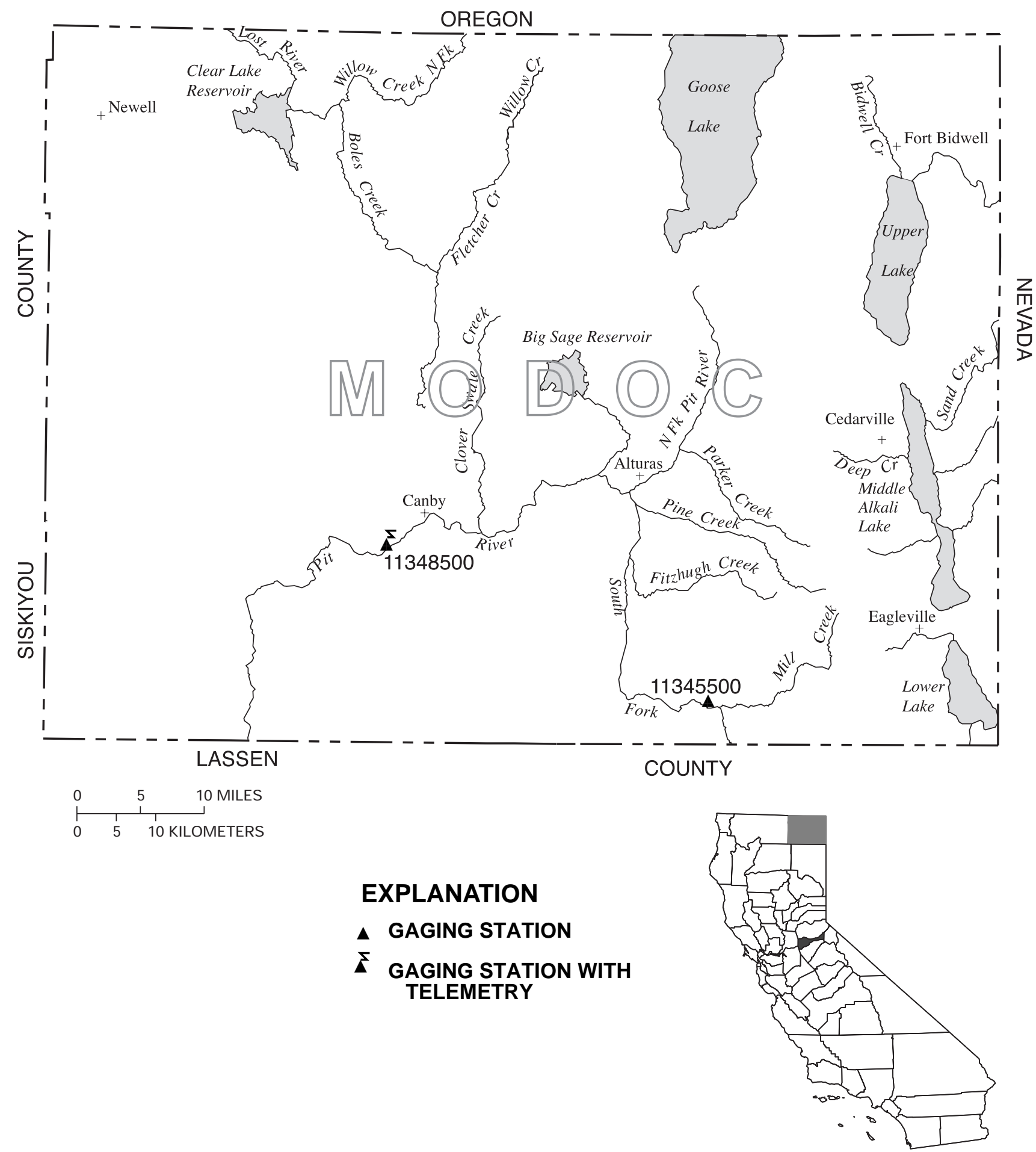

Figure 10. Location of discharge stations in Modoc County. 


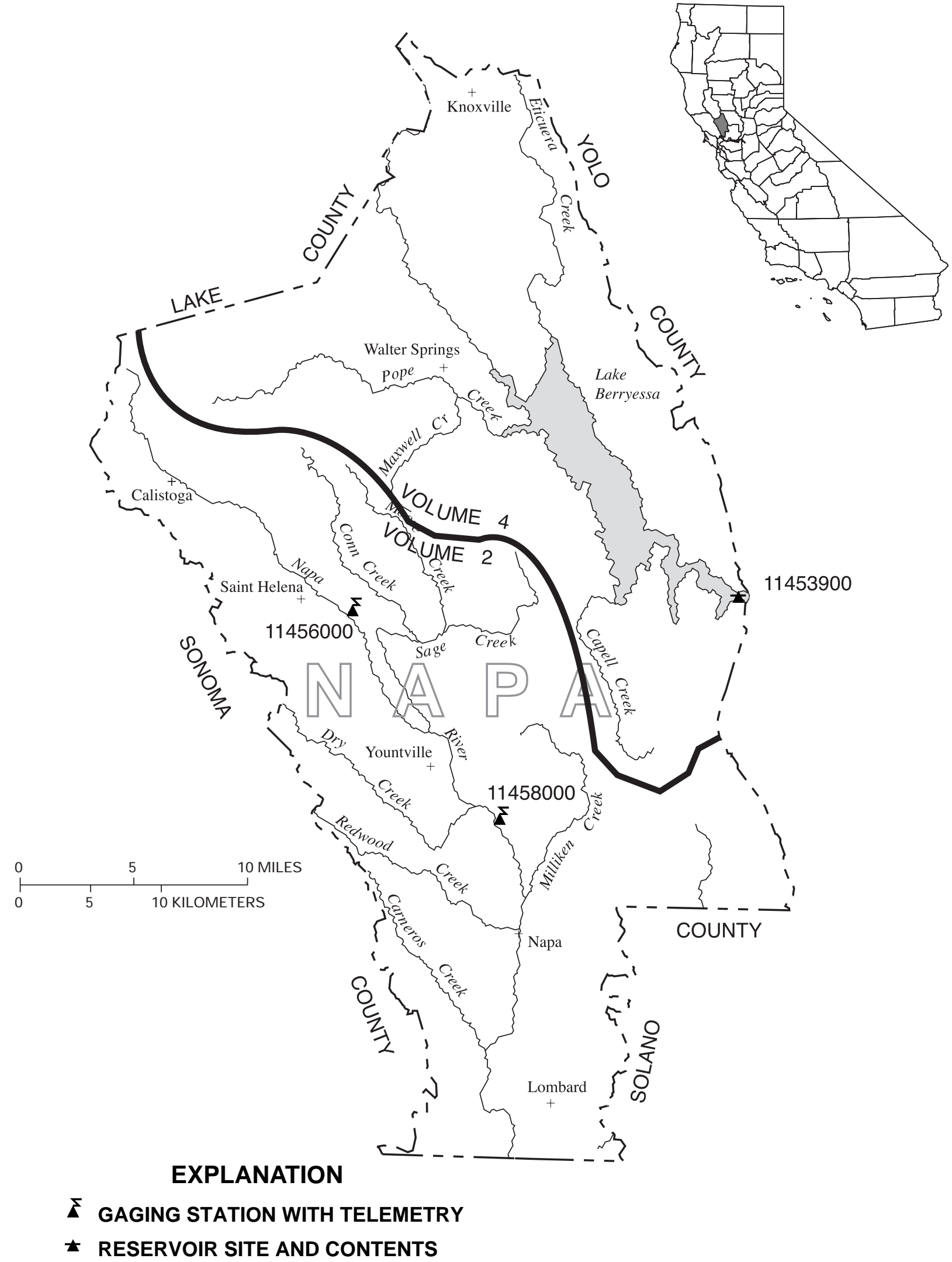

Figure 11. Location of discharge stations in Napa County.

(NOTE: Records for stations 11456000 and 11458000 published in volume 2.) 


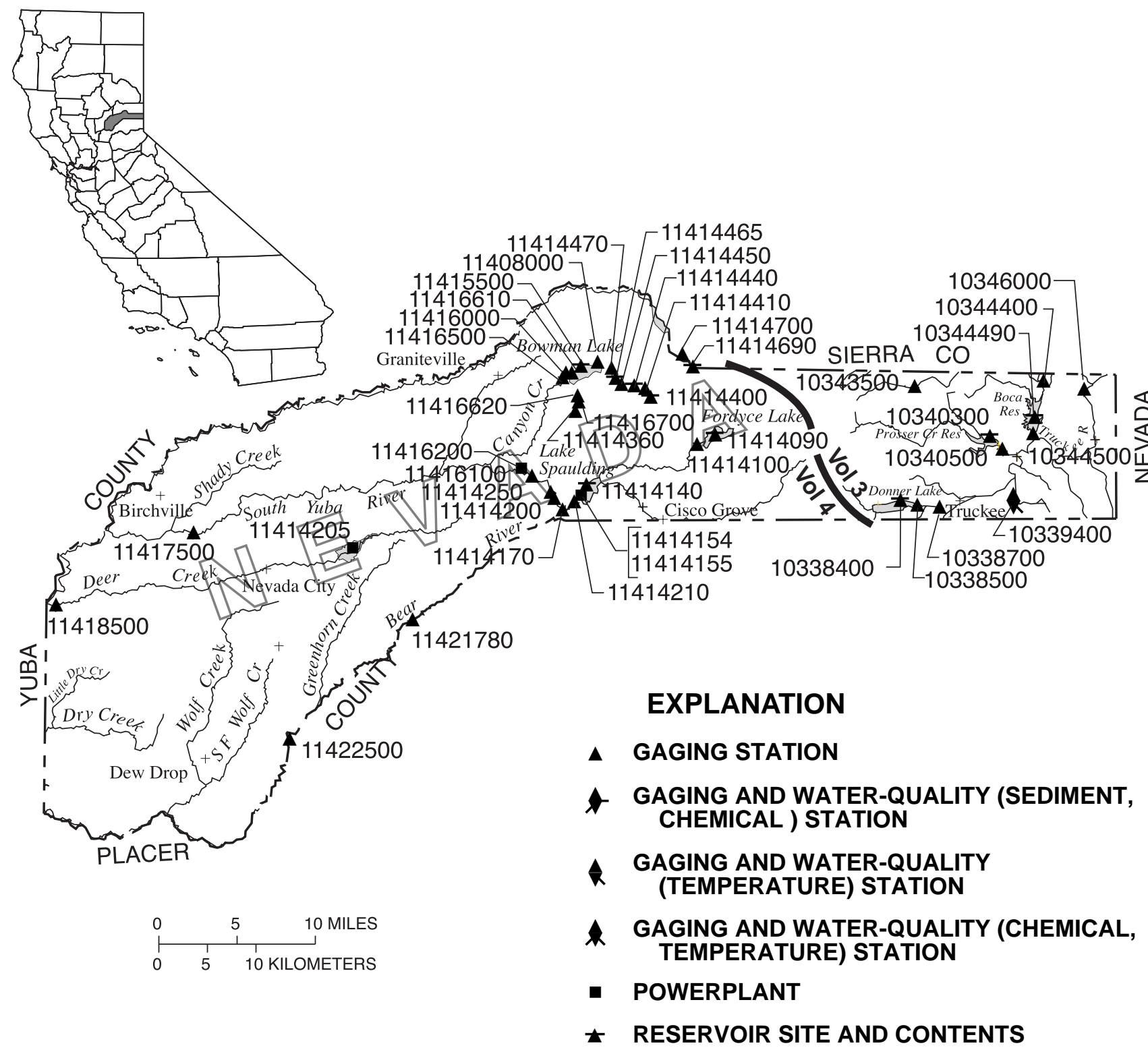

Figure 12. Location of discharge and water-quality stations in Nevada County. (NOTE: Records for stations 10338400 through 10346000 published in volume 3.) 


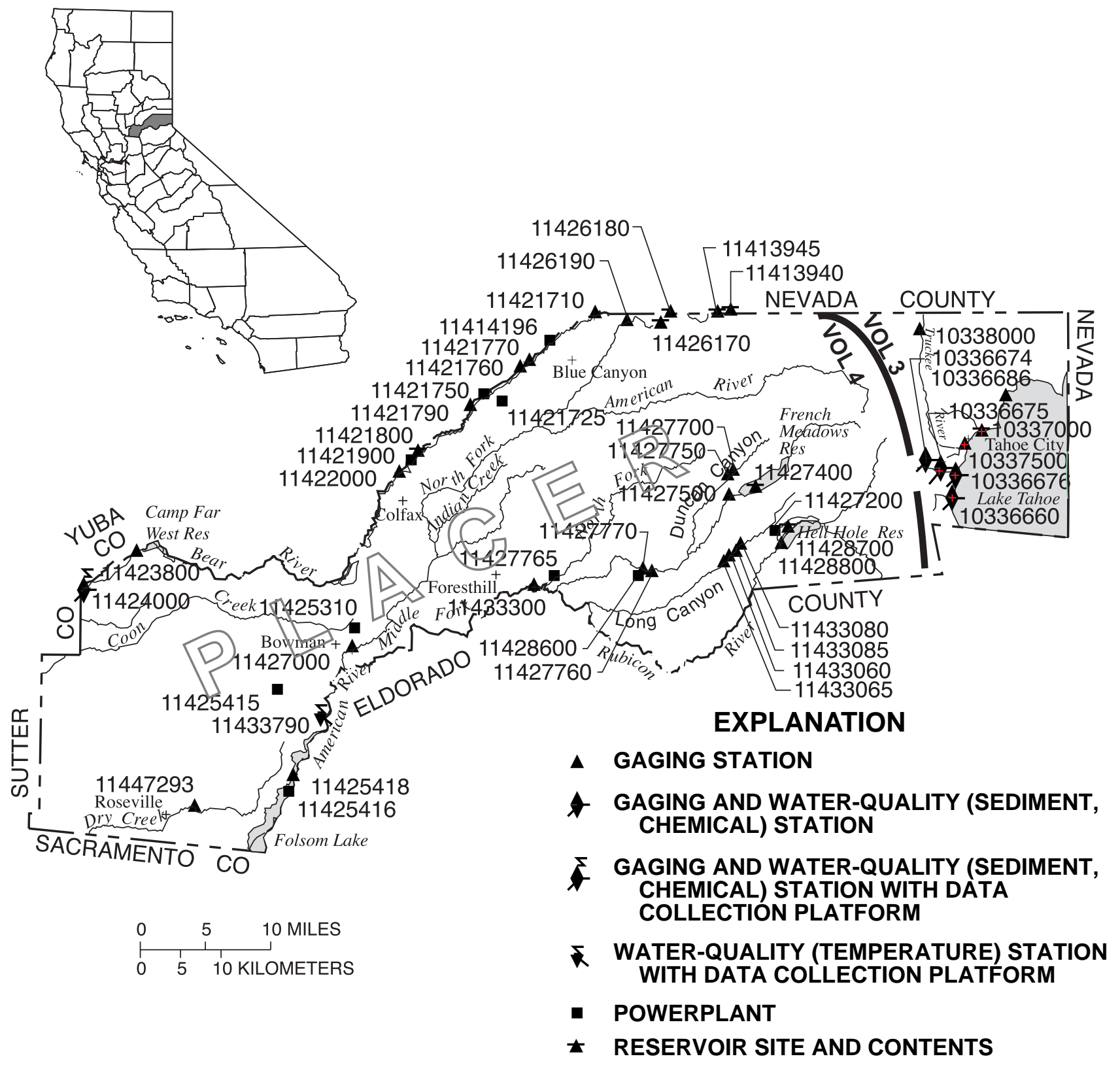

Figure 13. Location of discharge and water-quality stations in Placer County. (NOTE: Records for stations 10336660 through 10338000 published in volume 3.) 


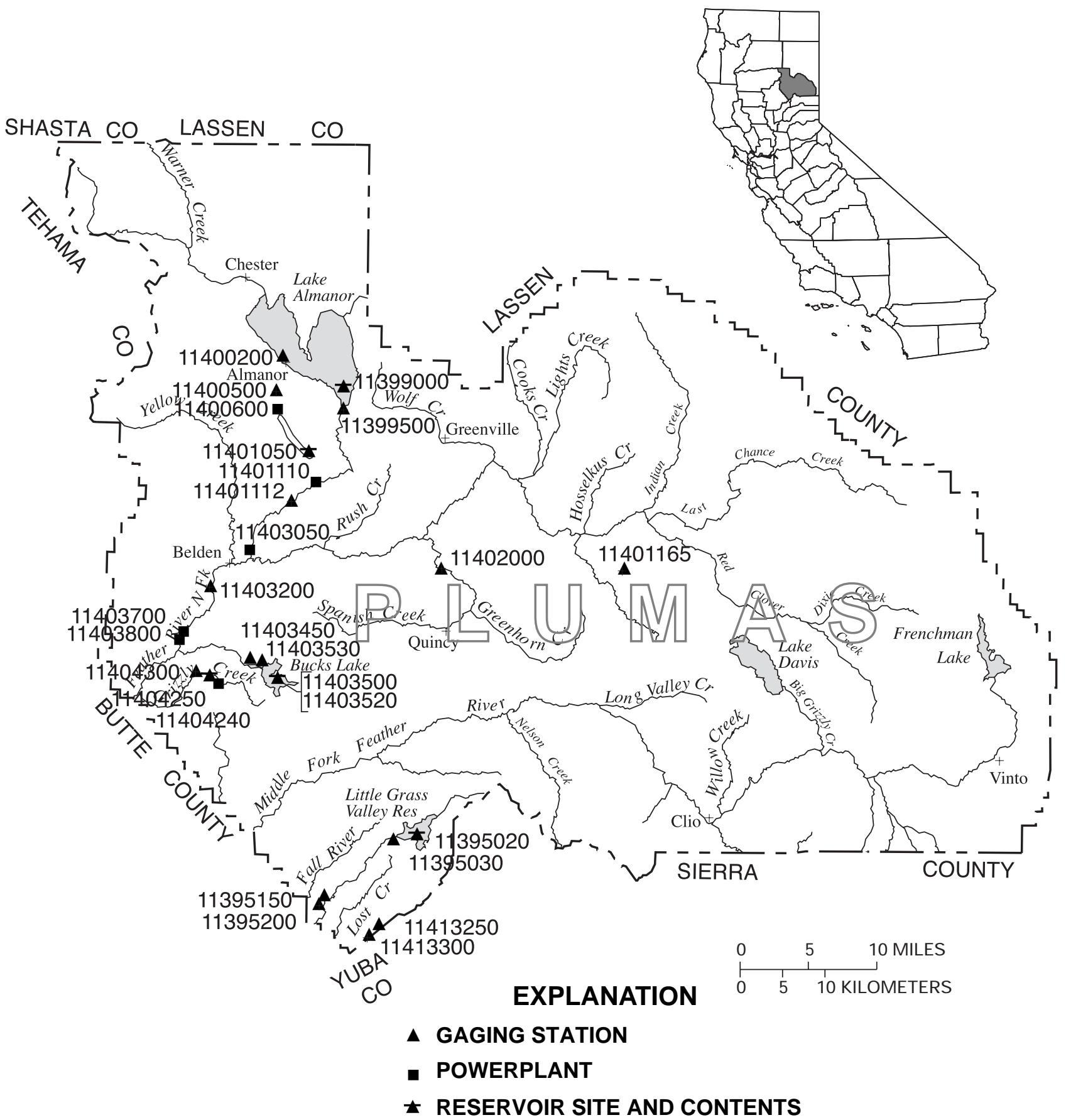

Figure 14. Location of discharge stations in Plumas County. 


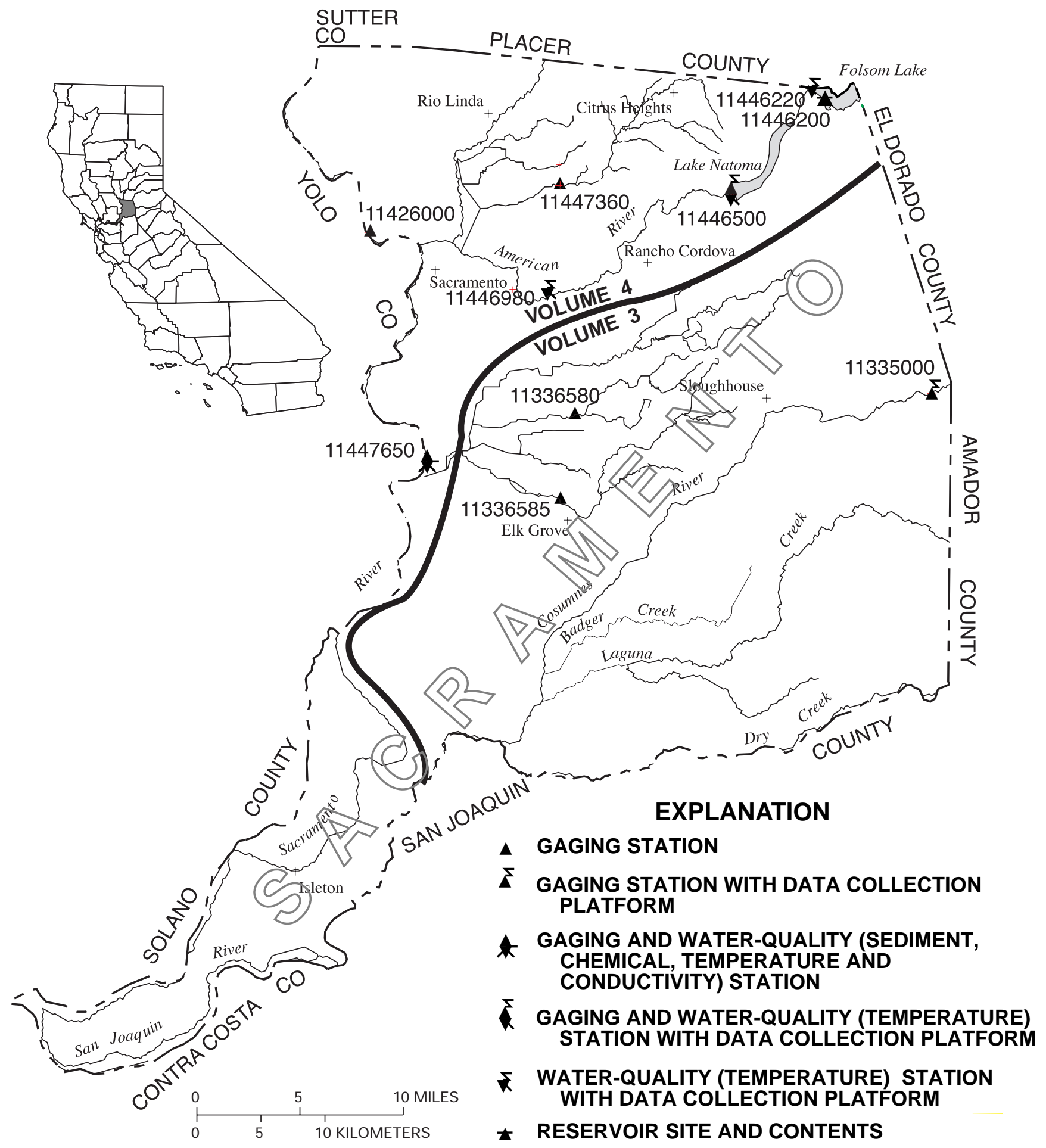

Figure 15. Location of discharge and water-quality stations in Sacramento County. (NOTE: Records for stations 11335000 through 11336585 published in volume 3.) 

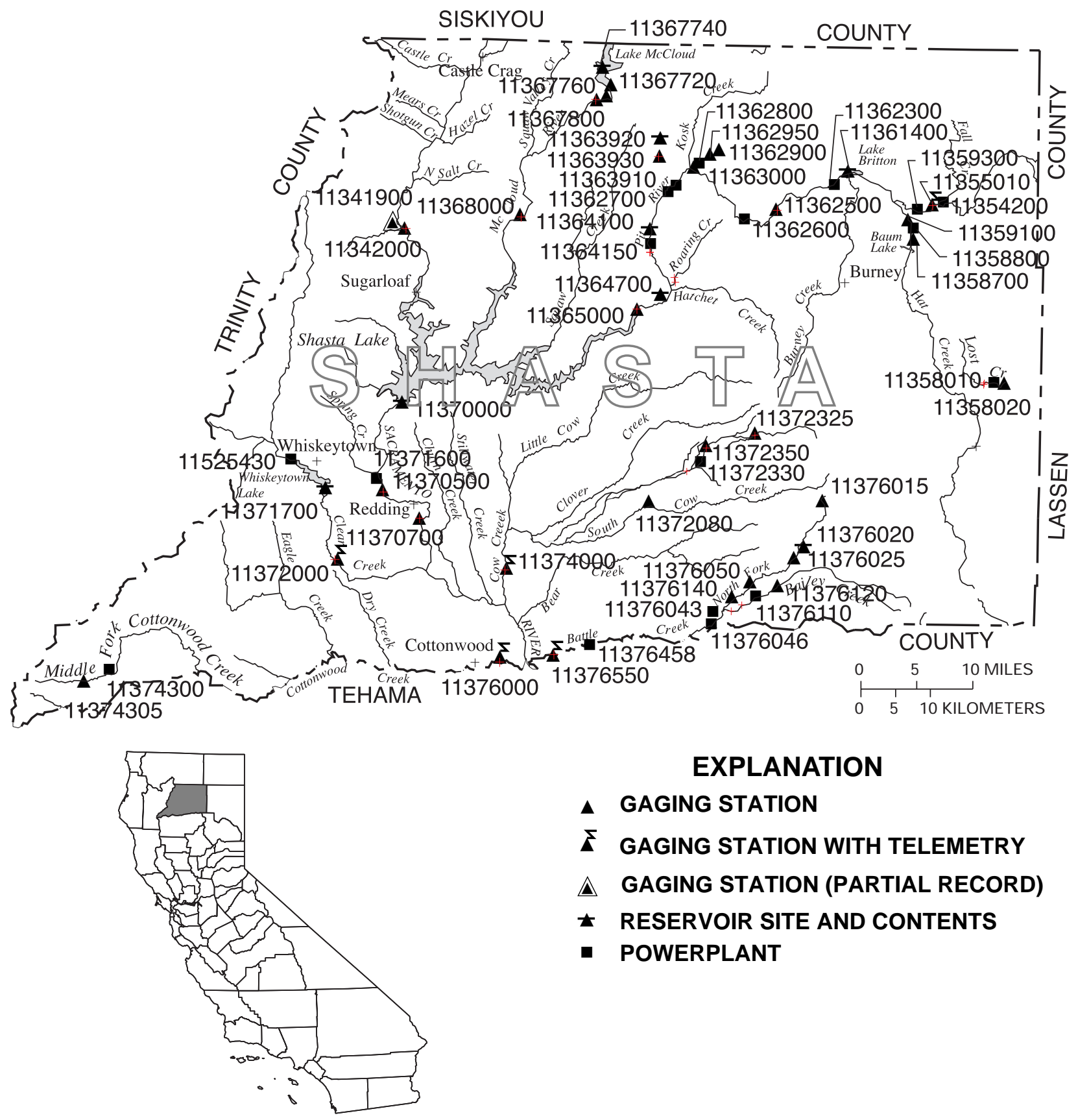

EXPLANATION

- GAGING STATION

$\Sigma$ GAGING STATION WITH TELEMETRY

$\triangle$ GAGING STATION (PARTIAL RECORD)

‡ RESERVOIR SITE AND CONTENTS

- POWERPLANT

Figure 16. Location of discharge stations in Shasta County. 


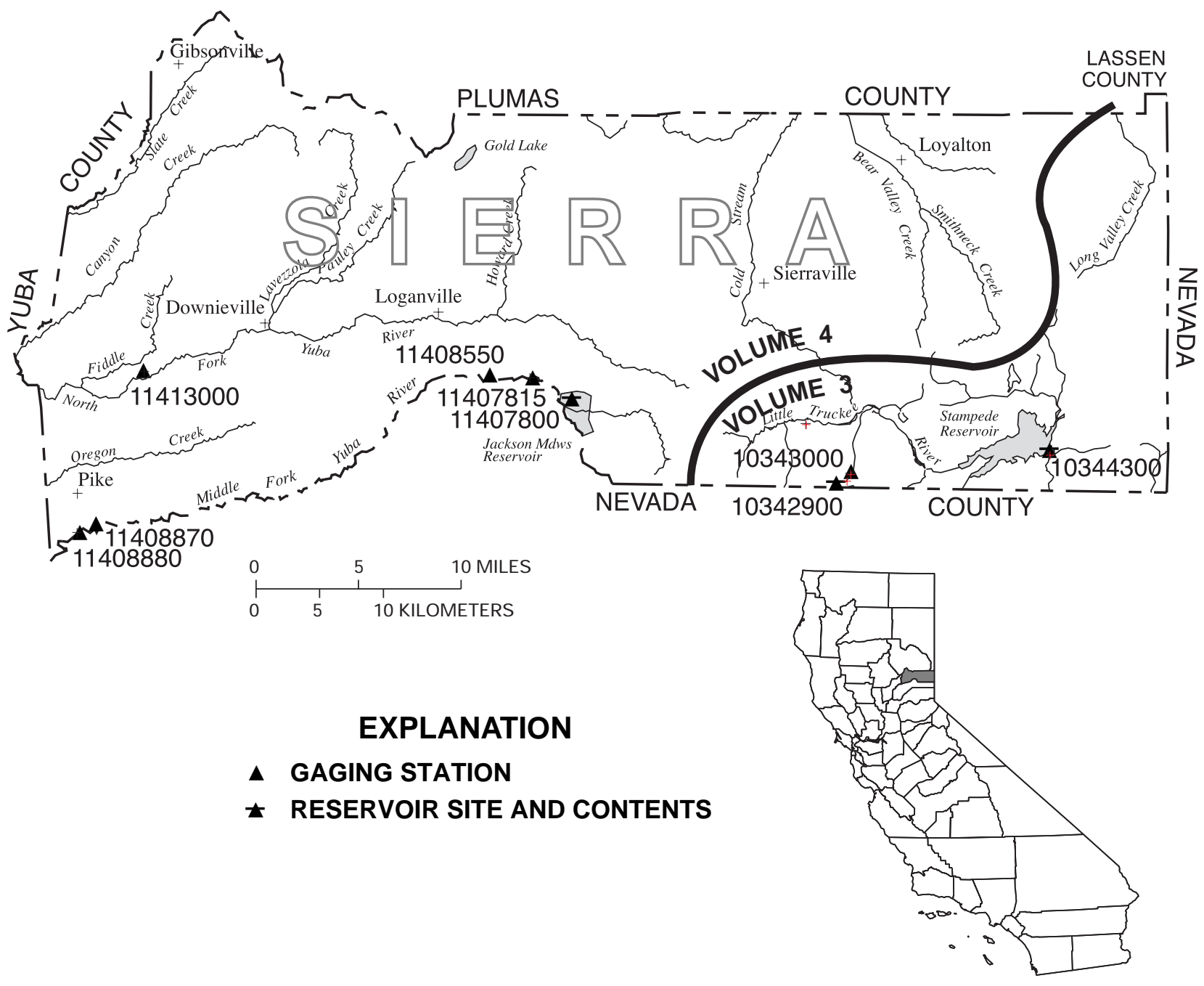

Figure 17. Location of discharge stations in Sierra County.

(NOTE: Records for stations 10342900 through 10344300 published in volume 3.) 


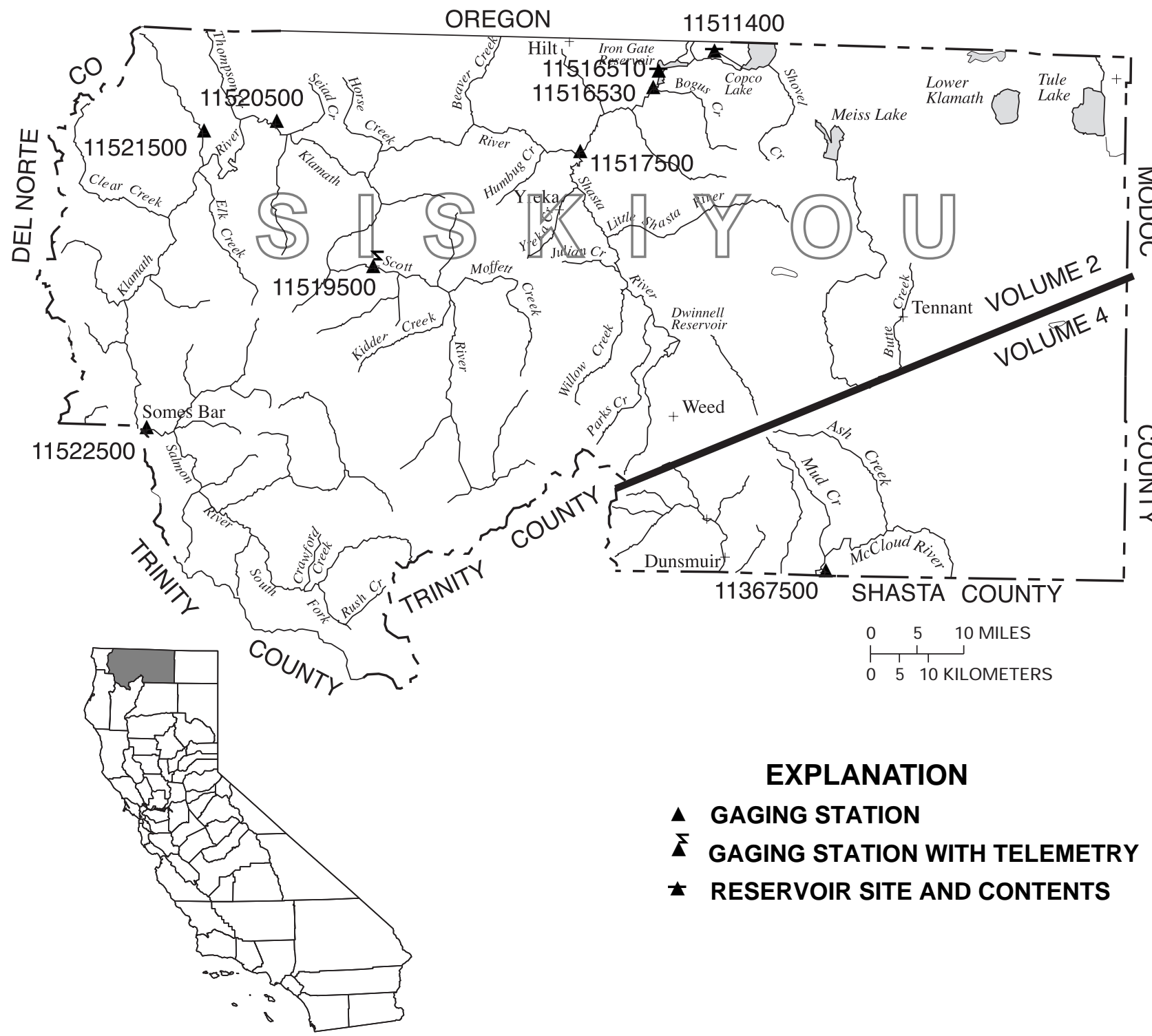

Figure 18. Location of discharge stations in Siskiyou County.

(NOTE: Records for stations 11511400 through 11522500 published in volume 2.) 


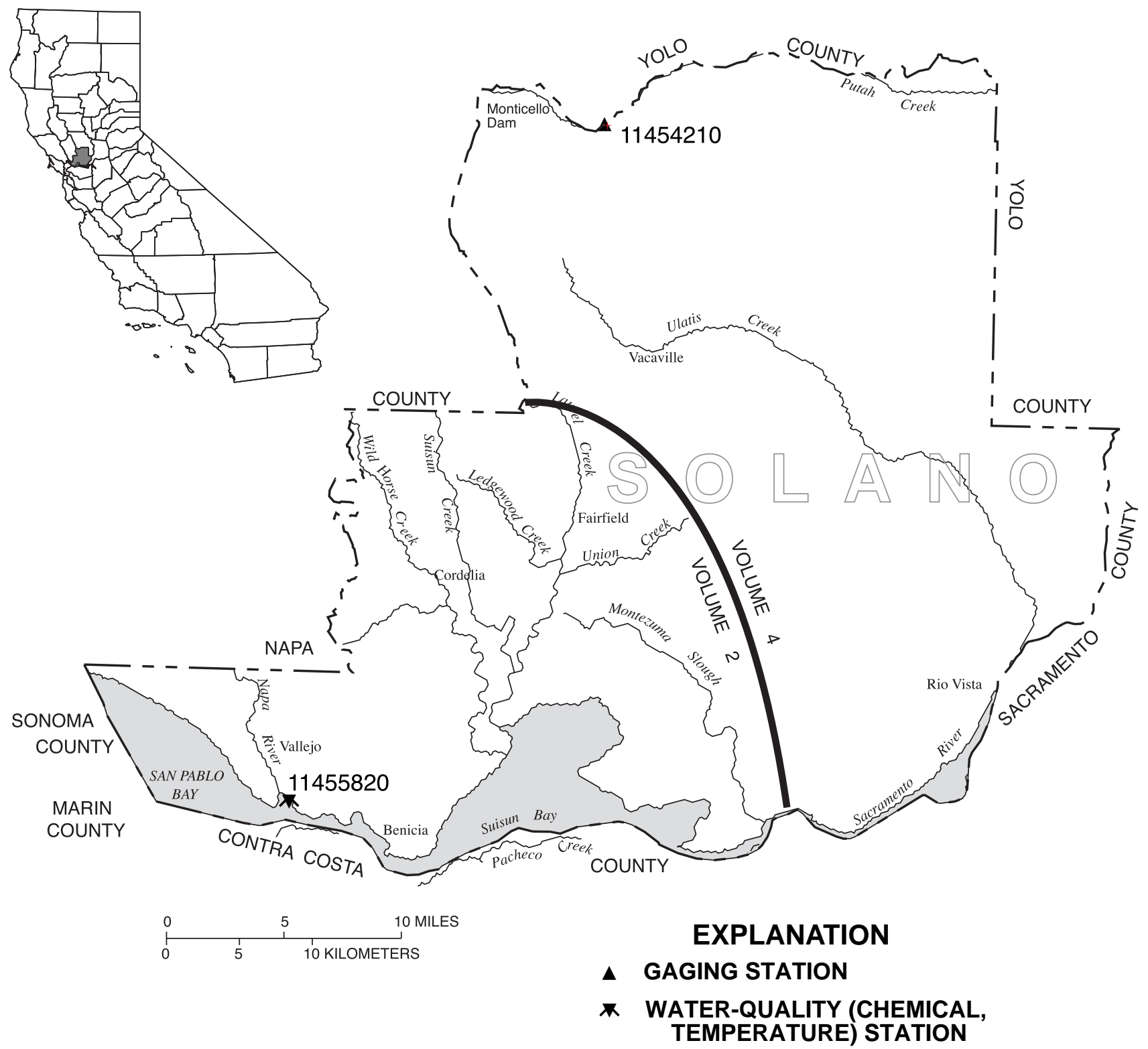

Figure 19. Location of discharge and water-quality stations in Solano County. (NOTE: Records for station 11455820 published in volume 2.) 


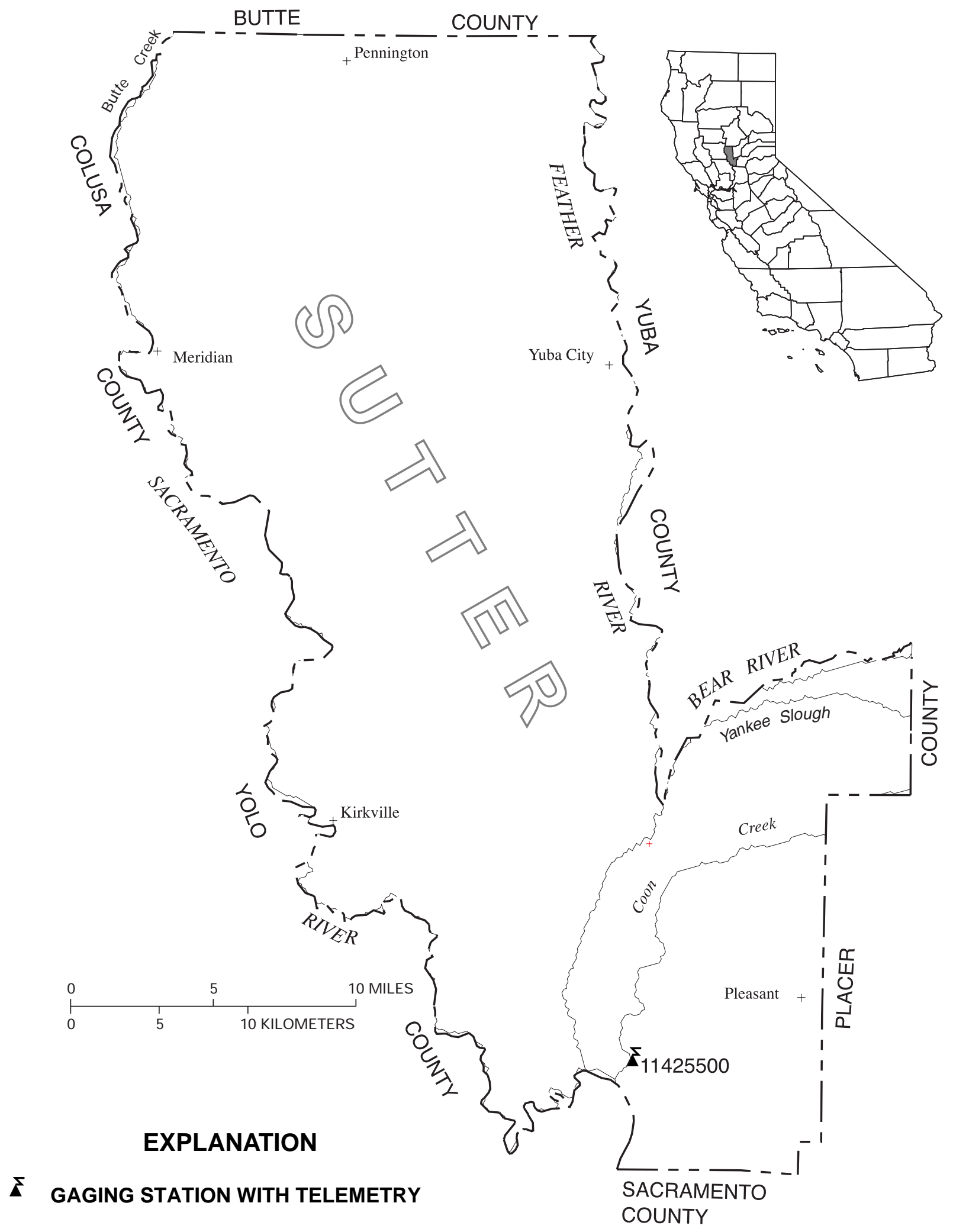

Figure 20. Location of discharge station in Sutter County. 


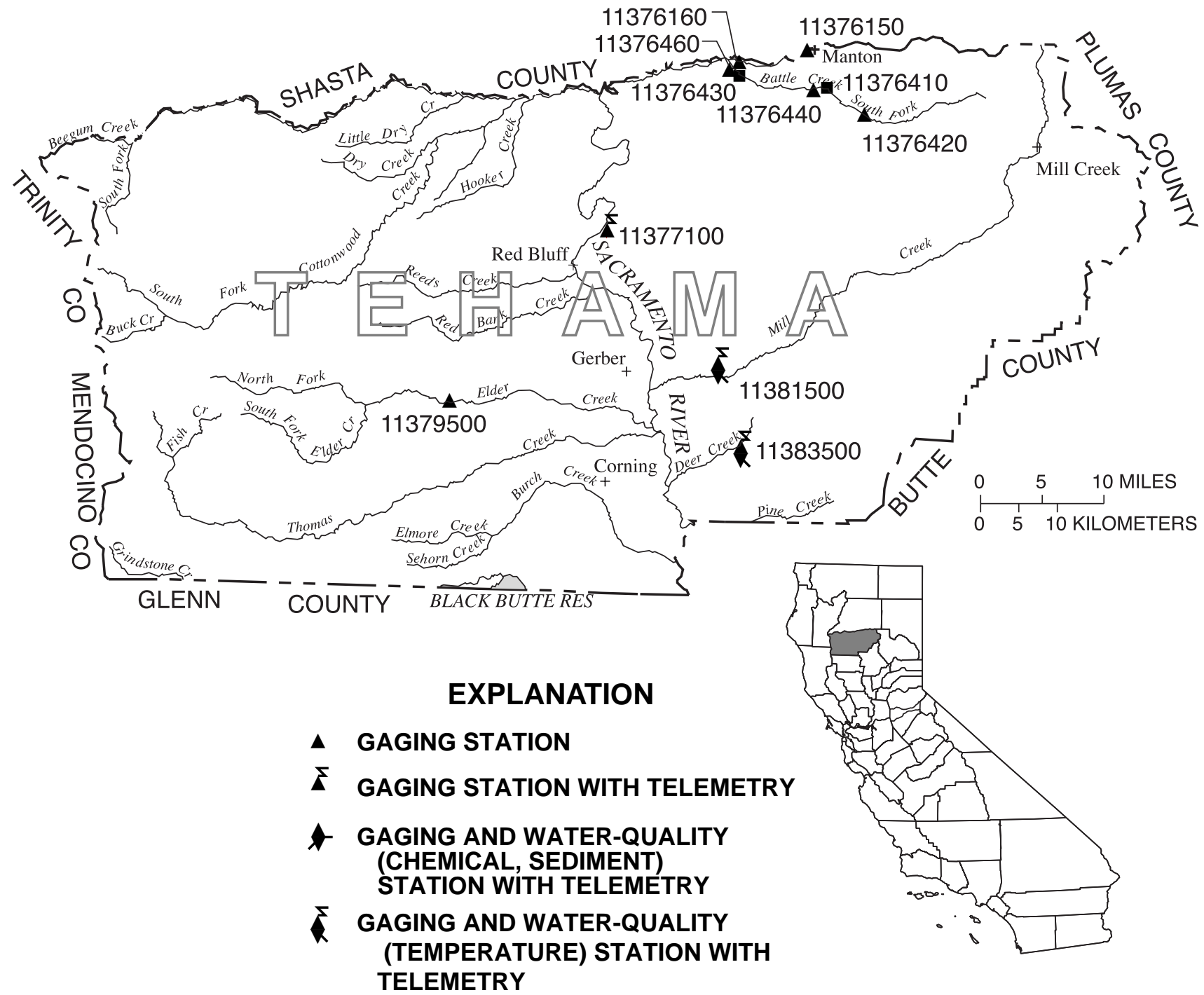

- POWERPLANT

Figure 21. Location of discharge and water-quality stations in Tehama County. 


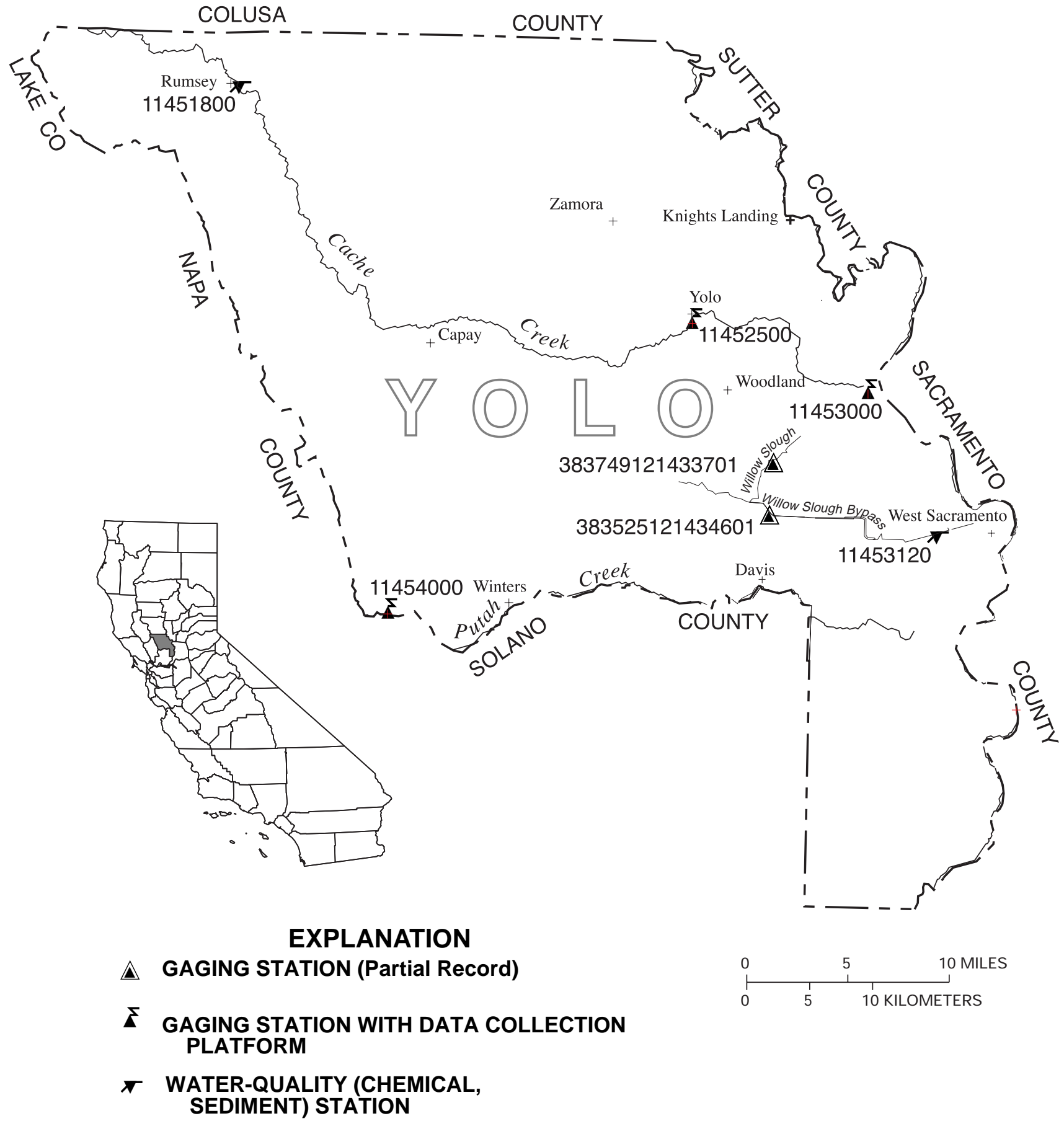

Figure 22. Location of discharge and water-quality stations in Yolo County. 


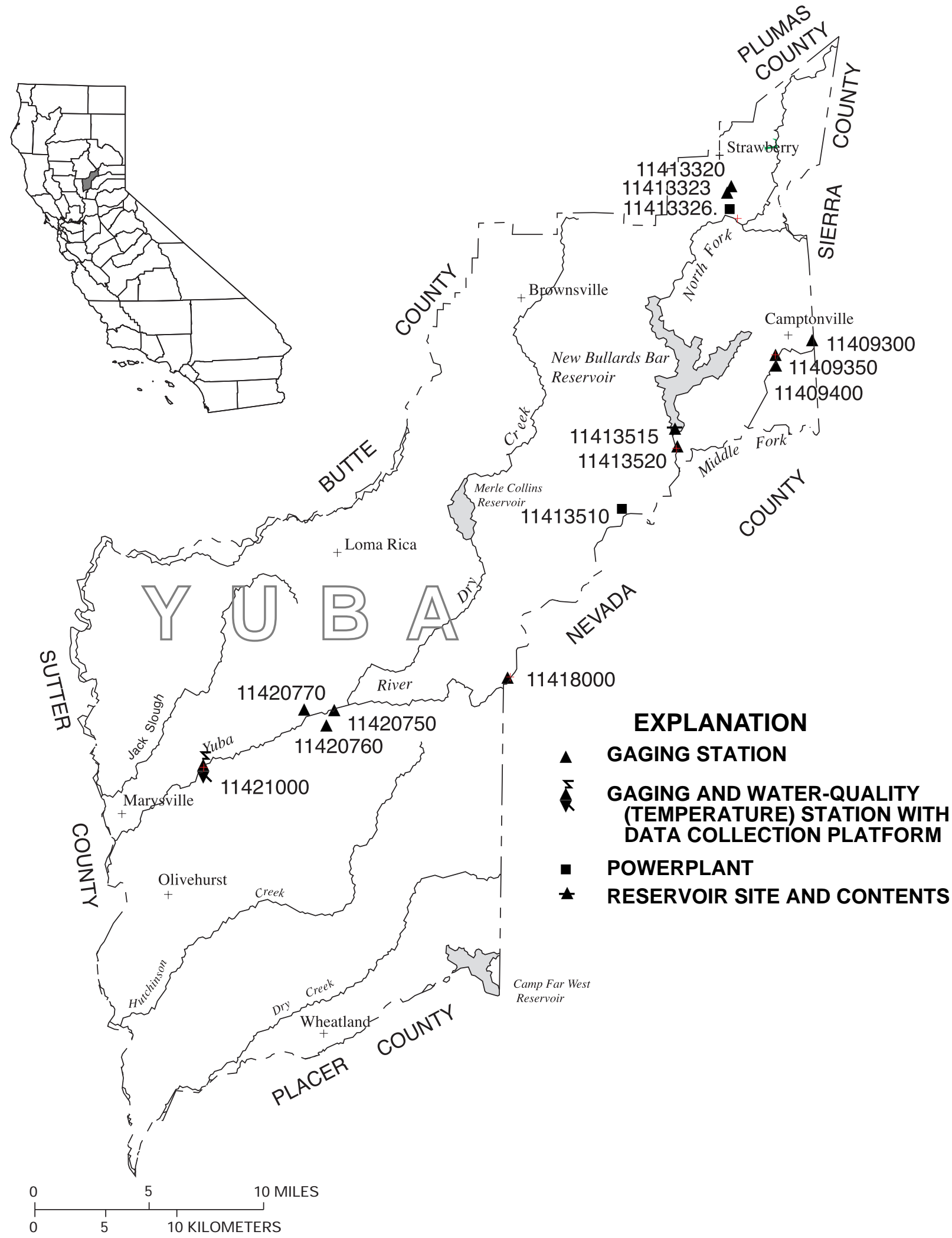

Figure 23. Location of discharge and water-quality stations in Yuba County. 


\section{SURFACE-WATER-DISCHARGE AND SURFACE-WATER-QUALITY RECORDS}

\section{Remark Codes}

The following remark codes may appear with the water-quality data in this report:

\begin{tabular}{ll} 
PRINTED OUTPUT & Estimated value. \\
\hline & Actual value is known to be greater than the value shown. \\
\hline & Actual value is known to be less than the value shown. \\
$\mathrm{K}$ & Results based on colony count outside the acceptable range (non-ideal colony count). \\
$\mathrm{L}$ & Biological organism count less than 0.5 percent (organism may be observed rather \\
than counted). & Biological organism count equal to or greater than 15 percent (dominant). \\
$\mathrm{D}$ & Not detected. \\
$\mathrm{ND}$ & Biological organism estimated as dominant. \\
$*$ & Instantaneous streamflow at the time of cross-sectional measurements. \\
$* *$ & Partial sampled width. \\
$\mathrm{l}$ & Laboratory value. \\
2 & Laboratory fixed-end point titration. \\
$\mathrm{A}$ & Samples collected by another agency. \\
$\mathrm{N}$ & Suspended-sediment concentration value determined from a sample collected and \\
$\mathrm{V}$ & processed according to National Water-Quality Assessment (NAWQA) protocol.
\end{tabular}

\section{Dissolved Trace-Element Concentrations}

NOTE: Traditionally, dissolved trace-element concentrations have been reported at the microgram per liter $(\mu \mathrm{g} / \mathrm{L})$ level. Recent evidence, mostly from large rivers, indicates that actual dissolved-phase concentrations for a number of trace elements are within the range of 10's to 100's of nanograms per liter (ng/L). Data above the $\mu \mathrm{g} / \mathrm{L}$ level should be viewed with caution. Such data may actually represent elevated environmental concentrations from natural or human causes; however, these data could reflect contamination introduced during sampling, processing, or analysis. To confidently produce dissolved trace-element data with insignificant contamination, the U.S. Geological Survey began using new trace-element protocols at some stations in water year 1994.

\section{Change in National Trends Network Procedures}

NOTE: Sample handling procedures at all National Trends Network stations were changed substantially on January 11, 1994, in order to reduce contamination from the sample shipping container. The data for samples before and after that date are different and not directly comparable. A tabular summary of the differences based on a special intercomparison study, is available from the NADP/NTN Coordination Office, Colorado State University, Fort Collins, CO 80523 (Telephone: 303-491-5643). 



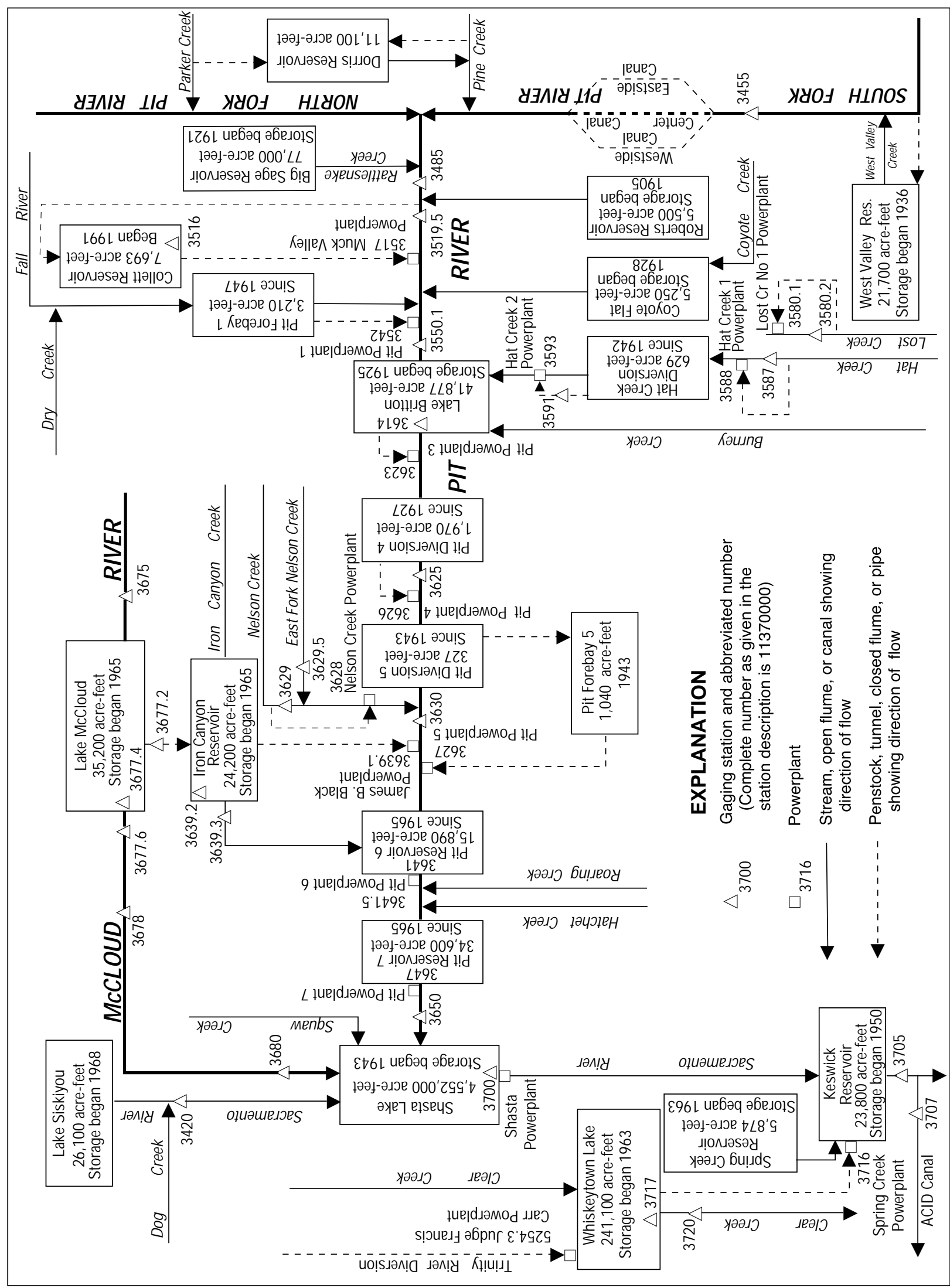


LOCATION.-Lat 4056'23", long 122²4'58", in SW 1/4 NW 1/4 sec.35, T.36 N., R.5 W, Shasta County, Hydrologic Unit 18020005, U.S. Bureau of Reclamation property, on left bank, 0.2 mi downstream from Dog Creek, 0.6 mi southeast of Delta, 2.8 mi south of Lamoine, and 29 mi downstream from Lake Siskiyou.

DRAINAGE AREA.- $425 \mathrm{mi}^{2}$.

PERIOD OF RECORD.-October 1944 to current year. Monthly discharge only for some periods, published in WSP 1315-A.

CHEMICAL DATA: Water years 1951-81.

WATER TEMPERATURE: Water years 1951, 1954-57, 1963-79.

REVISED RECORDS.-WSP 1395: 1951(M). WDR-CA-94-4: 1993(P).

GAGE.-Water-stage recorder. Datum of gage is $1,075.00 \mathrm{ft}$ above sea level (levels by U.S. Bureau of Reclamation).

REMARKS.- - Records excellent. Some regulation by Lake Siskiyou, capacity, 26,100 acre-ft, since December 1968. Some minor diversions for irrigation upstream from station. See schematic diagram of Pit and McCloud River Basins.

EXTREMES FOR PERIOD OF RECORD.-Maximum discharge, 69,800 ft 3 s, Jan. 16, 1974, gage height, $27.20 \mathrm{ft}$ in gage well, $28.7 \mathrm{ft}$ from floodmarks, from rating curve extended above $19,000 \mathrm{ft}^{3} / \mathrm{s}$ on basis of slope-area measurements at gage height $19.50 \mathrm{ft}$, and of peak flow; minimum daily, $117 \mathrm{ft}^{3} / \mathrm{s}$, Aug. 5, 6, 12-15, 1977.

EXTREMES FOR CURRENT YEAR.-Peak discharges greater than base discharge of $8,000 \mathrm{ft}^{3} / \mathrm{s}$, or maximum:

$\begin{array}{lccccccc}\text { Date } & \text { Time } & \begin{array}{c}\text { Discharge } \\ \left(\mathrm{ft}^{3} / \mathrm{s}\right)\end{array} & \begin{array}{c}\text { Gage height } \\ (\mathrm{ft})\end{array} & \text { Date } & \text { Time } & \begin{array}{c}\text { Discharge } \\ \left(\mathrm{ft}^{3} / \mathrm{s}\right)\end{array} & \begin{array}{c}\text { Gage height } \\ (\mathrm{ft})\end{array} \\ \text { Nov. 23 } & 1330 & 8,310 & 10.13 & \text { Nov. 30 } & 1230 & 13,000 & 11.59\end{array}$

DISCHARGE, CUBIC FEET PER SECOND, WATER YEAR OCTOBER 1998 TO SEPTEMBER 1999 DAILY MEAN VALUES

\begin{tabular}{|c|c|c|c|c|c|c|c|c|c|c|c|c|}
\hline DAY & $\mathrm{OCT}$ & NOV & $\mathrm{DEC}$ & JAN & FEB & MAR & APR & MAY & JUN & JUL & AUG & SEP \\
\hline 1 & 318 & 343 & 5130 & 555 & 905 & 4920 & 1850 & 2310 & 1670 & 475 & 292 & 247 \\
\hline 2 & 317 & 347 & 3450 & 539 & 857 & 3760 & 1700 & 2540 & 1550 & 449 & 291 & 245 \\
\hline 3 & 317 & 351 & 4320 & 528 & 823 & 4010 & 1580 & 2270 & 1310 & 435 & 288 & 242 \\
\hline 4 & 314 & 357 & 2880 & 517 & 801 & 3130 & 1540 & 2040 & 1170 & 431 & 286 & 238 \\
\hline 5 & 311 & 362 & 2240 & 505 & 765 & 2550 & 1500 & 1930 & 1160 & 433 & 285 & 237 \\
\hline 6 & 308 & 365 & 1870 & 499 & 1640 & 2160 & 1450 & 2070 & 1150 & 430 & 286 & 235 \\
\hline 7 & 304 & 629 & 1380 & 495 & 5140 & 1930 & 1440 & 2270 & 1060 & 414 & 297 & 233 \\
\hline 8 & 303 & 538 & 1130 & 494 & 3120 & 1920 & 2730 & 2110 & 996 & 402 & 301 & 231 \\
\hline 9 & 303 & 456 & 1050 & 486 & 3090 & 1780 & 2200 & 1930 & 946 & 393 & 288 & 230 \\
\hline 10 & 303 & 481 & 955 & 477 & 2390 & 1660 & 2050 & 1780 & 915 & 383 & 285 & 231 \\
\hline 11 & 303 & 522 & 895 & 473 & 1920 & 1630 & 1980 & 1740 & 893 & 379 & 292 & 229 \\
\hline 12 & 303 & 483 & 856 & 470 & 1620 & 1560 & 2040 & 1850 & 900 & 363 & 287 & 225 \\
\hline 13 & 303 & 453 & 934 & 468 & 1440 & 1520 & 2170 & 1870 & 904 & 356 & 280 & 225 \\
\hline 14 & 303 & 426 & 953 & 461 & 1350 & 1600 & 2270 & 1740 & 906 & 355 & 278 & 224 \\
\hline 15 & 301 & 423 & 863 & 477 & 1240 & 1620 & 2360 & 1610 & 887 & 345 & 276 & 222 \\
\hline 16 & 298 & 438 & 822 & 705 & 1620 & 1610 & 2500 & 1550 & 834 & 336 & 267 & 222 \\
\hline 17 & 297 & 620 & 829 & 1210 & 2140 & 1640 & 2850 & 1590 & 793 & 332 & 240 & 223 \\
\hline 18 & 295 & 524 & 836 & 2910 & 2530 & 1710 & 3340 & 1840 & 763 & 332 & 235 & 223 \\
\hline 19 & 292 & 438 & 794 & 2300 & 2630 & 1730 & 3670 & 1970 & 723 & 331 & 245 & 222 \\
\hline 20 & 291 & 394 & 758 & 2470 & 2150 & 1780 & 3500 & 2110 & 693 & 325 & 260 & 222 \\
\hline 21 & 289 & 1400 & 678 & 2300 & 2060 & 1770 & 3130 & 2150 & 665 & 322 & 255 & 222 \\
\hline 22 & 286 & 1370 & 665 & 2990 & 2130 & 1700 & 2770 & 2190 & 637 & 318 & 254 & 221 \\
\hline 23 & 286 & 4190 & 645 & 3900 & 2780 & 1880 & 2560 & 2360 & 628 & 315 & 254 & 221 \\
\hline 24 & 317 & 2280 & 637 & 2460 & 3110 & 3530 & 2650 & 2540 & 605 & 312 & 255 & 221 \\
\hline 25 & 400 & 1780 & 609 & 1890 & 5490 & 5820 & 2790 & 2640 & 577 & 310 & 251 & 220 \\
\hline 26 & 371 & 2090 & 598 & 1610 & 3550 & 4150 & 2920 & 2490 & 554 & 307 & 248 & 216 \\
\hline 27 & 331 & 1860 & 592 & 1340 & 2840 & 3200 & 2730 & 2420 & 536 & 304 & 249 & 216 \\
\hline 28 & 328 & 1190 & 582 & 1190 & 5380 & 2680 & 2340 & 2420 & 510 & 287 & 252 & 217 \\
\hline 29 & 330 & 1220 & 573 & 1090 & --- & 2360 & 2100 & 2280 & 494 & 280 & 249 & 217 \\
\hline 30 & 331 & 7780 & 566 & 1020 & --- & 2190 & 2100 & 1960 & 479 & 291 & 246 & 218 \\
\hline 31 & 333 & --- & 564 & 990 & --- & 2010 & --- & 1730 & --- & 293 & 247 & --- \\
\hline TOTAL & 9686 & 34110 & 39654 & 37819 & 65511 & 75510 & 70810 & 64300 & 25908 & 11038 & 8319 & 6795 \\
\hline MEAN & 312 & 1137 & 1279 & 1220 & 2340 & 2436 & 2360 & 2074 & 864 & 356 & 268 & 226 \\
\hline MAX & 400 & 7780 & 5130 & 3900 & 5490 & 5820 & 3670 & 2640 & 1670 & 475 & 301 & 247 \\
\hline MIN & 286 & 343 & 564 & 461 & 765 & 1520 & 1440 & 1550 & 479 & 280 & 235 & 216 \\
\hline $\mathrm{AC}-\mathrm{FT}$ & 19210 & 67660 & 78650 & 75010 & 129900 & 149800 & 140500 & 127500 & 51390 & 21890 & 16500 & 13480 \\
\hline
\end{tabular}


11342000 SACRAMENTO RIVER AT DELTA, CA—Continued

STATISTICS OF MONTHLY MEAN DATA FOR WATER YEARS 1945 - 1999, BY WATER YEAR (WY)

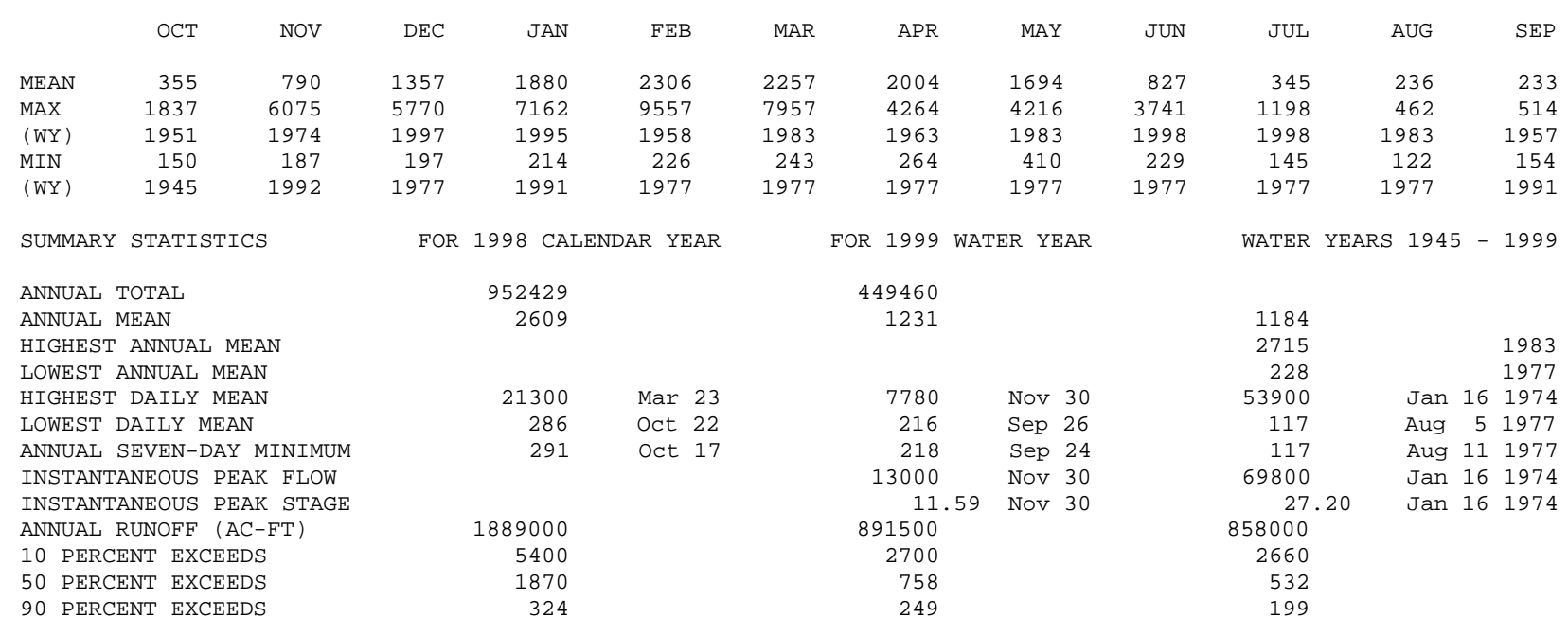




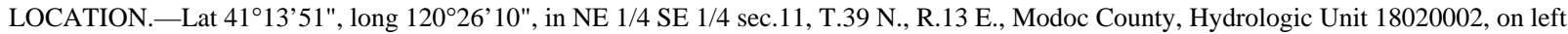
bank, $250 \mathrm{ft}$ downstream from highway bridge, $1.4 \mathrm{mi}$ downstream from West Valley Creek, and $3.5 \mathrm{mi}$ east of Likely.

DRAINAGE AREA.-247 $\mathrm{mi}^{2}$.

PERIOD OF RECORD.-October 1928 to current year. Monthly discharge only for some periods, published in WSP 1315-A.

CHEMICAL DATA: Water years 1951-79.

WATER TEMPERATURE: Water years 1965-79.

SEDIMENT DATA: Water years 1957-61, 1967-70.

REVISED RECORDS.-WSP 1931: Drainage area, 1932(M), 1938(M), 1952(M). WDR CA-88-4: 1983(M).

GAGE.-Water-stage recorder. Datum of gage is 4,507.74 ft above sea level. Prior to Oct. 1, 1931, at site 1,000 ft downstream at different datum.

REMARKS.-Records fair. Considerable regulation by West Valley Reservoir on West Valley Creek beginning in May 1937, usable capacity,

21,700 acre-ft. Diversions for irrigation of about 3,800 acres upstream from station. See schematic diagram of Pit and McCloud River Basins.

EXTREMES FOR PERIOD OF RECORD.-Maximum discharge, 1,620 ft ${ }^{3} / \mathrm{s}$, June 2, 1971, gage height, $6.05 \mathrm{ft}$; minimum, $0.2 \mathrm{ft}^{3} / \mathrm{s}$, Feb. $3,1941$.

DISCHARGE, CUBIC FEET PER SECOND, WATER YEAR OCTOBER 1998 TO SEPTEMBER 1999

DAILY MEAN VALUES

\begin{tabular}{|c|c|c|c|c|c|c|c|c|c|c|c|c|}
\hline DAY & OCT & NOV & $\mathrm{DEC}$ & JAN & FEB & MAR & APR & MAY & JUN & JUL & AUG & SEP \\
\hline 1 & 48 & 41 & 45 & e23 & e21 & 93 & 28 & 235 & 320 & 164 & 163 & 103 \\
\hline 2 & 47 & 42 & 39 & e21 & e27 & 66 & 30 & 261 & 315 & 151 & 171 & 110 \\
\hline 3 & 47 & 38 & 35 & e20 & e21 & 62 & 33 & 278 & 331 & 148 & 180 & 126 \\
\hline 4 & 46 & 25 & 35 & e19 & e19 & 53 & 26 & 265 & 361 & 145 & 191 & 147 \\
\hline 5 & 46 & 18 & e35 & e18 & e22 & 47 & 36 & 247 & 365 & 141 & 216 & 145 \\
\hline 6 & 45 & 17 & e30 & e17 & e19 & 45 & 52 & 240 & 351 & 140 & 220 & 145 \\
\hline 7 & 44 & 20 & e25 & e17 & e28 & 45 & 66 & 250 & 322 & 133 & 211 & 141 \\
\hline 8 & 44 & 25 & e30 & e17 & e 46 & 43 & 81 & 259 & 298 & 127 & 201 & 121 \\
\hline 9 & 43 & 25 & e35 & e17 & e57 & 40 & 92 & 260 & 270 & 125 & 186 & 99 \\
\hline 10 & 43 & 25 & e39 & e17 & e51 & 40 & 109 & 260 & 251 & 115 & 157 & 85 \\
\hline 11 & 42 & 26 & e 40 & e17 & e51 & 40 & 117 & 254 & 238 & 107 & 128 & 76 \\
\hline 12 & 42 & 25 & e 41 & e17 & e 42 & 42 & 118 & 256 & 224 & 112 & 95 & 74 \\
\hline 13 & 42 & 25 & 42 & e17 & e36 & 51 & 119 & 265 & 212 & 136 & 83 & 72 \\
\hline 14 & 41 & 26 & 45 & e17 & e34 & 48 & 124 & 262 & 205 & 155 & 75 & 64 \\
\hline 15 & 42 & 26 & 35 & e22 & e34 & 44 & 127 & 260 & 195 & 157 & 75 & 57 \\
\hline 16 & 42 & 28 & 31 & e29 & e34 & 37 & 129 & 255 & 190 & 175 & 72 & 59 \\
\hline 17 & 41 & 28 & 29 & e25 & e35 & 26 & 138 & 257 & 182 & 209 & 71 & 56 \\
\hline 18 & 41 & 29 & 26 & e35 & e35 & 27 & 158 & 270 & 178 & 209 & 87 & 57 \\
\hline 19 & 41 & 25 & e22 & e31 & e36 & 30 & 171 & 287 & 183 & 208 & 104 & 58 \\
\hline 20 & 40 & 25 & e18 & e31 & e36 & 32 & 193 & 300 & 212 & 196 & 119 & 56 \\
\hline 21 & 39 & 25 & e15 & e30 & e37 & 30 & 216 & 310 & 204 & 183 & 143 & 63 \\
\hline 22 & 39 & 33 & e14 & e27 & e37 & 27 & 207 & 321 & 194 & 177 & 145 & 81 \\
\hline 23 & 38 & 34 & e14 & e26 & e38 & 25 & 195 & 336 & 185 & 172 & 146 & 82 \\
\hline 24 & 38 & 35 & e15 & e26 & e 40 & 24 & 200 & 356 & 176 & 171 & 144 & 82 \\
\hline 25 & 42 & 33 & e17 & e35 & e 42 & 25 & 215 & 380 & 170 & 170 & 143 & 85 \\
\hline 26 & 42 & 29 & e19 & e36 & e 43 & 31 & 231 & 395 & 163 & 162 & 146 & 86 \\
\hline 27 & 42 & 35 & e24 & e31 & e 45 & 31 & 247 & 396 & 156 & 144 & 158 & 85 \\
\hline 28 & 43 & 46 & e30 & e28 & 66 & 28 & 242 & 392 & 145 & 141 & 154 & 86 \\
\hline 29 & 44 & 43 & e28 & e26 & --- & 27 & 225 & 379 & 147 & 148 & 149 & 81 \\
\hline 30 & 42 & 40 & e26 & e23 & --- & 27 & 221 & 359 & 173 & 164 & 132 & 73 \\
\hline 31 & 40 & --- & e24 & e24 & --- & 27 & --- & 336 & --- & 165 & 110 & --- \\
\hline TOTAL & 1316 & 892 & 903 & 739 & 1032 & 1213 & 4146 & 9181 & 6916 & 4850 & 4375 & 2655 \\
\hline MEAN & 42.5 & 29.7 & 29.1 & 23.8 & 36.9 & 39.1 & 138 & 296 & 231 & 156 & 141 & 88.5 \\
\hline MAX & 48 & 46 & 45 & 36 & 66 & 93 & 247 & 396 & 365 & 209 & 220 & 147 \\
\hline MIN & 38 & 17 & 14 & 17 & 19 & 24 & 26 & 235 & 145 & 107 & 71 & 56 \\
\hline $\mathrm{AC}-\mathrm{ET}$ & 2610 & 1770 & 1790 & 1470 & 2050 & 2410 & 8220 & 18210 & 13720 & 9620 & 8680 & 5270 \\
\hline
\end{tabular}

e Estimated. 
11345500 SOUTH FORK PIT RIVER NEAR LIKELY, CA—Continued

STATISTICS OF MONTHLY MEAN DATA FOR WATER YEARS 1929 - 1999, BY WATER YEAR (WY)

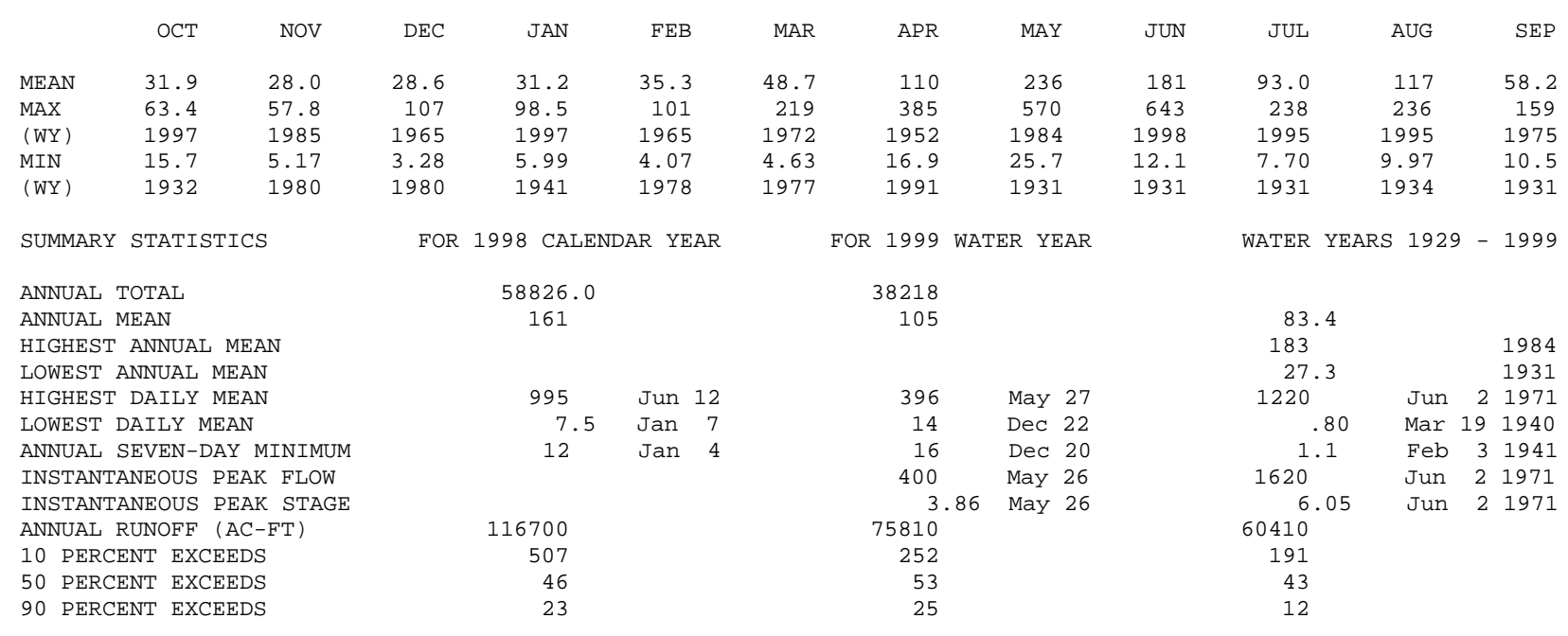




\section{PIT RIVER NEAR CANBY, CA}

LOCATION.-Lat 41 24'22", long 12055'36", in NW 1/4 SW 1/4 sec.10, T.41 N., R.9 E., Modoc County, Hydrologic Unit 18020002, on right bank, at lower end of Warm Spring Valley, and 3.9 mi southwest of Canby.

DRAINAGE AREA.-1,431 $\mathrm{mi}^{2}$, excluding Goose Lake Basin.

PERIOD OF RECORD._-January 1904 to December 1905, May 1929 to current year (1929-31 incomplete).

CHEMICAL DATA: Water years 1951-79.

WATER TEMPERATURE: Water years 1965-79.

SEDIMENT DATA: Water years 1957-61, 1967-70.

REVISED RECORDS.-WSP 1445: 1904, 1935(M), 1936, 1937(M). WSP 1931: Drainage area.

GAGE.-Water-stage recorder. Datum of gage is 4,266.0 ft above sea level. January 1904 to December 1905, nonrecording gage and May 6, 1929, to Sept. 30, 1931, water-stage recorder, at site $100 \mathrm{ft}$ upstream at different datum.

REMARKS.-Records good. Low flow regulated by many small reservoirs, total capacity about 144,000 acre-ft. Diversions for irrigation of about 39,000 acres upstream from station. See schematic diagram of Pit and McCloud River Basins.

EXTREMES FOR PERIOD OF RECORD.-Maximum discharge observed, 13,000 ft 3 /s, Mar. 8, 1904, gage height, $15.0 \mathrm{ft}$, site and datum then in use; minimum daily, $0.1 \mathrm{ft}^{3} / \mathrm{s}$, several days in April 1934 and August 1935.

EXTREMES FOR CURRENT YEAR.-Peak discharges greater than base discharge of $800 \mathrm{ft}^{3} / \mathrm{s}$, or maximum:

$\begin{array}{lccclcrc}\text { Date } & \text { Time } & \begin{array}{c}\text { Discharge } \\ \left(\mathrm{ft}^{3} / \mathrm{s}\right)\end{array} & \begin{array}{c}\text { Gage height } \\ (\mathrm{ft})\end{array} & \text { Date } & \text { Time } & \begin{array}{c}\text { Discharge } \\ \left(\mathrm{ft}^{3} / \mathrm{s}\right)\end{array} & \begin{array}{c}\text { Gage height } \\ (\mathrm{ft})\end{array} \\ \text { Dec. } 1 & 1915 & 1,320 & 5.43 & \text { Feb. 10 } & 1900 & 2,320 & 6.85 \\ \text { Dec. 14 } & 2015 & 1,140 & 5.14 & \text { Mar. 3 } & 0200 & 3,100 & 7.82 \\ \text { Jan. 24 } & 1500 & 836 & 4.59 & \text { Apr. 21 } & 2345 & 921 & 4.65\end{array}$

DISCHARGE, CUBIC FEET PER SECOND, WATER YEAR OCTOBER 1998 TO SEPTEMBER 1999 DAILY MEAN VALUES

\begin{tabular}{|c|c|c|c|c|c|c|c|c|c|c|c|c|}
\hline DAY & $\mathrm{OCT}$ & NOV & $\mathrm{DEC}$ & JAN & FEB & MAR & APR & MAY & JUN & JUL & AUG & SEP \\
\hline 1 & 386 & 246 & 1220 & 295 & 249 & 2180 & 591 & 568 & 461 & 59 & 51 & 86 \\
\hline 2 & 330 & 199 & 1070 & 234 & 223 & 3000 & 559 & 640 & 432 & 91 & 45 & 121 \\
\hline 3 & 247 & 198 & 708 & 157 & 225 & 3000 & 539 & 769 & 491 & 76 & 40 & 103 \\
\hline 4 & 237 & 193 & 600 & 143 & 253 & 2650 & 525 & 835 & 621 & 49 & 33 & 98 \\
\hline 5 & 241 & 178 & 528 & 165 & 265 & 1960 & 491 & 850 & 752 & 40 & 34 & 101 \\
\hline 6 & 243 & 152 & 413 & 149 & 275 & 1350 & 505 & 775 & 788 & 38 & 39 & 98 \\
\hline 7 & 251 & 154 & 286 & 145 & 751 & 1060 & 512 & 715 & 738 & 39 & 44 & 99 \\
\hline 8 & 239 & 175 & e275 & 135 & 1270 & 905 & 512 & 664 & 588 & 43 & 49 & 133 \\
\hline 9 & 238 & 193 & e280 & 120 & 1640 & 795 & 449 & 660 & 452 & 56 & 64 & 138 \\
\hline 10 & 245 & 195 & e 300 & 128 & 2010 & 742 & 446 & 665 & 301 & 36 & 74 & 160 \\
\hline 11 & 238 & 240 & e320 & 138 & 2160 & 701 & 450 & 620 & 320 & 35 & 92 & 210 \\
\hline 12 & 237 & 282 & e360 & 139 & 1540 & 659 & 461 & 603 & 272 & 32 & 143 & 201 \\
\hline 13 & 241 & 251 & 452 & 142 & 875 & 626 & 508 & 599 & 249 & 28 & 247 & 202 \\
\hline 14 & 245 & 246 & 961 & 148 & 669 & 661 & 457 & 584 & 258 & 29 & 225 & 194 \\
\hline 15 & 239 & 227 & 895 & 156 & 606 & 686 & 440 & 602 & 235 & 27 & 179 & 182 \\
\hline 16 & 238 & 222 & 598 & 190 & 570 & 647 & 480 & 488 & 166 & 26 & 138 & 175 \\
\hline 17 & 239 & 226 & 470 & 239 & 615 & 678 & 521 & 509 & 126 & 27 & 119 & 159 \\
\hline 18 & 234 & 246 & 411 & 417 & 928 & 688 & 598 & 399 & 107 & 28 & 114 & 144 \\
\hline 19 & 247 & 235 & 340 & 700 & 1180 & 688 & 677 & 301 & 132 & 37 & 95 & 140 \\
\hline 20 & 241 & 215 & 240 & 575 & 1000 & 687 & 776 & 232 & 147 & 32 & 69 & 139 \\
\hline 21 & 226 & 210 & 161 & 544 & 763 & 700 & 853 & 308 & 147 & 25 & 56 & 147 \\
\hline 22 & 225 & 295 & e150 & 497 & 630 & 668 & 889 & 364 & 100 & 15 & 41 & 129 \\
\hline 23 & 229 & 664 & e160 & 664 & 617 & 630 & 766 & 407 & 84 & 25 & 40 & 159 \\
\hline 24 & 247 & 843 & e180 & 798 & 781 & 601 & 661 & 425 & 62 & 57 & 43 & 155 \\
\hline 25 & 254 & 687 & e220 & 682 & 1290 & 593 & 656 & 461 & 60 & 59 & 41 & 159 \\
\hline 26 & 241 & 532 & 247 & 529 & 1410 & 606 & 701 & 501 & 69 & 74 & 44 & 148 \\
\hline 27 & 242 & 666 & 270 & 432 & 1100 & 636 & 734 & 547 & 54 & 81 & 47 & 156 \\
\hline 28 & 248 & 957 & 294 & 341 & 1150 & 614 & 661 & 585 & 26 & 85 & 47 & 158 \\
\hline 29 & 267 & 754 & 331 & 291 & --- & 565 & 656 & 499 & 16 & 76 & 43 & 156 \\
\hline 30 & 283 & 788 & 345 & 295 & --- & 567 & 607 & 490 & 18 & 65 & 44 & 122 \\
\hline 31 & 276 & --- & 307 & 285 & --- & 603 & --- & 508 & --- & 61 & 53 & -- \\
\hline TOTAL & 7794 & 10669 & 13392 & 9873 & 25045 & 31146 & 17681 & 17173 & 8272 & 1451 & 2393 & 4372 \\
\hline MEAN & 251 & 356 & 432 & 318 & 894 & 1005 & 589 & 554 & 276 & 46.8 & 77.2 & 146 \\
\hline MAX & 386 & 957 & 1220 & 798 & 2160 & 3000 & 889 & 850 & 788 & 91 & 247 & 210 \\
\hline MIN & 225 & 152 & 150 & 120 & 223 & 565 & 440 & 232 & 16 & 15 & 33 & 86 \\
\hline $\mathrm{AC}-\mathrm{FT}$ & 15460 & 21160 & 26560 & 19580 & 49680 & 61780 & 35070 & 34060 & 16410 & 2880 & 4750 & 8670 \\
\hline
\end{tabular}


11348500 PIT RIVER NEAR CANBY, CA—Continued

STATISTICS OF MONTHLY MEAN DATA FOR WATER YEARS 1904 - 1999, BY WATER YEAR (WY)

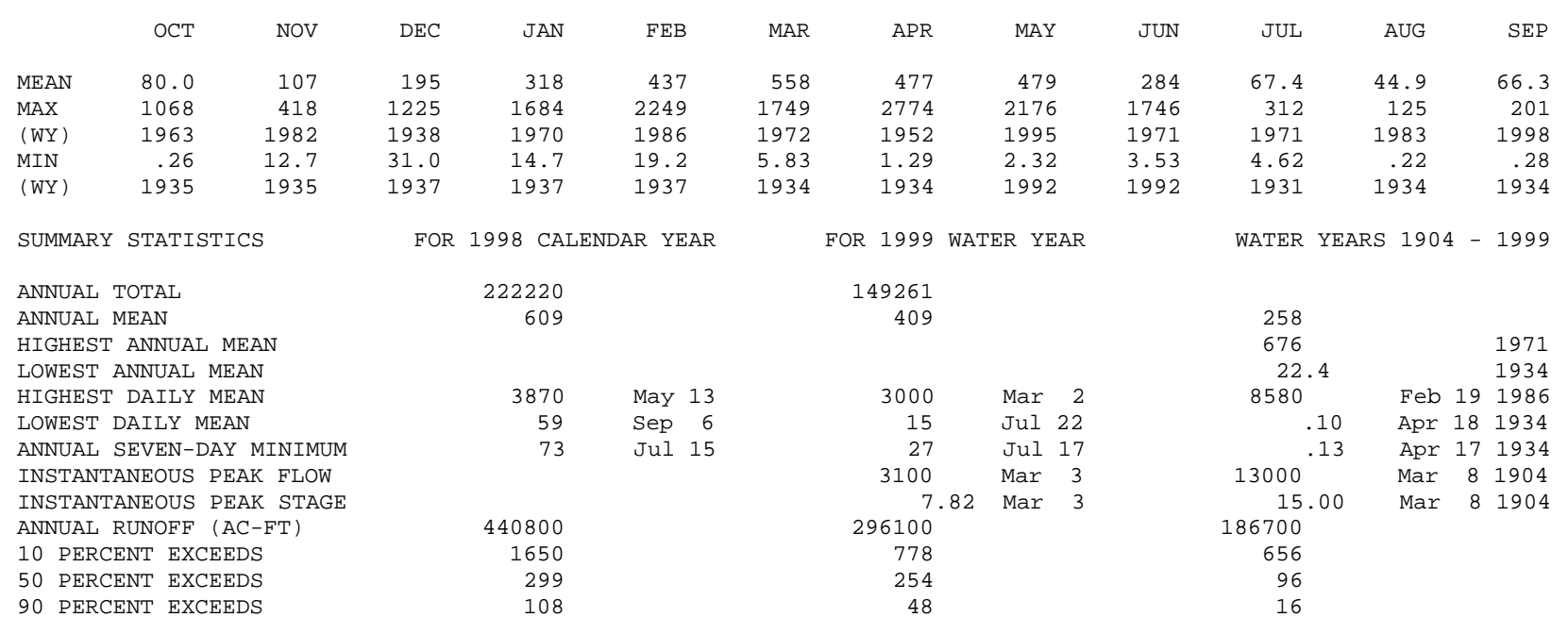


LOCATION.—Lat 40 $58^{\prime} 00^{\prime \prime}$, long $121^{\circ} 13^{\prime} 00^{\prime \prime}$, unsurveyed, Lassen County, Hydrologic Unit 18020003, on right bank, 1.9 mi east of Muck Valley Powerplant, 5.5 mi northwest of Little Valley, and 9.1 mi southwest of Nubieber.

PERIOD OF RECORD.-October 1991 to September 1992; October 1993 to current year.

GAGE.-Water-stage recorder. Datum of gage is sea level.

REMARKS.--Lake is formed by earth and rockfill dam. Storage began Dec. 31, 1990. Water is diverted from the Pit River through a tunnel to the reservoir. Operating pool from elevation 4,030 ft, capacity 155 acre-ft, to 4,065 ft, capacity 7,693 acre-ft. Crest of spillway is at elevation $4,065 \mathrm{ft}$. Reservoir is used for power generation. Figures given represent total contents. Data not published below the minimum operating level at elevation 4,030 ft, capacity 155 acre-ft. See schematic diagram of Pit and McCloud River Basins.

COOPERATION.-Records were provided by Malacha Hydro Limited Partnership, under general supervision of the U.S. Geological Survey, in connection with a Federal Energy Regulatory Commission project.

Capacity table (elevation, in feet, and contents, in acre-feet)

(Based on table provided by Malacha Hydro Limited Partnership, dated November 1991)

$\begin{array}{llll}4,030 & 155 & 4,040 & 1,899 \\ 4,032 & 395 & 4,050 & 4,052 \\ 4,035 & 931 & 4,065 & 7,693\end{array}$

RESERVOIR STORAGE (ACRE-FEET), WATER YEAR OCTOBER 1998 TO SEPTEMBER 1999 DAILY OBSERVATION AT 2400 HOURS

\begin{tabular}{|c|c|c|c|c|c|c|c|c|c|c|c|c|}
\hline DAY & $\mathrm{OCT}$ & NOV & $\mathrm{DEC}$ & JAN & FEB & MAR & $\mathrm{APR}$ & MAY & JUN & JUL & AUG & SEP \\
\hline 1 & 4035 & 6532 & 656 & 1788 & 5004 & 6186 & 6509 & 5960 & 6889 & 3655 & 399 & 297 \\
\hline 2 & 4433 & 6296 & 656 & 1788 & 4876 & 6300 & 6472 & 6253 & 6785 & 3362 & 389 & 269 \\
\hline 3 & 4984 & 5769 & 675 & 1788 & 4732 & 6383 & 6436 & 6309 & 6668 & 3362 & 383 & 255 \\
\hline 4 & 5457 & 5228 & 678 & 1748 & 4606 & 6347 & 6400 & 6201 & 6629 & 3362 & 347 & 255 \\
\hline 5 & 5712 & 4680 & 683 & 1674 & 4678 & 6314 & 6367 & 6150 & 7414 & 3362 & 297 & 243 \\
\hline 6 & 5877 & 4124 & 683 & 1599 & 5405 & 6282 & 6329 & 6049 & 7368 & 3061 & 271 & 237 \\
\hline 7 & 5972 & 4139 & 814 & 1541 & 5863 & 6269 & 6293 & 5974 & 7225 & 2782 & 271 & 237 \\
\hline 8 & 6011 & 4146 & 814 & 1477 & 5754 & 6238 & 6266 & 6257 & 7090 & 2542 & 271 & 237 \\
\hline 9 & 6058 & 3625 & 738 & 1477 & 5730 & 6213 & 6231 & 6533 & 6962 & 2300 & 257 & 237 \\
\hline 10 & 6463 & 3163 & 720 & 1477 & 5733 & 6180 & 6198 & 6535 & 6836 & 2300 & 257 & 237 \\
\hline 11 & 6878 & 3178 & 680 & 1509 & 5736 & 6147 & 6163 & 6417 & 6685 & 2300 & 257 & 237 \\
\hline 12 & 6651 & 2741 & 907 & 1524 & 5729 & 6110 & 6124 & 6308 & 6974 & 2081 & 257 & 237 \\
\hline 13 & 6403 & 2377 & 914 & 1518 & 5856 & 6075 & 6093 & 6198 & 7184 & 1869 & 257 & 237 \\
\hline 14 & 5995 & 2384 & 918 & 1514 & 6076 & 6043 & 6053 & 6122 & 7054 & 1655 & 257 & 255 \\
\hline 15 & 5648 & 2388 & 1060 & 1542 & 6365 & 6025 & 6018 & 6258 & 6913 & 1446 & 257 & 505 \\
\hline 16 & 5527 & 1954 & 1511 & 2168 & 6271 & 6127 & 5986 & 6384 & 6770 & 1218 & 257 & 726 \\
\hline 17 & 6019 & 1534 & 1787 & 2914 & 6239 & 6241 & 5952 & 6334 & 6488 & 1218 & 257 & 681 \\
\hline 18 & 6522 & 1155 & 1787 & 3113 & 6143 & 6344 & 5918 & 6228 & 6202 & 1218 & 257 & 681 \\
\hline 19 & 6373 & 781 & 1783 & 3057 & 6005 & 6450 & 5758 & 6164 & 6202 & 1099 & 257 & 681 \\
\hline 20 & 6171 & 531 & 1783 & 3079 & 5971 & 6726 & 5601 & 6514 & 6202 & 982 & 289 & 701 \\
\hline 21 & 6042 & 550 & 1783 & 3098 & 5948 & 6923 & 5449 & 6647 & 5967 & 867 & 319 & 660 \\
\hline 22 & 5855 & 556 & 1783 & 3183 & 5827 & 6881 & 5302 & 7167 & 5641 & 745 & 319 & 640 \\
\hline 23 & 5632 & 576 & 1783 & 4028 & 5941 & 6842 & 5208 & 7580 & 5331 & 603 & 335 & 664 \\
\hline 24 & 6066 & 576 & 1783 & 5097 & 6046 & 6805 & 5550 & 7509 & 5012 & 603 & 335 & 682 \\
\hline 25 & 6522 & 576 & 1783 & 5363 & 6166 & 6765 & 5880 & 7476 & 4688 & 603 & 335 & 682 \\
\hline 26 & 6361 & 592 & 1783 & 5386 & 6250 & 6728 & 6282 & 7349 & 4688 & 497 & 335 & 682 \\
\hline 27 & 5985 & 604 & 1783 & 5392 & 6217 & 6689 & 6104 & 7224 & 4688 & 425 & 297 & 688 \\
\hline 28 & 5793 & 604 & 1783 & 5348 & 6191 & 6646 & 5930 & 7101 & 4373 & 425 & 297 & 632 \\
\hline 29 & 5629 & 618 & 1783 & 5199 & --- & 6613 & 5763 & 7058 & 4079 & 399 & 297 & 586 \\
\hline 30 & 5473 & 650 & 1788 & 5199 & --- & 6584 & 5665 & 7028 & 3958 & 399 & 297 & 554 \\
\hline 31 & 5997 & --- & 1788 & 5199 & --- & 6548 & --- & 7002 & --- & 399 & 297 & --- \\
\hline MAX & 6878 & 6532 & 1788 & 5392 & 6365 & 6923 & 6509 & 7580 & 7414 & 3655 & 399 & 726 \\
\hline MIN & 4035 & 531 & 656 & 1477 & 4606 & 6025 & 5208 & 5960 & 3958 & 399 & 257 & 237 \\
\hline $\mathrm{a}$ & 12650 & 26090 & 32120 & 22671 & 31370 & 37320 & 36170 & 30680 & 15340 & 3700 & 1250 & 3400 \\
\hline
\end{tabular}

a Discharge, in acre-feet, for Muck Valley Powerplant (station 11351700), provided by Malacha Hydro Limited Partnership. 
LOCATION.-Lat $41^{\circ} 00^{\prime} 55^{\prime \prime}$, long $121^{\circ} 09^{\prime} 13^{\prime \prime}$, in NE 1/4 SW 1/4 sec.27, T.37 N., R.7 E., Lassen County, Hydrologic Unit 18020003, on right bank, 1.7 mi upstream from North Gulch, 2.2 mi upstream from Spring Gulch, and 7.4 mi south of Bieber.

DRAINAGE AREA.-2,475 $\mathrm{mi}^{2}$, excluding Goose Lake Basin.

PERIOD OF RECORD.—October 1994 to current year.

GAGE.-Acoustic velocity meter measures minimum bypass flow; water-stage recorder and Ogee weir for spillway. Elevation of gage is $4,120 \mathrm{ft}$ above sea level, from topographic map.

REMARKS.-Flow at this station has two components which are combined for publication: low-flow release (station 11351946) and flow over Ogee weir (station 11351948). Water is diverted upstream of weir through a tunnel to Collett Reservoir (station 11351600), for power generation. During powerplant operation, the minimum release is $50 \mathrm{ft}^{3} / \mathrm{s}$. See schematic diagram of Pit and McCloud River Basins.

COOPERATION.—Records were provided by Malacha Hydro Limited Partnership, under general supervision of the U.S. Geological Survey, in connection with a Federal Energy Regulatory commission project.

EXTREMES FOR PERIOD OF RECORD.—Maximum daily discharge, 16,800 ft³/s, Jan. 3, 1997; no flow many days during 1995 and 1997.

DISCHARGE, CUBIC FEET PER SECOND, WATER YEAR OCTOBER 1998 TO SEPTEMBER 1999

DAILY MEAN VALUES

\begin{tabular}{|c|c|c|c|c|c|c|c|c|c|c|c|c|}
\hline DAY & $\mathrm{OCT}$ & NOV & $\mathrm{DEC}$ & JAN & FEB & MAR & APR & MAY & JUN & JUL & AUG & SEP \\
\hline 1 & 53 & 53 & 1630 & 53 & 86 & 2750 & 931 & 470 & 54 & 11 & 2.0 & 2.0 \\
\hline 2 & 53 & 53 & 2110 & 53 & 55 & 3760 & 890 & 419 & 53 & 5.0 & 2.0 & 2.0 \\
\hline 3 & 53 & 53 & 2040 & 53 & 54 & 4590 & 786 & 558 & 53 & 2.0 & 2.0 & 2.0 \\
\hline 4 & 53 & 53 & 1630 & 53 & 54 & 5440 & 667 & 685 & 54 & 2.0 & 2.0 & 2.0 \\
\hline 5 & 53 & 53 & 1120 & 53 & 54 & 5320 & 667 & 766 & 134 & 2.0 & 2.0 & 2.0 \\
\hline 6 & 53 & 53 & 706 & 54 & 54 & 4200 & 631 & 667 & 102 & 2.0 & 2.0 & 2.0 \\
\hline 7 & 53 & 54 & 336 & 53 & 421 & 2950 & 685 & 595 & 267 & 2.0 & 2.0 & 12 \\
\hline 8 & 53 & 54 & 284 & 54 & 1460 & 2060 & 725 & 385 & 215 & 2.0 & 2.0 & 2.0 \\
\hline 9 & 53 & 54 & 54 & 54 & 2110 & 1790 & 705 & 282 & 102 & 2.0 & 2.0 & 2.0 \\
\hline 10 & 53 & 53 & 55 & 54 & 2390 & 1630 & 631 & 248 & 54 & 4.0 & 2.0 & 2.0 \\
\hline 11 & 53 & 53 & 54 & 54 & 3020 & 1460 & 649 & 265 & 54 & 2.0 & 2.0 & 2.0 \\
\hline 12 & 54 & 53 & 102 & 53 & 2950 & 1370 & 746 & 282 & 54 & 2.0 & 2.0 & 2.0 \\
\hline 13 & 53 & 53 & 232 & 54 & 2490 & 1330 & 631 & 164 & 54 & 2.0 & 2.0 & 2.0 \\
\hline 14 & 52 & 53 & 542 & 54 & 1900 & 1060 & 558 & 85 & 54 & 2.0 & 2.0 & 13 \\
\hline 15 & 52 & 53 & 913 & 53 & 1350 & 1150 & 453 & 53 & 54 & 2.0 & 2.0 & 54 \\
\hline 16 & 53 & 53 & 971 & 53 & 1240 & 1210 & 402 & 53 & 54 & 2.0 & 2.0 & 54 \\
\hline 17 & 53 & 53 & 665 & 53 & 1170 & 1330 & 282 & 53 & 53 & 2.0 & 2.0 & 52 \\
\hline 18 & 53 & 53 & 435 & 54 & 1490 & 1350 & 368 & 53 & 51 & 2.0 & 2.0 & 19 \\
\hline 19 & 53 & 53 & 214 & 632 & 1880 & 1390 & 505 & 54 & 12 & 2.0 & 2.0 & 27 \\
\hline 20 & 53 & 53 & 54 & 850 & 1970 & 1420 & 705 & 54 & 5.0 & 2.0 & 20 & 49 \\
\hline 21 & 53 & 53 & 54 & 996 & 1810 & 1370 & 828 & 54 & 21 & 2.0 & 24 & 34 \\
\hline 22 & 53 & 54 & 53 & 954 & 1580 & 1330 & 952 & 54 & 7.0 & 2.0 & 2.0 & 35 \\
\hline 23 & 53 & 55 & 53 & 1510 & 1330 & 1330 & 1040 & 54 & 21 & 2.0 & 22 & 38 \\
\hline 24 & 53 & 1020 & 53 & 1930 & 1440 & 1260 & 890 & 54 & 37 & 2.0 & 2.0 & 38 \\
\hline 25 & 53 & 1460 & 53 & 1830 & 1900 & 1210 & 685 & 54 & 15 & 2.0 & 16 & 12 \\
\hline 26 & 53 & 1100 & 53 & 1510 & 2510 & 1190 & 685 & 54 & 18 & 2.0 & 18 & 9.0 \\
\hline 27 & 53 & 1020 & 53 & 1020 & 2420 & 1190 & 725 & 54 & 12 & 16 & 2.0 & 27 \\
\hline 28 & 53 & 1260 & 53 & 707 & 2220 & 1120 & 766 & 54 & 9.0 & 24 & 2.0 & 27 \\
\hline 29 & 53 & 1330 & 53 & 455 & --- & 995 & 705 & 54 & 12 & 5.0 & 2.0 & 26 \\
\hline 30 & 53 & 1330 & 53 & 198 & --- & 911 & 667 & 54 & 24 & 18 & 17 & 28 \\
\hline 31 & 53 & --- & 53 & 182 & --- & 869 & --- & 54 & --- & 2.0 & 16 & --- \\
\hline TOTAL & 1642 & 9745 & 14731 & 13736 & 41408 & 60335 & 20560 & 6785 & 1709.0 & 131.0 & 181.0 & 578.0 \\
\hline MEAN & 53.0 & 325 & 475 & 443 & 1479 & 1946 & 685 & 219 & 57.0 & 4.23 & 5.84 & 19.3 \\
\hline MAX & 54 & 1460 & 2110 & 1930 & 3020 & 5440 & 1040 & 766 & 267 & 24 & 24 & 54 \\
\hline MIN & 52 & 53 & 53 & 53 & 54 & 869 & 282 & 53 & 5.0 & 2.0 & 2.0 & 2.0 \\
\hline $\mathrm{AC}-\mathrm{FT}$ & 3260 & 19330 & 29220 & 27250 & 82130 & 119700 & 40780 & 13460 & 3390 & 260 & 359 & 1150 \\
\hline
\end{tabular}


11351950 PIT RIVER BELOW DIVERSION TO MUCK VALLEY POWERPLANT, NEAR BIEBER, CA—Continued

STATISTICS OF MONTHLY MEAN DATA FOR WATER YEARS 1995 - 1999, BY WATER YEAR (WY)

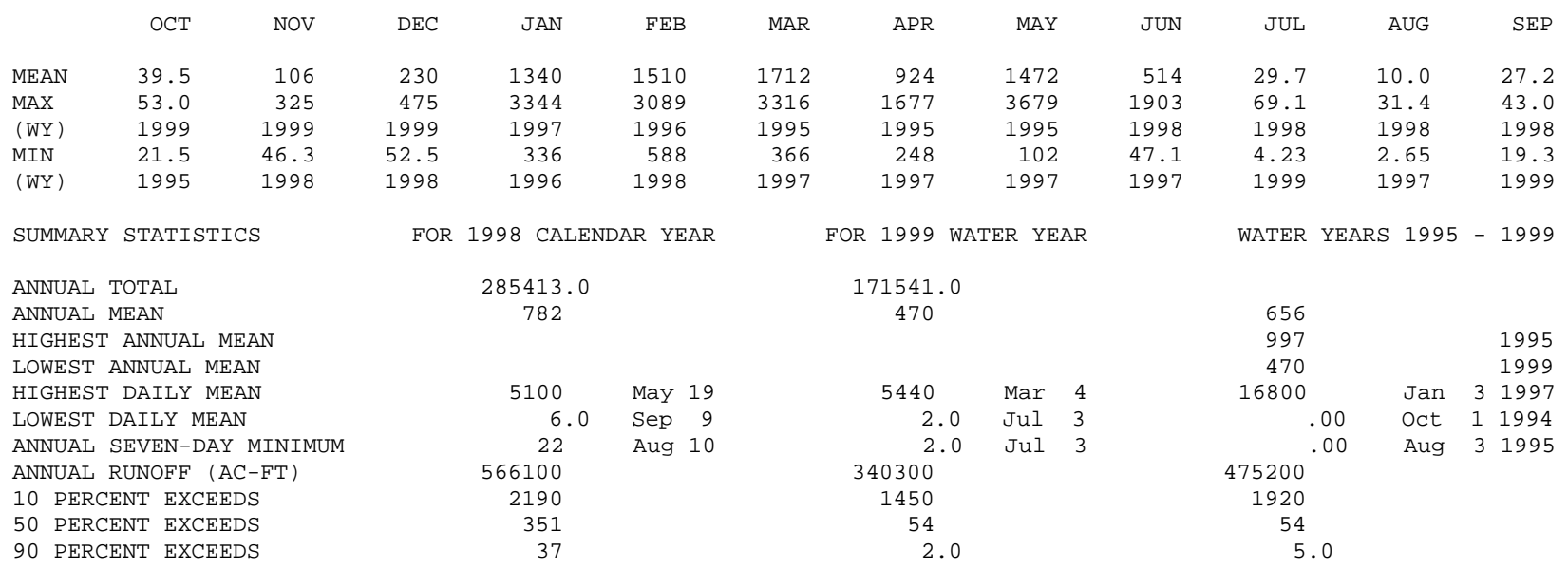


11354200 PIT NO. 1 POWERPLANT NEAR FALL RIVER MILLS, CA

LOCATION.-Lat 4059’28", long 121 '29’49", in SE 1/4 NE 1/4 sec.10, T.37 N., R.4 E., Shasta County, Hydrologic Unit 18020003, on right bank of Pit River, 2.3 mi downstream from Pit River Falls, and 3.2 mi southwest of Fall River Mills.

PERIOD OF RECORD.-October 1986 to current year. Unpublished records for water years 1973-86 available in files of the U.S. Geological Survey. Fragmentary record for water years 1922-72 available in files of the Pacific Gas \& Electric Co.

GAGE.-Discharge computed from powerplant output.

REMARKS.-Water is diverted from Fall River at Pit No. 1 Forebay at NW 1/4 SW 1/4 sec.25, T.37 N., R.4 E., through a tunnel to powerplant and then into Pit River. See schematic diagram of Pit and McCloud River Basins.

COOPERATION.--Records were collected by Pacific Gas \& Electric Co., under general supervision of the U.S. Geological Survey, in connection with a Federal Energy Regulatory Commission project.

EXTREMES FOR PERIOD OF RECORD.-Maximum daily discharge, 2,490 ft³/s, Mar. 13, 1995; no flow several days most years.

DISCHARGE, CUBIC FEET PER SECOND, WATER YEAR OCTOBER 1998 TO SEPTEMBER 1999 DAILY MEAN VALUES

\begin{tabular}{|c|c|c|c|c|c|c|c|c|c|c|c|c|}
\hline DAY & $\mathrm{OCT}$ & NOV & $\mathrm{DEC}$ & JAN & FEB & MAR & APR & MAY & JUN & JUL & AUG & SEP \\
\hline 1 & 1310 & 1530 & 1960 & 1480 & 1580 & 2080 & 1990 & 1800 & 1640 & 1440 & 1240 & .00 \\
\hline 2 & 1320 & 1340 & 1900 & 1500 & 1410 & 2060 & 1870 & 1670 & 1650 & 1320 & 1250 & 1260 \\
\hline 3 & 1260 & 1450 & 1940 & 1440 & 1230 & 2040 & 1840 & 1840 & 1790 & 1310 & 1100 & 1280 \\
\hline 4 & 1360 & 1370 & 2140 & 1430 & 1230 & 2120 & 1820 & 2090 & 1740 & 1380 & 412 & 1570 \\
\hline 5 & 1520 & 1420 & 1830 & 1420 & 1360 & 2120 & 1720 & 2010 & 1700 & 1410 & 1020 & 1240 \\
\hline 6 & 1370 & 1410 & 1890 & 1410 & 1410 & 2040 & 1800 & 1910 & 1660 & 1390 & 1480 & 1320 \\
\hline 7 & 1370 & 1510 & 1320 & 1450 & 1560 & 1920 & 1790 & 1800 & 1640 & 1170 & 1120 & 1510 \\
\hline 8 & 1460 & 1540 & 1120 & 1480 & 2130 & 1890 & 1740 & 1880 & 1520 & 1320 & 1320 & 1340 \\
\hline 9 & 1360 & 1560 & 997 & 1270 & 2140 & 1990 & 1850 & 1860 & 1290 & 1320 & 1660 & 1330 \\
\hline 10 & 1390 & 1500 & 1140 & 1580 & 2100 & 2000 & 1850 & 1780 & 1450 & 1380 & 1380 & 1310 \\
\hline 11 & 1370 & 1490 & 1120 & 1450 & 2060 & 2030 & 1840 & 1780 & 1580 & 1350 & 1380 & 1310 \\
\hline 12 & 1390 & 1510 & 1160 & 1440 & 1920 & 1870 & 1780 & 1780 & 1580 & 1360 & 1220 & 1290 \\
\hline 13 & 1330 & 1450 & 1790 & 1530 & 1820 & 1920 & 1750 & 1780 & 1510 & 1260 & 1500 & 1270 \\
\hline 14 & 1430 & 1380 & 1650 & 1400 & 1680 & 1860 & 1810 & 1960 & 1470 & 1200 & 1370 & 1340 \\
\hline 15 & 1460 & 1460 & 1590 & 1370 & 1910 & 1920 & 2020 & 2040 & 1550 & 1290 & 1300 & 1310 \\
\hline 16 & 1460 & 1330 & 1670 & 1440 & 1710 & 1890 & 2070 & 1820 & 1610 & 1280 & 1210 & 1290 \\
\hline 17 & 1390 & 1470 & 1360 & 1490 & 2000 & 1820 & 1790 & 1770 & 1010 & 1270 & 1270 & 1320 \\
\hline 18 & 1650 & 1410 & 1610 & 1610 & 2060 & 1790 & 1880 & 1760 & 1120 & 1290 & 1370 & 1200 \\
\hline 19 & 1340 & 1410 & 1510 & 1840 & 2100 & 1770 & 1830 & 1740 & 1470 & 1190 & 1300 & 1350 \\
\hline 20 & 1340 & 1440 & 1630 & 1960 & 2030 & 1820 & 1990 & 1770 & 1480 & 1360 & 1260 & 1410 \\
\hline 21 & 1250 & 1310 & 1360 & 2070 & 1960 & 1850 & 1850 & 1770 & 1480 & 1260 & 1330 & 1340 \\
\hline 22 & 1500 & 1510 & 1450 & 2160 & 1940 & 1860 & 1940 & 1820 & 1480 & 1210 & 1300 & 1280 \\
\hline 23 & 1430 & 1690 & 1490 & 2100 & 1950 & 1860 & 2080 & 1680 & 1430 & 1280 & 1340 & 1300 \\
\hline 24 & 1380 & 1790 & 1460 & 2140 & 1950 & 1840 & 1640 & 1660 & 1350 & 1200 & 1410 & 1350 \\
\hline 25 & 1390 & 1720 & 1440 & 1810 & 2040 & 1870 & 1790 & 1680 & 1370 & 1350 & 1380 & 1270 \\
\hline 26 & 1380 & 1720 & 1420 & 1810 & 2130 & 1850 & 1820 & 1720 & 1450 & 1270 & 1300 & 1440 \\
\hline 27 & 1370 & 1920 & 1610 & 1820 & 2130 & 1910 & 1880 & 1820 & 1450 & 1280 & 1400 & 1260 \\
\hline 28 & 1320 & 1740 & 1610 & 1770 & 2020 & 1820 & 1900 & 1660 & 1410 & 1330 & 1340 & 1160 \\
\hline 29 & 1470 & 1680 & 1530 & 1720 & --- & 1820 & 1920 & 1720 & 1480 & 1250 & 1230 & 1160 \\
\hline 30 & 1450 & 1750 & 1310 & 1670 & --- & 1800 & 1920 & 1720 & 1310 & 1300 & 1370 & 1250 \\
\hline 31 & 1390 & --- & 1520 & 1670 & --- & 1860 & --- & 1660 & --- & 1180 & 1250 & --- \\
\hline TOTAL & 43210 & 45810 & 47527 & 50730 & 51560 & 59290 & 55770 & 55750 & 44670 & 40200 & 39812 & 38060.00 \\
\hline MEAN & 1394 & 1527 & 1533 & 1636 & 1841 & 1913 & 1859 & 1798 & 1489 & 1297 & 1284 & 1269 \\
\hline MAX & 1650 & 1920 & 2140 & 2160 & 2140 & 2120 & 2080 & 2090 & 1790 & 1440 & 1660 & 1570 \\
\hline MIN & 1250 & 1310 & 997 & 1270 & 1230 & 1770 & 1640 & 1660 & 1010 & 1170 & 412 & .00 \\
\hline$A C-F T$ & 85710 & 90860 & 94270 & 100600 & 102300 & 117600 & 110600 & 110600 & 88600 & 79740 & 78970 & 75490 \\
\hline
\end{tabular}

STATISTICS OF MONTHLY MEAN DATA FOR WATER YEARS 1987 - 1999, BY WATER YEAR (WY)

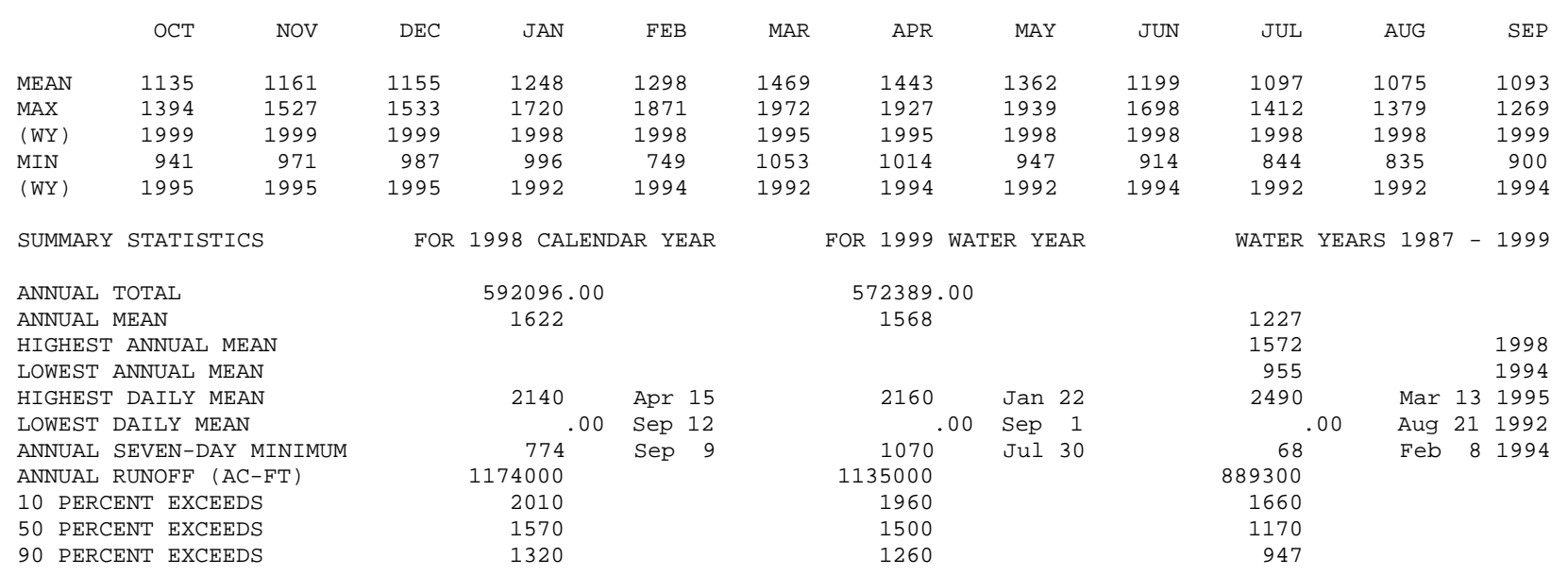


LOCATION.-Lat 4059'00", long 121³0’39", in NE 1/4 NW 1/4 sec.15, T.36 N., R.4 E., Shasta County, Hydrologic Unit 18020003, on left bank, 0.9 mi downstream from Pit No. 1 Powerplant, and 4 mi southwest of Fall River Mills.

DRAINAGE AREA.-3,761 $\mathrm{mi}^{2}$, excluding Goose Lake Basin.

PERIOD OF RECORD.-August 1975 to current year.

GAGE.-Water-stage recorder and crest-stage gage. Elevation of gage is 2,798.21 ft above sea level (levels by Pacific Gas and Electric Co.).

REMARKS.--Records good. Low flow regulated by many small reservoirs (total usable reservoir capacity, 210,000 acre-ft) and Pit No. 1

Powerplant. Many diversions upstream from station for irrigation. See schematic diagram of Pit and McCloud River Basins.

EXTREMES FOR PERIOD OF RECORD.-Maximum discharge, 30,000 $\mathrm{ft}^{3} / \mathrm{s}$, Feb. 20, 1986, gage height, $17.03 \mathrm{ft}$; minimum daily, $535 \mathrm{ft} 3 / \mathrm{s}$, Sept. 11, 1994

EXTREMES OUTSIDE PERIOD OF RECORD._Flood of January 1974 reached a stage of $14.8 \mathrm{ft}$, from floodmarks on right bank, discharge $22,600 \mathrm{ft}^{3} / \mathrm{s}$.

EXTREMES FOR CURRENT YEAR.-Peak discharges greater than base discharge of 4,000 $\mathrm{ft}^{3} / \mathrm{s}$, or maximum:

$\begin{array}{lcccccc}\text { Date } & \text { Time } & \begin{array}{c}\text { Discharge } \\ \left(\mathrm{ft}^{3} / \mathrm{s}\right)\end{array} & \begin{array}{c}\text { Gage height } \\ (\mathrm{ft})\end{array} & \text { Date } & \text { Time } & \begin{array}{c}\text { Discharge } \\ \left(\mathrm{ft}^{3} / \mathrm{s}\right)\end{array} \\ \begin{array}{l}\text { Nov. } 30 \\ \text { Feb. } 9\end{array} & 2245 & 6,390 & 9.50 & \text { Mar. } 5 & 0245 & 8,930 \\ (\mathrm{ft})\end{array}$

DISCHARGE, CUBIC FEET PER SECOND, WATER YEAR OCTOBER 1998 TO SEPTEMBER 1999 DAILY MEAN VALUES

\begin{tabular}{|c|c|c|c|c|c|c|c|c|c|c|c|c|}
\hline DAY & $\mathrm{OCT}$ & NOV & $\mathrm{DEC}$ & JAN & FEB & MAR & $\mathrm{APR}$ & MAY & JUN & JUL & AUG & SEP \\
\hline 1 & 1650 & 1700 & 5410 & 2210 & 2510 & 5940 & 3900 & 3140 & 2360 & 1740 & 1420 & 1180 \\
\hline 2 & 1690 & 1630 & 5230 & 2200 & 2350 & 6470 & 3780 & 2880 & 2440 & 1620 & 1400 & 1790 \\
\hline 3 & 1530 & 2140 & 5430 & 2110 & 2080 & 7390 & 3650 & 3110 & 2550 & 1570 & 1450 & 1460 \\
\hline 4 & 1550 & 2050 & 5220 & 2020 & 2110 & 8250 & 3540 & 3650 & 2520 & 1530 & 1440 & 1720 \\
\hline 5 & 1730 & 2030 & 4190 & 1970 & 2220 & 8580 & 3380 & 3660 & 2130 & 1550 & 1360 & 1440 \\
\hline 6 & 1690 & 2000 & 3720 & 1980 & 1910 & 7710 & 3450 & 3560 & 2360 & 1520 & 1590 & 1480 \\
\hline 7 & 1720 & 2000 & 2790 & 1980 & 2050 & 6470 & 3420 & 3450 & 2590 & 1580 & 1380 & 1640 \\
\hline 8 & 1770 & 1860 & 3120 & 1980 & 4140 & 5460 & 3390 & 3310 & 2660 & 1570 & 1460 & 1540 \\
\hline 9 & 1670 & 2030 & 2690 & 1720 & 6470 & 4990 & 3520 & 3090 & 2590 & 1580 & 1480 & 1520 \\
\hline 10 & 1670 & 2110 & 2670 & 2010 & 6030 & 4760 & 3470 & 3040 & 2400 & 1580 & 1530 & 1500 \\
\hline 11 & 1540 & 2030 & 2440 & 1880 & 6200 & 4590 & 3490 & 3150 & 2230 & 1510 & 1530 & 1500 \\
\hline 12 & 1720 & 2010 & 2480 & 1840 & 6170 & 4300 & 3490 & 3160 & 1910 & 1510 & 1410 & 1470 \\
\hline 13 & 1890 & 2090 & 2920 & 1970 & 5670 & 4200 & 3440 & 3130 & 1670 & 1440 & 1650 & 1460 \\
\hline 14 & 1940 & 2020 & 3250 & 1850 & 4930 & 4030 & 3420 & 3170 & 1680 & 1540 & 1500 & 1510 \\
\hline 15 & 1970 & 1900 & 3350 & 1830 & 4380 & 4040 & 3610 & 3190 & 1880 & 1550 & 1460 & 1490 \\
\hline 16 & 1980 & 1900 & 3550 & 1850 & 4000 & 4000 & 3500 & 2820 & 1940 & 1520 & 1390 & 1510 \\
\hline 17 & 1730 & 2120 & 3080 & 1730 & 4160 & 3940 & 3250 & 2790 & 1760 & 1510 & 1390 & 1670 \\
\hline 18 & 1790 & 2130 & 3090 & 1940 & 4610 & 3980 & 3320 & 2770 & 1750 & 1460 & 1540 & 1540 \\
\hline 19 & 1680 & 2070 & 2690 & 2930 & 5150 & 4010 & 3370 & 2600 & 1730 & 1370 & 1500 & 1560 \\
\hline 20 & 1880 & 2130 & 2570 & 3430 & 5200 & 4080 & 3670 & 2390 & 1610 & 1510 & 1440 & 1610 \\
\hline 21 & 1760 & 1840 & 2270 & 3790 & 5020 & 4120 & 3700 & 2210 & 1590 & 1440 & 1500 & 1680 \\
\hline 22 & 2020 & 2230 & 2350 & 3900 & 4750 & 4240 & 3830 & 2190 & 1690 & 1410 & 1520 & 1650 \\
\hline 23 & 1860 & 2690 & 2230 & 4090 & 4350 & 4190 & 4110 & 1890 & 1740 & 1460 & 1520 & 1630 \\
\hline 24 & 1780 & 3500 & 2100 & 4370 & 4240 & 4090 & 3500 & 2300 & 1630 & 1390 & 1640 & 1660 \\
\hline 25 & 1640 & 4100 & 2050 & 4280 & 4780 & 4050 & 3380 & 2260 & 1650 & 1510 & 1570 & 1600 \\
\hline 26 & 1710 & 3860 & 2030 & 4250 & 5680 & 3990 & 3090 & 2460 & 1690 & 1410 & 1520 & 1660 \\
\hline 27 & 1900 & 4110 & 2200 & 3690 & 5860 & 4060 & 3510 & 2600 & 1570 & 1410 & 1620 & 1410 \\
\hline 28 & 1970 & 3980 & 2230 & 3340 & 5490 & 3950 & 3670 & 2420 & 1520 & 1480 & 1560 & 1450 \\
\hline 29 & 1950 & 3900 & 2180 & 3060 & --- & 3880 & 3670 & 2550 & 1720 & 1530 & 1450 & 1580 \\
\hline 30 & 1900 & 4630 & 1580 & 2820 & --- & 3760 & 3510 & 2500 & 1560 & 1500 & 1590 & 1640 \\
\hline 31 & 1770 & --- & 2250 & 2670 & --- & 3740 & --- & 2350 & --- & 1400 & 1470 & --- \\
\hline TOTAL & 55050 & 74790 & 93360 & 81690 & 122510 & 151260 & 106030 & 87790 & 59120 & 46700 & 46280 & 46550 \\
\hline MEAN & 1776 & 2493 & 3012 & 2635 & 4375 & 4879 & 3534 & 2832 & 1971 & 1506 & 1493 & 1552 \\
\hline MAX & 2020 & 4630 & 5430 & 4370 & 6470 & 8580 & 4110 & 3660 & 2660 & 1740 & 1650 & 1790 \\
\hline MIN & 1530 & 1630 & 1580 & 1720 & 1910 & 3740 & 3090 & 1890 & 1520 & 1370 & 1360 & 1180 \\
\hline $\mathrm{AC}-\mathrm{FT}$ & 109200 & 148300 & 185200 & 162000 & 243000 & 300000 & 210300 & 174100 & 117300 & 92630 & 91800 & 92330 \\
\hline
\end{tabular}


11355010 PIT RIVER BELOW PIT NO. 1 POWERPLANT, NEAR FALL RIVER MILLS, CA—Continued

STATISTICS OF MONTHLY MEAN DATA FOR WATER YEARS 1975 - 1999, BY WATER YEAR (WY)

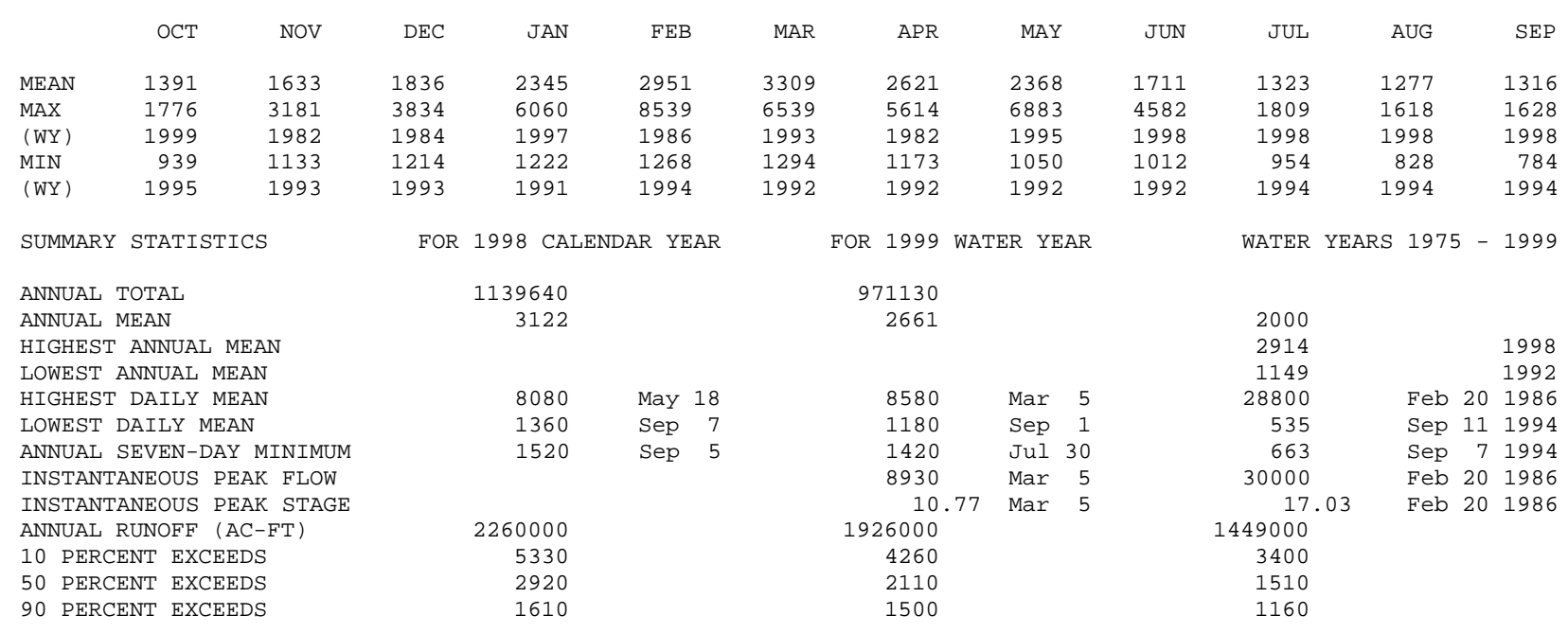


LOCATION.-Lat 4045'35", long 121²4'46", in NW 1/4 SW 1/4 sec.34, T.34 N., R.5 E., Shasta County, Hydrologic Unit 18020003, on right bank, 0.4 mi downstream from Lost Creek Diversion Dam, 2.5 mi downstream from Porcupine Reservoir, 6.0 mi north of Old Station, and 13.2 mi southeast of Cassel.

DRAINAGE AREA.-7.53 $\mathrm{mi}^{2}$.

PERIOD OF RECORD.-October 1989 to September 1997; October 1998 to September 1999 (operated as low-flow station only).

GAGE.-Water-stage recorder and sharp-crested weir. Elevation of gage is 3,900 ft above sea level, from topographic map.

REMARKS.-During times of powerplant operation, the minimum release requirement is $15 \mathrm{ft}^{3} / \mathrm{s}$; flow is computed to $80 \mathrm{ft}^{3} / \mathrm{s}$. See schematic diagram of Pit and McCloud River Basins.

COOPERATION.--Records were collected by Snow Mountain Hydro, under general supervision of the U.S. Geological Survey, in connection with a Federal Energy Regulatory Commission project.

DISCHARGE, CUBIC FEET PER SECOND, WATER YEAR OCTOBER 1998 TO SEPTEMBER 1999 DAILY MEAN VALUES

\begin{tabular}{|c|c|c|c|c|c|c|c|c|c|c|c|c|}
\hline DAY & $\mathrm{OCT}$ & NOV & $\mathrm{DEC}$ & JAN & FEB & MAR & APR & MAY & JUN & JUL & AUG & SEP \\
\hline 1 & 16 & 16 & 17 & 16 & 17 & 22 & 16 & 17 & 22 & 16 & 16 & 17 \\
\hline 2 & 16 & 17 & 16 & 16 & 16 & 17 & 16 & 16 & 17 & 17 & 16 & 16 \\
\hline 3 & 16 & 16 & 17 & 17 & 16 & 25 & 16 & 17 & 17 & 16 & 16 & 17 \\
\hline 4 & 17 & 17 & 22 & 20 & 17 & 17 & 16 & 16 & 17 & 17 & 16 & 16 \\
\hline 5 & 16 & 25 & 16 & 17 & 17 & 17 & 17 & 17 & 17 & 17 & 16 & 16 \\
\hline 6 & 16 & 17 & 17 & 17 & 17 & 20 & 17 & 16 & 17 & 17 & 23 & 17 \\
\hline 7 & 16 & 17 & 17 & 16 & 31 & 18 & 17 & 16 & 17 & 16 & 17 & 16 \\
\hline 8 & 16 & 22 & 17 & 17 & 17 & 16 & 17 & 16 & 19 & 19 & 17 & 17 \\
\hline 9 & 16 & 16 & 17 & 17 & 24 & 16 & 17 & 17 & 16 & 17 & 16 & 16 \\
\hline 10 & 16 & 16 & 17 & 17 & 17 & 17 & 16 & 17 & 16 & 17 & 19 & 17 \\
\hline 11 & 17 & 19 & 21 & 17 & 17 & 17 & 16 & 16 & 17 & 16 & 17 & 16 \\
\hline 12 & 17 & 17 & 17 & 16 & 17 & 16 & 17 & 16 & 17 & 17 & 17 & 17 \\
\hline 13 & 17 & 17 & 17 & 17 & 17 & 16 & 17 & 16 & 16 & 22 & 16 & 17 \\
\hline 14 & 19 & 16 & 16 & 17 & 17 & 17 & 17 & 17 & 17 & 19 & 17 & 17 \\
\hline 15 & 20 & 16 & 16 & 19 & 16 & 16 & 17 & 17 & 17 & 16 & 17 & 17 \\
\hline 16 & 16 & 16 & 16 & 17 & 17 & 16 & 17 & 17 & 17 & 16 & 16 & 17 \\
\hline 17 & 16 & 16 & 16 & 22 & 18 & 17 & 17 & 21 & 17 & 16 & 23 & 26 \\
\hline 18 & 16 & 17 & 16 & 17 & 17 & 17 & 17 & 16 & 16 & 16 & 17 & 17 \\
\hline 19 & 16 & 16 & 17 & 16 & 17 & 17 & 17 & 17 & 17 & 16 & 16 & 17 \\
\hline 20 & 17 & 16 & 16 & 17 & 17 & 21 & 17 & 19 & 16 & 17 & 36 & 17 \\
\hline 21 & 17 & 16 & 17 & 20 & 17 & 21 & 19 & 17 & 16 & 37 & 16 & 16 \\
\hline 22 & 17 & 17 & 17 & 17 & 17 & 17 & 16 & 16 & 16 & 25 & 16 & 22 \\
\hline 23 & 17 & 19 & 16 & 17 & 19 & 16 & 17 & 17 & 16 & 17 & 23 & 17 \\
\hline 24 & 16 & 16 & 16 & 16 & 18 & 16 & 16 & 17 & 16 & 16 & 19 & 16 \\
\hline 25 & 17 & 16 & 17 & 16 & 17 & 17 & 16 & 17 & 16 & 17 & 16 & 16 \\
\hline 26 & 17 & 17 & 17 & 16 & 17 & 17 & 16 & 17 & 16 & 21 & 17 & 16 \\
\hline 27 & 19 & 19 & 17 & 17 & 18 & 18 & 16 & 17 & 16 & 20 & 16 & 17 \\
\hline 28 & 21 & 16 & 16 & 17 & 27 & 17 & 16 & 17 & 16 & 17 & 19 & 17 \\
\hline 29 & 16 & 16 & 16 & 17 & --- & 16 & 17 & 17 & 18 & 17 & 16 & 17 \\
\hline 30 & 17 & 26 & 17 & 17 & --- & 17 & 16 & 17 & 18 & 17 & 24 & 17 \\
\hline 31 & 17 & --- & 16 & 16 & --- & 17 & --- & 17 & --- & 16 & 23 & --- \\
\hline TOTAL & 523 & 523 & 523 & 531 & 509 & 544 & 499 & 523 & 506 & 560 & 569 & 514 \\
\hline MEAN & 16.9 & 17.4 & 16.9 & 17.1 & 18.2 & 17.5 & 16.6 & 16.9 & 16.9 & 18.1 & 18.4 & 17.1 \\
\hline MAX & 21 & 26 & 22 & 22 & 31 & 25 & 19 & 21 & 22 & 37 & 36 & 26 \\
\hline MIN & 16 & 16 & 16 & 16 & 16 & 16 & 16 & 16 & 16 & 16 & 16 & 16 \\
\hline $\mathrm{AC}-\mathrm{FT}$ & 1040 & 1040 & 1040 & 1050 & 1010 & 1080 & 990 & 1040 & 1000 & 1110 & 1130 & 1020 \\
\hline$a$ & 3090 & 3010 & 3230 & 3210 & 3200 & 3710 & 3310 & 3160 & 3060 & 3040 & 2980 & 3020 \\
\hline
\end{tabular}

a Discharge, in acre-feet, for Lost Creek No. 1 Powerplant (station 11358010), provided by Snow Mountain Hydro. 
11358700 HAT CREEK BELOW HAT NO. 1 DIVERSION DAM, NEAR BURNEY, CA

LOCATION.-Lat 4055'08", long 121³3'02", in NW 1/4 SW 1/4 sec.5, T.36 N., R.4 E., Shasta County, Hydrologic Unit 18020003, on right bank, at Hat No. 1 Diversion Dam on Hat Creek, 6.5 mi northeast of Burney.

DRAINAGE AREA.-347 $\mathrm{mi}^{2}$.

PERIOD OF RECORD.-Oct. 1 to Dec. 8, 1987 (fragmentary), Dec. 9, 1987, to current year (operated as a low-flow station only). Unpublished fragmentary records for water years 1980-87 available in files of the U.S. Geological Survey.

GAGE.-Water-stage recorder and Cipolletti weir. Elevation of gage is 3,180 ft above sea level, from topographic map.

REMARKS.-This station records fishwater release only. The minimum release requirement is $2.0 \mathrm{ft}^{3} / \mathrm{s}$ at all times. Flow is computed to $9.0 \mathrm{ft}^{3} / \mathrm{s}$. See schematic diagram of Pit and McCloud River Basins.

COOPERATION.-Records were collected by Pacific Gas \& Electric Co., under general supervision of the U.S. Geological Survey, in connection with a Federal Energy Regulatory Commission project.

DISCHARGE, CUBIC FEET PER SECOND, WATER YEAR OCTOBER 1998 TO SEPTEMBER 1999 DAILY MEAN VALUES

\begin{tabular}{|c|c|c|c|c|c|c|c|c|c|c|c|c|}
\hline DAY & OCT & NOV & $\mathrm{DEC}$ & JAN & FEB & MAR & APR & MAY & JUN & JUL & AUG & SEP \\
\hline 1 & 3.2 & 3.1 & 3.1 & 3.3 & 3.1 & 2.9 & 3.2 & 3.2 & 3.0 & 3.0 & 2.8 & 3.0 \\
\hline 2 & 3.1 & 3.1 & 3.1 & 3.2 & 3.0 & 2.9 & 3.2 & 3.2 & 3.0 & 3.0 & 2.9 & 3.0 \\
\hline 3 & 3.0 & 3.1 & 3.1 & 3.2 & 3.0 & 2.9 & 3.2 & 3.2 & 3.0 & 3.0 & 3.0 & 3.0 \\
\hline 4 & 3.0 & 3.0 & 3.1 & 3.2 & 3.0 & 3.0 & 3.2 & 3.2 & 3.0 & 3.0 & 3.0 & 3.0 \\
\hline 5 & 3.1 & 3.0 & 3.1 & 3.2 & 3.1 & 2.9 & 3.2 & 3.0 & 3.0 & 3.0 & 2.9 & 3.0 \\
\hline 6 & 3.1 & 3.0 & 3.1 & 3.1 & 3.2 & 2.9 & 3.1 & 3.0 & 2.9 & 2.9 & 3.0 & 3.0 \\
\hline 7 & 3.1 & 3.0 & 3.1 & 3.1 & 3.2 & 2.9 & 3.2 & 3.0 & 2.9 & 2.9 & 3.0 & 3.0 \\
\hline 8 & 3.2 & 3.0 & 3.1 & 3.1 & 3.2 & 2.9 & 3.3 & 3.0 & 2.8 & 2.9 & 2.9 & 3.0 \\
\hline 9 & 3.2 & 3.0 & 3.1 & 3.2 & 3.2 & 3.0 & 3.4 & 3.0 & 2.8 & 2.9 & 3.1 & 3.0 \\
\hline 10 & 3.1 & 3.0 & 3.0 & 3.1 & 3.0 & 3.0 & 3.4 & 3.1 & 2.9 & 2.8 & 3.2 & 3.0 \\
\hline 11 & 3.2 & 3.0 & 3.0 & 3.1 & 2.9 & 2.9 & 3.3 & 3.1 & 2.9 & 2.8 & 3.2 & 3.0 \\
\hline 12 & 3.2 & 3.0 & 3.0 & 3.1 & 2.9 & 2.9 & 3.3 & 3.1 & 2.9 & 2.8 & 3.2 & 3.3 \\
\hline 13 & 3.2 & 3.0 & 3.0 & 3.1 & 2.8 & 2.9 & 3.2 & 3.2 & 2.9 & 3.4 & 3.2 & 3.5 \\
\hline 14 & 3.2 & 3.0 & 3.0 & 3.0 & 2.8 & 3.0 & 3.3 & 3.2 & 3.0 & 3.0 & 3.2 & 3.1 \\
\hline 15 & 3.2 & 3.0 & 3.0 & 3.0 & 2.8 & 5.9 & 3.2 & 3.2 & 3.3 & 3.1 & 3.2 & 3.0 \\
\hline 16 & 3.2 & 3.0 & 3.0 & 3.0 & 2.9 & 7.2 & 3.0 & 3.2 & --- & 3.2 & 3.1 & 2.8 \\
\hline 17 & 3.2 & 3.0 & 3.0 & 3.0 & 3.0 & 6.9 & 3.1 & 3.2 & 6.8 & 3.2 & 3.1 & 5.2 \\
\hline 18 & 3.2 & 3.0 & 3.0 & 3.0 & 3.0 & 5.5 & 3.1 & 3.1 & 3.3 & 3.2 & 3.0 & 6.2 \\
\hline 19 & 3.2 & 3.0 & 3.0 & 3.0 & 3.0 & 2.8 & 3.1 & 3.1 & 3.3 & 3.1 & 2.9 & 6.2 \\
\hline 20 & 3.2 & 3.0 & 3.0 & 3.0 & 2.9 & 2.8 & 3.1 & 3.2 & 3.2 & 3.1 & 2.9 & 4.9 \\
\hline 21 & 3.1 & 2.9 & 3.0 & 3.0 & 2.9 & 2.8 & 3.1 & 3.2 & 3.2 & 3.1 & 2.9 & 2.9 \\
\hline 22 & 3.0 & 3.0 & 3.0 & 3.0 & 3.0 & 3.0 & 3.1 & 3.1 & 3.2 & 3.1 & 2.9 & 3.0 \\
\hline 23 & 3.0 & 3.0 & 3.0 & 3.1 & 3.0 & 3.2 & 3.1 & 3.1 & 3.2 & 3.0 & --- & 3.0 \\
\hline 24 & 3.0 & 3.0 & 3.0 & 3.1 & 2.9 & 3.2 & 3.1 & 3.0 & 3.3 & 3.0 & 4.6 & 3.0 \\
\hline 25 & 3.0 & 3.0 & 3.1 & 3.0 & 2.9 & 3.2 & 3.0 & 2.9 & 3.3 & 3.0 & 3.0 & 2.9 \\
\hline 26 & 3.0 & 3.0 & 3.3 & 3.0 & 2.9 & 3.2 & 3.0 & 3.0 & 3.3 & 3.0 & 3.1 & 3.0 \\
\hline 27 & 3.0 & 3.0 & 3.3 & 3.0 & 2.9 & 3.2 & 3.0 & 3.0 & 3.3 & 2.9 & 3.1 & 3.0 \\
\hline 28 & 3.0 & 3.0 & 3.3 & 3.1 & 2.9 & 3.2 & 3.0 & 3.1 & 3.1 & 2.9 & 3.0 & 3.0 \\
\hline 29 & 3.1 & 3.0 & 3.3 & 3.1 & --- & 3.2 & 3.0 & 3.1 & 3.1 & 2.9 & 3.0 & 3.0 \\
\hline 30 & 3.0 & 3.1 & 3.3 & 3.1 & --- & 3.2 & 3.0 & 3.1 & 3.0 & 2.9 & 3.0 & 3.0 \\
\hline 31 & 3.1 & --- & 3.3 & 3.1 & --- & 3.2 & --- & 3.0 & --- & 2.8 & 3.0 & --- \\
\hline TOTAL & 96.4 & 90.3 & 95.8 & 95.6 & 83.4 & 106.7 & 94.5 & 96.1 & --- & 92.9 & --- & 101.0 \\
\hline MEAN & 3.11 & 3.01 & 3.09 & 3.08 & 2.98 & 3.44 & 3.15 & 3.10 & --- & 3.00 & --- & 3.37 \\
\hline MAX & 3.2 & 3.1 & 3.3 & 3.3 & 3.2 & 7.2 & 3.4 & 3.2 & --- & 3.4 & --- & 6.2 \\
\hline MIN & 3.0 & 2.9 & 3.0 & 3.0 & 2.8 & 2.8 & 3.0 & 2.9 & --- & 2.8 & --- & 2.8 \\
\hline $\mathrm{AC}-\mathrm{FT}$ & 191 & 179 & 190 & 190 & 165 & 212 & 187 & 191 & --- & 184 & --- & 200 \\
\hline
\end{tabular}


11358800 HAT CREEK NO. 1 POWERPLANT NEAR BURNEY, CA

LOCATION.-Lat 4055'45", long 121`32’37", in SW 1/4 SW 1/4 sec.32, T.36 N., R.4 E., Shasta County, Hydrologic Unit 18020003, on right bank of Hat Creek, at the upper end of Baum Lake, and 7.4 mi northeast of Burney.

PERIOD OF RECORD.-October 1986 to current year. Unpublished records for water years 1981-86 available in files of the U.S. Geological Survey. Fragmentary records for water years 1921-80 available in the files of the Pacific Gas \& Electric Co.

GAGE.-Discharge computed from powerplant output.

REMARKS.-Water is diverted from left bank of Hat Creek at NW 1/4 SW 1/4 sec.5, T.36 N., R.8 W., through a canal to powerplant and then into Hat Creek. See schematic diagram of Pit and McCloud River Basins.

COOPERATION.--Records were collected by Pacific Gas \& Electric Co., under general supervision of the U.S. Geological Survey, in connection with a Federal Energy Regulatory Commission project.

EXTREMES FOR PERIOD OF RECORD.-Maximum daily discharge, $518 \mathrm{ft}^{3}$ /s, Nov. 2, 1998; no flow several days most years.

DISCHARGE, CUBIC FEET PER SECOND, WATER YEAR OCTOBER 1998 TO SEPTEMBER 1999 DAILY MEAN VALUES

\begin{tabular}{|c|c|c|c|c|c|c|c|c|c|c|c|c|}
\hline DAY & OCT & NOV & DEC & JAN & FEB & MAR & APR & MAY & JUN & JUL & AUG & SEP \\
\hline 1 & 398 & 426 & 470 & 420 & 411 & 435 & 424 & 372 & 397 & 355 & 329 & 357 \\
\hline 2 & 398 & 518 & 457 & 415 & 410 & 438 & 425 & 358 & 394 & 352 & 335 & 354 \\
\hline 3 & 398 & 420 & 458 & 415 & 412 & 442 & 421 & 354 & 408 & 350 & 335 & 354 \\
\hline 4 & 398 & 420 & 455 & 414 & 413 & 438 & 422 & 342 & 419 & 351 & 341 & 351 \\
\hline 5 & 398 & 415 & 449 & 415 & 412 & 436 & 424 & 336 & 411 & 350 & 337 & 351 \\
\hline 6 & 398 & 420 & 443 & 415 & 417 & 433 & 426 & 320 & 406 & 340 & 341 & 348 \\
\hline 7 & 375 & 420 & 436 & 429 & 432 & 433 & 422 & 326 & 417 & 334 & 340 & 345 \\
\hline 8 & 375 & 432 & 435 & 413 & 431 & 434 & 421 & 335 & 391 & 336 & 338 & 338 \\
\hline 9 & 409 & 420 & 476 & 413 & 467 & 438 & 423 & 339 & 393 & 337 & 344 & 332 \\
\hline 10 & 409 & 443 & 430 & 413 & 453 & 435 & 423 & 337 & 387 & 336 & 336 & 339 \\
\hline 11 & 409 & 443 & 432 & 413 & 438 & 434 & 425 & 330 & 372 & 343 & 340 & 345 \\
\hline 12 & 387 & 426 & 434 & 413 & 433 & 397 & 423 & 340 & 370 & 342 & 338 & 340 \\
\hline 13 & 387 & 426 & 434 & 413 & 431 & 432 & 421 & 346 & 363 & 285 & 333 & 335 \\
\hline 14 & 364 & 432 & 437 & 411 & 432 & 431 & 420 & 350 & 368 & 341 & 333 & 335 \\
\hline 15 & 364 & 426 & 436 & 413 & 432 & 135 & 418 & 362 & 381 & 344 & 333 & 331 \\
\hline 16 & 387 & 422 & 433 & 418 & 432 & .00 & 418 & 360 & 370 & 352 & 332 & 343 \\
\hline 17 & 387 & 464 & 432 & 418 & 432 & .00 & 418 & 355 & 370 & 356 & 334 & 84 \\
\hline 18 & 387 & 464 & 431 & 424 & 434 & 136 & 419 & 345 & 375 & 360 & 340 & .00 \\
\hline 19 & 409 & 414 & 428 & 423 & 434 & 428 & 408 & 351 & 386 & 350 & 330 & .00 \\
\hline 20 & 398 & 424 & 429 & 421 & 430 & 430 & 396 & 329 & 381 & 357 & 331 & 94 \\
\hline 21 & 409 & 427 & 402 & 419 & 430 & 430 & 385 & 371 & 374 & 346 & 334 & 320 \\
\hline 22 & 409 & 435 & 417 & 418 & 430 & 429 & .00 & 340 & 381 & 345 & 332 & 334 \\
\hline 23 & 420 & 446 & 416 & 428 & 430 & 428 & 375 & 340 & 384 & 339 & 89 & 332 \\
\hline 24 & 420 & 450 & 415 & 423 & 432 & 429 & 392 & 339 & 395 & 337 & 105 & 332 \\
\hline 25 & 420 & 441 & 416 & 418 & 441 & 415 & 386 & 346 & 404 & 335 & 328 & 332 \\
\hline 26 & 432 & 438 & 419 & 420 & 436 & 431 & 368 & 349 & 405 & 331 & 330 & 326 \\
\hline 27 & 420 & 448 & 422 & 418 & 435 & 427 & 363 & 364 & 394 & 331 & 341 & 328 \\
\hline 28 & 420 & 445 & 423 & 415 & 435 & 426 & 370 & 375 & 391 & 330 & 344 & 332 \\
\hline 29 & 420 & 438 & 421 & 414 & --- & 426 & 382 & 373 & 391 & 330 & 350 & 328 \\
\hline 30 & 420 & 461 & 419 & 413 & --- & 430 & 384 & 381 & 369 & 327 & 361 & 325 \\
\hline 31 & 420 & --- & 410 & 414 & --- & 428 & --- & 388 & --- & 329 & 356 & --- \\
\hline TOTAL & 12445 & 13104 & 13415 & 12929 & 12055 & 11884.00 & 11802.00 & 10853 & 11647 & 10551 & 9990 & 8965.00 \\
\hline MEAN & 401 & 437 & 433 & 417 & 431 & 383 & 393 & 350 & 388 & 340 & 322 & 299 \\
\hline MAX & 432 & 518 & 476 & 429 & 467 & 442 & 426 & 388 & 419 & 360 & 361 & 357 \\
\hline MIN & 364 & 414 & 402 & 411 & 410 & .00 & .00 & 320 & 363 & 285 & 89 & .00 \\
\hline $\mathrm{AC}-\mathrm{FT}$ & 24680 & 25990 & 26610 & 25640 & 23910 & 23570 & 23410 & 21530 & 23100 & 20930 & 19820 & 17780 \\
\hline$a$ & 33290 & 33040 & 33230 & 33890 & 31070 & 33850 & 28970 & 30530 & 32020 & 29650 & 28820 & 27740 \\
\hline
\end{tabular}

a Discharge, in acre-feet, for Hat Creek No. 2 Powerplant (station 11359300), provided by Pacific Gas \& Electric Co. 
LOCATION.-Lat 4057'01", long 121`32’39", in SE 1/4 NW 1/4 sec.29, T.36 N., R.4 E., Shasta County, Hydrologic Unit 18020003, on right bank of Hat No. 2 Power Canal, $75 \mathrm{ft}$ downstream from Hat No. 2 Diversion Dam on Hat Creek, 7.9 mi northeast of Burney.

PERIOD OF RECORD.-Oct. 1 to Dec. 9, 1987 (fragmentary), Dec. 10, 1987, to current year (operated as a low-flow station only). Unpublished fragmentary records for water years 1979-87 available in files of the U.S. Geological Survey.

GAGE.-Water-stage recorder and Parshall flume. Elevation of gage is 2,980 ft sea level, from topographic map.

REMARKS.- This station records fishwater release only. The minimum release requirement is $8.0 \mathrm{ft}^{3} / \mathrm{s}$ at all times. Flow is computed to $15 \mathrm{ft}^{3} / \mathrm{s}$. See schematic diagram of Pit and McCloud River Basins.

COOPERATION.-Records were collected by Pacific Gas \& Electric Co., under general supervision of the U.S. Geological Survey, in connection with a Federal Energy Regulatory Commission project.

DISCHARGE, CUBIC FEET PER SECOND, WATER YEAR OCTOBER 1998 TO SEPTEMBER 1999 DAILY MEAN VALUES

\begin{tabular}{|c|c|c|c|c|c|c|c|c|c|c|c|c|}
\hline DAY & $\mathrm{OCT}$ & NOV & $\mathrm{DEC}$ & JAN & FEB & MAR & APR & MAY & JUN & JUL & AUG & SEP \\
\hline 1 & 9.7 & 9.5 & 9.5 & 10 & 9.4 & 8.7 & 9.6 & 9.7 & 9.7 & 9.0 & 9.3 & 9.3 \\
\hline 2 & 9.7 & 9.5 & 9.5 & 9.9 & 9.4 & 8.9 & 9.7 & 9.7 & 9.5 & 9.6 & 9.4 & 9.3 \\
\hline 3 & 9.7 & 9.6 & 9.5 & 9.9 & 9.4 & 8.9 & 9.6 & 9.8 & 9.5 & 9.7 & 9.5 & 9.4 \\
\hline 4 & 9.7 & 9.6 & 9.5 & 9.9 & 9.4 & 9.0 & 9.5 & 9.7 & 9.4 & 9.9 & 9.5 & 9.2 \\
\hline 5 & 9.6 & 9.6 & 9.5 & 9.6 & 9.4 & 9.1 & 9.5 & 9.6 & 9.3 & 9.7 & 9.3 & 9.1 \\
\hline 6 & 9.4 & 9.5 & 9.5 & 9.2 & 9.4 & 9.1 & 9.5 & 9.3 & 9.1 & 9.7 & 9.3 & 9.1 \\
\hline 7 & 9.4 & 9.6 & 9.4 & 9.2 & 9.6 & 9.1 & 9.4 & 9.5 & 9.2 & 10 & 9.1 & 9.2 \\
\hline 8 & 9.5 & 9.7 & 9.4 & 9.2 & 9.7 & 9.1 & 9.4 & 9.6 & 9.2 & 9.5 & 9.1 & 9.0 \\
\hline 9 & 9.6 & 9.6 & 9.4 & 9.2 & 9.5 & 9.1 & 9.6 & 9.7 & 9.1 & 9.0 & 9.4 & 9.3 \\
\hline 10 & 9.4 & 9.5 & 9.3 & 9.1 & 9.3 & 9.1 & 9.7 & 9.7 & 9.0 & 9.1 & 9.4 & 9.1 \\
\hline 11 & 9.5 & 9.6 & 9.3 & 9.1 & 9.4 & 9.1 & 9.5 & 9.5 & 8.9 & 9.3 & 9.4 & 9.1 \\
\hline 12 & 9.5 & 9.5 & 9.4 & 9.1 & 9.1 & 9.1 & 9.4 & 9.6 & 9.0 & 9.1 & 9.4 & 9.5 \\
\hline 13 & 9.5 & 9.5 & 9.4 & 9.1 & 9.1 & 9.1 & 9.4 & 9.4 & 8.7 & 9.0 & 9.4 & 9.6 \\
\hline 14 & 9.6 & 9.5 & 9.5 & 9.1 & 9.1 & 9.1 & 9.6 & 9.3 & 9.2 & 9.7 & 9.3 & 9.6 \\
\hline 15 & 9.6 & 9.4 & 9.5 & 9.3 & 9.1 & 9.1 & 9.5 & 9.5 & 9.4 & 9.3 & 9.2 & 9.6 \\
\hline 16 & 9.6 & 9.4 & --- & 9.4 & 9.1 & 9.2 & 9.6 & 9.6 & 9.2 & 9.3 & 9.0 & 9.7 \\
\hline 17 & 9.7 & 9.4 & 9.3 & 9.4 & 9.1 & 9.3 & 9.6 & 9.7 & 9.0 & 9.5 & 9.2 & 9.5 \\
\hline 18 & 9.7 & 9.4 & 9.2 & 9.4 & 9.1 & 9.2 & 9.5 & 9.6 & 9.1 & 9.7 & 9.5 & 9.7 \\
\hline 19 & 9.8 & 9.4 & 9.1 & 9.4 & 9.1 & 9.1 & --- & 9.6 & 9.5 & 9.8 & 9.3 & 9.6 \\
\hline 20 & 9.6 & 9.4 & 9.2 & 9.4 & 9.1 & 9.1 & --- & 9.5 & 9.3 & 9.7 & 9.3 & 9.7 \\
\hline 21 & 9.3 & 9.4 & 9.2 & 9.4 & 9.1 & 9.1 & --- & 9.4 & 9.1 & 8.8 & 9.1 & 9.7 \\
\hline 22 & 9.3 & 9.5 & 9.2 & 9.4 & 9.1 & 9.1 & --- & 9.2 & 9.0 & 9.2 & 9.1 & 9.9 \\
\hline 23 & 9.2 & 9.7 & 9.3 & 9.4 & 9.1 & 9.1 & 9.5 & 9.1 & 9.1 & 9.8 & 9.2 & 10 \\
\hline 24 & 9.3 & 9.7 & e9. 4 & 9.4 & 9.1 & 9.1 & 9.2 & 9.2 & 9.0 & 9.4 & 9.6 & 10 \\
\hline 25 & 9.4 & 9.6 & e9. 8 & 9.4 & 9.2 & 9.1 & 9.2 & 9.3 & 9.7 & 9.4 & 9.5 & 10 \\
\hline 26 & 9.4 & 9.5 & e9. 9 & 9.4 & 9.0 & 9.1 & 9.1 & 9.3 & 9.4 & 9.3 & 9.7 & 10 \\
\hline 27 & 9.4 & 9.7 & e9.9 & 9.4 & 8.8 & 9.1 & 9.1 & 9.5 & 9.2 & 9.2 & 9.5 & 10 \\
\hline 28 & 9.4 & 9.6 & 9.9 & 9.3 & 8.8 & 9.1 & 9.3 & 9.8 & 9.3 & 9.4 & 9.1 & 10 \\
\hline 29 & 9.4 & 9.5 & 9.9 & 9.4 & --- & 9.1 & 9.4 & 9.7 & 9.4 & 9.7 & 9.2 & 10 \\
\hline 30 & 9.4 & 9.7 & 9.8 & 9.4 & --- & 9.6 & 9.6 & 9.7 & 9.3 & 9.5 & 9.1 & 10 \\
\hline 31 & 9.5 & --- & 10 & 9.4 & --- & 9.4 & --- & 9.7 & --- & 9.4 & 9.2 & --- \\
\hline TOTAL & 294.8 & 286.1 & --- & 291.2 & 258.0 & 282.4 & --- & 295.5 & 276.8 & 292.7 & 288.6 & 287.2 \\
\hline MEAN & 9.51 & 9.54 & --- & 9.39 & 9.21 & 9.11 & --- & 9.53 & 9.23 & 9.44 & 9.31 & 9.57 \\
\hline MAX & 9.8 & 9.7 & --- & 10 & 9.7 & 9.6 & --- & 9.8 & 9.7 & 10 & 9.7 & 10 \\
\hline MIN & 9.2 & 9.4 & --- & 9.1 & 8.8 & 8.7 & --- & 9.1 & 8.7 & 8.8 & 9.0 & 9.0 \\
\hline $\mathrm{AC}-\mathrm{FT}$ & 585 & 567 & --- & 578 & 512 & 560 & --- & 586 & 549 & 581 & 572 & 570 \\
\hline
\end{tabular}

e Estimated.

NOTE: Canal out of service Dec. 16 and Apr. 19-22 and all flow remained in the natural channel. 


\section{LAKE BRITTON NEAR BURNEY}

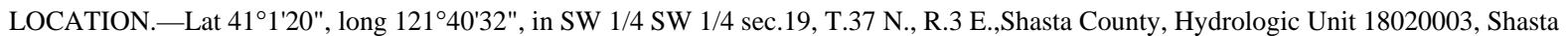
National Forest, at control house on right bank, $200 \mathrm{ft}$ upstream from dam on Pit River, 1.1 mi downstream from Clark Creek, 1.3 mi northwest of Burney Falls, and 9 mi north of Burney.

DRAINAGE AREA. - $4,607 \mathrm{mi}^{2}$, excluding Goose Lake Basin.

PERIOD OF RECORD, October 1965 to current year (monthend contents only). Fragmentary records for water years 1925-65 in files of the Pacific Gas \& Electric Co.

GAGE, remote telemark read once daily. Datum of gage is $19.53 \mathrm{ft}$ above sea level (levels by Pacific Gas \& Electric Co.). Monthend contents based on capacity table dated Dec. 1, 1976, provided by Pacific Gas \& Electric Co.

REMARKS.- - Reservoir is formed by gravity-type concrete dam. Storage began July 15,1925 . Usable capacity, 41,877 acre-ft between elevations $2,665.0 \mathrm{ft}$, invert of sluice gate, and 2,758.0 ft, top of flash boards. Dead storage, $30 \mathrm{acre}-\mathrm{ft}$. Normal operating pool is from elevation 2,744.0 $\mathrm{ft}$, capacity, 26,183 acre-ft, to 2,757.0 ft, capacity, 40,626 acre-ft. Figures given represent total contents. Lake is used for power generation and recreation. See schematic diagram of Pit and McCloud River Basins. Records prior to water year 1977 reported usable contents only.

COOPERATION.-Record of contents collected by Pacific Gas \& Electric Co., under general supervision of the U.S. Geological Survey, in connection with a Federal Energy Regulatory Commission project. Contents not rounded to U.S. Geological Survey standards.

EXTREMES (AT 2400 HOURS) FOR PERIOD OF RECORD.-Maximum total contents, 47,922 acre-ft, Feb. 20, 1986, elevation, 2,762.50 ft; minimum total contents, 26,755 acre-ft, Oct. 9, 1976, elevation, 2,744.60 ft.

EXTREMES (AT 2400 HOURS) FOR CURRENT YEAR.-Maximum contents, 49,427 acre-ft, Nov. 24, elevation, 2,756.84 ft; minimum, 30,482 acre-ft, Nov. 10, elevation, $2,748.29 \mathrm{ft}$.

\section{IRON CANYON RESERVOIR NEAR BIG BEND}

LOCATION.-Lat $41^{\circ} 02^{\prime} 41^{\prime \prime}$, long $121^{\circ} 58^{\prime} 52^{\prime \prime}$, in SW 1/4 SE 1/4 sec.21, T.37 N., R.1 W., Shasta County, Hydrologic Unit 18020003, Shasta National Forest, in control house on left bank, $500 \mathrm{ft}$ upstream from Iron Canyon Dam on Iron Canyon Creek, and 3.7 mi northwest of Big Bend.

DRAINAGE AREA, $11.1 \mathrm{mi}^{2}$. PERIOD OF RECORD, December 1965 to current year (monthend contents only).

GAGE, water-stage recorder. Datum of gage is sea level (levels by Pacific Gas \& Electric Co.). Monthend contents based on capacity table dated May 17, 1965, provided by Pacific Gas \& Electric Co.

REMARKS.-Reservoir is formed by a rockfill dam completed in 1965 . Usable capacity is 24,197 acre- $\mathrm{ft}$ between elevations $2,525.00 \mathrm{ft}$, invert of sluice pipe, and 2,665.00 ft, crest of spillway. Dead storage, 44 acre-ft. Normal operating pool is from elevation 2,565.0 ft, capacity, 990 acre-ft, to 2,664.0 ft, capacity, 23,738 acre-ft. Water is diverted from Lake McCloud (station 11367740) through a tunnel to Iron Canyon Reservoir and then into the Pit River via James B. Black Powerplant (station 11363910). Figures given represent total contents. Water is used for power generation and recreation. See schematic diagram of Pit and McCloud River Basins.

COOPERATION.-Record of contents collected by Pacific Gas \& Electric Co., under general supervision of the U.S. Geological Survey, in connection with a Federal Energy Regulatory Commission project. Contents not rounded to U.S. Geological Survey standards.

EXTREMES (AT 2400 HOURS) FOR PERIOD OF RECORD.-Maximum contents, 23,539 acre-ft, May 16, 22, 1977, elevation, 2,663.60 ft; normal minimum since reservoir first filled, 2,860 acre-ft, May 23, 24, 29, June 2, 7, 9, 14, 23, 24, 1966, elevation, 2,590.00 ft. Contents reduced to 195 acre-ft, elevation, 2,540.00 ft, Feb. 10, 1971, when reservoir was drained for inspection.

EXTREMES (AT 2400 HOURS) FOR CURRENT YEAR.-Maximum contents, 13,207 acre-ft, Oct. 11, elevation, 2,638.50 ft; minimum, 3,344 acre-ft, Jan. 30, elevation, 2,594.20 ft.

\section{LAKE McCLOUD NEAR McCLOUD}

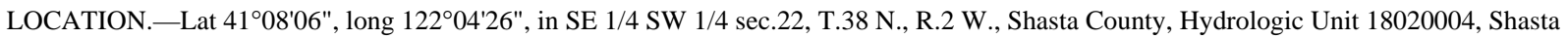
National Forest, on McCloud Dam near spillway on McCloud River, $200 \mathrm{ft}$ downstream from Panther Creek, and 8.8 mi southeast of McCloud.

DRAINAGE AREA, $403 \mathrm{mi}^{2}$. PERIOD OF RECORD, October 1965 to current year (monthend contents only).

GAGE, water-stage recorder. Datum of gage is sea level (levels by Pacific Gas \& Electric Co.). Monthend contents based on capacity table dated June 29, 1965, provided by Pacific Gas \& Electric Co.

REMARKS.-Reservoir is formed by a rockfill dam completed in 1965. Usable capacity, 35,231 acre-ft between elevations 2,471.30 ft, invert of sluice pipe, and 2,680.00 ft, maximum operational water surface. Dead storage, 3 acre- $\mathrm{ft}$. Normal operating pool is from elevation 2,635.00 ft, capacity, 16,425 acre-ft, to 2,680.00 ft, capacity, 35,234 acre-ft. Water is diverted from Lake McCloud (station 11367740) through a diversion tunnel to Iron Canyon Reservoir (station 11363920) and then into the Pit River via James B. Black Powerplant (station 11363910). Figures given represent total contents. Water is used for power generation and recreation. See schematic diagram of Pit and McCloud River Basins.

COOPERATION.-Record of contents collected by Pacific Gas \& Electric Co., under general supervision of the U.S. Geological Survey, in connection with a Federal Energy Regulatory Commission project. Contents not rounded to U.S. Geological Survey standards.

EXTREMES (AT 2400 HOURS) FOR PERIOD OF RECORD.-Maximum contents, 35,967 acre-ft, Jan. 15, 1974, elevation, 2,681.40 ft; minimum since reservoir first filled, 13,017 acre-ft, Oct. 14-22, 1981, elevation, 2,632.50 ft.

EXTREMES (AT 2400 HOURS) FOR CURRENT YEAR.-Maximum contents, 34,849 acre-ft, Apr. 25, elevation, 2,679.30 ft; minimum, 16,518 acre-ft, Nov. 13, elevation, 2,635.30 ft. 
RESERVOIRS IN PIT AND McCLOUD RIVER BASINS, CA—Continued

MONTHEND ELEVATION AND CONTENTS AT 2400 HOURS, WATER YEAR OCTOBER 1998 TO SEPTEMBER 1999

\begin{tabular}{|c|c|c|c|c|c|c|c|c|c|}
\hline & 113614 & LAKE BF & RITTON & 11363920 IR & ON CAN & YON RESERVOIR & 113677 & LAKE M & IcCLOUD \\
\hline Date & $\begin{array}{l}\text { Elevation } \\
\quad(\mathrm{ft})\end{array}$ & $\begin{array}{l}\text { Contents } \\
\text { (acre- } \\
\text { ft) }\end{array}$ & $\begin{array}{l}\text { Change in } \\
\text { contents } \\
\text { (acre- } \\
\mathrm{ft})\end{array}$ & $\begin{array}{c}\text { Elevation } \\
\text { (ft) }\end{array}$ & $\begin{array}{l}\text { Contents } \\
\text { (acre- } \\
\text { ft) }\end{array}$ & $\begin{array}{l}\text { Change in } \\
\text { contents } \\
\text { (acre- } \\
\text { ft) }\end{array}$ & $\begin{array}{l}\text { Elevation } \\
\quad(\mathrm{ft})\end{array}$ & $\begin{array}{l}\text { Contents } \\
\text { acre- } \\
\text { ft) }\end{array}$ & $\begin{array}{l}\text { Change in } \\
\text { contents } \\
\text { (acre- } \\
\mathrm{ft} \text { ) }\end{array}$ \\
\hline Sept. 30 . & $.2,752.50$ & 35,154 & - & $2,625.30$ & 9,290 & - & $2,660.40$ & 25,961 & - \\
\hline Oct. $31 \ldots$ & $.2,749.60$ & 31,889 & $-3,265$ & $2,606.70$ & 5,182 & $-4,108$ & $2,653.00$ & 22,898 & $-3,063$ \\
\hline Nov. $30 \ldots \ldots$ & $.2,755.84$ & 39,167 & $+7,278$ & $2,607.50$ & 5,325 & +143 & $2,656.10$ & 24,159 & $-1,261$ \\
\hline Dec. $31 \ldots$ & $.2,753.38$ & 36,185 & $-2,982$ & $2,612.10$ & 6,195 & +870 & $2,649.30$ & 21,454 & $-2,705$ \\
\hline CAL YR 1998 & 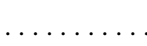 & 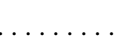 & $+2,350$ & & & $-2,394$ & & & +453 \\
\hline Jan. $31 \ldots$ & $.2,756.17$ & 39,581 & $+3,396$ & $2,598.70$ & 3,931 & $-2,264$ & $2,661.30$ & 26,365 & $+4,911$ \\
\hline Feb. $28 \ldots$ & $.2,756.04$ & 39,417 & -164 & $2,612.20$ & 6,221 & $+2,290$ & $2,661.70$ & 26,534 & +169 \\
\hline Mar. 31.... & $.2,755.27$ & 38,469 & -948 & $2,608.60$ & 5,530 & -691 & $2,673.70$ & 32,031 & $+5,497$ \\
\hline Apr. $30 \ldots$. & $.2,756.26$ & 39,694 & $+1,225$ & $2,619.30$ & 7,765 & $+2,235$ & $2,678.00$ & 34,217 & $+2,186$ \\
\hline May $31 . . .$. & $.2,755.90$ & 39,242 & -452 & $2,616.90$ & 7,226 & -539 & $2,677.70$ & 34,084 & -133 \\
\hline June $30 \ldots$ & $.2,753.34$ & 36,138 & $-3,104$ & $2,622.10$ & 8,460 & $+1,234$ & $2,671.90$ & 31,207 & $-2,877$ \\
\hline July $31 \ldots$ & $.2,753.64$ & 36,491 & +353 & $2,622.50$ & 8,559 & +99 & $2,660.10$ & 25,837 & $-5,370$ \\
\hline Aug. 31. . . . . & $.2,755.54$ & 38,792 & $+2,301$ & $2,627.40$ & 9,860 & $+1,301$ & $2,650.40$ & 21,874 & $-3,963$ \\
\hline Sept. $30 \ldots$. . & $.2,752.12$ & 34,717 & $-4,075$ & $2,616.40$ & 7,118 & $-2,742$ & $2,643.60$ & 19,338 & $-2,536$ \\
\hline WTR YR 1999 & & & -437 & & & $-2,172$ & & & $-6,623$ \\
\hline
\end{tabular}




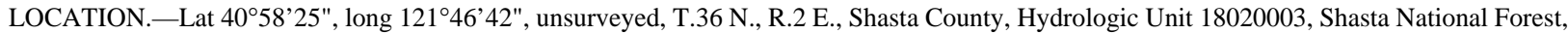
on right bank, 0.6 mi downstream from Ruling Creek, 1.3 mi downstream from Pit No. 4 Dam, and 2.7 mi downstream from Pit No. 3 Powerplant.

DRAINAGE AREA.-4,648 $\mathrm{mi}^{2}$, excluding Goose Lake Basin.

PERIOD OF RECORD.-May 1922 to current year. Monthly discharge only for some periods, published in WSP 1315-A. Published as "near Pecks Bridge" April to October 1922, and as "at Lindsay Flat" November 1922 to June 1927.

REVISED RECORDS.-WSP 843: 1935(M). WSP 1315-A: 1928(M). WDR CA-75-4: Drainage area.

GAGE.-Water-stage recorder. Elevation of gage is 2,358 ft above sea level, from river-profile map. Prior to November 1922, water-stage recorder at site at Pecks Bridge 7.4 mi upstream at different datum. November 1922 to June 20, 1927, at site at Lindsay Flat 1.8 mi upstream at different datum. June 20, 1927, to Sept. 5, 1990, at site $200 \mathrm{ft}$ downstream at datum $0.15 \mathrm{ft}$ lower.

REMARKS.- - Low flow completely regulated by small reservoirs and powerplants, total usable reservoir capacity, 253,000 acre-ft. Many diversions upstream from station; diversion to Pit No. 4 Powerplant began June 9, 1955. See schematic diagram of Pit and McCloud River Basins.

COOPERATION.-Records were collected by Pacific Gas \& Electric Co., under general supervision of the U.S. Geological Survey, in connection with a Federal Energy Regulatory Commission project.

EXTREMES FOR PERIOD OF RECORD.-Maximum discharge, $33,700 \mathrm{ft}^{3} / \mathrm{s}$, Feb. 20, 1986, gage height, $18.70 \mathrm{ft}$; minimum daily, prior to diversion to Pit No. 4 Powerplant in 1955, $234 \mathrm{ft}^{3} / \mathrm{s}$, Sept. 13, 1953. Minimum daily, since diversion to Pit No. 4 Powerplant, $22 \mathrm{ft}^{3} / \mathrm{s}$ Dec. 2-4, 1969.

DISCHARGE, CUBIC FEET PER SECOND, WATER YEAR OCTOBER 1998 TO SEPTEMBER 1999 DAILY MEAN VALUES

\begin{tabular}{|c|c|c|c|c|c|c|c|c|c|c|c|c|}
\hline DAY & $\mathrm{OCT}$ & NOV & $\mathrm{DEC}$ & JAN & FEB & MAR & APR & MAY & JUN & JUL & AUG & SEP \\
\hline 1 & 165 & 164 & 6150 & 163 & 162 & 3690 & 1160 & 407 & 173 & 180 & 181 & 178 \\
\hline 2 & 182 & 165 & 5220 & 165 & 162 & 4380 & 1020 & 406 & 176 & 182 & 186 & 177 \\
\hline 3 & 182 & 167 & 5510 & 163 & 162 & 5340 & 907 & 419 & 454 & 177 & 188 & 179 \\
\hline 4 & 177 & 166 & 6050 & 163 & 164 & 6190 & 774 & 851 & 635 & 178 & 185 & 179 \\
\hline 5 & 176 & 162 & 4580 & 162 & 163 & 6430 & 266 & 1630 & 461 & 177 & 181 & 177 \\
\hline 6 & 166 & 167 & 3610 & 162 & 169 & 5500 & 548 & 782 & 179 & 180 & 178 & 176 \\
\hline 7 & 172 & 166 & 2420 & 165 & 164 & 4560 & 802 & 617 & 181 & 189 & 180 & 180 \\
\hline 8 & 170 & 164 & 181 & 164 & 163 & 4650 & 947 & 389 & 185 & 185 & 186 & 182 \\
\hline 9 & 169 & 163 & 169 & 163 & 6140 & 5100 & 486 & 196 & 191 & 185 & 186 & 182 \\
\hline 10 & 176 & 174 & 172 & 160 & 4750 & 4190 & 163 & 161 & 191 & 189 & 184 & 180 \\
\hline 11 & 167 & 184 & 163 & 163 & 4170 & 2420 & 393 & 159 & 191 & 192 & 189 & 183 \\
\hline 12 & 169 & 168 & 169 & 167 & 4030 & 1950 & 1250 & 169 & 192 & 1470 & 187 & 181 \\
\hline 13 & 168 & 161 & 165 & 161 & 3440 & 1740 & 295 & 174 & 187 & 1460 & 190 & 182 \\
\hline 14 & 385 & 160 & 164 & 163 & 2780 & 1300 & 396 & 165 & 183 & 214 & 188 & 180 \\
\hline 15 & 1030 & 160 & 167 & 160 & 1730 & 2850 & 981 & 165 & 183 & 202 & 187 & 177 \\
\hline 16 & 161 & 160 & 627 & 162 & 1450 & 3600 & 448 & 163 & 186 & 203 & 190 & 182 \\
\hline 17 & 163 & 161 & 596 & 161 & 1090 & 3400 & 390 & 160 & 184 & 198 & 197 & 179 \\
\hline 18 & 164 & 162 & 166 & 169 & 2260 & 3340 & 482 & 163 & 181 & 199 & 203 & 179 \\
\hline 19 & 167 & 163 & 163 & 161 & 2800 & 1580 & 539 & 160 & 182 & 191 & 205 & 182 \\
\hline 20 & 165 & 163 & 164 & 1420 & 2810 & 1570 & 935 & 162 & 183 & 190 & 202 & 178 \\
\hline 21 & 164 & 175 & 161 & 1400 & 2740 & 1620 & 1260 & 160 & 182 & 191 & 196 & 185 \\
\hline 22 & 164 & 164 & 164 & 1900 & 2420 & 1350 & 1260 & 162 & 184 & 197 & 194 & 185 \\
\hline 23 & 164 & 166 & 165 & 1940 & 1850 & 1590 & 1450 & 164 & 186 & 192 & 196 & 184 \\
\hline 24 & 172 & 163 & 179 & 2250 & 1630 & 1420 & 1180 & 167 & 188 & 191 & 188 & 184 \\
\hline 25 & 169 & 2000 & 167 & 1600 & 2320 & 1290 & 875 & 553 & 189 & 184 & 181 & 181 \\
\hline 26 & 162 & 1950 & 163 & 1880 & 3390 & 1750 & 332 & 185 & 186 & 181 & 181 & 183 \\
\hline 27 & 168 & 1600 & 163 & 1020 & 3620 & 1480 & 156 & 171 & 183 & 189 & 181 & 182 \\
\hline 28 & 163 & 1610 & 164 & 661 & 3210 & 1660 & 510 & 612 & 181 & 184 & 189 & 188 \\
\hline 29 & 164 & 1470 & 163 & 265 & --- & 1960 & 819 & 181 & 181 & 187 & 189 & 183 \\
\hline 30 & 164 & 3240 & 167 & 167 & --- & 1270 & 631 & 172 & 179 & 181 & 180 & 178 \\
\hline 31 & 165 & --- & 163 & 164 & --- & 1230 & --- & 173 & --- & 180 & 175 & --- \\
\hline TOTAL & 6293 & 15838 & 38425 & 17764 & 59939 & 90400 & 21655 & 10198 & 6517 & 8398 & 5823 & 5426 \\
\hline MEAN & 203 & 528 & 1240 & 573 & 2141 & 2916 & 722 & 329 & 217 & 271 & 188 & 181 \\
\hline MAX & 1030 & 3240 & 6150 & 2250 & 6140 & 6430 & 1450 & 1630 & 635 & 1470 & 205 & 188 \\
\hline MIN & 161 & 160 & 161 & 160 & 162 & 1230 & 156 & 159 & 173 & 177 & 175 & 176 \\
\hline $\mathrm{AC}-\mathrm{FT}$ & 12480 & 31410 & 76220 & 35230 & 118900 & 179300 & 42950 & 20230 & 12930 & 16660 & 11550 & 10760 \\
\hline a & 133900 & 144800 & 170900 & 157400 & 158800 & 174300 & 162300 & 140700 & 142400 & 111800 & 110200 & 113000 \\
\hline $\mathrm{b}$ & 156900 & 173400 & 186100 & 199500 & 209500 & 210300 & 227900 & 203900 & 158300 & 120100 & 120300 & 125700 \\
\hline
\end{tabular}

a Discharge, in acre-feet, for Pit No. 3 Powerplant (station 11362300), provided by Pacific Gas \& Electric Co.

b Diversion, in acre-feet, to Pit No. 4 Powerplant (station 11362600), provided by Pacific Gas \& Electric Co. 
11362500 PIT RIVER BELOW PIT NO. 4 DAM, CA—Continued

STATISTICS OF MONTHLY MEAN DATA FOR WATER YEARS 1927 - 1954, BY WATER YEAR (WY)

\begin{tabular}{|c|c|c|c|c|c|c|c|c|c|c|c|c|}
\hline & $\mathrm{OCT}$ & NOV & DEC & JAN & FEB & MAR & APR & MAY & JUN & JUL & AUG & SEP \\
\hline MEAN & 1945 & 2102 & 2458 & 2700 & 3338 & 3799 & 3766 & 2877 & 2307 & 1925 & 1833 & 1865 \\
\hline MAX & 2385 & 2544 & 5968 & 5523 & 6872 & 8510 & 11400 & 5507 & 4096 & 2652 & 2146 & 2318 \\
\hline$(W Y)$ & 1954 & 1954 & 1938 & 1953 & 1942 & 1938 & 1952 & 1938 & 1953 & 1952 & 1954 & 1953 \\
\hline MIN & 1571 & 1666 & 1745 & 1698 & 1742 & 1895 & 1730 & 1635 & 1612 & 1569 & 1509 & 1541 \\
\hline (WY) & 1935 & 1934 & 1935 & 1937 & 1933 & 1934 & 1934 & 1934 & 1934 & 1934 & 1934 & 1934 \\
\hline
\end{tabular}

SUMMARY STATISTICS

ANNUAL MEAN

HIGHEST ANNUAL MEAN

LOWEST ANNUAL MEAN

HIGHEST DAILY MEAN

LOWEST DAILY MEAN

ANNUAL SEVEN-DAY MINIMUM

INSTANTANEOUS PEAK STAGE

ANNUAL RUNOFF (AC-FT)

10 PERCENT EXCEEDS

50 PERCENT EXCEEDS

90 PERCENT EXCEEDS
INSTANTANEOUS PEAK FLOW

WATER YEARS 1927 - 1954

\begin{tabular}{|c|c|c|}
\hline 2572 & & \\
\hline 4066 & & 1952 \\
\hline 1703 & & 1934 \\
\hline 26200 & Dec 12 & 1937 \\
\hline 234 & Sep 13 & 1953 \\
\hline 1450 & Aug 2 & 1936 \\
\hline a30200 & Dec 12 & 1937 \\
\hline 17.90 & Dec 12 & 1937 \\
\hline 1863000 & & \\
\hline 3810 & & \\
\hline 2170 & & \\
\hline 1630 & & \\
\hline
\end{tabular}

a From rating curve extended above $12,000 \mathrm{ft}^{3} / \mathrm{s}$ on basis of velocity-area studies.

STATISTICS OF MONTHLY MEAN DATA FOR WATER YEARS 1955 - 1999, BY WATER YEAR (WY)

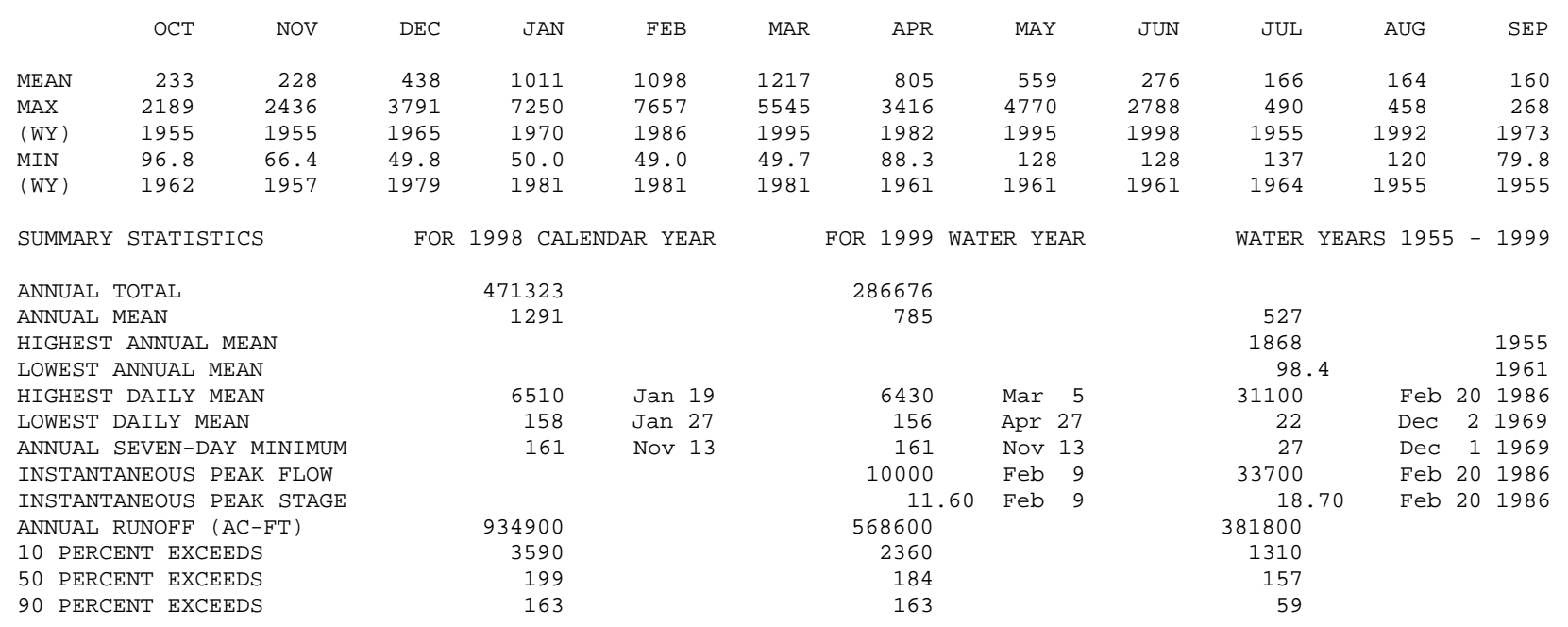




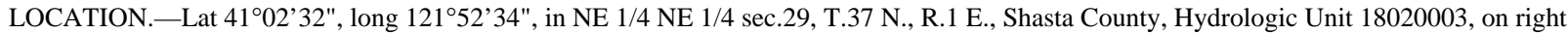
bank, $400 \mathrm{ft}$ upstream from Snowslide Creek, $0.3 \mathrm{mi}$ downstream from Bull Creek, and 2.3 mi northeast of Big Bend.

DRAINAGE AREA.- $-13.2 \mathrm{mi}^{2}$.

PERIOD OF RECORD.-October 1993 to September 1996; October 1996 to current year (operated as a low-flow station only).

GAGE.-Water-stage recorder and broad-crested weir; water-stage recorder and sharp-crested weir. Elevation of gages is 2,320 ft above sea level, from topographic map.

REMARKS.- - Records fair. Flow at this station has two components which are combined for publication: flow over a broad-crested weir (station 11362880) and flow over a sharp-crested weir (station 11362890). Water is diverted upstream of weirs through a tunnel to Nelson Creek Powerplant (station 11362800), returning to Nelson Creek at its confluence with the Pit River. Flow is computed to $100 \mathrm{ft}^{3} / \mathrm{s}$. See schematic diagram of Pit and McCloud River Basins.

EXTREMES FOR PERIOD OF RECORD.-Maximum discharge, $623 \mathrm{ft}^{3}$ /s, Feb. 19, 1996; minimum daily, $7.4 \mathrm{ft}^{3} / \mathrm{s}$, Sept. 8, $21,22,1994$.

DISCHARGE, CUBIC FEET PER SECOND, WATER YEAR OCTOBER 1998 TO SEPTEMBER 1999

\section{DAILY MEAN VALUES}

\begin{tabular}{|c|c|c|c|c|c|c|c|c|c|c|c|c|}
\hline DAY & $\mathrm{OCT}$ & NOV & $\mathrm{DEC}$ & JAN & FEB & MAR & APR & MAY & JUN & JUL & AUG & SEP \\
\hline 1 & 16 & 15 & --- & 17 & 26 & --- & 78 & 72 & 16 & 15 & 17 & 15 \\
\hline 2 & 16 & 15 & --- & 17 & 22 & --- & 78 & 75 & 18 & 15 & 17 & 15 \\
\hline 3 & 17 & 15 & --- & 17 & 17 & --- & 79 & --- & 16 & 15 & 17 & 14 \\
\hline 4 & 16 & 14 & 77 & 16 & 16 & --- & 69 & 83 & 15 & 15 & 17 & 14 \\
\hline 5 & 16 & 14 & 46 & 16 & 16 & --- & 69 & 77 & 15 & 15 & 16 & 14 \\
\hline 6 & 15 & 15 & 30 & 16 & 24 & --- & 64 & 75 & 15 & 15 & 17 & 14 \\
\hline 7 & 15 & 25 & 21 & 19 & --- & --- & 62 & 74 & 15 & 15 & 17 & 14 \\
\hline 8 & 15 & 20 & 23 & 16 & --- & --- & 71 & 70 & 16 & 15 & 17 & 14 \\
\hline 9 & 15 & 16 & 19 & 15 & --- & --- & 61 & 64 & 15 & 15 & 16 & 14 \\
\hline 10 & 15 & 17 & 18 & 16 & --- & --- & 60 & 57 & 16 & 15 & 16 & 14 \\
\hline 11 & 15 & 16 & 17 & 16 & --- & --- & 59 & 57 & 16 & 15 & 16 & 14 \\
\hline 12 & 15 & 17 & 15 & 16 & --- & --- & 62 & 51 & 16 & 15 & 16 & 14 \\
\hline 13 & 15 & 17 & 15 & 16 & 86 & --- & 68 & 48 & 16 & 15 & 16 & 14 \\
\hline 14 & 15 & 17 & 15 & 16 & 80 & --- & 74 & 43 & 15 & 15 & 16 & 14 \\
\hline 15 & 15 & 16 & 16 & 19 & 73 & --- & 78 & 40 & 16 & 15 & 16 & 14 \\
\hline 16 & 15 & 18 & 16 & 22 & 70 & --- & 81 & 40 & 15 & 15 & 16 & 14 \\
\hline 17 & 15 & 40 & 16 & 41 & 71 & --- & 87 & 41 & 15 & 15 & 15 & 14 \\
\hline 18 & 15 & 18 & 16 & 77 & 80 & --- & --- & 43 & 16 & 15 & 15 & 14 \\
\hline 19 & 15 & 15 & 16 & --- & 82 & --- & --- & 37 & 16 & 15 & 15 & 13 \\
\hline 20 & 15 & 17 & 16 & --- & 77 & 76 & --- & 34 & 16 & 15 & 15 & 13 \\
\hline 21 & 14 & 20 & 19 & --- & 75 & 76 & --- & 34 & 15 & 16 & 15 & 13 \\
\hline 22 & 14 & 72 & 20 & --- & 71 & 76 & --- & 43 & 15 & 15 & 15 & 13 \\
\hline 23 & 14 & --- & 18 & --- & 80 & 76 & 90 & 38 & 16 & 15 & 15 & 13 \\
\hline 24 & 15 & --- & 17 & --- & 90 & 90 & 83 & 34 & 15 & 15 & 15 & 13 \\
\hline 25 & 15 & 51 & 17 & --- & --- & --- & 85 & 32 & 15 & 15 & 15 & 13 \\
\hline 26 & 15 & 53 & 16 & --- & --- & --- & 88 & 31 & 15 & 15 & 15 & 13 \\
\hline 27 & 15 & 50 & 16 & 67 & --- & --- & 86 & 31 & 15 & 15 & 15 & 13 \\
\hline 28 & 16 & 23 & 16 & 54 & --- & --- & 80 & 26 & 15 & 15 & 15 & 13 \\
\hline 29 & 15 & 19 & 16 & 44 & --- & 90 & 74 & 22 & 15 & 15 & 15 & 13 \\
\hline 30 & 15 & --- & 16 & 38 & --- & 85 & 72 & 17 & 15 & 16 & 15 & 13 \\
\hline 31 & 15 & --- & 21 & 33 & --- & 83 & --- & 15 & --- & 17 & 15 & --- \\
\hline TOTAL & 469 & --- & --- & --- & --- & --- & --- & --- & 465 & 469 & 488 & 410 \\
\hline MEAN & 15.1 & --- & --- & --- & --- & --- & --- & --- & 15.5 & 15.1 & 15.7 & 13.7 \\
\hline MAX & 17 & --- & --- & --- & --- & --- & --- & --- & 18 & 17 & 17 & 15 \\
\hline MIN & 14 & --- & --- & --- & --- & --- & --- & --- & 15 & 15 & 15 & 13 \\
\hline$A C-F T$ & 930 & --- & --- & --- & --- & --- & --- & --- & 922 & 930 & 968 & 813 \\
\hline$a$ & .00 & 286 & 1300 & 1220 & 2740 & 2080 & 3020 & 3010 & 1380 & 283 & .00 & .00 \\
\hline
\end{tabular}

a Discharge, in acre-feet, for Nelson Creek Powerplant (station 11362800), provided by Sierra Pacific Industries. 
LOCATION.—Lat 41 $02^{\prime} 25^{\prime \prime}$, long $121^{\circ} 52$ '28", in NE 1/4 NE 1/4 sec.29, T.37 N., R.1 E., Shasta County, Hydrologic Unit 18020003, on right bank $700 \mathrm{ft}$ upstream from Nelson Creek and 2.3 mi northeast of Big Bend.

DRAINAGE AREA.- $8.18 \mathrm{mi}^{2}$.

PERIOD OF RECORD.-October 1993 to September 1996. October 1996 to current year (operated as a low-flow station only).

GAGE.-Water-stage recorder and broad-crested weir; water-stage recorder and sharp-crested weir. Elevation of gages is 2,360 ft above sea level, from topographic map.

REMARKS.--Records good. Flow at this station has two components which are combined for publication: flow over a broad-crested weir (station 11362940) and flow over a sharp-crested weir (station 11362945). Water is diverted upstream of weirs through a pipe to Nelson Creek (station 11362900). Flows computed to $50 \mathrm{ft}^{3} / \mathrm{s}$. See schematic diagram of Pit and McCloud River Basins.

EXTREMES FOR PERIOD OF RECORD.-Maximum discharge, $267 \mathrm{ft}^{3} / \mathrm{s}$, Mar. 15, 1995; minimum daily, $0.07 \mathrm{ft}^{3} / \mathrm{s}$, Aug. 12 to Sept. 23, 1994, and Oct. 11, 1994.

DISCHARGE, CUBIC FEET PER SECOND, WATER YEAR OCTOBER 1998 TO SEPTEMBER 1999

DAILY MEAN VALUES

\begin{tabular}{|c|c|c|c|c|c|c|c|c|c|c|c|c|}
\hline DAY & OCT & NOV & $\mathrm{DEC}$ & JAN & FEB & MAR & APR & MAY & JUN & JUL & AUG & SEP \\
\hline 1 & 6.1 & 5.7 & --- & 9.8 & 21 & 36 & 26 & 27 & 12 & 9.8 & 6.0 & 4.7 \\
\hline 2 & 6.0 & 5.6 & --- & 9.6 & 21 & 35 & 26 & 28 & 14 & 9.5 & 5.9 & 4.6 \\
\hline 3 & 5.9 & 5.5 & 36 & 9.4 & 21 & 35 & 26 & 31 & 14 & 9.4 & 5.8 & 4.6 \\
\hline 4 & 5.8 & 5.6 & 27 & 9.0 & 21 & 34 & 25 & 29 & 13 & 9.3 & 5.7 & 4.5 \\
\hline 5 & 5.6 & 5.6 & 20 & 8.9 & 21 & 32 & 26 & 28 & 12 & 9.0 & 5.6 & 4.5 \\
\hline 6 & 5.5 & 5.7 & 19 & 8.9 & 22 & 32 & 25 & 28 & 12 & 8.9 & 5.8 & 4.5 \\
\hline 7 & 5.5 & 6.1 & 19 & 8.9 & 35 & 31 & 25 & 28 & 11 & 8.7 & 6.0 & 4.3 \\
\hline 8 & 5.5 & 6.1 & 17 & 8.6 & 34 & 31 & 25 & 27 & 11 & 8.5 & 5.8 & 4.3 \\
\hline 9 & 5.5 & 5.8 & 16 & 8.6 & 45 & 30 & 24 & 26 & 11 & 8.2 & 5.5 & 4.2 \\
\hline 10 & 5.5 & 5.7 & 17 & 8.6 & 37 & 29 & 24 & 25 & 11 & 8.3 & 5.5 & 4.3 \\
\hline 11 & 5.5 & 5.0 & 16 & 8.6 & 33 & 28 & 24 & 25 & 11 & 7.9 & 5.6 & 4.0 \\
\hline 12 & 5.5 & 5.4 & 16 & 8.6 & 31 & 28 & 25 & 25 & 11 & 7.4 & 5.5 & 3.6 \\
\hline 13 & 5.5 & 5.4 & 15 & 8.4 & 30 & 27 & 25 & 24 & 11 & 7.3 & 5.5 & 3.5 \\
\hline 14 & 5.5 & 5.3 & 14 & 8.3 & 29 & 27 & 25 & 23 & 11 & 7.3 & 5.4 & 3.5 \\
\hline 15 & 5.5 & 5.6 & 14 & 9.2 & 27 & 27 & 26 & 22 & 11 & 7.2 & 5.3 & 3.4 \\
\hline 16 & 5.4 & 5.6 & 13 & 11 & 28 & 27 & 27 & 21 & 11 & 7.1 & 5.2 & 3.4 \\
\hline 17 & 5.4 & 9.3 & 13 & 16 & 29 & 27 & 27 & 20 & 11 & 7.1 & 5.2 & 3.3 \\
\hline 18 & 5.4 & 6.5 & 13 & 22 & 31 & 27 & 28 & 20 & 11 & 6.9 & 5.1 & 3.3 \\
\hline 19 & 5.6 & 5.8 & 13 & 26 & 29 & 27 & 29 & 20 & 11 & 6.8 & 5.0 & 3.3 \\
\hline 20 & 5.7 & 5.6 & 12 & 35 & 27 & 27 & 29 & 19 & 11 & 6.7 & 5.0 & 3.3 \\
\hline 21 & 5.8 & 12 & 12 & 33 & 27 & 27 & 29 & 19 & 11 & 6.8 & 4.9 & 3.3 \\
\hline 22 & 5.7 & 14 & 12 & 32 & 26 & 27 & 29 & 18 & 11 & 6.7 & 4.9 & 3.3 \\
\hline 23 & 5.6 & 21 & 12 & 37 & 27 & 27 & 28 & 18 & 11 & 6.6 & 4.8 & 3.3 \\
\hline 24 & 6.2 & --- & 12 & 33 & 27 & 29 & 28 & 17 & 11 & 6.5 & 4.9 & 3.2 \\
\hline 25 & 6.0 & 16 & 12 & 31 & 30 & 30 & 29 & 17 & 11 & 6.3 & 4.8 & 3.2 \\
\hline 26 & 5.7 & 15 & 11 & 29 & 28 & 30 & 30 & 16 & 11 & 6.2 & 4.8 & 3.1 \\
\hline 27 & 5.7 & 16 & 11 & 26 & 29 & 30 & 29 & 16 & 11 & 6.2 & 4.7 & 3.0 \\
\hline 28 & 6.0 & 13 & 11 & 24 & 35 & 30 & 28 & 15 & 10 & 6.2 & 4.7 & 3.0 \\
\hline 29 & 5.8 & 12 & 10 & 23 & --- & 28 & 27 & 14 & 10 & 6.1 & 4.5 & 3.0 \\
\hline 30 & 5.7 & 20 & 10 & 22 & --- & 28 & 27 & 13 & 9.9 & 5.9 & 4.7 & 3.0 \\
\hline 31 & 5.6 & --- & 10 & 22 & --- & 27 & --- & 12 & --- & 5.9 & 4.7 & --- \\
\hline TOTAL & 175.7 & --- & --- & 555.4 & 801 & 910 & 801 & 671 & 337.9 & 230.7 & 162.8 & 110.5 \\
\hline MEAN & 5.67 & --- & --- & 17.9 & 28.6 & 29.4 & 26.7 & 21.6 & 11.3 & 7.44 & 5.25 & 3.68 \\
\hline MAX & 6.2 & --- & --- & 37 & 45 & 36 & 30 & 31 & 14 & 9.8 & 6.0 & 4.7 \\
\hline MIN & 5.4 & --- & --- & 8.3 & 21 & 27 & 24 & 12 & 9.9 & 5.9 & 4.5 & 3.0 \\
\hline $\mathrm{AC}-\mathrm{FT}$ & 349 & --- & --- & 1100 & 1590 & 1800 & 1590 & 1330 & 670 & 458 & 323 & 219 \\
\hline
\end{tabular}




\section{PIT RIVER AT BIG BEND, CA}

LOCATION.-Lat 41 01 '10", long 121 '54'36", in NW 1/4 SW 1/4 sec.31, T.37 N., R.1 E., Shasta County, Hydrologic Unit 18020003, on left bank at Big Bend, 0.4 mi downstream from Nelson Creek, 1.5 mi upstream from Kosk Creek, and 3.1 mi downstream from Pit No. 5 Dam.

DRAINAGE AREA.-4,711 $\mathrm{mi}^{2}$, excluding Goose Lake Basin.

PERIOD OF RECORD.-October 1910 to current year. Monthly discharge only for some periods, published in WSP 1315-A. Published as "at Henderson" 1910-23.

REVISED RECORDS.-WSP 1345: 1911, 1914(M), 1916(M), 1917, 1928, 1935-36(M). WDR CA-75-4: Drainage area.

GAGE.-Water-stage recorder. Datum of gage is 1,674.47 ft above sea level. Prior to Dec. 28, 1912, nonrecording gage; Dec. 28, 1912, to June 21, 1924, water-stage recorder at same site, at datum $7.69 \mathrm{ft}$ higher. June 22, 1924, to Sept. 30, 1988, at site $200 \mathrm{ft}$ downstream at same datum.

REMARKS.-Low flow completely regulated by many reservoirs and powerplants, total usable reservoir capacity, about 253,000 acre-ft. Many diversions upstream from station; diversion to Pit No. 5 Powerplant (station 11362700) began May 1, 1944. See schematic diagram of Pit and McCloud River Basins.

COOPERATION.--Records were collected by Pacific Gas \& Electric Co., under general supervision of the U.S. Geological Survey, in connection with a Federal Energy Regulatory Commission project.

EXTREMES FOR PERIOD OF RECORD.-Maximum discharge, 49,000 ft 3 s, Jan. 25, 1970, gage height, $18.17 \mathrm{ft}$ in gage well, $19.0 \mathrm{ft}$ from floodmarks, site then in use, from rating curve extended above 17,000 ft $3 / \mathrm{s}$; maximum gage height, 18.70 ft, Feb. 20, 1986, site then in use; minimum daily, $692 \mathrm{ft}^{3} / \mathrm{s}$, July 9, 1925; since diversion to Pit No. 5 Powerplant, minimum daily, $34 \mathrm{ft}^{3} / \mathrm{s}, \mathrm{Mar}^{29}, 1955$.

EXTREMES FOR CURRENT YEAR.-Peak discharges greater than base discharge of 6,000 $\mathrm{ft}^{3} / \mathrm{s}$, or maximum:

$\begin{array}{cccccccc}\text { Date } & \text { Time } & \begin{array}{c}\text { Discharge } \\ \left(\mathrm{ft}^{3} / \mathrm{s}\right)\end{array} & \begin{array}{c}\text { Gage height } \\ (\mathrm{ft})\end{array} & \text { Date } & \text { Time } & \begin{array}{c}\text { Discharge } \\ \left(\mathrm{ft}^{3} / \mathrm{s}\right)\end{array} & \begin{array}{c}\text { Gage height } \\ (\mathrm{ft})\end{array} \\ \text { Dec. } 1 & 1015 & 9,060 & 12.15 & \text { Feb. } 17 & 0945 & 6,350 & 11.17 \\ \text { Feb. } 9 & 1745 & 13,900 & 13.56 & \text { Mar. } 4 & 1800 & 7,970\end{array}$

DISCHARGE, CUBIC FEET PER SECOND, WATER YEAR OCTOBER 1998 TO SEPTEMBER 1999 DAILY MEAN VALUES

\begin{tabular}{|c|c|c|c|c|c|c|c|c|c|c|c|c|}
\hline DAY & OCT & NOV & $\mathrm{DEC}$ & JAN & FEB & MAR & APR & MAY & JUN & JUL & AUG & SEP \\
\hline 1 & 150 & 152 & 4900 & 165 & 714 & 4620 & 1540 & 998 & 208 & 157 & 145 & 153 \\
\hline 2 & 151 & 161 & 4030 & 170 & 1280 & 5110 & 1480 & 767 & 217 & 156 & 144 & 143 \\
\hline 3 & 156 & 162 & 4650 & 173 & 1170 & 6060 & 1360 & 1080 & 216 & 161 & 145 & 146 \\
\hline 4 & 174 & 172 & 4320 & 171 & 1230 & 6990 & 1160 & 1270 & 207 & 154 & 144 & 144 \\
\hline 5 & 175 & 176 & 3260 & 163 & 1060 & 7130 & 683 & 1490 & 200 & 153 & 158 & 145 \\
\hline 6 & 148 & 184 & 2180 & 162 & 351 & 6240 & 769 & 1330 & 198 & 152 & 149 & 138 \\
\hline 7 & 144 & 203 & 1270 & 160 & 1120 & 5240 & 1240 & 1260 & 196 & 154 & 150 & 139 \\
\hline 8 & 148 & 185 & 437 & 162 & 1610 & 3900 & 1450 & 1040 & 195 & 154 & 149 & 138 \\
\hline 9 & 150 & 187 & 451 & 155 & 8150 & 3610 & 1170 & 654 & 187 & 157 & 145 & 139 \\
\hline 10 & 152 & 193 & 247 & 152 & 6950 & 3000 & 803 & 487 & 186 & 156 & 145 & 145 \\
\hline 11 & 177 & 187 & 212 & 163 & 5610 & 3210 & 845 & 434 & 185 & 147 & 146 & 137 \\
\hline 12 & 145 & 185 & 204 & 180 & 4670 & 2680 & 1710 & 494 & 186 & 148 & 145 & 142 \\
\hline 13 & 145 & 200 & 205 & 159 & 3960 & 1930 & 843 & 435 & 184 & 149 & 139 & 140 \\
\hline 14 & 142 & 190 & 239 & 175 & 3280 & 1940 & 707 & 581 & 176 & 150 & 144 & 157 \\
\hline 15 & 520 & 204 & 296 & 177 & 2300 & 1410 & 1430 & 620 & 181 & 150 & 140 & 164 \\
\hline 16 & 144 & 236 & 573 & 183 & 1840 & 1520 & 1180 & 532 & 178 & 150 & 145 & 148 \\
\hline 17 & 149 & 341 & 1320 & 262 & 1770 & 1370 & 944 & 335 & 181 & 152 & 147 & 142 \\
\hline 18 & 152 & 269 & 638 & 341 & 2560 & 1430 & 1040 & 245 & 176 & 147 & 140 & 146 \\
\hline 19 & 145 & 268 & 432 & 437 & 3280 & 1650 & 1120 & 250 & 170 & 148 & 147 & 144 \\
\hline 20 & 149 & 279 & 276 & 1690 & 3330 & 1600 & 1480 & 240 & 167 & 145 & 148 & 158 \\
\hline 21 & 142 & 434 & 227 & 2020 & 3290 & 1970 & 1640 & 233 & 170 & 150 & 140 & 150 \\
\hline 22 & 143 & 472 & 182 & 2490 & 2950 & 2060 & 1500 & 229 & 170 & 150 & 139 & 145 \\
\hline 23 & 144 & 608 & 180 & 2650 & 2520 & 2310 & 1700 & 227 & 169 & 150 & 139 & 157 \\
\hline 24 & 155 & 432 & 180 & 2800 & 2220 & 2010 & 1410 & 229 & 169 & 144 & 143 & 144 \\
\hline 25 & 146 & 2140 & 177 & 2100 & 2880 & 1910 & 1330 & 247 & 159 & 141 & 141 & 148 \\
\hline 26 & 146 & 2610 & 177 & 2310 & 4010 & 1920 & 826 & 225 & 154 & 145 & 143 & 144 \\
\hline 27 & 154 & 2190 & 182 & 1670 & 4190 & 1980 & 754 & 226 & 155 & 146 & 147 & 144 \\
\hline 28 & 153 & 2150 & 183 & 1160 & 4260 & 1710 & 1000 & 261 & 163 & 149 & 140 & 144 \\
\hline 29 & 140 & 2030 & 176 & 872 & --- & 1820 & 1220 & 216 & 156 & 146 & 143 & 152 \\
\hline 30 & 140 & 2290 & 172 & 505 & --- & 1660 & 1290 & 214 & 162 & 145 & 142 & 141 \\
\hline 31 & 145 & --- & 171 & 427 & --- & 1640 & --- & 210 & --- & 148 & 140 & --- \\
\hline TOTAL & 5024 & 19490 & 32147 & 24404 & 82555 & 91630 & 35624 & 17059 & 5421 & 4654 & 4472 & 4377 \\
\hline MEAN & 162 & 650 & 1037 & 787 & 2948 & 2956 & 1187 & 550 & 181 & 150 & 144 & 146 \\
\hline MAX & 520 & 2610 & 4900 & 2800 & 8150 & 7130 & 1710 & 1490 & 217 & 161 & 158 & 164 \\
\hline MIN & 140 & 152 & 171 & 152 & 351 & 1370 & 683 & 210 & 154 & 141 & 139 & 137 \\
\hline $\mathrm{AC}-\mathrm{FT}$ & 9970 & 38660 & 63760 & 48410 & 163700 & 181700 & 70660 & 33840 & 10750 & 9230 & 8870 & 8680 \\
\hline
\end{tabular}


11363000 PIT RIVER AT BIG BEND, CA—Continued

STATISTICS OF MONTHLY MEAN DATA FOR WATER YEARS 1911 - 1943, BY WATER YEAR (WY)

\begin{tabular}{|c|c|c|c|c|c|c|c|c|c|c|c|c|}
\hline & $\mathrm{OCT}$ & NOV & DEC & JAN & FEB & MAR & APR & MAY & JUN & JUL & AUG & SEP \\
\hline MEAN & 2206 & 2373 & 2676 & 3000 & 3927 & 4449 & 4446 & 3229 & 2520 & 2214 & 2100 & 2107 \\
\hline MAX & 3021 & 3186 & 6792 & 7675 & 7989 & 9953 & 11410 & 6216 & 3763 & 3218 & 2987 & 2975 \\
\hline$(W Y)$ & 1912 & 1912 & 1938 & 1914 & 1942 & 1938 & 1917 & 1938 & 1911 & 1911 & 1911 & 1911 \\
\hline MIN & 1607 & 1740 & 1764 & 1750 & 1746 & 2051 & 1860 & 1734 & 1672 & 1584 & 1526 & 1565 \\
\hline (WY) & 1935 & 1934 & 1935 & 1937 & 1933 & 1931 & 1934 & 1934 & 1934 & 1934 & 1934 & 1934 \\
\hline
\end{tabular}

SUMMARY STATISTICS

ANNUAL MEAN

HIGHEST ANNUAL MEAN

LOWEST ANNUAL MEAN

HIGHEST DAILY MEAN

LOWEST DAILY MEAN

ANNUAL SEVEN-DAY MINIMUM

INSTANTANEOUS PEAK FLOW

INSTANTANEOUS PEAK STAGE

ANNUAL RUNOFF (AC-FT)

10 PERCENT EXCEEDS

50 PERCENT EXCEEDS

90 PERCENT EXCEEDS
WATER YEARS 1911 - 1943

$\begin{array}{ccrr}2931 & & & \\ 4597 & & & 1938 \\ 1787 & & 1934 \\ 30300 & \text { Dec } 12 & 1937 \\ 692 & \text { Jul } & 9 & 1925 \\ 915 & \text { Jul } & 4 & 1925 \\ \text { a34200 } & \text { Dec } 12 & 1937 \\ 16.26 & \text { Dec } 12 & 1937 \\ 2123000 & & & \\ 4520 & & & \\ 2440 & & & \\ 1750 & & & \end{array}$

a From rating extended above $11,000 \mathrm{ft}^{3} / \mathrm{s}$ on basis of velocity-area studies.

STATISTICS OF MONTHLY MEAN DATA FOR WATER YEARS 1944 - 1999, BY WATER YEAR (WY)

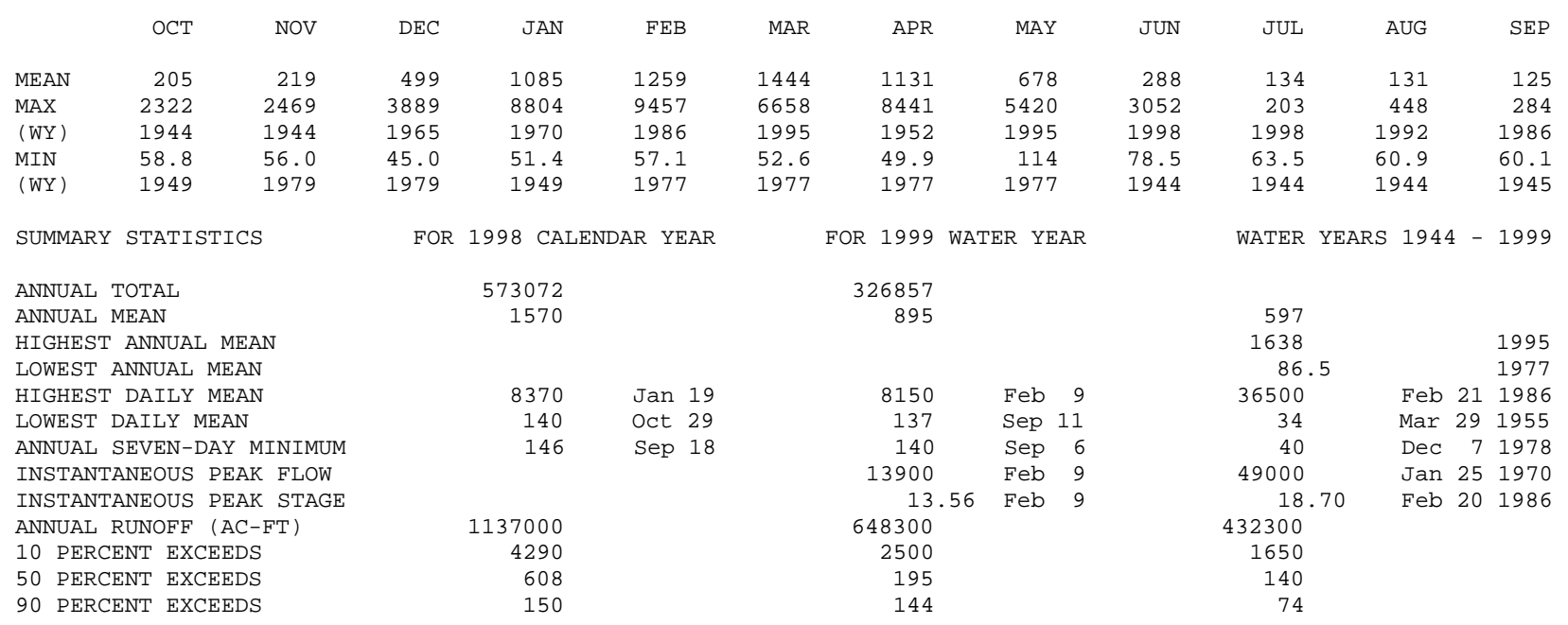


11363910 JAMES B. BLACK POWERPLANT NEAR BIG BEND, CA

LOCATION.-Lat 4059'12", long 121 58’35", in SW 1/4 SE 1/4 sec.9, T.36 N., R.1 W., Shasta County, Hydrologic Unit 18020003, at powerplant on right bank of Pit River and 5.8 mi downstream from Big Bend.

PERIOD OF RECORD.-December 1965 to current year.

GAGE.-Discharge computed from powerplant output.

REMARKS.-Water is diverted from Lake McCloud (station 11367740) at SE 1/4 SW 1/4 sec.22, T.38 N., R.2 W., through McCloud-Iron Canyon Diversion Tunnel (station 11367720) to Iron Canyon Reservoir (station 11363920), then through the penstock for powerplant and into the Pit River. Records are combined flow of diversion from McCloud River at McCloud Dam plus Iron Canyon Creek. See schematic diagram of Pit and McCloud River Basins.

COOPERATION.-Records were collected by Pacific Gas \& Electric Co., under general supervision of the U.S. Geological Survey, in connection with a Federal Energy Regulatory Commission project.

EXTREMES FOR PERIOD OF RECORD.-Maximum daily discharge, 2,420 ft³ s, July 15, 1966; no flow several days most years.

DISCHARGE, CUBIC FEET PER SECOND, WATER YEAR OCTOBER 1998 TO SEPTEMBER 1999 DAILY MEAN VALUES

\begin{tabular}{|c|c|c|c|c|c|c|c|c|c|c|c|c|}
\hline DAY & OCT & NOV & $\mathrm{DEC}$ & JAN & FEB & MAR & $A P R$ & MAY & JUN & JUL & AUG & SEP \\
\hline 1 & 1300 & 821 & 1590 & 1010 & 1290 & 1230 & 1660 & 1320 & 1170 & 1020 & 897 & 818 \\
\hline 2 & 1020 & 1270 & 1920 & 1100 & 1280 & 1500 & 1800 & 1590 & 1040 & 1110 & 1010 & 765 \\
\hline 3 & .00 & 1190 & 1920 & 1290 & 1260 & 1610 & 1450 & 1300 & 1200 & 114 & 1250 & 681 \\
\hline 4 & .00 & 1140 & 1420 & 521 & 1170 & 1630 & 992 & 1270 & 1200 & 688 & 760 & 820 \\
\hline 5 & 1320 & 1590 & 1910 & 1140 & 1530 & 1750 & 1380 & 1230 & 1330 & 1080 & 758 & 1360 \\
\hline 6 & 1500 & 1160 & 1280 & 1430 & 1410 & 1650 & 1380 & 1160 & 1200 & 868 & 727 & 1150 \\
\hline 7 & 1580 & 1050 & 1340 & 1360 & 1270 & 1560 & 1630 & 1130 & 1340 & 408 & 460 & 1090 \\
\hline 8 & 1300 & 1140 & 1260 & 1200 & 1430 & 1380 & 1560 & 1320 & 1130 & 1210 & 893 & 1100 \\
\hline 9 & 787 & 1150 & .00 & 1270 & 1430 & 1240 & 1250 & 1220 & 969 & 1290 & 1240 & 845 \\
\hline 10 & .00 & 1140 & $1110^{\circ}$ & 639 & 1460 & 1330 & 1480 & 1300 & 1390 & 1430 & 1080 & 850 \\
\hline 11 & .00 & 911 & 1130 & 687 & 1380 & 1280 & 1250 & 1420 & 1180 & 1540 & 1320 & 701 \\
\hline 12 & 1310 & 1010 & 1460 & 687 & 1340 & 1690 & 1300 & 1280 & 1020 & 1300 & 557 & 772 \\
\hline 13 & 1580 & 612 & 1340 & 918 & 1390 & 1390 & 1240 & 1320 & 634 & 1970 & 880 & 1110 \\
\hline 14 & 1200 & 617 & 1180 & 967 & 1330 & 1210 & 591 & 1300 & 1090 & 1970 & 578 & 1060 \\
\hline 15 & 480 & 38 & 1320 & 1240 & 1330 & 1320 & 1420 & 1390 & 907 & 1090 & 540 & 916 \\
\hline 16 & 1710 & 866 & 1360 & 953 & 958 & 1420 & 1410 & 1300 & 1380 & 188 & 1240 & 826 \\
\hline 17 & 1190 & 1110 & 1350 & 1140 & 1420 & 1320 & 1400 & 1090 & 1020 & 511 & 1130 & 442 \\
\hline 18 & 1310 & 965 & 1360 & 1160 & 1390 & 1450 & 1320 & 1160 & 1460 & 1010 & 1220 & 456 \\
\hline 19 & 1300 & 946 & 237 & 1340 & 1420 & 1290 & 1240 & 1050 & 1120 & 1320 & 1160 & 782 \\
\hline 20 & 1070 & 845 & 794 & 1480 & 1380 & 1400 & 224 & 1030 & 1000 & 1110 & 1250 & 875 \\
\hline 21 & 1190 & 899 & 1870 & 1330 & 1340 & 1500 & 620 & 1560 & 1030 & 1400 & 453 & 1210 \\
\hline 22 & 1230 & 928 & 1770 & 1740 & 1390 & 1300 & 1120 & 1500 & 1170 & 1110 & 625 & 1170 \\
\hline 23 & 1440 & 902 & 1360 & 1470 & 1210 & 1380 & 1840 & 1320 & 1430 & 775 & 985 & 956 \\
\hline 24 & 1350 & 1360 & 1100 & 1450 & 1320 & 1620 & 1360 & 1200 & 1390 & 644 & 887 & 699 \\
\hline 25 & 1250 & 1770 & 1170 & 1100 & 1050 & 1460 & 1260 & 1360 & 1170 & 848 & 1210 & 928 \\
\hline 26 & 1260 & 1560 & 1090 & 895 & 1060 & 1010 & 1340 & 1220 & 1250 & 1370 & 910 & 523 \\
\hline 27 & 1290 & 1470 & 1160 & 964 & 1170 & 1320 & 1220 & 1240 & 975 & 1440 & 873 & 928 \\
\hline 28 & 1160 & 1240 & 1210 & 1200 & 1110 & 1170 & 1360 & 1260 & 1100 & 1320 & 994 & 1020 \\
\hline 29 & 1200 & 705 & 941 & 1890 & --- & 1370 & 1130 & 1140 & 1180 & 1080 & 1110 & 789 \\
\hline 30 & 1070 & 1160 & 732 & 1820 & --- & 1620 & 1280 & 1180 & 1160 & 1570 & 402 & 900 \\
\hline 31 & 876 & --- & 792 & 1110 & --- & 1690 & --- & 1310 & --- & 346 & 351 & --- \\
\hline TOTAL3 & 33273.00 & 31565 & 38476.00 & 36501 & 36518 & 44090 & 38507 & 39470 & 34635 & 33130 & 27750 & 26542 \\
\hline MEAN & 1073 & 1052 & 1241 & 1177 & 1304 & 1422 & 1284 & 1273 & 1154 & 1069 & 895 & 885 \\
\hline MAX & 1710 & 1770 & 1920 & 1890 & 1530 & 1750 & 1840 & 1590 & 1460 & 1970 & 1320 & 1360 \\
\hline MIN & .00 & 38 & .00 & 521 & 958 & 1010 & 224 & 1030 & 634 & 114 & 351 & 442 \\
\hline $\mathrm{AC}-\mathrm{FT}$ & 66000 & 62610 & 76320 & 72400 & 72430 & 87450 & 76380 & 78290 & 68700 & 65710 & 55040 & 52650 \\
\hline a & 175600 & 194500 & 226300 & 215300 & 206800 & 247600 & 238500 & 231400 & 185800 & 144800 & 142500 & 146000 \\
\hline
\end{tabular}

a Discharge, in acre-feet, for Pit No. 5 Powerplant (station 11362700), provided by Pacific Gas \& Electric Co. 


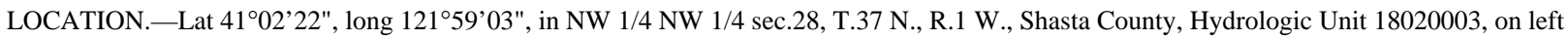
bank 0.2 mi downstream from Iron Canyon Dam and 4.2 mi west of Big Bend.

DRAINAGE AREA.- $-11.2 \mathrm{mi}^{2}$.

PERIOD OF RECORD.-August 1966 to current year (beginning October 1994, operated as a low-flow station only).

REVISED RECORDS.-WDR CA-95-4: Drainage area.

GAGE.-Water-stage recorder, $60^{\circ}$ sharp-crested V-notch weir, and concrete control with flashboards in 2- by 10-ft opening. Datum of gage is 2,461.52 ft above sea level (levels by Pacific Gas \& Electric Co.).

REMARKS.-Flow is completely regulated by Iron Canyon Reservoir (station 11363920). There is an interbasin diversion from Lake McCloud (station 11367740) to Iron Canyon Reservoir and then through a tunnel to James B. Black Powerplant on the Pit River (station 11363910). This station records fishwater release only. The minimum release requirement is $3.0 \mathrm{ft}^{3} / \mathrm{s}$ at all times. Flow is computed to $12.0 \mathrm{ft}^{3} / \mathrm{s}$. See schematic diagram of Pit and McCloud River Basins.

COOPERATION.-Records were collected by Pacific Gas \& Electric Co., under general supervision of the U.S. Geological Survey, in connection with a Federal Energy Regulatory Commission project.

EXTREMES FOR PERIOD OF RECORD.-Maximum discharge, $650 \mathrm{ft}^{3} / \mathrm{s}$, Feb. 5, 1986, gage height unknown (flashboards removed from weir), from equation for a 4- by 4-ft slide gate. Flow was the result of full travel test of slide gate at Iron Canyon Dam; maximum gage height, $3.24 \mathrm{ft}$, Feb. 25, 1978 (flashboards in weir), was the result of failure of the James B. Black Penstock; no flow, July 15-18, 1967.

DISCHARGE, CUBIC FEET PER SECOND, WATER YEAR OCTOBER 1998 TO SEPTEMBER 1999 DAILY MEAN VALUES

\begin{tabular}{|c|c|c|c|c|c|c|c|c|c|c|c|c|}
\hline DAY & $\mathrm{OCT}$ & NOV & DEC & JAN & FEB & MAR & APR & MAY & JUN & JUL & AUG & SEP \\
\hline 1 & 5.2 & 4.0 & 6.1 & 4.7 & 3.9 & 7.8 & 4.9 & 4.5 & 4.3 & 3.8 & 3.9 & 4.6 \\
\hline 2 & 4.9 & 4.2 & 5.6 & 4.7 & 3.9 & 7.2 & 4.2 & 4.6 & 4.5 & 3.9 & 3.9 & 4.3 \\
\hline 3 & 5.1 & 4.0 & 6.2 & 4.4 & 3.9 & 7.1 & 3.8 & 4.6 & 4.6 & 4.1 & 3.8 & 4.4 \\
\hline 4 & 5.7 & 3.9 & 5.2 & 4.7 & 3.9 & 6.5 & 4.0 & 4.5 & 4.5 & 4.2 & 3.8 & 4.5 \\
\hline 5 & 6.2 & 3.7 & 4.6 & 4.7 & 3.8 & 5.9 & 4.7 & 4.5 & 4.5 & 4.3 & 3.9 & 4.4 \\
\hline 6 & 5.9 & 3.5 & 3.9 & 4.4 & 4.9 & 5.5 & 4.7 & 4.5 & 4.4 & 4.3 & 4.0 & 4.2 \\
\hline 7 & 5.7 & 3.7 & 4.0 & 4.2 & 7.4 & 4.9 & 4.2 & 4.5 & 4.4 & 4.3 & 4.1 & 3.9 \\
\hline 8 & 5.5 & 3.6 & 4.0 & 3.9 & 6.7 & 4.9 & 4.6 & 4.5 & 4.4 & 4.4 & 4.1 & 3.7 \\
\hline 9 & 5.4 & 3.5 & 4.0 & 3.8 & 9.1 & 5.2 & 4.4 & 4.6 & 4.5 & 4.3 & 4.1 & 3.7 \\
\hline 10 & 5.7 & 3.5 & 4.3 & 3.9 & 7.2 & --- & 4.5 & 4.6 & 4.4 & 4.2 & 4.1 & 3.9 \\
\hline 11 & 6.3 & 3.3 & 4.6 & 4.3 & 6.1 & 5.1 & 4.2 & 4.5 & 4.4 & 4.1 & 4.0 & 3.9 \\
\hline 12 & 6.3 & 3.5 & 4.3 & 4.7 & 5.7 & 4.9 & e 4.2 & 4.5 & 4.4 & 4.0 & 3.9 & 4.0 \\
\hline 13 & 6.3 & 3.7 & 4.0 & 4.7 & 5.5 & 4.7 & 4.1 & 4.5 & 4.4 & 3.9 & 4.0 & 4.0 \\
\hline 14 & 6.1 & 3.9 & 4.0 & 4.7 & 5.0 & 4.8 & 4.4 & 4.5 & 4.2 & 3.9 & 4.0 & 3.9 \\
\hline 15 & 6.1 & 4.2 & 4.0 & 4.7 & 4.8 & 4.8 & 4.5 & 4.4 & 4.3 & 4.0 & 4.1 & 3.8 \\
\hline 16 & 6.2 & 4.7 & 4.1 & 4.6 & 5.0 & 4.8 & 4.6 & 4.4 & 4.3 & 4.2 & 4.1 & 3.8 \\
\hline 17 & 5.7 & 4.9 & 4.0 & 5.5 & 5.3 & 4.8 & 4.4 & 4.4 & 4.3 & 4.3 & 4.0 & 3.9 \\
\hline 18 & 5.5 & 4.6 & 3.8 & 6.4 & 5.6 & 4.7 & 4.5 & 4.4 & 4.3 & 4.3 & 3.9 & 4.1 \\
\hline 19 & 5.3 & 4.6 & 4.3 & 6.5 & 5.2 & 4.7 & 4.5 & 4.4 & 4.3 & 4.3 & 3.9 & 4.1 \\
\hline 20 & 5.2 & 4.6 & 4.9 & 6.9 & 4.9 & 4.7 & 4.8 & 4.5 & 4.3 & 4.2 & 3.7 & 4.1 \\
\hline 21 & 5.2 & 5.8 & 4.9 & 6.7 & 4.7 & 4.6 & 6.3 & 4.5 & 4.3 & 4.2 & 3.7 & 4.1 \\
\hline 22 & 4.9 & 5.5 & 4.3 & 6.7 & 4.7 & 4.7 & 5.1 & 4.3 & 4.2 & 4.1 & 3.8 & 3.9 \\
\hline 23 & 4.9 & --- & 3.9 & 6.6 & 5.1 & 4.7 & 4.5 & 4.2 & 4.0 & 4.1 & 3.9 & 3.8 \\
\hline 24 & 4.8 & 6.0 & 3.9 & 6.2 & 5.4 & 4.9 & 4.5 & 4.2 & 4.0 & 4.2 & 3.9 & 3.9 \\
\hline 25 & 4.3 & 4.9 & 3.9 & 5.7 & 6.1 & 4.9 & 4.5 & 4.2 & 4.0 & 4.2 & 3.8 & 3.8 \\
\hline 26 & 4.1 & 4.4 & 3.8 & 5.4 & 6.2 & 4.6 & 4.6 & 4.2 & 3.9 & 4.1 & 4.0 & 3.9 \\
\hline 27 & 3.9 & 3.8 & 3.9 & 5.2 & 6.3 & 4.9 & 4.6 & 4.2 & 4.0 & 4.0 & 4.2 & 4.2 \\
\hline 28 & 3.8 & 3.6 & 3.8 & 5.2 & 7.8 & 5.1 & 4.6 & 4.2 & 4.0 & 3.9 & 4.2 & 3.9 \\
\hline 29 & 3.9 & 3.9 & 3.9 & 5.0 & --- & 5.2 & 4.6 & 4.4 & 3.9 & 3.9 & 4.2 & 3.9 \\
\hline 30 & 3.8 & 7.2 & 4.0 & 4.3 & --- & 5.2 & 4.5 & 4.3 & 3.9 & 3.7 & 4.3 & 3.9 \\
\hline 31 & 4.0 & --- & 4.6 & 3.9 & --- & 5.4 & --- & 4.3 & --- & 3.7 & 4.5 & --- \\
\hline TOTAL & 161.9 & --- & 134.8 & 157.3 & 154.1 & --- & 136.0 & 136.9 & 127.9 & 127.1 & 123.8 & 120.5 \\
\hline MEAN & 5.22 & --- & 4.35 & 5.07 & 5.50 & --- & 4.53 & 4.42 & 4.26 & 4.10 & 3.99 & 4.02 \\
\hline MAX & 6.3 & --- & 6.2 & 6.9 & 9.1 & --- & 6.3 & 4.6 & 4.6 & 4.4 & 4.5 & 4.6 \\
\hline MIN & 3.8 & --- & 3.8 & 3.8 & 3.8 & --- & 3.8 & 4.2 & 3.9 & 3.7 & 3.7 & 3.7 \\
\hline $\mathrm{AC}-\mathrm{FT}$ & 321 & --- & 267 & 312 & 306 & --- & 270 & 272 & 254 & 252 & 246 & 239 \\
\hline
\end{tabular}




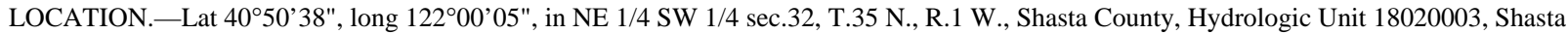
National Forest, on left bank 0.7 mi downstream from Pit No. 7 Dam and Powerplant, 1.4 mi upstream from Potem Creek, and 4.1 mi west of town of Montgomery Creek.

DRAINAGE AREA.—4,952 $\mathrm{mi}^{2}$, excluding Goose Lake Basin.

PERIOD OF RECORD.-October 1944 to current year (monthly discharge only December 1964 to May 1965). Monthly discharge only for some periods, published in WSP 1315-A.

CHEMICAL DATA: Water years 1951, 1953, 1955-81.

WATER TEMPERATURE: Water years 1951, 1954-57, 1959.

REVISED RECORDS.—WSP 1931: Drainage area. WDR CA-86-4: 1983 (M).

GAGE.-Water-stage recorder and crest-stage gage. Datum of gage is $1,000.00 \mathrm{ft}$ above sea level (levels by Pacific Gas \& Electric Co.). October 1944 to Feb. 17, 1963, at site 0.7 mi upstream at different datum. Feb. 17, 1963, to May 21, 1965, at site 1.5 mi upstream at different datum. May 21, 1965, to June 20, 1981, at site 0.9 mi downstream at datum 1,036.00 ft above sea level.

REMARKS.- Low flow completely regulated by many reservoirs and powerplants, total usable reservoir capacity, 337,000 acre-ft. Many diversions upstream from station for irrigation. Diversion from McCloud River to Iron Canyon Reservoir (station 11363920) began December 1965. See schematic diagram of Pit and McCloud River Basins.

COOPERATION.-Records were collected by Pacific Gas \& Electric Co., under general supervision of the U.S. Geological Survey, in connection with a Federal Energy Regulatory Commission project.

EXTREMES FOR PERIOD OF RECORD.-Maximum discharge, 73,000 ft 3 s, Jan. 24, 1970, gage height, $32.36 \mathrm{ft}$, site and datum then in use; maximum gage height, $74.65 \mathrm{ft}$, Feb. 19, 1986; minimum daily, $30 \mathrm{ft}^{3} / \mathrm{s}$, July 12, 27, 1975, result of construction work below Pit No. 7 Powerplant.

DISCHARGE, CUBIC FEET PER SECOND, WATER YEAR OCTOBER 1998 TO SEPTEMBER 1999 DAILY MEAN VALUES

\begin{tabular}{|c|c|c|c|c|c|c|c|c|c|c|c|c|}
\hline DAY & OCT & NOV & $\mathrm{DEC}$ & JAN & FEB & MAR & APR & MAY & JUN & JUL & AUG & SEP \\
\hline 1 & e 6870 & 5430 & 12900 & 3930 & 6170 & 14300 & 8030 & e7150 & e5950 & e 3260 & 2700 & 3460 \\
\hline 2 & 4760 & 2900 & 11600 & 3380 & 6550 & 14500 & 8800 & e 8680 & e 5020 & e 4310 & 4340 & 2930 \\
\hline 3 & 890 & 3060 & 15400 & 6090 & 5620 & 14500 & 8180 & e7610 & e 5620 & e2450 & 4140 & 3740 \\
\hline 4 & 1010 & 3990 & 11800 & 4900 & 5580 & 15500 & 7480 & e 7500 & e 5670 & e4000 & 3830 & 3780 \\
\hline 5 & 4550 & 4350 & 10700 & 4770 & 7580 & 15300 & 7240 & e7320 & e 6190 & e 4270 & 3370 & 4780 \\
\hline 6 & 4720 & 4930 & 9010 & 5670 & 6860 & 14000 & 7210 & e7420 & e 5640 & e 3120 & 3400 & 3930 \\
\hline 7 & 6320 & 5720 & 8460 & 6450 & 11100 & 12500 & 7280 & e 7530 & e 4880 & e3590 & 1560 & 3780 \\
\hline 8 & 6200 & 5430 & 6520 & 5230 & 9390 & 10800 & 8390 & e7050 & e 5210 & e 3220 & 3060 & 3870 \\
\hline 9 & 6170 & 5280 & 7230 & 4480 & 20700 & 10700 & e7660 & e 6380 & e 5120 & e4030 & 4960 & 3960 \\
\hline 10 & 232 & 5430 & 5430 & 3420 & 15200 & 9680 & e 8230 & e7000 & e 5530 & e4910 & 4140 & 3540 \\
\hline 11 & 602 & 5540 & 6560 & 4330 & 12700 & 9960 & e7810 & e 6700 & e5430 & e 4870 & 3660 & 3540 \\
\hline 12 & 3340 & 4250 & 6580 & 3690 & 12500 & 9580 & e 8280 & e 6230 & e 5610 & e 5220 & 3900 & 2890 \\
\hline 13 & 2480 & 3770 & 6160 & 4350 & 10800 & 9190 & e7540 & e 6590 & e 4170 & e 8240 & 3710 & 4050 \\
\hline 14 & 5070 & 3680 & 6680 & 4320 & 11300 & 7800 & e7280 & e7140 & e 5640 & e7860 & 2210 & 3920 \\
\hline 15 & 5130 & 3430 & 5780 & 4300 & 8860 & 8760 & e 7170 & e 6670 & e5540 & e4390 & 3810 & 3790 \\
\hline 16 & 4360 & 3950 & 7420 & 3790 & 8730 & 8680 & e 7430 & e 6420 & e 4730 & e1360 & 4830 & 3270 \\
\hline 17 & 5170 & 4490 & 7470 & 5550 & 9520 & 8300 & e 7750 & e 6530 & e4330 & e2990 & 4060 & 3160 \\
\hline 18 & 4940 & 4060 & 5750 & 7690 & 10300 & 7860 & e 9660 & e 4660 & e5390 & e4020 & 3300 & 2510 \\
\hline 19 & 5270 & 3730 & 4910 & 8300 & 10900 & 8350 & e7600 & e5510 & e3730 & e 3660 & 3710 & 1760 \\
\hline 20 & 4880 & 5390 & 5810 & 11400 & 11100 & 8480 & e9060 & e 6450 & e 3600 & e 3800 & 4350 & 3200 \\
\hline 21 & 4440 & 4860 & 7460 & 10500 & 10900 & 8930 & e5650 & e7060 & e 3660 & e 4570 & 2410 & 4770 \\
\hline 22 & 4530 & 4630 & 6840 & 10500 & 9760 & 7890 & e7720 & e 3780 & e 4850 & e5000 & 4250 & 5350 \\
\hline 23 & 4960 & 7950 & 5960 & 12000 & 11100 & 8470 & e7650 & e 6540 & e 4900 & e2920 & 4060 & 3030 \\
\hline 24 & 4600 & 8490 & 5220 & 10200 & 9410 & 9700 & e9790 & e4900 & e 4710 & e 1540 & 3720 & 3600 \\
\hline 25 & 3630 & 8550 & 5820 & 9080 & 12200 & 9520 & e7990 & e 5570 & e 4360 & 2730 & 3030 & 4280 \\
\hline 26 & 5480 & 8800 & 5460 & 8530 & 11600 & 8230 & e 7750 & e 5540 & e2990 & 4530 & 3180 & 4410 \\
\hline 27 & 5340 & 8970 & 5610 & 8000 & 11400 & 8770 & e 7170 & e5110 & e 3200 & 4420 & 4050 & 3430 \\
\hline 28 & 4200 & 8670 & 5290 & 7670 & 14500 & 8920 & e 6830 & e 5660 & e 4550 & 4630 & 2450 & 3810 \\
\hline 29 & 4860 & 7620 & 4360 & 7870 & --- & 7840 & e 6550 & e5790 & e3650 & 4630 & 1740 & 4130 \\
\hline 30 & 5240 & 11000 & 3890 & 7420 & --- & 8560 & e7550 & e 4660 & e 4300 & 5310 & 2870 & 3780 \\
\hline 31 & 6500 & --- & 3180 & 6470 & --- & 8780 & --- & e 5310 & --- & 1410 & 2590 & --- \\
\hline TOTAL & 136744 & 168350 & 221260 & 204280 & 292330 & 314350 & 232730 & 196460 & 144170 & 125260 & 107390 & 110450 \\
\hline MEAN & 4411 & 5612 & 7137 & 6590 & 10440 & 10140 & 7758 & 6337 & 4806 & 4041 & 3464 & 3682 \\
\hline MAX & 6870 & 11000 & 15400 & 12000 & 20700 & 15500 & 9790 & 8680 & 6190 & 8240 & 4960 & 5350 \\
\hline MIN & 232 & 2900 & 3180 & 3380 & 5580 & 7800 & 5650 & 3780 & 2990 & 1360 & 1560 & 1760 \\
\hline$A C-F T$ & 271200 & 333900 & 438900 & 405200 & 579800 & 623500 & 461600 & 389700 & 286000 & 248500 & 213000 & 219100 \\
\hline a & 14531 & 15407 & 15153 & 14634 & 14522 & 14115 & 15018 & 15255 & 15171 & 14515 & 14930 & 11568 \\
\hline $\mathrm{b}$ & 255300 & 295800 & 348400 & 342200 & 328000 & 413500 & 389000 & 356600 & 279400 & 237200 & 209400 & 209800 \\
\hline C & 32080 & 33058 & 33099 & 32317 & 33200 & 33523 & 33946 & 33578 & 31807 & 32030 & 33403 & 29770 \\
\hline
\end{tabular}

e Estimated.

a Contents, in acre-feet, at end of month for Pit No. 6 Reservoir (station 11364100), provided by Pacific Gas \& Electric Co.

b Discharge, in acre-feet, for Pit No. 6 Powerplant (station 11364150), provided by Pacific Gas \& Electric Co.

c Contents, in acre-feet, at end of month for Pit No. 7 Reservoir (station 11364700), provided by Pacific Gas \& Electric Co. 
11365000 PIT RIVER NEAR MONTGOMERY CREEK, CA—Continued

STATISTICS OF MONTHLY MEAN DATA FOR WATER YEARS 1945 - 1965, BY WATER YEAR (WY)

\begin{tabular}{|c|c|c|c|c|c|c|c|c|c|c|c|c|}
\hline & $\mathrm{OCT}$ & NOV & DEC & JAN & FEB & MAR & APR & MAY & JUN & JUL & AUG & SEP \\
\hline MEAN & 2643 & 2828 & 3821 & 4320 & 5592 & 5331 & 5711 & 4297 & 3127 & 2376 & 2231 & 2284 \\
\hline MAX & 5999 & 3710 & 9541 & 11240 & 12970 & 8212 & 13350 & 7380 & 5044 & 3037 & 2651 & 2744 \\
\hline$(W Y)$ & 1963 & 1951 & 1956 & 1956 & 1958 & 1956 & 1952 & 1952 & 1953 & 1958 & 1958 & 1959 \\
\hline MIN & 2112 & 2232 & 2219 & 2137 & 2500 & 3225 & 3404 & 2299 & 2353 & 1935 & 1971 & 1899 \\
\hline (WY) & 1950 & 1950 & 1950 & 1949 & 1948 & 1964 & 1947 & 1947 & 1950 & 1949 & 1947 & 1949 \\
\hline
\end{tabular}

SUMMARY STATISTICS

ANNUAL TOTAL

ANNUAL MEAN

HIGHEST ANNUAL MEAN

LOWEST ANNUAL MEAN

HIGHEST DAILY MEAN

LOWEST DAILY MEAN

ANNUAL SEVEN-DAY MINIMUM

INSTANTANEOUS PEAK FLOW

INSTANTANEOUS PEAK STAGE

ANNUAL RUNOFF (AC-FT)

10 PERCENT EXCEEDS

50 PERCENT EXCEEDS

90 PERCENT EXCEEDS
WATER YEARS 1945 - 1965

\begin{tabular}{|c|c|c|}
\hline \multicolumn{3}{|l|}{3704} \\
\hline 5529 & & 1956 \\
\hline 2658 & & 1947 \\
\hline 32100 & Dec 23 & 1955 \\
\hline 150 & Jul 19 & 1965 \\
\hline 1610 & Jul 19 & 1965 \\
\hline 37100 & Dec 23 & 1955 \\
\hline 14.12 & Dec 23 & 1955 \\
\hline \multicolumn{3}{|l|}{2684000} \\
\hline \multicolumn{3}{|l|}{6080} \\
\hline 3010 & & \\
\hline 1740 & & \\
\hline
\end{tabular}

STATISTICS OF MONTHLY MEAN DATA FOR WATER YEARS 1966 - 1999, BY WATER YEAR (WY)

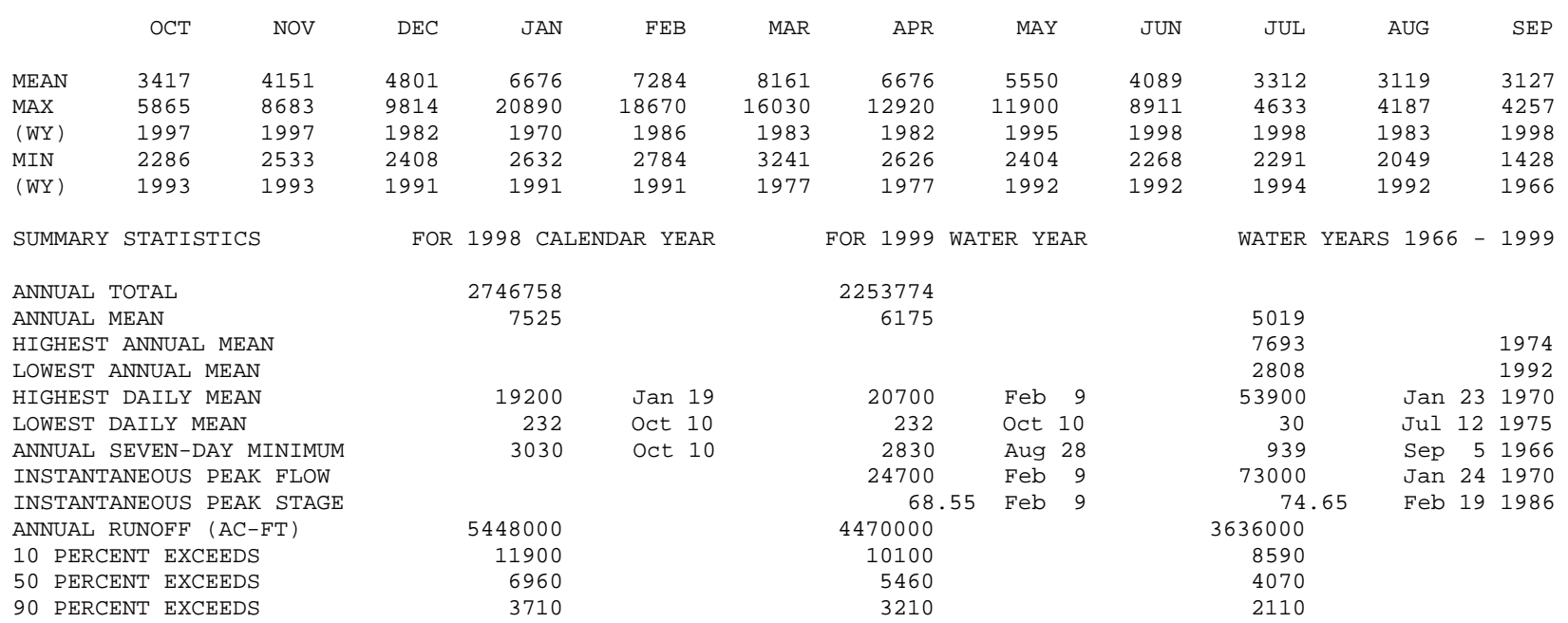


LOCATION.-Lat $41^{\circ} 11^{\prime} 18^{\prime \prime}$, long $122^{\circ} 03^{\prime} 52^{\prime \prime}$, in NW 1/4 NE 1/4 sec.34, T.39 N., R.2 W., Siskiyou County, Hydrologic Unit 18020004, on right bank 0.4 mi downstream from Angel Creek and 6 mi southeast of McCloud.

DRAINAGE AREA.-358 $\mathrm{mi}^{2}$.

PERIOD OF RECORD.-April 1931 to current year.

REVISED RECORDS.-WSP 843: 1936(M). WSP 1445: 1940(M). WSP 1931: Drainage area.

GAGE.-Water-stage recorder. Datum of gage is 2,711.2 ft above sea level, from river-profile map.

REMARKS.- Two small diversions upstream from station for irrigation and one 22-in. pipeline for town of McCloud. See schematic diagram of Pit and McCloud River Basins.

COOPERATION.-Records were collected by Pacific Gas \& Electric Co., under general supervision of the U.S. Geological Survey, in connection with a Federal Energy Regulatory Commission project.

EXTREMES FOR PERIOD OF RECORD.-Maximum discharge, 15,400 ft $3 / \mathrm{s}$, Jan. 1, 1997, gage height, $11.22 \mathrm{ft}$, from rating curve extended above $8,800 \mathrm{ft}^{3} / \mathrm{s}$ on basis of slope-area measurement of peak flow; minimum daily, $524 \mathrm{ft}^{3} / \mathrm{s}$, Nov. $23,24,1932$.

EXTREMES FOR CURRENT YEAR.-Peak discharges greater than base discharge of 1,500 $\mathrm{ft}^{3} / \mathrm{s}$, or maximum:

$\begin{array}{ccccccc}\text { Date } & \text { Time } & \begin{array}{c}\text { Discharge } \\ \left(\mathrm{ft}^{3} / \mathrm{s}\right)\end{array} & \begin{array}{c}\text { Gage height } \\ (\mathrm{ft})\end{array} & \text { Date } & \text { Time } & \begin{array}{c}\text { Discharge } \\ \left(\mathrm{ft}^{3} / \mathrm{s}\right)\end{array} \\ \text { Nov. 23 } & 2045 & 1,940 & 3.13 & \text { Mar. 1 } & 0145 & 1,500 \\ \text { Nov. 30 } & 2115 & 2,700 & 3.90 & \text { Apr. 27 } & 0530 & 1,600 \\ \text { Jan. 21 } & 0145 & 1,550 & 2.84 & & & 2.78 \\ & & & & & \end{array}$

DISCHARGE, CUBIC FEET PER SECOND, WATER YEAR OCTOBER 1998 TO SEPTEMBER 1999 DAILY MEAN VALUES

\begin{tabular}{|c|c|c|c|c|c|c|c|c|c|c|c|c|}
\hline DAY & OCT & NOV & $\mathrm{DEC}$ & JAN & FEB & MAR & APR & MAY & JUN & JUL & AUG & SEP \\
\hline 1 & 1010 & 989 & 2180 & 981 & 1040 & 1470 & 1190 & 1450 & 1340 & 1030 & 961 & 939 \\
\hline 2 & 1020 & 985 & 1690 & 977 & 1030 & 1370 & 1180 & 1500 & 1400 & 1030 & 960 & 939 \\
\hline 3 & 1010 & 984 & 2090 & 973 & 1020 & 1440 & 1170 & 1530 & 1360 & 1020 & 959 & 936 \\
\hline 4 & 1010 & 981 & 1750 & 972 & 1020 & 1380 & 1160 & 1500 & 1280 & 1020 & 959 & 934 \\
\hline 5 & 1010 & 980 & 1520 & 972 & 1020 & 1290 & 1170 & 1440 & 1260 & 1020 & 959 & 932 \\
\hline 6 & 1010 & 980 & 1420 & 972 & 1030 & 1260 & 1150 & 1430 & 1260 & 1010 & 958 & 932 \\
\hline 7 & 1010 & 993 & 1360 & 970 & 1110 & 1220 & 1150 & 1450 & 1240 & 1010 & 959 & 930 \\
\hline 8 & 1010 & 983 & 1310 & 964 & 1170 & 1200 & 1200 & 1430 & 1210 & 1010 & 958 & 929 \\
\hline 9 & 1010 & 979 & 1280 & 960 & 1130 & 1190 & 1170 & 1390 & 1200 & 1000 & 955 & 928 \\
\hline 10 & 1010 & 982 & 1260 & 960 & 1100 & 1160 & 1160 & 1360 & 1180 & 999 & 956 & 928 \\
\hline 11 & 1000 & 979 & 1240 & 960 & 1080 & 1130 & 1150 & 1350 & 1180 & 998 & 955 & 927 \\
\hline 12 & 1000 & 975 & 1270 & 959 & 1060 & 1120 & 1160 & 1350 & 1180 & 997 & 952 & 927 \\
\hline 13 & 1000 & 973 & 1300 & 959 & 1050 & 1110 & 1170 & 1360 & 1180 & 996 & 950 & 926 \\
\hline 14 & 1000 & 972 & 1300 & 959 & 1040 & 1110 & 1190 & 1340 & 1170 & 993 & 948 & 924 \\
\hline 15 & 1000 & 977 & 1280 & 963 & 1030 & 1110 & 1230 & 1310 & 1150 & 989 & 947 & 923 \\
\hline 16 & 1000 & 976 & 1270 & 982 & 1030 & 1110 & 1270 & 1300 & 1140 & 987 & 946 & 923 \\
\hline 17 & 999 & 994 & 1270 & 1020 & 1030 & 1110 & 1300 & 1300 & 1130 & 985 & 946 & 921 \\
\hline 18 & 998 & 979 & 1260 & 1350 & 1070 & 1110 & 1380 & 1330 & 1120 & 985 & 944 & 920 \\
\hline 19 & 996 & 972 & 1250 & 1350 & 1080 & 1130 & 1440 & 1360 & 1110 & 983 & 944 & 918 \\
\hline 20 & 994 & 972 & 1250 & 1450 & 1060 & 1130 & 1500 & 1360 & 1100 & 982 & 942 & 917 \\
\hline 21 & 994 & 1020 & 1240 & 1450 & 1050 & 1140 & 1510 & 1370 & 1090 & 980 & 941 & 916 \\
\hline 22 & 992 & 1080 & 1230 & 1300 & 1040 & 1150 & 1470 & 1370 & 1090 & 977 & 940 & 916 \\
\hline 23 & 992 & 1390 & 1130 & 1270 & 1040 & 1150 & 1440 & 1390 & 1080 & 975 & 941 & 915 \\
\hline 24 & 1010 & 1450 & 998 & 1210 & 1070 & 1200 & 1460 & 1420 & 1070 & 973 & 941 & 916 \\
\hline 25 & 999 & 1170 & 998 & 1160 & 1170 & 1280 & 1480 & 1450 & 1070 & 972 & 940 & 915 \\
\hline 26 & 992 & 1130 & 998 & 1130 & 1140 & 1290 & 1540 & 1430 & 1060 & 972 & 940 & 914 \\
\hline 27 & 992 & 1170 & 998 & 1100 & 1120 & 1280 & 1580 & 1430 & 1050 & 971 & 940 & 914 \\
\hline 28 & 993 & 1110 & 996 & 1090 & 1270 & 1250 & 1490 & 1440 & 1040 & 969 & 940 & 912 \\
\hline 29 & 991 & 1080 & 992 & 1070 & --- & 1240 & 1440 & 1430 & 1040 & 966 & 940 & 913 \\
\hline 30 & 989 & 1860 & 990 & 1060 & --- & 1240 & 1430 & 1380 & 1030 & 965 & 940 & 912 \\
\hline 31 & 989 & --- & 987 & 1050 & --- & 1220 & --- & 1350 & --- & 963 & 939 & --- \\
\hline TOTAL & 31030 & 32065 & 40107 & 33543 & 30100 & 37590 & 39330 & 43300 & 34810 & 30727 & 29400 & 27696 \\
\hline MEAN & 1001 & 1069 & 1294 & 1082 & 1075 & 1213 & 1311 & 1397 & 1160 & 991 & 948 & 923 \\
\hline MAX & 1020 & 1860 & 2180 & 1450 & 1270 & 1470 & 1580 & 1530 & 1400 & 1030 & 961 & 939 \\
\hline MIN & 989 & 972 & 987 & 959 & 1020 & 1110 & 1150 & 1300 & 1030 & 963 & 939 & 912 \\
\hline $\mathrm{AC}-\mathrm{FT}$ & 61550 & 63600 & 79550 & 66530 & 59700 & 74560 & 78010 & 85890 & 69050 & 60950 & 58310 & 54940 \\
\hline
\end{tabular}


11367500 McCLOUD RIVER NEAR McCLOUD, CA—Continued

STATISTICS OF MONTHLY MEAN DATA FOR WATER YEARS 1931 - 1999, BY WATER YEAR (WY)

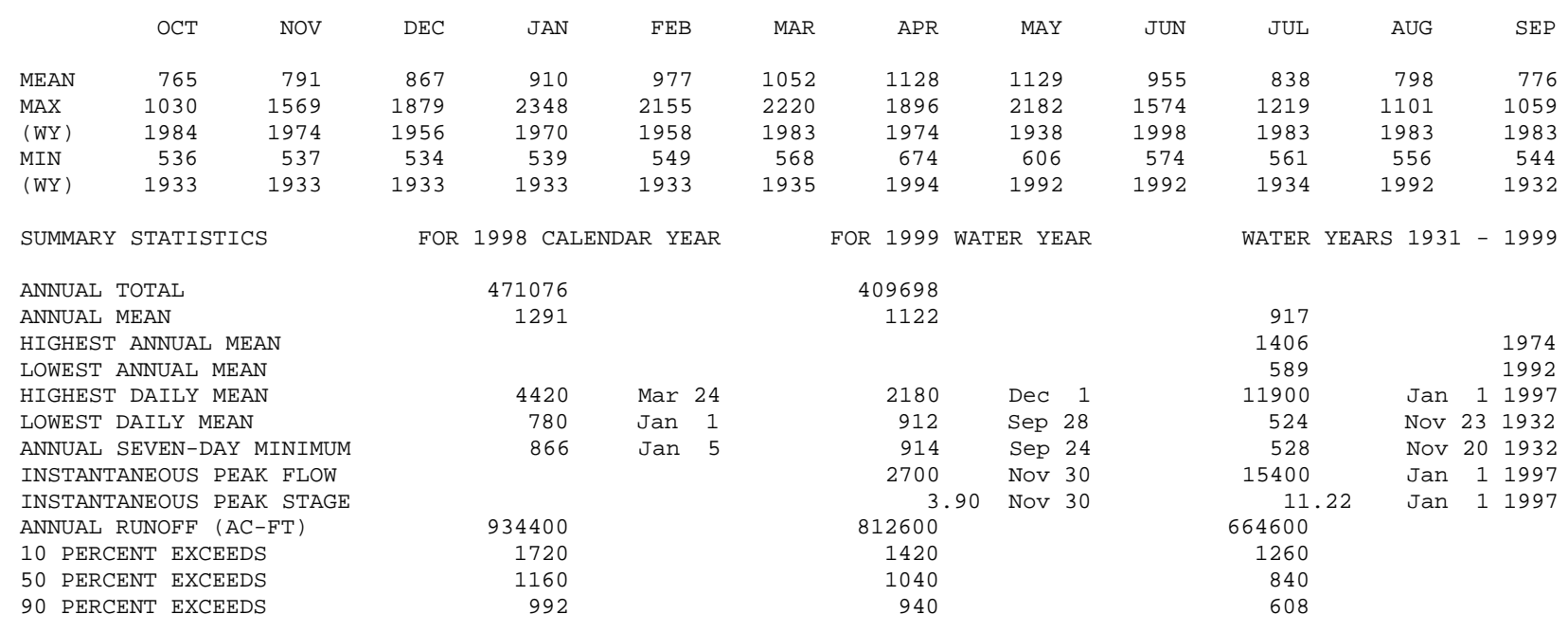




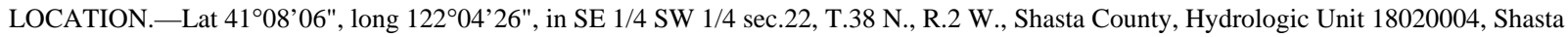
National Forest, on left bank of Lake McCloud, and 8.8 mi southeast of McCloud.

PERIOD OF RECORD.-December 1965 to current year.

REVISED RECORDS.-WDR CA-75-4: 1973.

GAGE.-None. Water-stage recorders on Iron Canyon Reservoir and Lake McCloud (stations 11363920 and 11367740) used to compute record.

REMARKS.-Water is diverted from Lake McCloud (station 11367740) via tunnel to Iron Canyon Reservoir (station 11363920) and then via penstock into James B. Black Powerplant (station 11363910) on the Pit River. Diversion began Dec. 1, 1965. See schematic diagram of Pit and McCloud River Basins.

COOPERATION.- Records were collected by Pacific Gas \& Electric Co., under general supervision of the U.S. Geological Survey, in connection with a Federal Energy Regulatory Commission project.

EXTREMES FOR PERIOD OF RECORD.-Maximum daily discharge, $1,890 \mathrm{ft}^{3} / \mathrm{s}$, several days during May and June 1967; no flow several days in 1965-68, 1971, 1978.

DISCHARGE, CUBIC FEET PER SECOND, WATER YEAR OCTOBER 1998 TO SEPTEMBER 1999 DAILY MEAN VALUES

\begin{tabular}{|c|c|c|c|c|c|c|c|c|c|c|c|c|}
\hline DAY & OCT & NOV & $\mathrm{DEC}$ & JAN & FEB & MAR & $A P R$ & MAY & JUN & JUL & AUG & SEP \\
\hline 1 & 942 & 934 & 1140 & 934 & 1210 & 1100 & 1280 & 1180 & 1180 & 1070 & 926 & 733 \\
\hline 2 & 929 & 965 & 1150 & 943 & 1200 & 1140 & 1340 & 1210 & 1160 & 1060 & 919 & 716 \\
\hline 3 & 825 & 974 & 1190 & 972 & 1190 & 1190 & 1350 & 1210 & 1160 & 971 & 955 & 701 \\
\hline 4 & 825 & 973 & 1210 & 972 & 1170 & 1230 & 1280 & 1210 & 1150 & 936 & 918 & 711 \\
\hline 5 & 791 & 1040 & 1300 & 923 & 1200 & 1290 & 1280 & 1190 & 1160 & 945 & 890 & 776 \\
\hline 6 & 855 & 1030 & 1270 & 984 & 1210 & 1320 & 1290 & 1190 & 1160 & 932 & 861 & 803 \\
\hline 7 & 915 & 1000 & 1270 & 1030 & 1180 & 1320 & 1330 & 1180 & 1170 & 878 & 813 & 830 \\
\hline 8 & 938 & 993 & 1250 & 1040 & 1200 & 1330 & 1350 & 1180 & 1170 & 909 & 813 & 858 \\
\hline 9 & 907 & 1000 & 1230 & 1050 & 1190 & 1300 & 1350 & 1170 & 1140 & 936 & 850 & 842 \\
\hline 10 & 805 & 1010 & 1230 & 958 & 1200 & 1300 & 1340 & 1180 & 1160 & 978 & 871 & 827 \\
\hline 11 & 717 & 954 & 1230 & 899 & 1200 & 1270 & 1310 & 1190 & 1160 & 1020 & 906 & 798 \\
\hline 12 & 717 & 932 & 1230 & 856 & 1190 & 1310 & 1300 & 1190 & 1150 & 1020 & 850 & 785 \\
\hline 13 & 717 & 853 & 1230 & 855 & 1200 & 1300 & 1280 & 1200 & 1100 & 1040 & 851 & 819 \\
\hline 14 & 717 & 793 & 1150 & 868 & 1200 & 1270 & 1270 & 1210 & 1080 & 1040 & 815 & 836 \\
\hline 15 & 821 & 661 & 1140 & 908 & 1200 & 1260 & 1270 & 1220 & 1060 & 965 & 778 & 829 \\
\hline 16 & 895 & 706 & 1160 & 907 & 1140 & 1270 & 1280 & 1220 & 1080 & 903 & 821 & 811 \\
\hline 17 & 918 & 756 & 1160 & 923 & 1160 & 1260 & 1290 & 1190 & 1080 & 860 & 846 & 746 \\
\hline 18 & 944 & 773 & 1160 & 980 & 1180 & 1270 & 1280 & 1190 & 1100 & 872 & 879 & 694 \\
\hline 19 & 960 & 782 & 1020 & 1040 & 1200 & 1260 & 1290 & 1170 & 1100 & 908 & 904 & 694 \\
\hline 20 & 948 & 781 & 976 & 1110 & 1210 & 1260 & 1280 & 1160 & 1080 & 912 & 933 & 707 \\
\hline 21 & 953 & 793 & 1070 & 1140 & 1200 & 1280 & 1230 & 1200 & 1070 & 949 & 862 & 772 \\
\hline 22 & 969 & 806 & 1150 & 1220 & 1210 & 1270 & 1180 & 1220 & 1080 & 957 & 827 & 823 \\
\hline 23 & 1000 & 806 & 1150 & 1220 & 1180 & 1270 & 1190 & 1220 & 1090 & 924 & 839 & 832 \\
\hline 24 & 1020 & 930 & 1150 & 1230 & 1190 & 1310 & 1210 & 1210 & 1110 & 894 & 849 & 814 \\
\hline 25 & 1040 & 1040 & 1150 & 1190 & 1140 & 1320 & 1210 & 1220 & 1110 & 887 & 881 & 828 \\
\hline 26 & 1030 & 1110 & 1150 & 1130 & 1110 & 1240 & 1210 & 1210 & 1100 & 922 & 873 & 788 \\
\hline 27 & 1050 & 1160 & 1150 & 1090 & 1100 & 1230 & 1200 & 1210 & 1080 & 965 & 861 & 812 \\
\hline 28 & 1050 & 1150 & 1070 & 1100 & 1090 & 1200 & 1200 & 1210 & 1080 & 986 & 867 & 834 \\
\hline 29 & 1050 & 1050 & 1030 & 1190 & --- & 1200 & 1190 & 1200 & 1090 & 986 & 887 & 798 \\
\hline 30 & 1050 & 1070 & 978 & 1270 & --- & 1190 & 1180 & 1190 & 1090 & 1040 & 803 & 799 \\
\hline 31 & 1050 & --- & 978 & 1220 & --- & 1240 & --- & 1200 & --- & 942 & 735 & -- \\
\hline TOTAL & 28348 & 27825 & 35722 & 32152 & 33050 & 39000 & 38040 & 37130 & 33500 & 29607 & 26683 & 23616 \\
\hline MEAN & 914 & 928 & 1152 & 1037 & 1180 & 1258 & 1268 & 1198 & 1117 & 955 & 861 & 787 \\
\hline MAX & 1050 & 1160 & 1300 & 1270 & 1210 & 1330 & 1350 & 1220 & 1180 & 1070 & 955 & 858 \\
\hline MIN & 717 & 661 & 976 & 855 & 1090 & 1100 & 1180 & 1160 & 1060 & 860 & 735 & 694 \\
\hline $\mathrm{AC}-\mathrm{FT}$ & 56230 & 55190 & 70850 & 63770 & 65550 & 77360 & 75450 & 73650 & 66450 & 58730 & 52930 & 46840 \\
\hline
\end{tabular}


LOCATION.-Lat 41 $07^{\prime} 44^{\prime \prime}$, long $122^{\circ} 04^{\prime} 08^{\prime \prime}$, in SW 1/4 NE 1/4 sec.27, T.38 N., R.2 W., Shasta County, Hydrologic Unit 18020004, Shasta National Forest, on left bank 0.1 mi downstream from Lizard Creek, 0.6 mi downstream from McCloud Dam, and 9 mi southeast of McCloud. DRAINAGE AREA.-404 $\mathrm{mi}^{2}$.

PERIOD OF RECORD.-April 1966 to current year (operated as a low-flow station only).

GAGE.-Water-stage recorder. Datum of gage is 2,398.76 ft above sea level (levels by Pacific Gas \& Electric Co.). Prior to Apr. 7, 1972, at datum $3.00 \mathrm{ft}$ higher.

REMARKS.-Low flow regulated by Lake McCloud (station 11367740) since November 1965. Most of McCloud River runoff is diverted from reservoir through tunnel to Iron Canyon Reservoir (station 11363920) in Pit River Basin. This station records fishwater release. The minimum release requirement is $40 \mathrm{ft}^{3} / \mathrm{s}$ at all times. Prior to water year 1974 , flow was computed up to $400 \mathrm{ft}^{3} / \mathrm{s}$. During water years $1975-81$, because of channel changes, flow was computed up to $200 \mathrm{ft}^{3} / \mathrm{s}$. Currently, because of maximum required release, flow is computed to $220 \mathrm{ft}^{3} / \mathrm{s}$. See schematic diagram of Pit and McCloud River Basins.

COOPERATION.--Records were collected by Pacific Gas \& Electric Co., under general supervision of the U.S. Geological Survey, in connection with a Federal Energy Regulatory Commission project.

DISCHARGE, CUBIC FEET PER SECOND, WATER YEAR OCTOBER 1998 TO SEPTEMBER 1999 DAILY MEAN VALUES

\begin{tabular}{|c|c|c|c|c|c|c|c|c|c|c|c|c|}
\hline DAY & $\mathrm{OCT}$ & NOV & DEC & JAN & FEB & MAR & APR & MAY & JUN & JUL & AUG & SEP \\
\hline 1 & 184 & 184 & 75 & 128 & 81 & 74 & 77 & --- & --- & 174 & 179 & 195 \\
\hline 2 & 185 & 184 & 64 & 123 & 84 & 73 & 77 & --- & --- & 174 & 179 & 194 \\
\hline 3 & 184 & 184 & 60 & 123 & 87 & 74 & 76 & --- & --- & 174 & 179 & 194 \\
\hline 4 & 185 & 184 & 68 & 123 & 87 & 74 & 75 & --- & --- & 174 & 179 & 194 \\
\hline 5 & 185 & 185 & 73 & 123 & 91 & 74 & 75 & --- & --- & 174 & 182 & 195 \\
\hline 6 & 185 & 184 & 78 & 124 & 91 & 73 & 81 & --- & 215 & 176 & 182 & 194 \\
\hline 7 & 185 & 184 & 92 & 128 & 60 & 72 & 83 & --- & 161 & 175 & 182 & 195 \\
\hline 8 & 185 & 183 & 110 & 129 & 57 & 72 & 84 & --- & 160 & 176 & 181 & 194 \\
\hline 9 & 184 & 183 & 123 & 133 & 59 & 72 & 84 & --- & 161 & 175 & 181 & 194 \\
\hline 10 & 184 & 186 & 136 & 133 & 56 & 71 & 84 & --- & 162 & 175 & 181 & 193 \\
\hline 11 & 185 & 187 & 138 & 133 & 54 & --- & 83 & --- & 162 & 177 & 181 & 194 \\
\hline 12 & 185 & 187 & 137 & 126 & 53 & 77 & 83 & --- & 164 & 181 & 181 & 193 \\
\hline 13 & 185 & 186 & 144 & 124 & 53 & 77 & 83 & 220 & 165 & 180 & 182 & 193 \\
\hline 14 & 185 & 186 & 149 & 131 & 53 & 76 & 84 & 220 & 165 & 180 & 181 & 193 \\
\hline 15 & 185 & 187 & 148 & 135 & 60 & 72 & 84 & 220 & 164 & 181 & 181 & 193 \\
\hline 16 & 185 & 187 & 113 & 131 & 62 & 76 & 84 & 220 & 165 & 180 & 182 & 193 \\
\hline 17 & 185 & 188 & 108 & 123 & 62 & 75 & 85 & 220 & 165 & 180 & 181 & 193 \\
\hline 18 & 185 & 188 & 112 & 58 & 64 & 75 & 85 & 219 & 165 & 180 & 181 & 193 \\
\hline 19 & 184 & 188 & 113 & 55 & 65 & 75 & 86 & 220 & 165 & 180 & 181 & 193 \\
\hline 20 & 184 & 188 & 114 & 56 & 64 & 75 & 86 & --- & 165 & 180 & 181 & 193 \\
\hline 21 & 184 & 190 & 121 & 56 & 63 & 75 & 94 & --- & 165 & 180 & 180 & 193 \\
\hline 22 & 184 & 190 & 131 & 57 & 63 & 75 & --- & --- & 164 & 180 & 181 & 193 \\
\hline 23 & 186 & 147 & 131 & 60 & 63 & 74 & --- & --- & 165 & 180 & 180 & 193 \\
\hline 24 & 186 & 81 & 131 & 57 & 64 & 77 & --- & --- & 166 & 180 & 180 & 192 \\
\hline 25 & 185 & 90 & 128 & 56 & 67 & 81 & --- & --- & 167 & 180 & 180 & 193 \\
\hline 26 & 185 & 112 & 130 & 55 & 66 & 80 & --- & --- & 169 & 180 & 181 & 193 \\
\hline 27 & 184 & 115 & 131 & 55 & 65 & 79 & --- & --- & 173 & 180 & 181 & 193 \\
\hline 28 & 183 & 121 & 130 & 55 & 71 & 78 & --- & --- & 173 & 179 & 181 & 193 \\
\hline 29 & 188 & 129 & 130 & 63 & --- & 78 & --- & --- & 173 & 179 & 182 & 194 \\
\hline 30 & 187 & 144 & 131 & 68 & --- & 78 & --- & --- & 173 & 179 & 181 & 194 \\
\hline 31 & 185 & --- & 131 & 74 & --- & 77 & --- & --- & --- & 178 & 186 & --- \\
\hline TOTAL & 5731 & 5032 & 3580 & 2995 & 1865 & --- & --- & --- & --- & 5521 & 5610 & 5804 \\
\hline MEAN & 185 & 168 & 115 & 96.6 & 66.6 & --- & --- & --- & --- & 178 & 181 & 193 \\
\hline MAX & 188 & 190 & 149 & 135 & 91 & --- & --- & --- & --- & 181 & 186 & 195 \\
\hline MIN & 183 & 81 & 60 & 55 & 53 & --- & --- & --- & --- & 174 & 179 & 192 \\
\hline $\mathrm{AC}-\mathrm{FT}$ & 11370 & 9980 & 7100 & 5940 & 3700 & --- & --- & --- & --- & 10950 & 11130 & 11510 \\
\hline
\end{tabular}


LOCATION.—Lat 41 $06^{\prime} 39^{\prime \prime}$, long $122^{\circ} 05^{\prime} 42^{\prime \prime}$, in NE 1/4 SW 1/4 sec.33, T.38 N., R.2 W., Shasta County, Hydrologic Unit 18020004, Shasta National Forest, on right bank at Ah-Di-Na, 1.8 mi downstream from Squirrel Creek, 3.9 mi downstream from McCloud Dam, and 9.6 mi south of McCloud.

DRAINAGE AREA.-427 $\mathrm{mi}^{2}$.

PERIOD OF RECORD.-October 1964 to current year.

REVISED RECORDS.-WDR CA-98-4: 1997 (m).

GAGE.-Water-stage recorder. Elevation of gage is 2,160 ft above sea level, from topographic map.

REMARKS.- - Low flow completely regulated by Lake McCloud (station 11367740) 3.9 mi upstream since November 1965. Diversion to Iron Canyon Reservoir (station 11363920) through McCloud-Iron Canyon diversion tunnel (station 11367720) started Dec. 1, 1965. This station records fishwater release. The minimum release requirements range from 160 to $210 \mathrm{ft}^{3} / \mathrm{s}$ per schedule outlined in Federal Energy Regulatory Commission License 2106. See schematic diagram of Pit and McCloud River Basins.

COOPERATION.-Records were collected by Pacific Gas \& Electric Co., under general supervision of the U.S. Geological Survey, in connection with a Federal Energy Regulatory Commission project.

EXTREMES FOR PERIOD OF RECORD.-Prior to completion of McCloud Dam in 1965, maximum discharge, 9,660 $\mathrm{ft}^{3} / \mathrm{s}$, Dec. 22, 1964, gage height, $9.43 \mathrm{ft}$, from rating curve extended above 2,500 ft $3 / \mathrm{s}$; minimum daily, $86 \mathrm{ft}^{3} / \mathrm{s}$, Oct. 1-26, 1964. Since completion of McCloud Dam, maximum discharge, $31,700 \mathrm{ft}^{3} / \mathrm{s}$, Jan. 1, 1997, gage height, $14.77 \mathrm{ft}$, from rating curve extended above $8,000 \mathrm{ft}^{3} / \mathrm{s}$ on basis of slope-area measurement of peak flow; minimum daily, $41 \mathrm{ft}^{3} / \mathrm{s}$, Dec. 18-20, 1971 (caused by valve malfunction at McCloud Dam).

EXTREMES OUTSIDE PERIOD OF RECORD.-Flood of Dec. 21, 1955, reached a stage of $12.5 \mathrm{ft}$, discharge, $17,800 \mathrm{ft}^{3} / \mathrm{s}$, from rating curve extended above $2,500 \mathrm{ft}^{3} / \mathrm{s}$

EXTREMES FOR CURRENT YEAR.-Peak discharges greater than base discharge of $3,000 \mathrm{ft}^{3} / \mathrm{s}$, or maximum:

\begin{tabular}{|c|c|c|c|c|c|c|}
\hline Date & Time & $\begin{array}{c}\text { Discharge } \\
\left(\mathrm{ft}^{3} / \mathrm{s}\right)\end{array}$ & $\begin{array}{c}\text { Gage height } \\
\text { (ft) }\end{array}$ & Date & Tim & $\begin{array}{l}\text { Gage height } \\
\text { (ft) }\end{array}$ \\
\hline Nov. 30 & 2115 & 1,330 & 3.75 & & & \\
\hline
\end{tabular}

DISCHARGE, CUBIC FEET PER SECOND, WATER YEAR OCTOBER 1998 TO SEPTEMBER 1999 DAILY MEAN VALUES

\begin{tabular}{|c|c|c|c|c|c|c|c|c|c|c|c|c|}
\hline DAY & $\mathrm{OCT}$ & NOV & $\mathrm{DEC}$ & JAN & FEB & MAR & APR & MAY & JUN & JUL & AUG & SEP \\
\hline 1 & 225 & 225 & 674 & 186 & 175 & 517 & 232 & 449 & 393 & 229 & 226 & 242 \\
\hline 2 & 225 & 225 & 397 & 179 & 175 & 403 & 220 & 449 & 382 & 228 & 226 & 242 \\
\hline 3 & 225 & 224 & 520 & 179 & 175 & 396 & 212 & 449 & 375 & 227 & 225 & 242 \\
\hline 4 & 225 & 223 & 361 & 179 & 174 & 355 & 203 & 449 & 372 & 227 & 246 & 242 \\
\hline 5 & 225 & 225 & 286 & 178 & 176 & 312 & 203 & 554 & 342 & 227 & 248 & 242 \\
\hline 6 & 225 & 225 & 246 & 178 & 203 & 282 & 200 & 572 & 290 & 228 & 249 & 242 \\
\hline 7 & 225 & 236 & 231 & 183 & 313 & 259 & 201 & 571 & 231 & 228 & 249 & 241 \\
\hline 8 & 225 & 228 & 235 & 184 & 286 & 247 & 238 & 566 & 227 & 227 & 249 & 239 \\
\hline 9 & 225 & 225 & 235 & 188 & 309 & 233 & 226 & 559 & 228 & 227 & 249 & 239 \\
\hline 10 & 225 & 230 & 241 & 186 & 271 & 216 & 220 & 491 & 229 & 226 & 249 & 239 \\
\hline 11 & 225 & 231 & 237 & 186 & 231 & 390 & 216 & 359 & 227 & 228 & 249 & 239 \\
\hline 12 & 225 & 229 & 231 & 179 & 206 & 204 & 218 & 354 & 228 & 233 & 249 & 239 \\
\hline 13 & 225 & 228 & 235 & 176 & 190 & 198 & 231 & 350 & 229 & 232 & 249 & 239 \\
\hline 14 & 225 & 228 & 238 & 182 & 179 & 195 & 250 & 347 & 229 & 233 & 249 & 239 \\
\hline 15 & 225 & 231 & 234 & 191 & 176 & 191 & 262 & 343 & 227 & 233 & 249 & 239 \\
\hline 16 & 225 & 231 & 193 & 189 & 179 & 196 & 276 & 339 & 229 & 233 & 248 & 239 \\
\hline 17 & 225 & 253 & 184 & 235 & 186 & 202 & 300 & 336 & 228 & 233 & 248 & 239 \\
\hline 18 & 225 & 237 & 185 & 319 & 229 & 210 & 334 & 336 & 227 & 230 & 247 & 239 \\
\hline 19 & 225 & 233 & 186 & 343 & 254 & 219 & 350 & 335 & 226 & 230 & 247 & 239 \\
\hline 20 & 225 & 231 & 186 & 405 & 234 & 227 & 349 & 394 & 225 & 230 & 246 & 239 \\
\hline 21 & 223 & 296 & 188 & 355 & 216 & 232 & 332 & 411 & 225 & 229 & 246 & 239 \\
\hline 22 & 222 & 285 & 201 & 335 & 204 & 233 & 589 & 521 & 224 & 229 & 246 & 239 \\
\hline 23 & 222 & 452 & 202 & 390 & 213 & 233 & 433 & 523 & 224 & 228 & 246 & 239 \\
\hline 24 & 222 & 279 & 198 & 305 & 240 & 300 & 509 & 521 & 225 & 228 & 245 & 239 \\
\hline 25 & 222 & 237 & 194 & 255 & 364 & 466 & 609 & 520 & 225 & 228 & 245 & 239 \\
\hline 26 & 222 & 238 & 193 & 223 & 317 & 391 & 721 & 516 & 227 & 228 & 245 & 239 \\
\hline 27 & 222 & 238 & 193 & 199 & 278 & 340 & 762 & 514 & 232 & 227 & 246 & 239 \\
\hline 28 & 224 & 225 & 193 & 190 & 470 & 302 & 661 & 514 & 231 & 227 & 246 & 239 \\
\hline 29 & 227 & 227 & 193 & 184 & --- & 278 & 449 & 514 & 230 & 226 & 246 & 239 \\
\hline 30 & 228 & 739 & 191 & 181 & --- & 263 & 449 & 514 & 230 & 225 & 247 & 239 \\
\hline 31 & 225 & --- & 191 & 181 & --- & 246 & --- & 422 & --- & 225 & 250 & --- \\
\hline TOTAL & 6959 & 7814 & 7672 & 7023 & 6623 & 8736 & 10455 & 14092 & 7617 & 7089 & 7605 & 7190 \\
\hline MEAN & 224 & 260 & 247 & 227 & 237 & 282 & 348 & 455 & 254 & 229 & 245 & 240 \\
\hline MAX & 228 & 739 & 674 & 405 & 470 & 517 & 762 & 572 & 393 & 233 & 250 & 242 \\
\hline MIN & 222 & 223 & 184 & 176 & 174 & 191 & 200 & 335 & 224 & 225 & 225 & 239 \\
\hline $\mathrm{AC}-\mathrm{FT}$ & 13800 & 15500 & 15220 & 13930 & 13140 & 17330 & 20740 & 27950 & 15110 & 14060 & 15080 & 14260 \\
\hline
\end{tabular}




\section{McCLOUD RIVER AT AH-DI-NA, NEAR McCLOUD, CA—Continued}

STATISTICS OF MONTHLY MEAN DATA FOR WATER YEARS 1965 - 1999, BY WATER YEAR (WY)

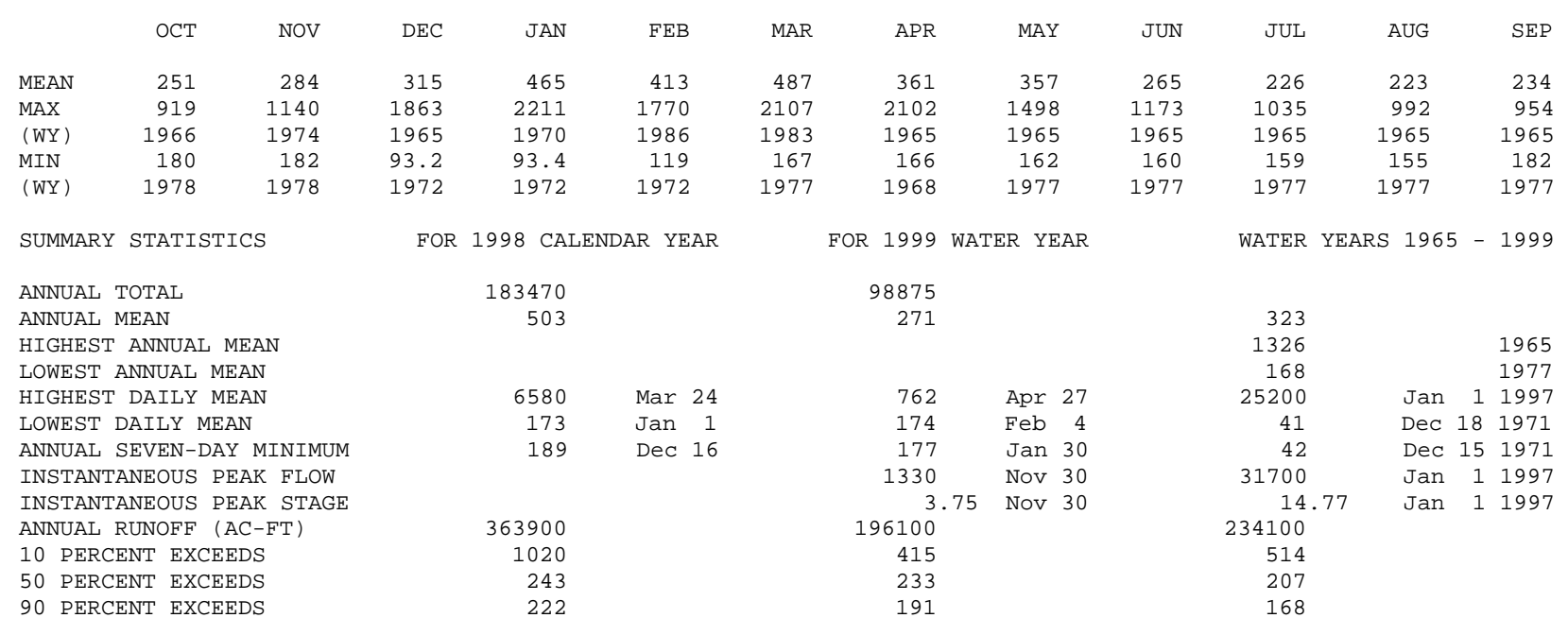




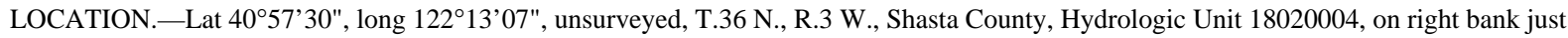
upstream from Shasta Lake, 0.2 mi downstream from Big Bollibokka Creek, and 11.3 mi east of Lamoine.

DRAINAGE AREA.-604 $\mathrm{mi}^{2}$.

PERIOD OF RECORD.-October 1945 to current year. Prior to 1950, published as "above Shasta Reservoir."

TEMPERATURE: Water years 1956-59.

REVISED RECORDS.—WSP 1445: 1953(M). WSP 1931: Drainage area. WDR CA-94-4: 1993(P).

GAGE.-Water-stage recorder. Datum of gage is $1,100.00 \mathrm{ft}$ above sea level (levels by U.S. Bureau of Reclamation).

REMARKS.- - Low flow completely regulated by Lake McCloud (station 11367740) 16.5 mi upstream since Nov. 3, 1965. Diversions to Iron Canyon Reservoir (station 11363920) began Dec. 1, 1965. See schematic diagram of Pit and McCloud River Basins.

COOPERATION.-Records were collected by Pacific Gas \& Electric Co., under general supervision of the U.S. Geological Survey, in connection with a Federal Energy Regulatory Commission project.

EXTREMES FOR PERIOD OF RECORD.-Maximum discharge, 51,300 ft $\mathrm{ft}^{3} / \mathrm{s}$, Jan. 1, 1997, gage height, $29.00 \mathrm{ft}$, from rating curve extended above $15,000 \mathrm{ft}^{3} / \mathrm{s}$ on basis of slope-area measurement of peak flow; minimum daily, $109 \mathrm{ft}^{3} / \mathrm{s}$, Dec. 16-20, 1971. Minimum prior to regulation by Lake McCloud, $825 \mathrm{ft}^{3} / \mathrm{s}$, Jan. 3, 1950 .

EXTREMES FOR CURRENT YEAR.-Peak discharges greater than base discharge of $4,500 \mathrm{ft}^{3} / \mathrm{s}$, or maximum:

\begin{tabular}{|c|c|c|c|c|c|c|c|}
\hline Date & Time & $\begin{array}{c}\text { Discharge } \\
\left(\mathrm{ft}^{3} / \mathrm{s}\right)\end{array}$ & $\begin{array}{c}\text { Gage height } \\
\text { (ft) }\end{array}$ & Date & Time & $\begin{array}{c}\text { Discharge } \\
\left(\mathrm{ft}^{3} / \mathrm{s}\right)\end{array}$ & $\begin{array}{c}\text { Gage height } \\
\text { (ft) }\end{array}$ \\
\hline Nov. 30 & 2100 & 7,750 & 17.06 & & & & \\
\hline
\end{tabular}

DISCHARGE, CUBIC FEET PER SECOND, WATER YEAR OCTOBER 1998 TO SEPTEMBER 1999 DAILY MEAN VALUES

\begin{tabular}{|c|c|c|c|c|c|c|c|c|c|c|c|c|}
\hline DAY & OCT & NOV & $\mathrm{DEC}$ & JAN & $\mathrm{FEB}$ & MAR & APR & MAY & JUN & JUL & AUG & SEP \\
\hline 1 & 354 & 354 & 4200 & 418 & 647 & 3240 & 1010 & 1260 & 637 & 402 & 340 & 349 \\
\hline 2 & 354 & 346 & 2340 & 404 & 615 & 2450 & 941 & 1090 & 654 & 397 & 340 & 348 \\
\hline 3 & 354 & 346 & 3100 & 398 & 594 & 2220 & 888 & 1040 & 649 & 394 & 339 & 343 \\
\hline 4 & 353 & 345 & 2000 & 391 & 577 & 1840 & 841 & 1110 & 631 & 393 & 338 & 343 \\
\hline 5 & 353 & 346 & 1380 & 389 & 561 & 1570 & 853 & 1100 & 609 & 388 & 340 & 343 \\
\hline 6 & 349 & 350 & 1080 & 386 & 1010 & 1380 & 812 & 1000 & 539 & 386 & 345 & 343 \\
\hline 7 & 346 & 472 & 916 & 386 & 3520 & 1230 & 811 & 988 & 474 & 383 & 352 & 340 \\
\hline 8 & 351 & 392 & 837 & 384 & 2430 & 1190 & 1260 & 973 & 451 & 379 & 352 & 339 \\
\hline 9 & 350 & 365 & 765 & 383 & 2910 & 1140 & 1200 & 952 & 443 & 378 & 344 & 338 \\
\hline 10 & 348 & 397 & 722 & 383 & 2200 & 1070 & 1100 & 894 & 441 & 376 & 345 & 340 \\
\hline 11 & 348 & 398 & 689 & 380 & 1560 & 1170 & 1040 & 768 & 437 & 374 & 352 & 336 \\
\hline 12 & 346 & 379 & 656 & 372 & 1260 & 960 & 1010 & 727 & 434 & 378 & 347 & 336 \\
\hline 13 & 352 & 369 & 652 & 362 & 1070 & 928 & 1010 & 717 & 433 & 375 & 341 & 336 \\
\hline 14 & 349 & 364 & 652 & 365 & 964 & 928 & 1030 & 704 & 427 & 376 & 338 & 335 \\
\hline 15 & 345 & 388 & 613 & 391 & 879 & 916 & 1020 & 688 & 428 & 374 & 339 & 334 \\
\hline 16 & 345 & 385 & 569 & 429 & 958 & 905 & 1020 & 677 & 427 & 373 & 336 & 334 \\
\hline 17 & 345 & 527 & 531 & 726 & 1170 & 906 & 1040 & 667 & 427 & 373 & 335 & 336 \\
\hline 18 & 345 & 423 & 523 & 2080 & 1470 & 918 & 1100 & 660 & 428 & 371 & 335 & 336 \\
\hline 19 & 340 & 388 & 512 & 1900 & 1670 & 924 & 1120 & 655 & 428 & 368 & 333 & 336 \\
\hline 20 & 336 & 376 & 502 & 2240 & 1440 & 936 & 1090 & 697 & 423 & 367 & 329 & 336 \\
\hline 21 & 336 & 791 & 479 & 1840 & 1320 & 941 & 1040 & 722 & 421 & 365 & 327 & 335 \\
\hline 22 & 336 & 806 & 484 & 1990 & 1360 & 927 & 1240 & 801 & 416 & 362 & 328 & 333 \\
\hline 23 & 338 & 2100 & 479 & 3040 & 1660 & 949 & 1070 & 822 & 412 & 361 & 326 & 331 \\
\hline 24 & 393 & 1330 & 471 & 1930 & 1820 & 1440 & 1100 & 821 & 411 & 358 & 328 & 332 \\
\hline 25 & 371 & 926 & 463 & 1410 & 2860 & 2910 & 1190 & 810 & 410 & 359 & 327 & 331 \\
\hline 26 & 352 & 867 & 457 & 1150 & 2280 & 2210 & 1290 & 799 & 407 & 357 & 324 & 331 \\
\hline 27 & 346 & 891 & 449 & 959 & 1770 & 1720 & 1350 & 795 & 413 & 354 & 324 & 331 \\
\hline 28 & 350 & 729 & 444 & 849 & 2880 & 1450 & 1240 & 790 & 409 & 355 & 325 & 331 \\
\hline 29 & 348 & 728 & 437 & 777 & --- & 1280 & 1050 & 800 & 405 & 350 & 326 & 331 \\
\hline 30 & 349 & 4450 & 430 & 729 & --- & 1180 & 1230 & 783 & 403 & 343 & 329 & 334 \\
\hline 31 & 347 & --- & 427 & 696 & --- & 1080 & --- & 701 & --- & 341 & 332 & --- \\
\hline TOTAL & 10829 & 21328 & 28259 & 28537 & 43455 & 42908 & 31996 & 26011 & 13927 & 11510 & 10416 & 10101 \\
\hline MEAN & 349 & 711 & 912 & 921 & 1552 & 1384 & 1067 & 839 & 464 & 371 & 336 & 337 \\
\hline MAX & 393 & 4450 & 4200 & 3040 & 3520 & 3240 & 1350 & 1260 & 654 & 402 & 352 & 349 \\
\hline MIN & 336 & 345 & 427 & 362 & 561 & 905 & 811 & 655 & 403 & 341 & 324 & 331 \\
\hline $\mathrm{AC}-\mathrm{FT}$ & 21480 & 42300 & 56050 & 56600 & 86190 & 85110 & 63460 & 51590 & 27620 & 22830 & 20660 & 20040 \\
\hline
\end{tabular}


11368000 McCLOUD RIVER ABOVE SHASTA LAKE, CA—Continued

STATISTICS OF MONTHLY MEAN DATA FOR WATER YEARS 1946 - 1965, BY WATER YEAR (WY)

\begin{tabular}{|c|c|c|c|c|c|c|c|c|c|c|c|c|}
\hline & OCT & NOV & $\mathrm{DEC}$ & JAN & FEB & MAR & $\mathrm{APR}$ & MAY & JUN & JUL & AUG & SEP \\
\hline MEAN & 1121 & 1252 & 2080 & 2077 & 2617 & 2177 & 2467 & 1965 & 1460 & 1159 & 1059 & 1020 \\
\hline MAX & 1899 & 2162 & 6513 & 4525 & 7493 & 3966 & 4599 & 2978 & 2248 & 1715 & 1489 & 1395 \\
\hline (WY) & 1951 & 1951 & 1956 & 1953 & 1958 & 1958 & 1963 & 1958 & 1958 & 1958 & 1958 & 1958 \\
\hline MIN & 856 & 870 & 856 & 903 & 1040 & 1265 & 1320 & 1085 & 1069 & 901 & 852 & 839 \\
\hline (WY) & 1950 & 1950 & 1950 & 1949 & 1948 & 1964 & 1964 & 1947 & 1949 & 1950 & 1950 & 1950 \\
\hline
\end{tabular}

SUMMARY STATISTICS

ANNUAL MEAN

HIGHEST ANNUAL MEAN

LOWEST ANNUAL MEAN

HIGHEST DAILY MEAN

LOWEST DAILY MEAN

ANNUAL SEVEN-DAY MINIMUM

INSTANTANEOUS PEAK FLOW

INSTANTANEOUS PEAK STAGE

ANNUAL RUNOFF (AC-FT)

10 PERCENT EXCEEDS

50 PERCENT EXCEEDS

90 PERCENT EXCEEDS
WATER YEARS 1946 - 1965

\begin{tabular}{|c|c|c|}
\hline 1699 & & \\
\hline 2703 & & 1958 \\
\hline 1213 & & 1950 \\
\hline 36100 & Dec 21 & 1955 \\
\hline 825 & $\operatorname{Jan} 3$ & 1950 \\
\hline 826 & Oct 9 & 1950 \\
\hline a 45200 & Dec 22 & 1955 \\
\hline 28.20 & Dec 22 & 1955 \\
\hline 1231000 & & \\
\hline 2670 & & \\
\hline 1270 & & \\
\hline 928 & & \\
\hline
\end{tabular}

a from rating curve extended above $6,400 \mathrm{ft}^{3} / \mathrm{s}$ on basis of slope-area measurement of peak flow.

STATISTICS OF MONTHLY MEAN DATA FOR WATER YEARS 1967 - 1999, BY WATER YEAR (WY)

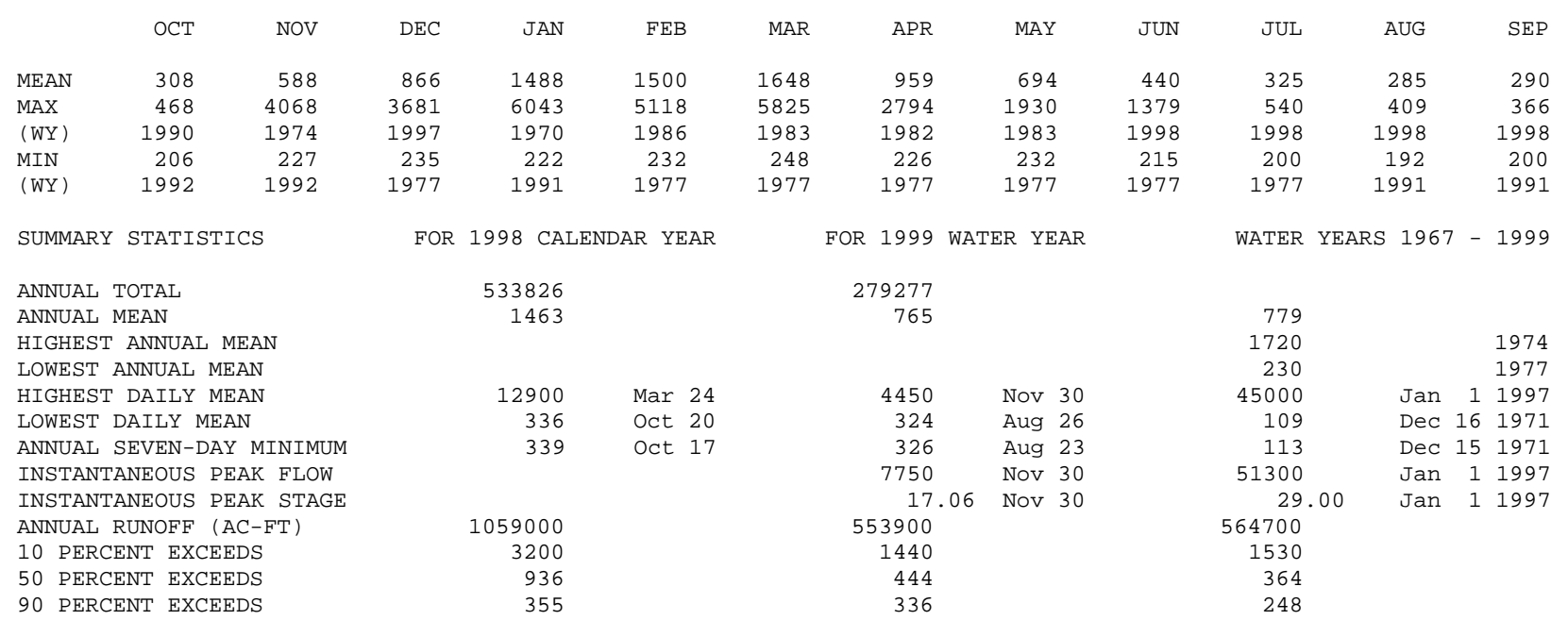




\section{SHASTA LAKE NEAR REDDING, CA}

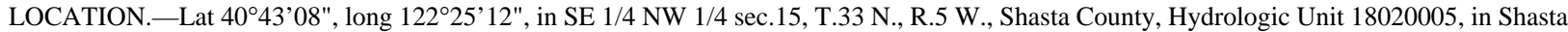
Dam on Sacramento River near right bank, 2 mi downstream from Squaw Creek, and 9.5 mi north of Redding.

DRAINAGE AREA.-6,421 $\mathrm{mi}^{2}$, excluding Goose Lake Basin.

PERIOD OF RECORD.- - November 1942 to current year. Prior to 1950, published as Shasta Reservoir near Redding.

CHEMICAL DATA: Water years 1978-80.

GAGE.-Water-stage recorder. Datum of gage is sea level (levels by U.S. Bureau of Reclamation). Prior to July 10, 1944, nonrecording gage at various sites near dam at same datum. Contents based on capacity table dated May 8, 1967, provided by U.S. Bureau of Reclamation.

REMARKS.-Lake is formed by concrete gravity-type dam completed in 1949; regulation began Dec. 30, 1943. Usable capacity, 4,436,400 acre-ft between elevations $737.75 \mathrm{ft}$, invert of lowest set of river outlets, and 1,067.0 ft, top of flashboard gates on drum-type spillway gates. Operating pool from elevation, $840.0 \mathrm{ft}$, capacity, 587,127 acre-ft to 1,067.0 ft, capacity, 4,552,090 acre-ft. Dead storage, 115,800 acre-ft. Installation of flashboard gates on top of drum gates completed Nov. 12, 1964. All water passes down the Sacramento River, most of which is through powerplant at dam. Figures given represent total contents at 2400 hours. Lake is used for flood control, power generation, irrigation, and recreation. See schematic diagram of Pit and McCloud River Basins.

COOPERATION.-Records were provided by U.S. Bureau of Reclamation, not rounded to U.S. Geological Survey standards.

EXTREMES (AT 2400 HOURS) FOR PERIOD OF RECORD.-Maximum contents, 4,550,300 acre-ft, May 19, 1967, elevation, 1,066.94 ft; minimum since first filling, 562,600 acre-ft, Sept. 13, 1977, elevation, $836.68 \mathrm{ft}$.

EXTREMES (AT 2400 HOURS) FOR CURRENT YEAR.-Maximum contents, 4,314,588 acre-ft, May 21, elevation, 1,058.89 ft; minimum, $3,248,975$ acre-ft, Dec. 21, elevation, $1018.42 \mathrm{ft}$.

Capacity table (elevation, in feet, and contents, in acre-feet)

(Based on table provided by U.S. Bureau of Reclamation, dated May 8, 1967)

$\begin{array}{rrrrrrrrrrrr}830 & 515,543 & 870 & 843,589 & 910 & 1,291,854 & 950 & 1,876,996 & 990 & 2,616,622 & 1,030 & 3,533,478 \\ 840 & 587,127 & 880 & 943,929 & 920 & 1,424,780 & 960 & 2,046,829 & 1,000 & 2,828,544 & 1,050 & 4,063,108 \\ 850 & 665,511 & 890 & 1,051,713 & 930 & 1,566,238 & 970 & 2,226,093 & 1,010 & 3,051,750 & 1,067 & 4,552,090 \\ 860 & 751,027 & 900 & 1,167,888 & 940 & 1,717,255 & 980 & 2,416,019 & 1,020 & 3,286,929 & & \end{array}$

RESERVOIR STORAGE (ACRE-FEET), WATER YEAR OCTOBER 1998 TO SEPTEMBER 1999 DAILY OBSERVATION AT 2400 HOURS

\begin{tabular}{|c|c|c|c|c|c|c|c|c|c|c|c|c|}
\hline DAY & OCT & NOV & $\mathrm{DEC}$ & JAN & $\mathrm{FEB}$ & MAR & APR & MAY & JUN & JUL & AUG & SEP \\
\hline 1 & 3443059 & 3406196 & 3472688 & 3277302 & 3593240 & 3722696 & 3880983 & 4263107 & 4303058 & 4104544 & 3701511 & 3430405 \\
\hline 2 & 439830 & 3403492 & 3494965 & 3284762 & 3598104 & 3722171 & 3899788 & 4273150 & 4300464 & 4091991 & 3690808 & 3422989 \\
\hline 3 & 3429663 & 3400787 & 3533985 & 3294399 & 3601431 & 3724534 & 3913820 & 4280611 & 4297872 & 4076157 & 3677285 & 3416318 \\
\hline 4 & 3419284 & 3399310 & 3542600 & 3299942 & 3599382 & 3721124 & 3924882 & 4287498 & 4295852 & 4064220 & 3664818 & 3411621 \\
\hline 5 & 415082 & 3398325 & 3542600 & 3305244 & 3590169 & 3711169 & 3941146 & 4293258 & 4295563 & 4053160 & 3653687 & 3407916 \\
\hline 6 & 410631 & 398325 & 3506509 & 3310303 & 3604502 & 3693157 & 3892 & 4297872 & 4294409 & 4039896 & 3643865 & 02260 \\
\hline 7 & 414092 & 3407178 & 3480428 & 3316356 & 3659890 & 3673648 & 7516 & 4302481 & 4291238 & 24746 & 3630710 & 97343 \\
\hline 8 & 416319 & 3407178 & 3451752 & 3320475 & 3686895 & 3657306 & 3989352 & 4306517 & 4288934 & 4011820 & 3620936 & 3393161 \\
\hline 9 & 409150 & 407178 & 3426450 & 323382 & 3745278 & 3640250 & 4007969 & 4307094 & 4285204 & 3999755 & 3615276 & 3388490 \\
\hline 10 & 399556 & 3412611 & 3396851 & 3324108 & 3772722 & 3615018 & 4026945 & 4308536 & 4282334 & 3989352 & 3609875 & 3383572 \\
\hline 11 & 195 & 587 & 584 & 773 & 2258 & 0937 & 35 & 960 & 0324 & 9230 & 1431 & 3924 \\
\hline 12 & 752 & 9892 & 232 & 742 & 789 & 3586 & 132 & 517 & 7455 & 3607 & 4264 & 2563 \\
\hline 13 & 839 & 2261 & 867 & & 697 & 2384 & 8107 & 940 & & & 8122 & 9382 \\
\hline 14 & 4554 & 3393161 & 3309099 & 3331376 & 3770074 & 3552990 & 6157 & 6517 & 4268561 & 3940061 & 3576642 & 66935 \\
\hline 15 & 385047 & 3384308 & 3296086 & 3334040 & 3746861 & 3553498 & 4085044 & 4305078 & 4264830 & 3927318 & 3569769 & 63266 \\
\hline 16 & & $\$ 233$ & & & 925 & & & & & & & \\
\hline 17 & 7013 & 368158 & 6821 & 4240 & 5876 & 9073 & 5103 & 7094 & 4253385 & 6563 & 8564 & 4240 \\
\hline 18 & 8490 & 355457 & 6473 & 5782 & 0205 & 3148 & 377 & 671 & 4248244 & 3403 & 0961 & 8640 \\
\hline 19 & 210 & 824 & 158 & 542 & 460 & 9514 & 3863 & 398 & 0536 & 15 & 2854 & 2068 \\
\hline 20 & 391441 & 3331133 & 3250172 & 3457713 & 3683778 & 3579698 & 4145399 & 4313146 & 4231114 & 3856622 & 3536519 & 37685 \\
\hline 21 & & & & & & & & & & & & \\
\hline 22 & 718 & 320717 & 9934 & 94965 & 3683778 & 8338 & 1864 & 8822 & 1500 & & 9609 & 8172 \\
\hline 23 & 457 & 349127 & 3047 & 27679 & 3687155 & 5564 & 2601 & 9687 & 4201275 & 52 & 2804 & 8416 \\
\hline 24 & 5620 & 3359108 & 680 & 1839 & 3691592 & 1702 & 9292 & 0264 & 0240 & 6090 & 4248 & 34766 \\
\hline 25 & 3393654 & 3364977 & 3259510 & 3547670 & 3713517 & 3708036 & 4212068 & 4311996 & 4179489 & 3791794 & 3495467 & 3333555 \\
\hline 26 & & 3372074 & & & 11949 & & & & & & & 3798 \\
\hline 27 & 397343 & 3375254 & 3264788 & 3557804 & 3704121 & 3771132 & 4236538 & 4311130 & 4151026 & 69279 & 3479428 & 28468 \\
\hline 28 & 3397343 & 3374765 & 3266953 & 3562638 & 3712733 & 3796561 & 4243963 & 4310842 & 4139514 & 3757140 & 3469442 & 3327499 \\
\hline 29 & 3397343 & 3382104 & 3268156 & 3571296 & --- & 3819060 & 4249673 & 4311130 & 4126342 & 45014 & 3456967 & 3327742 \\
\hline 30 & 3399310 & 3435606 & 3269361 & 9698 & --- & 3841938 & 56240 & 4306228 & 4116822 & & 7528 & 3327499 \\
\hline 31 & 3403984 & --- & 3269602 & 3588122 & --- & 3863036 & --- & 4304500 & --- & 3716927 & 3437843 & \\
\hline MAX & 443059 & 3435606 & & & 3782789 & & & & & & & 30405 \\
\hline MIN & 3382839 & 3320717 & 3248975 & 3277302 & 3590169 & 3552990 & 3880983 & 4263107 & 4116822 & 3716927 & 3437843 & 3327499 \\
\hline $\mathrm{a}$ & 1024.81 & 1026.09 & 1019.28 & 1032.15 & 1036.97 & 1042.65 & 1056.86 & 1058.54 & 1051.93 & 1037.13 & 1026.18 & 1021.68 \\
\hline b & -37089 & +31622 & -166004 & +318520 & +124611 & +150303 & +393204 & +48260 & -187678 & -399895 & -279084 & -110344 \\
\hline
\end{tabular}




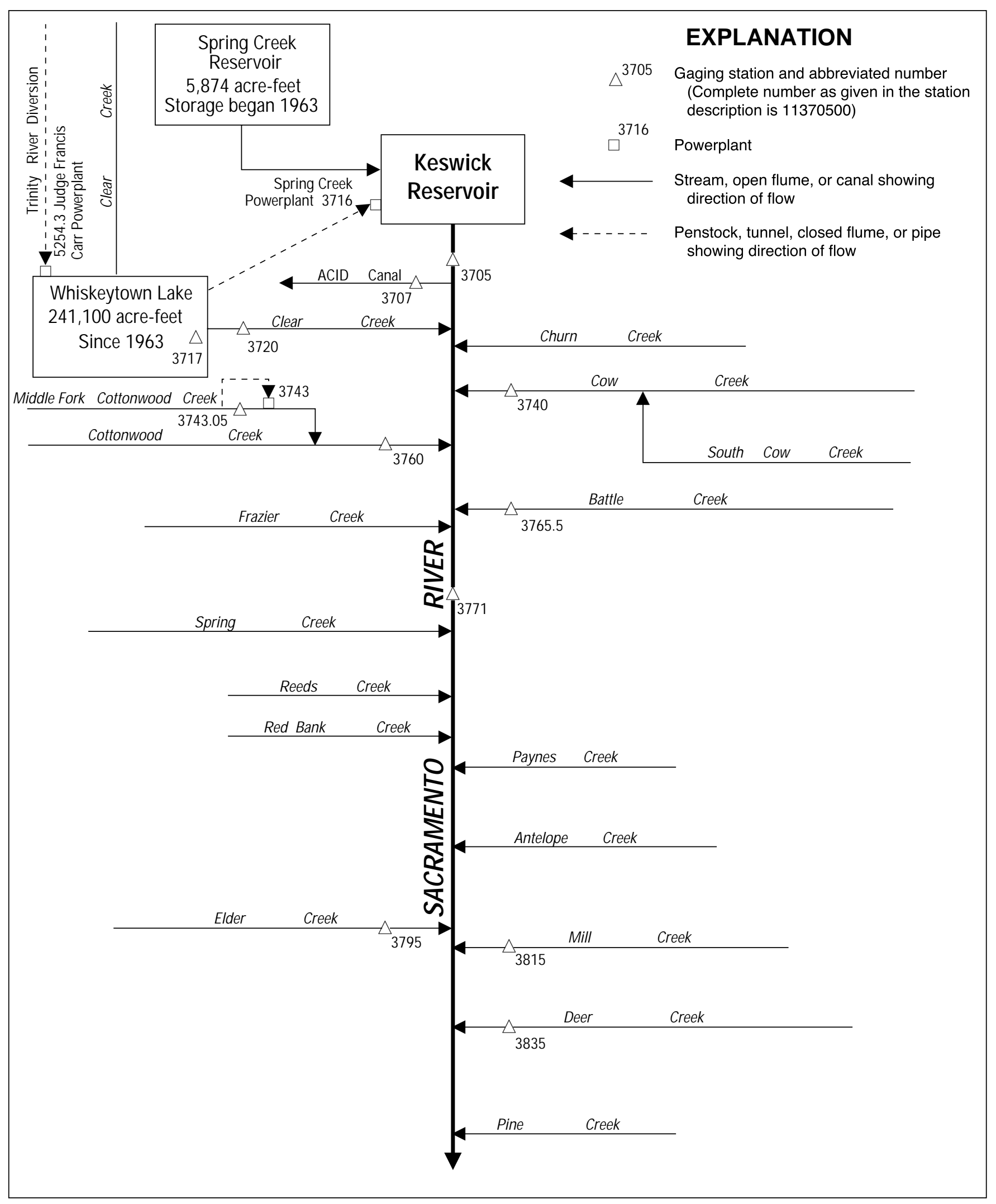

Figure 25. Diversions and storage in upper Sacramento River Basin. 
LOCATION.-Lat 40³6'04", 1ong 122²6’36", in SW 1/4 NW 1/4 sec.28, T.32 N., R.5 W., Shasta County, Hydrologic Unit 18020101, on right bank 0.4 mi upstream from Middle Creek, 0.8 mi downstream from Keswick Dam, 1.6 mi downstream from Keswick, and 10 mi downstream from Shasta Dam.

DRAINAGE AREA.-6,468 $\mathrm{mi}^{2}$, excluding Goose Lake Basin.

PERIOD OF RECORD.-October 1938 to current year. Monthly discharge only for some periods, published in WSP 1315-A.

CHEMICAL DATA: Water years 1951-94. Published as "near Keswick" in 1951 and 1953, and as "at Keswick Dam, near Keswick" in 1968-69.

BIOLOGICAL DATA: Water years 1979-81.

SPECIFIC CONDUCTANCE: Water years 1978-94.

WATER TEMPERATURE: Water years 1978-94.

SEDIMENT DATA: Water years 1978-94.

REVISED RECORDS.-WSP 1931: Drainage area.

GAGE.-Water-stage recorder. Datum of gage is $479.81 \mathrm{ft}$ above sea level. Prior to Oct. 1, 1939, at site $1.5 \mathrm{mi}$ upstream at datum $20.2 \mathrm{ft}$ higher and Oct. 1, 1939, to Apr. 30, 1942, at site 1.5 mi upstream at datum 15.2 ft higher. Aug. 20, 1960, to July 3, 1973, auxiliary water-stage recorder at city of Redding pumping plant $2.1 \mathrm{mi}$ downstream.

REMARKS.-Records excellent. Flow completely regulated by Shasta Lake (station 11370000) beginning Dec. 30, 1943. Minor regulation by Keswick Reservoir since 1950, total capacity, 23,800 acre-ft, operational capacity, 4,170 acre-ft, between normal operating elevations of $579.0 \mathrm{ft}$ and $586.0 \mathrm{ft}$. No diversion between Shasta Dam and station at Keswick. Since December 1963, water is released from Whiskeytown Lake (station 11371700), through a tunnel to Spring Creek Powerplant (station 11371600), and then into Keswick Reservoir. See schematic diagrams of upper Sacramento River Basin and Pit and McCloud River Basins.

EXTREMES FOR PERIOD OF RECORD.-Maximum discharge, 186,000 $\mathrm{ft}^{3} / \mathrm{s}$, Feb. 23, 1940, gage height, $47.2 \mathrm{ft}$, site and datum then in use, from rating curve extended above $75,000 \mathrm{ft}^{3} / \mathrm{s}$ on basis of peak discharge at Kennet, plus 4,000 $\mathrm{ft}^{3} / \mathrm{s}$ estimated inflow; minimum observed, $2,730 \mathrm{ft}^{3} / \mathrm{s}$, Aug. 22, 1939. Since regulation by Shasta Dam in 1943, maximum discharge, 81,400 ft ${ }^{3} / \mathrm{s}, \mathrm{Apr}$. 1, 1974, gage height, $31.92 \mathrm{ft}$; maximum gage height, $32.71 \mathrm{ft}$, Jan. 4, 1997; minimum discharge, $154 \mathrm{ft}^{3} / \mathrm{s}$, May 15, 1948.

DISCHARGE, CUBIC FEET PER SECOND, WATER YEAR OCTOBER 1998 TO SEPTEMBER 1999 DAILY MEAN VALUES

\begin{tabular}{|c|c|c|c|c|c|c|c|c|c|c|c|c|}
\hline DAY & $\mathrm{OCT}$ & NOV & $\mathrm{DEC}$ & JAN & FEB & MAR & $\mathrm{APR}$ & MAY & JUN & JUL & AUG & SEP \\
\hline 1 & 8130 & 6040 & 14700 & 5600 & 7530 & 27700 & 5540 & 9990 & 11100 & 13300 & 13100 & 9580 \\
\hline 2 & 8000 & 6000 & 14700 & 5560 & 7560 & 27800 & 5550 & 10000 & 11100 & 13200 & 13100 & 9560 \\
\hline 3 & 8040 & 5980 & 14800 & 5550 & 6470 & 27600 & 5560 & 10000 & 11200 & 13000 & 13100 & 9130 \\
\hline 4 & 8050 & 5960 & 18300 & 5560 & 11500 & 28900 & 5570 & 10000 & 11100 & 13100 & 12600 & 9130 \\
\hline 5 & 8060 & 5940 & 25100 & 5580 & 15900 & 30200 & 5560 & 10000 & 10600 & 13200 & 12100 & 9120 \\
\hline 6 & 8150 & 7090 & 25200 & 5580 & 16500 & 30100 & 5510 & 11000 & 10500 & 13300 & 11600 & 9080 \\
\hline 7 & 8070 & 7150 & 25200 & 5540 & 17500 & 30100 & 5570 & 11000 & 10600 & 13400 & 11000 & 8690 \\
\hline 8 & 7610 & 7020 & 25300 & 5410 & 16300 & 30000 & 5780 & 11000 & 10600 & 13300 & 11000 & 8690 \\
\hline 9 & 7770 & 6930 & 22700 & 5450 & 13800 & 30300 & 7220 & 11000 & 10600 & 13300 & 11000 & 8660 \\
\hline 10 & 7530 & 6970 & 22200 & 5550 & 14800 & 30300 & 8190 & 10900 & 10600 & 13300 & 10400 & 8610 \\
\hline 11 & 7040 & 6970 & 22200 & 5570 & 16700 & 29600 & 8020 & 11000 & 10700 & 13200 & 10400 & 8070 \\
\hline 12 & 6750 & 8260 & 21900 & 5570 & 19700 & 25400 & 7980 & 11000 & 10600 & 13400 & 10100 & 8070 \\
\hline 13 & 6580 & 9350 & 19000 & 5580 & 19800 & 21900 & 9850 & 10100 & 10600 & 14000 & 9950 & 8090 \\
\hline 14 & 6310 & 9550 & 16000 & 5600 & 19900 & 18600 & 9960 & 10400 & 11100 & 13500 & 10000 & 8080 \\
\hline 15 & 6100 & 9590 & 14700 & 5560 & 24900 & 15600 & 9950 & 11000 & 11200 & 13700 & 9580 & 8090 \\
\hline 16 & 5990 & 9750 & 14600 & 5570 & 25400 & 14500 & 9960 & 10100 & 11200 & 13600 & 9590 & 8090 \\
\hline 17 & 6010 & 14200 & 14800 & 5610 & 28200 & 14200 & 9960 & 9620 & 11300 & 13600 & 9600 & 8080 \\
\hline 18 & 6010 & 14700 & 14400 & 5570 & 30200 & 12200 & 9970 & 9280 & 11700 & 13500 & 9590 & 7580 \\
\hline 19 & 6030 & 14900 & 12900 & 5590 & 26200 & 10400 & 9940 & 9080 & 11800 & 13600 & 9590 & 7580 \\
\hline 20 & 6030 & 14700 & 11100 & 9690 & 25900 & 9070 & 8730 & 9410 & 12200 & 13500 & 9610 & 7600 \\
\hline 21 & 6050 & 14700 & 9690 & 15400 & 25700 & 7720 & 8540 & 9990 & 12200 & 13100 & 9580 & 7600 \\
\hline 22 & 6080 & 14400 & 8260 & 15300 & 22100 & 6700 & 8560 & 11000 & 12300 & 13100 & 9600 & 7120 \\
\hline 23 & 6090 & 14800 & 7140 & 15100 & 21300 & 6070 & 8580 & 11100 & 13000 & 13100 & 9600 & 7130 \\
\hline 24 & 6070 & 14800 & 6940 & 14800 & 21400 & 6190 & 8570 & 10200 & 13100 & 13300 & 9590 & 7100 \\
\hline 25 & 6100 & 14900 & 6960 & 14500 & 22000 & 6220 & 8560 & 10100 & 13300 & 13100 & 9600 & 7100 \\
\hline 26 & 6090 & 14800 & 6920 & 13000 & 27600 & 5690 & 8560 & 10600 & 13200 & 13100 & 9590 & 7080 \\
\hline 27 & 6090 & 14700 & 7000 & 12100 & 28200 & 5530 & 8580 & 11200 & 13700 & 13100 & 9570 & 7070 \\
\hline 28 & 6050 & 14600 & 7110 & 10300 & 28000 & 5550 & 9860 & 11600 & 13600 & 13200 & 9580 & 6600 \\
\hline 29 & 6070 & 14800 & 6120 & 9170 & -- & 5540 & 9930 & 11600 & 13600 & 13100 & 9530 & 6610 \\
\hline 30 & 6070 & 15100 & 5810 & 8030 & --- & 5560 & 9980 & 11400 & 13200 & 13400 & 9530 & 6620 \\
\hline 31 & 6080 & --- & 5660 & 7530 & --- & 5550 & --- & 11100 & --- & 13500 & 9580 & --- \\
\hline TOTAL & 209100 & 324650 & 447410 & 250520 & 561060 & 530790 & 244090 & 325770 & 351600 & 413100 & 322360 & 239610 \\
\hline MEAN & 6745 & 10820 & 14430 & 8081 & 20040 & 17120 & 8136 & 10510 & 11720 & 13330 & 10400 & 7987 \\
\hline MAX & 8150 & 15100 & 25300 & 15400 & 30200 & 30300 & 9980 & 11600 & 13700 & 14000 & 13100 & 9580 \\
\hline MIN & 5990 & 5940 & 5660 & 5410 & 6470 & 5530 & 5510 & 9080 & 10500 & 13000 & 9530 & 6600 \\
\hline $\mathrm{AC}-\mathrm{FT}$ & 414700 & 643900 & 887400 & 496900 & 1113000 & 1053000 & 484200 & 646200 & 697400 & 819400 & 639400 & 475300 \\
\hline
\end{tabular}


11370500 SACRAMENTO RIVER AT KESWICK, CA—Continued

STATISTICS OF MONTHLY MEAN DATA FOR WATER YEARS 1946 - 1962, BY WATER YEAR (WY)

\begin{tabular}{|c|c|c|c|c|c|c|c|c|c|c|c|c|}
\hline & $\mathrm{OCT}$ & NOV & $\mathrm{DEC}$ & JAN & $\mathrm{FEB}$ & MAR & APR & MAY & JUN & JUL & AUG & SEP \\
\hline MEAN & 5992 & 5603 & 6611 & 10610 & 11700 & 6564 & 6714 & 8212 & 8564 & 9951 & 10030 & 7331 \\
\hline MAX & 8572 & 8970 & 16680 & 32870 & 44170 & 14490 & 21180 & 13400 & 10300 & 11810 & 11870 & 10030 \\
\hline (WY) & 1959 & 1958 & 1951 & 1953 & 1958 & 1957 & 1958 & 1948 & 1948 & 1951 & 1958 & 1958 \\
\hline MIN & 4785 & 4064 & 3726 & 3234 & 3060 & 2546 & 2830 & 5247 & 6437 & 7480 & 7057 & 5239 \\
\hline (WY) & 1948 & 1952 & 1960 & 1962 & 1950 & 1950 & 1950 & 1951 & 1947 & 1947 & 1947 & 1947 \\
\hline
\end{tabular}

SUMMARY STATISTICS

ANNUAL MEAN

HIGHEST ANNUAL MEAN

LOWEST ANNUAL MEAN

HIGHEST DAILY MEAN

LOWEST DAILY MEAN

ANNUAL SEVEN-DAY MINIMUM

INSTANTANEOUS PEAK FLOW

INSTANTANEOUS PEAK STAGE

INSTANTANEOUS LOW FLOW

ANNUAL RUNOFF (AC-FT)

10 PERCENT EXCEEDS

50 PERCENT EXCEEDS

90 PERCENT EXCEEDS
WATER YEARS 1946 - 1962

$\begin{array}{rlll}8141 & & & \\ 13910 & & & 1958 \\ 5364 & & & 1950 \\ 75800 & \text { Feb } 21 & 1958 \\ 2360 & \text { Mar } 15 & 1950 \\ 2440 & \text { Mar } 9 & 1950 \\ 78800 & \text { Feb } 21 & 1958 \\ 31.55 & \text { Feb 21 } 1958 \\ 154 & \text { May } 15 & 1948 \\ 5898000 & & & \\ 11600 & & & \\ 7000 & & & \\ 3720 & & & \end{array}$

STATISTICS OF MONTHLY MEAN DATA FOR WATER YEARS 1964 - 1999, BY WATER YEAR (WY)

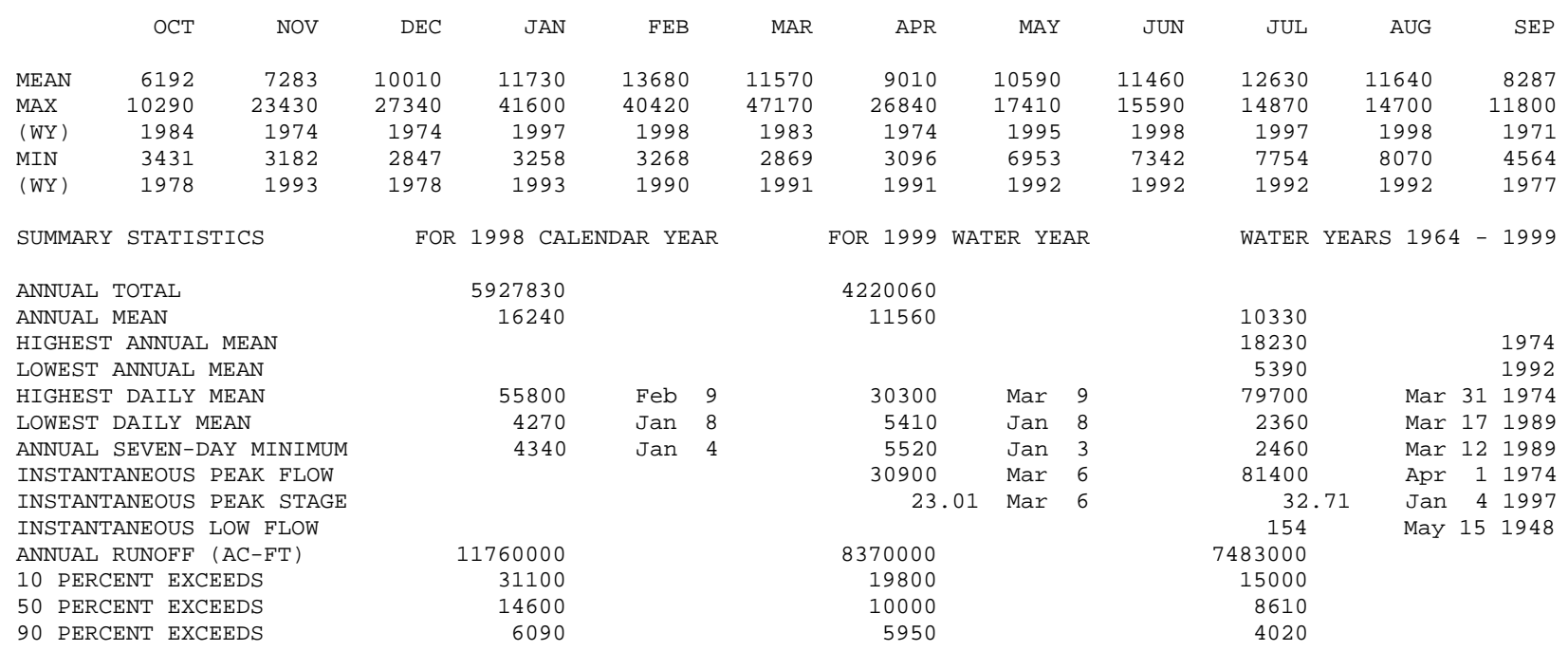


LOCATION.-Lat 40³4'08", 1ong 122²2’49", unsurveyed, Shasta County, Hydrologic Unit 18020101, on right bank of canal 10 ft upstream from Sharon Street, $900 \mathrm{ft}$ downstream from Parkview Avenue, and $0.75 \mathrm{mi}$ southwest of Mercy Hospital.

PERIOD OF RECORD.-April to September 1989, April 1991 to current year (beginning October 1994, irrigation season only).

GAGE.-Water-stage recorder and acoustic-velocity meter. Elevation of gage is $480 \mathrm{ft}$ above sea level, from topographic map.

REMARKS.-Records good. Canal diverts from Sacramento River 0.3 mi downstream from Southern Pacific Railroad bridge and 0.1 mi upstream from Highway 273; water is used for irrigation. See schematic diagrams for upper Sacramento River Basin and Pit and McCloud River Basins.

EXTREMES FOR PERIOD OF RECORD.-Maximum daily discharge, $370 \mathrm{ft}^{3}$ /s, June 9, 1989; no flow at times each year.

DISCHARGE, CUBIC FEET PER SECOND, WATER YEAR OCTOBER 1998 TO SEPTEMBER 1999 DAILY MEAN VALUES

\begin{tabular}{|c|c|c|c|c|c|c|c|c|c|c|c|c|}
\hline DAY & OCT & NOV & $\mathrm{DEC}$ & JAN & $\mathrm{FEB}$ & MAR & APR & MAY & JUN & JUL & AUG & SEP \\
\hline 1 & 241 & --- & --- & --- & --- & --- & --- & 295 & 331 & 245 & 281 & 249 \\
\hline 2 & 239 & --- & --- & --- & --- & --- & --- & 301 & 310 & 260 & 280 & 245 \\
\hline 3 & 237 & --- & --- & --- & --- & --- & --- & 304 & 219 & 278 & 279 & 239 \\
\hline 4 & 235 & --- & --- & --- & --- & --- & --- & 310 & 250 & 284 & 274 & 245 \\
\hline 5 & 233 & --- & --- & --- & --- & --- & --- & 308 & 253 & 285 & 270 & 246 \\
\hline 6 & 233 & --- & --- & --- & --- & --- & --- & 311 & 290 & 285 & 267 & 246 \\
\hline 7 & 232 & --- & --- & --- & --- & --- & --- & 314 & 297 & 282 & 267 & 246 \\
\hline 8 & 231 & --- & --- & --- & --- & --- & 24 & 322 & 312 & 278 & 265 & 245 \\
\hline 9 & 237 & --- & --- & --- & --- & --- & 59 & 321 & 316 & 277 & 268 & 243 \\
\hline 10 & 234 & --- & --- & --- & --- & --- & 64 & 319 & 315 & 281 & 274 & 244 \\
\hline 11 & 227 & --- & --- & --- & --- & --- & 61 & 314 & 312 & 284 & 269 & 241 \\
\hline 12 & 219 & --- & --- & --- & --- & --- & 104 & 306 & 315 & 288 & 265 & 240 \\
\hline 13 & 219 & --- & --- & --- & --- & --- & 175 & 294 & 322 & 295 & 262 & 239 \\
\hline 14 & 218 & --- & --- & --- & --- & --- & 176 & 282 & 331 & 290 & 264 & 235 \\
\hline 15 & 215 & --- & --- & --- & --- & --- & 175 & 289 & 325 & 285 & 258 & 231 \\
\hline 16 & 211 & --- & --- & --- & --- & --- & 175 & 289 & 299 & 282 & 256 & 235 \\
\hline 17 & 208 & --- & --- & --- & --- & --- & 173 & 297 & 300 & 281 & 253 & 236 \\
\hline 18 & 206 & --- & --- & --- & --- & --- & 167 & 297 & 303 & 285 & 252 & 231 \\
\hline 19 & 204 & --- & --- & --- & --- & --- & 215 & 296 & 295 & 285 & 251 & 229 \\
\hline 20 & 204 & --- & --- & --- & --- & --- & 238 & 296 & 322 & 279 & 248 & 239 \\
\hline 21 & 204 & --- & --- & --- & --- & --- & 249 & 299 & 325 & 275 & 251 & 248 \\
\hline 22 & 205 & --- & --- & --- & --- & --- & 248 & 303 & 320 & 275 & 252 & 246 \\
\hline 23 & 206 & --- & --- & --- & --- & --- & 252 & 306 & 314 & 272 & 257 & 248 \\
\hline 24 & 197 & --- & --- & --- & --- & --- & 269 & 297 & 327 & 276 & 265 & 248 \\
\hline 25 & 146 & --- & --- & --- & --- & --- & 269 & 292 & 325 & 278 & 263 & 246 \\
\hline 26 & e78 & --- & --- & --- & --- & --- & 270 & 290 & 328 & 279 & 261 & 245 \\
\hline 27 & --- & --- & --- & --- & --- & --- & 270 & 252 & 334 & 280 & 260 & 246 \\
\hline 28 & --- & --- & --- & --- & --- & --- & 286 & 286 & 254 & 280 & 260 & 245 \\
\hline 29 & --- & --- & --- & --- & --- & --- & 301 & 321 & 114 & 278 & 259 & 246 \\
\hline 30 & --- & --- & --- & --- & --- & --- & 296 & 324 & 250 & 280 & 258 & 245 \\
\hline 31 & --- & --- & --- & --- & --- & --- & --- & 329 & -- & 281 & 255 & --- \\
\hline TOTAL & --- & --- & --- & --- & --- & --- & --- & 9364 & 8908 & 8663 & 8144 & 7267 \\
\hline MEAN & --- & --- & --- & --- & --- & --- & --- & 302 & 297 & 279 & 263 & 242 \\
\hline MAX & --- & --- & --- & --- & --- & --- & --- & 329 & 334 & 295 & 281 & 249 \\
\hline MIN & --- & --- & --- & --- & --- & --- & --- & 252 & 114 & 245 & 248 & 229 \\
\hline $\mathrm{AC}-\mathrm{FT}$ & --- & --- & --- & --- & --- & --- & --- & 18570 & 17670 & 17180 & 16150 & 14410 \\
\hline
\end{tabular}


LOCATION.—Lat 40³8'49", long 122³7’34", Shasta County, Hydrologic Unit 18010212, at powerplant 1.6 mi downstream from Mill Creek and 3.8 mi south of French Gulch.

PERIOD OF RECORD.—April 1963 to current year.

GAGE.--Recorded powerplant output.

REMARKS.-Water is diverted from Trinity River at NW 1/4 SE 1/4 sec.8, T.33 N., R.8 W., through a tunnel to powerplant and then into

Whiskeytown Lake (station 11371700). See schematic diagram of upper Sacramento and Pit and McCloud River Basins.

COOPERATION.- - Records were provided by U.S. Bureau of Reclamation, not rounded to U.S. Geological Survey standards.

EXTREMES FOR PERIOD OF RECORD.-Maximum daily discharge, 4,000 ft ${ }^{3} / \mathrm{s}$, Oct. 18, 1987; no flow for many days most years.

DISCHARGE, CUBIC FEET PER SECOND, WATER YEAR OCTOBER 1998 TO SEPTEMBER 1999 DAILY MEAN VALUES

\begin{tabular}{|c|c|c|c|c|c|c|c|c|c|c|c|c|}
\hline DAY & OCT & NOV & DEC & JAN & FEB & MAR & APR & MAY & JUN & JUL & AUG & SEP \\
\hline 1 & 952 & 331 & 0 & 2820 & 1035 & 1651 & 0 & 2024 & 1869 & 2310 & 2243 & 1833 \\
\hline 2 & 967 & 301 & 0 & 3227 & 296 & 1652 & 0 & 2164 & 2023 & 2301 & 2165 & 2093 \\
\hline 3 & 946 & 292 & 0 & 3327 & 0 & 1685 & 468 & 2163 & 2184 & 2235 & 1966 & 1634 \\
\hline 4 & 972 & 328 & 0 & 2958 & 1686 & 1645 & 504 & 1568 & 1998 & 2289 & 2156 & 1896 \\
\hline 5 & 932 & 355 & 0 & 1714 & 1667 & 1654 & 499 & 1951 & 2099 & 2406 & 2161 & 1877 \\
\hline 6 & 1123 & 299 & 0 & 1708 & 1698 & 1659 & 1015 & 1158 & 1921 & 2376 & 2330 & 1766 \\
\hline 7 & 934 & 307 & 0 & 1143 & 1686 & 1670 & 1018 & 1681 & 2147 & 2271 & 2111 & 1751 \\
\hline 8 & 1089 & 334 & 698 & 1177 & 569 & 1680 & 1033 & 1677 & 2223 & 2451 & 2254 & 1530 \\
\hline 9 & 1055 & 330 & 527 & 1160 & 58 & 1602 & 936 & 1549 & 1976 & 2405 & 1869 & 1472 \\
\hline 10 & 986 & 328 & 0 & 1162 & 0 & 1626 & 988 & 1494 & 2023 & 2381 & 1909 & 1676 \\
\hline 11 & 1007 & 334 & 0 & 1143 & 0 & 1496 & 1002 & 1164 & 2188 & 2401 & 2083 & 1725 \\
\hline 12 & 945 & 300 & 0 & 1287 & 281 & 1695 & 892 & 617 & 2247 & 2520 & 2332 & 1940 \\
\hline 13 & 1037 & 324 & 0 & 983 & 0 & 1633 & 837 & 642 & 2035 & 2380 & 1547 & 1699 \\
\hline 14 & 943 & 300 & 2 & 1134 & 0 & 1636 & 857 & 759 & 2040 & 2454 & 1120 & 1748 \\
\hline 15 & 671 & 300 & 677 & 1148 & 0 & 1647 & 1406 & 803 & 1877 & 2293 & 1768 & 1317 \\
\hline 16 & 746 & 1348 & 1214 & 1134 & 0 & 1250 & 949 & 992 & 2024 & 2395 & 1716 & 1735 \\
\hline 17 & 753 & 3146 & 1310 & 1108 & 0 & 1621 & 844 & 874 & 2058 & 2342 & 1685 & 1740 \\
\hline 18 & 813 & 3113 & 1194 & 1121 & 0 & 1634 & 993 & 852 & 2211 & 2537 & 1169 & 1961 \\
\hline 19 & 748 & 3134 & 1178 & 1269 & 0 & 538 & 1567 & 1034 & 2370 & 2480 & 1367 & 1871 \\
\hline 20 & 758 & 3149 & 1366 & 1652 & 1012 & 505 & 1282 & 1075 & 2089 & 2535 & 1324 & 1599 \\
\hline 21 & 436 & 3140 & 1068 & 1668 & 1071 & 538 & 1352 & 998 & 1980 & 2359 & 1187 & 1580 \\
\hline 22 & 0 & 3098 & 1007 & 1666 & 1004 & 499 & 2881 & 1053 & 2149 & 2450 & 1179 & 1700 \\
\hline 23 & 743 & 3094 & 957 & 835 & 987 & 505 & 3093 & 1198 & 2115 & 2495 & 1200 & 1781 \\
\hline 24 & 749 & 3114 & 799 & 1001 & 1047 & 214 & 3019 & 1139 & 1796 & 2290 & 1235 & 1930 \\
\hline 25 & 798 & 3115 & 1108 & 1014 & 982 & 500 & 2672 & 1274 & 2486 & 2297 & 1135 & 1842 \\
\hline 26 & 798 & 3130 & 2119 & 1007 & 1681 & 352 & 1549 & 1730 & 2162 & 2328 & 1170 & 1275 \\
\hline 27 & 365 & 3141 & 1151 & 1047 & 1644 & 0 & 1769 & 1713 & 1987 & 1974 & 1294 & 1711 \\
\hline 28 & 327 & 3138 & 1134 & 1008 & 1633 & 10 & 1602 & 1738 & 2270 & 1955 & 1203 & 1779 \\
\hline 29 & 300 & 3117 & 772 & 1001 & --- & 0 & 1599 & 1462 & 2424 & 1973 & 1230 & 1398 \\
\hline 30 & 300 & 969 & 1111 & 1007 & --- & 0 & 2309 & 2030 & 2168 & 1999 & 1226 & 1704 \\
\hline 31 & 300 & --- & 1760 & 970 & --- & 0 & --- & 1849 & --- & 2499 & 892 & --- \\
\hline TOTAL & 23493 & 47709 & 21152 & 44599 & 20037 & 32797 & 38935 & 42425 & 63139 & 72381 & 50226 & 51563 \\
\hline MEAN & 758 & 1590 & 682 & 1439 & 716 & 1058 & 1298 & 1369 & 2105 & 2335 & 1620 & 1719 \\
\hline MAX & 1123 & 3149 & 2119 & 3327 & 1698 & 1695 & 3093 & 2164 & 2486 & 2537 & 2332 & 2093 \\
\hline MIN & 0 & 292 & 0 & 835 & 0 & 0 & 0 & 617 & 1796 & 1955 & 892 & 1275 \\
\hline $\mathrm{AC}-\mathrm{FT}$ & 46600 & 94630 & 41950 & 88460 & 39740 & 65050 & 77230 & 84150 & 125200 & 143600 & 99620 & 10230 \\
\hline
\end{tabular}

STATISTICS OF MONTHLY MEAN DATA FOR WATER YEARS 1963 - 1999, BY WATER YEAR (WY)

\begin{tabular}{|c|c|c|c|c|c|c|c|c|c|c|c|c|}
\hline & $\mathrm{OCT}$ & NOV & $\mathrm{DEC}$ & JAN & FEB & MAR & APR & MAY & JUN & JUL & AUG & SEP \\
\hline MEAN & 1320 & 842 & 672 & 607 & 798 & 864 & 1191 & 1341 & 1840 & 2371 & 2229 & 2074 \\
\hline MAX & 3363 & 2158 & 2891 & 2755 & 3223 & 3111 & 3220 & 3513 & 3662 & 3589 & 3236 & 3504 \\
\hline$(W Y)$ & 1988 & 1967 & 1979 & 1982 & 1974 & 1974 & 1970 & 1974 & 1969 & 1968 & 1977 & 1988 \\
\hline MIN & 166 & 18.0 & .16 & .000 & .34 & .000 & .000 & .097 & .63 & 253 & 507 & 415 \\
\hline$(W Y)$ & 1994 & 1992 & 1993 & 1986 & 1988 & 1988 & 1978 & 1991 & 1993 & 1978 & 1992 & 1997 \\
\hline
\end{tabular}

SUMMARY STATISTICS

ANNUAL TOTAL

ANNUAL MEAN

HIGHEST ANNUAL MEAN

LOWEST ANNUAL MEAN

HIGHEST DAILY MEAN

LOWEST DAILY MEAN

ANNUAL SEVEN-DAY MINIMUM

ANNUAL RUNOFF (AC-FT)

10 PERCENT EXCEEDS

50 PERCENT EXCEEDS

90 PERCENT EXCEEDS
FOR 1998 CALENDAR YEAR

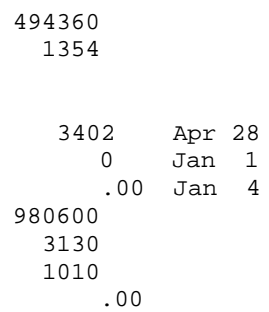

FOR 1999 WATER YEAR

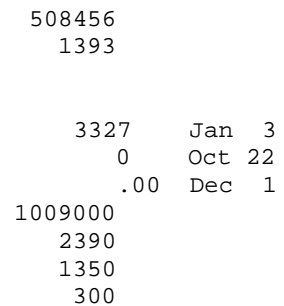

WATER YEARS 1963 - 1999

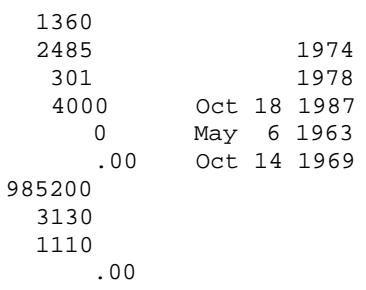


LOCATION.—Lat 40³7'41", long 122²7'59", in NE 1/4 SE 1/4 sec.18, T.32 N., R.5 W., Shasta County, Hydrologic Unit 18020112, at powerplant on Spring Creek, 0.4 mi northwest of Keswick, and 4.9 mi northwest of Redding.

PERIOD OF RECORD.—December 1963 to current year.

GAGE.-Discharge computed from powerplant output.

REMARKS.-Water is released from Whiskeytown Lake (station 11371700) through a tunnel to powerplant and then into Keswick Reservoir.

Spring Creek Reservoir releases into Keswick Reservoir at Spring Creek Powerplant. See schematic diagrams of upper Sacramento River and Pit and McCloud River Basins.

COOPERATION.-Records were provided by U.S. Bureau of Reclamation, not rounded to U.S. Geological Survey standards.

EXTREMES FOR PERIOD OF RECORD.-Maximum daily discharge, 4,800 ft 3 /s, May 2, 1983; no flow for many days most years.

DISCHARGE, CUBIC FEET PER SECOND, WATER YEAR OCTOBER 1998 TO SEPTEMBER 1999 DAILY MEAN VALUES

\begin{tabular}{|c|c|c|c|c|c|c|c|c|c|c|c|c|}
\hline DAY & $\mathrm{OCT}$ & NOV & $\mathrm{DEC}$ & JAN & FEB & MAR & APR & MAY & JUN & JUL & AUG & SEP \\
\hline 1 & 1114 & 937 & 2252 & 3871 & 1082 & 3293 & 2154 & 1951 & 1976 & 2438 & 2059 & 1595 \\
\hline 2 & 1152 & 905 & 276 & 3809 & 269 & 2109 & 2136 & 1967 & 2033 & 2344 & 2099 & 1597 \\
\hline 3 & 1004 & 898 & 1134 & 2728 & 263 & 2542 & 348 & 1995 & 2013 & 2184 & 2104 & 1573 \\
\hline 4 & 935 & 620 & 932 & 2068 & 1729 & 3999 & 234 & 1977 & 2124 & 2130 & 2012 & 1595 \\
\hline 5 & 888 & 530 & 1083 & 2447 & 1414 & 2259 & 266 & 1958 & 2136 & 2465 & 2003 & 1596 \\
\hline 6 & 973 & 492 & 684 & 1436 & 2607 & 1737 & 683 & 1984 & 2289 & 2286 & 2167 & 1593 \\
\hline 7 & 1729 & 688 & 274 & 969 & 3875 & 2068 & 427 & 1971 & 2255 & 2107 & 2077 & 1481 \\
\hline 8 & 0 & 886 & 595 & 1017 & 3244 & 2872 & 476 & 1960 & 1991 & 2377 & 1996 & 1546 \\
\hline 9 & 612 & 1078 & 166 & 958 & 1064 & 3410 & 2061 & 1722 & 2244 & 2362 & 2105 & 1545 \\
\hline 10 & 1224 & 1163 & 215 & 1078 & 818 & 2198 & 2076 & 1622 & 2178 & 2241 & 2142 & 1504 \\
\hline 11 & 1262 & 1131 & 281 & 1328 & 377 & 1854 & 2052 & 1168 & 2133 & 2437 & 2132 & 1536 \\
\hline 12 & 954 & 890 & 285 & 1024 & 661 & 1476 & 2070 & 1005 & 2131 & 2570 & 1811 & 1500 \\
\hline 13 & 960 & 762 & 91 & 1030 & 372 & 2399 & 2053 & 695 & 2389 & 2562 & 1550 & 1487 \\
\hline 14 & 1097 & 263 & 274 & 1088 & 349 & 2427 & 2072 & 802 & 2347 & 2548 & 1582 & 1512 \\
\hline 15 & 634 & 252 & 273 & 981 & 343 & 2259 & 2038 & 863 & 1741 & 2200 & 1577 & 1495 \\
\hline 16 & 1002 & 724 & 665 & 967 & 329 & 2535 & 1886 & 940 & 2226 & 2234 & 1555 & 1510 \\
\hline 17 & 1172 & 3421 & 1966 & 1538 & 517 & 2370 & 1891 & 892 & 1991 & 2272 & 1560 & 1490 \\
\hline 18 & 1335 & 3485 & 1452 & 2016 & 1450 & 2023 & 1933 & 1576 & 2102 & 2708 & 1363 & 1485 \\
\hline 19 & 1240 & 4124 & 1285 & 1188 & 733 & 1162 & 1899 & 1323 & 2616 & 2335 & 1063 & 1495 \\
\hline 20 & 1261 & 3563 & 1177 & 1859 & 1424 & 1132 & 1935 & 1395 & 2183 & 2664 & 1044 & 1462 \\
\hline 21 & 1134 & 2737 & 1088 & 3249 & 2278 & 1180 & 1954 & 1027 & 2010 & 2372 & 1085 & 1585 \\
\hline 22 & 627 & 2966 & 1136 & 1755 & 2286 & 1175 & 1948 & 1222 & 2019 & 2511 & 1100 & 1530 \\
\hline 23 & 676 & 4069 & 1266 & 1718 & 1817 & 1384 & 2097 & 1335 & 1920 & 2652 & 1087 & 1709 \\
\hline 24 & 972 & 4201 & 1332 & 1964 & 1419 & 1603 & 1987 & 1375 & 2008 & 2098 & 1079 & 1269 \\
\hline 25 & 944 & 4162 & 922 & 1350 & 3151 & 1838 & 1954 & 1331 & 2455 & 2036 & 1253 & 1558 \\
\hline 26 & 1339 & 4144 & 1084 & 1116 & 3530 & 2180 & 1999 & 1997 & 2536 & 2161 & 1080 & 1045 \\
\hline 27 & 1009 & 3165 & 1386 & 1164 & 3243 & 2157 & 1953 & 1943 & 1966 & 2034 & 1082 & 1668 \\
\hline 28 & 1046 & 4156 & 1390 & 1932 & 2687 & 1991 & 1963 & 1942 & 1984 & 2078 & 1092 & 1749 \\
\hline 29 & 611 & 3528 & 1063 & 1383 & --- & 2074 & 1934 & 2017 & 2530 & 2068 & 1054 & 1476 \\
\hline 30 & 922 & 3006 & 1054 & 1070 & --- & 2074 & 1939 & 1921 & 2462 & 2085 & 1138 & 1690 \\
\hline 31 & 887 & --- & 1366 & 1321 & --- & 2074 & --- & 2080 & --- & 2051 & 1121 & --- \\
\hline TOTAL & 30715 & 62946 & 28447 & 51422 & 43331 & 65854 & 50418 & 47956 & 64988 & 71610 & 48172 & 45876 \\
\hline MEAN & 991 & 2098 & 918 & 1659 & 1548 & 2124 & 1681 & 1547 & 2166 & 2310 & 1554 & 1529 \\
\hline MAX & 1729 & 4201 & 2252 & 3871 & 3875 & 3999 & 2154 & 2080 & 2616 & 2708 & 2167 & 1749 \\
\hline MIN & 0 & 252 & 91 & 958 & 263 & 1132 & 234 & 695 & 1741 & 2034 & 1044 & 1045 \\
\hline$A C-F T$ & 60920 & 124900 & 56420 & 102000 & 85950 & 130600 & 100000 & 95120 & 128900 & 142000 & 95550 & 91000 \\
\hline a & 171 & 958 & 1880 & 1100 & 4180 & 2750 & 3000 & 635 & 379 & 93 & 159 & 0 \\
\hline
\end{tabular}

STATISTICS OF MONTHLY MEAN DATA FOR WATER YEARS 1964 - 1999, BY WATER YEAR (WY)

\begin{tabular}{|c|c|c|c|c|c|c|c|c|c|c|c|c|}
\hline & $\mathrm{OCT}$ & NOV & $\mathrm{DEC}$ & JAN & FEB & MAR & $\mathrm{APR}$ & MAY & JUN & JUL & AUG & SEP \\
\hline MEAN & 1601 & 1283 & 1101 & 1373 & 1633 & 1648 & 1411 & 1571 & 2001 & 2434 & 2310 & 2208 \\
\hline MAX & 3691 & 3174 & 4032 & 4532 & 4498 & 4364 & 4405 & 4265 & 3866 & 3886 & 3654 & 3526 \\
\hline (WY) & 1989 & 1967 & 1974 & 1974 & 1974 & 1983 & 1983 & 1983 & 1969 & 1968 & 1977 & 1988 \\
\hline MIN & 265 & .87 & 1.55 & 2.10 & 3.36 & 86.6 & 5.23 & 5.45 & 158 & 250 & 467 & 416 \\
\hline$(W Y)$ & 1978 & 1992 & 1992 & 1991 & 1991 & 1988 & 1987 & 1991 & 1989 & 1978 & 1992 & 1992 \\
\hline
\end{tabular}

SUMMARY STATISTICS

ANNUAL TOTAL

ANNUAL MEAN

HIGHEST ANNUAL MEAN

LOWEST ANNUAL MEAN

HIGHEST DAILY MEAN

LOWEST DAILY MEAN

ANNUAL SEVEN-DAY MINIMUM

ANNUAL RUNOFF (AC-FT)

10 PERCENT EXCEEDS

50 PERCENT EXCEEDS

90 PERCENT EXCEEDS
FOR 1998 CALENDAR YEAR

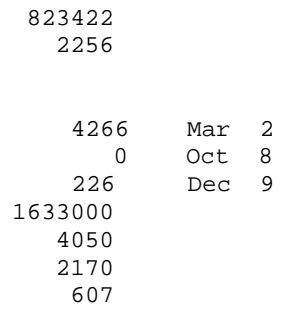

FOR 1999 WATER YEAR

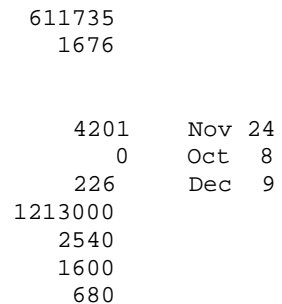

WATER YEARS 1964 - 1999

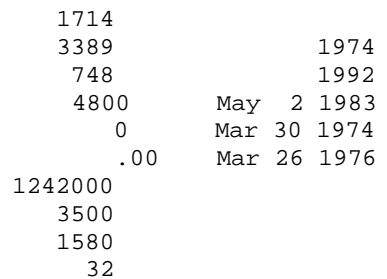

a Discharge, in acre-feet, from Spring Creek Reservoir, provided by U.S. Bureau of Reclamation. 
LOCATION.-Lat 40³7’03", long 122³1’31", unsurveyed, Shasta County, Hydrologic Unit 18010112, Whiskeytown-Shasta-Trinity National Recreation Area, at outlet works to Spring Creek Powerplant on Clear Creek, 1.8 mi downstream from Whiskey Creek, and 7.8 mi northeast of Igo.

DRAINAGE AREA.-200 $\mathrm{mi}^{2}$.

PERIOD OF RECORD.-May 1963 to current year. Prior to October 1964 published as Whiskeytown Reservoir near Igo.

GAGE.-Water-stage recorder. Datum of gage is sea level (levels by U.S. Bureau of Reclamation). Contents based on capacity table dated April 1962 provided by U.S. Bureau of Reclamation.

REMARKS.- - Lake is formed by earth and rockfill dam. Storage began in May 1963. Usable capacity, 241,088 acre-ft between elevations $972.0 \mathrm{ft}$, invert of sluice pipe, and 1,210.00 ft, crest of glory hole spillway. Dead storage 8 acre-ft. Normal operating pool is from elevation 1,197.0 ft, capacity, 201,288 acre-ft, to 1,210.0 ft, capacity, 241,096 acre-ft. Transbasin water enters the reservoir through Judge Francis Carr Powerplant (station 11525430) and is released through Spring Creek Tunnel to Spring Creek Powerplant (station 11371600) and Keswick Reservoir. Figures given represent total contents at 2400 hours. Lake is used for power generation and recreation. See schematic diagrams of upper Sacramento River Basin and Pit and McCloud River Basins.

COOPERATION.--Records were provided by U.S. Bureau of Reclamation, not rounded to U.S. Geological Survey standards.

EXTREMES (AT 2400 HOURS) FOR PERIOD OF RECORD.-Maximum contents, 258,600 acre-ft, Mar. 2, 1983, elevation, 1,215.34 ft; minimum since first filling, 145,562 acre-ft, Dec. 27, 1992, elevation, $1,176.05 \mathrm{ft}$.

EXTREMES (AT 2400 HOURS) FOR CURRENT YEAR.-Maximum contents, 239,143 acre-ft, Oct.. 9, elevation, 1,209.39 ft; minimum, 204,063 acre ft, Dec. 7, elevation, 1,197.95 ft.

Capacity table (elevation, in feet, and contents, in acre-feet)

(Based on survey by U.S. Bureau of Reclamation in 1962)

$\begin{array}{rrrrrrrr}1,015 & 714 & 1,040 & 3,055 & 1,080 & 15,076 & 1,140 & 73,960 \\ 1,020 & 994 & 1,050 & 4,898 & 1,100 & 27,542 & 1,180 & 155,276 \\ 1,030 & 1,797 & 1,060 & 7,418 & 1,120 & 46,701 & 1,220 & 274,389\end{array}$

RESERVOIR STORAGE (ACRE-FEET), WATER YEAR OCTOBER 1998 TO SEPTEMBER 1999 DAILY OBSERVATION AT 2400 HOURS

\begin{tabular}{|c|c|c|c|c|c|c|c|c|c|c|c|c|}
\hline DAY & OCT & NOV & $\mathrm{DEC}$ & JAN & FEB & MAR & $\mathrm{APR}$ & MAY & JUN & JUL & AUG & SEP \\
\hline 1 & 238023 & 218598 & 205329 & 205417 & 204946 & 204680 & 207957 & 235925 & 237831 & 237704 & 237609 & 237069 \\
\hline 2 & 237831 & 217139 & 206625 & 204621 & 205181 & 206389 & 205211 & 237260 & 238311 & 237514 & 237577 & 237768 \\
\hline 3 & 237895 & 215804 & 206448 & 205888 & 204739 & 206978 & 206713 & 238279 & 238759 & 237418 & 237450 & 237577 \\
\hline 4 & 238119 & 215110 & 205947 & 206006 & 204769 & 204268 & 208313 & 238375 & 238823 & 237863 & 237609 & 237927 \\
\hline 5 & 238183 & 214628 & 204916 & 204621 & 205564 & 204828 & 210155 & 239047 & 238951 & 237831 & 238087 & 238343 \\
\hline 6 & 238343 & 214175 & 204151 & 204975 & 207541 & 206124 & 212221 & 238215 & 238311 & 238087 & 238119 & 238439 \\
\hline 7 & 236624 & 214296 & 204063 & 205328 & 207274 & 206625 & 214869 & 238183 & 238215 & 238247 & 238439 & 238631 \\
\hline 8 & 238503 & 213179 & 204828 & 205564 & 204651 & 206477 & 218263 & 237863 & 238759 & 238375 & 238823 & 238279 \\
\hline 9 & 239143 & 211532 & 205829 & 205800 & 205004 & 204504 & 218051 & 238087 & 238471 & 238439 & 238279 & 237736 \\
\hline 10 & 238311 & 210215 & 205682 & 205829 & 204916 & 204769 & 218415 & 238471 & 238215 & 238727 & 237768 & 237673 \\
\hline 11 & 237641 & 208640 & 205299 & 205387 & 205270 & 205446 & 218841 & 238855 & 238439 & 238599 & 237577 & 237545 \\
\hline 12 & 237482 & 207274 & 204946 & 205653 & 205446 & 206919 & 218871 & 238471 & 238823 & 238599 & 238759 & 238023 \\
\hline 13 & 237418 & 206301 & 205004 & 205417 & 205623 & 206507 & 218871 & 238567 & 238215 & 238119 & 238791 & 238087 \\
\hline 14 & 236878 & 206301 & 204739 & 205505 & 205682 & 206242 & 218658 & 238759 & 237673 & 237863 & 237545 & 238151 \\
\hline 15 & 236687 & 206301 & 205653 & 205829 & 205594 & 206389 & 219726 & 238727 & 237641 & 238119 & 237609 & 237418 \\
\hline 16 & 235893 & 207541 & 207067 & 206183 & 206124 & 205270 & 219757 & 239047 & 237355 & 238503 & 237641 & 237450 \\
\hline 17 & 234844 & 207274 & 206212 & 206183 & 206566 & 205240 & 219267 & 239015 & 237704 & 238567 & 237736 & 237482 \\
\hline 18 & 233708 & 206772 & 206036 & 205417 & 205653 & 206036 & 219084 & 238087 & 238215 & 238407 & 237132 & 237959 \\
\hline 19 & 232540 & 204946 & 205829 & 206389 & 205888 & 206065 & 220278 & 237895 & 237959 & 238567 & 237545 & 238247 \\
\hline 20 & 231280 & 204209 & 206507 & 206860 & 206860 & 206212 & 220553 & 237673 & 237895 & 238439 & 237959 & 238023 \\
\hline 21 & 229652 & 205888 & 206566 & 204739 & 206301 & 206330 & 220798 & 237927 & 237895 & 238247 & 238023 & 237545 \\
\hline 22 & 228214 & 206860 & 206743 & 205947 & 205358 & 206360 & 223842 & 237927 & 238343 & 238055 & 237959 & 237386 \\
\hline 23 & 228151 & 209086 & 206419 & 206242 & 205800 & 206448 & 226877 & 238023 & 238759 & 237704 & 238023 & 237164 \\
\hline 24 & 228027 & 208254 & 205564 & 205417 & 207126 & 210245 & 229715 & 237959 & 238247 & 237831 & 238247 & 237959 \\
\hline 25 & 227561 & 206890 & 206006 & 205741 & 206418 & 215834 & 231941 & 238375 & 238471 & 238151 & 237863 & 238055 \\
\hline 26 & 226349 & 205888 & 206094 & 206360 & 205741 & 217352 & 232224 & 238631 & 237736 & 238407 & 237736 & 238055 \\
\hline 27 & 224891 & 206802 & 205770 & 206654 & 204710 & 216926 & 233076 & 238375 & 237673 & 238247 & 237704 & 237673 \\
\hline 28 & 223349 & 205358 & 205446 & 205270 & 205299 & 215714 & 233423 & 238055 & 238407 & 237673 & 237641 & 237260 \\
\hline 29 & 222670 & 206124 & 205034 & 204857 & --- & 214145 & 233423 & 237069 & 238471 & 237228 & 237704 & 236846 \\
\hline 30 & 221135 & 207214 & 205211 & 205034 & --- & 212341 & 235035 & 237577 & 237959 & 236815 & 237514 & 236433 \\
\hline 31 & 219879 & --- & 206654 & 204739 & --- & 210275 & --- & 237800 & --- & 237418 & 236878 & --- \\
\hline a & 1203.23 & 1199.02 & 1198.83 & 1198.18 & 1198.37 & 1200.05 & 1208.10 & 1208.97 & 1209.02 & 1208.85 & 1208.68 & 1208.54 \\
\hline $\mathrm{b}$ & -18432 & -12665 & -560 & -1915 & +560 & +4976 & +24760 & +2765 & +159 & -541 & -540 & -445 \\
\hline MAX & 239143 & 218598 & 207067 & 206860 & 207541 & 217352 & 235035 & 239047 & 238951 & 238727 & 238823 & 238631 \\
\hline MIN & 219879 & 204209 & 204063 & 204621 & 204651 & 204268 & 205211 & 235925 & 237355 & 236815 & 236878 & 236433 \\
\hline
\end{tabular}

a Elevation, in feet, at end of month.

b Change in contents, in acre-feet. 
LOCATION.-Lat 40³0'48", long 122³1'23", unsurveyed, Shasta County, Hydrologic Unit 18020112, on left bank at old highway bridge on Redding-Igo Road, 1.0 mi northeast of Igo, 7.0 mi downstream from Whiskeytown Dam, 8.3 mi southwest of Redding, and 10.4 mi upstream from mouth.

DRAINAGE AREA.-228 $\mathrm{mi}^{2}$.

PERIOD OF RECORD.-October 1940 to current year.

CHEMICAL DATA: Water years 1958-79.

WATER TEMPERATURE: Water years 1965-79.

REVISED RECORDS._-WSP 1345: Drainage area. WSP 1395: 1941(M).

GAGE.-Water-stage recorder. Datum of gage is $672.99 \mathrm{ft}$ above sea level.

REMARKS.-Records excellent. Low flow completely regulated by Whiskeytown Lake (station 11371700) since May 1963. Transbasin diversion from Trinity River through Judge Francis Carr Powerplant (station 11525430) to Whiskeytown Lake began in April 1963. Diversions from Whiskeytown Lake to Spring Creek Powerplant (station 11371600) began in December 1963. See schematic diagrams of upper Sacramento River and Pit and McCloud River Basins.

EXTREMES FOR PERIOD OF RECORD.-Maximum discharge, 24,500 ft 3 /s, Dec. 21, 1955, gage height, $13.75 \mathrm{ft}^{3}$ minimum daily, $9.0 \mathrm{ft}^{3} / \mathrm{s}$, Sept. 4-7, 1950. Since completion of Whiskeytown Dam in 1963, maximum discharge, 19,200 ft $3 / \mathrm{s}$, Mar. 3, 1983 , gage height, $12.73 \mathrm{ft}$, from rating curve extended above $12,000 \mathrm{ft}^{3} / \mathrm{s}$ on basis of slope-area measurement of peak flow; minimum daily, $30 \mathrm{ft} / \mathrm{s}, \mathrm{Oct}$. $10,11,1977$.

DISCHARGE, CUBIC FEET PER SECOND, WATER YEAR OCTOBER 1998 TO SEPTEMBER 1999

DAILY MEAN VALUES

\begin{tabular}{|c|c|c|c|c|c|c|c|c|c|c|c|c|}
\hline DAY & $\mathrm{OCT}$ & NOV & DEC & JAN & FEB & MAR & APR & MAY & JUN & JUL & AUG & SEP \\
\hline 1 & 50 & 204 & 466 & 219 & 252 & 399 & 320 & 260 & 201 & 153 & 148 & 152 \\
\hline 2 & 50 & 203 & 468 & 218 & 248 & 375 & 307 & 267 & 176 & 154 & 148 & 152 \\
\hline 3 & 50 & 203 & 595 & 217 & 245 & 355 & 298 & 264 & 177 & 154 & 148 & 152 \\
\hline 4 & 50 & 203 & 359 & 217 & 243 & 331 & 291 & 259 & 174 & 153 & 148 & 152 \\
\hline 5 & 119 & 205 & 305 & 217 & 239 & 316 & 331 & 254 & 172 & 152 & 149 & 152 \\
\hline 6 & 203 & 212 & 280 & 216 & 998 & 306 & 315 & 252 & 174 & 152 & 149 & 152 \\
\hline 7 & 203 & 284 & 267 & 216 & 1500 & 296 & 304 & 252 & 171 & 151 & 149 & 173 \\
\hline 8 & 205 & 226 & 262 & 216 & 600 & 391 & 348 & 249 & 170 & 151 & 148 & 200 \\
\hline 9 & 207 & 216 & 252 & 216 & 496 & 433 & 317 & 246 & 168 & 151 & 148 & 200 \\
\hline 10 & 206 & 238 & 247 & 216 & 402 & 368 & 392 & 245 & 167 & 151 & 152 & 227 \\
\hline 11 & 205 & 235 & 242 & 216 & 354 & 340 & 512 & 245 & 167 & 151 & 153 & 251 \\
\hline 12 & 206 & 218 & 239 & 216 & 327 & 322 & 448 & 245 & 167 & 151 & 154 & 252 \\
\hline 13 & 206 & 214 & 239 & 216 & 314 & 313 & 392 & 241 & 166 & 150 & 154 & 252 \\
\hline 14 & 206 & 213 & 237 & 217 & 307 & 327 & 358 & 242 & 165 & 150 & 155 & 252 \\
\hline 15 & 206 & 212 & 233 & 219 & 293 & 321 & 338 & 240 & 165 & 149 & 152 & 252 \\
\hline 16 & 206 & 212 & 230 & 222 & 485 & 306 & 325 & 239 & 164 & 149 & 152 & 252 \\
\hline 17 & 206 & 219 & 230 & 299 & 605 & 300 & 318 & 237 & 164 & 149 & 152 & 252 \\
\hline 18 & 205 & 214 & 227 & 364 & 479 & 295 & 310 & 238 & 160 & 150 & 152 & 252 \\
\hline 19 & 204 & 213 & 227 & 299 & 412 & 289 & 303 & 235 & 160 & 149 & 152 & 252 \\
\hline 20 & 204 & 211 & 226 & 301 & 455 & 316 & 297 & 234 & 161 & 150 & 152 & 252 \\
\hline 21 & 203 & 238 & 224 & 287 & 509 & 309 & 291 & 233 & 162 & 150 & 152 & 252 \\
\hline 22 & 203 & 233 & 223 & 655 & 503 & 305 & 286 & 230 & 160 & 149 & 152 & 252 \\
\hline 23 & 203 & 828 & 222 & 620 & 487 & 366 & 280 & 229 & 160 & 149 & 152 & 252 \\
\hline 24 & 214 & 305 & 222 & 378 & 454 & 942 & 277 & 229 & 160 & 149 & 152 & 252 \\
\hline 25 & 206 & 259 & 222 & 319 & 524 & 867 & 275 & 228 & 162 & 149 & 152 & 252 \\
\hline 26 & 204 & 260 & 222 & 303 & 432 & 566 & 273 & 227 & 160 & 149 & 152 & 252 \\
\hline 27 & 205 & 251 & 222 & 280 & 390 & 460 & 269 & 226 & 160 & 149 & 152 & 252 \\
\hline 28 & 205 & 239 & 220 & 270 & 418 & 404 & 266 & 225 & 159 & 149 & 152 & 252 \\
\hline 29 & 204 & 482 & 219 & 262 & --- & 372 & 263 & 226 & 155 & 148 & 152 & 252 \\
\hline 30 & 203 & 723 & 219 & 258 & --- & 354 & 261 & 225 & 149 & 148 & 152 & 252 \\
\hline 31 & 204 & --- & 219 & 262 & --- & 336 & --- & 224 & --- & 148 & 152 & --- \\
\hline TOTAL & 5651 & 8173 & 8265 & 8631 & 12971 & 11980 & 9565 & 7446 & 4976 & 4657 & 4687 & 6751 \\
\hline MEAN & 182 & 272 & 267 & 278 & 463 & 386 & 319 & 240 & 166 & 150 & 151 & 225 \\
\hline MAX & 214 & 828 & 595 & 655 & 1500 & 942 & 512 & 267 & 201 & 154 & 155 & 252 \\
\hline MIN & 50 & 203 & 219 & 216 & 239 & 289 & 261 & 224 & 149 & 148 & 148 & 152 \\
\hline $\mathrm{AC}-\mathrm{FT}$ & 11210 & 16210 & 16390 & 17120 & 25730 & 23760 & 18970 & 14770 & 9870 & 9240 & 9300 & 13390 \\
\hline
\end{tabular}


11372000 CLEAR CREEK NEAR IGO, CA—Continued

STATISTICS OF MONTHLY MEAN DATA FOR WATER YEARS 1941 - 1962, BY WATER YEAR (WY)

\begin{tabular}{|c|c|c|c|c|c|c|c|c|c|c|c|c|}
\hline & OCT & NOV & $\mathrm{DEC}$ & JAN & $\mathrm{FEB}$ & MAR & $\mathrm{APR}$ & MAY & JUN & JUL & AUG & SEP \\
\hline MEAN & 76.7 & 150 & 597 & 807 & 1226 & 834 & 676 & 347 & 161 & 63.4 & 35.1 & 32.8 \\
\hline MAX & 373 & 427 & 2336 & 2513 & 5753 & 2595 & 2431 & 773 & 289 & 126 & 64.6 & 89.7 \\
\hline (WY) & 1951 & 1951 & 1956 & 1941 & 1958 & 1941 & 1941 & 1957 & 1953 & 1941 & 1941 & 1957 \\
\hline MIN & 25.8 & 39.0 & 47.0 & 65.5 & 142 & 168 & 172 & 87.6 & 66.5 & 24.3 & 14.3 & 13.4 \\
\hline (WY) & 1950 & 1960 & 1950 & 1947 & 1948 & 1955 & 1944 & 1947 & 1950 & 1950 & 1950 & 1944 \\
\hline
\end{tabular}

SUMMARY STATISTICS

ANNUAL MEAN

HIGHEST ANNUAL MEAN

LOWEST ANNUAL MEAN

HIGHEST DAILY MEAN

LOWEST DAILY MEAN

ANNUAL SEVEN-DAY MINIMUM

INSTANTANEOUS PEAK FLOW

INSTANTANEOUS PEAK STAGE

ANNUAL RUNOFF (AC-FT)

10 PERCENT EXCEEDS

50 PERCENT EXCEEDS

90 PERCENT EXCEEDS
WATER YEARS 1941 - 1962

$\begin{array}{cccr}413 & & & \\ 1092 & & & 1941 \\ 128 & & & 1944 \\ 15100 & & \text { Mar } 1 & 1941 \\ 9.0 & \text { Sep } 4 & 1950 \\ 9.5 & \text { Sep } 1 & 1950 \\ 24500 & \text { Dec } 21 & 1955 \\ 13.75 & \text { Dec 21 } & 1955 \\ 299000 & & & \\ 929 & & & \\ 133 & & & \\ 27 & & & \end{array}$

STATISTICS OF MONTHLY MEAN DATA FOR WATER YEARS 1965 - 1999, BY WATER YEAR (WY)

\begin{tabular}{|c|c|c|c|c|c|c|c|c|c|c|c|c|}
\hline & $\mathrm{OCT}$ & NOV & $\mathrm{DEC}$ & JAN & FEB & MAR & APR & MAY & JUN & JUL & AUG & SEP \\
\hline MEAN & 73.2 & 145 & 203 & 304 & 305 & 339 & 163 & 102 & 72.1 & 59.7 & 57.3 & 57.0 \\
\hline MAX & 317 & 299 & 625 & 1358 & 1612 & 3437 & 668 & 419 & 249 & 150 & 151 & 225 \\
\hline$(W Y)$ & 1993 & 1974 & 1965 & 1970 & 1998 & 1983 & 1974 & 1982 & 1993 & 1999 & 1999 & 1999 \\
\hline MIN & 38.8 & 70.7 & 94.2 & 54.3 & 49.8 & 51.3 & 50.7 & 48.6 & 42.9 & 39.2 & 37.9 & 37.9 \\
\hline (WY) & 1978 & 1969 & 1977 & 1977 & 1977 & 1977 & 1977 & 1966 & 1966 & 1966 & 1966 & 1977 \\
\hline
\end{tabular}

SUMMARY STATISTICS

ANNUAL TOTAL

ANNUAL MEAN

HIGHEST ANNUAL MEAN

LOWEST ANNUAL MEAN

HIGHEST DAILY MEAN

LOWEST DAILY MEAN

ANNUAL SEVEN-DAY MINIMUM

INSTANTANEOUS PEAK FLOW

INSTANTANEOUS PEAK STAGE

ANNUAL RUNOFF (AC-FT)

10 PERCENT EXCEEDS

50 PERCENT EXCEEDS

90 PERCENT EXCEEDS
FOR 1998 CALENDAR YEAR

$\begin{array}{rrr}127304 & & \\ 349 & & \\ & & \\ 8150 & \text { Feb } & 8 \\ 50 & \text { Sep } 13 \\ 50 & \text { Sep } 13 \\ & & \\ & & \\ 252500 & & \\ 659 & \\ 230 & \\ 51 & \end{array}$

FOR 1999 WATER YEAR

$$
93753
$$

$\begin{array}{rrr}1500 & \text { Feb } & 7 \\ 50 & \text { Oct } & 1 \\ 104 & \text { Oct } & 1 \\ 2710 & \text { Feb } & 6 \\ 6.94 & \text { Feb } & 6\end{array}$

186000

391

227
WATER YEARS 1965 - 1999

$\begin{array}{rlrr}157 & & & \\ 570 & & & 1983 \\ 57.9 & & 1977 \\ 15000 & \text { Mar } 3 & 1983 \\ 30 & \text { Oct } 10 & 1977 \\ 31 & \text { Oct } 5 & 1977 \\ 19200 & \text { Mar } 3 & 1983 \\ 12.73 & \text { Mar } 3 & 1983 \\ 114100 & & & \\ 256 & & & \\ 73 & & & \\ 49 & & & \end{array}$




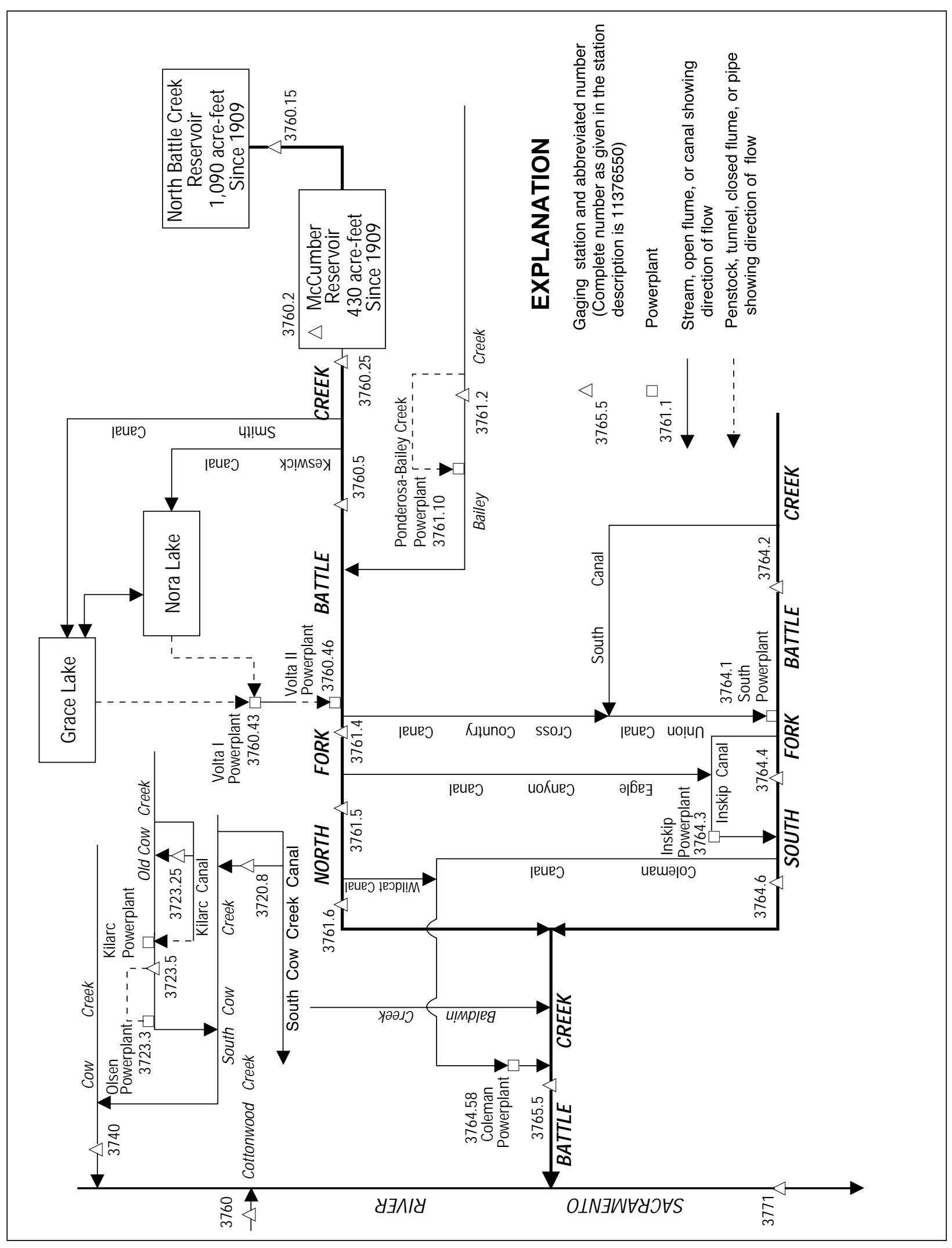


LOCATION.-Lat 40³5'35", long 121 '58'53", in NE 1/4 NW 1/4 sec.33, T.32 N., R.1 W., Shasta County, Hydrologic Unit 18020118, on left bank, 2.5 mi northeast of Cow Creek Powerplant, and 4.3 mi southwest of Whitmore.

PERIOD OF RECORD.-October 1986 to current year (operated as a low-flow station only). Unpublished records for water years $1984-86$ available in files of the U.S. Geological Survey.

GAGE.-Water-stage recorder. Elevation of gage is 1,560 ft above sea level, from topographic map.

REMARKS.-This station records fishwater release only. The minimum release requirements are $2.0 \mathrm{ft}^{3} / \mathrm{s} \mathrm{during} \mathrm{dry}$ years and $4.0 \mathrm{ft}^{3} / \mathrm{s}$ during normal years. Flow is computed to $7.8 \mathrm{ft}^{3} / \mathrm{s}$. See schematic diagram of Battle Creek and Cow Creek Basins.

COOPERATION.-Records were collected by Pacific Gas \& Electric Co., under general supervision of the U.S. Geological Survey, in connection with a Federal Energy Regulatory Commission project.

DISCHARGE, CUBIC FEET PER SECOND, WATER YEAR OCTOBER 1998 TO SEPTEMBER 1999 DAILY MEAN VALUES

\begin{tabular}{|c|c|c|c|c|c|c|c|c|c|c|c|c|}
\hline DAY & $\mathrm{OCT}$ & NOV & $\mathrm{DEC}$ & JAN & FEB & MAR & APR & MAY & JUN & JUL & AUG & SEP \\
\hline 1 & 5.5 & 5.5 & 5.6 & 5.6 & 6.5 & --- & 6.2 & 6.6 & 6.3 & 5.5 & 5.4 & 5.4 \\
\hline 2 & 5.5 & 5.5 & 5.7 & 5.6 & 6.4 & 7.3 & 6.2 & 6.9 & 6.7 & 5.5 & 5.4 & 5.3 \\
\hline 3 & 5.5 & 5.5 & 6.9 & 5.6 & 6.3 & 7.3 & 6.1 & 6.9 & 6.5 & 5.5 & 5.4 & 5.3 \\
\hline 4 & 5.5 & 5.5 & 5.8 & 5.6 & 6.3 & 6.6 & 6.1 & 6.8 & 6.4 & 5.5 & 5.4 & 5.3 \\
\hline 5 & 5.5 & 5.5 & 5.5 & 5.6 & 5.9 & 6.3 & 6.2 & 6.6 & 6.3 & 5.5 & 5.4 & 5.3 \\
\hline 6 & 5.5 & 5.5 & 5.5 & 5.6 & 6.4 & 6.1 & 6.1 & 6.6 & 6.2 & 5.4 & 5.4 & 5.3 \\
\hline 7 & 5.5 & 6.8 & 5.6 & 5.6 & 6.9 & 6.1 & 6.0 & 6.6 & 6.2 & 5.3 & 5.4 & 5.3 \\
\hline 8 & 5.5 & 6.5 & 5.9 & 5.6 & 6.7 & 6.3 & 6.6 & 6.5 & 6.2 & 5.7 & 5.4 & 5.3 \\
\hline 9 & 5.5 & 5.5 & 6.1 & 5.6 & --- & 6.4 & 6.3 & 6.4 & 6.3 & 7.1 & 5.4 & 5.4 \\
\hline 10 & 5.5 & 5.8 & 6.0 & 5.6 & 6.7 & 6.1 & 6.5 & 6.4 & 6.2 & 6.0 & 5.4 & 5.4 \\
\hline 11 & 5.5 & 5.8 & 5.9 & 5.6 & 6.8 & 6.0 & 6.5 & 6.3 & 6.2 & --- & 5.4 & 5.4 \\
\hline 12 & 5.5 & 5.5 & 5.9 & 5.6 & 6.3 & 5.9 & 6.4 & 6.4 & 6.2 & 7.8 & 5.4 & 5.4 \\
\hline 13 & 5.4 & 5.5 & 5.9 & 5.6 & 6.1 & 5.9 & 6.4 & 6.4 & 6.1 & 7.4 & 5.4 & 5.4 \\
\hline 14 & 5.5 & 5.5 & 6.2 & 5.6 & 6.1 & 5.8 & 6.3 & 6.4 & 6.1 & 7.5 & 5.4 & 5.4 \\
\hline 15 & 5.5 & 5.5 & 5.9 & 6.2 & 5.8 & 5.8 & 6.3 & 6.3 & 6.1 & 6.7 & 5.4 & 5.4 \\
\hline 16 & 5.5 & 5.5 & 5.8 & 6.9 & 6.5 & 6.1 & 6.4 & 6.3 & 6.1 & 5.6 & 5.4 & 5.4 \\
\hline 17 & 5.5 & 6.2 & 5.7 & 7.2 & 7.2 & 6.3 & 6.5 & 6.2 & 6.0 & 5.6 & 5.4 & 5.4 \\
\hline 18 & 5.5 & 5.6 & 5.6 & 7.4 & 6.8 & 6.3 & 6.6 & 6.1 & 5.9 & 5.6 & 5.4 & 5.4 \\
\hline 19 & 5.5 & 5.5 & 5.6 & 6.8 & 6.6 & 6.3 & 6.7 & 6.2 & 5.8 & 5.6 & 5.6 & 5.4 \\
\hline 20 & 5.5 & 5.5 & 5.6 & 7.0 & 6.8 & 6.3 & 6.7 & 6.2 & 5.7 & 5.6 & 5.4 & 5.4 \\
\hline 21 & 5.5 & 6.5 & 5.5 & 7.0 & 6.8 & 6.3 & 6.7 & 6.3 & 5.7 & 5.6 & 5.4 & 5.4 \\
\hline 22 & 5.5 & 5.6 & 5.5 & 6.9 & 6.4 & 6.3 & 6.6 & 6.3 & 5.6 & 5.6 & 5.4 & 5.4 \\
\hline 23 & 5.4 & 6.8 & 5.7 & --- & 6.3 & 6.3 & 6.6 & 6.5 & 5.6 & 5.6 & 5.4 & 5.4 \\
\hline 24 & 5.4 & 5.9 & 6.1 & 7.5 & 6.5 & 6.6 & 6.6 & 6.5 & 5.6 & 5.6 & 5.4 & 5.4 \\
\hline 25 & 6.0 & 5.8 & 6.2 & 7.1 & --- & 6.8 & 6.6 & 6.6 & 5.6 & 5.6 & 5.4 & 5.3 \\
\hline 26 & 5.5 & 6.3 & 6.2 & 7.2 & 6.6 & 6.6 & 6.7 & 6.6 & 5.6 & 5.6 & 5.4 & 5.4 \\
\hline 27 & 5.5 & 6.7 & 6.3 & 7.0 & 5.9 & 6.5 & 6.7 & 6.6 & 5.5 & 5.6 & 5.4 & 5.4 \\
\hline 28 & 5.5 & 5.6 & 6.2 & 6.8 & 7.1 & 6.4 & 6.6 & 6.6 & 5.5 & 5.5 & 5.4 & 5.4 \\
\hline 29 & 5.5 & 5.9 & 6.0 & 6.6 & --- & 6.4 & 6.5 & 6.5 & 5.5 & 5.4 & 5.4 & 5.5 \\
\hline 30 & 5.5 & 5.2 & 5.8 & 6.5 & --- & 6.4 & 6.6 & 6.4 & 5.5 & 5.4 & 5.4 & 5.4 \\
\hline 31 & 5.5 & --- & 5.9 & 6.6 & --- & 6.4 & --- & 6.4 & --- & 5.4 & 5.4 & --- \\
\hline TOTAL & 170.7 & 174.0 & 182.1 & --- & --- & --- & 193.3 & 200.4 & 179.2 & --- & 167.6 & 161.3 \\
\hline MEAN & 5.51 & 5.80 & 5.87 & --- & --- & --- & 6.44 & 6.46 & 5.97 & --- & 5.41 & 5.38 \\
\hline MAX & 6.0 & 6.8 & 6.9 & --- & --- & --- & 6.7 & 6.9 & 6.7 & --- & 5.6 & 5.5 \\
\hline MIN & 5.4 & 5.2 & 5.5 & --- & --- & --- & 6.0 & 6.1 & 5.5 & --- & 5.4 & 5.3 \\
\hline $\mathrm{AC}-\mathrm{FT}$ & 339 & 345 & 361 & --- & --- & --- & 383 & 397 & 355 & --- & 332 & 320 \\
\hline
\end{tabular}


11372325 KILARC CANAL DIVERSION TO OLD COW CREEK, NEAR WHITMORE, CA

LOCATION.-Lat 4041'13", long 121 48'27", in SW 1/4 NE 1/4 sec.25, T.32 N., R.1 E., Shasta County, Hydrologic Unit 18020118, on right bank of Kilarc Canal, 3.6 mi upstream of Kilarc Powerplant, and 6.9 mi northeast of Whitmore.

PERIOD OF RECORD.-October 1986 to current year (operated as a low-flow station only). Unpublished records for water years $1983-86$ available in files of the U.S. Geological Survey.

GAGE.-Water-stage recorder and Cipolletti weir. Elevation of gage is 3,840 ft above sea level, from topographic map.

REMARKS.- This station records fishwater release only. The minimum release requirement is $2.0 \mathrm{ft}^{3} / \mathrm{s}$ during dry or normal years. Flow is computed to $5.0 \mathrm{ft}^{3} / \mathrm{s}$. See schematic diagram of Battle Creek and Cow Creek Basins.

COOPERATION.-Records were collected by Pacific Gas \& Electric Co., under general supervision of the U.S. Geological Survey, in connection with a Federal Energy Regulatory Commission project.

DISCHARGE, CUBIC FEET PER SECOND, WATER YEAR OCTOBER 1998 TO SEPTEMBER 1999 DAILY MEAN VALUES

\begin{tabular}{|c|c|c|c|c|c|c|c|c|c|c|c|c|}
\hline DAY & $\mathrm{OCT}$ & NOV & $\mathrm{DEC}$ & JAN & FEB & MAR & APR & MAY & JUN & JUL & AUG & SEP \\
\hline 1 & 2.9 & 2.9 & 4.0 & 3.2 & 3.3 & 3.7 & 3.5 & 3.9 & 3.6 & --- & 2.8 & 3.0 \\
\hline 2 & 2.9 & 2.9 & 4.1 & 3.1 & 3.2 & 3.6 & 3.5 & 3.9 & 3.7 & 3.0 & 2.8 & 3.0 \\
\hline 3 & 2.9 & 2.9 & 4.2 & 3.1 & 3.3 & 3.6 & 3.4 & 3.9 & 3.7 & 3.0 & 3.0 & 3.1 \\
\hline 4 & 2.9 & 2.9 & 3.8 & 3.1 & 3.5 & 3.5 & 3.4 & 3.8 & 3.6 & 3.0 & 3.1 & 3.1 \\
\hline 5 & 2.9 & 2.9 & 3.7 & 3.1 & 3.5 & 3.5 & 3.4 & 3.8 & 3.5 & 2.9 & 3.1 & 3.1 \\
\hline 6 & 2.9 & 2.9 & 3.7 & 3.0 & 3.6 & 3.4 & 3.4 & 3.8 & 3.5 & 2.9 & 3.0 & 3.1 \\
\hline 7 & 2.9 & 3.0 & 3.7 & 3.0 & 3.7 & 3.4 & 3.4 & 3.8 & 3.5 & 2.9 & 3.1 & 3.1 \\
\hline 8 & 2.9 & 2.9 & 3.7 & 3.0 & 3.6 & 3.3 & 3.4 & 3.8 & 3.5 & 2.9 & 3.1 & 3.1 \\
\hline 9 & 2.9 & 2.9 & 3.5 & 3.0 & 3.7 & 3.3 & 3.4 & 3.8 & 3.6 & 2.9 & 3.1 & 3.1 \\
\hline 10 & 2.9 & 2.9 & 3.5 & 3.0 & 3.5 & 3.3 & 3.4 & 3.8 & 3.7 & 2.9 & 3.0 & 3.1 \\
\hline 11 & 2.9 & 2.9 & 3.3 & 3.0 & 3.6 & 3.2 & 3.4 & 3.7 & 3.7 & 2.9 & 3.0 & 3.1 \\
\hline 12 & --- & 2.9 & 3.0 & 2.9 & 3.7 & 3.2 & 3.4 & 3.8 & 3.7 & 2.9 & 3.0 & 3.1 \\
\hline 13 & --- & 2.9 & 3.2 & 2.9 & 3.6 & 3.2 & 3.4 & 3.6 & 3.7 & 3.0 & 3.0 & 3.1 \\
\hline 14 & --- & 2.9 & 3.1 & 2.9 & 3.6 & 3.2 & 3.5 & 3.7 & 3.6 & 3.0 & 3.0 & 3.1 \\
\hline 15 & --- & 2.9 & 3.0 & 3.8 & 3.6 & 3.2 & 3.5 & 3.6 & 3.6 & 3.0 & 3.1 & 3.1 \\
\hline 16 & 3.3 & 2.9 & 3.0 & 3.7 & 3.6 & 3.3 & 3.6 & 3.7 & 3.6 & 3.1 & --- & 3.1 \\
\hline 17 & 2.9 & 2.9 & 3.1 & 3.5 & 3.8 & 3.5 & 3.7 & 3.7 & 3.6 & 3.0 & --- & 3.1 \\
\hline 18 & 2.9 & 2.9 & 3.0 & 3.7 & 3.7 & 3.5 & 3.7 & 3.7 & 3.5 & 3.0 & 3.1 & 3.0 \\
\hline 19 & 2.9 & 2.9 & 2.9 & 3.5 & 3.6 & 3.5 & 3.8 & 3.7 & 3.5 & 3.1 & 3.0 & 3.3 \\
\hline 20 & 2.9 & 2.9 & 2.9 & 3.5 & 3.6 & 3.5 & 3.8 & 3.7 & 3.4 & 3.0 & 3.0 & 3.1 \\
\hline 21 & 2.9 & 3.1 & 3.0 & 3.5 & 3.6 & 3.5 & 3.8 & 3.7 & 3.4 & 3.0 & 3.1 & 3.1 \\
\hline 22 & 2.9 & 4.0 & 3.0 & 3.5 & 3.5 & 3.5 & 3.7 & 3.7 & 3.3 & 3.1 & 3.1 & 3.1 \\
\hline 23 & 2.9 & 3.9 & 3.0 & 3.6 & 3.5 & 3.5 & 3.7 & 3.7 & 3.3 & 3.0 & 3.1 & 3.1 \\
\hline 24 & 3.0 & 3.8 & 3.0 & 3.5 & 3.5 & 3.5 & 3.8 & 3.7 & 3.2 & 3.0 & 3.1 & 3.1 \\
\hline 25 & 3.2 & 3.8 & 3.0 & 3.4 & 3.5 & 3.5 & 3.8 & 3.7 & 3.2 & 3.0 & 3.1 & 3.0 \\
\hline 26 & 2.9 & 3.8 & 3.2 & 3.4 & 3.4 & 3.6 & 3.9 & 3.7 & 3.1 & 3.0 & 3.1 & 3.1 \\
\hline 27 & 2.9 & 4.0 & 3.3 & 3.3 & 3.4 & 3.5 & 3.8 & 3.7 & 3.0 & 3.0 & 3.1 & 3.1 \\
\hline 28 & 2.9 & 3.7 & 3.3 & 3.3 & 3.8 & 3.5 & 3.8 & 3.7 & --- & 2.9 & 3.2 & 3.2 \\
\hline 29 & 2.9 & 3.3 & 3.2 & 3.3 & --- & 3.5 & 3.8 & 3.6 & --- & 2.9 & 3.1 & 3.1 \\
\hline 30 & 2.9 & 4.0 & 3.2 & 3.3 & --- & 3.5 & 3.9 & 3.5 & --- & 2.9 & 3.1 & 3.1 \\
\hline 31 & 2.9 & --- & 3.3 & 3.3 & --- & 3.5 & --- & 3.5 & --- & 2.8 & 3.1 & --- \\
\hline TOTAL & --- & 95.5 & 102.9 & 101.4 & 99.5 & 106.5 & 108.0 & 115.4 & --- & --- & --- & 92.9 \\
\hline MEAN & --- & 3.18 & 3.32 & 3.27 & 3.55 & 3.44 & 3.60 & 3.72 & --- & --- & --- & 3.10 \\
\hline MAX & --- & 4.0 & 4.2 & 3.8 & 3.8 & 3.7 & 3.9 & 3.9 & --- & --- & --- & 3.3 \\
\hline MIN & --- & 2.9 & 2.9 & 2.9 & 3.2 & 3.2 & 3.4 & 3.5 & --- & --- & --- & 3.0 \\
\hline $\mathrm{AC}-\mathrm{FT}$ & --- & 189 & 204 & 201 & 197 & 211 & 214 & 229 & --- & --- & --- & 184 \\
\hline
\end{tabular}

NOTE: Canal out of service Oct. 12-15, June 28 to July 1, and Aug. 16-17 and all flow remained in the natural channel. 
LOCATION.-Lat 4040'10", long 121 53'27", in NW 1/4 SW 1/4 sec.32, T.33 N., R.1 E., Shasta County, Hydrologic Unit 18020118, on right bank, 1.2 mi downstream from Kilarc Powerhouse, 2.2 mi upstream from Glendenning Creek, and 3.0 mi north of Whitmore.

DRAINAGE AREA.-32.6 $\mathrm{mi}^{2}$.

PERIOD OF RECORD._-January 1990 to September 1992, (operated as low-flow station only). October 1996 to September 1997 ; October 1998 to September 1999.

GAGE.-Water-stage recorder. Elevation of gage is 2,340 ft above sea level, from topographic map.

REMARKS.-This station records regulated bypass flow or natural flow only. During times of powerplant operation the minimum release requirement is $30 \mathrm{ft}^{3} / \mathrm{s}$. See schematic diagram of Battle Creek and Cow Creek Basins.

COOPERATION.--Records were collected by Synergics Incorporated, under general supervision of the U.S. Geological Survey, in connection with a Federal Energy Regulatory Commission project.

EXTREMES FOR PERIOD OF RECORD.-Maximum discharge, 2,280 $\mathrm{ft}^{3} / \mathrm{s}$, Jan. 1, 1997, gage-height, $7.29 \mathrm{ft}$; minimum daily, $6.9 \mathrm{ft}$ /s, Aug. 7, 9, 1997.

DISCHARGE, CUBIC FEET PER SECOND, WATER YEAR OCTOBER 1998 TO SEPTEMBER 1999 DAILY MEAN VALUES

\begin{tabular}{|c|c|c|c|c|c|c|c|c|c|c|c|c|}
\hline DAY & OCT & NOV & $\mathrm{DEC}$ & JAN & FEB & MAR & APR & MAY & JUN & JUL & AUG & SEP \\
\hline 1 & 41 & 37 & 109 & 34 & 35 & 189 & 40 & 63 & 47 & 40 & 44 & 38 \\
\hline 2 & 41 & 37 & 68 & 34 & 35 & 118 & 39 & 90 & 71 & 40 & 44 & 38 \\
\hline 3 & 40 & 36 & 160 & 34 & 35 & 138 & 39 & 94 & 59 & 40 & 43 & 38 \\
\hline 4 & 40 & 36 & 59 & 34 & 34 & 97 & 40 & 68 & 44 & 40 & 43 & 37 \\
\hline 5 & 39 & 36 & 37 & 34 & 35 & 77 & 43 & 60 & 43 & 40 & 43 & 38 \\
\hline 6 & 37 & 38 & 36 & 35 & 35 & 62 & 42 & 60 & 43 & 40 & 44 & 37 \\
\hline 7 & 38 & 45 & 36 & 35 & 37 & 47 & 41 & 63 & 44 & 40 & 45 & 37 \\
\hline 8 & 39 & 42 & 36 & 34 & 38 & 40 & 45 & 54 & 44 & 40 & 43 & 36 \\
\hline 9 & 35 & 38 & 36 & 34 & 160 & 41 & 43 & 48 & 44 & 40 & 44 & 37 \\
\hline 10 & 35 & 38 & 39 & 34 & 69 & 55 & 44 & 44 & 43 & 40 & 43 & 38 \\
\hline 11 & 36 & 36 & 35 & 35 & 42 & 42 & 42 & 42 & 40 & 40 & 43 & 36 \\
\hline 12 & 36 & 37 & 35 & 35 & 40 & 40 & 41 & 44 & 42 & 40 & 45 & 36 \\
\hline 13 & 33 & 37 & 35 & 34 & 40 & 38 & 42 & 40 & 42 & 40 & 42 & 37 \\
\hline 14 & 35 & 37 & 35 & 34 & 40 & 38 & 41 & 38 & 46 & 39 & 41 & 36 \\
\hline 15 & 35 & 38 & 34 & 35 & 40 & 38 & 43 & 41 & 51 & 47 & 41 & 36 \\
\hline 16 & 36 & 38 & 34 & 36 & 44 & 39 & 41 & 43 & 46 & 44 & 36 & 36 \\
\hline 17 & 35 & 45 & 34 & 37 & 62 & 40 & 60 & 43 & 41 & 42 & 41 & 35 \\
\hline 18 & 36 & 37 & 34 & 39 & 43 & 40 & 63 & 43 & 40 & 44 & 40 & 34 \\
\hline 19 & 35 & 36 & 35 & 35 & 40 & 39 & 97 & 43 & 40 & 43 & 40 & 36 \\
\hline 20 & 35 & 37 & 35 & 36 & 42 & 40 & 90 & 43 & 41 & 44 & 40 & 36 \\
\hline 21 & 36 & 47 & 40 & 36 & 41 & 40 & 105 & 43 & 41 & 46 & 39 & 35 \\
\hline 22 & 35 & 50 & 35 & 36 & 40 & 41 & 97 & 42 & 41 & 44 & 40 & 35 \\
\hline 23 & 36 & 94 & 34 & 54 & 39 & 40 & 103 & 43 & 40 & 45 & 37 & 35 \\
\hline 24 & 36 & 75 & 35 & 35 & 40 & 41 & 85 & 43 & 40 & 43 & 42 & 35 \\
\hline 25 & 36 & 38 & 34 & 35 & 45 & 40 & 58 & 43 & 40 & 45 & 39 & 30 \\
\hline 26 & 39 & 42 & 36 & 36 & 41 & 40 & 83 & 43 & 40 & 43 & 38 & 36 \\
\hline 27 & 37 & 64 & 33 & 35 & 40 & 41 & 77 & 45 & 40 & 43 & 39 & 35 \\
\hline 28 & 38 & 38 & 35 & 35 & 122 & 40 & 63 & 46 & 40 & 45 & 38 & 34 \\
\hline 29 & 38 & 40 & 35 & 35 & --- & 40 & 54 & 45 & 40 & 46 & 38 & 35 \\
\hline 30 & 36 & 103 & 34 & 35 & --- & 40 & 60 & 44 & 40 & 45 & 40 & 35 \\
\hline 31 & 37 & --- & 34 & 35 & --- & 41 & --- & 43 & --- & 45 & 39 & --- \\
\hline TOTAL & 1141 & 1352 & 1347 & 1105 & 1354 & 1702 & 1761 & 1544 & 1313 & 1313 & 1274 & 1077 \\
\hline MEAN & 36.8 & 45.1 & 43.5 & 35.6 & 48.4 & 54.9 & 58.7 & 49.8 & 43.8 & 42.4 & 41.1 & 35.9 \\
\hline MAX & 41 & 103 & 160 & 54 & 160 & 189 & 105 & 94 & 71 & 47 & 45 & 38 \\
\hline MIN & 33 & 36 & 33 & 34 & 34 & 38 & 39 & 38 & 40 & 39 & 36 & 30 \\
\hline $\mathrm{AC}-\mathrm{FT}$ & 2260 & 2680 & 2670 & 2190 & 2690 & 3380 & 3490 & 3060 & 2600 & 2600 & 2530 & 2140 \\
\hline a & 0 & 783 & 2100 & 2190 & 4370 & 5640 & 4770 & 6750 & 2860 & 256 & 0 & 0 \\
\hline
\end{tabular}

a Discharge, in acre-feet, for Olsen Powerplant (station 11372330), provided by Synergics Incorporated. 
LOCATION.-Lat 40³0'19", long 122 $13^{\prime} 56^{\prime \prime}$, in NE 1/4 NW 1/4 sec.32, T.31 N., R.3 W., Shasta County, Hydrologic Unit 18020101, on right bank, 2.9 mi upstream from mouth, 4.2 mi southwest of Millville, and 4.3 mi downstream from Little Cow Creek.

DRAINAGE AREA. $-425 \mathrm{mi}^{2}$.

PERIOD OF RECORD.-October 1949 to current year.

CHEMICAL DATA: Water years 1959-66.

WATER TEMPERATURE: Water years 1966-71, 1973-76, 1978-79.

SEDIMENT DATA: Water year 1978

REVISED RECORDS.-WSP 1931: Drainage area.

GAGE.-Water-stage recorder and crest-stage gage. Datum of gage is $385.7 \mathrm{ft}$ above sea level. Prior to June 11, 1987, at datum $3.00 \mathrm{ft}$ higher.

REMARKS.-Records good. Numerous small diversions upstream from station for irrigation. See schematic diagrams of upper Sacramento River Basin and Battle Creek and Cow Creek Basins.

EXTREMES FOR PERIOD OF RECORD.-Maximum discharge, 48,700 ft $3 / \mathrm{s}$, Nov. 16, 1981, gage height, $24.22 \mathrm{ft}$, present datum; maximum gage height, $24.55 \mathrm{ft}$, Dec. 27, 1951, present datum; minimum daily, $0.02 \mathrm{ft}^{3} / \mathrm{s}$, July 29, 1977 .

EXTREMES OUTSIDE PERIOD OF RECORD.-Flood of 1937 or 1940 reached a stage of $26.8 \mathrm{ft}$ from floodmarks, present datum; probable backwater effect from high flows on the Sacramento River.

EXTREMES FOR CURRENT YEAR.-Peak discharges greater than base discharge of 13,900 $\mathrm{ft}^{3} / \mathrm{s}$, or maximum:

$\begin{array}{lccccccc}\text { Date } & \text { Time } & \begin{array}{c}\text { Discharge } \\ \left(\mathrm{ft}^{3} / \mathrm{s}\right)\end{array} & \begin{array}{c}\text { Gage height } \\ (\mathrm{ft})\end{array} & \text { Date } & \text { Time } & \begin{array}{c}\text { Discharge } \\ \left(\mathrm{ft}^{3} / \mathrm{s}\right)\end{array} & \begin{array}{c}\text { Gage height } \\ (\mathrm{ft})\end{array} \\ \text { Nov. 30 } & 0245 & 18,100 & 15.41 & \text { Feb. } 9 & 0745 & 31,800 & 20.01\end{array}$

DISCHARGE, CUBIC FEET PER SECOND, WATER YEAR OCTOBER 1998 TO SEPTEMBER 1999 DAILY MEAN VALUES

\begin{tabular}{|c|c|c|c|c|c|c|c|c|c|c|c|c|}
\hline DAY & OCT & NOV & $\mathrm{DEC}$ & JAN & $\mathrm{FEB}$ & MAR & APR & MAY & JUN & JUL & AUG & SEP \\
\hline 1 & 137 & 173 & 3660 & 296 & 585 & 4120 & 922 & 650 & 410 & 129 & 74 & 65 \\
\hline 2 & 141 & 168 & 3640 & 279 & 512 & 2560 & 819 & 732 & 494 & 124 & 74 & 64 \\
\hline 3 & 141 & 171 & 6910 & 271 & 486 & 2620 & 760 & 780 & 579 & 117 & 70 & 62 \\
\hline 4 & 135 & 168 & 2310 & 266 & 487 & 1950 & 709 & 705 & 477 & 120 & 67 & 61 \\
\hline 5 & 135 & 168 & 1420 & 263 & 455 & 1620 & 786 & 642 & 420 & 119 & 66 & 54 \\
\hline 6 & 130 & 173 & 1150 & 258 & 1940 & 1420 & 828 & 615 & 383 & 119 & 65 & 57 \\
\hline 7 & 127 & 338 & 991 & 252 & 5590 & 1270 & 736 & 622 & 354 & 111 & 85 & 57 \\
\hline 8 & 134 & 412 & 1050 & 241 & 4250 & 1170 & 937 & 592 & 332 & 108 & 81 & 57 \\
\hline 9 & 140 & 274 & 841 & 233 & 14800 & 2090 & 941 & 547 & 318 & 98 & 79 & 57 \\
\hline 10 & 141 & 284 & 692 & 231 & 3710 & 1580 & 985 & 529 & 304 & 109 & 80 & 58 \\
\hline 11 & 139 & 548 & 620 & 230 & 2250 & 1300 & 1710 & 511 & 288 & 101 & 91 & 65 \\
\hline 12 & 140 & 270 & 568 & 231 & 1680 & 1150 & 1030 & 495 & 277 & 102 & 89 & 56 \\
\hline 13 & 142 & 225 & 584 & 229 & 1340 & 1050 & 887 & 501 & 275 & 90 & 90 & 60 \\
\hline 14 & 135 & 208 & 1020 & 230 & 1450 & 984 & 814 & 499 & 270 & 83 & 86 & 62 \\
\hline 15 & 138 & 198 & 639 & 254 & 1190 & 916 & 781 & 474 & 262 & 81 & 83 & 59 \\
\hline 16 & 140 & 200 & 545 & 462 & 3850 & 849 & 774 & 431 & 254 & 88 & 76 & 63 \\
\hline 17 & 137 & 1050 & 503 & 2560 & 5460 & 804 & 782 & 411 & 243 & 90 & 56 & 62 \\
\hline 18 & 135 & 487 & 470 & 3380 & 2950 & 787 & 791 & 406 & 237 & 84 & 54 & 63 \\
\hline 19 & 131 & 304 & 430 & 1900 & 2200 & 760 & 792 & 416 & 227 & 90 & 53 & 59 \\
\hline 20 & 127 & 245 & 407 & 2310 & 3230 & 789 & 814 & 425 & 213 & 82 & 54 & 60 \\
\hline 21 & 121 & 976 & 353 & 1870 & 6400 & 781 & 803 & 432 & 216 & 87 & 51 & 59 \\
\hline 22 & 124 & 2160 & 361 & 1180 & 2930 & 812 & 760 & 437 & 204 & 83 & 45 & 59 \\
\hline 23 & 127 & 3090 & 334 & 2780 & 2150 & 808 & 728 & 461 & 186 & 85 & 47 & 57 \\
\hline 24 & 202 & 2000 & 323 & 1570 & 1740 & 2400 & 725 & 473 & 181 & 80 & 47 & 61 \\
\hline 25 & 266 & 816 & 322 & 1080 & 3480 & 2970 & 729 & 494 & 178 & 84 & 53 & 64 \\
\hline 26 & 180 & 2000 & 321 & 1040 & 2100 & 1550 & 758 & 500 & 176 & 76 & 49 & 59 \\
\hline 27 & 171 & 3890 & 311 & 885 & 1760 & 1180 & 813 & 496 & 168 & 74 & 53 & 59 \\
\hline 28 & 171 & 1220 & 301 & 730 & 3870 & 1000 & 744 & 489 & 163 & 69 & 52 & 51 \\
\hline 29 & 178 & 4070 & 294 & 648 & --- & 925 & 668 & 483 & 150 & 71 & 53 & 53 \\
\hline 30 & 166 & 9440 & 288 & 591 & --- & 961 & 649 & 461 & 128 & 72 & 51 & 58 \\
\hline 31 & 165 & --- & 296 & 614 & --- & 1030 & --- & 425 & --- & 75 & 58 & --- \\
\hline TOTAL & 4596 & 35726 & 31954 & 27364 & 82845 & 44206 & 24975 & 16134 & 8367 & 2901 & 2032 & 1781 \\
\hline MEAN & 148 & 1191 & 1031 & 883 & 2959 & 1426 & 832 & 520 & 279 & 93.6 & 65.5 & 59.4 \\
\hline MAX & 266 & 9440 & 6910 & 3380 & 14800 & 4120 & 1710 & 780 & 579 & 129 & 91 & 65 \\
\hline MIN & 121 & 168 & 288 & 229 & 455 & 760 & 649 & 406 & 128 & 69 & 45 & 51 \\
\hline $\mathrm{AC}-\mathrm{FT}$ & 9120 & 70860 & 63380 & 54280 & 164300 & 87680 & 49540 & 32000 & 16600 & 5750 & 4030 & 3530 \\
\hline
\end{tabular}


11374000 COW CREEK NEAR MILLVILLE, CA-Continued

STATISTICS OF MONTHLY MEAN DATA FOR WATER YEARS 1950 - 1999, BY WATER YEAR (WY)

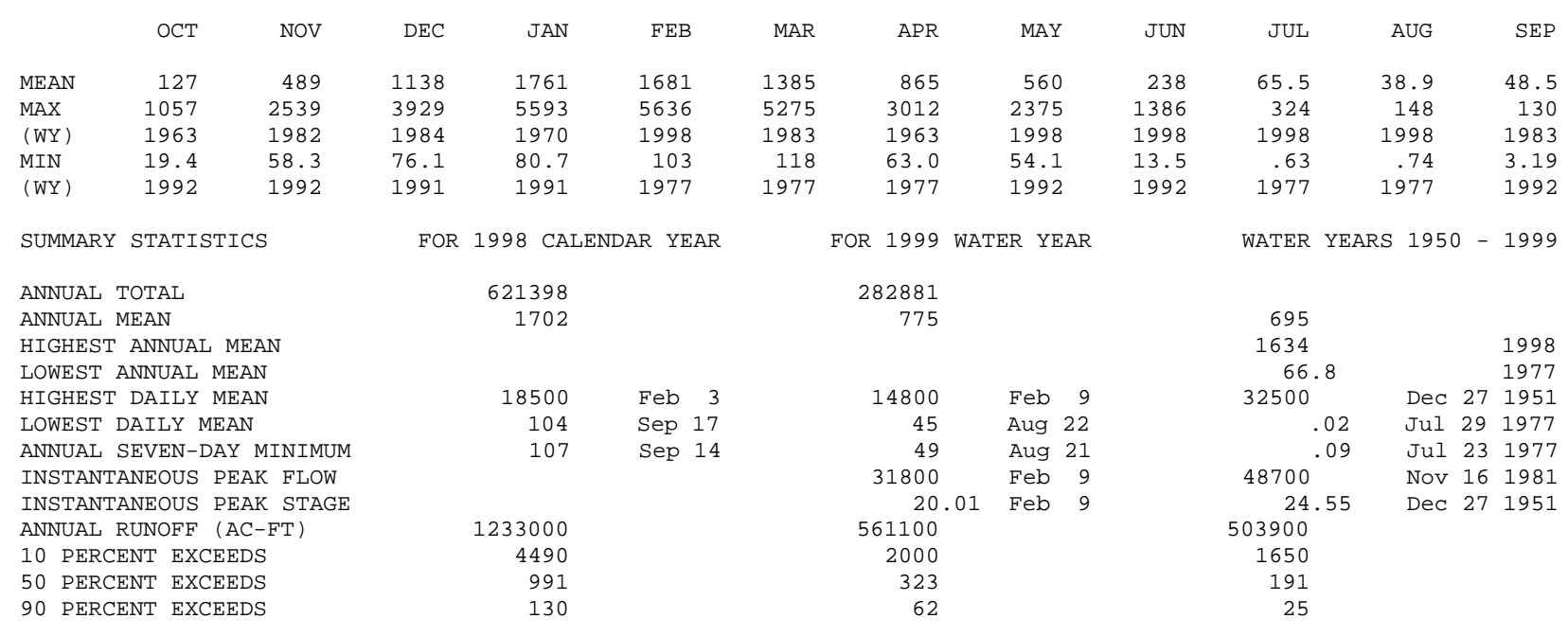


LOCATION.-Lat 40²4’35", long 12252’52", in NW 1/4 SE 1/4 sec.4, T.29 N., R.9 W., Shasta County, Hydrologic Unit 18020113, on left bank, 1.2 mi downstream from Cow Gulch, 1.0 mi upstream from Knob Gulch, and 2.4 mi northeast of the town of Platina.

DRAINAGE AREA.- $-46.0 \mathrm{mi}^{2}$.

PERIOD OF RECORD.- October 1997 to current year (low-flow records only, collected only seasonally during period of upstream diversion for power generation).

GAGE.-Water-stage recorder and V-notched weir. Elevation of gage is 2,050 ft above sea level, from topographic map.

REMARKS.-No records computed above $32 \mathrm{ft}^{3} / \mathrm{s}$. Record is only collected during the part of the year when flow is generally high enough to allow for upstream diversion of water to Arbuckle Mountain Powerplant (station 11374300). This year, record was collected Nov. 24, 1998, to June 6, 1999. Flow was above $32 \mathrm{ft}^{3} / \mathrm{s}$ for many days during this period. During times of powerplant operation, the minimum release requirement is $5.0 \mathrm{ft}^{3} / \mathrm{s}$. See schematic diagram of upper Sacramento River Basin.

COOPERATION.--Records were collected by Arbuckle Mountain Hydro, LLC, under general supervision of the U.S. Geological Survey, in connection with a Federal Energy Regulatory Commission Project.

DISCHARGE, CUBIC FEET PER SECOND, WATER YEAR OCTOBER 1998 TO SEPTEMBER 1999 DAILY MEAN VALUES

\begin{tabular}{|c|c|c|c|c|c|c|c|c|c|c|c|c|}
\hline DAY & $\mathrm{OCT}$ & NOV & $\mathrm{DEC}$ & JAN & $\mathrm{FEB}$ & MAR & $A P R$ & MAY & JUN & JUL & AUG & SEP \\
\hline 1 & --- & --- & --- & 20 & 12 & --- & --- & 12 & 16 & --- & --- & --- \\
\hline 2 & --- & --- & 28 & 15 & 12 & --- & --- & 12 & 27 & --- & --- & --- \\
\hline 3 & --- & --- & --- & 18 & 12 & --- & 26 & 12 & --- & --- & --- & --- \\
\hline 4 & --- & --- & --- & 18 & 12 & --- & 14 & 11 & 20 & --- & --- & --- \\
\hline 5 & --- & --- & --- & 16 & 12 & --- & 13 & 9.4 & 16 & --- & --- & --- \\
\hline 6 & --- & --- & --- & 14 & 24 & --- & 13 & 7.0 & 18 & --- & --- & --- \\
\hline 7 & --- & --- & --- & 15 & --- & --- & 11 & 8.1 & --- & --- & --- & --- \\
\hline 8 & --- & --- & --- & 17 & --- & --- & 12 & 6.6 & --- & --- & --- & --- \\
\hline 9 & --- & --- & 28 & 15 & --- & --- & 13 & 24 & --- & --- & --- & --- \\
\hline 10 & --- & --- & 10 & 13 & --- & 19 & 16 & --- & --- & --- & --- & --- \\
\hline 11 & --- & --- & 9.0 & 13 & --- & 13 & --- & 31 & --- & --- & --- & --- \\
\hline 12 & --- & --- & 6.9 & 14 & 15 & 13 & --- & 14 & --- & --- & --- & --- \\
\hline 13 & --- & --- & 7.9 & 14 & 27 & 17 & --- & --- & --- & --- & --- & --- \\
\hline 14 & --- & --- & 12 & 13 & 12 & 26 & --- & --- & --- & --- & --- & --- \\
\hline 15 & --- & --- & 8.6 & 10 & 11 & --- & --- & --- & --- & --- & --- & --- \\
\hline 16 & --- & --- & 7.5 & 10 & 24 & --- & --- & 30 & --- & --- & --- & --- \\
\hline 17 & --- & --- & 5.8 & 16 & --- & --- & --- & 24 & --- & --- & --- & --- \\
\hline 18 & --- & --- & 5.9 & 24 & --- & --- & --- & 23 & --- & --- & --- & --- \\
\hline 19 & --- & --- & 12 & 12 & --- & --- & 32 & 24 & --- & --- & --- & --- \\
\hline 20 & --- & --- & 21 & 12 & --- & --- & 18 & 22 & --- & --- & --- & --- \\
\hline 21 & --- & --- & 21 & 11 & --- & --- & 11 & 20 & --- & --- & --- & --- \\
\hline 22 & --- & --- & 19 & 17 & --- & --- & 16 & 18 & --- & --- & --- & --- \\
\hline 23 & --- & --- & 19 & --- & --- & --- & 11 & 16 & --- & --- & --- & --- \\
\hline 24 & --- & --- & 17 & 14 & --- & --- & 11 & 19 & --- & --- & --- & --- \\
\hline 25 & --- & 22 & 17 & 11 & --- & --- & 11 & --- & --- & --- & --- & --- \\
\hline 26 & --- & 18 & 17 & 15 & --- & --- & 12 & 31 & --- & --- & --- & --- \\
\hline 27 & --- & 14 & 17 & 11 & --- & --- & 12 & --- & --- & --- & --- & --- \\
\hline 28 & --- & 12 & 18 & 12 & --- & --- & 12 & 14 & --- & --- & --- & --- \\
\hline 29 & --- & 17 & 16 & 12 & --- & --- & 25 & 29 & --- & --- & --- & --- \\
\hline 30 & --- & --- & 15 & 12 & --- & --- & 5.8 & 30 & --- & --- & --- & --- \\
\hline 31 & --- & --- & 15 & 12 & --- & --- & --- & 15 & --- & --- & --- & --- \\
\hline TOTAL & --- & --- & --- & --- & --- & --- & --- & --- & --- & --- & --- & --- \\
\hline MEAN & --- & --- & --- & --- & --- & --- & --- & --- & --- & --- & --- & --- \\
\hline MAX & --- & --- & --- & --- & --- & --- & --- & --- & --- & --- & --- & --- \\
\hline MIN & --- & --- & --- & --- & --- & --- & --- & --- & --- & --- & --- & --- \\
\hline $\mathrm{AC}-\mathrm{FT}$ & --- & --- & --- & --- & --- & --- & --- & --- & --- & --- & --- & --- \\
\hline a & --- & 367 & 698 & 1330 & 2350 & 5480 & 5200 & 1450 & 129 & --- & --- & --- \\
\hline
\end{tabular}

a Discharge, in acre-feet, for Arbuckle Mountain Powerplant (station 11374300), provided by Arbuckle Mountain Hydro, LLC. 
LOCATION.—Lat 40²3'14", long 122 $14^{\prime} 15^{\prime \prime}$, in NE 1/4 NE 1/4 sec.7, T.29 N., R.3 W., Shasta County, Hydrologic Unit 18020102, on left bank, 2.2 mi east of Cottonwood, and 2.5 mi upstream from mouth.

DRAINAGE AREA.-927 $\mathrm{mi}^{2}$.

PERIOD OF RECORD.—October 1940 to current year.

CHEMICAL DATA: Water years 1982-85.

WATER TEMPERATURE: Water years 1963-67, 1977-85.

SEDIMENT DATA: Water years 1957-67, 1977-85.

REVISED RECORDS.-WSP 1345: 1943, 1944(M), 1946-47, 1949(M), 1951-52. WSP 1931: Drainage area.

GAGE.-Water-stage recorder and crest-stage gage. Datum of gage is $363.80 \mathrm{ft}$ above sea level (levels by U.S. Army Corps of Engineers). Prior to July 26, 1963, on right bank at datum $3.59 \mathrm{ft}$ higher. July 26, 1963, to Sept. 13, 1972, at site $250 \mathrm{ft}$ downstream on right bank at present datum. Sept. 21, 1967, to Jan. 14, 1968, supplementary gage at a site 1,450 ft downstream on right bank at datum $2.35 \mathrm{ft}$ higher.

REMARKS.--Records fair. Small diversions for irrigation upstream from station. At times during irrigation season, Cottonwood Creek receives water from the Sacramento River by way of Anderson-Cottonwood Irrigation District Canal. See schematic diagrams of upper Sacramento River Basin and Battle Creek and Cow Creek Basins.

EXTREMES FOR PERIOD OF RECORD.-Maximum discharge, 86,000 ft $\mathrm{ft}^{3} / \mathrm{s}$, Mar. 1, 1983, gage height, $21.59 \mathrm{ft}$ from rating curve extended above $34,000 \mathrm{ft}^{3} / \mathrm{s}$ on basis of runoff comparisons with upstream stations then in use; minimum, $15 \mathrm{ft}^{3} / \mathrm{s}$ several days during September 1945 .

EXTREMES FOR CURRENT YEAR.-Peak discharges greater than base discharge of $11,000 \mathrm{ft}^{3} / \mathrm{s}$, or maximum:

\begin{tabular}{lccccccc}
\multicolumn{1}{c}{ Date } & Time & $\begin{array}{c}\text { Discharge } \\
\left(\mathrm{ft}^{3} / \mathrm{s}\right)\end{array}$ & $\begin{array}{c}\text { Gage height } \\
(\mathrm{ft})\end{array}$ & Date & Time & $\begin{array}{c}\text { Discharge } \\
\left(\mathrm{ft}^{3} / \mathrm{s}\right)\end{array}$ & $\begin{array}{c}\text { Gage height } \\
(\mathrm{ft})\end{array}$ \\
Nov. 30 & Unknown & $\mathrm{e} 11,000$ & Unknown & Mar. 24 & 2100 & 12,900 & 10.96 \\
Feb. 7 & 0500 & 11,300 & 10.51 & & & &
\end{tabular}

DISCHARGE, CUBIC FEET PER SECOND, WATER YEAR OCTOBER 1998 TO SEPTEMBER 1999 DAILY MEAN VALUES

\begin{tabular}{|c|c|c|c|c|c|c|c|c|c|c|c|c|}
\hline DAY & $\mathrm{OCT}$ & NOV & $\mathrm{DEC}$ & JAN & FEB & MAR & APR & MAY & JUN & JUL & AUG & SEP \\
\hline 1 & 126 & 131 & e 3200 & 300 & 671 & 3700 & 2110 & 1240 & 566 & 178 & 84 & 61 \\
\hline 2 & 128 & 135 & e 3500 & 289 & 565 & 2940 & 1890 & 1260 & 586 & 168 & 86 & 60 \\
\hline 3 & 134 & 134 & e 5400 & 280 & 527 & 2780 & 1710 & 1250 & 622 & 159 & 83 & 62 \\
\hline 4 & 131 & 129 & e3600 & 273 & 503 & 2460 & 1560 & 1170 & 559 & 156 & 83 & 65 \\
\hline 5 & 121 & 129 & e2000 & 268 & 473 & 2100 & 1630 & 1090 & 510 & 164 & 77 & 68 \\
\hline 6 & 122 & 131 & e1300 & 266 & 2650 & 1850 & 2470 & 1050 & 473 & 165 & 76 & 69 \\
\hline 7 & 125 & 194 & e1020 & 265 & 8990 & 1640 & 1930 & 1030 & 437 & 149 & 80 & 70 \\
\hline 8 & 126 & e430 & 825 & 259 & 4500 & 1610 & 1780 & 1010 & 415 & 142 & 87 & 66 \\
\hline 9 & 117 & e340 & 733 & 252 & 4480 & 2470 & 1620 & 963 & 408 & 146 & 102 & 62 \\
\hline 10 & 120 & e 310 & 655 & 248 & 2930 & 1710 & 1600 & 908 & 403 & 146 & 93 & 60 \\
\hline 11 & 129 & e 480 & 607 & 245 & 2020 & 1480 & 5180 & 907 & 390 & 147 & 103 & 57 \\
\hline 12 & 121 & e310 & 575 & 238 & 1590 & 1320 & 3250 & 903 & 381 & 140 & 104 & 56 \\
\hline 13 & 125 & e270 & 579 & 231 & 1330 & 1240 & 2770 & 913 & 374 & 134 & 94 & 63 \\
\hline 14 & 121 & e260 & 664 & 227 & 1420 & 1360 & 2690 & 858 & 363 & 122 & 87 & 57 \\
\hline 15 & 130 & e260 & 572 & 238 & 1210 & 2700 & 2600 & 788 & 364 & 119 & 78 & 54 \\
\hline 16 & 121 & e280 & 512 & 291 & 2940 & 1900 & 2540 & 757 & 365 & 111 & 71 & 54 \\
\hline 17 & 124 & e 440 & 486 & 511 & 6150 & 1740 & 2580 & 734 & 338 & 110 & 70 & 57 \\
\hline 18 & 121 & e960 & 471 & 1310 & 3760 & 1670 & 2710 & 703 & 323 & 116 & 65 & 64 \\
\hline 19 & 112 & e 470 & 455 & 1450 & 4140 & 1600 & 2690 & 726 & 308 & 125 & 71 & 60 \\
\hline 20 & 105 & e 410 & 433 & 979 & 3510 & 1990 & 2530 & 717 & 297 & 116 & 75 & 61 \\
\hline 21 & 110 & e780 & 402 & 1020 & 4730 & 2050 & 2280 & 693 & 293 & 116 & 70 & 59 \\
\hline 22 & 117 & e1050 & 369 & 911 & 3020 & 1850 & 2080 & 667 & 275 & 118 & 64 & 54 \\
\hline 23 & 116 & e2500 & 364 & 3400 & 2460 & 1880 & 1880 & 667 & 262 & 117 & 68 & 56 \\
\hline 24 & 266 & e1600 & 349 & 1950 & 2190 & 5900 & 1760 & 690 & 252 & 114 & 87 & 55 \\
\hline 25 & 311 & e1150 & 371 & 1310 & 2980 & 9220 & 1700 & 717 & 238 & 115 & 94 & 56 \\
\hline 26 & 269 & e 1750 & 354 & 1100 & 2630 & 5520 & 1730 & 713 & 232 & 105 & 74 & 54 \\
\hline 27 & 167 & e 3400 & 339 & 926 & 2320 & 4140 & 1650 & 686 & 221 & 100 & 62 & 58 \\
\hline 28 & 140 & e2400 & 326 & 760 & 2510 & 3340 & 1530 & 661 & 214 & 88 & 59 & 53 \\
\hline 29 & 132 & e1700 & 317 & 709 & --- & 2840 & 1380 & 634 & 207 & 106 & 55 & 55 \\
\hline 30 & 126 & e 7200 & 308 & 624 & --- & 2570 & 1290 & 602 & 190 & 93 & 54 & 54 \\
\hline 31 & 125 & --- & 304 & 654 & --- & 2390 & --- & 585 & --- & 84 & 61 & --- \\
\hline TOTAL & 4338 & 29733 & 31390 & 21784 & 77199 & 81960 & 65120 & 26292 & 10866 & 3969 & 2417 & 1780 \\
\hline MEAN & 140 & 991 & 1013 & 703 & 2757 & 2644 & 2171 & 848 & 362 & 128 & 78.0 & 59.3 \\
\hline MAX & 311 & 7200 & 5400 & 3400 & 8990 & 9220 & 5180 & 1260 & 622 & 178 & 104 & 70 \\
\hline MIN & 105 & 129 & 304 & 227 & 473 & 1240 & 1290 & 585 & 190 & 84 & 54 & 53 \\
\hline$A C-F T$ & 8600 & 58980 & 62260 & 43210 & 153100 & 162600 & 129200 & 52150 & 21550 & 7870 & 4790 & 3530 \\
\hline
\end{tabular}




\section{COTTONWOOD CREEK NEAR COTTONWOOD, CA—Continued}

STATISTICS OF MONTHLY MEAN DATA FOR WATER YEARS 1941 - 1999, BY WATER YEAR (WY)

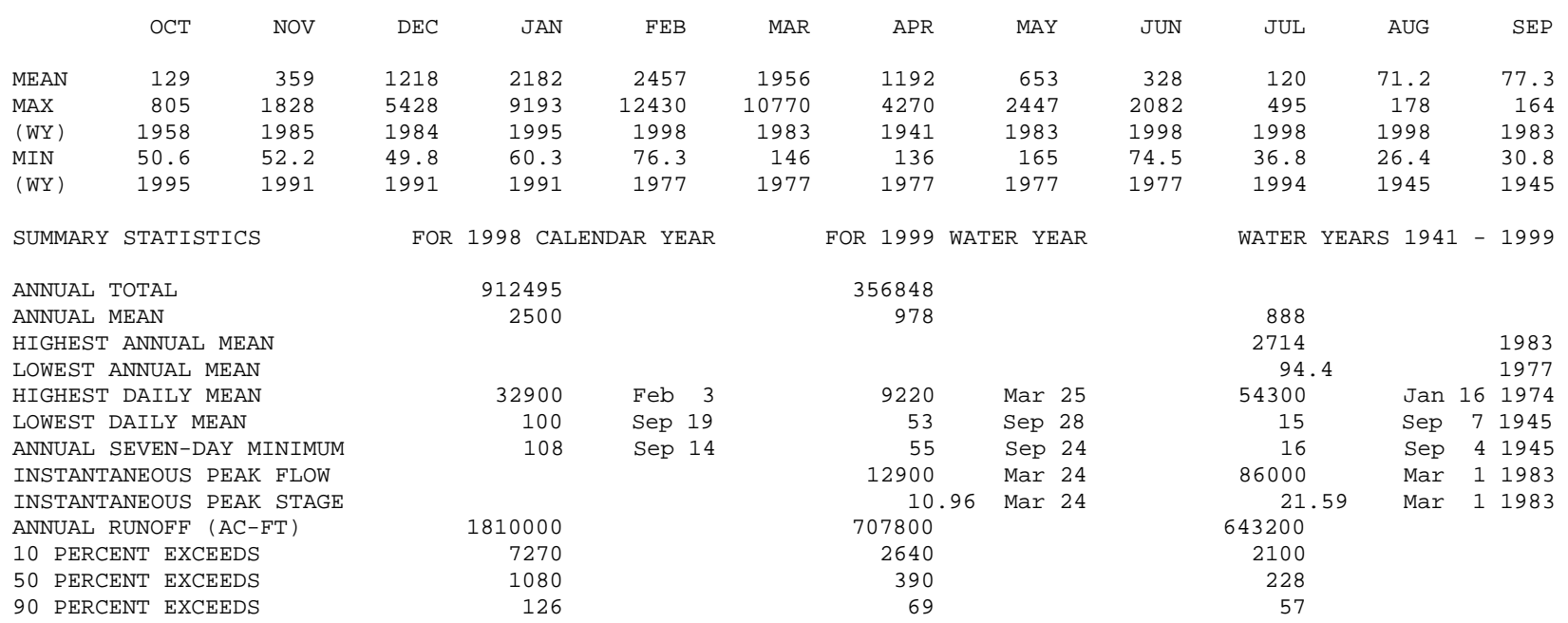


11376015 NORTH FORK BATTLE CREEK BELOW NORTH BATTLE CREEK DAM, NEAR MANZANITA LAKE, CA

LOCATION.-Lat 40³6’10", long 121³9’17", in SE 1/4 SE 1/4 sec.20, T.32 N., R.3 E., Shasta County, Hydrologic Unit 18020118, Lassen National Forest, on left bank, $300 \mathrm{ft}$ downstream from North Battle Creek Dam, and 6.7 mi northwest of Manzanita Lake.

DRAINAGE AREA.-6.40 $\mathrm{mi}^{2}$

PERIOD OF RECORD.-October 1987 to current year (operated as a low-flow station only). Unpublished records for water years $1978-87$ available in files of the U.S. Geological Survey. Fragmentary records for water years 1920-77 in files of the Pacific Gas \& Electric Co.

GAGE.-Water-stage recorder and a compound weir consisting of a 5-ft rectangular and V-notch weir. Elevation of gage is 5,560 ft above sea level, from topographic map.

REMARKS.- This station records fishwater release only. The minimum release requirement is $0.30 \mathrm{ft}^{3} / \mathrm{s}$ Oct. 1-31 and Apr. 1 to Sept. 30 . No license requirement Nov. 1 to Mar. 31, records not computed. Each fall, North Battle Creek Reservoir is drafted and flows may exceed the rated limits of the weirs; flow is computed to $60 \mathrm{ft}^{3} / \mathrm{s}$. See schematic diagram of Battle Creek and Cow Creek Basins.

COOPERATION.-Records were collected by Pacific Gas \& Electric Co., under general supervision of the U.S. Geological Survey, in connection with a Federal Energy Regulatory Commission project.

DISCHARGE, CUBIC FEET PER SECOND, WATER YEAR OCTOBER 1998 TO SEPTEMBER 1999

DAILY MEAN VALUES

\begin{tabular}{|c|c|c|c|c|c|c|c|c|c|c|c|c|}
\hline DAY & OCT & NOV & $\mathrm{DEC}$ & JAN & $\mathrm{FEB}$ & MAR & APR & MAY & JUN & JUL & AUG & SEP \\
\hline 1 & 3.6 & --- & --- & --- & --- & --- & 4.6 & 18 & 30 & 1.7 & 1.1 & 1.3 \\
\hline 2 & 5.0 & --- & --- & --- & --- & --- & 3.9 & 22 & 37 & 1.4 & 1.1 & 1.2 \\
\hline 3 & 5.1 & --- & --- & --- & --- & --- & 4.0 & 19 & 31 & 1.4 & 1.3 & 1.2 \\
\hline 4 & 5.1 & --- & --- & --- & --- & --- & 3.7 & 15 & 26 & 1.2 & 1.6 & 1.1 \\
\hline 5 & 5.2 & --- & --- & --- & --- & --- & 4.7 & 14 & 23 & 1.1 & 1.9 & 1.1 \\
\hline 6 & 5.0 & --- & --- & --- & --- & --- & 4.2 & 16 & 22 & 1.3 & 1.7 & 1.1 \\
\hline 7 & 4.8 & --- & --- & --- & --- & --- & 3.7 & 19 & 20 & 1.3 & 1.5 & 1.1 \\
\hline 8 & 4.8 & --- & --- & --- & --- & --- & 5.5 & 19 & 9.8 & 1.2 & 1.5 & 1.1 \\
\hline 9 & 4.8 & --- & --- & --- & --- & --- & 4.5 & 18 & 4.1 & 1.3 & 1.4 & 2.9 \\
\hline 10 & 4.8 & --- & --- & --- & --- & --- & 4.2 & 17 & 4.1 & 1.3 & 1.3 & 4.6 \\
\hline 11 & 4.8 & --- & --- & --- & --- & --- & 4.0 & 17 & 4.1 & 1.6 & .94 & 4.4 \\
\hline 12 & 5.0 & --- & --- & --- & --- & --- & 3.9 & 19 & 4.1 & 1.7 & .84 & 7.3 \\
\hline 13 & 11 & --- & --- & --- & --- & --- & 4.4 & 20 & 2.6 & 1.6 & .77 & 9.3 \\
\hline 14 & 15 & --- & --- & --- & --- & --- & 5.1 & 19 & 1.6 & 1.6 & .75 & 9.2 \\
\hline 15 & 15 & --- & --- & --- & --- & --- & 5.5 & 18 & 1.5 & 1.5 & .73 & 9.1 \\
\hline 16 & 15 & --- & --- & --- & --- & --- & 6.4 & 18 & 1.5 & 1.4 & .71 & 8.9 \\
\hline 17 & 15 & --- & --- & --- & --- & --- & 7.9 & 19 & 1.3 & 1.3 & 1.4 & 8.8 \\
\hline 18 & 15 & --- & --- & --- & --- & --- & 9.2 & 22 & 1.1 & 1.2 & 1.5 & 8.8 \\
\hline 19 & 15 & --- & --- & --- & --- & --- & 10 & 24 & 1.1 & 1.3 & 1.6 & 8.8 \\
\hline 20 & 15 & --- & --- & --- & --- & --- & 12 & 25 & 1.2 & 1.6 & 1.5 & 8.8 \\
\hline 21 & 15 & --- & --- & --- & --- & --- & 12 & 26 & 1.2 & 1.6 & 1.3 & 8.8 \\
\hline 22 & 14 & --- & --- & --- & --- & --- & 12 & 30 & 1.5 & 1.6 & 1.2 & 9.5 \\
\hline 23 & 14 & --- & --- & --- & --- & --- & 12 & 32 & 1.7 & 1.6 & 1.3 & 10 \\
\hline 24 & 14 & --- & --- & --- & --- & --- & 13 & 35 & 1.7 & 1.5 & 1.4 & 10 \\
\hline 25 & 21 & --- & --- & --- & --- & --- & 15 & 36 & 1.5 & 1.5 & 1.5 & 10 \\
\hline 26 & 26 & --- & --- & --- & --- & --- & 21 & 37 & 1.5 & 1.4 & 1.5 & 10 \\
\hline 27 & 25 & --- & --- & --- & --- & --- & 21 & 37 & 1.5 & 1.4 & 1.3 & 18 \\
\hline 28 & 25 & --- & --- & --- & --- & --- & 17 & 36 & 1.3 & 1.5 & 1.3 & 22 \\
\hline 29 & 24 & --- & --- & --- & --- & --- & 15 & 35 & 1.3 & 1.3 & 1.1 & 22 \\
\hline 30 & 24 & --- & --- & --- & --- & --- & 16 & 32 & 1.7 & 1.1 & 1.2 & 22 \\
\hline 31 & 23 & --- & --- & --- & --- & --- & --- & 29 & --- & 1.1 & 1.3 & --- \\
\hline TOTAL & 399.0 & --- & --- & --- & --- & --- & 265.4 & 743 & 242.0 & 43.6 & 39.54 & 242.4 \\
\hline MEAN & 12.9 & --- & --- & --- & --- & --- & 8.85 & 24.0 & 8.07 & 1.41 & 1.28 & 8.08 \\
\hline MAX & 26 & --- & --- & --- & --- & --- & 21 & 37 & 37 & 1.7 & 1.9 & 22 \\
\hline MIN & 3.6 & --- & --- & --- & --- & --- & 3.7 & 14 & 1.1 & 1.1 & .71 & 1.1 \\
\hline $\mathrm{AC}-\mathrm{FT}$ & 791 & --- & --- & --- & --- & --- & 526 & 1470 & 480 & 86 & 78 & 481 \\
\hline
\end{tabular}




\section{NORTH FORK BATTLE CREEK BELOW MCCUMBER DAM, NEAR MANZANITA LAKE, CA}

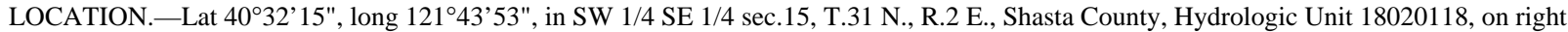
bank, $300 \mathrm{ft}$ downstream from McCumber Dam, $3.0 \mathrm{mi}$ northwest of Viola, and $9.0 \mathrm{mi}$ west of Manzanita Lake.

DRAINAGE AREA.-27.6 $\mathrm{mi}^{2}$.

PERIOD OF RECORD.-October 1987 to current year (operated as a low-flow station only). Unpublished records for water years 1978-87 available in files of the U.S. Geological Survey.

GAGE.-Water-stage recorder and V-notch weir. Elevation of gage is 4,080 ft above sea level, from topographic map.

REMARKS.-This station records fishwater release only. Prior to water year $1995 \mathrm{flow}$ computed to $211 \mathrm{ft}^{3} / \mathrm{s}$. The minimum release requirement is $0.30 \mathrm{ft}^{3} / \mathrm{s}$ at all times; flow is computed to $800 \mathrm{ft}^{3} / \mathrm{s}$. See schematic diagram of Battle Creek and Cow Creek Basins.

COOPERATION.-Records were collected by Pacific Gas \& Electric Co., under general supervision of the U.S. Geological Survey, in connection with a Federal Energy Regulatory Commission project.

DISCHARGE, CUBIC FEET PER SECOND, WATER YEAR OCTOBER 1998 TO SEPTEMBER 1999 DAILY MEAN VALUES

\begin{tabular}{|c|c|c|c|c|c|c|c|c|c|c|c|c|}
\hline DAY & OCT & NOV & $\mathrm{DEC}$ & JAN & FEB & MAR & APR & MAY & JUN & JUL & AUG & SEP \\
\hline 1 & 62 & 76 & 86 & 57 & 80 & 343 & 100 & 119 & 100 & 48 & 41 & 40 \\
\hline 2 & 64 & 76 & 90 & 59 & 78 & 284 & 97 & 132 & 116 & 48 & 41 & 39 \\
\hline 3 & 64 & 76 & 91 & 60 & 73 & 304 & 96 & 128 & 113 & 48 & 41 & 39 \\
\hline 4 & 64 & 75 & 92 & 59 & 70 & 265 & 89 & 117 & 102 & 47 & 41 & 39 \\
\hline 5 & 63 & 75 & 91 & 55 & 69 & 228 & 96 & 111 & 94 & 47 & 41 & 38 \\
\hline 6 & 63 & 75 & 90 & 52 & 69 & 155 & 95 & 108 & 91 & 46 & 42 & 36 \\
\hline 7 & 62 & 74 & 88 & 53 & 71 & 134 & 92 & 110 & 84 & 47 & 43 & 36 \\
\hline 8 & 61 & 68 & 86 & 53 & 80 & 123 & 96 & 108 & 81 & 47 & 42 & 36 \\
\hline 9 & 61 & 64 & 83 & 53 & 89 & 126 & 92 & 104 & 70 & 47 & 41 & 35 \\
\hline 10 & 60 & 64 & 77 & 53 & 136 & 114 & 91 & 102 & 68 & 46 & 43 & 39 \\
\hline 11 & 60 & 64 & 74 & 54 & 107 & 106 & 95 & 100 & 67 & 45 & 42 & 40 \\
\hline 12 & 60 & 63 & 71 & 55 & 94 & 104 & 99 & 102 & 65 & 45 & 41 & 52 \\
\hline 13 & 60 & 61 & 66 & 55 & 90 & 104 & 99 & 104 & 64 & 44 & 40 & 55 \\
\hline 14 & 60 & 61 & 66 & 55 & 90 & 102 & 100 & 102 & 60 & 44 & 40 & 54 \\
\hline 15 & 60 & 57 & 66 & 55 & 89 & 100 & 103 & 99 & 57 & 44 & 40 & 53 \\
\hline 16 & 61 & 57 & 66 & 56 & 88 & 98 & 108 & 97 & 57 & 44 & 40 & 53 \\
\hline 17 & 61 & 60 & 66 & 60 & 88 & 99 & 113 & 97 & 57 & 43 & 40 & 52 \\
\hline 18 & 61 & 63 & 66 & 70 & 101 & 98 & 121 & 101 & 56 & 43 & 40 & 52 \\
\hline 19 & 61 & 63 & 65 & 80 & 102 & 97 & 124 & 103 & 55 & 43 & 40 & 52 \\
\hline 20 & 62 & 64 & 64 & 83 & 92 & 98 & 126 & 105 & 55 & 43 & 40 & 51 \\
\hline 21 & 62 & 59 & 62 & 83 & 94 & 99 & 126 & 105 & 54 & 43 & 40 & 51 \\
\hline 22 & 66 & 58 & 60 & 83 & 90 & 99 & 118 & 107 & 53 & 43 & 40 & 51 \\
\hline 23 & 70 & 64 & 60 & 85 & 90 & 98 & 111 & 109 & 54 & 43 & 40 & 57 \\
\hline 24 & 70 & 74 & 61 & 87 & 90 & 99 & 117 & 112 & 54 & 44 & 40 & 60 \\
\hline 25 & 74 & 79 & 61 & 87 & 90 & 99 & 121 & 113 & 53 & 43 & 39 & 53 \\
\hline 26 & 77 & 77 & 61 & 86 & 90 & 90 & 132 & 113 & 52 & 43 & 39 & 52 \\
\hline 27 & 77 & 77 & 60 & 86 & 90 & 86 & 135 & 111 & 51 & 43 & 40 & 61 \\
\hline 28 & 77 & 77 & 60 & 85 & 220 & 89 & 122 & 110 & 49 & 43 & 40 & 67 \\
\hline 29 & 77 & 76 & 60 & 84 & --- & 102 & 115 & 109 & 45 & 42 & 39 & 58 \\
\hline 30 & 77 & 78 & 60 & 83 & --- & 105 & 115 & 105 & 48 & 41 & 39 & 51 \\
\hline 31 & 76 & --- & 60 & 81 & --- & 104 & -- & 100 & --- & 41 & 40 & --- \\
\hline TOTAL & 2033 & 2055 & 2209 & 2107 & 2610 & 4152 & 3244 & 3343 & 2025 & 1378 & 1255 & 1452 \\
\hline MEAN & 65.6 & 68.5 & 71.3 & 68.0 & 93.2 & 134 & 108 & 108 & 67.5 & 44.5 & 40.5 & 48.4 \\
\hline MAX & 77 & 79 & 92 & 87 & 220 & 343 & 135 & 132 & 116 & 48 & 43 & 67 \\
\hline MIN & 60 & 57 & 60 & 52 & 69 & 86 & 89 & 97 & 45 & 41 & 39 & 35 \\
\hline $\mathrm{AC}-\mathrm{FT}$ & 4030 & 4080 & 4380 & 4180 & 5180 & 8240 & 6430 & 6630 & 4020 & 2730 & 2490 & 2880 \\
\hline$a$ & 299 & 191 & 110 & 207 & 428 & 436 & 436 & 436 & 436 & 436 & 436 & 122 \\
\hline
\end{tabular}

a Contents, in acre-feet, at end of month for McCumber Reservoir (station 11376020), provided by Pacific Gas \& Electric Co. 
POWERPLANTS IN BATTLE CREEK AND COW CREEK BASINS

11376043 VOLTA NO. 1 POWERPLANT NEAR MANTON, CA, in NW 1/4 NE 1/4 sec.16, T.30 N., R.1 E., Shasta County, Hydrologic Unit $18020118,1.7 \mathrm{mi}$ north of Manton. Powerplant consists of one unit with a total of 8,550 KW normal operating capacity. See schematic diagram of Battle Creek and Cow Creek Basins.

11376046 VOLTA NO. 2 POWERPLANT NEAR MANTON, CA, in NE 1/4 SW 1/4 sec.16, T.30 N., R.1 E., Shasta County, Hydrologic Unit $18020118,1.2$ mi northeast of Manton. Powerplant consists of one unit with a total of $956 \mathrm{KW}$ normal operating capacity. See schematic diagram of Battle Creek and Cow Creek Basins.

11376410 SOUTH POWERPLANT NEAR MANTON, CA, in NE 1/4 SE 1/4 sec.5, T.29 N., R.1 E., Tehama County, Hydrologic Unit 18020118, $2.7 \mathrm{mi}$ south of Manton. Powerplant consists of one unit with a total of 6,750 KW normal operating capacity. See schematic diagram of Battle Creek and Cow Creek Basins.

11376430 INSKIP POWERPLANT NEAR MANTON, CA, in NE 1/4 NW 1/4 sec.3, T.29 N., R.1 W., Tehama County, Hydrologic Unit 18020118, $5.5 \mathrm{mi}$ southwest of Manton. Powerplant consists of one unit with a total of 7,650 KW normal operating capacity. See schematic diagram of Battle Creek and Cow Creek Basins.

11376458 COLEMAN POWERPLANT NEAR COTTONWOOD, CA, in SW 1/4 SW 1/4 sec.32, T.30 N., R.2 W., Shasta County, Hydrologic Unit $18020006,8.5 \mathrm{mi}$ east of Cottonwood. Powerplant consists of one unit with a total of 12,150 KW normal operating capacity. See schematic diagram of Battle Creek and Cow Creek Basins.

MONTHLY DISCHARGE, IN ACRE-FEET, WATER YEAR OCTOBER 1998 TO SEPTEMBER 1999

\begin{tabular}{|c|c|c|c|c|c|}
\hline Date & Volta No. 1 & Volta No. 2 & South & Inskip & Coleman \\
\hline 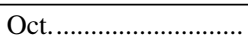 & 6,500 & 7,240 & 13,580 & 17,020 & 17,850 \\
\hline 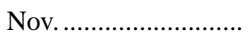 & 6,100 & 6,310 & 12,540 & 16,150 & 13,150 \\
\hline Dec. & 6,730 & 6,770 & 12,900 & 16,680 & 14,980 \\
\hline Jan. & 6,800 & 6,850 & 13,160 & 17,150 & 19,110 \\
\hline Feb. & 6,460 & 6,250 & 11,890 & 15,330 & 17,280 \\
\hline Mar. ............................... & 7,050 & 6,860 & 11,900 & 14,820 & 19,200 \\
\hline 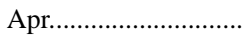 & 7,050 & 6,600 & 12,840 & 16,660 & 16,380 \\
\hline 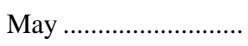 & 5,670 & 5,560 & 12,920 & 17,180 & 19,170 \\
\hline 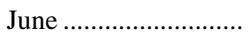 & 6,730 & 5,990 & 12,750 & 16,540 & 17,630 \\
\hline 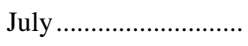 & 6,040 & 5,920 & 13,090 & 16,730 & 17,830 \\
\hline Aug & 5,350 & 5,910 & 12,580 & 15,020 & 15,420 \\
\hline 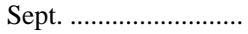 & 5,260 & 5,830 & 11,620 & 13,740 & 14,090 \\
\hline
\end{tabular}

Note.-Records were provided by Pacific Gas \& Electric Co., in connection with a Federal Energy Regulatory Commission project. Unpublished records for water years 1979-86 available in files of U.S.Geolgoical Survey. Fragmentary records prior to water year 1979 available in files of Pacific Gas \& Electric. 
LOCATION.—Lat 40³0'00", long 121 $48^{\prime} 29^{\prime \prime}$, in NW 1/4 NE 1/4 sec.36, T.31 N., R.1 E., Shasta County, Hydrologic Unit 18020118, on right bank, 4.2 mi east of Shingletown, and 5.5 mi northeast of Manton.

PERIOD OF RECORD.-October 1986 to current year (operated as a low-flow station only). Unpublished records for water years 1978-86 available in files of the U.S. Geological Survey.

GAGE.-Water-stage recorder and Parshall flume. Elevation of gage is 3,600 ft above sea level, from topographic map.

REMARKS.-This station records fishwater release only. The minimum release requirement is $3.0 \mathrm{ft}^{3} / \mathrm{s}$ at all times; flow is computed to $5.6 \mathrm{ft}^{3} / \mathrm{s}$. See schematic diagram of Battle Creek and Cow Creek Basins.

COOPERATION.-Records were collected by Pacific Gas \& Electric Co., under general supervision of the U.S. Geological Survey, in connection with a Federal Energy Regulatory Commission project.

DISCHARGE, CUBIC FEET PER SECOND, WATER YEAR OCTOBER 1998 TO SEPTEMBER 1999 DAILY MEAN VALUES

\begin{tabular}{|c|c|c|c|c|c|c|c|c|c|c|c|c|}
\hline DAY & $\mathrm{OCT}$ & NOV & $\mathrm{DEC}$ & JAN & FEB & MAR & APR & MAY & JUN & JUL & AUG & SEP \\
\hline 1 & 3.6 & 3.6 & 3.6 & 3.7 & 3.7 & 4.1 & 4.6 & 4.9 & 3.6 & 3.7 & 3.5 & 3.5 \\
\hline 2 & 3.7 & 3.6 & 3.6 & 3.7 & 3.7 & 4.6 & 4.7 & 4.9 & 3.5 & 3.6 & 3.5 & 3.5 \\
\hline 3 & 3.7 & 3.5 & 3.6 & 3.7 & 3.7 & 4.4 & 4.7 & --- & 3.4 & 3.5 & 3.5 & 3.4 \\
\hline 4 & 3.7 & 3.5 & 3.6 & 3.7 & 3.7 & 4.5 & 4.7 & --- & 3.4 & 3.5 & 3.5 & 3.4 \\
\hline 5 & 3.7 & 3.5 & 3.6 & 3.7 & 3.7 & 4.6 & 4.7 & --- & 3.4 & 3.5 & 3.5 & 3.4 \\
\hline 6 & 3.7 & 3.5 & 3.6 & 3.7 & 3.7 & 4.3 & 4.7 & --- & 3.4 & 3.5 & 3.5 & 3.4 \\
\hline 7 & 3.6 & 3.4 & 3.6 & 3.7 & 3.6 & 4.3 & 4.7 & --- & 3.5 & 3.5 & 3.5 & 3.4 \\
\hline 8 & 3.6 & 3.5 & 3.6 & 3.7 & 3.6 & 4.4 & 4.7 & --- & 3.5 & 3.5 & 3.5 & 3.4 \\
\hline 9 & 3.7 & 3.8 & 3.6 & 3.7 & 3.6 & 4.4 & 4.7 & --- & 3.5 & 3.5 & 3.6 & 3.4 \\
\hline 10 & 3.7 & 3.9 & 3.6 & 3.7 & 3.7 & 4.4 & 4.7 & --- & 3.5 & 3.5 & 3.6 & 3.5 \\
\hline 11 & 3.7 & 3.9 & 3.6 & 3.7 & 3.7 & 4.5 & 4.7 & --- & 3.7 & 3.5 & 3.6 & 3.5 \\
\hline 12 & 3.6 & 3.9 & 3.6 & 3.7 & 3.7 & 4.6 & 4.7 & --- & 3.8 & 3.5 & 3.6 & 3.6 \\
\hline 13 & 3.6 & 3.9 & 3.6 & 3.7 & 3.7 & 4.6 & 4.7 & --- & 3.8 & 3.5 & 3.6 & 3.5 \\
\hline 14 & 3.6 & 3.9 & 3.6 & 3.7 & 3.7 & 4.8 & 4.7 & 3.6 & 3.7 & 3.5 & 3.6 & 3.5 \\
\hline 15 & 3.6 & 3.9 & 3.7 & 3.7 & 3.7 & 5.4 & 4.8 & 3.6 & 3.7 & 3.5 & 3.6 & 3.5 \\
\hline 16 & 3.6 & 3.9 & 3.7 & 3.7 & 3.7 & 5.5 & 4.8 & 3.6 & 3.7 & 3.5 & 3.5 & 3.5 \\
\hline 17 & 3.6 & 3.9 & 3.7 & 3.7 & 3.7 & 5.3 & 4.8 & 3.6 & 3.7 & 3.5 & 3.5 & 3.6 \\
\hline 18 & 3.6 & 3.9 & 3.7 & 3.7 & 3.7 & 5.3 & 4.8 & 3.5 & 3.7 & 3.4 & 3.5 & 3.6 \\
\hline 19 & 3.6 & 3.9 & 3.7 & 3.7 & 3.7 & 5.4 & 4.8 & 3.5 & 3.7 & 3.4 & 3.5 & 3.6 \\
\hline 20 & 3.6 & 3.9 & 3.7 & 3.7 & 3.7 & 5.6 & 4.9 & 3.5 & 3.7 & 3.4 & 3.5 & 3.6 \\
\hline 21 & 3.6 & 3.9 & 3.6 & 3.7 & 3.7 & --- & 4.9 & 3.5 & 3.7 & 3.4 & 3.5 & 3.6 \\
\hline 22 & 3.6 & 3.8 & 3.6 & 3.7 & 3.7 & 5.6 & 4.8 & 3.5 & 3.6 & 3.4 & 3.5 & 3.6 \\
\hline 23 & 3.6 & 3.8 & 3.6 & 3.7 & 3.8 & 5.6 & 4.9 & 3.4 & 3.7 & 3.5 & 3.5 & 3.6 \\
\hline 24 & 3.5 & 3.8 & 3.7 & 3.7 & 3.7 & 5.6 & 4.9 & 3.5 & 3.7 & 3.6 & 3.5 & 3.7 \\
\hline 25 & 3.5 & 3.8 & 3.7 & 3.7 & 3.7 & 5.6 & 4.9 & 3.4 & 3.7 & 3.6 & 3.5 & 3.6 \\
\hline 26 & 3.5 & 3.8 & 3.6 & 3.7 & 3.8 & 5.6 & 4.9 & 3.4 & 3.7 & 3.5 & 3.5 & 3.6 \\
\hline 27 & 3.5 & 3.8 & 3.6 & 3.7 & 3.8 & 5.5 & 4.8 & 3.5 & 3.7 & 3.5 & 3.5 & 3.6 \\
\hline 28 & 3.5 & 3.8 & 3.6 & 3.7 & 3.7 & 5.1 & 4.9 & 3.6 & 3.6 & 3.5 & 3.5 & 3.6 \\
\hline 29 & 3.5 & 3.8 & 3.6 & 3.7 & --- & 4.7 & 4.9 & 3.6 & 3.6 & 3.4 & 3.5 & 3.6 \\
\hline 30 & 3.5 & 3.7 & 3.7 & 3.7 & --- & 4.7 & 4.9 & 3.6 & 3.7 & 3.4 & 3.5 & 3.5 \\
\hline 31 & 3.6 & --- & 3.7 & 3.7 & --- & 4.6 & --- & 3.6 & --- & 3.4 & 3.5 & --- \\
\hline TOTAL & 111.7 & 112.8 & 112.6 & 114.7 & 103.6 & --- & 143.4 & --- & 108.6 & 108.2 & 109.2 & 105.8 \\
\hline MEAN & 3.60 & 3.76 & 3.63 & 3.70 & 3.70 & --- & 4.78 & --- & 3.62 & 3.49 & 3.52 & 3.53 \\
\hline MAX & 3.7 & 3.9 & 3.7 & 3.7 & 3.8 & --- & 4.9 & --- & 3.8 & 3.7 & 3.6 & 3.7 \\
\hline MIN & 3.5 & 3.4 & 3.6 & 3.7 & 3.6 & --- & 4.6 & --- & 3.4 & 3.4 & 3.5 & 3.4 \\
\hline $\mathrm{AC}-\mathrm{FT}$ & 222 & 224 & 223 & 228 & 205 & --- & 284 & --- & 215 & 215 & 217 & 210 \\
\hline
\end{tabular}

NOTE: Canal was out of service May 3-13 and all flow remained in the natural channel. 
LOCATION.-Lat 40²7'59", long 12159'20", in NE 1/4 SE 1/4 sec.11, T.30 N., R.1 E., Shasta County, Hydrologic Unit 18020118, on right bank, $250 \mathrm{ft}$ downstream from Spring Creek, 0.4 mi upstream from Ponderosa Way, 3.3 mi northeast of Manton, and 3.9 mi southeast of Shingletown.

DRAINAGE AREA.-29.6 $\mathrm{mi}^{2}$.

PERIOD OF RECORD.- January 1990 to current year (operated as a low-flow station only).

GAGE.-Water-stage recorder and V-notch weir. Elevation of gage is 2,650 ft above sea level, from topographic map.

REMARKS.-During times of powerplant operation the minimum release requirement is $17 \mathrm{ft}^{3} / \mathrm{s}$; flow is computed to $109 \mathrm{ft}^{3} / \mathrm{s}$. See $\mathrm{schematic}$ diagram of Battle Creek and Cow Creek Basins.

COOPERATION.-Records were collected by Snow Mountain Hydro, under general supervision of the U.S. Geological Survey, in connection with a Federal Energy Regulatory Commission project.

DISCHARGE, CUBIC FEET PER SECOND, WATER YEAR OCTOBER 1997 TO SEPTEMBER 1998

DAILY MEAN VALUES

(NOT PREVIOUSLY PUBLISHED)

\begin{tabular}{|c|c|c|c|c|c|c|c|c|c|c|c|c|}
\hline DAY & $\mathrm{OCT}$ & NOV & $\mathrm{DEC}$ & JAN & FEB & MAR & APR & MAY & JUN & JUL & AUG & SEP \\
\hline 1 & 16 & 15 & 21 & 22 & 19 & 18 & 18 & 21 & 47 & 89 & 19 & 14 \\
\hline 2 & 16 & 15 & 20 & 19 & 23 & 20 & 18 & 52 & 58 & 93 & 16 & 14 \\
\hline 3 & 16 & 15 & 20 & 18 & --- & 21 & 18 & 32 & 103 & 95 & 16 & 14 \\
\hline 4 & 16 & 15 & 20 & 18 & 29 & 18 & 18 & 28 & 83 & 104 & 17 & 14 \\
\hline 5 & 16 & 15 & 20 & 21 & 19 & 18 & 18 & 29 & 83 & 95 & 17 & 14 \\
\hline 6 & 16 & 15 & 21 & 25 & 23 & 18 & 18 & 31 & 78 & 98 & 17 & 14 \\
\hline 7 & 16 & 14 & 20 & 26 & 20 & 18 & 19 & 28 & 82 & 68 & 17 & 14 \\
\hline 8 & 16 & 15 & 18 & 23 & 18 & 18 & 18 & 67 & 93 & 55 & 15 & 14 \\
\hline 9 & 18 & 16 & 21 & 25 & 18 & 18 & 18 & --- & 78 & 70 & 15 & 14 \\
\hline 10 & 19 & 16 & 22 & 21 & 18 & 18 & 18 & 80 & --- & 82 & 14 & 14 \\
\hline 11 & 18 & 16 & 22 & 31 & 17 & 18 & 18 & 58 & --- & 75 & 14 & 14 \\
\hline 12 & 17 & 16 & 21 & 76 & 18 & 18 & 18 & 54 & --- & 72 & 14 & 14 \\
\hline 13 & 16 & 16 & 21 & 21 & 22 & 18 & 18 & 39 & --- & 80 & 14 & 18 \\
\hline 14 & 16 & 16 & 23 & 22 & 29 & 18 & 18 & 34 & --- & 84 & 14 & 20 \\
\hline 15 & 16 & 16 & 25 & 55 & 18 & 18 & 18 & 41 & 102 & 83 & 14 & 24 \\
\hline 16 & 16 & 16 & 24 & 23 & 18 & 18 & 18 & 73 & --- & 79 & 14 & 24 \\
\hline 17 & 16 & 16 & 19 & 102 & 18 & 18 & 18 & 61 & 79 & 93 & 14 & 24 \\
\hline 18 & 16 & 17 & 18 & 93 & 18 & 18 & 18 & 43 & 80 & 93 & 14 & 24 \\
\hline 19 & 16 & 19 & 22 & 68 & 18 & 18 & 18 & 30 & --- & 79 & 14 & 24 \\
\hline 20 & 15 & 20 & 24 & 18 & 18 & 18 & 18 & 30 & --- & 82 & 14 & 24 \\
\hline 21 & 15 & 20 & 23 & 18 & 19 & 18 & 18 & 21 & --- & 84 & 14 & 23 \\
\hline 22 & 16 & 20 & 20 & 19 & 19 & 83 & 18 & 18 & --- & 85 & 14 & 23 \\
\hline 23 & 15 & 22 & 21 & 18 & 19 & 71 & 18 & 18 & 109 & 72 & 14 & 23 \\
\hline 24 & 15 & 20 & 22 & 18 & 19 & 78 & 18 & 21 & 107 & 76 & 14 & 23 \\
\hline 25 & 15 & 18 & 20 & 18 & 18 & 64 & 16 & 83 & --- & 74 & 14 & 23 \\
\hline 26 & 15 & 23 & 20 & 24 & 18 & 39 & 18 & 67 & --- & 70 & 14 & 24 \\
\hline 27 & 16 & 23 & 21 & 23 & 18 & 30 & 18 & 66 & 104 & 66 & 14 & 24 \\
\hline 28 & 15 & 21 & 21 & 18 & 18 & 26 & 18 & 96 & 93 & 57 & 14 & 24 \\
\hline 29 & 15 & 21 & 22 & 19 & --- & 21 & 18 & 79 & 98 & 51 & 14 & 23 \\
\hline 30 & 15 & 23 & 21 & 18 & --- & 20 & 18 & 54 & 94 & 39 & 14 & 23 \\
\hline 31 & 15 & --- & 21 & 17 & --- & 22 & --- & 46 & --- & 23 & 14 & --- \\
\hline TOTAL & 494 & 530 & 654 & 937 & 684 & 837 & 539 & --- & --- & 2366 & 457 & 583 \\
\hline MEAN & 15.9 & 17.7 & 21.1 & 30.2 & 24.4 & 27.0 & 18.0 & --- & --- & 76.3 & 14.7 & 19.4 \\
\hline MAX & 19 & 23 & 25 & 102 & 155 & 83 & 19 & --- & --- & 104 & 19 & 24 \\
\hline MIN & 15 & 14 & 18 & 17 & 17 & 18 & 16 & --- & --- & 23 & 14 & 14 \\
\hline $\mathrm{AC}-\mathrm{FT}$ & 980 & 1050 & 1300 & 1860 & 1360 & 1660 & 1070 & --- & --- & 4690 & 906 & 1160 \\
\hline$a$ & 0 & 24 & 63 & 2280 & 2950 & 2820 & 3220 & 4050 & 3720 & 4040 & 2610 & 228 \\
\hline
\end{tabular}

a Discharge, in acre-feet, for Ponderosa-Bailey Creek Powerplant (station 11376110), provided by Snow Mountain Hydro. 
11376120 BAILEY CREEK BELOW DIVERSION TO PONDEROSA-BAILEY CREEK POWERPLANT, NEAR MANTON, CA—Continued DISCHARGE, CUBIC FEET PER SECOND, WATER YEAR OCTOBER 1998 TO SEPTEMBER 1999

\section{DAILY MEAN VALUES}

\begin{tabular}{|c|c|c|c|c|c|c|c|c|c|c|c|c|}
\hline DAY & $\mathrm{OCT}$ & NOV & $\mathrm{DEC}$ & JAN & FEB & MAR & APR & MAY & JUN & JUL & AUG & SEP \\
\hline 1 & 23 & 22 & 18 & 18 & 18 & 50 & 18 & 18 & 23 & 18 & 23 & e19 \\
\hline 2 & 23 & 22 & 18 & 18 & 18 & 21 & 18 & 18 & 58 & 18 & 23 & e19 \\
\hline 3 & 23 & 22 & 27 & 18 & 18 & 26 & 18 & 18 & 22 & 17 & 23 & e18 \\
\hline 4 & 23 & 23 & 18 & 18 & 18 & 18 & 18 & 18 & 18 & 17 & e22 & e19 \\
\hline 5 & 22 & 23 & 18 & 18 & 18 & 18 & 18 & 18 & 18 & 17 & e22 & e19 \\
\hline 6 & 22 & 23 & 18 & 18 & 18 & 18 & 18 & 18 & 18 & 17 & e22 & e19 \\
\hline 7 & 22 & 25 & 19 & 18 & 18 & 18 & 18 & 18 & 19 & 17 & e 21 & e18 \\
\hline 8 & 22 & 25 & 18 & 18 & 18 & 20 & 18 & 18 & 18 & 17 & e21 & e18 \\
\hline 9 & 22 & 24 & 18 & 18 & 25 & 18 & 18 & 18 & 18 & 17 & e21 & e18 \\
\hline 10 & 22 & 32 & 18 & 18 & 18 & 18 & 18 & 18 & 18 & 17 & e20 & e18 \\
\hline 11 & 22 & 32 & 18 & 18 & 18 & 18 & 18 & 18 & 18 & 17 & e20 & e18 \\
\hline 12 & 22 & 21 & 18 & 18 & 18 & 18 & 18 & 18 & 21 & 17 & e20 & e18 \\
\hline 13 & 22 & 18 & 18 & 18 & 18 & 18 & 18 & 18 & 18 & 17 & e20 & e18 \\
\hline 14 & 22 & 18 & 18 & 18 & 18 & 18 & 18 & 18 & 18 & 17 & e20 & e18 \\
\hline 15 & 22 & 18 & 18 & 18 & 18 & 18 & 18 & 18 & 18 & 17 & e19 & e18 \\
\hline 16 & 22 & e19 & 18 & 18 & 18 & 18 & 18 & 18 & 18 & 17 & e19 & e18 \\
\hline 17 & 22 & e31 & 18 & 18 & 18 & 18 & 18 & 18 & 18 & 17 & e19 & e18 \\
\hline 18 & 22 & 18 & 17 & 18 & 18 & 18 & 18 & 18 & 19 & 17 & e19 & e17 \\
\hline 19 & 22 & 18 & 18 & 18 & 18 & 18 & 18 & 18 & 20 & 17 & e19 & 17 \\
\hline 20 & 21 & 18 & 18 & 18 & 18 & 18 & 18 & 18 & 19 & 17 & e19 & e17 \\
\hline 21 & e21 & 20 & 22 & 18 & 18 & 18 & 18 & 18 & 18 & 17 & e19 & e18 \\
\hline 22 & e20 & 18 & 18 & 18 & 18 & 18 & 18 & 18 & 21 & 17 & e19 & e17 \\
\hline 23 & 20 & 24 & 18 & 18 & 18 & 18 & 18 & 18 & 23 & 17 & e19 & e18 \\
\hline 24 & 24 & 19 & 18 & 18 & 18 & 18 & 18 & 19 & 26 & 17 & e19 & e18 \\
\hline 25 & 23 & 18 & 18 & 18 & 18 & 18 & 18 & 31 & 27 & 17 & e19 & e18 \\
\hline 26 & 17 & 18 & 18 & 18 & 18 & 18 & 18 & 41 & 20 & 17 & e19 & e17 \\
\hline 27 & 19 & 18 & 18 & 18 & 18 & 18 & 20 & 41 & 17 & 17 & e19 & e17 \\
\hline 28 & 24 & 18 & 18 & 18 & 37 & 18 & 18 & 37 & 17 & 17 & e19 & e17 \\
\hline 29 & 23 & 18 & 18 & 18 & --- & 18 & 18 & 36 & 17 & 17 & e19 & e17 \\
\hline 30 & 21 & 29 & 18 & 18 & --- & 18 & 18 & 24 & 17 & 16 & e19 & e17 \\
\hline 31 & 21 & --- & 18 & 18 & --- & 18 & --- & 22 & -- & 17 & e19 & -- \\
\hline TOTAL & 676 & 652 & 571 & 558 & 530 & 603 & 542 & 665 & 620 & 528 & 621 & 536 \\
\hline MEAN & 21.8 & 21.7 & 18.4 & 18.0 & 18.9 & 19.5 & 18.1 & 21.5 & 20.7 & 17.0 & 20.0 & 17.9 \\
\hline MAX & 24 & 32 & 27 & 18 & 37 & 50 & 20 & 41 & 58 & 18 & 23 & 19 \\
\hline MIN & 17 & 18 & 17 & 18 & 18 & 18 & 18 & 18 & 17 & 16 & 19 & 17 \\
\hline $\mathrm{AC}-\mathrm{FT}$ & 1340 & 1290 & 1130 & 1110 & 1050 & 1200 & 1080 & 1320 & 1230 & 1050 & 1230 & 1060 \\
\hline a & 0 & 625 & 1250 & 768 & 1750 & 2240 & 1890 & 2570 & 3520 & 1760 & 0 & 0 \\
\hline
\end{tabular}

e Estimated.

a Discharge, in acre-feet, for Ponderosa-Bailey Creek Powerplant (station 11376110), provided by Snow Mountain Hydro. 
11376140 NORTH FORK BATTLE CREEK BELOW DIVERSION TO CROSS COUNTRY CANAL, NEAR MANTON, CA

LOCATION.-Lat 40²7'16", long 121'51'35", in SW 1/4 NW 1/4 sec.15, T.30 N., R.1 E., Shasta County, Hydrologic Unit 18020118, on left bank, at diversion dam $800 \mathrm{ft}$ downstream (revised) from Volta No. 2 Powerplant, and 1.4 mi northeast of Manton.

DRAINAGE AREA.- $133 \mathrm{mi}^{2}$.

PERIOD OF RECORD.-October 1987 to current year (operated as a low-flow station only). Unpublished records for water years 1978-87 available in files of the U.S. Geological Survey. Fragmentary records for water year 1977 in files of Pacific Gas \& Electric Co.

GAGE.-Water-stage recorder and metal Alaskan fishladder. Elevation of gage is 2,240 ft above sea level, from topographic map.

REMARKS.-This station records fishwater release only. The minimum release requirement is $3.0 \mathrm{ft}^{3} / \mathrm{s}$ at all times; flow is computed to $6.8 \mathrm{ft}^{3} / \mathrm{s}$. See schematic diagram of Battle Creek and Cow Creek Basins.

COOPERATION.--Records were collected by Pacific Gas \& Electric Co., under general supervision of the U.S. Geological Survey, in connection with a Federal Energy Regulatory Commission project.

DISCHARGE, CUBIC FEET PER SECOND, WATER YEAR OCTOBER 1998 TO SEPTEMBER 1999 DAILY MEAN VALUES

\begin{tabular}{|c|c|c|c|c|c|c|c|c|c|c|c|c|}
\hline DAY & OCT & NOV & $\mathrm{DEC}$ & JAN & $\mathrm{FEB}$ & MAR & APR & MAY & JUN & JUL & AUG & SEP \\
\hline 1 & --- & --- & --- & --- & --- & --- & --- & --- & --- & --- & --- & 4.8 \\
\hline 2 & --- & --- & --- & --- & --- & --- & --- & --- & --- & --- & --- & 4.8 \\
\hline 3 & --- & --- & --- & --- & --- & --- & --- & --- & --- & --- & 5.7 & 4.8 \\
\hline 4 & --- & --- & --- & --- & --- & --- & --- & --- & --- & --- & 5.3 & 4.8 \\
\hline 5 & --- & --- & --- & --- & --- & --- & --- & --- & --- & --- & 5.3 & 4.9 \\
\hline 6 & --- & --- & --- & --- & --- & --- & --- & --- & --- & --- & 6.1 & 4.9 \\
\hline 7 & --- & --- & --- & --- & --- & --- & --- & --- & --- & --- & 6.7 & 4.8 \\
\hline 8 & --- & --- & --- & --- & --- & --- & --- & --- & --- & --- & 6.1 & 4.8 \\
\hline 9 & --- & --- & --- & --- & --- & --- & --- & --- & --- & --- & 6.0 & 4.8 \\
\hline 10 & --- & --- & --- & --- & --- & --- & --- & --- & --- & --- & 6.1 & 4.8 \\
\hline 11 & --- & --- & --- & --- & --- & --- & --- & --- & --- & --- & 6.2 & 4.8 \\
\hline 12 & --- & --- & --- & --- & --- & --- & --- & --- & --- & --- & 6.0 & 5.3 \\
\hline 13 & --- & --- & --- & --- & --- & --- & --- & --- & --- & --- & 5.8 & 5.0 \\
\hline 14 & --- & --- & --- & --- & --- & --- & --- & --- & --- & --- & 5.6 & 4.9 \\
\hline 15 & --- & --- & --- & --- & --- & --- & --- & --- & --- & --- & 5.5 & 4.8 \\
\hline 16 & --- & --- & --- & --- & --- & --- & --- & --- & --- & --- & 5.3 & 5.0 \\
\hline 17 & --- & --- & --- & --- & --- & --- & --- & --- & --- & --- & 5.1 & 5.0 \\
\hline 18 & --- & --- & --- & --- & --- & --- & --- & --- & --- & --- & 4.9 & 5.0 \\
\hline 19 & --- & --- & --- & --- & --- & --- & --- & --- & --- & --- & 4.9 & 5.1 \\
\hline 20 & --- & --- & --- & --- & --- & --- & --- & --- & --- & --- & 4.9 & 5.0 \\
\hline 21 & --- & --- & --- & --- & --- & --- & --- & --- & --- & --- & 5.3 & 5.0 \\
\hline 22 & --- & --- & --- & --- & --- & --- & --- & --- & --- & --- & -- & 5.0 \\
\hline 23 & --- & --- & --- & --- & --- & --- & --- & --- & --- & --- & --- & 5.0 \\
\hline 24 & --- & --- & --- & --- & --- & --- & --- & --- & --- & --- & --- & 5.7 \\
\hline 25 & --- & --- & --- & --- & --- & --- & --- & --- & --- & --- & 6.4 & 4.9 \\
\hline 26 & --- & --- & --- & --- & --- & --- & --- & --- & --- & --- & 5.9 & 5.0 \\
\hline 27 & --- & --- & --- & --- & --- & --- & --- & --- & --- & --- & 6.0 & 5.6 \\
\hline 28 & --- & --- & --- & --- & --- & --- & --- & --- & --- & --- & 5.9 & --- \\
\hline 29 & --- & --- & --- & --- & --- & --- & --- & --- & --- & --- & 5.8 & 6.0 \\
\hline 30 & --- & --- & --- & --- & --- & --- & --- & --- & --- & --- & 5.3 & 4.9 \\
\hline 31 & --- & --- & --- & --- & --- & --- & --- & --- & --- & --- & 4.8 & --- \\
\hline TOTAL & --- & --- & --- & --- & --- & --- & --- & --- & --- & --- & --- & --- \\
\hline MEAN & --- & --- & --- & --- & --- & --- & --- & --- & --- & --- & --- & --- \\
\hline MAX & --- & --- & --- & --- & --- & --- & --- & --- & --- & --- & --- & --- \\
\hline MIN & --- & --- & --- & --- & --- & --- & --- & --- & --- & --- & --- & --- \\
\hline $\mathrm{AC}-\mathrm{FT}$ & --- & --- & --- & --- & --- & --- & --- & --- & --- & --- & --- & --- \\
\hline
\end{tabular}


11376150 NORTH FORK BATTLE CREEK BELOW DIVERSION TO EAGLE CANYON CANAL, NEAR MANTON, CA

LOCATION.-Lat 40²5'26", long 121 ${ }^{\circ} 55^{\prime} 09^{\prime \prime}$, in NW 1/4 SE 1/4 sec.25, T.30 N., R.1 W., Tehama County, Hydrologic Unit 18020118, on left bank, at diversion dam to Eagle Canyon Canal, and 2.8 mi southwest of Manton.

DRAINAGE AREA.- $186 \mathrm{mi}^{2}$.

PERIOD OF RECORD.-October 1987 to current year (operated as a low-flow station only). Unpublished records for water years 1978-87 available in files of the U.S. Geological Survey. Fragmentary records for water year 1977 available in files of Pacific Gas \& Electric Co.

GAGE.-Water-stage recorder and metal Alaskan fishladder. Elevation of gage is 1,400 ft above sea level, from topographic map.

REMARKS.-This station records fishwater release only. Prior to water year 1996 flow computed to $7.2 \mathrm{ft}^{3} / \mathrm{s}$. The minimum release requirement is $3.0 \mathrm{ft}^{3} / \mathrm{s}$ at all times; flow is computed to $50 \mathrm{ft}^{3} / \mathrm{s}$. See schematic diagram of Battle Creek and Cow Creek Basins.

COOPERATION.-Records were collected by Pacific Gas \& Electric Co., under general supervision of the U.S. Geological Survey, in connection with a Federal Energy Regulatory Commission project.

DISCHARGE, CUBIC FEET PER SECOND, WATER YEAR OCTOBER 1998 TO SEPTEMBER 1999 DAILY MEAN VALUES

\begin{tabular}{|c|c|c|c|c|c|c|c|c|c|c|c|c|}
\hline DAY & OCT & NOV & DEC & JAN & FEB & MAR & APR & MAY & JUN & JUL & AUG & $\operatorname{SEP}$ \\
\hline 1 & --- & --- & --- & --- & --- & --- & --- & --- & --- & --- & e33 & e33 \\
\hline 2 & --- & --- & --- & --- & --- & --- & --- & --- & --- & --- & e33 & e33 \\
\hline 3 & --- & --- & --- & --- & --- & --- & --- & --- & --- & --- & e 34 & e33 \\
\hline 4 & --- & --- & --- & --- & --- & --- & --- & --- & --- & --- & e 34 & e33 \\
\hline 5 & --- & --- & --- & --- & --- & --- & --- & --- & --- & --- & e 34 & e33 \\
\hline 6 & --- & --- & --- & --- & --- & --- & --- & --- & --- & --- & e34 & e33 \\
\hline 7 & --- & --- & --- & --- & --- & --- & --- & --- & --- & --- & e34 & e33 \\
\hline 8 & --- & --- & --- & --- & --- & --- & --- & --- & --- & --- & e 32 & e33 \\
\hline 9 & --- & --- & --- & --- & --- & --- & --- & --- & --- & --- & e 33 & e33 \\
\hline 10 & --- & --- & --- & --- & --- & --- & --- & --- & --- & --- & e33 & e33 \\
\hline 11 & --- & --- & --- & --- & --- & --- & --- & --- & --- & --- & e33 & e33 \\
\hline 12 & --- & --- & --- & --- & --- & --- & --- & --- & --- & --- & e33 & e33 \\
\hline 13 & --- & --- & --- & --- & --- & --- & --- & --- & --- & --- & e33 & e 33 \\
\hline 14 & --- & --- & --- & --- & --- & --- & --- & --- & --- & --- & e31 & e 32 \\
\hline 15 & --- & --- & --- & --- & --- & --- & --- & --- & --- & --- & e 31 & e33 \\
\hline 16 & --- & --- & --- & --- & --- & --- & --- & --- & --- & --- & e32 & e33 \\
\hline 17 & --- & --- & --- & --- & --- & --- & --- & --- & --- & --- & e 32 & e33 \\
\hline 18 & --- & --- & --- & --- & --- & --- & --- & --- & --- & e 46 & e 32 & e33 \\
\hline 19 & --- & --- & --- & --- & --- & --- & --- & --- & --- & e 41 & e 32 & e33 \\
\hline 20 & -- & --- & --- & -- & --- & --- & --- & --- & --- & e37 & e 32 & e33 \\
\hline 21 & --- & --- & --- & --- & --- & --- & --- & --- & --- & e36 & e33 & e33 \\
\hline 22 & --- & --- & --- & --- & --- & --- & --- & --- & --- & e33 & e33 & e33 \\
\hline 23 & --- & --- & --- & --- & --- & --- & --- & --- & --- & e 32 & e33 & e 34 \\
\hline 24 & --- & --- & --- & --- & --- & --- & --- & --- & --- & e 32 & e 31 & e 34 \\
\hline 25 & --- & --- & --- & --- & --- & --- & --- & --- & --- & e31 & e31 & e34 \\
\hline 26 & --- & --- & --- & --- & --- & --- & --- & --- & --- & e29 & e 32 & e 34 \\
\hline 27 & --- & --- & --- & --- & --- & --- & --- & --- & --- & e 31 & e 32 & e35 \\
\hline 28 & --- & --- & --- & --- & --- & --- & --- & --- & --- & e 36 & e 32 & e 34 \\
\hline 29 & --- & --- & --- & --- & --- & --- & --- & --- & --- & e 34 & e 32 & e 36 \\
\hline 30 & --- & --- & --- & --- & --- & --- & --- & --- & --- & e 34 & e 32 & e 35 \\
\hline 31 & --- & --- & --- & --- & --- & --- & --- & --- & --- & e 34 & e33 & --- \\
\hline TOTAL & --- & --- & --- & --- & --- & --- & --- & --- & --- & --- & 1009 & 1001 \\
\hline MEAN & --- & --- & --- & --- & --- & --- & --- & --- & --- & --- & 32.5 & 33.4 \\
\hline MAX & --- & --- & --- & --- & --- & --- & --- & --- & --- & --- & 34 & 36 \\
\hline MIN & --- & --- & --- & --- & --- & --- & --- & --- & --- & --- & 31 & 32 \\
\hline$A C-F T$ & --- & --- & --- & --- & --- & --- & --- & --- & --- & --- & 2000 & 1990 \\
\hline
\end{tabular}


11376160 NORTH FORK BATTLE CREEK BELOW DIVERSION TO WILDCAT CANAL, NEAR MANTON, CA

LOCATION.-Lat 40²5'14", long 121 ${ }^{\circ} 57^{\prime} 36^{\prime \prime}$, in SE 1/4 SW 1/4 sec.27, T.30 N., R.1 W., Tehama County, Hydrologic Unit 18020118, on left bank, at diversion dam to Wildcat Canal, and 4.9 mi west of Manton.

DRAINAGE AREA.- $189 \mathrm{mi}^{2}$.

PERIOD OF RECORD.-October 1987 to current year (operated as a low-flow station only). Unpublished records for water years 1978-87 available in files of the U.S. Geological Survey. Fragmentary records for water year 1977 available in files of Pacific Gas \& Electric Co.

GAGE.-Water-stage recorder and metal Alaskan fishladder. Elevation of gage is 1,080 ft above sea level, from topographic map.

REMARKS.-This station records fishwater release only. The minimum release requirement is $3.0 \mathrm{ft}^{3} / \mathrm{s}$ at all times; flow is computed to $60 \mathrm{ft}^{3} / \mathrm{s}$. See schematic diagram of Battle Creek and Cow Creek Basins.

COOPERATION.-Records were collected by Pacific Gas \& Electric Co., under general supervision of the U.S. Geological Survey, in connection with a Federal Energy Regulatory Commission project.

DISCHARGE, CUBIC FEET PER SECOND, WATER YEAR OCTOBER 1998 TO SEPTEMBER 1999 DAILY MEAN VALUES

\begin{tabular}{|c|c|c|c|c|c|c|c|c|c|c|c|c|}
\hline DAY & OCT & NOV & $\mathrm{DEC}$ & JAN & FEB & MAR & APR & MAY & JUN & JUL & AUG & SEP \\
\hline 1 & --- & --- & --- & --- & --- & --- & --- & --- & --- & --- & 36 & 36 \\
\hline 2 & --- & --- & --- & --- & --- & --- & --- & --- & --- & --- & 36 & 36 \\
\hline 3 & --- & --- & --- & --- & --- & --- & --- & --- & --- & --- & 37 & 36 \\
\hline 4 & --- & --- & --- & --- & --- & --- & --- & --- & --- & --- & 37 & 36 \\
\hline 5 & --- & --- & --- & --- & --- & --- & --- & --- & --- & --- & 37 & 36 \\
\hline 6 & --- & --- & --- & 58 & --- & --- & --- & --- & --- & --- & 37 & 36 \\
\hline 7 & --- & --- & --- & 58 & --- & --- & --- & --- & --- & --- & 37 & 36 \\
\hline 8 & --- & --- & --- & 56 & --- & --- & --- & --- & --- & --- & 36 & 36 \\
\hline 9 & --- & --- & --- & 54 & --- & --- & --- & --- & --- & --- & 36 & 36 \\
\hline 10 & --- & --- & --- & 55 & --- & --- & --- & --- & --- & --- & 37 & 36 \\
\hline 11 & --- & --- & --- & 54 & --- & --- & --- & --- & --- & 59 & 36 & 36 \\
\hline 12 & --- & --- & --- & 56 & --- & --- & --- & --- & --- & --- & 36 & 36 \\
\hline 13 & 60 & --- & --- & 56 & --- & --- & --- & --- & --- & --- & 36 & 36 \\
\hline 14 & --- & --- & --- & 54 & --- & --- & --- & --- & --- & --- & 36 & 35 \\
\hline 15 & --- & --- & --- & --- & --- & --- & --- & --- & --- & --- & 36 & 36 \\
\hline 16 & --- & --- & --- & --- & --- & --- & --- & --- & --- & --- & 36 & 36 \\
\hline 17 & 60 & --- & --- & --- & --- & --- & --- & --- & --- & 54 & 36 & 36 \\
\hline 18 & 60 & --- & --- & --- & --- & --- & --- & --- & --- & 49 & 35 & 36 \\
\hline 19 & --- & --- & --- & --- & --- & --- & --- & --- & --- & 44 & 36 & 36 \\
\hline 20 & --- & --- & --- & --- & --- & --- & --- & --- & --- & 40 & 36 & 36 \\
\hline 21 & 59 & --- & --- & --- & --- & --- & --- & --- & --- & 39 & 36 & 36 \\
\hline 22 & --- & --- & --- & --- & --- & --- & --- & --- & --- & 36 & 36 & 36 \\
\hline 23 & --- & --- & --- & --- & --- & --- & --- & --- & --- & 35 & 36 & 37 \\
\hline 24 & --- & --- & --- & --- & --- & --- & --- & --- & --- & 35 & 34 & 37 \\
\hline 25 & --- & --- & --- & --- & --- & --- & --- & --- & --- & 34 & 34 & 37 \\
\hline 26 & --- & --- & --- & --- & --- & --- & --- & --- & --- & 32 & 35 & 37 \\
\hline 27 & --- & --- & --- & --- & --- & --- & --- & --- & --- & 34 & 35 & 38 \\
\hline 28 & --- & --- & --- & --- & --- & --- & --- & --- & --- & 39 & 35 & 37 \\
\hline 29 & --- & --- & --- & --- & --- & --- & --- & --- & --- & 37 & 35 & 39 \\
\hline 30 & --- & --- & --- & --- & --- & --- & --- & --- & --- & 37 & 35 & 38 \\
\hline 31 & --- & --- & --- & --- & --- & --- & --- & --- & --- & 37 & 36 & --- \\
\hline TOTAL & --- & --- & --- & --- & --- & --- & --- & --- & --- & --- & 1112 & 1091 \\
\hline MEAN & --- & --- & --- & --- & --- & --- & --- & --- & --- & --- & 35.9 & 36.4 \\
\hline MAX & --- & --- & --- & --- & --- & --- & --- & --- & --- & --- & 37 & 39 \\
\hline MIN & --- & --- & --- & --- & --- & --- & --- & --- & --- & --- & 34 & 35 \\
\hline $\mathrm{AC}-\mathrm{FT}$ & --- & --- & --- & --- & --- & --- & --- & --- & --- & --- & 2210 & 2160 \\
\hline
\end{tabular}




\section{SOUTH FORK BATTLE CREEK'BELOW DIVERSION TO SOUTH BATTLE CREEK CANAL, NEAR MANTON, 'CA}

LOCATION.-Lat $40^{\circ} 22^{\prime} 08^{\prime \prime}$, long $121^{\circ} 47^{\prime} 48^{\prime \prime}$, in SW 1/4 NW 1/4 sec.18, T.29 N., R.2 E., Tehama County, Hydrologic Unit 18020118, on right bank, at diversion dam to South Battle Creek Canal, and 5.9 mi southeast of Manton.

DRAINAGE AREA.- $-66.7 \mathrm{mi}^{2}$.

PERIOD OF RECORD.-October 1987 to current year (operated as a low-flow station only). Unpublished records for water years 1978-87 available in files of the U.S. Geological Survey. Fragmentary records for water years 1976-77 in files of Pacific Gas \& Electric Co.

GAGE.-Water-stage recorder and metal Alaskan fishladder. Elevation of gage is 2,040 ft above sea level, from topographic map.

REMARKS.-This station records fishwater release only. The minimum release requirement is $5.0 \mathrm{ft}^{3} / \mathrm{s}$ at all times; flow is computed to $8.9 \mathrm{ft} / \mathrm{s}$. See schematic diagram of Battle Creek and Cow Creek Basins.

COOPERATION.-Records were collected by Pacific Gas \& Electric Co., under general supervision of the U.S. Geological Survey, in connection with a Federal Energy Regulatory Commission project.

DISCHARGE, CUBIC FEET PER SECOND, WATER YEAR OCTOBER 1998 TO SEPTEMBER 1999 DAILY MEAN VALUES

\begin{tabular}{|c|c|c|c|c|c|c|c|c|c|c|c|c|}
\hline DAY & $\mathrm{OCT}$ & NOV & $\mathrm{DEC}$ & JAN & FEB & MAR & APR & MAY & JUN & JUL & AUG & SEP \\
\hline 1 & 6.8 & 6.8 & --- & 8.7 & --- & --- & --- & --- & --- & --- & 6.5 & 6.5 \\
\hline 2 & 6.7 & 6.7 & --- & 7.3 & --- & --- & --- & --- & --- & --- & 6.5 & 6.5 \\
\hline 3 & 6.7 & 6.7 & --- & 7.1 & --- & --- & --- & --- & --- & --- & 6.5 & 6.5 \\
\hline 4 & 6.7 & 6.8 & --- & 6.9 & --- & --- & --- & --- & --- & --- & 6.5 & 6.5 \\
\hline 5 & 6.8 & 6.8 & --- & 6.8 & --- & --- & --- & --- & --- & 8.8 & 6.5 & 6.5 \\
\hline 6 & 6.9 & 6.8 & --- & 6.8 & --- & --- & --- & --- & --- & 8.4 & 6.5 & 6.5 \\
\hline 7 & 6.8 & 8.5 & --- & 6.8 & --- & --- & --- & --- & --- & 7.4 & 6.5 & 6.5 \\
\hline 8 & 6.8 & --- & --- & 7.0 & --- & --- & --- & --- & --- & 7.0 & 6.4 & 6.5 \\
\hline 9 & 6.8 & 6.7 & --- & 6.8 & --- & --- & --- & --- & --- & 6.9 & 6.5 & 6.5 \\
\hline 10 & 6.8 & 6.7 & --- & 6.8 & --- & --- & --- & --- & --- & 7.0 & 6.5 & 6.5 \\
\hline 11 & 6.8 & 6.8 & --- & 6.8 & --- & --- & --- & --- & --- & 7.0 & 6.4 & 6.5 \\
\hline 12 & 6.8 & 6.8 & --- & 6.8 & --- & --- & --- & --- & --- & 6.9 & 6.5 & 6.5 \\
\hline 13 & 6.8 & 6.8 & --- & 6.8 & --- & --- & --- & --- & --- & 7.0 & 6.5 & 6.5 \\
\hline 14 & 6.8 & 6.8 & --- & 6.8 & --- & --- & --- & --- & --- & 6.9 & 6.5 & 6.5 \\
\hline 15 & 6.8 & 6.9 & --- & --- & --- & --- & --- & --- & --- & 7.0 & 6.5 & 6.5 \\
\hline 16 & 6.8 & 6.8 & --- & --- & --- & --- & --- & --- & --- & 6.9 & 6.5 & 6.5 \\
\hline 17 & 6.8 & --- & --- & --- & --- & --- & --- & --- & --- & 6.9 & 6.5 & 6.5 \\
\hline 18 & 6.8 & --- & --- & --- & --- & --- & --- & --- & --- & 6.9 & 6.5 & 6.5 \\
\hline 19 & 6.8 & 6.8 & --- & --- & --- & --- & --- & --- & --- & 6.8 & 6.5 & 6.5 \\
\hline 20 & 6.8 & 6.8 & --- & --- & --- & --- & --- & --- & --- & 6.5 & 6.5 & 6.5 \\
\hline 21 & 6.9 & --- & --- & --- & --- & --- & --- & --- & --- & 6.5 & 6.5 & 6.5 \\
\hline 22 & 6.8 & --- & --- & --- & --- & --- & --- & --- & --- & 6.5 & 6.5 & 6.5 \\
\hline 23 & 6.8 & --- & --- & --- & --- & --- & --- & --- & --- & 6.5 & 6.5 & 6.5 \\
\hline 24 & --- & --- & --- & --- & --- & --- & --- & --- & --- & 6.5 & 6.5 & 6.5 \\
\hline 25 & --- & --- & --- & --- & --- & --- & --- & --- & --- & 6.5 & 6.5 & 6.5 \\
\hline 26 & 6.8 & --- & --- & --- & --- & --- & --- & --- & --- & 6.5 & 6.5 & 6.5 \\
\hline 27 & 6.8 & --- & --- & --- & --- & --- & --- & --- & --- & 6.5 & 6.5 & 6.5 \\
\hline 28 & 6.8 & --- & --- & --- & --- & --- & --- & --- & --- & 6.5 & 6.5 & 6.5 \\
\hline 29 & 6.7 & --- & --- & --- & --- & --- & --- & --- & --- & 6.5 & 6.5 & 6.5 \\
\hline 30 & 6.7 & --- & 8.1 & --- & --- & --- & --- & --- & --- & 6.5 & 6.5 & 6.5 \\
\hline 31 & 6.8 & --- & --- & --- & --- & --- & --- & --- & --- & 6.5 & 6.5 & --- \\
\hline TOTAL & --- & --- & --- & --- & --- & --- & --- & --- & --- & --- & 201.3 & 195.0 \\
\hline MEAN & --- & --- & --- & --- & --- & --- & --- & --- & --- & --- & 6.49 & 6.50 \\
\hline MAX & --- & --- & --- & --- & --- & --- & --- & --- & --- & --- & 6.5 & 6.5 \\
\hline MIN & --- & --- & --- & --- & --- & --- & --- & --- & --- & --- & 6.4 & 6.5 \\
\hline $\mathrm{AC}-\mathrm{FT}$ & --- & --- & --- & --- & --- & --- & --- & --- & --- & --- & 399 & 387 \\
\hline
\end{tabular}


LOCATION.—Lat 40²3'43", long 121 '52' $57^{\prime \prime}$, in NW 1/4 SE 1/4 sec.5, T.29 N., R.1 E., Tehama County, Hydrologic Unit 18020118, on left bank, at diversion dam to Inskip Canal, and $2.8 \mathrm{mi}$ south of Manton.

DRAINAGE AREA.- $88.3 \mathrm{mi}^{2}$.

PERIOD OF RECORD.-October 1987 to current year (operated as a low-flow station only). Unpublished records for water years 1978-87 available in files of the U.S. Geological Survey. Fragmentary records for water year 1977 available in files of Pacific Gas \& Electric Co.

GAGE.-Water-stage recorder and metal Alaskan fishladder. Elevation of gage is 1,440 ft above sea level, from topographic map.

REMARKS.-This station records fishwater release only. The minimum release requirement is $5.0 \mathrm{ft}^{3} / \mathrm{s}$ at all times. Prior to Feb. 6, 1998, flow computed to $12 \mathrm{ft}^{3} / \mathrm{s}$; flow computed to $60 \mathrm{ft}^{3} / \mathrm{s}$. See schematic diagram of Battle Creek and Cow Creek Basins.

COOPERATION.-Records were collected by Pacific Gas \& Electric Co., under general supervision of the U.S. Geological Survey, in connection with a Federal Energy Regulatory Commission project.

DISCHARGE, CUBIC FEET PER SECOND, WATER YEAR OCTOBER 1998 TO SEPTEMBER 1999 DAILY MEAN VALUES

\begin{tabular}{|c|c|c|c|c|c|c|c|c|c|c|c|c|}
\hline DAY & $\mathrm{OCT}$ & NOV & $\mathrm{DEC}$ & JAN & FEB & MAR & APR & MAY & JUN & JUL & AUG & SEP \\
\hline 1 & 17 & 20 & --- & 36 & --- & --- & --- & --- & --- & 30 & 16 & 13 \\
\hline 2 & 17 & 19 & --- & 31 & e57 & --- & --- & --- & --- & 30 & 16 & 13 \\
\hline 3 & 18 & 17 & --- & 29 & e58 & --- & --- & --- & --- & 31 & 16 & 12 \\
\hline 4 & 17 & 17 & --- & 28 & e56 & --- & --- & --- & --- & 30 & 15 & 12 \\
\hline 5 & 17 & 18 & --- & e28 & e51 & --- & --- & --- & --- & 31 & 15 & 12 \\
\hline 6 & 16 & 18 & --- & e25 & --- & --- & --- & --- & --- & 28 & 15 & 12 \\
\hline 7 & 17 & 30 & --- & e28 & --- & --- & --- & --- & --- & 27 & 16 & 12 \\
\hline 8 & 15 & 54 & --- & e26 & --- & --- & --- & --- & --- & 47 & 15 & 12 \\
\hline 9 & 14 & 24 & --- & e23 & --- & --- & --- & --- & --- & 24 & 15 & 12 \\
\hline 10 & 14 & 24 & --- & e24 & --- & --- & --- & --- & --- & 22 & 17 & 11 \\
\hline 11 & 14 & 25 & --- & e22 & --- & --- & --- & --- & --- & 21 & 18 & 11 \\
\hline 12 & 14 & 22 & --- & e23 & --- & --- & --- & --- & --- & 21 & 15 & 11 \\
\hline 13 & 14 & 22 & --- & e20 & --- & --- & --- & --- & --- & 21 & 15 & 11 \\
\hline 14 & 14 & 22 & --- & e20 & --- & --- & --- & --- & --- & 20 & 15 & 11 \\
\hline 15 & 14 & 24 & --- & e28 & --- & --- & --- & --- & --- & 20 & 14 & 11 \\
\hline 16 & 14 & 27 & --- & --- & --- & --- & --- & --- & --- & 19 & 14 & 11 \\
\hline 17 & 13 & 50 & --- & --- & --- & --- & --- & --- & --- & 19 & 14 & 11 \\
\hline 18 & 13 & 41 & --- & --- & --- & --- & --- & --- & --- & 19 & 14 & 11 \\
\hline 19 & 14 & 26 & --- & --- & --- & --- & --- & --- & --- & 19 & 14 & 11 \\
\hline 20 & 14 & 22 & 56 & --- & --- & --- & --- & --- & --- & 18 & 14 & 11 \\
\hline 21 & 14 & 60 & 42 & --- & --- & --- & --- & --- & 60 & 18 & 13 & 11 \\
\hline 22 & 14 & --- & 42 & --- & --- & --- & --- & --- & 59 & 17 & 14 & 11 \\
\hline 23 & 14 & --- & 37 & --- & --- & --- & --- & --- & 56 & 17 & 14 & 11 \\
\hline 24 & 29 & --- & 39 & --- & --- & --- & --- & --- & 53 & 17 & 14 & 11 \\
\hline 25 & 36 & --- & 40 & --- & --- & --- & --- & --- & 49 & 17 & 13 & 11 \\
\hline 26 & 23 & --- & 43 & --- & --- & --- & --- & --- & 47 & 17 & 13 & 11 \\
\hline 27 & 21 & --- & 41 & --- & --- & --- & --- & --- & 45 & 16 & 13 & 10 \\
\hline 28 & 19 & --- & 41 & --- & --- & --- & --- & --- & 41 & 16 & 13 & 7.7 \\
\hline 29 & 19 & --- & 34 & --- & --- & --- & --- & --- & 40 & 16 & 12 & 6.8 \\
\hline 30 & 18 & --- & 33 & --- & --- & --- & --- & --- & 38 & 16 & 13 & 7.0 \\
\hline 31 & 18 & --- & 39 & --- & --- & --- & --- & --- & --- & 16 & 13 & --- \\
\hline TOTAL & 525 & --- & --- & --- & --- & --- & --- & --- & --- & 680 & 448 & 328.5 \\
\hline MEAN & 16.9 & --- & --- & --- & --- & --- & --- & --- & --- & 21.9 & 14.5 & 10.9 \\
\hline MAX & 36 & --- & --- & --- & --- & --- & --- & --- & --- & 47 & 18 & 13 \\
\hline MIN & 13 & --- & --- & --- & --- & --- & --- & --- & --- & 16 & 12 & 6.8 \\
\hline $\mathrm{AC}-\mathrm{FT}$ & 1040 & --- & --- & --- & --- & --- & --- & --- & --- & 1350 & 889 & 652 \\
\hline
\end{tabular}


LOCATION.-Lat 40²4'10", long 121 $58^{\prime} 02^{\prime \prime}$, in NW 1/4 NW 1/4 sec.3, T.29 N., R.1 W., Tehama County, Hydrologic Unit 18020118, on right bank , 7.5 mi southwest of Shingletown, and 5.7 mi southwest of Manton.

DRAINAGE AREA.-102 $\mathrm{mi}^{2}$.

PERIOD OF RECORD.-October 1987 to current year (operated as a low-flow station only). Unpublished records for water years 1978-86 available in files of the U.S. Geological Survey. Fragmentary records for water year 1977 available in files of Pacific Gas \& Electric Co.

GAGE.-Water-stage recorder and metal Alaskan fishladder. Elevation of gage is $980 \mathrm{ft}$ above sea level, from topographic map.

REMARKS.-This station records fishwater release only. Prior to water year 1996 flow computed to $10 \mathrm{ft}^{3} / \mathrm{s}$. The minimum release requirement is $5.0 \mathrm{ft}^{3} / \mathrm{s}$ at all times; flow is computed to $45 \mathrm{ft}^{3} / \mathrm{s}$. See schematic diagram of Battle Creek and Cow Creek Basins.

COOPERATION.- - Records were collected by the Pacific Gas \& Electric Co., under general supervision of the U.S. Geological Survey, in connection with a Federal Energy Regulatory Commission project.

DISCHARGE, CUBIC FEET PER SECOND, WATER YEAR OCTOBER 1998 TO SEPTEMBER 1999 DAILY MEAN VALUES

\begin{tabular}{|c|c|c|c|c|c|c|c|c|c|c|c|c|}
\hline DAY & OCT & NOV & $\mathrm{DEC}$ & JAN & $\mathrm{FEB}$ & MAR & $\mathrm{APR}$ & MAY & JUN & JUL & AUG & SEP \\
\hline 1 & 34 & 32 & --- & 34 & 43 & --- & --- & --- & --- & 37 & 36 & 35 \\
\hline 2 & 34 & 32 & --- & 34 & 41 & --- & --- & --- & --- & 37 & 36 & 35 \\
\hline 3 & 33 & 32 & --- & 34 & 39 & --- & --- & --- & --- & 37 & 36 & 35 \\
\hline 4 & 33 & 32 & --- & 34 & 37 & --- & --- & --- & --- & 37 & 36 & 35 \\
\hline 5 & 33 & 32 & --- & 34 & 35 & --- & --- & --- & --- & 37 & 36 & 35 \\
\hline 6 & 33 & 32 & --- & 34 & --- & --- & --- & --- & --- & 38 & 37 & 35 \\
\hline 7 & 33 & 33 & --- & 35 & --- & --- & --- & --- & --- & --- & 38 & 35 \\
\hline 8 & 33 & 31 & --- & 35 & --- & --- & --- & --- & --- & 40 & 39 & 35 \\
\hline 9 & 33 & 31 & --- & 35 & --- & --- & --- & --- & --- & 35 & 37 & 35 \\
\hline 10 & 33 & 33 & --- & 35 & --- & --- & --- & --- & --- & 36 & 37 & 35 \\
\hline 11 & 33 & 33 & --- & 35 & --- & --- & --- & --- & --- & 36 & 37 & 35 \\
\hline 12 & 33 & 33 & --- & 35 & --- & --- & --- & --- & --- & 35 & 36 & 35 \\
\hline 13 & 33 & 33 & --- & 35 & --- & --- & --- & --- & --- & 35 & 36 & 35 \\
\hline 14 & 33 & 33 & --- & 35 & --- & --- & --- & --- & --- & 35 & 36 & 35 \\
\hline 15 & 32 & 33 & --- & 37 & --- & --- & --- & --- & --- & 35 & 36 & 35 \\
\hline 16 & 32 & 33 & --- & --- & --- & --- & --- & --- & --- & 35 & 37 & 35 \\
\hline 17 & 32 & 35 & --- & --- & --- & --- & --- & --- & --- & 36 & 38 & 35 \\
\hline 18 & 32 & 33 & --- & --- & --- & --- & --- & --- & 40 & 36 & 37 & 35 \\
\hline 19 & 32 & 33 & 44 & --- & --- & --- & --- & --- & 37 & 35 & 35 & 35 \\
\hline 20 & 32 & 32 & 35 & --- & --- & --- & --- & --- & --- & 36 & 35 & 35 \\
\hline 21 & 32 & --- & 32 & --- & --- & --- & --- & --- & 42 & 36 & 36 & 35 \\
\hline 22 & 32 & --- & 32 & --- & --- & --- & --- & --- & 37 & 35 & 36 & 35 \\
\hline 23 & 32 & --- & 31 & --- & --- & --- & --- & --- & 37 & 35 & 36 & 35 \\
\hline 24 & 34 & --- & --- & --- & --- & --- & --- & --- & 37 & 36 & 36 & 35 \\
\hline 25 & 35 & --- & 41 & --- & --- & --- & --- & --- & 37 & 36 & 35 & 35 \\
\hline 26 & 32 & --- & 34 & --- & --- & --- & --- & --- & 38 & 36 & 34 & 35 \\
\hline 27 & 32 & --- & 34 & --- & --- & --- & --- & --- & 37 & 36 & 35 & 35 \\
\hline 28 & 32 & --- & 34 & --- & --- & --- & --- & --- & 39 & 36 & 35 & 35 \\
\hline 29 & 32 & --- & 34 & --- & --- & --- & --- & --- & 38 & 36 & 35 & 35 \\
\hline 30 & 32 & --- & 34 & --- & --- & --- & --- & --- & 38 & 36 & 35 & 35 \\
\hline 31 & 32 & --- & 34 & --- & --- & --- & --- & --- & --- & 36 & 35 & --- \\
\hline TOTAL & 1013 & --- & --- & --- & --- & --- & --- & --- & --- & --- & 1119 & 1050 \\
\hline MEAN & 32.7 & --- & --- & --- & --- & --- & --- & --- & --- & --- & 36.1 & 35.0 \\
\hline MAX & 35 & --- & --- & --- & --- & --- & --- & --- & --- & --- & 39 & 35 \\
\hline MIN & 32 & --- & --- & --- & --- & --- & --- & --- & --- & --- & 34 & 35 \\
\hline $\mathrm{AC}-\mathrm{FT}$ & 2010 & --- & --- & --- & --- & --- & --- & --- & --- & --- & 2220 & 2080 \\
\hline
\end{tabular}


11376550 BATTLE CREEK BELOW COLEMAN FISH HATCHERY, NEAR COTTONWOOD, CA

LOCATION.-Lat 40²3'54", long 12208'43", in SW 1/4 NE 1/4 sec.1, T.29 N., R.3 W., Shasta County, Hydrologic Unit 18020101, U.S. Fish and Wildlife Service land, on right bank, $3.7 \mathrm{mi}$ downstream from Spring Branch, 5.7 mi upstream from mouth, and 7.0 mi east of Cottonwood. DRAINAGE AREA.-357 $\mathrm{mi}^{2}$.

PERIOD OF RECORD.-O October 1961 to September 1996. October 1996 to September 1997 (operated as a low flow station only). October 1997 to current year. October 1940 to September 1961 at site 0.6 mi upstream published as "near Cottonwood"; low-flow records not equivalent owing to Coleman Fish Hatchery diversion, maximum flows considered equivalent.

CHEMICAL DATA: Water years 1962-66.

WATER TEMPERATURE: Water years 1966-79.

SEDIMENT DATA: Water years 1962-70.

GAGE.-Water-stage recorder. Elevation of gage is $415 \mathrm{ft}$ above sea level, from topographic map.

REMARKS.--Records excellent. Some regulation at low flows by five small powerplants, several small reservoirs, and Coleman Fish Hatchery. Coleman Fish Hatchery diverts from 50 to $90 \mathrm{ft}^{3} / \mathrm{s}$ and pumps ground water for temperature control, which is returned above the station. At times, $10 \mathrm{ft}^{3} / \mathrm{s}$ diverted upstream from station for irrigation. Flow is computed to $540 \mathrm{ft}^{3} / \mathrm{s}$. See schematic diagrams of Battle Creek and Cow Creek Basins and upper Sacramento River Basin.

EXTREMES FOR PERIOD OF RECORD.-Maximum discharge, 24,300 ft 3 /s, Jan. 24, 1970, gage height, $14.75 \mathrm{ft}$, from rating curve extended above $4,200 \mathrm{ft}^{3} / \mathrm{s}$ on basis of slope-area measurement of peak flow; minimum, $52 \mathrm{ft}^{3} / \mathrm{s}$, Aug. $8,1962$.

EXTREMES OUTSIDE PERIOD OF RECORD.-Maximum stage known, $15.8 \mathrm{ft}$, Dec. 11, 1937, from floodmarks, site and datum then in use, discharge, $35,000 \mathrm{ft}^{3} / \mathrm{s}$ by slope-area measurement.

EXTREMES FOR CURRENT YEAR.-Peak discharges greater than base discharge of 3,100 $\mathrm{ft}^{3} / \mathrm{s}$, or maximum:

$\begin{array}{ccccccc}\text { Date } & \text { Time } & \begin{array}{c}\text { Discharge } \\ \left(\mathrm{ft}^{3} / \mathrm{s}\right)\end{array} & \begin{array}{c}\text { Gage height } \\ (\mathrm{ft})\end{array} & \text { Date } & \text { Time } & \begin{array}{c}\text { Discharge } \\ \left(\mathrm{ft}^{3} / \mathrm{s}\right)\end{array} \\ \text { Nov. 30 } & 1615 & 4,230 & 5.94 & \text { Feb. } 9 & 0545 & 6,600\end{array}$

DISCHARGE, CUBIC FEET PER SECOND, WATER YEAR OCTOBER 1998 TO SEPTEMBER 1999 DAILY MEAN VALUES

\begin{tabular}{|c|c|c|c|c|c|c|c|c|c|c|c|c|}
\hline DAY & OCT & NOV & $\mathrm{DEC}$ & JAN & $\mathrm{FEB}$ & MAR & APR & MAY & JUN & JUL & AUG & SEP \\
\hline 1 & 430 & 439 & 1640 & 470 & 548 & 1890 & 693 & 742 & 713 & 450 & 329 & 304 \\
\hline 2 & 429 & 419 & 944 & 455 & 528 & 1350 & 667 & 787 & 812 & 445 & 326 & 303 \\
\hline 3 & 433 & 427 & 2070 & 457 & 519 & 1540 & 650 & 779 & 776 & 441 & 329 & 301 \\
\hline 4 & 418 & 420 & 1060 & 452 & 512 & 1240 & 628 & 702 & 701 & 435 & 330 & 299 \\
\hline 5 & 415 & 418 & 791 & 449 & 504 & 1060 & 657 & 668 & 652 & 424 & 332 & 298 \\
\hline 6 & 429 & 432 & 793 & 445 & 825 & 977 & 633 & 677 & 643 & 414 & 331 & 296 \\
\hline 7 & 428 & 488 & 720 & 440 & 1000 & 916 & 601 & 707 & 617 & 407 & 344 & 297 \\
\hline 8 & 432 & 520 & 689 & 437 & 896 & 878 & 651 & 695 & 590 & 394 & 335 & 297 \\
\hline 9 & 438 & 461 & 628 & 433 & 3200 & 909 & 640 & 678 & 571 & 391 & 335 & 297 \\
\hline 10 & 435 & 469 & 588 & 433 & 1300 & 870 & 654 & 661 & 548 & 385 & 336 & 300 \\
\hline 11 & 431 & 499 & 560 & 433 & 948 & 820 & 696 & 649 & 543 & 385 & 345 & 302 \\
\hline 12 & 436 & 441 & 545 & 433 & 819 & 789 & 690 & 663 & 544 & 383 & 334 & 301 \\
\hline 13 & 445 & 429 & 585 & 435 & 747 & 768 & 699 & 684 & 543 & 386 & 330 & 318 \\
\hline 14 & 434 & 430 & 883 & 430 & 736 & 765 & 706 & 665 & 543 & 389 & 327 & 314 \\
\hline 15 & 435 & 434 & 626 & 455 & 696 & 738 & 711 & 637 & 541 & 389 & 326 & 312 \\
\hline 16 & 443 & 436 & 579 & 548 & 1320 & 726 & 721 & 621 & 527 & 386 & 323 & 311 \\
\hline 17 & 432 & 489 & 573 & 585 & 1850 & 726 & 735 & 625 & 522 & 380 & 318 & 316 \\
\hline 18 & 432 & 523 & 553 & 806 & 1300 & 726 & 771 & 643 & 519 & 374 & 317 & 315 \\
\hline 19 & 436 & 461 & 539 & 654 & 1140 & 731 & 796 & 662 & 516 & 369 & 317 & 317 \\
\hline 20 & 429 & 445 & 520 & 632 & 1220 & 747 & 815 & 667 & 512 & 364 & 314 & 319 \\
\hline 21 & 422 & 603 & 493 & 658 & 1630 & 734 & 809 & 656 & 492 & 361 & 309 & 318 \\
\hline 22 & 418 & 883 & 486 & 578 & 1050 & 708 & 778 & 675 & 503 & 357 & 311 & 320 \\
\hline 23 & 415 & 1240 & 477 & 948 & 920 & 697 & 746 & 724 & 499 & 354 & 312 & 322 \\
\hline 24 & 487 & 983 & 468 & 786 & 869 & 766 & 752 & 760 & 497 & 356 & 315 & 332 \\
\hline 25 & 501 & 596 & 489 & 666 & 1150 & 1020 & 773 & 801 & 496 & 355 & 308 & 323 \\
\hline 26 & 460 & 897 & 486 & 666 & 1010 & 821 & 861 & 812 & 480 & 352 & 305 & 318 \\
\hline 27 & 447 & 1190 & 483 & 613 & 923 & 762 & 887 & 799 & 463 & 345 & 305 & 319 \\
\hline 28 & 456 & 676 & 481 & 582 & 1220 & 710 & 793 & 789 & 458 & 339 & 306 & 334 \\
\hline 29 & 464 & 717 & 477 & 555 & --- & 705 & 738 & 773 & 453 & 342 & 303 & 334 \\
\hline 30 & 442 & 2220 & 471 & 546 & --- & 708 & 723 & 747 & 448 & 334 & 301 & 324 \\
\hline 31 & 435 & --- & 472 & 562 & --- & 711 & --- & 718 & --- & 331 & 302 & --- \\
\hline TOTAL & 13587 & 19085 & 21169 & 17042 & 29380 & 27508 & 21674 & 21866 & 16722 & 11817 & 9955 & 9361 \\
\hline MEAN & 438 & 636 & 683 & 550 & 1049 & 887 & 722 & 705 & 557 & 381 & 321 & 312 \\
\hline MAX & 501 & 2220 & 2070 & 948 & 3200 & 1890 & 887 & 812 & 812 & 450 & 345 & 334 \\
\hline MIN & 415 & 418 & 468 & 430 & 504 & 697 & 601 & 621 & 448 & 331 & 301 & 296 \\
\hline $\mathrm{AC}-\mathrm{FT}$ & 26950 & 37860 & 41990 & 33800 & 58280 & 54560 & 42990 & 43370 & 33170 & 23440 & 19750 & 18570 \\
\hline
\end{tabular}




\section{BATTLE CREEK BELOW COLEMAN FISH HATCHERY, NEAR COTTONWOOD, CA—Continued}

STATISTICS OF MONTHLY MEAN DATA FOR WATER YEARS 1962 - 1999, BY WATER YEAR (WY)

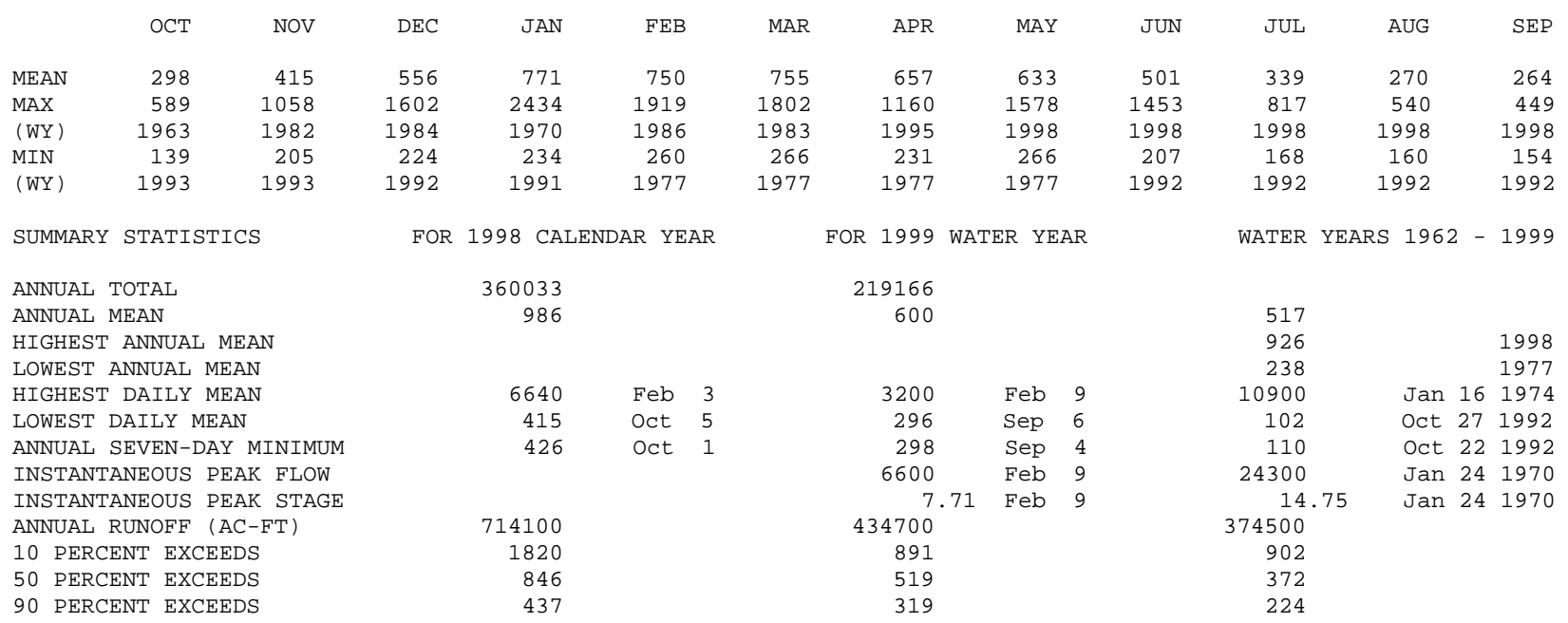




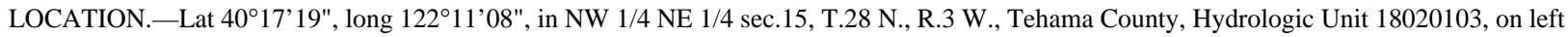
bank, 2.7 mi upstream from Bend Bridge, and 8.1 mi northeast of Red Bluff.

DRAINAGE AREA.- $-8,900 \mathrm{mi}^{2}$, excluding Goose Lake Basin.

PERIOD OF RECORD._-1879-88 annual observed maximums only, published in WSP 1315-A. January 1892 to current year. Monthly discharges only for some periods and yearly estimates for some incomplete years, published in WSP 1315-A. Published as "at Red Bluff" 1894-96, as "at Jellys Ferry" 1895-1902, and as "near Red Bluff" 1903-68 (station 11378000).

CHEMICAL DATA: Water years 1955-81, 1996-98.

SPECIFIC CONDUCTANCE: Water years 1955-63.

WATER TEMPERATURE: Water years 1955-80.

SEDIMENT DATA: Water year 1958-70, 1996-98 .

REVISED RECORDS.-WSP 861: 1904, 1907, 1909, 1914-15, 1927-28. WSP 1315-A: 1916(M), 1918(M), 1941(M). WSP 1931: Drainage area. WDR CA-69-2: 1965.

GAGE.-Water-stage recorder and crest-stage gage. Datum of gage is $285.77 \mathrm{ft}$ above sea level. See WSP 2131 for history of changes prior to September 1968

REMARKS.--Records excellent. Flow completely regulated by Shasta Lake (station 11370000), 52 mi upstream, since Dec. 30, 1943. Diversions, in addition to those on tributaries, for irrigation of about 22,000 acres between stations at Keswick and above Bend Bridge. Transbasin diversion from Trinity River to Whiskeytown Lake (station 11371700) via Judge Francis Carr Powerplant (station 11525430) started in April 1963. See schematic diagrams of upper Sacramento River Basin and Battle Creek and Cow Creek Basins.

EXTREMES FOR PERIOD OF RECORD.-Maximum discharge, 291,000 ft $\mathrm{ft}^{3} / \mathrm{s}$, Feb. 28, 1940, gage height, $38.9 \mathrm{ft}$, site and datum then in use, from rating curve extended above $170,000 \mathrm{ft}^{3} / \mathrm{s}$ on basis of velocity-area studies; minimum (water years 1892-1999), 2,000 $\mathrm{ft}^{3} / \mathrm{s}, \mathrm{Mar}^{2}$. 29, 1944. Since regulation by Shasta Lake in 1943, maximum discharge, 170,000 ft $3 / \mathrm{s}$, Dec. 22, 1964, gage height, $28.15 \mathrm{ft}$, site and datum then in use; maximum gage height, $36.60 \mathrm{ft}$, Jan. 24, 1970.

DISCHARGE, CUBIC FEET PER SECOND, WATER YEAR OCTOBER 1998 TO SEPTEMBER 1999 DAILY MEAN VALUES

\begin{tabular}{|c|c|c|c|c|c|c|c|c|c|c|c|c|}
\hline DAY & $\mathrm{OCT}$ & NOV & DEC & JAN & FEB & MAR & APR & MAY & JUN & JUL & AUG & SEP \\
\hline 1 & 9190 & 7510 & 28900 & 7750 & 10200 & 41200 & 10300 & 12500 & 12600 & 13800 & 13400 & 9690 \\
\hline 2 & 8910 & 7570 & 22900 & 7690 & 9900 & 36800 & 9900 & 12600 & 12700 & 13700 & 13200 & 9670 \\
\hline 3 & 8940 & 7590 & 32700 & 7630 & 9280 & 36800 & 9630 & 12800 & 13100 & 13400 & 13200 & 9410 \\
\hline 4 & 8930 & 7560 & 25000 & 7610 & 10500 & 35700 & 9410 & 12600 & 12800 & 13400 & 12900 & 9280 \\
\hline 5 & 8880 & 7510 & 28600 & 7610 & 18200 & 36400 & 9410 & 12400 & 12300 & 13600 & 12400 & 9270 \\
\hline 6 & 8990 & 8060 & 29900 & 7610 & 22000 & 35600 & 10500 & 12900 & 11900 & 13600 & 11900 & 9270 \\
\hline 7 & 8990 & 8940 & 29200 & 7600 & 43900 & 35400 & 9630 & 13300 & 11800 & 13700 & 11400 & 9030 \\
\hline 8 & 8810 & 9190 & 29300 & 7420 & 30100 & 34700 & 9750 & 13100 & 11700 & 13600 & 11200 & 8950 \\
\hline 9 & 8670 & 8660 & 27400 & 7400 & 48200 & 38400 & 10300 & 13100 & 11600 & 13600 & 11200 & 8940 \\
\hline 10 & 8660 & 8610 & 25400 & 7460 & 26900 & 36500 & 11800 & 12900 & 11600 & 13600 & 10900 & 8920 \\
\hline 11 & 8390 & 9380 & 25300 & 7510 & 23100 & 35300 & 17200 & 12900 & 11600 & 13600 & 10600 & 8660 \\
\hline 12 & 8100 & 9220 & 24800 & 7500 & 25900 & 31900 & 14100 & 13000 & 11500 & 13500 & 10400 & 8510 \\
\hline 13 & 7940 & 10100 & 23200 & 7480 & 25100 & 28000 & 14100 & 12400 & 11500 & 14200 & 10100 & 8550 \\
\hline 14 & 7750 & 10500 & 22000 & 7490 & 25200 & 24500 & 14600 & 12000 & 11800 & 13800 & 10200 & 8520 \\
\hline 15 & 7530 & 10600 & 18400 & 7540 & 27800 & 23000 & 14400 & 12700 & 12100 & 14200 & 9920 & 8510 \\
\hline 16 & 7360 & 10500 & 17800 & 7900 & 34100 & 20100 & 14200 & 12100 & 12100 & 13900 & 9750 & 8480 \\
\hline 17 & 7280 & e16100 & 17600 & 10100 & 47800 & 19200 & 14200 & 11400 & 12000 & 13900 & 9770 & 8510 \\
\hline 18 & 7260 & e 16800 & 17200 & 13700 & 40600 & 17500 & 14300 & 11100 & 12400 & 13800 & 9730 & 8240 \\
\hline 19 & 7240 & e16500 & 15900 & 12600 & 37800 & 15500 & 14200 & 10700 & 12500 & 13900 & 9710 & 8100 \\
\hline 20 & 7230 & e16300 & 14000 & 12700 & 34900 & 14300 & 13300 & 10900 & 12900 & 13800 & 9730 & 8100 \\
\hline 21 & 7200 & e17900 & 12300 & 21400 & 47200 & 13200 & 12400 & 11400 & 12900 & 13400 & 9710 & 8100 \\
\hline 22 & 7230 & e18900 & 10600 & 19700 & 34500 & 11800 & 12100 & 12200 & 12900 & 13300 & 9700 & 7850 \\
\hline 23 & 7260 & e20000 & 9500 & 26500 & 30300 & 11000 & 11800 & 12800 & 13400 & 13300 & 9720 & 7710 \\
\hline 24 & 7740 & e19100 & 8940 & 22000 & 28700 & 15600 & 11600 & 12200 & 13800 & 13400 & 9700 & 7670 \\
\hline 25 & 7900 & 18800 & 8920 & 19400 & 32900 & 26900 & 11600 & 12000 & 13800 & 13400 & 9720 & 7680 \\
\hline 26 & 7740 & 19300 & 8890 & 18000 & 34100 & 16700 & 11700 & 12200 & 13800 & 13300 & 9720 & 7650 \\
\hline 27 & 7670 & 23800 & 8880 & 16200 & 35100 & 13600 & 11700 & 12700 & 14100 & 13300 & 9670 & 7640 \\
\hline 28 & 7570 & 18500 & 8940 & 14000 & 36300 & 12100 & 12300 & 13200 & 14300 & 13400 & 9700 & 7390 \\
\hline 29 & 7560 & 20900 & 8530 & 12400 & --- & 11300 & 12600 & 13300 & 14300 & 13200 & 9680 & 7210 \\
\hline 30 & 7520 & 38300 & 8010 & 10900 & --- & 10900 & 12500 & 13200 & 13700 & 13500 & 9650 & 7230 \\
\hline 31 & 7520 & --- & 7870 & 10300 & --- & 10800 & --- & 12600 & --- & 13600 & 9690 & --- \\
\hline TOTAL & 247960 & 422700 & 576880 & 361100 & 830580 & 750700 & 365530 & 385200 & 379500 & 421700 & 328270 & 252740 \\
\hline MEAN & 7999 & 14090 & 18610 & 11650 & 29660 & 24220 & 12180 & 12430 & 12650 & 13600 & 10590 & 8425 \\
\hline MAX & 9190 & 38300 & 32700 & 26500 & 48200 & 41200 & 17200 & 13300 & 14300 & 14200 & 13400 & 9690 \\
\hline MIN & 7200 & 7510 & 7870 & 7400 & 9280 & 10800 & 9410 & 10700 & 11500 & 13200 & 9650 & 7210 \\
\hline$A C-F T$ & 491800 & 838400 & 1144000 & 716200 & 1647000 & 1489000 & 725000 & 764000 & 752700 & 836400 & 651100 & 501300 \\
\hline
\end{tabular}


11377100 SACRAMENTO RIVER ABOVE BEND BRIDGE, NEAR RED BLUFF, CA—Continued

STATISTICS OF MONTHLY MEAN DATA FOR WATER YEARS 1892 - 1943, BY WATER YEAR (WY)

\begin{tabular}{|c|c|c|c|c|c|c|c|c|c|c|c|c|}
\hline & OCT & NOV & $\mathrm{DEC}$ & JAN & $\mathrm{FEB}$ & MAR & APR & MAY & JUN & JUL & AUG & SEP \\
\hline MEAN & 4853 & 7538 & 11940 & 18960 & 24760 & 22210 & 18280 & 12310 & 7635 & 5127 & 4381 & 4404 \\
\hline MAX & 10910 & 21420 & 42780 & 72340 & 69240 & 73280 & 38810 & 27910 & 17640 & 10170 & 9050 & 8481 \\
\hline (WY) & 1905 & 1904 & 1893 & 1909 & 1902 & 1904 & 1904 & 1896 & 1906 & 1893 & 1893 & 1893 \\
\hline MIN & 2847 & 3300 & 3618 & 4142 & 4778 & 4434 & 4014 & 3253 & 2969 & 2622 & 2505 & 2551 \\
\hline$(W Y)$ & 1933 & 1937 & 1937 & 1937 & 1920 & 1924 & 1924 & 1924 & 1924 & 1931 & 1931 & 1934 \\
\hline
\end{tabular}

SUMMARY STATISTICS

ANNUAL MEAN

HIGHEST ANNUAL MEAN

LOWEST ANNUAL MEAN

HIGHEST DAILY MEAN

LOWEST DAILY MEAN

ANNUAL SEVEN-DAY MINIMUM

INSTANTANEOUS PEAK FLOW

INSTANTANEOUS PEAK STAGE

ANNUAL RUNOFF (AC-FT)

10 PERCENT EXCEEDS

50 PERCENT EXCEEDS

90 PERCENT EXCEEDS
WATER YEARS $1892-1943$

\begin{tabular}{|c|c|c|}
\hline 11800 & & \\
\hline 22180 & & 1904 \\
\hline 4096 & & 1924 \\
\hline 261000 & Feb 28 & 1940 \\
\hline 2400 & Aug 13 & 1931 \\
\hline 2470 & Aug 7 & 1931 \\
\hline 291000 & Feb 28 & 1940 \\
\hline 38.9 & Feb 28 & 1940 \\
\hline 8545000 & & \\
\hline 24000 & & \\
\hline 6500 & & \\
\hline 3520 & & \\
\hline
\end{tabular}

STATISTICS OF MONTHLY MEAN DATA FOR WATER YEARS 1946 - 1962, BY WATER YEAR (WY)

\begin{tabular}{|c|c|c|c|c|c|c|c|c|c|c|c|c|}
\hline & $\mathrm{OCT}$ & NOV & $\mathrm{DEC}$ & JAN & $\mathrm{FEB}$ & MAR & APR & MAY & JUN & JUL & AUG & SEP \\
\hline MEAN & 6501 & 6932 & 11440 & 16840 & 19340 & 11950 & 10210 & 10260 & 9469 & 10030 & 10030 & 7510 \\
\hline MAX & 10490 & 11180 & 29530 & 52620 & 76870 & 24840 & 32420 & 17830 & 12930 & 11630 & 11800 & 10230 \\
\hline (WY) & 1958 & 1958 & 1956 & 1956 & 1958 & 1958 & 1958 & 1948 & 1948 & 1951 & 1958 & 1958 \\
\hline MIN & 5468 & 4681 & 4336 & 5104 & 4579 & 4727 & 5335 & 6788 & 7253 & 7476 & 7080 & 5289 \\
\hline (WY) & 1960 & 1960 & 1960 & 1957 & 1948 & 1955 & 1950 & 1947 & 1947 & 1947 & 1947 & 1947 \\
\hline
\end{tabular}

SUMMARY STATISTICS

ANNUAL MEAN

HIGHEST ANNUAL MEAN

LOWEST ANNUAL MEAN

HIGHEST DAILY MEAN

LOWEST DAILY MEAN

ANNUAL SEVEN-DAY MINIMUM

INSTANTANEOUS PEAK FLOW

INSTANTANEOUS PEAK STAGE

ANNUAL RUNOFF (AC-FT)

10 PERCENT EXCEEDS

50 PERCENT EXCEEDS

90 PERCENT EXCEEDS
WATER YEARS 1946 - 1962

\begin{tabular}{|c|c|c|}
\hline 10840 & & \\
\hline 20330 & & 1958 \\
\hline 6690 & & 1947 \\
\hline 125000 & Feb 19 & 1958 \\
\hline 3640 & $\operatorname{Jan} 31$ & 1949 \\
\hline 3830 & Feb 27 & 1948 \\
\hline 139000 & Feb 19 & 1958 \\
\hline 24.98 & Feb 19 & 1958 \\
\hline 7852000 & & \\
\hline 16900 & & \\
\hline 8430 & & \\
\hline 5190 & & \\
\hline
\end{tabular}

STATISTICS OF MONTHLY MEAN DATA FOR WATER YEARS 1964 - 1999, BY WATER YEAR (WY)

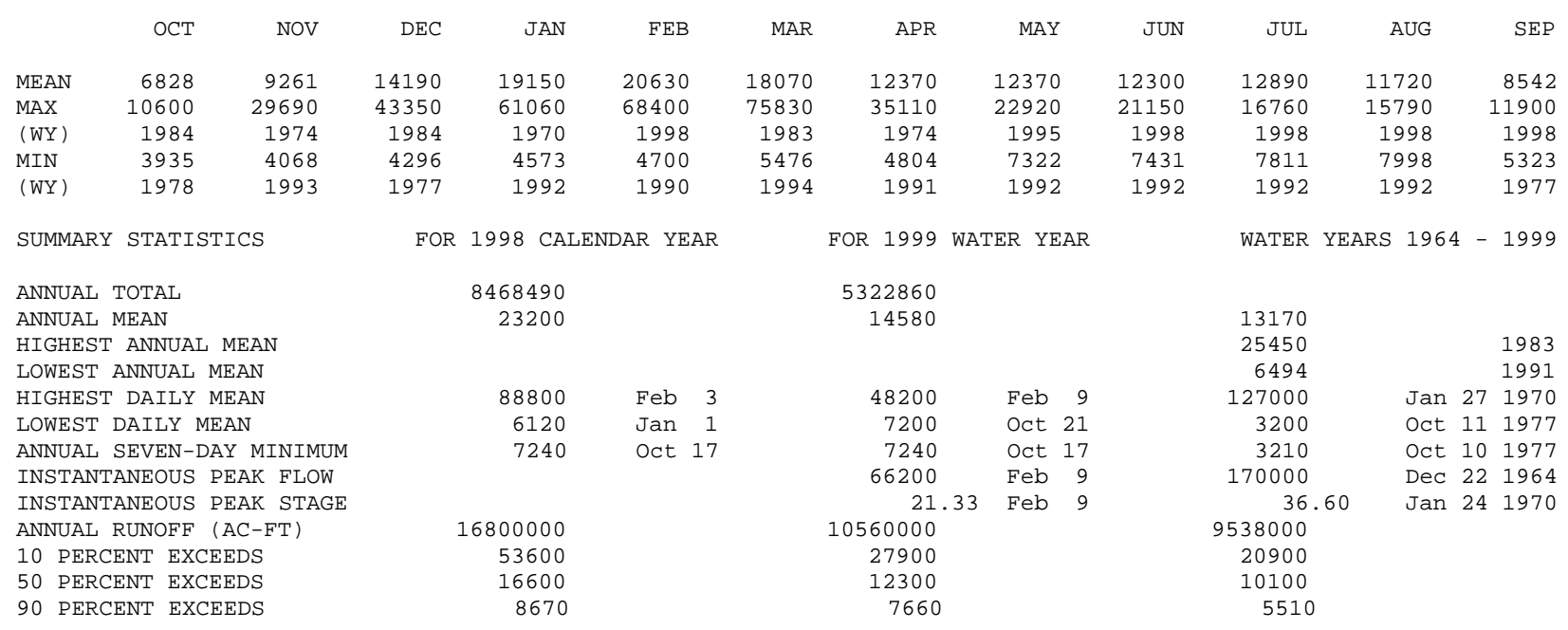


LOCATION.-Lat 4001'29", long 122³0'31", in SE 1/4 NW 1/4 sec.14, T.25 N., R.6 W., Tehama County, Hydrologic Unit 18020103, on left bank, 2.5 mi downstream from South Fork Elder Creek, 8.2 mi northwest of Flournoy, and 10 mi north of Paskenta.

DRAINAGE AREA.- $92.4 \mathrm{mi}^{2}$.

PERIOD OF RECORD.-October 1948 to current year. Monthly discharge only for some periods, published in WSP 1315-A.

CHEMICAL DATA: Water years 1959-66.

WATER TEMPERATURE: Water year 1963.

SEDIMENT DATA: Water years 1963-70.

REVISED RECORDS.-WSP 1515: 1956. WDR CA-70-2: 1967(P). WDR CA-75-4: 1966-67(P), 1969-71(P), 1973(P). WDR CA-78-4: Drainage area. WDR CA-94-4: 1993(P).

GAGE.-Water-stage recorder. Datum of gage is $718.1 \mathrm{ft}$ above sea level. Prior to Aug. 13, 1965, water-stage recorder at site $300 \mathrm{ft}$ downstream at datum $5.13 \mathrm{ft}$ lower.

REMARKS.- - Records good. No regulation or large diversion upstream from station. See schematic diagram of upper Sacramento River Basin.

EXTREMES FOR PERIOD OF RECORD.-Maximum discharge, 17,700 ft 3 s, Feb. 28, 1983, gage height, $12.10 \mathrm{ft}$, from rating curve extended above $5,200 \mathrm{ft}^{3} / \mathrm{s}$ on basis of slope-area measurements at gage height $11.34 \mathrm{ft}$ and of peak flow; maximum gage height, $13.90 \mathrm{ft}, \mathrm{Feb} .24,1958$, site and datum then in use; no flow at times some years.

EXTREMES FOR CURRENT YEAR.-Peak discharges greater than base discharge of 2,000 $\mathrm{ft}^{3} / \mathrm{s}$, or maximum:

\begin{tabular}{|c|c|c|c|c|c|c|c|}
\hline Date & Time & $\begin{array}{c}\text { Discharge } \\
\left(\mathrm{ft}^{3} / \mathrm{s}\right)\end{array}$ & $\begin{array}{l}\text { Gage height } \\
\text { (ft) }\end{array}$ & Date & Time & $\begin{array}{c}\text { Discharge } \\
\left(\mathrm{ft}^{3} / \mathrm{s}\right)\end{array}$ & $\begin{array}{l}\text { Gage height } \\
\text { (ft) }\end{array}$ \\
\hline Mar. 24 & Unknown & 2,600 & 6.38 & & & & \\
\hline
\end{tabular}

DISCHARGE, CUBIC FEET PER SECOND, WATER YEAR OCTOBER 1998 TO SEPTEMBER 1999 DAILY MEAN VALUES

\begin{tabular}{|c|c|c|c|c|c|c|c|c|c|c|c|c|}
\hline DAY & $\mathrm{OCT}$ & NOV & $\mathrm{DEC}$ & JAN & FEB & MAR & APR & MAY & JUN & JUL & AUG & SEP \\
\hline 1 & 9.3 & 12 & 170 & 22 & 42 & 302 & 178 & 165 & 45 & 14 & 6.1 & 3.3 \\
\hline 2 & 9.7 & 12 & 104 & 21 & 39 & 234 & 163 & 171 & 60 & 13 & 6.2 & 3.4 \\
\hline 3 & 10 & 12 & 142 & 20 & 37 & 251 & 152 & 157 & 61 & 13 & 6.3 & 3.4 \\
\hline 4 & 9.8 & 12 & 105 & 20 & 36 & 211 & 141 & 144 & 53 & 13 & 5.9 & 3.3 \\
\hline 5 & 9.1 & 12 & 79 & 20 & 34 & 181 & 166 & 134 & 46 & 13 & 6.0 & 3.1 \\
\hline 6 & 8.4 & 13 & 68 & 19 & 652 & 163 & 197 & 139 & 42 & 12 & 6.5 & 3.0 \\
\hline 7 & 8.4 & 33 & 60 & 19 & 996 & 147 & 177 & 144 & 39 & 12 & 8.0 & 3.0 \\
\hline 8 & 8.7 & 23 & 74 & 19 & 308 & 315 & 191 & 132 & 38 & 11 & 7.6 & 2.9 \\
\hline 9 & 9.3 & 17 & 61 & 19 & 464 & 252 & 164 & 120 & 36 & 11 & 6.9 & 2.7 \\
\hline 10 & 9.3 & 16 & 51 & 19 & 231 & 175 & 287 & 112 & 35 & 10 & 6.6 & 2.8 \\
\hline 11 & 9.2 & 22 & 47 & 18 & 164 & 149 & 895 & 112 & 34 & 9.5 & 7.5 & 3.2 \\
\hline 12 & 9.1 & 17 & 45 & 18 & 133 & 135 & 380 & 116 & 32 & 8.5 & 7.3 & 2.8 \\
\hline 13 & 9.4 & 15 & 45 & 18 & 116 & 129 & 346 & 110 & 31 & 8.1 & 6.8 & 2.5 \\
\hline 14 & 9.8 & 15 & 47 & 18 & 111 & 240 & 333 & 103 & 30 & 8.3 & 6.4 & 2.7 \\
\hline 15 & 9.5 & 16 & 41 & 19 & 96 & 251 & 309 & 96 & 29 & 8.3 & 6.0 & 2.4 \\
\hline 16 & 9.3 & 16 & 37 & 27 & 269 & 188 & 299 & 89 & 29 & 8.5 & 5.5 & 2.4 \\
\hline 17 & 9.2 & 24 & 36 & 26 & 395 & 170 & 321 & 85 & 27 & 8.7 & 5.2 & 2.2 \\
\hline 18 & 9.1 & 21 & 36 & 81 & 318 & 161 & 336 & 86 & 26 & 8.7 & 4.9 & 2.1 \\
\hline 19 & 8.9 & 17 & 34 & 59 & 279 & 153 & 332 & 87 & 24 & 8.2 & 4.7 & 2.4 \\
\hline 20 & 8.4 & 16 & 32 & 56 & 260 & 166 & 306 & 87 & 23 & 8.0 & 4.5 & 2.4 \\
\hline 21 & 8.4 & 18 & 30 & 66 & 227 & 160 & 266 & 82 & 22 & 8.3 & 4.2 & 2.5 \\
\hline 22 & 8.4 & 28 & 32 & 76 & 180 & 157 & 240 & 78 & 21 & 8.2 & 3.8 & 2.3 \\
\hline 23 & 8.6 & 258 & 34 & 266 & 162 & 192 & 215 & 75 & 19 & 7.8 & 3.4 & 2.3 \\
\hline 24 & 15 & 148 & 37 & 127 & 158 & e1130 & 209 & 74 & 19 & 7.7 & 3.3 & 2.3 \\
\hline 25 & 16 & 59 & 28 & 93 & 202 & e1270 & 216 & 70 & 18 & 7.7 & 3.4 & 2.2 \\
\hline 26 & 12 & 49 & 26 & 77 & 166 & 530 & 226 & 66 & 17 & 7.4 & 3.2 & 2.1 \\
\hline 27 & 11 & 58 & 25 & 62 & 151 & 358 & 210 & 62 & 17 & 7.2 & 3.1 & 1.7 \\
\hline 28 & 11 & 41 & 25 & 54 & 257 & 281 & 185 & 57 & 16 & 7.0 & 3.4 & 1.5 \\
\hline 29 & 11 & 104 & 23 & 48 & --- & 241 & 166 & 54 & 15 & 6.6 & 3.1 & 1.5 \\
\hline 30 & 11 & 466 & 23 & 45 & --- & 219 & 158 & 50 & 14 & 6.3 & 3.2 & 1.8 \\
\hline 31 & 11 & --- & 23 & 48 & --- & 200 & --- & 46 & --- & 6.2 & 3.2 & --- \\
\hline TOTAL & 307.3 & 1570 & 1620 & 1500 & 6483 & 8711 & 7764 & 3103 & 918 & 287.2 & 162.2 & 76.2 \\
\hline MEAN & 9.91 & 52.3 & 52.3 & 48.4 & 232 & 281 & 259 & 100 & 30.6 & 9.26 & 5.23 & 2.54 \\
\hline MAX & 16 & 466 & 170 & 266 & 996 & 1270 & 895 & 171 & 61 & 14 & 8.0 & 3.4 \\
\hline MIN & 8.4 & 12 & 23 & 18 & 34 & 129 & 141 & 46 & 14 & 6.2 & 3.1 & 1.5 \\
\hline $\mathrm{AC}-\mathrm{FT}$ & 610 & 3110 & 3210 & 2980 & 12860 & 17280 & 15400 & 6150 & 1820 & 570 & 322 & 151 \\
\hline
\end{tabular}

e Estimated. 


\section{ELDER CREEK NEAR PASKENTA, CA—Continued}

STATISTICS OF MONTHLY MEAN DATA FOR WATER YEARS 1949 - 1999, BY WATER YEAR (WY)

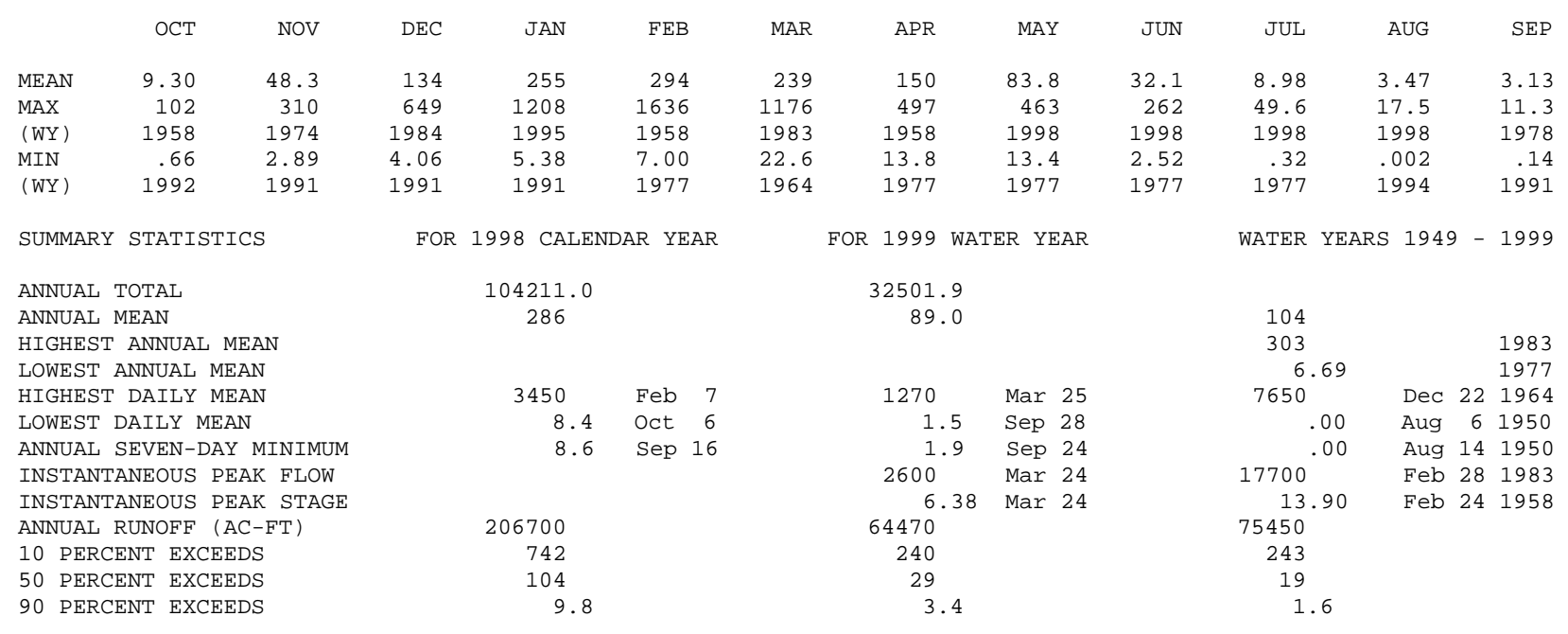


11381500 MILL CREEK NEAR LOS MOLINOS, CA

LOCATION.-Lat 4003'17", long 122 01'23", in NE 1/4 NW 1/4 sec.6, T.25 N., R.1 W., Tehama County, Hydrologic Unit 18020103, on right bank 4.5 mi northeast of Los Molinos, and 5.5 mi upstream from mouth.

DRAINAGE AREA.- $-131 \mathrm{mi}^{2}$.

\section{WATER-DISCHARGE RECORDS}

PERIOD OF RECORD.-September 1909 to August 1913 (fragmentary), October 1928 to current year.

REVISED RECORDS._WSP 1315-A: 1929(M). WSP 1931: Drainage area. WSP 2131: 1938(M).

GAGE.-Water-stage recorder. Elevation of gage is $385 \mathrm{ft}$ above sea level, from topographic map. Prior to September 1913, nonrecording gage at site $0.3 \mathrm{mi}$ downstream at different datum.

REMARKS.--Records good. No storage or large diversion upstream from station. See schematic diagram of upper Sacramento River Basin.

EXTREMES FOR PERIOD OF RECORD (water years 1929-99).-Maximum discharge, 36,400 ft 3 s, Dec. 11, 1937, gage height, $23.4 \mathrm{ft}$, from floodmarks, from rating curve extended above $14,000 \mathrm{ft}^{3} / \mathrm{s}$ on basis of step-backwater computation and slope-area measurement of peak flow; minimum, $49 \mathrm{ft}^{3} / \mathrm{s}$, Dec. 13, 1932.

EXTREMES FOR CURRENT YEAR.-Peak discharges greater than base discharge of 2,400 $\mathrm{ft}^{3} / \mathrm{s}$, or maximum:

\begin{tabular}{|c|c|c|c|c|c|c|c|}
\hline Date & Time & $\begin{array}{c}\text { Discharge } \\
\left(\mathrm{ft}^{3} / \mathrm{s}\right)\end{array}$ & $\begin{array}{l}\text { Gage height } \\
\text { (ft) }\end{array}$ & Date & Time & $\begin{array}{c}\text { Discharge } \\
\left(\mathrm{ft}^{3} / \mathrm{s}\right)\end{array}$ & $\begin{array}{c}\text { Gage height } \\
\text { (ft) }\end{array}$ \\
\hline Nov. 23 & 1930 & 2,650 & 6.59 & Feb. 9 & 1030 & 5,120 & 8.85 \\
\hline Nov. 30 & 1515 & 3,960 & 7.86 & & & & \\
\hline
\end{tabular}

DISCHARGE, CUBIC FEET PER SECOND, WATER YEAR OCTOBER 1998 TO SEPTEMBER 1999 DAILY MEAN VALUES

\begin{tabular}{|c|c|c|c|c|c|c|c|c|c|c|c|c|}
\hline DAY & OCT & NOV & $\mathrm{DEC}$ & JAN & FEB & MAR & APR & MAY & JUN & JUL & AUG & SEP \\
\hline 1 & 159 & 157 & 1160 & 175 & 287 & 1300 & 352 & 477 & 516 & 311 & 156 & 129 \\
\hline 2 & 159 & 154 & 595 & 170 & 270 & 920 & 335 & 514 & 552 & 303 & 154 & 129 \\
\hline 3 & 160 & 151 & 1410 & 168 & 262 & 1210 & 323 & 491 & 481 & 287 & 154 & 127 \\
\hline 4 & 161 & 150 & 796 & 166 & 257 & 919 & 309 & 435 & 417 & 277 & 153 & 126 \\
\hline 5 & 155 & 152 & 516 & 164 & 241 & 709 & 312 & 399 & 405 & 263 & 152 & 126 \\
\hline 6 & 152 & 154 & 434 & 164 & 397 & 595 & 303 & 408 & 438 & 251 & 152 & 126 \\
\hline 7 & 150 & 182 & 365 & 164 & 864 & 518 & 295 & 464 & 421 & 243 & 154 & 125 \\
\hline 8 & 149 & 205 & 334 & 162 & 761 & 468 & 368 & 450 & 400 & 226 & 152 & 124 \\
\hline 9 & 149 & 179 & 300 & 160 & 2720 & 527 & 360 & 428 & 385 & 220 & 152 & 124 \\
\hline 10 & 150 & 173 & 272 & 160 & 1170 & 467 & 348 & 410 & 373 & 214 & 153 & 124 \\
\hline 11 & 149 & 179 & 257 & 161 & 719 & 427 & 367 & 400 & 380 & 210 & 156 & 124 \\
\hline 12 & 148 & 171 & 245 & 159 & 557 & 398 & 375 & 427 & 390 & 208 & 151 & 122 \\
\hline 13 & 148 & 168 & 305 & 157 & 473 & 380 & 390 & 454 & 396 & 206 & 149 & 122 \\
\hline 14 & 150 & 168 & 479 & 153 & 431 & 374 & 417 & 426 & 406 & 204 & 147 & 122 \\
\hline 15 & 148 & 168 & 307 & 188 & 385 & 357 & 425 & 393 & 419 & 197 & 147 & 122 \\
\hline 16 & 146 & 169 & 270 & 262 & 599 & 350 & 435 & 379 & 404 & 190 & 147 & 124 \\
\hline 17 & 144 & 210 & 257 & 257 & 979 & 351 & 458 & 383 & 386 & 185 & 145 & 124 \\
\hline 18 & 144 & 198 & 248 & 605 & 842 & 356 & 521 & 414 & 396 & 181 & 144 & 124 \\
\hline 19 & 143 & 175 & 238 & 475 & 749 & 363 & 547 & 438 & 397 & 178 & 144 & 124 \\
\hline 20 & 142 & 169 & 221 & 528 & 851 & 372 & 572 & 446 & 383 & 174 & 137 & 125 \\
\hline 21 & 141 & 201 & 199 & 571 & 1240 & 368 & 567 & 440 & 370 & 173 & 132 & 129 \\
\hline 22 & 140 & 444 & 197 & 438 & 664 & 357 & 526 & 482 & 384 & 170 & 131 & 125 \\
\hline 23 & 139 & 1080 & 188 & 1100 & 541 & 345 & 486 & 522 & 385 & 168 & 131 & 124 \\
\hline 24 & 167 & 722 & 188 & 769 & 493 & 387 & 492 & 568 & 377 & 166 & 132 & 125 \\
\hline 25 & 191 & 342 & 183 & 561 & 985 & 632 & 513 & 629 & 372 & 165 & 129 & 124 \\
\hline 26 & 168 & 321 & 184 & 470 & 626 & 501 & 608 & 648 & 337 & 164 & 130 & 126 \\
\hline 27 & 165 & 544 & 181 & 395 & 530 & 479 & 622 & 626 & 316 & 162 & 130 & 124 \\
\hline 28 & 164 & 384 & 179 & 345 & 879 & 430 & 519 & 605 & 307 & 162 & 132 & 124 \\
\hline 29 & 165 & 414 & 177 & 313 & --- & 401 & 462 & 575 & 304 & 160 & 129 & 123 \\
\hline 30 & 156 & 1980 & 175 & 291 & --- & 385 & 444 & 530 & 308 & 158 & 128 & 125 \\
\hline 31 & 150 & --- & 176 & 303 & --- & 378 & --- & 524 & --- & 157 & 129 & --- \\
\hline TOTAL & 4752 & 9864 & 11036 & 10154 & 19772 & 16024 & 13051 & 14785 & 11805 & 6333 & 4432 & 3742 \\
\hline MEAN & 153 & 329 & 356 & 328 & 706 & 517 & 435 & 477 & 394 & 204 & 143 & 125 \\
\hline MAX & 191 & 1980 & 1410 & 1100 & 2720 & 1300 & 622 & 648 & 552 & 311 & 156 & 129 \\
\hline MIN & 139 & 150 & 175 & 153 & 241 & 345 & 295 & 379 & 304 & 157 & 128 & 122 \\
\hline $\mathrm{AC}-\mathrm{FT}$ & 9430 & 19570 & 21890 & 20140 & 39220 & 31780 & 25890 & 29330 & 23420 & 12560 & 8790 & 7420 \\
\hline
\end{tabular}




\section{MILL CREEK NEAR LOS MOLINOS, CA—Continued}

STATISTICS OF MONTHLY MEAN DATA FOR WATER YEARS 1929 - 1999, BY WATER YEAR (WY)

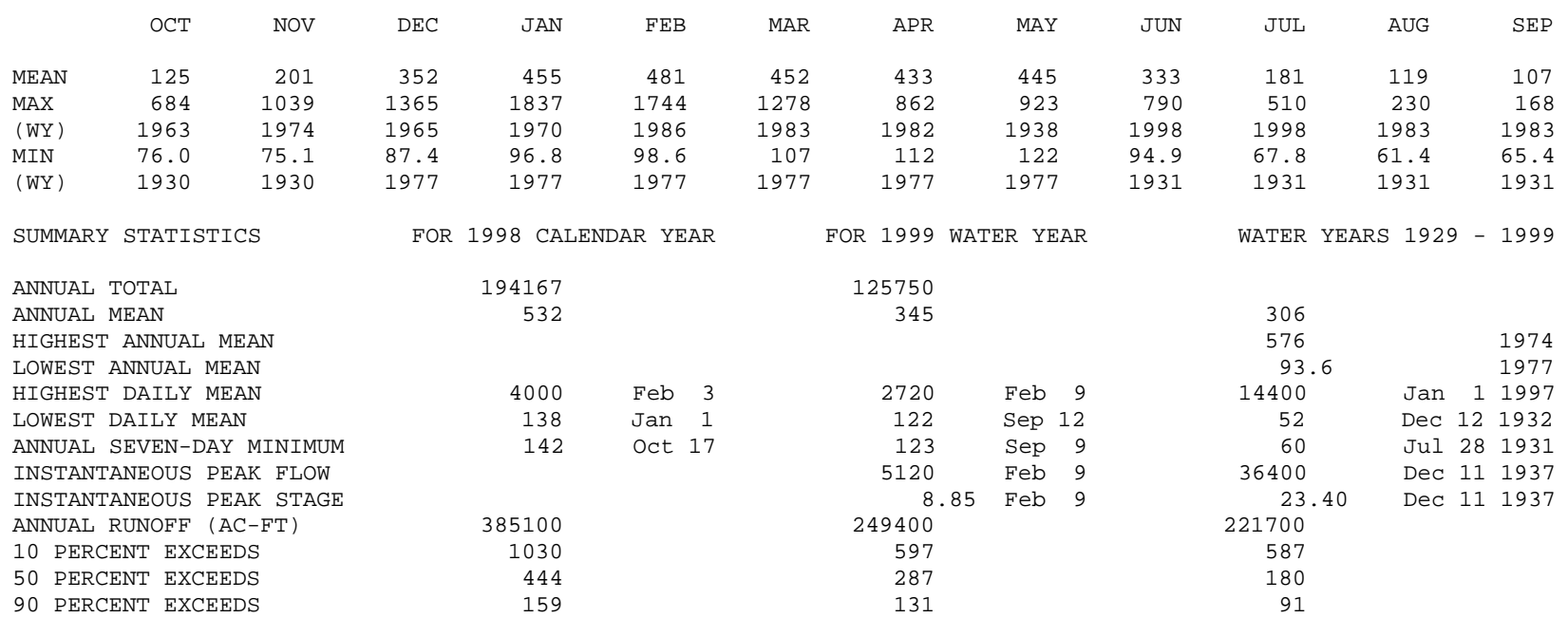




\section{WATER-QUALITY RECORDS}

PERIOD OF RECORD.-October 1998 to September 1999.

WATER TEMPERATURE: October 1998 to September 1999.

PERIOD OF DAILY RECORD.-October 1998 to September 1999.

WATER TEMPERATURE: October 1998 to September 1999.

INSTRUMENTATION.-Temperature recorder since Oct. 5, 1998.

EXTREMES FOR PERIOD OF DAILY RECORD.-

WATER TEMPERATURE: Maximum recorded, 23.5 ${ }^{\circ} \mathrm{C}$, July 13, 14, and Aug. 23, 1999; minimum recorded, 0.5 ${ }^{\circ} \mathrm{C}$, Dec. $23,1998$.

EXTREME FOR CURRENT YEAR.-

WATER TEMPERATURE: Maximum recorded, $23.5^{\circ} \mathrm{C}$, July 13, 14, and Aug. 23; minimum recorded, 0.5 ${ }^{\circ}$ C, Dec. 23.

TEMPERATURE, WATER (DEG. C), WATER YEAR OCTOBER 1998 TO SEPTEMBER 1999

\begin{tabular}{|c|c|c|c|c|c|c|c|c|c|c|c|c|}
\hline DAY & MAX & MIN & MAX & MIN & MAX & MIN & MAX & MIN & MAX & MIN & MAX & MIN \\
\hline & \multicolumn{2}{|c|}{ OCTOBER } & \multicolumn{2}{|c|}{ NOVEMBER } & \multicolumn{2}{|c|}{ DECEMBER } & \multicolumn{2}{|c|}{ JANUARY } & \multicolumn{2}{|c|}{ FEBRUARY } & \multicolumn{2}{|c|}{ MARCH } \\
\hline 1 & --- & --- & 11.0 & 9.5 & 9.0 & 8.5 & 7.0 & 6.0 & 6.0 & 5.0 & 9.0 & 7.5 \\
\hline 2 & --- & --- & 10.5 & 9.5 & 10.0 & 8.5 & 6.5 & 5.5 & 6.0 & 5.0 & 8.5 & 7.0 \\
\hline 3 & --- & --- & 11.0 & 9.5 & 10.0 & 8.0 & 7.0 & 6.0 & 6.5 & 5.5 & 9.0 & 7.5 \\
\hline 4 & --- & --- & 11.0 & 10.0 & 8.0 & 6.0 & 6.5 & 6.0 & 7.0 & 6.5 & 7.5 & 6.5 \\
\hline 5 & 14.0 & 12.5 & 10.5 & 9.5 & 6.0 & 4.5 & 6.0 & 5.5 & 7.0 & 6.0 & 7.5 & 6.0 \\
\hline 6 & 14.5 & 12.5 & 9.5 & 9.0 & 4.5 & 3.5 & 5.5 & 5.0 & 7.0 & 6.5 & 7.5 & 6.5 \\
\hline 7 & 14.5 & 13.0 & 9.0 & 8.5 & 5.0 & 3.5 & 5.5 & 5.0 & 8.5 & 7.0 & 7.5 & 6.0 \\
\hline 8 & 15.0 & 13.0 & 8.5 & 7.5 & 6.0 & 5.0 & 5.5 & 5.0 & 8.0 & 7.0 & 7.5 & 6.5 \\
\hline 9 & 14.5 & 13.0 & 7.5 & 6.5 & 6.0 & 5.0 & 6.0 & 5.5 & 9.0 & 6.0 & 6.5 & 6.0 \\
\hline 10 & 13.0 & 11.5 & 8.0 & 6.5 & 6.0 & 5.0 & 6.0 & 5.5 & 6.0 & 5.0 & 7.0 & 5.0 \\
\hline 11 & 12.5 & 11.0 & 8.5 & 7.5 & 6.5 & 5.5 & 6.0 & 5.5 & 5.5 & 4.5 & 8.0 & 5.5 \\
\hline 12 & 12.5 & 11.0 & 8.0 & 7.0 & 8.0 & 6.5 & 6.5 & 5.5 & 6.5 & 5.0 & 8.5 & 6.5 \\
\hline 13 & 14.0 & 11.5 & 8.5 & 7.5 & 8.5 & 8.0 & 7.0 & 6.0 & 7.0 & 6.0 & 9.5 & 7.5 \\
\hline 14 & 13.5 & 12.5 & 8.5 & 7.5 & 8.5 & 7.5 & 7.5 & 6.5 & 8.0 & 7.0 & 9.5 & 8.5 \\
\hline 15 & 12.5 & 11.0 & 9.5 & 8.0 & 8.5 & 7.0 & 9.0 & 7.0 & 7.5 & 6.5 & 10.0 & 7.5 \\
\hline 16 & 11.5 & 10.0 & 9.0 & 9.0 & 9.0 & 8.0 & 9.5 & 9.0 & 8.0 & 7.0 & 10.5 & 8.5 \\
\hline 17 & 11.5 & 9.5 & 10.0 & 9.0 & 9.0 & 8.5 & 9.5 & 8.5 & 9.0 & 8.0 & 11.0 & 9.5 \\
\hline 18 & 11.5 & 10.0 & 9.5 & 8.0 & 8.5 & 7.0 & 9.5 & 8.5 & 9.0 & 8.0 & 11.0 & 9.5 \\
\hline 19 & 13.0 & 10.5 & 8.0 & 7.5 & 7.0 & 5.0 & 8.5 & 8.0 & 8.0 & 6.5 & 10.5 & 9.0 \\
\hline 20 & 13.0 & 11.5 & 8.0 & 7.0 & 5.5 & 3.5 & 8.5 & 8.0 & 7.5 & 6.5 & 10.0 & 9.5 \\
\hline 21 & 13.0 & 11.5 & 10.0 & 8.0 & 3.5 & 1.5 & 8.0 & 6.5 & 7.5 & 6.5 & 10.0 & 8.0 \\
\hline 22 & 13.0 & 11.5 & 10.0 & 9.5 & 1.5 & 1.0 & 8.0 & 7.0 & 8.0 & 7.0 & 9.0 & 8.0 \\
\hline 23 & 12.5 & 11.0 & 11.5 & 9.5 & 1.5 & .5 & 8.0 & 6.5 & 9.5 & 7.5 & 9.0 & 8.0 \\
\hline 24 & 12.0 & 11.5 & 10.0 & 8.5 & 2.0 & 1.0 & 6.5 & 4.5 & 9.5 & 8.5 & 9.5 & 8.5 \\
\hline 25 & 12.0 & 11.0 & 9.0 & 8.5 & 4.5 & 2.0 & 6.0 & 4.5 & 9.0 & 7.5 & 11.0 & 9.0 \\
\hline 26 & 12.0 & 11.0 & 9.5 & 8.0 & 5.5 & 4.5 & 6.5 & 6.0 & 8.5 & 6.5 & 11.5 & 10.0 \\
\hline 27 & 12.5 & 11.0 & 10.0 & 9.0 & 5.5 & 5.0 & 6.5 & 5.0 & 9.0 & 8.0 & 10.5 & 8.5 \\
\hline 28 & 12.5 & 11.5 & 9.0 & 8.5 & 6.0 & 5.5 & 6.0 & 5.0 & 10.0 & 8.5 & 9.0 & 7.0 \\
\hline 29 & 12.5 & 11.5 & 9.0 & 8.0 & 6.0 & 5.0 & 6.0 & 4.5 & --- & --- & 9.0 & 7.5 \\
\hline 30 & 11.5 & 10.0 & 11.0 & 8.5 & 6.0 & 5.0 & 5.5 & 4.5 & --- & --- & 8.5 & 7.5 \\
\hline 31 & 10.0 & 9.5 & --- & --- & 7.0 & 6.0 & 6.0 & 5.5 & --- & --- & 8.0 & 6.0 \\
\hline MONTH & --- & --- & 11.5 & 6.5 & 10.0 & .5 & 9.5 & 4.5 & 10.0 & 4.5 & 11.5 & 5.0 \\
\hline
\end{tabular}


11381500 MILL CREEK NEAR LOS MOLINOS, CA—Continued

TEMPERATURE, WATER (DEG. C), WATER YEAR OCTOBER 1998 TO SEPTEMBER 1999

\begin{tabular}{|c|c|c|c|c|c|c|c|c|c|c|c|c|}
\hline \multirow[t]{2}{*}{ DAY } & MAX & MIN & MAX & MIN & MAX & MIN & MAX & MIN & MAX & MIN & MAX & MIN \\
\hline & \multicolumn{2}{|c|}{ APRIL } & \multicolumn{2}{|c|}{ MAY } & \multicolumn{2}{|c|}{ JUNE } & \multicolumn{2}{|c|}{ JULY } & \multicolumn{2}{|c|}{ AUGUST } & \multicolumn{2}{|c|}{ SEPTEMBER } \\
\hline 1 & 8.5 & 6.0 & 14.0 & 12.0 & 15.5 & 14.5 & 22.0 & 19.0 & 22.0 & 19.5 & 17.5 & 15.5 \\
\hline 2 & 9.5 & 6.5 & 13.5 & 9.5 & 14.5 & 11.0 & 21.5 & 18.5 & 22.5 & 20.0 & 18.0 & 15.0 \\
\hline 3 & 9.5 & 8.0 & 11.0 & 9.0 & 11.0 & 10.0 & 20.0 & 17.0 & 23.0 & 20.0 & 18.5 & 15.5 \\
\hline 4 & 8.5 & 6.5 & 11.5 & 8.0 & 14.5 & 9.5 & 18.5 & 16.0 & 23.0 & 20.5 & 19.0 & 16.0 \\
\hline 5 & 8.5 & 7.5 & 13.5 & 9.5 & 17.0 & 13.5 & 19.0 & 16.0 & 21.5 & 20.0 & 19.0 & 16.5 \\
\hline 6 & 9.0 & 6.5 & 15.0 & 12.0 & 16.5 & 14.5 & 20.0 & 17.0 & 20.0 & 19.0 & 20.0 & 17.0 \\
\hline 7 & 10.0 & 7.5 & 14.0 & 12.0 & 15.0 & 12.5 & 19.5 & 18.0 & 20.5 & 18.0 & 20.0 & 17.5 \\
\hline 8 & 10.0 & 7.0 & 12.5 & 10.5 & 15.0 & 12.0 & 20.5 & 18.0 & 20.5 & 17.5 & 20.5 & 17.5 \\
\hline 9 & 8.0 & 5.0 & 12.0 & 10.0 & 14.5 & 12.0 & 21.0 & 18.5 & 21.0 & 18.5 & 20.0 & 18.0 \\
\hline 10 & 8.0 & 6.5 & 12.0 & 10.0 & 16.0 & 12.5 & 21.5 & 19.0 & 20.5 & 19.0 & 20.0 & 18.0 \\
\hline 11 & 10.5 & 7.0 & 15.0 & 11.0 & 17.0 & 13.5 & 22.5 & 20.0 & 21.5 & 19.0 & 21.0 & 18.0 \\
\hline 12 & 12.0 & 9.0 & 15.0 & 13.0 & 17.5 & 14.5 & 23.0 & 21.5 & 22.0 & 19.5 & 20.5 & 18.0 \\
\hline 13 & 12.5 & 10.0 & 14.0 & 11.0 & 18.0 & 14.5 & 23.5 & 22.0 & 21.5 & 19.0 & 20.0 & 17.5 \\
\hline 14 & 13.0 & 10.5 & 12.5 & 10.0 & 18.5 & 15.5 & 23.5 & 22.0 & 21.0 & 18.5 & 20.5 & 18.0 \\
\hline 15 & 13.0 & 10.0 & 12.5 & 9.5 & 18.0 & 16.0 & 22.5 & 21.0 & 21.0 & 18.0 & 20.0 & 17.5 \\
\hline 16 & 13.0 & 11.0 & 13.5 & 10.5 & 18.5 & 15.0 & 21.5 & 20.0 & 21.0 & 18.0 & 20.0 & 17.5 \\
\hline 17 & 14.0 & 11.5 & 14.5 & 11.5 & 18.5 & 15.5 & 21.0 & 19.5 & 21.5 & 18.5 & 19.5 & 17.0 \\
\hline 18 & 13.5 & 11.5 & 15.5 & 12.5 & 19.0 & 16.0 & 20.5 & 18.5 & 21.5 & 18.5 & 18.5 & 17.0 \\
\hline 19 & 13.0 & 11.5 & 15.0 & 12.5 & 19.0 & 15.5 & 20.0 & 18.5 & 21.0 & 18.5 & 19.5 & 17.0 \\
\hline 20 & 12.5 & 11.5 & 14.5 & 12.5 & 18.5 & 16.0 & 20.5 & 18.0 & 21.0 & 18.0 & 20.0 & 17.5 \\
\hline 21 & 12.0 & 10.5 & 15.5 & 13.0 & 20.0 & 16.0 & 20.0 & 18.0 & 22.0 & 18.5 & 20.5 & 18.0 \\
\hline 22 & 12.0 & 10.0 & 16.0 & 13.5 & 20.5 & 17.0 & 20.5 & 18.0 & 23.0 & 19.5 & 20.5 & 18.0 \\
\hline 23 & 12.5 & 9.5 & 16.5 & 14.5 & 21.0 & 17.5 & 21.0 & 18.5 & 23.5 & 20.5 & 20.5 & 18.0 \\
\hline 24 & 13.5 & 11.0 & 16.0 & 15.0 & 20.5 & 18.0 & 21.0 & 18.5 & 23.0 & 21.0 & 20.5 & 18.0 \\
\hline 25 & 13.0 & 11.5 & 16.5 & 14.5 & 19.5 & 16.5 & 21.0 & 18.0 & 22.0 & 20.0 & 20.0 & 18.0 \\
\hline 26 & 13.5 & 12.0 & 16.0 & 14.5 & 18.5 & 15.5 & 22.5 & 19.5 & 21.5 & 19.5 & 18.5 & 16.5 \\
\hline 27 & 12.0 & 10.0 & 16.5 & 15.0 & 19.5 & 15.5 & 21.5 & 19.5 & 21.0 & 19.5 & 17.5 & 15.5 \\
\hline 28 & 10.5 & 8.5 & 16.0 & 14.5 & 20.5 & 16.5 & 21.5 & 19.0 & 22.0 & 19.0 & 16.5 & 14.5 \\
\hline 29 & 12.0 & 8.0 & 15.5 & 14.0 & 21.5 & 17.5 & 22.0 & 19.5 & 22.5 & 20.0 & 16.5 & 14.0 \\
\hline 30 & 13.0 & 10.5 & 16.0 & 13.0 & 22.0 & 19.0 & 22.0 & 19.0 & 21.0 & 19.0 & 17.0 & 15.0 \\
\hline 31 & --- & --- & 15.5 & 14.5 & --- & --- & 21.5 & 19.0 & 19.0 & 16.5 & --- & --- \\
\hline MONTH & 14.0 & 5.0 & 16.5 & 8.0 & 22.0 & 9.5 & 32.5 & 16.0 & 23.5 & 16.5 & 21.0 & 14.0 \\
\hline
\end{tabular}


11383500 DEER CREEK NEAR VINA, CA

LOCATION.-Lat 4000'51", long 121 56'50", in NW 1/4 NE 1/4 sec.23, T.25 N., R.1 W., Tehama County, Hydrologic Unit 18020103, on left bank 0.5 mi upstream from irrigation diversion dam and 7.9 mi northeast of Vina.

DRAINAGE AREA.-208 $\mathrm{mi}^{2}$.

WATER-DISCHARGE RECORDS

PERIOD OF RECORD.—October 1911 to September 1915, March 1920 to current year. December 1937 to January 1939 first published in WDR CA-94-4. Monthly discharge only for some periods, published in WSP 1315-A.

REVISED RECORDS.-WSP 1315-A: 1940-42(M). WSP 1931: Drainage area.

GAGE.-Water-stage recorder. Datum of gage is $479.2 \mathrm{ft}$ above sea level, from river-profile survey. Prior to Oct. 9, 1928, nonrecording gage at site $0.8 \mathrm{mi}$ downstream at different datum. Oct. 9, 1928, to Jan. 19, 1939, water-stage recorder at present site at datum $2.64 \mathrm{ft}$ higher.

REMARKS.-Records good. No storage or large diversions upstream from station. See schematic diagram of upper Sacramento River Basin.

EXTREMES FOR PERIOD OF RECORD.-Maximum discharge, 24,000 ft $3 / \mathrm{s}$, Jan. 1, 1997, gage height, $15.56 \mathrm{ft}$, from rating curve extended above $9,200 \mathrm{ft}^{3} / \mathrm{s}$; maximum gage height, $19.20 \mathrm{ft}$, Dec. 10, 1937; minimum, $43 \mathrm{ft}^{3} / \mathrm{s}$, Dec. 13, 1932.

EXTREMES FOR CURRENT YEAR.-Peak discharges greater than base discharge of 2,500 $\mathrm{ft}^{3} / \mathrm{s}$, or maximum:

$\begin{array}{lccccccc}\text { Date } & \text { Time } & \begin{array}{c}\text { Discharge } \\ \left(\mathrm{ft}^{3} / \mathrm{s}\right)\end{array} & \begin{array}{c}\text { Gage height } \\ (\mathrm{ft})\end{array} & \text { Date } & \text { Time } & \begin{array}{c}\text { Discharge } \\ \left(\mathrm{ft}^{3} / \mathrm{s}\right)\end{array} & \begin{array}{c}\text { Gage height } \\ (\mathrm{ft})\end{array} \\ \text { Nov. 23 } & 1700 & 2,710 & 6.52 & \text { Feb. } 9 & 0730 & 7,120 & 9.57 \\ \text { Nov. 30 } & 1345 & 5,190 & 8.39 & \text { Feb. 21 } & 0315 & 2,570 & 6.40\end{array}$

DISCHARGE, CUBIC FEET PER SECOND, WATER YEAR OCTOBER 1998 TO SEPTEMBER 1999 DAILY MEAN VALUES

\begin{tabular}{|c|c|c|c|c|c|c|c|c|c|c|c|c|}
\hline DAY & OCT & NOV & $\mathrm{DEC}$ & JAN & $\mathrm{FEB}$ & MAR & $A P R$ & MAY & JUN & JUL & AUG & SEP \\
\hline 1 & 148 & 140 & 1510 & 191 & 348 & 2000 & 505 & 517 & 271 & 145 & 127 & 125 \\
\hline 2 & 148 & 140 & 741 & 182 & 321 & 1510 & 479 & 529 & 285 & 144 & 128 & 127 \\
\hline 3 & 148 & 136 & 1510 & 176 & 309 & 1780 & 456 & 552 & 286 & 143 & 128 & 127 \\
\hline 4 & 148 & 136 & 984 & 174 & 301 & 1460 & 428 & 526 & 273 & 142 & 127 & 125 \\
\hline 5 & 144 & 134 & 632 & 168 & 290 & 1170 & 434 & 469 & 251 & 142 & 127 & 123 \\
\hline 6 & 141 & 138 & 512 & 168 & 521 & 1000 & 418 & 455 & 239 & 140 & 127 & 121 \\
\hline 7 & 141 & 183 & 427 & 165 & 1280 & 856 & 410 & 462 & 227 & 139 & 132 & 123 \\
\hline 8 & 141 & 208 & 379 & 162 & 1140 & 773 & 542 & 445 & 223 & 139 & 132 & 122 \\
\hline 9 & 141 & 172 & 340 & 159 & 4480 & 850 & 510 & 428 & 216 & 137 & 131 & 122 \\
\hline 10 & 141 & 156 & 305 & 158 & 2140 & 734 & 500 & 410 & 209 & 135 & 131 & 123 \\
\hline 11 & 140 & 165 & 284 & 158 & 1290 & 659 & 528 & 399 & 203 & 135 & 142 & 125 \\
\hline 12 & 138 & 153 & 273 & 156 & 966 & 614 & 582 & 400 & 197 & 135 & 135 & 123 \\
\hline 13 & 137 & 147 & 355 & 155 & 786 & 587 & 596 & 400 & 193 & 133 & 131 & 123 \\
\hline 14 & 138 & 145 & 474 & 155 & 695 & 572 & 597 & 388 & 188 & 132 & 128 & 123 \\
\hline 15 & 138 & 143 & 333 & 180 & 604 & 541 & 594 & 370 & 182 & 131 & 127 & 123 \\
\hline 16 & 136 & 150 & 299 & 263 & 704 & 533 & 599 & 356 & 180 & 131 & 126 & 124 \\
\hline 17 & 136 & 192 & 286 & 252 & 1210 & 528 & 612 & 347 & 176 & 132 & 126 & 124 \\
\hline 18 & 136 & 211 & 273 & 528 & 1180 & 532 & 661 & 345 & 172 & 132 & 125 & 124 \\
\hline 19 & 134 & 167 & 264 & 502 & 1140 & 533 & 677 & 343 & 168 & 132 & 125 & 125 \\
\hline 20 & 131 & 151 & 251 & 679 & 1240 & 530 & 697 & 336 & 168 & 133 & 124 & 123 \\
\hline 21 & 129 & 158 & 229 & 820 & 1710 & 523 & 695 & 329 & 168 & 132 & 125 & 122 \\
\hline 22 & 129 & 397 & 222 & 578 & 1050 & 506 & 643 & 326 & 164 & 134 & 125 & 120 \\
\hline 23 & 129 & 1230 & 210 & 1430 & 889 & 488 & 594 & 328 & 159 & 134 & 126 & 120 \\
\hline 24 & 158 & 883 & 210 & 1010 & 821 & 543 & 589 & 330 & 156 & 133 & 127 & 121 \\
\hline 25 & 187 & 351 & 206 & 762 & 1460 & 818 & 581 & 333 & 155 & 134 & 125 & 120 \\
\hline 26 & 151 & 282 & 205 & 641 & 1020 & 690 & 655 & 332 & 154 & 133 & 125 & 117 \\
\hline 27 & 141 & 492 & 203 & 530 & 866 & 674 & 689 & 327 & 153 & 131 & 125 & 115 \\
\hline 28 & 136 & 375 & 200 & 461 & 1160 & 606 & 600 & 319 & 149 & 133 & 127 & 114 \\
\hline 29 & 137 & 373 & 197 & 411 & --- & 566 & 548 & 309 & 147 & 132 & 124 & 114 \\
\hline 30 & 136 & 2630 & 190 & 375 & --- & 550 & 519 & 300 & 145 & 130 & 123 & 116 \\
\hline 31 & 134 & --- & 189 & 377 & --- & 536 & --- & 283 & --- & 129 & 127 & --- \\
\hline TOTAL & 4372 & 10338 & 12693 & 12126 & 29921 & 24262 & 16938 & 11993 & 5857 & 4187 & 3958 & 3654 \\
\hline MEAN & 141 & 345 & 409 & 391 & 1069 & 783 & 565 & 387 & 195 & 135 & 128 & 122 \\
\hline MAX & 187 & 2630 & 1510 & 1430 & 4480 & 2000 & 697 & 552 & 286 & 145 & 142 & 127 \\
\hline MIN & 129 & 134 & 189 & 155 & 290 & 488 & 410 & 283 & 145 & 129 & 123 & 114 \\
\hline $\mathrm{AC}-\mathrm{FT}$ & 8670 & 20510 & 25180 & 24050 & 59350 & 48120 & 33600 & 23790 & 11620 & 8300 & 7850 & 7250 \\
\hline
\end{tabular}




\section{DEER CREEK NEAR VINA, CA—Continued}

STATISTICS OF MONTHLY MEAN DATA FOR WATER YEARS 1912 - 1999, BY WATER YEAR (WY)

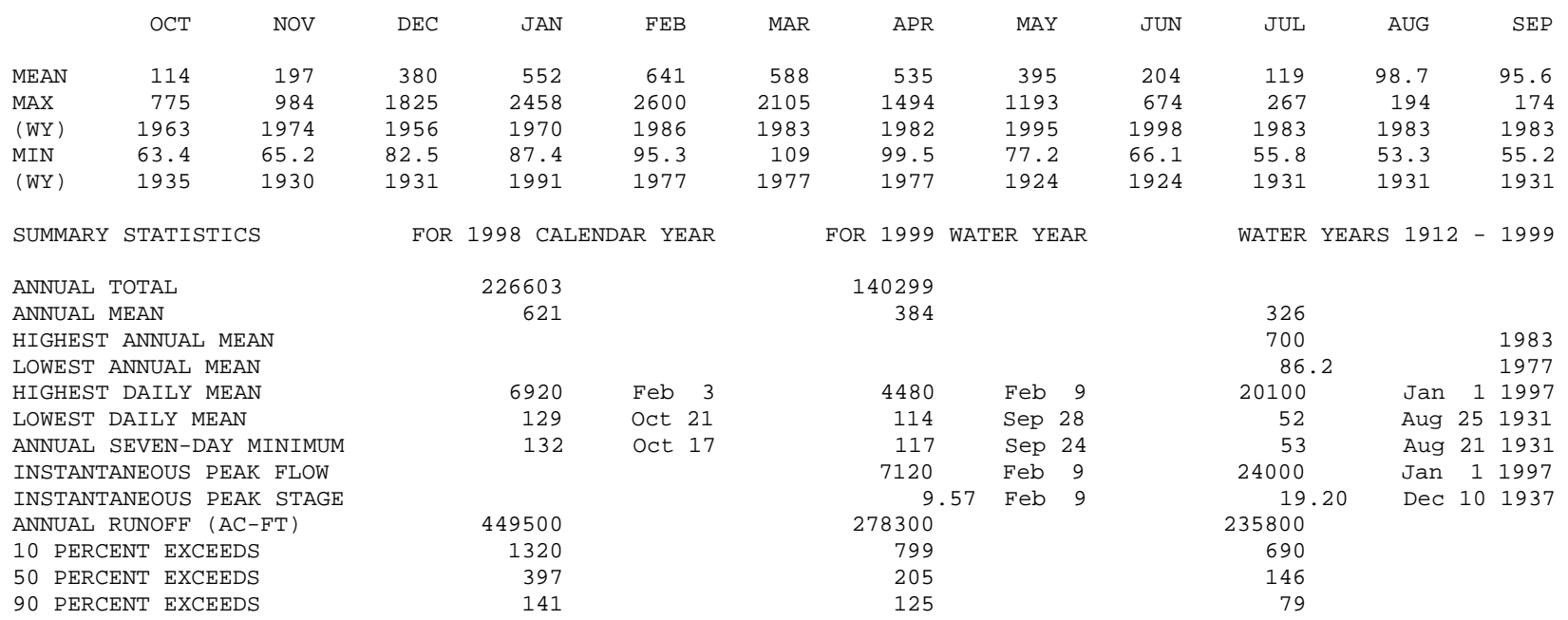




\section{WATER-QUALITY RECORDS}

PERIOD OF RECORD.—October 1998 to September 1999.

WATER TEMPERATURE: October 1998 to September 1999.

PERIOD OF DAILY RECORD.-October 1998 to September 1999.

WATER TEMPERATURE: October 1998 to September 1999.

INSTRUMENTATION.--Temperature recorder since Oct. 5, 1998.

REMARKS.-Interruption in record due to malfunction of the recording equipment.

EXTREMES FOR PERIOD OF DAILY RECORD.-

WATER TEMPERATURE: Maximum recorded, $25.5^{\circ} \mathrm{C}$, July 12-14, 1999; minimum recorded, 0.5 ${ }^{\circ} \mathrm{C}$, Dec. 23, 24, 1998.

EXTREME FOR CURRENT YEAR.-

WATER TEMPERATURE: Maximum recorded, $25.5^{\circ} \mathrm{C}$, July 12-14; minimum recorded, $0.5 \infty^{\circ} \mathrm{C}$, Dec. $23,24$.

\section{TEMPERATURE, WATER (DEG. C), WATER YEAR OCTOBER 1998 TO SEPTEMBER 1999}

\begin{tabular}{|c|c|c|c|c|c|c|c|c|c|c|c|c|}
\hline \multirow[t]{2}{*}{ DAY } & $\operatorname{MAX}$ & MIN & MAX & MIN & MAX & MIN & MAX & MIN & MAX & MIN & $\operatorname{MAX}$ & MIN \\
\hline & \multicolumn{2}{|c|}{ OCTOBER } & \multicolumn{2}{|c|}{ NOVEMBER } & \multicolumn{2}{|c|}{ DECEMBER } & \multicolumn{2}{|c|}{ JANUARY } & \multicolumn{2}{|c|}{ FEBRUARY } & \multicolumn{2}{|c|}{ MARCH } \\
\hline 1 & --- & --- & 11.0 & 9.5 & 8.5 & 8.0 & --- & --- & 5.5 & 4.5 & 8.5 & 7.5 \\
\hline 2 & --- & --- & 10.5 & 9.5 & 9.5 & 8.5 & --- & --- & 5.5 & 4.5 & 8.5 & 7.0 \\
\hline 3 & --- & --- & 11.0 & 9.5 & 9.5 & 7.0 & --- & --- & 6.0 & 5.0 & 8.5 & 7.5 \\
\hline 4 & --- & --- & 11.0 & 10.0 & 7.0 & 6.0 & --- & --- & 7.0 & 6.0 & 7.5 & 6.5 \\
\hline 5 & 14.5 & 13.0 & 10.5 & 9.5 & 6.0 & 5.0 & --- & --- & 6.5 & 5.5 & 7.0 & 6.0 \\
\hline 6 & 14.5 & 13.0 & 9.5 & 9.0 & 5.0 & 4.0 & --- & --- & 7.0 & 6.0 & 7.0 & 6.5 \\
\hline 7 & 14.5 & 12.5 & 9.5 & 9.0 & 4.5 & 3.5 & 5.5 & 4.5 & 8.5 & 7.0 & 7.5 & 6.0 \\
\hline 8 & 15.0 & 13.0 & 9.0 & 8.0 & 5.5 & 4.5 & 5.5 & 4.0 & 8.0 & 7.5 & 7.0 & 6.5 \\
\hline 9 & 14.5 & 13.0 & 8.0 & 7.0 & 5.5 & 4.5 & 5.5 & 4.5 & 8.5 & 5.5 & 7.0 & 6.0 \\
\hline 10 & 13.5 & 12.0 & 8.0 & 7.0 & 5.5 & 5.0 & 5.0 & 5.0 & 5.5 & 5.0 & 7.0 & 5.0 \\
\hline 11 & 12.5 & 11.5 & 8.5 & 7.5 & 6.0 & 5.0 & 5.5 & 4.5 & 5.5 & 5.0 & 7.5 & 5.5 \\
\hline 12 & 12.5 & 11.5 & 8.0 & 7.0 & 7.0 & 6.0 & 6.0 & 4.5 & 6.5 & 5.0 & 8.0 & 6.0 \\
\hline 13 & 14.0 & 12.0 & 8.0 & 7.0 & 8.5 & 7.0 & 6.0 & 5.5 & 6.5 & 6.0 & 9.0 & 7.0 \\
\hline 14 & 13.0 & 12.0 & 8.0 & 7.0 & 8.5 & 7.5 & 6.5 & 5.5 & 7.5 & 6.5 & 9.0 & 8.0 \\
\hline 15 & 12.5 & 11.0 & 9.0 & 8.0 & 7.5 & 6.5 & 8.0 & 6.5 & 7.0 & 6.0 & 9.5 & 7.5 \\
\hline 16 & 11.5 & 10.5 & 9.0 & 8.5 & 8.5 & 7.5 & 9.0 & 8.0 & 7.5 & 6.5 & 10.0 & 8.0 \\
\hline 17 & 11.5 & 10.0 & 9.5 & 9.0 & 9.0 & 8.0 & 9.0 & 8.5 & 8.0 & 7.5 & 11.0 & 9.0 \\
\hline 18 & 11.5 & 9.5 & 9.5 & 8.0 & 8.0 & 6.5 & 9.5 & 8.5 & 8.0 & 7.5 & 10.5 & 9.0 \\
\hline 19 & 12.5 & 10.5 & 8.0 & 7.0 & 6.5 & 5.0 & 8.5 & 8.0 & 7.5 & 6.5 & 10.0 & 9.0 \\
\hline 20 & 12.5 & 11.5 & 8.0 & 6.5 & 5.0 & 3.5 & 8.5 & 7.5 & 7.0 & 6.5 & 10.0 & 9.0 \\
\hline 21 & 12.5 & 11.5 & 9.5 & 8.0 & 3.5 & 1.5 & 7.5 & 6.5 & 7.0 & 6.0 & 9.5 & 8.0 \\
\hline 22 & 12.5 & 11.5 & 9.5 & 9.0 & 1.5 & 1.0 & 7.5 & 6.5 & 8.0 & 6.5 & 9.0 & 7.5 \\
\hline 23 & 12.0 & 11.0 & 11.0 & 9.5 & 1.5 & .5 & 7.5 & 6.0 & 9.0 & 7.0 & 9.0 & 8.0 \\
\hline 24 & 12.0 & 11.5 & 9.5 & 8.5 & 2.0 & .5 & 6.0 & 5.0 & 8.5 & 8.0 & 9.5 & 8.5 \\
\hline 25 & 12.0 & 11.0 & 9.0 & 8.5 & 4.0 & 1.5 & 5.5 & 4.5 & 8.5 & 7.5 & 10.5 & 9.0 \\
\hline 26 & 12.0 & 10.5 & 9.5 & 8.0 & 4.5 & 4.0 & 6.5 & 5.5 & 8.0 & 6.5 & 11.5 & 9.5 \\
\hline 27 & 12.0 & 11.0 & 9.5 & 9.0 & 5.0 & 4.0 & 6.0 & 5.0 & 9.0 & 7.5 & 10.0 & 8.0 \\
\hline 28 & 12.5 & 11.5 & 9.0 & 8.5 & 5.5 & 5.0 & 5.5 & 5.0 & 10.0 & 8.5 & 9.0 & 7.0 \\
\hline 29 & 12.0 & 11.0 & 8.5 & 8.0 & 5.0 & 4.5 & 5.5 & 4.5 & --- & --- & 9.0 & 7.0 \\
\hline 30 & 11.5 & 10.5 & 10.5 & 8.5 & 5.5 & 4.0 & 5.0 & 4.0 & --- & --- & 8.5 & 7.0 \\
\hline 31 & 10.5 & 9.5 & --- & --- & 6.5 & 5.5 & 5.5 & 5.0 & --- & --- & 8.0 & 6.0 \\
\hline MONTH & --- & --- & 11.0 & 6.5 & 9.5 & .5 & --- & --- & 10.0 & 4.5 & 11.5 & 5.0 \\
\hline
\end{tabular}




\section{DEER CREEK NEAR VINA, CA—Continued}

\section{TEMPERATURE, WATER (DEG. C), WATER YEAR OCTOBER 1998 TO SEPTEMBER 1999}

\begin{tabular}{|c|c|c|c|c|c|c|c|c|c|c|c|c|}
\hline DAY & MAX & MIN & MAX & MIN & MAX & MIN & MAX & MIN & MAX & MIN & MAX & MIN \\
\hline & \multicolumn{2}{|c|}{ APRIL } & \multicolumn{2}{|c|}{ MAY } & \multicolumn{2}{|c|}{ JUNE } & \multicolumn{2}{|c|}{ JULY } & \multicolumn{2}{|c|}{ AUGUST } & \multicolumn{2}{|c|}{ SEPTEMBER } \\
\hline 1 & 8.0 & 6.0 & 15.0 & 11.5 & 18.0 & 16.0 & 24.5 & 22.0 & 22.5 & 20.0 & 18.0 & 16.5 \\
\hline 2 & 9.0 & 6.0 & 13.5 & 11.0 & 17.0 & 14.0 & 24.0 & 21.5 & 23.0 & 20.5 & 18.0 & 15.5 \\
\hline 3 & 9.0 & 7.5 & 11.5 & 9.5 & 14.0 & 12.0 & 23.0 & 20.5 & 23.5 & 20.5 & 18.5 & 16.0 \\
\hline 4 & 8.5 & 6.0 & 11.5 & 8.0 & 15.0 & 11.0 & 21.5 & 19.0 & 23.5 & 21.0 & 19.0 & 16.5 \\
\hline 5 & 8.5 & 7.5 & 13.5 & 9.5 & 17.5 & 14.0 & 21.5 & 18.5 & 23.0 & 21.0 & 19.0 & 17.0 \\
\hline 6 & 9.0 & 6.5 & 15.0 & 11.5 & 18.5 & 15.5 & 22.0 & 19.0 & 21.5 & 20.0 & 19.5 & 17.5 \\
\hline 7 & 9.5 & 7.0 & 14.5 & 12.0 & 17.0 & 15.0 & 22.0 & 19.5 & 21.5 & 19.0 & 20.0 & 17.5 \\
\hline 8 & 9.5 & 7.0 & 13.5 & 10.5 & 17.0 & 14.0 & 22.5 & 19.5 & 21.0 & 18.5 & 20.0 & 18.0 \\
\hline 9 & 7.5 & 5.0 & 13.0 & 10.0 & 16.0 & 14.0 & 23.0 & 20.0 & 21.5 & 19.0 & 20.0 & 18.5 \\
\hline 10 & 7.0 & 6.0 & 12.5 & 10.0 & 17.5 & 14.0 & 23.5 & 20.5 & 20.5 & 19.0 & 20.0 & 18.0 \\
\hline 11 & 10.0 & 6.5 & 15.0 & 11.0 & 18.5 & 15.0 & 24.5 & 21.5 & 22.0 & 19.5 & 21.0 & 18.5 \\
\hline 12 & 11.0 & 8.0 & 15.5 & 13.0 & 19.0 & 16.0 & 25.5 & 22.5 & 22.0 & 19.5 & 20.5 & 18.5 \\
\hline 13 & 12.0 & 9.0 & 14.5 & 11.5 & 20.0 & 17.0 & 25.5 & 23.5 & 21.5 & 19.5 & 20.0 & 18.5 \\
\hline 14 & 12.5 & 10.0 & 13.5 & 10.5 & 21.0 & 17.5 & 25.5 & 23.0 & 21.5 & 19.0 & 20.5 & 18.0 \\
\hline 15 & 13.0 & 10.0 & 13.5 & 10.0 & 20.5 & 18.0 & 24.5 & 22.0 & 21.0 & 18.5 & 20.0 & 18.0 \\
\hline 16 & 13.5 & 10.5 & 14.0 & 10.5 & 21.0 & 17.5 & 23.5 & 21.5 & 21.5 & 18.5 & 20.0 & 18.0 \\
\hline 17 & 14.0 & 11.0 & 14.5 & 11.5 & 21.0 & 18.0 & 22.5 & 20.5 & 21.5 & 19.0 & 19.5 & 17.5 \\
\hline 18 & 14.0 & 11.5 & 16.0 & 13.0 & 21.0 & 18.5 & 22.0 & 19.5 & 21.5 & 19.0 & 18.5 & 17.5 \\
\hline 19 & 13.5 & 11.0 & 16.0 & 13.5 & 21.5 & 18.5 & 22.0 & 19.5 & 21.5 & 18.5 & 19.5 & 17.0 \\
\hline 20 & 13.5 & 11.0 & 16.0 & 13.5 & 21.0 & 18.5 & 21.5 & 19.0 & 21.0 & 18.5 & 20.0 & 18.0 \\
\hline 21 & 12.5 & 10.5 & 17.0 & 13.5 & 22.5 & 19.0 & 21.5 & 19.0 & 21.5 & 19.0 & 20.5 & 18.0 \\
\hline 22 & 12.0 & 9.5 & 18.0 & 14.5 & 23.0 & 20.0 & 21.5 & 19.0 & 22.5 & 20.0 & 20.0 & 18.0 \\
\hline 23 & 13.0 & 9.5 & 19.0 & 15.5 & 24.0 & 21.0 & 21.5 & 19.0 & 23.5 & 21.0 & 20.5 & 18.5 \\
\hline 24 & 13.5 & 10.5 & 19.0 & 16.0 & 23.5 & 21.0 & 21.5 & 19.0 & 23.5 & 21.5 & 20.5 & 18.5 \\
\hline 25 & 13.5 & 11.0 & 20.0 & 17.0 & 22.5 & 20.0 & 22.0 & 19.0 & 22.5 & 20.5 & 20.0 & 18.5 \\
\hline 26 & 14.5 & 12.0 & 20.5 & 17.5 & 21.5 & 19.0 & 23.0 & 20.0 & 21.5 & 20.0 & 19.0 & 17.0 \\
\hline 27 & 13.0 & 10.5 & 20.5 & 17.5 & 21.5 & 19.0 & 22.5 & 20.0 & 21.5 & 20.5 & 17.5 & 16.0 \\
\hline 28 & 11.0 & 8.5 & 20.0 & 17.0 & 22.5 & 19.5 & 22.5 & 19.5 & 22.5 & 20.0 & 17.0 & 15.5 \\
\hline 29 & 12.0 & 8.0 & 19.0 & 16.5 & 23.5 & 20.5 & 22.5 & 20.0 & 22.5 & 20.5 & 17.5 & 15.5 \\
\hline 30 & 13.5 & 10.0 & 18.5 & 15.5 & 24.5 & 21.5 & 22.5 & 20.0 & 21.5 & 19.5 & 17.5 & 15.5 \\
\hline 31 & --- & --- & 19.0 & 16.0 & --- & --- & 22.5 & 19.5 & 19.5 & 17.5 & --- & --- \\
\hline MONTH & 14.5 & 5.0 & 20.5 & 8.0 & 24.5 & 11.0 & 25.5 & 18.5 & 23.5 & 17.5 & 21.0 & 15.5 \\
\hline
\end{tabular}




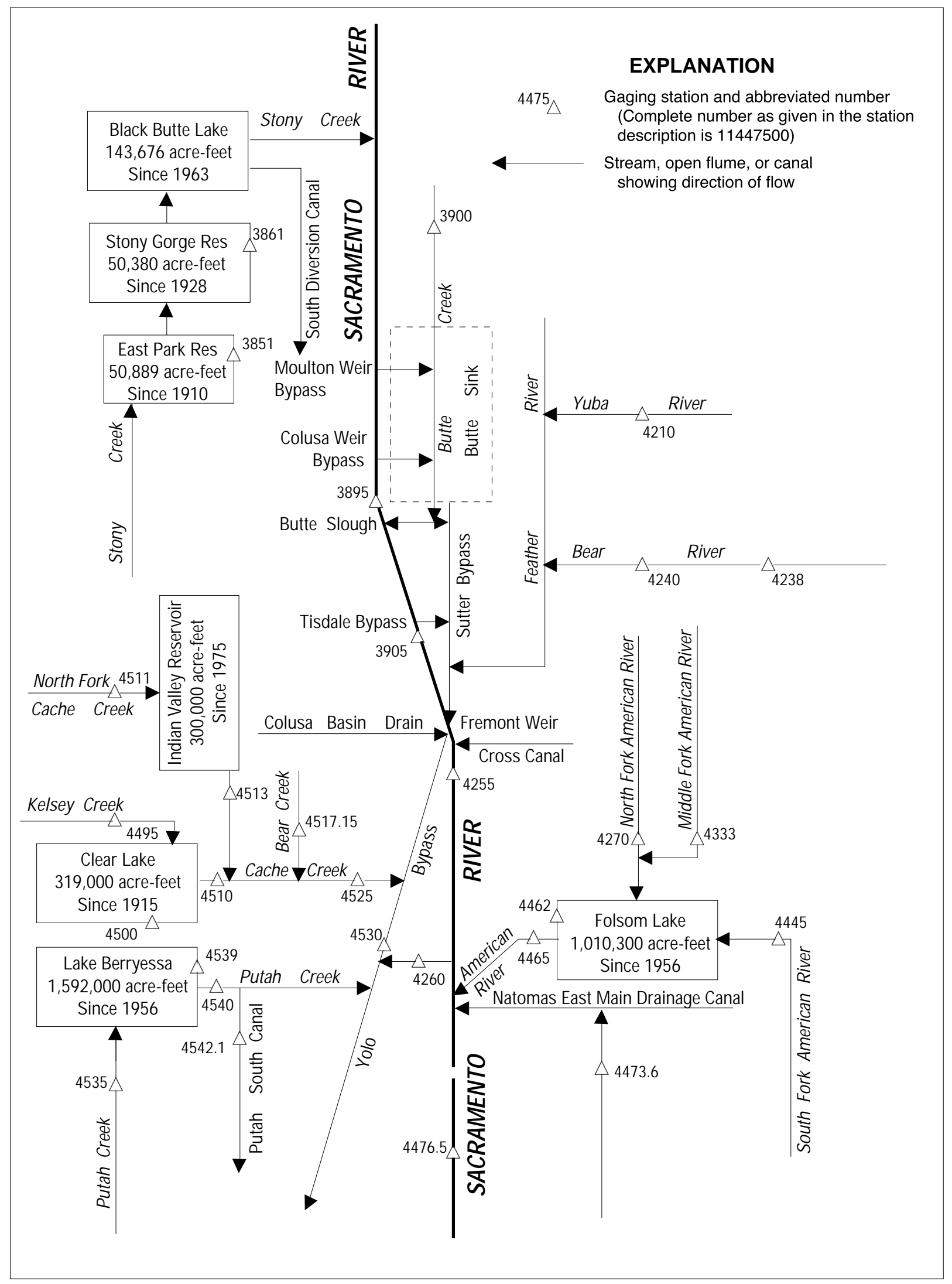

Figure 27. Diversions and storage in lower Sacramento River Basin. 


\section{EAST PARK RESERVOIR NEAR STONYFORD}

LOCATION.-Lat 39²1'24", long 122³0'53", in SW 1/4 NE 1/4 sec.3, T.17 N., R.6 W., Colusa County, Hydrologic Unit 18020115, near south side of spillway section on East Park Dam on Little Stony Creek, 1.9 mi southeast of Stonyford.

DRAINAGE AREA.-98.2 $\mathrm{mi}^{2}$.

PERIOD OF RECORD.-October 1969 to current year.

GAGE.-Nonrecording gage read once daily. Datum of gage is sea level (levels by U.S. Bureau of Reclamation).

REMARKS.--Reservoir is formed by a concrete arch-type dam. Storage began in 1910. Capacity, 48,210 acre-ft, between elevations 1,131.68 ft, invert of sluice pipe, and 1,198.18 ft, crest of spillway. Capacity increased to 50,889 acre-ft with the addition of flashboards to an elevation of 1,199.68 ft. Dead storage, 279 acre-ft. Records of contents provided by U.S. Bureau of Reclamation. See schematic diagram of lower Sacramento River Basin.

EXTREMES FOR PERIOD OF RECORD.-Maximum contents, 53,500 acre-ft, Mar. 30, 1974, elevation, 1,201.10 ft; minimum, 280 acre-ft, Aug. 8 to Oct. 31, 1972, Apr. 30 to Nov. 1, 1977, elevation, 1,131.68 ft.

EXTREMES FOR CURRENT YEAR. -Maximum contents, 49,990 acre-ft, Feb. 9, elevation, 1,199.18 ft; minimum, 42,680 acre-ft, Sept. 30, elevation, $1,194.92 \mathrm{ft}$.

\section{STONY GORGE RESERVOIR NEAR ELK CREEK}

LOCATION.-Lat 39³5’09", long 122³1'54", in NE 1/4 SE 1/4 sec.16, T.20 N., R.6 W., Glenn County, Hydrologic Unit 18020115, on south end of Stony Gorge Dam on Stony Creek, 1.3 mi southeast of Elk Creek.

DRAINAGE AREA.-301 $\mathrm{mi}^{2}$.

PERIOD OF RECORD.-October 1969 to current year.

GAGE.-Nonrecording gage read once daily. Datum of gage is sea level (levels by U.S. Bureau of Reclamation).

REMARKS.--Reservoir is formed by slab and buttress-type dam. Storage began in 1928. Capacity, 50,380 acre- $\mathrm{ft}$ between elevations $728.0 \mathrm{ft}$, top of low intake, and $841.0 \mathrm{ft}$, crest of spillway. No dead storage. Records of contents provided by U.S. Bureau of Reclamation. See schematic diagram of lower Sacramento River Basin.

EXTREMES FOR PERIOD OF RECORD.-Maximum contents, 54,630 acre-ft, Mar. 26, 1971, elevation, $844.20 \mathrm{ft}$; minimum, 3,810 acre-ft, Nov. 6, 1971, elevation, $779.20 \mathrm{ft}$.

EXTREMES FOR CURRENT YEAR.-Maximum contents, 50,383 acre-ft, Apr. 19, elevation, 841.00 ft; minimum, 35,055 acre-ft, Sept. 29, elevation, $828.01 \mathrm{ft}$.

MONTHEND ELEVATION AND CONTENTS AT 0800 HOURS, WATER YEAR OCTOBER 1998 TO SEPTEMBER 1999

$\begin{array}{cccccc}\text { Elevation } & \text { Change in } & \text { Contents } \\ (\mathrm{ft}) & (\text { acre-ft) } & \begin{array}{c}\text { contents } \\ (\text { acre-ft })\end{array} & \begin{array}{c}\text { Elevation } \\ (\mathrm{ft})\end{array} & \begin{array}{c}\text { Contents } \\ \text { (acre-ft) }\end{array} & \begin{array}{c}\text { Change in } \\ \text { contents } \\ (\text { acre-ft })\end{array}\end{array}$

\section{EAST PARK RESERVOIR}

Sept. $30 \ldots \ldots \ldots \ldots \ldots$. $1,196.64$

Oct. $31 \ldots \ldots \ldots \ldots \ldots$. $1,196.14$

Nov. 30. . . . . . . . . . 1, 196.69

Dec. $31 \ldots \ldots \ldots \ldots \ldots$. $1,198.22$

CAL YR 1998

Jan. $31 \ldots \ldots \ldots \ldots \ldots$. 1,198.33

Feb. $28 \ldots \ldots \ldots \ldots$. 1,198.51

Mar. $31 \ldots \ldots \ldots \ldots \ldots$. . . . . . . . . . .

Apr. $30 \ldots \ldots \ldots \ldots \ldots \quad 1,198.35$

May $31 \ldots \ldots \ldots \ldots \ldots$. . . . . . . . . . . . .

June $30 \ldots \ldots \ldots \ldots$. . . . 1,197.78

July $31 \ldots \ldots \ldots \ldots \ldots$. $1,196.72$

Aug. 31 . . . . . . . . . . 1,195.86

Sept. $30 \ldots \ldots \ldots \ldots \quad 1,194.92$
45,553

44,705

45,637

48,284

$\begin{array}{rr}- & 25,251 \\ 48,479 & 195 \\ 48,798 & 319 \\ 48,798 & 0 \\ 48,514 & -284 \\ 48,178 & -336 \\ 47,514 & -664 \\ 45,688 & -1,826 \\ 44,236 & -1,452 \\ 42,680 & -1,556\end{array}$

$-1,199$
-848
932
2,647

11386100 STONY GORGE RESERVOIR

829.34

829.59

830.11

832.31

36,485
36,758
37,326

37,326
39,791

$-$

831.40

833.90

838.97

840.44

840.40

837.81

838.31

838.50

828.03

$\begin{array}{rr}- & 6,771 \\ 38,761 & \\ 41,626 & -1,030 \\ 47,785 & 2,865 \\ 49,661 & 6,159 \\ 49,610 & 1,876 \\ 46,336 & -51 \\ 46,958 & -3,274 \\ 47,197 & 622 \\ 35,076 & -12,121\end{array}$

WTR YR 1999.

$-2,873$

$-1,409$ 
11389500 SACRAMENTO RIVER AT COLUSA, CA

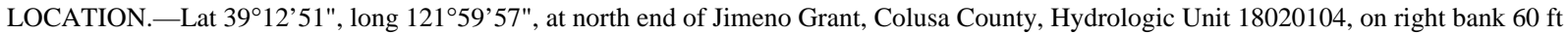
downstream from highway bridge at Colusa and at mile 89.4 upstream from Sacramento.

DRAINAGE AREA.-12,090 $\mathrm{mi}^{2}$.

\section{WATER-DISCHARGE RECORDS}

PERIOD OF RECORD.-April 1921 to current year (prior to October 1940, low-water periods only).

REVISED RECORDS.-WSP 1345: 1952. WDR CA-77-4: Drainage area

GAGE.-Water-stage recorder and crest-stage gage. Datum of gage is $2.95 \mathrm{ft}$ below sea level. Prior to December 1930 , water-stage recorder in center fender pier $50 \mathrm{ft}$ upstream from bridge at same datum.

REMARKS.-Records good. Natural flow of stream affected by storage reservoirs, including Shasta Lake (station 11370000) since 1943, power development, bypassing for flood control, diversions for irrigation, and return flow from irrigated areas. When discharge exceeds about $30,000 \mathrm{ft}^{3} / \mathrm{s}$, flow begins over Colusa Weir, $2.5 \mathrm{mi}$ upstream on left bank, into Butte Sink and Sutter Bypass. See schematic diagram of lower Sacramento River Basin.

EXTREMES FOR PERIOD OF RECORD.-Maximum discharge (water years 1941-99), 51,800 ft³/s, Mar. 4, 1983, gage height, 68.50 ft; maximum gage height, $69.20 \mathrm{ft}$, Feb. 18, 1942; minimum recorded, $820 \mathrm{ft}^{3} / \mathrm{s}$, Ju1y 25, 26, 1931, gage height, $34.79 \mathrm{ft}$.

DISCHARGE, CUBIC FEET PER SECOND, WATER YEAR OCTOBER 1998 TO SEPTEMBER 1999 DAILY MEAN VALUES

\begin{tabular}{|c|c|c|c|c|c|c|c|c|c|c|c|c|}
\hline DAY & $\mathrm{OCT}$ & NOV & $\mathrm{DEC}$ & JAN & FEB & MAR & APR & MAY & JUN & JUL & AUG & SEP \\
\hline 1 & 9150 & 6610 & 35400 & 9730 & 13400 & 35300 & 15300 & 12100 & 10700 & 9480 & 9560 & 6920 \\
\hline 2 & 9020 & 7040 & 34900 & 9510 & 12900 & 37300 & 14400 & 12000 & 10500 & 9130 & 9590 & 7070 \\
\hline 3 & 8660 & 7030 & 29700 & 9360 & 12400 & 36500 & 13700 & 12100 & 10900 & 8920 & 9330 & 7070 \\
\hline 4 & 8560 & 6820 & 34100 & 9200 & 12000 & 36200 & 13100 & 12100 & 11400 & 8720 & 9270 & 7030 \\
\hline 5 & 8490 & 6690 & 32100 & 9050 & 11700 & 35700 & 12800 & 11800 & 11700 & 8770 & 9130 & 6900 \\
\hline 6 & 8420 & 6600 & 31400 & 8960 & 16000 & 35300 & 12700 & 11400 & 11500 & 8910 & 8680 & 7170 \\
\hline 7 & 8370 & 7140 & 32100 & 8900 & 22400 & 34800 & 13500 & 11400 & 10900 & 8920 & 8370 & 7210 \\
\hline 8 & 8280 & 7940 & 31600 & 8830 & 36000 & 34500 & 13100 & 11700 & 10500 & 8900 & 8050 & 7010 \\
\hline 9 & 8160 & 8480 & 31400 & 8720 & 35200 & 34300 & 13100 & 11500 & 10200 & 8850 & 7900 & 6930 \\
\hline 10 & 7910 & 8190 & 30400 & 8590 & 39700 & 35200 & 13600 & 11300 & 9930 & 8710 & 7910 & 7200 \\
\hline 11 & 7870 & 7920 & 27900 & 8560 & 37800 & 35000 & 14600 & 11100 & 9620 & 8730 & 7810 & 7310 \\
\hline 12 & 7710 & 8760 & 26900 & 8580 & 32800 & 34300 & 20100 & 10900 & 9170 & 8920 & 7520 & 7130 \\
\hline 13 & 7430 & 8530 & 26500 & 8560 & 31900 & 33200 & 20800 & 11000 & 8960 & 8850 & 7450 & 6860 \\
\hline 14 & 7230 & 9050 & 26400 & 8540 & 31000 & 31600 & 18900 & 10900 & 8840 & 9110 & 7250 & 6910 \\
\hline 15 & 7000 & 9680 & 27300 & 8560 & 29800 & 29400 & 18800 & 10500 & 8770 & 9140 & 7240 & 6910 \\
\hline 16 & e 6850 & e 9940 & 23300 & 8760 & 30200 & 27500 & 18500 & 9640 & 9000 & 9120 & 7100 & 7350 \\
\hline 17 & e 6650 & e10100 & 21000 & 9100 & 34100 & 25000 & 18600 & 9810 & 8940 & 9190 & 6800 & 7400 \\
\hline 18 & e 6500 & e10800 & 19900 & 9960 & 38800 & 23400 & 18600 & 9490 & 8920 & 9310 & 6600 & 7370 \\
\hline 19 & e 6400 & e13900 & 19300 & 14200 & 38300 & 22100 & 18200 & 9460 & 9050 & 9530 & 6530 & 7240 \\
\hline 20 & e 6350 & e14700 & 18400 & 14900 & 37300 & 20100 & 17700 & 9390 & 9160 & 9660 & 6570 & 6980 \\
\hline 21 & 6260 & 14600 & 16700 & 15000 & 36300 & 18500 & 17300 & 9450 & 9240 & 9630 & 6570 & 7000 \\
\hline 22 & 6160 & 15300 & 15000 & 20000 & 39500 & 17500 & 16000 & 9840 & 9370 & 9530 & 6540 & 6950 \\
\hline 23 & 6050 & 18700 & 13500 & 21700 & 37000 & 16000 & 14900 & 10400 & 9300 & 9120 & 6450 & 6880 \\
\hline 24 & e 6100 & 22800 & 12400 & 27700 & 34300 & 15100 & 14000 & 11000 & 9380 & 9100 & 6510 & 6670 \\
\hline 25 & e 6500 & 27500 & 11600 & 27600 & 33200 & 19500 & 13200 & 10800 & 9760 & 9180 & 6610 & 6690 \\
\hline 26 & e 7150 & 22100 & 11300 & 24500 & 34700 & 33000 & 12900 & 10400 & 9540 & 9310 & 6670 & 6700 \\
\hline 27 & e 7100 & 20900 & 11200 & 22500 & 34800 & 30000 & 12800 & 10300 & 9570 & 9330 & 6730 & 6600 \\
\hline 28 & e 7100 & 25400 & 11000 & 20300 & 34800 & 25200 & 12800 & 10700 & 9730 & 9290 & 6720 & 6520 \\
\hline 29 & 6770 & 22500 & 11000 & 17900 & --- & 21800 & 12500 & 10900 & 9970 & 9360 & 6760 & 6360 \\
\hline 30 & 6740 & 22600 & 10700 & 15800 & --- & 18900 & 12500 & 11000 & 9910 & 9350 & 6870 & 6080 \\
\hline 31 & 6570 & --- & 10000 & 14400 & --- & 16400 & --- & 11100 & --- & 9460 & 6840 & --- \\
\hline TOTAL & 227510 & 388320 & 694400 & 417970 & 838300 & 868600 & 459000 & 335480 & 294430 & 283530 & 231930 & 208420 \\
\hline MEAN & 7339 & 12940 & 22400 & 13480 & 29940 & 28020 & 15300 & 10820 & 9814 & 9146 & 7482 & 6947 \\
\hline MAX & 9150 & 27500 & 35400 & 27700 & 39700 & 37300 & 20800 & 12100 & 11700 & 9660 & 9590 & 7400 \\
\hline MIN & 6050 & 6600 & 10000 & 8540 & 11700 & 15100 & 12500 & 9390 & 8770 & 8710 & 6450 & 6080 \\
\hline $\mathrm{AC}-\mathrm{FT}$ & 451300 & 770200 & 1377000 & 829000 & 1663000 & 1723000 & 910400 & 665400 & 584000 & 562400 & 460000 & 413400 \\
\hline
\end{tabular}

e Estimated. 


\section{SACRAMENTO RIVER AT COLUSA, CA—Continued}

STATISTICS OF MONTHLY MEAN DATA FOR WATER YEARS 1946 - 1999, BY WATER YEAR (WY)

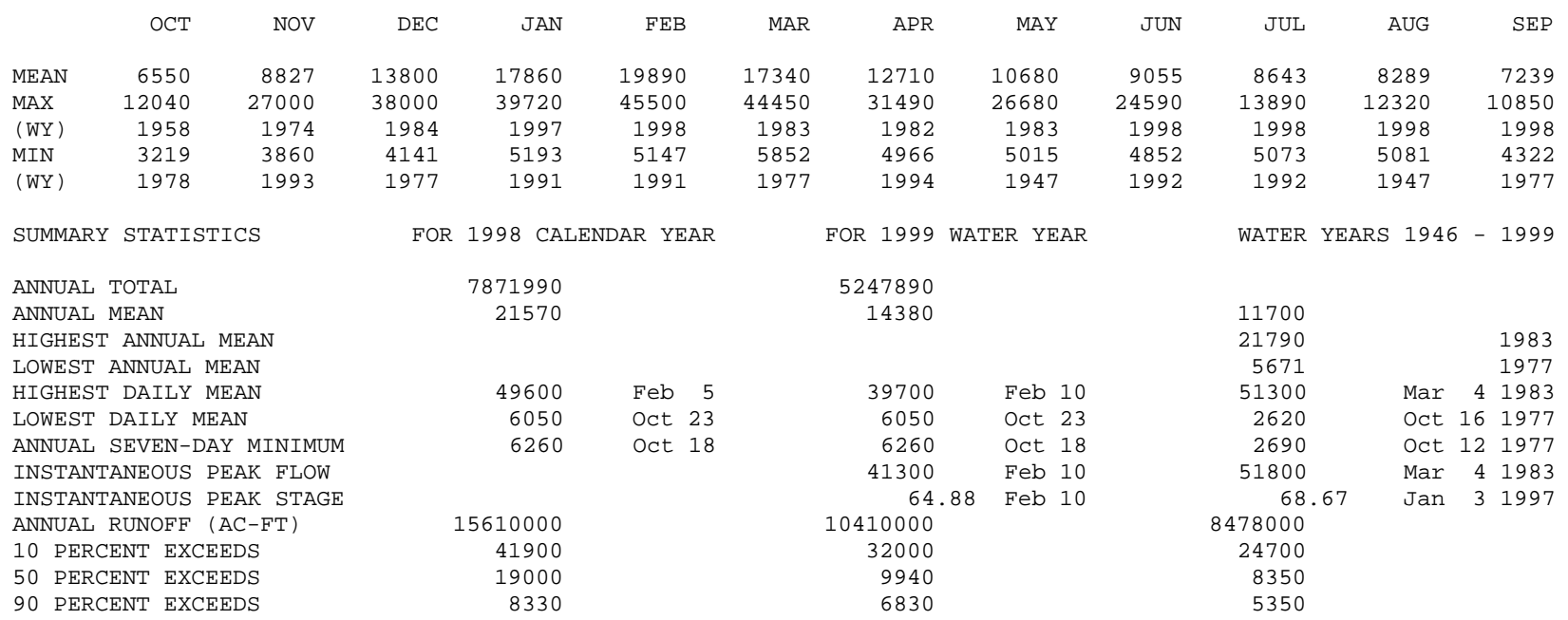




\section{WATER-QUALITY RECORDS}

PERIOD OF RECORD.-Water years 1959-66, 1973-80, 1996 to current year.

CHEMICAL DATA: Water years 1959-66, 1996 to current year.

SPECIFIC CONDUCTANCE: Water years 1995-98.

WATER TEMPERATURE: Water years 1975, 1977-80, 1995-98.

SEDIMENT: Water years 1973-80, 1996 to current year.

PERIOD OF DAILY RECORD.-

SPECIFIC CONDUCTANCE: Water years 1995-98.

WATER TEMPERATURE: Water years 1995-98.

INSTRUMENTATION.-Water-quality monitor since October 1995.

WATER-QUALITY DATA, WATER YEAR OCTOBER 1998 TO SEPTEMBER 1999

\begin{tabular}{|c|c|c|c|c|c|c|c|c|c|}
\hline DATE & TIME & $\begin{array}{c}\text { DIS- } \\
\text { CHARGE, } \\
\text { INST. } \\
\text { CUBIC } \\
\text { FEET } \\
\text { PER } \\
\text { SECOND } \\
(00061)\end{array}$ & $\begin{array}{l}\text { SPE- } \\
\text { CIFIC } \\
\text { CON- } \\
\text { DUCT- } \\
\text { ANCE } \\
(\text { US /CM) } \\
(00095)\end{array}$ & $\begin{array}{c}\text { PH } \\
\text { WATER } \\
\text { WHOLE } \\
\text { FIELD } \\
\text { (STAND- } \\
\text { ARD } \\
\text { UNITS) } \\
(00400)\end{array}$ & $\begin{array}{c}\text { TEMPER- } \\
\text { ATURE } \\
\text { WATER } \\
(\text { DEG C) } \\
(00010)\end{array}$ & $\begin{array}{l}\text { BARO- } \\
\text { METRIC } \\
\text { PRES- } \\
\text { SURE } \\
\text { (MM } \\
\text { OF } \\
\text { HG) } \\
(00025)\end{array}$ & $\begin{array}{c}\text { OXYGEN, } \\
\text { DIS- } \\
\text { SOLVED } \\
(\text { MG } / L) \\
(00300)\end{array}$ & $\begin{array}{c}\text { OXYGEN, } \\
\text { DIS- } \\
\text { SOLVED } \\
\text { (PER- } \\
\text { CENT } \\
\text { SATUR- } \\
\text { ATION) } \\
\text { (00301) }\end{array}$ & $\begin{array}{c}\text { ALKA- } \\
\text { LINITY } \\
\text { WAT.DIS } \\
\text { GRAN T. } \\
\text { FIELD } \\
\text { CACO3 } \\
\text { (MG/L) } \\
(29802)\end{array}$ \\
\hline OCT & & & & & & & & & \\
\hline$\stackrel{21}{21} \ldots$ & 1200 & 6250 & 143 & 7.9 & 14.6 & 758 & 10.2 & 101 & 64 \\
\hline$\underset{\mathrm{DEC}}{12 \ldots}$ & 1020 & 8920 & 137 & 8.0 & 11.3 & 767 & 10.6 & 96 & 50 \\
\hline$\underset{\text { JAN }}{29} \ldots$ & 1130 & 11000 & 156 & 7.9 & 8.8 & 763 & 11.7 & 101 & 59 \\
\hline$\underset{F E B}{20}$ & 1100 & 15100 & 121 & 7.9 & 10.6 & 756 & 10.8 & 98 & 41 \\
\hline$\underset{M A R}{17} \cdots$ & 1100 & 33500 & 126 & 7.6 & 8.8 & 766 & 11.3 & 97 & 51 \\
\hline$\underset{A P R}{11 \cdots}$ & 1040 & 35100 & 120 & 7.8 & 8.7 & 761 & 11.5 & 99 & 48 \\
\hline$\underset{M A Y}{08}$ & 1030 & 13200 & 170 & 7.9 & 11.2 & 765 & 10.9 & 99 & 62 \\
\hline $\begin{array}{l}06 \ldots \\
\text { JUN }\end{array}$ & 1030 & 11400 & 137 & 7.8 & 15.5 & 758 & -- & -- & 54 \\
\hline${ }_{\text {JUL }}^{03 \ldots}$ & 1040 & 10800 & 124 & 7.5 & 14.7 & 760 & 8.9 & 88 & 48 \\
\hline $\begin{array}{c}20 \ldots \\
\text { AUG }\end{array}$ & 1100 & 9670 & 117 & 8.0 & 17.6 & 761 & 10.1 & 106 & 45 \\
\hline $\operatorname{SEP}_{\operatorname{SEP}}^{17} \cdots$ & 1000 & 6820 & 131 & 8.1 & 19.4 & 762 & 4.6 & 50 & 58 \\
\hline $09 \ldots$ & 1130 & 6920 & 127 & 8.1 & 18.5 & 756 & 7.7 & 83 & 52 \\
\hline
\end{tabular}

PARTICLE-SIZE DISTRIBUTION, WATER YEAR OCTOBER 1998 TO SEPTEMBER 1999

\begin{tabular}{|c|c|c|c|c|c|c|}
\hline DATE & TIME & $\begin{array}{c}\text { DIS- } \\
\text { CHARGE, } \\
\text { INST. } \\
\text { CUBIC } \\
\text { FEET } \\
\text { PER } \\
\text { SECOND } \\
(00061)\end{array}$ & $\begin{array}{c}\text { TEMPER- } \\
\text { ATURE } \\
\text { WATER } \\
(\text { DEG C) } \\
(00010)\end{array}$ & $\begin{array}{l}\text { SEDI- } \\
\text { MENT, } \\
\text { SUS- } \\
\text { PENDED } \\
(\text { MG/L) } \\
(80154)\end{array}$ & $\begin{array}{c}\text { SEDI- } \\
\text { MENT, } \\
\text { DIS- } \\
\text { CHARGE, } \\
\text { SUS- } \\
\text { PENDED } \\
\text { (T/DAY) } \\
(80155)\end{array}$ & $\begin{array}{c}\text { SED. } \\
\text { SUSP. } \\
\text { SIEVE } \\
\text { DIAM. } \\
\text { O FINER } \\
\text { THAN } \\
.062 \text { MM } \\
(70331)\end{array}$ \\
\hline OCT & & & & & & \\
\hline$\underset{\text { NOV }}{21 \ldots N}$ & 1200 & 6250 & 14.6 & 41 & 692 & 85 \\
\hline$\underset{\mathrm{DEC}}{12 \ldots \mathrm{N}}$ & 1020 & 8920 & 11.3 & 57 & 1370 & 77 \\
\hline$\underset{\text { JAN }}{29 \ldots N}$ & 1130 & 11000 & 8.8 & 50 & 1480 & 87 \\
\hline$\underset{F E B}{20 \ldots N}$ & 1100 & 15100 & 10.6 & 123 & 5010 & 75 \\
\hline$\underset{M A R}{17 \ldots N}$ & 1100 & 33500 & 8.8 & 52 & 4700 & 90 \\
\hline$\underset{A P R}{11 \ldots N}$ & 1040 & 35100 & 8.7 & 54 & 5120 & 79 \\
\hline$\underset{M A Y}{08 \ldots N}$ & 1030 & 13200 & 11.2 & 83 & 2960 & 70 \\
\hline$\underset{\text { JUN }}{06 \ldots \mathrm{N}}$ & 1030 & 11400 & 15.5 & 50 & 1540 & 85 \\
\hline$\underset{\text { JUL }}{03 \ldots \mathrm{N}}$ & 1040 & 10800 & 14.7 & 50 & 1460 & 60 \\
\hline$\underset{\text { AUG }}{20 \ldots N}$ & 1100 & 9670 & 17.6 & 28 & 731 & 62 \\
\hline$\underset{\operatorname{SEP}}{17 \ldots N}$ & 1000 & 6820 & 19.4 & 34 & 626 & 76 \\
\hline $09 \ldots \mathrm{N}$ & 1130 & 6920 & 18.5 & 37 & 691 & 58 \\
\hline
\end{tabular}

N Suspended-sediment concentration value determined from a sample collected and processed according to National Water-Quality Assessment (NAWQA) protocol. 


\section{BUTTE CREEK BELOW DIVERSION DAM, NEAR STIRLING CITY, CA}

LOCATION.-Lat 3958'53", long 121³5'15", unsurveyed, T.25 N., R.3 E., Butte County, Hydrologic Unit 18020120, on left bank $400 \mathrm{ft}$ downstream from diversion dam, 0.1 mi upstream from Haw Creek, and 6.2 mi northwest of Stirling City.

DRAINAGE AREA.- $-61.3 \mathrm{mi}^{2}$.

PERIOD OF RECORD.—January to February 1986, June 1986 to current year (low-flow records only).

GAGE.-Water-stage recorder. Elevation of gage is 2,840 ft above sea level, from topographic map.

REMARKS. - No records computed above $40 \mathrm{ft}^{3} / \mathrm{s}$. Flow regulated by diversion dam $400 \mathrm{ft}$ upstream. Most of the water is diverted at diversion dam to Butte Creek Canal and then to De Sabla Powerplant (station 11389750).

COOPERATION.--Records were collected by Pacific Gas \& Electric Co., under general supervision of the U.S. Geological Survey, in connection with a Federal Energy Regulatory Commission project.

DISCHARGE, CUBIC FEET PER SECOND, WATER YEAR OCTOBER 1998 TO SEPTEMBER 1999 DAILY MEAN VALUES

\begin{tabular}{|c|c|c|c|c|c|c|c|c|c|c|c|c|}
\hline DAY & OCT & NOV & DEC & JAN & FEB & MAR & $\mathrm{APR}$ & MAY & JUN & JUL & AUG & SEP \\
\hline 1 & 19 & 20 & --- & --- & --- & --- & --- & --- & --- & 22 & 20 & 21 \\
\hline 2 & 19 & 20 & --- & --- & --- & --- & --- & --- & --- & 23 & 20 & 21 \\
\hline 3 & 20 & 20 & --- & 40 & --- & --- & --- & --- & --- & 23 & 20 & 21 \\
\hline 4 & 20 & 20 & --- & 34 & --- & --- & --- & --- & --- & 23 & 20 & 21 \\
\hline 5 & 19 & 20 & --- & 28 & --- & --- & --- & --- & --- & 24 & 20 & 21 \\
\hline 6 & 19 & 30 & --- & 25 & --- & --- & --- & --- & --- & 24 & 20 & 21 \\
\hline 7 & 19 & --- & --- & 24 & --- & --- & --- & --- & --- & 23 & 20 & 21 \\
\hline 8 & 19 & --- & --- & 23 & --- & --- & --- & --- & --- & 22 & 20 & 20 \\
\hline 9 & 19 & --- & --- & 22 & --- & --- & --- & --- & --- & 20 & 20 & 20 \\
\hline 10 & 20 & 40 & --- & 22 & --- & --- & --- & --- & --- & 20 & 20 & 20 \\
\hline 11 & 19 & 40 & --- & 21 & --- & --- & --- & --- & --- & 19 & 21 & 21 \\
\hline 12 & 19 & 31 & --- & 21 & --- & --- & --- & --- & --- & 19 & 21 & 22 \\
\hline 13 & 20 & 25 & --- & 20 & --- & --- & --- & --- & --- & 19 & 21 & 22 \\
\hline 14 & 20 & 25 & --- & 21 & --- & --- & --- & --- & --- & 20 & 21 & 22 \\
\hline 15 & 20 & 25 & --- & --- & --- & --- & --- & --- & --- & 20 & 21 & 22 \\
\hline 16 & 20 & 26 & --- & --- & --- & --- & --- & --- & --- & 20 & 20 & 22 \\
\hline 17 & 20 & --- & --- & --- & --- & --- & --- & --- & --- & 20 & 20 & 22 \\
\hline 18 & 20 & 38 & --- & --- & --- & --- & --- & --- & --- & 20 & 20 & 22 \\
\hline 19 & 19 & 30 & --- & --- & --- & --- & --- & --- & --- & 20 & 20 & 22 \\
\hline 20 & 19 & 27 & --- & --- & --- & --- & --- & --- & --- & 20 & 20 & 22 \\
\hline 21 & 19 & --- & --- & --- & --- & --- & --- & --- & --- & 20 & 20 & 22 \\
\hline 22 & 19 & --- & --- & --- & --- & --- & --- & --- & --- & 20 & 20 & 22 \\
\hline 23 & 19 & --- & --- & --- & --- & --- & --- & --- & 34 & 20 & 20 & 22 \\
\hline 24 & 34 & --- & --- & --- & --- & --- & --- & --- & 29 & 20 & 21 & 22 \\
\hline 25 & --- & --- & --- & --- & --- & --- & --- & --- & 27 & 22 & 21 & 22 \\
\hline 26 & 36 & --- & --- & --- & --- & --- & --- & --- & 26 & 20 & 20 & 21 \\
\hline 27 & 29 & --- & --- & --- & --- & --- & --- & --- & 25 & 20 & 20 & 21 \\
\hline 28 & 21 & --- & --- & --- & --- & --- & --- & --- & 24 & 20 & 20 & 21 \\
\hline 29 & 20 & --- & --- & --- & --- & --- & --- & --- & 21 & 20 & 20 & 22 \\
\hline 30 & 20 & --- & --- & --- & --- & --- & --- & --- & 22 & 20 & 21 & 22 \\
\hline 31 & 20 & --- & --- & --- & --- & --- & --- & --- & --- & 20 & 20 & --- \\
\hline TOTAL & --- & --- & --- & --- & --- & --- & --- & --- & --- & 643 & 628 & 643 \\
\hline MEAN & --- & --- & --- & --- & --- & --- & --- & --- & --- & 20.7 & 20.3 & 21.4 \\
\hline MAX & --- & --- & --- & --- & --- & --- & --- & --- & --- & 24 & 21 & 22 \\
\hline MIN & --- & --- & --- & --- & --- & --- & --- & --- & --- & 19 & 20 & 20 \\
\hline $\mathrm{AC}-\mathrm{FT}$ & --- & --- & --- & --- & --- & --- & --- & --- & --- & 1280 & 1250 & 1280 \\
\hline
\end{tabular}


LOCATION.-Lat 3954'05", long 121³7’24", in NW 1/4 NE 1/4 sec.34, T.24 N., R.3 E., Butte County, Hydrologic Unit 18020120, on left bank $30 \mathrm{ft}$ downstream from diversion dam, $0.2 \mathrm{mi}$ upstream from American Ravine, and 2.0 mi north of De Sabla.

DRAINAGE AREA.- $-96.4 \mathrm{mi}^{2}$.

PERIOD OF RECORD.-April 1992 to current year (low-flow records only).

GAGE.-Water-stage recorder and concrete control. Elevation of gage is 1,900 ft above sea level, from topographic map.

REMARKS.-No records computed above $60 \mathrm{ft}^{3} / \mathrm{s}$. Flow regulated by Forks of Butte Diversion Dam $30 \mathrm{ft}$ upstream. Water is diverted out of creek to Butte Canal 7.4 mi upstream by Pacific Gas and Electric Co. Water is diverted $30 \mathrm{ft}$ upstream to Forks of Butte Powerplant (station 11389747).

COOPERATION.--Records were collected by Energy Growth Partnership I, under general supervision of the U.S. Geological Survey, in connection with a Federal Energy Regulatory Commission project.

DISCHARGE, CUBIC FEET PER SECOND, WATER YEAR OCTOBER 1998 TO SEPTEMBER 1999

DAILY MEAN VALUES

\begin{tabular}{|c|c|c|c|c|c|c|c|c|c|c|c|c|}
\hline DAY & $\mathrm{OCT}$ & NOV & $\mathrm{DEC}$ & JAN & FEB & MAR & $\mathrm{APR}$ & MAY & JUN & JUL & AUG & SEP \\
\hline 1 & 44 & 43 & --- & 48 & 48 & --- & --- & --- & 48 & 53 & 41 & 38 \\
\hline 2 & 44 & 43 & --- & 48 & 48 & --- & 58 & --- & 48 & 54 & 53 & 38 \\
\hline 3 & 47 & 42 & --- & 48 & 48 & --- & 50 & --- & 48 & 54 & 41 & 38 \\
\hline 4 & 45 & 42 & --- & 48 & 48 & --- & 48 & --- & 48 & 53 & 42 & 38 \\
\hline 5 & 44 & 43 & --- & 48 & 48 & --- & 58 & --- & 48 & 54 & 42 & e38 \\
\hline 6 & 43 & 47 & --- & 48 & 47 & --- & 48 & --- & 48 & 53 & 43 & e38 \\
\hline 7 & 43 & 48 & 53 & 48 & --- & --- & 48 & --- & 48 & 52 & 43 & 37 \\
\hline 8 & 42 & 48 & 48 & 48 & --- & --- & --- & --- & 48 & 51 & 43 & 37 \\
\hline 9 & 42 & 48 & 48 & 60 & --- & --- & --- & --- & 48 & 48 & 43 & 37 \\
\hline 10 & 43 & 48 & 48 & --- & --- & --- & --- & --- & 48 & 47 & 44 & 37 \\
\hline 11 & 43 & 48 & 48 & --- & --- & --- & --- & --- & 48 & 46 & 44 & 37 \\
\hline 12 & 43 & 49 & 48 & --- & --- & --- & --- & --- & 48 & 46 & 43 & 38 \\
\hline 13 & 42 & 50 & 48 & 60 & --- & --- & --- & --- & 48 & 46 & 40 & 38 \\
\hline 14 & 43 & 49 & 48 & 59 & --- & --- & --- & --- & 48 & 45 & 40 & 37 \\
\hline 15 & 42 & 48 & 48 & 50 & --- & --- & --- & --- & 48 & 45 & 40 & 37 \\
\hline 16 & 42 & 51 & 48 & 48 & --- & --- & --- & --- & 48 & 45 & 39 & 37 \\
\hline 17 & 42 & 49 & 48 & 53 & --- & --- & --- & --- & 48 & 45 & 39 & 37 \\
\hline 18 & 42 & 48 & 48 & --- & --- & --- & --- & --- & 48 & 44 & 39 & 37 \\
\hline 19 & 41 & 59 & 48 & --- & --- & --- & --- & --- & 48 & 44 & 39 & 37 \\
\hline 20 & 41 & 53 & 48 & --- & --- & --- & --- & --- & 48 & 44 & 39 & 37 \\
\hline 21 & 41 & 51 & 49 & --- & --- & --- & --- & 51 & 48 & 44 & 38 & 37 \\
\hline 22 & 41 & 50 & 48 & --- & --- & --- & --- & 48 & 48 & 44 & 38 & 37 \\
\hline 23 & 41 & --- & 48 & --- & --- & --- & --- & 48 & 48 & 44 & 50 & 37 \\
\hline 24 & 47 & --- & 48 & --- & --- & --- & --- & 48 & 54 & 44 & 38 & 37 \\
\hline 25 & 48 & 48 & 48 & --- & --- & --- & --- & 50 & 52 & 44 & 38 & 37 \\
\hline 26 & 58 & 48 & 48 & --- & --- & --- & --- & 49 & --- & 43 & 38 & 37 \\
\hline 27 & 56 & 48 & 48 & --- & --- & --- & --- & 48 & 60 & 43 & 39 & 36 \\
\hline 28 & 45 & 48 & 48 & --- & --- & --- & --- & 48 & 58 & 43 & 38 & 36 \\
\hline 29 & 43 & 57 & 48 & 49 & --- & --- & --- & 48 & 55 & 42 & 38 & 36 \\
\hline 30 & 42 & --- & 48 & 48 & --- & --- & --- & 48 & 54 & 42 & 38 & 36 \\
\hline 31 & 42 & --- & 48 & 48 & --- & --- & --- & 48 & --- & 42 & 38 & --- \\
\hline TOTAL & 1362 & --- & --- & --- & --- & --- & --- & --- & --- & 1444 & 1268 & 1114 \\
\hline MEAN & 43.9 & --- & --- & --- & --- & --- & --- & --- & --- & 46.6 & 40.9 & 37.1 \\
\hline MAX & 58 & --- & --- & --- & --- & --- & --- & --- & --- & 54 & 53 & 38 \\
\hline MIN & 41 & --- & --- & --- & --- & --- & --- & --- & --- & 42 & 38 & 36 \\
\hline $\mathrm{AC}-\mathrm{FT}$ & 2700 & --- & --- & --- & --- & --- & --- & --- & --- & 2860 & 2520 & 2210 \\
\hline a & 87 & 3810 & 8810 & 7690 & 13030 & 15370 & 14750 & 14120 & 3530 & 0 & 0 & 0 \\
\hline
\end{tabular}

e Estimated.

a Diversion, in acre-feet, to Forks of Butte Powerplant, provided by Energy Growth Partnership I. 


\section{BUTTE CREEK BELOW CENTERVILLE DIVERSION DAM, NEAR PARADISE, CA}

LOCATION.-Lat 3952'01", long 121'37' 58", in SW 1/4 NW 1/4 sec.10, T.23 N., R.3 E., Butte County, Hydrologic Unit 18020120, on left bank $400 \mathrm{ft}$ downstream from Centerville Diversion Dam, 0.2 mi downstream from De Sabla Powerplant, and 6.8 mi north of Paradise.

DRAINAGE AREA.- $101 \mathrm{mi}^{2}$.

PERIOD OF RECORD.-November 1985 to February 1986, June 1986 to current year (low-flow records only).

GAGE.-Water-stage recorder. Elevation of gage is $1,130 \mathrm{ft}$ above sea level, from topographic map.

REMARKS.- No records computed above $60 \mathrm{ft}^{3} / \mathrm{s}$. Flow regulated by several reservoirs and diversions upstream. Most of the water is diverted at Centerville Diversion Dam to the Centerville Powerplant (station 11389775).

COOPERATION.--Records were collected by Pacific Gas \& Electric Co., under general supervision of the U.S. Geological Survey, in connection with a Federal Energy Regulatory Commission project.

DISCHARGE, CUBIC FEET PER SECOND, WATER YEAR OCTOBER 1998 TO SEPTEMBER 1999 DAILY MEAN VALUES

\begin{tabular}{|c|c|c|c|c|c|c|c|c|c|c|c|c|}
\hline DAY & OCT & NOV & $\mathrm{DEC}$ & JAN & FEB & MAR & APR & MAY & JUN & JUL & AUG & SEP \\
\hline 1 & 46 & --- & --- & --- & --- & --- & --- & --- & --- & 56 & 47 & 45 \\
\hline 2 & 46 & --- & --- & --- & --- & --- & --- & --- & --- & 56 & 47 & 45 \\
\hline 3 & 47 & --- & --- & --- & --- & --- & --- & --- & --- & 56 & 51 & 45 \\
\hline 4 & 46 & --- & --- & --- & --- & --- & --- & --- & --- & 56 & 51 & 45 \\
\hline 5 & 46 & --- & --- & --- & --- & --- & --- & --- & --- & 56 & 50 & 45 \\
\hline 6 & 46 & --- & --- & --- & --- & --- & --- & --- & --- & 56 & 50 & 45 \\
\hline 7 & 46 & --- & --- & --- & --- & --- & --- & --- & --- & 51 & 51 & 45 \\
\hline 8 & 46 & --- & --- & --- & --- & --- & --- & --- & --- & 48 & 50 & 45 \\
\hline 9 & 46 & --- & --- & --- & --- & --- & --- & --- & --- & 48 & 50 & 45 \\
\hline 10 & 46 & --- & --- & --- & --- & --- & --- & --- & --- & 48 & 51 & 45 \\
\hline 11 & 46 & --- & --- & --- & --- & --- & --- & --- & --- & 46 & 52 & 45 \\
\hline 12 & 46 & --- & --- & --- & --- & --- & --- & --- & --- & 48 & 52 & 45 \\
\hline 13 & 46 & --- & --- & --- & --- & --- & --- & --- & --- & 52 & 51 & 45 \\
\hline 14 & 46 & --- & --- & --- & --- & --- & --- & --- & --- & 46 & 51 & 45 \\
\hline 15 & 46 & --- & --- & --- & --- & --- & --- & --- & --- & 46 & 51 & 45 \\
\hline 16 & 46 & --- & --- & --- & --- & --- & --- & --- & --- & 46 & 51 & 45 \\
\hline 17 & 46 & --- & --- & --- & --- & --- & --- & --- & --- & 46 & --- & 45 \\
\hline 18 & 46 & --- & --- & --- & --- & --- & --- & --- & --- & 46 & --- & 45 \\
\hline 19 & 46 & --- & --- & --- & --- & --- & --- & --- & --- & 46 & 49 & 45 \\
\hline 20 & 46 & --- & --- & --- & --- & --- & --- & --- & --- & 45 & 45 & 49 \\
\hline 21 & 46 & --- & --- & --- & --- & --- & --- & --- & --- & 46 & 45 & 45 \\
\hline 22 & 46 & --- & --- & --- & --- & --- & --- & --- & --- & 47 & 45 & 45 \\
\hline 23 & --- & --- & --- & --- & --- & --- & --- & --- & --- & 47 & 45 & 45 \\
\hline 24 & --- & --- & --- & --- & --- & --- & --- & --- & --- & 47 & 45 & 45 \\
\hline 25 & --- & --- & --- & --- & --- & --- & --- & --- & --- & 47 & 45 & 45 \\
\hline 26 & --- & --- & --- & --- & --- & --- & --- & --- & --- & 47 & 45 & 45 \\
\hline 27 & --- & --- & --- & --- & --- & --- & --- & --- & --- & 47 & 45 & 45 \\
\hline 28 & --- & --- & --- & --- & --- & --- & --- & --- & 57 & 47 & 45 & 45 \\
\hline 29 & --- & --- & --- & --- & --- & --- & --- & --- & 58 & 47 & 45 & 45 \\
\hline 30 & --- & --- & --- & --- & --- & --- & --- & --- & 57 & 47 & 45 & 45 \\
\hline 31 & --- & --- & --- & --- & --- & --- & --- & --- & --- & 47 & 45 & --- \\
\hline TOTAL & --- & --- & --- & --- & --- & --- & --- & --- & --- & 1514 & --- & 1354 \\
\hline MEAN & --- & --- & --- & --- & --- & --- & --- & --- & --- & 48.8 & --- & 45.1 \\
\hline MAX & --- & --- & --- & --- & --- & --- & --- & --- & --- & 56 & --- & 49 \\
\hline MIN & --- & --- & --- & --- & --- & --- & --- & --- & --- & 45 & --- & 45 \\
\hline $\mathrm{AC}-\mathrm{FT}$ & --- & --- & --- & --- & --- & --- & --- & --- & --- & 3000 & --- & 2690 \\
\hline $\mathrm{a}$ & 6260 & 1860 & 7890 & 6130 & 4370 & 4610 & 6750 & 7820 & 8230 & 6800 & 6250 & 5290 \\
\hline
\end{tabular}

CAL YR 1998 a 83300

WTR YR 1999 a 72270

a Diversion, in acre-feet, to Centerville Powerplant, provided by Pacific Gas \& Electric Co. 
11389800 TOADTOWN CANAL ${ }^{-}$ABOVE BUTTE CANAL, NEAR STIRLING CITY, CA

LOCATION.-Lat 3953'09", long 121³6’35", in NE 1/4 NW 1/4 sec.2, T.23 N., R.3 E., Butte County, Hydrologic Unit 18020120, on right bank $600 \mathrm{ft}$ upstream from Butte Canal and $4.6 \mathrm{mi}$ west of Stirling City.

PERIOD OF RECORD.-October 1986 to current year. Monthly discharges for water years 1931-86 are published as a line item to Butte Creek near Chico (station 11390000).

GAGE.-Water-stage recorder and Parshall flume. Elevation of gage is 2,790 ft above sea level, from topographic map.

REMARKS.-Canal diverts from right bank of West Branch Feather River, in sec.16, T.24 N., R.4 E. at Hendricks Diversion Dam to Hendricks Canal, flows through tunnel down Long Ravine to Toadtown Canal, and discharges into Butte Canal. Butte Canal flows to De Sabla Powerplant (station 11389750) on Butte Creek.

COOPERATION.-Records were collected by Pacific Gas \& Electric Co., under general supervision of the U.S. Geological Survey, in connection with a Federal Energy Regulatory Commission project.

EXTREMES FOR PERIOD OF RECORD.-Maximum daily discharge, $127 \mathrm{ft}^{3}$ /s, Feb. 12, May 20, 1995, no flow at times in most years.

DISCHARGE, CUBIC FEET PER SECOND, WATER YEAR OCTOBER 1998 TO SEPTEMBER 1999

DAILY MEAN VALUES

\begin{tabular}{|c|c|c|c|c|c|c|c|c|c|c|c|c|}
\hline DAY & $\mathrm{OCT}$ & NOV & $\mathrm{DEC}$ & JAN & FEB & MAR & APR & MAY & JUN & JUL & AUG & SEP \\
\hline 1 & 86 & 43 & 68 & 92 & 89 & 90 & 96 & 102 & 112 & 79 & 66 & 66 \\
\hline 2 & 86 & 42 & 90 & 90 & 95 & 93 & 102 & 102 & 112 & 77 & 65 & 66 \\
\hline 3 & 92 & 42 & 104 & 88 & 95 & 97 & 102 & 45 & 111 & 75 & 62 & 67 \\
\hline 4 & 88 & 42 & 101 & 86 & 95 & 102 & 96 & 2.0 & 110 & 73 & 59 & 66 \\
\hline 5 & 86 & 43 & 98 & 84 & 85 & 108 & 90 & 2.3 & 109 & 71 & 58 & 66 \\
\hline 6 & 85 & 44 & 72 & 83 & 70 & 107 & 89 & 6.0 & 109 & 69 & 58 & 65 \\
\hline 7 & 86 & 68 & 77 & 82 & 75 & 107 & 99 & 4.5 & 110 & 68 & 59 & 66 \\
\hline 8 & 86 & 66 & 88 & 80 & 74 & 103 & 78 & 4.4 & 111 & 67 & 58 & 69 \\
\hline 9 & 83 & 52 & 94 & 78 & 76 & 97 & 75 & 4.4 & 114 & 65 & 58 & 69 \\
\hline 10 & 83 & 50 & 99 & 78 & 80 & 96 & 72 & 4.3 & 115 & 64 & 61 & 69 \\
\hline 11 & 82 & 50 & 99 & 77 & 78 & 103 & 72 & 4.2 & 112 & 63 & 71 & 68 \\
\hline 12 & 81 & 48 & 93 & 76 & 87 & 109 & 91 & 4.1 & 114 & 62 & 67 & 67 \\
\hline 13 & 81 & 47 & 90 & 75 & 90 & 103 & 116 & 4.0 & 113 & 89 & 66 & 67 \\
\hline 14 & 78 & 46 & 100 & 75 & 90 & 97 & 115 & 4.3 & 113 & 89 & 65 & 66 \\
\hline 15 & 76 & 46 & 109 & 87 & 90 & 106 & 115 & 4.4 & 113 & 87 & 65 & 66 \\
\hline 16 & 71 & 48 & 109 & 85 & 85 & 113 & 115 & 4.3 & 113 & 62 & 65 & 68 \\
\hline 17 & 62 & 71 & 109 & 87 & 84 & 109 & 115 & 4.2 & 113 & 60 & 64 & 67 \\
\hline 18 & 61 & 58 & 110 & 87 & 81 & 105 & 114 & 4.6 & 114 & 60 & 64 & 67 \\
\hline 19 & 59 & 50 & 99 & 81 & 77 & 105 & 115 & 58 & 114 & 59 & 63 & 67 \\
\hline 20 & 57 & 48 & 87 & 73 & 80 & 100 & 115 & 118 & 114 & 59 & 67 & 46 \\
\hline 21 & 61 & 53 & 83 & 70 & 72 & 96 & 115 & 112 & 113 & 60 & 67 & 34 \\
\hline 22 & 60 & 80 & 70 & 72 & 83 & 96 & 109 & 112 & 107 & 60 & 67 & 33 \\
\hline 23 & 48 & 97 & 70 & 84 & 97 & 96 & 104 & 111 & 101 & 59 & 69 & 33 \\
\hline 24 & 57 & 74 & 66 & 73 & 98 & 97 & 103 & 111 & 99 & 59 & 67 & 33 \\
\hline 25 & 59 & 88 & 67 & 84 & 100 & 105 & 103 & 111 & 98 & 58 & 68 & 33 \\
\hline 26 & 46 & 90 & 67 & 99 & 96 & 109 & 103 & 112 & 96 & 58 & 68 & 33 \\
\hline 27 & 43 & 93 & 67 & 97 & 95 & 107 & 108 & 112 & 93 & 70 & 68 & 32 \\
\hline 28 & 43 & 91 & 79 & 97 & 97 & 108 & 108 & 112 & 88 & 69 & 67 & 32 \\
\hline 29 & 43 & 92 & 96 & 98 & --- & 109 & 102 & 112 & 85 & 69 & 66 & 32 \\
\hline 30 & 42 & 77 & 95 & 92 & --- & 103 & 102 & 112 & 81 & 68 & 66 & 32 \\
\hline 31 & 41 & --- & 95 & 85 & --- & 96 & --- & 112 & --- & 67 & 66 & --- \\
\hline TOTAL & 2112 & 1839 & 2751 & 2595 & 2414 & 3172 & 3039 & 1716.0 & 3207 & 2095 & 2000 & 1645 \\
\hline MEAN & 68.1 & 61.3 & 88.7 & 83.7 & 86.2 & 102 & 101 & 55.4 & 107 & 67.6 & 64.5 & 54.8 \\
\hline MAX & 92 & 97 & 110 & 99 & 100 & 113 & 116 & 118 & 115 & 89 & 71 & 69 \\
\hline MIN & 41 & 42 & 66 & 70 & 70 & 90 & 72 & 2.0 & 81 & 58 & 58 & 32 \\
\hline $\mathrm{AC}-\mathrm{FT}$ & 4190 & 3650 & 5460 & 5150 & 4790 & 6290 & 6030 & 3400 & 6360 & 4160 & 3970 & 3260 \\
\hline a & 7540 & 7070 & 7660 & 8480 & 7350 & 9160 & 9170 & 4800 & 10010 & 7690 & 6900 & 5730 \\
\hline
\end{tabular}

a Discharge, in acre-feet, at De Sabla Powerplant provided by Pacific Gas \& Electric Co. 


\section{TOADTOWN CANAL ABOVE BUTTE CANAL, NEAR STIRLING CITY, CA—Continued}

STATISTICS OF MONTHLY MEAN DATA FOR WATER YEARS 1987 - 1999, BY WATER YEAR (WY)

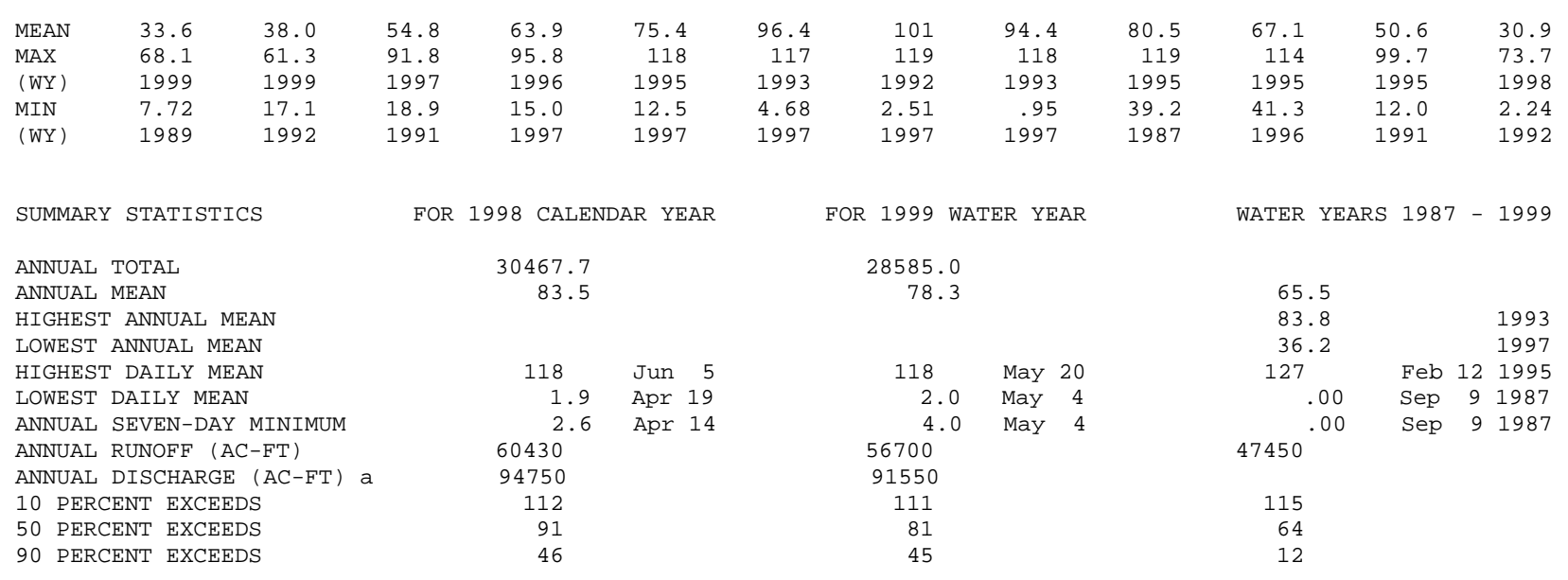

a Discharge, in acre-feet, at De Sabla Powerplant provided by Pacific Gas \& Electric Co. 


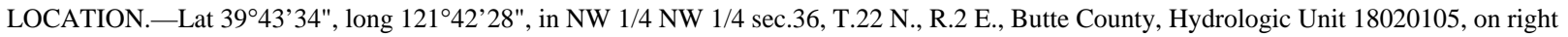
bank 0.7 mi downstream from Little Butte Creek and 7.5 mi east of Chico.

DRAINAGE AREA.-147 $\mathrm{mi}^{2}$.

WATER-DISCHARGE RECORDS

PERIOD OF RECORD.- October 1930 to current year. Monthly discharge only for some periods, published in WSP 1315-A.

REVISED RECORDS.-WSP 1445: 1953(M). WSP 1931: Drainage area.

GAGE.-Water-stage recorder and crest-stage gage. Elevation of gage is $320 \mathrm{ft}$ above sea level, from topographic map. Prior to Aug. 13, 1944, water-stage recorder at site 0.4 mi upstream at different datum. Aug. 13, 1944, to June 5, 1986, at datum $3.00 \mathrm{ft}$ higher.

REMARKS.-Records excellent. Flow slightly regulated by storage in Magalia Reservoir, usable capacity, 2,640 acre-ft, and since 1957 by Paradise Reservoir, usable capacity, 11,500 acre-ft. Diversions upstream from station for irrigation and domestic use of about 7,000 acre-ft annually. Butte Creek receives water above station from West Branch Feather River by way of Toadtown Canal (11389800).

EXTREMES FOR PERIOD OF RECORD.-Maximum discharge, 35,600 ft ${ }^{3} / \mathrm{s}$, Jan. 1, 1997, gage height, $15.06 \mathrm{ft}$, in gage well, $15.7 \mathrm{ft}$ from floodmarks, on basis of slope-area measurement of peak flow; maximum gage height, $17.52 \mathrm{ft}$, Feb. 17, 1986, present datum; minimum discharge, $10 \mathrm{ft}^{3} / \mathrm{s}$, Nov. 29, 1952.

EXTREMES FOR CURRENT YEAR.-Peak discharges greater than base discharge of 2,700 $\mathrm{ft}^{3} / \mathrm{s}$, or maximum:

\begin{tabular}{|c|c|c|c|c|c|c|c|}
\hline Date & Time & $\begin{array}{l}\text { Discharge } \\
\left(\mathrm{ft}^{3} / \mathrm{s}\right)\end{array}$ & $\begin{array}{l}\text { Gage height } \\
\text { (ft) }\end{array}$ & Date & Time & $\begin{array}{c}\text { Discharge } \\
\left(\mathrm{ft}^{3} / \mathrm{s}\right)\end{array}$ & $\begin{array}{l}\text { Gage height } \\
\text { (ft) }\end{array}$ \\
\hline Dec. 3 & 1000 & 2,860 & 3.89 & Feb. 9 & 0800 & 8,270 & 6.96 \\
\hline Jan. 23 & 0445 & 2,720 & 3.78 & & & & \\
\hline
\end{tabular}

DISCHARGE, CUBIC FEET PER SECOND, WATER YEAR OCTOBER 1998 TO SEPTEMBER 1999 DAILY MEAN VALUES

\begin{tabular}{|c|c|c|c|c|c|c|c|c|c|c|c|c|}
\hline DAY & $\mathrm{OCT}$ & NOV & $\mathrm{DEC}$ & JAN & FEB & MAR & APR & MAY & JUN & JUL & AUG & SEP \\
\hline 1 & 204 & 172 & e1600 & 271 & 460 & 2080 & 592 & 586 & 458 & 229 & 188 & 167 \\
\hline 2 & 205 & 169 & e995 & 264 & 441 & 1650 & 573 & 596 & 465 & 218 & 196 & 168 \\
\hline 3 & 224 & 165 & 1700 & 264 & 426 & 2060 & 553 & 551 & 443 & 206 & 205 & 168 \\
\hline 4 & 213 & 166 & 1080 & 257 & 413 & 1650 & 528 & 455 & 445 & 207 & 191 & 167 \\
\hline 5 & 205 & 170 & 711 & 255 & 390 & 1390 & 560 & 420 & 416 & 219 & 190 & 164 \\
\hline 6 & 200 & 170 & 560 & 255 & 639 & 1220 & 539 & 407 & 394 & 230 & 203 & 164 \\
\hline 7 & 199 & 324 & 464 & 254 & 2030 & 1080 & 535 & 425 & 372 & 224 & 193 & 163 \\
\hline 8 & 202 & 277 & 428 & 246 & 1620 & 1020 & 702 & 439 & 373 & 209 & 170 & 168 \\
\hline 9 & 199 & 208 & 397 & 237 & 4960 & 1050 & 666 & 437 & 357 & 206 & 168 & 167 \\
\hline 10 & 200 & 195 & 374 & 240 & 2430 & 917 & 619 & 437 & 365 & 200 & 174 & 169 \\
\hline 11 & 198 & 212 & 356 & 237 & 1640 & 845 & 688 & 434 & 353 & 195 & 196 & 166 \\
\hline 12 & 193 & 191 & 344 & 237 & 1320 & 795 & 711 & 453 & 357 & 194 & 186 & 164 \\
\hline 13 & 192 & 176 & 341 & 232 & 1110 & 751 & 750 & 427 & 337 & 223 & 181 & 164 \\
\hline 14 & 191 & 172 & 376 & 226 & 995 & 737 & 757 & 417 & 325 & 228 & 177 & 163 \\
\hline 15 & 188 & 168 & 354 & 242 & 872 & 711 & 728 & 385 & 316 & 226 & 176 & 161 \\
\hline 16 & 185 & 172 & 347 & 294 & 1010 & 699 & 713 & 380 & 308 & 199 & 173 & 164 \\
\hline 17 & 170 & 262 & 334 & 364 & 1740 & 669 & 703 & 375 & 302 & 187 & 168 & 164 \\
\hline 18 & 164 & 231 & 326 & 819 & 1550 & 637 & 709 & 386 & 310 & 179 & 174 & 163 \\
\hline 19 & 160 & 193 & 321 & 764 & 1520 & 602 & 702 & 409 & 302 & 173 & 168 & 166 \\
\hline 20 & 154 & 182 & 299 & 1180 & 1410 & 619 & 710 & 495 & 303 & 175 & 171 & 147 \\
\hline 21 & 160 & 197 & 290 & 1020 & 1720 & 613 & 713 & 477 & 302 & 182 & 172 & 124 \\
\hline 22 & 159 & 385 & 294 & 780 & 1350 & 595 & 689 & 466 & 289 & 180 & 171 & 124 \\
\hline 23 & 148 & e1220 & 282 & 2070 & 1170 & 584 & 644 & 483 & 276 & 179 & 174 & 121 \\
\hline 24 & 189 & e870 & 277 & 1250 & 1050 & 638 & 628 & 474 & 262 & 174 & 172 & 121 \\
\hline 25 & 236 & 404 & 269 & 886 & 1520 & 977 & 645 & 507 & 256 & 180 & 169 & 119 \\
\hline 26 & 185 & 338 & 265 & 785 & 1230 & 846 & 703 & 499 & 262 & 174 & 169 & 120 \\
\hline 27 & 181 & 465 & 260 & 661 & 1080 & 763 & 709 & 504 & 266 & 179 & 170 & 121 \\
\hline 28 & 171 & 395 & 264 & 582 & 1720 & 683 & 640 & 489 & 255 & 186 & 170 & 118 \\
\hline 29 & 171 & 461 & 282 & 527 & --- & 648 & 595 & 468 & 236 & 186 & 166 & 117 \\
\hline 30 & 164 & e2300 & 267 & 490 & --- & 636 & 579 & 450 & 233 & 186 & 166 & 117 \\
\hline 31 & 159 & --- & 277 & 495 & --- & 614 & --- & 453 & --- & 189 & 168 & --- \\
\hline TOTAL & 5769 & 11010 & 14734 & 16684 & 37816 & 28779 & 19583 & 14184 & 9938 & 6122 & 5515 & 4489 \\
\hline MEAN & 186 & 367 & 475 & 538 & 1351 & 928 & 653 & 458 & 331 & 197 & 178 & 150 \\
\hline MAX & 236 & 2300 & 1700 & 2070 & 4960 & 2080 & 757 & 596 & 465 & 230 & 205 & 169 \\
\hline MIN & 148 & 165 & 260 & 226 & 390 & 584 & 528 & 375 & 233 & 173 & 166 & 117 \\
\hline$A C-F T$ & 11440 & 21840 & 29220 & 33090 & 75010 & 57080 & 38840 & 28130 & 19710 & 12140 & 10940 & 8900 \\
\hline
\end{tabular}

e Estimated. 


\section{BUTTE CREEK NEAR CHICO, CA—Continued}

STATISTICS OF MONTHLY MEAN DATA FOR WATER YEARS 1931 - 1999, BY WATER YEAR (WY)

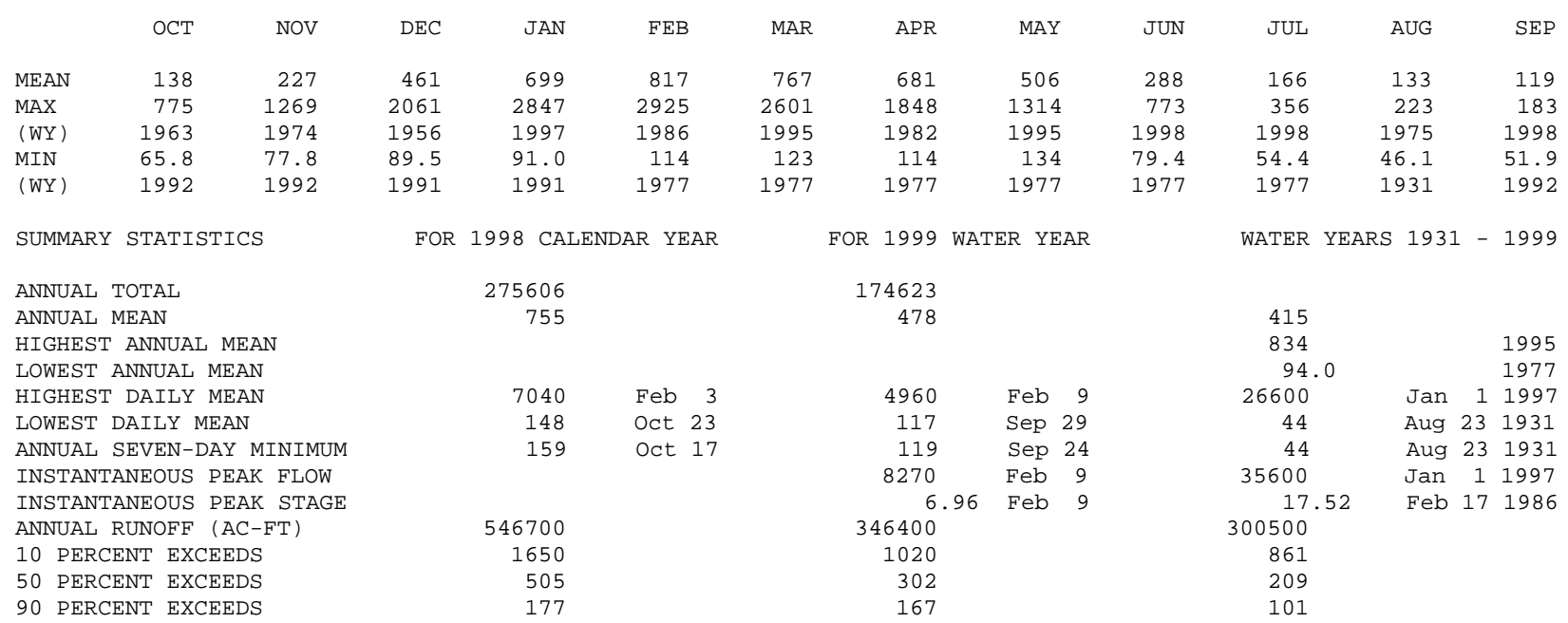




\section{WATER-QUALITY RECORDS}

PERIOD OF RECORD.-Water years 1953-79, October 1998 to September 1999.

CHEMICAL DATA: Water years 1953-79.

WATER TEMPERATURE: Water years 1962-79, October 1998 to September 1999.

PERIOD OF DAILY RECORD.-

WATER TEMPERATURE: November 1961 to January 1979, October 1998 to September 1999.

INSTRUMENTATION.-Temperature recorder since October 1998.

EXTREMES FOR PERIOD OF DAILY RECORD.-

WATER TEMPERATURE: Maximum recorded, $26.0^{\circ} \mathrm{C}$, July $21,22,1966$, and on several days in 1977 ; minimum recorded, $0.5^{\circ} \mathrm{C}$, Dec. 8,31 , 1978, Jan. 1, 1979.

EXTREME FOR CURRENT YEAR.-

WATER TEMPERATURE: Maximum recorded, $24.5^{\circ} \mathrm{C}$, July 12,13 ; minimum recorded, $1.0^{\circ} \mathrm{C}$, Dec. $22-24$.

TEMPERATURE, WATER (DEG. C), WATER YEAR OCTOBER 1998 TO SEPTEMBER 1999

\begin{tabular}{|c|c|c|c|c|c|c|c|c|c|c|c|c|}
\hline DAY & MAX & MIN & MAX & MIN & MAX & MIN & MAX & MIN & MAX & MIN & MAX & MIN \\
\hline & \multicolumn{2}{|c|}{ OCTOBER } & \multicolumn{2}{|c|}{ NOVEMBER } & \multicolumn{2}{|c|}{ DECEMBER } & \multicolumn{2}{|c|}{ JANUARY } & \multicolumn{2}{|c|}{ FEBRUARY } & \multicolumn{2}{|c|}{ MARCH } \\
\hline 1 & 17.0 & 13.5 & 11.5 & 10.0 & 9.5 & 9.0 & 6.5 & 4.5 & 7.0 & 5.0 & 10.0 & 9.0 \\
\hline 2 & 15.5 & 14.0 & 11.0 & 8.5 & 9.5 & 9.0 & 6.5 & 4.0 & 7.0 & 4.5 & 9.5 & 8.0 \\
\hline 3 & 16.0 & 13.0 & 11.0 & 9.5 & 9.5 & 7.5 & 6.5 & 4.5 & 7.0 & 5.0 & 10.0 & 8.5 \\
\hline 4 & 15.0 & 11.5 & 11.0 & 9.0 & 7.5 & 6.0 & 6.5 & 4.5 & 8.0 & 6.0 & 9.0 & 7.5 \\
\hline 5 & 15.0 & 11.0 & 11.0 & 9.0 & 6.0 & 5.0 & 5.5 & 4.0 & 7.5 & 5.5 & 8.5 & 6.5 \\
\hline 6 & 14.0 & 11.0 & 10.0 & 9.0 & 5.5 & 4.5 & 5.5 & 4.5 & 7.5 & 6.5 & 8.5 & 7.0 \\
\hline 7 & 14.5 & 11.0 & 9.5 & 8.5 & 5.0 & 4.0 & 5.5 & 4.5 & 8.5 & 7.5 & 9.0 & 7.0 \\
\hline 8 & 15.0 & 11.5 & 9.0 & 8.0 & 6.5 & 5.0 & 6.0 & 3.5 & 8.5 & 8.5 & 7.5 & 7.0 \\
\hline 9 & 14.5 & 11.5 & 9.0 & 7.0 & 6.0 & 4.5 & 5.5 & 4.0 & 9.0 & 7.0 & 7.5 & 6.0 \\
\hline 10 & 13.5 & 10.5 & 8.0 & 7.5 & 6.0 & 4.5 & 5.5 & 4.5 & 7.5 & 6.5 & 7.5 & 6.0 \\
\hline 11 & 13.0 & 10.0 & 9.0 & 7.5 & 6.0 & 5.0 & 6.0 & 4.0 & 7.5 & 6.0 & 9.0 & 6.0 \\
\hline 12 & 12.5 & 10.0 & 8.5 & 6.5 & 7.0 & 5.5 & 6.5 & 4.0 & 7.5 & 6.5 & 9.0 & 6.5 \\
\hline 13 & 14.0 & 11.0 & 9.0 & 6.5 & 7.5 & 6.5 & 7.0 & 4.5 & 7.5 & 6.5 & 9.5 & 7.5 \\
\hline 14 & 13.5 & 10.5 & 9.0 & 6.5 & 8.0 & 6.5 & 6.5 & 5.0 & 8.5 & 7.5 & 9.5 & 8.0 \\
\hline 15 & 13.0 & 9.5 & 9.5 & 7.5 & 8.0 & 6.0 & 7.5 & 6.0 & 8.5 & 7.0 & 10.0 & 7.5 \\
\hline 16 & 12.0 & 9.0 & 8.5 & 7.5 & 9.0 & 6.5 & 8.5 & 7.5 & 8.5 & 7.5 & 10.5 & 8.0 \\
\hline 17 & 12.0 & 8.5 & 9.5 & 8.0 & 8.5 & 7.0 & 8.5 & 8.0 & 9.5 & 8.5 & 11.0 & 9.0 \\
\hline 18 & 12.0 & 8.5 & 9.0 & 7.0 & 8.0 & 6.5 & 9.5 & 8.5 & 9.5 & 8.5 & 11.0 & 9.0 \\
\hline 19 & 13.0 & 9.0 & 8.5 & 6.0 & 7.0 & 5.5 & 9.0 & 8.5 & 9.0 & 7.5 & 10.5 & 9.0 \\
\hline 20 & 13.0 & 9.5 & 8.0 & 6.0 & 6.0 & 3.5 & 9.0 & 8.5 & 8.5 & 7.5 & 10.5 & 8.5 \\
\hline 21 & 13.0 & 9.5 & 9.0 & 7.5 & 3.5 & 2.0 & 8.5 & 7.5 & 8.5 & 7.5 & 10.0 & 8.0 \\
\hline 22 & 13.0 & 10.0 & 9.5 & 8.5 & 3.0 & 1.0 & 8.5 & 7.5 & 8.5 & 7.5 & 9.0 & 7.5 \\
\hline 23 & 13.0 & 9.5 & 10.0 & 8.5 & 2.5 & 1.0 & 9.0 & 8.0 & 10.0 & 8.0 & 9.5 & 8.0 \\
\hline 24 & 11.5 & 11.0 & 9.5 & 8.5 & 2.5 & 1.0 & 8.0 & 6.5 & 9.0 & 8.5 & 9.0 & 8.0 \\
\hline 25 & 12.5 & 10.5 & 9.0 & 7.5 & 4.0 & 2.0 & 7.5 & 6.0 & 9.0 & 8.0 & 11.0 & 8.5 \\
\hline 26 & 12.5 & 10.0 & 8.5 & 7.5 & 5.0 & 3.5 & 7.5 & 6.5 & 8.5 & 7.5 & 12.0 & 9.5 \\
\hline 27 & 12.5 & 10.5 & 9.0 & 8.5 & 5.5 & 4.0 & 7.0 & 5.5 & 9.5 & 8.5 & 10.5 & 8.0 \\
\hline 28 & 12.0 & 11.0 & 9.5 & 8.5 & 6.0 & 4.5 & 7.0 & 5.0 & 9.5 & 9.0 & 10.0 & 7.0 \\
\hline 29 & 12.5 & 10.5 & 9.0 & 8.0 & 6.0 & 4.0 & 6.5 & 5.0 & --- & --- & 9.0 & 7.5 \\
\hline 30 & 12.0 & 9.5 & 10.0 & 8.5 & 6.0 & 4.0 & 6.0 & 4.5 & --- & --- & 9.0 & 7.0 \\
\hline 31 & 10.5 & 9.0 & --- & --- & 6.5 & 5.0 & 6.5 & 5.5 & --- & --- & 8.0 & 6.0 \\
\hline MONTH & 17.0 & 8.5 & 11.5 & 6.0 & 9.5 & 1.0 & 9.5 & 3.5 & 10.0 & 4.5 & 12.0 & 6.0 \\
\hline
\end{tabular}




\section{BUTTE CREEK NEAR CHICO, CA—Continued}

\section{TEMPERATURE, WATER (DEG. C), WATER YEAR OCTOBER 1998 TO SEPTEMBER 1999}

\begin{tabular}{|c|c|c|c|c|c|c|c|c|c|c|c|c|}
\hline \multirow[t]{2}{*}{ DAY } & MAX & MIN & $\operatorname{MAX}$ & MIN & MAX & MIN & MAX & MIN & MAX & MIN & MAX & MIN \\
\hline & \multicolumn{2}{|c|}{ APRIL } & \multicolumn{2}{|c|}{ MAY } & \multicolumn{2}{|c|}{ JUNE } & \multicolumn{2}{|c|}{ JULY } & \multicolumn{2}{|c|}{ AUGUST } & \multicolumn{2}{|c|}{ SEPTEMBER } \\
\hline 1 & 9.0 & 5.5 & 14.5 & 10.5 & 16.5 & 14.0 & 23.5 & 19.0 & 22.0 & 17.0 & 18.5 & 14.5 \\
\hline 2 & 9.5 & 5.5 & 12.0 & 9.5 & 15.0 & 12.5 & 23.0 & 19.0 & 22.5 & 17.5 & 19.0 & 14.0 \\
\hline 3 & 8.5 & 6.5 & 12.0 & 9.0 & 12.5 & 11.0 & 21.5 & 18.5 & 23.0 & 17.5 & 19.0 & 14.0 \\
\hline 4 & 9.5 & 5.5 & 13.0 & 8.5 & 14.0 & 10.0 & 21.0 & 17.0 & 23.0 & 18.0 & 19.0 & 14.0 \\
\hline 5 & 7.5 & 6.0 & 14.0 & 9.0 & 15.5 & 11.5 & 21.5 & 16.5 & 21.5 & 18.0 & 19.5 & 14.5 \\
\hline 6 & 9.0 & 5.5 & 15.5 & 11.0 & 17.0 & 13.0 & 21.5 & 16.0 & 20.5 & 18.0 & 20.0 & 15.0 \\
\hline 7 & 9.0 & 6.0 & 15.0 & 11.0 & 16.0 & 13.0 & 21.5 & 16.5 & 21.0 & 16.5 & 20.0 & 15.0 \\
\hline 8 & 8.0 & 6.0 & 14.0 & 9.5 & 16.0 & 12.0 & 22.0 & 16.5 & 21.0 & 16.5 & 20.5 & 15.5 \\
\hline 9 & 9.0 & 5.0 & 13.5 & 9.5 & 15.0 & 12.0 & 22.5 & 17.0 & 20.5 & 17.0 & 19.0 & 16.5 \\
\hline 10 & 7.0 & 6.0 & 13.0 & 9.5 & 16.0 & 12.0 & 23.0 & 17.5 & 19.0 & 17.0 & 20.5 & 16.0 \\
\hline 11 & 10.5 & 6.5 & 15.0 & 10.0 & 17.0 & 12.5 & 23.5 & 18.5 & 21.5 & 17.0 & 20.0 & 16.0 \\
\hline 12 & 12.0 & 7.5 & 16.0 & 12.0 & 17.5 & 13.5 & 24.5 & 19.5 & 22.0 & 16.5 & 21.0 & 16.0 \\
\hline 13 & 12.5 & 8.5 & 14.5 & 11.0 & 18.5 & 14.5 & 24.5 & 20.5 & 21.5 & 16.5 & 20.5 & 16.5 \\
\hline 14 & 13.0 & 9.0 & 14.0 & 10.0 & 18.5 & 15.0 & 24.0 & 20.0 & 21.5 & 16.0 & 21.0 & 16.0 \\
\hline 15 & 13.0 & 9.5 & 14.0 & 9.5 & 18.5 & 15.5 & 23.5 & 19.5 & 21.0 & 15.5 & 20.5 & 16.0 \\
\hline 16 & 13.5 & 10.0 & 14.0 & 9.5 & 19.0 & 15.5 & 23.0 & 18.5 & 21.5 & 15.5 & 20.0 & 16.0 \\
\hline 17 & 14.5 & 11.0 & 14.5 & 10.5 & 19.0 & 15.5 & 22.5 & 18.0 & 22.0 & 16.0 & 20.0 & 15.5 \\
\hline 18 & 14.0 & 11.0 & 16.5 & 11.5 & 19.5 & 16.0 & 22.0 & 17.0 & 21.5 & 16.5 & 19.0 & 15.5 \\
\hline 19 & 14.0 & 11.0 & 16.0 & 11.5 & 20.0 & 16.0 & 21.5 & 17.0 & 21.5 & 15.5 & 20.0 & 16.0 \\
\hline 20 & 14.0 & 11.0 & 15.5 & 12.0 & 19.5 & 16.0 & 21.5 & 16.5 & 21.5 & 15.5 & 20.0 & 15.5 \\
\hline 21 & 13.0 & 10.0 & 16.5 & 11.5 & 20.5 & 16.5 & 21.5 & 16.5 & 22.0 & 16.0 & 21.0 & 16.5 \\
\hline 22 & 13.0 & 9.5 & 17.5 & 12.5 & 21.0 & 17.0 & 21.5 & 16.5 & 22.5 & 17.0 & 21.0 & 16.5 \\
\hline 23 & 13.0 & 9.0 & 18.0 & 13.0 & 22.0 & 17.5 & 21.5 & 16.5 & 23.5 & 18.5 & 21.0 & 17.0 \\
\hline 24 & 14.0 & 10.0 & 18.0 & 13.5 & 21.5 & 18.0 & 21.5 & 16.5 & 23.0 & 18.5 & 21.0 & 17.0 \\
\hline 25 & 14.0 & 10.5 & 18.0 & 14.0 & 21.5 & 18.0 & 21.5 & 16.5 & 22.0 & 18.0 & 21.0 & 16.5 \\
\hline 26 & 14.5 & 11.0 & 19.0 & 14.5 & 20.5 & 17.0 & 22.5 & 17.0 & 20.5 & 17.5 & 20.0 & 15.5 \\
\hline 27 & 13.0 & 9.5 & 19.0 & 15.0 & 20.5 & 16.5 & 21.5 & 17.0 & 22.5 & 18.0 & 19.0 & 15.0 \\
\hline 28 & 10.0 & 8.0 & 18.5 & 14.5 & 21.5 & 16.5 & 22.0 & 17.5 & 23.0 & 18.0 & 18.0 & 14.5 \\
\hline 29 & 12.0 & 7.5 & 17.5 & 14.0 & 22.0 & 17.5 & 22.0 & 17.0 & 23.0 & 18.0 & 19.0 & 14.5 \\
\hline 30 & 13.5 & 9.0 & 17.5 & 13.5 & 23.0 & 18.5 & 22.0 & 17.0 & 21.0 & 17.5 & 19.0 & 14.5 \\
\hline 31 & -- & -- & 18.0 & 13.5 & -- & -- & 22.0 & 17.0 & 20.0 & 15.5 & -- & -- \\
\hline MONTH & 14.5 & 5.0 & 19.0 & 8.5 & 23.0 & 10.0 & 24.5 & 16.0 & 23.5 & 15.5 & 21.0 & 14.0 \\
\hline
\end{tabular}


11390500 SACRAMENTO RIVER BELOW WILKINS SLOUGH, NEAR GRIMES, CA

LOCATION.-Lat 3900’36", long 12149'25", in NW 1/4 NE 1/4 sec.2, T.13 N., R.1 E., Colusa County, Hydrologic Unit 18020104, on right bank 1,200 ft downstream from Wilkins Slough, $5.8 \mathrm{mi}$ southeast of Grimes, and at mile 62.9 upstream from Sacramento.

DRAINAGE AREA.-12,926 $\mathrm{mi}^{2}$.

\section{WATER-DISCHARGE RECORDS}

PERIOD OF RECORD.-August 1931 to current year (prior to October 1938, low-water periods only). Monthly discharge only for some periods, published in WSP 1315-A. Prior to October 1965, published as "below Wilkins Slough."

GAGE.-Water-stage recorder and crest-stage gage. Datum of gage is $3.00 \mathrm{ft}$ below sea level.

REMARKS.-Records good. Natural flow of stream affected by storage reservoirs, power development, bypassing for flood control, diversions for irrigation, and return flow from irrigated areas. When discharge exceeds about $23,000 \mathrm{ft}^{3} / \mathrm{s}$, flow begins over Tisdale Weir, $1.0 \mathrm{mi}$ upstream on left bank, into Sutter Bypass. Records tabulated below do not include flow over Tisdale Weir. See schematic diagram of lower Sacramento River Basin.

EXTREMES FOR PERIOD OF RECORD.-Maximum discharge (water years 1939-99), 32,700 ft ${ }^{3} / \mathrm{s}$, Feb. 20, 1986, gage height, 52.50 ft; maximum gage height, $52.75 \mathrm{ft}$, Mar. 1, 1940; minimum daily, $645 \mathrm{ft}^{3} / \mathrm{s}$, Aug. 9, 1939.

DISCHARGE, CUBIC FEET PER SECOND, WATER YEAR OCTOBER 1998 TO SEPTEMBER 1999 DAILY MEAN VALUES

\begin{tabular}{|c|c|c|c|c|c|c|c|c|c|c|c|c|}
\hline DAY & $\mathrm{OCT}$ & NOV & $\mathrm{DEC}$ & JAN & FEB & MAR & APR & MAY & JUN & JUL & AUG & SEP \\
\hline 1 & 9150 & 6620 & 25600 & 9680 & 14200 & 26600 & 15400 & 10800 & 9660 & 8130 & e 8080 & 6210 \\
\hline 2 & 9050 & 6680 & 26700 & 9490 & 13600 & 27100 & 14600 & 10600 & 9350 & 7660 & e 8100 & 6500 \\
\hline 3 & 8840 & 6600 & 25100 & 9320 & 13000 & 27100 & 13800 & 10700 & 9630 & 7480 & e8000 & 6620 \\
\hline 4 & 8650 & 6620 & 25900 & 9210 & 12500 & 26900 & 13200 & 10900 & 10300 & 7310 & 7960 & 6630 \\
\hline 5 & 8530 & 6690 & 26100 & 9050 & 11900 & 26900 & 12700 & 10600 & 10800 & 7270 & 7960 & 6500 \\
\hline 6 & 8450 & 6610 & 25600 & 8960 & 14300 & 26700 & 12500 & 10100 & 10800 & 7340 & 7610 & 6770 \\
\hline 7 & 8390 & 6540 & 25900 & 8920 & 18500 & 26600 & 13000 & 9700 & 10200 & 7410 & 7340 & 6950 \\
\hline 8 & 8310 & 7420 & 25800 & 8860 & 26100 & 26500 & 13100 & 9940 & 9750 & 7330 & 7030 & 6910 \\
\hline 9 & 8250 & 8220 & 25700 & 8790 & 26800 & 26400 & 12800 & 9920 & 9270 & 7330 & 6840 & 6780 \\
\hline 10 & 8060 & 8270 & 25400 & 8690 & 27800 & 26600 & 13300 & 9560 & 8800 & 7260 & 6840 & 7100 \\
\hline 11 & 7950 & 7940 & 24500 & 8640 & 28200 & 26600 & 13900 & 9240 & 8510 & 7240 & 6830 & 7310 \\
\hline 12 & 7830 & 8340 & 24000 & 8660 & 26600 & 26400 & 17100 & 8950 & 8080 & 7400 & 6600 & 7270 \\
\hline 13 & 7580 & 8550 & 23800 & 8650 & 26100 & 26100 & 19900 & 8980 & 7760 & 7420 & 6490 & 7010 \\
\hline 14 & 7340 & 8690 & 23700 & 8630 & 25900 & 25700 & 18600 & 9090 & 7610 & 7480 & 6370 & 7010 \\
\hline 15 & 7060 & 9320 & 24100 & 8640 & 25400 & 24900 & 18200 & 8750 & 7360 & 7720 & 6260 & 7040 \\
\hline 16 & 6900 & 9680 & 22800 & 8780 & 25400 & 24200 & 17900 & 8280 & 7540 & 7540 & 6250 & 7120 \\
\hline 17 & 6680 & 9850 & 20800 & 9020 & 26300 & 23300 & 17800 & 8180 & 7570 & 7690 & 5960 & 7400 \\
\hline 18 & 6540 & 10500 & 19500 & 9550 & 27700 & 22500 & 17900 & 8020 & 7500 & 7820 & 5630 & 7440 \\
\hline 19 & 6450 & 13500 & 18900 & 12700 & 27700 & 21700 & 17700 & 7910 & 7470 & 8010 & 5460 & 7340 \\
\hline 20 & 6380 & 14400 & 18200 & 14900 & 27500 & 20000 & 17000 & 7930 & 7710 & 8120 & 5400 & 7140 \\
\hline 21 & 6300 & 14500 & 16900 & 15100 & 27100 & 18500 & 16800 & 8000 & 7710 & 8180 & 5370 & 7080 \\
\hline 22 & 6230 & 14600 & 15400 & 17900 & 27900 & 17500 & 15900 & 8350 & 7850 & 8120 & 5370 & 7060 \\
\hline 23 & 6100 & 17100 & 13900 & 20500 & 27500 & 16200 & 14700 & 8770 & 7820 & 7810 & 5380 & 7060 \\
\hline 24 & 6150 & 19300 & 12700 & 23600 & 26700 & 15100 & 13500 & 9280 & 7790 & 7670 & 5460 & 6870 \\
\hline 25 & 6500 & 23700 & 11700 & 24400 & 26200 & 16100 & 12600 & 9440 & 8130 & 7700 & 5510 & 6870 \\
\hline 26 & 7150 & 21700 & 11200 & 23400 & 26400 & 24600 & 12100 & 9020 & 8080 & 7890 & 5590 & 6910 \\
\hline 27 & 7080 & 19500 & 11000 & 22400 & 26500 & 24800 & 11800 & 8850 & 8040 & 7880 & 5720 & 6830 \\
\hline 28 & 6990 & 22400 & 10800 & 20800 & 26600 & 23300 & 11800 & 9060 & 8070 & 7810 & 5850 & 6660 \\
\hline 29 & 6840 & 21900 & 10700 & 18700 & --- & 21500 & 11500 & 9400 & 8250 & 7890 & 5890 & 6500 \\
\hline 30 & 6780 & 20500 & 10600 & 16800 & --- & 19100 & 11300 & 9720 & 8240 & 7960 & 6010 & 6260 \\
\hline 31 & 6670 & --- & 10100 & 15400 & --- & 16700 & --- & 9920 & --- & 7930 & 6150 & --- \\
\hline TOTAL & 229180 & 366240 & 613100 & 408140 & 660400 & 722200 & 442400 & 287960 & 255650 & 237800 & 199310 & 207150 \\
\hline MEAN & 7393 & 12210 & 19780 & 13170 & 23590 & 23300 & 14750 & 9289 & 8522 & 7671 & 6429 & 6905 \\
\hline MAX & 9150 & 23700 & 26700 & 24400 & 28200 & 27100 & 19900 & 10900 & 10800 & 8180 & 8100 & 7440 \\
\hline MIN & 6100 & 6540 & 10100 & 8630 & 11900 & 15100 & 11300 & 7910 & 7360 & 7240 & 5370 & 6210 \\
\hline$A C-F T$ & 454600 & 726400 & 1216000 & 809500 & 1310000 & 1432000 & 877500 & 571200 & 507100 & 471700 & 395300 & 410900 \\
\hline
\end{tabular}


11390500 SACRAMENTO RIVER BELOW WILKINS SLOUGH, NEAR GRIMES, CA—Continued

STATISTICS OF MONTHLY MEAN DATA FOR WATER YEARS 1946 - 1999, BY WATER YEAR (WY)

\begin{tabular}{|c|c|c|c|c|c|c|c|c|c|c|c|c|}
\hline OCT & NOV & $\mathrm{DEC}$ & & JAN & FEB & MAR & APR & MAY & JUN & \multicolumn{2}{|r|}{ AUG } & SEP \\
\hline MEAN & 8548 & 12450 & 150 & 990 & 6850 & 15460 & 11550 & 9448 & 7889 & 7395 & 7300 & 7238 \\
\hline 11800 & 20510 & 27430 & 273 & 310 & 9090 & 29490 & 24920 & 23110 & 20670 & 12500 & 10940 & 10620 \\
\hline 1958 & 1974 & 1984 & & 997 & 1998 & 1983 & 1982 & 1983 & 1998 & 1998 & 1998 & 1967 \\
\hline 3330 & 3839 & 4103 & & 281 & 5012 & 5152 & 4201 & 3397 & 3451 & 3784 & 4086 & 4065 \\
\hline 1978 & 1993 & 1977 & 19 & 91 & 1991 & 1977 & 1994 & 1992 & 1992 & 1992 & 1947 & 1977 \\
\hline \multicolumn{2}{|c|}{ SUMMARY STATISTICS } & $\mathrm{FOR}$ & 1998 & CALENDAR & R YEAR & & FOR 1999 WAT & WATER YEAR & & WATER YEARS & 1946 & $5-1999$ \\
\hline ANNUAL TOTAL & & & 63591 & 30 & & & 4629530 & & & & & \\
\hline ANNUAL MEAN & & & 174 & 20 & & & 12680 & & & 10450 & & \\
\hline HIGHEST ANNUA & MEAN & & & & & & & & & 17980 & & 1983 \\
\hline LOWEST ANNUAL & IEAN & & & & & & & & & 5109 & & 1977 \\
\hline HIGHEST DAILY & IEAN & & 310 & 00 & Eeb 9 & & 28200 & Feb 11 & & 32600 & Feb 2 & 201986 \\
\hline LOWEST DAILY 1 & AN & & 61 & 00 & bet 23 & & 5370 & Aug 21 & & 2720 & May & 71992 \\
\hline ANNUAL SEVEN-I & AY MINIMUM & & 63 & 300 & oct 19 & & 5420 & Aug 19 & & 2880 & Oct & 121977 \\
\hline INSTANTANEOUS & EAK FLOW & & & & & & 28700 & Feb 11 & & 32700 & Feb 2 & 201986 \\
\hline INSTANTANEOUS & EAK STAGE & & & & & & 49.35 & Feb 11 & & 52.68 & Jan & 41997 \\
\hline ANNUAL RUNOFF & $(\mathrm{AC}-\mathrm{FT})$ & & 126100 & & & & 9183000 & & & 7572000 & & \\
\hline 10 PERCENT EX & EDS & & 281 & & & & 25800 & & & 22200 & & \\
\hline 50 PERCENT EX & EDS & & 177 & & & & 9050 & & & 8010 & & \\
\hline 90 PERCENT EX & EDS & & 82 & & & & 6620 & & & 5030 & & \\
\hline
\end{tabular}




\section{WATER-QUALITY RECORDS}

PERIOD OF RECORD.-October 1966 to current year.

PERIOD OF DAILY RECORD.-

WATER TEMPERATURE: October 1966 to current year.

INSTRUMENTATION.-Water-temperature recorder since October 1966.

REMARKS.-Interruption in record due to malfunction of the recording instrument.

EXTREMES FOR PERIOD OF DAILY RECORD.-

WATER TEMPERATURE: Maximum recorded, $25.5^{\circ} \mathrm{C}$, Sept. 6-8, 1977, June 3-5, 1992; minimum recorded, 3.5 ${ }^{\circ} \mathrm{C}$, Dec. $23-25,1990$.

EXTREMES FOR CURRENT YEAR.-

WATER TEMPERATURE: Maximum recorded, $21.0^{\circ} \mathrm{C}$, many days during June, July, and August; minimum recorded, $7.5^{\circ} \mathrm{C}$, Dec. $24,25$.

TEMPERATURE, WATER (DEG. C), WATER YEAR OCTOBER 1998 TO SEPTEMBER 1999

\begin{tabular}{|c|c|c|c|c|c|c|c|c|c|c|c|c|}
\hline DAY & MAX & MIN & MAX & MIN & MAX & MIN & MAX & MIN & MAX & MIN & MAX & MIN \\
\hline & \multicolumn{2}{|c|}{ OCTOBER } & \multicolumn{2}{|c|}{ NOVEMBER } & \multicolumn{2}{|c|}{ DECEMBER } & \multicolumn{2}{|c|}{ JANUARY } & \multicolumn{2}{|c|}{ FEBRUARY } & \multicolumn{2}{|c|}{$\mathrm{MARCH}$} \\
\hline 1 & --- & --- & 13.0 & 13.0 & 12.5 & 12.5 & 10.0 & 10.0 & 9.5 & 8.5 & 10.5 & 9.5 \\
\hline 2 & --- & --- & 13.0 & 12.5 & 12.5 & 12.5 & 10.5 & 10.0 & 9.5 & 9.0 & 10.5 & 10.0 \\
\hline 3 & --- & --- & 13.0 & 12.5 & 13.0 & 12.5 & 10.0 & 10.0 & 9.5 & 9.0 & 10.5 & 10.0 \\
\hline 4 & --- & --- & 13.5 & 12.5 & 13.0 & 12.5 & 10.0 & 10.0 & 9.5 & 9.0 & 10.5 & 10.0 \\
\hline 5 & --- & --- & 13.5 & 13.0 & 12.5 & 11.0 & 10.0 & 9.5 & 9.5 & 9.0 & 10.0 & 9.5 \\
\hline 6 & --- & --- & 13.0 & 13.0 & 11.0 & 10.5 & 9.5 & 9.0 & 9.5 & 9.0 & 9.5 & 9.0 \\
\hline 7 & --- & --- & 13.0 & 12.5 & 11.0 & 10.5 & 9.0 & 8.5 & 9.5 & 9.0 & 9.5 & 9.0 \\
\hline 8 & --- & --- & 12.5 & 12.0 & 11.5 & 11.0 & 9.0 & 8.5 & 9.5 & 9.0 & 9.0 & 9.0 \\
\hline 9 & --- & --- & 12.0 & 11.5 & 11.5 & 11.0 & 9.0 & 8.5 & 10.0 & 9.0 & 9.0 & 8.5 \\
\hline 10 & --- & --- & 12.0 & 12.0 & 11.5 & 11.5 & 8.5 & 8.5 & 10.0 & 9.0 & 9.0 & 9.0 \\
\hline 11 & --- & --- & 12.0 & 11.5 & 12.0 & 11.5 & 8.5 & 8.5 & 9.0 & 8.5 & 9.0 & 8.5 \\
\hline 12 & --- & --- & 12.0 & 11.5 & 12.0 & 12.0 & 9.0 & 8.5 & 8.5 & 8.0 & 9.5 & 9.0 \\
\hline 13 & --- & --- & 12.5 & 12.0 & 12.5 & 12.0 & 9.5 & 9.0 & 8.5 & 8.0 & 10.0 & 9.5 \\
\hline 14 & --- & --- & 12.5 & 12.0 & 12.5 & 12.5 & 9.5 & 9.0 & 9.0 & 8.5 & 10.0 & 10.0 \\
\hline 15 & --- & --- & 13.0 & 12.0 & 12.5 & 12.0 & 10.0 & 9.5 & 9.0 & 9.0 & 10.5 & 10.0 \\
\hline 16 & --- & --- & 13.0 & 12.5 & 12.5 & 12.0 & 10.5 & 10.0 & 9.5 & 9.0 & 10.5 & 10.0 \\
\hline 17 & --- & --- & 13.0 & 12.5 & 12.5 & 12.5 & 11.0 & 10.5 & 9.5 & 9.0 & 11.0 & 10.5 \\
\hline 18 & --- & --- & 13.5 & 12.5 & 12.5 & 12.5 & 12.0 & 11.0 & 9.5 & 9.0 & 11.5 & 11.0 \\
\hline 19 & --- & --- & 13.0 & 13.0 & 12.5 & 12.0 & 12.0 & 11.5 & 10.0 & 9.0 & 11.5 & 11.0 \\
\hline 20 & --- & --- & 13.0 & 12.5 & 12.0 & 10.5 & 12.0 & 11.5 & 9.5 & 9.0 & 11.5 & 11.0 \\
\hline 21 & --- & --- & 13.0 & 12.5 & 10.5 & 8.5 & 11.5 & 11.5 & 9.0 & 8.5 & 11.5 & 11.0 \\
\hline 22 & --- & --- & 13.0 & 13.0 & 8.5 & 8.0 & 11.5 & 10.5 & 9.0 & 8.5 & 12.0 & 11.5 \\
\hline 23 & --- & --- & 13.5 & 13.0 & 8.0 & 8.0 & 10.5 & 10.0 & 9.5 & 9.0 & 11.5 & 11.0 \\
\hline 24 & --- & --- & 13.5 & 13.0 & 8.0 & 7.5 & 10.5 & 10.0 & 10.0 & 9.5 & 11.0 & 10.5 \\
\hline 25 & --- & --- & 13.0 & 12.5 & 8.0 & 7.5 & 10.0 & 9.0 & 10.0 & 10.0 & 11.5 & 10.5 \\
\hline 26 & --- & --- & 12.5 & 12.5 & 8.5 & 8.0 & 9.0 & 8.5 & 10.0 & 9.5 & 11.5 & 11.0 \\
\hline 27 & --- & --- & 13.0 & 12.5 & 9.0 & 8.5 & 8.5 & 8.5 & 9.5 & 9.5 & 12.5 & 11.5 \\
\hline 28 & 14.0 & 14.0 & 13.0 & 13.0 & 10.0 & 9.0 & 9.0 & 8.5 & 9.5 & 9.5 & 12.5 & 12.0 \\
\hline 29 & 14.5 & 14.0 & 13.0 & 12.5 & 10.0 & 10.0 & 9.0 & 9.0 & --- & --- & 12.0 & 11.5 \\
\hline 30 & 14.0 & 13.5 & 12.5 & 12.5 & 10.0 & 10.0 & 9.0 & 8.5 & --- & --- & 12.0 & 11.0 \\
\hline 31 & 13.5 & 13.0 & --- & --- & 10.5 & 10.0 & 9.0 & 8.5 & --- & --- & 11.5 & 11.0 \\
\hline MONTH & --- & --- & 13.5 & 11.5 & 13.0 & 7.5 & 12.0 & 8.5 & 10.0 & 8.0 & 12.5 & 8.5 \\
\hline
\end{tabular}


11390500 SACRAMENTO RIVER BELOW WILKINS SLOUGH, NEAR GRIMES, CA—Continued

TEMPERATURE, WATER (DEG. C), WATER YEAR OCTOBER 1998 TO SEPTEMBER 1999

\begin{tabular}{|c|c|c|c|c|c|c|c|c|c|c|c|c|}
\hline DAY & MAX & MIN & MAX & MIN & MAX & MIN & MAX & MIN & MAX & MIN & MAX & MIN \\
\hline & \multicolumn{2}{|c|}{ APRIL } & \multicolumn{2}{|c|}{ MAY } & \multicolumn{2}{|c|}{ JUNE } & \multicolumn{2}{|c|}{ JULY } & \multicolumn{2}{|c|}{ AUGUST } & \multicolumn{2}{|c|}{ SEPTEMBER } \\
\hline 1 & 11.5 & 10.5 & 16.0 & 14.0 & 19.0 & 18.0 & 21.0 & 19.5 & 19.5 & 18.5 & 19.0 & 18.5 \\
\hline 2 & 11.5 & 10.5 & 16.0 & 15.0 & 18.0 & 17.0 & 21.0 & 20.0 & 19.5 & 18.5 & 18.5 & 18.0 \\
\hline 3 & 12.0 & 11.0 & 16.0 & 15.0 & 17.0 & 15.5 & 21.0 & 19.5 & 20.0 & 19.0 & 18.0 & 17.5 \\
\hline 4 & 11.5 & 11.0 & 15.5 & 14.5 & 15.5 & 15.0 & 20.5 & 19.0 & 20.5 & 18.0 & 18.0 & 17.5 \\
\hline 5 & 11.5 & 11.0 & 15.5 & 14.5 & 16.5 & 15.0 & 20.0 & 19.0 & 20.5 & 19.5 & 18.5 & 18.0 \\
\hline 6 & 11.5 & 10.5 & 16.5 & 15.0 & 17.5 & 16.0 & 20.0 & 19.0 & 20.0 & 18.5 & 18.5 & 18.0 \\
\hline 7 & 11.5 & 9.5 & 17.0 & 15.5 & 18.5 & 17.0 & 20.0 & 19.0 & 19.5 & 18.5 & 19.0 & 18.5 \\
\hline 8 & 12.0 & 11.0 & 17.0 & 16.0 & 19.0 & 17.5 & 20.0 & 19.0 & 19.0 & 18.0 & 19.0 & 18.5 \\
\hline 9 & 12.0 & 11.0 & 16.0 & 15.0 & 19.0 & 17.5 & 20.5 & 19.5 & 19.0 & 18.5 & 19.0 & 19.0 \\
\hline 10 & 12.0 & 11.0 & 15.5 & 14.5 & 19.0 & 18.0 & 20.5 & 19.5 & 19.0 & 18.5 & 19.0 & 19.0 \\
\hline 11 & 11.5 & 10.5 & 16.5 & 14.5 & 19.5 & 18.0 & 21.0 & 19.5 & 18.5 & 18.0 & 19.0 & 19.0 \\
\hline 12 & 12.0 & 11.0 & 17.5 & 15.5 & 19.5 & 18.5 & 21.0 & 20.0 & 19.0 & 18.0 & 19.0 & 19.0 \\
\hline 13 & 13.0 & 11.5 & 18.0 & 16.0 & 20.0 & 19.0 & 21.0 & 20.0 & 19.5 & 19.0 & 19.0 & 19.0 \\
\hline 14 & 14.5 & 13.0 & 17.0 & 16.0 & 20.5 & 19.5 & 21.0 & 20.0 & 20.0 & 19.5 & 19.5 & 19.0 \\
\hline 15 & 15.0 & 14.0 & 16.5 & 15.0 & 20.5 & 19.5 & 21.0 & 20.0 & 20.0 & 19.5 & 19.5 & 19.0 \\
\hline 16 & 15.0 & 14.5 & 16.5 & 14.5 & 21.0 & 19.5 & 20.5 & 19.5 & 20.0 & 20.0 & 19.5 & 19.0 \\
\hline 17 & 15.5 & 14.5 & 17.0 & 15.5 & 21.0 & 20.0 & 20.5 & 19.0 & 20.5 & 18.5 & 19.0 & 18.5 \\
\hline 18 & 16.0 & 15.0 & 17.5 & 16.5 & 21.0 & 19.0 & 20.0 & 19.0 & 20.5 & 20.0 & 19.0 & 18.0 \\
\hline 19 & 16.0 & 15.5 & 18.0 & 17.0 & 20.5 & 19.5 & 20.0 & 18.5 & 20.0 & 19.5 & 18.5 & 18.0 \\
\hline 20 & 16.5 & 15.5 & 18.0 & 17.5 & 20.5 & 19.5 & 19.5 & 18.5 & 20.0 & 20.0 & 18.5 & 18.0 \\
\hline 21 & 16.0 & 15.5 & 18.5 & 17.5 & 20.5 & 19.5 & 19.0 & 18.0 & 20.0 & 19.5 & 18.5 & 18.0 \\
\hline 22 & 16.0 & 15.0 & 19.0 & 18.0 & 20.5 & 19.5 & 19.5 & 18.0 & 20.5 & 20.0 & 19.0 & 18.5 \\
\hline 23 & 15.0 & 14.5 & 19.0 & 18.0 & 21.0 & 19.5 & 19.5 & 18.5 & 20.5 & 20.0 & 19.0 & 19.0 \\
\hline 24 & 15.5 & 14.5 & 19.5 & 18.0 & 21.0 & 20.0 & 19.5 & 18.0 & 21.0 & 20.5 & 19.0 & 19.0 \\
\hline 25 & 16.0 & 15.0 & 19.5 & 18.0 & 21.0 & 19.0 & 19.5 & 18.5 & 21.0 & 20.0 & 19.5 & 19.0 \\
\hline 26 & 16.0 & 15.5 & 20.5 & 18.5 & 21.0 & 19.5 & 19.5 & 18.5 & 21.0 & 20.5 & 19.5 & 18.5 \\
\hline 27 & 16.5 & 15.5 & 20.5 & 19.0 & 20.5 & 19.5 & 19.5 & 18.5 & 20.5 & 20.0 & 19.0 & 17.5 \\
\hline 28 & 16.0 & 15.0 & 20.5 & 19.0 & 20.5 & 19.0 & 19.5 & 18.5 & 20.0 & 19.5 & 17.5 & 16.5 \\
\hline 29 & 15.0 & 14.0 & 20.0 & 19.0 & 21.0 & 19.0 & 19.5 & 18.5 & 20.0 & 20.0 & 17.0 & 16.0 \\
\hline 30 & 15.0 & 13.5 & 19.5 & 18.5 & 21.0 & 19.5 & 19.5 & 18.5 & 20.0 & 19.0 & 17.0 & 16.5 \\
\hline 31 & --- & --- & 19.5 & 18.0 & --- & --- & 19.5 & 18.5 & 19.5 & 19.0 & --- & --- \\
\hline MONTH & 16.5 & 9.5 & 20.5 & 14.0 & 21.0 & 15.0 & 21.0 & 18.0 & 21.0 & 18.0 & 19.5 & 16.0 \\
\hline
\end{tabular}




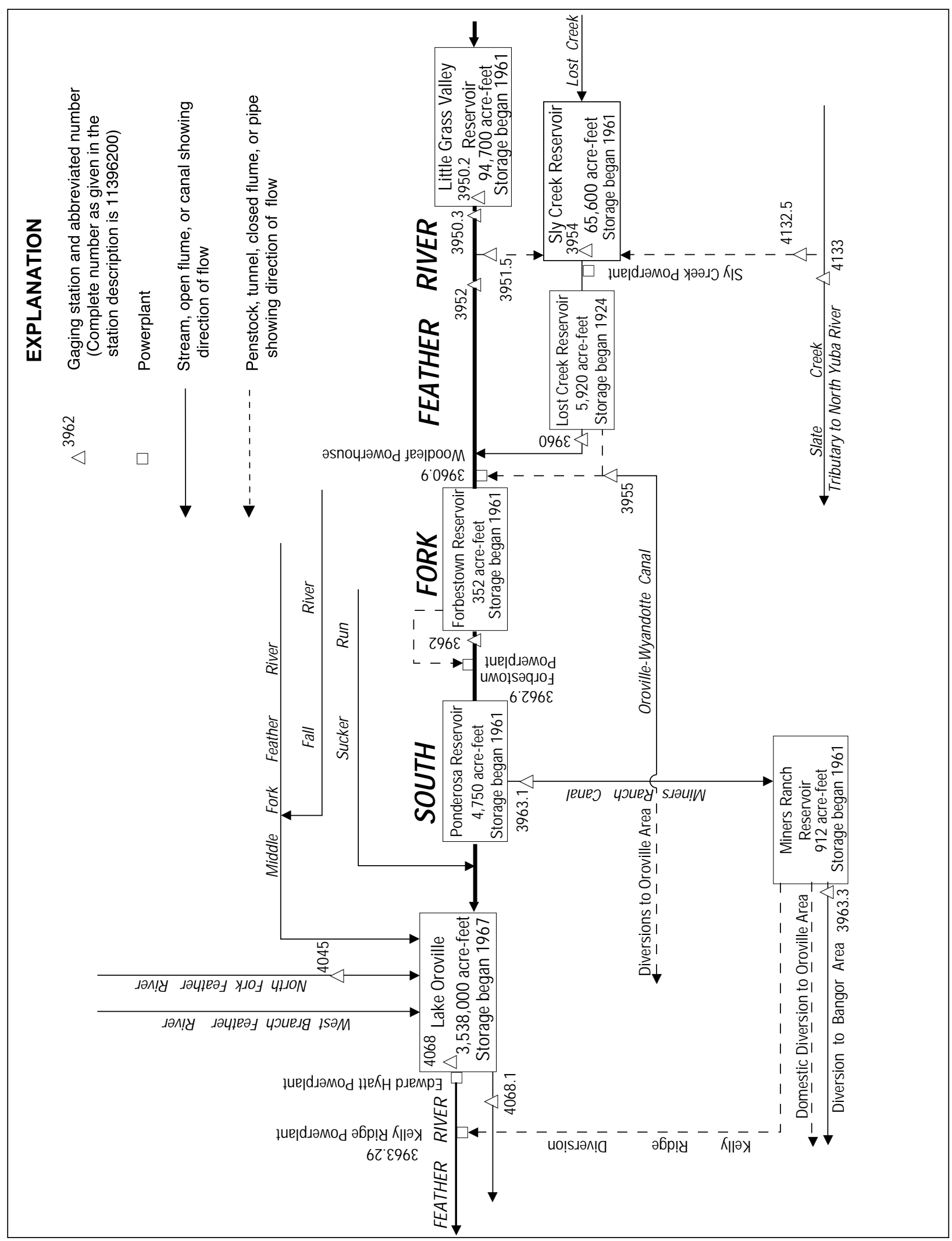

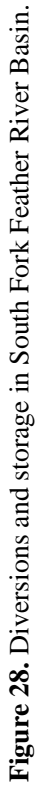




\section{LITTLE GRASS VALLEY RESERVOIR NEAR LA PORTE, CA}

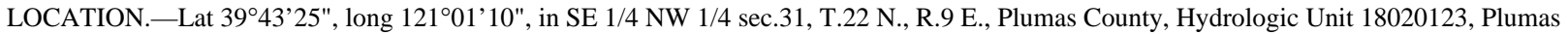
National Forest, on right bank $300 \mathrm{ft}$ upstream from dam on South Fork Feather River, and $3.3 \mathrm{mi}$ northwest of La Porte.

DRAINAGE AREA.- $-25.8 \mathrm{mi}^{2}$.

PERIOD OF RECORD.-October 1961 to current year. Monthend elevation and contents only, October 1961 to October 1962.

GAGE.-Water-stage recorder. Datum of gage is sea level (levels by Oroville-Wyandotte Irrigation District). Prior to Nov. 1, 1962, in valve chamber in dam at same datum.

REMARKS.- Reservoir is formed by rockfill dam. Storage began in October 1961. Total capacity, 94,700 acre-ft between elevations 4,876 ft, invert of release valve, and 5,047 ft, top of spillway gates, all of which is available for release. Water is released down South Fork Feather River for power development and irrigation. Records represent total contents at 2400 hours. See schematic diagram of South Fork Feather River Basin.

COOPERATION.- Records provided by Oroville-Wyandotte Irrigation District, under general supervision of the U.S. Geological Survey, in connection with a Federal Energy Regulatory Commission project.

EXTREMES FOR PERIOD OF RECORD.-Maximum contents, 98,000 acre-ft, May 1, 1995, and May 17, 1996, elevation, 5,049.0 ft; minimum since reservoir first filled, 30,300 acre-ft, many days during 1977, elevation, 4,994.8 ft.

EXTREMES FOR CURRENT YEAR.—Maximum contents, 84,400 acre-ft, several days in June, elevation, 5,040.6 ft; minimum, 47,600 acre-ft, Nov. 2-6, elevation, 5,012.8 ft.

Capacity table (elevation, in feet, and contents, in acre-feet)

(Based on survey by Pacific Gas \& Electric Co. in 1963)

$\begin{array}{llll}4,990 & 26,300 & 5,030 & 68,900 \\ 5,000 & 34,600 & 5,040 & 83,500 \\ 5,010 & 44,400 & 5,048 & 96,300 \\ 5,020 & 55,900 & 5,049 & 98,000\end{array}$

RESERVOIR STORAGE (ACRE-FEET), WATER YEAR OCTOBER 1998 TO SEPTEMBER 1999

DAILY OBSERVATION AT 2400 HOURS

\begin{tabular}{|c|c|c|c|c|c|c|c|c|c|c|c|c|}
\hline DAY & $\mathrm{OCT}$ & NOV & $\mathrm{DEC}$ & JAN & FEB & MAR & APR & MAY & JUN & JUL & AUG & SEP \\
\hline 1 & 59300 & 47800 & 53700 & 61100 & 69300 & 72700 & 71100 & 70600 & 83300 & 82000 & 75500 & 68400 \\
\hline 2 & 58700 & 47600 & 54300 & 61100 & 69500 & 73300 & 70800 & 71200 & 83500 & 81700 & 75200 & 68100 \\
\hline 3 & 58400 & 47600 & 56100 & 61200 & 69600 & 74000 & 70300 & 71800 & 83600 & 81400 & 75000 & 67800 \\
\hline 4 & 58000 & 47600 & 56900 & 61200 & 69600 & 74400 & 70100 & 72200 & 84000 & 81300 & 74700 & 67700 \\
\hline 5 & 57400 & 47600 & 57400 & 61100 & 69600 & 74900 & 69900 & 72700 & 84100 & 81100 & 74400 & 67600 \\
\hline 6 & 57100 & 47600 & 57800 & 60800 & 69800 & 75000 & 69600 & 73000 & 84100 & 81000 & 74100 & 67300 \\
\hline 7 & 56700 & 47900 & 58200 & 60600 & 70500 & 75300 & 69200 & 73400 & 84100 & 80700 & 74000 & 67100 \\
\hline 8 & 56300 & 47900 & 58500 & 60300 & 71100 & 75500 & 69000 & 73900 & 84300 & 80600 & 73900 & 66900 \\
\hline 9 & 55900 & 47900 & 58700 & 60000 & 72500 & 75500 & 68600 & 74300 & 84300 & 80400 & 73600 & 66700 \\
\hline 10 & 55500 & 47900 & 58900 & 59800 & 73100 & 75500 & 68400 & 74600 & 84400 & 80100 & 73300 & 66500 \\
\hline 11 & 55100 & 47900 & 59000 & 59500 & 73300 & 75300 & 68100 & 75000 & 84400 & 80000 & 73100 & 66300 \\
\hline 12 & 54700 & 47900 & 59100 & 59300 & 73300 & 75300 & 67800 & 75300 & 84400 & 79800 & 72800 & 66000 \\
\hline 13 & 54300 & 47900 & 59400 & 59000 & 73000 & 75200 & 67500 & 75700 & 84400 & 79700 & 72700 & 65900 \\
\hline 14 & 53900 & 47900 & 59500 & 58700 & 72800 & 75000 & 67200 & 76200 & 84400 & 79400 & 72500 & 65600 \\
\hline 15 & 53600 & 47900 & 59700 & 58700 & 72700 & 75000 & 66900 & 76500 & 84400 & 79200 & 72200 & 65400 \\
\hline 16 & 53100 & 47900 & 59800 & 58600 & 72700 & 75000 & 66800 & 76900 & 84400 & 79000 & 72000 & 65200 \\
\hline 17 & 52700 & 48100 & 59900 & 58900 & 72700 & 74900 & 66700 & 77200 & 84300 & 78700 & 71800 & 65000 \\
\hline 18 & 52200 & 48100 & 59900 & 60000 & 72700 & 74400 & 66700 & 77600 & 84100 & 78500 & 71500 & 64700 \\
\hline 19 & 51900 & 48100 & 60000 & 61200 & 72500 & 74100 & 66700 & 78100 & 84000 & 78200 & 71200 & 64600 \\
\hline 20 & 51500 & 48100 & 60200 & 62900 & 72500 & 73900 & 66800 & 78500 & 83800 & 78100 & 71100 & 64300 \\
\hline 21 & 51100 & 48300 & 60300 & 63800 & 72400 & 73600 & 66900 & 79000 & 83800 & 77800 & 70800 & 64200 \\
\hline 22 & 50600 & 48400 & 60400 & 65000 & 72200 & 73300 & 67100 & 79400 & 83700 & 77600 & 70600 & 63900 \\
\hline 23 & 50300 & 49700 & 60400 & 66200 & 72000 & 73000 & 67200 & 79800 & 83500 & 77400 & 70300 & 63800 \\
\hline 24 & 49900 & 49900 & 60600 & 66800 & 71700 & 72800 & 67600 & 80400 & 83200 & 77200 & 70200 & 63600 \\
\hline 25 & 49600 & 50200 & 60600 & 67300 & 71500 & 72500 & 68000 & 81000 & 83000 & 76900 & 69900 & 63300 \\
\hline 26 & 49100 & 50400 & 60700 & 67700 & 71400 & 72400 & 68500 & 81400 & 82900 & 76800 & 69800 & 63200 \\
\hline 27 & 48800 & 50700 & 60800 & 68000 & 71400 & 72100 & 69000 & 82000 & 82800 & 76500 & 69500 & 62900 \\
\hline 28 & 48300 & 51000 & 60800 & 68200 & 72100 & 71800 & 69600 & 82300 & 82600 & 76300 & 69300 & 62600 \\
\hline 29 & 48000 & 51400 & 60800 & 68500 & --- & 71700 & 69900 & 82600 & 82500 & 76000 & 69000 & 62500 \\
\hline 30 & 47800 & 53000 & 61000 & 68600 & --- & 71500 & 70300 & 82900 & 82300 & 75900 & 68900 & 62300 \\
\hline 31 & 47800 & --- & 61100 & 69000 & --- & 71200 & --- & 83200 & --- & 75600 & 68600 & -- \\
\hline MAX & 59300 & 53000 & 61100 & 69000 & 73300 & 75500 & 71100 & 83200 & 84400 & 82000 & 75500 & 68400 \\
\hline MIN & 47800 & 47600 & 53700 & 58600 & 69300 & 71200 & 66700 & 70600 & 82300 & 75600 & 68600 & 62300 \\
\hline$a$ & 5012.9 & 5017.5 & 5024.0 & 5030.1 & 5032.2 & 5031.6 & 5031.0 & 5039.8 & 5039.2 & 5034.6 & 5029.8 & 5024.9 \\
\hline $\mathrm{b}$ & -11900 & +5200 & +8100 & +7900 & +3100 & -900 & -900 & +12900 & -900 & -6700 & -7000 & -6300 \\
\hline
\end{tabular}

CAL YR 1998 b +5600

WTR YR 1999 b +2600

a Elevation, in feet, at end of month.

b change in contents, in acre-feet. 
11395030 SOUTH FORK FEATHER RIVER BELOW LITTLE GRASS VALLEY DAM, CA

LOCATION.-Lat 3943'26", long 12101'16", in SW 1/4 NW 1/4 sec.31, T.22 N., R.9 E., Plumas County, Hydrologic Unit 18020123, Plumas National Forest, on left bank 0.1 mi downstream from Little Grass Valley Dam and 3.5 mi northwest of La Porte.

DRAINAGE AREA.- $-25.9 \mathrm{mi}^{2}$.

PERIOD OF RECORD.-O October 1927 to September 1933 (published as "near La Porte"), October 1960 to current year.

REVISED RECORDS.-WSP 1931: Drainage area.

GAGE.-Water-stage recorder. Datum of gage is 4,809.0 ft above sea level. Prior to Oct. 1, 1960, at site 0.4 mi upstream at different datum. Oct. 1, 1960, to Oct. 30, 1962, at present site and datum. Nov. 1, 1962, to May 31, 1966, at site on outlet works at base of Little Grass Valley Dam $0.1 \mathrm{mi}$ upstream at datum $4,850.00 \mathrm{ft}$ above sea level.

REMARKS.-Flow regulated by Little Grass Valley Reservoir (station 11395020) beginning in October 1961. No diversion upstream from station. See schematic diagram of South Fork Feather River Basin.

COOPERATION.--Records provided by Oroville-Wyandotte Irrigation District, under general supervision of the U.S. Geological Survey, in connection with a Federal Energy Regulatory Commission project.

EXTREMES FOR PERIOD OF RECORD.-Maximum discharge, 7,370 $\mathrm{ft}^{3} / \mathrm{s}$, Jan. 1, 1997, gage height, $14.80 \mathrm{ft}$; minimum daily, $0.2 \mathrm{ft}^{3} / \mathrm{s}$, Oct. 28-31, Nov. 2, 1961.

DISCHARGE, CUBIC FEET PER SECOND, WATER YEAR OCTOBER 1998 TO SEPTEMBER 1999 DAILY MEAN VALUES

\begin{tabular}{|c|c|c|c|c|c|c|c|c|c|c|c|c|}
\hline DAY & OCT & NOV & $\mathrm{DEC}$ & JAN & FEB & MAR & $A P R$ & MAY & JUN & JUL & AUG & SEP \\
\hline 1 & 204 & 15 & 20 & 16 & 9.9 & 119 & 260 & 112 & 67 & 113 & 112 & 112 \\
\hline 2 & 204 & 15 & 21 & 16 & 9.9 & 116 & 260 & 112 & 66 & 113 & 113 & 112 \\
\hline 3 & 204 & 15 & 32 & 16 & 9.9 & 119 & 259 & 112 & 66 & 113 & 113 & 112 \\
\hline 4 & 204 & 15 & 20 & 16 & 68 & 116 & 259 & 112 & 66 & 113 & 113 & 112 \\
\hline 5 & 203 & 15 & 18 & 100 & 113 & 116 & 260 & 112 & 66 & 113 & 113 & 112 \\
\hline 6 & 203 & 15 & 17 & 165 & 114 & 136 & 259 & 112 & 66 & 113 & 113 & 112 \\
\hline 7 & 203 & 16 & 17 & 165 & 117 & 158 & 260 & 113 & 66 & 113 & 112 & 112 \\
\hline 8 & 203 & 16 & 17 & 164 & 116 & 174 & 259 & 113 & 66 & 113 & 113 & 112 \\
\hline 9 & 203 & 16 & 17 & 165 & 123 & 203 & 259 & 112 & 66 & 113 & 113 & 112 \\
\hline 10 & 203 & 16 & 17 & 165 & 117 & 190 & 259 & 112 & 66 & 113 & 113 & 112 \\
\hline 11 & 203 & 16 & 16 & 164 & 194 & 178 & 259 & 113 & 66 & 113 & 113 & 112 \\
\hline 12 & 203 & 16 & 16 & 165 & 275 & 166 & 259 & 114 & 66 & 113 & 113 & 112 \\
\hline 13 & 203 & 16 & 17 & 166 & 274 & 155 & 259 & 114 & 66 & 113 & 113 & 112 \\
\hline 14 & 203 & 16 & 17 & 166 & 273 & 145 & 259 & 84 & 66 & 113 & 113 & 112 \\
\hline 15 & 204 & 16 & 17 & 166 & 273 & 136 & 259 & 65 & 94 & 113 & 113 & 112 \\
\hline 16 & 205 & 16 & 17 & 168 & 273 & 127 & 259 & 65 & 113 & 114 & 113 & 112 \\
\hline 17 & 204 & 16 & 17 & 169 & 277 & 199 & 261 & 65 & 113 & 113 & 113 & 112 \\
\hline 18 & 203 & 16 & 17 & 177 & 275 & 261 & 261 & 66 & 113 & 113 & 113 & 112 \\
\hline 19 & 203 & 16 & 17 & 174 & 274 & 261 & 262 & 66 & 113 & 112 & 113 & 112 \\
\hline 20 & 204 & 16 & 17 & 178 & 274 & 261 & 262 & 66 & 113 & 112 & 113 & 110 \\
\hline 21 & 203 & 16 & 16 & 83 & 273 & 261 & 262 & 67 & 113 & 112 & 113 & 110 \\
\hline 22 & 203 & 18 & 16 & 12 & 273 & 261 & 261 & 68 & 113 & 112 & 112 & 110 \\
\hline 23 & 203 & 25 & 16 & 15 & 273 & 261 & 172 & 68 & 113 & 112 & 112 & 110 \\
\hline 24 & 203 & 18 & 16 & 12 & 273 & 261 & 109 & 69 & 113 & 112 & 111 & 110 \\
\hline 25 & 203 & 17 & 16 & 11 & 273 & 261 & 110 & 69 & 113 & 113 & 111 & 110 \\
\hline 26 & 204 & 16 & 16 & 11 & 203 & 261 & 111 & 69 & 113 & 113 & 112 & 110 \\
\hline 27 & 202 & 18 & 16 & 10 & 113 & 261 & 111 & 68 & 113 & 113 & 112 & 110 \\
\hline 28 & 202 & 17 & 16 & 10 & 118 & 260 & 111 & 68 & 113 & 113 & 112 & 110 \\
\hline 29 & 202 & 17 & 16 & 10 & -- & 261 & 110 & 67 & 113 & 113 & 112 & 110 \\
\hline 30 & 95 & 30 & 16 & 9.9 & --- & 261 & 111 & 67 & 113 & 113 & 112 & 110 \\
\hline 31 & 15 & --- & 16 & 9.9 & --- & 261 & --- & 67 & --- & 113 & 112 & --- \\
\hline TOTAL & 6004 & 506 & 540 & 2874.8 & 5258.7 & 6206 & 6662 & 2687 & 2714 & 3498 & 3489 & 3338 \\
\hline MEAN & 194 & 16.9 & 17.4 & 92.7 & 188 & 200 & 222 & 86.7 & 90.5 & 113 & 113 & 111 \\
\hline MAX & 205 & 30 & 32 & 178 & 277 & 261 & 262 & 114 & 113 & 114 & 113 & 112 \\
\hline MIN & 15 & 15 & 16 & 9.9 & 9.9 & 116 & 109 & 65 & 66 & 112 & 111 & 110 \\
\hline $\mathrm{AC}-\mathrm{FT}$ & 11910 & 1000 & 1070 & 5700 & 10430 & 12310 & 13210 & 5330 & 5380 & 6940 & 6920 & 6620 \\
\hline
\end{tabular}


11395030 SOUTH FORK FEATHER RIVER BELOW LITTLE GRASS VALLEY DAM, CA—Continued

STATISTICS OF MONTHLY MEAN DATA FOR WATER YEARS 1928 - 1933, BY WATER YEAR (WY)

\begin{tabular}{|c|c|c|c|c|c|c|c|c|c|c|c|c|}
\hline & OCT & NOV & $\mathrm{DEC}$ & JAN & FEB & MAR & APR & MAY & JUN & JUL & AUG & SEP \\
\hline MEAN & 2.56 & 19.5 & 47.6 & 26.3 & 45.2 & 134 & 181 & 201 & 78.8 & 7.70 & 1.74 & 1.35 \\
\hline MAX & 6.62 & 94.5 & 206 & 51.3 & 94.7 & 386 & 301 & 384 & 169 & 13.7 & 2.54 & 1.72 \\
\hline (WY) & 1932 & 1928 & 1930 & 1928 & 1930 & 1928 & 1930 & 1932 & 1933 & 1932 & 1932 & 1930 \\
\hline MIN & 1.43 & 1.67 & 2.65 & 3.60 & 3.55 & 14.5 & 106 & 48.9 & 13.8 & 2.38 & 1.06 & 1.04 \\
\hline (WY) & 1929 & 1930 & 1933 & 1933 & 1933 & 1933 & 1933 & 1931 & 1931 & 1931 & 1931 & 1931 \\
\hline
\end{tabular}

SUMMARY STATISTICS

ANNUAL MEAN

HIGHEST ANNUAL MEAN

LOWEST ANNUAL MEAN

HIGHEST DAILY MEAN

LOWEST DAILY MEAN

ANNUAL SEVEN-DAY MINIMUM

INSTANTANEOUS PEAK FLOW

INSTANTANEOUS PEAK STAGE

ANNUAL RUNOFF (AC-FT)

10 PERCENT EXCEEDS

50 PERCENT EXCEEDS

90 PERCENT EXCEEDS
WATER YEARS 1928 - 1933

\begin{tabular}{|c|c|c|}
\hline 62.3 & & \\
\hline 85.6 & & 1932 \\
\hline 28.0 & & 1931 \\
\hline 1800 & Mar 25 & 1928 \\
\hline .90 & Aug 25 & 1931 \\
\hline .90 & Sep 1 & 1931 \\
\hline 2600 & Mar 26 & 1928 \\
\hline 7.00 & Mar 26 & 1928 \\
\hline 45140 & & \\
\hline 202 & & \\
\hline 10 & & \\
\hline 1.4 & & \\
\hline
\end{tabular}

STATISTICS OF MONTHLY MEAN DATA FOR WATER YEARS 1963 - 1999, BY WATER YEAR (WY)

\begin{tabular}{|c|c|c|c|c|c|c|c|c|c|c|c|c|}
\hline & OCT & NOV & $\mathrm{DEC}$ & JAN & $\mathrm{FEB}$ & MAR & APR & MAY & JUN & JUL & AUG & SEP \\
\hline MEAN & 100 & 74.0 & 72.1 & 104 & 111 & 110 & 84.2 & 138 & 101 & 117 & 146 & 167 \\
\hline MAX & 305 & 404 & 420 & 725 & 694 & 586 & 317 & 489 & 403 & 350 & 344 & \\
\hline (WY) & 1970 & 1982 & 1982 & 1997 & 1986 & 1995 & 1989 & 1995 & 1998 & 1983 & 1968 & \\
\hline MIN & 13.0 & 2.94 & 4.01 & 2.36 & 2.25 & 3.70 & 4.31 & 4.38 & 3.99 & 3.71 & 7.43 & 10. \\
\hline (WY) & 1986 & 1976 & 1979 & 1964 & 1976 & 1964 & 1964 & 1977 & 1977 & 1977 & 1976 & 198 \\
\hline
\end{tabular}

SUMMARY STATISTICS

ANNUAL TOTAL

ANNUAL MEAN

HIGHEST ANNUAL MEAN

LOWEST ANNUAL MEAN

HIGHEST DAILY MEAN

LOWEST DAILY MEAN

ANNUAL SEVEN-DAY MINIMUM

INSTANTANEOUS PEAK FLOW

INSTANTANEOUS PEAK STAGE

ANNUAL RUNOFF (AC-FT)

10 PERCENT EXCEEDS

50 PERCENT EXCEEDS

90 PERCENT EXCEEDS
FOR 1998 CALENDAR YEAR

64704
177
703
12
12

128300
383
195
16

FOR 1999 WATER YEAR

$\begin{array}{ccc}43777.5 & & \\ 120 & & \\ & & \\ & & \\ 277 & \text { Feb } 17 \\ 9.9 & \text { Jan } 30 \\ 9.9 & \text { Jan } 28 \\ 284 & \text { Feb } 17 \\ 9.10 & \text { Feb } 17 \\ 86830 & & \\ 260 & & \\ 113 & & \\ 16 & & \end{array}$

WATER YEARS 1963 - 1999

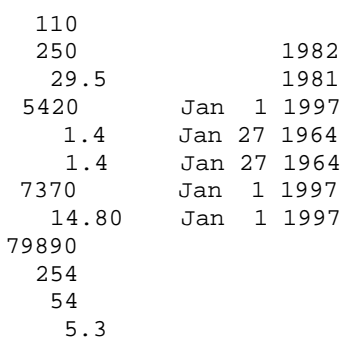


11395150 SOUTH FORK TUNNEL NEAR STRAWBERRY VALLEY, CA

LOCATION.-Lat $39^{\circ} 38^{\prime} 55^{\prime \prime}$, long $12^{\circ} 07^{\prime} 00^{\prime \prime}$, in NW 1/4 SW 1/4 sec.29, T.21 N., R.8 E., Plumas County, Hydrologic Unit 18020123, Plumas National Forest, 3.2 mi upstream from Rock Creek, and 5.8 mi north of Strawberry Valley.

PERIOD OF RECORD.-October 1973 to current year. Records of daily discharge for November 1961 to September 1973 are in files of the U.S. Geological Survey. Monthly diversion used to adjust South Fork Feather River below diversion dam near Strawberry Valley (station 11395200) since October 1961.

GAGE.-Water-stage recorder. Datum of gage is sea level.

REMARKS.--Tunnel diverts water from South Fork Feather River to Sly Creek Reservoir (station 11395400) for power development. See schematic diagram of South Fork Feather River Basin.

COOPERATION.-Records provided by Oroville-Wyandotte Irrigation District, under general supervision of the U.S. Geological Survey, in connection with a Federal Energy Regulatory Commission project.

EXTREMES FOR PERIOD OF RECORD.-Maximum daily discharge, $570 \mathrm{ft}^{3} / \mathrm{s}$, Mar. 13, May 25-29, June 3, 1983; no flow many days in 1980-82, Mar. 11-28, 1995 and Jan. 1-9, 1997.

DISCHARGE, CUBIC FEET PER SECOND, WATER YEAR OCTOBER 1998 TO SEPTEMBER 1999

DAILY MEAN VALUES

\begin{tabular}{|c|c|c|c|c|c|c|c|c|c|c|c|c|}
\hline DAY & OCT & NOV & $\mathrm{DEC}$ & JAN & FEB & MAR & APR & MAY & JUN & JUL & AUG & SEP \\
\hline 1 & 212 & 8.7 & 303 & 31 & 70 & 448 & 326 & 182 & 80 & 108 & 112 & 107 \\
\hline 2 & 212 & 7.7 & 208 & 30 & 66 & 357 & 322 & 185 & 80 & 109 & 111 & 108 \\
\hline 3 & 215 & 7.6 & 459 & 29 & 63 & 440 & 317 & 223 & 80 & 109 & 111 & 108 \\
\hline 4 & 212 & 7.3 & 273 & 28 & 93 & 353 & 313 & 208 & 79 & 109 & 110 & 108 \\
\hline 5 & 211 & 8.6 & 174 & 75 & 159 & 306 & 316 & 194 & 77 & 110 & 110 & 109 \\
\hline 6 & 211 & 10 & 131 & 177 & 165 & 282 & 310 & 193 & 76 & 109 & 110 & 108 \\
\hline 7 & 211 & 31 & 102 & 176 & 348 & 276 & 308 & 194 & 75 & 109 & 111 & 107 \\
\hline 8 & 212 & 24 & 89 & 175 & 364 & 279 & 311 & 186 & 74 & 109 & 110 & 107 \\
\hline 9 & 211 & 16 & 76 & 174 & 527 & 303 & 304 & 178 & 73 & 109 & 110 & 108 \\
\hline 10 & 210 & 15 & 68 & 174 & 439 & 278 & 303 & 172 & 73 & 109 & 109 & 107 \\
\hline 11 & 209 & 16 & 62 & 173 & 397 & 255 & 308 & 168 & 72 & 109 & 110 & 107 \\
\hline 12 & 209 & 14 & 58 & 173 & 438 & 235 & 312 & 168 & 72 & 108 & 109 & 107 \\
\hline 13 & 209 & 14 & 57 & 174 & 400 & 225 & 320 & 166 & 72 & 109 & 108 & 106 \\
\hline 14 & 210 & 14 & 56 & 174 & 382 & 211 & 333 & 145 & 71 & 109 & 108 & 105 \\
\hline 15 & 208 & 14 & 54 & 187 & 367 & 200 & 342 & 111 & 85 & 109 & 107 & 105 \\
\hline 16 & 207 & 14 & 55 & 216 & 380 & 190 & 349 & 106 & 114 & 109 & 108 & 104 \\
\hline 17 & 206 & 34 & 58 & 248 & 483 & 245 & 363 & 103 & 114 & 109 & 109 & 104 \\
\hline 18 & 205 & 22 & 56 & 448 & 467 & 326 & 376 & 102 & 113 & 109 & 108 & 104 \\
\hline 19 & 205 & 17 & 55 & 446 & 459 & 326 & 380 & 101 & 112 & 109 & 108 & 104 \\
\hline 20 & 205 & 15 & 53 & 544 & 426 & 329 & 382 & 100 & 113 & 109 & 108 & 104 \\
\hline 21 & 205 & 22 & 47 & 427 & 400 & 329 & 377 & 98 & 113 & 110 & 108 & 103 \\
\hline 22 & 208 & 74 & 44 & 256 & 382 & 325 & 366 & 97 & 112 & 111 & 107 & 104 \\
\hline 23 & 211 & 175 & 42 & 451 & 371 & 324 & 300 & 96 & 111 & 112 & 108 & 104 \\
\hline 24 & 219 & 125 & 41 & 281 & 367 & 327 & 213 & 95 & 111 & 112 & 107 & 104 \\
\hline 25 & 212 & 67 & 40 & 204 & 388 & 350 & 213 & 93 & 111 & 111 & 107 & 104 \\
\hline 26 & 210 & 51 & 38 & 161 & 313 & 349 & 224 & 91 & 110 & 111 & 107 & 104 \\
\hline 27 & 212 & 106 & 36 & 129 & 204 & 348 & 219 & 89 & 110 & 110 & 109 & 103 \\
\hline 28 & 211 & 94 & 34 & 108 & 361 & 343 & 205 & 87 & 110 & 111 & 107 & 102 \\
\hline 29 & 208 & 86 & 33 & 94 & --- & 337 & 191 & 85 & 110 & 111 & 107 & 103 \\
\hline 30 & 161 & 400 & 32 & 83 & --- & 336 & 183 & 83 & 109 & 112 & 107 & 103 \\
\hline 31 & 12 & --- & 32 & 79 & --- & 331 & --- & 81 & --- & 112 & 107 & --- \\
\hline TOTAL & 6259 & 1509.9 & 2866 & 6125 & 9279 & 9563 & 9086 & 4180 & 2812 & 3402 & 3368 & 3161 \\
\hline MEAN & 202 & 50.3 & 92.5 & 198 & 331 & 308 & 303 & 135 & 93.7 & 110 & 109 & 105 \\
\hline MAX & 219 & 400 & 459 & 544 & 527 & 448 & 382 & 223 & 114 & 112 & 112 & 109 \\
\hline MIN & 12 & 7.3 & 32 & 28 & 63 & 190 & 183 & 81 & 71 & 108 & 107 & 102 \\
\hline $\mathrm{AC}-\mathrm{FT}$ & 12410 & 2990 & 5680 & 12150 & 18400 & 18970 & 18020 & 8290 & 5580 & 6750 & 6680 & 6270 \\
\hline
\end{tabular}

STATISTICS OF MONTHLY MEAN DATA FOR WATER YEARS 1974 - 1999, BY WATER YEAR (WY)

\begin{tabular}{|c|c|c|c|c|c|c|c|c|c|c|c|c|}
\hline MEAN & 87.8 & 99.9 & 107 & 129 & 153 & 182 & 152 & 168 & 113 & 117 & 130 & 148 \\
\hline MAX & 202 & 377 & 462 & 381 & 406 & 454 & 429 & 520 & 421 & 363 & 327 & 390 \\
\hline (WY) & 1999 & 1982 & 1982 & 1974 & 1996 & 1983 & 1989 & 1993 & 1983 & 1983 & 1983 & 1978 \\
\hline MIN & 6.21 & 4.14 & 3.36 & 5.99 & 8.49 & 9.71 & 8.68 & 16.4 & 7.22 & 4.43 & 4.03 & .000 \\
\hline (WY) & 1986 & 1977 & 1977 & 1977 & 1977 & 1977 & 1977 & 1977 & 1977 & 1977 & 1981 & 1981 \\
\hline
\end{tabular}

SUMMARY STATISTICS

ANNUAL TOTAL

ANNUAL MEAN

HIGHEST ANNUAL MEAN

LOWEST ANNUAL MEAN

HIGHEST DAILY MEAN

LOWEST DAILY MEAN

ANNUAL SEVEN-DAY MINIMUM

ANNUAL RUNOFF (AC-FT)

10 PERCENT EXCEEDS

50 PERCENT EXCEEDS

90 PERCENT EXCEEDS
FOR 1998 CALENDAR YEAR

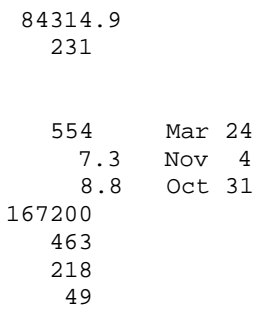

FOR 1999 WATER YEAR

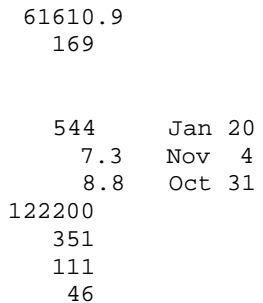

WATER YEARS 1974 - 1999

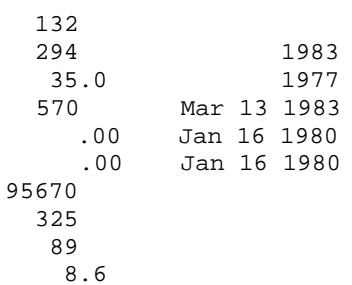


LOCATION.-Lat 39³8'51", long 12107'04", in NE 1/4 SE 1/4 sec.30, T.21 N., R.8 E., Plumas County, Hydrologic Unit 18020123, Plumas National Forest, on left bank $0.1 \mathrm{mi}$ downstream from diversion dam, $3.1 \mathrm{mi}$ upstream from Rock Creek, and $5.8 \mathrm{mi}$ north of Strawberry Valley. DRAINAGE AREA.- $-37.7 \mathrm{mi}^{2}$.

PERIOD OF RECORD.-October 1960 to current year.

REVISED RECORDS.-WDR CA-80-4: 1976(M).

GAGE.-Water-stage recorder and since May 8, 1987, sharp crested rectangular weir. Datum of gage is 3,535.02 ft above sea level (levels by Oroville-Wyandotte Irrigation District).

REMARKS.-Flow regulated by Little Grass Valley Reservoir (station 11395020) since October 1961. South Fork Diversion Tunnel, maximum capacity, about $600 \mathrm{ft}^{3} / \mathrm{s} 500 \mathrm{ft}$ upstream, diverts to Sly Creek Reservoir (station 11395400); diversion began in November 1961. See schematic diagram of South Fork Feather River Basin.

COOPERATION.-Records provided by Oroville-Wyandotte Irrigation District, under general supervision of the U.S. Geological Survey, in connection with a Federal Energy Regulatory Commission project.

EXTREMES FOR PERIOD OF RECORD.-Maximum discharge, 11,300 $\mathrm{ft}^{3} / \mathrm{s}$, Jan. 1, 1997, gage height unknown, from computation of peak flow over diversion dam; minimum daily, $0.3 \mathrm{ft}^{3} / \mathrm{s}$, Dec. 25, 1962, to Jan. 2, 1963, Mar. 1-3, 1963.

DISCHARGE, CUBIC FEET PER SECOND, WATER YEAR OCTOBER 1998 TO SEPTEMBER 1999

\begin{tabular}{|c|c|c|c|c|c|c|c|c|c|c|c|c|}
\hline DAY & $\mathrm{OCT}$ & NOV & $\mathrm{DEC}$ & JAN & FEB & MAR & $\mathrm{APR}$ & MAY & JUN & JUL & AUG & SEP \\
\hline 1 & 12 & 10 & 6.4 & 6.0 & 6.2 & 5.8 & 5.8 & 11 & 11 & 11 & 11 & 11 \\
\hline 2 & 12 & 10 & 6.3 & 6.0 & 6.2 & 5.8 & 5.8 & 11 & 11 & 11 & 11 & 11 \\
\hline 3 & 12 & 10 & 27 & 6.0 & 6.2 & 5.9 & 5.8 & 11 & 11 & 11 & 11 & 11 \\
\hline 4 & 12 & 10 & 6.4 & 6.0 & 5.9 & 5.8 & 5.8 & 11 & 11 & 11 & 11 & 11 \\
\hline 5 & 12 & 10 & 6.2 & 6.1 & 5.8 & 5.8 & 5.8 & 11 & 11 & 11 & 11 & 11 \\
\hline 6 & 12 & 7.7 & 6.2 & 6.2 & 6.0 & 5.8 & 5.8 & 11 & 11 & 11 & 11 & 11 \\
\hline 7 & 12 & 5.9 & 6.2 & 6.2 & 6.5 & 5.8 & 5.8 & 11 & 11 & 11 & 11 & 11 \\
\hline 8 & 12 & 5.8 & 6.2 & 6.2 & 6.6 & 5.8 & 5.9 & 11 & 11 & 11 & 11 & 11 \\
\hline 9 & 12 & 5.8 & 6.2 & 6.2 & 226 & 5.8 & 5.8 & 11 & 11 & 11 & 11 & 11 \\
\hline 10 & 12 & 5.8 & 6.2 & 6.2 & 31 & 5.8 & 5.8 & 11 & 11 & 11 & 11 & 11 \\
\hline 11 & 12 & 5.8 & 6.2 & 6.2 & 5.1 & 5.8 & 5.8 & 11 & 11 & 11 & 11 & 11 \\
\hline 12 & 12 & 5.8 & 6.2 & 6.2 & 5.5 & 5.8 & 5.8 & 11 & 11 & 11 & 11 & 11 \\
\hline 13 & 12 & 5.8 & 6.2 & 6.2 & 5.8 & 5.8 & 5.8 & 11 & 11 & 11 & 11 & 11 \\
\hline 14 & 12 & 5.8 & 6.1 & 6.2 & 5.8 & 5.8 & 5.8 & 11 & 11 & 11 & 11 & 11 \\
\hline 15 & 12 & 5.8 & 6.0 & 6.3 & 5.8 & 5.8 & 5.8 & 11 & 11 & 11 & 11 & 11 \\
\hline 16 & 12 & 5.8 & 6.0 & 6.2 & 5.9 & 5.8 & 5.8 & 11 & 11 & 11 & 11 & 11 \\
\hline 17 & 12 & 5.9 & 6.0 & 6.3 & 6.1 & 5.8 & 5.8 & 11 & 11 & 11 & 11 & 11 \\
\hline 18 & 12 & 5.8 & 6.0 & 6.7 & 6.0 & 5.8 & 5.8 & 11 & 11 & 11 & 11 & 11 \\
\hline 19 & 12 & 5.8 & 6.0 & 7.4 & 5.9 & 5.8 & 5.8 & 11 & 11 & 11 & 11 & 11 \\
\hline 20 & 12 & 5.8 & 6.0 & 73 & 5.8 & 5.8 & 5.8 & 11 & 11 & 11 & 11 & 11 \\
\hline 21 & 12 & 6.0 & 6.0 & 6.7 & 5.8 & 5.8 & 5.8 & 11 & 11 & 11 & 11 & 11 \\
\hline 22 & 12 & 6.0 & 6.0 & 6.5 & 5.8 & 5.8 & 5.8 & 11 & 11 & 11 & 11 & 11 \\
\hline 23 & 12 & 6.4 & 6.0 & 7.6 & 5.8 & 5.8 & 5.8 & 11 & 11 & 11 & 11 & 11 \\
\hline 24 & 12 & 6.2 & 6.0 & 6.5 & 5.8 & 5.8 & 5.6 & 11 & 11 & 11 & 11 & 11 \\
\hline 25 & 12 & 6.0 & 6.0 & 6.2 & 5.8 & 5.9 & 5.6 & 11 & 11 & 11 & 11 & 11 \\
\hline 26 & 12 & 5.9 & 6.0 & 6.2 & 5.8 & 5.8 & 5.8 & 11 & 11 & 11 & 11 & 11 \\
\hline 27 & 12 & 6.2 & 6.0 & 6.2 & 5.8 & 5.8 & 5.8 & 11 & 11 & 11 & 11 & 11 \\
\hline 28 & 12 & 6.2 & 6.0 & 6.2 & 5.8 & 5.8 & 5.7 & 11 & 11 & 11 & 11 & 11 \\
\hline 29 & 12 & 6.2 & 6.0 & 6.2 & --- & 5.8 & 5.6 & 11 & 11 & 11 & 11 & 11 \\
\hline 30 & 11 & 6.9 & 6.0 & 6.2 & --- & 5.9 & 8.8 & 11 & 11 & 11 & 11 & 11 \\
\hline 31 & 10 & --- & 6.0 & 6.2 & --- & 5.8 & --- & 11 & --- & 11 & 11 & --- \\
\hline TOTAL & 369 & 201.1 & 210.0 & 262.5 & 410.5 & 180.1 & 176.4 & 341 & 330 & 341 & 341 & 330 \\
\hline MEAN & 11.9 & 6.70 & 6.77 & 8.47 & 14.7 & 5.81 & 5.88 & 11.0 & 11.0 & 11.0 & 11.0 & 11.0 \\
\hline MAX & 12 & 10 & 27 & 73 & 226 & 5.9 & 8.8 & 11 & 11 & 11 & 11 & 11 \\
\hline MIN & 10 & 5.8 & 6.0 & 6.0 & 5.1 & 5.8 & 5.6 & 11 & 11 & 11 & 11 & 11 \\
\hline $\mathrm{AC}-\mathrm{FT}$ & 732 & 399 & 417 & 521 & 814 & 357 & 350 & 676 & 655 & 676 & 676 & 655 \\
\hline
\end{tabular}

STATISTICS OF MONTHLY MEAN DATA FOR WATER YEARS 1964 - 1999, BY WATER YEAR (WY)

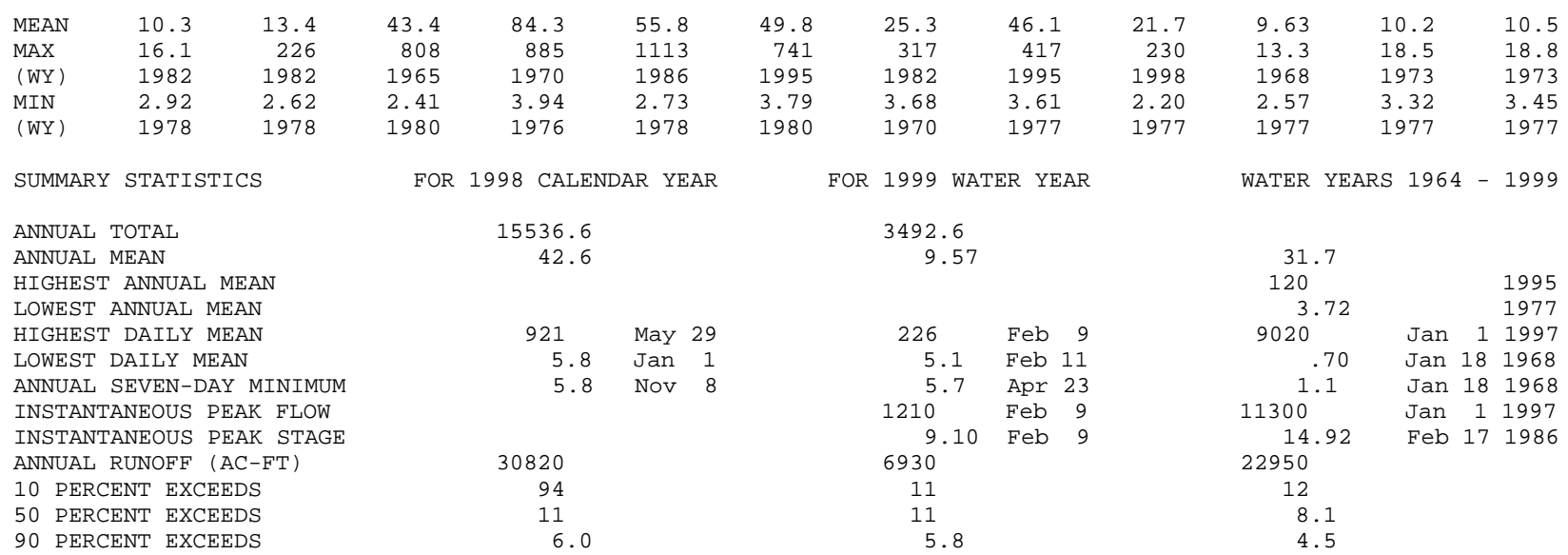


11395400 SLY CREEK RESERVOIR NEAR STRAWBERRY VALLEY, CA

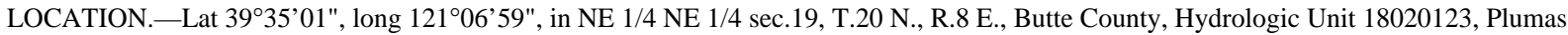
National Forest, on right bank $100 \mathrm{ft}$ upstream from dam on Lost Creek, and 1.4 mi northwest of Strawberry Valley.

DRAINAGE AREA.-24.0 $\mathrm{mi}^{2}$.

PERIOD OF RECORD.-November 1961 to current year (fragmentary prior to Mar. 14, 1962).

GAGE.-Water-stage recorder. Datum of gage is sea level (levels by Oroville-Wyandotte Irrigation District). Prior to Sept. 30, 1966, water-stage recorder in valve chamber inside dam at same datum. Oct. 1, 1966, to December 1974, nonrecording gage read once daily.

REMARKS.--Reservoir is formed by earthfill dam. Storage began in November 1961. Tota1 capacity, 65,600 acre-ft between elevations 3,285 ft, invert of outlet, and 3,531 ft, top of spillway gate, all of which is available for release. Water is diverted into reservoir from South Fork Feather River through South Fork Diversion Tunnel and from North Yuba River Basin through Slate Creek Tunnel (station 11413250). Records represent total contents at 2400 hours. See schematic diagram of South Fork Feather River Basin.

COOPERATION.--Records provided by Oroville-Wyandotte Irrigation District, under general supervision of the U.S. Geological Survey, in connection with a Federal Energy Regulatory Commission project.

EXTREMES FOR PERIOD OF RECORD.-Maximum contents, 65,600 acre-ft, June 22, 1978, elevation, 3,530.9 ft; minimum observed under normal operating conditions since reservoir first filled, 860 acre-ft, Feb. 11, 1976, elevation, 3,320.0 ft. Reservoir completely drained for powerplant construction, Sept. 12 to Oct. $17,1981$.

EXTREMES FOR CURRENT YEAR.-Maximum contents, 57,500 acre-ft, Mar. 3, elevation, 3,517.2 ft; minimum, 10,800 acre-ft, Jan. 14, elevation, unknown.

Capacity table (elevation, in feet, and contents, in acre-feet)

(Based on survey by Pacific Gas \& Electric Co. in 1946)

$\begin{array}{rrrrrr}3,310 & 450 & 3,360 & 4,300 & 3,450 & 26,300 \\ 3,315 & 655 & 3,380 & 7,360 & 3,480 & 38,500 \\ 3,320 & 860 & 3,400 & 11,500 & 3,510 & 53,400 \\ 3,340 & 2,150 & 3,420 & 16,600 & 3,531 & 65,600\end{array}$

RESERVOIR STORAGE (ACRE-FEET), WATER YEAR OCTOBER 1998 TO SEPTEMBER 1999

DAILY OBSERVATION AT 2400 HOURS

\begin{tabular}{|c|c|c|c|c|c|c|c|c|c|c|c|c|}
\hline DAY & $\mathrm{OCT}$ & NOV & $\mathrm{DEC}$ & JAN & FEB & MAR & APR & MAY & JUN & JUL & AUG & SEP \\
\hline 1 & 25900 & 20900 & 23800 & e13800 & 33000 & 57200 & 49300 & 50000 & 56700 & 51900 & 41300 & 27000 \\
\hline 2 & 26400 & 20500 & 25100 & e13200 & 32500 & 57300 & 49000 & 50300 & 56600 & 51400 & 41100 & 26600 \\
\hline 3 & 26800 & 20600 & 27800 & e 12500 & 32100 & 57500 & 48600 & 51100 & 56700 & 51400 & 40600 & 26300 \\
\hline 4 & 27200 & 20700 & 29400 & e11700 & 31800 & 57400 & 48100 & 51500 & 56600 & 51300 & 39700 & 26000 \\
\hline 5 & 27600 & 20800 & 30200 & e11500 & 31500 & 57100 & 48400 & e51900 & 56600 & 51300 & 39500 & 26000 \\
\hline 6 & 28000 & 20400 & 30300 & e11400 & 31400 & 56900 & 47700 & e 52300 & 56700 & 50800 & 39300 & 26200 \\
\hline 7 & 28400 & 19300 & 30200 & e11100 & 33100 & 56700 & 47400 & 52900 & 56600 & 50400 & 39000 & 26000 \\
\hline 8 & 28800 & 19100 & 29900 & e11000 & 35200 & 56600 & 47300 & 53200 & 56500 & 50000 & 39200 & 25200 \\
\hline 9 & 28800 & 17900 & 29500 & e 11300 & e39500 & 56500 & 47000 & 53400 & 56400 & 49500 & 38500 & 24700 \\
\hline 10 & 28000 & 17200 & 29000 & e 11600 & 42300 & 56300 & 46900 & 53600 & 56200 & 49100 & 38600 & 24400 \\
\hline 11 & 27600 & 16100 & 28400 & e11400 & 44100 & 55700 & 46500 & 53800 & 56200 & 48900 & 38500 & 24200 \\
\hline 12 & 26800 & 15300 & 27800 & e 11100 & 45500 & 54900 & 46400 & 53900 & 56200 & 48300 & 37800 & 24400 \\
\hline 13 & 26100 & 14300 & 27100 & e11000 & 46500 & 54000 & 46200 & 54200 & 56300 & 47700 & 37100 & 24000 \\
\hline 14 & 25800 & 13700 & 26400 & e10800 & 47300 & 53100 & 46200 & 54200 & 56200 & 47200 & 36700 & 23500 \\
\hline 15 & 25600 & 13500 & 25800 & e11000 & 48000 & 52200 & 46200 & 54100 & 56400 & 47200 & 36300 & 23300 \\
\hline 16 & 25700 & 13100 & 25200 & e11300 & 48900 & 51300 & 46200 & 53900 & 56200 & 47100 & 35900 & 23200 \\
\hline 17 & 25800 & 13200 & 24600 & e12400 & 50900 & 50500 & 46300 & 53800 & 56000 & 46700 & 35200 & 23400 \\
\hline 18 & 25800 & 13300 & 24000 & e14700 & 52300 & 49900 & 46500 & 53900 & 55500 & 47000 & 35000 & 23600 \\
\hline 19 & 25800 & 13200 & 23300 & e18000 & 53600 & 49300 & 46400 & 54100 & 55400 & 46300 & 34600 & 23800 \\
\hline 20 & 25900 & 13100 & 22500 & e21600 & 54300 & 49500 & 46600 & 54300 & 55800 & 46100 & 34100 & 23900 \\
\hline 21 & 26000 & 12800 & e21900 & e24000 & 54800 & 49600 & 46400 & 54500 & 55500 & 45500 & 33700 & 23900 \\
\hline 22 & 26400 & 13300 & e21100 & e27200 & e 55100 & 49600 & 46300 & 55200 & 55100 & 45100 & 33200 & 23600 \\
\hline 23 & 26000 & 15000 & e20300 & e29900 & 55200 & 49500 & 46500 & 55600 & 54700 & 44400 & 32300 & 23400 \\
\hline 24 & 25400 & 16400 & e19700 & e 31700 & 55300 & 49200 & 46900 & 55700 & 54300 & 44300 & 31600 & 23600 \\
\hline 25 & 25200 & 16800 & e19100 & 33200 & 55700 & 49300 & 47400 & 55800 & 53900 & 44600 & 30800 & 23500 \\
\hline 26 & 24600 & 16800 & e18500 & 33800 & 55700 & 49300 & 48100 & 55900 & 53800 & 44000 & 30400 & 23400 \\
\hline 27 & 24000 & 17500 & e17700 & 34000 & 55400 & 49400 & 48800 & 55700 & 54000 & 43400 & 29800 & 23000 \\
\hline 28 & 23400 & 18100 & e16900 & 34100 & 56300 & 49600 & 49400 & 55800 & 53700 & 42900 & 29200 & 22700 \\
\hline 29 & 22700 & 18700 & e15900 & 34000 & --- & 49600 & 49600 & 55800 & 53200 & 42500 & 28700 & 22200 \\
\hline 30 & 22100 & 21600 & e 15100 & 33700 & --- & 49600 & 49700 & 56300 & 52800 & 42200 & 28100 & 22000 \\
\hline 31 & 21600 & --- & e 14400 & 33300 & --- & 49500 & --- & 56400 & --- & 41900 & 27800 & --- \\
\hline MAX & 28800 & 21600 & 30300 & 34100 & 56300 & 57500 & 49700 & 56400 & 56700 & 51900 & 41300 & 27000 \\
\hline MIN & 21600 & 12800 & 14400 & 10800 & 31400 & 49200 & 46200 & 50000 & 52800 & 41900 & 27800 & 22000 \\
\hline$a$ & 3436.3 & 3436.4 & 3411.8 & 3468.0 & 3415.1 & 3502.5 & 3503.0 & 3515.3 & 3508.7 & 3487.2 & 3453.9 & 3437.6 \\
\hline $\mathrm{b}$ & -3900 & 0 & -7200 & +18900 & +23000 & -6800 & +200 & +6700 & -3600 & -10900 & -14100 & -5800 \\
\hline
\end{tabular}

CAL YR 1998 b -2100

WTR YR 1999 b -3500

e Estimated.

a Elevation, in feet, at end of month.

b Change in contents, in acre-feet. 


\section{OROVILLE-WYANDOTTE CANAL NEAR CLIPPER MILLS, CA}

LOCATION.—Lat 39³3'15", long 12111'31", in NW 1/4 NE 1/4 sec.33, T.20 N., R.7 E., Butte County, Hydrologic Unit 18020123, in concrete valve house at head of canal and $2.5 \mathrm{mi}$ north of Clipper Mills.

PERIOD OF RECORD.-October 1927 to September 1941 (published as Forbestown Ditch), October 1953 to current year. Monthly discharge only for October 1953 to September 1961, published with records for Lost Creek near Clipper Mills.

GAGE.-Water-stage recorder and Parshall flume. Datum of gage is 3,166.0 ft above sea level (levels by Oroville-Wyandotte Irrigation District). Prior to Sept. 30, 1941, nonrecording gages and Oct. 1, 1941, to Nov. 16, 1962, water-stage recorder at sites at different datums 4 mi upstream in abandoned part of canal, 0.3 mi downstream from Lost Creek Dam.

REMARKS.-Water is discharged to canal through valve in Woodleaf Penstock. Prior to Nov. 16, 1962, canal diverted from Lost Creek Dam. Water is used for irrigation and domestic supply. Demand for water reduced when a large lumber mill closed at Woodleaf in 1962. See schematic diagram of South Fork Feather River Basin.

COOPERATION.--Records provided by Oroville-Wyandotte Irrigation District, under general supervision of the U.S. Geological Survey, in connection with a Federal Energy Regulatory Commission project.

EXTREMES FOR PERIOD OF RECORD.-Maximum daily discharge, $43 \mathrm{ft}^{3} / \mathrm{s}$, Aug. 9 to Sept. 9, 1937, Aug. 13-15, 1977; no flow at times in many years.

DISCHARGE, CUBIC FEET PER SECOND, WATER YEAR OCTOBER 1998 TO SEPTEMBER 1999 DAILY MEAN VALUES

\begin{tabular}{|c|c|c|c|c|c|c|c|c|c|c|c|c|}
\hline DAY & OCT & NOV & $\mathrm{DEC}$ & JAN & FEB & MAR & APR & MAY & JUN & JUL & AUG & SEP \\
\hline 1 & 19 & .00 & .00 & .00 & .00 & .00 & .00 & .00 & 7.7 & 18 & 16 & 24 \\
\hline 2 & 19 & .00 & .00 & .00 & .00 & .00 & .00 & .00 & 7.8 & 18 & 16 & 24 \\
\hline 3 & 19 & .00 & .00 & .00 & .00 & .00 & .00 & .00 & 7.9 & 18 & 19 & 24 \\
\hline 4 & 19 & .00 & .00 & .00 & .00 & .00 & .00 & .00 & 7.9 & 18 & 21 & 24 \\
\hline 5 & 14 & .00 & .00 & .00 & .00 & .00 & .00 & .00 & 7.8 & 18 & 21 & 24 \\
\hline 6 & 11 & .00 & .00 & .00 & .00 & .00 & .00 & .00 & 7.7 & 18 & 21 & 24 \\
\hline 7 & 11 & .00 & .00 & .00 & .00 & .00 & .00 & .00 & 7.8 & 18 & 21 & 23 \\
\hline 8 & 11 & .00 & .00 & .00 & .00 & .00 & .00 & .00 & 7.8 & 18 & 21 & 24 \\
\hline 9 & 11 & .00 & .00 & .00 & .00 & .00 & .00 & .00 & 7.8 & 18 & 21 & 23 \\
\hline 10 & 11 & .00 & .00 & .00 & .00 & .00 & .00 & .00 & 7.8 & 18 & 21 & 24 \\
\hline 11 & 11 & .00 & .00 & .00 & .00 & .00 & .00 & .00 & 7.7 & 18 & 21 & 24 \\
\hline 12 & 11 & .00 & .00 & .00 & .00 & .00 & .00 & 1.5 & 7.7 & 18 & 21 & 24 \\
\hline 13 & 11 & .00 & .00 & .00 & .00 & .00 & .00 & 2.4 & 7.8 & 18 & 21 & 24 \\
\hline 14 & 7.9 & .00 & .00 & 5.9 & .00 & .00 & .00 & 5.7 & 7.8 & 18 & 21 & 24 \\
\hline 15 & 3.8 & .00 & .00 & 9.1 & .00 & .00 & .00 & 8.0 & 7.7 & 17 & 21 & 24 \\
\hline 16 & .00 & 7.0 & .00 & 4.1 & .00 & .00 & .00 & 7.8 & 7.8 & 16 & 21 & 24 \\
\hline 17 & .00 & 11 & .00 & .00 & .00 & .00 & .00 & 7.7 & 7.8 & 16 & 22 & 22 \\
\hline 18 & .00 & 11 & .00 & .00 & .00 & .00 & .00 & 7.8 & 7.8 & 16 & 24 & 21 \\
\hline 19 & .00 & 11 & .00 & .00 & .00 & .00 & .00 & 7.8 & 7.7 & 16 & 23 & 21 \\
\hline 20 & .00 & 3.7 & .00 & .00 & .00 & .00 & .00 & 7.8 & 7.7 & 16 & 24 & 21 \\
\hline 21 & .00 & .00 & .00 & .00 & .00 & .00 & .00 & 7.8 & 7.8 & 16 & 23 & 21 \\
\hline 22 & .00 & .00 & .00 & .00 & .00 & .00 & .00 & 7.8 & 7.7 & 16 & 23 & 21 \\
\hline 23 & .00 & .00 & .00 & .00 & .00 & .00 & .00 & 7.7 & 9.0 & 16 & 23 & 21 \\
\hline 24 & .00 & .00 & .00 & .00 & .00 & .00 & .00 & 7.7 & 9.6 & 16 & 23 & 21 \\
\hline 25 & .00 & .00 & .00 & .00 & .00 & .00 & .00 & 7.7 & 11 & 16 & 24 & 21 \\
\hline 26 & 5.5 & .00 & .00 & .00 & .00 & .00 & .00 & 7.8 & 11 & 16 & 24 & 21 \\
\hline 27 & 11 & .00 & .00 & .00 & .00 & .00 & .00 & 7.7 & 11 & 16 & 24 & 21 \\
\hline 28 & 4.6 & .00 & 5.3 & .00 & .00 & .00 & .00 & 7.8 & 11 & 16 & 24 & 22 \\
\hline 29 & .00 & .00 & 11 & .00 & --- & .00 & .00 & 7.7 & 16 & 16 & 24 & 24 \\
\hline 30 & .00 & .00 & 11 & .00 & --- & .00 & .00 & 7.7 & 18 & 16 & 24 & 24 \\
\hline 31 & .00 & --- & 5.3 & .00 & --- & .00 & --- & 7.8 & --- & 16 & 24 & --- \\
\hline TOTAL & 210.80 & 43.70 & 32.60 & 19.10 & 0.00 & 0.00 & 0.00 & 141.70 & 267.6 & 525 & 677 & 684 \\
\hline MEAN & 6.80 & 1.46 & 1.05 & .62 & .000 & .000 & .000 & 4.57 & 8.92 & 16.9 & 21.8 & 22.8 \\
\hline MAX & 19 & 11 & 11 & 9.1 & .00 & .00 & .00 & 8.0 & 18 & 18 & 24 & 24 \\
\hline MIN & .00 & .00 & .00 & .00 & .00 & .00 & .00 & .00 & 7.7 & 16 & 16 & 21 \\
\hline & 418 & 87 & 65 & 38 & 00 & 00 & 00 & 281 & 531 & 1040 & 1340 & 1360 \\
\hline
\end{tabular}

STATISTICS OF MONTHLY MEAN DATA FOR WATER YEARS 1963 - 1999, BY WATER YEAR (WY)

\begin{tabular}{|c|c|c|c|c|c|c|c|c|c|c|c|}
\hline \multirow{2}{*}{$\begin{array}{l}\text { MEAN } \\
\text { MAX }\end{array}$} & 12.5 & 5.57 & 2.25 & 1.32 & .76 & .96 & 1.77 & 5.57 & 11.9 & 17.2 & 20.6 \\
\hline & 20.2 & 16.5 & 8.64 & 6.89 & 5.34 & 6.70 & 11.4 & 20.2 & 29.3 & 26.4 & 37.4 \\
\hline (WY) & 1967 & 1968 & 1977 & 1968 & 1977 & 1964 & 1977 & 1977 & 1963 & 1976 & 1977 \\
\hline MIN & 3.75 & .84 & .000 & .000 & .000 & .000 & .000 & .000 & .88 & 7.60 & 9.47 \\
\hline (WY) & 1990 & 1992 & 1982 & 1980 & 1963 & 1963 & 1963 & 1975 & 1998 & 1998 & 1965 \\
\hline
\end{tabular}

SUMMARY STATISTICS

ANNUAL TOTAL

ANNUAL MEAN

HIGHEST ANNUAL MEAN

LOWEST ANNUAL MEAN

HIGHEST DAILY MEAN

LOWEST DAILY MEAN

ANNUAL SEVEN-DAY MINIMUM

ANNUAL RUNOFF (AC-FT)

10 PERCENT EXCEEDS

50 PERCENT EXCEEDS

90 PERCENT EXCEEDS
FOR 1998 CALENDAR YEAR

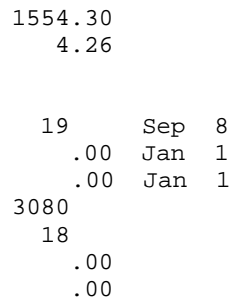

FOR 1999 WATER YEAR

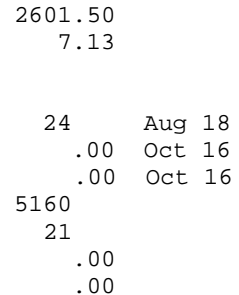

WATER YEARS 1963 - 1999

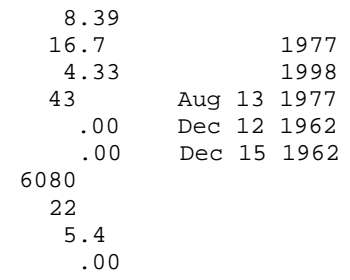


11396000 LOST CREEK NEAR CLIPPER MILLS, CA

LOCATION.-Lat 39³4’25", long 12108’26", in SE 1/4 SW 1/4 sec.24, T.20 N., R.7 E., Butte County, Hydrologic Unit 18020123, Plumas National Forest, on left bank 0.3 mi downstream from Lost Creek Reservoir, and 2.8 mi north of Clipper Mills.

DRAINAGE AREA.- $-30.0 \mathrm{mi}^{2}$.

PERIOD OF RECORD.-October 1927 to September 1941, October 1948 to current year. Records for Woodleaf Powerplant from February 1963 to September 1966 in files of the U.S. Geological Survey.

REVISED RECORDS._WSP 1395: 1954. WSP 1931: Drainage area.

GAGE.-Water-stage recorder. Sharp crested weir for low-water control since June 20, 1987. Elevation of gage is $3,170 \mathrm{ft}$ above sea level, from topographic map. Prior to June 20,1987, at site $100 \mathrm{ft}$ downstream at same datum.

REMARKS.-Flow regulated by Sly Creek Reservoir (station 11395400) 1.5 mi upstream and Lost Creek Reservoir 0.3 mi upstream, usable capacity, 5,920 acre-ft with flashboards. Water is diverted into Sly Creek Reservoir through South Fork Diversion Tunnel from South Fork Feather River and through Slate Creek Tunnel (station 11413250) from North Yuba River Basin. Woodleaf Tunnel diverts from Lost Creek Reservoir to Woodleaf Powerplant. Oroville-Wyandotte Canal (station 11395500) diverts from Woodleaf Penstock for irrigation and domestic use. Records represent seepage, release, and spill from Lost Creek Reservoir to Lost Creek. See schematic diagram of South Fork Feather River Basin.

COOPERATION.--Records provided by Oroville-Wyandotte Irrigation District, under general supervision of the U.S. Geological Survey, in connection with a Federal Energy Regulatory Commission project.

EXTREMES FOR PERIOD OF RECORD.-Maximum discharge, 5,760 ft ${ }^{3} / \mathrm{s}$, Jan.1, 1997, gage height, $13.50 \mathrm{ft}$; no flow at times in some years.

DISCHARGE, CUBIC FEET PER SECOND, WATER YEAR OCTOBER 1998 TO SEPTEMBER 1999

DAILY MEAN VALUES

\begin{tabular}{|c|c|c|c|c|c|c|c|c|c|c|c|c|}
\hline DAY & $\mathrm{OCT}$ & NOV & $\mathrm{DEC}$ & JAN & FEB & MAR & $A P R$ & MAY & JUN & JUL & AUG & SEP \\
\hline 1 & 8.4 & 9.6 & 6.5 & 5.5 & 5.9 & 547 & 8.6 & 12 & 8.6 & 8.8 & 8.5 & 8.2 \\
\hline 2 & 8.4 & 9.6 & 5.9 & 5.5 & 5.9 & 756 & 8.6 & 13 & 8.6 & 8.8 & 8.5 & 8.4 \\
\hline 3 & 8.4 & 9.0 & 7.7 & 5.5 & 5.9 & 895 & 8.7 & 13 & 8.6 & 8.7 & 8.4 & 8.7 \\
\hline 4 & 8.4 & 8.4 & 6.3 & 5.5 & 5.9 & 873 & 8.8 & 14 & 8.6 & 8.6 & 8.4 & 8.8 \\
\hline 5 & 8.4 & 8.3 & 6.0 & 5.5 & 5.8 & 754 & 9.0 & 14 & 8.9 & 8.5 & 8.5 & 8.7 \\
\hline 6 & 8.4 & 8.2 & 5.8 & 5.5 & 6.5 & 639 & 9.0 & 12 & 9.1 & 8.4 & 8.6 & 8.3 \\
\hline 7 & 8.4 & 8.8 & 5.6 & 5.5 & 9.4 & 529 & 9.0 & 9.8 & 9.6 & 8.4 & 8.7 & 8.2 \\
\hline 8 & 8.4 & 8.5 & 5.5 & 5.5 & 9.2 & 447 & 9.1 & 8.7 & 9.8 & 8.4 & 8.7 & 8.3 \\
\hline 9 & 8.4 & 7.1 & 5.5 & 5.5 & 14 & 409 & 9.1 & 8.6 & 9.6 & 8.4 & 8.4 & 8.5 \\
\hline 10 & 8.9 & 5.5 & 5.5 & 5.5 & 9.7 & 339 & 9.1 & 8.6 & 9.3 & 8.4 & 8.5 & 9.4 \\
\hline 11 & 9.2 & 5.9 & 5.5 & 5.5 & 8.0 & 304 & 9.3 & 8.6 & 8.9 & 8.4 & 8.6 & 9.8 \\
\hline 12 & 10 & 5.7 & 5.5 & 5.5 & 7.2 & 297 & 9.3 & 8.6 & 8.7 & 8.4 & 8.6 & 9.3 \\
\hline 13 & 12 & 6.1 & 5.6 & 5.5 & 6.9 & 293 & 9.4 & 8.6 & 8.6 & 8.5 & 8.6 & 8.4 \\
\hline 14 & 10 & 6.7 & 5.5 & 5.5 & 6.7 & 288 & 9.3 & 8.6 & 8.6 & 8.5 & 9.2 & 8.8 \\
\hline 15 & 8.5 & 6.7 & 5.5 & 5.6 & 6.4 & 284 & 9.3 & 9.1 & 8.6 & 8.6 & 9.0 & 9.5 \\
\hline 16 & 8.3 & 6.8 & 5.5 & 5.5 & 6.9 & 279 & 9.3 & 12 & 8.6 & 8.6 & 8.7 & 9.3 \\
\hline 17 & 8.2 & 6.9 & 5.5 & 6.0 & 7.8 & 274 & 9.1 & 11 & 8.6 & 8.5 & 8.6 & 8.5 \\
\hline 18 & 8.2 & 6.7 & 5.5 & 6.7 & 11 & 270 & 9.1 & 10 & 8.6 & 8.4 & 8.5 & 8.2 \\
\hline 19 & 8.3 & 6.7 & 5.5 & 8.1 & 206 & 269 & 9.1 & 9.2 & 8.7 & 8.3 & 8.2 & 8.2 \\
\hline 20 & 8.9 & 6.7 & 5.5 & 8.8 & 365 & 64 & 9.1 & 8.8 & 8.9 & 8.4 & 8.4 & 8.2 \\
\hline 21 & 11 & 6.9 & 5.5 & 7.3 & 361 & 6.0 & 9.4 & 8.6 & 8.6 & 8.4 & 8.6 & 8.2 \\
\hline 22 & 10 & 6.8 & 5.5 & 7.0 & 357 & 5.9 & 12 & 8.6 & 8.6 & 8.4 & 8.6 & 8.2 \\
\hline 23 & 8.4 & 8.1 & 5.6 & 9.1 & 341 & 5.9 & 14 & 8.4 & 8.6 & 8.7 & 8.6 & 8.4 \\
\hline 24 & 8.6 & 7.2 & 5.5 & 7.4 & 315 & 5.9 & 19 & 8.4 & 8.7 & 8.9 & 9.1 & 8.6 \\
\hline 25 & 8.6 & 6.0 & 5.5 & 6.7 & 332 & 6.0 & 21 & 8.4 & 8.7 & 8.5 & 10 & 8.6 \\
\hline 26 & 8.6 & 5.5 & 5.5 & 6.4 & 302 & 5.9 & 20 & 8.4 & 8.9 & 8.3 & 10 & 8.5 \\
\hline 27 & 8.5 & 6.1 & 5.5 & 6.1 & 297 & 5.9 & 16 & 8.5 & 8.8 & 8.2 & 9.3 & 8.4 \\
\hline 28 & 8.4 & 5.8 & 5.4 & 6.0 & 344 & 5.9 & 11 & 8.7 & 8.6 & 8.3 & 9.1 & 8.3 \\
\hline 29 & 8.5 & 6.1 & 5.4 & 5.9 & --- & 5.7 & 11 & 8.6 & 8.6 & 8.4 & 9.1 & 8.2 \\
\hline 30 & 9.2 & 7.7 & 5.4 & 5.9 & --- & 5.8 & 12 & 8.7 & 8.7 & 8.3 & 8.8 & 8.2 \\
\hline 31 & 9.3 & --- & 5.5 & 5.9 & --- & 7.0 & --- & 8.6 & --- & 8.2 & 8.4 & --- \\
\hline TOTAL & 275.2 & 214.1 & 175.7 & 191.4 & 3359.1 & 8875.9 & 326.7 & 302.1 & 264.3 & 262.6 & 271.2 & 257.3 \\
\hline MEAN & 8.88 & 7.14 & 5.67 & 6.17 & 120 & 286 & 10.9 & 9.75 & 8.81 & 8.47 & 8.75 & 8.58 \\
\hline MAX & 12 & 9.6 & 7.7 & 9.1 & 365 & 895 & 21 & 14 & 9.8 & 8.9 & 10 & 9.8 \\
\hline MIN & 8.2 & 5.5 & 5.4 & 5.5 & 5.8 & 5.7 & 8.6 & 8.4 & 8.6 & 8.2 & 8.2 & 8.2 \\
\hline $\mathrm{AC}-\mathrm{FT}$ & 546 & 425 & 349 & 380 & 6660 & 17610 & 648 & 599 & 524 & 521 & 538 & 510 \\
\hline $\mathrm{a}$ & 16040 & 15430 & 34720 & 22680 & 30890 & 34410 & 33290 & 31350 & 18070 & 19370 & 19620 & 12210 \\
\hline
\end{tabular}

a Diversion, acre-feet, through Woodleaf Powerplant (station 11396090), provided by Oroville-Wyandotte Irrigation District. 


\section{LOST CREEK NEAR CLIPPER MILLS, CA—Continued}

STATISTICS OF MONTHLY MEAN DATA FOR WATER YEARS 1928 - 1961, BY WATER YEAR (WY)

\begin{tabular}{|c|c|c|c|c|c|c|c|c|c|c|c|c|}
\hline & OCT & NOV & $\mathrm{DEC}$ & JAN & $\mathrm{FEB}$ & MAR & $\mathrm{APR}$ & MAY & JUN & JUL & AUG & SEP \\
\hline MEAN & 3.78 & 8.61 & 66.0 & 93.4 & 170 & 175 & 191 & 129 & 29.9 & 6.42 & 4.23 & 5.13 \\
\hline MAX & 13.4 & 121 & 544 & 485 & 562 & 467 & 423 & 441 & 153 & 34.7 & 10.2 & 15.3 \\
\hline (WY) & 1928 & 1951 & 1956 & 1956 & 1958 & 1938 & 1938 & 1952 & 1952 & 1952 & 1961 & 1960 \\
\hline MIN & .20 & .000 & .000 & .15 & .50 & 25.7 & 4.68 & 1.21 & 1.33 & .20 & .10 & .10 \\
\hline$(W Y)$ & 1935 & 1960 & 1960 & 1960 & 1937 & 1933 & 1931 & 1931 & 1934 & 1939 & 1934 & 1934 \\
\hline
\end{tabular}

SUMMARY STATISTICS

ANNUAL MEAN

HIGHEST ANNUAL MEAN

LOWEST ANNUAL MEAN

HIGHEST DAILY MEAN

LOWEST DAILY MEAN

ANNUAL SEVEN-DAY MINIMUM

INSTANTANEOUS PEAK FLOW

INSTANTANEOUS PEAK STAGE

ANNUAL RUNOFF (AC-FT)

10 PERCENT EXCEEDS

50 PERCENT EXCEEDS

90 PERCENT EXCEEDS
WATER YEARS 1928 - 1961

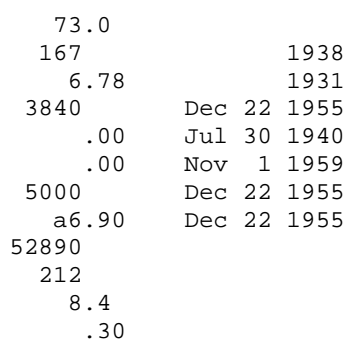

STATISTICS OF MONTHLY MEAN DATA FOR WATER YEARS 1962 - 1999, BY WATER YEAR (WY)

\begin{tabular}{|c|c|c|c|c|c|c|c|c|c|c|c|c|}
\hline & $\mathrm{OCT}$ & NOV & $\mathrm{DEC}$ & JAN & $\mathrm{FEB}$ & MAR & APR & MAY & JUN & JUL & AUG & SEP \\
\hline MEAN & 13.1 & 6.88 & 43.9 & 57.2 & 73.6 & 82.8 & 56.5 & 48.0 & 41.4 & 3.98 & 3.50 & 3.80 \\
\hline MAX & 392 & 179 & 417 & 674 & 512 & 573 & 410 & 454 & 750 & 16.0 & 22.2 & 34.4 \\
\hline (WY) & 1963 & 1963 & 1998 & 1997 & 1986 & 1983 & 1993 & 1995 & 1995 & 1962 & 1966 & 1997 \\
\hline MIN & .006 & .029 & .094 & .10 & .35 & .33 & .22 & .13 & .097 & .10 & .000 & .000 \\
\hline (WY) & 1965 & 1975 & 1975 & 1962 & 1964 & 1964 & 1968 & 1968 & 1966 & 1963 & 1964 & 1963 \\
\hline
\end{tabular}

SUMMARY STATISTICS

ANNUAL TOTAL

ANNUAL MEAN

HIGHEST ANNUAL MEAN

LOWEST ANNUAL MEAN

HIGHEST DAILY MEAN

LOWEST DAILY MEAN

ANNUAL SEVEN-DAY MINIMUM

INSTANTANEOUS PEAK FLOW

INSTANTANEOUS PEAK STAGE

ANNUAL RUNOFF (AC-FT)

TOTAL DIVERSION (AC-FT) b

10 PERCENT EXCEEDS

50 PERCENT EXCEEDS

90 PERCENT EXCEEDS
FOR 1998 CALENDAR YEAR

\begin{tabular}{|c|c|}
\hline $\begin{array}{c}46730.1 \\
128\end{array}$ & \\
\hline 750 & $\operatorname{Mar} 25$ \\
\hline 5.4 & Dec 28 \\
\hline 5.5 & Dec 24 \\
\hline 92690 & \\
\hline 327300 & \\
\hline 412 & \\
\hline 9.3 & \\
\hline 6.0 & \\
\hline
\end{tabular}

FOR 1999 WATER YEAR

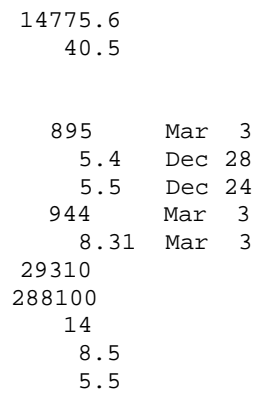

WATER YEARS 1962 - 1999

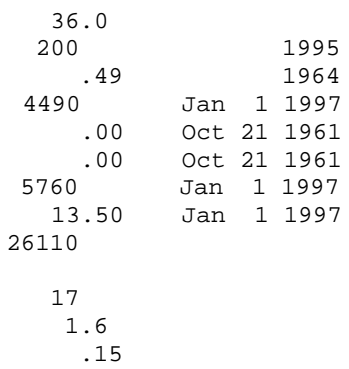

a Site then in use.

b Diversion, acre-feet, through Woodleaf Powerplant (station 11396090), provided by Oroville-Wyandotte Irrigation District. 
LOCATION.-Lat 39³3'05", long 121¹2’30", in SE 1/4 NE 1/4 sec.32, T.20 N., R.7 E., Butte County, Hydrologic Unit 18020123, Plumas National Forest, on right bank $500 \mathrm{ft}$ downstream from Forbestown Dam, 0.4 mi upstream from Oroleve Creek, and 4.0 mi northeast of Forbestown.

DRAINAGE AREA.- $-87.5 \mathrm{mi}^{2}$.

PERIOD OF RECORD._-July 1962 to current year. Records for Forbestown Powerplant from February 1963 to September 1966 in files of the U.S. Geological Survey.

GAGE.-Water-stage recorder. Elevation of gage is 1,690 ft above sea level, from topographic map.

REMARKS.-Flow regulated by Little Grass Valley Reservoir (station 11395020), Sly Creek Reservoir (station 11395400), and smaller reservoirs. Water from North Yuba River Basin is imported through Slate Creek Tunnel (station 11413250) to Sly Creek Reservoir. Oroville-Wyandotte Canal (station 11395500) diverts upstream from station. Tunnel $600 \mathrm{ft}$ upstream from station diverts most flow through Forbestown Powerplant (station 11396290) except fishwater releases and uncontrolled spill over Forbestown Dam. See schematic diagram of South Fork Feather River Basin.

COOPERATION.-Records provided by Oroville-Wyandotte Irrigation District, under general supervision of the U.S. Geological Survey, in connection with a Federal Energy Regulatory Commission project.

EXTREMES FOR PERIOD OF RECORD.-Maximum discharge, 21,800 ft 3 /s, Jan. 1, 1997, gage height, $17.64 \mathrm{ft}$, from rating curve extended above $5,400 \mathrm{ft}^{3} / \mathrm{s}$ on basis of flow-over-dam measurement of peak flow; minimum daily, $0.6 \mathrm{ft}^{3} / \mathrm{s}, \mathrm{Apr}^{4}, 1963$.

DISCHARGE, CUBIC FEET PER SECOND, WATER YEAR OCTOBER 1998 TO SEPTEMBER 1999 DAILY MEAN VALUES

\begin{tabular}{|c|c|c|c|c|c|c|c|c|c|c|c|c|}
\hline DAY & $\mathrm{OCT}$ & NOV & $\mathrm{DEC}$ & JAN & FEB & MAR & APR & MAY & JUN & JUL & AUG & SEP \\
\hline 1 & 39 & 11 & 6.1 & 6.3 & 6.2 & 768 & 6.3 & 11 & 11 & 10 & 10 & 10 \\
\hline 2 & 38 & 11 & 6.1 & 6.3 & 13 & 946 & 6.1 & 11 & 10 & 10 & 10 & 10 \\
\hline 3 & 39 & 11 & 6.4 & 6.3 & 25 & 1150 & 6.1 & 11 & 10 & 10 & 10 & 10 \\
\hline 4 & 39 & 11 & 6.1 & 6.2 & 6.3 & 1100 & 6.2 & 11 & 10 & 10 & 10 & 10 \\
\hline 5 & 38 & 11 & 6.1 & 6.2 & 6.3 & 937 & 6.3 & 11 & 10 & 10 & 10 & 10 \\
\hline 6 & 38 & 11 & 6.1 & 6.2 & 8.0 & 783 & 6.1 & 11 & 10 & 10 & 10 & 10 \\
\hline 7 & 21 & 11 & 6.1 & 6.3 & 237 & 645 & 6.1 & 12 & 10 & 10 & 10 & 10 \\
\hline 8 & 11 & 11 & 6.1 & 6.2 & 227 & 553 & 6.3 & 12 & 10 & 10 & 10 & 10 \\
\hline 9 & 11 & 8.2 & 6.1 & 6.2 & 1030 & 513 & 6.4 & 12 & 10 & 10 & 10 & 10 \\
\hline 10 & 11 & 5.9 & 6.1 & 6.2 & 360 & 431 & 6.5 & 12 & 10 & 10 & 10 & 10 \\
\hline 11 & 11 & 6.1 & 6.1 & 6.3 & 189 & 384 & 6.5 & 11 & 10 & 10 & 13 & 10 \\
\hline 12 & 11 & 6.1 & 6.1 & 6.3 & 130 & 369 & 6.5 & 11 & 10 & 10 & 10 & 10 \\
\hline 13 & 11 & 6.1 & 6.1 & 6.3 & 90 & 360 & 6.5 & 11 & 10 & 10 & 10 & 10 \\
\hline 14 & 11 & 6.0 & 6.2 & 6.3 & 69 & 352 & 6.5 & 10 & 10 & 10 & 10 & 10 \\
\hline 15 & 11 & 6.1 & 6.2 & 6.3 & 52 & 342 & 6.4 & 10 & 10 & 10 & 10 & 10 \\
\hline 16 & 11 & 6.0 & 6.1 & 6.3 & 87 & 333 & 6.3 & 11 & 10 & 10 & 10 & 10 \\
\hline 17 & 11 & 6.1 & 6.2 & 6.4 & 233 & 325 & 6.4 & 11 & 10 & 10 & 10 & 10 \\
\hline 18 & 11 & 6.1 & 6.2 & 6.5 & 163 & 318 & 6.3 & 11 & 10 & 10 & 10 & 10 \\
\hline 19 & 11 & 6.1 & 6.1 & 6.8 & 291 & 309 & 6.3 & 11 & 10 & 10 & 10 & 10 \\
\hline 20 & 11 & 6.1 & 6.1 & 6.6 & 443 & 26 & 6.3 & 11 & 10 & 10 & 10 & 22 \\
\hline 21 & 11 & 6.1 & 6.1 & 7.9 & 449 & 6.1 & 6.3 & 11 & 10 & 10 & 10 & 35 \\
\hline 22 & 11 & 6.1 & 6.3 & 6.4 & 418 & 6.1 & 6.3 & 10 & 10 & 10 & 10 & 110 \\
\hline 23 & 11 & 6.3 & 6.3 & 6.6 & 405 & 6.1 & 13 & 11 & 10 & 10 & 10 & 35 \\
\hline 24 & 11 & 6.1 & 6.3 & 6.3 & 389 & 6.3 & 6.5 & 11 & 10 & 10 & 10 & 21 \\
\hline 25 & 11 & 6.1 & 6.3 & 6.3 & 472 & 6.2 & 6.5 & 11 & 10 & 10 & 10 & 10 \\
\hline 26 & 11 & 6.0 & 6.2 & 6.3 & 411 & 6.1 & 6.5 & 11 & 10 & 10 & 10 & 10 \\
\hline 27 & 11 & 6.2 & 6.2 & 6.3 & 388 & 6.1 & 6.5 & 10 & 10 & 10 & 10 & 10 \\
\hline 28 & 11 & 6.1 & 6.3 & 6.3 & 517 & 6.1 & 6.5 & 10 & 10 & 10 & 10 & 10 \\
\hline 29 & 11 & 6.1 & 6.2 & 13 & --- & 6.1 & 6.4 & 11 & 10 & 10 & 10 & 10 \\
\hline 30 & 11 & 6.4 & 6.3 & 6.3 & --- & 6.1 & 8.8 & 10 & 10 & 10 & 10 & 10 \\
\hline 31 & 11 & --- & 6.3 & 6.3 & --- & 6.3 & --- & 11 & --- & 10 & 10 & --- \\
\hline TOTAL & 516 & 224.4 & 191.5 & 204.5 & 7114.8 & 11011.6 & 199.7 & 339 & 301 & 310 & 313 & 473 \\
\hline MEAN & 16.6 & 7.48 & 6.18 & 6.60 & 254 & 355 & 6.66 & 10.9 & 10.0 & 10.0 & 10.1 & 15.8 \\
\hline MAX & 39 & 11 & 6.4 & 13 & 1030 & 1150 & 13 & 12 & 11 & 10 & 13 & 110 \\
\hline MIN & 11 & 5.9 & 6.1 & 6.2 & 6.2 & 6.1 & 6.1 & 10 & 10 & 10 & 10 & 10 \\
\hline $\mathrm{AC}-\mathrm{FT}$ & 1020 & 445 & 380 & 406 & 14110 & 21840 & 396 & 672 & 597 & 615 & 621 & 938 \\
\hline $\mathrm{a}$ & 16600 & 16520 & 38790 & 28350 & 36230 & 40010 & 38270 & 34300 & 19590 & 20410 & 20330 & 12220 \\
\hline
\end{tabular}

a Diversion, in acre-feet, to Forbestown Powerplant (station 11396290), provided by Oroville-Wyandotte Irrigation District. 


\section{SOUTH FORK FEATHER RIVER BELOW FORBESTOWN DAM, CA—Continued}

STATISTICS OF MONTHLY MEAN DATA FOR WATER YEARS 1962 - 1999, BY WATER YEAR (WY)

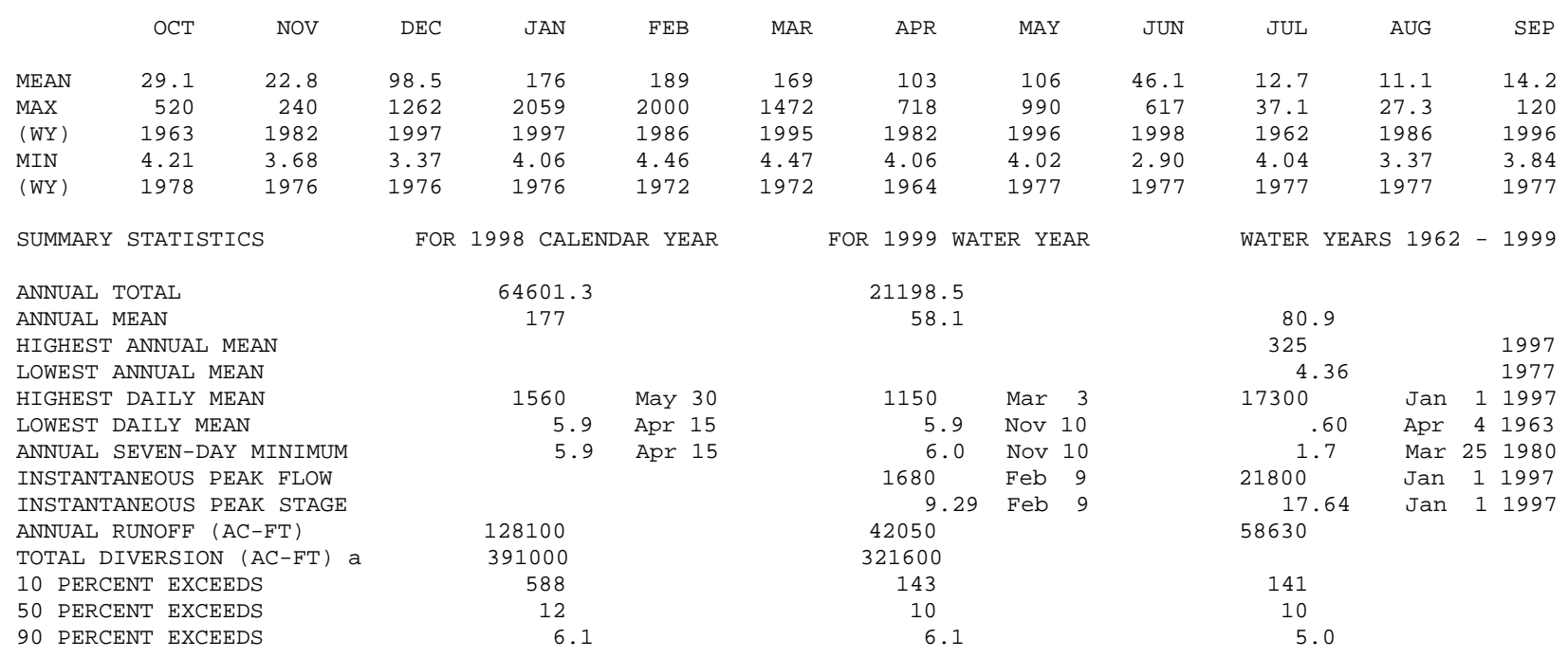

a Diversion, in acre-feet, to Forbestown Powerplant (station 11396290), provided by Oroville-Wyandotte Irrigation District. 


\section{MINERS RANCH CANAL BELOW PONDEROSA DAM, NEAR FORBESTOWN, CA}

LOCATION.-Lat 39³3'00", long 121¹8'20", in SE 1/4 NW 1/4 sec.33, T.20 N., R.6 E., Butte County, Hydrologic Unit 18020123, on right bank $800 \mathrm{ft}$ downstream from Ponderosa Dam and 3 mi northwest of Forbestown.

PERIOD OF RECORD.-October 1962 to current year.

REVISED RECORDS.-WDR CA-88-4: diversion only.

GAGE.-Water-stage recorder and concrete control. Elevation of gage is $975 \mathrm{ft}$ above sea level, from topographic map.

REMARKS.-Canal diverts from South Fork Feather River at Ponderosa Dam. Water is used for power development and irrigation. See schematic diagram of South Fork Feather River Basin.

COOPERATION.-Records provided by Oroville-Wyandotte Irrigation District, under general supervision of the U.S. Geological Survey, in connection with a Federal Energy Regulatory Commission project.

EXTREMES FOR PERIOD OF RECORD.-Maximum daily discharge, $314 \mathrm{ft}^{3} / \mathrm{s}$, May 13, 1984; no flow at times in most years.

DISCHARGE, CUBIC FEET PER SECOND, WATER YEAR OCTOBER 1998 TO SEPTEMBER 1999

DAILY MEAN VALUES

\begin{tabular}{|c|c|c|c|c|c|c|c|c|c|c|c|c|}
\hline DAY & OCT & NOV & $\mathrm{DEC}$ & JAN & FEB & MAR & APR & MAY & JUN & JUL & AUG & SEP \\
\hline 1 & 19 & 282 & 261 & 270 & 261 & 263 & 265 & 278 & 282 & 285 & 285 & 285 \\
\hline 2 & 147 & 281 & 261 & 269 & 261 & 263 & 266 & 278 & 279 & 285 & 285 & 285 \\
\hline 3 & .00 & 281 & 261 & 269 & 261 & 261 & 267 & 279 & 257 & 285 & 285 & 285 \\
\hline 4 & .00 & 281 & 261 & 270 & 266 & 260 & 269 & 278 & 277 & 285 & 285 & 285 \\
\hline 5 & .00 & 255 & 261 & 267 & 269 & 261 & 268 & 278 & 277 & 285 & 285 & 285 \\
\hline 6 & .00 & 281 & 260 & 266 & 267 & 265 & 269 & 278 & 278 & 285 & 285 & 285 \\
\hline 7 & .00 & 281 & 262 & 266 & 264 & 266 & 269 & 278 & 279 & 285 & 285 & 285 \\
\hline 8 & .00 & 279 & 218 & 265 & 262 & 266 & 269 & 276 & 279 & 285 & 285 & 285 \\
\hline 9 & 176 & 241 & 266 & 265 & 254 & 266 & 268 & 273 & 282 & 285 & 285 & 285 \\
\hline 10 & 266 & 275 & 266 & 261 & 251 & 265 & 269 & 276 & 284 & 285 & 284 & 285 \\
\hline 11 & 261 & 276 & 205 & 266 & 233 & 263 & 195 & 247 & 285 & 285 & 276 & 285 \\
\hline 12 & 277 & 272 & 277 & 270 & 266 & 263 & 127 & 282 & 285 & 249 & 276 & 284 \\
\hline 13 & 278 & 269 & 277 & 270 & 267 & 263 & 270 & 282 & 284 & 285 & 285 & 284 \\
\hline 14 & 279 & 269 & 279 & 270 & 266 & 263 & 270 & 282 & 285 & 285 & 285 & 283 \\
\hline 15 & 280 & 269 & 279 & 269 & 266 & 266 & 272 & 282 & 285 & 285 & 285 & 260 \\
\hline 16 & 282 & 269 & 271 & 265 & 267 & 268 & 272 & 282 & 221 & 285 & 285 & 282 \\
\hline 17 & 186 & 269 & 265 & 262 & 265 & 267 & 272 & 284 & 285 & 285 & 285 & 285 \\
\hline 18 & 122 & 269 & 266 & 268 & 261 & 267 & 272 & 286 & 285 & 285 & 285 & 285 \\
\hline 19 & 122 & 268 & 268 & 225 & 262 & 267 & 272 & 284 & 285 & 285 & 285 & 128 \\
\hline 20 & 197 & 235 & 268 & 266 & 262 & 267 & 274 & 283 & 285 & 285 & 285 & 5.0 \\
\hline 21 & 263 & 268 & 266 & 269 & 261 & 267 & 276 & 283 & 285 & 285 & 285 & 20 \\
\hline 22 & 216 & 267 & 264 & 229 & 263 & 267 & 274 & 283 & 285 & 285 & 285 & 30 \\
\hline 23 & 262 & 267 & 266 & 271 & 225 & 266 & 273 & 283 & 285 & 285 & 272 & 77 \\
\hline 24 & 271 & 275 & 268 & 271 & 260 & 266 & 273 & 283 & 285 & 285 & 285 & 75 \\
\hline 25 & 279 & 238 & 268 & 271 & 273 & 265 & 273 & 282 & 285 & 285 & 283 & .00 \\
\hline 26 & 282 & 261 & 269 & 271 & 269 & 264 & 274 & 283 & 285 & 256 & 285 & .00 \\
\hline 27 & 282 & 260 & 270 & 271 & 264 & 264 & 275 & 283 & 285 & 285 & 285 & .00 \\
\hline 28 & 282 & 269 & 270 & 269 & 263 & 264 & 276 & 282 & 250 & 285 & 285 & .00 \\
\hline 29 & 282 & 265 & 271 & 268 & --- & 265 & 244 & 283 & 285 & 285 & 285 & .00 \\
\hline 30 & 282 & 261 & 270 & 266 & --- & 265 & 278 & 283 & 285 & 285 & 285 & 84 \\
\hline 31 & 281 & --- & 269 & 239 & --- & 265 & --- & 283 & --- & 285 & 285 & --- \\
\hline TOTAL & 5874.00 & 8033 & 8183 & 8194 & 7309 & 8208 & 7891 & 8677 & 8374 & 8770 & 8801 & 5517.00 \\
\hline MEAN & 189 & 268 & 264 & 264 & 261 & 265 & 263 & 280 & 279 & 283 & 284 & 184 \\
\hline MAX & 282 & 282 & 279 & 271 & 273 & 268 & 278 & 286 & 285 & 285 & 285 & 285 \\
\hline MIN & .00 & 235 & 205 & 225 & 225 & 260 & 127 & 247 & 221 & 249 & 272 & .00 \\
\hline $\mathrm{AC}-\mathrm{FT}$ & 11650 & 15930 & 16230 & 16250 & 14500 & 16280 & 15650 & 17210 & 16610 & 17400 & 17460 & 10940 \\
\hline a & 9430 & 15140 & 15370 & 15620 & 13730 & 15600 & 14680 & 15630 & 14730 & 15110 & 15210 & 9410 \\
\hline
\end{tabular}

STATISTICS OF MONTHLY MEAN DATA FOR WATER YEARS 1963 - 1999, BY WATER YEAR (WY)

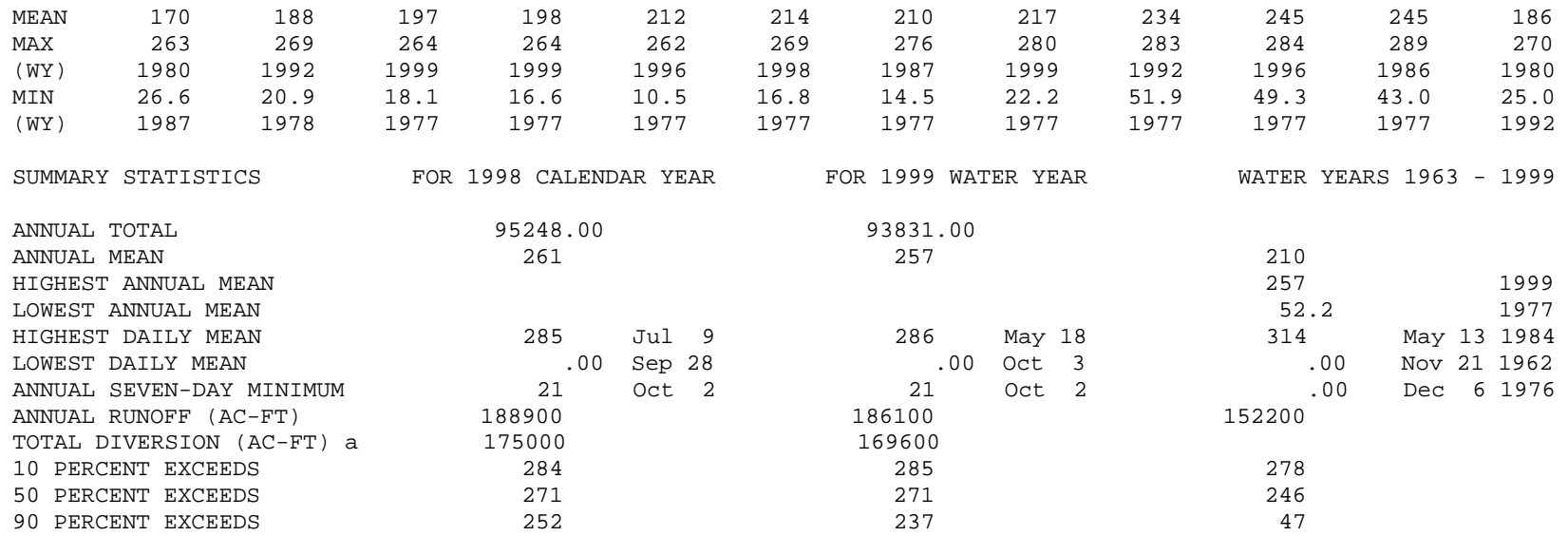

a Discharge, in acre-feet, through Kelly Ridge Powerplant (station 11396329), provided by Oroville-Wyandotte Irrigation District. 


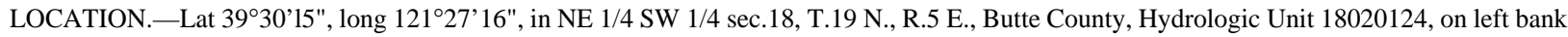
$400 \mathrm{ft}$ downstream from outlet at Miners Ranch Dam and 5 mi east of Oroville.

PERIOD OF RECORD.—January 1963 to current year.

GAGE.-Water-stage recorder and Parshall flume. Elevation of gage is $815 \mathrm{ft}$ above sea level, from topographic map.

REMARKS.-Flow regulated by Miners Ranch Reservoir, capacity, 912 acre-ft. Canal completed in November 1962. Water is used for irrigation. See schematic diagram of South Fork Feather River Basin.

COOPERATION.-Records provided by Oroville-Wyandotte Irrigation District, under general supervision of the U.S. Geological Survey, in connection with a Federal Energy Regulatory Commission project.

EXTREMES FOR PERIOD OF RECORD.-Maximum daily discharge, $65 \mathrm{ft}^{3}$ /s, Aug. 17-20, 1963; no flow for several days in $1965,1969$.

DISCHARGE, CUBIC FEET PER SECOND, WATER YEAR OCTOBER 1998 TO SEPTEMBER 1999 DAILY MEAN VALUES

\begin{tabular}{|c|c|c|c|c|c|c|c|c|c|c|c|c|}
\hline DAY & OCT & NOV & $\mathrm{DEC}$ & JAN & $\mathrm{FEB}$ & MAR & APR & MAY & JUN & JUL & AUG & SEP \\
\hline 1 & 19 & 14 & 7.0 & 8.8 & 7.0 & 7.4 & 7.7 & 11 & 13 & 17 & 18 & 18 \\
\hline 2 & 19 & 14 & 7.0 & 8.8 & 6.8 & 7.5 & 7.7 & 11 & 13 & 17 & 18 & 19 \\
\hline 3 & 19 & 14 & 7.0 & 8.8 & 6.7 & 7.3 & 7.7 & 11 & 13 & 17 & 18 & 19 \\
\hline 4 & 19 & 13 & 7.0 & 8.8 & 8.2 & 7.1 & 7.7 & 11 & 13 & 17 & 18 & 18 \\
\hline 5 & 19 & 11 & 7.0 & 7.9 & 8.3 & 7.0 & 8.0 & 11 & 13 & 17 & 18 & 18 \\
\hline 6 & 19 & 10 & 7.0 & 7.2 & 7.2 & 7.0 & 8.0 & 11 & 13 & 17 & 18 & 18 \\
\hline 7 & 19 & 10 & 6.5 & 7.2 & 7.5 & 7.2 & 8.0 & 11 & 13 & 17 & 18 & 18 \\
\hline 8 & 19 & 10 & 6.1 & 7.2 & 7.5 & 7.7 & 8.0 & 11 & 13 & 17 & 18 & 17 \\
\hline 9 & 19 & 10 & 6.1 & 7.2 & 7.5 & 7.7 & 7.9 & 11 & 13 & 17 & 18 & 17 \\
\hline 10 & 19 & 9.1 & 6.2 & 7.2 & 7.5 & 7.7 & 7.7 & 11 & 13 & 17 & 18 & 17 \\
\hline 11 & 19 & 8.2 & 6.0 & 7.2 & 7.3 & 7.7 & 7.8 & 11 & 13 & 17 & 18 & 17 \\
\hline 12 & 19 & 8.2 & 5.8 & 7.2 & 7.2 & 7.7 & 7.8 & 11 & 13 & 17 & 18 & 17 \\
\hline 13 & 18 & 8.3 & 6.0 & 7.2 & 7.2 & 7.7 & 7.7 & 11 & 13 & 17 & 18 & 17 \\
\hline 14 & 17 & 8.4 & 6.0 & 7.2 & 7.2 & 7.7 & 7.7 & 12 & 14 & 17 & 18 & 17 \\
\hline 15 & 17 & 8.2 & 6.0 & 7.2 & 7.2 & 7.7 & 7.9 & 13 & 15 & 18 & 18 & 17 \\
\hline 16 & 17 & 8.2 & 6.0 & 7.2 & 7.2 & 7.7 & 7.7 & 13 & 15 & 18 & 18 & 17 \\
\hline 17 & 17 & 8.2 & 6.0 & 7.2 & 7.5 & 7.7 & 7.7 & 13 & 17 & 18 & 18 & 17 \\
\hline 18 & 17 & 8.2 & 6.0 & 7.2 & 7.5 & 7.7 & 8.0 & 13 & 20 & 18 & 18 & 17 \\
\hline 19 & 17 & 8.2 & 6.0 & 7.3 & 7.5 & 8.0 & 7.9 & 13 & 20 & 18 & 18 & 17 \\
\hline 20 & 17 & 8.2 & 5.8 & 7.2 & 7.5 & 8.0 & 9.1 & 13 & 20 & 19 & 18 & 17 \\
\hline 21 & 18 & 8.2 & 5.8 & 7.2 & 7.5 & 8.0 & 10 & 13 & 20 & 18 & 18 & 17 \\
\hline 22 & 17 & 8.2 & 6.7 & 7.1 & 7.2 & 8.0 & 11 & 13 & 19 & 18 & 18 & 17 \\
\hline 23 & 17 & 7.7 & 8.1 & 7.0 & 7.2 & 8.0 & 13 & 13 & 19 & 18 & 18 & 17 \\
\hline 24 & 17 & 7.0 & 8.5 & 7.1 & 7.2 & 8.0 & 13 & 13 & 18 & 18 & 18 & 17 \\
\hline 25 & 17 & 7.2 & 8.5 & 7.2 & 7.2 & 8.0 & 14 & 13 & 18 & 18 & 18 & 17 \\
\hline 26 & 17 & 7.2 & 8.5 & 7.0 & 7.5 & 8.0 & 13 & 13 & 18 & 18 & 18 & 17 \\
\hline 27 & 18 & 7.2 & 8.5 & 7.0 & 7.4 & 7.8 & 13 & 13 & 18 & 18 & 18 & 17 \\
\hline 28 & 16 & 7.2 & 8.5 & 7.0 & 7.2 & 7.7 & 12 & 13 & 18 & 18 & 18 & 17 \\
\hline 29 & 14 & 7.1 & 8.5 & 7.0 & --- & 7.7 & 11 & 14 & 19 & 18 & 18 & 17 \\
\hline 30 & 14 & 7.0 & 8.5 & 7.0 & --- & 7.7 & 11 & 14 & 18 & 18 & 18 & 17 \\
\hline 31 & 14 & --- & 8.7 & 7.0 & --- & 7.7 & --- & 14 & --- & 18 & 18 & --- \\
\hline TOTAL & 544 & 271.4 & 215.3 & 228.8 & 205.9 & 237.8 & 278.7 & 379 & 475 & 545 & 558 & 519 \\
\hline MEAN & 17.5 & 9.05 & 6.95 & 7.38 & 7.35 & 7.67 & 9.29 & 12.2 & 15.8 & 17.6 & 18.0 & 17.3 \\
\hline MAX & 19 & 14 & 8.7 & 8.8 & 8.3 & 8.0 & 14 & 14 & 20 & 19 & 18 & 19 \\
\hline MIN & 14 & 7.0 & 5.8 & 7.0 & 6.7 & 7.0 & 7.7 & 11 & 13 & 17 & 18 & 17 \\
\hline $\mathrm{AC}-\mathrm{FT}$ & 1080 & 538 & 427 & 454 & 408 & 472 & 553 & 752 & 942 & 1080 & 1110 & 1030 \\
\hline
\end{tabular}

STATISTICS OF MONTHLY MEAN DATA FOR WATER YEARS 1963 - 1999, BY WATER YEAR (WY)

\begin{tabular}{|c|c|c|c|c|c|c|c|c|c|c|c|c|}
\hline MEAN & 16.9 & 7.92 & 5.58 & 4.60 & 4.20 & 4.59 & 8.69 & 16.1 & 21.7 & 24.2 & 24.4 & 22.1 \\
\hline MAX & 29.7 & 14.3 & 11.2 & 12.0 & 7.68 & 8.27 & 20.3 & 27.8 & 42.0 & 56.4 & 53.4 & 36.2 \\
\hline (WY) & 1965 & 1972 & 1975 & 1963 & 1980 & 1988 & 1970 & 1970 & 1963 & 1963 & 1963 & 1963 \\
\hline MIN & 5.42 & 1.47 & .035 & .30 & .25 & .20 & 2.65 & 6.41 & 11.0 & 16.0 & 17.1 & 14.4 \\
\hline (WY) & 1985 & 1969 & 1966 & 1966 & 1966 & 1966 & 1983 & 1995 & 1998 & 1982 & 1992 & 1993 \\
\hline
\end{tabular}

SUMMARY STATISTICS

ANNUAL TOTAL

ANNUAL MEAN

HIGHEST ANNUAL MEAN

LOWEST ANNUAL MEAN

HIGHEST DAILY MEAN

LOWEST DAILY MEAN

ANNUAL SEVEN-DAY MINIMUM

ANNUAL RUNOFF (AC-FT)

10 PERCENT EXCEEDS

50 PERCENT EXCEEDS

90 PERCENT EXCEEDS
FOR 1998 CALENDAR YEAR

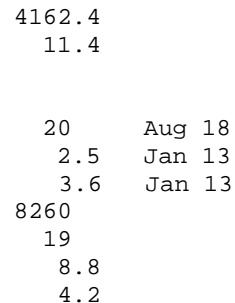

FOR 1999 WATER YEAR

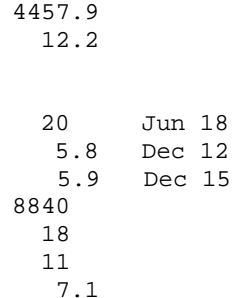

WATER YEARS 1963 - 1999

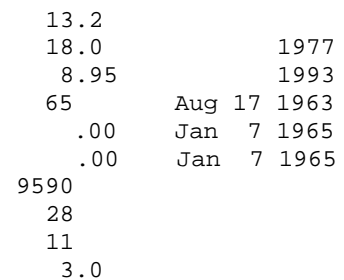




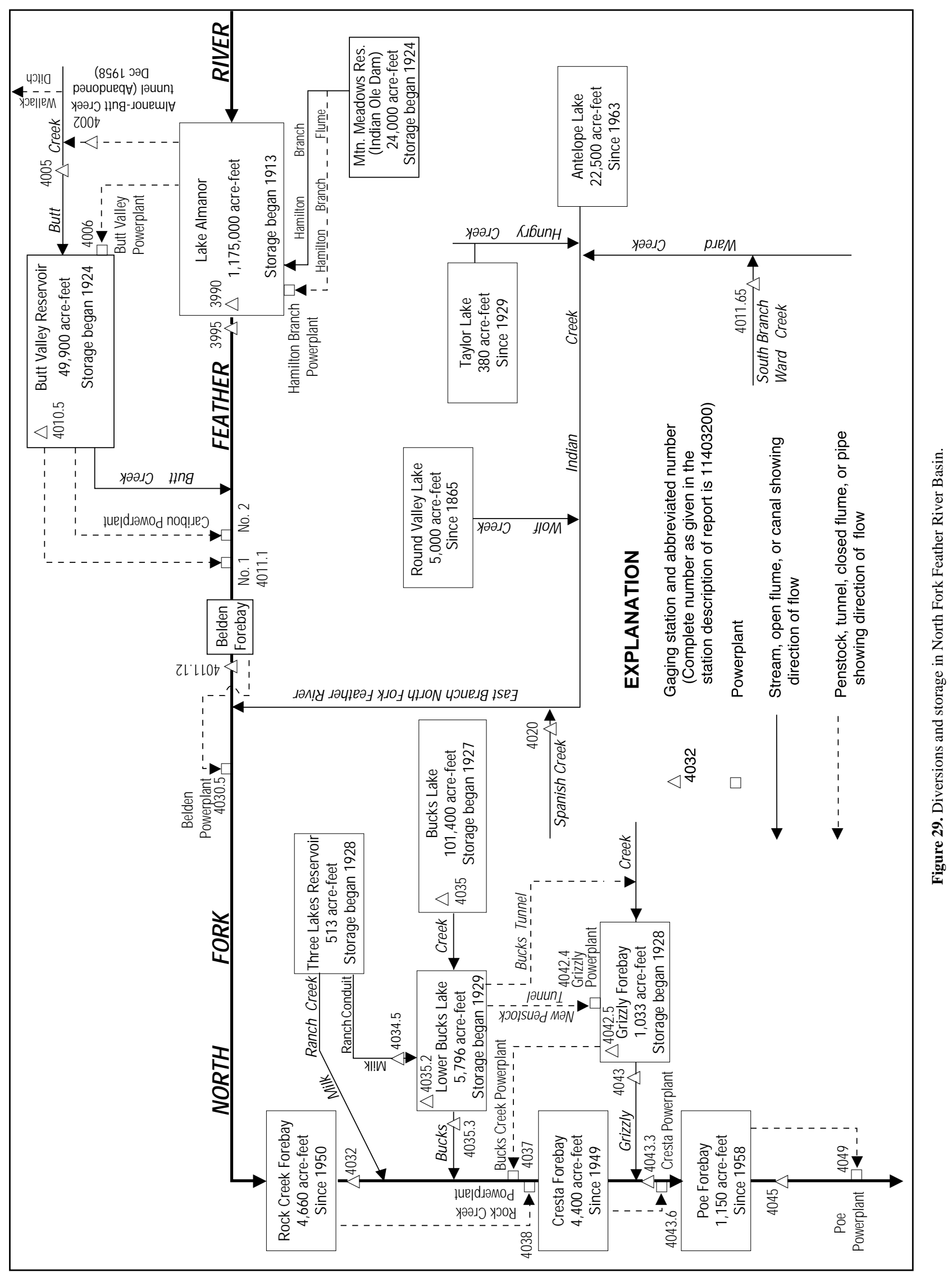


LOCATION.-Lat 40¹2’46", long 12109’43", in SW 1/4 NE 1/4 sec.11, T.27 N., R.7 E., Plumas County, Hydrologic Unit 18020121, Lassen National Forest, at intake tower to Butt Valley Tunnel at Prattville, 4.7 mi northwest of Lake Almanor Dam, and 5.6 mi northwest of Canyondam.

DRAINAGE AREA.- $491 \mathrm{mi}^{2}$.

PERIOD OF RECORD.—July 1913 to current year. Monthly contents only for some periods, published in WSP 1315-A. Published as "near Prattville" 1937-60. Prior to October 1964, records published as usable contents.

REVISED RECORDS.-WSP 1931: Drainage area.

GAGE.-Nonrecording gage read once daily. Datum of gage is $10.23 \mathrm{ft}$ below sea level (levels by Pacific Gas \& Electric Co.). Prior to June 1, 1965, nonrecording gage at site $4.7 \mathrm{mi}$ southeast at same datum.

REMARKS.-Lake is formed by earthfill dam; storage began in July 1913; dam raised to gage height 4,455 ft in 1917 and 4,515 ft in 1927 . Usable capacity, 1,174,887 acre-ft between gage heights 4,422 ft, invert of outlet, and 4,495.5 ft, maximum storage limit. Dead storage, 8,948 acre-ft. Water is diverted by tunnel and penstock to Butt Valley Powerplant (station 11400600) and then is used for power development in the North Fork Feather River. Figures given, including extremes, represent total contents at 2400 hours. See schematic diagram of North Fork Feather River Basin.

COOPERATION.-Records were collected by Pacific Gas \& Electric Co., under general supervision of the U.S. Geological Survey, in connection with a Federal Energy Regulatory Commission project. Contents not rounded to U.S. Geological Survey standards.

EXTREMES FOR PERIOD OF RECORD.—Maximum contents, 1,142,960 acre-ft, June 8, 1982, gage height, 4,494.00 ft; minimum, 5,230 acre-ft, Feb. 5, 1918, gage height, 4,416.1 ft.

EXTREMES FOR CURRENT YEAR.—Maximum contents, 1,109,782 acre-ft, June 16, gage height, 4,492.77 ft; minimum, 821,074 acre-ft, Jan. 16, gage height, $4,481.42 \mathrm{ft}$.

Capacity table (gage height, in feet, and contents, in acre-feet)

(Based on surveys by Pacific Gas \& Electric Co. in 1924 and 1926)

$\begin{array}{rrrrrr}4,422 & 8,948 & 4,434 & 49,510 & 4,460 & 376,686 \\ 4,424 & 10,067 & 4,437 & 74,189 & 4,470 & 565,519 \\ 4,426 & 11,260 & 4,440 & 101,869 & 4,480 & 787,304 \\ 4,428 & 13,480 & 4,445 & 156,414 & 4,490 & 1,036,269 \\ 4,430 & 21,200 & 4,450 & 220,848 & 4,495.5 & 1,183,835 \\ 4,432 & 34,173 & 4,455 & 94,531 & & \end{array}$

RESERVOIR STORAGE (ACRE-FEET), WATER YEAR OCTOBER 1998 TO SEPTEMBER 1999 DAILY OBSERVATION AT 2400 HOURS

\begin{tabular}{|c|c|c|c|c|c|c|c|c|c|c|c|c|}
\hline DAY & OCT & NOV & $\mathrm{DEC}$ & JAN & FEB & MAR & APR & MAY & JUN & JUL & AUG & SEP \\
\hline 1 & 951399 & 924945 & 903487 & 839832 & 860444 & 883952 & 954439 & 1012757 & 1080914 & 1102002 & 1053617 & 974781 \\
\hline 2 & 948362 & 922190 & 903735 & 838383 & 860200 & 888634 & 956214 & 1015619 & 1084914 & 1100128 & 1050978 & 975293 \\
\hline 3 & 945834 & 919438 & 905475 & 837418 & 860444 & 892091 & 957229 & 1017963 & 1088923 & 1101199 & 1048869 & 976317 \\
\hline 4 & 943308 & 916439 & 903984 & 835731 & 860444 & 895306 & 958498 & 1019527 & 1091853 & 1100664 & 1046500 & 976830 \\
\hline 5 & 940785 & 913194 & 903239 & 833803 & 863125 & 898525 & 960785 & 1020831 & 1093985 & 1098257 & 1042982 & 977599 \\
\hline 6 & 938516 & 912446 & 901501 & 832840 & 867030 & 901005 & 962565 & 1022657 & 1096654 & 1096921 & 1040197 & 978624 \\
\hline 7 & 937005 & 911698 & 899021 & 830675 & 870208 & 903487 & 964092 & 1024746 & 1098806 & 1095853 & 1037316 & 978624 \\
\hline 8 & 935494 & 911698 & 897286 & 829713 & 873391 & 905972 & 968170 & 1026314 & 1100931 & 1093027 & 1034165 & 977599 \\
\hline 9 & 933984 & 909456 & 894069 & 829232 & 878297 & 909954 & 969701 & 1028668 & 1102994 & 1091415 & 1032850 & 977599 \\
\hline 10 & 931973 & 908211 & 891597 & 828992 & 878297 & 911698 & 971745 & 1030761 & 1104949 & 1091146 & 1030487 & 976830 \\
\hline 11 & 929963 & 905475 & 888881 & 828031 & 877560 & 913693 & 973535 & 1031809 & 1107096 & 1088215 & 1028652 & 976573 \\
\hline 12 & 927955 & 902494 & 886415 & 826110 & 877560 & 915690 & 974814 & 1032071 & 1107096 & 1087854 & 1025771 & 975805 \\
\hline 13 & 926199 & 899764 & 883706 & 822991 & 878542 & 917438 & 977120 & 1032071 & 1108707 & 84381 & 1022633 & 974781 \\
\hline 14 & 924945 & 899021 & 881737 & 822512 & 880754 & 919438 & 978658 & 1032071 & 1108976 & 1081980 & 1019761 & 973502 \\
\hline 15 & 923192 & 896543 & 879279 & 822033 & 881983 & 921189 & 980967 & 1033381 & 1109244 & 1082513 & 1017155 & 972735 \\
\hline 16 & 920188 & 894069 & 878051 & 821074 & 882229 & 923192 & 982509 & 1032857 & 1109782 & 1080381 & 1014292 & 970946 \\
\hline 17 & 918438 & 892832 & 876333 & 824190 & 881737 & 923442 & 984308 & 1034168 & 1108976 & 1078251 & 1011953 & 969159 \\
\hline 18 & 919688 & 890115 & 873636 & 826830 & 882229 & 925697 & 986367 & 1036528 & 1108438 & 1075858 & 1008061 & 968904 \\
\hline 19 & 920438 & 887401 & 870942 & 831637 & 881000 & 927453 & 987654 & 1038891 & 1108170 & 1075061 & 1005730 & 967119 \\
\hline 20 & 921438 & 884444 & 868252 & 834767 & 881000 & 930214 & 990231 & 1042044 & 1107901 & 1075326 & 1002110 & 966101 \\
\hline 21 & 922941 & 881983 & 865565 & 837177 & 880016 & 931973 & 992294 & 4410 & 07633 & 73998 & 00045 & 964319 \\
\hline 22 & 923943 & 881983 & 861418 & 840314 & 877560 & 934488 & 994875 & 1045200 & 1107364 & 72671 & 996933 & 963810 \\
\hline 23 & 925196 & 884937 & 860687 & 844907 & 875842 & 936501 & 996167 & 48622 & 1106291 & 71611 & 993308 & 961777 \\
\hline 24 & 927704 & 888141 & 860931 & 847086 & 874616 & 939524 & 999011 & 1052577 & 1104145 & 1069490 & 991240 & 962793 \\
\hline 25 & 928708 & 888141 & 854363 & 849267 & 874126 & 941542 & 1000046 & 1056008 & 1104345 & 1069755 & 988916 & 961777 \\
\hline 26 & 930214 & 891597 & 850964 & 850964 & 875842 & 943308 & 1003412 & 1060500 & 1103073 & 1068166 & 986853 & 960761 \\
\hline 27 & 931470 & 893821 & 848297 & 852905 & 877560 & 945076 & 1005486 & 1064204 & 1103264 & 1065786 & 983248 & 959746 \\
\hline 28 & 932476 & 893574 & 845875 & 853877 & 881000 & 946592 & 1008080 & 1068444 & 1103609 & 1063672 & 980678 & 959239 \\
\hline 29 & 933733 & 895553 & 842489 & 854849 & --- & 948109 & 1008859 & 1071363 & 1103073 & 1061820 & 978624 & 957971 \\
\hline 30 & 930968 & 902990 & 840314 & 856307 & --- & 950385 & 1014418 & 1075348 & 1103073 & 1059435 & 976061 & 957211 \\
\hline 31 & 928206 & --- & 839107 & 857523 & --- & 952665 & --- & 1078539 & --- & 1056755 & 975549 & \\
\hline MAX & 951399 & 924945 & 905475 & 857523 & 882229 & 952665 & 1014418 & 78539 & 1109782 & 2002 & 1053617 & 978624 \\
\hline MIN & 918438 & 881983 & 839107 & 821074 & 860200 & 883952 & 954439 & 1012757 & 1080914 & 1056755 & 975549 & 957211 \\
\hline$a$ & 4485.79 & 4484.78 & 4482.17 & 4482.93 & 4483.89 & 4486.76 & 4489.01 & 4491.60 & 4492.52 & 4490.77 & 4487.66 & 4486.94 \\
\hline $\mathrm{b}$ & -24966 & -25216 & -63883 & +18416 & +23477 & +71665 & +61753 & +64121 & +24534 & -46318 & -81206 & -18338 \\
\hline
\end{tabular}

CAL YR 1998 MAX 1123508 MIN 625736 b +214881

WTR YR 1999 MAX 1109782 MIN 821074 b $\quad+4039$

a Gage height, in feet, at end of month.

b Change in contents, in acre-feet. 
LOCATION.-Lat 40¹0'06", long 12105'31", in NE 1/4 SW 1/4 sec.28, T.27 N., R.8 E., Plumas County, Hydrologic Unit 18020121, Plumas National Forest, on left bank 0.4 mi downstream from Almanor Dam, 4.5 mi southeast of Prattville, and 9 mi upstream from Butt Creek.

DRAINAGE AREA.-493 $\mathrm{mi}^{2}$.

PERIOD OF RECORD._June 1905 to current year. Published as "below Prattville" prior to 1911. No record for January, February, or March 1911. Estimated mean discharge for water year 1911 published in WSP 1315-A.

REVISED RECORDS.-WSP 1245: 1951 (yearly summaries). WSP 1285: 1952 (yearly summaries). WDR CA-88-4: 1987 (monthly and yearly totals for Butt Valley Powerplant).

GAGE.-Water-stage recorder and broad-crested weir. Datum of gage is 4,379.86 ft above sea level. Prior to Oct. 1, 1936, nonrecording gages or water-stage recorders at several sites within $0.5 \mathrm{mi}$ of present site at various datums.

REMARKS.-Flow regulated since 1913 by Lake Almanor (station 11399000) 0.5 mi upstream and since 1924 by Mountain Meadows Reservoir, capacity, 24,000 acre-ft, $12 \mathrm{mi}$ upstream on Hamilton Branch. Water diverted from Lake Almanor to Butt Valley Reservoir (station 11401050) through old Almanor-Butt Creek Tunnel from May 1921 to December 1958, for use at Caribou Powerplant. Old tunnel closed Dec. 30, 1958, and diversion began Dec. 31, 1958, to Butt Valley Powerplant (station 11400600) at upstream end of Butt Valley Reservoir. See schematic diagram of North Fork Feather River Basin.

COOPERATION.--Records were collected by Pacific Gas \& Electric Co., under general supervision of the U.S. Geological Survey, in connection with a Federal Energy Regulatory Commission project.

EXTREMES FOR PERIOD OF RECORD.-Maximum discharge, 10,000 ft 3 $/ \mathrm{s}$, Mar. 19, 1907, before construction of dam, gage height, $16.2 \mathrm{ft}$, at former site, from rating curve extended above $3,700 \mathrm{ft}^{3} / \mathrm{s}$; no flow at times during 1914, 1919, 1923.

DISCHARGE, CUBIC FEET PER SECOND, WATER YEAR OCTOBER 1998 TO SEPTEMBER 1999 DAILY MEAN VALUES

\begin{tabular}{|c|c|c|c|c|c|c|c|c|c|c|c|c|}
\hline DAY & $\mathrm{OCT}$ & NOV & $\mathrm{DEC}$ & JAN & FEB & MAR & APR & MAY & JUN & JUL & AUG & SEP \\
\hline 1 & 38 & 35 & 38 & 38 & 38 & 38 & 40 & 34 & 37 & 38 & 37 & 37 \\
\hline 2 & 38 & 35 & 38 & 38 & 38 & 38 & 40 & 36 & 37 & 38 & 37 & 37 \\
\hline 3 & 38 & 35 & 38 & 38 & 38 & 39 & 40 & 36 & 37 & 38 & 37 & 37 \\
\hline 4 & 38 & 35 & 38 & 38 & 38 & 39 & 40 & 36 & 37 & 38 & 37 & 37 \\
\hline 5 & 38 & 35 & 38 & 38 & 38 & 39 & 40 & 36 & 37 & 38 & 37 & 37 \\
\hline 6 & 38 & 35 & 38 & 38 & 38 & 39 & 40 & 36 & 37 & 37 & 37 & 37 \\
\hline 7 & 37 & 35 & 38 & 38 & 38 & 39 & 40 & 36 & 37 & 37 & 37 & 37 \\
\hline 8 & 37 & 35 & 38 & 38 & 38 & 39 & 40 & 36 & 37 & 37 & 37 & 37 \\
\hline 9 & 37 & 37 & 38 & 38 & 39 & 39 & 40 & 36 & 37 & 37 & 37 & 37 \\
\hline 10 & 37 & 38 & 38 & 38 & 39 & 39 & 40 & 36 & 37 & 37 & 37 & 37 \\
\hline 11 & 37 & 38 & 38 & 38 & 39 & 39 & 40 & 36 & 37 & 37 & 37 & 37 \\
\hline 12 & 37 & 38 & 38 & 38 & 39 & 39 & 40 & 36 & 37 & 37 & 37 & 37 \\
\hline 13 & 37 & 38 & 38 & 38 & 39 & 39 & 40 & 36 & 37 & 37 & 37 & 37 \\
\hline 14 & 37 & 38 & 38 & 38 & 38 & 39 & 40 & 37 & 38 & 37 & 37 & 37 \\
\hline 15 & 36 & 38 & 38 & 38 & 38 & 40 & 40 & 37 & 38 & 37 & 37 & 37 \\
\hline 16 & 36 & 38 & 38 & 38 & 38 & 40 & 40 & 37 & 38 & 37 & 37 & 37 \\
\hline 17 & 36 & 38 & 38 & 38 & 38 & 40 & 40 & 37 & 38 & 37 & 37 & 36 \\
\hline 18 & 36 & 38 & 38 & 38 & 38 & 40 & 40 & 37 & 38 & 37 & 37 & 36 \\
\hline 19 & 36 & 38 & 38 & 38 & 38 & 40 & 40 & 37 & 38 & 37 & 37 & 36 \\
\hline 20 & 36 & 38 & 38 & 38 & 38 & 40 & 40 & 36 & 38 & 37 & 37 & 36 \\
\hline 21 & 36 & 38 & 38 & 38 & 38 & 40 & 40 & 37 & 38 & 37 & 37 & 36 \\
\hline 22 & 36 & 38 & 38 & 38 & 38 & 40 & 40 & 37 & 38 & 37 & 37 & 36 \\
\hline 23 & 36 & 38 & 38 & 39 & 38 & 40 & 41 & 37 & 37 & 37 & 37 & 36 \\
\hline 24 & 36 & 38 & 38 & 38 & 38 & 40 & 41 & 37 & 37 & 37 & 37 & 36 \\
\hline 25 & 36 & 38 & 38 & 38 & 38 & 40 & 41 & 37 & 37 & 37 & 37 & 36 \\
\hline 26 & 36 & 38 & 38 & 38 & 38 & 40 & 41 & 37 & 37 & 37 & 37 & 36 \\
\hline 27 & 36 & 38 & 38 & 38 & 38 & 40 & 39 & 37 & 37 & 37 & 37 & 36 \\
\hline 28 & 35 & 38 & 38 & 38 & 38 & 40 & 35 & 37 & 37 & 37 & 37 & 36 \\
\hline 29 & 35 & 38 & 38 & 38 & --- & 40 & 35 & 37 & 37 & 37 & 37 & 36 \\
\hline 30 & 35 & 39 & 38 & 38 & --- & 40 & 34 & 37 & 37 & 37 & 37 & 36 \\
\hline 31 & 35 & --- & 38 & 38 & --- & 40 & --- & 37 & --- & 37 & 37 & --- \\
\hline TOTAL & 1132 & 1116 & 1178 & 1179 & 1069 & 1224 & 1187 & 1131 & 1119 & 1152 & 1147 & 1096 \\
\hline MEAN & 36.5 & 37.2 & 38.0 & 38.0 & 38.2 & 39.5 & 39.6 & 36.5 & 37.3 & 37.2 & 37.0 & 36.5 \\
\hline MAX & 38 & 39 & 38 & 39 & 39 & 40 & 41 & 37 & 38 & 38 & 37 & 37 \\
\hline MIN & 35 & 35 & 38 & 38 & 38 & 38 & 34 & 34 & 37 & 37 & 37 & 36 \\
\hline $\mathrm{AC}-\mathrm{FT}$ & 2250 & 2210 & 2340 & 2340 & 2120 & 2430 & 2350 & 2240 & 2220 & 2280 & 2280 & 2170 \\
\hline a & 68830 & 102800 & 131900 & 42310 & 42800 & 417 & 12330 & 37040 & 42840 & 82170 & 107800 & 41950 \\
\hline
\end{tabular}

a Diversion, in acre-feet, to Butt Valley Powerplant, provided by Pacific Gas \& Electric Co. 


\section{NORTH FORK FEATHER RIVER NEAR PRATTVILLE, CA—Continued}

STATISTICS OF MONTHLY MEAN DATA FOR WATER YEARS 1925 - 1958, BY WATER YEAR (WY)

\begin{tabular}{|c|c|c|c|c|c|c|c|c|c|c|c|c|}
\hline MEAN & 498 & 393 & 371 & 282 & 349 & 272 & 318 & 327 & 349 & 479 & 602 & 569 \\
\hline MAX & 1607 & 1414 & 1418 & 1489 & 2124 & 1609 & 1852 & 2206 & 1065 & 1280 & 1755 & 1762 \\
\hline (WY) & 1931 & 1931 & 1938 & 1946 & 1938 & 1929 & 1938 & 1938 & 1935 & 1929 & 1929 & 1929 \\
\hline MIN & 3.80 & 3.32 & 3.41 & 3.20 & 3.20 & 3.61 & 2.63 & 2.02 & 2.11 & 8.02 & 3.72 & 3.16 \\
\hline (WY) & 1942 & 1940 & 1937 & 1944 & 1944 & 1944 & 1939 & 1939 & 1939 & 1943 & 1937 & 1937 \\
\hline
\end{tabular}

SUMMARY STATISTICS

ANNUAL TOTAL

ANNUAL MEAN

HIGHEST ANNUAL MEAN

LOWEST ANNUAL MEAN

HIGHEST DAILY MEAN

LOWEST DAILY MEAN

ANNUAL SEVEN-DAY MINIMUM

INSTANTANEOUS PEAK FLOW

INSTANTANEOUS PEAK STAGE

ANNUAL RUNOFF (AC-FT)

10 PERCENT EXCEEDS

50 PERCENT EXCEEDS

90 PERCENT EXCEEDS
WATER YEARS $1925-1958$

\begin{tabular}{|c|c|c|}
\hline 401 & & \\
\hline 1061 & & 1929 \\
\hline 27.1 & & 1937 \\
\hline 2670 & May 17 & 1942 \\
\hline .50 & Apr 28 & 1949 \\
\hline .87 & Apr 25 & 1949 \\
\hline 2710 & May 22 & 1941 \\
\hline 6.95 & May 22 & 1941 \\
\hline 290600 & & \\
\hline 1060 & & \\
\hline 60 & & \\
\hline 4.4 & & \\
\hline
\end{tabular}

STATISTICS OF MONTHLY MEAN DATA FOR WATER YEARS 1960 - 1999, BY WATER YEAR (WY)

\begin{tabular}{|c|c|c|c|c|c|c|c|c|c|c|c|c|}
\hline MEAN & 47.3 & 44.9 & 33.1 & 80.2 & 78.7 & 37.4 & 42.0 & 49.9 & 68.1 & 63.3 & 59.6 & 44.8 \\
\hline MAX & 510 & 546 & 59.6 & 1901 & 1800 & 163 & 293 & 352 & 660 & 688 & 596 & 415 \\
\hline (WY) & 1997 & 1997 & 1997 & 1997 & 1997 & 1997 & 1983 & 1996 & 1996 & 1996 & 1996 & 1996 \\
\hline MIN & 17.3 & 8.65 & 7.47 & 8.67 & 10.0 & 9.90 & 10.1 & 15.7 & 16.0 & 15.4 & 14.9 & 15.0 \\
\hline (WY) & 1978 & 1960 & 1960 & 1960 & 1962 & 1964 & 1964 & 1977 & 1977 & 1977 & 1977 & 1977 \\
\hline
\end{tabular}

SUMMARY STATISTICS

ANNUAL TOTAL

ANNUAL MEAN

HIGHEST ANNUAL MEAN

LOWEST ANNUAL MEAN

HIGHEST DAILY MEAN

LOWEST DAILY MEAN

ANNUAL SEVEN-DAY MINIMUM

INSTANTANEOUS PEAK FLOW

INSTANTANEOUS PEAK STAGE

ANNUAL RUNOFF (AC-ET)

ANNUAL DIVERSION (AC-FT) a

10 PERCENT EXCEEDS

50 PERCENT EXCEEDS

90 PERCENT EXCEEDS
FOR 1998 CALENDAR YEAR

\begin{tabular}{|c|c|}
\hline $\begin{array}{r}13955 \\
38 .\end{array}$ & \\
\hline 43 & Jan 17 \\
\hline 35 & Jul 1 \\
\hline 35 & Jul 1 \\
\hline 27680 & \\
\hline 85600 & \\
\hline 41 & \\
\hline 38 & \\
\hline 36 & \\
\hline
\end{tabular}

FOR 1999 WATER YEAR

$$
\begin{aligned}
& 13730 \\
& 37.6 \\
& 41 \text { Apr } 23 \\
& \text { Apr } 30 \\
& \text { Oct } 28 \\
& 41 \text { Apr } 22 \\
& 2.57 \text { Apr } 22 \\
& 27230 \\
& 713100 \\
& 40 \\
& \begin{array}{l}
38 \\
36
\end{array}
\end{aligned}
$$

WATER YEARS $1960-1999$

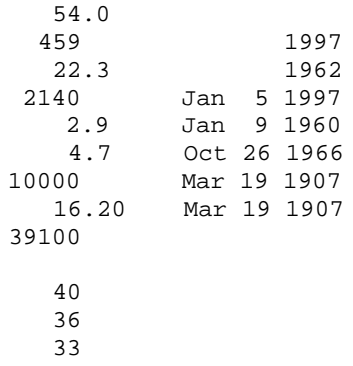

a Diversion, in acre-feet, to Butt Valley Powerplant, provided by Pacific Gas \& Electric Co. 


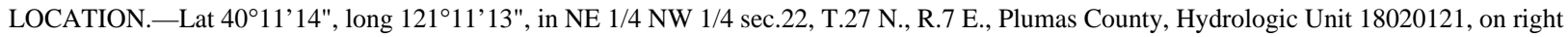
bank $500 \mathrm{ft}$ downstream from outlet of old Almanor-Butt Creek Tunnel, and 2.2 mi southwest of Prattville.

DRAINAGE AREA. $-69.3 \mathrm{mi}^{2}$.

PERIOD OF RECORD.-October 1936 to September 1959, October 1964 to current year. Published as "below tunnel No. 1" 1938-40. Records for water years 1937-38 published in WSP 1515. Records prior to 1964 not equivalent owing to inflow from Almanor-Butt Creek Tunnel.

GAGE.-Water-stage recorder and concrete control. Elevation of gage is 4,300 ft above sea level, from topographic map. Prior to Oct. 5, 1937, at site $200 \mathrm{ft}$ downstream at datum $4 \mathrm{ft}$ lower.

REMARKS.- - No regulation upstream from station. Howell-Bunger valve in conduit from Lake Almanor (station 11399000) to Butt Valley Powerplant (station 11400600) is opened for short periods several times a year, causing sharp peaks. Wallack Ditch upstream from station diverts about $3 \mathrm{ft}^{3} / \mathrm{s}$ during each irrigation season into Yellow Creek Basin. Some inflow $500 \mathrm{ft}$ upstream that is the leakage from the abandoned Almanor-Butt Creek Tunnel at Outlet (station 11400200) is included in the table below. See schematic diagram of North Fork Feather River Basin.

COOPERATION.-Records were collected by Pacific Gas \& Electric Co., under general supervision of the U.S. Geological Survey, in connection with a Federal Energy Regulatory Commission project.

EXTREMES FOR PERIOD OF RECORD.-Maximum discharge, 4,080 ft 3 s, Jan. 1, 1997, gage height, $6.22 \mathrm{ft}$, from rating curve extended above $1,400 \mathrm{ft}^{3} / \mathrm{s}$; minimum daily, $26 \mathrm{ft}^{3} / \mathrm{s}$, several days during May and June 1976.

DISCHARGE, CUBIC FEET PER SECOND, WATER YEAR OCTOBER 1998 TO SEPTEMBER 1999

DAILY MEAN VALUES

\begin{tabular}{|c|c|c|c|c|c|c|c|c|c|c|c|c|}
\hline DAY & $\mathrm{OCT}$ & NOV & DEC & JAN & FEB & MAR & APR & MAY & JUN & JUL & AUG & SEP \\
\hline 1 & 62 & 64 & 181 & 69 & 84 & 224 & 133 & 215 & 155 & 72 & 58 & 56 \\
\hline 2 & 62 & 63 & 143 & 68 & 82 & 178 & 127 & 220 & 160 & 71 & 57 & 57 \\
\hline 3 & 62 & 63 & 262 & 67 & 82 & 252 & 124 & 212 & 152 & 71 & 57 & 55 \\
\hline 4 & 62 & 63 & 147 & 67 & 82 & 196 & 119 & 192 & 140 & 71 & 58 & 55 \\
\hline 5 & 62 & 63 & 114 & 66 & 80 & 169 & 121 & 181 & 133 & 71 & 58 & 55 \\
\hline 6 & 62 & 64 & 101 & 66 & 79 & 155 & 119 & 190 & 128 & 71 & 59 & 54 \\
\hline 7 & 62 & 73 & 96 & 66 & 136 & 143 & 116 & 202 & 122 & 70 & 59 & 53 \\
\hline 8 & 62 & 72 & 92 & 65 & 131 & 136 & 118 & 191 & 118 & 69 & 58 & 53 \\
\hline 9 & 62 & 66 & 86 & 65 & 287 & 131 & 116 & 183 & 115 & 66 & 59 & 52 \\
\hline 10 & 62 & 66 & 83 & 65 & 198 & 123 & 113 & 178 & 109 & 66 & 63 & 53 \\
\hline 11 & 62 & 66 & 81 & 65 & 152 & 120 & 121 & 180 & 103 & 66 & 63 & 51 \\
\hline 12 & 62 & 65 & 81 & 65 & 134 & 118 & 137 & 192 & 99 & 65 & 61 & 50 \\
\hline 13 & 63 & 65 & 81 & 65 & 123 & 121 & 140 & 192 & 97 & 65 & 59 & 50 \\
\hline 14 & 62 & 65 & 83 & 66 & 117 & 120 & 146 & 180 & 96 & 64 & 58 & 50 \\
\hline 15 & 62 & 66 & 79 & 81 & 110 & 119 & 152 & 170 & 91 & 64 & 58 & 50 \\
\hline 16 & 62 & 66 & 77 & 114 & 113 & 123 & 160 & 167 & 88 & 63 & 57 & 50 \\
\hline 17 & 62 & 81 & 77 & 98 & 156 & 131 & 180 & 166 & 87 & 63 & 56 & 50 \\
\hline 18 & 62 & 69 & 77 & 170 & 146 & 139 & 202 & 171 & 86 & 63 & 55 & 50 \\
\hline 19 & 62 & 65 & 74 & 131 & 135 & 141 & 214 & 173 & 84 & 64 & 55 & 50 \\
\hline 20 & 62 & 64 & 71 & 163 & 123 & 144 & 225 & 172 & 83 & 62 & 55 & 51 \\
\hline 21 & 62 & 74 & 63 & 148 & 119 & 141 & 226 & 172 & 82 & 59 & 55 & 53 \\
\hline 22 & 62 & 132 & 69 & 123 & 113 & 137 & 214 & 184 & 80 & 59 & 54 & 52 \\
\hline 23 & 62 & 193 & 72 & 186 & 111 & 137 & 206 & 193 & 77 & 60 & 55 & 50 \\
\hline 24 & 69 & 134 & 73 & 131 & 112 & 152 & 211 & 205 & 78 & 59 & 54 & 50 \\
\hline 25 & 68 & 86 & 76 & 116 & 118 & 168 & 219 & 214 & 77 & 58 & 54 & 50 \\
\hline 26 & 65 & 87 & 78 & 109 & 106 & 170 & 320 & 212 & 77 & 58 & 54 & 50 \\
\hline 27 & 63 & 134 & 78 & 100 & 106 & 165 & 264 & 207 & 76 & 59 & 55 & 50 \\
\hline 28 & 63 & 97 & 75 & 95 & 165 & 152 & 223 & 195 & 75 & 59 & 56 & 50 \\
\hline 29 & 63 & 88 & 74 & 91 & --- & 148 & 204 & 184 & 75 & 60 & 56 & 50 \\
\hline 30 & 63 & 285 & 72 & 88 & --- & 148 & 204 & 172 & 74 & 59 & 57 & 50 \\
\hline 31 & 62 & --- & 72 & 88 & --- & 141 & --- & 161 & --- & 58 & 57 & --- \\
\hline TOTAL & 1943 & 2639 & 2888 & 2957 & 3500 & 4642 & 5174 & 5826 & 3017 & 1985 & 1770 & 1550 \\
\hline MEAN & 62.7 & 88.0 & 93.2 & 95.4 & 125 & 150 & 172 & 188 & 101 & 64.0 & 57.1 & 51.7 \\
\hline MAX & 69 & 285 & 262 & 186 & 287 & 252 & 320 & 220 & 160 & 72 & 63 & 57 \\
\hline MIN & 62 & 63 & 63 & 65 & 79 & 118 & 113 & 161 & 74 & 58 & 54 & 50 \\
\hline $\mathrm{AC}-\mathrm{FT}$ & 3850 & 5230 & 5730 & 5870 & 6940 & 9210 & 10260 & 11560 & 5980 & 3940 & 3510 & 3070 \\
\hline $\mathrm{a}$ & 526 & 498 & 501 & 498 & 462 & 517 & 511 & 542 & 527 & 538 & 530 & 499 \\
\hline
\end{tabular}

a Inflow, in acre-feet, from Almanor-Butt Creek Tunnel at Outlet, provided by Pacific Gas \& Electric Co. 


\section{BUTT CREEK BELOW ALMANOR-BUTT CREEK TUNNEL, NEAR PRATTVILLE, CA—Continued}

STATISTICS OF MONTHLY MEAN DATA FOR WATER YEARS 1937 - 1999, BY WATER YEAR (WY)

\begin{tabular}{|c|c|c|c|c|c|c|c|c|c|c|c|c|}
\hline MEAN & 372 & 345 & 357 & 303 & 300 & 334 & 341 & 379 & 371 & 390 & 382 & 378 \\
\hline MAX & 995 & 1073 & 1419 & 1098 & 1025 & 1050 & 1178 & 1176 & 1092 & 1038 & 1019 & 990 \\
\hline (WY) & 1943 & 1938 & 1959 & 1953 & 1941 & 1953 & 1952 & 1956 & 1958 & 1953 & 1953 & 1953 \\
\hline MIN & 32.3 & 39.2 & 39.3 & 39.4 & 38.0 & 47.8 & 47.5 & 42.7 & 32.9 & 28.7 & 27.8 & 29.4 \\
\hline (WY) & 1989 & 1992 & 1991 & 1992 & 1937 & 1977 & 1977 & 1976 & 1976 & 1977 & 1977 & 1992 \\
\hline
\end{tabular}

SUMMARY STATISTICS

ANNUAL TOTAL

ANNUAL MEAN

HIGHEST ANNUAL MEAN

LOWEST ANNUAL MEAN

HIGHEST DAILY MEAN

LOWEST DAILY MEAN

ANNUAL SEVEN-DAY MINIMUM

INSTANTANEOUS PEAK FLOW

INSTANTANEOUS PEAK STAGE

ANNUAL RUNOFF (AC-FT)

ANNUAL INFLOW (AC-FT) a

10 PERCENT EXCEEDS

50 PERCENT EXCEEDS

90 PERCENT EXCEEDS
FOR 1998 CALENDAR YEAR

$\begin{array}{rrr}53539 & & \\ 147 & & \\ & & \\ 715 & & \text { Mar } 24 \\ 52 & \text { Jan } & 1 \\ 59 & \text { Jan } & 4 \\ & & \\ 106200 & & \\ 6210 & & \\ 334 & & \\ 88 & & \\ 62 & & \end{array}$

FOR 1999 WATER YEAR

$\begin{array}{ccc}37891 & & \\ 104 & & \\ & & \\ 320 & \text { Apr } 26 \\ 50 & \text { Sep } 12 \\ 50 & \text { Sep } 12 \\ 567 & \text { Nov } 30 \\ 2.23 & \text { Nov } 30 \\ 75160 & & \\ 6150 & & \\ 188 & & \\ 79 & & \\ 55 & & \end{array}$

WATER YEARS 1937 - 1999

\begin{tabular}{|c|c|c|}
\hline 355 & & \\
\hline 974 & & 1953 \\
\hline 40.1 & & 1977 \\
\hline 2830 & Feb 17 & 1986 \\
\hline 26 & May 26 & 1976 \\
\hline 26 & May 30 & 1976 \\
\hline 4080 & Jan 1 & 1997 \\
\hline 6.22 & Jan & 1997 \\
\hline 57000 & & \\
\hline $\begin{array}{l}989 \\
101\end{array}$ & & \\
\hline 43 & & \\
\hline
\end{tabular}

a Inflow, in acre-feet, from Almanor-Butt Creek Tunnel at Outlet, provided by Pacific Gas \& Electric Co. 
11401050 BUTT VALLEY RESERVOIR NEAR CARIBOU, CA

LOCATION.-Lat $40^{\circ} 06^{\prime} 59^{\prime \prime}$, long $121^{\circ} 08^{\prime} 42^{\prime \prime}$, in SE 1/4 SW 1/4 sec.12, T.26 N., R.7 E., Plumas County, Hydrologic Unit 18020121, on center intake tower in Butt Valley Reservoir, 2.5 mi north of Caribou, and 5.4 mi southwest of Canyon Dam.

DRAINAGE AREA.- $-83.5 \mathrm{mi}^{2}$.

PERIOD OF RECORD.-OCtober 1985 to current year. Unpublished records for water years 1983-85 available in files of the U.S. Geological Survey.

GAGE.-Water-stage recorder. Datum of gage is $10.23 \mathrm{ft}$ below sea level (levels by Great Western Power Co.).

REMARKS.-Lake is formed by earthfill dam. Storage began in 1924. Usable capacity, 49,930 acre-ft between elevations 4,075.9 ft, invert of outlet tunnel, and 4,132.1 ft, crest of spillway. Water is diverted by tunnel and penstock to Caribou Powerplants (station 11401110). Figures given, including extremes, represent total contents at 2400 hours. See schematic diagram of North Fork Feather River Basin.

COOPERATION.-Records were collected by Pacific Gas \& Electric Co., under general supervision of the U.S. Geological Survey, in connection with a Federal Energy Regulatory Commission project. Contents not rounded to U.S. Geological Survey standards.

EXTREMES FOR PERIOD OF RECORD.—Maximum contents, 52,667 acre-ft, Feb. 18, 19, 1986, elevation, 4,133.80 ft; minimum, 4,284 acre-ft, Mar. 3, 1997, elevation, 4,094.95 ft.

EXTREMES FOR CURRENT YEAR.-Maximum contents, 49,529 acre-ft, Sept. 1, elevation, 4,131.85 ft; minimum, 24,536 acre-ft, Feb. 26, elevation, $4,114.86 \mathrm{ft}$.

Capacity table (elevation, in feet, and contents, in acre-feet)

(Based on surveys by Great Western Power Co. in 1923 and 1924)

$\begin{array}{rrrr}4,090 & 1,754 & 4,120 & 31,592 \\ 4,100 & 8,024 & 4,130 & 46,591 \\ 4,110 & 18,395 & 4,137 & 57,891\end{array}$

RESERVOIR STORAGE (ACRE-FEET), WATER YEAR OCTOBER 1998 TO SEPTEMBER 1999 DAILY OBSERVATION AT 2400 HOURS

\begin{tabular}{|c|c|c|c|c|c|c|c|c|c|c|c|c|}
\hline DAY & $\mathrm{OCT}$ & NOV & $\mathrm{DEC}$ & JAN & FEB & MAR & APR & MAY & JUN & JUL & AUG & SEP \\
\hline 1 & 45178 & 44710 & 41620 & 42233 & 37919 & 26330 & 32519 & 29891 & 46544 & 43173 & 40949 & 49529 \\
\hline 2 & 45021 & 44632 & 41544 & 41544 & 40248 & 27036 & 32162 & 29557 & 46560 & 42418 & 40491 & 48876 \\
\hline 3 & 44710 & 45492 & 42772 & 40324 & 37919 & 27828 & 31877 & 29139 & 46371 & 41650 & 39841 & 48126 \\
\hline 4 & 44476 & 46120 & 43080 & 39358 & 35394 & 28447 & 31451 & 28861 & 46765 & 41376 & 39841 & 46293 \\
\hline 5 & 44165 & 47066 & 43157 & 38516 & 32176 & 28958 & 31522 & 28861 & 46765 & 41696 & 39826 & 45209 \\
\hline 6 & 44554 & 45806 & 43080 & 38666 & 29515 & 29417 & 31451 & 29209 & 46702 & 41727 & 40248 & 43142 \\
\hline 7 & 44710 & 46041 & 42772 & 39358 & 28420 & 29849 & 31169 & 30634 & 46860 & 41833 & 40476 & 42757 \\
\hline 8 & 44710 & 45335 & 42541 & 39267 & 26466 & 30465 & 31169 & 31550 & 46876 & 42803 & 40128 & 42495 \\
\hline 9 & 45021 & 45413 & 43157 & 39191 & 27966 & 30887 & 31028 & 31820 & 46908 & 43342 & 39629 & 42541 \\
\hline 10 & 46120 & 45649 & 43157 & 38741 & 28255 & 31254 & 30887 & 32775 & 47414 & 43419 & 40037 & 42341 \\
\hline 11 & 46120 & 45884 & 43003 & 38591 & 28516 & 31592 & 30817 & 33912 & 46876 & 43590 & 40082 & 41849 \\
\hline 12 & 46434 & 45413 & 43157 & 38965 & 28722 & 31948 & 30747 & 36141 & 46277 & 43808 & 39795 & 42094 \\
\hline 13 & 47224 & 45099 & 43932 & 39418 & 27828 & 32290 & 30592 & 38696 & 45932 & 42880 & 39478 & 42464 \\
\hline 14 & 47303 & 43854 & 44554 & 39569 & 27691 & 32633 & 30394 & 40399 & 46010 & 43127 & 40644 & 42772 \\
\hline 15 & 47699 & 43311 & 44477 & 39493 & 27457 & 32975 & 30338 & 41772 & 45947 & 42957 & 41513 & 43327 \\
\hline 16 & 48016 & 42772 & 44399 & 39946 & 27622 & 33263 & 30507 & 43204 & 45822 & 42418 & 41987 & 44181 \\
\hline 17 & 48413 & 42695 & 44943 & 38591 & 27650 & 33522 & 30507 & 44025 & 45869 & 42849 & 42880 & 44725 \\
\hline 18 & 45492 & 42772 & 45099 & 39071 & 26873 & 33811 & 31550 & 43745 & 45994 & 42865 & 43388 & 44492 \\
\hline 19 & 46670 & 43003 & 45413 & 39403 & 26262 & 34186 & 32219 & 43265 & 46230 & 42295 & 44337 & 45492 \\
\hline 20 & 46434 & 42926 & 45492 & 40188 & 25791 & 33897 & 33090 & 42680 & 46465 & 41330 & 44072 & 46293 \\
\hline 21 & 46434 & 43080 & 44865 & 40022 & 25724 & 33782 & 32875 & 42772 & 46860 & 41544 & 44445 & 47240 \\
\hline 22 & 46434 & 43080 & 44865 & 40399 & 26262 & 33594 & 31806 & 44134 & 46971 & 40598 & 44928 & 46923 \\
\hline 23 & 46512 & 43621 & 45696 & 41162 & 26466 & 33594 & 31240 & 44508 & 46908 & 40537 & 46497 & 48349 \\
\hline 24 & 46591 & 43699 & 45492 & 41544 & 25590 & 33450 & 31169 & 45194 & 47240 & 41681 & 46214 & 47129 \\
\hline 25 & 46670 & 41620 & 45649 & 41849 & 25804 & 33378 & 30887 & 46246 & 46955 & 41528 & 45256 & 46828 \\
\hline 26 & 46749 & 38889 & 46041 & 42618 & 24536 & 33450 & 31042 & 46591 & 46340 & 40857 & 44368 & 46844 \\
\hline 27 & 46749 & 39342 & 46120 & 43080 & 24908 & 33306 & 30831 & 46797 & 46246 & 40689 & 43917 & 46828 \\
\hline 28 & 46591 & 39418 & 46355 & 42387 & 25590 & 33017 & 30239 & 46987 & 45476 & 41112 & 43652 & 45900 \\
\hline 29 & 46277 & 39493 & 46670 & 42156 & --- & 32889 & 30408 & 47003 & 44399 & 39811 & 45775 & 46387 \\
\hline 30 & 44943 & 40248 & 46670 & 42079 & --- & 32832 & 30352 & 46844 & 43435 & 39539 & 47905 & 46512 \\
\hline 31 & 45256 & --- & 45413 & 42002 & --- & 32690 & --- & 46860 & --- & 40445 & 48142 & --- \\
\hline MAX & 48413 & 47066 & 46670 & 43080 & 40248 & 34186 & 33090 & 47003 & 47414 & 43808 & 48142 & 49529 \\
\hline MIN & 44165 & 38889 & 41544 & 38516 & 24536 & 26330 & 30239 & 28861 & 43435 & 39539 & 39478 & 41849 \\
\hline$a$ & 4129.15 & 4125.90 & 4129.25 & 4127.05 & 4115.65 & 4120.77 & 4119.12 & 4130.17 & 4127.98 & 4126.03 & 4130.98 & 4129.95 \\
\hline $\mathrm{b}$ & +78 & -5008 & +5165 & -3411 & -16412 & +7100 & -2338 & +16508 & -3425 & -2990 & +7697 & -1630 \\
\hline $\mathrm{C}$ & 18090 & 30160 & 34480 & 17450 & 17730 & 258 & 1680 & 2570 & 9810 & 23880 & 33220 & 296 \\
\hline
\end{tabular}

CAL YR 1998 MAX 49370 MIN 33811 b +5618 c 645900 WTR YR 1999 MAX 49529 MIN 24536 b +1334 c 189600

a Elevation, in feet, at end of month.

b Change in contents, in acre-feet.

c Discharge, in acre-feet, through Caribou Powerplants, provided by Pacific Gas \& Electric Co. 
LOCATION.-Lat 4004'17", long 121 $09^{\prime} 49^{\prime \prime}$, in NE 1/4 NW 1/4 sec.35, T.26 N., R.7 E., Plumas County, Hydrologic Unit 18020121, Plumas National Forest, on left bank 0.4 mi downstream from Belden Dam, 0.5 mi upstream from Deadwood Canyon, and 6.4 mi northeast of Belden. DRAINAGE AREA.-612 $\mathrm{mi}^{2}$.

PERIOD OF RECORD.—October 1969 to current year. July 1959 to September 1969 in files of Pacific Gas \& Electric Co.

REVISED RECORDS.-WDR CA-78-4: 1977 (monthly and yearly summaries).

GAGE.-Water-stage recorder. Datum of gage is 2,800.77 ft above sea level (levels by Pacific Gas \& Electric Co.).

REMARKS.-Flow regulated by Butt Valley Reservoir (station 11401050), Lake Almanor (station 11399000), Belden Reservoir, and Mountain Meadows Reservoir, combined capacity, 1,267,000 acre-ft. Diversion to Belden Powerplant (station 11403050) began on Aug. 27, 1969. See schematic diagram of North Fork Feather River Basin.

COOPERATION.-Records were collected by Pacific Gas \& Electric Co., under general supervision of the U.S. Geological Survey, in connection with a Federal Energy Regulatory Commission project.

EXTREMES FOR PERIOD OF RECORD.-Maximum discharge, 3,460 ft $\mathrm{ft}^{3} / \mathrm{s}$, Jan. 1, 1997, gage height, $9.17 \mathrm{ft}$; minimum daily, $2.3 \mathrm{ft}^{3} / \mathrm{s}$, Oct. 25 , 1981.

DISCHARGE, CUBIC FEET PER SECOND, WATER YEAR OCTOBER 1998 TO SEPTEMBER 1999 DAILY MEAN VALUES

\begin{tabular}{|c|c|c|c|c|c|c|c|c|c|c|c|c|}
\hline DAY & $\mathrm{OCT}$ & NOV & $\mathrm{DEC}$ & JAN & FEB & MAR & APR & MAY & JUN & JUL & AUG & SEP \\
\hline 1 & 63 & 64 & 63 & 63 & 63 & 157 & 407 & 144 & 144 & 146 & 143 & 143 \\
\hline 2 & 64 & 64 & 62 & 63 & 67 & 162 & 405 & 144 & 143 & 142 & 144 & 143 \\
\hline 3 & 64 & 65 & 63 & 63 & 69 & 254 & 399 & 144 & 143 & 143 & 143 & 143 \\
\hline 4 & 64 & 65 & 63 & 63 & 67 & 292 & 417 & 145 & 143 & 142 & 143 & 143 \\
\hline 5 & 63 & 64 & 62 & 63 & 66 & 242 & 398 & 144 & 143 & 143 & 143 & 143 \\
\hline 6 & 63 & 64 & 62 & 63 & 66 & 197 & 386 & 144 & 143 & 143 & 143 & 119 \\
\hline 7 & 64 & 64 & 62 & 64 & 65 & 175 & 399 & 144 & 143 & 144 & 143 & 62 \\
\hline 8 & 64 & 64 & 62 & 63 & 65 & 156 & 405 & 144 & 143 & 144 & 144 & 62 \\
\hline 9 & 64 & 65 & 62 & 63 & 105 & 191 & 395 & 145 & 143 & 143 & 144 & 63 \\
\hline 10 & 63 & 63 & 62 & 62 & 68 & 170 & 400 & 144 & 143 & 143 & 143 & 62 \\
\hline 11 & 64 & 61 & 62 & 63 & 66 & 150 & 407 & 144 & 142 & 142 & 143 & 63 \\
\hline 12 & 64 & 61 & 62 & 63 & 65 & 152 & 402 & 144 & 144 & 143 & 143 & 62 \\
\hline 13 & 64 & 61 & 63 & 63 & 66 & 153 & 405 & 144 & 145 & 144 & 143 & 63 \\
\hline 14 & 63 & 61 & 63 & 62 & 65 & 152 & 399 & 144 & 142 & 143 & 144 & 63 \\
\hline 15 & 64 & 61 & 62 & 62 & 65 & 151 & 392 & 144 & 143 & 143 & 143 & 63 \\
\hline 16 & 64 & 62 & 63 & 63 & 65 & 151 & 397 & 144 & 143 & 143 & 143 & 63 \\
\hline 17 & 65 & 61 & 63 & 64 & 65 & 151 & 405 & 144 & 143 & 143 & 143 & 63 \\
\hline 18 & 72 & 62 & 63 & 64 & 65 & 151 & 399 & 144 & 143 & 144 & 144 & 64 \\
\hline 19 & 146 & 62 & 62 & 64 & 66 & 210 & 397 & 144 & 143 & 143 & 143 & 63 \\
\hline 20 & 147 & 61 & 63 & 65 & 64 & 359 & 251 & 144 & 143 & 143 & 143 & 64 \\
\hline 21 & 149 & 62 & 63 & 64 & 65 & 405 & 148 & 144 & 142 & 144 & 143 & 64 \\
\hline 22 & 150 & 62 & 63 & 64 & 65 & 398 & 148 & 145 & 143 & 143 & 144 & 63 \\
\hline 23 & 160 & 62 & 64 & 64 & 65 & 391 & 148 & 144 & 143 & 144 & 148 & 64 \\
\hline 24 & 162 & 66 & 63 & 63 & 65 & 381 & 146 & 145 & 144 & 143 & 144 & 63 \\
\hline 25 & 155 & 65 & 64 & 63 & 65 & 397 & 146 & 144 & 143 & 143 & 144 & 64 \\
\hline 26 & 153 & 64 & 64 & 64 & 127 & 403 & 148 & 144 & 143 & 144 & 143 & 63 \\
\hline 27 & 150 & 65 & 64 & 63 & 153 & 387 & 146 & 143 & 142 & 143 & 144 & 63 \\
\hline 28 & 150 & 62 & 64 & 62 & 154 & 399 & 145 & 145 & 143 & 142 & 144 & 62 \\
\hline 29 & 149 & 61 & 63 & 63 & --- & 402 & 144 & 144 & 144 & 142 & 144 & 63 \\
\hline 30 & 87 & 63 & 63 & 63 & --- & 399 & 147 & 144 & 143 & 143 & 143 & 64 \\
\hline 31 & 64 & --- & 63 & 63 & --- & 392 & --- & 144 & --- & 143 & 143 & --- \\
\hline TOTAL & 2978 & 1887 & 1947 & 1959 & 2112 & 8130 & 9331 & 4468 & 4292 & 4438 & 4449 & 2347 \\
\hline MEAN & 96.1 & 62.9 & 62.8 & 63.2 & 75.4 & 262 & 311 & 144 & 143 & 143 & 144 & 78.2 \\
\hline MAX & 162 & 66 & 64 & 65 & 154 & 405 & 417 & 145 & 145 & 146 & 148 & 143 \\
\hline MIN & 63 & 61 & 62 & 62 & 63 & 150 & 144 & 143 & 142 & 142 & 143 & 62 \\
\hline $\mathrm{AC}-\mathrm{FT}$ & 5910 & 3740 & 3860 & 3890 & 4190 & 16130 & 18510 & 8860 & 8510 & 8800 & 8820 & 4660 \\
\hline a & 64050 & 106200 & 118000 & 59490 & 86320 & 0 & 16620 & 30240 & 46350 & 81130 & 94740 & 48720 \\
\hline
\end{tabular}

a Diversion, in acre-feet, to Belden Powerplant, provided by Pacific Gas \& Electric Co. 
11401112 NORTH FORK FEATHER RIVER BELOW BELDEN DAM, CA—Continued

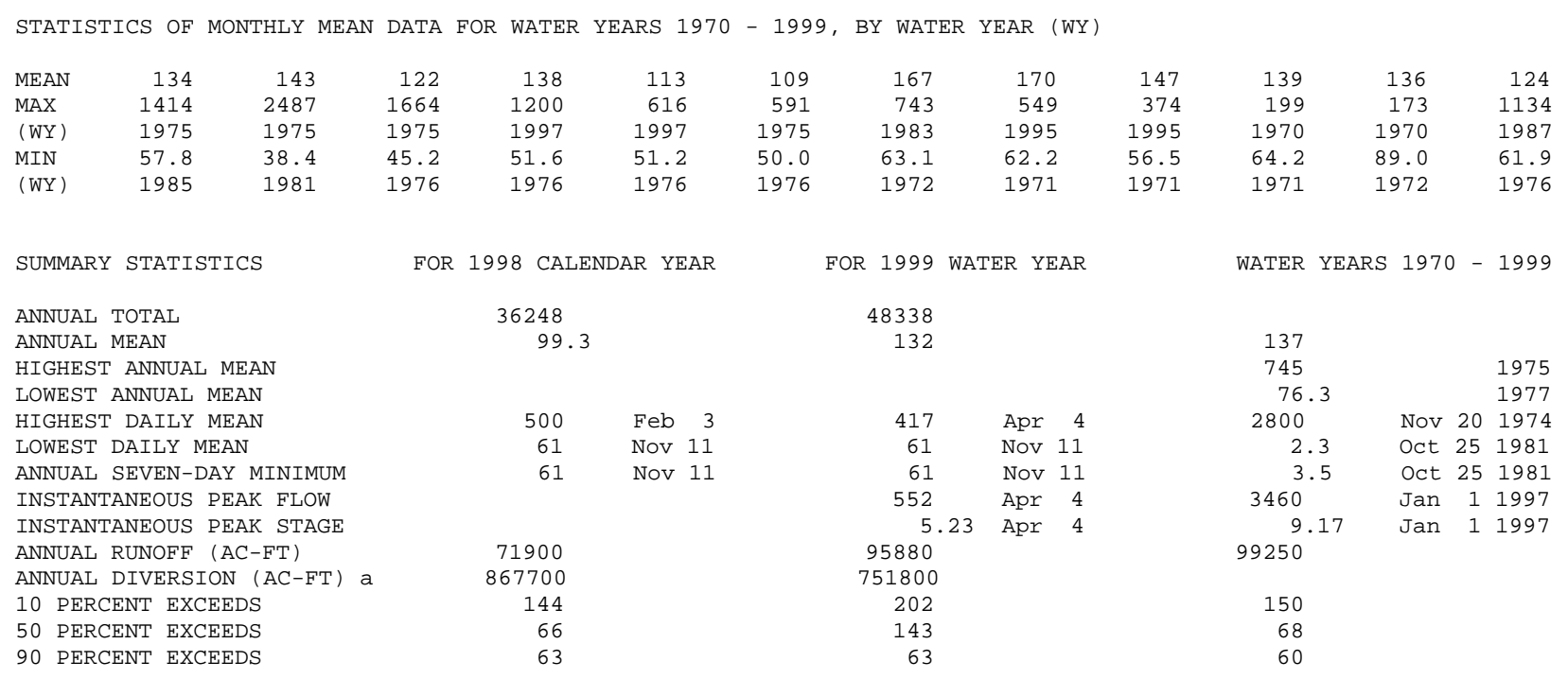

a Diversion, in acre-feet, to Belden Powerplant, provided by Pacific Gas \& Electric Co. 


\section{SOUTH BRANCH WARD CREEK BELOW DIVERSION DAM, NEAR GENESEE, CA}

LOCATION.-Lat 4000'07", long 12042'07", in SE 1/4 NE 1/4 sec.26, T.25 N., R.11 E., Plumas County, Hydrologic Unit 18020122, on left bank $20 \mathrm{ft}$ downstream from diversion dam, $30 \mathrm{ft}$ downstream from Nye Creek, $3.5 \mathrm{mi}$ upstream from Indian Creek, and 3.8 mi southeast of Genesee.

DRAINAGE AREA.-6.74 $\mathrm{mi}^{2}$.

PERIOD OF RECORD.-October 1990 to current year (low-flow records only).

GAGE.-Water-stage recorder and V-notch sharp-crested weir in concrete control. Elevation of gage is 5,300 ft above sea level, from topographic map.

REMARKS.-No records computed above $12 \mathrm{ft}^{3} / \mathrm{s}$. Flow regulated at diversion dam $20 \mathrm{ft}$ upstream. Some water is diverted to Five Bears Powerplant and bypasses this gage. See schematic diagram of North Fork Feather River Basin.

COOPERATION.-Records were collected by Five Bears Hydro, Inc., under general supervision of the U.S. Geological Survey, in connection with a Federal Energy Regulatory Commission project.

\section{DISCHARGE, CUBIC FEET PER SECOND, WATER YEAR OCTOBER 1998 TO SEPTEMBER 1999} DAILY MEAN VALUES

\begin{tabular}{|c|c|c|c|c|c|c|c|c|c|c|c|c|}
\hline DAY & $\mathrm{OCT}$ & NOV & $\mathrm{DEC}$ & JAN & FEB & MAR & APR & MAY & JUN & JUL & AUG & SEP \\
\hline 1 & 3.2 & 3.2 & 3.9 & 3.2 & 3.2 & 4.9 & 10 & 12 & --- & 3.3 & 3.2 & 3.2 \\
\hline 2 & 3.2 & 3.2 & 3.9 & 3.2 & 3.2 & 11 & 10 & 12 & 12 & 3.3 & 3.2 & 3.2 \\
\hline 3 & 3.2 & 3.2 & 6.9 & 3.2 & 3.2 & 11 & 11 & 11 & 10 & 3.3 & 3.2 & 3.2 \\
\hline 4 & 3.2 & 3.2 & 6.0 & 3.2 & 3.2 & 10 & 10 & 11 & 10 & 3.2 & 3.2 & 3.2 \\
\hline 5 & 3.2 & 3.2 & 5.4 & 3.2 & 3.2 & 10 & 10 & 11 & 10 & 3.2 & 3.2 & 3.2 \\
\hline 6 & 3.2 & 3.2 & 4.9 & 3.2 & 3.2 & 10 & 9.5 & 12 & 11 & 3.2 & 3.2 & 3.2 \\
\hline 7 & 3.2 & 3.3 & 4.7 & 3.2 & 3.5 & 10 & 9.4 & 12 & 11 & 3.2 & 3.2 & 3.2 \\
\hline 8 & 3.2 & 3.2 & 4.6 & 3.2 & 3.4 & 10 & 9.3 & 12 & 11 & 3.3 & 3.2 & 3.2 \\
\hline 9 & 3.2 & 3.2 & 4.5 & 3.2 & 4.3 & 10 & 8.8 & 12 & 11 & 3.2 & 3.2 & 3.2 \\
\hline 10 & 3.2 & 3.2 & 4.4 & 3.2 & 4.0 & 9.4 & 8.7 & 11 & 11 & 3.2 & 3.2 & 3.2 \\
\hline 11 & 3.2 & 3.2 & 4.4 & 3.2 & 3.8 & 9.0 & 9.0 & 11 & 10 & 3.2 & 3.2 & 3.2 \\
\hline 12 & 3.2 & 3.2 & 4.4 & 3.2 & 3.7 & 8.7 & 9.6 & 12 & 10 & 3.2 & 3.2 & 3.2 \\
\hline 13 & 3.2 & 3.2 & 4.4 & 3.2 & 3.6 & 8.8 & 11 & 12 & 10 & 3.2 & 3.2 & 3.2 \\
\hline 14 & 3.2 & 3.2 & 4.3 & 3.2 & 3.6 & 8.8 & 11 & 11 & 10 & 3.2 & 3.2 & 3.2 \\
\hline 15 & 3.2 & 3.2 & 4.1 & 3.2 & 3.6 & 9.0 & 11 & 11 & 10 & 3.2 & 3.2 & 3.2 \\
\hline 16 & 3.2 & 3.2 & 4.1 & 3.3 & 3.6 & 9.2 & 11 & 11 & 10 & 3.2 & 3.2 & 3.2 \\
\hline 17 & 3.2 & 3.2 & 4.7 & 3.3 & 3.9 & 9.6 & 11 & 11 & 5.1 & 3.3 & 3.2 & 3.2 \\
\hline 18 & 3.2 & 3.2 & 4.7 & 3.6 & 3.9 & 10 & 12 & 11 & 3.6 & 3.3 & 3.2 & 3.2 \\
\hline 19 & 3.2 & 3.2 & 3.9 & 3.7 & 3.8 & 11 & --- & 12 & 3.7 & 3.3 & 3.2 & 3.2 \\
\hline 20 & 3.2 & 3.2 & 3.2 & 4.2 & 3.8 & 11 & --- & 12 & 3.7 & 3.2 & 3.2 & 3.2 \\
\hline 21 & 3.2 & 3.7 & 4.2 & 3.6 & 4.2 & 11 & --- & 12 & 3.6 & 3.2 & 3.2 & 3.2 \\
\hline 22 & 3.2 & 4.2 & 5.0 & 3.5 & 3.5 & 11 & 12 & --- & 3.6 & 3.2 & 3.2 & 3.2 \\
\hline 23 & 3.2 & 4.4 & 3.4 & 3.7 & 3.5 & 11 & 11 & --- & 3.6 & 3.2 & 3.4 & 3.2 \\
\hline 24 & 3.2 & 3.7 & 3.2 & 3.6 & 3.6 & 11 & 12 & --- & 4.3 & 3.3 & 3.2 & 3.2 \\
\hline 25 & 3.2 & 3.4 & 3.2 & 3.5 & 3.8 & 11 & 12 & --- & 3.5 & 3.2 & 3.2 & 3.2 \\
\hline 26 & 3.2 & 3.4 & 3.2 & 3.4 & 3.7 & 11 & --- & --- & 3.4 & 3.2 & 3.2 & 3.2 \\
\hline 27 & 3.2 & 3.4 & 3.2 & 3.3 & 3.6 & 10 & --- & --- & 3.3 & 3.2 & 3.2 & 3.2 \\
\hline 28 & 3.2 & 4.1 & 3.2 & 3.3 & 3.8 & 10 & --- & --- & 4.1 & 3.2 & 3.2 & 3.2 \\
\hline 29 & 3.2 & 3.4 & 3.2 & 3.3 & --- & 10 & 11 & --- & 4.9 & 3.2 & 3.2 & 3.2 \\
\hline 30 & 3.2 & 3.9 & 3.2 & 3.2 & --- & 10 & 11 & --- & 3.5 & 3.2 & 3.2 & 3.6 \\
\hline 31 & 3.2 & --- & 3.2 & 3.2 & --- & 10 & --- & --- & --- & 3.2 & 3.2 & --- \\
\hline TOTAL & 99.2 & 101.7 & 129.6 & 103.7 & 101.4 & 307.4 & --- & --- & --- & 100.0 & 99.4 & 96.4 \\
\hline MEAN & 3.20 & 3.39 & 4.18 & 3.35 & 3.62 & 9.92 & --- & --- & --- & 3.23 & 3.21 & 3.21 \\
\hline MAX & 3.2 & 4.4 & 6.9 & 4.2 & 4.3 & 11 & --- & --- & --- & 3.3 & 3.4 & 3.6 \\
\hline MIN & 3.2 & 3.2 & 3.2 & 3.2 & 3.2 & 4.9 & --- & --- & --- & 3.2 & 3.2 & 3.2 \\
\hline $\mathrm{AC}-\mathrm{FT}$ & 197 & 202 & 257 & 206 & 201 & 610 & --- & --- & --- & 198 & 197 & 191 \\
\hline
\end{tabular}


LOCATION.-Lat 4000'11", long 12057'12", in SE 1/4 NE 1/4 sec.27, T.25 N., R.9 E., Plumas County, Hydrologic Unit 18020122, on right bank $200 \mathrm{ft}$ upstream from Blackhawk Creek and 0.9 mi southeast of Keddie.

DRAINAGE AREA.-184 $\mathrm{mi}^{2}$.

PERIOD OF RECORD.-October 1933 to current year.

REVISED RECORDS.-WSP 1041: 1938(M).

GAGE.-Water-stage recorder. Datum of gage is 3,129.86 ft above sea level.

REMARKS.-Records good. Low flow regulated by five small reservoirs having a combined capacity of 800 acre-ft. Approximately 4,600 acres irrigated upstream from station (from information provided by U.S. Forest Service). City of Quincy diverts about 450 acre-ft annually for municipal supply. See schematic diagram of North Fork Feather River Basin.

EXTREMES FOR PERIOD OF RECORD.-Maximum discharge, 22,100 ft 3 /s, Jan. 2, 1997, gage height, $15.68 \mathrm{ft}$, from rating curve extended above $5,200 \mathrm{ft}^{3} / \mathrm{s}$ on basis of slope-area measurement of peak flow; minimum daily, $3.0 \mathrm{ft}^{3} / \mathrm{s}$, Sept. 4, 5, 1988 .

EXTREMES FOR CURRENT YEAR.-Peak discharges greater than base discharge of 1,700 $\mathrm{ft}^{3} / \mathrm{s}$, or maximum:

$\begin{array}{lccccrrr}\text { Date } & \text { Time } & \begin{array}{c}\text { Discharge } \\ \left(\mathrm{ft}^{3} / \mathrm{s}\right)\end{array} & \begin{array}{c}\text { Gage height } \\ (\mathrm{ft})\end{array} & \text { Date } & \text { Time } & \begin{array}{r}\text { Discharge } \\ \left(\mathrm{ft}^{3} / \mathrm{s}\right)\end{array} & \begin{array}{r}\text { Gage height } \\ (\mathrm{ft})\end{array} \\ \text { Nov. 30 } & 1900 & 3,170 & 6.43 & \text { Feb. } 9 & 1115 & 8,340 & \mathrm{a} 10.08 \\ \text { Jan. 20 } & 2015 & 2,750 & 6.05 & \text { Feb. } 28 & 2315 & 1,970\end{array}$

a From high-water mark in well.

DISCHARGE, CUBIC FEET PER SECOND, WATER YEAR OCTOBER 1998 TO SEPTEMBER 1999 DAILY MEAN VALUES

\begin{tabular}{|c|c|c|c|c|c|c|c|c|c|c|c|c|}
\hline DAY & $\mathrm{OCT}$ & NOV & $\mathrm{DEC}$ & JAN & $\mathrm{FEB}$ & MAR & APR & MAY & JUN & JUL & AUG & SEP \\
\hline 1 & 62 & 66 & 1260 & 124 & 265 & 1580 & 343 & 486 & 309 & 76 & 42 & 34 \\
\hline 2 & 61 & 65 & 560 & 119 & 245 & 1150 & 322 & 537 & 292 & 74 & 39 & 43 \\
\hline 3 & 66 & 64 & 1600 & 116 & 234 & 1600 & 310 & 492 & 294 & 72 & 36 & 44 \\
\hline 4 & 67 & 63 & 911 & 113 & 228 & 1190 & 291 & 444 & 290 & 78 & 31 & 47 \\
\hline 5 & 63 & 65 & 497 & 113 & 217 & 912 & 302 & 406 & 256 & 76 & 35 & 41 \\
\hline 6 & 58 & 69 & 373 & 112 & 262 & 756 & 299 & 424 & 246 & 67 & 40 & 35 \\
\hline 7 & 59 & 95 & 295 & 111 & 1710 & 643 & 297 & 489 & 235 & 60 & 48 & 39 \\
\hline 8 & 60 & 123 & 261 & 110 & 1700 & 569 & 340 & 471 & 220 & 64 & 44 & 30 \\
\hline 9 & 60 & 89 & 232 & 106 & 5390 & 556 & 325 & 436 & 205 & 62 & 43 & 30 \\
\hline 10 & 61 & 81 & 210 & 103 & 2040 & 473 & 316 & 410 & 201 & 56 & 36 & 39 \\
\hline 11 & 60 & 81 & 197 & 104 & 1180 & 430 & 357 & 398 & 191 & 63 & 57 & 32 \\
\hline 12 & 59 & 77 & 189 & 104 & 850 & 400 & 414 & 422 & 189 & 59 & 52 & 32 \\
\hline 13 & 56 & 76 & 182 & 104 & 675 & 386 & 450 & 441 & 184 & 52 & 47 & 39 \\
\hline 14 & 60 & 75 & 190 & 103 & 565 & 383 & 502 & 397 & 175 & 56 & 46 & 34 \\
\hline 15 & 60 & 74 & 178 & 122 & 473 & 374 & 514 & 362 & 168 & 50 & 46 & 40 \\
\hline 16 & 59 & 73 & 171 & 211 & 483 & 368 & 525 & 343 & 164 & 52 & 46 & 35 \\
\hline 17 & 59 & 124 & 168 & 218 & 1170 & 369 & 589 & 343 & 152 & 58 & 41 & 39 \\
\hline 18 & 59 & 109 & 166 & 992 & 1060 & 387 & 684 & 350 & 140 & 57 & 30 & 36 \\
\hline 19 & 57 & 87 & 164 & 1000 & 1040 & 406 & 713 & 366 & 137 & 53 & 30 & 38 \\
\hline 20 & 51 & 80 & 157 & 2040 & 794 & 414 & 732 & 375 & 136 & 45 & 35 & 37 \\
\hline 21 & 54 & 78 & 133 & 1540 & 761 & 390 & 719 & 376 & e132 & 43 & 37 & 35 \\
\hline 22 & 57 & 200 & 147 & 874 & 627 & 369 & 615 & 399 & 134 & 49 & 38 & 40 \\
\hline 23 & 61 & 635 & 136 & 1960 & 579 & 358 & 537 & 432 & 117 & 52 & 37 & 39 \\
\hline 24 & 68 & 609 & 156 & 1130 & 553 & 359 & 535 & 450 & 118 & 48 & 36 & 35 \\
\hline 25 & 89 & 251 & 141 & 758 & 934 & 422 & 573 & 481 & 110 & 48 & 34 & 34 \\
\hline 26 & 71 & 180 & 141 & 575 & 727 & 431 & 694 & 481 & 112 & 48 & 30 & 35 \\
\hline 27 & 66 & 411 & 139 & 444 & 603 & 444 & 667 & 449 & 106 & 44 & 35 & 40 \\
\hline 28 & 68 & 336 & 137 & 375 & 1030 & 403 & 571 & 409 & 96 & 48 & 41 & 33 \\
\hline 29 & 65 & 290 & 132 & 334 & --- & 376 & 473 & 377 & 90 & 42 & 43 & 38 \\
\hline 30 & 65 & 1630 & 128 & 303 & --- & 375 & 441 & 359 & 85 & 42 & 37 & 36 \\
\hline 31 & 64 & --- & 128 & 288 & --- & 369 & --- & 337 & --- & 43 & 30 & --- \\
\hline TOTAL & 1925 & 6256 & 9479 & 14706 & 26395 & 17642 & 14450 & 12942 & 5284 & 1737 & 1222 & 1109 \\
\hline MEAN & 62.1 & 209 & 306 & 474 & 943 & 569 & 482 & 417 & 176 & 56.0 & 39.4 & 37.0 \\
\hline MAX & 89 & 1630 & 1600 & 2040 & 5390 & 1600 & 732 & 537 & 309 & 78 & 57 & 47 \\
\hline MIN & 51 & 63 & 128 & 103 & 217 & 358 & 291 & 337 & 85 & 42 & 30 & 30 \\
\hline $\mathrm{AC}-\mathrm{FT}$ & 3820 & 12410 & 18800 & 29170 & 52350 & 34990 & 28660 & 25670 & 10480 & 3450 & 2420 & 2200 \\
\hline
\end{tabular}




\section{SPANISH CREEK ABOVE BLACKHAWK CREEK, AT KEDDIE, CA—Continued}

STATISTICS OF MONTHLY MEAN DATA FOR WATER YEARS 1934 - 1999, BY WATER YEAR (WY)

\begin{tabular}{|c|c|c|c|c|c|c|c|c|c|c|c|c|}
\hline MEAN & 58.8 & 131 & 288 & 447 & 527 & 568 & 566 & 433 & 176 & 53.2 & 29.2 & 30.9 \\
\hline MAX & 702 & 1015 & 1498 & 2657 & 2843 & 2043 & 1715 & 1301 & 755 & 187 & 74.6 & 63.8 \\
\hline (WY) & 1963 & 1982 & 1956 & 1997 & 1986 & 1995 & 1952 & 1938 & 1983 & 1983 & 1983 & 1983 \\
\hline MIN & 18.4 & 34.9 & 35.3 & 37.5 & 50.5 & 56.1 & 44.3 & 50.6 & 18.6 & 10.8 & 5.10 & 7.57 \\
\hline (WY) & 1989 & 1991 & 1977 & 1937 & 1991 & 1977 & 1977 & 1977 & 1977 & 1934 & 1934 & 1934 \\
\hline
\end{tabular}

SUMMARY STATISTICS

ANNUAL TOTAL

ANNUAL MEAN

HIGHEST ANNUAL MEAN

LOWEST ANNUAL MEAN

HIGHEST DAILY MEAN

LOWEST DAILY MEAN

ANNUAL SEVEN-DAY MINIMUM

INSTANTANEOUS PEAK FLOW

INSTANTANEOUS PEAK STAGE

ANNUAL RUNOFF (AC-FT)

10 PERCENT EXCEEDS

50 PERCENT EXCEEDS

90 PERCENT EXCEEDS
FOR 1998 CALENDAR YEAR

$\begin{array}{rrr}169823 & & \\ 465 & & \\ & & \\ 4510 & \text { Feb } & 3 \\ 41 & \text { Sep } 15 \\ 46 & \text { Sep } 14 \\ & & \\ & & \\ 336800 & & \\ 1000 & \\ 323 & & \\ 54 & \end{array}$

FOR 1999 WATER YEAR

$\begin{array}{rrr}113147 & & \\ 310 & & \\ & & \\ 5390 & \text { Feb } & 9 \\ 30 & \text { Aug } & 18 \\ 34 & \text { Sep } & 8 \\ 8340 & \text { Feb } & 9 \\ 10.08 & \text { Feb } & 9 \\ 224400 & & \\ 688 & & \\ 152 & & \\ 39 & & \end{array}$

WATER YEARS 1934 - 1999

\begin{tabular}{|c|c|c|c|}
\hline 274 & & & \\
\hline 641 & & & 1995 \\
\hline 34.1 & & & 1977 \\
\hline 18000 & Jan & 2 & 1997 \\
\hline 3.0 & Sep & 4 & 1988 \\
\hline 4.4 & Aug 1 & 18 & 1934 \\
\hline 22100 & Jan & 2 & 1997 \\
\hline 15.68 & Jan & 2 & 1997 \\
\hline 98800 & & & \\
\hline 656 & & & \\
\hline 90 & & & \\
\hline 24 & & & \\
\hline
\end{tabular}


11403200 NORTH FORK FEATHER RIVER BELOW ROCK CREEK DIVERSION DAM, CA

LOCATION.-Lat 3958'49", long 121¹6’33", in SW 1/4 NW 1/4 sec.35, T.25 N., R.6 E., Plumas County, Hydrologic Unit 18020121, Plumas National Forest, on left bank 0.7 mi downstream from Rock Creek Diversion Dam and 5.0 mi northeast of Storrie.

DRAINAGE AREA.- $-1,773 \mathrm{mi}^{2}$.

PERIOD OF RECORD.-October 1985 to February 1986, October 1986 to current year. Unpublished records for water years $1982-85$ available in files of the U.S. Geological Survey.

GAGE.-Water-stage recorder. Elevation of gage is 2,120 ft above sea level, from topographic map.

REMARKS.-Low and medium flow regulated by Rock Creek Forebay 0.7 mi upstream. Most of the flow is diverted to Rock Creek Powerplant (station 11403800). Diversion to Rock Creek Powerplant began Feb. 28, 1950. See schematic diagram of North Fork Feather River Basin.

COOPERATION.-Records were collected by Pacific Gas \& Electric Co., under general supervision of the U.S. Geological Survey, in connection with a Federal Energy Regulatory Commission project.

EXTREMES FOR PERIOD OF RECORD._Maximum discharge, 91,600 ft 3 /s, Jan. 2, 1997, gage height, $31.85 \mathrm{ft}$; minimum daily, $50 \mathrm{ft}{ }^{3} / \mathrm{s}$, Feb. 7 , 1989.

DISCHARGE, CUBIC FEET PER SECOND, WATER YEAR OCTOBER 1998 TO SEPTEMBER 1999 DAILY MEAN VALUES

\begin{tabular}{|c|c|c|c|c|c|c|c|c|c|c|c|c|}
\hline DAY & $\mathrm{OCT}$ & NOV & $\mathrm{DEC}$ & JAN & FEB & MAR & $\mathrm{APR}$ & MAY & JUN & JUL & AUG & SEP \\
\hline 1 & 146 & 146 & 3420 & 83 & 84 & 3270 & 831 & 794 & 174 & 132 & 125 & 164 \\
\hline 2 & 146 & 118 & 1220 & 81 & 82 & 2490 & 96 & 889 & 175 & 139 & 125 & 164 \\
\hline 3 & 155 & 80 & 4210 & 84 & 82 & 3730 & 93 & 788 & 168 & 140 & 128 & 170 \\
\hline 4 & 155 & 81 & 3200 & 82 & 81 & 3160 & 97 & 406 & 168 & 137 & 131 & 245 \\
\hline 5 & 149 & 81 & 1670 & 82 & 80 & 1830 & 93 & 236 & 446 & 132 & 131 & 209 \\
\hline 6 & 142 & 79 & 1110 & 85 & 80 & 1120 & 92 & 233 & 953 & 130 & 133 & 170 \\
\hline 7 & 145 & 81 & 710 & 85 & 1650 & 578 & 93 & 223 & 493 & 131 & 129 & 151 \\
\hline 8 & 146 & 81 & 506 & 84 & 4610 & 231 & 98 & 218 & 230 & 131 & 130 & 165 \\
\hline 9 & 150 & 79 & 235 & 84 & 15400 & 193 & 95 & 203 & 143 & 135 & 129 & 175 \\
\hline 10 & 145 & 76 & 90 & 82 & 9220 & 128 & 90 & 189 & 148 & 132 & 130 & 188 \\
\hline 11 & 149 & 74 & 93 & 81 & 5150 & 70 & 89 & 176 & 145 & 124 & 125 & 191 \\
\hline 12 & 149 & 72 & 95 & 81 & 3650 & 140 & 97 & 186 & 152 & 131 & 122 & 176 \\
\hline 13 & 148 & 73 & 93 & 83 & 2050 & 211 & 96 & 186 & 152 & 132 & 120 & 180 \\
\hline 14 & 151 & 73 & 88 & 80 & 121 & 212 & 94 & 176 & 152 & 136 & 124 & 170 \\
\hline 15 & 158 & 76 & 87 & 82 & 72 & 176 & 84 & 179 & 151 & 135 & 120 & 170 \\
\hline 16 & 150 & 74 & 88 & 84 & 767 & 153 & 95 & 170 & 143 & 134 & 123 & 149 \\
\hline 17 & 160 & 83 & 86 & 86 & 2040 & 154 & 133 & 172 & 149 & 135 & 121 & 184 \\
\hline 18 & 151 & 78 & 84 & 90 & 2590 & 152 & 680 & 181 & 148 & 126 & 120 & 194 \\
\hline 19 & 161 & 77 & 87 & 86 & 2830 & 166 & 1060 & 182 & 147 & 128 & 122 & 180 \\
\hline 20 & 155 & 77 & 83 & 2440 & 2120 & 164 & 1170 & 177 & 139 & 132 & 119 & 196 \\
\hline 21 & 140 & 75 & 82 & 2880 & 1940 & 165 & 1680 & 179 & 146 & 131 & 123 & 226 \\
\hline 22 & 127 & 80 & 81 & 457 & 1500 & 157 & 1340 & 185 & 148 & 124 & 120 & 246 \\
\hline 23 & 138 & 860 & 82 & 2740 & 1390 & 138 & 1130 & 179 & 146 & 131 & 125 & 502 \\
\hline 24 & 178 & 528 & 85 & 1440 & 1460 & 126 & 1030 & 177 & 146 & 130 & 120 & 311 \\
\hline 25 & 175 & 391 & 84 & 225 & 2360 & 124 & 1070 & 181 & 141 & 130 & 118 & 181 \\
\hline 26 & 170 & 435 & 84 & 88 & 1470 & 109 & 2800 & 190 & 147 & 128 & 122 & 181 \\
\hline 27 & 150 & 291 & 84 & 86 & 667 & 380 & 3060 & 190 & 144 & 129 & 114 & 178 \\
\hline 28 & 138 & 80 & 84 & 84 & 815 & 397 & 1160 & 174 & 141 & 127 & 107 & 179 \\
\hline 29 & 134 & 81 & 84 & 86 & --- & 774 & 885 & 168 & 137 & 129 & 115 & 188 \\
\hline 30 & 158 & 2290 & 84 & 85 & --- & 1360 & 687 & 183 & 127 & 125 & 307 & 198 \\
\hline 31 & 156 & --- & 83 & 84 & --- & 1210 & --- & 179 & --- & 125 & 607 & --- \\
\hline TOTAL & 4675 & 6770 & 18172 & 12280 & 64361 & 23268 & 20118 & 7949 & 5999 & 4061 & 4485 & 5981 \\
\hline MEAN & 151 & 226 & 586 & 396 & 2299 & 751 & 671 & 256 & 200 & 131 & 145 & 199 \\
\hline MAX & 178 & 2290 & 4210 & 2880 & 15400 & 3730 & 3060 & 889 & 953 & 140 & 607 & 502 \\
\hline MIN & 127 & 72 & 81 & 80 & 72 & 70 & 84 & 168 & 127 & 124 & 107 & 149 \\
\hline $\mathrm{AC}-\mathrm{FT}$ & 9270 & 13430 & 36040 & 24360 & 127700 & 46150 & 39900 & 15770 & 11900 & 8050 & 8900 & 11860 \\
\hline $\mathrm{a}$ & 91450 & 154000 & 182100 & 155800 & 147000 & 161800 & 156900 & 173000 & 115100 & 107900 & 117800 & 64940 \\
\hline
\end{tabular}

a Diversion, in acre-feet, to Rock Creek Powerplant, provided by Pacific Gas \& Electric Co. 


\section{NORTH FORK FEATHER RIVER BELOW ROCK CREEK DIVERSION DAM, CA—Continued}

STATISTICS OF MONTHLY MEAN DATA FOR WATER YEARS 1987 - 1999, BY WATER YEAR (WY)

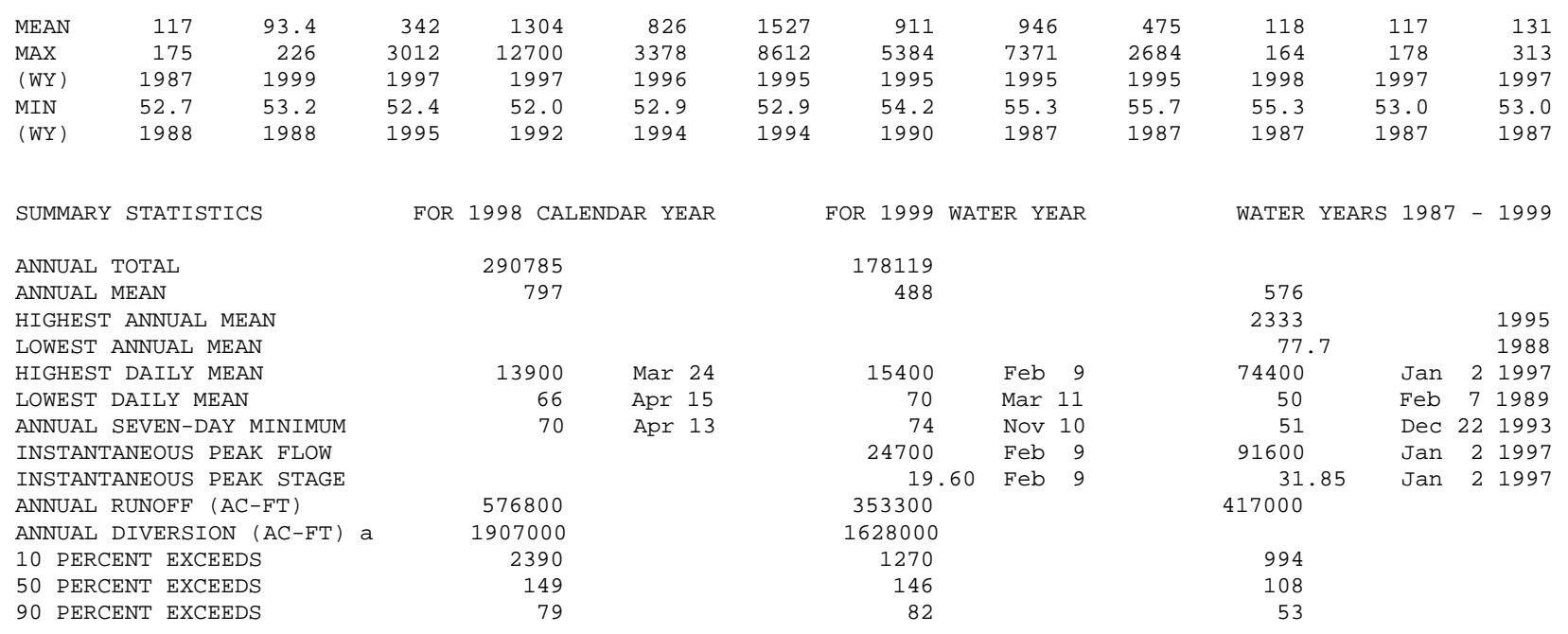

a Diversion, in acre-feet, to Rock Creek Powerplant, provided by Pacific Gas \& Electric Co. 
11403450 MILK RANCH CONDUIT AT OUTLET, NEAR BUCKS LODGE, CA

LOCATION.-Lat 3954'09", long 121¹3’36", in SW 1/4 SW 1/4 sec.29, T.24 N., R.7 E., Plumas County, Hydrologic Unit 18020121, Plumas National Forest, on left bank $150 \mathrm{ft}$ upstream from right abutment of Lower Bucks Lake Dam, $200 \mathrm{ft}$ upstream from outlet, and 3.4 mi northwest of Bucks Lodge.

PERIOD OF RECORD.-October 1986 to current year. Unpublished records for water years 1981-84 available in files of the U.S. Geological Survey.

GAGE.-Water-stage recorder in 3-ft steel pipe. Elevation of gage is 5,050 ft above sea level.

REMARKS.-Conduit diverts from channel below Three Lakes Reservoir, capacity, 513 acre-ft, and from 12 additional diversions along the conduit. Water is used for power at Bucks Creek Powerplant (station 11403700) and Grizzly Powerplant (station 11404240). See schematic diagram of North Fork Feather River Basin.

COOPERATION.--Records were collected by Pacific Gas \& Electric Co., under general supervision of the U.S. Geological Survey, in connection with a Federal Energy Regulatory Commission project.

EXTREMES FOR PERIOD OF RECORD.-Maximum daily discharge, $71 \mathrm{ft}^{3}$ /s, Apr. 29, 1995, May 17, 1996; minimum daily, no flow for many days in the 1997,1998 , and 1999 water years.

DISCHARGE, CUBIC FEET PER SECOND, WATER YEAR OCTOBER 1998 TO SEPTEMBER 1999

DAILY MEAN VALUES

\begin{tabular}{|c|c|c|c|c|c|c|c|c|c|c|c|c|}
\hline DAY & OCT & NOV & $\mathrm{DEC}$ & JAN & FEB & MAR & APR & MAY & JUN & JUL & AUG & SEP \\
\hline 1 & .00 & 9.6 & .02 & .00 & .00 & .00 & .00 & .00 & .00 & .00 & .00 & .00 \\
\hline 2 & .00 & 9.4 & .00 & .00 & .00 & .00 & .00 & .00 & .00 & .00 & .00 & .00 \\
\hline 3 & .00 & 9.5 & .00 & .00 & .00 & .00 & .00 & .00 & .00 & .00 & .00 & .00 \\
\hline 4 & .00 & 9.6 & .00 & .00 & .00 & .00 & .00 & .00 & .00 & .00 & .00 & .00 \\
\hline 5 & .00 & 9.6 & .00 & .00 & .00 & .00 & .00 & .00 & .00 & .00 & .00 & .00 \\
\hline 6 & .00 & 9.4 & .00 & .00 & .00 & .00 & .00 & .00 & .00 & .00 & .00 & .00 \\
\hline 7 & .00 & 10 & .00 & .00 & .00 & .00 & .00 & .00 & .00 & .00 & .00 & .00 \\
\hline 8 & .00 & 10 & .00 & .00 & .00 & .00 & .00 & .00 & .00 & .00 & .00 & .00 \\
\hline 9 & .00 & 9.6 & .00 & .00 & .00 & .00 & .00 & .00 & .00 & .00 & .00 & .00 \\
\hline 10 & .00 & 9.4 & .00 & .00 & .00 & .00 & .00 & .00 & .00 & .00 & .00 & .00 \\
\hline 11 & .00 & 9.3 & .00 & .00 & .00 & .00 & .00 & .00 & .00 & .00 & .00 & .00 \\
\hline 12 & .00 & 9.3 & .00 & .00 & .00 & .00 & .00 & .00 & .00 & .00 & .00 & .00 \\
\hline 13 & .00 & 9.3 & .00 & .00 & .00 & .00 & .00 & .00 & .00 & .00 & .00 & .00 \\
\hline 14 & .00 & 9.2 & .00 & .00 & .00 & .00 & .00 & .00 & .00 & .00 & .00 & .00 \\
\hline 15 & .00 & 9.1 & .00 & .00 & .00 & .00 & .00 & .00 & .00 & .00 & .00 & .00 \\
\hline 16 & .00 & 9.2 & .00 & .00 & .00 & .00 & .00 & .00 & .00 & .00 & .00 & .00 \\
\hline 17 & .00 & 9.7 & .00 & .00 & .00 & .00 & .00 & .00 & .00 & .00 & .00 & .00 \\
\hline 18 & .00 & 9.0 & .00 & .00 & .00 & .00 & .00 & .00 & .00 & .00 & .00 & .00 \\
\hline 19 & .00 & 8.9 & .00 & .00 & .00 & .00 & .00 & .00 & .00 & .00 & .00 & .00 \\
\hline 20 & .00 & 8.7 & .00 & .00 & .00 & .00 & .00 & .00 & .00 & .00 & .00 & .00 \\
\hline 21 & .00 & 11 & .00 & .00 & .00 & .00 & .00 & .00 & .00 & .00 & .00 & .00 \\
\hline 22 & .00 & 13 & .00 & .00 & .00 & .00 & .00 & .00 & .00 & .00 & .00 & .00 \\
\hline 23 & .00 & 24 & .00 & .00 & .00 & .00 & .00 & .00 & .00 & .00 & .00 & .00 \\
\hline 24 & .00 & .02 & .00 & .00 & .00 & .00 & .00 & .00 & .00 & .00 & .00 & .00 \\
\hline 25 & .00 & .00 & .00 & .00 & .00 & .00 & .00 & .00 & .00 & .00 & .00 & .00 \\
\hline 26 & .00 & .00 & .00 & .00 & .00 & .00 & .00 & .00 & .00 & .00 & .00 & .00 \\
\hline 27 & .00 & .02 & .00 & .00 & .00 & .00 & .00 & .00 & .00 & .00 & .00 & .00 \\
\hline 28 & .10 & .02 & .00 & .00 & .00 & .00 & .00 & .00 & .00 & .00 & .00 & .00 \\
\hline 29 & 3.1 & .01 & .00 & .00 & --- & .00 & .00 & .00 & .00 & .00 & .00 & .00 \\
\hline 30 & 9.7 & .02 & .00 & .00 & --- & .00 & .00 & .00 & .00 & .00 & .00 & .00 \\
\hline 31 & 9.6 & --- & .00 & .00 & --- & .00 & --- & .00 & --- & .00 & .00 & --- \\
\hline TOTAL & 22.50 & 235.89 & 0.02 & 0.00 & 0.00 & 0.00 & 0.00 & 0.00 & 0.00 & 0.00 & 0.00 & 0.00 \\
\hline MEAN & .73 & 7.86 & .001 & .000 & .000 & .000 & .000 & .000 & .000 & .000 & .000 & .000 \\
\hline MAX & 9.7 & 24 & .02 & .00 & .00 & .00 & .00 & .00 & .00 & .00 & .00 & .00 \\
\hline MIN & .00 & .00 & .00 & .00 & .00 & .00 & .00 & .00 & .00 & .00 & .00 & .00 \\
\hline $\mathrm{AC}-\mathrm{FT}$ & 45 & 468 & .04 & .00 & .00 & .00 & .00 & .00 & .00 & .00 & .00 & .00 \\
\hline
\end{tabular}

STATISTICS OF MONTHLY MEAN DATA FOR WATER YEARS 1987 - 1999, BY WATER YEAR (WY)

\begin{tabular}{|c|c|c|c|c|c|c|c|c|c|c|c|c|}
\hline MEAN & 3.08 & 4.01 & 6.19 & 5.76 & 8.99 & 16.5 & 26.9 & 28.1 & 14.3 & 6.14 & 2.97 & 2.96 \\
\hline MAX & 6.96 & 8.15 & 27.5 & 19.2 & 38.7 & 42.7 & 59.6 & 66.6 & 57.3 & 30.5 & 7.35 & 6.82 \\
\hline (WY) & 1994 & 1990 & 1997 & 1995 & 1996 & 1989 & 1989 & 1993 & 1993 & 1995 & 1992 & 1990 \\
\hline MIN & .000 & .000 & .000 & .000 & .000 & .000 & .000 & .000 & .000 & .000 & .000 & 0 \\
\hline (WY) & 1998 & 1998 & 1998 & 1998 & 1997 & 1997 & 1997 & 1997 & 1997 & 1997 & 1997 & 19 \\
\hline
\end{tabular}

SUMMARY STATISTICS

ANNUAL TOTAL

ANNUAL MEAN

HIGHEST ANNUAL MEAN

LOWEST ANNUAL MEAN

HIGHEST DAILY MEAN

LOWEST DAILY MEAN

ANNUAL SEVEN-DAY MINIMUM

ANNUAL RUNOFF (AC-FT)

10 PERCENT EXCEEDS

50 PERCENT EXCEEDS

90 PERCENT EXCEEDS
FOR 1998 CALENDAR YEAR

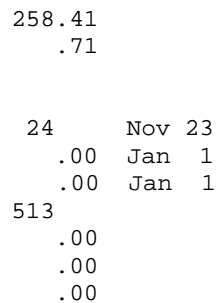

FOR 1999 WATER YEAR

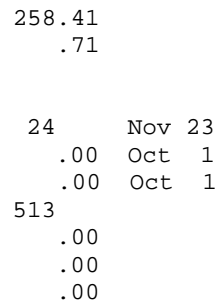

WATER YEARS 1987 - 1999

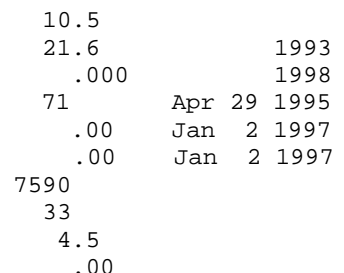


LOCATION.-Lat 3953'45", long 12112'08", in SE 1/4 NW 1/4 sec.33, T.24 N., R.7 E., Plumas County, Hydrologic Unit 18020121, Plumas National Forest, in outlet structure $100 \mathrm{ft}$ upstream from dam on Bucks Creek, 2.0 mi northwest of Bucks Lodge, and 15 mi west of Quincy. DRAINAGE AREA.- $-28.6 \mathrm{mi}^{2}$.

PERIOD OF RECORD.-1927-28 (year-end contents only, published in WSP 1315-A), October 1928 to current year. Prior to October 1954, published as Bucks Creek Reservoir near Bucks Ranch.

GAGE.-Water-stage recorder. Datum of gage is $3.50 \mathrm{ft}$ below sea level (levels by Feather River Power Co.).

REMARKS.--Reservoir is formed by concrete-faced, rockfill dam, completed in 1927; storage began in May 1927. Capacity, 101,400 acre-ft between elevations $5,064.75 \mathrm{ft}$, sill of outlet gate, and 5,154.85 ft, spillway crest. Storage of 274 acre- $\mathrm{ft}$ is not available for release. Released water flows down Bucks Creek to Lower Bucks Lake (station 11403520), where most of the water is diverted to Bucks Creek Tunnel or Grizzly Powerplant (station 11304240), which discharges into Grizzly Creek. Figures given, including extremes, represent total contents at 2400 hours. See schematic diagram of North Fork Feather River Basin.

COOPERATION.-Records were collected by Pacific Gas \& Electric Co., under general supervision of the U.S. Geological Survey, in connection with a Federal Energy Regulatory Commission project. Contents not rounded to U.S. Geological Survey standards.

EXTREMES FOR PERIOD OF RECORD.-Maximum contents, 107,278 acre-ft, May 17, 1996, elevation, 5,157.9 ft; minimum, 12,330 acre-ft, Feb. 27, 1929, elevation, 5,090.7 ft.

EXTREMES FOR CURRENT YEAR.-Maximum contents, 100,833 acre-ft, July 2, 3, elevation, 5,154.4 ft; minimum, 49,814 acre-ft, Jan. 16, elevation, $5,123.1 \mathrm{ft}$

Capacity table (elevation, in feet, and contents, in acre-feet)

(Based on survey by Feather River Power Co. in 1927)

$\begin{array}{llrr}5,090 & 11,742 & 5,130 & 59,997 \\ 5,095 & 16,183 & 5,140 & 75,894 \\ 5,100 & 21,180 & 5,150 & 92,950 \\ 5,110 & 32,519 & 5,160 & 111,220\end{array}$

$5,120 \quad 45,472$

RESERVOIR STORAGE (ACRE-FEET), WATER YEAR OCTOBER 1998 TO SEPTEMBER 1999 DAILY OBSERVATION AT 2400 HOURS

\begin{tabular}{|c|c|c|c|c|c|c|c|c|c|c|c|c|}
\hline DAY & $\mathrm{OCT}$ & NOV & $\mathrm{DEC}$ & JAN & FEB & MAR & $A P R$ & MAY & JUN & JUL & AUG & SEP \\
\hline 1 & 74580 & 61994 & 58782 & 54007 & 55483 & 63233 & 64952 & 71001 & 91719 & 100651 & 89795 & 75730 \\
\hline 2 & 74089 & 61530 & 59238 & 53714 & 55632 & 63857 & 64637 & 71649 & 92071 & 100833 & 89273 & 75730 \\
\hline 3 & 73763 & 61223 & 60917 & 53422 & 55632 & 64481 & 64481 & 72297 & 92598 & 100833 & 88752 & 75730 \\
\hline 4 & 73273 & 60764 & 61223 & 53130 & 55483 & 64794 & 64481 & 72784 & 93127 & 100651 & 88752 & 75730 \\
\hline 5 & 72947 & 60304 & 61070 & 52691 & 55188 & 65109 & 64325 & 73273 & 93658 & 100287 & 87885 & 75730 \\
\hline 6 & 72459 & 59997 & 60917 & 52401 & 55335 & 65109 & 64169 & 73763 & 94189 & 99743 & 87367 & 75730 \\
\hline 7 & 71973 & 59997 & 60610 & 51967 & 56079 & 65266 & 63857 & 74416 & 94720 & 99382 & 86851 & 75730 \\
\hline 8 & 71487 & 59845 & 60304 & 51678 & 56973 & 65581 & 64013 & 74909 & 95076 & 99201 & 86334 & 75730 \\
\hline 9 & 71001 & 59541 & 59997 & 51389 & 58328 & 65581 & 63857 & 75566 & 95433 & 99020 & 85818 & 75730 \\
\hline 10 & 70679 & 58934 & 59845 & 51100 & 58630 & 65738 & 63857 & 76059 & 95789 & 98659 & 85475 & 75894 \\
\hline 11 & 70197 & 58630 & 59541 & 51100 & 58934 & 65424 & 64013 & 76556 & 96324 & 98118 & 84962 & 75730 \\
\hline 12 & 69714 & 58177 & 59238 & 50814 & 59238 & 65424 & 63857 & 77217 & 96682 & 97759 & 84619 & 75730 \\
\hline 13 & 69232 & 57726 & 58934 & 50386 & 59238 & 65266 & 63545 & 77881 & 97041 & 97220 & 84107 & 75894 \\
\hline 14 & 68913 & 57425 & 58934 & 50100 & 59541 & 65266 & 63545 & 78381 & 97400 & 96861 & 83597 & 75730 \\
\hline 15 & 68434 & 56973 & 58782 & 49957 & 59693 & 65424 & 63545 & 78880 & 97759 & 96324 & 83087 & 75894 \\
\hline 16 & 67955 & 56675 & 58782 & 49814 & 60304 & 65266 & 63701 & 79548 & 97938 & 96324 & 82577 & 75894 \\
\hline 17 & 67478 & 56526 & 58478 & 50386 & 60610 & 65266 & 64013 & 80051 & 98297 & 95433 & 82239 & 75730 \\
\hline 18 & 67003 & 56079 & 58177 & 51389 & 61070 & 65266 & 64481 & 80721 & 98478 & 94898 & 81733 & 75894 \\
\hline 19 & 66527 & 56632 & 57726 & 51823 & 61223 & 65109 & 64952 & 81227 & 98659 & 94366 & 81227 & 75894 \\
\hline 20 & 66053 & 55335 & 57726 & 52837 & 61685 & 65109 & 65424 & 81902 & 98839 & 94012 & 80721 & 75894 \\
\hline 21 & 65738 & 55188 & 57565 & 53130 & 61994 & 65109 & 65895 & 82747 & 99201 & 93825 & 80386 & 75894 \\
\hline 22 & 65266 & 55040 & 57124 & 53714 & 62149 & 64952 & 66210 & 83427 & 99382 & 93481 & 79883 & 75894 \\
\hline 23 & 64794 & 56377 & 56824 & 54450 & 61839 & 64794 & 66686 & 84277 & 99563 & 93304 & 79381 & 75894 \\
\hline 24 & 64637 & 56824 & 56526 & 54893 & 61839 & 64637 & 67161 & 85304 & 99743 & 93127 & 78880 & 75894 \\
\hline 25 & 64325 & 56675 & 56228 & 54893 & 61839 & 64637 & 67795 & 86334 & 99924 & 92774 & 78381 & 75894 \\
\hline 26 & 64325 & 56526 & 55930 & 54745 & 61530 & 64637 & 68434 & 87195 & 100105 & 92423 & 78048 & 75894 \\
\hline 27 & 64013 & 56675 & 55632 & 54745 & 61685 & 64794 & 69073 & 88059 & 100105 & 91895 & 77548 & 75566 \\
\hline 28 & 63701 & 56675 & 55483 & 54893 & 62613 & 64952 & 69553 & 89099 & 100469 & 91719 & 77052 & 75566 \\
\hline 29 & 63233 & 56526 & 55188 & 55188 & --- & 64794 & 69875 & 89795 & 100469 & 91192 & 76556 & 75401 \\
\hline 30 & 62922 & 58328 & 54745 & 55335 & --- & 65109 & 70357 & 90668 & 100651 & 90668 & 76059 & 74909 \\
\hline 31 & 62458 & --- & 54450 & 55483 & --- & 64952 & --- & 91368 & --- & 90319 & 75730 & --- \\
\hline MAX & 74580 & 61994 & 61223 & 55483 & 62613 & 65738 & 70357 & 91368 & 100651 & 100833 & 89795 & 75894 \\
\hline MIN & 62458 & 55040 & 54450 & 49814 & 55188 & 63233 & 63545 & 71001 & 91719 & 90319 & 75730 & 74909 \\
\hline$a$ & 5131.6 & 5128.9 & 5126.3 & 5127.0 & 5131.7 & 5133.2 & 5136.6 & 5149.1 & 5154.3 & 5148.5 & 5139.9 & 5139.4 \\
\hline $\mathrm{b}$ & -12615 & -4130 & -3878 & +1033 & +7130 & +2339 & +5405 & +21011 & +9283 & -10332 & -14589 & -821 \\
\hline
\end{tabular}

CAL YR 1998 MAX 104867 MIN 45611 b +8839

WTR YR 1999 MAX 100833 MIN 49814 b $\quad-164$ 
LOCATION.-Lat 3953'59", long 121¹3’32", in NE 1/4 NW 1/4 sec.32, T.24 N., R.7 E., Plumas County, Hydrologic Unit 18020121, Plumas National Forest, in outlet tower for Bucks Creek Tunnel $900 \mathrm{ft}$ upstream from Buck Diversion Dam, 1.3 mi downstream from Bucks Lake Dam, and 3.2 mi northwest of Bucks Lodge.

DRAINAGE AREA.- $-31.3 \mathrm{mi}^{2}$.

PERIOD OF RECORD.-October 1985 to current year. Unpublished records for water years 1981-85 available in files of the U.S. Geological Survey.

GAGE.-Water-stage recorder. Datum of gage is $3.50 \mathrm{ft}$ below sea level (levels by Pacific Gas \& Electric Co.).

REMARKS.-Lake is formed by concrete dam. Storage began in October 1929. Usable capacity, 5,796 acre-ft between elevations 4,952 ft, point of lowest drawdown, and 5,021.95 ft, crest of spillway. Water is received from Bucks Lake (station 11403500) and from Milk Ranch Conduit (station 11403450). Most of the water is diverted through Bucks Creek Tunnel or Grizzly Powerplant (station 11404240) and discharges into Grizzly Creek for power development downstream. Figures given, including extremes, represent total contents at 2400 hours. See schematic diagram of North Fork Feather River Basin.

COOPERATION.-Records were collected by Pacific Gas \& Electric Co., under general supervision of the U.S. Geological Survey, in connection with a Federal Energy Regulatory Commission project. Contents not rounded to U.S. Geological Survey standards.

EXTREMES FOR PERIOD OF RECORD.-Maximum contents, 6,203 acre-ft, May 18, 1996, elevation, 5,024.6 ft; minimum, 99 acre-ft, Sept. 9, 1993, elevation, 4,956.1 ft.

EXTREMES FOR CURRENT YEAR.-Maximum contents, 5,414 acre-ft, July 20, elevation, 5,018.8 ft; minimum, 3,524 acre-ft, July 3, elevation, $5,003.2 \mathrm{ft}$.

Capacity table (elevation, in feet, and contents, in acre-feet)

(Based on survey by Feather River Power Co. in 1928)

$\begin{array}{lrll}4,950 & 24 & 5,000 & 3,175 \\ 4,960 & 194 & 5,010 & 4,307 \\ 4,970 & 624 & 5,020 & 5,573 \\ 4,980 & 1,314 & 5,030 & 6,981 \\ 4,990 & 2,171 & & \end{array}$

RESERVOIR STORAGE (ACRE-FEET), WATER YEAR OCTOBER 1998 TO SEPTEMBER 1999 DAILY OBSERVATION AT 2400 HOURS

\begin{tabular}{|c|c|c|c|c|c|c|c|c|c|c|c|c|}
\hline DAY & OCT & NOV & $\mathrm{DEC}$ & JAN & FEB & MAR & APR & MAY & JUN & JUL & AUG & SEP \\
\hline 1 & 4118 & 4001 & 4785 & 4355 & 4660 & 4710 & 4404 & 3805 & 3817 & 3579 & 4685 & 5166 \\
\hline 2 & 4036 & 3931 & 4562 & 4319 & 4673 & 4735 & 4513 & 3828 & 4071 & 3557 & 4673 & 5166 \\
\hline 3 & 3955 & 3989 & 4648 & 4452 & 4319 & 4685 & 4428 & 3862 & 4071 & 3524 & 4501 & 5166 \\
\hline 4 & 3955 & 4071 & 4428 & 4428 & 4200 & 4598 & 4440 & 3862 & 4048 & 3897 & 4464 & 5153 \\
\hline 5 & 3920 & 4094 & 4452 & 4392 & 4379 & 4464 & 4404 & 3862 & 4048 & 4236 & 4488 & 5153 \\
\hline 6 & 3908 & 4416 & 4379 & 4355 & 4685 & 4537 & 4355 & 3885 & 4048 & 4525 & 4623 & 5140 \\
\hline 7 & 3897 & 4513 & 4296 & 4260 & 4735 & 4416 & 4525 & 3908 & 4036 & 4860 & 4697 & 5127 \\
\hline 8 & 3851 & 4440 & 4200 & 4319 & 4773 & 4452 & 4416 & 3920 & 4024 & 4611 & 4623 & 5127 \\
\hline 9 & 3794 & 4355 & 4094 & 4392 & 4872 & 4501 & 4440 & 3931 & 4013 & 4488 & 4673 & 5114 \\
\hline 10 & 3943 & 4392 & 3989 & 4635 & 4885 & 4343 & 4513 & 3943 & 3760 & 4537 & 4697 & 5114 \\
\hline 11 & 3943 & 4416 & 3874 & 4379 & 4885 & 4488 & 4379 & 3966 & 3760 & 4697 & 4574 & 5101 \\
\hline 12 & 3978 & 4189 & 4153 & 4319 & 4440 & 4440 & 4452 & 3989 & 3748 & 4660 & 4598 & 5101 \\
\hline 13 & 3931 & 4094 & 4598 & 4549 & 5025 & 4598 & 4404 & 4013 & 3737 & 4722 & 4452 & 5089 \\
\hline 14 & 3897 & 4001 & 4525 & 4331 & 5037 & 4476 & 4513 & 4024 & 3680 & 4673 & 4367 & 5089 \\
\hline 15 & 3874 & 3828 & 4586 & 4284 & 5037 & 4343 & 4367 & 3966 & 3658 & 4697 & 4284 & 5076 \\
\hline 16 & 3828 & 3726 & 4392 & 4611 & 5050 & 4501 & 4404 & 3931 & 3658 & 4885 & 4260 & 5063 \\
\hline 17 & 3680 & 3658 & 4367 & 4476 & 4999 & 4440 & 4416 & 3862 & 3647 & 4986 & 4200 & 5063 \\
\hline 18 & 3851 & 3613 & 4331 & 4476 & 5025 & 4319 & 4428 & 3874 & 3726 & 5114 & 4212 & 5050 \\
\hline 19 & 3714 & 3591 & 4513 & 4598 & 4923 & 4392 & 4440 & 3839 & 3714 & 5231 & 4106 & 5050 \\
\hline 20 & 3636 & 3591 & 4488 & 4598 & 4722 & 4416 & 4452 & 3760 & 3692 & 5414 & 3978 & 5037 \\
\hline 21 & 3579 & 3874 & 4236 & 4611 & 4452 & 4307 & 4464 & 3760 & 3680 & 5153 & 4106 & 5037 \\
\hline 22 & 3613 & 4165 & 4272 & 4635 & 4319 & 4452 & 4379 & 3771 & 3680 & 5140 & 3989 & 5025 \\
\hline 23 & 3955 & 4673 & 4307 & 4673 & 4476 & 4488 & 4296 & 3771 & 3692 & 5166 & 4416 & 4999 \\
\hline 24 & 4404 & 4476 & 4392 & 4685 & 4440 & 4537 & 4236 & 3760 & 3703 & 4872 & 4537 & 4974 \\
\hline 25 & 4513 & 4367 & 4452 & 4537 & 4464 & 4428 & 4260 & 3624 & 3703 & 5089 & 4355 & 4948 \\
\hline 26 & 4416 & 4392 & 4501 & 4598 & 4623 & 4673 & 4212 & 3624 & 3703 & 4860 & 4212 & 4936 \\
\hline 27 & 4549 & 4549 & 4611 & 4489 & 4673 & 4549 & 4236 & 3624 & 3714 & 5025 & 4331 & 5114 \\
\hline 28 & 4319 & 4319 & 4464 & 4452 & 4735 & 4476 & 4249 & 3624 & 3714 & 4885 & 4118 & 4986 \\
\hline 29 & 4236 & 4612 & 4416 & 4452 & --- & 4574 & 3771 & 3624 & 3726 & 4697 & 4331 & 4936 \\
\hline 30 & 4141 & 4772 & 4392 & 4440 & --- & 4416 & 3782 & 3624 & 3726 & 4785 & 4797 & 5140 \\
\hline 31 & 4094 & --- & 4367 & 4440 & --- & 4537 & --- & 3591 & --- & 4722 & 5179 & --- \\
\hline MAX & 4549 & 4772 & 4785 & 4685 & 5050 & 4735 & 4525 & 4024 & 4071 & 5414 & 5179 & 5166 \\
\hline MIN & 3579 & 3591 & 3874 & 4260 & 4200 & 4307 & 3771 & 3591 & 3647 & 3524 & 3978 & 4936 \\
\hline$a$ & 5008.2 & 5013.8 & 5010.5 & 5011.1 & 5013.5 & 5011.9 & 5005.5 & 5003.8 & 5005.0 & 5013.4 & 5017.0 & 5016.7 \\
\hline $\mathrm{b}$ & -36 & +678 & -405 & +73 & +295 & -198 & -755 & -191 & +135 & +996 & +457 & -39 \\
\hline
\end{tabular}

CAL YR 1998 MAX 5884 MIN $3579 \quad$ b $\quad+273$ WTR YR 1999 MAX 5414 MIN 3524 b +1010

a Elevation, in feet, at end of month.

b Change in contents, in acre-feet. 
11403530 BUCKS CREEK BELOW DIVERSION DAM, NEAR BUCKS LODGE, CA

LOCATION.-Lat 3954'16", long 121¹3'47", in NW 1/4 SW 1/4 sec.29, T.24 N., R.7 E., Plumas County, Hydrologic Unit 18020121, Plumas National Forest, on left bank $20 \mathrm{ft}$ upstream from unnamed tributary, 0.2 mi downstream from diversion dam, and 3.6 mi northwest of Bucks Lodge.

DRAINAGE AREA.-31.5 $\mathrm{mi}^{2}$.

PERIOD OF RECORD.-October 1990 to current year. Unpublished records for water years 1981-90 available in files of the U.S. Geological Survey.

GAGE.-Water-stage recorder and concrete control with V-notch sharp-crested weir Sept. 19, 1990, to Sept. 24, 1998. Ultrasonic velocity meter since Sept. 24, 1998. Elevation of gage is 4,850 ft above sea level, from topographic map.

REMARKS.-Flow regulated by diversion dam at lower Bucks Lake 0.2 mi upstream, where most of the flow is diverted to Grizzly Creek via Bucks Creek Tunnel outlet or Grizzly Powerplant (station 11404240). Prior to Sept. 19, 1990, low flows regulated by fixed-plate orifice at outlet of diversion dam. See schematic diagram of North Fork Feather River Basin.

COOPERATION.--Records were collected by Pacific Gas \& Electric Co., under general supervision of the U.S. Geological Survey, in connection with a Federal Energy Regulatory Commission project.

\section{DISCHARGE, CUBIC FEET PER SECOND, WATER YEAR OCTOBER 1998 TO SEPTEMBER 1999}

\section{DAILY MEAN VALUES}

\begin{tabular}{|c|c|c|c|c|c|c|c|c|c|c|c|c|}
\hline DAY & $\mathrm{OCT}$ & NOV & $\mathrm{DEC}$ & JAN & FEB & MAR & $\mathrm{APR}$ & MAY & JUN & JUL & AUG & SEP \\
\hline 1 & 3.7 & 3.7 & 2.9 & 1.5 & 1.5 & 1.5 & 2.7 & 2.6 & 3.6 & 3.6 & 3.8 & 3.9 \\
\hline 2 & 3.7 & 3.6 & 1.5 & 1.5 & 1.5 & 1.5 & 2.7 & 2.6 & 3.6 & 3.5 & 3.8 & 3.9 \\
\hline 3 & 3.7 & 3.6 & 1.5 & 1.5 & 1.5 & 1.5 & 2.7 & 2.6 & 3.7 & 3.5 & 3.8 & 3.9 \\
\hline 4 & 3.6 & 3.7 & 1.5 & 1.5 & 1.5 & 1.5 & 2.7 & 2.6 & 3.7 & 3.6 & 3.8 & 3.9 \\
\hline 5 & 3.6 & 3.7 & 1.5 & 1.5 & 1.5 & 1.5 & 2.7 & 2.6 & 3.7 & 3.7 & 3.8 & 3.9 \\
\hline 6 & 3.6 & 3.7 & 1.5 & 1.5 & 1.5 & 1.5 & 2.7 & 2.6 & 3.7 & 3.7 & 3.8 & 3.9 \\
\hline 7 & 3.6 & 3.8 & 1.5 & 1.5 & 1.5 & 1.5 & 2.7 & 2.6 & 3.7 & 3.8 & 3.8 & 3.9 \\
\hline 8 & 3.6 & 3.8 & 1.5 & 1.5 & 1.5 & 1.5 & 2.7 & 2.6 & 3.7 & 3.8 & 3.8 & 3.9 \\
\hline 9 & 3.6 & 3.8 & 1.5 & 1.5 & 1.5 & 1.5 & 2.7 & 2.6 & 3.7 & 3.8 & 3.8 & 3.9 \\
\hline 10 & 3.6 & 3.7 & 1.4 & 1.5 & 1.5 & 1.5 & 2.8 & 2.6 & 3.6 & 3.8 & 3.8 & 3.9 \\
\hline 11 & 3.6 & 3.8 & 1.4 & 1.5 & 1.5 & 1.5 & 2.7 & 2.6 & 3.6 & 3.8 & 3.8 & 3.9 \\
\hline 12 & 3.7 & 3.7 & 1.4 & 1.5 & 1.5 & 1.5 & 2.7 & 3.3 & 3.6 & 3.8 & 3.8 & 3.9 \\
\hline 13 & 3.6 & 3.7 & 1.5 & 1.5 & 1.5 & 1.5 & 2.7 & 3.6 & 3.6 & 3.8 & 3.8 & 3.9 \\
\hline 14 & 3.6 & 3.7 & 1.5 & 1.5 & 1.5 & 1.5 & 2.7 & 3.6 & 3.6 & 3.8 & 3.8 & 3.9 \\
\hline 15 & 3.6 & 3.6 & 1.5 & 1.5 & 1.5 & 1.5 & 2.7 & 3.6 & 3.6 & 3.8 & 3.7 & 3.9 \\
\hline 16 & 3.6 & 3.6 & 1.5 & 1.5 & 1.5 & 1.5 & 2.7 & 3.6 & 3.6 & 3.9 & 3.7 & 3.9 \\
\hline 17 & 3.6 & 3.6 & 1.5 & 1.5 & 1.5 & 1.5 & 2.7 & 3.6 & 3.6 & 3.9 & 3.7 & 3.9 \\
\hline 18 & 3.6 & 3.6 & 1.5 & 1.5 & 1.5 & 1.5 & 2.7 & 3.6 & 3.6 & 3.9 & 3.7 & 3.9 \\
\hline 19 & 3.6 & 3.6 & 1.5 & 1.5 & 1.5 & 1.5 & 2.7 & 3.6 & 3.6 & 3.9 & 3.7 & 3.9 \\
\hline 20 & 3.6 & 3.6 & 1.5 & 1.5 & 1.5 & 1.5 & 2.7 & 3.6 & 3.6 & 3.9 & 3.7 & 3.9 \\
\hline 21 & 3.6 & 3.6 & 1.5 & 1.5 & 1.5 & 1.5 & 2.7 & 3.6 & 3.6 & 3.9 & 3.7 & 3.9 \\
\hline 22 & 3.6 & 3.7 & 1.5 & 1.5 & 1.5 & 1.5 & 2.7 & 3.6 & 3.6 & 3.9 & 3.7 & 3.9 \\
\hline 23 & 3.6 & 3.8 & 1.5 & 1.5 & 1.5 & 1.5 & 2.7 & 3.6 & 3.6 & 3.9 & 3.7 & 3.9 \\
\hline 24 & 3.7 & 3.8 & 1.5 & 1.5 & 1.5 & 1.5 & 2.7 & 3.6 & 3.6 & 3.9 & 3.8 & 3.9 \\
\hline 25 & 3.8 & 3.7 & 1.5 & 1.5 & 1.5 & 1.5 & 2.7 & 3.6 & 3.6 & 3.9 & 3.8 & 3.9 \\
\hline 26 & 3.8 & 3.7 & 1.5 & 1.5 & 1.5 & 1.5 & 2.7 & 3.5 & 3.6 & 3.9 & 3.7 & 3.9 \\
\hline 27 & 3.8 & 3.8 & 1.5 & 1.5 & 1.5 & 1.5 & 2.7 & 3.5 & 3.6 & 3.9 & 3.7 & 3.9 \\
\hline 28 & 3.8 & 3.8 & 1.5 & 1.5 & 1.5 & 1.5 & 2.7 & 3.5 & 3.6 & 3.9 & 3.7 & 3.9 \\
\hline 29 & 3.7 & 3.8 & 1.5 & 1.5 & --- & 1.5 & 2.7 & 3.5 & 3.6 & 3.8 & 3.7 & 3.9 \\
\hline 30 & 3.7 & 3.8 & 1.5 & 1.5 & --- & 1.5 & 2.6 & 3.5 & 3.6 & 3.8 & 3.8 & 3.9 \\
\hline 31 & 3.7 & --- & 1.5 & 1.5 & --- & 2.1 & --- & 3.5 & --- & 3.9 & 3.9 & --- \\
\hline TOTAL & 113.2 & 111.1 & 47.6 & 46.5 & 42.0 & 47.1 & 81.0 & 99.7 & 108.7 & 118.0 & 116.6 & 117.0 \\
\hline MEAN & 3.65 & 3.70 & 1.54 & 1.50 & 1.50 & 1.52 & 2.70 & 3.22 & 3.62 & 3.81 & 3.76 & 3.90 \\
\hline MAX & 3.8 & 3.8 & 2.9 & 1.5 & 1.5 & 2.1 & 2.8 & 3.6 & 3.7 & 3.9 & 3.9 & 3.9 \\
\hline MIN & 3.6 & 3.6 & 1.4 & 1.5 & 1.5 & 1.5 & 2.6 & 2.6 & 3.6 & 3.5 & 3.7 & 3.9 \\
\hline $\mathrm{AC}-\mathrm{FT}$ & 225 & 220 & 94 & 92 & 83 & 93 & 161 & 198 & 216 & 234 & 231 & 232 \\
\hline a & 12850 & 11210 & 10740 & 7540 & 3640 & 7440 & 7270 & 593 & 377 & 10310 & 13870 & 649 \\
\hline
\end{tabular}

CAL YR 1998 a 126000

WTR YR 1999 a 86480

a Diversion, in acre-feet, to Grizzly Powerplant, provided by Pacific Gas \& Electric Co. 
LOCATION.-Lat 3953’32", long 121¹7’25", in SW 1/4 NE 1/4 sec.34, T.24 N., R.6 E., Plumas County, Hydrologic Unit 18020121, Plumas National Forest, in outlet tower for Bucks Creek Powerplant $100 \mathrm{ft}$ upstream from Grizzly Diversion Dam, $2.4 \mathrm{mi}$ southeast of Storrie, and 6.2 mi west of Bucks Lodge.

DRAINAGE AREA.- $-14.4 \mathrm{mi}^{2}$.

PERIOD OF RECORD.-October 1986 to current year. Unpublished records for water years 1981-86 available in files of the U.S. Geological Survey.

GAGE.-Water-stage recorder. Datum of gage is $3.50 \mathrm{ft}$ below sea level (levels by Pacific Gas \& Electric Co.).

REMARKS.-Lake is formed by concrete dam. Storage began in July 1928 . Usable capacity, 1,033 acre-ft between elevations $4,271 \mathrm{ft}$, bottom of diversion tunnel, and 4,316.0 ft, crest of spillway. Water is received from Bucks Creek via Bucks Creek Tunnel and Grizzly Powerplant (station 11404240) which enter Grizzly Creek upstream. Most of the water is diverted through tunnel to Bucks Creek Powerplant (station 11403700) for power development downstream on North Fork Feather River. Figures given, including extremes, represent total contents. See schematic diagram of North Fork Feather River Basin.

COOPERATION.-Records were collected by Pacific Gas \& Electric Co., under general supervision of the U.S. Geological Survey, in connection with a Federal Energy Regulatory Commission project. Contents not rounded to U.S. Geological Survey standards.

EXTREMES FOR PERIOD OF RECORD.-Maximum contents, 1,329 acre-ft, Dec. 30, 1996, elevation, 4,321.5 ft; minimum, 216 acre-ft, Sept. 20, 1991, elevation, 4,282.8 ft.

EXTREMES FOR CURRENT YEAR.-Maximum contents, 1,143 acre-ft, Nov. 30, elevation, 4,316.8 ft; minimum, 721 acre-ft, June 30, elevation, $4,304.5 \mathrm{ft}$.

Capacity table (elevation, in feet, and contents, in acre-feet)

(Based on survey by Feather River Power Co. in 1928)

$\begin{array}{rrrr}4,290 & 350 & 4,305 & 736 \\ 4,295 & 464 & 4,310 & 898 \\ 4,300 & 592 & 4,320 & 1,268\end{array}$

RESERVOIR STORAGE (ACRE-FEET), WATER YEAR OCTOBER 1998 TO SEPTEMBER 1999 DAILY OBSERVATION AT 2400 HOURS

\begin{tabular}{|c|c|c|c|c|c|c|c|c|c|c|c|c|}
\hline DAY & $\mathrm{OCT}$ & NOV & $\mathrm{DEC}$ & JAN & FEB & MAR & APR & MAY & JUN & JUL & AUG & SEP \\
\hline 1 & 970 & 871 & 932 & 981 & 792 & 1027 & 877 & 967 & 847 & 864 & 901 & 1053 \\
\hline 2 & 999 & 1027 & 928 & 1017 & 789 & 770 & 877 & 949 & 851 & 854 & 871 & 1060 \\
\hline 3 & 1042 & 1038 & 1097 & 942 & 844 & 953 & 824 & 991 & 792 & 877 & 1009 & 1068 \\
\hline 4 & 999 & 1020 & 1017 & 977 & 891 & 953 & 821 & 946 & 821 & 911 & 1049 & 1071 \\
\hline 5 & 963 & 1060 & 1009 & 984 & 824 & 995 & 918 & 918 & 818 & 956 & 1049 & 1079 \\
\hline 6 & 1017 & 844 & 1009 & 1002 & 749 & 988 & 1020 & 904 & 837 & 956 & 1057 & 1082 \\
\hline 7 & 1009 & 894 & 1013 & 1057 & 981 & 949 & 928 & 908 & 767 & 811 & 1006 & 1090 \\
\hline 8 & 1038 & 871 & 1006 & 977 & 755 & 981 & 967 & 991 & 742 & 861 & 1027 & 1094 \\
\hline 9 & 1049 & 921 & 984 & 1002 & 1112 & 984 & 887 & 960 & 758 & 1024 & 981 & 1101 \\
\hline 10 & 828 & 891 & 956 & 1031 & 887 & 984 & 764 & 911 & 963 & 1068 & 932 & 1109 \\
\hline 11 & 834 & 724 & 939 & 1017 & 960 & 956 & 767 & 871 & 877 & 991 & 967 & 1112 \\
\hline 12 & 811 & 871 & 1031 & 1046 & 946 & 908 & 844 & 877 & 792 & 1068 & 894 & 1116 \\
\hline 13 & 861 & 837 & 898 & 925 & 967 & 871 & 1006 & 881 & 783 & 999 & 901 & 1116 \\
\hline 14 & 884 & 815 & 921 & 960 & 977 & 824 & 887 & 795 & 874 & 1060 & 915 & 1116 \\
\hline 15 & 901 & 821 & 736 & 1035 & 1027 & 808 & 953 & 789 & 932 & 1006 & 908 & 1120 \\
\hline 16 & 908 & 805 & 761 & 1020 & 970 & 828 & 864 & 761 & 932 & 977 & 932 & 1057 \\
\hline 17 & 991 & 844 & 828 & 1120 & 891 & 799 & 881 & 795 & 898 & 1017 & 1002 & 1006 \\
\hline 18 & 911 & 770 & 871 & 1068 & 761 & 877 & 799 & 799 & 925 & 1046 & 942 & 1013 \\
\hline 19 & 831 & 783 & 960 & 1009 & 854 & 942 & 783 & 953 & 953 & 1027 & 1020 & 1017 \\
\hline 20 & 861 & 789 & 981 & 1120 & 767 & 854 & 932 & 1046 & 981 & 1042 & 1082 & 1024 \\
\hline 21 & 921 & 824 & 901 & 999 & 828 & 828 & 967 & 984 & 1006 & 1071 & 1006 & 1027 \\
\hline 22 & 871 & 1024 & 908 & 1009 & 871 & 815 & 960 & 999 & 988 & 1038 & 1060 & 1035 \\
\hline 23 & 799 & 1131 & 921 & 1027 & 857 & 871 & 967 & 1002 & 956 & 925 & 871 & 1053 \\
\hline 24 & 851 & 970 & 851 & 755 & 956 & 939 & 967 & 970 & 904 & 942 & 956 & 1071 \\
\hline 25 & 921 & 963 & 928 & 811 & 1046 & 1038 & 991 & 1053 & 908 & 891 & 967 & 1090 \\
\hline 26 & 928 & 963 & 967 & 730 & 977 & 1042 & 1049 & 1009 & 881 & 915 & 1002 & 1049 \\
\hline 27 & 752 & 960 & 1006 & 884 & 1006 & 877 & 911 & 999 & 861 & 821 & 828 & 1035 \\
\hline 28 & 789 & 908 & 1013 & 908 & 1116 & 945 & 758 & 881 & 824 & 828 & 995 & 1079 \\
\hline 29 & 792 & 761 & 1017 & 928 & --- & 956 & 1009 & 877 & 736 & 1031 & 1035 & 1086 \\
\hline 30 & 808 & 1143 & 928 & 939 & --- & 898 & 901 & 942 & 721 & 939 & 1042 & 1057 \\
\hline 31 & 844 & --- & 981 & 884 & --- & 908 & --- & 867 & --- & 963 & 1049 & --- \\
\hline MAX & 1049 & 1143 & 1097 & 1120 & 1116 & 1042 & 1049 & 1053 & 1006 & 1071 & 1082 & 1120 \\
\hline MIN & 752 & 724 & 736 & 730 & 749 & 770 & 758 & 761 & 721 & 811 & 828 & 1006 \\
\hline$a$ & 4308.4 & 4316.8 & 4312.4 & 4309.6 & 4316.1 & 4310.3 & 4310.1 & 4309.1 & 4304.5 & 4311.9 & 4314.3 & 4314.5 \\
\hline $\mathrm{b}$ & -109 & +299 & -162 & -97 & +232 & -208 & -7 & -34 & -146 & +242 & +86 & +8 \\
\hline
\end{tabular}

CAL YR 1998 MAX 1197 MIN $706 \quad$ b +192

WTR YR 1999 MAX 1143 MIN 721 b +104 
LOCATION.-Lat 3953'29", long 121¹7’35", in SW 1/4 NE 1/4 sec.34, T.24 N., R.6 E., Plumas County, Hydrologic Unit 18020121, Plumas National Forest, on right bank 0.2 mi downstream from diversion dam, and 2.4 mi southeast of Storrie.

DRAINAGE AREA.-14.4 $\mathrm{mi}^{2}$.

PERIOD OF RECORD.-October 1985 to current year. Unpublished records for water years 1976-85 available in files of the U.S. Geological Survey.

GAGE.-Water-stage recorder and concrete control with V-notch sharp-crested weir, since Oct. 8, 1987. Elevation of gage is 4,320 ft above sea level, from topographic map. Prior to Oct. 8, 1987, at datum $1.79 \mathrm{ft}$ higher.

REMARKS.-Flow regulated by diversion dam 0.2 mi upstream. There is considerable inflow upstream from the diversion dam from Bucks Creek Tunnel outlet and Grizzly Powerplant (station 11404240). Most of the flow is diverted to Bucks Creek Powerplant (station 11403700) on North Fork Feather River. See schematic diagram of North Fork Feather River Basin.

COOPERATION.- Records were collected by Pacific Gas \& Electric Co., under general supervision of the U.S. Geological Survey, in connection with a Federal Energy Regulatory Commission project.

EXTREMES FOR PERIOD OF RECORD.-Maximum discharge, 6,300 ft 3 /s, Jan. 1, 1997, gage height, $7.33 \mathrm{ft}$, from rating curve extended above $260 \mathrm{ft}^{3} / \mathrm{s}$ on basis of computation of peak flow over dam; maximum gage height, $9.54 \mathrm{ft}$, Feb. 17, 1986, datum then in use; minimum daily, $1.9 \mathrm{ft}^{3} / \mathrm{s}$, June 14,1988 .

DISCHARGE, CUBIC FEET PER SECOND, WATER YEAR OCTOBER 1998 TO SEPTEMBER 1999 DAILY MEAN VALUES

\begin{tabular}{|c|c|c|c|c|c|c|c|c|c|c|c|c|}
\hline DAY & OCT & NOV & $\mathrm{DEC}$ & JAN & FEB & MAR & APR & MAY & JUN & JUL & AUG & SEP \\
\hline 1 & 4.6 & 4.5 & 34 & 2.4 & 2.5 & 29 & 3.5 & 3.4 & 4.7 & 4.5 & 4.6 & 4.8 \\
\hline 2 & 4.7 & 4.5 & 2.9 & 2.4 & 2.5 & 3.1 & 3.4 & 3.4 & 4.7 & 4.6 & 4.6 & 4.8 \\
\hline 3 & 4.7 & 4.7 & 273 & 2.4 & 2.4 & 3.2 & 3.4 & 3.5 & 4.7 & 4.6 & 4.7 & 4.8 \\
\hline 4 & 4.7 & 4.7 & 3.1 & 2.4 & 2.5 & 3.2 & 3.3 & 3.4 & 4.7 & 4.6 & 4.7 & 4.8 \\
\hline 5 & 4.7 & 4.7 & 2.9 & 2.4 & 2.5 & 3.1 & 3.4 & 3.4 & 4.7 & 4.7 & 4.8 & 4.8 \\
\hline 6 & 4.6 & 4.7 & 2.8 & 2.4 & 2.5 & 3.1 & 3.5 & 3.3 & 4.7 & 4.7 & 4.8 & 4.8 \\
\hline 7 & 4.7 & 4.8 & 2.7 & 2.4 & 2.9 & 3.0 & 3.5 & 3.3 & 4.7 & 4.6 & 4.7 & 4.8 \\
\hline 8 & 4.7 & 4.6 & 2.6 & 2.4 & 3.0 & 3.0 & 3.5 & 3.4 & 4.6 & 4.5 & 4.7 & 4.8 \\
\hline 9 & 4.7 & 4.5 & 2.6 & 2.4 & 70 & 3.0 & 3.4 & 3.4 & 4.6 & 4.7 & 4.8 & 4.9 \\
\hline 10 & 4.6 & 4.6 & 2.5 & 2.4 & 3.8 & 2.9 & 3.4 & 3.4 & 4.6 & 4.8 & 4.7 & 4.9 \\
\hline 11 & 4.5 & 4.5 & 2.5 & 2.4 & 3.4 & 2.9 & 3.3 & 3.4 & 4.8 & 4.8 & 4.7 & 4.9 \\
\hline 12 & 4.4 & 4.5 & 2.5 & 2.4 & 3.2 & 2.8 & 3.4 & 4.3 & 4.7 & 4.7 & 4.6 & 7.2 \\
\hline 13 & 4.5 & 4.5 & 2.6 & 2.4 & 3.1 & 2.7 & 3.5 & 4.8 & 4.6 & 4.7 & 4.6 & 10 \\
\hline 14 & 4.5 & 4.4 & 2.5 & 2.4 & 3.1 & 2.7 & 3.5 & 4.8 & 4.6 & 4.7 & 4.6 & 11 \\
\hline 15 & 4.5 & 4.4 & 2.5 & 2.4 & 3.0 & 2.7 & 3.5 & 4.7 & 4.7 & 4.8 & 4.6 & 11 \\
\hline 16 & 4.5 & 4.5 & 2.4 & 2.5 & 3.0 & 2.7 & 3.5 & 4.7 & 4.7 & 4.7 & 4.6 & 8.3 \\
\hline 17 & 4.6 & 4.6 & 2.4 & 2.5 & 3.0 & 2.7 & 3.5 & 4.7 & 4.7 & 4.7 & 4.7 & 4.7 \\
\hline 18 & 4.6 & 4.5 & 2.4 & 198 & 3.0 & 2.7 & 3.5 & 4.7 & 4.7 & 4.8 & 4.7 & 4.7 \\
\hline 19 & 4.5 & 4.4 & 2.4 & 2.7 & 2.9 & 2.7 & 3.4 & 4.8 & 4.7 & 4.8 & 4.7 & 4.7 \\
\hline 20 & 4.5 & 4.4 & 2.5 & 19 & 2.9 & 2.7 & 3.4 & 4.9 & 4.8 & 4.8 & 4.8 & 4.7 \\
\hline 21 & 4.5 & 4.5 & 2.5 & 4.1 & 2.9 & 2.6 & 3.5 & 4.9 & 4.8 & 4.8 & 4.8 & 4.7 \\
\hline 22 & 4.6 & 4.7 & 2.5 & 2.9 & 2.8 & 2.6 & 3.5 & 4.9 & 4.8 & 4.8 & 4.8 & 4.7 \\
\hline 23 & 4.5 & 321 & 2.5 & 36 & 2.8 & 2.6 & 3.5 & 4.9 & 4.8 & 4.7 & 4.8 & 4.7 \\
\hline 24 & 4.6 & 9.7 & 2.5 & 2.8 & 2.8 & 2.7 & 3.5 & 4.9 & 4.7 & 4.7 & 4.6 & 4.8 \\
\hline 25 & 4.6 & 5.0 & 2.5 & 2.7 & 2.9 & 2.8 & 3.5 & 4.9 & 4.7 & 4.6 & 4.7 & 4.8 \\
\hline 26 & 4.6 & 5.0 & 2.5 & 2.7 & 2.9 & 2.8 & 3.5 & 4.9 & 4.7 & 4.6 & 4.7 & 4.8 \\
\hline 27 & 4.5 & 5.0 & 2.5 & 2.6 & 2.8 & 2.7 & 3.4 & 4.9 & 4.7 & 4.6 & 4.7 & 4.8 \\
\hline 28 & 4.4 & 4.9 & 2.4 & 2.6 & 3.1 & 2.7 & 3.3 & 4.9 & 4.6 & 4.5 & 4.7 & 4.7 \\
\hline 29 & 4.4 & 4.9 & 2.4 & 2.6 & --- & 2.7 & 3.3 & 4.8 & 4.6 & 4.7 & 4.8 & 4.8 \\
\hline 30 & 4.4 & 136 & 2.4 & 2.6 & --- & 2.7 & 3.4 & 4.8 & 4.4 & 4.8 & 4.8 & 4.8 \\
\hline 31 & 4.5 & --- & 2.4 & 2.6 & --- & 3.0 & --- & 4.8 & --- & 4.7 & 4.8 & --- \\
\hline TOTAL & 141.4 & 591.7 & 380.9 & 324.9 & 148.2 & 113.8 & 103.2 & 133.3 & 140.5 & 145.3 & 145.9 & 167.0 \\
\hline MEAN & 4.56 & 19.7 & 12.3 & 10.5 & 5.29 & 3.67 & 3.44 & 4.30 & 4.68 & 4.69 & 4.71 & 5.57 \\
\hline MAX & 4.7 & 321 & 273 & 198 & 70 & 29 & 3.5 & 4.9 & 4.8 & 4.8 & 4.8 & 11 \\
\hline MIN & 4.4 & 4.4 & 2.4 & 2.4 & 2.4 & 2.6 & 3.3 & 3.3 & 4.4 & 4.5 & 4.6 & 4.7 \\
\hline $\mathrm{AC}-\mathrm{FT}$ & 280 & 1170 & 756 & 644 & 294 & 226 & 205 & 264 & 279 & 288 & 289 & 331 \\
\hline a & 13500 & 14540 & 15770 & 13700 & 11230 & 15320 & 13540 & 10970 & 4100 & 10950 & 14180 & 910 \\
\hline
\end{tabular}

a Diversion, in acre-feet, to Bucks Creek Powerplant, provided by Pacific Gas \& Electric Co. 


\section{GRIZZLY CREEK BELOW DIVERSION DAM, NEAR STORRIE, CA—Continued}

STATISTICS OF MONTHLY MEAN DATA FOR WATER YEARS 1986 - 1999, BY WATER YEAR (WY)

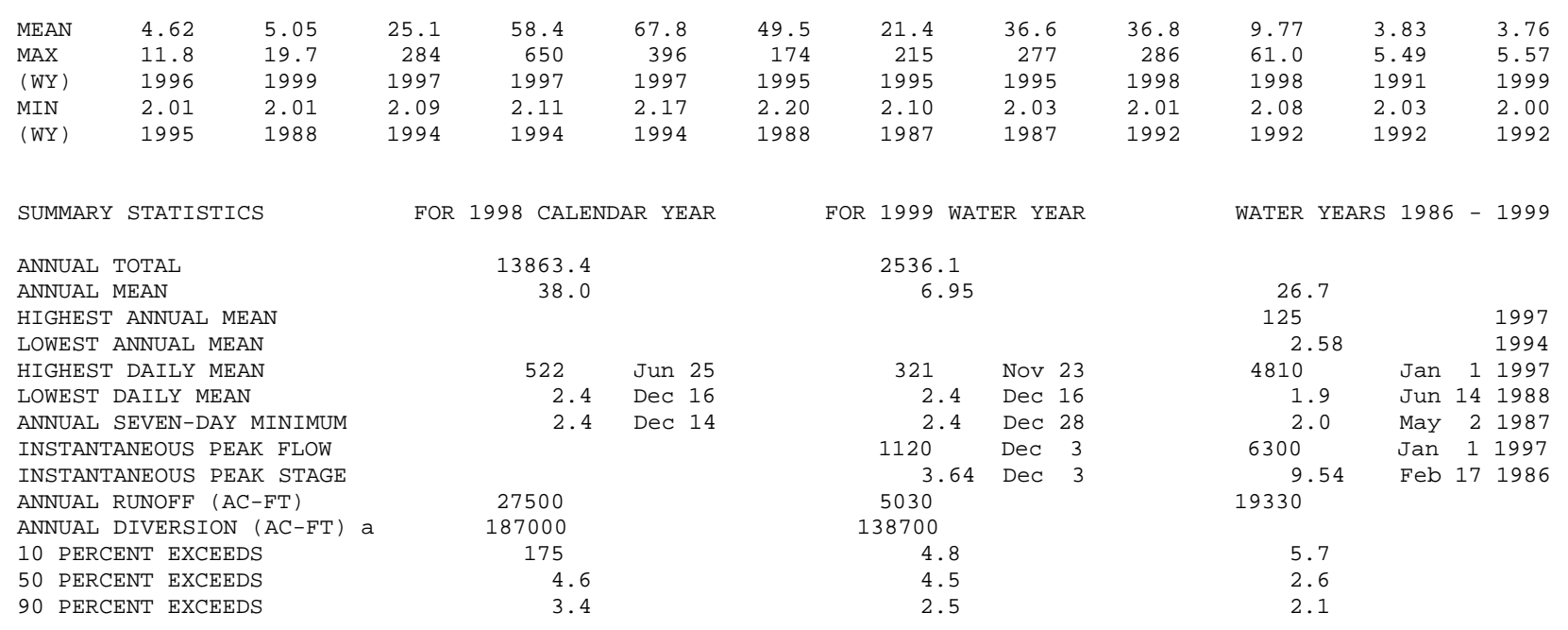

a Diversion, in acre-feet, to Bucks Creek Powerplant, provided by Pacific Gas \& Electric Co. 
LOCATION.-Lat 3951'09", long 121²3'29", in NE 1/4 NW 1/4 sec.14, T.23 N., R.5 E., Butte County, Hydrologic Unit 18020121, Lassen National Forest, on left bank 0.7 mi upstream from Bear Ranch Creek, 1.6 mi downstream from Grizzly Creek, and 2.1 mi downstream from Cresta Dam.

DRAINAGE AREA.- $-1,914 \mathrm{mi}^{2}$.

PERIOD OF RECORD.-October 1985 to February 1986, October 1986 to current year. Unpublished records for water years $1982-85$ available in files of the U.S. Geological Survey.

GAGE.-Water-stage recorder. Elevation of gage is 1,480 ft above sea level, from topographic map.

REMARKS.-Flow regulated by numerous reservoirs upstream, combined capacity, 1,386,000 acre-ft. Most of the flow bypasses this station through Cresta Powerplant (station 11404360). Diversion through Cresta Powerplant began in 1949. See schematic diagram of North Fork Feather River Basin.

COOPERATION.--Records were collected by Pacific Gas \& Electric Co., under general supervision of the U.S. Geological Survey, in connection with a Federal Energy Regulatory Commission project.

EXTREMES FOR PERIOD OF RECORD.-Maximum discharge, 115,000 $\mathrm{ft}^{3} / \mathrm{s}$, Jan. 1, 1997, gage height, $29.97 \mathrm{ft}$; minimum daily, $37 \mathrm{ft}^{3} / \mathrm{s}$, July 25, 1994.

DISCHARGE, CUBIC FEET PER SECOND, WATER YEAR OCTOBER 1998 TO SEPTEMBER 1999 DAILY MEAN VALUES

\begin{tabular}{|c|c|c|c|c|c|c|c|c|c|c|c|c|}
\hline DAY & OCT & NOV & $\mathrm{DEC}$ & JAN & $\mathrm{FEB}$ & MAR & $A P R$ & MAY & JUN & JUL & AUG & SEP \\
\hline 1 & 68 & 79 & 4880 & 98 & 173 & 4990 & 299 & 1040 & 90 & 79 & 104 & 209 \\
\hline 2 & 71 & 365 & 2250 & 97 & 164 & 3590 & 279 & 1240 & 91 & 82 & 94 & 554 \\
\hline 3 & 73 & 841 & 7000 & 99 & 156 & 5150 & 279 & 1230 & 94 & 81 & 100 & 124 \\
\hline 4 & 73 & 818 & 4100 & 101 & 156 & 4290 & 281 & 774 & 559 & 77 & 105 & 164 \\
\hline 5 & 68 & 868 & 2230 & 91 & 154 & 2960 & 312 & 608 & 681 & 72 & 105 & 299 \\
\hline 6 & 69 & 865 & 2840 & 93 & 185 & 1840 & 281 & 413 & 86 & 73 & 102 & 135 \\
\hline 7 & 66 & 835 & 1350 & 94 & 2190 & 1230 & 264 & 266 & 84 & 76 & 107 & 143 \\
\hline 8 & 64 & 713 & 685 & 95 & 4330 & 736 & 305 & 249 & 82 & 74 & 104 & 170 \\
\hline 9 & 63 & 648 & 464 & 88 & 15500 & 434 & 289 & 230 & 76 & 70 & 105 & 178 \\
\hline 10 & 67 & 600 & 287 & 88 & 9240 & 388 & 286 & 218 & 76 & 72 & 110 & 155 \\
\hline 11 & 62 & 191 & 232 & 85 & 5060 & 339 & 282 & 220 & 76 & 69 & 102 & 164 \\
\hline 12 & 65 & 83 & 152 & 97 & 2910 & 336 & 297 & 232 & 72 & 66 & 103 & 172 \\
\hline 13 & 66 & 77 & 149 & 100 & 1830 & 349 & 300 & 236 & 71 & 70 & 103 & 173 \\
\hline 14 & 66 & 70 & 141 & 98 & 912 & 344 & 311 & 216 & 70 & 69 & 104 & 193 \\
\hline 15 & 63 & 74 & 128 & 101 & 446 & 325 & 304 & 197 & 71 & 71 & 99 & 208 \\
\hline 16 & 64 & 76 & 127 & 117 & 764 & 269 & 297 & 198 & 69 & 71 & 100 & 206 \\
\hline 17 & 62 & 123 & 126 & 123 & 2910 & 210 & 345 & 203 & 68 & 72 & 101 & 201 \\
\hline 18 & 62 & 88 & 126 & 864 & 3350 & 206 & 1040 & 201 & 68 & 66 & 99 & 210 \\
\hline 19 & 64 & 77 & 122 & 914 & 3480 & 202 & 1510 & 198 & 68 & 68 & 102 & 201 \\
\hline 20 & 67 & 72 & 114 & 1620 & 2800 & 211 & 1470 & 196 & 64 & 69 & 100 & 213 \\
\hline 21 & 66 & 90 & 108 & 3420 & 2390 & 208 & 2080 & 191 & 64 & 67 & 94 & 226 \\
\hline 22 & 66 & 146 & 108 & 1170 & 1980 & 203 & 1770 & 196 & 62 & 66 & 95 & 233 \\
\hline 23 & 62 & 3040 & 107 & 4210 & 1840 & 184 & 1470 & 193 & 60 & 66 & 106 & 253 \\
\hline 24 & 281 & 1140 & 107 & 2380 & 1650 & 207 & 1410 & 155 & 60 & 67 & 102 & 413 \\
\hline 25 & 727 & 167 & 107 & 713 & 2780 & 260 & 1480 & 120 & 59 & 66 & 98 & 296 \\
\hline 26 & 438 & 150 & 106 & 301 & 1990 & 236 & 2020 & 139 & 58 & 63 & 97 & 286 \\
\hline 27 & 87 & 256 & 101 & 229 & 816 & 612 & 2290 & 113 & 59 & 65 & 104 & 287 \\
\hline 28 & 74 & 197 & 103 & 212 & 2150 & 732 & 1540 & 106 & 58 & 63 & 91 & 310 \\
\hline 29 & 74 & 186 & 107 & 197 & --- & 322 & 1150 & 99 & 59 & 72 & 80 & 349 \\
\hline 30 & 79 & 4720 & 107 & 188 & --- & 311 & 945 & 97 & 66 & 100 & 81 & 323 \\
\hline 31 & 83 & --- & 101 & 179 & --- & 282 & --- & 93 & --- & 100 & 82 & --- \\
\hline TOTAL & 3360 & 17655 & 28665 & 18262 & 72306 & 31956 & 25186 & 9867 & 3221 & 2242 & 3079 & 7048 \\
\hline MEAN & 108 & 588 & 925 & 589 & 2582 & 1031 & 840 & 318 & 107 & 72.3 & 99.3 & 235 \\
\hline MAX & 727 & 4720 & 7000 & 4210 & 15500 & 5150 & 2290 & 1240 & 681 & 100 & 110 & 554 \\
\hline MIN & 62 & 70 & 101 & 85 & 154 & 184 & 264 & 93 & 58 & 63 & 80 & 124 \\
\hline $\mathrm{AC}-\mathrm{FT}$ & 6660 & 35020 & 56860 & 36220 & 143400 & 63380 & 49960 & 19570 & 6390 & 4450 & 6110 & 13980 \\
\hline a & 106800 & 169800 & 202100 & 177900 & 193800 & 206600 & 188500 & 214400 & 138400 & 126600 & 136200 & 65900 \\
\hline
\end{tabular}

a Diversion, in acre-feet, to Cresta Powerplant, provided by Pacific Gas \& Electric Co. 
11404330 NORTH FORK FEATHER RIVER BELOW GRIZZLY CREEK, CA—Continued

STATISTICS OF MONTHLY MEAN DATA FOR WATER YEARS 1986 - 1999, BY WATER YEAR (WY)

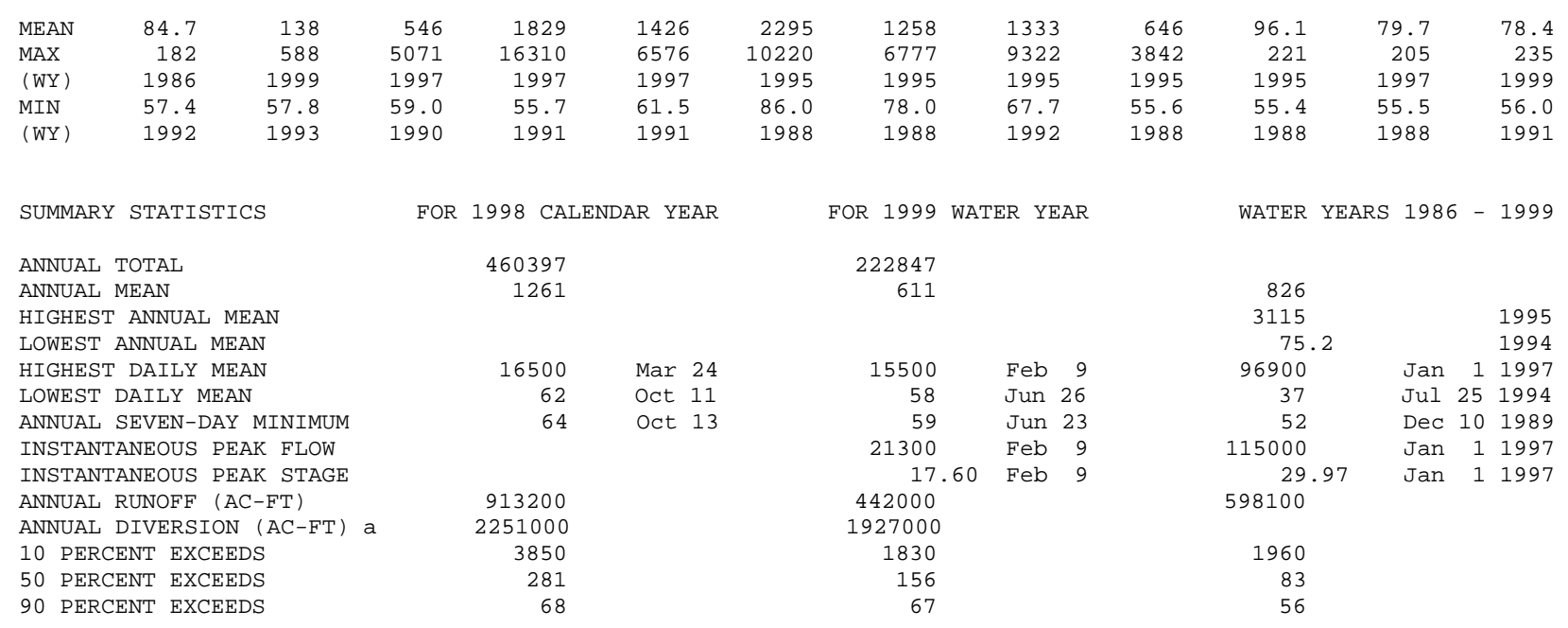

a Diversion, in acre-feet, to Cresta Powerplant, provided by Pacific Gas \& Electric Co. 


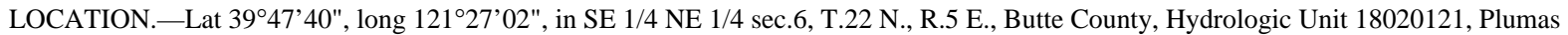
National Forest, on left bank between railroad and highway bridges, $0.6 \mathrm{mi}$ downstream from Flea Valley Creek and Pulga, and $1.6 \mathrm{mi}$ downstream from Poe Dam.

DRAINAGE AREA.-1,953 $\mathrm{mi}^{2}$.

PERIOD OF RECORD.-October 1910 to current year. Monthly discharge only for some periods and yearly estimates for water years 1911 and 1938, published in WSP 1315-A. Prior to October 1960, published as "at Big Bar."

CHEMICAL DATA: Water years 1963-66, 1972, 1977.

WATER TEMPERATURE: Water years 1963-83.

REVISED RECORDS.-WSP 931: 1938(M), 1940. WSP 1515: 1935. WDR CA-77-4: 1976 (yearly summaries).

GAGE.-Water-stage recorder. Datum of gage is $1,305.62 \mathrm{ft}$ above sea level. Prior to Oct. 1, 1937, at site 1.1 mi upstream at different datum. Oct. 1, 1937, to Sept. 30, 1958, at present site at datum $5.00 \mathrm{ft}$ higher.

REMARKS.-Flow regulated by Lake Almanor, Bucks Lake, Butt Valley Reservoir (stations 11399000, 11403500, 11401050), Mountain Meadows Reservoir, and five forebays, combined capacity, 1,386,000 acre-ft. Diversion through Poe Powerplant (station 11404900) began on May 29, 1958. See schematic diagram of North Fork Feather River Basin.

COOPERATION.-Records were collected by Pacific Gas \& Electric Co., under general supervision of the U.S. Geological Survey, in connection with a Federal Energy Regulatory Commission project.

EXTREMES FOR PERIOD OF RECORD.-Maximum discharge, 105,400 ft 3 /s, Jan. 1, 1997, gage height, $41.65 \mathrm{ft}$, from rating curve extended above $32,000 \mathrm{ft}^{3} / \mathrm{s}$ on basis of slope area measurement of peak discharge; minimum daily, $5.4 \mathrm{ft}^{3} / \mathrm{s}$, Sept. 18, 1977 .

DISCHARGE, CUBIC FEET PER SECOND, WATER YEAR OCTOBER 1998 TO SEPTEMBER 1999 DAILY MEAN VALUES

\begin{tabular}{|c|c|c|c|c|c|c|c|c|c|c|c|c|}
\hline DAY & OCT & NOV & $\mathrm{DEC}$ & JAN & FEB & MAR & APR & MAY & JUN & JUL & AUG & SEP \\
\hline 1 & 122 & 118 & 6020 & 121 & 149 & 8070 & 2310 & 3430 & 1370 & 112 & 90 & 89 \\
\hline 2 & 124 & 119 & 2650 & 117 & 148 & 6400 & 2350 & 3610 & 1180 & 112 & 88 & 96 \\
\hline 3 & 131 & 120 & 8050 & 119 & 147 & 8140 & 1790 & 3650 & 940 & 111 & 90 & 91 \\
\hline 4 & 129 & 118 & 4640 & 119 & 145 & 7110 & 1830 & 3100 & 540 & 111 & 93 & 92 \\
\hline 5 & 124 & 117 & 2500 & 116 & 139 & 5360 & 1800 & 2870 & 622 & 110 & 92 & 93 \\
\hline 6 & 122 & 117 & 1690 & 118 & 163 & 4230 & 1740 & 2870 & 800 & 111 & 91 & 90 \\
\hline 7 & 122 & 141 & 1180 & 120 & 2650 & 3540 & 1380 & 2660 & 377 & 114 & 91 & 95 \\
\hline 8 & 126 & 121 & 1030 & 119 & 5090 & 3410 & 1530 & 2560 & 643 & 112 & 92 & 98 \\
\hline 9 & 124 & 120 & 877 & 114 & 19300 & 2990 & 1560 & 2630 & 300 & 114 & 91 & 99 \\
\hline 10 & 126 & 124 & 674 & 115 & 10700 & 2660 & 1570 & 2430 & 116 & 113 & 93 & 99 \\
\hline 11 & 122 & 122 & 585 & 115 & 5400 & 2200 & 1630 & 2350 & 118 & 111 & 93 & 99 \\
\hline 12 & 122 & 116 & 196 & 117 & 3510 & 1950 & 1880 & 2310 & 117 & 104 & 90 & 100 \\
\hline 13 & 123 & 120 & 132 & 117 & 2370 & 1750 & 2180 & 2470 & 117 & 92 & 90 & 100 \\
\hline 14 & 123 & 121 & 132 & 115 & 1220 & 1790 & 2350 & 2450 & 115 & 91 & 92 & 100 \\
\hline 15 & 122 & 121 & 130 & 118 & 823 & 2050 & 2510 & 2300 & 116 & 91 & 91 & 100 \\
\hline 16 & 123 & 409 & 126 & 123 & 1200 & 1860 & 2500 & 2230 & 115 & 92 & 92 & 101 \\
\hline 17 & 123 & 1600 & 128 & 145 & 3570 & 1990 & 2720 & 2040 & 113 & 91 & 91 & 103 \\
\hline 18 & 118 & 1390 & 125 & 1700 & 4060 & 1890 & 3490 & 2100 & 113 & 90 & 93 & 101 \\
\hline 19 & 115 & 1300 & 126 & 1300 & 4020 & 2150 & 3880 & 2300 & 114 & 90 & 94 & 101 \\
\hline 20 & 112 & 1300 & 126 & 4130 & 3260 & 2610 & 3870 & 2320 & 115 & 92 & 91 & 99 \\
\hline 21 & 116 & 854 & 123 & 4350 & 3190 & 2400 & 4380 & 2310 & 116 & 90 & 92 & 99 \\
\hline 22 & 115 & 2240 & 120 & 1700 & 2740 & 2460 & 4110 & 2360 & 114 & 90 & 92 & 97 \\
\hline 23 & 113 & 5600 & 123 & 5270 & 2450 & 2210 & 3840 & 2420 & 111 & 90 & 88 & 100 \\
\hline 24 & 122 & 3590 & 123 & 3210 & 2210 & 2250 & 3730 & 2320 & 113 & 90 & 90 & 100 \\
\hline 25 & 128 & 1460 & 119 & 1350 & 4370 & 2480 & 3820 & 2370 & 113 & 91 & 90 & 99 \\
\hline 26 & 122 & 171 & 122 & 739 & 4370 & 2600 & 4270 & 2400 & 114 & 91 & 92 & 98 \\
\hline 27 & 123 & 170 & 121 & 173 & 2780 & 2760 & 4570 & 2430 & 113 & 90 & 92 & 99 \\
\hline 28 & 121 & 144 & 121 & 163 & 4810 & 2550 & 3840 & 2390 & 114 & 91 & 93 & 96 \\
\hline 29 & 111 & 154 & 121 & 159 & --- & 2440 & 3580 & 1660 & 111 & 91 & 91 & 95 \\
\hline 30 & 116 & 5230 & 119 & 156 & --- & 2670 & 3300 & 1600 & 113 & 89 & 89 & 96 \\
\hline 31 & 121 & --- & 121 & 156 & --- & 2620 & --- & 1640 & --- & 90 & 91 & --- \\
\hline TOTAL & 3761 & 27427 & 32450 & 26584 & 94984 & 99590 & 84310 & 76580 & 9173 & 3057 & 2828 & 2925 \\
\hline MEAN & 121 & 914 & 1047 & 858 & 3392 & 3213 & 2810 & 2470 & 306 & 98.6 & 91.2 & 97.5 \\
\hline MAX & 131 & 5600 & 8050 & 5270 & 19300 & 8140 & 4570 & 3650 & 1370 & 114 & 94 & 103 \\
\hline MIN & 111 & 116 & 119 & 114 & 139 & 1750 & 1380 & 1600 & 111 & 89 & 88 & 89 \\
\hline $\mathrm{AC}-\mathrm{FT}$ & 7460 & 54400 & 64360 & 52730 & 188400 & 197500 & 167200 & 151900 & 18190 & 6060 & 5610 & 5800 \\
\hline a & 98380 & 144600 & 195100 & 168300 & 172800 & 110400 & 103600 & 110300 & 121400 & 115300 & 124500 & 71270 \\
\hline
\end{tabular}

a Diversion, in acre-feet, to Poe Powerplant, provided by Pacific Gas \& Electric Co. 


\section{NORTH FORK FEATHER RIVER AT PULGA, CA—Continued}

STATISTICS OF MONTHLY MEAN DATA FOR WATER YEARS 1911 - 1999, BY WATER YEAR (WY)

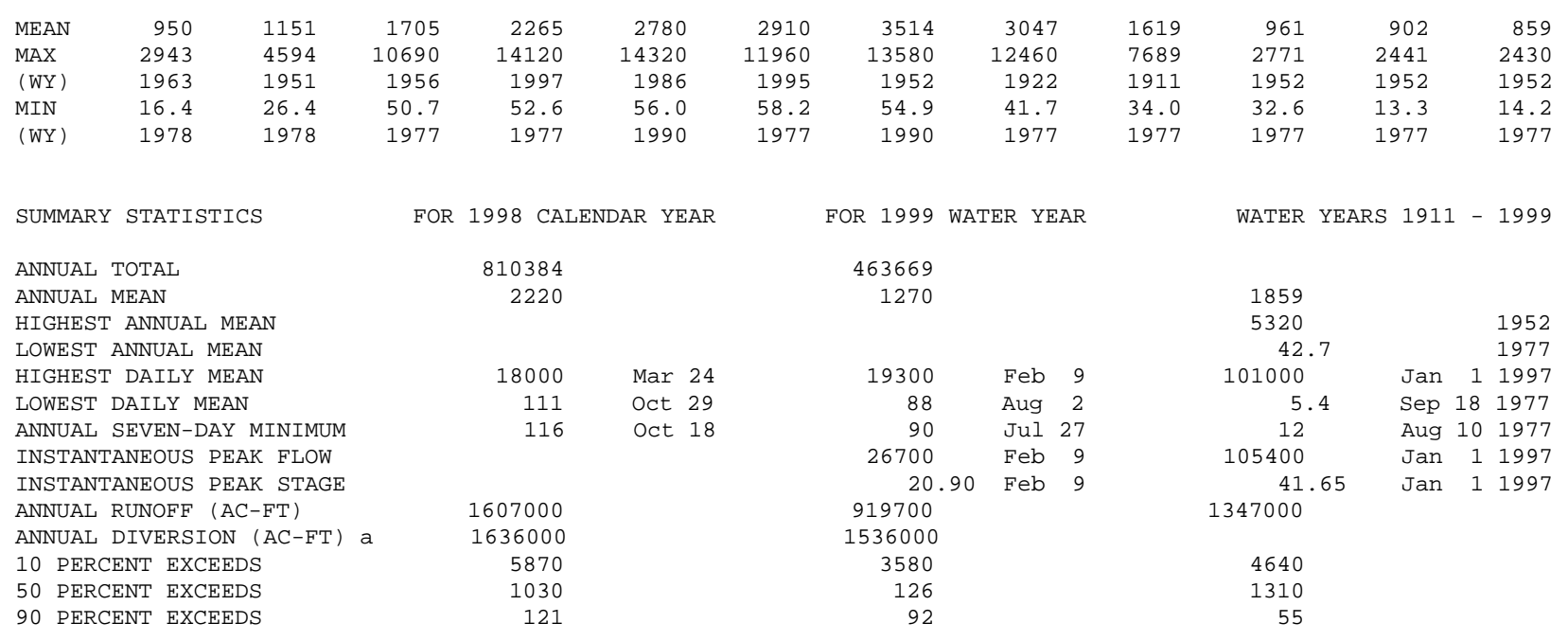

a Diversion, in acre-feet, to Poe Powerplant, provided by Pacific Gas \& Electric Co. 
LOCATION.-Lat 4001'48", long 12128'36", unsurveyed, T.25 N., R.4 E., Butte County, Hydrologic Unit 18020121, Lassen National Forest, on right bank $500 \mathrm{ft}$ downstream from outlet structure on Philbrook Dam, and 5.4 mi southeast of Butte Meadows.

DRAINAGE AREA. $-5.05 \mathrm{mi}^{2}$.

PERIOD OF RECORD.-_July 1989 to current year (no winter records). Unpublished records for water years 1986-89 available in files of the U.S. Geological Survey.

GAGE.-Water-stage recorder, Parshall flume, and V-notch sharp-crested weir. Elevation of gage is 5,490 ft above sea level, from topographic map. October 1985 to July 1989, nonrecording gage at same site and datum. In June 1989, V-notch sharp-crested weir installed in flume to be used at low flows.

REMARKS.- Records not computed for winter months. Flow completely regulated by Philbrook Reservoir, usable capacity, 5,370 acre-ft, $500 \mathrm{ft}$ upstream. Spillwater from Philbrook Reservoir bypasses this station.

COOPERATION.-Records were collected by Pacific Gas \& Electric Co., under general supervision of the U.S. Geological Survey, in connection with a Federal Energy Regulatory Commission project.

DISCHARGE, CUBIC FEET PER SECOND, WATER YEAR OCTOBER 1998 TO SEPTEMBER 1999

DAILY MEAN VALUES

\begin{tabular}{|c|c|c|c|c|c|c|c|c|c|c|c|c|}
\hline DAY & OCT & NOV & $\mathrm{DEC}$ & JAN & FEB & MAR & $\mathrm{APR}$ & MAY & JUN & JUL & AUG & SEP \\
\hline 1 & 42 & 2.7 & --- & --- & --- & --- & 4.2 & 4.5 & 4.4 & 3.5 & 23 & 39 \\
\hline 2 & 41 & 2.7 & --- & --- & --- & --- & 4.2 & 4.5 & 4.3 & 3.5 & 23 & 40 \\
\hline 3 & 41 & 2.7 & --- & --- & --- & --- & 4.2 & 4.5 & 4.4 & 3.5 & 23 & 40 \\
\hline 4 & 41 & 2.7 & --- & --- & --- & --- & 4.2 & 4.5 & 4.4 & 3.5 & 23 & 40 \\
\hline 5 & 40 & 2.7 & --- & --- & --- & --- & 4.2 & 2.4 & 4.4 & 3.5 & 23 & 40 \\
\hline 6 & 40 & 2.7 & --- & --- & --- & --- & 4.2 & 4.2 & 4.4 & 3.5 & 23 & 40 \\
\hline 7 & 45 & 2.7 & --- & --- & --- & --- & 4.2 & 4.3 & 3.8 & 3.5 & 23 & 43 \\
\hline 8 & 48 & 2.7 & --- & --- & --- & --- & 4.2 & 4.3 & 3.2 & 3.5 & 23 & 45 \\
\hline 9 & 47 & 2.7 & --- & --- & --- & --- & 4.2 & 4.3 & 3.2 & 3.5 & 23 & 45 \\
\hline 10 & 47 & 2.7 & --- & --- & --- & --- & 4.2 & 4.3 & 3.3 & 3.5 & 29 & 44 \\
\hline 11 & 46 & 2.7 & --- & --- & --- & --- & 4.2 & 4.3 & 3.3 & 3.5 & 34 & 44 \\
\hline 12 & 46 & 2.7 & --- & --- & --- & --- & 4.2 & 4.3 & 3.3 & 16 & 34 & 43 \\
\hline 13 & 45 & 2.7 & --- & --- & --- & --- & 4.2 & 4.3 & 3.3 & 39 & 34 & 43 \\
\hline 14 & 44 & 2.7 & --- & --- & --- & --- & 4.2 & 4.3 & 3.3 & 39 & 34 & 42 \\
\hline 15 & 44 & 2.7 & --- & --- & --- & --- & 4.3 & 4.3 & 3.4 & 26 & 34 & 43 \\
\hline 16 & 34 & 2.7 & --- & --- & --- & 4.2 & 4.5 & 4.3 & 3.4 & 7.9 & 34 & 45 \\
\hline 17 & 28 & 2.7 & --- & --- & --- & 4.2 & 4.5 & 4.3 & 3.4 & 7.9 & 33 & 44 \\
\hline 18 & 27 & 2.7 & --- & --- & --- & 4.2 & 4.5 & 4.3 & 3.4 & 7.9 & 33 & 43 \\
\hline 19 & 27 & --- & --- & --- & --- & 4.2 & 4.5 & 4.4 & 3.4 & 7.9 & 36 & 40 \\
\hline 20 & 27 & --- & --- & --- & --- & 4.2 & 4.5 & 4.4 & 3.4 & 7.9 & 39 & 3.9 \\
\hline 21 & 26 & --- & --- & --- & --- & 4.2 & 4.5 & 4.4 & 3.4 & 7.9 & 39 & 3.9 \\
\hline 22 & 24 & --- & --- & --- & --- & 4.2 & 4.5 & 4.4 & 3.4 & 7.9 & 39 & 4.0 \\
\hline 23 & 2.6 & --- & --- & --- & --- & 4.2 & 4.5 & 4.4 & 3.4 & 7.9 & 39 & 4.0 \\
\hline 24 & 2.7 & --- & --- & --- & --- & 4.2 & 4.5 & 4.4 & 3.4 & 7.9 & 39 & 4.0 \\
\hline 25 & 2.7 & --- & --- & --- & --- & 4.2 & 4.5 & 4.5 & 3.4 & 7.9 & 40 & 4.0 \\
\hline 26 & 2.7 & --- & --- & --- & --- & 4.2 & 4.5 & 4.6 & 3.4 & 15 & 40 & 4.0 \\
\hline 27 & 2.7 & --- & --- & --- & --- & 4.2 & 4.5 & 4.6 & 3.5 & 23 & 40 & 4.0 \\
\hline 28 & 2.7 & --- & --- & --- & --- & 4.2 & 4.5 & 4.5 & 3.5 & 23 & 40 & 4.0 \\
\hline 29 & 2.7 & --- & --- & --- & --- & 4.2 & 4.5 & 4.4 & 3.5 & 23 & 40 & 4.0 \\
\hline 30 & 2.7 & --- & --- & --- & --- & 4.2 & 4.5 & 4.4 & 3.5 & 23 & 39 & 4.0 \\
\hline 31 & 2.7 & --- & --- & --- & --- & 4.2 & --- & 4.4 & --- & 23 & 39 & --- \\
\hline TOTAL & 874.2 & --- & --- & --- & --- & --- & 130.6 & 134.0 & 107.8 & 367.5 & 1015 & 846.8 \\
\hline MEAN & 28.2 & --- & --- & --- & --- & --- & 4.35 & 4.32 & 3.59 & 11.9 & 32.7 & 28.2 \\
\hline MAX & 48 & --- & --- & --- & --- & --- & 4.5 & 4.6 & 4.4 & 39 & 40 & 45 \\
\hline MIN & 2.6 & --- & --- & --- & --- & --- & 4.2 & 2.4 & 3.2 & 3.5 & 23 & 3.9 \\
\hline $\mathrm{AC}-\mathrm{FT}$ & 1730 & --- & --- & --- & --- & --- & 259 & 266 & 214 & 729 & 2010 & 1680 \\
\hline
\end{tabular}


LOCATION.-Lat 3956'03", long 121³1'43", in NW 1/4 SE 1/4 sec.16, T.24 N., R.4 E., Butte County, Hydrologic Unit 18020121, on right bank $200 \mathrm{ft}$ upstream from road bridge, $1,800 \mathrm{ft}$ downstream from Hendricks Diversion Dam, and 1.9 mi north of Stirling City.

DRAINAGE AREA.-46.1 $\mathrm{mi}^{2}$.

PERIOD OF RECORD.-August 1986 to current year (low-flow records only).

GAGE.-Water-stage recorder. Elevation of gage is 3,210 ft above sea level, from topographic map.

REMARKS.-No records computed above $40 \mathrm{ft}^{3} / \mathrm{s}$. Most of the water is diverted at Hendricks Diversion Dam to the Hendricks Canal and Toadtown Canal (station 11389800) and then to De Sabla Powerplant (station 11389750) on Butte Creek.

COOPERATION.--Records were collected by Pacific Gas \& Electric Co., under general supervision of the U.S. Geological Survey, in connection with a Federal Energy Regulatory Commission project.

DISCHARGE, CUBIC FEET PER SECOND, WATER YEAR OCTOBER 1998 TO SEPTEMBER 1999 DAILY MEAN VALUES

\begin{tabular}{|c|c|c|c|c|c|c|c|c|c|c|c|c|}
\hline DAY & OCT & NOV & $\mathrm{DEC}$ & JAN & $\mathrm{FEB}$ & MAR & APR & MAY & JUN & JUL & AUG & SEP \\
\hline 1 & 21 & 20 & --- & 20 & --- & --- & --- & --- & --- & 23 & 19 & 21 \\
\hline 2 & 21 & 20 & --- & 20 & --- & --- & --- & --- & --- & 24 & 19 & 21 \\
\hline 3 & 21 & 20 & --- & 20 & --- & --- & --- & --- & --- & 24 & 19 & 21 \\
\hline 4 & 21 & 20 & --- & 20 & --- & --- & --- & --- & --- & 24 & 19 & 21 \\
\hline 5 & 20 & 20 & --- & 20 & --- & --- & --- & --- & --- & 24 & 19 & 20 \\
\hline 6 & 20 & 20 & --- & 20 & --- & --- & --- & --- & --- & 24 & 19 & 20 \\
\hline 7 & 20 & 39 & --- & 20 & --- & --- & --- & --- & --- & 23 & 19 & 21 \\
\hline 8 & 20 & 23 & --- & 20 & --- & --- & --- & --- & --- & 23 & 19 & 21 \\
\hline 9 & 20 & 21 & --- & 20 & --- & --- & --- & --- & --- & 23 & 19 & 21 \\
\hline 10 & 20 & 21 & --- & 20 & --- & --- & --- & --- & --- & 23 & 19 & 21 \\
\hline 11 & 20 & 21 & --- & 20 & --- & --- & --- & --- & --- & 23 & 19 & 21 \\
\hline 12 & 20 & 20 & --- & 20 & --- & --- & --- & --- & --- & 23 & 19 & 22 \\
\hline 13 & 20 & 20 & --- & 20 & --- & --- & --- & --- & --- & 25 & 19 & 22 \\
\hline 14 & 20 & 20 & --- & 20 & --- & --- & --- & --- & --- & 24 & 19 & 21 \\
\hline 15 & 20 & 21 & --- & 24 & --- & --- & --- & --- & --- & 24 & 19 & 21 \\
\hline 16 & 19 & 21 & --- & --- & --- & --- & --- & --- & --- & 23 & 19 & 21 \\
\hline 17 & 19 & 27 & --- & --- & --- & --- & --- & --- & --- & 23 & 19 & 21 \\
\hline 18 & 19 & 22 & --- & --- & --- & --- & --- & --- & --- & 23 & 19 & 21 \\
\hline 19 & 19 & 21 & --- & --- & --- & --- & --- & --- & 34 & 23 & 19 & 22 \\
\hline 20 & 19 & 21 & --- & --- & --- & --- & --- & --- & 26 & 22 & 19 & 21 \\
\hline 21 & 19 & 26 & --- & --- & --- & --- & --- & --- & 23 & 20 & 20 & 21 \\
\hline 22 & 19 & --- & --- & --- & --- & --- & --- & --- & 24 & 19 & 20 & 21 \\
\hline 23 & 20 & --- & --- & --- & --- & --- & --- & --- & 25 & 19 & 20 & 21 \\
\hline 24 & 21 & --- & 40 & --- & --- & --- & --- & --- & 25 & 19 & 20 & 21 \\
\hline 25 & 21 & --- & 38 & --- & --- & --- & --- & --- & 25 & 19 & 20 & 21 \\
\hline 26 & 20 & --- & 36 & --- & --- & --- & --- & --- & 25 & 19 & 20 & 20 \\
\hline 27 & 20 & --- & 33 & --- & --- & --- & --- & --- & 24 & 19 & 20 & 20 \\
\hline 28 & 20 & --- & 27 & --- & --- & --- & --- & --- & 24 & 19 & 20 & 20 \\
\hline 29 & 20 & 39 & 22 & --- & --- & --- & --- & --- & 24 & 19 & 20 & 20 \\
\hline 30 & 20 & --- & 21 & --- & --- & --- & --- & --- & 24 & 19 & 20 & 21 \\
\hline 31 & 20 & --- & 21 & --- & --- & --- & --- & --- & --- & 19 & 20 & --- \\
\hline TOTAL & 619 & --- & --- & --- & --- & --- & --- & --- & --- & 678 & 600 & 627 \\
\hline MEAN & 20.0 & --- & --- & --- & --- & --- & --- & --- & --- & 21.9 & 19.4 & 20.9 \\
\hline MAX & 21 & --- & --- & --- & --- & --- & --- & --- & --- & 25 & 20 & 22 \\
\hline MIN & 19 & --- & --- & --- & --- & --- & --- & --- & --- & 19 & 19 & 20 \\
\hline $\mathrm{AC}-\mathrm{FT}$ & 1230 & --- & --- & --- & --- & --- & --- & --- & --- & 1340 & 1190 & 1240 \\
\hline
\end{tabular}




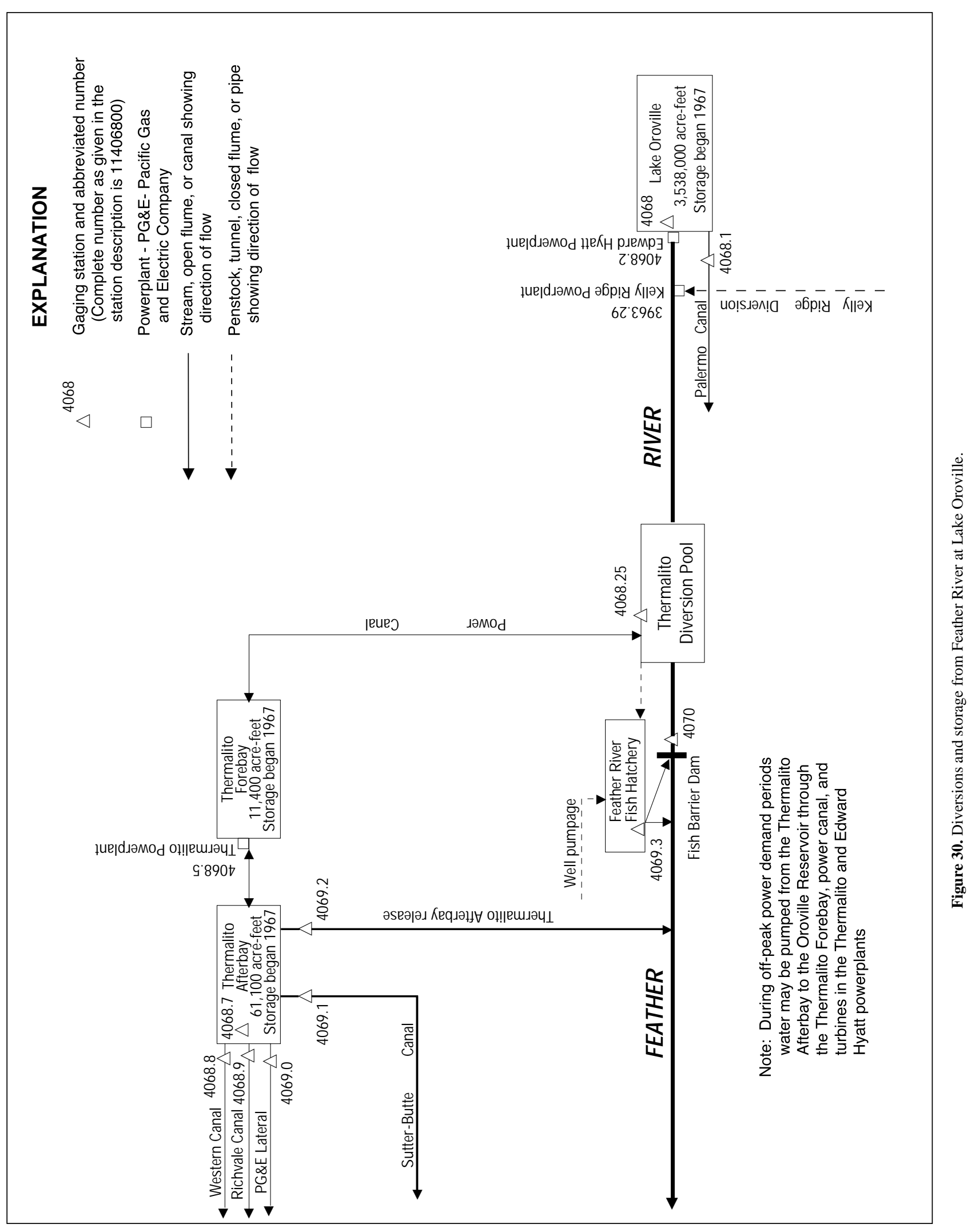


LOCATION.-Lat $39^{\circ} 32^{\prime} 06^{\prime \prime}$, long $121^{\circ} 28^{\prime} 25^{\prime \prime}$, in NE 1/4 SW 1/4 sec.1, T.19 N., R.4 E., Butte County, Hydrologic Unit 18020123, near intake structure at left end of Oroville Dam on Feather River, $1.0 \mathrm{mi}$ downstream from North Fork Feather River, and 4.2 mi east of Oroville.

DRAINAGE AREA.-3,607 $\mathrm{mi}^{2}$.

PERIOD OF RECORD.--November 1967 to current year.

GAGE.-Water-stage recorder. Datum of gage is $0.47 \mathrm{ft}$ above sea level (levels by California Department of Water Resources). Contents based on capacity table in use since Sept. 21, 1967.

REMARKS.--Reservoir is formed by an earthfill dam with concrete chute-type sidehill spillway completed May 13, 1968; storage began Nov. 14, 1967. Usable capacity, 2,685,385 acre-ft between elevations $640.0 \mathrm{ft}$, minimum power pool, and $900.0 \mathrm{ft}$, normal maximum pool. Dead storage, 852,192 acre-ft. Total capacity at normal maximum pool, 3,537,577 acre-ft; temporary detention storage occurred at times during construction; maximum was 155,200 acre-ft, Dec. 23, 1964. Water is released to Edward Hyatt Powerplant (station 11406820) through penstock in left abutment of dam and to Palermo Canal (station 11406810) through concrete tunnel also in left abutment of dam. Three of the total of six turbines in the Edward Hyatt Powerplant are reversible and during periods of low power demand water is pumped at times from the river back into Lake Oroville. Records, including extremes, represent total contents at 2400 hours. Maximum inflow of 266,000 $\mathrm{ft}^{3} / \mathrm{s} \mathrm{during}$ a 2 -hour period Feb. 17, 1986. See schematic diagram showing diversions and storage from Feather River at Lake Oroville.

COOPERATION.--Records were collected by California Department of Water Resources, under general supervision of the U.S. Geological Survey, in connection with a Federal Energy Regulatory Commission project. Contents not rounded to U.S. Geological Survey standards.

EXTREMES FOR PERIOD OF RECORD.—Maximum contents, 3,536,000 acre-ft, June 4, 1973, gage height, $899.88 \mathrm{ft}$; minimum since initial storage began, 882,395 acre-ft, Sept. 7, 1977, gage height, $645.11 \mathrm{ft}$.

EXTREMES FOR CURRENT YEAR.-Maximum contents, 3,481,007 acre-ft, June, 13, gage height, $896.40 \mathrm{ft}$; minimum, 2,427,271 acre-ft, Sept. 30, gage height, $820.14 \mathrm{ft}$.

Capacity table (gage height, in feet, and contents, in acre-feet)

(Based on table provided by California Department of Water Resources, dated Sept. 21, 1967)

$\begin{array}{lrrrrrrr}640 & 852,192 & 710 & 1,332,547 & 780 & 1,974,240 & 850 & 2,808,349 \\ 650 & 911,975 & 720 & 1,413,685 & 790 & 2,080,969 & 860 & 2,944,741 \\ 660 & 974,560 & 730 & 1,498,175 & 800 & 2,191,742 & 870 & 3,085,747 \\ 670 & 1,040,003 & 740 & 1,586,086 & 810 & 2,306,597 & 880 & 3,231,454 \\ 680 & 1,108,406 & 750 & 1,677,554 & 820 & 2,425,571 & 890 & 3,382,038 \\ 690 & 1,179,915 & 760 & 1,772,690 & 830 & 2,548,850 & 900 & 3,537,577 \\ 700 & 1,254,634 & 770 & 1,871,511 & 840 & 2,676,446 & & \end{array}$

RESERVOIR STORAGE (ACRE-FEET), WATER YEAR OCTOBER 1998 TO SEPTEMBER 1999

\section{DAILY OBSERVATION AT 2400 HOURS}

\begin{tabular}{|c|c|c|c|c|c|c|c|c|c|c|c|c|}
\hline DAY & $\mathrm{OCT}$ & NOV & $\mathrm{DEC}$ & JAN & FEB & MAR & APR & MAY & JUN & JUL & AUG & SEP \\
\hline 1 & 2819227 & 724076 & 2903896 & 2692041 & 2762339 & 2810360 & 2950990 & 3261626 & 3455296 & 3404282 & 2852178 & 2569983 \\
\hline 2 & 2809421 & 726835 & 2900190 & & 2759821 & 2816941 & & & 3454519 & & & 2560785 \\
\hline 3 & 811971 & 727229 & 928412 & 2697122 & 758761 & 2832024 & 2972573 & 3283600 & 3454830 & 3366605 & 2818420 & 553242 \\
\hline 4 & 816538 & 727886 & 930484 & 695298 & 53731 & 839182 & 985436 & 287947 & 52965 & 3348335 & 04594 & 544337 \\
\hline 5 & 2804862 & 727097 & 919030 & 692432 & 738809 & 835129 & 2994127 & 3287048 & 3461361 & 3333619 & 2789480 & 541206 \\
\hline 6 & 2796829 & 2727492 & 2904858 & 2690609 & 724864 & 824745 & 3000587 & 3285698 & 3470238 & 3319553 & 2775487 & 2539205 \\
\hline 7 & 787344 & 731436 & 2885399 & 689958 & 729200 & 2813045 & 3005367 & 3286448 & 3470238 & 3304174 & 2767248 & 529585 \\
\hline 8 & 779880 & 735516 & 871343 & 690609 & 741314 & 798702 & 015509 & 300413 & 71641 & 3288547 & 2763002 & 517006 \\
\hline 9 & 772961 & 2734989 & 855163 & 689958 & 823533 & 2794021 & 023273 & 315175 & 468524 & 3268192 & 751614 & 506826 \\
\hline 10 & 776152 & 2733015 & 2842021 & 2690870 & 53263 & 2787878 & 36007 & 17892 & 54474 & 3249711 & 739337 & 2498654 \\
\hline 11 & 780013 & 2737360 & 2825688 & & & & & & & & & 2497293 \\
\hline 12 & 5019 & 8019 & 434 & & 45 & & & & & & & 3605 \\
\hline 13 & 772030 & 2739205 & & & & & & & & & & 5439 \\
\hline 14 & 523 & 3821 & & & & & & & & & & 0006 \\
\hline 15 & 472 & 6857 & & & & & & & & & & 5197 \\
\hline 16 & 821 & 537 & 879 & & 5 & 5934 & & & & & & 0763 \\
\hline 17 & 764992 & 2746065 & 9996 & 764 & 7965 & 09957 & 6940 & 3436 & 1018 & 4764 & 41 & 7196 \\
\hline 18 & 7514 & 2747782 & 1068 & & 5823 & 14522 & 6672 & 6 & 4629 & & 46 & 76582 \\
\hline 19 & 5788 & 6593 & 384 & 556 & 5060 & 1783 & 2580 & 7 & 9615 & & 591 & 2486059 \\
\hline 20 & 762472 & 8310 & 632 & & 91 & 669 & 091 & & 137 & & 58 & $24^{\circ}$ \\
\hline 21 & 146 & 805 & & & & & & & & & & 546 \\
\hline 22 & 759556 & 2762339 & 6993 & 99772 & 74822 & 2857199 & 7633 & 6 & & & & 54556 \\
\hline 23 & 753201 & 2787478 & & & 5354 & & & & & & & 7458 \\
\hline 24 & & 2802048 & & & & & & & & & & 1717 \\
\hline 25 & & 2811837 & & & & & & & & & & 8179 \\
\hline 26 & & & & & & & & & & & & 54281 \\
\hline 27 & 305 & 4319 & & & 9214 & 9366 & 07 & & & 18 & 11 & 46960 \\
\hline 28 & 14 & 5945 & 0 & & 91482 & & & & & & & 1845 \\
\hline 29 & 717648 & 2858828 & 269 & & --- & 2917927 & 42132 & 4 & 3432814 & 7721 & 29 & 2435765 \\
\hline 30 & 717386 & 2889913 & 269 & & --- & 2928689 & 46589 & 34439 & 3419375 & 28 & 89208 & 2427271 \\
\hline 31 & 2721845 & --- & 2687876 & 2767779 & --- & 2941141 & --- & & & 65217 & 77812 & \\
\hline & & & & & & & & & & & & 9990 \\
\hline MIN & 2717386 & 2724076 & 2686446 & 2689958 & 2724864 & 2787878 & 2950990 & 3261626 & 19375 & 55217 & $\$ 77812$ & 2427271 \\
\hline $\mathrm{a}$ & 843.48 & 856.02 & 840.838 & 846.96 & 848.74 & 859.74 & 881.02 & 894.70 & 892.43 & 854.21 & 832.30 & 820.14 \\
\hline b & -109640 & +168068 & -202037 & +79903 & +23703 & +149659 & +305448 & +207930 & -35144 & -554158 & -287405 & -150541 \\
\hline C & 5945 & 1706 & 1442 & 901 & 1032 & 2334 & 5064 & & 9594 & 11085 & 9184 & 7692 \\
\hline $\mathrm{d}$ & 256900 & 166800 & 652200 & 433900 & 741300 & 505700 & 253600 & 328900 & 296000 & 717200 & 456400 & 245100 \\
\hline
\end{tabular}

CAL YR 1998 b +4637504

WTR YR 1999 b -404214

ANNUAL DIVERSION (AC-FT) CAL YR 1998 d 6078000

ANNUAL DIVERSION (AC-FT) WTR YR 1999 d 5054000

a Gage height, in feet, at end of month.

b Change in contents, in acre-feet.

c Total evaporation, in acre-feet, provided by California Department of Water Resources; not reviewed by the U.S. Geological Survey.

d Diversion, in acre-feet, to Edward Hyatt Powerplant, provided by California Department of Water Resources. 


\section{PALERMO CANAL NEAR OROVILLE, CA}

LOCATION.-Lat 39³1'59", long 121²8'54", in SW 1/4 SW 1/4 sec.1, T.19 N., R.4 E., Butte County, Hydrologic Unit 18020106, on right bank $50 \mathrm{ft}$ downstream from Oroville Dam and $4.4 \mathrm{mi}$ east of Oroville.

PERIOD OF RECORD.—Apri1 1965 to current year. Daily discharge records of diversion from Kelly Ridge Penstock for period April 1965 to October 1968, when Kelly Ridge Penstock supplied the entire flow of Palermo Canal, are in files of the U.S. Geological Survey.

GAGE.-Water-stage recorder and Parshall flume. Datum of gage is $547.67 \mathrm{ft}$ above sea level (levels by California Department of Water Resources). April 1965 to October 1968, water-stage recorder and Parshall flume at site of diversion from Kelly Ridge Penstock, $0.4 \mathrm{mi}$ downstream at different datum.

REMARKS.-Canal diverts from left end of Oroville Dam. Water is used for irrigation near Oroville. During period of construction of Oroville Dam, water was released from Kelly Ridge Penstock to meet irrigation requirements. See schematic diagram showing diversions and storage from Feather River at Lake Oroville.

COOPERATION.-Records were provided by California Department of Water Resources, under general supervision of the U.S. Geological Survey, in connection with a Federal Energy Regulatory Commission project.

EXTREMES FOR PERIOD OF RECORD.-Maximum daily discharge, $28 \mathrm{ft}^{3} / \mathrm{s}$, several days during July to September 1967; no flow at times in some years.

DISCHARGE, CUBIC FEET PER SECOND, WATER YEAR OCTOBER 1998 TO SEPTEMBER 1999 DAILY MEAN VALUES

\begin{tabular}{|c|c|c|c|c|c|c|c|c|c|c|c|c|}
\hline DAY & $\mathrm{OCT}$ & NOV & $\mathrm{DEC}$ & JAN & FEB & MAR & APR & MAY & JUN & JUL & AUG & SEP \\
\hline 1 & 20 & 10 & 1.7 & 2.8 & 2.8 & 2.8 & 2.9 & 7.1 & 18 & 20 & 19 & 19 \\
\hline 2 & 20 & 10 & 1.7 & 2.8 & 2.8 & 2.8 & 2.9 & 7.1 & 18 & 20 & 19 & 19 \\
\hline 3 & 20 & 10 & 1.7 & 2.8 & 2.8 & 2.9 & 2.9 & 7.1 & 18 & 20 & 19 & 19 \\
\hline 4 & 20 & 8.1 & 1.7 & 2.8 & 2.8 & 2.9 & 3.0 & 7.1 & 18 & 20 & 19 & 19 \\
\hline 5 & 20 & 7.1 & 1.7 & 2.7 & 2.8 & 3.1 & 3.0 & 9.0 & 18 & 20 & 19 & 19 \\
\hline 6 & 20 & 7.1 & 1.7 & 2.8 & 2.8 & 3.1 & 3.0 & 9.9 & 18 & 20 & 19 & 19 \\
\hline 7 & 20 & 7.1 & 1.7 & 2.7 & 2.8 & 3.1 & 3.0 & 13 & 18 & 20 & 19 & 19 \\
\hline 8 & 20 & 7.1 & 1.7 & 2.7 & 2.8 & 3.0 & 3.0 & 15 & 18 & 20 & 19 & 19 \\
\hline 9 & 20 & 5.8 & 1.7 & 2.7 & 2.8 & 3.1 & 3.0 & 15 & 18 & 20 & 19 & 19 \\
\hline 10 & 20 & 5.1 & 2.4 & 2.7 & 2.9 & 3.1 & 3.0 & 15 & 18 & 20 & 19 & 19 \\
\hline 11 & 20 & 5.1 & 3.0 & 2.7 & 2.9 & 3.1 & 3.0 & 15 & 18 & 20 & 19 & 19 \\
\hline 12 & 20 & 5.1 & 3.0 & 2.7 & 2.9 & 3.2 & 3.0 & 15 & 18 & 19 & 19 & 19 \\
\hline 13 & 17 & 4.9 & 3.0 & 2.7 & 2.9 & 3.0 & 3.0 & 16 & 18 & 19 & 19 & 19 \\
\hline 14 & 15 & 5.0 & 2.9 & 2.7 & 2.9 & 2.8 & 3.0 & 17 & 18 & 19 & 19 & 19 \\
\hline 15 & 15 & 5.1 & 2.9 & 2.7 & 2.8 & 2.9 & 3.0 & 17 & 18 & 19 & 19 & 19 \\
\hline 16 & 15 & 5.1 & 2.9 & 2.7 & 2.8 & 2.9 & 3.0 & 17 & 18 & 19 & 19 & 19 \\
\hline 17 & 15 & 5.1 & 2.8 & 2.7 & 2.9 & 2.9 & 3.0 & 17 & 18 & 19 & 19 & 19 \\
\hline 18 & 15 & 5.1 & 2.9 & 2.7 & 2.9 & 2.9 & 3.0 & 17 & 18 & 19 & 19 & 19 \\
\hline 19 & 15 & 5.1 & 2.9 & 2.8 & 2.9 & 2.8 & 3.0 & 17 & 18 & 19 & 19 & 19 \\
\hline 20 & 15 & 5.1 & 2.9 & 2.8 & 2.9 & 2.7 & 3.0 & 17 & 18 & 19 & 19 & 19 \\
\hline 21 & 14 & 5.2 & 2.8 & 2.8 & 2.9 & 2.7 & 3.9 & 18 & 18 & 19 & 19 & 19 \\
\hline 22 & 13 & 5.1 & 2.8 & 2.8 & 2.9 & 2.7 & 4.3 & 18 & 18 & 19 & 19 & 18 \\
\hline 23 & 13 & 4.9 & 2.8 & 2.8 & 2.8 & 2.7 & 4.3 & 18 & 18 & 19 & 19 & 18 \\
\hline 24 & 13 & 4.9 & 2.8 & 2.8 & 2.9 & 2.6 & 4.3 & 18 & 18 & 19 & 19 & 18 \\
\hline 25 & 13 & 5.0 & 2.8 & 2.8 & 2.9 & .00 & 3.8 & 18 & 18 & 19 & 19 & 18 \\
\hline 26 & 13 & 5.0 & 2.8 & 2.8 & 2.9 & 1.1 & 3.6 & 18 & 18 & 19 & 19 & 18 \\
\hline 27 & 13 & 5.1 & 2.8 & 2.8 & 2.9 & 2.7 & 3.7 & 18 & 18 & 19 & 19 & 18 \\
\hline 28 & 11 & 5.1 & 2.8 & 2.8 & 2.8 & 2.7 & 3.7 & 18 & 18 & 19 & 19 & 18 \\
\hline 29 & 10 & 5.1 & 2.8 & 2.8 & --- & 2.0 & 3.7 & 18 & 19 & 19 & 19 & 18 \\
\hline 30 & 10 & 2.8 & 2.8 & 2.8 & --- & 2.9 & 5.9 & 18 & 20 & 19 & 19 & 18 \\
\hline 31 & 10 & --- & 2.8 & 2.8 & --- & 2.9 & --- & 18 & --- & 19 & 19 & --- \\
\hline TOTAL & 495 & 176.3 & 77.7 & 85.5 & 79.9 & 84.10 & 100.9 & 468.3 & 543 & 600 & 589 & 561 \\
\hline MEAN & 16.0 & 5.88 & 2.51 & 2.76 & 2.85 & 2.71 & 3.36 & 15.1 & 18.1 & 19.4 & 19.0 & 18.7 \\
\hline MAX & 20 & 10 & 3.0 & 2.8 & 2.9 & 3.2 & 5.9 & 18 & 20 & 20 & 19 & 19 \\
\hline MIN & 10 & 2.8 & 1.7 & 2.7 & 2.8 & .00 & 2.9 & 7.1 & 18 & 19 & 19 & 18 \\
\hline$A C-F T$ & 982 & 350 & 154 & 170 & 158 & 167 & 200 & 929 & 1080 & 1190 & 1170 & 1110 \\
\hline
\end{tabular}

STATISTICS OF MONTHLY MEAN DATA FOR WATER YEARS 1969 - 1999, BY WATER YEAR (WY)

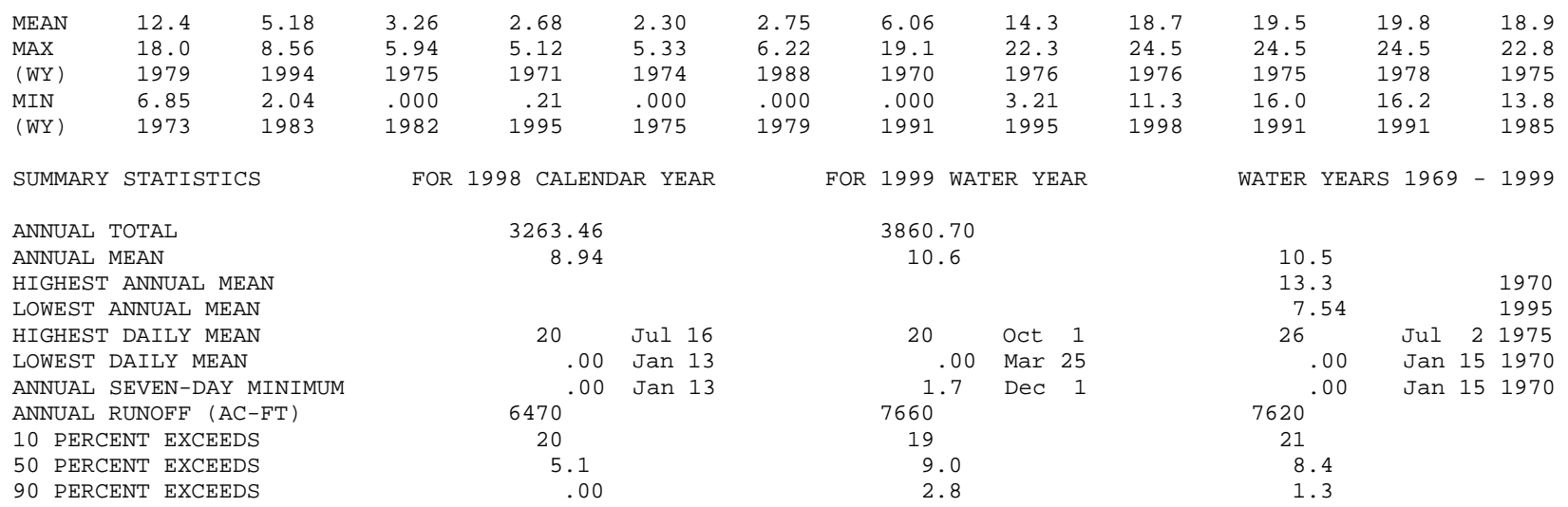


LOCATION.-Lat 39²7’30", long 121³8'17", in NE 1/4 SE 1/4 sec.33, T.19 N., R.3 E., Butte County, Hydrologic Unit 18020106, at dam 195 ft northeast of centerline of outlet structure and $5.7 \mathrm{mi}$ southwest of Oroville.

PERIOD OF RECORD.-October 1967 to current year.

GAGE.-Water-stage recorder. Datum of gage is $100.47 \mathrm{ft}$ above sea level (levels by California Department of Water Resources). Auxiliary waterstage recorder $90 \mathrm{ft}$ southwest of centerline of Western Canal outlet and $7.2 \mathrm{mi}$ west of Oroville.

REMARKS.-Reservoir is formed by an earthfill dam completed in 1967. Diversion from the reservoir began Oct. 12, 1967. Usable capacity, 61,144 acre-ft between gage heights 120.0 and $139.0 \mathrm{ft}$, extreme operating levels. Normal operating range is from 123 to $136.5 \mathrm{ft}$. Water is released to four canals (stations 11406880, 11406890, 11406900, and 11406910) and to the Feather River (station 11406920) from the reservoir. Total maximum release to the four canals is approximately 4,000 $\mathrm{ft}^{3} / \mathrm{s}$. Water is pumped, at times, from Thermalito Afterbay back into Thermalito Forebay (station 11406840) during off-peak periods to be re-released through Thermalito Powerplant (station 11406850) for power generation during peak-demand periods. Records, including extremes, represent total contents at 2400 hours. See schematic diagram showing diversions and storage from Feather River at Lake Oroville.

COOPERATION.-Records were collected by California Department of Water Resources, under general supervision of the U.S. Geological Survey, in connection with a Federal Energy Regulatory Commission project. Contents not rounded to U.S. Geological Survey standards.

EXTREMES FOR PERIOD OF RECORD.-Maximum contents, 57,300 acre-ft, May 24, 1969, gage height, $136.56 \mathrm{ft}$; minimum since initial operation began, 5,590 acre-ft, Mar. 1, 1968, gage height, $119.09 \mathrm{ft}$.

EXTREMES FOR CURRENT YEAR.-Maximum contents, 50,331 acre-ft, Apr. 16, gage height, $134.90 \mathrm{ft}$; minimum, 19,826 acre-ft, Dec. 14, gage height, $125.87 \mathrm{ft}$.

Capacity table (gage height, in feet, and contents, in acre-feet)

(Based on table provided by California Department of Water Resources, dated Oct. 10, 1968)

$\begin{array}{rrrrrr}119 & 5,465 & 124 & 15,157 & 130 & 32,150 \\ 120 & 7,054 & 126 & 20,171 & 134 & 46,719 \\ 122 & 10,792 & 128 & 25,832 & 139 & 68,198\end{array}$

RESERVOIR STORAGE (ACRE-FEET), WATER YEAR OCTOBER 1998 TO SEPTEMBER 1999 DAILY OBSERVATION AT 2400 HOURS

\begin{tabular}{|c|c|c|c|c|c|c|c|c|c|c|c|c|}
\hline DAY & OCT & NOV & $\mathrm{DEC}$ & JAN & FEB & MAR & APR & MAY & JUN & JUL & AUG & SEP \\
\hline 1 & 37998 & 34346 & 38106 & 26433 & 27534 & 28531 & 43644 & 35556 & 31260 & 29449 & 26707 & 31589 \\
\hline 2 & 41897 & 31064 & 34038 & 24383 & 28062 & 34758 & 44568 & 25264 & 35940 & 30672 & 27937 & 32019 \\
\hline 3 & 34966 & 30478 & 31556 & 22327 & 29385 & 41185 & 39705 & 27411 & 39778 & 30737 & 30380 & 31589 \\
\hline 4 & 24412 & 29545 & 28720 & 24441 & 33122 & 43951 & 35625 & 29067 & 43452 & 32819 & 29929 & 33392 \\
\hline 5 & 27906 & 30770 & 28374 & 27906 & 36397 & 43912 & 36998 & 35625 & 38938 & 32786 & 31457 & 31227 \\
\hline 6 & 29865 & 30966 & 28374 & 29194 & 37460 & 44028 & 39705 & 41148 & 33291 & 30025 & 31556 & 26798 \\
\hline 7 & 33358 & 29672 & 28531 & 29801 & 37496 & 44298 & 42918 & 45658 & 34346 & 29897 & 28155 & 31293 \\
\hline 8 & 36432 & 27073 & 27534 & 30510 & 38322 & 44568 & 43797 & 36256 & 35625 & 27906 & 22299 & 37461 \\
\hline 9 & 40036 & 28625 & 25801 & 32585 & 39156 & 41972 & 45854 & 24704 & 37747 & 31064 & 26798 & 42804 \\
\hline 10 & 35451 & 31031 & 25741 & 31919 & 38575 & 41972 & 40257 & 24646 & 42463 & 32752 & 30900 & 45932 \\
\hline 11 & 30025 & 31787 & 23401 & 32485 & 37247 & 39375 & 34586 & 28657 & 43605 & 30770 & 32052 & 42463 \\
\hline 12 & 33426 & 33257 & 22946 & 33122 & 36221 & 37926 & 35695 & 33494 & 36185 & 32385 & 34586 & 33156 \\
\hline 13 & 37175 & 34758 & 22974 & 32954 & 35277 & 44220 & 37782 & 40812 & 29321 & 33528 & 40331 & 36679 \\
\hline 14 & 42766 & 31886 & 19826 & 34517 & 34243 & 34896 & 42160 & 45269 & 30672 & 33021 & 35347 & 37318 \\
\hline 15 & 44105 & 30998 & 20600 & 35800 & 33358 & 37890 & 47353 & 40072 & 32485 & 32719 & 27288 & 37318 \\
\hline 16 & 45619 & 33392 & 20897 & 31820 & 33629 & 41148 & 50331 & 31391 & 35695 & 33257 & 27380 & 37998 \\
\hline 17 & 40738 & 36115 & 22351 & 25651 & 31820 & 41897 & 45776 & 35208 & 40405 & 36714 & 28594 & 38034 \\
\hline 18 & 35277 & 37854 & 22748 & 22188 & 32285 & 42010 & 39120 & 37890 & 44995 & 38466 & 29704 & 33799 \\
\hline 19 & 33257 & 40887 & 20279 & 22216 & 32118 & 40924 & 38322 & 41222 & 39705 & 35556 & 29897 & 21524 \\
\hline 20 & 31721 & 41859 & 21278 & 24558 & 32652 & 32987 & 38430 & 43644 & 33325 & 36679 & 33223 & 23344 \\
\hline 21 & 29004 & 40553 & 28000 & 22077 & 32585 & 25057 & 40183 & 43720 & 35625 & 35139 & 29226 & 26373 \\
\hline 22 & 25771 & 37353 & 31064 & 23602 & 32418 & 27196 & 42918 & 36962 & 36856 & 36750 & 28500 & 28563 \\
\hline 23 & 26828 & 37425 & 34209 & 24675 & 32351 & 31391 & 38647 & 31688 & 40924 & 36573 & 29385 & 32451 \\
\hline 24 & 25891 & 38034 & 32351 & 25472 & 31886 & 42311 & 32585 & 35277 & 40961 & 31260 & 35765 & 35625 \\
\hline 25 & 34209 & 35451 & 27442 & 26011 & 31391 & 47711 & 25443 & 37247 & 42690 & 24998 & 41334 & 34380 \\
\hline 26 & 31622 & 31787 & 25353 & 26464 & 30542 & 49802 & 27288 & 39631 & 38070 & 25146 & 45345 & 26252 \\
\hline 27 & 33528 & 29641 & 22439 & 26646 & 26828 & 48070 & 31985 & 41822 & 32418 & 26252 & 45424 & 26524 \\
\hline 28 & 37282 & 26373 & 23373 & 28000 & 25532 & 43912 & 37175 & 46129 & 29067 & 29449 & 36538 & 24529 \\
\hline 29 & 38974 & 24005 & 24412 & 29194 & --- & 43223 & 43337 & 39266 & 28783 & 32152 & 27504 & 23717 \\
\hline 30 & 39412 & 32451 & 25861 & 30933 & --- & 43567 & 42576 & 32853 & 28062 & 30445 & 27380 & 27012 \\
\hline 31 & 36045 & --- & 29131 & 28688 & --- & 42501 & --- & 26433 & --- & 30478 & 29823 & --- \\
\hline MAX & 45619 & 41859 & 38106 & 35800 & 39156 & 49802 & 50331 & 46129 & 44995 & 38466 & 45424 & 45932 \\
\hline MIN & 24412 & 24005 & 19826 & 22077 & 25532 & 25057 & 25443 & 24646 & 28062 & 24998 & 22299 & 21524 \\
\hline$a$ & 131.14 & 130.09 & 129.07 & 128.93 & 127.90 & 132.91 & 132.93 & 128.20 & 128.73 & 129.49 & 129.29 & 128.39 \\
\hline $\mathrm{b}$ & +2075 & -3594 & -3320 & -443 & -3156 & +16969 & +75 & -16143 & +1629 & +2416 & -655 & -2811 \\
\hline C & 1329 & 508 & 325 & 184 & 275 & 647 & 1473 & 1981 & 2132 & 2271 & 1975 & 1662 \\
\hline d & 233400 & 149800 & 644200 & 420000 & 718900 & 470500 & 235000 & 811000 & 279500 & 702900 & 437900 & 219200 \\
\hline
\end{tabular}

CAL YR 1998 b -7760

WTY YR 1999 b -6958

ANNUAL DIVERSION (AC-FT) CAL YR 1998 d 5908000

ANNUAL DIVERSION (AC-FT) WTR YR 1999 d 5322000

a Gage height, in feet, at end of month.

b Change in contents, in acre-feet.

c Total evaporation, in acre-feet, provided by California Department of Water Resources; not reviewed by the U.S. Geological Survey.

d Diversion, in acre-feet, to Thermalito Powerplant, provided by California Department of Water Resources. 
LOCATION.-Lat 39³0'19", long 121 $41^{\prime} 06^{\prime \prime}$, in SW 1/4 NW 1/4 sec.18, T.19 N., R.3 E., Butte County, Hydrologic Unit 18020105, on left bank $500 \mathrm{ft}$ downstream from Thermalito Afterbay Dam and $7.3 \mathrm{mi}$ west of Oroville.

PERIOD OF RECORD.-October 1967 to current year.

GAGE.-Water-stage recorder. Datum of gage is $100.47 \mathrm{ft}$ above sea level (levels by California Department of Water Resources).

REMARKS.-Water is diverted from Thermalito Afterbay (station 11406870) and is used for irrigation. See schematic diagram showing diversions and storage from Feather River at Lake Oroville.

COOPERATION.-Records collected by California Department of Water Resources, under general supervision of the U.S. Geological Survey, in connection with a Federal Energy Regulatory Commission project.

EXTREMES FOR PERIOD OF RECORD.-Maximum daily discharge, 1,210 ft³/s, May 18, 1999; no flow at times each year.

DISCHARGE, CUBIC FEET PER SECOND, WATER YEAR OCTOBER 1998 TO SEPTEMBER 1999 DAILY MEAN VALUES

\begin{tabular}{|c|c|c|c|c|c|c|c|c|c|c|c|c|}
\hline DAY & OCT & NOV & $\mathrm{DEC}$ & JAN & FEB & MAR & APR & MAY & JUN & JUL & AUG & SEP \\
\hline 1 & 69 & 663 & 50 & 179 & .00 & .00 & .00 & 597 & 575 & 1080 & 905 & 489 \\
\hline 2 & 88 & 623 & 47 & 179 & .00 & .00 & .00 & 643 & 562 & 1050 & 905 & 448 \\
\hline 3 & 152 & 541 & 61 & 178 & .00 & .00 & .00 & 683 & 528 & 1040 & 905 & 411 \\
\hline 4 & 167 & 519 & 86 & 179 & .00 & .00 & .00 & 775 & 471 & 1040 & 884 & 363 \\
\hline 5 & 168 & 538 & 98 & 179 & .00 & .00 & .00 & 936 & 422 & 1030 & 870 & 316 \\
\hline 6 & 168 & 538 & 98 & 184 & .00 & .00 & .00 & 1110 & 399 & 1020 & 870 & 287 \\
\hline 7 & 168 & 538 & 155 & 188 & .00 & .00 & .00 & 1200 & 428 & 1030 & 858 & 278 \\
\hline 8 & 200 & 538 & 189 & 189 & .00 & .00 & .00 & 1200 & 495 & 1040 & 851 & 259 \\
\hline 9 & 237 & 538 & 159 & 188 & .00 & .00 & .00 & 1200 & 567 & 1040 & 849 & 235 \\
\hline 10 & 247 & 538 & 149 & 188 & .00 & .00 & .00 & 1200 & 628 & 1030 & 844 & 211 \\
\hline 11 & 256 & 538 & 148 & 65 & .00 & .00 & .00 & 1200 & 712 & 1030 & 830 & 181 \\
\hline 12 & 307 & 513 & 148 & .00 & .00 & .00 & .00 & 1200 & 794 & 1030 & 811 & 171 \\
\hline 13 & 318 & 498 & 147 & .00 & .00 & .00 & .00 & 1200 & 823 & 1040 & 791 & 164 \\
\hline 14 & 318 & 482 & 148 & .00 & .00 & .00 & .00 & 1200 & 824 & 1040 & 784 & 142 \\
\hline 15 & 326 & 473 & 149 & .00 & .00 & .00 & .00 & 1200 & 815 & 1040 & 783 & 121 \\
\hline 16 & 350 & 449 & 149 & .00 & .00 & .00 & .00 & 1200 & 799 & 1030 & 783 & 113 \\
\hline 17 & 358 & 409 & 151 & .00 & .00 & .00 & .00 & 1200 & 798 & 996 & 784 & 112 \\
\hline 18 & 358 & 398 & 150 & .00 & .00 & .00 & .00 & 1210 & 816 & 977 & 772 & 112 \\
\hline 19 & 383 & 399 & 149 & .00 & .00 & .00 & 52 & 1160 & 847 & 977 & 749 & 111 \\
\hline 20 & 485 & 345 & 149 & .00 & .00 & .00 & 97 & 1050 & 848 & 966 & 743 & 112 \\
\hline 21 & 614 & 298 & 154 & .00 & .00 & .00 & 98 & 959 & 851 & 946 & 743 & 112 \\
\hline 22 & 648 & 299 & 159 & .00 & .00 & .00 & 99 & 881 & 849 & 947 & 743 & 105 \\
\hline 23 & 648 & 245 & 160 & .00 & .00 & .00 & 97 & 792 & 877 & 953 & 719 & 97 \\
\hline 24 & 648 & 199 & 159 & .00 & .00 & .00 & 114 & 760 & 898 & 945 & 687 & 98 \\
\hline 25 & 648 & 179 & 158 & .00 & .00 & .00 & 139 & 773 & 944 & 938 & 678 & 98 \\
\hline 26 & 623 & 168 & 158 & .00 & .00 & .00 & 232 & 757 & 981 & 938 & 666 & 96 \\
\hline 27 & 631 & 169 & 158 & .00 & .00 & .00 & 307 & 687 & 997 & 938 & 636 & 122 \\
\hline 28 & 658 & 169 & 167 & .00 & .00 & .00 & 352 & 636 & 1030 & 937 & 605 & 176 \\
\hline 29 & 674 & 168 & 179 & .00 & --- & .00 & 398 & 602 & 1060 & 935 & 598 & 208 \\
\hline 30 & 680 & 120 & 179 & .00 & --- & .00 & 485 & 573 & 1080 & 934 & 573 & 217 \\
\hline 31 & 663 & --- & 179 & .00 & --- & .00 & --- & 573 & --- & 924 & 528 & --- \\
\hline TOTAL & 12258 & 12092 & 4390 & 1896.00 & 0.00 & 0.00 & 2470.00 & 29357 & 22718 & 30861 & 23747 & 5965 \\
\hline MEAN & 395 & 403 & 142 & 61.2 & .000 & .000 & 82.3 & 947 & 757 & 996 & 766 & 199 \\
\hline MAX & 680 & 663 & 189 & 189 & .00 & .00 & 485 & 1210 & 1080 & 1080 & 905 & 489 \\
\hline MIN & 69 & 120 & 47 & .00 & .00 & .00 & .00 & 573 & 399 & 924 & 528 & 96 \\
\hline $\mathrm{AC}-\mathrm{FT}$ & 24310 & 23980 & 8710 & 3760 & .00 & .00 & 4900 & 58230 & 45060 & 61210 & 47100 & 11830 \\
\hline
\end{tabular}

STATISTICS OF MONTHLY MEAN DATA FOR WATER YEARS 1968 - 1999, BY WATER YEAR (WY)

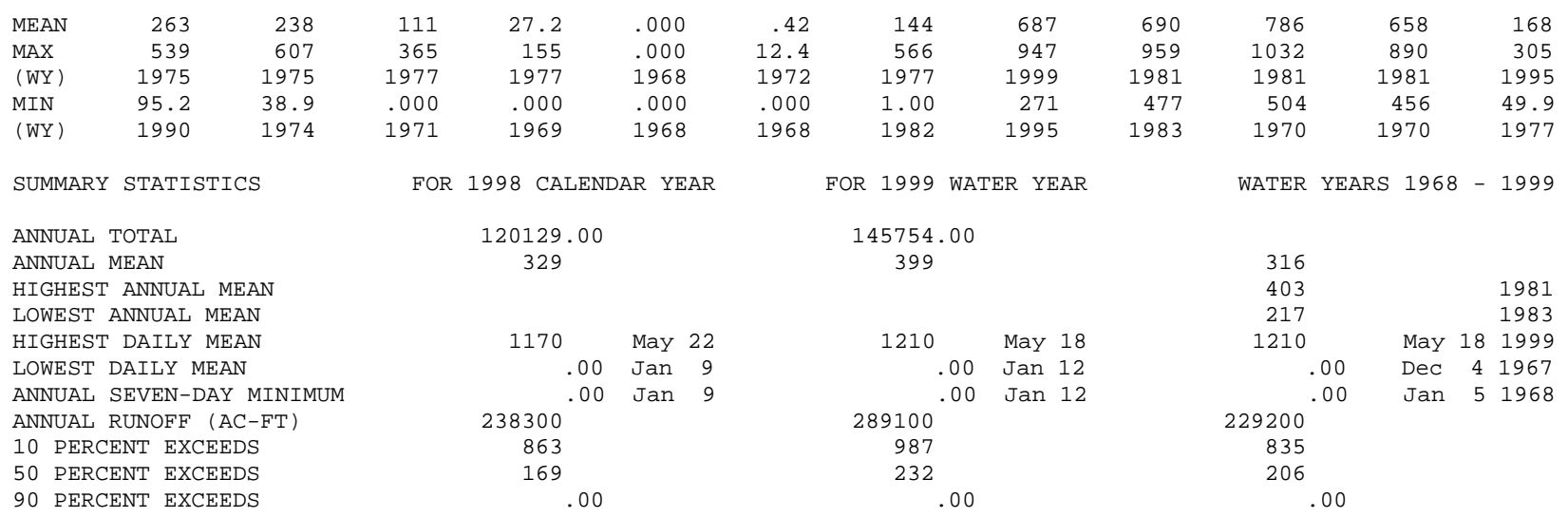


11406890 RICHVALE CANAL AT INTAKE, NEAR OROVILLE, CA

LOCATION.-Lat 39³0'19", long 12141'06", in SW 1/4 NW 1/4 sec.18, T.19 N., R.3 E., Butte County, Hydrologic Unit 18020105, on right bank $500 \mathrm{ft}$ downstream from axis of Thermalito Afterbay Dam and $7.3 \mathrm{mi}$ west of Oroville.

PERIOD OF RECORD.-April 1968 to current year.

REVISED RECORDS.-WDR CA-91-4: 1990.

GAGE.-Water-stage recorder. Datum of gage is $100.47 \mathrm{ft}$ above sea level (levels by California Department of Water Resources).

REMARKS.-Canal diverts from Thermalito Afterbay (station 11406870); water is used for irrigation. See schematic diagram showing diversions and storage from Feather River at Lake Oroville.

COOPERATION.--Records collected by California Department of Water Resources, under general supervision of the U.S. Geological Survey, in connection with a Federal Energy Regulatory Commission project.

EXTREMES FOR PERIOD OF RECORD.-Maximum daily discharge, $511 \mathrm{ft}^{3} / \mathrm{s}$, May 16, 1974; no flow for many days each year.

DISCHARGE, CUBIC FEET PER SECOND, WATER YEAR OCTOBER 1998 TO SEPTEMBER 1999

DAILY MEAN VALUES

\begin{tabular}{|c|c|c|c|c|c|c|c|c|c|c|c|c|}
\hline DAY & OCT & NOV & $\mathrm{DEC}$ & JAN & FEB & MAR & $A P R$ & MAY & JUN & JUL & AUG & SEP \\
\hline 1 & 32 & 238 & 220 & 232 & .00 & .00 & .00 & 320 & 249 & 443 & 437 & 281 \\
\hline 2 & 33 & 238 & 213 & 232 & .00 & .00 & .00 & 347 & 250 & 450 & 437 & 254 \\
\hline 3 & 30 & 239 & 213 & 232 & .00 & .00 & .00 & 349 & 237 & 454 & 436 & 243 \\
\hline 4 & 28 & 265 & 213 & 234 & .00 & .00 & .00 & 358 & 230 & 454 & 437 & 220 \\
\hline 5 & 27 & 278 & 214 & 234 & .00 & .00 & .00 & 376 & 230 & 454 & 436 & 201 \\
\hline 6 & 38 & 312 & 214 & 234 & .00 & .00 & .00 & 379 & 202 & 453 & 437 & 198 \\
\hline 7 & 53 & 328 & 226 & 233 & .00 & .00 & .00 & 378 & 190 & 453 & 437 & 199 \\
\hline 8 & 71 & 328 & 234 & 233 & .00 & .00 & .00 & 411 & 219 & 452 & 438 & 185 \\
\hline 9 & 95 & 328 & 232 & 234 & .00 & .00 & .00 & 448 & 230 & 452 & 435 & 151 \\
\hline 10 & 98 & 328 & 233 & 232 & .00 & .00 & .00 & 458 & 231 & 452 & 428 & 118 \\
\hline 11 & 98 & 329 & 232 & 234 & .00 & .00 & .00 & 458 & 260 & 458 & 425 & 102 \\
\hline 12 & 104 & 308 & 233 & 234 & .00 & .00 & .00 & 469 & 330 & 458 & 426 & 98 \\
\hline 13 & 115 & 281 & 232 & 234 & .00 & .00 & .00 & 473 & 358 & 456 & 428 & 98 \\
\hline 14 & 132 & 273 & 232 & 234 & .00 & .00 & .00 & 474 & 398 & 450 & 424 & 85 \\
\hline 15 & 139 & 273 & 233 & 71 & .00 & .00 & .00 & 473 & 418 & 445 & 409 & 78 \\
\hline 16 & 138 & 274 & 233 & .00 & .00 & .00 & .00 & 473 & 418 & 446 & 403 & 69 \\
\hline 17 & 139 & 274 & 234 & .00 & .00 & .00 & .00 & 474 & 419 & 446 & 396 & 64 \\
\hline 18 & 138 & 246 & 235 & .00 & .00 & .00 & .00 & 443 & 418 & 439 & 393 & 52 \\
\hline 19 & 139 & 233 & 234 & .00 & .00 & .00 & 15 & 393 & 428 & 438 & 393 & 40 \\
\hline 20 & 139 & 234 & 234 & .00 & .00 & .00 & 70 & 379 & 425 & 437 & 393 & 35 \\
\hline 21 & 139 & 233 & 234 & .00 & .00 & .00 & 112 & 358 & 420 & 436 & 393 & 20 \\
\hline 22 & 170 & 233 & 234 & .00 & .00 & .00 & 124 & 310 & 419 & 435 & 380 & 10 \\
\hline 23 & 184 & 234 & 234 & .00 & .00 & .00 & 141 & 283 & 436 & 433 & 374 & 17 \\
\hline 24 & 183 & 233 & 233 & .00 & .00 & .00 & 140 & 278 & 443 & 433 & 374 & 23 \\
\hline 25 & 183 & 233 & 233 & .00 & .00 & .00 & 138 & 288 & 444 & 432 & 374 & 21 \\
\hline 26 & 183 & 233 & 233 & .00 & .00 & .00 & 186 & 294 & 443 & 433 & 353 & 18 \\
\hline 27 & 197 & 234 & 233 & .00 & .00 & .00 & 215 & 350 & 443 & 432 & 344 & 18 \\
\hline 28 & 204 & 233 & 233 & .00 & .00 & .00 & 214 & 406 & 443 & 431 & 343 & 19 \\
\hline 29 & 204 & 233 & 233 & .00 & --- & .00 & 231 & 390 & 443 & 431 & 322 & 20 \\
\hline 30 & 227 & 234 & 234 & .00 & --- & .00 & 258 & 320 & 443 & 431 & 314 & 21 \\
\hline 31 & 239 & --- & 234 & .00 & --- & .00 & --- & 262 & --- & 433 & 297 & --- \\
\hline TOTAL & 3899 & 7940 & 7112 & 3337.00 & 0.00 & 0.00 & 1844.00 & 11872 & 10517 & 13750 & 12316 & 2958 \\
\hline MEAN & 126 & 265 & 229 & 108 & .000 & .000 & 61.5 & 383 & 351 & 444 & 397 & 98.6 \\
\hline MAX & 239 & 329 & 235 & 234 & .00 & .00 & 258 & 474 & 444 & 458 & 438 & 281 \\
\hline MIN & 27 & 233 & 213 & .00 & .00 & .00 & .00 & 262 & 190 & 431 & 297 & 10 \\
\hline $\mathrm{AC}-\mathrm{FT}$ & 7730 & 15750 & 14110 & 6620 & .00 & .00 & 3660 & 23550 & 20860 & 27270 & 24430 & 5870 \\
\hline
\end{tabular}

STATISTICS OF MONTHLY MEAN DATA FOR WATER YEARS 1968 - 1999, BY WATER YEAR (WY)

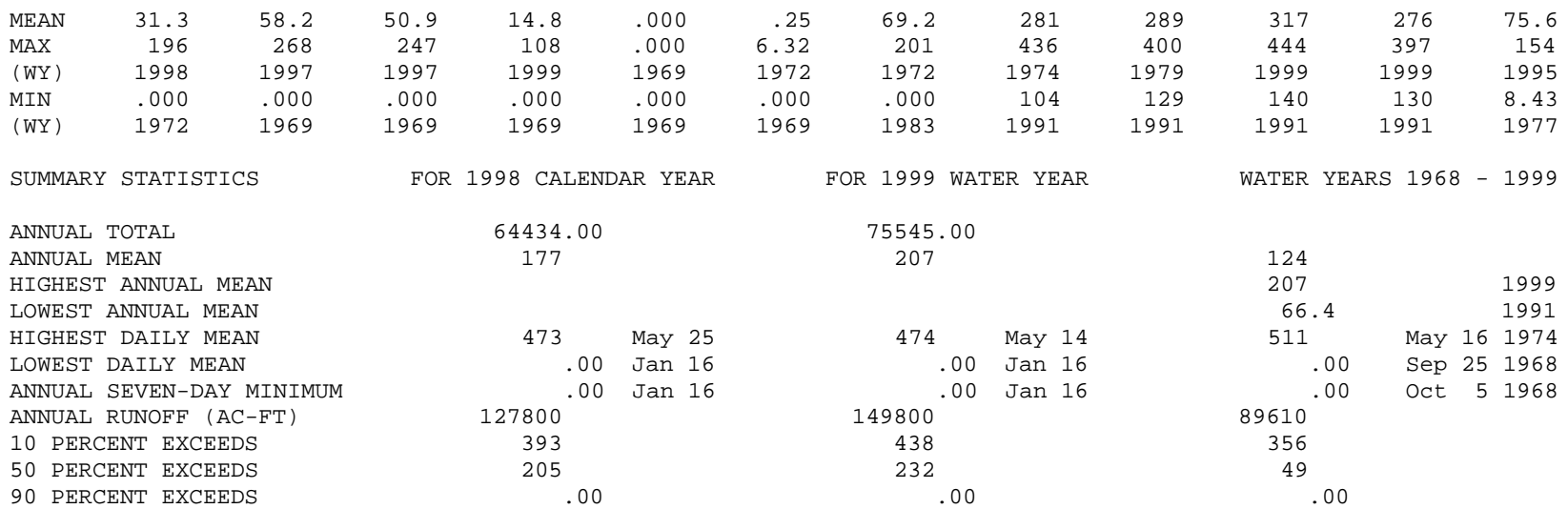




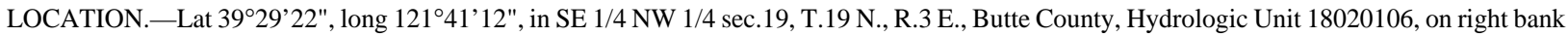
$82 \mathrm{ft}$ downstream from axis of Thermalito Afterbay Dam and $7.2 \mathrm{mi}$ west of Oroville.

PERIOD OF RECORD.-April 1968 to current year.

GAGE.-Water-stage recorder. Datum of gage is $113.47 \mathrm{ft}$ above sea level (levels by California Department of Water Resources).

REMARKS.-Flow regulated at outlet works from Thermalito Afterbay (station 11406870); water is used for irrigation. See schematic diagram showing diversions and storage from Feather River at Lake Oroville.

COOPERATION.-Records collected by California Department of Water Resources, under general supervision of the U.S. Geological Survey, in connection with a Federal Energy Regulatory Commission project.

EXTREMES FOR PERIOD OF RECORD.-Maximum daily discharge, $46 \mathrm{ft}^{3}$ /s, Apr. 24, 1977, May 16, 1978; no flow for many days each year.

DISCHARGE, CUBIC FEET PER SECOND, WATER YEAR OCTOBER 1998 TO SEPTEMBER 1999 DAILY MEAN VALUES

\begin{tabular}{|c|c|c|c|c|c|c|c|c|c|c|c|c|}
\hline DAY & $\mathrm{OCT}$ & NOV & $\mathrm{DEC}$ & JAN & FEB & MAR & APR & MAY & JUN & JUL & AUG & SEP \\
\hline 1 & .00 & 22 & .00 & 1.3 & .00 & .00 & .00 & 33 & 4.5 & 15 & 16 & 11 \\
\hline 2 & .00 & 23 & .00 & 1.3 & .00 & .00 & .00 & 38 & 1.9 & 15 & 16 & 9.5 \\
\hline 3 & .00 & 25 & .00 & 1.2 & .00 & .00 & .00 & 29 & 2.0 & 14 & 16 & 7.5 \\
\hline 4 & .00 & 25 & .00 & 1.1 & .00 & .00 & .00 & 26 & 2.0 & 15 & 16 & 6.2 \\
\hline 5 & .00 & 11 & .00 & 1.2 & .00 & .00 & .00 & 25 & 2.1 & 15 & 16 & 6.2 \\
\hline 6 & .00 & 3.4 & .00 & 1.3 & .00 & .00 & .00 & 25 & 2.0 & 15 & 16 & 5.9 \\
\hline 7 & .00 & 3.4 & .63 & 1.3 & .00 & .00 & .00 & 19 & 1.9 & 15 & 16 & 5.1 \\
\hline 8 & .00 & 3.4 & 1.0 & 1.3 & .00 & .00 & .00 & 14 & 3.7 & 16 & 16 & 2.4 \\
\hline 9 & .00 & 3.3 & 1.0 & 1.3 & .00 & .00 & .00 & 15 & 5.6 & 17 & 16 & .00 \\
\hline 10 & .00 & 3.7 & 1.0 & 1.3 & .00 & .00 & .00 & 18 & 8.8 & 18 & 16 & .00 \\
\hline 11 & .00 & 4.0 & 1.0 & .43 & .00 & .00 & .00 & 20 & 9.9 & 18 & 15 & .00 \\
\hline 12 & .00 & 2.9 & 1.0 & .00 & .00 & .00 & .00 & 13 & 9.5 & 18 & 15 & .00 \\
\hline 13 & .00 & 2.0 & 1.0 & .00 & .00 & .00 & .00 & 7.3 & 9.7 & 18 & 15 & .00 \\
\hline 14 & .00 & 2.0 & .90 & .00 & .00 & .00 & .00 & 5.6 & 12 & 18 & 14 & .00 \\
\hline 15 & .00 & 2.0 & .90 & .00 & .00 & .00 & .00 & 5.5 & 13 & 18 & 14 & .00 \\
\hline 16 & .00 & 2.0 & .90 & .00 & .00 & .00 & .00 & 5.7 & 12 & 18 & 15 & .00 \\
\hline 17 & .00 & 2.0 & 1.5 & .00 & .00 & .00 & .00 & 5.5 & 20 & 18 & 15 & .00 \\
\hline 18 & .00 & 2.2 & 1.9 & .00 & .00 & .00 & .00 & 7.4 & 28 & 18 & 15 & .00 \\
\hline 19 & .00 & 2.2 & 1.9 & .00 & .00 & .00 & .00 & 10 & 30 & 18 & 15 & .00 \\
\hline 20 & .00 & 2.2 & 1.9 & .00 & .00 & .00 & .00 & 11 & 28 & 17 & 15 & .00 \\
\hline 21 & .00 & 2.2 & 2.0 & .00 & .00 & .00 & .00 & 9.1 & 26 & 17 & 15 & .00 \\
\hline 22 & .00 & 2.2 & 2.1 & .00 & .00 & .00 & .00 & 7.8 & 26 & 17 & 15 & .00 \\
\hline 23 & .00 & 2.2 & 1.3 & .00 & .00 & .00 & .00 & 7.6 & 23 & 17 & 15 & .00 \\
\hline 24 & .00 & 2.2 & .69 & .00 & .00 & .00 & .00 & 7.4 & 19 & 17 & 15 & .00 \\
\hline 25 & .00 & 2.2 & .91 & .00 & .00 & .00 & .00 & 8.1 & 16 & 17 & 15 & .00 \\
\hline 26 & .00 & 2.1 & 1.3 & .00 & .00 & .00 & .00 & 7.4 & 16 & 17 & 15 & .00 \\
\hline 27 & .00 & 2.0 & 1.2 & .00 & .00 & .00 & .00 & 7.1 & 16 & 17 & 15 & .00 \\
\hline 28 & .00 & 2.0 & 1.1 & .00 & .00 & .00 & 13 & 8.0 & 16 & 17 & 14 & .00 \\
\hline 29 & 8.4 & 1.9 & 1.1 & .00 & --- & .00 & 19 & 8.2 & 15 & 17 & 14 & .00 \\
\hline 30 & 19 & 1.1 & 1.2 & .00 & --- & .00 & 23 & 7.6 & 15 & 16 & 14 & .00 \\
\hline 31 & 22 & --- & 1.3 & .00 & --- & .00 & --- & 7.6 & --- & 16 & 13 & --- \\
\hline TOTAL & 49.40 & 166.8 & 30.73 & 13.03 & 0.00 & 0.00 & 55.00 & 418.9 & 394.6 & 519 & 468 & 53.80 \\
\hline MEAN & 1.59 & 5.56 & .99 & .42 & .000 & .000 & 1.83 & 13.5 & 13.2 & 16.7 & 15.1 & 1.79 \\
\hline MAX & 22 & 25 & 2.1 & 1.3 & .00 & .00 & 23 & 38 & 30 & 18 & 16 & 11 \\
\hline MIN & .00 & 1.1 & .00 & .00 & .00 & .00 & .00 & 5.5 & 1.9 & 14 & 13 & .00 \\
\hline $\mathrm{AC}-\mathrm{FT}$ & 98 & 331 & 61 & 26 & .00 & .00 & 109 & 831 & 783 & 1030 & 928 & 107 \\
\hline
\end{tabular}

STATISTICS OF MONTHLY MEAN DATA FOR WATER YEARS 1968 - 1999, BY WATER YEAR (WY)

\begin{tabular}{|c|c|c|c|c|c|c|c|c|c|c|c|c|}
\hline MEAN & .33 & 1.80 & .68 & .077 & .000 & .000 & 3.59 & 12.7 & 12.4 & 13.5 & 10.8 & 1.26 \\
\hline MAX & 3.47 & 6.58 & 3.49 & .51 & .000 & .000 & 14.8 & 23.2 & 18.3 & 17.1 & 15.1 & 2.62 \\
\hline (WY) & 1997 & 1996 & 1987 & 1994 & 1969 & 1969 & 1977 & 1975 & 1981 & 1981 & 1999 & 1972 \\
\hline MIN & .000 & .000 & .000 & .000 & .000 & .000 & .000 & 6.55 & 8.40 & 9.37 & 7.12 & .000 \\
\hline (WY) & 1969 & 1969 & 1969 & 1969 & 1969 & 1969 & 1974 & 1994 & 1998 & 1970 & 1988 & 1994 \\
\hline
\end{tabular}

SUMMARY STATISTICS

ANNUAL TOTAL

ANNUAL MEAN

HIGHEST ANNUAL MEAN

LOWEST ANNUAL MEAN

HIGHEST DAILY MEAN

LOWEST DAILY MEAN

ANNUAL SEVEN-DAY MINIMUM

ANNUAL RUNOFF (AC-FT)

10 PERCENT EXCEEDS

50 PERCENT EXCEEDS

90 PERCENT EXCEEDS
FOR 1998 CALENDAR YEAR

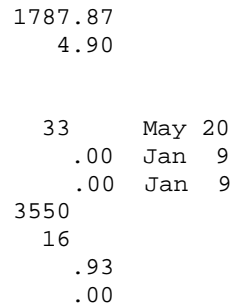

FOR 1999 WATER YEAR

2169.26

$$
5.94
$$$$
38 \text { May } 2
$$$$
\begin{array}{rrr}
.00 & \text { Oct } 1 \\
.00 & \text { Oct } & 1
\end{array}
$$$$
4300
$$$$
17
$$$$
\begin{array}{r}
1.2 \\
.00
\end{array}
$$

WATER YEARS 1968 - 1999

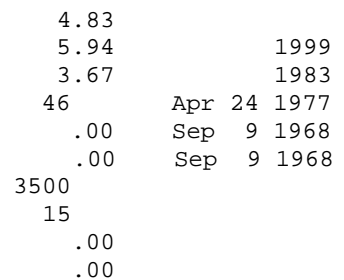


11406910 SUTTER-BUTTE CANAL AT INTAKE, NEAR OROVILLE, CA

LOCATION.-Lat 39²7’01", long 121³9’27", in NW corner of Boga Fernandez Grant, T.18 N., R.3 E., Butte County, Hydrologic Unit 18020105, on left bank $675 \mathrm{ft}$ downstream from Thermalito Afterbay Dam and 6.8 mi southwest of Oroville.

PERIOD OF RECORD.-November 1967 to current year.

GAGE.-Water-stage recorder. Datum of gage is $109.97 \mathrm{ft}$ above sea level (levels by California Department of Water Resources). Prior to May 1, 1970, at datum $109.50 \mathrm{ft}$ lower.

REMARKS.-Water is diverted from Thermalito Afterbay and is used for irrigation. See schematic diagram showing diversions and storage from Feather River at Lake Oroville.

COOPERATION.-Records collected by California Department of Water Resources, under general supervision of the U.S. Geological Survey, in connection with a Federal Energy Regulatory Commission project.

EXTREMES FOR PERIOD OF RECORD.-Maximum daily discharge, 2,110 ft 3 /s, Apr. 22-24, 1968; no flow for many days each year.

DISCHARGE, CUBIC FEET PER SECOND, WATER YEAR OCTOBER 1998 TO SEPTEMBER 1999 DAILY MEAN VALUES

\begin{tabular}{|c|c|c|c|c|c|c|c|c|c|c|c|c|}
\hline DAY & OCT & NOV & $\mathrm{DEC}$ & JAN & FEB & MAR & APR & MAY & JUN & JUL & AUG & SEP \\
\hline 1 & 473 & 522 & 465 & 439 & .00 & .00 & .00 & 1490 & 1450 & 1590 & 1520 & 1220 \\
\hline 2 & 464 & 532 & 438 & 439 & .00 & .00 & .00 & 1640 & 1460 & 1570 & 1500 & 1190 \\
\hline 3 & 445 & 543 & 415 & 439 & .00 & .00 & .00 & 1680 & 1430 & 1550 & 1500 & 1140 \\
\hline 4 & 445 & 557 & 395 & 441 & .00 & .00 & .00 & 1690 & 1360 & 1550 & 1510 & 1100 \\
\hline 5 & 462 & 580 & 402 & 434 & .00 & .00 & .00 & 1720 & 1280 & 1550 & 1490 & 1020 \\
\hline 6 & 468 & 597 & 407 & 431 & .00 & .00 & .00 & 1740 & 1210 & 1550 & 1480 & 984 \\
\hline 7 & 477 & 580 & 407 & 431 & .00 & .00 & .00 & 1750 & 1220 & 1550 & 1480 & 964 \\
\hline 8 & 493 & 570 & 406 & 432 & .00 & .00 & .00 & 1780 & 1260 & 1560 & 1490 & 947 \\
\hline 9 & 497 & 556 & 410 & 431 & .00 & .00 & .00 & 1800 & 1330 & 1560 & 1500 & 929 \\
\hline 10 & 495 & 551 & 417 & 430 & .00 & .00 & .00 & 1800 & 1410 & 1560 & 1490 & 905 \\
\hline 11 & 491 & 541 & 416 & 433 & .00 & .00 & .00 & 1810 & 1450 & 1550 & 1480 & 873 \\
\hline 12 & 469 & 523 & 424 & 433 & .00 & .00 & .00 & 1810 & 1500 & 1550 & 1480 & 849 \\
\hline 13 & 470 & 522 & 429 & 434 & .00 & .00 & .00 & 1820 & 1520 & 1570 & 1460 & 829 \\
\hline 14 & 472 & 530 & 430 & 435 & .00 & .00 & .00 & 1820 & 1540 & 1570 & 1460 & 799 \\
\hline 15 & 487 & 549 & 432 & 132 & .00 & .00 & .00 & 1810 & 1590 & 1570 & 1460 & 785 \\
\hline 16 & 503 & 550 & 438 & .00 & .00 & .00 & .00 & 1800 & 1590 & 1570 & 1450 & 783 \\
\hline 17 & 536 & 516 & 441 & .00 & .00 & .00 & .00 & 1800 & 1570 & 1570 & 1430 & 766 \\
\hline 18 & 546 & 528 & 444 & .00 & .00 & .00 & .00 & 1750 & 1560 & 1570 & 1420 & 750 \\
\hline 19 & 548 & 516 & 441 & .00 & .00 & .00 & 166 & 1670 & 1540 & 1570 & 1410 & 749 \\
\hline 20 & 559 & 486 & 441 & .00 & .00 & .00 & 236 & 1630 & 1540 & 1580 & 1400 & 739 \\
\hline 21 & 554 & 490 & 449 & .00 & .00 & .00 & 291 & 1560 & 1530 & 1580 & 1420 & 705 \\
\hline 22 & 565 & 497 & 446 & .00 & .00 & .00 & 343 & 1500 & 1540 & 1580 & 1420 & 702 \\
\hline 23 & 597 & 486 & 443 & .00 & .00 & .00 & 477 & 1490 & 1560 & 1570 & 1450 & 701 \\
\hline 24 & 596 & 473 & 441 & .00 & .00 & .00 & 656 & 1490 & 1570 & 1560 & 1410 & 701 \\
\hline 25 & 578 & 466 & 441 & .00 & .00 & .00 & 713 & 1510 & 1590 & 1550 & 1380 & 689 \\
\hline 26 & 565 & 474 & 440 & .00 & .00 & .00 & 886 & 1530 & 1590 & 1570 & 1380 & 664 \\
\hline 27 & 508 & 475 & 441 & .00 & .00 & .00 & 927 & 1530 & 1590 & 1580 & 1340 & 646 \\
\hline 28 & 513 & 474 & 441 & .00 & .00 & .00 & 1000 & 1490 & 1590 & 1570 & 1320 & 594 \\
\hline 29 & 537 & 475 & 442 & .00 & --- & .00 & 1100 & 1510 & 1590 & 1550 & 1330 & 555 \\
\hline 30 & 544 & 477 & 441 & .00 & --- & .00 & 1280 & 1500 & 1590 & 1540 & 1310 & 548 \\
\hline 31 & 486 & --- & 442 & .00 & --- & .00 & --- & 1470 & --- & 1540 & 1240 & --- \\
\hline TOTAL & 15843 & 15636 & 13365 & 6214.00 & 0.00 & 0.00 & 8075.00 & 51390 & 44550 & 48450 & 44410 & 24826 \\
\hline MEAN & 511 & 521 & 431 & 200 & .000 & .000 & 269 & 1658 & 1485 & 1563 & 1433 & 828 \\
\hline MAX & 597 & 597 & 465 & 441 & .00 & .00 & 1280 & 1820 & 1590 & 1590 & 1520 & 1220 \\
\hline MIN & 445 & 466 & 395 & .00 & .00 & .00 & .00 & 1470 & 1210 & 1540 & 1240 & 548 \\
\hline $\mathrm{AC}-\mathrm{FT}$ & 31420 & 31010 & 26510 & 12330 & .00 & .00 & 16020 & 101900 & 88360 & 96100 & 88090 & 49240 \\
\hline
\end{tabular}

STATISTICS OF MONTHLY MEAN DATA FOR WATER YEARS 1968 - 1999, BY WATER YEAR (WY)

\begin{tabular}{|c|c|c|c|c|c|c|c|c|c|c|c|c|}
\hline MEAN & 383 & 146 & 110 & 30.0 & 23.5 & 94.9 & 538 & 1396 & 1380 & 1477 & 1358 & 728 \\
\hline MAX & 661 & 527 & 431 & 216 & 374 & 571 & 1294 & 1815 & 1643 & 1709 & 1608 & 893 \\
\hline (WY) & 1975 & 1996 & 1999 & 1996 & 1977 & 1976 & 1968 & 1975 & 1975 & 1981 & 1982 & 1995 \\
\hline MIN & 77.2 & .000 & .000 & .000 & .000 & .000 & .000 & 519 & 826 & 834 & 776 & 283 \\
\hline (WY) & 1978 & 1975 & 1971 & 1969 & 1969 & 1978 & 1983 & 1977 & 1992 & 1991 & 1991 & 1977 \\
\hline
\end{tabular}

SUMMARY STATISTICS

ANNUAL TOTAL

ANNUAL MEAN

HIGHEST ANNUAL MEAN

LOWEST ANNUAL MEAN

HIGHEST DAILY MEAN

LOWEST DAILY MEAN

ANNUAL SEVEN-DAY MINIMUM

ANNUAL RUNOFF (AC-FT)

10 PERCENT EXCEEDS

50 PERCENT EXCEEDS

90 PERCENT EXCEEDS
FOR 1998 CALENDAR YEAR

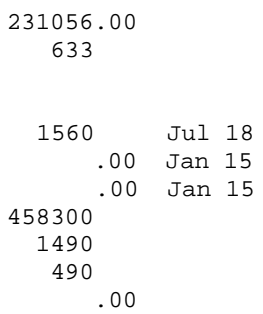

FOR 1999 WATER YEAR

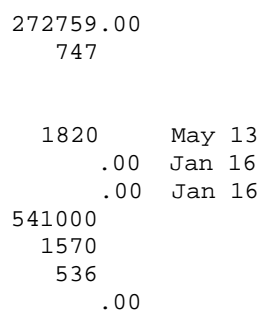

WATER YEARS 1968 - 1999

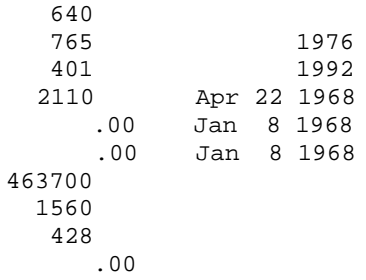


11406920 THERMALITO AFTERBAY RELEASE TO FEATHER RIVER, NEAR OROVILLE, CA

LOCATION.-Lat 39²7’23", long 12138'10", in NW 1/4 SE 1/4 sec.33, T.19 N., R.3 E., Butte County, Hydrologic Unit 18020106, on left bank of outlet channel $955 \mathrm{ft}$ downstream from centerline of Thermalito Afterbay Dam and 5.7 mi southwest of Oroville.

PERIOD OF RECORD.--November 1967 to current year.

WATER TEMPERATURE: Water years 1969-92.

GAGE.-Water-stage recorder. Datum of gage is $113.47 \mathrm{ft}$ above sea level (levels by California Department of Water Resources). Prior to May 1, 1970, at datum $13.00 \mathrm{ft}$ lower.

REMARKS.-Flow regulated by gates of Thermalito Afterbay outlet $955 \mathrm{ft}$ upstream. See schematic diagram showing diversions and storage from Feather River at Lake Oroville.

COOPERATION.- Records collected by California Department of Water Resources, under general supervision of the U.S. Geological Survey, in connection with a Federal Energy Regulatory Commission project.

EXTREMES FOR PERIOD OF RECORD.-Maximum discharge, 21,600 ft $3 / \mathrm{s}, \mathrm{Jan} .28,1970$, gage height, $23.30 \mathrm{ft}$, datum then in use, 21,600 ft $3 /$ s, Jan. 2, 1997, gage height, $11.45 \mathrm{ft}$; no flow for many days during 1968.

DISCHARGE, CUBIC FEET PER SECOND, WATER YEAR OCTOBER 1998 TO SEPTEMBER 1999 DAILY MEAN VALUES

\begin{tabular}{|c|c|c|c|c|c|c|c|c|c|c|c|c|}
\hline DAY & OCT & NOV & $\mathrm{DEC}$ & JAN & FEB & MAR & APR & MAY & JUN & JUL & AUG & SEP \\
\hline 1 & 5410 & 1830 & 7720 & 2890 & 8960 & 13500 & 2390 & 4390 & 1260 & 7430 & 7930 & 2890 \\
\hline 2 & 5410 & 1680 & 14500 & 2890 & 6680 & 13500 & 2390 & 4400 & 1260 & 7430 & 7930 & 2890 \\
\hline 3 & 5410 & 1480 & 14500 & 2890 & 6430 & 13500 & 2390 & 4400 & 1260 & 7430 & 7430 & 2890 \\
\hline 4 & 5400 & 1280 & 15500 & 2900 & 6890 & 15600 & 2390 & 3670 & 1260 & 7430 & 6920 & 2890 \\
\hline 5 & 4970 & 1180 & 16500 & 2900 & 10900 & 17100 & 2390 & 3410 & 1260 & 7430 & 6430 & 2890 \\
\hline 6 & 4460 & 1180 & 16500 & 2660 & 15500 & 17100 & 2390 & 3400 & 1260 & 7430 & 5930 & 2890 \\
\hline 7 & 3960 & 1180 & 16500 & 2150 & 16500 & 17100 & 2390 & 3400 & 1260 & 7680 & 5420 & 2890 \\
\hline 8 & 3450 & 1180 & 15500 & 1790 & 14800 & 17100 & 2400 & 3390 & 1260 & 7940 & 4910 & 2390 \\
\hline 9 & 2950 & 1180 & 13500 & 1600 & 12700 & 16000 & 2670 & 3390 & 1260 & 7940 & 4170 & 2390 \\
\hline 10 & 2440 & 1180 & 13500 & 1430 & 15700 & 13500 & 3380 & 3400 & 1260 & 7930 & 3410 & 2390 \\
\hline 11 & 1940 & 1180 & 13500 & 1380 & 17100 & 10500 & 3390 & 3030 & 1260 & 7930 & 3400 & 2380 \\
\hline 12 & 1890 & 1180 & 13500 & 1390 & 17100 & 7450 & 3390 & 2520 & 1260 & 7930 & 3400 & 2380 \\
\hline 13 & 1880 & 1180 & 13500 & 1370 & 17100 & 7430 & 3400 & 2020 & 1260 & 8180 & 3400 & 2390 \\
\hline 14 & 1860 & 1170 & 13500 & 1380 & 17100 & 7420 & 3400 & 1780 & 1260 & 8330 & 3390 & 2390 \\
\hline 15 & 1840 & 1180 & 12000 & 1380 & 17100 & 6090 & 3400 & 1580 & 1260 & 7540 & 3400 & 2390 \\
\hline 16 & 1840 & 1180 & 9960 & 2340 & 17100 & 5410 & 3400 & 1370 & 1260 & 8430 & 3150 & 2380 \\
\hline 17 & 1830 & 1180 & 7950 & 3370 & 17100 & 5410 & 3390 & 1260 & 1260 & 8440 & 2640 & 2390 \\
\hline 18 & 1830 & 1170 & 7430 & 4390 & 17100 & 5410 & 3390 & 1260 & 1260 & 8440 & 2400 & 2390 \\
\hline 19 & 1830 & 1170 & 7430 & 5420 & 17100 & 5410 & 3400 & 1260 & 1260 & 8690 & 2640 & 2380 \\
\hline 20 & 1830 & 1180 & 7440 & 6820 & 17100 & 5170 & 3660 & 1260 & 1250 & 8940 & 3140 & 2390 \\
\hline 21 & 1830 & 1270 & 7450 & 13200 & 17100 & 4660 & 4400 & 1260 & 1260 & 8940 & 3650 & 2390 \\
\hline 22 & 1830 & 1670 & 7450 & 13500 & 16900 & 4150 & 4400 & 1260 & 1260 & 8930 & 3900 & 2390 \\
\hline 23 & 1840 & 1780 & 6440 & 13500 & 13500 & 3660 & 4400 & 1260 & 1260 & 8940 & 3900 & 1800 \\
\hline 24 & 1830 & 1780 & 5420 & 13500 & 13500 & 3410 & 4400 & 1260 & 1260 & 8930 & 3900 & 1780 \\
\hline 25 & 1830 & 1780 & 4420 & 13500 & 13500 & 3390 & 4390 & 1260 & 1260 & 8930 & 3900 & 1780 \\
\hline 26 & 1830 & 1780 & 4410 & 13500 & 13500 & 3140 & 4400 & 1260 & 1820 & 8940 & 4150 & 2060 \\
\hline 27 & 1830 & 1790 & 4400 & 13500 & 13500 & 2640 & 4410 & 1260 & 3880 & 8930 & 4890 & 3140 \\
\hline 28 & 1840 & 1790 & 4400 & 13500 & 13500 & 2390 & 4400 & 1260 & 5910 & 8940 & 4910 & 3400 \\
\hline 29 & 1840 & 1790 & 3650 & 13500 & --- & 2390 & 4400 & 1260 & 6670 & 8690 & 4900 & 3400 \\
\hline 30 & 1840 & 1790 & 2910 & 12000 & --- & 2390 & 4400 & 1260 & 7170 & 8180 & 4660 & 2900 \\
\hline 31 & 1840 & --- & 2900 & 9980 & --- & 2390 & --- & 1260 & --- & 7930 & 3680 & --- \\
\hline TOTAL & 82610 & 42340 & 304280 & 196520 & 401060 & 254310 & 103400 & 68450 & 56940 & 255200 & 137880 & 76300 \\
\hline MEAN & 2665 & 1411 & 9815 & 6339 & 14320 & 8204 & 3447 & 2208 & 1898 & 8232 & 4448 & 2543 \\
\hline MAX & 5410 & 1830 & 16500 & 13500 & 17100 & 17100 & 4410 & 4400 & 7170 & 8940 & 7930 & 3400 \\
\hline MIN & 1830 & 1170 & 2900 & 1370 & 6430 & 2390 & 2390 & 1260 & 1250 & 7430 & 2400 & 1780 \\
\hline$A C-F T$ & 163900 & 83980 & 603500 & 389800 & 795500 & 504400 & 205100 & 135800 & 112900 & 506200 & 273500 & 151300 \\
\hline
\end{tabular}

STATISTICS OF MONTHLY MEAN DATA FOR WATER YEARS 1968 - 1999, BY WATER YEAR (WY)

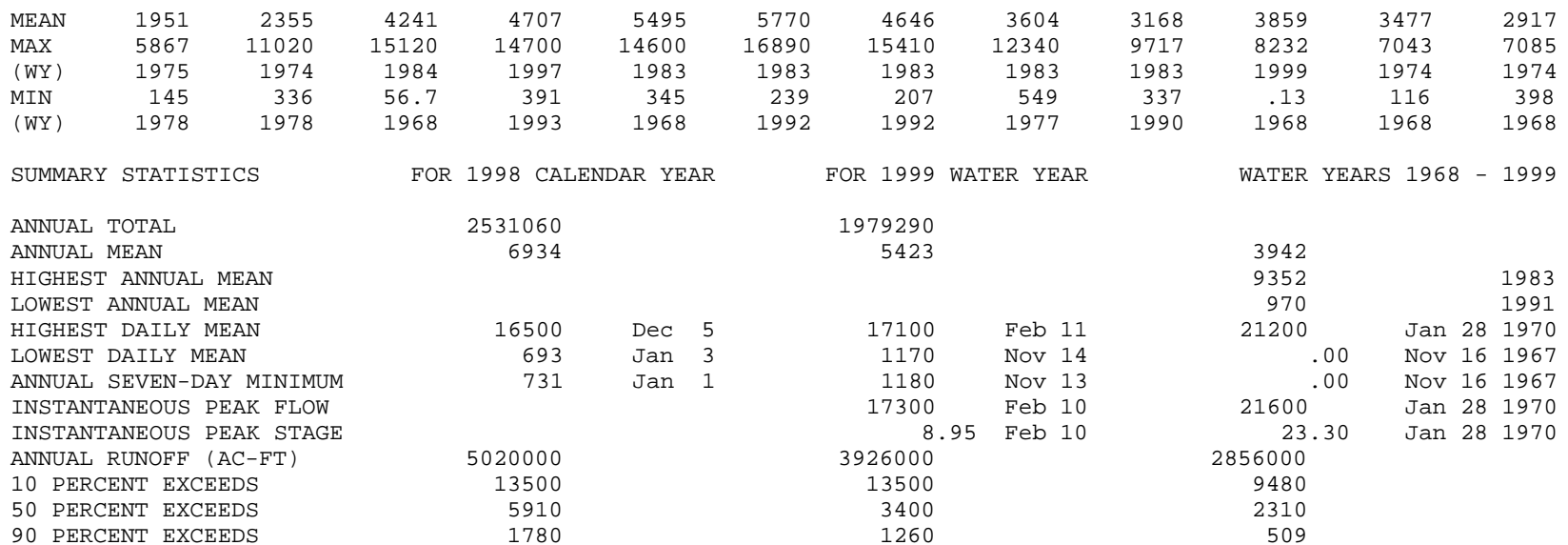


LOCATION.-Lat 39³1'18", long 121³2'48", in Boga Fernandez Grant, T.19 N., R.4 E., Butte County, Hydrologic Unit 18020106, on right bank $300 \mathrm{ft}$ upstream from fish barrier dam on Feather River, 0.4 mi downstream from Thermalito Diversion Dam, 0.8 mi northeast of Oroville Post Office, and 4.8 mi downstream from Oroville Dam.

DRAINAGE AREA.-3,624 $\mathrm{mi}^{2}$.

PERIOD OF RECORD.-October 1901 to current year. Monthly discharge only for some periods, published in WSP 1315-A. October 1934 to September 1961 pub1ished as "near Oroville."

CHEMICAL DATA: Water years 1906-07, 1951-77.

SPECIFIC CONDUCTANCE: Water years 1972-78.

WATER TEMPERATURE: Water years 1954-92.

SEDIMENT DATA: Water years 1957-79.

REVISED RECORDS.-WSP 843: 1907(M), 1909(M), 1914-15(M), 1919(M), 1927-28(M). WSP 881: $1913-28$ (yearly summaries). WSP 1515: 1906-8. WSP 1931: Drainage area. WDR CA-74-2: 1968-70, adjusted monthly discharge.

GAGE.-Water-stage recorder. Datum of gage is $148.97 \mathrm{ft}$ above sea level (levels by California Department of Water Resources). See WSP 1931 for history of changes prior to Oct. 1, 1964.

REMARKS.-Flow completely regulated by Lake Oroville (station 11406800), beginning November 1967, and Thermalito Diversion Pool (station 11406825), capacity 13,500 acre-ft. Diversions upstream from station for power and irrigation. Feather River Fish Hatchery (station 11406930) diverts up to $120 \mathrm{ft}^{3} / \mathrm{s}$ at Thermalito Diversion Dam 0.4 mi upstream from gage. Daily figures shown are combined figures of river flow and diversion to fish hatchery. See schematic diagram showing diversions and storage from Feather River at Lake Oroville.

COOPERATION.-Records were collected by California Department of Water Resources, under general supervision of the U.S. Geological Survey, in connection with a Federal Energy Regulatory Commission project.

EXTREMES FOR PERIOD OF RECORD._Prior to completion of Oroville Dam: Maximum discharge observed, $230,000 \mathrm{ft}^{3} / \mathrm{s}$ Mar. 19,1907 , elevation, $167.5 \mathrm{ft}$ above sea level, site and datum then in use, maximum discharge (since completion of Oroville Dam), $161,000 \mathrm{ft}^{3} / \mathrm{s}$, Jan. 2, 1997, gage height $25.45 \mathrm{ft}$; minimum, $300 \mathrm{ft}^{3} / \mathrm{s}$, estimated, Nov. 9, 1931.

Combined flow (since completion of Oroville Dam): Maximum daily discharge, 132,000 $\mathrm{ft}^{3} / \mathrm{s}, \mathrm{Feb}$. 18, 1986; minimum daily, $222 \mathrm{ft}^{3} / \mathrm{s}$, Sept. 19, 1972

EXTREMES FOR CURRENT YEAR.-River only: Maximum discharge, $8,100 \mathrm{ft}^{3} / \mathrm{s}$, Feb. 21, gage height, $4.39 \mathrm{ft}$; maximum gage height, $4.39 \mathrm{ft}$, Feb. 18, 21; minimum daily, $441 \mathrm{ft}^{3} / \mathrm{s}$, June 14 . Combined flow: Maximum daily discharge, 8,080 ft³/s, Feb. 19; minimum daily, $559 \mathrm{ft}^{3} / \mathrm{s}$, June 14.

DISCHARGE, CUBIC FEET PER SECOND, WATER YEAR OCTOBER 1998 TO SEPTEMBER 1999 DAILY MEAN VALUES

\begin{tabular}{|c|c|c|c|c|c|c|c|c|c|c|c|c|}
\hline DAY & OCT & NOV & $\mathrm{DEC}$ & JAN & FEB & MAR & APR & MAY & JUN & JUL & AUG & SEP \\
\hline 1 & 626 & 637 & 631 & 623 & 622 & 645 & 619 & 620 & 610 & 620 & 624 & 667 \\
\hline 2 & 630 & 636 & 624 & 619 & 623 & 636 & 623 & 626 & 626 & 627 & 625 & 660 \\
\hline 3 & 629 & 629 & 627 & 623 & 623 & 644 & 620 & 625 & 609 & 632 & 619 & 665 \\
\hline 4 & 627 & 631 & 628 & 622 & 624 & 1370 & 624 & 623 & 616 & 630 & 621 & 664 \\
\hline 5 & 617 & 635 & 638 & 624 & 621 & 3000 & 629 & 620 & 609 & 627 & 613 & 662 \\
\hline 6 & 632 & 640 & 630 & 623 & 627 & 3000 & 635 & 617 & 604 & 627 & 618 & 663 \\
\hline 7 & 636 & 640 & 634 & 622 & 629 & 3000 & 627 & 618 & 619 & 628 & 617 & 668 \\
\hline 8 & 637 & 643 & 630 & 616 & 632 & 2040 & 628 & 620 & 619 & 627 & 604 & 664 \\
\hline 9 & 633 & 637 & 621 & 636 & 634 & 623 & 627 & 620 & 615 & 627 & 651 & 669 \\
\hline 10 & 631 & 642 & 624 & 631 & 616 & 644 & 628 & 620 & 573 & 626 & 638 & 672 \\
\hline 11 & 631 & 638 & 625 & 625 & 626 & 626 & 625 & 615 & 575 & 623 & 639 & 663 \\
\hline 12 & 634 & 635 & 626 & 624 & 623 & 614 & 623 & 615 & 570 & 620 & 626 & 661 \\
\hline 13 & 632 & 638 & 627 & 621 & 625 & 628 & 621 & 620 & 561 & 620 & 620 & 669 \\
\hline 14 & 633 & 639 & 628 & 620 & 642 & 626 & 619 & 621 & 559 & 627 & 601 & 668 \\
\hline 15 & 635 & 643 & 620 & 619 & 643 & 627 & 617 & 622 & 565 & 629 & 603 & 671 \\
\hline 16 & 637 & 653 & 616 & 615 & 708 & 633 & 611 & 616 & 607 & 630 & 618 & 669 \\
\hline 17 & 634 & 646 & 613 & 615 & 2270 & 722 & 613 & 615 & 658 & 629 & 616 & 695 \\
\hline 18 & 631 & 639 & 618 & 614 & 7860 & 626 & 614 & 620 & 648 & 628 & 623 & 703 \\
\hline 19 & 631 & 638 & 624 & 617 & 8080 & 627 & 616 & 622 & 646 & 624 & 627 & 676 \\
\hline 20 & 627 & 634 & 626 & 618 & 8030 & 620 & 617 & 627 & 654 & 629 & 635 & 669 \\
\hline 21 & 631 & 636 & 627 & 616 & 8040 & 622 & 619 & 626 & 654 & 627 & 621 & 654 \\
\hline 22 & 631 & 630 & 620 & 618 & 5870 & 626 & 613 & 618 & 661 & 624 & 630 & 633 \\
\hline 23 & 636 & 640 & 620 & 617 & 703 & 625 & 615 & 625 & 650 & 625 & 638 & 629 \\
\hline 24 & 625 & 633 & 627 & 627 & 682 & 631 & 614 & 626 & 663 & 626 & 639 & 636 \\
\hline 25 & 628 & 629 & 629 & 627 & 655 & 627 & 618 & 626 & 628 & 624 & 655 & 635 \\
\hline 26 & 624 & 629 & 627 & 628 & 650 & 622 & 619 & 624 & 627 & 623 & 652 & 701 \\
\hline 27 & 626 & 631 & 626 & 625 & 647 & 633 & 621 & 623 & 626 & 624 & 640 & 622 \\
\hline 28 & 624 & 628 & 625 & 632 & 664 & 632 & 623 & 625 & 621 & 624 & 626 & 624 \\
\hline 29 & 633 & 635 & 624 & 625 & --- & 623 & 621 & 625 & 620 & 625 & 606 & 626 \\
\hline 30 & 630 & 638 & 627 & 627 & --- & 624 & 614 & 627 & 618 & 624 & 648 & 623 \\
\hline 31 & 633 & --- & 627 & 627 & --- & 623 & --- & 628 & --- & 624 & 665 & --- \\
\hline TOTAL & 19544 & 19102 & 19389 & 19296 & 54269 & 28839 & 18613 & 19275 & 18511 & 19400 & 19458 & 19781 \\
\hline MEAN & 630 & 637 & 625 & 622 & 1938 & 930 & 620 & 622 & 617 & 626 & 628 & 659 \\
\hline MAX & 637 & 653 & 638 & 636 & 8080 & 3000 & 635 & 628 & 663 & 632 & 665 & 703 \\
\hline MIN & 617 & 628 & 613 & 614 & 616 & 614 & 611 & 615 & 559 & 620 & 601 & 622 \\
\hline $\mathrm{AC}-\mathrm{FT}$ & 38770 & 37890 & 38460 & 38270 & 107600 & 57200 & 36920 & 38230 & 36720 & 38480 & 38590 & 39240 \\
\hline MEAN a & 2751 & 6061 & 7933 & 8647 & 16679 & 11908 & 9768 & 9157 & 4779 & 3173 & 3234 & 1958 \\
\hline $\mathrm{AC}-\mathrm{FTa}$ & 169200 & 360600 & 487800 & 531700 & 926300 & 732200 & 581200 & 563000 & 284400 & 195100 & 198900 & 116500 \\
\hline
\end{tabular}

a Adjusted for unreviewed evaporation, change in contents, and diversions in and out of Lake Oroville, Thermalito Diversion Pool, Thermalito Forebay, and Thermalito Afterbay (station 11406870). 
11407000 FEATHER RIVER AT OROVILLE, CA—Continued

STATISTICS OF MONTHLY MEAN DATA FOR WATER YEARS 1902 - 1967, BY WATER YEAR (WY)

\begin{tabular}{|c|c|c|c|c|c|c|c|c|c|c|c|c|}
\hline & $\mathrm{OCT}$ & NOV & $\mathrm{DEC}$ & JAN & FEB & MAR & $\mathrm{APR}$ & MAY & JUN & JUL & AUG & SEP \\
\hline MEAN & 2085 & 3069 & 5296 & 6790 & 9463 & 10080 & 12120 & 9930 & 5176 & 2505 & 1980 & 1792 \\
\hline MAX & 12370 & 19710 & 28410 & 39860 & 28030 & 39760 & 30100 & 25150 & 15650 & 5999 & 3265 & 2883 \\
\hline (WY) & 1963 & 1904 & 1956 & 1909 & 1904 & 1904 & 1911 & 1938 & 1911 & 1907 & 1967 & 1967 \\
\hline MIN & 745 & 853 & 1102 & 1350 & 1714 & 1564 & 2146 & 1246 & 924 & 852 & 956 & 992 \\
\hline (WY) & 1933 & 1933 & 1950 & 1947 & 1933 & 1924 & 1924 & 1924 & 1924 & 1924 & 1924 & 1924 \\
\hline
\end{tabular}

SUMMARY STATISTICS

ANNUAL MEAN

HIGHEST ANNUAL MEAN

LOWEST ANNUAL MEAN

HIGHEST DAILY MEAN

LOWEST DAILY MEAN

ANNUAL SEVEN-DAY MINIMUM

INSTANTANEOUS PEAK FLOW

INSTANTANEOUS PEAK STAGE

ANNUAL RUNOFF (AC-FT)

10 PERCENT EXCEEDS

50 PERCENT EXCEEDS

90 PERCENT EXCEEDS
WATER YEARS 1902 - 1967

\begin{tabular}{|c|c|c|}
\hline 5834 & & \\
\hline 12860 & & 1907 \\
\hline 1623 & & 1924 \\
\hline 187000 & Mar 19 & 1907 \\
\hline 577 & Oct 3 & 1932 \\
\hline 652 & Sep 30 & 1932 \\
\hline 230000 & Mar 19 & 1907 \\
\hline 167.5 & Mar 19 & 1907 \\
\hline \multicolumn{3}{|l|}{4226000} \\
\hline \multicolumn{3}{|l|}{13300} \\
\hline \multicolumn{3}{|l|}{2870} \\
\hline 1470 & & \\
\hline
\end{tabular}

STATISTICS OF MONTHLY MEAN DATA FOR WATER YEARS 1969 - 1999, BY WATER YEAR (WY)

\begin{tabular}{|c|c|c|c|c|c|c|c|c|c|c|c|c|}
\hline & OCT & NOV & $\mathrm{DEC}$ & JAN & FEB & MAR & APR & MAY & JUN & JUL & AUG & SEP \\
\hline MEAN & 559 & 754 & 1217 & 3163 & 2366 & 2129 & 1011 & 770 & 510 & 506 & 494 & 496 \\
\hline MAX & 1580 & 3313 & 7728 & 26750 & 25180 & 18870 & 7064 & 7916 & 998 & 775 & 640 & 659 \\
\hline (WY) & 1996 & 1982 & 1997 & 1997 & 1986 & 1995 & 1982 & 1995 & 1989 & 1992 & 1997 & 1999 \\
\hline MIN & 399 & 397 & 392 & 401 & 399 & 404 & 401 & 387 & 405 & 404 & 393 & 389 \\
\hline (WY) & 1969 & 1979 & 1979 & 1976 & 1978 & 1978 & 1977 & 1969 & 1974 & 1981 & 1979 & 1972 \\
\hline
\end{tabular}

SUMMARY STATISTICS

ANNUAL TOTAL

ANNUAL MEAN

ANNUAL MEAN ADJUSTED a

HIGHEST ANNUAL MEAN

LOWEST ANNUAL MEAN

HIGHEST DAILY MEAN

LOWEST DAILY MEAN

ANNUAL SEVEN-DAY MINIMUM

INSTANTANEOUS PEAK FLOW

INSTANTANEOUS PEAK STAGE

ANNUAL RUNOFF (AC-FT)

ANNUAL RUNOFE (AC-EI)

10 PERCENT EXCEEDS

50 PERCENT EXCEEDS

90 PERCENT EXCEEDS
FOR 1998 CALENDAR YEAR

FOR 1999 WATER YEAR $\begin{array}{rr}400703 & 275477 \\ 1098 & 755 \\ 9931 & 7109\end{array}$

$10200 \quad$ Feb $6 \quad 8080 \quad$ Feb 19

$602 \quad$ Aug $25 \quad 559$ Jun 14

a Adjusted for unreviewed evaporation, change in contents, and diversions in and out of Lake Oroville, Thermalito Diversion Pool, Thermalito Forebay, and Thermalito Afterbay (station 11406870).

b Includes water year 1968. 


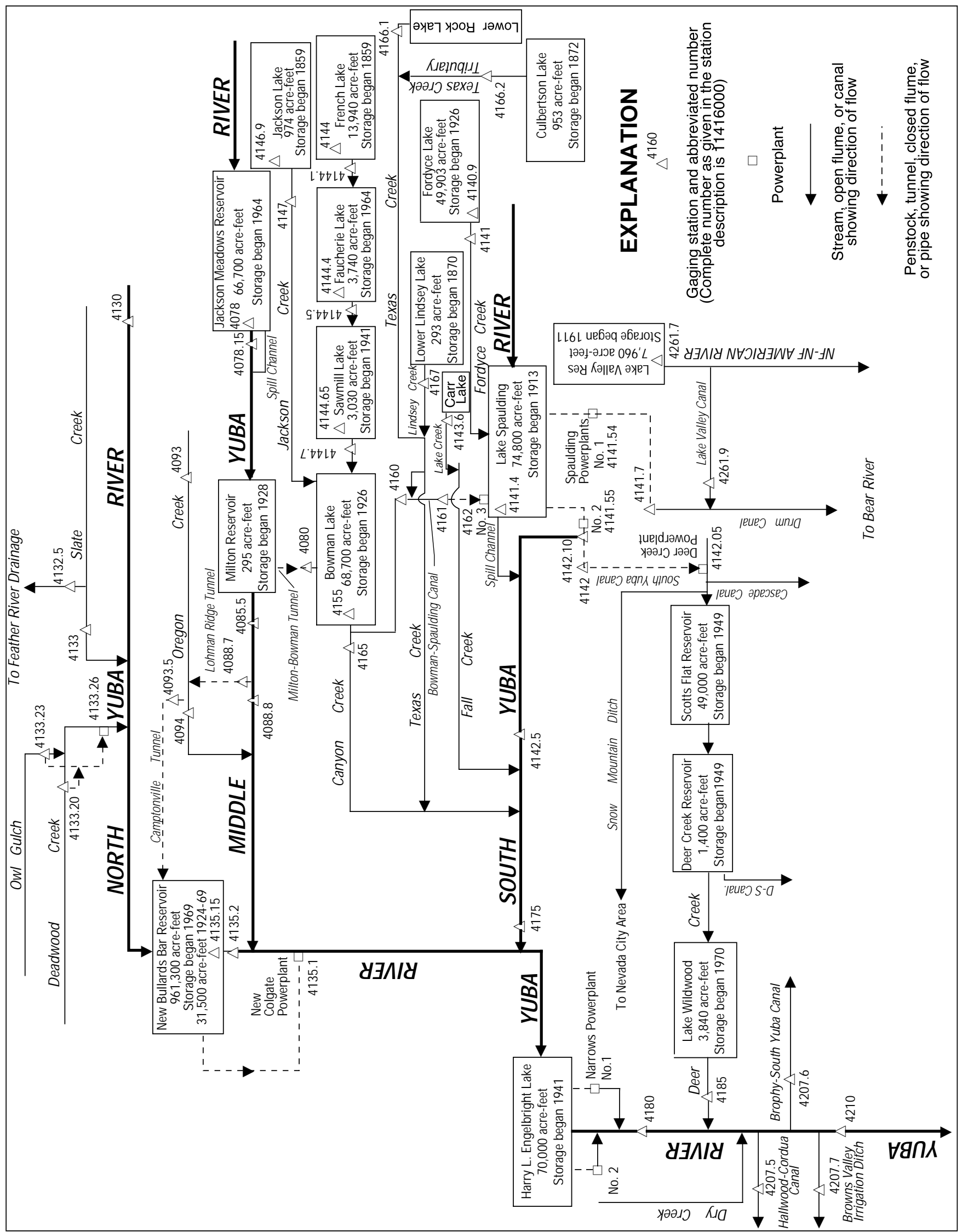

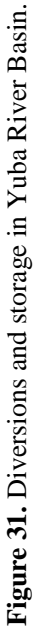


LOCATION.-Lat 39³0'33", long 120³3’08", in NW 1/4 SE 1/4 sec.18, T.19 N., R.13 E., Sierra County, Hydrologic Unit 18020125, Tahoe National Forest, on right bank at Jackson Meadows Dam on Middle Yuba River, 0.7 mi downstream from Pass Creek, and 5.7 mi southeast of Sierra City.

DRAINAGE AREA.- $-37.6 \mathrm{mi}^{2}$.

PERIOD OF RECORD.-November 1964 to current year.

GAGE.-Water-stage recorder. Datum of gage is sea level (levels by Nevada Irrigation District).

REMARKS.-Reservoir is formed by an earthfill dam. Storage began Nov. 9, 1964. Usable capacity, 66,700 acre-ft between elevations 5,933.0 ft, bottom of intake tower, and 6,036.0 ft, top of radial spillway gates. Dead contents, 2,500 acre-ft. Records, including extremes, represent total contents. See schematic diagram of Yuba River Basin.

COOPERATION.--Records were collected by Nevada Irrigation District, under general supervision of the U.S. Geological Survey, in connection with a Federal Energy Regulatory Commission project.

EXTREMES FOR PERIOD OF RECORD.—Maximum contents, 71,100 acre-ft, May 31 and June 1, 1993, elevation, 6,037.78 ft; minimum since reservoir first filled, 2,500 acre-ft, Sept. 27-29, 1976, elevation, 5,933.1 ft.

EXTREMES FOR CURRENT YEAR.-Maximum contents, 68,300 acre-ft, June 29-July 3, maximum elevation, 6,035.15 ft, July 1, 2; minimum, 32,300 acre-ft, Nov. 20, elevation, 5,996.22 ft.

Capacity table (elevation, in feet, and contents, in acre-feet)

(Based on table provided by Nevada Irrigation District, dated February 1965)

$\begin{array}{rrrr}5,930 & 2,000 & 5,990 & 27,600 \\ 5,940 & 3,920 & 6,000 & 35,300 \\ 5,950 & 6,760 & 6,010 & 43,900 \\ 5,960 & 10,600 & 6,020 & 53,200 \\ 5,970 & 15,400 & 6,030 & 63,000 \\ 5,980 & 21,000 & 6,040 & 73,500\end{array}$

RESERVOIR STORAGE (ACRE-FEET), WATER YEAR OCTOBER 1998 TO SEPTEMBER 1999 DAILY OBSERVATION AT 2400 HOURS

\begin{tabular}{|c|c|c|c|c|c|c|c|c|c|c|c|c|}
\hline DAY & OCT & NOV & $\mathrm{DEC}$ & JAN & $\mathrm{FEB}$ & MAR & $A P R$ & MAY & JUN & JUL & AUG & SEP \\
\hline 1 & 52700 & 39900 & 33600 & e 35100 & e38400 & 43400 & 42100 & 42000 & 55600 & 68300 & 63900 & 57700 \\
\hline 2 & 52300 & 39500 & 33700 & 35100 & e38600 & 43600 & e 41800 & 42400 & 55500 & 68300 & 63700 & 57500 \\
\hline 3 & 52000 & 39000 & 34000 & 35200 & e38800 & 44000 & e41600 & 42600 & 55200 & 68300 & 63500 & 57200 \\
\hline 4 & 51600 & 38600 & 34200 & 35200 & e39000 & 44200 & e41300 & 42600 & 55200 & 68200 & 63300 & 57000 \\
\hline 5 & 51200 & 38100 & 34300 & 35200 & e39200 & 44300 & e 41100 & 42600 & 55600 & 68200 & 63100 & 56800 \\
\hline 6 & 50900 & 37700 & 34300 & 35200 & e39300 & 44500 & e 40800 & 42900 & 56200 & 68100 & 62900 & 56600 \\
\hline 7 & 50500 & 37400 & 34400 & e35200 & e39500 & 44600 & e40600 & 43500 & 56700 & 68000 & 62700 & 56400 \\
\hline 8 & 50100 & 36900 & 34400 & 35200 & e39700 & 44700 & e40400 & 44000 & 57200 & 67900 & 62500 & 56100 \\
\hline 9 & 49800 & 36500 & 34400 & 35200 & e39900 & 44900 & e40100 & 44500 & 57700 & 67800 & 62300 & 55900 \\
\hline 10 & 49400 & 36100 & 34500 & 35300 & e40000 & 45000 & e39900 & 44900 & 58200 & 67700 & 62200 & 55700 \\
\hline 11 & 49100 & 35600 & 34500 & 35300 & e 40200 & 44900 & e39600 & 45400 & 58800 & 67500 & 62000 & 55500 \\
\hline 12 & 48700 & 35200 & 34500 & e 35300 & e40400 & 44700 & e 39400 & e46000 & 59400 & 67400 & 61800 & 55300 \\
\hline 13 & 48400 & 34700 & 34600 & 35300 & e40600 & 44600 & e39200 & e 46600 & 60100 & 67300 & 61600 & 55100 \\
\hline 14 & 48000 & 34300 & 34600 & 35300 & e40800 & 44400 & e38900 & e 47200 & 60800 & 67100 & 61400 & 54900 \\
\hline 15 & 47500 & 33900 & 34600 & e 35400 & e40900 & 44300 & 38700 & e 47800 & 61600 & 67000 & 61200 & 54600 \\
\hline 16 & 47000 & 33400 & 34700 & 35600 & e41100 & 44100 & e39000 & e48400 & 62500 & 66800 & 61000 & 54400 \\
\hline 17 & 46600 & 33100 & 34700 & e 35700 & e 41300 & 44000 & e39200 & e49000 & 63200 & 66600 & 60800 & 54200 \\
\hline 18 & 46100 & 32700 & 34700 & e35900 & e 41500 & 43900 & e39300 & 49800 & 63900 & 66500 & 60600 & 54000 \\
\hline 19 & 45700 & 32400 & 34800 & e36100 & e41700 & 43800 & e39500 & 50700 & 64500 & 66300 & 60400 & 53800 \\
\hline 20 & 45200 & 32300 & 34900 & e 36300 & e 41800 & 43700 & e 39600 & 51600 & 65000 & 66100 & 60200 & 53500 \\
\hline 21 & 44800 & 32400 & 34900 & e 36500 & e42000 & 43500 & e39800 & 52600 & 65500 & 65900 & 60000 & 53300 \\
\hline 22 & 44300 & 32400 & 34900 & e 36700 & e 42200 & 43400 & e 40000 & 53800 & 66100 & 65800 & 59800 & 53100 \\
\hline 23 & 43900 & 32700 & 34900 & e36800 & e42300 & 43300 & 40100 & 55200 & 66600 & 65600 & 59600 & 52700 \\
\hline 24 & 43500 & 32800 & 34900 & e 37000 & 42400 & 43200 & 40200 & 55900 & 67000 & 65400 & 59400 & 52400 \\
\hline 25 & 43100 & 32800 & 35000 & e 37200 & 42600 & 43100 & 40500 & 56200 & 67400 & 65200 & 59200 & 52100 \\
\hline 26 & 42600 & 32800 & 35000 & e 37400 & 42700 & 43000 & 41100 & 56200 & 67700 & 65000 & 59000 & 51800 \\
\hline 27 & 42100 & 32900 & 35000 & e 37500 & 42800 & 42900 & 41400 & 56200 & 67900 & 64900 & 58800 & 51400 \\
\hline 28 & 41700 & 32900 & 35000 & e 37700 & 43000 & 42900 & 41600 & 56000 & 68100 & 64700 & 58600 & 51100 \\
\hline 29 & 41200 & 33000 & 35000 & e 37900 & --- & 42800 & 41700 & 55700 & 68300 & 64500 & 58300 & 50800 \\
\hline 30 & 40800 & 33500 & 35100 & e 38100 & --- & 42600 & 41800 & 55600 & 68300 & 64300 & 58100 & 50500 \\
\hline 31 & 40300 & --- & 35100 & e 38200 & --- & 42300 & --- & 55600 & --- & 64100 & 57900 & --- \\
\hline MAX & 52700 & 39900 & 35100 & 38200 & 43000 & 45000 & 42100 & 56200 & 68300 & 68300 & 63900 & 57700 \\
\hline MIN & 40300 & 32300 & 33600 & 35100 & 38400 & 42300 & 38700 & 42000 & 55200 & 64100 & 57900 & 50500 \\
\hline$a$ & 6005.94 & 5997.68 & 5999.69 & & 6009.00 & 6008.18 & 6007.57 & 6022.51 & 6035.14 & 6031.09 & 6024.90 & 6017.18 \\
\hline $\mathrm{b}$ & -12800 & -6800 & +1600 & +3100 & +4800 & -700 & -500 & +13800 & +12700 & -4200 & -6200 & -7400 \\
\hline
\end{tabular}

CAL YR 1998 MAX $69300 \quad$ MIN $32300 \quad$ b +2400

WTR YR 1999 MAX 68300 MIN 32300 b -2600

e Estimated.

a Elevation, in feet, at end of month.

b Change in contents, in acre-feet. 
LOCATION.-Lat 39³0’36", long 120³3'15", in NW 1/4 SE 1/4 sec.18, T.19 N., R.13 E., Sierra County, Hydrologic Unit 18020125, Tahoe National Forest, in outlet structure near right bank below Jackson Meadows Dam on Middle Yuba River, 0.7 mi downstream from Pass Creek, and 5.7 mi southeast of Sierra City.

DRAINAGE AREA.-37.6 $\mathrm{mi}^{2}$.

PERIOD OF RECORD.-July 1994 to current year.

GAGE.-Ultrasonic meter measures flow in two outlet pipes. Elevation of gage is 5,910 ft above sea level, from topographic map.

REMARKS.-Flow regulated by Jackson Meadows Reservoir (station 11407800). Flow over the spillway bypasses this station. See schematic diagram of Yuba River Basin.

COOPERATION.-Records were collected by Nevada Irrigation District, under general supervision of the U.S. Geological Survey, in connection with a Federal Energy Regulatory Commission project.

EXTREMES FOR PERIOD OF RECORD.-Maximum daily discharge, $415 \mathrm{ft}^{3} / \mathrm{s}$, May 23, 28, 1996; minimum daily, $7.9 \mathrm{ft}^{3} / \mathrm{s}$, several days November 1994

DISCHARGE, CUBIC FEET PER SECOND, WATER YEAR OCTOBER 1998 TO SEPTEMBER 1999 DAILY MEAN VALUES

\begin{tabular}{|c|c|c|c|c|c|c|c|c|c|c|c|c|}
\hline DAY & OCT & NOV & $\mathrm{DEC}$ & JAN & $\mathrm{FEB}$ & MAR & $\mathrm{APR}$ & MAY & JUN & JUL & AUG & SEP \\
\hline 1 & 183 & 233 & 9.1 & 9.2 & 9.6 & 9.6 & 206 & 207 & 222 & 113 & e 98 & e109 \\
\hline 2 & 183 & 233 & 9.0 & 9.2 & 9.4 & 9.6 & 207 & 207 & 222 & 101 & e98 & e109 \\
\hline 3 & 183 & 232 & 9.1 & 9.2 & 9.5 & 9.7 & 207 & 207 & 222 & 101 & e98 & e109 \\
\hline 4 & 182 & 231 & 9.1 & 9.3 & 9.4 & 9.7 & 206 & 208 & 221 & 101 & e98 & 109 \\
\hline 5 & 182 & 231 & 9.1 & 9.1 & 9.4 & 9.7 & 206 & 208 & 221 & 101 & e 98 & 108 \\
\hline 6 & 182 & 230 & 9.1 & 9.3 & 9.4 & 9.7 & 206 & 208 & 222 & 100 & e98 & 108 \\
\hline 7 & 181 & 230 & 9.1 & 9.3 & 9.4 & 9.7 & 205 & 208 & 222 & 99 & 97 & 108 \\
\hline 8 & 181 & 229 & 9.1 & 9.3 & 9.4 & 9.7 & 205 & 210 & 176 & 100 & 97 & 108 \\
\hline 9 & 181 & 229 & 9.1 & 9.3 & 9.5 & 9.7 & 205 & 210 & 136 & 100 & 97 & 108 \\
\hline 10 & 181 & 231 & 9.1 & 9.3 & 9.5 & 45 & 205 & 211 & 122 & 100 & 97 & 108 \\
\hline 11 & 180 & 233 & 9.1 & 9.3 & 9.6 & 113 & 204 & 211 & 105 & 100 & 97 & 108 \\
\hline 12 & 180 & 232 & 9.1 & 9.3 & 9.8 & 135 & 204 & 212 & 105 & 100 & 97 & 108 \\
\hline 13 & 179 & 232 & 9.1 & 9.3 & 9.7 & 135 & 204 & 213 & 106 & 99 & 97 & 108 \\
\hline 14 & 207 & 231 & 9.1 & 9.3 & 9.5 & 135 & 203 & 214 & 106 & 100 & 97 & 108 \\
\hline 15 & 235 & 231 & 9.1 & 9.3 & 9.5 & 135 & 203 & 214 & 66 & 100 & 97 & 110 \\
\hline 16 & 235 & 230 & 9.1 & 9.3 & 9.7 & 134 & 203 & 214 & 26 & 99 & 97 & 114 \\
\hline 17 & 234 & 229 & 9.1 & 9.4 & 9.6 & 135 & 203 & 215 & 27 & 100 & 97 & 114 \\
\hline 18 & 234 & 229 & 9.1 & 9.4 & 9.9 & 134 & 203 & 215 & 26 & 99 & 97 & 114 \\
\hline 19 & 233 & 153 & 9.1 & 9.4 & 9.7 & 133 & 203 & 216 & 27 & e100 & 97 & 113 \\
\hline 20 & 232 & 35 & 9.1 & 9.4 & 9.5 & 133 & 204 & 217 & 26 & e100 & 97 & 113 \\
\hline 21 & 234 & 9.0 & 9.1 & 9.3 & 9.5 & 134 & 204 & 218 & 27 & e100 & 97 & 113 \\
\hline 22 & 234 & 9.0 & 9.1 & 9.3 & 9.5 & 133 & 204 & 219 & 26 & e100 & 96 & 135 \\
\hline 23 & 234 & 9.0 & 9.1 & 9.3 & 9.7 & 133 & 205 & 221 & 27 & e99 & 103 & 159 \\
\hline 24 & 232 & 9.0 & 9.1 & 9.3 & 9.6 & 133 & 205 & 222 & 26 & e99 & 110 & 158 \\
\hline 25 & 231 & 9.0 & 9.1 & 9.3 & 9.6 & 133 & 205 & 222 & 27 & e99 & 109 & 158 \\
\hline 26 & 231 & 9.0 & 9.1 & 9.4 & 9.6 & 133 & 205 & 223 & 27 & e98 & 109 & 158 \\
\hline 27 & 230 & 9.0 & 9.1 & 9.4 & 9.6 & 133 & 206 & 222 & 27 & e98 & 109 & 158 \\
\hline 28 & 229 & 9.0 & 9.1 & 9.4 & 9.6 & 133 & 206 & 222 & 27 & e98 & 109 & 158 \\
\hline 29 & 229 & 9.0 & 9.1 & 9.3 & --- & 163 & 206 & 222 & 70 & e 98 & 109 & 157 \\
\hline 30 & 231 & 9.0 & 9.2 & 9.4 & --- & 206 & 207 & 222 & 127 & e98 & e109 & 157 \\
\hline 31 & 234 & --- & 9.1 & 9.4 & --- & 206 & --- & 222 & --- & e 98 & e109 & --- \\
\hline TOTAL & 6517 & 4434.0 & 282.1 & 288.7 & 267.7 & 3094.1 & 6145 & 6660 & 3017 & 3098 & 3115 & 3705 \\
\hline MEAN & 210 & 148 & 9.10 & 9.31 & 9.56 & 99.8 & 205 & 215 & 101 & 99.9 & 100 & 124 \\
\hline MAX & 235 & 233 & 9.2 & 9.4 & 9.9 & 206 & 207 & 223 & 222 & 113 & 110 & 159 \\
\hline MIN & 179 & 9.0 & 9.0 & 9.1 & 9.4 & 9.6 & 203 & 207 & 26 & 98 & 96 & 108 \\
\hline $\mathrm{AC}-\mathrm{ET}$ & 12930 & 8790 & 560 & 573 & 531 & 6140 & 12190 & 13210 & 5980 & 6140 & 6180 & 7350 \\
\hline
\end{tabular}

CAL YR 1998 TOTAL 48153.5 MEAN 132 MAX 284 MIN 9.0 AC-FT 95510 WTR YR 1999 TOTAL 40623.6 MEAN 111 MAX 235 MIN 9.0 AC-FT 80580 


\section{MILTON-BOWMAN TUNNEL OUTLET NEAR GRANITEVILLE, CA}

LOCATION.-Lat 39²7'37", long 120³6'37", in NW 1/4 NE 1/4 sec.3, T.18 N., R.12 E., Nevada County, Hydrologic Unit 18020125, on right bank $100 \mathrm{ft}$ downstream from tunnel outlet near upper end of Bowman Lake, and 6.9 mi east of Graniteville.

PERIOD OF RECORD.-May 1928 to September 1930, February 1931 to current year. Monthly discharge only for some periods, published in WSP 1315-A. Prior to October 1962, published as "Milton-Bowman tunnel at outlet."

GAGE.-Water-stage recorder and Parshall flume. Datum of gage is 5,592.51 ft above sea level. Prior to Sept. 22, 1964, at datum $0.56 \mathrm{ft}$ higher.

REMARKS.-Tunnel diverts from Middle Yuba River at Milton Reservoir, in sec.12, T.19 N., R.12 E., and discharges into Bowman Lake. Nearly the entire flow of Middle Yuba River is diverted during low and medium flows. Middle Yuba River is regulated by Jackson Meadows Reservoir (station 11407800) since November 1964. See schematic diagram of Yuba River Basin.

COOPERATION.-Records were collected by Nevada Irrigation District, under general supervision of the U.S. Geological Survey, in connection with a Federal Energy Regulatory Commission project.

EXTREMES FOR PERIOD OF RECORD.-Maximum daily discharge, $492 \mathrm{ft}^{3} / \mathrm{s}$, Feb. 11, 1941; minimum daily, $0.4 \mathrm{ft}^{3} / \mathrm{s}$, Oct. $7,1944$.

DISCHARGE, CUBIC FEET PER SECOND, WATER YEAR OCTOBER 1998 TO SEPTEMBER 1999 DAILY MEAN VALUES

\begin{tabular}{|c|c|c|c|c|c|c|c|c|c|c|c|c|}
\hline DAY & $\mathrm{OCT}$ & NOV & DEC & JAN & FEB & MAR & APR & MAY & JUN & JUL & AUG & SEP \\
\hline 1 & 180 & 234 & e11 & e11 & 17 & 51 & 216 & 244 & 412 & 117 & 96 & 106 \\
\hline 2 & 179 & 231 & e11 & e12 & 17 & 38 & 214 & 247 & 412 & 101 & 96 & 105 \\
\hline 3 & 179 & 231 & e22 & e12 & 16 & 43 & 214 & 240 & 402 & 100 & 96 & 105 \\
\hline 4 & 179 & 230 & e11 & e12 & 16 & 35 & 212 & 234 & 382 & 100 & 96 & 105 \\
\hline 5 & 177 & 230 & e11 & e11 & 16 & 30 & 214 & 235 & 258 & 100 & 96 & 105 \\
\hline 6 & 177 & 230 & e12 & e12 & 16 & 27 & 211 & 242 & 243 & 100 & 96 & 104 \\
\hline 7 & 178 & 233 & e12 & 11 & 35 & 25 & 210 & 251 & 242 & 99 & 96 & 104 \\
\hline 8 & 177 & 229 & e12 & 10 & 33 & 24 & 212 & 250 & 208 & 99 & 96 & 104 \\
\hline 9 & 177 & 227 & e12 & 10 & 51 & 23 & 208 & 247 & 150 & 99 & 96 & 104 \\
\hline 10 & 177 & 228 & e12 & 10 & 35 & 38 & 208 & 246 & 142 & 99 & 97 & 104 \\
\hline 11 & 176 & 232 & e12 & 10 & 28 & 112 & 208 & 250 & 121 & 99 & 96 & 104 \\
\hline 12 & 176 & 230 & e12 & 10 & 25 & 141 & 209 & 259 & 121 & 99 & 95 & 103 \\
\hline 13 & 176 & 230 & e12 & 10 & 23 & 141 & 211 & 260 & 121 & 99 & 95 & 104 \\
\hline 14 & 189 & 229 & e12 & 10 & 22 & 141 & 213 & 251 & 120 & 99 & 95 & 103 \\
\hline 15 & 229 & 228 & e12 & 12 & 20 & 140 & 216 & 246 & 100 & 98 & 95 & 104 \\
\hline 16 & 232 & 227 & e12 & 17 & 22 & 140 & 220 & 246 & 43 & 98 & 95 & 108 \\
\hline 17 & 232 & 232 & e12 & 18 & 38 & 141 & 226 & 250 & 40 & 98 & 94 & 108 \\
\hline 18 & 232 & 227 & e12 & 22 & 30 & 142 & 233 & 256 & 38 & 98 & 94 & 109 \\
\hline 19 & 231 & 180 & e12 & 23 & 25 & 142 & 239 & 259 & 37 & 98 & 94 & 109 \\
\hline 20 & 230 & 56 & e12 & 24 & 23 & 143 & 244 & 259 & 36 & 98 & 94 & 109 \\
\hline 21 & 230 & 12 & e11 & 29 & 23 & 142 & 243 & 261 & 35 & 97 & 94 & 108 \\
\hline 22 & 232 & 15 & e11 & 34 & 20 & 141 & 237 & 269 & 34 & 97 & 94 & 120 \\
\hline 23 & 232 & 26 & e11 & 55 & 19 & 142 & 236 & 291 & 34 & 97 & 98 & 150 \\
\hline 24 & 234 & 24 & e11 & 35 & 19 & 142 & 239 & 409 & 32 & 97 & 106 & 150 \\
\hline 25 & 234 & 13 & e11 & 29 & 20 & 143 & 245 & 422 & 31 & 97 & 106 & 150 \\
\hline 26 & 231 & 11 & e11 & 26 & 18 & 145 & 258 & 425 & 30 & 97 & 106 & 150 \\
\hline 27 & 229 & 12 & e11 & 23 & 17 & 147 & 252 & 424 & 30 & 97 & 106 & 149 \\
\hline 28 & 228 & 13 & e11 & 21 & 25 & 146 & 244 & 423 & 29 & 96 & 106 & 149 \\
\hline 29 & 228 & 14 & e11 & 20 & --- & 164 & 236 & 420 & 49 & 96 & 106 & 149 \\
\hline 30 & 237 & e21 & e12 & 19 & --- & 220 & 236 & 416 & 125 & 96 & 106 & 149 \\
\hline 31 & 233 & --- & e11 & 18 & --- & 219 & --- & 414 & --- & 96 & 106 & --- \\
\hline TOTAL & 6431 & 4535 & 368 & 576 & 669 & 3468 & 6764 & 9146 & 4057 & 3061 & 3042 & 3531 \\
\hline MEAN & 207 & 151 & 11.9 & 18.6 & 23.9 & 112 & 225 & 295 & 135 & 98.7 & 98.1 & 118 \\
\hline MAX & 237 & 234 & 22 & 55 & 51 & 220 & 258 & 425 & 412 & 117 & 106 & 150 \\
\hline MIN & 176 & 11 & 11 & 10 & 16 & 23 & 208 & 234 & 29 & 96 & 94 & 103 \\
\hline $\mathrm{AC}-\mathrm{FT}$ & 12760 & 9000 & 730 & 1140 & 1330 & 6880 & 13420 & 18140 & 8050 & 6070 & 6030 & 7000 \\
\hline
\end{tabular}


11408000 MILTON-BOWMAN TUNNEL OUTLET NEAR GRANITEVILLE, CA—Continued

STATISTICS OF MONTHLY MEAN DATA FOR WATER YEARS 1928 - 1964, BY WATER YEAR (WY)

\begin{tabular}{|c|c|c|c|c|c|c|c|c|c|c|c|c|}
\hline MEAN & 8.00 & 14.6 & 31.4 & 35.3 & 51.6 & 72.9 & 176 & 242 & 142 & 28.6 & 6.77 & 3.88 \\
\hline MAX & 101 & 65.4 & 118 & 124 & 143 & 213 & 294 & 414 & 272 & 90.9 & 26.8 & 10.1 \\
\hline (WY) & 1963 & 1951 & 1956 & 1942 & 1963 & 1940 & 1936 & 1937 & 1933 & 1938 & 1952 & 1952 \\
\hline MIN & .50 & .50 & .70 & 1.00 & 4.28 & 9.19 & 19.7 & 45.6 & 24.8 & 4.21 & 2.06 & 1.00 \\
\hline (WY) & 1931 & 1931 & 1931 & 1931 & 1931 & 1933 & 1938 & 1936 & 1934 & 1939 & 1964 & 1931 \\
\hline
\end{tabular}

SUMMARY STATISTICS

ANNUAL MEAN

HIGHEST ANNUAL MEAN

LOWEST ANNUAL MEAN

HIGHEST DAILY MEAN

LOWEST DAILY MEAN

ANNUAL SEVEN-DAY MINIMUM

ANNUAL RUNOFF (AC-FT)

10 PERCENT EXCEEDS

50 PERCENT EXCEEDS

90 PERCENT EXCEEDS
WATER YEARS $1928-1964$

\begin{tabular}{|c|c|c|c|}
\hline 67.9 & & & \\
\hline 97.2 & & & 1930 \\
\hline 33.5 & & & 1949 \\
\hline 492 & Feb & 11 & 1941 \\
\hline .40 & Oct & 7 & 1944 \\
\hline .50 & Oct & 1 & 1930 \\
\hline 49180 & & & \\
\hline 220 & & & \\
\hline 20 & & & \\
\hline 3.0 & & & \\
\hline
\end{tabular}

STATISTICS OF MONTHLY MEAN DATA FOR WATER YEARS 1966 - 1999, BY WATER YEAR (WY)

\begin{tabular}{|c|c|c|c|c|c|c|c|c|c|c|c|c|}
\hline MEAN & 152 & 129 & 59.0 & 37.6 & 36.6 & 55.5 & 57.1 & 98.6 & 81.5 & 64.7 & 88.3 & 154 \\
\hline MAX & 310 & 368 & 357 & 211 & 197 & 265 & 225 & 333 & 280 & 174 & 253 & 300 \\
\hline (WY) & 1981 & 1973 & 1973 & 1985 & 1985 & 1986 & 1999 & 1969 & 1998 & 1976 & 1968 & 1974 \\
\hline MIN & 1.52 & 1.34 & 1.25 & 1.17 & 1.20 & 1.68 & 5.38 & 7.69 & 5.23 & 3.95 & 2.20 & 1.72 \\
\hline (WY) & 1977 & 1977 & 1977 & 1977 & 1977 & 1977 & 1977 & 1986 & 1976 & 1977 & 1993 & 1981 \\
\hline
\end{tabular}

SUMMARY STATISTICS

ANNUAL TOTAL

ANNUAL MEAN

HIGHEST ANNUAL MEAN

LOWEST ANNUAL MEAN

HIGHEST DAILY MEAN

LOWEST DAILY MEAN

ANNUAL SEVEN-DAY MINIMUM

ANNUAL RUNOFF (AC-FT)

10 PERCENT EXCEEDS

50 PERCENT EXCEEDS

90 PERCENT EXCEEDS
FOR 1998 CALENDAR YEAR

$\begin{array}{rrr}50777.8 & & \\ 139 & & \\ & & \\ 394 & \text { Jun } & 14 \\ 9.0 & \text { Jan } & 1 \\ 9.7 & \text { Jan } & 1 \\ 100700 & & \\ 282 & & \\ 165 & & \\ 11 & & \end{array}$

FOR 1999 WATER YEAR

\begin{tabular}{|c|c|}
\hline $\begin{array}{r}45648 \\
125\end{array}$ & \\
\hline 425 & May 26 \\
\hline 10 & Jan 8 \\
\hline 10 & Jan \\
\hline 90540 & \\
\hline 244 & \\
\hline 104 & \\
\hline 12 & \\
\hline
\end{tabular}

WATER YEARS 1966 - 1999

\begin{tabular}{|c|c|c|}
\hline \multicolumn{3}{|l|}{84.6} \\
\hline 133 & & 1998 \\
\hline 14.5 & & 1977 \\
\hline 438 & Nov & 1972 \\
\hline 1.1 & Dec 11 & 1976 \\
\hline 1.1 & Dec 26 & 1976 \\
\hline \multicolumn{3}{|l|}{61310} \\
\hline \multicolumn{3}{|l|}{257} \\
\hline 26 & & \\
\hline 5.3 & & \\
\hline
\end{tabular}


LOCATION.—Lat 39³1'19", long 120³4'57", in SW 1/4 SW 1/4 sec.12, T.19 N., R.12 E., Sierra County, Hydrologic Unit 18020125, Tahoe National Forest, on right bank $350 \mathrm{ft}$ downstream from Milton Dam, and $4.1 \mathrm{mi}$ southeast of Sierra City.

DRAINAGE AREA.- $-39.9 \mathrm{mi}^{2}$.

PERIOD OF RECORD.-OCtober 1987 to current year. Unpublished records for water years 1965-87 available in files of the U.S. Geological Survey.

REVISED RECORDS.-WDR CA-88-4: Drainage area.

GAGE.-Water-stage recorder, sharp-crested weir, and crest-stage gage. Elevation of gage is 5,690 ft above sea level, from topographic map. Prior to October 1987, nonrecording gage $450 \mathrm{ft}$ downstream at different datum.

REMARKS.-Middle Yuba River is regulated by Jackson Meadows Reservoir (station 11407800) since November 1964 and Milton Reservoir. Tunnel diverts from Middle Yuba River at Milton Dam, in sec.12, T.19 N., R.12 E., and discharges into Bowman Lake via Milton-Bowman Tunnel (station 11408000). Practically the entire flow of Middle Yuba River is diverted during low and medium flows. See schematic diagram of Yuba River Basin.

COOPERATION.-Records were collected by Nevada Irrigation District, under general supervision of the U.S. Geological Survey, in connection with a Federal Energy Regulatory Commission project.

EXTREMES FOR PERIOD OF RECORD.-Maximum discharge, 8,610 ft³/s, Jan. 2, 1997, gage height, $17.1 \mathrm{ft}$, from flood marks; minimum daily, $0.77 \mathrm{ft}^{3} / \mathrm{s}$, Nov. 3, 1990.

DISCHARGE, CUBIC FEET PER SECOND, WATER YEAR OCTOBER 1998 TO SEPTEMBER 1999 DAILY MEAN VALUES

\begin{tabular}{|c|c|c|c|c|c|c|c|c|c|c|c|c|}
\hline DAY & OCT & NOV & $\mathrm{DEC}$ & JAN & FEB & MAR & APR & MAY & JUN & JUL & AUG & SEP \\
\hline 1 & 3.7 & 3.7 & 3.4 & 3.5 & 3.5 & 3.7 & 3.8 & 3.8 & 324 & 3.7 & 3.6 & 3.6 \\
\hline 2 & 3.7 & 3.7 & 3.4 & 3.4 & 3.5 & 3.6 & 3.8 & 3.8 & 335 & 3.7 & 3.5 & 3.6 \\
\hline 3 & 3.7 & 3.7 & 3.6 & 3.4 & 3.5 & 3.7 & 3.8 & 3.9 & 222 & 3.7 & 3.5 & 3.6 \\
\hline 4 & 3.7 & 3.7 & 3.4 & 3.4 & 3.5 & 3.6 & 3.8 & 3.8 & 86 & 3.6 & 3.5 & 3.6 \\
\hline 5 & 4.9 & 3.7 & 3.4 & 3.4 & 3.5 & 3.6 & 3.9 & 3.8 & 4.6 & 3.6 & 3.6 & 3.6 \\
\hline 6 & 3.7 & 3.7 & 3.4 & 3.4 & 3.5 & 3.6 & 3.8 & 3.8 & 4.3 & 3.6 & 3.6 & 3.6 \\
\hline 7 & 3.7 & 3.8 & 3.3 & 3.4 & 3.7 & 3.5 & 3.8 & 3.8 & 4.1 & 3.6 & 3.6 & 3.6 \\
\hline 8 & 3.7 & 3.7 & 3.3 & 3.4 & 3.6 & 3.5 & 3.8 & 3.8 & 3.9 & 3.6 & 3.6 & 3.6 \\
\hline 9 & 3.7 & 3.7 & 3.3 & 3.4 & 3.8 & 3.5 & 3.8 & 3.8 & 3.8 & 3.6 & 3.6 & 3.6 \\
\hline 10 & 3.8 & 3.7 & 3.3 & 3.4 & 3.6 & 3.6 & 3.8 & 3.8 & 3.8 & 3.6 & 3.6 & 3.6 \\
\hline 11 & 3.8 & 3.7 & 3.3 & 3.4 & 3.6 & 3.8 & 3.8 & 3.8 & 3.8 & 3.6 & 3.6 & 3.6 \\
\hline 12 & 3.8 & 3.7 & 3.3 & 3.4 & 3.6 & 3.8 & 3.8 & 3.8 & 3.8 & 3.6 & 3.6 & 3.6 \\
\hline 13 & 3.8 & 3.7 & 3.3 & 3.4 & 3.5 & 3.8 & 3.8 & 3.8 & 3.8 & 3.6 & 3.6 & 3.6 \\
\hline 14 & 3.8 & 3.7 & 3.3 & 3.4 & 3.5 & 3.8 & 3.8 & 3.8 & 3.7 & 3.6 & 3.6 & 3.6 \\
\hline 15 & 3.9 & 3.7 & 3.3 & 3.5 & 3.5 & 3.8 & 3.8 & 3.8 & 3.7 & 3.6 & 3.6 & 3.6 \\
\hline 16 & 3.9 & 3.7 & 3.3 & 3.5 & 3.6 & 3.8 & 3.9 & 3.8 & 3.6 & 3.6 & 3.6 & 3.6 \\
\hline 17 & 3.8 & 3.8 & 3.3 & 3.6 & 3.7 & 3.8 & 3.9 & 3.8 & 3.6 & 3.6 & 3.6 & 3.6 \\
\hline 18 & 3.8 & 3.7 & 3.3 & 3.8 & 3.6 & 3.8 & 3.9 & 3.8 & 3.6 & 3.6 & 3.6 & 3.6 \\
\hline 19 & 3.8 & 3.7 & 3.3 & 3.7 & 3.6 & 3.8 & 3.9 & 3.8 & 3.6 & 3.6 & 3.6 & 3.6 \\
\hline 20 & 3.8 & 3.4 & 3.3 & 3.9 & 3.5 & 3.8 & 3.9 & 3.8 & 3.6 & 3.6 & 3.6 & 3.6 \\
\hline 21 & 3.8 & 3.4 & 3.4 & 3.6 & 3.5 & 3.8 & 3.9 & 3.8 & 3.6 & 3.6 & 3.6 & 3.6 \\
\hline 22 & 3.8 & 3.4 & 3.4 & 3.6 & 3.5 & 3.8 & 3.9 & 3.8 & 3.5 & 3.6 & 3.6 & 3.6 \\
\hline 23 & 3.8 & 3.6 & 3.4 & 3.7 & 3.5 & 3.8 & 3.9 & 3.9 & 3.5 & 3.6 & 3.6 & 3.6 \\
\hline 24 & 3.9 & 3.4 & 3.4 & 3.6 & 3.5 & 3.8 & 3.9 & 290 & 3.4 & 3.6 & 3.6 & 3.7 \\
\hline 25 & 3.8 & 3.3 & 3.4 & 3.5 & 3.5 & 3.8 & 3.9 & 590 & 3.5 & 3.6 & 3.6 & 3.7 \\
\hline 26 & 3.8 & 3.3 & 3.4 & 3.5 & 3.5 & 3.8 & 3.9 & 674 & 3.5 & 3.6 & 3.6 & 3.7 \\
\hline 27 & 3.8 & 3.4 & 3.4 & 3.5 & 3.5 & 3.8 & 3.9 & 666 & 3.5 & 3.6 & 3.6 & 3.7 \\
\hline 28 & 3.8 & 3.3 & 3.4 & 3.5 & 3.6 & 3.8 & 3.9 & 609 & 3.5 & 3.6 & 3.6 & 3.7 \\
\hline 29 & 3.8 & 3.4 & 3.4 & 3.5 & --- & 3.8 & 3.8 & 508 & 3.5 & 3.6 & 3.6 & 3.7 \\
\hline 30 & 3.7 & 3.6 & 3.4 & 3.5 & --- & 3.9 & 3.8 & 365 & 3.7 & 3.6 & 3.6 & 3.7 \\
\hline 31 & 3.7 & --- & 3.4 & 3.5 & --- & 3.8 & --- & 339 & --- & 3.6 & 3.6 & --- \\
\hline TOTAL & 118.2 & 108.0 & 104.2 & 108.7 & 99.5 & 115.8 & 115.4 & 4128.6 & 1063.5 & 111.9 & 111.3 & 108.7 \\
\hline MEAN & 3.81 & 3.60 & 3.36 & 3.51 & 3.55 & 3.74 & 3.85 & 133 & 35.5 & 3.61 & 3.59 & 3.62 \\
\hline MAX & 4.9 & 3.8 & 3.6 & 3.9 & 3.8 & 3.9 & 3.9 & 674 & 335 & 3.7 & 3.6 & 3.7 \\
\hline MIN & 3.7 & 3.3 & 3.3 & 3.4 & 3.5 & 3.5 & 3.8 & 3.8 & 3.4 & 3.6 & 3.5 & 3.6 \\
\hline $\mathrm{AC}-\mathrm{FT}$ & 234 & 214 & 207 & 216 & 197 & 230 & 229 & 8190 & 2110 & 222 & 221 & 216 \\
\hline
\end{tabular}


11408550 MIDDLE YUBA RIVER BELOW MILTON DAM, NEAR SIERRA CITY, CA—Continued

STATISTICS OF MONTHLY MEAN DATA FOR WATER YEARS 1988 - 1999, BY WATER YEAR (WY)

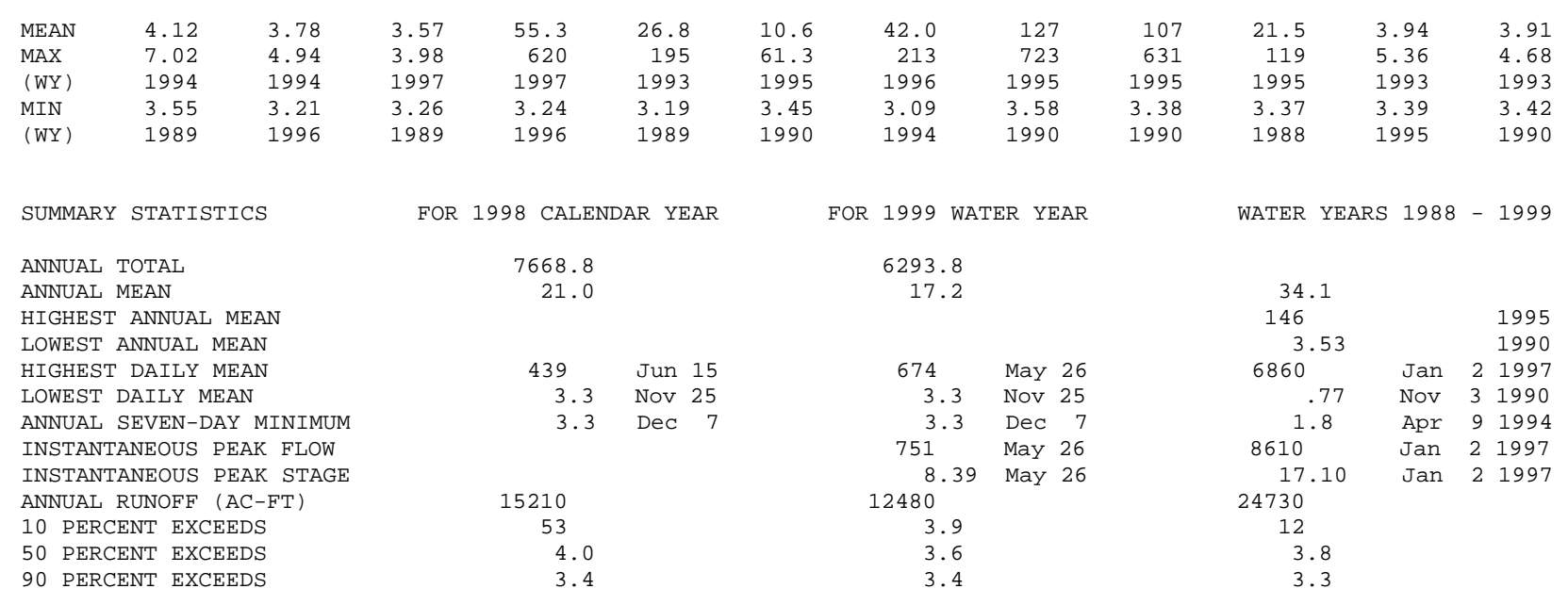


LOCATION.-Lat $39^{\circ} 24^{\prime} 25^{\prime \prime}$, long 12059'43", in SW 1/4 NE 1/4 sec.20, T.18 N., R.8 E., Sierra County, Hydrologic Unit 18020125, Tahoe National Forest, at tunnel intake at Our House Dam and 4.0 mi southeast of Camptonville.

PERIOD OF RECORD.-October 1988 to current year. Records of monthly diversion published with Middle Yuba River below Our House Dam, near Camptonville (station 11408880), for water years 1969-88.

GAGE.-Water-stage recorder. Datum of gage is $2,014.77 \mathrm{ft}$ above sea level.

REMARKS.--Records good except for estimated daily discharges, which are fair. Tunnel diverts water from Middle Yuba River to New Bullards Bar Reservoir (station 11413515) for power development. See schematic diagram of Yuba River Basin.

EXTREMES FOR PERIOD OF RECORD.-Maximum daily discharge, $839 \mathrm{ft}^{3}$ /s, Mar. 25, 1989; no flow for many days in most years.

DISCHARGE, CUBIC FEET PER SECOND, WATER YEAR OCTOBER 1998 TO SEPTEMBER 1999 DAILY MEAN VALUES

\begin{tabular}{|c|c|c|c|c|c|c|c|c|c|c|c|c|}
\hline DAY & $\mathrm{OCT}$ & NOV & $\mathrm{DEC}$ & JAN & $\mathrm{FEB}$ & MAR & APR & MAY & JUN & JUL & AUG & SEP \\
\hline 1 & 23 & 23 & 599 & 84 & 284 & 763 & 310 & 438 & 685 & 80 & 20 & 6.2 \\
\hline 2 & 23 & 23 & 381 & 80 & 256 & 736 & 293 & 483 & 673 & 75 & 19 & 5.5 \\
\hline 3 & 22 & 19 & 642 & 77 & 238 & e750 & 289 & 564 & 566 & 70 & 19 & 5.5 \\
\hline 4 & 23 & 18 & 502 & 74 & 230 & e735 & 270 & 486 & 420 & 67 & 18 & 5.1 \\
\hline 5 & 21 & 20 & 419 & 72 & 218 & 718 & 284 & 444 & 268 & 63 & 18 & 4.0 \\
\hline 6 & e20 & 21 & 321 & 70 & 258 & e700 & 271 & 452 & 279 & 59 & 17 & 3.4 \\
\hline 7 & e20 & 68 & 236 & 68 & 773 & e 615 & 265 & 496 & 277 & 55 & 19 & 2.8 \\
\hline 8 & e17 & 73 & 208 & 66 & 809 & 556 & 303 & 510 & 247 & 52 & 18 & 2.9 \\
\hline 9 & e18 & 35 & 191 & 63 & 695 & 540 & 285 & 474 & 226 & 49 & 17 & 2.8 \\
\hline 10 & 18 & 28 & 173 & 61 & 626 & 483 & 274 & 453 & 215 & 46 & 19 & 3.3 \\
\hline 11 & 17 & 39 & 161 & 60 & 662 & 449 & 306 & 447 & 204 & 44 & 20 & 2.7 \\
\hline 12 & 17 & 29 & 153 & 59 & e 690 & 425 & 345 & 493 & 212 & 42 & 17 & 1.8 \\
\hline 13 & 16 & 25 & 159 & 58 & e 660 & 408 & 380 & 536 & 211 & 39 & 16 & 1.6 \\
\hline 14 & 17 & 23 & 174 & 56 & 571 & 402 & 441 & 496 & 209 & 38 & 14 & 2.8 \\
\hline 15 & 16 & 21 & 160 & 77 & 505 & 378 & 457 & 429 & 209 & 37 & 13 & 1.9 \\
\hline 16 & 16 & 21 & 163 & 199 & 552 & 364 & 463 & 400 & 208 & 34 & 12 & 1.3 \\
\hline 17 & 15 & 69 & 174 & 255 & 773 & 359 & 496 & 399 & 204 & 33 & 12 & 1.1 \\
\hline 18 & 15 & 65 & 167 & 705 & 752 & 365 & 537 & 437 & 188 & 32 & 11 & 1.2 \\
\hline 19 & 14 & 39 & 159 & 741 & 741 & 371 & 571 & 458 & 176 & 30 & 9.9 & 1.8 \\
\hline 20 & 13 & 31 & 151 & 670 & 722 & 377 & 585 & 470 & 166 & 30 & 9.2 & 1.5 \\
\hline 21 & 13 & 32 & 129 & 572 & 709 & 359 & 585 & 469 & 155 & 31 & 9.2 & 1.6 \\
\hline 22 & 13 & 174 & 122 & 554 & 643 & 338 & 524 & 515 & 146 & 31 & 8.9 & 1.1 \\
\hline 23 & 13 & e240 & 113 & 575 & 587 & 329 & 481 & 560 & 141 & 29 & 8.7 & .99 \\
\hline 24 & 24 & 460 & 111 & 551 & e 560 & 324 & 481 & 700 & 132 & 28 & 7.5 & .92 \\
\hline 25 & 42 & 160 & 105 & 608 & 702 & 331 & 499 & 770 & 122 & 27 & 6.5 & .88 \\
\hline 26 & 24 & 107 & 101 & 587 & 611 & 337 & 581 & 780 & 112 & 27 & 6.2 & .54 \\
\hline 27 & 19 & 155 & 97 & 488 & 546 & e350 & 579 & 805 & 102 & 25 & 8.9 & .28 \\
\hline 28 & 18 & e200 & 94 & 427 & 609 & e 343 & 511 & 800 & 94 & 24 & 7.2 & .25 \\
\hline 29 & 19 & 223 & 90 & e370 & --- & 332 & 447 & 785 & 89 & 23 & 6.1 & .23 \\
\hline 30 & 23 & 707 & 88 & e340 & --- & 341 & 408 & 775 & 84 & 22 & 5.4 & .25 \\
\hline 31 & 19 & --- & 88 & 317 & --- & 340 & --- & 733 & --- & 21 & 6.4 & --- \\
\hline TOTAL & 588 & 3148 & 6431 & 8984 & 15982 & 14218 & 12521 & 17057 & 7020 & 1263 & 399.1 & 66.24 \\
\hline MEAN & 19.0 & 105 & 207 & 290 & 571 & 459 & 417 & 550 & 234 & 40.7 & 12.9 & 2.21 \\
\hline MAX & 42 & 707 & 642 & 741 & 809 & 763 & 585 & 805 & 685 & 80 & 20 & 6.2 \\
\hline MIN & 13 & 18 & 88 & 56 & 218 & 324 & 265 & 399 & 84 & 21 & 5.4 & .23 \\
\hline $\mathrm{AC}-\mathrm{FT}$ & 1170 & 6240 & 12760 & 17820 & 31700 & 28200 & 24840 & 33830 & 13920 & 2510 & 792 & 131 \\
\hline
\end{tabular}

STATISTICS OF MONTHLY MEAN DATA FOR WATER YEARS 1989 - 1999, BY WATER YEAR (WY)

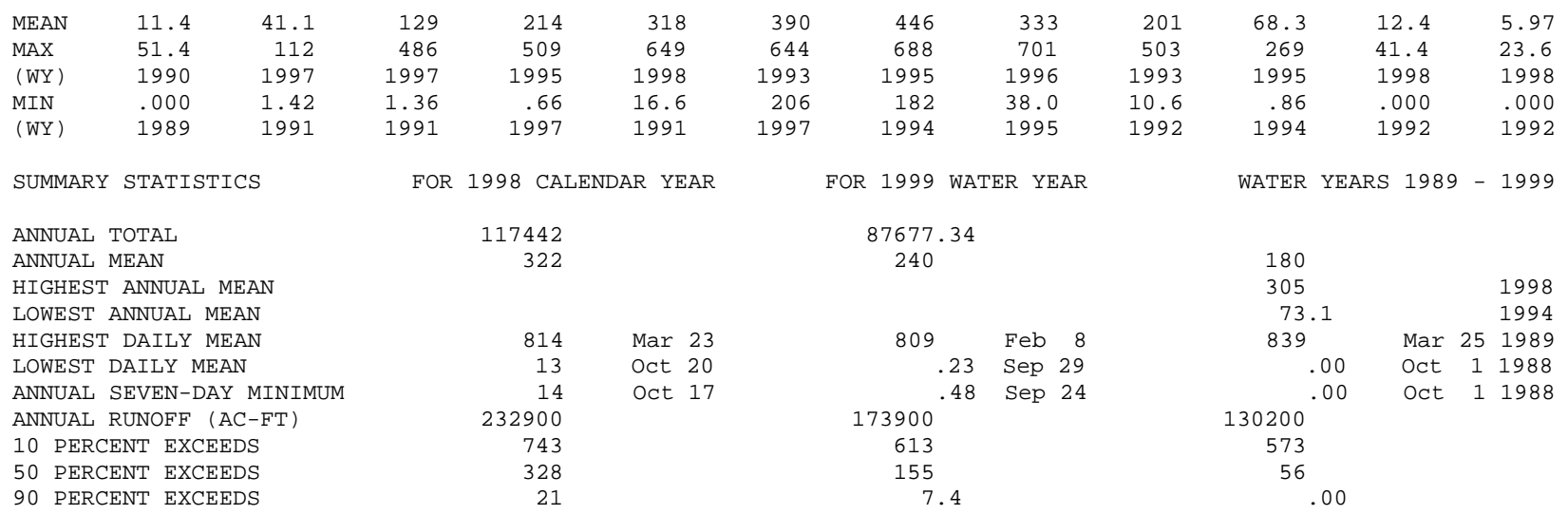

e Estimated. 
LOCATION.-Lat 39²4'42", long 12059’49", in SW 1/4 NW 1/4 sec.20, T.18 N., R.9 E., Sierra County, Hydrologic Unit 18020125, Tahoe National Forest, on right bank $300 \mathrm{ft}$ downstream from Our House Dam and $4.0 \mathrm{mi}$ southeast of Camptonville.

DRAINAGE AREA.-145 $\mathrm{mi}^{2}$.

PERIOD OF RECORD.-October 1968 to current year.

GAGE.-Water-stage recorder, sharp-crested weir since Oct. 16, 1990, and crest-stage gage. Datum of gage is $1,957.51 \mathrm{ft}$ above sea level. Prior to Nov. 4, 1970, water-stage recorder at datum $10 \mathrm{ft}$ higher. Prior to Oct. 1, 1987, at site $75 \mathrm{ft}$ downstream.

REMARKS.--Records good except for periods of spill and estimated daily discharges, which are fair. Natural flow of stream affected by Jackson Meadows Reservoir (station 11407800), Milton Bowman Tunnel (station 11408000), which diverts upstream from station to Bowman Lake (station 11415500), and Lohman Ridge Tunnel (station 11408870), which diverts $300 \mathrm{ft}$ upstream to Oregon Creek and then to New Bullards Bar Reservoir (station 11413515) via Camptonville Tunnel (station 11409350). Other small diversions upstream from station. See schematic diagram of Yuba River Basin.

EXTREMES FOR PERIOD OF RECORD.-Maximum discharge, 27,500 ft $3 / \mathrm{s}$, Jan. 2, 1997, gage height, $30.7 \mathrm{ft}$, from floodmark, present datum, from rating curve extended above $8,600 \mathrm{ft}^{3} / \mathrm{s}$ on basis of theoretical rating of Our House Dam spillway; minimum daily, $2.1 \mathrm{ft}^{3} / \mathrm{s}, \mathrm{Jan} .10,1982$.

DISCHARGE, CUBIC FEET PER SECOND, WATER YEAR OCTOBER 1998 TO SEPTEMBER 1999

\begin{tabular}{|c|c|c|c|c|c|c|c|c|c|c|c|c|}
\hline DAY & OCT & NOV & $\mathrm{DEC}$ & JAN & FEB & MAR & APR & MAY & JUN & JUL & AUG & SEP \\
\hline 1 & 33 & 33 & 153 & 34 & 41 & 787 & 40 & 55 & 58 & 38 & 37 & 37 \\
\hline 2 & 33 & 33 & 41 & 34 & 40 & 368 & 40 & 56 & 58 & 38 & 37 & 37 \\
\hline 3 & 33 & 33 & 608 & 33 & 40 & 650 & 40 & 58 & 55 & 38 & 37 & 37 \\
\hline 4 & 33 & 33 & 238 & 34 & 40 & 350 & 40 & 57 & 55 & 38 & 37 & 37 \\
\hline 5 & 33 & 33 & 40 & 33 & 39 & 162 & 39 & 56 & 54 & 38 & 37 & 37 \\
\hline 6 & 33 & 33 & 38 & 33 & 39 & 60 & 39 & 56 & 54 & 38 & 37 & 37 \\
\hline 7 & 33 & 34 & 37 & 33 & 708 & 43 & 39 & 57 & 55 & 38 & 37 & 37 \\
\hline 8 & 33 & 34 & 37 & 33 & 761 & 42 & 40 & 57 & 56 & 38 & 37 & 37 \\
\hline 9 & 33 & 33 & 36 & 33 & e 3400 & 42 & 40 & 57 & 55 & 38 & 37 & 37 \\
\hline 10 & 33 & 33 & 36 & 33 & e1350 & 41 & 39 & 56 & 55 & 38 & 37 & 37 \\
\hline 11 & 33 & 33 & 35 & 33 & e 530 & 41 & 40 & 56 & 55 & 38 & 37 & 37 \\
\hline 12 & 33 & 33 & 35 & 33 & e250 & 41 & 41 & 56 & 55 & 38 & 37 & 37 \\
\hline 13 & 33 & 33 & 35 & 33 & e 54 & 41 & 41 & 55 & 55 & 38 & 37 & 37 \\
\hline 14 & 33 & 33 & 35 & 33 & e 40 & 41 & 49 & 54 & 55 & 37 & 37 & 37 \\
\hline 15 & 33 & 35 & 35 & 34 & e 40 & 41 & 54 & 53 & 55 & 37 & 37 & 37 \\
\hline 16 & 33 & 35 & 35 & 36 & e40 & 41 & 53 & 53 & 46 & 37 & 37 & 37 \\
\hline 17 & 33 & 35 & 35 & 36 & e 910 & 41 & 53 & 53 & 37 & 37 & 37 & 37 \\
\hline 18 & 33 & 34 & 35 & 634 & e457 & 40 & 53 & 54 & 37 & 37 & 37 & 37 \\
\hline 19 & 33 & 34 & 35 & 854 & e334 & 41 & 53 & 55 & 37 & 37 & 37 & 37 \\
\hline 20 & 33 & 34 & 35 & 2310 & e185 & 40 & 52 & 55 & 37 & 37 & 37 & 37 \\
\hline 21 & 33 & 34 & 34 & 1080 & e141 & 40 & 52 & 55 & 37 & 37 & 37 & 37 \\
\hline 22 & 33 & 36 & 34 & 445 & e47 & 40 & 51 & 56 & 37 & 37 & 37 & 37 \\
\hline 23 & 33 & 64 & 34 & 1290 & e 45 & 40 & 51 & 57 & 37 & 37 & 37 & 37 \\
\hline 24 & 33 & 71 & 34 & 560 & 42 & 40 & 51 & 105 & 37 & 37 & 37 & 37 \\
\hline 25 & 33 & 38 & 34 & 203 & 102 & 40 & 52 & 424 & 37 & 37 & 37 & 37 \\
\hline 26 & 33 & 38 & 34 & 47 & 43 & 40 & 53 & 565 & 37 & 37 & 37 & 37 \\
\hline 27 & 33 & 38 & 34 & 46 & 42 & 41 & 53 & 500 & 38 & 37 & 37 & 37 \\
\hline 28 & 33 & 39 & 34 & 45 & 155 & 40 & 52 & 392 & 39 & 37 & 37 & 37 \\
\hline 29 & 33 & 39 & 34 & 44 & --- & 40 & 52 & 244 & 38 & 37 & 37 & 36 \\
\hline 30 & 33 & 498 & 34 & 43 & --- & 40 & 53 & 91 & 38 & 37 & 37 & 37 \\
\hline 31 & 33 & --- & 34 & 43 & --- & 40 & --- & 62 & --- & 37 & 37 & --- \\
\hline TOTAL & 1023 & 1566 & 1988 & 8215 & 9915 & 3394 & 1405 & 3660 & 1399 & 1160 & 1147 & 1109 \\
\hline MEAN & 33.0 & 52.2 & 64.1 & 265 & 354 & 109 & 46.8 & 118 & 46.6 & 37.4 & 37.0 & 37.0 \\
\hline MAX & 33 & 498 & 608 & 2310 & 3400 & 787 & 54 & 565 & 58 & 38 & 37 & 37 \\
\hline MIN & 33 & 33 & 34 & 33 & 39 & 40 & 39 & 53 & 37 & 37 & 37 & 36 \\
\hline $\mathrm{AC}-\mathrm{FT}$ & 2030 & 3110 & 3940 & 16290 & 19670 & 6730 & 2790 & 7260 & 2770 & 2300 & 2280 & 2200 \\
\hline
\end{tabular}

STATISTICS OF MONTHLY MEAN DATA FOR WATER YEARS 1969 - 1999, BY WATER YEAR (WY)

\begin{tabular}{|c|c|c|c|c|c|c|c|c|c|}
\hline MEAN & 31.0 & 76.9 & 166 & 383 & 239 & 245 & 161 & 224 & 121 \\
\hline MAX & 52.7 & 462 & 1040 & 2973 & 1521 & 1228 & 1368 & 1697 & 994 \\
\hline (WY) & 1983 & 1982 & 1982 & 1997 & 1986 & 1995 & 1982 & 1995 & 1995 \\
\hline MIN & 16.6 & 20.4 & 20.7 & 7.10 & 28.0 & 31.3 & 33.9 & 32.5 & 28.8 \\
\hline (WY) & 1978 & 1978 & 1987 & 1987 & 1977 & 1976 & 1970 & 1970 & 1977 \\
\hline
\end{tabular}

$\begin{array}{lll}33.6 & 30.1 & 29.9 \\ 49.6 & 42.1 & 39.6 \\ 1983 & 1984 & 1986 \\ 17.5 & 13.0 & 14.3 \\ 1977 & 1977 & 1977\end{array}$

SUMMARY STATISTICS

ANNUAL TOTAL

ANNUAL MEAN

HIGHEST ANNUAL MEAN

LOWEST ANNUAL MEAN

HIGHEST DAILY MEAN

LOWEST DAILY MEAN

ANNUAL SEVEN-DAY MINIMUM

INSTANTANEOUS PEAK FLOW

INSTANTANEOUS PEAK STAGE

ANNUAL RUNOFF (AC-FT)

10 PERCENT EXCEEDS

50 PERCENT EXCEEDS

90 PERCENT EXCEEDS

e Estimated.
FOR 1998 CALENDAR YEAR

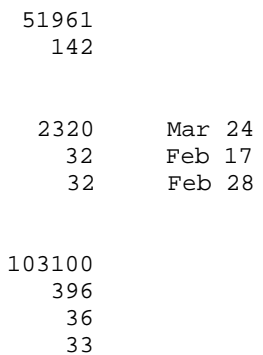

FOR 1999 WATER YEAR

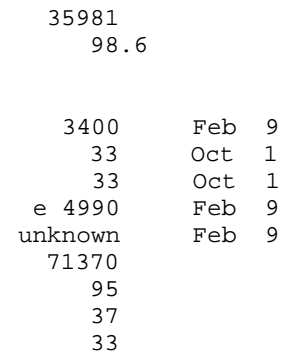

WATER YEARS 1969 - 1999

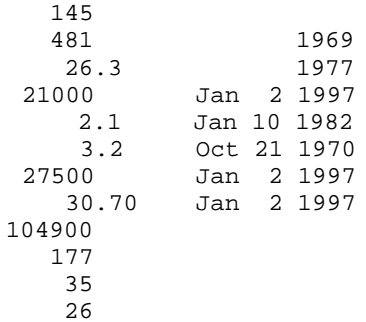


LOCATION.-Lat $39^{\circ} 26^{\prime} 46^{\prime \prime}$, long $121^{\circ} 02^{\prime} 43^{\prime \prime}$, in SE 1/4 NE 1/4 sec.11, T.18 N., R.8 E., Yuba County, Hydrologic Unit 18020125, Tahoe

National Forest, on right bank $25 \mathrm{ft}$ downstream from county bridge, $0.5 \mathrm{mi}$ southeast of Camptonville, and $5.5 \mathrm{mi}$ upstream from mouth.

DRAINAGE AREA.-23.0 $\mathrm{mi}^{2}$.

PERIOD OF RECORD.-August 1967 to current year.

GAGE.-Water-stage recorder. Elevation of gage is 2,230 ft above sea level, from topographic map.

REMARKS.- - Records good. No regulation or diversion upstream from station. See schematic diagram of Yuba River Basin.

EXTREMES FOR PERIOD OF RECORD.-Maximum discharge, 5,170 ft $3 / \mathrm{s}$, Jan. 1, 1997, gage height, $11.31 \mathrm{ft}$, from rating curve extended above $4,000 \mathrm{ft}^{3} / \mathrm{s}$, maximum gage height, $11.56 \mathrm{ft}$, Feb. 17, 1986; minimum daily, $0.53 \mathrm{ft}^{3} / \mathrm{s}$, Aug. 14-16, 1977, Sept. 6, 1988.

EXTREMES FOR CURRENT YEAR.-Peak discharges greater than base discharge of $500 \mathrm{ft}^{3} / \mathrm{s}$, or maximum:

\begin{tabular}{|c|c|c|c|c|c|c|c|}
\hline Date & Time & $\begin{array}{c}\text { Discharge } \\
\left(\mathrm{ft}^{3} / \mathrm{s}\right)\end{array}$ & $\begin{array}{l}\text { Gage height } \\
\text { (ft) }\end{array}$ & Date & Time & $\begin{array}{l}\text { Discharge } \\
\left(\mathrm{ft}^{3} / \mathrm{s}\right)\end{array}$ & $\begin{array}{l}\text { Gage height } \\
\text { (ft) }\end{array}$ \\
\hline Dec. 3 & 1200 & 661 & 6.71 & Feb. 17 & 0245 & 827 & 7.04 \\
\hline Jan. 20 & 1830 & 1,540 & 8.15 & Feb. 28 & 2245 & 661 & 6.71 \\
\hline Feb. 9 & 1015 & 1,800 & 8.46 & & & & \\
\hline
\end{tabular}

\section{DISCHARGE, CUBIC FEET PER SECOND, WATER YEAR OCTOBER 1998 TO SEPTEMBER 1999}

\begin{tabular}{|c|c|c|c|c|c|c|c|c|c|c|c|c|}
\hline DAY & $\mathrm{OCT}$ & NOV & $\mathrm{DEC}$ & JAN & FEB & MAR & APR & MAY & JUN & JUL & AUG & SEP \\
\hline 1 & 4.5 & 4.8 & 228 & 21 & 97 & 502 & 70 & 62 & 21 & 7.3 & 3.6 & 2.6 \\
\hline 2 & 4.4 & 4.7 & 117 & 20 & 90 & 364 & 68 & 64 & 21 & 7.0 & 4.7 & 2.6 \\
\hline 3 & 4.5 & 4.4 & 384 & 20 & 84 & 472 & 67 & 120 & 21 & 7.0 & 4.6 & 2.6 \\
\hline 4 & 4.6 & 4.3 & 226 & 19 & 81 & 368 & 64 & 109 & 21 & 6.8 & 3.4 & 2.6 \\
\hline 5 & 4.4 & 4.7 & 135 & 19 & 76 & 294 & 67 & 92 & 20 & 6.8 & 3.3 & 2.5 \\
\hline 6 & 4.1 & 5.0 & 100 & 18 & 84 & 244 & 63 & 84 & 19 & 6.5 & 3.4 & 2.5 \\
\hline 7 & 4.0 & 17 & 79 & 18 & 583 & 202 & 63 & 81 & 18 & 6.3 & 3.5 & 2.5 \\
\hline 8 & 3.9 & 13 & 68 & 18 & 610 & 175 & 71 & 78 & 18 & 6.2 & 3.6 & 2.4 \\
\hline 9 & 3.9 & 7.2 & 58 & 17 & 1320 & 160 & 66 & 75 & 17 & 6.4 & 3.5 & 2.4 \\
\hline 10 & 4.0 & 6.1 & 51 & 17 & 650 & 139 & 67 & 69 & 17 & 7.1 & 3.6 & 2.4 \\
\hline 11 & 4.0 & 7.3 & 45 & 16 & 405 & 127 & 80 & 65 & 16 & 7.5 & 3.6 & 2.4 \\
\hline 12 & 3.9 & 6.2 & 42 & 16 & 301 & 117 & 97 & 61 & 16 & 9.0 & 3.5 & 2.3 \\
\hline 13 & 3.9 & 5.6 & 43 & 15 & 239 & 112 & 104 & 58 & 15 & 8.1 & 3.3 & 2.3 \\
\hline 14 & 3.9 & 5.3 & 49 & 15 & 201 & 108 & 113 & 56 & 15 & 5.3 & 3.1 & 2.3 \\
\hline 15 & 3.9 & 5.1 & 48 & 18 & 169 & 103 & 117 & 53 & 14 & 5.1 & 3.1 & 2.3 \\
\hline 16 & 3.8 & 5.0 & 48 & 45 & 215 & 98 & 113 & 50 & 13 & 5.0 & 3.0 & 2.2 \\
\hline 17 & 3.7 & 11 & 46 & 87 & 555 & 94 & 110 & 47 & 13 & 4.9 & 2.9 & 2.2 \\
\hline 18 & 3.7 & 12 & 43 & 405 & 395 & 90 & 106 & 45 & 12 & 5.0 & 2.9 & 2.1 \\
\hline 19 & 3.6 & 7.6 & 40 & 519 & 345 & 87 & 104 & 44 & 11 & 5.1 & 2.9 & 2.3 \\
\hline 20 & 3.5 & 6.3 & 36 & 1010 & 287 & 87 & 101 & 42 & 11 & 4.9 & 2.9 & 2.3 \\
\hline 21 & 3.4 & 6.8 & 31 & 673 & 261 & 86 & 97 & 40 & 11 & 4.8 & 2.8 & 2.3 \\
\hline 22 & 3.4 & 33 & 29 & 394 & 220 & 84 & 91 & 37 & 10 & 4.6 & 2.8 & 2.2 \\
\hline 23 & 3.4 & 75 & 28 & 952 & 200 & 82 & 85 & 35 & 9.9 & 4.5 & 2.7 & 2.2 \\
\hline 24 & 4.8 & 91 & 27 & 497 & 191 & 81 & 81 & 34 & 9.5 & 4.3 & 2.6 & 2.2 \\
\hline 25 & 6.5 & 32 & 26 & 329 & 291 & 83 & 78 & 31 & 9.1 & 4.3 & 2.6 & 2.2 \\
\hline 26 & 4.8 & 21 & 26 & 246 & 225 & 80 & 80 & 29 & 9.0 & 4.3 & 2.6 & 2.2 \\
\hline 27 & 4.2 & 45 & 25 & 187 & 192 & 78 & 79 & 27 & 8.7 & 4.1 & 2.8 & 2.1 \\
\hline 28 & 4.1 & 68 & 24 & 154 & 303 & 76 & 76 & 25 & 8.3 & 4.0 & 2.8 & 2.0 \\
\hline 29 & 4.1 & 70 & 23 & 133 & --- & 74 & 70 & 24 & 8.0 & 3.9 & 2.6 & 2.0 \\
\hline 30 & 4.6 & 348 & 22 & 118 & --- & 76 & 65 & 23 & 7.7 & 3.8 & 2.5 & 2.0 \\
\hline 31 & 4.4 & --- & 22 & 110 & --- & 76 & --- & 22 & --- & 3.7 & 2.6 & --- \\
\hline TOTAL & 127.9 & 932.4 & 2169 & 6126 & 8670 & 4819 & 2513 & 1682 & 420.2 & 173.6 & 97.8 & 69.2 \\
\hline MEAN & 4.13 & 31.1 & 70.0 & 198 & 310 & 155 & 83.8 & 54.3 & 14.0 & 5.60 & 3.15 & 2.31 \\
\hline MAX & 6.5 & 348 & 384 & 1010 & 1320 & 502 & 117 & 120 & 21 & 9.0 & 4.7 & 2.6 \\
\hline MIN & 3.4 & 4.3 & 22 & 15 & 76 & 74 & 63 & 22 & 7.7 & 3.7 & 2.5 & 2.0 \\
\hline $\mathrm{AC}-\mathrm{FT}$ & 254 & 1850 & 4300 & 12150 & 17200 & 9560 & 4980 & 3340 & 833 & 344 & 194 & 137 \\
\hline
\end{tabular}

STATISTICS OF MONTHLY MEAN DATA FOR WATER YEARS 1968 - 1999, BY WATER YEAR (WY)

\begin{tabular}{|c|c|c|c|c|c|c|c|c|c|c|c|c|}
\hline MEAN & 5.24 & 33.3 & 84.9 & 167 & 163 & 170 & 112 & 64.0 & 19.9 & 6.04 & 2.90 & 2.80 \\
\hline MAX & 16.9 & 214 & 407 & 555 & 664 & 453 & 391 & 198 & 92.3 & 17.9 & 6.16 & 9.12 \\
\hline (WY) & 1982 & 1974 & 1984 & 1997 & 1986 & 1989 & 1982 & 1995 & 1998 & 1998 & 1998 & 1983 \\
\hline MIN & .84 & 3.03 & 2.30 & 3.88 & 6.28 & 10.8 & 7.64 & 9.45 & 3.61 & 1.11 & .68 & .67 \\
\hline (WY) & 1989 & 1991 & 1977 & 1991 & 1991 & 1977 & 1977 & 1987 & 1987 & 1977 & 1977 & 1988 \\
\hline
\end{tabular}

SUMMARY STATISTICS

ANNUAL TOTAL

ANNUAL MEAN

HIGHEST ANNUAL MEAN

LOWEST ANNUAL MEAN

HIGHEST DAILY MEAN

LOWEST DAILY MEAN

ANNUAL SEVEN-DAY MINIMUM

INSTANTANEOUS PEAK FLOW

INSTANTANEOUS PEAK STAGE

ANNUAL RUNOFF (AC-FT)

10 PERCENT EXCEEDS

50 PERCENT EXCEEDS

90 PERCENT EXCEEDS
FOR 1998 CALENDAR YEAR

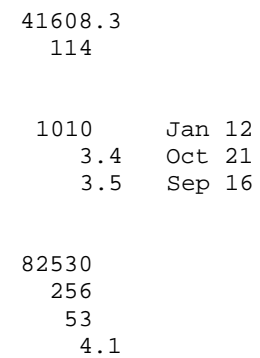

FOR 1999 WATER YEAR

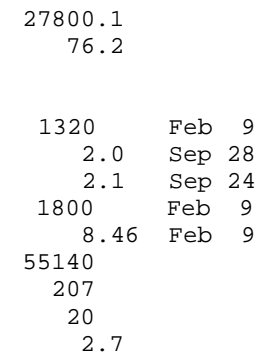

WATER YEARS 1968 - 1999

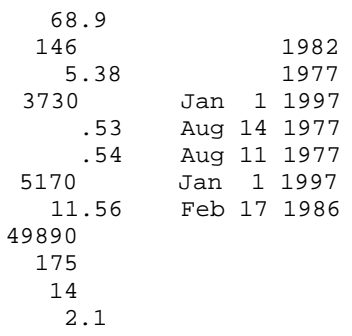


11409350 CAMPTONVILLE TUNNEL AT INTAKE, NEAR CAMPTONVILLE, CA

LOCATION.-Lat 39²6’25", long 12103’30", in NW 1/4 SW 1/4 sec.11, T.18 N., R.8 E., Yuba County, Hydrologic Unit 18020125, Tahoe National Forest, at tunnel intake at Log Cabin Dam 1.0 mi southwest of town of Camptonville.

PERIOD OF RECORD.-October 1988 to current year. Records of monthly diversion published with Oregon Creek below Log Cabin Dam near Camptonville (station 11409400) for water years 1969-88.

GAGE.-Water-stage recorder. Datum of gage is 1,952.00 ft above sea level (from contractor's drawings).

REMARKS.--Records fair. Water is diverted to Oregon Creek from the Middle Yuba River through Lohman Ridge Tunnel (station 11408870) $1,000 \mathrm{ft}$ upstream. Camptonville Tunnel diverts water from Oregon Creek to New Bullards Bar Reservoir (station 11413515) for power development. See schematic diagram of Yuba River Basin.

EXTREMES FOR PERIOD OF RECORD.-Maximum daily discharge, 1,090 $\mathrm{ft}^{3}$ /s, Mar. 25, 1989, and Feb. 3, 1998; no flow for many days each year.

DISCHARGE, CUBIC FEET PER SECOND, WATER YEAR OCTOBER 1998 TO SEPTEMBER 1999 DAILY MEAN VALUES

\begin{tabular}{|c|c|c|c|c|c|c|c|c|c|c|c|c|}
\hline DAY & $\mathrm{OCT}$ & NOV & $\mathrm{DEC}$ & JAN & FEB & MAR & APR & MAY & JUN & JUL & AUG & SEP \\
\hline 1 & 19 & 18 & 794 & 98 & 418 & 1030 & 420 & 524 & 709 & 83 & 16 & 2.0 \\
\hline 2 & 18 & 19 & 530 & 93 & 375 & 963 & 404 & 572 & 703 & 79 & 15 & 1.7 \\
\hline 3 & 18 & 16 & 867 & 90 & 357 & 1000 & 397 & 695 & 629 & 74 & 15 & 1.8 \\
\hline 4 & 19 & 14 & 719 & 87 & 345 & 961 & 371 & 624 & 478 & 69 & 14 & 1.5 \\
\hline 5 & 18 & 16 & 579 & 84 & 332 & 885 & 386 & 560 & 324 & 66 & 14 & .54 \\
\hline 6 & 16 & 17 & 452 & 82 & 370 & 845 & 372 & 562 & 325 & 60 & 13 & .08 \\
\hline 7 & 16 & 74 & 348 & 80 & 984 & 782 & 366 & 604 & 324 & 57 & 15 & .12 \\
\hline 8 & 13 & 81 & 307 & 77 & 1050 & 729 & 416 & 606 & 295 & 53 & 15 & .00 \\
\hline 9 & 14 & 34 & 262 & 74 & 1070 & 712 & 391 & 576 & 272 & 49 & 14 & .04 \\
\hline 10 & 14 & 24 & 222 & 71 & 1020 & 654 & 380 & 548 & 250 & 47 & 16 & .05 \\
\hline 11 & 13 & 36 & 201 & 70 & 959 & 617 & 429 & 537 & 248 & 44 & 17 & .16 \\
\hline 12 & 13 & 27 & 186 & 68 & 867 & 582 & 486 & 579 & 253 & 41 & 14 & .00 \\
\hline 13 & 12 & 22 & 191 & 67 & 823 & 562 & 520 & 624 & 248 & 38 & 12 & .00 \\
\hline 14 & 13 & 20 & 231 & 65 & 756 & 556 & 580 & 584 & 244 & e34 & 11 & .00 \\
\hline 15 & 12 & 18 & 208 & 86 & 687 & 531 & 604 & 510 & 242 & e33 & 9.7 & .00 \\
\hline 16 & 11 & 17 & 211 & 258 & 733 & 513 & 607 & 477 & 242 & e32 & 9.1 & .00 \\
\hline 17 & 11 & 67 & 221 & 361 & 1040 & 500 & 631 & 472 & 239 & e 30 & 8.5 & .00 \\
\hline 18 & 11 & 72 & 210 & 931 & 993 & 500 & 661 & 508 & 216 & e30 & 7.4 & .00 \\
\hline 19 & 9.9 & 38 & 196 & 1010 & 956 & 501 & 688 & 531 & 198 & e28 & 6.7 & .00 \\
\hline 20 & 8.8 & 29 & 185 & 997 & 884 & 506 & 701 & 545 & 182 & e27 & 6.0 & .00 \\
\hline 21 & 8.6 & 27 & 158 & 804 & 871 & 485 & 695 & 541 & 169 & 26 & 5.7 & .00 \\
\hline 22 & 8.3 & 214 & 149 & 769 & 815 & 461 & 636 & 588 & 159 & 25 & 5.3 & .00 \\
\hline 23 & 8.6 & 272 & 136 & 774 & 774 & 450 & 593 & 630 & 154 & 25 & 5.0 & .00 \\
\hline 24 & 18 & 583 & 133 & 925 & 747 & 443 & 585 & 720 & 143 & 23 & 3.9 & .00 \\
\hline 25 & 41 & 216 & 125 & 842 & 888 & 452 & 601 & 757 & 131 & 22 & 3.2 & .00 \\
\hline 26 & 21 & 130 & 121 & 795 & 801 & 452 & 673 & 766 & 119 & 22 & 2.4 & .00 \\
\hline 27 & 15 & 208 & 115 & 673 & 735 & 469 & 676 & 772 & 106 & 20 & 5.1 & .00 \\
\hline 28 & 14 & 315 & 111 & 596 & 815 & 461 & 613 & 768 & 97 & 20 & 4.0 & .00 \\
\hline 29 & 14 & 300 & 105 & 534 & --- & 443 & 544 & 762 & 92 & 19 & 2.6 & .00 \\
\hline 30 & 19 & 886 & 102 & 484 & --- & 449 & 502 & 747 & 88 & 18 & 1.5 & .00 \\
\hline 31 & 15 & --- & 101 & 459 & --- & 452 & --- & 733 & --- & 17 & 2.3 & -- \\
\hline TOTAL & 462.2 & 3810 & 8476 & 12404 & 21465 & 18946 & 15928 & 19022 & 7879 & 1211 & 289.4 & 7.99 \\
\hline MEAN & 14.9 & 127 & 273 & 400 & 767 & 611 & 531 & 614 & 263 & 39.1 & 9.34 & .27 \\
\hline MAX & 41 & 886 & 867 & 1010 & 1070 & 1030 & 701 & 772 & 709 & 83 & 17 & 2.0 \\
\hline MIN & 8.3 & 14 & 101 & 65 & 332 & 443 & 366 & 472 & 88 & 17 & 1.5 & .00 \\
\hline$A C-F T$ & 917 & 7560 & 16810 & 24600 & 42580 & 37580 & 31590 & 37730 & 15630 & 2400 & 574 & 16 \\
\hline
\end{tabular}

STATISTICS OF MONTHLY MEAN DATA FOR WATER YEARS 1989 - 1999, BY WATER YEAR (WY)

\begin{tabular}{|c|c|c|c|c|c|c|c|c|c|c|c|c|}
\hline MEAN & 10.8 & 48.5 & 155 & 299 & 425 & 547 & 539 & 388 & 219 & 77.5 & 9.74 & 3.74 \\
\hline MAX & 54.9 & 127 & 628 & 695 & 865 & 793 & 867 & 820 & 542 & 347 & 37.8 & 19.8 \\
\hline (WY) & 1990 & 1999 & 1997 & 1995 & 1998 & 1993 & 1995 & 1996 & 1993 & 1995 & 1998 & 1998 \\
\hline MIN & .000 & 1.28 & .83 & 1.16 & 16.7 & 308 & 173 & 53.2 & 7.22 & .11 & .000 & .000 \\
\hline (WY) & 1989 & 1991 & 1991 & 1991 & 1991 & 1994 & 1994 & 1992 & 1992 & 1994 & 1992 & 1991 \\
\hline
\end{tabular}

SUMMARY STATISTICS

ANNUAL TOTAL

ANNUAL MEAN

HIGHEST ANNUAL MEAN

LOWEST ANNUAL MEAN

HIGHEST DAILY MEAN

LOWEST DAILY MEAN

ANNUAL SEVEN-DAY MINIMUM

ANNUAL RUNOFF (AC-FT)

10 PERCENT EXCEEDS

50 PERCENT EXCEEDS

90 PERCENT EXCEEDS
FOR 1998 CALENDAR YEAR

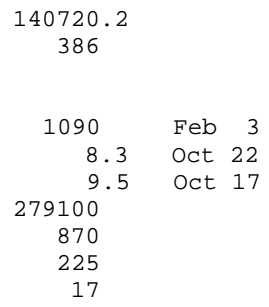

FOR 1999 WATER YEAR

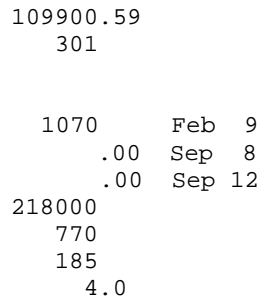

WATER YEARS 1989 - 1999

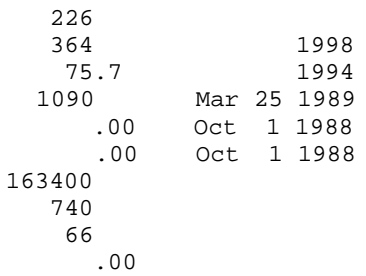




\section{OREGON CREEK BELOW LOG CABIN DAM, NEAR CAMPTONVILLE, CA}

LOCATION.—Lat 39²6'22", long 12103'29", in SW 1/4 SW 1/4 sec.11, T.18 N., R.8 E., Yuba County, Hydrologic Unit 18020125, Tahoe National Forest, on left bank $500 \mathrm{ft}$ downstream from Log Cabin Dam, $670 \mathrm{ft}$ upstream from High Point Ravine, and 1.1 mi southwest of Camptonville.

DRAINAGE AREA.-29.1 $\mathrm{mi}^{2}$.

PERIOD OF RECORD.-August 1968 to current year.

WATER TEMPERATURE: Water years 1972-79.

REVISED RECORDS.-WDR CA-81-4: 1980(M).

GAGE.-Water-stage recorder, sharp-crested weir since Nov. 13, 1990, and crest-stage gage. Datum of gage is 1,912.73 ft above sea level (levels by Yuba County Water Agency). Prior to July 24, 1973, at site $470 \mathrm{ft}$ downstream at datum $8.40 \mathrm{ft}$ lower. July 24, 1973, to Sept. 30, 1986, at site on right bank. Oct. 1, 1986, to Nov. 13, 1990, a sharp-crested weir was put in at same location and gage house located on left bank. The weir was deemed too shallow so a new sharp-crested weir was put in $70 \mathrm{ft}$ downstream at a datum $7.24 \mathrm{ft}$ lower.

REMARKS.--Records fair. Lohman Ridge Tunnel (station 11408870) diverts water into the basin from the Middle Yuba River. Camptonville Tunnel (station 11409350), maximum capacity, about 1,000 ft $3 / \mathrm{s}, 520 \mathrm{ft}$ upstream, diverts water out of the basin to New Bullards Bar Reservoir (station 11413515); diversion began October 1968. See schematic diagram of Yuba River Basin.

EXTREMES FOR PERIOD OF RECORD.-Maximum discharge, 6,400 ft $3 / \mathrm{s}$, Feb. 17, 1986, gage height, $11.24 \mathrm{ft}$, datum then in use, from rating curve extended above $50 \mathrm{ft}^{3} / \mathrm{s}$ based on flow-over-dam computation, maximum gage height $15.70 \mathrm{ft}$ (from floodmark), Jan. 1, 1997; minimum daily, $0.34 \mathrm{ft}^{3} / \mathrm{s}$, Sept. 18,1972 .

DISCHARGE, CUBIC FEET PER SECOND, WATER YEAR OCTOBER 1998 TO SEPTEMBER 1999 DAILY MEAN VALUES

\begin{tabular}{|c|c|c|c|c|c|c|c|c|c|c|c|c|}
\hline DAY & OCT & NOV & $\mathrm{DEC}$ & JAN & FEB & MAR & APR & MAY & JUN & JUL & AUG & SEP \\
\hline 1 & 10 & 11 & 17 & 12 & 18 & 173 & 18 & 13 & 15 & e10 & 9.0 & 8.4 \\
\hline 2 & 10 & 11 & 16 & 12 & 17 & 28 & 18 & 13 & e15 & e10 & e8. 9 & 8.4 \\
\hline 3 & 10 & 11 & 96 & 12 & 16 & 137 & 18 & 14 & e15 & 10 & 8.9 & 8.4 \\
\hline 4 & 10 & 11 & 20 & 12 & 16 & 31 & 17 & 14 & e14 & 10 & e8.9 & 8.4 \\
\hline 5 & 10 & 11 & 18 & 12 & 15 & e23 & 18 & 13 & e13 & 10 & e8.9 & 8.2 \\
\hline 6 & 9.9 & 11 & 17 & 12 & 15 & e22 & 17 & 13 & e13 & e10 & 8.9 & e 7.8 \\
\hline 7 & 10 & 13 & e16 & 12 & 328 & 21 & 17 & 14 & e13 & 10 & 9.0 & 7.2 \\
\hline 8 & 11 & 13 & e16 & 12 & 285 & 21 & 18 & 14 & 13 & e10 & e9.1 & 7.1 \\
\hline 9 & 11 & 12 & 15 & 12 & 1060 & 20 & e18 & 13 & e13 & e9. 8 & e9.0 & 7.2 \\
\hline 10 & 11 & 12 & 15 & 12 & 191 & 20 & 18 & 13 & e13 & 9.8 & 9.0 & 7.2 \\
\hline 11 & 11 & 12 & 15 & 12 & 30 & 20 & 18 & 13 & 13 & 9.8 & 9.0 & 7.2 \\
\hline 12 & 11 & 12 & 14 & 12 & 22 & 19 & 17 & 13 & 13 & 9.8 & e9.0 & 6.6 \\
\hline 13 & 11 & 11 & 14 & 12 & 22 & 19 & 19 & 14 & 14 & 9.8 & 8.8 & 5.9 \\
\hline 14 & 11 & 11 & 14 & 12 & 21 & 19 & 16 & 13 & 13 & 9.7 & 8.8 & 6.0 \\
\hline 15 & 11 & 11 & 14 & 12 & 20 & 19 & 14 & 13 & 13 & 9.7 & e8. 8 & 6.6 \\
\hline 16 & 11 & 11 & 14 & e14 & e20 & 19 & 14 & 13 & 12 & 9.7 & e 8.8 & 5.9 \\
\hline 17 & 11 & 12 & 14 & e15 & e221 & 19 & 14 & 13 & 9.9 & 9.7 & e8. 8 & 5.4 \\
\hline 18 & 11 & 13 & 14 & e106 & 52 & 18 & 14 & 13 & 9.7 & 9.7 & e 8.8 & 5.2 \\
\hline 19 & 11 & e12 & 14 & 160 & 27 & 18 & 14 & 13 & 9.6 & 9.7 & e9.0 & 5.6 \\
\hline 20 & 11 & 12 & 13 & 687 & e22 & 18 & 13 & 13 & 9.5 & 9.7 & e8.9 & e 5.9 \\
\hline 21 & 11 & 18 & 13 & 439 & 22 & 18 & 13 & 13 & 9.4 & 9.6 & e8. 6 & e 5.7 \\
\hline 22 & 11 & 14 & 13 & 168 & 21 & 18 & 13 & 14 & 9.3 & 9.6 & e8. 6 & 5.6 \\
\hline 23 & 11 & 14 & 13 & e1000 & e21 & e18 & 13 & 14 & 9.3 & 9.2 & e8. 6 & e 5.1 \\
\hline 24 & 11 & 15 & 13 & e26 & 21 & 18 & 12 & 15 & 9.2 & 9.0 & e8. 6 & e 4.9 \\
\hline 25 & 12 & e83 & 13 & 22 & 22 & 18 & 13 & 15 & 9.1 & 9.1 & 8.4 & e 4.9 \\
\hline 26 & 11 & e27 & 13 & 21 & 21 & 18 & 13 & e15 & 9.0 & 9.4 & e8. 4 & e 4.7 \\
\hline 27 & 11 & e21 & 13 & 20 & 20 & 18 & 13 & e15 & e10 & 9.4 & e8. 8 & 4.2 \\
\hline 28 & 11 & e19 & 13 & 19 & 63 & 18 & 13 & 15 & 10 & 9.2 & 8.6 & e3. 9 \\
\hline 29 & 11 & 96 & 13 & 19 & --- & 18 & 12 & 15 & e9.8 & e9. 2 & 8.5 & 3.9 \\
\hline 30 & 11 & e18 & 13 & 18 & --- & 18 & 12 & e15 & 9.8 & e 9.2 & e8. 3 & e 3.9 \\
\hline 31 & 11 & --- & 13 & 18 & --- & 18 & --- & e15 & --- & e9.0 & e8. 6 & --- \\
\hline TOTAL & 334.9 & 558 & 529 & 2932 & 2629 & 882 & 457 & 426 & 348.6 & 298.8 & 272.3 & 185.4 \\
\hline MEAN & 10.8 & 18.6 & 17.1 & 94.6 & 93.9 & 28.5 & 15.2 & 13.7 & 11.6 & 9.64 & 8.78 & 6.18 \\
\hline MAX & 12 & 96 & 96 & 1000 & 1060 & 173 & 19 & 15 & 15 & 10 & 9.1 & 8.4 \\
\hline MIN & 9.9 & 11 & 13 & 12 & 15 & 18 & 12 & 13 & 9.0 & 9.0 & 8.3 & 3.9 \\
\hline $\mathrm{AC}-\mathrm{ET}$ & 664 & 1110 & 1050 & 5820 & 5210 & 1750 & 906 & 845 & 691 & 593 & 540 & 368 \\
\hline
\end{tabular}


11409400 OREGON CREEK BELOW LOG CABIN DAM, NEAR CAMPTONVILLE, CA—Continued

STATISTICS OF MONTHLY MEAN DATA FOR WATER YEARS 1968 - 1999, BY WATER YEAR (WY)

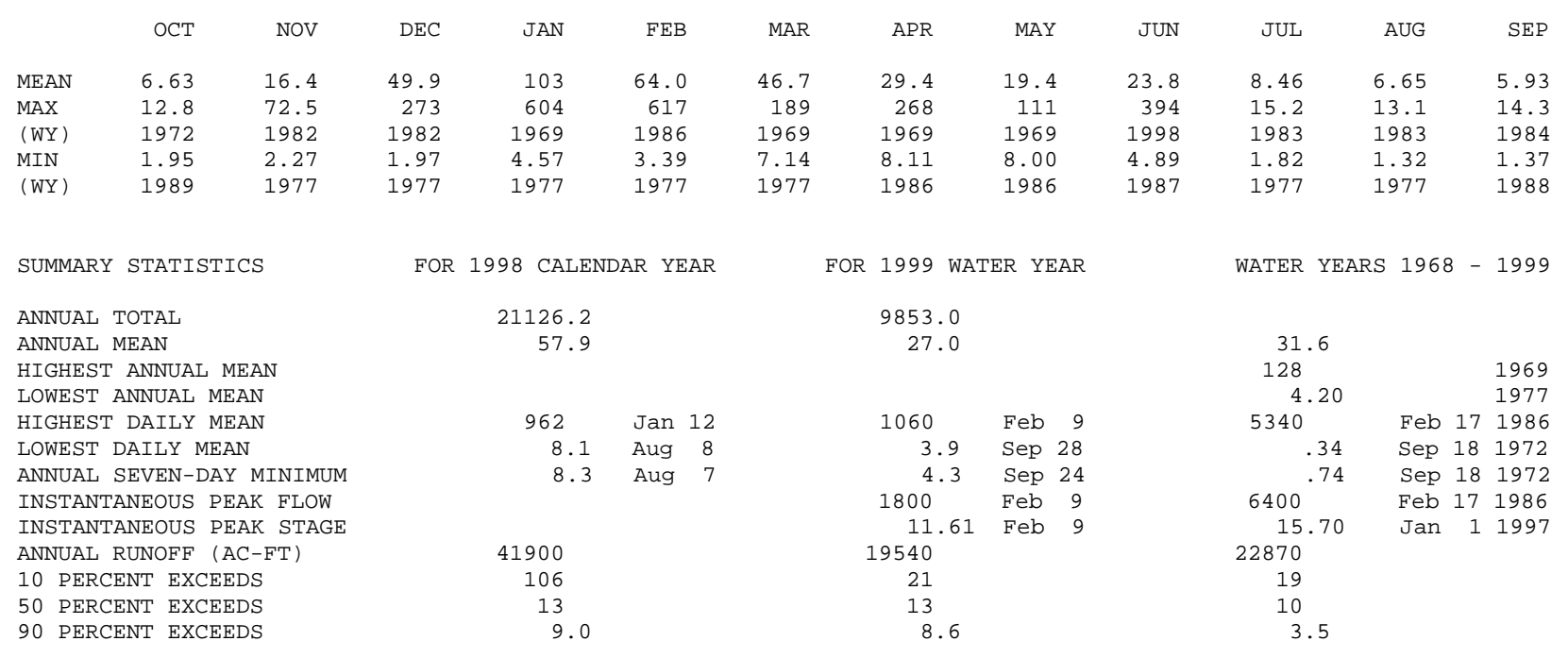




\section{NORTH YUBA RIVER BELOW GOODYEARS BAR, CA}

LOCATION.-Lat 39³1'30", long 12056'13", in NE 1/4 SW 1/4 sec.11, T.19 N., R.9 E., Sierra County, Hydrologic Unit 18020125, Tahoe National Forest, on right bank $200 \mathrm{ft}$ downstream from St. Catherine Creek, $3.1 \mathrm{mi}$ southwest of Goodyears Bar, and 6.4 mi southwest of Downieville.

DRAINAGE AREA.-250 $\mathrm{mi}^{2}$.

PERIOD OF RECORD.- October 1930 to current year. Prior to October 1949, published as North Fork Yuba River below Goodyears Bar. Monthly and yearly discharge only for some periods, published in WSP 1315-A.

REVISED RECORDS.-WSP 1041: 1944. WSP 1931: Drainage area.

GAGE.-Water-stage recorder. Datum of gage is 2,453 ft above sea level (river-profile survey).

REMARKS.- - Records good. Several small diversions upstream from station for irrigation and mining. See schematic diagram of Yuba River Basin. EXTREMES FOR PERIOD OF RECORD.-Maximum discharge, 45,500 ft $3 / \mathrm{s}$, Jan. 2, 1997, gage height, $25.65 \mathrm{ft}$, from rating curve extended above $11,900 \mathrm{ft}^{3} / \mathrm{s}$ on basis of one float measurement at $17,900 \mathrm{ft}^{3} / \mathrm{s}$ and slope-area measurements at gage heights 19.15 and $23.8 \mathrm{ft} ;$ minimum daily, $60 \mathrm{ft}^{3} / \mathrm{s}$, Sept. 7-14, 1977.

EXTREMES FOR CURRENT YEAR.-Peak discharges greater than base discharge of $3,200 \mathrm{ft}^{3} / \mathrm{s}$, or maximum:

$\begin{array}{lccccccc}\text { Date } & \text { Time } & \begin{array}{c}\text { Discharge } \\ \left(\mathrm{ft}^{3} / \mathrm{s}\right)\end{array} & \begin{array}{c}\text { Gage height } \\ (\mathrm{ft})\end{array} & \text { Date } & \text { Time } & \begin{array}{c}\text { Discharge } \\ \left(\mathrm{ft}^{3} / \mathrm{s}\right)\end{array} & \begin{array}{c}\text { Gage height } \\ (\mathrm{ft})\end{array} \\ \text { Nov. 23 } & 2045 & 3,320 & 7.96 & \text { Feb. } 9 & 0930 & 8,130 & 11.46 \\ \text { Nov. 30 } & 1730 & 3,620 & 8.24 & \text { Mar. 1 } & 0130 & 3,240 & 7.88 \\ \text { Dec. 3 } & 1130 & 4,470 & 8.99 & \text { May 25 } & 2330 & 4,370 & 8.91 \\ \text { Jan. 20 } & 1800 & 5,640 & 9.89 & & & & \end{array}$

DISCHARGE, CUBIC FEET PER SECOND, WATER YEAR OCTOBER 1998 TO SEPTEMBER 1999 DAILY MEAN VALUES

\begin{tabular}{|c|c|c|c|c|c|c|c|c|c|c|c|c|}
\hline DAY & OCT & NOV & $\mathrm{DEC}$ & JAN & $\mathrm{FEB}$ & MAR & APR & MAY & JUN & JUL & AUG & SEP \\
\hline 1 & 240 & 223 & 1630 & 345 & 773 & 2770 & 896 & 1600 & 2580 & 758 & 269 & 203 \\
\hline 2 & 236 & 221 & 1020 & 336 & 730 & 2120 & 848 & 1850 & 2490 & 722 & 266 & 203 \\
\hline 3 & 239 & 210 & 2910 & 330 & 706 & 2640 & 831 & 1720 & 2060 & 682 & 263 & 202 \\
\hline 4 & 240 & 207 & 1640 & 324 & 694 & 2110 & 789 & 1500 & 1690 & 633 & 258 & 199 \\
\hline 5 & 231 & 214 & 1090 & 320 & 669 & 1770 & 807 & 1400 & 1720 & 588 & 255 & 195 \\
\hline 6 & 225 & 218 & 885 & 319 & 731 & 1560 & 774 & 1540 & 1940 & 553 & 254 & 193 \\
\hline 7 & 222 & 329 & 734 & 315 & 2620 & 1400 & 749 & 1900 & 1900 & 521 & 258 & 190 \\
\hline 8 & 219 & 307 & 678 & 308 & 2520 & 1310 & 800 & 1950 & 1740 & 496 & 258 & 189 \\
\hline 9 & 218 & 242 & 602 & 303 & 5870 & 1260 & 754 & 1850 & 1650 & 477 & 254 & 187 \\
\hline 10 & 218 & 232 & 554 & 301 & 2980 & 1140 & 731 & 1780 & 1600 & 460 & 289 & 187 \\
\hline 11 & 216 & 242 & 528 & 299 & 2000 & 1070 & 763 & 1830 & 1650 & 443 & 279 & 187 \\
\hline 12 & 214 & 227 & 508 & 296 & 1620 & 1010 & 824 & 2170 & 1720 & 430 & 261 & 184 \\
\hline 13 & 213 & 225 & 517 & 293 & 1380 & 986 & 919 & 2390 & 1780 & 416 & 251 & 183 \\
\hline 14 & 212 & 228 & 526 & 292 & 1250 & 979 & 1080 & 2140 & 1790 & 422 & 244 & 184 \\
\hline 15 & 211 & 227 & 492 & 364 & 1120 & 949 & 1150 & 1780 & 1800 & 394 & 240 & 183 \\
\hline 16 & 210 & 227 & 504 & 775 & 1190 & 930 & 1220 & 1750 & 1770 & 377 & 238 & 180 \\
\hline 17 & 208 & 338 & 536 & 825 & 2280 & 929 & 1420 & 1860 & 1670 & 360 & 231 & 180 \\
\hline 18 & 208 & 274 & 526 & 3080 & 1870 & 954 & 1640 & 2080 & 1570 & 352 & 226 & 177 \\
\hline 19 & 205 & 243 & 509 & 2830 & 1730 & 990 & 1830 & 2250 & 1480 & 346 & 223 & 178 \\
\hline 20 & 203 & 234 & 484 & 4990 & 1510 & 1000 & 1940 & 2330 & 1390 & 338 & 221 & 177 \\
\hline 21 & 200 & 244 & 441 & 3210 & 1400 & 965 & 1940 & 2430 & 1330 & 332 & 219 & 179 \\
\hline 22 & 200 & 532 & 432 & 2060 & 1260 & 921 & 1700 & 2750 & 1300 & 325 & 217 & 178 \\
\hline 23 & 200 & 1430 & 416 & 3810 & 1190 & 905 & 1540 & 3050 & 1260 & 317 & 216 & 177 \\
\hline 24 & 239 & 1220 & 412 & 2280 & 1150 & 903 & 1570 & 3300 & 1210 & 310 & 213 & 175 \\
\hline 25 & 265 & 580 & 398 & 1690 & 1450 & 921 & 1740 & 3620 & 1110 & 304 & 209 & 175 \\
\hline 26 & 233 & 444 & 389 & 1390 & 1260 & 970 & 2160 & 3660 & 993 & 298 & 209 & 174 \\
\hline 27 & 227 & 539 & 377 & 1170 & 1150 & 1040 & 2060 & 3540 & 900 & 293 & 210 & 171 \\
\hline 28 & 220 & 580 & 370 & 1040 & 1570 & 1020 & 1790 & 3360 & 846 & 287 & 210 & 170 \\
\hline 29 & 223 & 598 & 360 & 946 & --- & 989 & 1520 & 2920 & 813 & 281 & 206 & 168 \\
\hline 30 & 223 & 2590 & 354 & 880 & --- & 991 & 1420 & 2590 & 786 & 275 & 203 & 168 \\
\hline 31 & 213 & --- & 355 & 842 & --- & 964 & --- & 2660 & --- & 272 & 203 & --- \\
\hline TOTAL & 6831 & 13625 & 21177 & 36563 & 44673 & 38466 & 38205 & 71550 & 46538 & 13062 & 7353 & 5496 \\
\hline MEAN & 220 & 454 & 683 & 1179 & 1595 & 1241 & 1274 & 2308 & 1551 & 421 & 237 & 183 \\
\hline MAX & 265 & 2590 & 2910 & 4990 & 5870 & 2770 & 2160 & 3660 & 2580 & 758 & 289 & 203 \\
\hline MIN & 200 & 207 & 354 & 292 & 669 & 903 & 731 & 1400 & 786 & 272 & 203 & 168 \\
\hline $\mathrm{AC}-\mathrm{FT}$ & 13550 & 27030 & 42000 & 72520 & 88610 & 76300 & 75780 & 141900 & 92310 & 25910 & 14580 & 10900 \\
\hline
\end{tabular}


11413000 NORTH YUBA RIVER BELOW GOODYEARS BAR, CA—Continued

STATISTICS OF MONTHLY MEAN DATA FOR WATER YEARS 1931 - 1999, BY WATER YEAR (WY)

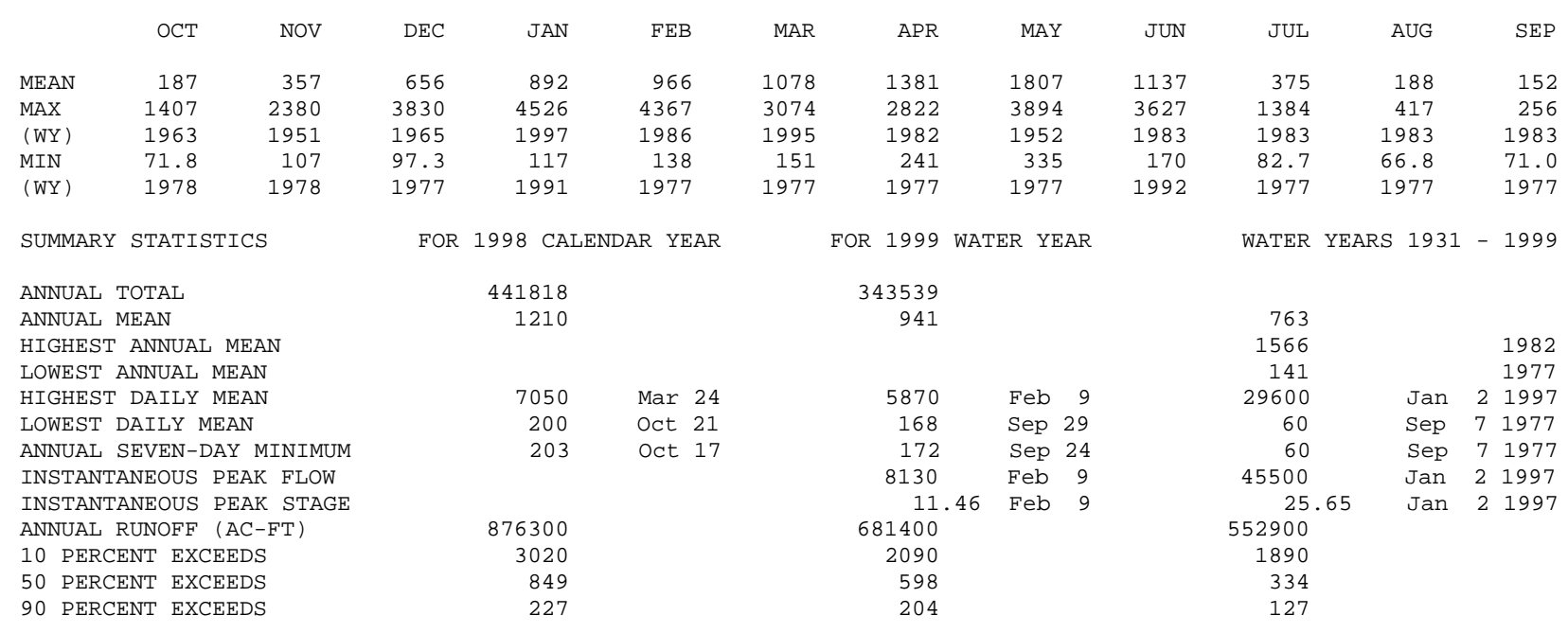




\section{SLATE CREEK TUNNEL NEAR STRAWBERRY VALLEY, CA}

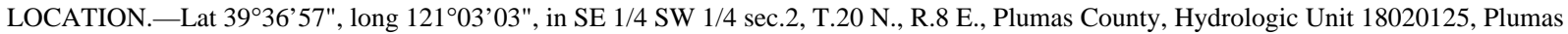
National Forest, on right bank $30 \mathrm{ft}$ upstream from diversion dam on Slate Creek, $0.3 \mathrm{mi}$ upstream from Feney Ravine, and 4.5 mi northeast of town of Strawberry Valley.

PERIOD OF RECORD.-February 1962 to current year. Monthly discharge only published as adjustment to Slate Creek below diversion dam near Strawberry Valley (station 11413300) February 1962 to September 1966; records of daily discharge are in files of the U.S. Geological Survey.

GAGE.-Water-stage recorder. Datum of gage is sea level.

REMARKS.-Tunnel diverts water from Slate Creek to Sly Creek Reservoir (station 11395400) for power development. See schematic diagrams of South Fork Feather River and Yuba River Basins.

COOPERATION.--Records provided by Oroville-Wyandotte Irrigation District, under general supervision of the U.S. Geological Survey, in connection with a Federal Energy Regulatory Commission project.

EXTREMES FOR PERIOD OF RECORD.-Maximum daily discharge, $863 \mathrm{ft}^{3} / \mathrm{s}$, Apr. 6, 1963; no flow for many days in each year.

DISCHARGE, CUBIC FEET PER SECOND, WATER YEAR OCTOBER 1998 TO SEPTEMBER 1999 DAILY MEAN VALUES

\begin{tabular}{|c|c|c|c|c|c|c|c|c|c|c|c|c|}
\hline DAY & OCT & NOV & DEC & JAN & FEB & MAR & APR & MAY & JUN & JUL & AUG & SEP \\
\hline 1 & .00 & .00 & 743 & 64 & 184 & 831 & 6.5 & 403 & 238 & 38 & .00 & .00 \\
\hline 2 & .00 & .00 & 602 & 61 & 168 & 823 & 6.5 & 439 & 221 & 35 & .00 & .00 \\
\hline 3 & .00 & .00 & 759 & 59 & 162 & 830 & 6.5 & 538 & 190 & 33 & .00 & .00 \\
\hline 4 & .00 & .00 & 768 & 55 & 159 & 823 & 6.5 & 464 & 164 & 30 & .00 & .00 \\
\hline 5 & .00 & .00 & 519 & 54 & 149 & 794 & 6.5 & 404 & 161 & 27 & .00 & .00 \\
\hline 6 & .00 & .00 & 351 & 53 & 152 & 525 & 6.5 & 413 & 166 & 25 & .00 & .00 \\
\hline 7 & .00 & .00 & 259 & 52 & 667 & 411 & 6.4 & 458 & 155 & 23 & .00 & .00 \\
\hline 8 & .00 & .00 & 216 & 49 & 813 & 361 & 6.2 & 429 & 137 & 21 & .00 & .00 \\
\hline 9 & .00 & .00 & 177 & 47 & 826 & 321 & 6.2 & 382 & 127 & 19 & .00 & .00 \\
\hline 10 & .00 & .00 & 153 & 46 & 816 & 272 & 6.2 & 353 & 118 & 18 & .00 & .00 \\
\hline 11 & .00 & 7.1 & 137 & 45 & 748 & 98 & 6.2 & 349 & 117 & 16 & .00 & .00 \\
\hline 12 & .00 & 10 & 124 & 44 & 548 & 6.5 & 6.2 & 393 & 117 & 15 & .00 & .00 \\
\hline 13 & .00 & 8.8 & 122 & 43 & 426 & 6.5 & 6.2 & 409 & 114 & 14 & .00 & .00 \\
\hline 14 & .00 & 8.8 & 120 & 43 & 362 & 6.5 & 6.2 & 357 & 111 & 13 & .00 & .00 \\
\hline 15 & .00 & 8.5 & 114 & 75 & 307 & 6.5 & 6.2 & 304 & 110 & 12 & .00 & .00 \\
\hline 16 & .00 & 9.0 & 133 & 300 & 321 & 6.5 & 6.2 & 287 & 106 & 11 & .00 & .00 \\
\hline 17 & .00 & 72 & 148 & 372 & 794 & 6.5 & 6.2 & 288 & 98 & 10 & .00 & .00 \\
\hline 18 & .00 & 43 & 142 & 818 & 730 & 6.5 & 6.2 & 308 & 91 & 9.6 & .00 & .00 \\
\hline 19 & .00 & 24 & 133 & 819 & 709 & 6.5 & 6.2 & 327 & 85 & 9.2 & .00 & .00 \\
\hline 20 & .00 & 18 & 122 & 814 & 507 & 6.5 & 6.2 & 324 & 79 & 8.6 & .00 & .00 \\
\hline 21 & .00 & 22 & 104 & 793 & 420 & 6.5 & 6.2 & 337 & 74 & 8.2 & .00 & .00 \\
\hline 22 & .00 & 212 & 96 & 810 & 347 & 6.5 & 6.2 & 380 & 71 & 7.9 & .00 & .00 \\
\hline 23 & .00 & 400 & 88 & 827 & 305 & 6.5 & 269 & 414 & 68 & 2.6 & .00 & .00 \\
\hline 24 & .00 & 427 & 89 & 808 & 287 & 6.5 & 452 & 435 & 64 & .00 & .00 & .00 \\
\hline 25 & .00 & 203 & 86 & 623 & 375 & 6.5 & 481 & 444 & 59 & .00 & .00 & .00 \\
\hline 26 & .00 & 131 & 80 & 462 & 301 & 6.5 & 594 & 434 & 53 & .00 & .00 & .00 \\
\hline 27 & .00 & 287 & 75 & 356 & 278 & 6.5 & 555 & 402 & 48 & .00 & .00 & .00 \\
\hline 28 & .00 & 253 & 72 & 296 & 572 & 6.5 & 451 & 349 & 44 & .00 & .00 & .00 \\
\hline 29 & .00 & 226 & 68 & 256 & --- & 6.5 & 379 & 297 & 42 & .00 & .00 & .00 \\
\hline 30 & .00 & 778 & 67 & 228 & --- & 6.5 & 369 & 264 & 40 & .00 & .00 & .00 \\
\hline 31 & .00 & --- & 68 & 210 & --- & 6.5 & --- & 251 & --- & .00 & .00 & --- \\
\hline TOTAL & 0.00 & 3148.20 & 6735 & 9582 & 12433 & 6219.0 & 3688.4 & 11636 & 3268 & 406.10 & 0.00 & 0.00 \\
\hline MEAN & .000 & 105 & 217 & 309 & 444 & 201 & 123 & 375 & 109 & 13.1 & .000 & .000 \\
\hline MAX & .00 & 778 & 768 & 827 & 826 & 831 & 594 & 538 & 238 & 38 & .00 & .00 \\
\hline MIN & .00 & .00 & 67 & 43 & 149 & 6.5 & 6.2 & 251 & 40 & .00 & .00 & .00 \\
\hline $\mathrm{AC}-\mathrm{FT}$ & .00 & 6240 & 13360 & 19010 & 24660 & 12340 & 7320 & 23080 & 6480 & 805 & .00 & .00 \\
\hline
\end{tabular}

STATISTICS OF MONTHLY MEAN DATA FOR WATER YEARS 1963 - 1999, BY WATER YEAR (WY)

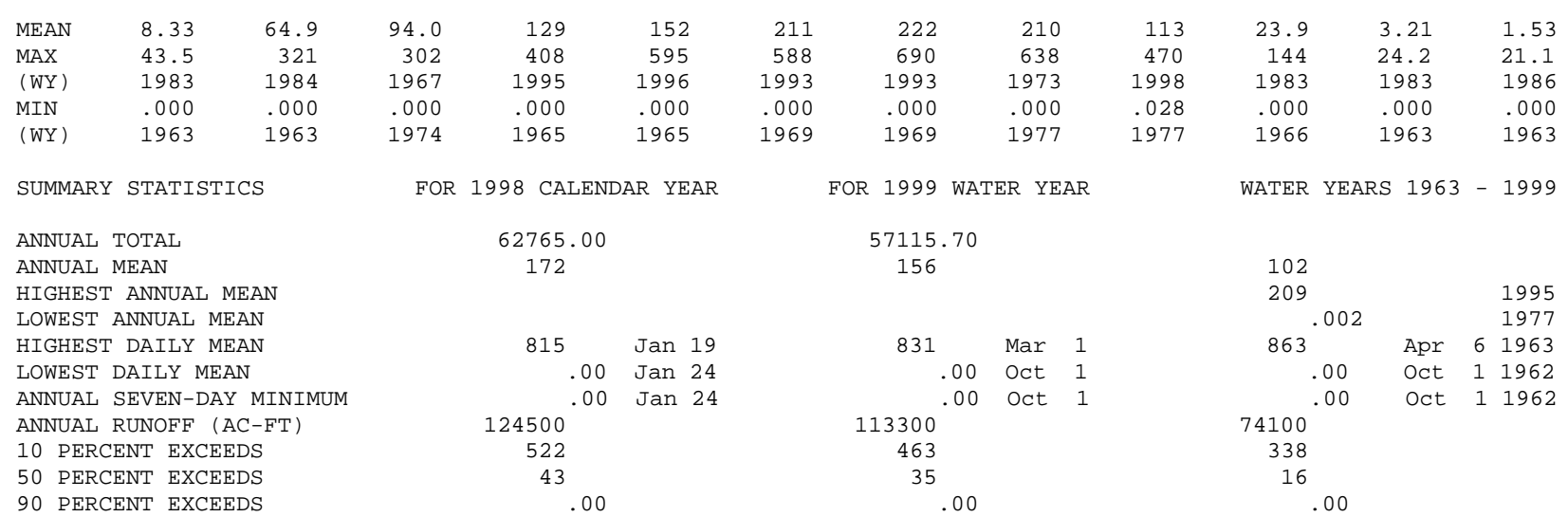




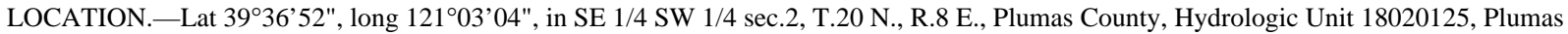
National Forest, on right bank $300 \mathrm{ft}$ downstream from diversion dam, 0.2 mi upstream from Feney Ravine, and 4.5 mi northeast of town of Strawberry Valley.

DRAINAGE AREA.- $-49.4 \mathrm{mi}^{2}$.

PERIOD OF RECORD.-October 1960 to current year.

GAGE.-Water-stage recorder and $130^{\circ}$ V-notch weir since October 1982. Elevation of gage is 3,570 ft above sea level, from topographic map.

REMARKS.-Slate Creek Tunnel (station 11413250) diverts up to $900 \mathrm{ft}^{3} / \mathrm{s}$ from Slate Creek Reservoir, capacity, 223 acre-ft, at diversion dam $300 \mathrm{ft}$ upstream, to Sly Creek Reservoir (station 11395400). Diversion began in February 1962. See schematic diagrams of South Fork Feather River and Yuba River Basins.

COOPERATION.-Records provided by Oroville-Wyandotte Irrigation District, under general supervision of the U.S. Geological Survey, in connection with a Federal Energy Regulatory Commission project.

EXTREMES FOR PERIOD OF RECORD.-Creek only: Maximum discharge, 17,300 ft 3 /s, Jan. 1, 1997, gage height, $17.20 \mathrm{ft}$, from rating curve extended above $5,500 \mathrm{ft}^{3} / \mathrm{s}$ on basis of computed flow over dam at gage heights $12.75,15.90,16.89$ and $17.20 \mathrm{ft}$; minimum, $0.3 \mathrm{ft}^{3} / \mathrm{s}$, Mar. 4, 5, 1962.

DISCHARGE, CUBIC FEET PER SECOND, WATER YEAR OCTOBER 1998 TO SEPTEMBER 1999 DAILY MEAN VALUES

\begin{tabular}{|c|c|c|c|c|c|c|c|c|c|c|c|c|}
\hline DAY & $\mathrm{OCT}$ & NOV & $\mathrm{DEC}$ & JAN & FEB & MAR & APR & MAY & JUN & JUL & AUG & SEP \\
\hline 1 & 16 & 16 & 320 & 14 & 13 & 612 & 211 & 11 & 10 & 11 & 16 & 11 \\
\hline 2 & 16 & 16 & 19 & 14 & 13 & 193 & 195 & 11 & 10 & 11 & 15 & 11 \\
\hline 3 & 21 & 15 & 1150 & 14 & 12 & 501 & 185 & 11 & 10 & 11 & 15 & 11 \\
\hline 4 & 21 & 14 & 142 & 15 & 11 & 179 & 172 & 11 & 10 & 11 & 15 & 11 \\
\hline 5 & 17 & 16 & 15 & 15 & 12 & 30 & 175 & 11 & 10 & 11 & 14 & 11 \\
\hline 6 & 16 & 17 & 14 & 15 & 12 & 11 & 167 & 11 & 10 & 11 & 14 & 10 \\
\hline 7 & 15 & 60 & 14 & 15 & 269 & 11 & 156 & 11 & 10 & 11 & 15 & 10 \\
\hline 8 & 15 & 64 & 14 & 15 & 219 & 11 & 158 & 11 & 11 & 11 & 15 & 10 \\
\hline 9 & 15 & 31 & 14 & 15 & 1860 & 11 & 146 & 11 & 11 & 11 & 14 & 10 \\
\hline 10 & 15 & 25 & 14 & 14 & 424 & 11 & 139 & 11 & 11 & 11 & 15 & 10 \\
\hline 11 & 15 & 18 & 14 & 14 & 31 & 149 & 150 & 11 & 11 & 11 & 15 & 10 \\
\hline 12 & 14 & 12 & 14 & 14 & 12 & 226 & 176 & 11 & 11 & 11 & 14 & 9.8 \\
\hline 13 & 14 & 12 & 14 & 14 & 12 & 219 & 218 & 11 & 11 & 11 & 14 & 9.7 \\
\hline 14 & 14 & 12 & 14 & 15 & 11 & 215 & 294 & 11 & 11 & 11 & 13 & 9.7 \\
\hline 15 & 14 & 12 & 14 & 15 & 11 & 202 & 341 & 11 & 11 & 11 & 13 & 9.7 \\
\hline 16 & 14 & 12 & 14 & 15 & 11 & 204 & 385 & 11 & 11 & 11 & 13 & 9.6 \\
\hline 17 & 14 & 12 & 14 & 15 & 86 & 216 & 476 & 10 & 11 & 11 & 13 & 9.5 \\
\hline 18 & 13 & 12 & 14 & 512 & 48 & 231 & 542 & 10 & 11 & 11 & 12 & 9.6 \\
\hline 19 & 13 & 12 & 14 & 547 & 15 & 241 & 578 & 10 & 11 & 11 & 12 & 9.8 \\
\hline 20 & 13 & 12 & 14 & 1580 & 12 & 247 & 594 & 10 & 11 & 11 & 12 & 9.8 \\
\hline 21 & 13 & 12 & 14 & 627 & 11 & 236 & 569 & 10 & 11 & 11 & 12 & 10 \\
\hline 22 & 13 & 12 & 14 & 112 & 11 & 220 & 486 & 10 & 11 & 11 & 12 & 9.9 \\
\hline 23 & 13 & 624 & 14 & 806 & 11 & 218 & 182 & 10 & 11 & 16 & 12 & 9.7 \\
\hline 24 & 25 & 115 & 14 & 116 & 11 & 223 & 11 & 10 & 11 & 18 & 11 & 9.6 \\
\hline 25 & 37 & 15 & 14 & 15 & 12 & 300 & 11 & 10 & 11 & 18 & 11 & 9.6 \\
\hline 26 & 19 & 15 & 14 & 15 & 11 & 315 & 11 & 10 & 11 & 18 & 11 & 9.5 \\
\hline 27 & 17 & 15 & 14 & 14 & 11 & 324 & 11 & 10 & 11 & 17 & 12 & 9.2 \\
\hline 28 & 16 & 15 & 14 & 14 & 287 & 294 & 11 & 10 & 11 & 17 & 11 & 9.1 \\
\hline 29 & 16 & 15 & 14 & 14 & --- & 268 & 11 & 10 & 11 & 17 & 11 & 9.1 \\
\hline 30 & 15 & 1090 & 14 & 13 & --- & 258 & 11 & 10 & 11 & 16 & 11 & 9.1 \\
\hline 31 & 15 & --- & 14 & 13 & --- & 237 & --- & 10 & --- & 16 & 11 & --- \\
\hline TOTAL & 504 & 2328 & 2010 & 4646 & 3459 & 6613 & 6772 & 326 & 323 & 395 & 404 & 297.0 \\
\hline MEAN & 16.3 & 77.6 & 64.8 & 150 & 124 & 213 & 226 & 10.5 & 10.8 & 12.7 & 13.0 & 9.90 \\
\hline MAX & 37 & 1090 & 1150 & 1580 & 1860 & 612 & 594 & 11 & 11 & 18 & 16 & 11 \\
\hline MIN & 13 & 12 & 14 & 13 & 11 & 11 & 11 & 10 & 10 & 11 & 11 & 9.1 \\
\hline $\mathrm{AC}-\mathrm{FT}$ & 1000 & 4620 & 3990 & 9220 & 6860 & 13120 & 13430 & 647 & 641 & 783 & 801 & 589 \\
\hline
\end{tabular}

STATISTICS OF MONTHLY MEAN DATA FOR WATER YEARS 1963 - 1999, BY WATER YEAR (WY)

\begin{tabular}{|c|c|c|c|c|c|c|c|c|c|c|c|c|}
\hline MEAN & 24.9 & 55.7 & 152 & 257 & 200 & 221 & 190 & 195 & 49.6 & 12.1 & 11.1 & 10.4 \\
\hline MAX & 437 & 545 & 1303 & 1334 & 1415 & 901 & 753 & 795 & 481 & 21.3 & 19.3 & 17.7 \\
\hline (WY) & 1963 & 1974 & 1965 & 1970 & 1986 & 1983 & 1982 & 1983 & 1983 & 1998 & 1965 & 1998 \\
\hline MIN & 5.85 & 7.51 & 5.80 & 9.04 & 8.49 & 6.61 & 6.12 & 6.15 & 6.95 & 5.17 & 3.82 & 6.13 \\
\hline (WY) & 1971 & 1977 & 1977 & 1975 & 1973 & 1968 & 1968 & 1968 & 1973 & 1977 & 1977 & 1987 \\
\hline
\end{tabular}

SUMMARY STATISTICS

ANNUAL TOTAL

ANNUAL MEAN

HIGHEST ANNUAL MEAN

LOWEST ANNUAL MEAN

HIGHEST DAILY MEAN

LOWEST DAILY MEAN

ANNUAL SEVEN-DAY MINIMUM

INSTANTANEOUS PEAK FLOW

INSTANTANEOUS PEAK STAGE

ANNUAL RUNOFF (AC-FT)

10 PERCENT EXCEEDS

50 PERCENT EXCEEDS

90 PERCENT EXCEEDS
FOR 1998 CALENDAR YEAR

\begin{tabular}{|c|c|}
\hline $\begin{array}{r}64185 \\
176\end{array}$ & \\
\hline 2440 & Mar 24 \\
\hline 11 & Feb 9 \\
\hline 11 & Jul 31 \\
\hline 127300 & \\
\hline 523 & \\
\hline 22 & \\
\hline 12 & \\
\hline
\end{tabular}

FOR 1999 WATER YEAR

$$
\begin{array}{rrr}
28077.0 & & \\
76.9 & & \\
& & \\
1860 & \text { Feb } & 9 \\
9.1 & \text { Sep } & 28 \\
9.3 & \text { Sep } & 24 \\
3330 & \text { Feb } & 9 \\
10.63 & \text { Feb } & 9 \\
55690 & & \\
224 & & \\
14 & & \\
10 & &
\end{array}
$$

WATER YEARS 1963 - 1999

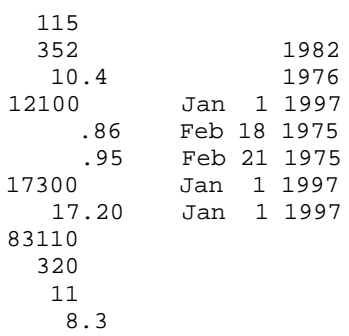




\section{DEADWOOD CREEK NEAR STRAWBERRY VALLEY, CA}

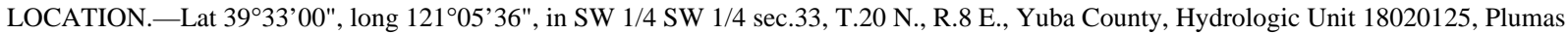
National Forest, on right bank $250 \mathrm{ft}$ upstream of confluence with Owl Gulch and $1.3 \mathrm{mi}$ southeast of Strawberry Valley.

DRAINAGE AREA.- $-3.16 \mathrm{mi}^{2}$.

PERIOD OF RECORD.-October 1994 to current year.

GAGE.-Water-stage recorder and $120^{\circ} \mathrm{V}$-notch weir. Elevation of gage is 3,275 ft above sea level, from topographic map.

REMARKS.-Water from creek is diverted at gage to Deadwood Creek Powerplant (station 11413326). See schematic diagram of Yuba River Basin.

COOPERATION.-Records provided by Yuba County Water Agency, under general supervision of the U.S. Geological Survey, in connection with a Federal Energy Regulatory Commission project.

EXTREMES FOR PERIOD OF RECORD.—Maximum daily discharge, $400 \mathrm{ft}^{3} / \mathrm{s}$, Jan. 1, 1997; minimum daily, $1.7 \mathrm{ft}^{3} / \mathrm{s}$, several days in February and March 1997.

DISCHARGE, CUBIC FEET PER SECOND, WATER YEAR OCTOBER 1998 TO SEPTEMBER 1999 DAILY MEAN VALUES

\begin{tabular}{|c|c|c|c|c|c|c|c|c|c|c|c|c|}
\hline DAY & OCT & NOV & $\mathrm{DEC}$ & JAN & FEB & MAR & APR & MAY & JUN & JUL & AUG & SEP \\
\hline 1 & 5.6 & 4.5 & 3.2 & 2.8 & 2.8 & 14 & 2.7 & 2.9 & 2.4 & 2.6 & 2.6 & 4.4 \\
\hline 2 & 5.6 & 4.5 & 11 & 2.8 & 2.8 & 10 & 2.7 & 2.9 & 2.3 & 2.6 & 2.6 & 4.4 \\
\hline 3 & 6.6 & 4.5 & 4.2 & 2.8 & 2.8 & 12 & 2.7 & 2.9 & 3.2 & 2.6 & 2.6 & 4.4 \\
\hline 4 & 4.8 & 4.5 & 3.0 & 2.8 & 2.8 & 7.6 & 2.7 & 2.6 & 2.4 & 2.6 & 2.6 & 4.3 \\
\hline 5 & 4.7 & 4.5 & 2.9 & 2.8 & 2.8 & 6.0 & 2.7 & 2.6 & 2.4 & 2.6 & 2.6 & 4.9 \\
\hline 6 & 4.5 & 4.5 & 2.9 & 2.8 & 4.1 & 4.9 & 2.7 & 2.6 & 2.4 & 2.6 & 2.6 & 4.3 \\
\hline 7 & 4.5 & 3.2 & 2.9 & 2.8 & 20 & 4.9 & 2.7 & 2.6 & 2.4 & 2.5 & 2.6 & 4.3 \\
\hline 8 & 4.5 & 3.2 & 2.8 & 2.8 & 19 & 4.2 & 2.8 & 2.6 & 2.4 & 2.5 & 2.6 & 4.2 \\
\hline 9 & 4.5 & 3.1 & 2.8 & 2.8 & 46 & 3.9 & 2.8 & 2.6 & 2.4 & 2.5 & 2.6 & 4.2 \\
\hline 10 & 4.5 & 3.1 & 2.8 & 2.8 & 33 & 3.4 & 2.8 & 2.6 & 2.5 & 2.5 & 2.6 & 4.3 \\
\hline 11 & 4.5 & 4.6 & 2.8 & 2.8 & 29 & 3.2 & 2.8 & 2.6 & 2.5 & 2.5 & 2.6 & 4.1 \\
\hline 12 & 4.5 & 3.2 & 2.8 & 2.8 & 28 & 3.2 & 2.8 & 2.6 & 2.5 & 2.5 & 2.6 & 4.1 \\
\hline 13 & 4.7 & 3.2 & 2.8 & 2.8 & 26 & 3.1 & 2.8 & 2.6 & 2.5 & 2.5 & 2.6 & 4.1 \\
\hline 14 & 4.7 & 2.9 & 5.6 & 2.8 & 25 & 3.0 & 2.8 & 2.6 & 2.5 & 2.5 & 2.6 & 4.1 \\
\hline 15 & 4.7 & 2.9 & 2.8 & 2.8 & 23 & 2.8 & 2.7 & 2.6 & 2.5 & 2.5 & 2.6 & 4.0 \\
\hline 16 & 4.7 & 3.0 & 2.8 & 2.8 & 31 & 2.6 & 2.8 & 2.6 & 2.6 & 2.6 & 2.6 & 4.0 \\
\hline 17 & 4.7 & 4.9 & 2.8 & 2.9 & 33 & 2.6 & 2.7 & 2.7 & 2.6 & 2.6 & 2.6 & 4.0 \\
\hline 18 & 4.7 & 2.9 & 2.8 & 6.2 & 31 & 2.6 & 2.7 & 2.7 & 2.6 & 2.6 & 2.6 & 4.1 \\
\hline 19 & 4.7 & 2.9 & 2.8 & 13 & 27 & 2.6 & 2.7 & 2.7 & 2.6 & 2.6 & 4.8 & 4.1 \\
\hline 20 & 4.7 & 3.6 & 2.8 & 22 & 28 & 2.5 & 2.7 & 2.7 & 2.6 & 2.6 & 4.3 & 4.0 \\
\hline 21 & 4.7 & 3.2 & 2.8 & 5.5 & 26 & 2.5 & 2.7 & 2.7 & 2.6 & 2.6 & 4.7 & 4.0 \\
\hline 22 & 4.7 & 4.2 & 2.8 & 9.4 & 21 & 2.5 & 2.7 & 2.7 & 2.6 & 2.6 & 4.3 & 3.9 \\
\hline 23 & 4.7 & 4.9 & 2.8 & 23 & 3.2 & 2.5 & 2.7 & 2.7 & 2.6 & 2.6 & 4.4 & 3.9 \\
\hline 24 & 5.1 & 3.0 & 2.8 & 5.9 & 7.4 & 2.5 & 2.7 & 2.6 & 2.6 & 2.6 & 4.4 & 3.9 \\
\hline 25 & 4.5 & 2.9 & 2.8 & 3.6 & 5.1 & 10 & 2.7 & 2.6 & 2.6 & 2.6 & 4.4 & 3.8 \\
\hline 26 & 4.5 & 3.0 & 2.8 & 3.0 & 4.2 & 17 & 2.7 & 2.6 & 2.6 & 2.6 & 4.4 & 3.8 \\
\hline 27 & 4.5 & 3.0 & 2.8 & 2.9 & 3.4 & 17 & 2.7 & 2.5 & 2.6 & 2.6 & 4.3 & 3.7 \\
\hline 28 & 4.5 & 5.4 & 2.8 & 2.8 & 13 & 6.0 & 2.7 & 2.5 & 2.6 & 2.6 & 4.4 & 3.7 \\
\hline 29 & 4.5 & 12 & 2.8 & 2.9 & --- & 8.0 & 2.7 & 2.5 & 2.6 & 2.6 & 4.3 & 3.7 \\
\hline 30 & 4.5 & 7.5 & 2.8 & 2.8 & --- & 2.6 & 2.7 & 2.5 & 2.6 & 2.6 & 4.4 & 3.7 \\
\hline 31 & 4.5 & --- & 2.8 & 2.9 & --- & 2.7 & --- & 2.4 & --- & 2.6 & 4.4 & --- \\
\hline TOTAL & 147.1 & 122.8 & 100.1 & 153.6 & 500.4 & 172.4 & 81.8 & 81.6 & 76.3 & 79.7 & 104.3 & 122.4 \\
\hline MEAN & 4.75 & 4.09 & 3.23 & 4.95 & 17.9 & 5.56 & 2.73 & 2.63 & 2.54 & 2.57 & 3.36 & 4.08 \\
\hline MAX & 6.6 & 12 & 11 & 23 & 46 & 17 & 2.8 & 2.9 & 3.2 & 2.6 & 4.8 & 4.9 \\
\hline MIN & 4.5 & 2.9 & 2.8 & 2.8 & 2.8 & 2.5 & 2.7 & 2.4 & 2.3 & 2.5 & 2.6 & 3.7 \\
\hline $\mathrm{AC}-\mathrm{FT}$ & 292 & 244 & 199 & 305 & 993 & 342 & 162 & 162 & 151 & 158 & 207 & 243 \\
\hline a & .00 & 363 & 698 & 999 & 773 & 1650 & 1310 & 876 & 486 & 230 & 63 & .00 \\
\hline
\end{tabular}

STATISTICS OF MONTHLY MEAN DATA FOR WATER YEARS 1995 - 1999, BY WATER YEAR (WY)

\begin{tabular}{|c|c|c|c|c|c|c|c|c|c|c|c|c|}
\hline MEAN & 3.89 & 3.87 & 6.42 & 15.8 & 12.2 & 8.42 & 4.68 & 4.63 & 2.95 & 3.15 & 3.29 & 3.66 \\
\hline MAX & 4.75 & 4.73 & 17.7 & 42.4 & 20.3 & 22.8 & 10.7 & 10.7 & 3.44 & 4.16 & 4.13 & 4.35 \\
\hline (WY) & 1999 & 1997 & 1997 & 1997 & 1998 & 1995 & 1995 & 1995 & 1995 & 1997 & 1997 & 1996 \\
\hline MIN & 2.04 & 3.09 & 2.75 & 4.32 & 4.64 & 3.53 & 2.73 & 2.63 & 2.54 & 2.57 & 2.87 & 3.02 \\
\hline (WY) & 1995 & 1995 & 1998 & 1996 & 1997 & 1997 & 1999 & 1999 & 1999 & 1999 & 1998 & 1998 \\
\hline
\end{tabular}

SUMMARY STATISTICS

ANNUAL TOTAL

ANNUAL MEAN

HIGHEST ANNUAL MEAN

LOWEST ANNUAL MEAN

HIGHEST DAILY MEAN

LOWEST DAILY MEAN

ANNUAL SEVEN-DAY MINIMUM

ANNUAL RUNOFF (AC-FT)

10 PERCENT EXCEEDS

50 PERCENT EXCEEDS

90 PERCENT EXCEEDS
FOR 1998 CALENDAR YEAR

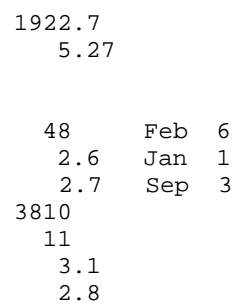

FOR 1999 WATER YEAR

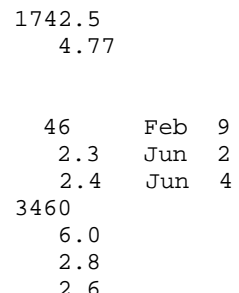

WATER YEARS 1995 - 1999

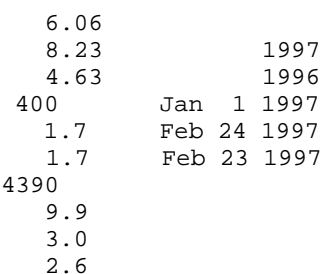

a Diversion, in acre-feet, to Deadwood Creek Powerplant, provided by Yuba County Water Agency. 
11413323 OWL GULCH NEAR STRAWBERRY VALLEY, CA

LOCATION.-Lat 39³2’44", long 12105’39", in SW 1/4 SW 1/4 sec.33, T.20 N., R.8 E., Yuba County, Hydrologic Unit 18020125, Plumas National Forest, on left bank $250 \mathrm{ft}$ upstream from Deadwood Creek and $1.3 \mathrm{mi}$ southeast of Strawberry Valley.

DRAINAGE AREA.-2.07 $\mathrm{mi}^{2}$.

PERIOD OF RECORD.-October 1994 to current year.

GAGE.-Water-stage recorder and $120^{\circ} \mathrm{V}$-notch weir. Elevation of gage is 3,050 ft above sea level, from topographic map.

REMARKS.-Water from creek is diverted at gage to Deadwood Creek Powerplant (station 11413326). See schematic diagram of Yuba River Basin.

COOPERATION.--Records provided by Yuba County Water Agency, under general supervision of the U.S. Geological Survey, in connection with a Federal Energy Regulatory Commission project.

EXTREMES FOR PERIOD OF RECORD.-Maximum daily discharge, $346 \mathrm{ft}^{3} / \mathrm{s}$, Jan. 1, 1997; minimum daily, $0.58 \mathrm{ft}^{3} / \mathrm{s}$, Sept. $17-22,1997$.

DISCHARGE, CUBIC FEET PER SECOND, WATER YEAR OCTOBER 1998 TO SEPTEMBER 1999 DAILY MEAN VALUES

\begin{tabular}{|c|c|c|c|c|c|c|c|c|c|c|c|c|}
\hline DAY & $\mathrm{OCT}$ & NOV & $\mathrm{DEC}$ & JAN & FEB & MAR & APR & MAY & JUN & JUL & AUG & SEP \\
\hline 1 & 2.7 & 2.9 & 1.9 & 1.7 & 2.0 & 15 & 2.0 & 2.9 & 2.1 & 1.7 & 1.6 & 1.5 \\
\hline 2 & 2.8 & 2.8 & 2.3 & 1.7 & 2.0 & 15 & 2.0 & 2.6 & 2.4 & 1.7 & 1.6 & 1.5 \\
\hline 3 & 3.1 & 2.8 & 2.9 & 1.7 & 1.9 & 13 & 2.0 & 2.3 & 1.4 & 1.7 & 1.6 & 1.5 \\
\hline 4 & 3.0 & 2.7 & 1.9 & 1.7 & 1.9 & 12 & 2.0 & 2.6 & 2.6 & 1.7 & 1.6 & 1.5 \\
\hline 5 & 2.8 & 2.5 & 1.8 & 1.7 & 1.9 & 12 & 2.0 & 2.1 & 2.6 & 1.7 & 1.6 & 1.5 \\
\hline 6 & 2.8 & 2.3 & 1.8 & 1.7 & 2.8 & 11 & 2.0 & 2.0 & 2.7 & 1.7 & 1.6 & 1.4 \\
\hline 7 & 2.8 & 2.4 & 1.7 & 1.7 & 13 & 8.2 & 2.0 & 2.0 & 2.7 & 1.7 & 1.5 & 1.5 \\
\hline 8 & 2.8 & 2.0 & 1.7 & 1.7 & 23 & 6.9 & 2.0 & 1.9 & 2.7 & 1.7 & 1.5 & 1.5 \\
\hline 9 & 2.8 & 2.0 & 1.7 & 1.7 & 36 & 3.7 & 2.0 & 1.9 & 1.8 & 1.7 & 1.5 & 1.4 \\
\hline 10 & 2.8 & 2.0 & 1.7 & 1.7 & 26 & 2.7 & 2.0 & 1.9 & 1.7 & 1.7 & 1.5 & 1.4 \\
\hline 11 & 2.8 & 2.2 & 1.7 & 1.7 & 13 & 2.0 & 2.0 & 2.0 & 1.7 & 1.7 & 1.5 & 1.4 \\
\hline 12 & 2.8 & 1.8 & 1.7 & 1.7 & 15 & 1.5 & 2.0 & 2.0 & 1.7 & 1.7 & 1.5 & 1.4 \\
\hline 13 & 2.9 & 1.8 & 1.7 & 1.7 & 15 & 1.5 & 2.0 & 2.0 & 1.7 & 1.7 & 1.5 & 1.4 \\
\hline 14 & 2.9 & 1.7 & 1.7 & 1.8 & 14 & 1.4 & 2.0 & 2.0 & 1.7 & 1.7 & 1.5 & 1.3 \\
\hline 15 & 2.9 & 1.7 & 1.7 & 1.8 & 14 & 1.2 & 2.0 & 2.0 & 1.7 & 1.7 & 1.5 & 1.4 \\
\hline 16 & 2.9 & 1.9 & 1.7 & 1.8 & 17 & 1.1 & 2.0 & 1.9 & 1.7 & 1.7 & 1.5 & 1.3 \\
\hline 17 & 2.9 & 2.6 & 1.7 & 2.0 & 12 & 1.0 & 2.0 & 1.8 & 1.7 & 1.7 & 1.6 & 1.4 \\
\hline 18 & 2.9 & 1.8 & 2.1 & 6.0 & 12 & 1.2 & 2.0 & 1.8 & 1.7 & 1.7 & 1.7 & 1.4 \\
\hline 19 & 2.9 & 1.8 & 1.7 & 6.2 & 12 & 2.1 & 2.0 & 1.8 & 1.7 & 1.7 & 1.7 & 1.4 \\
\hline 20 & 2.9 & 1.7 & 1.7 & 4.9 & 12 & 2.2 & 2.0 & 1.8 & 1.7 & 1.7 & 1.7 & 1.4 \\
\hline 21 & 2.9 & 2.0 & 1.7 & 8.2 & 12 & 2.3 & 2.0 & 1.8 & 1.7 & 1.7 & 1.7 & 1.3 \\
\hline 22 & 2.9 & 2.2 & 1.7 & 7.5 & 12 & 2.2 & 2.0 & 1.8 & 1.7 & 1.7 & 1.7 & 1.3 \\
\hline 23 & 2.9 & 2.5 & 1.7 & 6.6 & 8.0 & 2.2 & 2.6 & 1.8 & 1.7 & 1.7 & 1.6 & 1.3 \\
\hline 24 & 2.8 & 1.9 & 1.7 & 6.6 & 6.6 & 2.3 & 2.6 & 1.8 & 1.7 & 1.7 & 1.6 & 1.4 \\
\hline 25 & 2.7 & 1.8 & 1.7 & 6.6 & 8.4 & 1.4 & 2.6 & 1.8 & 1.7 & 1.7 & 1.7 & 1.3 \\
\hline 26 & 2.8 & 1.7 & 1.7 & 3.1 & 7.2 & 1.3 & 2.6 & 1.8 & 1.7 & 1.7 & 1.7 & 1.3 \\
\hline 27 & 2.8 & 1.8 & 1.7 & 2.4 & 31 & 1.3 & 2.6 & 1.8 & 1.7 & 1.7 & 1.6 & 1.3 \\
\hline 28 & 2.8 & 1.8 & 1.7 & 2.3 & 11 & 2.6 & 2.6 & 1.8 & 1.7 & 1.6 & 1.6 & 1.3 \\
\hline 29 & 2.9 & 2.7 & 1.7 & 2.3 & --- & 9.8 & 2.7 & 1.8 & 1.7 & 1.6 & 1.6 & 1.3 \\
\hline 30 & 2.9 & 3.2 & 1.7 & 2.0 & --- & 6.5 & 2.8 & 1.8 & 1.7 & 1.6 & 1.5 & 1.3 \\
\hline 31 & 2.9 & --- & 1.7 & 2.0 & --- & 3.8 & --- & 1.8 & --- & 1.6 & 1.5 & --- \\
\hline TOTAL & 88.5 & 65.0 & 55.5 & 96.2 & 342.7 & 150.4 & 65.1 & 61.1 & 56.7 & 52.3 & 49.1 & 41.6 \\
\hline MEAN & 2.85 & 2.17 & 1.79 & 3.10 & 12.2 & 4.85 & 2.17 & 1.97 & 1.89 & 1.69 & 1.58 & 1.39 \\
\hline MAX & 3.1 & 3.2 & 2.9 & 8.2 & 36 & 15 & 2.8 & 2.9 & 2.7 & 1.7 & 1.7 & 1.5 \\
\hline MIN & 2.7 & 1.7 & 1.7 & 1.7 & 1.9 & 1.0 & 2.0 & 1.8 & 1.4 & 1.6 & 1.5 & 1.3 \\
\hline $\mathrm{AC}-\mathrm{FT}$ & 176 & 129 & 110 & 191 & 680 & 298 & 129 & 121 & 112 & 104 & 97 & 83 \\
\hline
\end{tabular}

STATISTICS OF MONTHLY MEAN DATA FOR WATER YEARS 1995 - 1999, BY WATER YEAR (WY)

\begin{tabular}{|c|c|c|c|c|c|c|c|c|c|c|c|c|}
\hline MEAN & 1.80 & 1.85 & 4.39 & 13.6 & 11.7 & 6.99 & 4.03 & 4.21 & 2.18 & 1.89 & 1.57 & 1.56 \\
\hline MAX & 2.85 & 2.17 & 14.2 & 35.3 & 23.7 & 16.3 & 8.74 & 10.6 & 2.87 & 2.06 & 1.90 & 2.09 \\
\hline (WY) & 1999 & 1999 & 1997 & 1997 & 1998 & 1995 & 1995 & 1995 & 1998 & 1997 & 1998 & 1998 \\
\hline MIN & .99 & 1.56 & 1.72 & 3.10 & 3.97 & 2.46 & 2.13 & 1.97 & 1.79 & 1.69 & .77 & .79 \\
\hline (WY) & 1995 & 1995 & 1998 & 1999 & 1995 & 1997 & 1997 & 1999 & 1996 & 1999 & 1997 & 1997 \\
\hline
\end{tabular}

SUMMARY STATISTICS

ANNUAL TOTAL

ANNUAL MEAN

HIGHEST ANNUAL MEAN

LOWEST ANNUAL MEAN

HIGHEST DAILY MEAN

LOWEST DAILY MEAN

ANNUAL SEVEN-DAY MINIMUM

ANNUAL RUNOFF (AC-FT)

10 PERCENT EXCEEDS

50 PERCENT EXCEEDS

90 PERCENT EXCEEDS
FOR 1998 CALENDAR YEAR

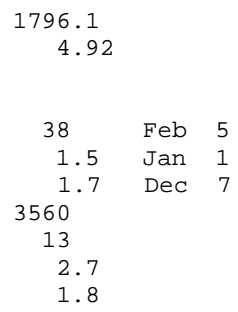

FOR 1999 WATER YEAR

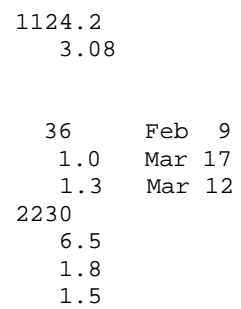

WATER YEARS 1995 - 1999

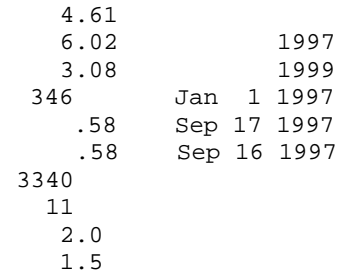




\section{NEW COLGATE POWERPLANT NEAR FRENCH CORRAL, CA}

LOCATION.—Lat 39¹9'51", long 121 ${ }^{\circ} 11^{\prime} 23^{\prime \prime}$, in NE 1/4 SE 1/4 sec.16, T.17 N., R.7 E., Yuba County, Hydrologic Unit 18020125, at powerplant on right bank of Yuba River, 0.3 mi upstream from Dobbins Creek, and 2.3 mi northwest of French Corral.

PERIOD OF RECORD.—October 1966 to current year. Prior to October 1969, published as "Colgate Powerplant."

GAGE.-Recorded output from powerplant turbines.

REMARKS.-Water is diverted from North Yuba River at New Bullards Bar Reservoir (station 11413515). Colgate Powerplant was rebuilt during the 1970 water year with an increased capacity. Prior to Oct. 31, 1973, Browns Valley Ditch diverted up to $10 \mathrm{ft}^{3} / \mathrm{s}$ at times from the head of the penstock for use in irrigation. See schematic diagram of Yuba River Basin.

COOPERATION.- - Records provided by Yuba County Water Agency, under general supervision of the U.S. Geological Survey, in connection with a Federal Energy Regulatory Commission project.

DISCHARGE, CUBIC FEET PER SECOND, WATER YEAR OCTOBER 1998 TO SEPTEMBER 1999 DAILY MEAN VALUES

\begin{tabular}{|c|c|c|c|c|c|c|c|c|c|c|c|c|}
\hline DAY & $\mathrm{OCT}$ & NOV & $\mathrm{DEC}$ & JAN & FEB & MAR & APR & MAY & JUN & JUL & AUG & SEP \\
\hline 1 & 1150 & 653 & 1240 & 3120 & 2030 & 3480 & 2780 & 2110 & 541 & 2200 & 2460 & 2040 \\
\hline 2 & 1280 & 1550 & 970 & 2700 & 2030 & 3520 & 3040 & 2570 & 1770 & 2120 & 2680 & 2310 \\
\hline 3 & 1160 & 1540 & 817 & 2510 & 3090 & 3380 & 3010 & 2770 & 2280 & 1930 & 2780 & 1300 \\
\hline 4 & 1050 & 1330 & 931 & 2920 & 2820 & 3510 & 2230 & 2760 & 2140 & 1810 & 2710 & 1870 \\
\hline 5 & 1050 & 1020 & 1040 & 3180 & 2820 & 3520 & 3060 & 2140 & 2360 & 2030 & 2550 & 979 \\
\hline 6 & 888 & 904 & 371 & 1670 & 3150 & 3320 & 3010 & 2390 & 2390 & 2150 & 2880 & 1600 \\
\hline 7 & 915 & 674 & 998 & 1970 & 3160 & 3390 & 2960 & 2350 & 2360 & 2310 & 2720 & 1680 \\
\hline 8 & 1380 & 865 & 1320 & 2300 & 3270 & 3520 & 2730 & 2620 & 2340 & 2220 & 2850 & 1120 \\
\hline 9 & 1370 & 1350 & 2190 & 2690 & 3480 & 3460 & 2930 & 1110 & 2260 & 2310 & 2880 & 1100 \\
\hline 10 & 1460 & 1490 & 2410 & 2930 & 3470 & 3520 & 2610 & 2410 & 1850 & 2410 & 2910 & 1030 \\
\hline 11 & 1140 & 2510 & 2450 & 2700 & 3370 & 3490 & 3500 & 2580 & 2260 & 2270 & 2850 & 629 \\
\hline 12 & 1150 & 2080 & 2300 & 2520 & 3360 & 3520 & 3520 & 2480 & 1460 & 2350 & 2740 & 1780 \\
\hline 13 & 1240 & 1850 & 1630 & 2860 & 3410 & 3530 & 2980 & 2610 & 1120 & 2290 & 2810 & 1220 \\
\hline 14 & 1360 & 827 & 2100 & 1890 & 3410 & 3450 & 3040 & 2270 & 1190 & 2530 & 2300 & 1220 \\
\hline 15 & 1140 & 877 & 2210 & 2100 & 3500 & 3480 & 3000 & 915 & 1330 & 2160 & 2720 & 1020 \\
\hline 16 & 1360 & 1870 & 1950 & 2130 & 3400 & 3510 & 3140 & 1210 & 1890 & 2330 & 2730 & 1100 \\
\hline 17 & 986 & 1620 & 2740 & 1410 & 3310 & 3550 & 3010 & 2440 & 1590 & 1710 & 2790 & 858 \\
\hline 18 & 933 & 1860 & 2640 & 1070 & 3320 & 3270 & 2470 & 2180 & 1810 & 2250 & 2880 & 1240 \\
\hline 19 & 1120 & 1770 & 2800 & 1680 & 3410 & 3290 & 2550 & 2600 & 958 & 2480 & 2970 & 1200 \\
\hline 20 & 1120 & 1860 & 3260 & 1270 & 3440 & 3190 & 2440 & 1780 & 565 & 2950 & 2830 & 1350 \\
\hline 21 & 1030 & 1660 & 3190 & 1610 & 3290 & 3110 & 2410 & 2310 & 2030 & 2990 & 2480 & 1280 \\
\hline 22 & 1220 & 1250 & 2880 & 2510 & 3420 & 3140 & 2480 & 1520 & 1780 & 2800 & 2590 & 1370 \\
\hline 23 & 1240 & 1540 & 2910 & 2440 & 3460 & 3130 & 2990 & 1600 & 2110 & 2640 & 2800 & 1190 \\
\hline 24 & 1080 & 1440 & 2360 & 2360 & 3500 & 3290 & 2040 & 2460 & 2190 & 1870 & 2890 & 943 \\
\hline 25 & 887 & 1530 & 2040 & 2050 & 3520 & 3180 & 2070 & 2490 & 1790 & 1780 & 2990 & 564 \\
\hline 26 & 1220 & 1670 & 2060 & 2220 & 3580 & 3070 & 2470 & 1460 & 1350 & 2650 & 2910 & 920 \\
\hline 27 & 1300 & 1630 & 2120 & 2160 & 3260 & 2560 & 2440 & 1140 & 1550 & 2650 & 2710 & 1510 \\
\hline 28 & 1440 & 1450 & 2150 & 2590 & 3240 & 2460 & 2380 & 176 & 2120 & 2570 & 2980 & 1180 \\
\hline 29 & 1350 & 1250 & 2170 & 2670 & --- & 2780 & 2010 & 463 & 2110 & 2670 & 2440 & 1330 \\
\hline 30 & 1390 & 1190 & 3000 & 2780 & --- & 2730 & 2800 & 192 & 3030 & 2900 & 1670 & 1180 \\
\hline 31 & 1280 & --- & 2910 & 2080 & --- & 2760 & --- & 838 & --- & 2910 & 1810 & --- \\
\hline TOTAL & 36689 & 43110 & 64157 & 71090 & 90520 & 101110 & 82100 & 58944 & 54524 & 73240 & 83310 & 38113 \\
\hline MEAN & 1184 & 1437 & 2070 & 2293 & 3233 & 3262 & 2737 & 1901 & 1817 & 2363 & 2687 & 1270 \\
\hline MAX & 1460 & 2510 & 3260 & 3180 & 3580 & 3550 & 3520 & 2770 & 3030 & 2990 & 2990 & 2310 \\
\hline MIN & 887 & 653 & 371 & 1070 & 2030 & 2460 & 2010 & 176 & 541 & 1710 & 1670 & 564 \\
\hline $\mathrm{AC}-\mathrm{FT}$ & 72770 & 85510 & 127300 & 141000 & 179500 & 200600 & 162800 & 116900 & 108100 & 145300 & 165200 & 75600 \\
\hline
\end{tabular}

STATISTICS OF MONTHLY MEAN DATA FOR WATER YEARS 1971 - 1999, BY WATER YEAR (WY)

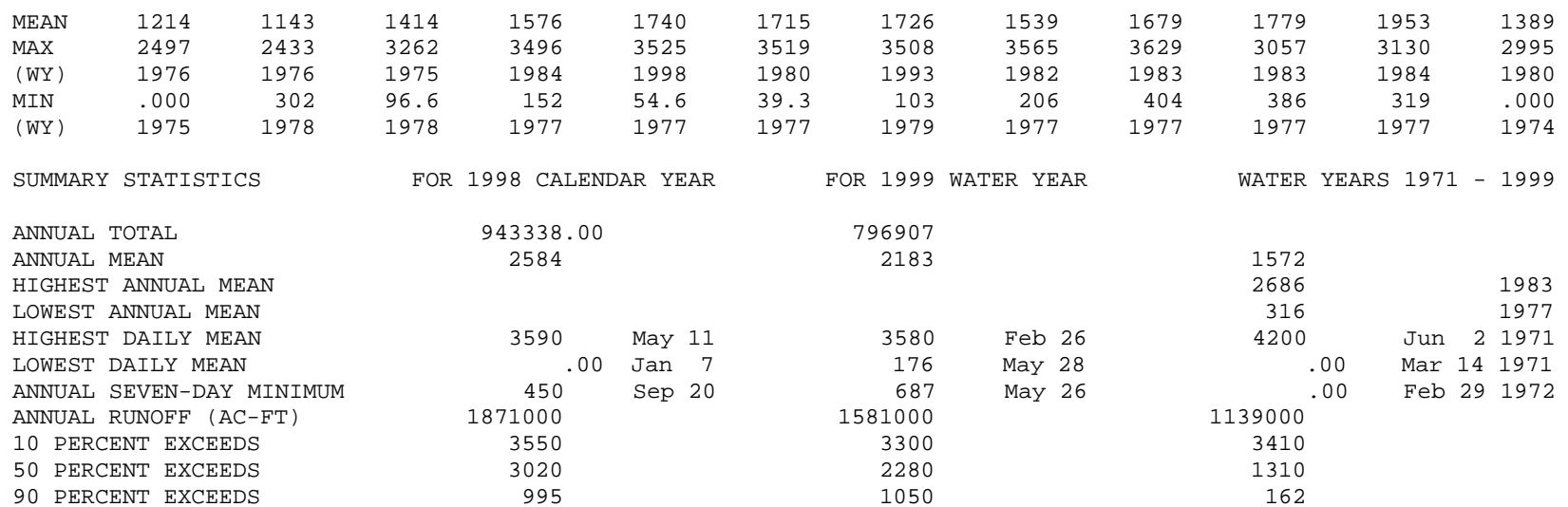




\section{NEW BULLARDS BAR RESERVOIR NEAR NORTH SAN JUAN, CA}

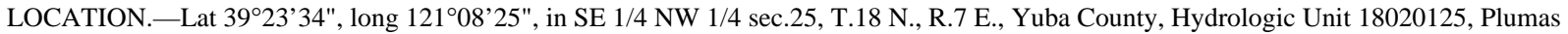
National Forest, in center of dam on North Yuba River, 2.2 mi upstream from Middle Yuba River, and 2.4 mi northwest of North San Juan. DRAINAGE AREA.-489 $\mathrm{mi}^{2}$.

PERIOD OF RECORD.- January 1969 to current year.

GAGE.-Water-stage recorder. Datum of gage is sea level (levels by Yuba County Water Agency).

REMARKS.- Reservoir is formed by concrete-arch dam with a concrete-sidehill spillway. Spill controlled by three 30 -ft by 53 -ft radial gates. Storage began in January 1969. Usable capacity, 727,380 acre-ft between elevations 1,732.0 ft, minimum power poo1, and 1,955.0 ft, normal gross pool. Dead storage, 233,920 acre-ft. Total capacity at normal gross pool, 961,300 acre-ft, elevation, 1,955.0 ft. Water is released to New Colgate Powerplant (station 11413510) through a tunnel at the dam. Water is diverted into the reservoir from Middle Yuba River via Lohman Ridge Tunnel to Oregon Creek then via Camptonville Tunnel (stations 11408870 and 11409350). Records, including extremes, represent total contents at 2400 hours. See schematic diagram of Yuba River Basin.

COOPERATION.- Records provided by Yuba County Water Agency, under general supervision of the U.S. Geological Survey, in connection with a Federal Energy Regulatory Commission project. Contents not rounded to U.S. Geological Survey standards.

EXTREMES FOR PERIOD OF RECORD.-Maximum contents, 972,224 acre-ft, June 27, 1995, elevation, 1,957.27 ft; minimum since reservoir first filled, 178,230 acre-ft, Dec. 29, 1980, elevation, 1,700.00 ft.

EXTREMES FOR CURRENT YEAR.-Maximum contents, 926,561 acre-ft, June 20, elevation, 1,947.66 ft; minimum, 580,709 acre-ft, Jan. 16, elevation, $1,862.12 \mathrm{ft}$.

Capacity table (elevation, in feet, and contents, in acre-feet)

(Based on survey by Yuba County Water Agency in 1969)

$\begin{array}{rrrr}1,600 & 64,900 & 1,750 & 270,110 \\ 1,630 & 90,570 & 1,800 & 389,977 \\ 1,660 & 122,993 & 1,850 & 539,748 \\ 1,690 & 162,983 & 1,900 & 721,130 \\ 1,720 & 211,768 & 1,960 & 985,471\end{array}$

RESERVOIR STORAGE (ACRE-FEET), WATER YEAR OCTOBER 1998 TO SEPTEMBER 1999 DAILY OBSERVATION AT 2400 HOURS

\begin{tabular}{|c|c|c|c|c|c|c|c|c|c|c|c|c|}
\hline DAY & OCT & NOV & $\mathrm{DEC}$ & JAN & FEB & MAR & APR & MAY & JUN & JUL & AUG & SEP \\
\hline 1 & 706827 & 658710 & 656505 & 629905 & 693480 & 798598 & 761441 & 783365 & 900648 & 913680 & 807520 & 670071 \\
\hline 2 & 705106 & 656431 & 660546 & 626237 & 693171 & 794667 & 760005 & 785207 & 904778 & 911506 & 803179 & 666145 \\
\hline 3 & 703700 & 654194 & 677017 & 622800 & 690699 & 794287 & 758365 & 787219 & 906802 & 909337 & 798683 & 664187 \\
\hline 4 & 702490 & 652297 & 684507 & 618619 & 688734 & 790664 & 757628 & 788268 & 908000 & 907539 & 794245 & 661107 \\
\hline 5 & 701204 & 651033 & 688503 & 613811 & 687156 & 786087 & 756442 & 789822 & 908092 & 905192 & 790075 & 659683 \\
\hline 6 & 700192 & 649995 & 692205 & 611987 & 685658 & 781610 & 754685 & 790874 & 908829 & 902574 & 785333 & 657103 \\
\hline 7 & 699142 & 650625 & 693906 & 609562 & 696191 & 777940 & 752849 & 792980 & 909475 & 899504 & 780900 & 654418 \\
\hline 8 & 697200 & 650440 & 694564 & 606395 & 706358 & 777108 & 752360 & 794794 & 909705 & 896623 & 776193 & 652743 \\
\hline 9 & 695261 & 648809 & 693249 & 602601 & 735854 & 777732 & 750812 & 798980 & 909844 & 893475 & 771380 & 651033 \\
\hline 10 & 693171 & 646959 & 691162 & 598225 & 747115 & 777316 & 749755 & 800463 & 910582 & 890107 & 766709 & 649439 \\
\hline 11 & 691626 & 643120 & 688849 & 594323 & 752604 & 776733 & 747602 & 801608 & 910629 & 886974 & 762140 & 648587 \\
\hline 12 & 690083 & 639993 & 686810 & 590683 & 755829 & 775985 & 746142 & 803774 & 912338 & 883712 & 757628 & 645665 \\
\hline 13 & 688426 & 637279 & 686081 & 586430 & 757628 & 774946 & 745049 & 806327 & 914744 & 880458 & 753053 & 643710 \\
\hline 14 & 686503 & 636436 & 684584 & 584034 & 758365 & 773992 & 745292 & 808971 & 917155 & 876762 & 749267 & 641868 \\
\hline 15 & 684890 & 635449 & 682706 & 581574 & 758160 & 772705 & 745899 & 812989 & 919245 & 873659 & 744765 & 640287 \\
\hline 16 & 682898 & 632673 & 681252 & 709 & 759759 & 1214 & 746385 & 816292 & 920175 & 870295 & 740281 & 638598 \\
\hline 17 & 681520 & 631142 & 678465 & 582370 & 767370 & 769600 & 747805 & 817408 & 921524 & 867967 & 735613 & 637388 \\
\hline 18 & 680296 & 628850 & 675799 & 595692 & 772788 & 768649 & 751057 & 819430 & 922175 & 864617 & 730724 & 635449 \\
\hline 19 & 678732 & 626383 & 672760 & 608672 & 776692 & 767741 & 756319 & 821240 & 924180 & 860920 & 725260 & 633622 \\
\hline 20 & 677093 & 623631 & 668748 & 634461 & 780065 & 767163 & 758775 & 824736 & 926561 & 856170 & 720892 & 631470 \\
\hline 21 & 675685 & 621645 & 664601 & 648550 & 783156 & 766503 & 762879 & 827464 & 926234 & 851391 & 716816 & 629504 \\
\hline 22 & 673899 & 621790 & 660957 & 655461 & 784830 & 765596 & 765967 & 832457 & 926187 & 846983 & 712519 & 627361 \\
\hline 23 & 672115 & 627725 & 657178 & 673140 & 785668 & 764442 & 766874 & 838082 & 925487 & 842810 & 707728 & 625440 \\
\hline 24 & 671093 & 632089 & 654492 & 681787 & 786254 & 763085 & 769021 & 842985 & 924413 & 840137 & 702802 & 623993 \\
\hline 25 & 670374 & 632016 & 652297 & 687349 & 789150 & 762304 & 771587 & 848348 & 923807 & 837645 & 697704 & 623270 \\
\hline 26 & 668748 & 630923 & 650032 & 691008 & 790285 & 761605 & 774739 & 855904 & 923947 & 833502 & 692785 & 621970 \\
\hline 27 & 667012 & 631142 & 647861 & 693249 & 791126 & 762304 & 777649 & 863726 & 923340 & 829328 & 688233 & 619411 \\
\hline 28 & 664940 & 631652 & 645149 & 693944 & 794709 & 762879 & 779773 & 872806 & 921524 & 825212 & 683128 & 617540 \\
\hline 29 & 663097 & 633147 & 642604 & 693751 & --- & 762551 & 781610 & 880549 & 919710 & 820938 & 678999 & 615352 \\
\hline 30 & 661145 & 648290 & 638341 & 692862 & --- & 762551 & 781652 & 887881 & 915949 & 816249 & 676331 & 613525 \\
\hline 31 & 659384 & --- & 634425 & 693442 & --- & 762263 & --- & 894114 & --- & 811449 & 673443 & --- \\
\hline MAX & 706827 & 658710 & 694564 & 693944 & 794709 & 798598 & 781652 & 894114 & 926561 & 913680 & 807520 & 670071 \\
\hline MIN & 659384 & 621645 & 634425 & 580709 & 685658 & 761605 & 745049 & 783365 & 900648 & 811449 & 673443 & 613525 \\
\hline a & 1883.98 & 1881.00 & 1877.23 & 1892.93 & 1917.98 & 1910.19 & 1914.87 & 1940.63 & 1945.38 & 1921.92 & 1887.71 & 1871.45 \\
\hline $\mathrm{b}$ & -48854 & -11094 & -13865 & +59017 & +101267 & -32446 & +19389 & +112462 & +21835 & -104500 & -138006 & -59918 \\
\hline
\end{tabular}

CAL YR 1998 b +87114

WTR YR 1999 b -94713

a Elevation, in feet, at end of month.

b Change in contents, in acre-feet. 
11413520 NORTH YUBA RIVER BELOW NEW BULLARDS BAR DAM, NEAR NORTH SAN JUAN, CA

LOCATION.-Lat 39²3'26", long 12108'36", in SE 1/4 NW 1/4 sec.25, T.18 N., R.7 E., Yuba County, Hydrologic Unit 18020125, Plumas National Forest, on right bank at old Colgate Dam, 0.2 mi downstream from New Bullards Bar Dam, and 2.5 mi northwest of North San Juan. DRAINAGE AREA.-490 $\mathrm{mi}^{2}$.

PERIOD OF RECORD.-_August 1966 to current year.

GAGE.-Water-stage recorder, and sharp-crested low-water control since Oct. 1, 1986. Elevation of gage is $1,350 \mathrm{ft}$ above sea level, from topographic map. Auxiliary water-stage recorder for high flow 0.9 mi downstream at different datum

REMARKS.-Records good. Flow regulated by New Bullards Bar Reservoir (station 11413515) since 1969. Prior to 1969, flow regulated by Bullards Bar Reservoir (usable capacity, 31,500 acre-ft). New Colgate Powerplant (station 11413510) diverts at New Bullards Bar Dam 0.2 mi upstream. Water is diverted to Feather River Basin through Slate Creek Tunnel (station 11413250). Camptonville Tunnel (station 11409350) diverts water from Middle Yuba River to New Bullards Bar Reservoir. Records include flow over New Bullards Bar Reservoir spillway. See schematic diagram of Yuba River Basin.

EXTREMES FOR PERIOD OF RECORD.-Maximum discharge, $56,200 \mathrm{ft}^{3} / \mathrm{s}$, Jan. 22, 1970, gage height, $35.29 \mathrm{ft}$, at auxiliary gage, from rating curve extended above $40,000 \mathrm{ft}^{3} / \mathrm{s}$ on basis of computation of flow over old Colgate Dam; minimum daily, $0.42 \mathrm{ft}^{3} / \mathrm{s}, \mathrm{Nov} .5,1966$.

EXTREMES OUTSIDE PERIOD OF RECORD._-Flood of Dec. 22, 1964, reached a stage of $49.8 \mathrm{ft}$, from floodmarks, discharge, $91,600 \mathrm{ft}$ /s, at auxiliary gage, from computation of flow over old Colgate Dam.

DISCHARGE, CUBIC FEET PER SECOND, WATER YEAR OCTOBER 1998 TO SEPTEMBER 1999 DAILY MEAN VALUES

\begin{tabular}{|c|c|c|c|c|c|c|c|c|c|c|c|c|}
\hline DAY & OCT & NOV & $\mathrm{DEC}$ & JAN & FEB & MAR & $\mathrm{APR}$ & MAY & JUN & JUL & AUG & SEP \\
\hline 1 & 6.8 & 6.8 & 6.7 & 6.6 & 6.5 & 2780 & 7.6 & 7.0 & 7.9 & 7.6 & 7.2 & 6.8 \\
\hline 2 & 6.8 & 6.8 & 7.0 & 6.6 & 6.8 & 4510 & 7.0 & 7.1 & 7.9 & 7.6 & 7.2 & 6.8 \\
\hline 3 & 6.8 & 6.8 & 8.4 & 6.6 & 6.8 & 4430 & 7.0 & 7.2 & 7.8 & 7.6 & 7.2 & 6.8 \\
\hline 4 & 6.8 & 6.8 & 6.7 & 6.6 & 6.8 & 4360 & 7.0 & 7.1 & 7.9 & 7.6 & 7.3 & 6.8 \\
\hline 5 & 6.8 & 6.8 & 6.5 & 6.6 & 6.8 & 3790 & 7.0 & 7.0 & 7.8 & 7.6 & 7.4 & 6.8 \\
\hline 6 & 6.8 & 6.7 & 6.5 & 6.6 & 7.3 & 3380 & 6.7 & 7.0 & 7.9 & 7.6 & 7.3 & 6.8 \\
\hline 7 & 6.8 & 6.2 & 6.4 & 6.6 & 11 & 2510 & 7.0 & 7.0 & 8.0 & 7.6 & 7.2 & 6.8 \\
\hline 8 & 6.8 & 5.8 & 6.5 & 6.6 & 8.2 & 870 & 7.3 & 7.0 & 8.0 & 7.6 & 7.2 & 6.8 \\
\hline 9 & 6.8 & 6.1 & 6.8 & 6.6 & 9.6 & 8.5 & 7.0 & 7.0 & 8.1 & 7.6 & 7.2 & 6.8 \\
\hline 10 & 6.8 & 6.9 & 6.8 & 6.6 & 7.2 & 8.2 & 6.7 & 7.0 & 8.0 & 7.6 & 7.2 & 6.8 \\
\hline 11 & 6.8 & 7.0 & 6.8 & 6.6 & 7.0 & 8.2 & 6.3 & 7.0 & 7.9 & 7.6 & 7.2 & 6.8 \\
\hline 12 & 6.8 & 6.8 & 6.8 & 6.6 & 8.4 & 8.2 & 6.6 & 7.0 & 7.8 & 7.6 & 7.2 & 6.8 \\
\hline 13 & 6.8 & 6.8 & 6.5 & 6.6 & 7.1 & 8.2 & 7.0 & 7.9 & 7.8 & 7.6 & 7.2 & 6.8 \\
\hline 14 & 6.8 & 6.8 & 6.2 & 6.6 & 7.0 & 8.2 & 7.0 & 7.2 & 8.0 & 7.6 & 7.2 & 6.8 \\
\hline 15 & 6.8 & 6.8 & 6.1 & 6.8 & 7.0 & 8.2 & 7.0 & 7.2 & 8.1 & 7.6 & 7.2 & 6.8 \\
\hline 16 & 6.8 & 6.8 & 6.3 & 6.9 & 8.0 & 8.2 & 7.0 & 7.2 & 8.0 & 7.6 & 7.2 & 6.8 \\
\hline 17 & 6.8 & 7.0 & 8.8 & 7.1 & 8.9 & 8.2 & 7.0 & 7.2 & 8.0 & 7.6 & 7.2 & 6.8 \\
\hline 18 & 6.8 & 6.8 & 6.7 & 8.0 & 7.0 & 8.2 & 7.0 & 7.2 & 8.0 & 7.6 & 7.2 & 6.8 \\
\hline 19 & 6.8 & 6.8 & 6.6 & 8.1 & 6.5 & 8.2 & 7.0 & 7.2 & 8.0 & 7.6 & 7.2 & 6.8 \\
\hline 20 & 6.8 & 6.6 & 6.6 & 7.9 & 7.5 & 8.2 & 7.0 & 7.2 & 8.0 & 7.5 & 7.2 & 6.8 \\
\hline 21 & 6.8 & 6.7 & 6.6 & 6.5 & 10 & 8.2 & 7.0 & 7.3 & 8.0 & 7.4 & 7.0 & 6.8 \\
\hline 22 & 6.8 & 6.6 & 6.6 & 7.0 & 7.6 & 8.2 & 7.0 & 7.2 & 8.0 & 7.5 & 7.0 & 6.8 \\
\hline 23 & 6.8 & 7.2 & 6.6 & 9.7 & 6.3 & 8.2 & 7.0 & 7.2 & 8.0 & 7.5 & 7.1 & 6.8 \\
\hline 24 & 7.1 & 6.5 & 6.6 & 6.9 & 6.4 & 8.2 & 7.0 & 7.4 & 8.0 & 7.6 & 7.0 & 6.8 \\
\hline 25 & 7.3 & 6.4 & 6.6 & 7.0 & 6.6 & 8.3 & 7.0 & 7.4 & 8.0 & 7.6 & 7.0 & 6.8 \\
\hline 26 & 6.8 & 6.4 & 6.6 & 6.9 & 5.6 & 8.1 & 7.0 & 7.4 & 7.9 & 7.6 & 7.0 & 6.8 \\
\hline 27 & 6.8 & 7.1 & 6.6 & 6.6 & 5.6 & 8.0 & 7.0 & 7.6 & 7.8 & 7.6 & 7.0 & 6.8 \\
\hline 28 & 6.8 & 6.6 & 6.6 & 6.8 & 356 & 8.0 & 7.0 & 7.8 & 7.8 & 7.6 & 7.0 & 6.8 \\
\hline 29 & 6.8 & 7.1 & 6.6 & 6.8 & --- & 8.0 & 7.0 & 7.8 & 7.8 & 7.6 & 6.9 & 6.8 \\
\hline 30 & 6.8 & 7.7 & 6.5 & 6.8 & --- & 8.2 & 7.0 & 7.8 & 7.8 & 7.6 & 6.8 & 6.8 \\
\hline 31 & 6.8 & --- & 6.6 & 6.8 & --- & 8.2 & --- & 7.9 & --- & 7.5 & 6.8 & --- \\
\hline TOTAL & 211.6 & 202.2 & 208.2 & 215.0 & 555.5 & 26818.3 & 209.2 & 225.5 & 238.0 & 235.0 & 221.0 & 204.0 \\
\hline MEAN & 6.83 & 6.74 & 6.72 & 6.94 & 19.8 & 865 & 6.97 & 7.27 & 7.93 & 7.58 & 7.13 & 6.80 \\
\hline MAX & 7.3 & 7.7 & 8.8 & 9.7 & 356 & 4510 & 7.6 & 7.9 & 8.1 & 7.6 & 7.4 & 6.8 \\
\hline MIN & 6.8 & 5.8 & 6.1 & 6.5 & 5.6 & 8.0 & 6.3 & 7.0 & 7.8 & 7.4 & 6.8 & 6.8 \\
\hline $\mathrm{AC}-\mathrm{FT}$ & 420 & 401 & 413 & 426 & 1100 & 53190 & 415 & 447 & 472 & 466 & 438 & 405 \\
\hline
\end{tabular}


11413520 NORTH YUBA RIVER BELOW NEW BULLARDS BAR DAM, NEAR NORTH SAN JUAN, CA—Continued

STATISTICS OF MONTHLY MEAN DATA FOR WATER YEARS 1966 - 1999, BY WATER YEAR (WY)

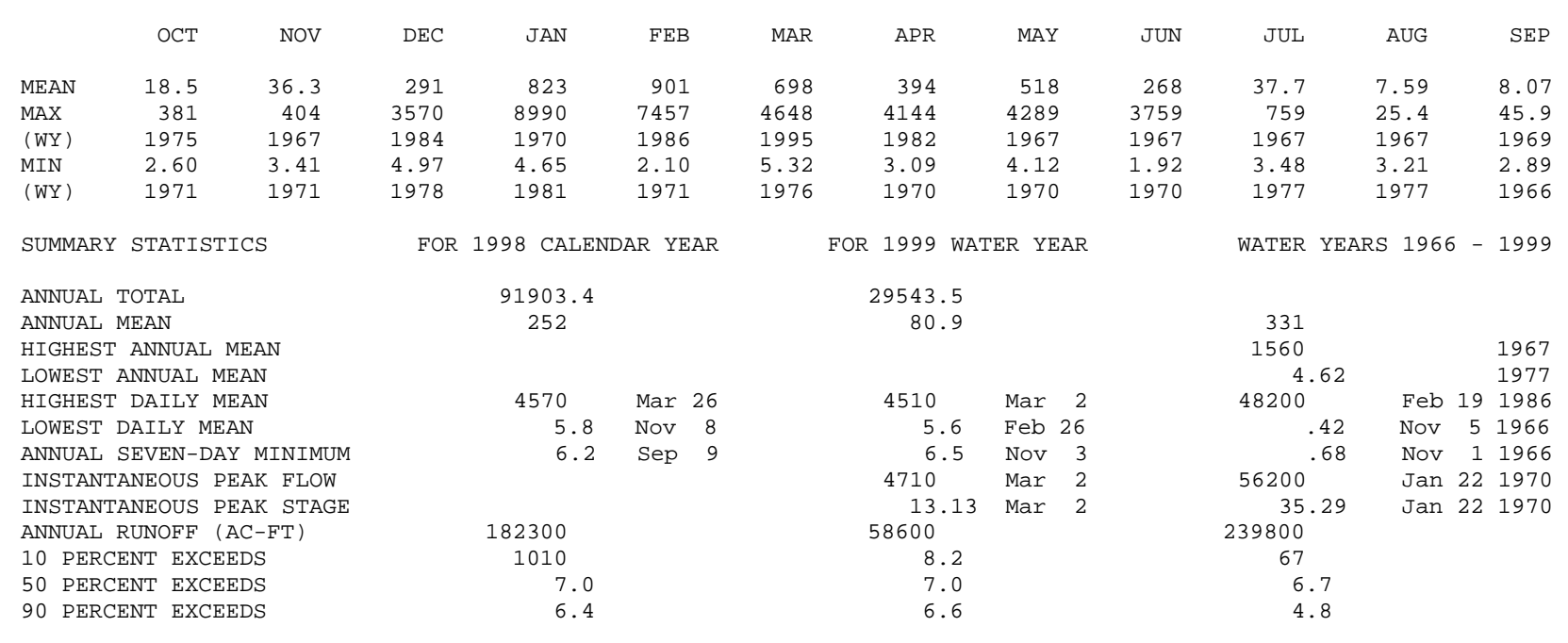




\section{KIDD LAKE NEAR SODA SPRINGS, CA}

LOCATION.—Lat 39¹8'41", long 120²5'54", in SW 1/4 NW 1/4 sec.29, T.17 N., R.14 E., Placer County, Hydrologic Unit 18020125, on outlet structure on Kidd Lake Dam and 3.0 mi west of Soda Springs.

DRAINAGE AREA.- $-1.00 \mathrm{mi}^{2}$.

PERIOD OF RECORD.-July 1991 to current year. Unpublished records for water years 1966-91 available in files of the U.S. Geological Survey.

GAGE.-Water-stage recorder. Datum of gage is 6,600.3 ft above sea level (levels by Pacific Gas \& Electric Co.). Prior to July 1991, nonrecording gage at same site and datum.

REMARKS.--Records not collected during winter months. Reservoir is formed on natural lake by rock-fill dam completed in 1855 . Usable capacity, 1,505 acre-ft between gage heights $0.0 \mathrm{ft}$, invert of outlet, and $27.3 \mathrm{ft}$, crest of spillway. Water is used for power development downstream. Records represent usable contents at 2400 hours.

COOPERATION.-Records were collected by Pacific Gas \& Electric Co., under general supervision of the U.S. Geological Survey, in connection with a Federal Energy Regulatory Commission project.

Capacity table (gage height, in feet, and contents, in acre-feet)

(Based on survey by Pacific Gas \& Electric Co., dated April 1965)

$\begin{array}{rrrr}0 & 0 & 16 & 654 \\ 4 & 117 & 20 & 918 \\ 8 & 259 & 28 & 1,568\end{array}$

RESERVOIR STORAGE (ACRE-FEET), WATER YEAR OCTOBER 1998 TO SEPTEMBER 1999

DAILY OBSERVATION AT 2400 HOURS

\begin{tabular}{|c|c|c|c|c|c|c|c|c|c|c|c|c|}
\hline DAY & OCT & NOV & $\mathrm{DEC}$ & JAN & $\mathrm{FEB}$ & MAR & $\mathrm{APR}$ & MAY & JUN & JUL & AUG & SEP \\
\hline 1 & e1290 & e415 & --- & --- & --- & --- & --- & --- & 1554 & 1515 & 1385 & 1065 \\
\hline 2 & e1270 & e388 & --- & --- & --- & --- & --- & --- & 1555 & 1513 & 1371 & 1064 \\
\hline 3 & e1250 & 360 & --- & --- & --- & --- & --- & --- & 1550 & 1509 & 1360 & 1061 \\
\hline 4 & e1230 & --- & --- & --- & --- & --- & --- & --- & 1546 & 1507 & 1351 & 1059 \\
\hline 5 & 1209 & --- & --- & --- & --- & --- & --- & --- & 1548 & 1503 & 1341 & 1058 \\
\hline 6 & e1178 & --- & --- & --- & --- & --- & --- & --- & 1551 & 1500 & 1331 & 1055 \\
\hline 7 & e1147 & --- & --- & --- & --- & --- & --- & --- & 1550 & 1498 & 1318 & 1054 \\
\hline 8 & e1116 & --- & --- & --- & --- & --- & --- & --- & 1550 & 1494 & 1300 & 1053 \\
\hline 9 & e1085 & --- & --- & --- & --- & --- & --- & --- & 1550 & 1492 & 1285 & 1051 \\
\hline 10 & e1054 & --- & --- & --- & --- & --- & --- & --- & 1551 & 1490 & 1274 & 1049 \\
\hline 11 & e1023 & --- & --- & --- & --- & --- & --- & --- & 1552 & 1467 & 1261 & 1040 \\
\hline 12 & e992 & --- & --- & --- & --- & --- & --- & --- & 1552 & 1445 & 1248 & 1021 \\
\hline 13 & 962 & --- & --- & --- & --- & --- & --- & --- & 1552 & 1447 & 1236 & 1001 \\
\hline 14 & e937 & --- & --- & --- & --- & --- & --- & --- & 1550 & 1450 & 1222 & 982 \\
\hline 15 & e912 & --- & --- & --- & --- & --- & --- & --- & 1549 & 1452 & 1210 & 960 \\
\hline 16 & e887 & --- & --- & --- & --- & --- & --- & --- & 1546 & 1453 & 1197 & 947 \\
\hline 17 & e862 & --- & --- & --- & --- & --- & --- & --- & 1543 & 1453 & 1184 & 946 \\
\hline 18 & 834 & --- & --- & --- & --- & --- & --- & --- & 1540 & 1451 & 1172 & 945 \\
\hline 19 & e804 & --- & --- & --- & --- & --- & --- & --- & 1539 & 1449 & 1158 & 944 \\
\hline 20 & e785 & --- & --- & --- & --- & --- & --- & 1335 & 1537 & 1447 & 1146 & 943 \\
\hline 21 & e755 & --- & --- & --- & --- & --- & --- & 1358 & 1536 & 1446 & 1132 & 942 \\
\hline 22 & e725 & --- & --- & --- & --- & --- & --- & 1384 & 1536 & 1445 & 1117 & 940 \\
\hline 23 & e695 & --- & --- & --- & --- & --- & --- & 1414 & 1535 & 1441 & 1102 & 940 \\
\hline 24 & e 665 & --- & --- & --- & --- & --- & --- & 1449 & 1531 & 1441 & 1087 & 937 \\
\hline 25 & e635 & --- & --- & --- & --- & --- & --- & 1484 & 1530 & 1439 & 1078 & 936 \\
\hline 26 & e 600 & --- & --- & --- & --- & --- & --- & 1515 & 1527 & 1434 & 1077 & 934 \\
\hline 27 & e 565 & --- & --- & --- & --- & --- & --- & 1543 & 1525 & 1432 & 1076 & 931 \\
\hline 28 & 525 & --- & --- & --- & --- & --- & --- & 1555 & 1523 & 1428 & 1075 & 928 \\
\hline 29 & e498 & --- & --- & --- & --- & --- & --- & 1554 & 1520 & 1420 & 1071 & 927 \\
\hline 30 & e471 & --- & --- & --- & --- & --- & --- & 1555 & 1518 & 1409 & 1070 & 926 \\
\hline 31 & e 443 & --- & --- & --- & --- & --- & --- & 1555 & --- & 1394 & 1068 & --- \\
\hline MAX & 1290 & --- & --- & --- & --- & --- & --- & --- & 1555 & 1515 & 1385 & 1065 \\
\hline MIN & 443 & --- & --- & --- & --- & --- & --- & --- & 1518 & 1394 & 1068 & 926 \\
\hline a & & & & & & & & 27.86 & 27.45 & 26.02 & 22.02 & 20.11 \\
\hline $\mathrm{b}$ & -868 & & & & & & & & -37 & -124 & -326 & -142 \\
\hline
\end{tabular}

WTR YR $1999 \quad b-385$

e Estimated.

a Gage height, in feet, at end of month.

b Change in contents, in acre-feet. 
11413945 LOWER CASCADE LAKE NEAR SODA SPRINGS, CA

LOCATION.-Lat $39^{\circ} 18^{\prime} 12^{\prime \prime}$, long 120²6'19", in SE 1/4 SE 1/4 sec.30, T.17 N., R.14 E., Placer County, Hydrologic Unit 18020125, Tahoe National Forest, on outlet structure on Lower Cascade Lake Dam and 3.6 mi southwest of Soda Springs.

DRAINAGE AREA.-1.02 $\mathrm{mi}^{2}$.

PERIOD OF RECORD._-July 1991 to current year. Unpublished records for water years 1966-90 available in files of the U.S. Geological Survey.

GAGE.-Water-stage recorder. Datum of gage is 6,560.4 ft above sea level (levels by Pacific Gas \& Electric Co.). Prior to July 1991, nonrecording gage at same site and datum.

REMARKS.-Except for instantaneous observations, there were no usable data for the entire year due to equipment malfunction. Reservoir is formed on natural lake by rock-fill dam completed in 1860. Usable capacity, 484 acre-ft between gage heights $0.0 \mathrm{ft}$, invert of outlet, and $21.5 \mathrm{ft}$, crest of spillway. Water is used for power development downstream.

COOPERATION.-Records were collected by Pacific Gas \& Electric Co., under general supervision of the U.S. Geological Survey, in connection with a Federal Energy Regulatory Commission project.

Capacity table (gage height, in feet, and contents, in acre-feet)

(Based on survey by Pacific Gas \& Electric Co., dated April 1965)

$\begin{array}{rrrr}0 & 0 & 16 & 318 \\ 4 & 62 & 20 & 435 \\ 8 & 133 & 22 & 500 \\ 12 & 218 & 23 & 530\end{array}$

RESERVOIR STORAGE (ACRE-FEET), WATER YEAR OCTOBER 1998 TO SEPTEMBER 1999 DAILY INSTANTANEOUS VALUES

\begin{tabular}{|c|c|c|c|c|c|c|c|c|c|c|c|c|}
\hline DAY & OCT & NOV & DEC & JAN & FEB & MAR & APR & MAY & JUN & JUL & AUG & SEP \\
\hline 1 & --- & --- & --- & --- & --- & --- & --- & --- & --- & --- & 394 & --- \\
\hline 2 & --- & --- & --- & --- & --- & --- & --- & --- & --- & --- & --- & --- \\
\hline 3 & --- & 7 & --- & --- & --- & --- & --- & --- & --- & --- & --- & --- \\
\hline 4 & --- & --- & --- & --- & --- & --- & --- & --- & --- & --- & --- & --- \\
\hline 5 & 352 & --- & --- & --- & --- & --- & --- & --- & --- & 462 & --- & 174 \\
\hline 6 & --- & --- & --- & --- & --- & --- & --- & --- & --- & --- & --- & --- \\
\hline 7 & --- & --- & --- & --- & --- & --- & --- & --- & --- & --- & 382 & --- \\
\hline 8 & --- & --- & --- & --- & --- & --- & --- & --- & --- & --- & --- & --- \\
\hline 9 & --- & --- & --- & -- & -- & --- & --- & --- & --- & --- & --- & --- \\
\hline 10 & --- & --- & --- & --- & --- & --- & --- & --- & --- & --- & --- & --- \\
\hline 11 & --- & --- & --- & --- & --- & --- & --- & --- & --- & 445 & --- & --- \\
\hline 12 & --- & --- & --- & --- & --- & --- & --- & --- & --- & --- & --- & 76 \\
\hline 13 & --- & --- & --- & --- & --- & --- & --- & --- & --- & --- & --- & --- \\
\hline 14 & --- & --- & --- & --- & --- & --- & --- & --- & --- & --- & 369 & --- \\
\hline 15 & --- & --- & -- & --- & -- & --- & --- & --- & --- & --- & --- & --- \\
\hline 16 & --- & --- & --- & --- & --- & --- & --- & --- & --- & --- & --- & --- \\
\hline 17 & --- & --- & --- & --- & --- & --- & --- & 378 & --- & --- & --- & --- \\
\hline 18 & 289 & --- & --- & --- & --- & --- & --- & --- & --- & 423 & --- & 145 \\
\hline 19 & --- & --- & --- & --- & --- & --- & --- & --- & --- & --- & --- & --- \\
\hline 20 & --- & --- & --- & --- & --- & --- & --- & --- & --- & --- & --- & --- \\
\hline 21 & --- & --- & --- & --- & --- & --- & --- & --- & --- & --- & 355 & --- \\
\hline 22 & --- & --- & --- & --- & --- & --- & --- & --- & --- & --- & --- & --- \\
\hline 23 & --- & --- & --- & --- & --- & --- & --- & --- & --- & --- & --- & --- \\
\hline 24 & --- & --- & --- & --- & --- & --- & --- & --- & --- & --- & --- & --- \\
\hline 25 & --- & --- & --- & -- & -- & --- & --- & --- & --- & 409 & 345 & --- \\
\hline 26 & --- & --- & --- & --- & --- & --- & --- & --- & --- & --- & --- & 223 \\
\hline 27 & --- & --- & --- & --- & --- & --- & --- & --- & --- & --- & --- & --- \\
\hline 28 & 107 & --- & --- & --- & --- & --- & --- & --- & --- & --- & --- & --- \\
\hline 29 & --- & --- & --- & --- & --- & --- & --- & --- & --- & --- & 293 & --- \\
\hline 30 & --- & --- & --- & --- & --- & --- & --- & --- & --- & --- & --- & --- \\
\hline 31 & --- & --- & --- & --- & --- & --- & --- & --- & --- & --- & --- & --- \\
\hline MAX & --- & --- & --- & --- & --- & --- & --- & --- & --- & --- & --- & --- \\
\hline MIN & --- & --- & --- & --- & --- & --- & --- & --- & --- & --- & --- & --- \\
\hline
\end{tabular}




\section{FORDYCE LAKE NEAR CISCO, CA}

LOCATION.-Lat 39²2'44", long 120²9'40", in NE 1/4 SE 1/4 sec.34, T.18 N., R.12 E., Nevada County, Hydrologic Unit 18020125, near left abutment of Fordyce Dam on Fordyce Creek and 5.3 mi northeast of Cisco.

DRAINAGE AREA.- $-31.7 \mathrm{mi}^{2}$.

PERIOD OF RECORD.—October 1977 to current year. Periodic gage heights only for October 1965 to September 1976 and daily contents for water year 1977 are in the files of the U.S. Geological Survey.

GAGE.-Water-stage recorder. Datum of gage is 6,290.5 ft above sea level (levels by Pacific Gas \& Electric Co.). Prior to Nov. 29, 1976, nonrecording gage on upstream side of dam at same datum.

REMARKS.-Lake is formed by a rockfill dam; storage began in 1926. In 1980 the capacity of Fordyce Lake was increased by the addition of $3 \mathrm{ft}$ of flashboards. Capacity, 49,903 acre-ft between gage heights $0.85 \mathrm{ft}$, bottom of outlet valve, and $114.6 \mathrm{ft}$, top of flashboards in spillway. Released water flows down Fordyce Creek (station 11414100) to Lake Spaulding (station 11414140) for use in a power and irrigation system. See schematic diagram of Yuba River Basin.

COOPERATION.--Records were collected by Pacific Gas \& Electric Co., under general supervision of the U.S. Geological Survey, in connection with a Federal Energy Regulatory Commission project. Contents not rounded to U.S. Geological Survey standards.

EXTREMES FOR PERIOD OF RECORD.—Maximum contents, 50,025 acre-ft, June 28, 1999, gage height, $114.77 \mathrm{ft}$; minimum, 250 acre-ft, Oct. 31 to Nov. 7, 1979.

EXTREMES FOR CURRENT YEAR.-Maximum contents, 50,025 acre-ft, June 28, gage height, 114.77 ft; minimum, 746 acre-ft, Sept. 30 , gage height, $9.78 \mathrm{ft}$.

Capacity table (gage height, in feet, and contents, in acre-feet)

\begin{tabular}{|c|c|c|c|c|c|c|c|}
\hline 4 & 219 & 20 & 2,608 & 40 & 8,183 & 80 & 26,770 \\
\hline 5 & 278 & 25 & 3,827 & 50 & 11,797 & 90 & 32,820 \\
\hline 10 & 774 & 30 & 5,170 & 60 & 16,174 & 100 & 39,342 \\
\hline 15 & 1,570 & 35 & 6,628 & 70 & 21,196 & 114.6 & 49,903 \\
\hline
\end{tabular}

RESERVOIR STORAGE (ACRE-FEET), WATER YEAR OCTOBER 1998 TO SEPTEMBER 1999 DAILY OBSERVATION AT 2400 HOURS

\begin{tabular}{|c|c|c|c|c|c|c|c|c|c|c|c|c|}
\hline DAY & OCT & NOV & $\mathrm{DEC}$ & JAN & FEB & MAR & $\mathrm{APR}$ & MAY & JUN & JUL & AUG & SEP \\
\hline 1 & 25224 & 20220 & 15466 & 10790 & 10475 & 10052 & 10411 & 17236 & 41689 & 49854 & 25687 & 5984 \\
\hline 2 & 25088 & 20054 & 15373 & 10609 & 10419 & 10152 & 10407 & 17800 & 42278 & 49739 & 24804 & 5351 \\
\hline 3 & 24940 & 19889 & 15503 & 10434 & 10370 & 10237 & 10400 & 18137 & 42313 & 49570 & 23944 & 4718 \\
\hline 4 & 24787 & 19719 & 15415 & 10255 & 10348 & 10255 & 10389 & 18326 & 42137 & 49302 & 23075 & 4119 \\
\hline 5 & 24628 & 19555 & 15290 & 10141 & 10303 & 10263 & 10415 & 18532 & 42538 & 48990 & 22208 & 3537 \\
\hline 6 & 24482 & 19386 & 15142 & 10078 & 10311 & 10252 & 10389 & 19025 & 43410 & 48480 & 21345 & 2963 \\
\hline 7 & 24319 & 19223 & 14977 & 10008 & 10359 & 10226 & 10367 & 19807 & 44084 & 47659 & 20491 & 2408 \\
\hline 8 & 24173 & 19056 & 14809 & 9939 & 10434 & 10226 & 10393 & 20512 & 44582 & 46866 & 19647 & 1876 \\
\hline 9 & 24022 & 18894 & 14637 & 9870 & 10508 & 10215 & 10355 & 21143 & 45091 & 46051 & 18843 & 1382 \\
\hline 10 & 23866 & 18727 & 14457 & 9802 & 10489 & 10185 & 10326 & 21756 & 45603 & 45230 & 18047 & 1072 \\
\hline 11 & 23710 & 18572 & 14278 & 9735 & 10452 & 10133 & 10296 & 22572 & 46412 & 44408 & 17241 & 997 \\
\hline 12 & 23549 & 18411 & 14105 & 9667 & 10411 & 10093 & 10289 & 23727 & 47224 & 43589 & 16440 & 964 \\
\hline 13 & 23389 & 18222 & 13954 & 9600 & 10367 & 10056 & 10359 & 24770 & 48102 & 42771 & 15657 & 956 \\
\hline 14 & 23223 & 17988 & 13771 & 9544 & 10333 & 10030 & 10501 & 25481 & 48450 & 41955 & 14868 & 884 \\
\hline 15 & 23058 & 17830 & 13602 & 9583 & 10285 & 10001 & 10677 & 25975 & 48503 & 41103 & 14105 & 845 \\
\hline 16 & 22883 & 17628 & 13442 & 9643 & 10292 & 9975 & 10916 & 26578 & 48647 & 40229 & 13348 & 831 \\
\hline 17 & 22708 & 17451 & 13305 & 9760 & 10259 & 9953 & 11283 & 27449 & 49142 & 39349 & 13007 & 821 \\
\hline 18 & 22528 & 17227 & 13164 & 10196 & 10244 & 9964 & 11757 & 28492 & 49555 & 38465 & 12952 & 817 \\
\hline 19 & 22349 & 16998 & 13020 & 10407 & 10200 & 9994 & 12254 & 29608 & 49816 & 37571 & 12901 & 814 \\
\hline 20 & 22176 & 16771 & 12889 & 10602 & 10181 & 10019 & 12817 & 30719 & 49769 & 36633 & 12842 & 809 \\
\hline 21 & 21998 & 16584 & 12718 & 10639 & 10155 & 10041 & 13327 & 32058 & 49762 & 35699 & 12792 & 801 \\
\hline 22 & 21809 & 16393 & 12553 & 10688 & 10104 & 10038 & 13671 & 33658 & 49915 & 34768 & 12751 & 798 \\
\hline 23 & 21627 & 16402 & 12376 & 10752 & 10049 & 10038 & 13959 & 35478 & 49944 & 33830 & 12278 & 786 \\
\hline 24 & 21547 & 16236 & 12201 & 10748 & 10012 & 10005 & 14336 & 37537 & 49915 & 32908 & 11549 & 782 \\
\hline 25 & 21446 & 16042 & 12022 & 10726 & 9994 & 10001 & 14909 & 37590 & 49746 & 31989 & 10813 & 779 \\
\hline 26 & 21223 & 15839 & 11853 & 10707 & 9939 & 10067 & 15522 & 37200 & 49723 & 31075 & 10074 & 775 \\
\hline 27 & 21053 & 15680 & 11671 & 10669 & 9895 & 10159 & 15966 & 41752 & 49862 & 30165 & 9337 & 766 \\
\hline 28 & 20890 & 15484 & 11498 & 10632 & 9924 & 10255 & 16293 & 41871 & 50025 & 29264 & 8626 & 754 \\
\hline 29 & 20721 & 15382 & 11322 & 10591 & --- & 10315 & 16512 & 41528 & 49981 & 28356 & 7935 & 749 \\
\hline 30 & 20554 & 15540 & 11139 & 10546 & --- & 10389 & 16747 & 41486 & 49915 & 27455 & 7269 & 746 \\
\hline 31 & 20387 & --- & 10977 & 10516 & --- & 10411 & --- & 41458 & --- & 26572 & 6610 & --- \\
\hline MAX & 25224 & 20220 & 15503 & 10790 & 10508 & 10411 & 16747 & 41871 & 50025 & 49854 & 25687 & 5984 \\
\hline MIN & 20387 & 15382 & 10977 & 9544 & 9895 & 9953 & 10289 & 17236 & 41689 & 26572 & 6610 & 746 \\
\hline a & 68.46 & 58.65 & 47.90 & 46.68 & 45.08 & 46.40 & 61.20 & 103.08 & 114.62 & 79.66 & 34.94 & 9.78 \\
\hline $\mathrm{b}$ & -4974 & -4847 & -4563 & -461 & -592 & +487 & +6336 & +24711 & +8457 & -23343 & -19962 & -5864 \\
\hline
\end{tabular}

CAL YR 1998 MAX 49900 MIN 8556 b -2123 WTR YR 1999 MAX 50025 MIN 746 b -24615 
11414100 FORDYCE CREEK BELOW FORDYCE DAM, NEAR CISCO, CA

LOCATION.-Lat 39²2’48", long 120²9'54", in NW 1/4 SE 1/4 sec.34, T.18 N., R.13 E., Nevada County, Hydrologic Unit 18020125, Tahoe National Forest, on right bank $850 \mathrm{ft}$ downstream from Fordyce Dam, and $5.3 \mathrm{mi}$ northeast of Cisco.

DRAINAGE AREA.-31.7 $\mathrm{mi}^{2}$.

PERIOD OF RECORD.-June 1966 to current year.

GAGE.-Water-stage recorder. Elevation of gage is 6,250 ft above sea level, from topographic map.

REMARKS.-Flow regulated by Fordyce Lake (station 11414090). See schematic diagram of Yuba River Basin.

COOPERATION.--Records were collected by Pacific Gas \& Electric Co., under general supervision of the U.S. Geological Survey, in connection with a Federal Energy Regulatory Commission project.

EXTREMES FOR PERIOD OF RECORD.-Maximum discharge, 4,660 ft 3 /s, July 9, 1974, gage height, $7.90 \mathrm{ft}$ in gage well, $6.82 \mathrm{ft}$ from highwater marks, from rating curve extended above $1,000 \mathrm{ft}^{3} / \mathrm{s}$ on basis of slope-area measurement of peak flow; minimum daily, $3.5 \mathrm{ft}^{3} / \mathrm{s}$,

Jan. 2-9, 1979.

DISCHARGE, CUBIC FEET PER SECOND, WATER YEAR OCTOBER 1998 TO SEPTEMBER 1999 DAILY MEAN VALUES

\begin{tabular}{|c|c|c|c|c|c|c|c|c|c|c|c|c|}
\hline DAY & $\mathrm{OCT}$ & NOV & $\mathrm{DEC}$ & JAN & FEB & MAR & $\mathrm{APR}$ & MAY & JUN & JUL & AUG & SEP \\
\hline 1 & 132 & 125 & 118 & 106 & 46 & 49 & 47 & 59 & 654 & 333 & 441 & 330 \\
\hline 2 & 132 & 125 & 118 & 106 & 46 & 47 & 46 & 59 & 358 & 321 & 438 & 324 \\
\hline 3 & 131 & 124 & 120 & 104 & 46 & 47 & 46 & 59 & 359 & 312 & 434 & 318 \\
\hline 4 & 132 & 124 & 117 & 105 & 46 & 47 & 46 & 59 & 358 & 305 & 430 & 311 \\
\hline 5 & 131 & 124 & 116 & 73 & 46 & 47 & 46 & 60 & 321 & 301 & 431 & 303 \\
\hline 6 & 131 & 123 & 117 & 46 & 46 & 47 & 46 & 62 & 230 & 404 & 433 & 295 \\
\hline 7 & 130 & 123 & 117 & 46 & 47 & 47 & 46 & 62 & 232 & 490 & 428 & 286 \\
\hline 8 & 130 & 123 & 116 & 45 & 47 & 46 & 46 & 63 & 234 & 486 & 425 & 275 \\
\hline 9 & 130 & 123 & 115 & 45 & 48 & 46 & 46 & 63 & 235 & 481 & 420 & 263 \\
\hline 10 & 130 & 123 & 115 & 45 & 47 & 46 & 46 & 64 & 235 & 480 & 416 & 164 \\
\hline 11 & 129 & 122 & 115 & 45 & 46 & 46 & 46 & 66 & 237 & 477 & 412 & 40 \\
\hline 12 & 129 & 122 & 115 & 45 & 46 & 46 & 47 & 67 & 241 & 473 & 407 & 15 \\
\hline 13 & 129 & 122 & 114 & 45 & 46 & 46 & 48 & 68 & 359 & 471 & 403 & 12 \\
\hline 14 & 129 & 121 & 114 & 45 & 46 & 46 & 48 & 68 & 649 & 470 & 398 & 10 \\
\hline 15 & 129 & 121 & 114 & 46 & 46 & 46 & 48 & 69 & 781 & 468 & 393 & 10 \\
\hline 16 & 129 & 121 & 114 & 46 & 46 & 46 & 49 & 70 & 675 & 466 & 389 & 7.7 \\
\hline 17 & 128 & 121 & 113 & 47 & 46 & 46 & 50 & 71 & 507 & 463 & 171 & 6.6 \\
\hline 18 & 128 & 121 & 112 & 50 & 46 & 46 & 51 & 72 & 405 & 461 & 17 & 6.6 \\
\hline 19 & 128 & 120 & 110 & 47 & 46 & 46 & 52 & 73 & 502 & 471 & 17 & 6.4 \\
\hline 20 & 127 & 120 & 110 & 49 & 46 & 46 & 53 & 74 & 567 & 485 & 17 & 6.4 \\
\hline 21 & 127 & 119 & 111 & 47 & 46 & 46 & 53 & 76 & 558 & 481 & 17 & 6.3 \\
\hline 22 & 127 & 119 & 108 & 47 & 46 & 46 & 53 & 78 & 532 & 478 & 17 & 6.2 \\
\hline 23 & 127 & 122 & 110 & 47 & 46 & 46 & 54 & 80 & 571 & 473 & 238 & 6.2 \\
\hline 24 & 127 & 119 & 107 & 47 & 46 & 46 & 55 & 82 & 568 & 470 & 375 & 6.1 \\
\hline 25 & 126 & 119 & 109 & 47 & 46 & 47 & 56 & 146 & 481 & 466 & 369 & 6.0 \\
\hline 26 & 126 & 118 & 109 & 46 & 45 & 48 & 58 & 459 & 331 & 462 & 364 & 5.9 \\
\hline 27 & 126 & 118 & 108 & 47 & 45 & 47 & 56 & 797 & 228 & 459 & 358 & 5.8 \\
\hline 28 & 125 & 118 & 107 & 46 & 47 & 47 & 56 & 918 & 235 & 454 & 353 & 5.6 \\
\hline 29 & 125 & 118 & 108 & 46 & --- & 47 & 56 & 886 & 344 & 452 & 347 & 5.5 \\
\hline 30 & 125 & 122 & 105 & 46 & --- & 47 & 58 & 774 & 345 & 449 & 341 & 5.4 \\
\hline 31 & 125 & --- & 107 & 46 & --- & 47 & --- & 799 & --- & 444 & 336 & --- \\
\hline TOTAL & 3980 & 3640 & 3489 & 1698 & 1292 & 1443 & 1508 & 6403 & 12332 & 13706 & 10035 & 3048.7 \\
\hline MEAN & 128 & 121 & 113 & 54.8 & 46.1 & 46.5 & 50.3 & 207 & 411 & 442 & 324 & 102 \\
\hline MAX & 132 & 125 & 120 & 106 & 48 & 49 & 58 & 918 & 781 & 490 & 441 & 330 \\
\hline MIN & 125 & 118 & 105 & 45 & 45 & 46 & 46 & 59 & 228 & 301 & 17 & 5.4 \\
\hline $\mathrm{AC}-\mathrm{FT}$ & 7890 & 7220 & 6920 & 3370 & 2560 & 2860 & 2990 & 12700 & 24460 & 27190 & 19900 & 6050 \\
\hline
\end{tabular}

STATISTICS OF MONTHLY MEAN DATA FOR WATER YEARS 1966 - 1999, BY WATER YEAR (WY)

\begin{tabular}{|c|c|c|c|c|c|c|c|c|c|c|c|c|}
\hline MEAN & 83.7 & 43.1 & 28.7 & 37.2 & 57.0 & 73.4 & 69.5 & 190 & 368 & 295 & 217 & 141 \\
\hline MAX & 428 & 236 & 173 & 278 & 328 & 353 & 315 & 727 & 957 & 542 & 403 & 497 \\
\hline (WY) & 1976 & 1977 & 1982 & 1997 & 1984 & 1984 & 1986 & 1996 & 1995 & 1995 & 1983 & 1980 \\
\hline MIN & 4.35 & 3.90 & 3.75 & 4.76 & 4.78 & 5.07 & 9.21 & 17.0 & 36.4 & 21.7 & 11.4 & 4.84 \\
\hline (WY) & 1978 & 1979 & 1979 & 1981 & 1977 & 1977 & 1977 & 1977 & 1976 & 1981 & 1987 & 1977 \\
\hline
\end{tabular}

SUMMARY STATISTICS

ANNUAL TOTAL

ANNUAL MEAN

HIGHEST ANNUAL MEAN

LOWEST ANNUAL MEAN

HIGHEST DAILY MEAN

LOWEST DAILY MEAN

ANNUAL SEVEN-DAY MINIMUM

INSTANTANEOUS PEAK FLOW

INSTANTANEOUS PEAK STAGE

ANNUAL RUNOFF (AC-FT)

10 PERCENT EXCEEDS

50 PERCENT EXCEEDS

90 PERCENT EXCEEDS
FOR 1998 CALENDAR YEAR

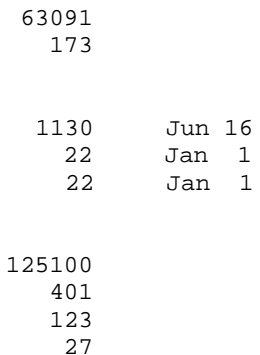

FOR 1999 WATER YEAR

$$
\begin{array}{ccc}
62574.7 & & \\
171 & & \\
& & \\
918 & \text { May } 28 \\
5.4 & \text { Sep } 30 \\
5.8 & \text { Sep } 24 \\
988 & \text { May 29 } \\
4.55 & \text { May 29 } \\
124100 & & \\
461 & & \\
112 & & \\
46 & &
\end{array}
$$

WATER YEARS 1966 - 1999

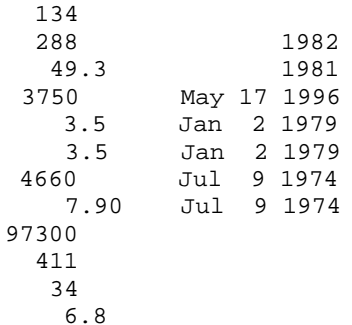




\section{LAKE SPAULDING NEAR EMIGRANT GAP, CA}

LOCATION.—Lat 39¹9’35", long 120³8’32", in SE 1/4 NE 1/4 sec.20, T.17 N., R.12 E., Nevada County, Hydrologic Unit 18020125, near center of Spaulding Dam on South Yuba River and 2.5 mi northeast of Emigrant Gap.

DRAINAGE AREA.-118 $\mathrm{mi}^{2}$.

PERIOD OF RECORD.—October 1964 to current year.

GAGE.-Water-stage recorder. Datum of gage is 4,809.6 ft above sea level (levels by Pacific Gas \& Electric Co.). Prior to July 1968, nonrecording gage at same site and datum.

REMARKS.- - Lake is formed by three concrete-arch dams with spillway on the middle arch. Storage began in 1913. Capacity, 74,773 acre-ft between gage heights $0.6 \mathrm{ft}$, bottom of outlet, and $205.0 \mathrm{ft}$, top of radial gates. Released water flows through Spaulding Powerplants Nos. 1 and 2 (stations 11414154 and 11414155). Flow through Powerplant No. 1 is transported out of Yuba River Basin by Drum Canal to Bear River Basin. See schematic diagrams of Yuba and Bear River Basins.

COOPERATION.—Records were collected by Pacific Gas \& Electric Co., under general supervision of the U.S. Geological Survey, in connection with a Federal Energy Regulatory Commission project. Contents not rounded to U.S. Geological Survey standards.

EXTREMES FOR PERIOD OF RECORD.-Maximum contents, 75,100 acre-ft, July 13, 1967, gage height, 205.5 ft; minimum, 914 acre-ft, Feb. 28, 1976, gage height, $25.5 \mathrm{ft}$.

EXTREMES FOR CURRENT YEAR.-Maximum contents, 74,836 acre-ft, June 22, gage height, 205.09 ft; minimum, 8,648 acre-ft, Apr. 13, gage height, $66.61 \mathrm{ft}$.

Capacity table (gage height, in feet, and contents, in acre-feet)

(Based on survey by Pacific Gas \& Electric Co., dated Apr. 23, 1965)

$\begin{array}{rrrr}20 & 566 & 70 & 9,632 \\ 25 & 874 & 100 & 19,541 \\ 30 & 1,352 & 150 & 41,545 \\ 40 & 2,742 & 200 & 71,329 \\ 50 & 4,578 & 206 & 75,473\end{array}$

RESERVOIR STORAGE (ACRE-FEET), WATER YEAR OCTOBER 1998 TO SEPTEMBER 1999 DAILY OBSERVATION AT 2400 HOURS

\begin{tabular}{|c|c|c|c|c|c|c|c|c|c|c|c|c|}
\hline DAY & $\mathrm{OCT}$ & NOV & $\mathrm{DEC}$ & JAN & FEB & MAR & APR & MAY & JUN & JUL & AUG & SEP \\
\hline 1 & 49089 & 41359 & 41504 & 30527 & 33257 & 31087 & 14782 & 23607 & 69746 & 74342 & 62171 & 56133 \\
\hline 2 & 48536 & 41111 & 41685 & 29831 & 32890 & 31561 & 14032 & 24821 & 69954 & 74272 & 61888 & 55876 \\
\hline 3 & 47964 & 40859 & 44310 & 29150 & 32733 & 32429 & 13269 & 25185 & 70048 & 74099 & 61592 & 55608 \\
\hline 4 & 47373 & 40593 & 44834 & 28443 & 32664 & 32383 & 12568 & 25132 & 69987 & 73829 & 61292 & 55655 \\
\hline 5 & 46747 & 40363 & 44915 & 27689 & 32424 & 31975 & 12056 & 25149 & 70525 & 73469 & 60992 & 55685 \\
\hline 6 & 46169 & 40179 & 44802 & 26881 & 32236 & 31435 & 11509 & 25932 & 71574 & 73234 & 60743 & 55685 \\
\hline 7 & 45588 & 40123 & 44743 & 26205 & 33443 & 30782 & 10960 & 27485 & 72258 & 73193 & 60986 & 55322 \\
\hline 8 & 45001 & 39909 & 44315 & 25430 & 34448 & 30118 & 10440 & 28742 & 72615 & 73110 & 61223 & 54949 \\
\hline 9 & 44679 & 39661 & 43709 & 25230 & 37138 & 29386 & 9927 & 29610 & 72904 & 73000 & 61354 & 54530 \\
\hline 10 & 45275 & 39443 & 43112 & 25047 & 37542 & 28491 & 9418 & 30389 & 73200 & 72752 & 61429 & 53978 \\
\hline 11 & 45870 & 39237 & 42524 & 24250 & 37300 & 27476 & 8947 & 31666 & 73787 & 72128 & 61454 & 53580 \\
\hline 12 & 45442 & 39005 & 41939 & 23449 & 36859 & 26442 & 8651 & 33784 & 74113 & 71390 & 61379 & 53090 \\
\hline 13 & 44856 & 38789 & 41504 & 22842 & 36308 & 25467 & 8648 & 35694 & 74474 & 70660 & 61285 & 52163 \\
\hline 14 & 44267 & 38529 & 41055 & 22223 & 35743 & 24611 & 9004 & 36727 & 74613 & 69880 & 61492 & 51205 \\
\hline 15 & 43682 & 38280 & 40531 & 22146 & 35105 & 23737 & 9480 & 37104 & 74578 & 69137 & 61674 & 50260 \\
\hline 16 & 43349 & 38051 & 40235 & 23351 & 34828 & 22928 & 10164 & 37675 & 74613 & 68452 & 61586 & 49189 \\
\hline 17 & 43852 & 37957 & 39970 & 24583 & 35411 & 22265 & 11255 & 38834 & 74703 & 68312 & 61229 & 48263 \\
\hline 18 & 44416 & 37784 & 39590 & 28224 & 35416 & 21726 & 12616 & 40485 & 74738 & 67546 & 60463 & 48286 \\
\hline 19 & 44278 & 37547 & 39126 & 30265 & 35057 & 21274 & 14141 & 42294 & 74766 & 66798 & 59683 & 48742 \\
\hline 20 & 44065 & 37286 & 38589 & 33424 & 34553 & 20753 & 15673 & 44049 & 74794 & 66054 & 58920 & 49201 \\
\hline 21 & 43772 & 37443 & 37982 & 34099 & 34056 & 20064 & 16928 & 46125 & 74815 & 65307 & 58405 & 49656 \\
\hline 22 & 43497 & 37784 & 37340 & 34425 & 33312 & 19270 & 17565 & 48904 & 74836 & 64546 & 57882 & 50113 \\
\hline 23 & 43280 & 38915 & 36678 & 35989 & 32498 & 18508 & 17901 & 52129 & 74808 & 63777 & 57343 & 50561 \\
\hline 24 & 43154 & 39423 & 36008 & 36168 & 31729 & 17802 & 18562 & 55489 & 74745 & 62988 & 57216 & 51022 \\
\hline 25 & 42970 & 39206 & 35344 & 36139 & 31128 & 17183 & 19791 & 59615 & 74509 & 62203 & 57083 & 51462 \\
\hline 26 & 42786 & 38809 & 34666 & 36158 & 30376 & 16914 & 21650 & 63458 & 74272 & 61411 & 56926 & 51910 \\
\hline 27 & 42571 & 38504 & 33986 & 35921 & 29544 & 16893 & 22504 & 66857 & 73988 & 60824 & 56770 & 52360 \\
\hline 28 & 42335 & 38186 & 33299 & 35430 & 29562 & 16712 & 22822 & 68951 & 73780 & 60955 & 56836 & 52776 \\
\hline 29 & 42106 & 38220 & 32599 & 34828 & --- & 16361 & 22714 & 69278 & 73932 & 61373 & 56872 & 53236 \\
\hline 30 & 41835 & 40957 & 31898 & 34198 & --- & 15968 & 22741 & 69365 & 74168 & 61756 & 56631 & 53673 \\
\hline 31 & 41576 & --- & 31218 & 33611 & --- & 15469 & --- & 69438 & --- & 61982 & 56385 & --- \\
\hline MAX & 49089 & 41359 & 44915 & 36168 & 37542 & 32429 & 22822 & 69438 & 74836 & 74342 & 62171 & 56133 \\
\hline MIN & 41576 & 37286 & 31218 & 22146 & 29544 & 15469 & 8648 & 23607 & 69746 & 60824 & 56385 & 48263 \\
\hline$a$ & 150.06 & 148.86 & 128.69 & 133.90 & 124.97 & 88.45 & 108.48 & 197.19 & 204.13 & 185.70 & 176.59 & 172.01 \\
\hline $\mathrm{b}$ & -8085 & -619 & -9739 & +2393 & -4049 & -14093 & +7272 & +46697 & +4730 & -12186 & -5597 & -2712 \\
\hline C & 31000 & 28480 & 33850 & 26600 & 27100 & 15370 & 1720 & 27950 & 40210 & 40030 & 41780 & 20640 \\
\hline $\mathrm{d}$ & 3360 & 2910 & 2230 & 3450 & 4100 & 8210 & 4830 & 8650 & 9000 & 5810 & 4070 & 4370 \\
\hline
\end{tabular}

CAL YR 1998 MAX 74787 MIN 18436 b +11702 c 474000 d 67640

WTR YR 1999 MAX 74836 MIN 8648 b +4012 c 334700 d 61000

a Gage height, in feet, at end of month

b Change in contents, in acre-feet.

c Diversion, in acre-feet, to Spaulding No. 1 Powerplant, provided by Pacific Gas \& Electric Co.

d Diversion, in acre-feet, to Spaulding No. 2 Powerplant, provided by Pacific Gas \& Electric Co. 
11414170 DRUM CANAL AT TUNNEL OUTLET, NEAR EMIGRANT GAP, CA

LOCATION.-Lat 39¹9'03", long 120³9'08", in SE 1/4 SW 1/4 sec.20, T.17 N., R.12 E., Nevada County, Hydrologic Unit 18020125, Tahoe National Forest, $100 \mathrm{ft}$ downstream from tunnel outlet, $1.0 \mathrm{mi}$ downstream from Spaulding No. 1 Powerplant, and 1.7 mi northeast of Emigrant Gap.

PERIOD OF RECORD.-October 1964 to current year. Prior to October 1972, published as "Drum Canal at intake."

GAGE.-Water-stage recorder. Elevation of gage is $4,880 \mathrm{ft}$ above sea level, from topographic map. Prior to Oct. 1,1968 , in powerplant 0.7 mi upstream at different datum.

REMARKS.-Canal diverts from Spaulding No. 1 Powerplant (station 11414154) at Lake Spaulding Dam. Most of the water from Drum Canal enters the Bear River via Drum No. 1 and 2 Powerplants (station 11414196) at Drum Afterbay. Some of the water is diverted out of Drum Forebay to Alta Powerplant (station 11421725). See schematic diagrams of Yuba and Bear River Basins.

COOPERATION.-Records were collected by Pacific Gas \& Electric Co., under general supervision of the U.S. Geological Survey, in connection with a Federal Energy Regulatory Commission project.

EXTREMES FOR PERIOD OF RECORD.-Maximum daily discharge, $864 \mathrm{ft}^{3} / \mathrm{s}$, May 1, 1998; no flow for several days in most years.

DISCHARGE, CUBIC FEET PER SECOND, WATER YEAR OCTOBER 1998 TO SEPTEMBER 1999

DAILY MEAN VALUES

\begin{tabular}{|c|c|c|c|c|c|c|c|c|c|c|c|c|}
\hline DAY & OCT & NOV & $\mathrm{DEC}$ & JAN & FEB & MAR & APR & MAY & JUN & JUL & AUG & SEP \\
\hline 1 & 707 & 530 & 575 & 750 & 594 & 686 & 846 & 847 & 849 & 806 & 600 & 732 \\
\hline 2 & 703 & 529 & 596 & 744 & 588 & 746 & 845 & 844 & 838 & 849 & 851 & 731 \\
\hline 3 & 710 & 528 & 504 & 740 & 495 & 654 & 844 & 835 & 847 & 853 & 852 & 727 \\
\hline 4 & 725 & 527 & 548 & 747 & 477 & 711 & 843 & 846 & 846 & 853 & 856 & 550 \\
\hline 5 & 726 & 515 & 562 & 749 & 543 & 729 & 826 & 842 & 849 & 856 & 855 & 553 \\
\hline 6 & 722 & 505 & 572 & 742 & 541 & 733 & 823 & 845 & 851 & 856 & 828 & 553 \\
\hline 7 & 719 & 505 & 494 & 625 & 505 & 744 & 822 & 842 & 846 & 854 & 559 & 743 \\
\hline 8 & 727 & 504 & 693 & 569 & 525 & 750 & 809 & 845 & 848 & 853 & 563 & 741 \\
\hline 9 & 591 & 503 & 741 & 281 & 385 & 763 & 791 & 841 & 849 & 853 & 633 & 743 \\
\hline 10 & 116 & 502 & 738 & 268 & 528 & 815 & 777 & 844 & 850 & 855 & 657 & 753 \\
\hline 11 & 116 & 501 & 736 & 555 & 726 & 855 & 774 & 845 & 851 & 854 & 674 & 536 \\
\hline 12 & 635 & 500 & 744 & 613 & 789 & 856 & 768 & 848 & 851 & 854 & 718 & 548 \\
\hline 13 & 723 & 500 & 712 & 604 & 792 & 853 & 759 & 848 & 855 & 853 & 720 & 743 \\
\hline 14 & 718 & 507 & 701 & 604 & 791 & 843 & 745 & 842 & 848 & 854 & 559 & 745 \\
\hline 15 & 717 & 506 & 744 & 549 & 793 & 845 & 726 & 848 & 839 & 851 & 557 & 740 \\
\hline 16 & 582 & 505 & 743 & 233 & 668 & 833 & 719 & 847 & 849 & 832 & 700 & 733 \\
\hline 17 & 138 & 505 & 747 & 238 & 640 & 827 & 778 & 844 & 854 & 511 & 710 & 735 \\
\hline 18 & 114 & 504 & 754 & 545 & 647 & 846 & 790 & 850 & 855 & 854 & 713 & 248 \\
\hline 19 & 487 & 503 & 755 & 553 & 680 & 843 & 824 & 847 & 852 & 855 & 710 & .00 \\
\hline 20 & 519 & 455 & 753 & 444 & 705 & 843 & 848 & 851 & 852 & 854 & 702 & .00 \\
\hline 21 & 518 & 255 & 751 & 525 & 664 & 841 & 851 & 854 & 858 & 854 & 572 & .00 \\
\hline 22 & 517 & 262 & 752 & 554 & 750 & 839 & 845 & 845 & 857 & 855 & 581 & .00 \\
\hline 23 & 519 & 499 & 755 & 491 & 771 & 840 & 841 & 843 & 853 & 852 & 727 & .00 \\
\hline 24 & 523 & 440 & 753 & 579 & 753 & 824 & 848 & 844 & 850 & 852 & 717 & .00 \\
\hline 25 & 523 & 572 & 747 & 584 & 690 & 808 & 842 & 847 & 854 & 853 & 716 & .00 \\
\hline 26 & 523 & 594 & 751 & 532 & 707 & 845 & 847 & 850 & 853 & 853 & 720 & .00 \\
\hline 27 & 529 & 617 & 747 & 611 & 757 & 846 & 846 & 845 & 854 & 852 & 720 & .00 \\
\hline 28 & 533 & 613 & 749 & 712 & 655 & 847 & 844 & 846 & 857 & 622 & 587 & .00 \\
\hline 29 & 533 & 591 & 755 & 755 & --- & 842 & 844 & 849 & 855 & 443 & 594 & .00 \\
\hline 30 & 532 & 532 & 754 & 764 & --- & 838 & 844 & 850 & 857 & 498 & 735 & .00 \\
\hline 31 & 531 & --- & 757 & 739 & --- & 832 & --- & 846 & --- & 591 & 733 & --- \\
\hline TOTAL & 16976 & 15109 & 21683 & 17999 & 18159 & 24977 & 24409 & 26220 & 25527 & 24785 & 21419 & 11854.00 \\
\hline MEAN & 548 & 504 & 699 & 581 & 649 & 806 & 814 & 846 & 851 & 800 & 691 & 395 \\
\hline MAX & 727 & 617 & 757 & 764 & 793 & 856 & 851 & 854 & 858 & 856 & 856 & 753 \\
\hline MIN & 114 & 255 & 494 & 233 & 385 & 654 & 719 & 835 & 838 & 443 & 557 & .00 \\
\hline $\mathrm{AC}-\mathrm{FT}$ & 33670 & 29970 & 43010 & 35700 & 36020 & 49540 & 48420 & 52010 & 50630 & 49160 & 42480 & 23510 \\
\hline a & 31560 & 30630 & 42300 & 36620 & 37910 & 47960 & 47310 & 49470 & 46790 & 44560 & 40240 & 21320 \\
\hline $\mathrm{b}$ & 1060 & 885 & 351 & 659 & 980 & 700 & 516 & 113 & 657 & 1280 & 1260 & 1120 \\
\hline
\end{tabular}

a Discharge, in acre-feet, to Drum No. 1 and 2 Powerplants, provided by Pacific Gas \& Electric Co.

b Discharge, in acre-feet, to Alta Powerplant, provided by Pacific Gas \& Electric Co. 


\section{DRUM CANAL AT TUNNEL OUTLET, NEAR EMIGRANT GAP, CA—Continued}

STATISTICS OF MONTHLY MEAN DATA FOR WATER YEARS 1965 - 1999, BY WATER YEAR (WY)

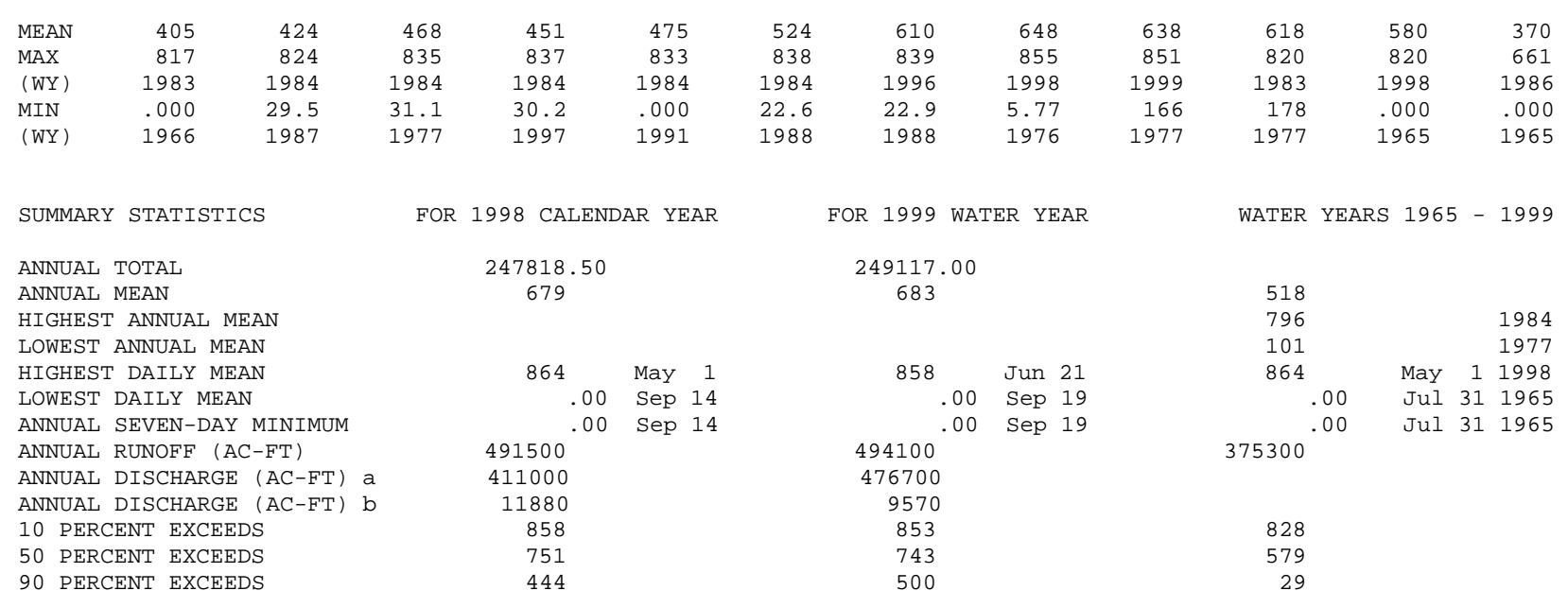

a Discharge, in acre-feet, to Drum No. 1 and 2 Powerplants, provided by Pacific Gas \& Electric Co.

b Discharge, in acre-feet, to Alta Powerplant, provided by Pacific Gas \& Electric Co. 
11414200 SOUTH YUBA CANAL NEAR EMIGRANT GAP, CA

LOCATION.-Lat 39¹8'49", long 120³9'43", in SE 1/4 NE 1/4 sec.30, T.17 N., R.12 E., Nevada County, Hydrologic Unit 18020125, on left bank of concrete flume $400 \mathrm{ft}$ downstream from Bowman Lake Road and 2.5 mi northeast of Emigrant Gap.

PERIOD OF RECORD.-October 1964 to current year.

GAGE.-Water-stage recorder and concrete control. Elevation of gage is 4,590 ft above sea level, from topographic map.

REMARKS. - Canal diverts from Spaulding No. 2 Powerplant (station 11414155) at Lake Spaulding Dam. Downstream from the gage, some flow is diverted to Bear River. The remainder of the water enters Deer Creek at Deer Creek Powerplant (station 11414205). See schematic diagrams of Yuba and Bear River Basins.

COOPERATION.--Records were collected by Pacific Gas \& Electric Co., under general supervision of the U.S. Geological Survey, in connection with a Federal Energy Regulatory Commission project.

EXTREMES FOR PERIOD OF RECORD.-Maximum daily discharge, $165 \mathrm{ft}^{3} / \mathrm{s}$, Aug. 3, 1965; no flow at times in some years.

DISCHARGE, CUBIC FEET PER SECOND, WATER YEAR OCTOBER 1998 TO SEPTEMBER 1999

DAILY MEAN VALUES

\begin{tabular}{|c|c|c|c|c|c|c|c|c|c|c|c|c|}
\hline DAY & $\mathrm{OCT}$ & NOV & $\mathrm{DEC}$ & JAN & FEB & MAR & APR & MAY & JUN & JUL & AUG & SEP \\
\hline 1 & 54 & 53 & 33 & e39 & e37 & 137 & 125 & 134 & 129 & 91 & 67 & 68 \\
\hline 2 & 52 & 53 & 36 & e39 & 37 & 138 & 125 & 137 & 129 & 130 & 68 & 68 \\
\hline 3 & 52 & 53 & 36 & e39 & 39 & 142 & 123 & 140 & 129 & 129 & 67 & 68 \\
\hline 4 & 51 & 53 & e33 & e39 & 39 & 139 & 66 & 141 & 128 & 129 & 70 & 69 \\
\hline 5 & 51 & 53 & e33 & e 40 & 39 & 138 & 28 & 140 & 128 & 130 & 70 & 68 \\
\hline 6 & 50 & 53 & e37 & e 40 & 39 & 138 & 18 & 137 & 129 & 130 & 69 & 68 \\
\hline 7 & 50 & 54 & 35 & e 40 & 33 & 136 & 13 & 140 & 129 & 130 & 66 & 68 \\
\hline 8 & 51 & 48 & 36 & e 40 & 24 & 136 & 17 & 141 & 129 & 130 & 67 & 68 \\
\hline 9 & 52 & 56 & e31 & e39 & 21 & 135 & 17 & 141 & 130 & 129 & 62 & 70 \\
\hline 10 & 49 & 55 & e30 & e 42 & 22 & 136 & 17 & 141 & 131 & 130 & 61 & 70 \\
\hline 11 & 52 & 53 & e30 & e76 & 21 & 137 & 17 & 141 & 131 & 130 & 68 & 69 \\
\hline 12 & 52 & 52 & e38 & e 80 & 25 & 137 & 8.3 & 141 & 131 & 130 & 64 & 69 \\
\hline 13 & 51 & 52 & e38 & e84 & 37 & 138 & 42 & 141 & 128 & 129 & 63 & 69 \\
\hline 14 & 51 & 52 & e 38 & e 84 & 36 & 141 & 120 & 141 & 126 & 129 & 63 & 69 \\
\hline 15 & 52 & 52 & e38 & e78 & 37 & 145 & 121 & 140 & 126 & 96 & 63 & 70 \\
\hline 16 & 53 & 52 & e38 & e73 & 68 & 143 & 122 & 139 & 126 & 68 & 62 & 71 \\
\hline 17 & 55 & 53 & e 38 & e78 & 132 & 141 & 122 & 135 & 126 & 73 & 63 & 71 \\
\hline 18 & 54 & 46 & e38 & e 57 & 138 & 139 & 125 & 133 & 126 & 71 & 64 & 72 \\
\hline 19 & 53 & 42 & e38 & e 65 & 137 & 137 & 127 & 136 & 126 & 68 & 61 & 70 \\
\hline 20 & 53 & 41 & e34 & e39 & 138 & 136 & 129 & 137 & 126 & 67 & 64 & 74 \\
\hline 21 & 53 & 41 & e31 & e50 & 138 & 135 & 127 & 136 & 126 & 69 & 63 & 76 \\
\hline 22 & 53 & 40 & e39 & e 65 & 136 & 134 & 129 & 135 & 126 & 68 & 63 & 76 \\
\hline 23 & 53 & 42 & e38 & e 49 & 138 & 132 & 129 & 134 & 126 & 69 & 64 & 77 \\
\hline 24 & 53 & 41 & e38 & e 67 & 137 & 132 & 128 & 135 & 126 & 68 & 63 & 75 \\
\hline 25 & 53 & 39 & e38 & e 42 & 136 & 131 & 129 & 136 & 127 & 67 & 67 & 75 \\
\hline 26 & 53 & 39 & e38 & e 42 & 134 & 129 & 132 & 119 & 127 & 67 & 68 & 75 \\
\hline 27 & 53 & 39 & e 38 & e37 & 133 & 128 & 133 & 134 & 127 & 67 & 68 & 75 \\
\hline 28 & 53 & 38 & e38 & e35 & 118 & 128 & 134 & 125 & 106 & 67 & 66 & 74 \\
\hline 29 & 53 & 38 & e39 & e36 & --- & 128 & 133 & 131 & 75 & 66 & 68 & 72 \\
\hline 30 & 53 & 34 & e39 & e37 & --- & 127 & 133 & 132 & 40 & 66 & 68 & 89 \\
\hline 31 & 53 & --- & e39 & e37 & --- & 126 & --- & 131 & --- & 67 & 68 & --- \\
\hline TOTAL & 1621 & 1417 & 1123 & 1608 & 2169 & 4199 & 2789.3 & 4224 & 3669 & 2960 & 2028 & 2153 \\
\hline MEAN & 52.3 & 47.2 & 36.2 & 51.9 & 77.5 & 135 & 93.0 & 136 & 122 & 95.5 & 65.4 & 71.8 \\
\hline MAX & 55 & 56 & 39 & 84 & 138 & 145 & 134 & 141 & 131 & 130 & 70 & 89 \\
\hline MIN & 49 & 34 & 30 & 35 & 21 & 126 & 8.3 & 119 & 40 & 66 & 61 & 68 \\
\hline $\mathrm{AC}-\mathrm{FT}$ & 3220 & 2810 & 2230 & 3190 & 4300 & 8330 & 5530 & 8380 & 7280 & 5870 & 4020 & 4270 \\
\hline a & 2890 & 2520 & 2230 & 3310 & 2200 & 2340 & 325 & 2690 & 4330 & 3560 & 3780 & 3470 \\
\hline
\end{tabular}

STATISTICS OF MONTHLY MEAN DATA FOR WATER YEARS 1965 - 1999, BY WATER YEAR (WY)

\begin{tabular}{|c|c|c|c|c|c|c|c|c|c|c|c|c|}
\hline MEAN & 78.5 & 67.7 & 71.3 & 74.3 & 78.7 & 83.5 & 75.4 & 109 & 111 & 97.2 & 91.8 & 88.5 \\
\hline MAX & 158 & 157 & 157 & 155 & 151 & 147 & 146 & 156 & 163 & 160 & 155 & 152 \\
\hline (WY) & 1966 & 1966 & 1966 & 1984 & 1984 & 1980 & 1967 & 1980 & 1965 & 1965 & 1965 & 1965 \\
\hline MIN & 35.9 & 14.7 & 33.4 & 18.2 & 11.4 & 15.6 & 11.3 & 27.2 & 46.9 & 46.1 & 41.7 & 38.0 \\
\hline (WY) & 1978 & 1995 & 1978 & 1997 & 1997 & 1997 & 1979 & 1977 & 1977 & 1977 & 1977 & 1977 \\
\hline
\end{tabular}

SUMMARY STATISTICS

ANNUAL TOTAL

ANNUAL MEAN

HIGHEST ANNUAL MEAN

LOWEST ANNUAL MEAN

HIGHEST DAILY MEAN

LOWEST DAILY MEAN

ANNUAL SEVEN-DAY MINIMUM

ANNUAL RUNOFF (AC-FT)

ANNUAL DISCHARGE (AC-FT) a

10 PERCENT EXCEEDS

50 PERCENT EXCEEDS

90 PERCENT EXCEEDS
FOR 1998 CALENDAR YEAR

31270.5

$$
85.7
$$

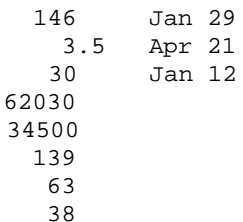

FOR 1999 WATER YEAR

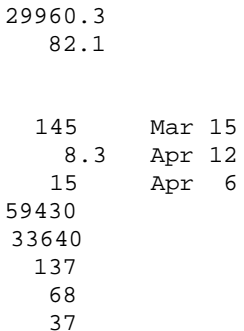

WATER YEARS 1965 - 1999

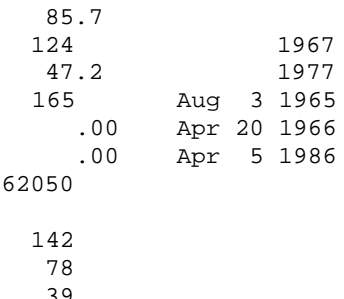

e Estimated.

a Discharge, in acre-feet, to Deer Creek Powerplant, provided by Pacific Gas \& Electric Co. 
LOCATION.-Lat 39¹9'28", long 120³8'42", in NE 1/4 SE 1/4 sec.20, T.17 N., R.12 E., Nevada County, Hydrologic Unit 18020125, on left bank $200 \mathrm{ft}$ downstream from Spaulding No. 2 Powerplant, $0.2 \mathrm{mi}$ downstream from Spaulding Dam, and 2.3 mi northeast of Emigrant Gap. DRAINAGE AREA.-118 $\mathrm{mi}^{2}$.

PERIOD OF RECORD.-October 1985 to current year. Unpublished records for water years 1965-85 in files of the U.S. Geological Survey.

GAGE.-Water-stage recorder and V-notch sharp-crested weir and steel-lipped rectangular weir. Elevation of gage is 4,670 ft above sea level, from topographic map. Prior to June 1988, at same site and different datum.

REMARKS.-Flow regulated by Lake Spaulding (station 11414140) 0.2 mi upstream. Water is released at the intake to South Yuba Canal (station 11414200) $100 \mathrm{ft}$ upstream. See schematic diagrams of Yuba and Bear River Basins.

COOPERATION.-Records were collected by Pacific Gas \& Electric Co., under general supervision of the U.S. Geological Survey, in connection with a Federal Energy Regulatory Commission project.

EXTREMES FOR PERIOD OF RECORD.-Maximum discharge, $194 \mathrm{ft}^{3} / \mathrm{s}$, Apr. 14, June 8, 1986, gage height, $3.37 \mathrm{ft}$, from rating curve extended above $45 \mathrm{ft}^{3} / \mathrm{s}$, on basis of weir formula; minimum daily, $0.09 \mathrm{ft}^{3} / \mathrm{s}$, Nov. 5-7, 1985 .

DISCHARGE, CUBIC FEET PER SECOND, WATER YEAR OCTOBER 1998 TO SEPTEMBER 1999 DAILY MEAN VALUES

\begin{tabular}{|c|c|c|c|c|c|c|c|c|c|c|c|c|}
\hline DAY & $\mathrm{OCT}$ & NOV & $\mathrm{DEC}$ & JAN & FEB & MAR & APR & MAY & JUN & JUL & AUG & SEP \\
\hline 1 & 5.6 & 6.2 & 1.8 & 2.0 & 1.7 & 22 & 3.3 & 1.8 & 41 & 2.4 & 6.1 & 7.2 \\
\hline 2 & 5.6 & 6.2 & 1.8 & 2.3 & 1.8 & 23 & 5.0 & 1.9 & 41 & 2.6 & 5.9 & 7.2 \\
\hline 3 & 5.6 & 6.1 & 4.2 & 2.7 & 1.9 & 25 & 4.9 & 4.5 & 41 & 2.6 & 5.9 & 7.2 \\
\hline 4 & 5.6 & 6.0 & 1.4 & 2.7 & 1.9 & 23 & 3.6 & 6.6 & 41 & 2.6 & 5.9 & 7.2 \\
\hline 5 & 5.6 & 5.8 & 1.2 & 2.7 & 1.9 & 23 & 2.8 & 8.4 & 41 & 2.5 & 5.9 & 7.2 \\
\hline 6 & 5.6 & 6.2 & 1.2 & 2.7 & 1.9 & 23 & 2.8 & 11 & 42 & 2.4 & 5.9 & 7.2 \\
\hline 7 & 5.6 & 6.8 & 1.1 & 2.7 & 6.4 & 23 & 2.6 & 11 & 42 & 2.4 & 5.9 & 7.4 \\
\hline 8 & 5.6 & 6.0 & 1.6 & 2.9 & 4.8 & 23 & 2.5 & 11 & 42 & 2.3 & 5.9 & 7.4 \\
\hline 9 & 5.7 & 5.8 & 2.1 & 3.1 & 5.2 & 23 & 2.4 & 12 & 42 & 2.3 & 5.6 & 7.2 \\
\hline 10 & 5.6 & 5.7 & 2.1 & 3.1 & 2.0 & 20 & 2.4 & 11 & 42 & 3.7 & 6.0 & 7.2 \\
\hline 11 & 5.6 & 6.4 & 2.1 & 3.2 & 1.9 & 17 & 3.3 & 11 & 42 & 4.5 & 6.5 & 7.2 \\
\hline 12 & 5.8 & 6.3 & 2.1 & 3.3 & 1.9 & 15 & 3.5 & 11 & 43 & 4.4 & 6.5 & 7.2 \\
\hline 13 & 5.9 & 6.2 & 2.2 & 3.3 & 1.9 & 13 & 2.8 & 15 & 44 & 4.4 & 6.5 & 7.2 \\
\hline 14 & 6.0 & 6.3 & 2.2 & 3.3 & 2.0 & 8.9 & 2.6 & 18 & 44 & 4.5 & 6.5 & 7.2 \\
\hline 15 & 6.2 & 7.2 & 2.1 & 3.7 & 1.9 & 3.1 & 2.5 & 18 & 43 & 3.9 & 6.5 & 7.2 \\
\hline 16 & 6.1 & 6.9 & 2.1 & 3.5 & 3.7 & 2.7 & 2.5 & 18 & 43 & 3.4 & 6.5 & 7.2 \\
\hline 17 & 5.9 & 8.1 & 2.1 & 2.2 & 11 & 2.4 & 2.4 & 22 & 43 & 3.3 & 6.5 & 7.2 \\
\hline 18 & 5.9 & 8.1 & 2.1 & 3.7 & 20 & 2.3 & 2.3 & 27 & 43 & 3.4 & 6.5 & 7.2 \\
\hline 19 & 5.9 & 7.8 & 2.0 & 4.4 & 14 & 2.2 & 2.1 & 27 & 43 & 3.4 & 6.1 & 7.4 \\
\hline 20 & 6.2 & 7.8 & 2.0 & 4.2 & 2.9 & 2.3 & 1.9 & 27 & 43 & 3.4 & 6.2 & 7.6 \\
\hline 21 & 6.2 & 7.6 & 1.9 & 1.4 & 2.8 & 2.2 & 1.9 & 27 & 43 & 3.7 & 6.2 & 7.6 \\
\hline 22 & 6.2 & 7.6 & 1.9 & 2.3 & 2.8 & 2.0 & 1.7 & 28 & 43 & 4.0 & 6.6 & 7.6 \\
\hline 23 & 6.2 & 10 & 1.9 & 3.6 & 2.9 & 1.9 & 1.6 & 30 & 43 & 4.2 & 7.2 & 7.6 \\
\hline 24 & 6.3 & 7.6 & 1.9 & .97 & 2.9 & 1.9 & 1.7 & 31 & 43 & 5.2 & 7.2 & 7.6 \\
\hline 25 & 6.2 & 7.1 & 1.9 & .76 & 13 & 1.9 & 1.8 & 33 & 43 & 5.9 & 7.2 & 7.6 \\
\hline 26 & 6.2 & 6.7 & 1.9 & .71 & 26 & 1.9 & 2.0 & 51 & 43 & 5.9 & 7.2 & 7.6 \\
\hline 27 & 6.2 & 7.3 & 1.9 & .70 & 26 & 1.9 & 2.0 & 34 & 43 & 5.9 & 7.2 & 6.7 \\
\hline 28 & 6.2 & 5.1 & 1.9 & .67 & 23 & 1.7 & 2.0 & 34 & 21 & 5.9 & 7.1 & 5.3 \\
\hline 29 & 6.2 & 4.0 & 1.8 & .67 & --- & 1.7 & 2.0 & 41 & 2.3 & 6.2 & 7.2 & 5.3 \\
\hline 30 & 6.2 & 4.1 & 1.7 & .67 & --- & 1.9 & 1.9 & 41 & 2.1 & 6.2 & 7.2 & 5.4 \\
\hline 31 & 6.2 & --- & 1.8 & 1.0 & --- & 1.9 & --- & 41 & --- & 6.2 & 7.2 & --- \\
\hline TOTAL & 183.9 & 199.0 & 60.0 & 75.15 & 190.1 & 317.8 & 76.8 & 664.2 & 1172.4 & 123.7 & 200.8 & 213.3 \\
\hline MEAN & 5.93 & 6.63 & 1.94 & 2.42 & 6.79 & 10.3 & 2.56 & 21.4 & 39.1 & 3.99 & 6.48 & 7.11 \\
\hline MAX & 6.3 & 10 & 4.2 & 4.4 & 26 & 25 & 5.0 & 51 & 44 & 6.2 & 7.2 & 7.6 \\
\hline MIN & 5.6 & 4.0 & 1.1 & .67 & 1.7 & 1.7 & 1.6 & 1.8 & 2.1 & 2.3 & 5.6 & 5.3 \\
\hline $\mathrm{AC}-\mathrm{ET}$ & 365 & 395 & 119 & 149 & 377 & 630 & 152 & 1320 & 2330 & 245 & 398 & 423 \\
\hline
\end{tabular}

STATISTICS OF MONTHLY MEAN DATA FOR WATER YEARS 1986 - 1999, BY WATER YEAR (WY)

\begin{tabular}{|c|c|c|c|c|c|c|c|c|c|c|c|c|}
\hline MEAN & 4.37 & 4.25 & 4.39 & 4.27 & 10.5 & 17.0 & 23.7 & 25.3 & 25.8 & 6.89 & 4.68 & 4.75 \\
\hline MAX & 6.80 & 6.63 & 21.2 & 17.7 & 61.4 & 111 & 118 & 85.8 & 111 & 29.1 & 8.84 & 8.22 \\
\hline (WY) & 1998 & 1999 & 1997 & 1995 & 1986 & 1986 & 1986 & 1986 & 1986 & 1998 & 1997 & 1997 \\
\hline MIN & 1.50 & 1.52 & 1.72 & 1.70 & 2.13 & 1.95 & 2.05 & 1.75 & 1.71 & 1.71 & 1.55 & 1.58 \\
\hline (WY) & 1986 & 1986 & 1987 & 1989 & 1989 & 1988 & 1987 & 1987 & 1987 & 1986 & 1986 & 1987 \\
\hline
\end{tabular}

SUMMARY STATISTICS

ANNUAL TOTAL

ANNUAL MEAN

HIGHEST ANNUAL MEAN

LOWEST ANNUAL MEAN

HIGHEST DAILY MEAN

LOWEST DAILY MEAN

ANNUAL SEVEN-DAY MINIMUM

INSTANTANEOUS PEAK FLOW

INSTANTANEOUS PEAK STAGE

ANNUAL RUNOFF (AC-FT)

10 PERCENT EXCEEDS

50 PERCENT EXCEEDS

90 PERCENT EXCEEDS
FOR 1998 CALENDAR YEAR

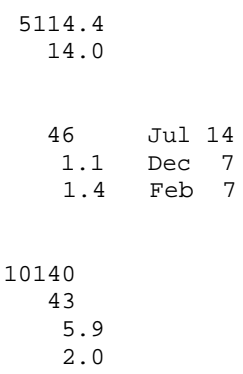

FOR 1999 WATER YEAR

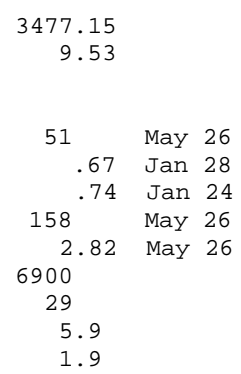

WATER YEARS 1986 - 1999

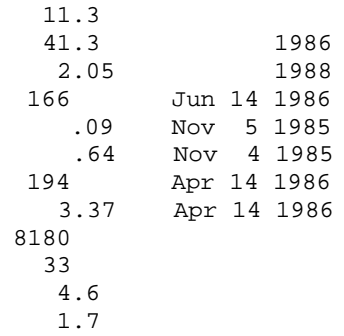


11414250 SOUTH YUBA RIVER AT LANGS CROSSING, NEAR EMIGRANT GAP, CA

LOCATION.-Lat 39¹9'07", long 120³9'24", in SW 1/4 SW 1/4 sec.20, T.17 N., R.12 E., Nevada County, Hydrologic Unit 18020125, on right bank $50 \mathrm{ft}$ downstream from road bridge, $0.8 \mathrm{mi}$ downstream from Spaulding Nos. 1 and 2 Powerplants, and 1.6 mi northeast of Emigrant Gap. DRAINAGE AREA.- $120 \mathrm{mi}^{2}$.

PERIOD OF RECORD.--December 1965 to current year.

GAGE.-Water-stage recorder. Datum of gage is 4,432.44 ft above sea level (levels by Pacific Gas \& Electric Co.).

REMARKS.-Flow regulated by Lake Spaulding (station 11414140) 0.8 mi upstream. Lake Spaulding receives water from Canyon Creek via the Bowman-Spaulding Canal (station 11416100). Most of the water is diverted out of the Yuba River just downstream from Spaulding Dam via Drum Canal (station 11414170) and South Yuba Canal (station 11414200). See schematic diagrams of Yuba and Bear River Basins.

COOPERATION.-Records were collected by Pacific Gas \& Electric Co., under general supervision of the U.S. Geological Survey, in connection with a Federal Energy Regulatory Commission project.

EXTREMES FOR PERIOD OF RECORD.-Maximum discharge, about 34,200 ft $\mathrm{ft}^{3} / \mathrm{s}$, Jan. 1, 1997, gage height, $23.60 \mathrm{ft}$, from rating curve extended above $8,800 \mathrm{ft}^{3} / \mathrm{s}$ on basis of spillway rating at Spaulding Dam; minimum daily, $2.1 \mathrm{ft}^{3} / \mathrm{s}$, on several days during July and September 1977.

DISCHARGE, CUBIC FEET PER SECOND, WATER YEAR OCTOBER 1998 TO SEPTEMBER 1999 DAILY MEAN VALUES

\begin{tabular}{|c|c|c|c|c|c|c|c|c|c|c|c|c|}
\hline DAY & $\mathrm{OCT}$ & NOV & $\mathrm{DEC}$ & JAN & FEB & MAR & APR & MAY & JUN & JUL & AUG & SEP \\
\hline 1 & 6.6 & 8.3 & 27 & 5.3 & 9.1 & 60 & 11 & 7.0 & e1020 & 6.9 & 5.5 & 7.0 \\
\hline 2 & 6.5 & 7.4 & 20 & 5.3 & 8.9 & 44 & 12 & 8.9 & e728 & 7.4 & 5.5 & 7.1 \\
\hline 3 & 6.5 & 7.1 & 56 & 5.7 & 8.9 & 67 & 12 & 19 & e 401 & 7.2 & 5.6 & 7.1 \\
\hline 4 & 6.4 & 6.9 & 23 & 5.6 & 9.2 & 42 & 11 & 16 & e274 & 6.9 & 5.6 & 7.0 \\
\hline 5 & 6.2 & 7.0 & 15 & 5.4 & 9.0 & 35 & 11 & 15 & e274 & 6.5 & 5.7 & 7.1 \\
\hline 6 & 6.2 & 7.3 & 12 & 5.3 & 9.6 & 32 & 11 & 16 & e229 & 6.2 & 5.8 & 7.0 \\
\hline 7 & 6.3 & 14 & 11 & 5.2 & 75 & 30 & 11 & 16 & e247 & 6.0 & 5.8 & 6.8 \\
\hline 8 & 6.3 & 11 & 10 & 5.2 & 68 & 29 & 11 & 16 & e253 & 5.7 & 5.8 & 6.8 \\
\hline 9 & 6.4 & 9.0 & 10 & 5.6 & 121 & 28 & 11 & 16 & e259 & 5.5 & 5.6 & 6.8 \\
\hline 10 & 6.2 & 8.6 & 9.4 & 5.6 & 34 & 26 & 10 & 15 & e260 & 6.4 & 6.2 & 6.7 \\
\hline 11 & 6.3 & 9.1 & 9.3 & 5.6 & 22 & 23 & 14 & 15 & e268 & 12 & 6.5 & 6.6 \\
\hline 12 & 6.7 & 8.3 & 9.5 & 5.6 & 17 & 22 & 18 & 15 & e 558 & 29 & 6.4 & 6.5 \\
\hline 13 & 6.8 & 7.9 & 10 & 5.6 & 15 & 21 & 21 & 17 & e 568 & 30 & 6.2 & 6.4 \\
\hline 14 & 6.8 & 7.7 & 10 & 5.7 & 15 & 18 & 22 & 19 & e383 & 28 & 6.2 & 6.4 \\
\hline 15 & 6.7 & 7.4 & 9.8 & 7.5 & 13 & 13 & 22 & 19 & e771 & 27 & 6.0 & 6.2 \\
\hline 16 & 6.6 & 7.3 & 9.7 & 12 & 24 & 12 & 20 & 18 & e 800 & 26 & 6.0 & 6.2 \\
\hline 17 & 6.3 & 14 & 9.1 & 13 & 57 & 12 & 17 & 22 & e370 & 26 & 6.0 & 6.2 \\
\hline 18 & 6.3 & 10 & 8.4 & 49 & 48 & 12 & 15 & 26 & e 633 & 25 & 6.0 & 7.9 \\
\hline 19 & 6.9 & 9.2 & 7.9 & 62 & 36 & 12 & 14 & 26 & e 633 & 19 & 5.7 & 8.8 \\
\hline 20 & 6.9 & 8.4 & 7.6 & 92 & 20 & 13 & 14 & 26 & e370 & 6.8 & 5.9 & 8.8 \\
\hline 21 & 6.9 & 9.3 & 7.0 & 40 & 18 & 12 & 12 & 28 & e 503 & 5.5 & 5.9 & 8.6 \\
\hline 22 & 6.9 & 16 & 6.6 & 30 & 16 & 12 & 11 & 30 & e463 & 5.4 & 6.0 & 8.4 \\
\hline 23 & 6.9 & 38 & 6.2 & 76 & 16 & 11 & 9.6 & 32 & e311 & 5.1 & 6.5 & 8.1 \\
\hline 24 & 9.2 & 25 & 5.9 & 26 & 16 & 11 & 9.3 & 35 & e417 & 5.5 & 6.4 & 8.1 \\
\hline 25 & 8.5 & 15 & 5.8 & 18 & 26 & 11 & 9.1 & 37 & e368 & 6.1 & 6.3 & 8.1 \\
\hline 26 & 12 & 12 & 5.8 & 15 & 33 & 11 & 12 & 61 & e188 & 5.9 & 6.3 & 8.0 \\
\hline 27 & 9.6 & 14 & 5.6 & 13 & 34 & 11 & 11 & 380 & e 91 & 5.6 & 6.4 & 7.3 \\
\hline 28 & 8.2 & 14 & 5.6 & 11 & 48 & 10 & 8.9 & 1120 & e21 & 5.5 & 6.4 & 5.1 \\
\hline 29 & 8.0 & 26 & 5.3 & 10 & --- & 9.6 & 8.0 & 1630 & e 8.2 & 5.6 & 7.0 & 5.2 \\
\hline 30 & 7.5 & 57 & 5.1 & 9.3 & --- & 10 & 7.3 & 1580 & e 6.8 & 5.5 & 7.0 & 5.4 \\
\hline 31 & 7.4 & --- & 5.2 & 9.0 & --- & 11 & --- & 1710 & --- & 5.5 & 7.0 & --- \\
\hline TOTAL & 221.0 & 402.2 & 348.8 & 569.5 & 826.7 & 670.6 & 386.2 & 6990.9 & 11676.0 & 354.7 & 189.2 & 211.7 \\
\hline MEAN & 7.13 & 13.4 & 11.3 & 18.4 & 29.5 & 21.6 & 12.9 & 226 & 389 & 11.4 & 6.10 & 7.06 \\
\hline MAX & 12 & 57 & 56 & 92 & 121 & 67 & 22 & 1710 & 1020 & 30 & 7.0 & 8.8 \\
\hline MIN & 6.2 & 6.9 & 5.1 & 5.2 & 8.9 & 9.6 & 7.3 & 7.0 & 6.8 & 5.1 & 5.5 & 5.1 \\
\hline$A C-F T$ & 438 & 798 & 692 & 1130 & 1640 & 1330 & 766 & 13870 & 23160 & 704 & 375 & 420 \\
\hline
\end{tabular}


11414250 SOUTH YUBA RIVER AT LANGS CROSSING, NEAR EMIGRANT GAP, CA—Continued

STATISTICS OF MONTHLY MEAN DATA FOR WATER YEARS 1966 - 1999, BY WATER YEAR (WY)

\begin{tabular}{|c|c|c|c|c|c|c|c|c|c|c|c|c|}
\hline MEAN & 7.14 & 42.4 & 49.7 & 123 & 95.4 & 91.0 & 87.1 & 328 & 442 & 71.4 & 6.12 & 6.41 \\
\hline MAX & 18.8 & 683 & 685 & 2465 & 1626 & 1304 & 620 & 1734 & 2613 & 822 & 9.44 & 10.3 \\
\hline (WY) & 1972 & 1984 & 1982 & 1997 & 1986 & 1986 & 1982 & 1996 & 1983 & 1983 & 1971 & 1986 \\
\hline MIN & 2.68 & 4.51 & 5.44 & 4.51 & 5.58 & 5.10 & 3.41 & 5.29 & 3.05 & 2.34 & 2.43 & 2.73 \\
\hline (WY) & 1978 & 1978 & 1977 & 1976 & 1977 & 1977 & 1977 & 1977 & 1977 & 1977 & 1977 & 1977 \\
\hline
\end{tabular}

SUMMARY STATISTICS

ANNUAL TOTAL

ANNUAL MEAN

HIGHEST ANNUAL MEAN

LOWEST ANNUAL MEAN

HIGHEST DAILY MEAN

LOWEST DAILY MEAN

ANNUAL SEVEN-DAY MINIMUM

INSTANTANEOUS PEAK FLOW

INSTANTANEOUS PEAK STAGE

ANNUAL RUNOFF (AC-FT)

10 PERCENT EXCEEDS

50 PERCENT EXCEEDS

90 PERCENT EXCEEDS
FOR 1998 CALENDAR YEAR

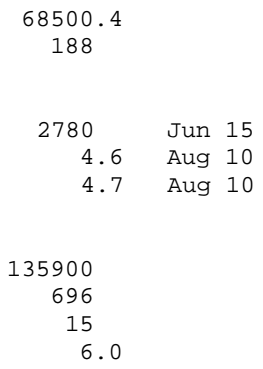

FOR 1999 WATER YEAR

\begin{tabular}{|c|c|}
\hline $\begin{array}{r}22847.5 \\
62.6\end{array}$ & \\
\hline 1710 & May 31 \\
\hline 5.1 & Dec 30 \\
\hline 5.3 & Dec 27 \\
\hline 1830 & May 31 \\
\hline 8.24 & May 31 \\
\hline 45320 & \\
\hline 71 & \\
\hline 9.7 & \\
\hline 5.7 & \\
\hline
\end{tabular}

WATER YEARS 1966 - 1999

$\begin{array}{ccrr}115 & & & \\ 448 & & & 1997 \\ 4.35 & & & 1977 \\ 25400 & \text { Jan } 1 & 1997 \\ 2.1 & \text { Jul } 15 & 1977 \\ 2.1 & \text { Sep } 22 & 1977 \\ 34200 & \text { Jan } & 1 & 1997 \\ 23.60 & \text { Jan } 1 & 1997 \\ 83470 & & & \\ 96 & & & \\ 7.6 & & & \\ 5.3 & & & \end{array}$


11414360 LAKE CREEK BELOW CARR LAKE, NEAR GRANITEVILLE, CA

LOCATION.-Lat 39²3'57", long 120³8'31", in SE 1/4 NE 1/4 sec.29, T.18 N., R.12 E., Nevada County, Hydrologic Unit 18020125, Tahoe National Forest, on right bank $65 \mathrm{ft}$ downstream from Carr Lake, $2.0 \mathrm{mi}$ upstream from Fall Creek, and $5.8 \mathrm{mi}$ southeast of Graniteville.

DRAINAGE AREA. $-0.48 \mathrm{mi}^{2}$.

PERIOD OF RECORD.-OCtober 1995 to current year. Unpublished records for water years 1965-95 available in files of the U.S. Geological Survey.

GAGE.-Water-stage recorder and compound rectangular weir. Elevation of gage is 6,650 ft above sea level (levels by Pacific Gas \& Electric Co). August 1965 to November 1975, nonrecording gage at site $65 \mathrm{ft}$ upstream at different datum. November 1975 to July 1984, nonrecording gage at same site but different datum. July 1984 to September 1995, nonrecording gage at same site and datum.

REMARKS.-Records not computed for winter months. Flow regulated by Carr Lake. See schematic diagram of Yuba River Basin.

COOPERATION.-Records were collected by Pacific Gas \& Electric Co., under general supervision of the U.S. Geological Survey, in connection with a Federal Energy Regulatory Commission project.

DISCHARGE, CUBIC FEET PER SECOND, WATER YEAR OCTOBER 1998 TO SEPTEMBER 1999

DAILY MEAN VALUES

\begin{tabular}{|c|c|c|c|c|c|c|c|c|c|c|c|c|}
\hline DAY & OCT & NOV & DEC & JAN & FEB & MAR & APR & MAY & JUN & JUL & AUG & SEP \\
\hline 1 & .64 & 10 & --- & --- & --- & --- & --- & --- & --- & 2.4 & e.35 & e. 94 \\
\hline 2 & .64 & 10 & --- & --- & --- & --- & --- & --- & --- & 2.1 & e.41 & e. 64 \\
\hline 3 & .64 & 10 & --- & --- & --- & --- & --- & --- & --- & 1.5 & e.41 & e. 64 \\
\hline 4 & .60 & 10 & --- & --- & --- & --- & --- & --- & --- & 1.3 & e.41 & e. 73 \\
\hline 5 & .95 & 10 & --- & --- & --- & --- & --- & --- & --- & 1.3 & e.41 & e. 73 \\
\hline 6 & 1.6 & 9.8 & --- & --- & --- & --- & --- & --- & --- & 1.3 & e.48 & e.73 \\
\hline 7 & 1.5 & 9.7 & --- & --- & --- & --- & --- & --- & --- & 1.3 & e.41 & e.73 \\
\hline 8 & 1.5 & 9.6 & --- & --- & --- & --- & --- & --- & --- & 1.3 & e.41 & e.73 \\
\hline 9 & 1.5 & 9.2 & --- & --- & --- & --- & --- & --- & --- & 1.3 & e.41 & e.73 \\
\hline 10 & 1.5 & 9.2 & --- & --- & --- & --- & --- & --- & --- & 1.3 & e.41 & e1.1 \\
\hline 11 & 1.5 & 9.0 & --- & --- & --- & --- & --- & --- & --- & 1.2 & e.41 & e1.2 \\
\hline 12 & 1.5 & 8.6 & --- & --- & --- & --- & --- & --- & --- & 1.3 & e.41 & e2.0 \\
\hline 13 & 1.3 & --- & --- & --- & --- & --- & --- & --- & --- & 1.4 & e.41 & e3.1 \\
\hline 14 & 1.3 & --- & --- & --- & --- & --- & --- & --- & --- & 1.2 & e.41 & e 4.0 \\
\hline 15 & 1.3 & --- & --- & --- & -- & --- & --- & --- & --- & .69 & e.41 & e3.1 \\
\hline 16 & 1.1 & --- & --- & --- & --- & --- & --- & --- & --- & .40 & e.41 & e2.6 \\
\hline 17 & 2.7 & --- & --- & --- & --- & --- & --- & --- & 7.4 & .31 & e.41 & e2.2 \\
\hline 18 & 5.2 & --- & --- & --- & --- & --- & --- & --- & 6.9 & .36 & e.41 & el.8 \\
\hline 19 & 5.3 & --- & --- & --- & --- & --- & --- & --- & 6.5 & .50 & e.41 & el. 5 \\
\hline 20 & 5.3 & --- & --- & --- & --- & --- & --- & --- & 6.0 & .83 & e.41 & e2.2 \\
\hline 21 & 5.4 & --- & --- & --- & --- & --- & --- & --- & 5.5 & .94 & e.41 & e2.9 \\
\hline 22 & 5.5 & --- & --- & --- & --- & --- & --- & --- & 5.2 & .85 & e.41 & e2.4 \\
\hline 23 & 5.4 & --- & --- & --- & --- & --- & --- & --- & 5.5 & .79 & e.41 & e2.0 \\
\hline 24 & 5.8 & --- & --- & --- & --- & --- & --- & --- & 5.8 & .57 & e.41 & e1.6 \\
\hline 25 & 5.8 & --- & --- & --- & -- & --- & --- & --- & 5.1 & .51 & e.41 & $\mathrm{e} 1.6$ \\
\hline 26 & 5.8 & --- & --- & --- & --- & --- & --- & --- & 4.2 & .48 & e.41 & e1.6 \\
\hline 27 & 6.0 & --- & --- & --- & --- & --- & --- & --- & 3.1 & .44 & e.41 & e1.6 \\
\hline 28 & 7.6 & --- & --- & --- & --- & --- & --- & --- & 2.6 & .37 & e.41 & e1.6 \\
\hline 29 & 8.9 & --- & --- & --- & --- & --- & --- & --- & 2.4 & .34 & e.56 & e2.6 \\
\hline 30 & 7.1 & --- & --- & --- & --- & --- & --- & --- & 2.2 & .30 & e.73 & e4.0 \\
\hline 31 & 8.2 & --- & --- & --- & --- & --- & --- & --- & --- & .30 & e.83 & --- \\
\hline TOTAL & 109.07 & --- & --- & --- & --- & --- & --- & --- & --- & 29.18 & 13.61 & 53.30 \\
\hline MEAN & 3.52 & --- & --- & --- & --- & --- & --- & --- & --- & .94 & .44 & 1.78 \\
\hline MAX & 8.9 & --- & --- & --- & --- & --- & --- & --- & --- & 2.4 & .83 & 4.0 \\
\hline MIN & .60 & --- & --- & --- & --- & --- & --- & --- & --- & .30 & .35 & .64 \\
\hline $\mathrm{AC}-\mathrm{FT}$ & 216 & --- & --- & --- & --- & --- & --- & --- & --- & 58 & 27 & 106 \\
\hline
\end{tabular}


LOCATION.-Lat 39²5'16", long 120³2'28", in SE 1/4 SW 1/4 sec.17, T.18 N., R.13 E., Nevada County, Hydrologic Unit 18020125, Tahoe National Forest, on right bank near French Lake Dam on Canyon Creek, 0.5 mi upstream from Weil Lake, and 8.2 mi north of Cisco.

DRAINAGE AREA. $-4.60 \mathrm{mi}^{2}$.

PERIOD OF RECORD.-October 1986 to current year. Unpublished records for water years 1966-86 available in the files of the U.S. Geological Survey.

GAGE.-Water-stage recorder. Datum of gage is sea level (levels by Nevada Irrigation District).

REMARKS.--Reservoir is formed on natural lake by rock-filled dam completed in 1859. Usable capacity, 13,940 acre-ft between elevations $6,594.90 \mathrm{ft}$, invert of outlet gate, and 6,660.28 ft, crest of spillway. Figures given represent usable contents. Released water is used for hydroelectric power and irrigation downstream. See schematic diagram of Yuba River Basin.

COOPERATION.-Records were collected by Nevada Irrigation District, under general supervision of the U.S. Geological Survey, in connection with a Federal Energy Regulatory Commission project.

EXTREMES FOR PERIOD OF RECORD.-Maximum contents, 14,300 acre-ft, June 19, 1998, elevation, $6661.26 \mathrm{ft}$; minimum, 6140 acre-ft, Nov. 16, 1998, elevation, $6632.07 \mathrm{ft}$.

EXTREMES FOR CURRENT YEAR.-Maximum contents, 14,200 acre-ft, many days, maximum elevation, 6661.17, June 16; minimum, 6,140 acre-ft, Nov. 16, elevation, $6632.07 \mathrm{ft}$.

Capacity table (elevation, in feet, and contents, in acre-feet) (Based on survey by Nevada Irrigation District in 1964)

$\begin{array}{llrr}6,610 & 1,805 & 6,640 & 8,006 \\ 6,620 & 3,636 & 6,650 & 10,701 \\ 6,630 & 5,677 & 6,662 & 14,542\end{array}$

RESERVOIR STORAGE (ACRE-FEET), WATER YEAR OCTOBER 1998 TO SEPTEMBER 1999 DAILY OBSERVATION AT 2400 HOURS

\begin{tabular}{|c|c|c|c|c|c|c|c|c|c|c|c|c|}
\hline DAY & $\mathrm{OCT}$ & NOV & $\mathrm{DEC}$ & JAN & FEB & MAR & APR & MAY & JUN & JUL & AUG & SEP \\
\hline 1 & 6450 & 6190 & 6690 & 7120 & 7830 & 8480 & 8880 & 10400 & 14200 & e14000 & 13500 & 9710 \\
\hline 2 & 6440 & 6190 & 6720 & 7120 & 7830 & 8530 & 8880 & 10500 & 14100 & e14000 & 13400 & 9560 \\
\hline 3 & 6430 & 6180 & 6910 & 7120 & 7830 & 8580 & 8890 & 10600 & 14100 & e14000 & 13300 & 9410 \\
\hline 4 & 6410 & 6170 & 6950 & 7120 & 7840 & 8590 & 8890 & 10700 & 14000 & e14000 & 13300 & 9260 \\
\hline 5 & 6410 & 6160 & 6970 & 7120 & 7840 & 8600 & 8920 & 10700 & 14100 & e14000 & 13200 & 9130 \\
\hline 6 & 6400 & 6160 & 6990 & 7120 & 7890 & 8600 & 8930 & 10800 & 14200 & e14000 & 13100 & 8990 \\
\hline 7 & 6390 & 6200 & 6990 & 7120 & 7960 & 8600 & 8930 & 11000 & 14100 & e14000 & 13000 & 8850 \\
\hline 8 & 6370 & 6180 & 7000 & 7110 & 8040 & 8620 & 8980 & 11100 & 14100 & e14000 & 12900 & 8710 \\
\hline 9 & 6360 & 6180 & 7000 & 7110 & 8130 & 8660 & 8980 & 11200 & 14100 & e14000 & 12800 & 8570 \\
\hline 10 & 6350 & 6190 & 7000 & 7110 & 8130 & 8660 & 8980 & 11300 & 14100 & e14000 & 12700 & 8430 \\
\hline 11 & 6340 & 6180 & 7010 & 7110 & 8130 & 8660 & 8980 & 11400 & 14200 & e14000 & 12600 & 8290 \\
\hline 12 & 6330 & 6170 & 7010 & 7100 & 8130 & 8660 & 8990 & 11700 & 14200 & e14000 & 12500 & 8150 \\
\hline 13 & 6320 & 6170 & 7020 & 7100 & 8130 & 8650 & 9010 & 11800 & 14200 & 14000 & 12400 & 8010 \\
\hline 14 & 6310 & 6160 & 7030 & 7110 & 8140 & 8660 & 9050 & 11900 & 14200 & 14000 & 12200 & 7890 \\
\hline 15 & 6300 & 6160 & 7030 & 7150 & 8140 & 8660 & 9090 & 12000 & e14200 & 14000 & 12200 & 7810 \\
\hline 16 & 6280 & 6140 & 7050 & 7200 & 8190 & 8660 & 9140 & 12100 & e 14200 & 14000 & 12100 & 7800 \\
\hline 17 & 6280 & 6180 & 7070 & 7270 & 8210 & 8670 & 9220 & 12200 & e14200 & 14000 & 12000 & 7800 \\
\hline 18 & 6270 & 6180 & 7080 & 7490 & 8230 & 8680 & 9320 & 12400 & e14200 & 13900 & 11900 & 7790 \\
\hline 19 & 6250 & 6170 & 7100 & 7600 & 8230 & 8700 & 9450 & 12700 & e14200 & 13900 & 11700 & 7780 \\
\hline 20 & 6250 & 6170 & 7120 & 7670 & 8250 & 8710 & 9580 & 12900 & e 14200 & 13900 & 11600 & 7770 \\
\hline 21 & 6230 & 6180 & 7120 & 7700 & 8280 & 8720 & 9680 & 13100 & e14200 & 13900 & 11400 & 7760 \\
\hline 22 & 6220 & 6190 & 7120 & 7740 & 8270 & 8730 & 9750 & 13400 & e14100 & 13900 & 11200 & 7760 \\
\hline 23 & 6210 & 6310 & 7130 & 7790 & 8270 & 8740 & 9790 & 13700 & e14100 & 13900 & 11100 & 7750 \\
\hline 24 & 6230 & 6340 & 7130 & 7800 & 8270 & 8740 & 9870 & 14000 & e14100 & 13900 & 10900 & 7740 \\
\hline 25 & 6230 & 6360 & 7120 & 7800 & 8300 & 8740 & 9980 & 14200 & e14100 & 13800 & 10800 & 7730 \\
\hline 26 & 6220 & 6350 & 7120 & 7810 & 8300 & 8760 & 10100 & 14200 & e14100 & 13800 & 10600 & 7720 \\
\hline 27 & 6210 & 6370 & 7120 & 7810 & 8300 & 8790 & 10200 & 14200 & e14100 & 13800 & 10500 & 7710 \\
\hline 28 & 6200 & 6370 & 7120 & 7820 & 8380 & 8810 & 10300 & 14200 & e14100 & 13800 & 10300 & 7700 \\
\hline 29 & 6210 & 6420 & 7120 & 7810 & --- & 8830 & 10300 & 14100 & e14100 & 13700 & 10200 & 7700 \\
\hline 30 & 6190 & 6640 & 7110 & 7820 & --- & 8860 & 10300 & 14200 & e14000 & 13600 & 10000 & 7690 \\
\hline 31 & 6190 & --- & 7120 & 7830 & --- & 8870 & --- & 14200 & --- & 13600 & 9860 & --- \\
\hline MAX & 6450 & 6640 & 7130 & 7830 & 8380 & 8870 & 10300 & 14200 & 14200 & 14000 & 13500 & 9710 \\
\hline MIN & 6190 & 6140 & 6690 & 7100 & 7830 & 8480 & 8880 & 10400 & 14000 & 13600 & 9860 & 7690 \\
\hline $\mathrm{a}$ & 6632.26 & 6634.28 & 6636.29 & 6639.27 & 6641.44 & 6643.34 & 6648.64 & 6660.95 & & 6659.14 & 6647.01 & 6638.67 \\
\hline $\mathrm{b}$ & -270 & +450 & +480 & +710 & +550 & +490 & +1430 & +3900 & -200 & -400 & -3740 & -2170 \\
\hline
\end{tabular}

WTR YR 1999 MAX 14200 MIN $6140 \quad$ b +1230

e Estimated.

a Elevation, in feet, at end of month.

b Change in contents, in acre-feet. 
11414410 CANYON CREEK BELOW FRENCH LAKE, NEAR CISCO, CA

LOCATION.-Lat 39²5'16", long 120³2'30", in SE 1/4 SW 1/4 sec.17, T.18 N., R.13 E., Nevada County, Hydrologic Unit 18020125, Tahoe National Forest, on left bank $10 \mathrm{ft}$ downstream from outlet at French Lake Dam on Canyon Creek, $0.5 \mathrm{mi}$ upstream from Weil Lake, and $8.2 \mathrm{mi}$ north of Cisco.

DRAINAGE AREA.- $-4.60 \mathrm{mi}^{2}$.

PERIOD OF RECORD._-January 1989 to current year (low-flow records only). Unpublished records for water years 1967-88 available in files of the U.S. Geological Survey.

GAGE.-Water-stage recorder and V-notch sharp-crested weir. Elevation of gage is 6,590 ft above sea level, from topographic map. Prior to January 1989, nonrecording gages at three sites and datums.

REMARKS.-No records computed above $3.2 \mathrm{ft}^{3} / \mathrm{s}$. Flow regulated by French Lake (station 11414400). Flow over the spillway bypasses this station. See schematic diagram of Yuba River Basin.

COOPERATION.—Records were collected by Nevada Irrigation District, under general supervision of the U.S. Geological Survey, in connection with a Federal Energy Regulatory Commission project.

DISCHARGE, CUBIC FEET PER SECOND, WATER YEAR OCTOBER 1998 TO SEPTEMBER 1999

DAILY MEAN VALUES

\begin{tabular}{|c|c|c|c|c|c|c|c|c|c|c|c|c|}
\hline DAY & OCT & NOV & $\mathrm{DEC}$ & JAN & $\mathrm{FEB}$ & MAR & $A P R$ & MAY & JUN & JUL & AUG & SEP \\
\hline 1 & --- & --- & --- & --- & --- & --- & --- & --- & --- & --- & --- & --- \\
\hline 2 & --- & --- & --- & --- & --- & --- & --- & --- & --- & --- & --- & --- \\
\hline 3 & --- & --- & --- & --- & --- & --- & --- & --- & --- & --- & --- & --- \\
\hline 4 & --- & --- & --- & --- & --- & --- & --- & --- & --- & --- & --- & --- \\
\hline 5 & --- & --- & --- & --- & --- & --- & --- & --- & --- & --- & --- & --- \\
\hline 6 & --- & --- & --- & --- & --- & --- & --- & --- & --- & --- & --- & --- \\
\hline 7 & --- & --- & --- & --- & --- & --- & --- & --- & --- & --- & --- & --- \\
\hline 8 & --- & --- & --- & --- & --- & --- & --- & --- & --- & --- & --- & --- \\
\hline 9 & --- & --- & --- & --- & --- & --- & --- & --- & --- & --- & --- & --- \\
\hline 10 & --- & --- & --- & --- & --- & --- & --- & --- & --- & --- & --- & --- \\
\hline 11 & --- & --- & --- & --- & --- & --- & --- & --- & --- & --- & --- & --- \\
\hline 12 & --- & --- & --- & --- & --- & --- & --- & --- & --- & --- & --- & --- \\
\hline 13 & --- & --- & --- & --- & --- & --- & --- & --- & --- & --- & --- & --- \\
\hline 14 & --- & --- & --- & --- & --- & --- & --- & --- & --- & --- & --- & --- \\
\hline 15 & --- & --- & --- & --- & --- & --- & --- & --- & --- & --- & --- & --- \\
\hline 16 & --- & --- & --- & --- & --- & --- & --- & --- & --- & --- & --- & --- \\
\hline 17 & --- & --- & --- & --- & --- & --- & --- & --- & --- & --- & --- & --- \\
\hline 18 & --- & --- & --- & --- & --- & --- & --- & --- & --- & --- & --- & --- \\
\hline 19 & --- & --- & --- & --- & --- & --- & --- & --- & --- & --- & --- & --- \\
\hline 20 & --- & --- & --- & --- & --- & --- & --- & --- & --- & --- & --- & --- \\
\hline 21 & --- & --- & --- & --- & --- & --- & --- & --- & --- & --- & --- & --- \\
\hline 22 & --- & --- & --- & --- & --- & --- & --- & --- & --- & --- & --- & --- \\
\hline 23 & --- & --- & --- & --- & --- & --- & --- & --- & --- & --- & --- & --- \\
\hline 24 & --- & --- & --- & --- & --- & --- & --- & --- & --- & --- & --- & --- \\
\hline 25 & --- & --- & --- & --- & --- & --- & --- & --- & --- & --- & --- & --- \\
\hline 26 & --- & --- & --- & --- & --- & --- & --- & --- & --- & --- & --- & --- \\
\hline 27 & --- & --- & --- & --- & --- & --- & --- & --- & --- & --- & --- & --- \\
\hline 28 & --- & --- & --- & --- & --- & --- & --- & --- & --- & --- & --- & --- \\
\hline 29 & --- & --- & --- & --- & --- & --- & --- & --- & --- & --- & --- & --- \\
\hline 30 & --- & --- & --- & --- & --- & --- & --- & --- & --- & --- & --- & --- \\
\hline 31 & --- & --- & --- & --- & --- & --- & --- & --- & --- & --- & --- & --- \\
\hline TOTAL & --- & --- & --- & --- & --- & --- & --- & --- & --- & --- & --- & --- \\
\hline MEAN & --- & --- & --- & --- & --- & --- & --- & --- & --- & --- & --- & --- \\
\hline MAX & --- & --- & --- & --- & --- & --- & --- & --- & --- & --- & --- & --- \\
\hline MIN & --- & --- & --- & --- & --- & --- & --- & --- & --- & --- & --- & --- \\
\hline $\mathrm{AC}-\mathrm{FT}$ & --- & --- & --- & --- & --- & --- & --- & --- & --- & --- & --- & --- \\
\hline
\end{tabular}




\section{FAUCHERIE LAKE NEAR CISCO, CA}

LOCATION.-Lat 39²5'45", long 120³4'04", in SE 1/4 NE 1/4 sec.13, T.18 N., R.12 E., Nevada County, Hydrologic Unit 18020125, Tahoe National Forest, near right bank end of Faucherie Dam on Canyon Creek, 8.5 mi north of Cisco.

DRAINAGE AREA.- $-8.97 \mathrm{mi}^{2}$.

PERIOD OF RECORD.-OCtober 1986 to current year. Unpublished records for water years 1965-86 available in files of the U.S. Geological Survey.

GAGE.-Staff gages, observed approximately weekly during the summer months. Datum of gage is sea level (levels by Nevada Irrigation District).

REMARKS.- Reservoir is formed on natural lake by earth-filled dam initially constructed prior to 1880 and enlarged in 1964. Usable capacity, 3,740 acre- $\mathrm{ft}$ between elevations 6,090.00 ft, invert of outlet gate, and 6,123.00 ft, crest of spillway. Dead storage, below elevation 6,090 ft, 240 acre-ft. Figures given represent total contents. Released water is used for hydroelectric power and irrigation downstream. See schematic diagram of Yuba River Basin.

COOPERATION.-Records were collected by Nevada Irrigation District, under general supervision of the U.S. Geological Survey, in connection with a Federal Energy Regulatory Commission project.

Capacity table (elevation, in feet, and contents, in acre-feet)

(Based on survey by Nevada Irrigation District in 1964)

$\begin{array}{rrrr}6,090 & 240 & 6,110 & 2,216 \\ 6,095 & 628 & 6,115 & 2,854 \\ 6,100 & 1,095 & 6,120 & 3,540 \\ 6,105 & 1,629 & 6,125 & 4,280\end{array}$

\section{RESERVOIR STORAGE (ACRE-FEET), WATER YEAR OCTOBER 1998 TO SEPTEMBER 1999} DAILY INSTANTANEOUS VALUES

\begin{tabular}{|c|c|c|c|c|c|c|c|c|c|c|c|c|}
\hline DAY & OCT & NOV & $\mathrm{DEC}$ & JAN & $\mathrm{FEB}$ & MAR & $\mathrm{APR}$ & MAY & JUN & JUL & AUG & SEP \\
\hline 1 & 3970 & --- & --- & --- & --- & --- & --- & --- & --- & --- & --- & --- \\
\hline 2 & --- & --- & --- & --- & --- & --- & --- & --- & --- & --- & --- & --- \\
\hline 3 & --- & --- & --- & --- & --- & --- & --- & --- & --- & --- & --- & --- \\
\hline 4 & --- & 3980 & --- & --- & --- & --- & --- & --- & --- & --- & --- & --- \\
\hline 5 & --- & --- & --- & --- & --- & --- & --- & --- & --- & --- & --- & --- \\
\hline 6 & --- & --- & --- & --- & --- & --- & --- & --- & --- & --- & --- & --- \\
\hline 7 & 3970 & --- & --- & --- & --- & --- & --- & --- & --- & --- & --- & --- \\
\hline 8 & 3970 & --- & --- & --- & --- & --- & --- & --- & --- & --- & --- & 3970 \\
\hline 9 & --- & --- & 4010 & --- & --- & --- & --- & --- & --- & --- & --- & --- \\
\hline 10 & --- & --- & --- & --- & --- & --- & --- & 4020 & --- & --- & --- & --- \\
\hline 11 & --- & --- & --- & --- & --- & --- & --- & --- & --- & --- & --- & --- \\
\hline 12 & --- & --- & --- & --- & --- & --- & --- & --- & --- & --- & --- & --- \\
\hline 13 & --- & --- & --- & --- & --- & --- & --- & --- & --- & --- & --- & --- \\
\hline 14 & --- & --- & --- & --- & --- & --- & --- & --- & --- & --- & --- & --- \\
\hline 15 & 3980 & --- & --- & --- & --- & --- & --- & --- & --- & --- & --- & --- \\
\hline 16 & --- & --- & --- & --- & --- & --- & --- & --- & --- & --- & --- & 3070 \\
\hline 17 & --- & --- & --- & --- & --- & --- & --- & --- & --- & --- & --- & --- \\
\hline 18 & --- & --- & --- & --- & --- & --- & --- & --- & --- & --- & --- & --- \\
\hline 19 & --- & --- & --- & --- & --- & --- & --- & --- & --- & --- & --- & --- \\
\hline 20 & --- & --- & --- & --- & --- & --- & --- & --- & --- & --- & --- & 2250 \\
\hline 21 & 3980 & --- & --- & --- & --- & --- & --- & --- & 4040 & --- & --- & --- \\
\hline 22 & --- & --- & --- & --- & --- & --- & --- & --- & --- & 3980 & --- & --- \\
\hline 23 & --- & --- & --- & --- & --- & --- & --- & --- & --- & --- & --- & 1660 \\
\hline 24 & --- & --- & --- & --- & --- & --- & --- & --- & --- & --- & --- & --- \\
\hline 25 & --- & --- & --- & --- & --- & --- & --- & --- & --- & --- & 4020 & --- \\
\hline 26 & --- & --- & --- & --- & --- & --- & --- & --- & --- & --- & --- & --- \\
\hline 27 & --- & --- & --- & --- & --- & --- & --- & --- & --- & --- & --- & --- \\
\hline 28 & --- & --- & --- & --- & --- & --- & --- & --- & --- & --- & --- & --- \\
\hline 29 & --- & --- & --- & --- & --- & --- & --- & --- & 3960 & 4000 & --- & --- \\
\hline 30 & --- & --- & --- & --- & --- & --- & --- & --- & --- & --- & --- & --- \\
\hline 31 & --- & --- & --- & --- & --- & --- & --- & --- & --- & --- & --- & --- \\
\hline MAX & --- & --- & --- & --- & --- & --- & --- & --- & --- & --- & --- & --- \\
\hline MIN & --- & --- & --- & --- & --- & --- & --- & --- & --- & --- & --- & --- \\
\hline
\end{tabular}


11414450 CANYON CREEK BELOW FAUCHERIE LAKE, NEAR CISCO, CA

LOCATION.-Lat 39²5'46", long 120³4'06", in SE 1/4 NE 1/4 sec.13, T.18 N., R.12 E., Nevada County, Hydrologic Unit 18020125, Tahoe National Forest, on left bank $80 \mathrm{ft}$ downstream from Faucherie Dam on Canyon Creek, 8.5 mi north of Cisco.

DRAINAGE AREA.- $-8.97 \mathrm{mi}^{2}$.

PERIOD OF RECORD._-January 1989 to current year (low-flow records only). Unpublished records for water years $1965-88$ available in files of the U.S. Geological Survey.

GAGE.-Water-stage recorder and V-notch sharp-crested weir. Elevation of gage is 6,080 ft above sea level, from topographic map. October 1964 to July 1988, nonrecording gage at site $10 \mathrm{ft}$ downstream at different datum. July 1988 to January 1989, nonrecording gage at same site and datum.

REMARKS.-No records computed above $3.2 \mathrm{ft}^{3} / \mathrm{s}$. Flow regulated by Faucherie Lake (station 11414440). Flow over the spillway bypasses this station. See schematic diagram of Yuba River Basin.

COOPERATION.- - Records were collected by Nevada Irrigation District, under general supervision of the U.S. Geological Survey, in connection with a Federal Energy Regulatory Commission project.

DISCHARGE, CUBIC FEET PER SECOND, WATER YEAR OCTOBER 1998 TO SEPTEMBER 1999

DAILY MEAN VALUES

\begin{tabular}{|c|c|c|c|c|c|c|c|c|c|c|c|c|}
\hline DAY & OCT & NOV & $\mathrm{DEC}$ & JAN & FEB & MAR & $\mathrm{APR}$ & MAY & JUN & JUL & AUG & SEP \\
\hline 1 & 3.0 & 2.9 & 2.9 & e2. 8 & 2.9 & 2.9 & e2.9 & e2.9 & e2.8 & 2.8 & 2.9 & 2.9 \\
\hline 2 & 3.0 & 2.9 & 2.9 & e2. 8 & 2.9 & 2.9 & e2.9 & e2. 9 & e2. 8 & 2.8 & 2.9 & 2.9 \\
\hline 3 & 3.0 & 2.9 & 3.0 & e2. 8 & 2.9 & 2.9 & e2. 9 & e2. 9 & e2.8 & 2.8 & 2.9 & 2.9 \\
\hline 4 & 3.0 & 2.9 & e3.0 & e2. 8 & 2.9 & 2.9 & e2.9 & e2. 9 & e2.8 & 2.8 & 2.9 & 2.9 \\
\hline 5 & 3.0 & 2.9 & e3.0 & e2. 8 & 2.9 & 2.9 & e2.9 & e2. 8 & $\mathrm{e} 2.8$ & 2.8 & 2.9 & 2.9 \\
\hline 6 & 3.0 & 2.9 & e 3.0 & 2.8 & 2.9 & 2.9 & e2.9 & e2. 8 & e2. 8 & 2.8 & 2.9 & 2.9 \\
\hline 7 & 3.0 & 2.9 & e2.9 & 2.8 & 2.9 & 2.9 & e2.9 & e2.8 & e2.8 & 2.8 & 2.9 & --- \\
\hline 8 & 3.0 & 2.9 & e2. 9 & 2.8 & 2.9 & 2.9 & e2. 9 & e2. 8 & e2. 8 & 2.8 & 2.9 & --- \\
\hline 9 & 3.0 & 2.9 & e2. 9 & 2.8 & 2.9 & 2.9 & e2.9 & e2. 8 & e2. 8 & 2.8 & 2.9 & --- \\
\hline 10 & 3.0 & 2.9 & e2.9 & 2.8 & 2.9 & 2.9 & e2.9 & e2. 8 & e2. 8 & 2.8 & 2.9 & --- \\
\hline 11 & 2.9 & 2.9 & 2.9 & 2.8 & 2.9 & e2.9 & e2.9 & e2. 8 & e2. 8 & 2.8 & 2.9 & --- \\
\hline 12 & 2.9 & 2.9 & 2.9 & 2.8 & 2.9 & e2. 9 & e2.9 & e2. 8 & e2.8 & 2.8 & 2.9 & --- \\
\hline 13 & 2.9 & 2.9 & 2.9 & 2.8 & 2.9 & e2.9 & e2.9 & e2.8 & e2.8 & 2.8 & 2.9 & --- \\
\hline 14 & 2.9 & 2.9 & 2.9 & 2.8 & 2.9 & e2.9 & e2.9 & e2. 8 & e2.8 & 2.8 & 2.9 & --- \\
\hline 15 & 2.9 & 2.9 & 2.9 & 2.9 & 2.9 & $\mathrm{e} 2.9$ & e2.9 & e2. 8 & e2. 8 & 2.8 & 2.9 & --- \\
\hline 16 & 2.9 & 2.9 & 2.9 & 2.9 & 2.9 & e2. 9 & e2.9 & e2. 8 & e2.8 & 2.8 & 2.9 & --- \\
\hline 17 & 2.9 & 2.9 & 2.9 & 2.9 & 2.9 & e2.9 & e2.9 & e2. 8 & e2. 8 & 2.8 & 2.9 & --- \\
\hline 18 & 2.9 & 2.9 & 2.9 & 2.9 & 2.9 & e2.9 & e2.9 & e2. 8 & e2. 8 & 2.8 & 2.9 & --- \\
\hline 19 & 2.9 & 2.9 & e2.9 & 2.9 & 2.9 & e2.9 & e2.9 & e2. 8 & e2. 8 & 2.8 & 2.9 & --- \\
\hline 20 & 2.9 & 2.9 & e2.9 & 2.9 & 2.9 & e2.9 & e2.9 & e2. 8 & e2. 8 & 2.8 & 2.9 & --- \\
\hline 21 & 2.9 & 2.9 & e2.9 & 2.9 & 2.9 & e2.9 & e2.9 & e2. 8 & e2. 8 & 2.8 & 2.9 & --- \\
\hline 22 & 2.9 & 2.9 & e2.9 & 2.9 & 2.9 & e2.9 & e2.9 & e2.8 & e2. 8 & 2.8 & 2.9 & --- \\
\hline 23 & 2.9 & 3.0 & e2. 9 & 2.9 & 2.9 & e2.9 & e2. 9 & e2. 8 & e2. 8 & 2.8 & 2.9 & --- \\
\hline 24 & 2.9 & 2.9 & e2.9 & 2.9 & 2.9 & e2.9 & e2.9 & e2.8 & e2.8 & 2.8 & 2.9 & e3.2 \\
\hline 25 & 2.9 & 2.9 & e2.9 & 2.9 & 2.9 & e2.9 & e2. 9 & e2. 8 & e2. 8 & 2.8 & 2.9 & e 3.2 \\
\hline 26 & 2.9 & 2.9 & e2.9 & 2.9 & 2.9 & e2.9 & e2.9 & e2. 8 & e2. 8 & 2.8 & 2.9 & e3.2 \\
\hline 27 & 2.9 & 2.9 & e2.9 & 2.9 & 2.9 & e2.9 & e2.9 & e2. 8 & e2.8 & 2.9 & 2.9 & --- \\
\hline 28 & 2.9 & 2.9 & e2. 8 & 2.9 & 2.9 & e2.9 & e2.9 & e2. 8 & e2. 8 & 2.9 & 2.9 & --- \\
\hline 29 & 2.9 & 2.9 & e2. 8 & 2.9 & --- & e2.9 & e2.9 & e2. 8 & e2. 8 & 2.9 & 2.9 & --- \\
\hline 30 & 2.9 & 3.0 & e2. 8 & 2.9 & --- & e2. 9 & e2.9 & e2. 8 & 2.8 & 2.9 & 2.9 & --- \\
\hline 31 & 2.9 & --- & e2. 8 & 2.9 & --- & e2.9 & --- & e2. 8 & --- & 2.9 & 2.9 & --- \\
\hline TOTAL & 90.9 & 87.2 & 89.9 & 88.5 & 81.2 & 89.9 & 87.0 & 87.2 & 84.0 & 87.3 & 89.9 & --- \\
\hline MEAN & 2.93 & 2.91 & 2.90 & 2.85 & 2.90 & 2.90 & 2.90 & 2.81 & 2.80 & 2.82 & 2.90 & --- \\
\hline MAX & 3.0 & 3.0 & 3.0 & 2.9 & 2.9 & 2.9 & 2.9 & 2.9 & 2.8 & 2.9 & 2.9 & --- \\
\hline MIN & 2.9 & 2.9 & 2.8 & 2.8 & 2.9 & 2.9 & 2.9 & 2.8 & 2.8 & 2.8 & 2.9 & --- \\
\hline $\mathrm{AC}-\mathrm{FT}$ & 180 & 173 & 178 & 176 & 161 & 178 & 173 & 173 & 167 & 173 & 178 & --- \\
\hline
\end{tabular}

e Estimated. 
LOCATION.-Lat 39²6'44", long 120³6'02", in NW 1/4 NW 1/4 sec.11, T.18 N., R.12 E., Nevada County, Hydrologic Unit 18020125, Tahoe National Forest, near right bank end of Sawmill Lake Dam on Canyon Creek, 0.8 mi upstream from Bowman Lake, and 7.2 mi east of Graniteville.

DRAINAGE AREA.- $-16.4 \mathrm{mi}^{2}$.

PERIOD OF RECORD.-October 1986 to current year. Unpublished records for water years 1966-86 available in files of the U.S. Geological Survey.

GAGE.-Staff gages, observed approximately weekly during the summer months. Datum of gage is sea level (levels by Nevada Irrigation District).

REMARKS.-Reservoir is formed by a rock-filled dam initially constructed prior to 1880 and enlarged in 1941 . Usable capacity, 3,030 acre-ft between elevations 5,805 ft, base of dam, and 5,860 ft, crest of spillway. Figures given represent usable contents. Released water is used for hydroelectric power and irrigation downstream. See schematic diagram of Yuba River Basin.

COOPERATION.—Records were collected by Nevada Irrigation District, under general supervision of the U.S. Geological Survey, in connection with a Federal Energy Regulatory Commission project.

Capacity table (elevation, in feet, and contents, in acre-feet)

(Based on survey by Nevada Irrigation District in 1964)

$\begin{array}{rrrr}5,805 & 0 & 5,850 & 2,000 \\ 5,820 & 110 & 5,860 & 3,030 \\ 5,830 & 430 & 5,863 & 3,375\end{array}$

RESERVOIR STORAGE (ACRE-FEET), WATER YEAR OCTOBER 1998 TO SEPTEMBER 1999

DAILY INSTANTANEOUS VALUES

\begin{tabular}{|c|c|c|c|c|c|c|c|c|c|c|c|c|}
\hline DAY & OCT & NOV & $\mathrm{DEC}$ & JAN & FEB & MAR & APR & MAY & JUN & JUL & AUG & SEP \\
\hline 1 & 1610 & --- & --- & --- & --- & --- & --- & --- & --- & --- & --- & --- \\
\hline 2 & --- & --- & --- & --- & --- & --- & --- & --- & --- & --- & --- & --- \\
\hline 3 & --- & --- & --- & --- & --- & --- & --- & --- & --- & --- & --- & --- \\
\hline 4 & --- & 1560 & --- & --- & --- & --- & --- & --- & --- & --- & --- & --- \\
\hline 5 & --- & --- & --- & --- & --- & --- & --- & --- & --- & --- & --- & --- \\
\hline 6 & --- & --- & --- & --- & --- & --- & --- & --- & --- & 3080 & --- & --- \\
\hline 7 & --- & --- & --- & --- & --- & --- & --- & --- & --- & --- & --- & --- \\
\hline 8 & 1570 & --- & --- & --- & --- & --- & --- & --- & --- & --- & --- & --- \\
\hline 9 & --- & --- & 3090 & --- & --- & --- & --- & --- & --- & --- & --- & --- \\
\hline 10 & --- & --- & --- & --- & --- & --- & --- & 3100 & 3110 & --- & --- & --- \\
\hline 11 & --- & --- & --- & --- & --- & --- & --- & --- & --- & --- & --- & --- \\
\hline 12 & --- & --- & --- & --- & --- & --- & --- & --- & --- & --- & --- & --- \\
\hline 13 & --- & --- & --- & --- & --- & --- & --- & --- & --- & --- & --- & --- \\
\hline 14 & --- & --- & --- & --- & --- & --- & --- & --- & --- & --- & --- & --- \\
\hline 15 & 1530 & --- & -- & --- & -- & --- & --- & --- & --- & --- & --- & --- \\
\hline 16 & --- & --- & --- & --- & --- & --- & --- & --- & --- & --- & --- & --- \\
\hline 17 & --- & --- & --- & --- & --- & --- & --- & --- & --- & --- & --- & --- \\
\hline 18 & --- & --- & --- & --- & --- & --- & --- & --- & --- & --- & --- & --- \\
\hline 19 & 1500 & 1870 & --- & --- & --- & --- & --- & --- & --- & --- & --- & --- \\
\hline 20 & --- & --- & --- & --- & --- & --- & --- & --- & --- & --- & --- & --- \\
\hline 21 & 1500 & --- & --- & --- & --- & --- & --- & --- & 3110 & --- & --- & --- \\
\hline 22 & --- & --- & --- & --- & --- & --- & --- & --- & --- & 3060 & --- & --- \\
\hline 23 & --- & --- & --- & --- & --- & --- & --- & --- & --- & --- & --- & --- \\
\hline 24 & --- & --- & --- & --- & --- & --- & --- & --- & --- & --- & --- & --- \\
\hline 25 & --- & --- & --- & --- & --- & --- & --- & --- & --- & --- & 3090 & --- \\
\hline 26 & --- & --- & --- & --- & --- & --- & --- & --- & --- & --- & --- & --- \\
\hline 27 & --- & --- & --- & --- & --- & --- & --- & --- & --- & --- & --- & --- \\
\hline 28 & --- & --- & --- & --- & --- & --- & --- & --- & --- & --- & --- & 2270 \\
\hline 29 & --- & --- & --- & --- & --- & --- & --- & --- & --- & --- & --- & --- \\
\hline 30 & --- & --- & --- & --- & --- & --- & --- & --- & 3090 & --- & --- & --- \\
\hline 31 & --- & --- & --- & --- & --- & --- & --- & --- & --- & --- & --- & --- \\
\hline MAX & --- & --- & --- & --- & --- & --- & --- & --- & --- & --- & --- & -- \\
\hline MIN & --- & --- & --- & --- & --- & --- & --- & --- & --- & --- & --- & --- \\
\hline
\end{tabular}


11414470 CANYON CREEK BELOW SAWMILL LAKE, NEAR GRANITEVILLE, CA

LOCATION.-Lat $39^{\circ} 26^{\prime} 44^{\prime \prime}$, long 120³6’05", in NW 1/4 NW 1/4 sec.11, T.18 N., R.12 E., Nevada County, Hydrologic Unit 18020125, Tahoe National Forest, on right bank $130 \mathrm{ft}$ downstream from outlet at Sawmill Lake Dam on Canyon Creek, 0.8 mi upstream from Bowman Lake, and 7.2 mi east of Graniteville.

DRAINAGE AREA.- $-16.4 \mathrm{mi}^{2}$.

PERIOD OF RECORD.-October 1989 to current year. Unpublished records for water years 1965-89 available in files of the U.S. Geological Survey.

GAGE.-Water-stage recorder and V-notch sharp-crested weir in concrete control. Elevation of gage is 5,790 ft above sea level, from topographic map. September 1964 to July 6, 1988, nonrecording gage at two sites $470 \mathrm{ft}$ downstream at different datum. July 7, 1988, to January 1989, nonrecording gage at same site and datum.

REMARKS.—No records computed above $2.6 \mathrm{ft}^{3} / \mathrm{s}$. Flow completely regulated by Sawmill Lake (station 11414465). Flow over the spillway bypasses this station. See schematic diagram of Yuba River Basin.

COOPERATION.-Records were collected by Nevada Irrigation District, under general supervision of the U.S. Geological Survey, in connection with a Federal Energy Regulatory Commission project.

DISCHARGE, CUBIC FEET PER SECOND, WATER YEAR OCTOBER 1998 TO SEPTEMBER 1999 DAILY MEAN VALUES

\begin{tabular}{|c|c|c|c|c|c|c|c|c|c|c|c|c|}
\hline DAY & $\mathrm{OCT}$ & NOV & $\mathrm{DEC}$ & JAN & $\mathrm{FEB}$ & MAR & APR & MAY & JUN & JUL & AUG & SEP \\
\hline 1 & --- & --- & 2.5 & --- & --- & --- & --- & --- & --- & --- & --- & --- \\
\hline 2 & --- & --- & 2.5 & --- & --- & --- & --- & --- & --- & --- & --- & --- \\
\hline 3 & --- & --- & 2.4 & --- & --- & --- & --- & --- & --- & --- & --- & --- \\
\hline 4 & --- & --- & 2.3 & --- & --- & --- & --- & --- & --- & --- & --- & --- \\
\hline 5 & --- & --- & 2.3 & --- & --- & --- & --- & --- & --- & --- & --- & --- \\
\hline 6 & --- & --- & 2.4 & --- & --- & --- & --- & --- & --- & --- & --- & --- \\
\hline 7 & --- & --- & 2.4 & --- & --- & --- & --- & --- & --- & --- & --- & --- \\
\hline 8 & --- & --- & 2.4 & --- & --- & --- & --- & --- & --- & --- & --- & --- \\
\hline 9 & --- & --- & --- & --- & --- & --- & --- & --- & --- & --- & --- & --- \\
\hline 10 & --- & --- & --- & --- & --- & --- & --- & --- & --- & --- & --- & --- \\
\hline 11 & --- & --- & --- & --- & --- & --- & --- & --- & --- & --- & --- & --- \\
\hline 12 & --- & --- & --- & --- & --- & --- & --- & --- & --- & --- & --- & --- \\
\hline 13 & --- & --- & --- & --- & --- & --- & --- & --- & --- & --- & --- & --- \\
\hline 14 & --- & --- & --- & --- & --- & --- & --- & --- & --- & --- & --- & --- \\
\hline 15 & --- & --- & --- & --- & --- & --- & --- & --- & --- & --- & --- & --- \\
\hline 16 & --- & --- & --- & --- & --- & --- & --- & --- & --- & --- & --- & --- \\
\hline 17 & --- & --- & --- & --- & --- & --- & --- & --- & --- & --- & --- & --- \\
\hline 18 & --- & --- & --- & --- & --- & --- & --- & --- & --- & --- & --- & --- \\
\hline 19 & --- & --- & --- & --- & --- & --- & --- & --- & --- & --- & --- & --- \\
\hline 20 & --- & --- & --- & --- & --- & --- & --- & --- & --- & --- & --- & --- \\
\hline 21 & --- & --- & --- & --- & --- & --- & --- & --- & --- & --- & --- & --- \\
\hline 22 & --- & --- & --- & --- & --- & --- & --- & --- & --- & --- & --- & --- \\
\hline 23 & --- & --- & --- & --- & --- & --- & --- & --- & --- & --- & --- & --- \\
\hline 24 & --- & --- & --- & --- & --- & --- & --- & --- & --- & --- & --- & --- \\
\hline 25 & --- & --- & --- & --- & --- & --- & --- & --- & --- & --- & --- & --- \\
\hline 26 & --- & --- & --- & --- & --- & --- & --- & --- & --- & --- & --- & --- \\
\hline 27 & --- & --- & --- & --- & --- & --- & --- & --- & --- & --- & --- & --- \\
\hline 28 & --- & --- & --- & --- & --- & --- & --- & --- & --- & --- & --- & --- \\
\hline 29 & --- & --- & --- & --- & --- & --- & --- & --- & --- & --- & --- & --- \\
\hline 30 & --- & --- & --- & --- & --- & --- & --- & --- & --- & --- & --- & --- \\
\hline 31 & --- & --- & --- & --- & --- & --- & --- & --- & --- & --- & --- & --- \\
\hline TOTAL & --- & --- & --- & --- & --- & --- & --- & --- & --- & --- & --- & --- \\
\hline MEAN & --- & --- & --- & --- & --- & --- & --- & --- & --- & --- & --- & --- \\
\hline MAX & --- & --- & --- & --- & --- & --- & --- & --- & --- & --- & --- & --- \\
\hline MIN & --- & --- & --- & --- & --- & --- & --- & --- & --- & --- & --- & --- \\
\hline $\mathrm{AC}-\mathrm{FT}$ & --- & --- & --- & --- & --- & --- & --- & --- & --- & --- & --- & --- \\
\hline
\end{tabular}




\section{JACKSON LAKE NEAR SIERRA CITY, CA}

LOCATION.-Lat 39²7'52", long 120³3'44", in SW 1/4 SW 1/4 sec.31, T.19 N., R.13 E., Nevada County, Hydrologic Unit 18020125, Tahoe National Forest, on outlet structure on Jackson Lake Dam on Jackson Creek, 3.0 mi upstream from Bowman Lake, and 8.0 mi southeast of Sierra City.

DRAINAGE AREA.- $-0.65 \mathrm{mi}^{2}$.

PERIOD OF RECORD.-October 1986 to current year. Unpublished records for water years 1965-86 available in files of U.S. Geological Survey.

GAGE.-Water-stage recorder installed Nov. 2, 1998. Records prior to Nov. 2 are instantaneous values. Datum of gage is 6,570 ft above sea level (levels by Nevada Irrigation District).

REMARKS.-Data for Jan. 28 to Mar. 29 missing due to equipment failure. Reservoir is formed on natural lake by earth-filled dam completed in 1859. Usable capacity, 974 acre-ft between gage height $0.0 \mathrm{ft}$, invert of outlet, and $22.67 \mathrm{ft}$, crest of spillway. Dead storage below gage height $0.0 \mathrm{ft}, 360$ acre-ft. Figures given represent total contents. Released water is used for hydroelectric power and irrigation downstream. See schematic diagram of Yuba River Basin.

COOPERATION.-Records were collected by Nevada Irrigation District, under general supervision of the U.S. Geological Survey, in connection with a Federal Energy Regulatory Commission project.

EXTREMES FOR PERIOD OF RECORD.-Maximum contents recorded, 1,350 acre-ft, several days during June and July 1999, maximum elevation, 6,593.03 ft, June 14, 1999; minimum recorded, 428 acre-ft, Nov. 21, 22, 1998, elevation, $6571.80 \mathrm{ft}$.

Capacity table (gage height, in feet, and contents, in acre-feet) (Based on survey by Nevada Irrigation District in 1964)

$\begin{array}{rrrr}0 & 360 & 15 & 958 \\ 5 & 545 & 20 & 1,185 \\ 10 & 730 & 24 & 1,407\end{array}$

RESERVOIR STORAGE (ACRE-FEET), WATER YEAR OCTOBER 1998 TO SEPTEMBER 1999 DAILY OBSERVATION AT 2400 HOURS

\begin{tabular}{|c|c|c|c|c|c|c|c|c|c|c|c|c|}
\hline DAY & $\mathrm{OCT}$ & NOV & $\mathrm{DEC}$ & JAN & FEB & MAR & APR & MAY & JUN & JUL & AUG & SEP \\
\hline 1 & --- & --- & 482 & 468 & --- & --- & 508 & 630 & 1160 & 1350 & 1170 & 1070 \\
\hline 2 & --- & 444 & 486 & 466 & --- & --- & 506 & 644 & 1180 & 1340 & 1170 & 1070 \\
\hline 3 & --- & 442 & 498 & 465 & --- & --- & 506 & 649 & 1190 & 1340 & 1160 & 1070 \\
\hline 4 & --- & 440 & 500 & 463 & --- & --- & 505 & 651 & 1200 & 1340 & 1160 & 1060 \\
\hline 5 & --- & 439 & 501 & 461 & --- & --- & 507 & 653 & 1210 & 1340 & 1150 & 1060 \\
\hline 6 & --- & 438 & 501 & 460 & --- & --- & 506 & 663 & 1230 & 1340 & 1140 & 1060 \\
\hline 7 & --- & 440 & 500 & 458 & --- & --- & 505 & 676 & 1250 & 1340 & 1140 & 1060 \\
\hline 8 & --- & 440 & 498 & 456 & --- & --- & 508 & 684 & 1260 & 1340 & 1130 & 1050 \\
\hline 9 & --- & 439 & 498 & 455 & --- & --- & 506 & 692 & 1270 & 1330 & 1130 & 1050 \\
\hline 10 & --- & 438 & 497 & 453 & --- & --- & 506 & 698 & 1290 & 1320 & 1120 & 1050 \\
\hline 11 & --- & 437 & 496 & 451 & --- & --- & 505 & 710 & 1300 & 1320 & 1120 & 1050 \\
\hline 12 & --- & 435 & 494 & 450 & --- & --- & 504 & 731 & 1320 & 1310 & 1120 & 1040 \\
\hline 13 & --- & 434 & 492 & 448 & --- & --- & 505 & 750 & 1340 & 1310 & 1120 & 1040 \\
\hline 14 & --- & 431 & 493 & 447 & --- & --- & 506 & 760 & 1350 & 1300 & 1110 & 1040 \\
\hline 15 & --- & 431 & 493 & 452 & --- & --- & 508 & 764 & 1350 & 1290 & 1110 & 1040 \\
\hline 16 & --- & 429 & 491 & 455 & --- & --- & 512 & 770 & 1350 & 1290 & 1110 & 1030 \\
\hline 17 & --- & 432 & 490 & 458 & --- & --- & 520 & 782 & 1350 & 1280 & 1110 & 1030 \\
\hline 18 & --- & 431 & 490 & 479 & --- & --- & 529 & 799 & 1350 & 1270 & 1100 & 1030 \\
\hline 19 & --- & 430 & 488 & 492 & --- & --- & 540 & 816 & 1350 & 1270 & 1100 & 1030 \\
\hline 20 & --- & 432 & 490 & 500 & --- & --- & 552 & 832 & 1350 & 1260 & 1100 & 1030 \\
\hline 21 & --- & 428 & 489 & 502 & --- & --- & 563 & 853 & 1350 & 1250 & 1100 & 1020 \\
\hline 22 & --- & 428 & 488 & 506 & --- & --- & 570 & 882 & 1350 & 1240 & 1090 & 1020 \\
\hline 23 & --- & 445 & 486 & 509 & --- & --- & 572 & 917 & 1350 & 1240 & 1090 & 1020 \\
\hline 24 & --- & 446 & 483 & 508 & --- & --- & 578 & 951 & 1350 & 1230 & 1090 & 1020 \\
\hline 25 & --- & 447 & 482 & 507 & --- & --- & 589 & 987 & 1350 & 1220 & 1090 & 1010 \\
\hline 26 & --- & 445 & 479 & 507 & --- & --- & 606 & 1020 & 1350 & 1210 & 1090 & 1010 \\
\hline 27 & --- & 443 & 477 & 506 & --- & --- & 613 & 1050 & 1350 & 1210 & 1080 & 1010 \\
\hline 28 & --- & 442 & 476 & --- & --- & --- & 618 & 1070 & 1350 & 1200 & 1080 & 1010 \\
\hline 29 & --- & 451 & 473 & --- & --- & --- & 620 & 1090 & 1350 & 1190 & 1080 & 1000 \\
\hline 30 & --- & 478 & 471 & --- & --- & 508 & 622 & 1120 & 1350 & 1190 & 1080 & 1000 \\
\hline 31 & --- & --- & 470 & --- & --- & 508 & --- & 1140 & --- & 1180 & 1070 & --- \\
\hline MAX & --- & --- & 501 & --- & --- & --- & 622 & 1140 & 1350 & 1350 & 1170 & 1070 \\
\hline MIN & --- & --- & 470 & --- & --- & --- & 504 & 630 & 1160 & 1180 & 1070 & 1000 \\
\hline a & & 6573.17 & 6572.98 & & & 6574.01 & 6577.08 & 6588.96 & 6592.91 & 6589.88 & 6587.56 & 6585.96 \\
\hline $\mathrm{b}$ & & & -8 & & & & +114 & +518 & +210 & -170 & -110 & -70 \\
\hline
\end{tabular}

a Elevation, in feet, at end of month. b Change in contents, in acre-feet. 
LOCATION.-Lat 39²7'53", long 120³3'46", in SW 1/4 SW 1/4 sec.31, T.19 N., R.13 E., Nevada County, Hydrologic Unit 18020125, Tahoe National Forest, on left bank $75 \mathrm{ft}$ downstream from Jackson Lake Dam on Jackson Creek, 3.0 mi upstream from Bowman Lake, and $8.0 \mathrm{mi}$ southeast of Sierra City.

DRAINAGE AREA.- $-0.65 \mathrm{mi}^{2}$.

PERIOD OF RECORD._-January 1989 to September 1992, April 1993 to current year (low-flow records only). Unpublished records for water years $1965-88$ available in files of the U.S. Geological Survey.

GAGE.-Water-stage recorder and V-notch sharp-crested weir. Elevation of gage is 6,570 ft above sea level, from topographic map. October 1964 to October 1986, nonrecording gage at site $25 \mathrm{ft}$ downstream at different datum. October 1986 to January 1989, nonrecording gage at same site and datum.

REMARKS.-No records computed above $2.9 \mathrm{ft}^{3} / \mathrm{s}$. Flow regulated by Jackson Lake (station 11414690). Flow over the spillway bypasses this station. See schematic diagram of Yuba River Basin.

COOPERATION.--Records were collected by Nevada Irrigation District, under general supervision of the U.S. Geological Survey, in connection with a Federal Energy Regulatory Commission project.

DISCHARGE, CUBIC FEET PER SECOND, WATER YEAR OCTOBER 1998 TO SEPTEMBER 1999 DAILY MEAN VALUES

\begin{tabular}{|c|c|c|c|c|c|c|c|c|c|c|c|c|}
\hline DAY & $\mathrm{OCT}$ & NOV & $\mathrm{DEC}$ & JAN & FEB & MAR & $\mathrm{APR}$ & MAY & JUN & JUL & AUG & SEP \\
\hline 1 & --- & --- & 1.3 & 1.2 & e1. 4 & e1. 4 & 1.4 & 2.1 & .91 & 1.2 & --- & 1.1 \\
\hline 2 & --- & --- & 1.3 & 1.2 & e1. 4 & e1. 4 & 1.4 & 2.2 & 1.2 & 1.2 & --- & 1.1 \\
\hline 3 & --- & .88 & 1.4 & 1.2 & e1. 4 & e1. 4 & 1.4 & 2.2 & 1.2 & 1.1 & --- & 1.1 \\
\hline 4 & --- & .89 & 1.4 & 1.2 & e1. 4 & e1.4 & 1.4 & 2.3 & 1.2 & 1.1 & --- & 1.1 \\
\hline 5 & --- & .87 & 1.4 & 1.2 & e1. 4 & e1. 4 & 1.4 & 2.3 & 1.2 & 1.1 & --- & 1.1 \\
\hline 6 & --- & .86 & 1.4 & 1.2 & e1. 4 & e1. 4 & 1.4 & 2.3 & 1.2 & 1.1 & --- & 1.1 \\
\hline 7 & --- & .81 & 1.3 & 1.2 & e1. 4 & e1. 4 & 1.4 & 2.4 & 1.2 & 1.1 & --- & 1.1 \\
\hline 8 & --- & .77 & 1.3 & 1.1 & e1. 4 & e1.4 & 1.4 & 2.4 & 1.2 & --- & --- & 1.0 \\
\hline 9 & --- & .77 & 1.3 & 1.1 & e1. 4 & e1.4 & 1.4 & 2.5 & 1.2 & --- & --- & 1.0 \\
\hline 10 & --- & .75 & 1.3 & 1.1 & e1. 4 & e1.4 & 1.4 & 2.5 & 1.2 & --- & --- & 1.1 \\
\hline 11 & --- & .76 & 1.3 & 1.1 & e1. 4 & e1. 4 & 1.4 & 2.6 & 1.3 & --- & 1.4 & 1.0 \\
\hline 12 & --- & .74 & 1.2 & 1.1 & e1. 4 & 1.5 & 1.4 & 2.7 & 1.2 & --- & 1.2 & 1.0 \\
\hline 13 & --- & .93 & 1.1 & 1.1 & e1. 4 & 1.5 & 1.4 & 2.7 & 1.2 & --- & .99 & 1.0 \\
\hline 14 & --- & 1.2 & 1.1 & 1.1 & e1. 4 & 1.5 & 1.4 & 2.7 & 1.2 & --- & .91 & 1.0 \\
\hline 15 & --- & 1.1 & 1.2 & 1.1 & $\mathrm{e} 1.4$ & 1.5 & 1.4 & 2.8 & 1.2 & --- & .84 & 1.0 \\
\hline 16 & --- & 1.1 & 1.1 & 1.1 & e1. 4 & 1.5 & 1.4 & --- & 1.2 & --- & .88 & 1.0 \\
\hline 17 & --- & 1.1 & 1.2 & 1.1 & e1. 4 & 1.5 & 1.4 & --- & 1.2 & --- & 1.0 & 1.0 \\
\hline 18 & --- & 1.1 & 1.3 & 1.3 & e1. 4 & 1.5 & 1.5 & --- & 1.2 & --- & 1.0 & 1.0 \\
\hline 19 & --- & 1.1 & 1.3 & 1.3 & e1. 4 & 1.5 & 1.6 & --- & 1.2 & --- & .99 & 1.0 \\
\hline 20 & --- & 1.1 & 1.3 & 1.4 & e1. 4 & 1.5 & 1.6 & --- & 1.2 & --- & 1.0 & 1.0 \\
\hline 21 & --- & 1.0 & 1.3 & 1.4 & e1. 4 & 1.4 & 1.7 & --- & 1.2 & --- & 1.0 & 1.0 \\
\hline 22 & --- & 1.1 & 1.3 & 1.4 & e1. 4 & 1.4 & 1.7 & --- & 1.1 & --- & 1.0 & 1.0 \\
\hline 23 & --- & 1.1 & 1.3 & 1.5 & e1. 4 & 1.4 & 1.8 & --- & 1.2 & --- & .99 & 1.0 \\
\hline 24 & --- & 1.1 & 1.3 & 1.5 & e1. 4 & 1.4 & 1.8 & --- & 1.2 & --- & .99 & 1.0 \\
\hline 25 & --- & 1.1 & 1.3 & 1.5 & e1. 4 & 1.4 & 1.9 & --- & 1.2 & --- & .99 & 1.0 \\
\hline 26 & --- & 1.1 & 1.3 & 1.5 & e1.4 & 1.4 & 1.9 & --- & 1.2 & --- & .97 & 1.0 \\
\hline 27 & --- & 1.1 & 1.3 & 1.4 & e1. 4 & 1.4 & 2.0 & --- & 1.2 & --- & .97 & 1.0 \\
\hline 28 & --- & 1.1 & 1.3 & e1.4 & e1. 4 & 1.4 & 2.0 & .83 & 1.2 & --- & .96 & 1.0 \\
\hline 29 & --- & 1.1 & 1.3 & e1. 4 & --- & 1.4 & 2.1 & .80 & 1.2 & --- & .96 & 1.0 \\
\hline 30 & --- & 1.2 & 1.3 & e1. 4 & --- & 1.4 & 2.1 & .80 & 1.2 & --- & .96 & 1.0 \\
\hline 31 & --- & --- & 1.3 & e1. 4 & --- & 1.4 & --- & .78 & --- & --- & 1.0 & --- \\
\hline TOTAL & --- & --- & 39.8 & 39.2 & 39.2 & 44.3 & 47.5 & --- & 35.71 & --- & --- & 30.8 \\
\hline MEAN & --- & --- & 1.28 & 1.26 & 1.40 & 1.43 & 1.58 & --- & 1.19 & --- & --- & 1.03 \\
\hline MAX & --- & --- & 1.4 & 1.5 & 1.4 & 1.5 & 2.1 & --- & 1.3 & --- & --- & 1.1 \\
\hline MIN & --- & --- & 1.1 & 1.1 & 1.4 & 1.4 & 1.4 & --- & .91 & --- & --- & 1.0 \\
\hline $\mathrm{AC}-\mathrm{FT}$ & --- & --- & 79 & 78 & 78 & 88 & 94 & --- & 71 & --- & --- & 61 \\
\hline
\end{tabular}


11415500 BOWMAN LAKE NEAR GRANITEVILLE, CA

LOCATION.—Lat 39²7'01", long 120³9'09", in SE 1/4 SW 1/4 sec.5, T.18 N., R.12 E., Nevada County, Hydrologic Unit 18020125, on right bank near rockfill portion of Bowman Dam on Canyon Creek, 4.6 mi east of Graniteville, and 8 mi south of Sierra City.

DRAINAGE AREA.-27.1 $\mathrm{mi}^{2}$.

PERIOD OF RECORD.-December 1926 to current year.

REVISED RECORDS.-WSP 1931: Drainage area.

GAGE.-Water-stage recorder. Datum of gage is sea level (levels by Nevada Irrigation District). Prior to Oct. 8, 1964, nonrecording gage at same site and datum.

REMARKS.-_Lake is formed by one rockfill and one concrete-arch dam; storage began in November 1926. Total capacity, 68,700 acre-ft between elevations 5,400 ft, bottom of outlet tunnel, and 5,563.6 ft, top of radial spillway gates and crest of concrete-arch dam. Flashboards are occasionally added, increasing elevation to $5,565.8 \mathrm{ft}$ and capacity to 70,400 acre-ft, all of which is available for release. Lake receives water from Middle Yuba River via Milton-Bowman Tunnel (station 11408000), and releases it through Bowman-Spaulding Canal (station 11416000) which conveys it to reservoirs of Pacific Gas \& Electric Co. Water is eventually used for irrigation by Nevada Irrigation District. Records, including extremes, represent total contents. See schematic diagram of Yuba River Basin.

COOPERATION.- Records were collected by Nevada Irrigation District, under general supervision of the U.S. Geological Survey, in connection with a Federal Energy Regulatory Commission project.

EXTREMES FOR PERIOD OF RECORD.-Maximum contents, 71,000 acre-ft, May 30, 1965, elevation, 5,566.5 ft; lake completely drained for inspection and repair Nov. 25 to Dec. 9, 1949, Oct. 1-20, 1966, Oct. 4-29, 1972, and Sept. 21-30, 1981.

EXTREMES FOR CURRENT YEAR.-Maximum contents, 64,600 acre-ft, June 24, elevation, 5558.93 ft; minimum, 28,900 acre-ft, Apr. 11-13, elevation, $5508.52 \mathrm{ft}$.

Capacity table (elevation, in feet, and contents, in acre-feet)

(Based on table dated Nov. 24, 1926)

$\begin{array}{lrrr}5,419.6 & 0 & 5,470 & 10,200 \\ 5,430 & 900 & 5,480 & 14,200 \\ 5,440 & 2,100 & 5,510 & 30,000 \\ 5,450 & 4,100 & 5,540 & 49,800 \\ 5,460 & 6,900 & 5,570 & 73,800\end{array}$

RESERVOIR STORAGE (ACRE-FEET), WATER YEAR OCTOBER 1998 TO SEPTEMBER 1999

DAILY OBSERVATION AT 2400 HOURS

\begin{tabular}{|c|c|c|c|c|c|c|c|c|c|c|c|c|}
\hline DAY & $\mathrm{OCT}$ & NOV & DEC & JAN & FEB & MAR & APR & MAY & JUN & JUL & AUG & SEP \\
\hline 1 & 48900 & 44600 & 41600 & 34400 & 33400 & 32200 & 29300 & 35600 & 58100 & 63500 & 62700 & 53800 \\
\hline 2 & 48700 & 44500 & 41600 & 34000 & 33100 & 32400 & 29300 & 36200 & 59200 & 63400 & 62300 & 53600 \\
\hline 3 & 48500 & 44400 & 42300 & 33700 & 32900 & 32700 & 29200 & 36500 & 59900 & 63200 & 62000 & 53200 \\
\hline 4 & 48300 & 44300 & 42300 & 33400 & 32600 & 32700 & 29200 & 36700 & 60300 & 63000 & 61600 & 53100 \\
\hline 5 & 48100 & 44200 & 42200 & 33000 & 32300 & 32600 & 29200 & 36800 & 60700 & 62700 & 61300 & 52800 \\
\hline 6 & 47900 & 44100 & 42000 & 32700 & 32200 & 32500 & 29200 & 37200 & 61200 & 62500 & 61000 & 51700 \\
\hline 7 & 47700 & 44100 & 41800 & 32400 & 32700 & 32300 & 29100 & 37800 & 61800 & 62200 & 60700 & 52300 \\
\hline 8 & 47500 & 44100 & 41600 & 32300 & 33100 & 32200 & 29100 & 38300 & 62100 & 61900 & 60400 & 52100 \\
\hline 9 & 47300 & 44000 & 41300 & 32200 & 34000 & 32100 & 29100 & 38700 & 62200 & 61600 & 60100 & 51900 \\
\hline 10 & 47100 & 43900 & 41100 & 32100 & 34100 & 31900 & 29000 & 39100 & 62300 & 61700 & 59800 & 51800 \\
\hline 11 & 46900 & 43800 & 40800 & 32100 & 34000 & 31700 & 28900 & 39600 & 62400 & 61900 & 59500 & 51700 \\
\hline 12 & 46700 & 43700 & 40500 & 31900 & 33800 & 31500 & 28900 & 40300 & 62600 & 62200 & 59200 & 51500 \\
\hline 13 & 46500 & 43600 & 40200 & 31500 & 33700 & 31300 & 28900 & 41000 & 62800 & 62400 & 58900 & 50100 \\
\hline 14 & 46300 & 43500 & 39900 & 31100 & 33500 & 31100 & 29000 & 41500 & 63000 & 62700 & 58500 & 51400 \\
\hline 15 & 46200 & 43400 & 39600 & 30900 & 33300 & 30800 & 29100 & 41700 & 63300 & 62900 & 58200 & 51400 \\
\hline 16 & 46000 & 43300 & 39300 & 30900 & 33200 & 30600 & 29200 & 42000 & 63600 & 63100 & 57900 & 51400 \\
\hline 17 & 45900 & 43300 & 39100 & 30900 & 33400 & 30400 & 29500 & 42400 & 63900 & 63300 & 57600 & 50900 \\
\hline 18 & 45800 & 43200 & 38800 & 32300 & 33400 & 30300 & 30000 & 43000 & 64100 & 63500 & 57300 & 51100 \\
\hline 19 & 45700 & 43100 & 38600 & 33200 & 33300 & 30100 & 30500 & 43600 & 64300 & 63700 & 57000 & 51000 \\
\hline 20 & 45600 & 42800 & 38300 & 34400 & 33100 & 30000 & 31200 & 44200 & 64400 & 63900 & 56700 & 50800 \\
\hline 21 & 45700 & 42500 & 38000 & 34800 & 33000 & 29900 & 31700 & 44900 & 64500 & 64100 & 56500 & 50700 \\
\hline 22 & 45500 & 42200 & 37700 & 34900 & 32800 & 29700 & 32100 & 45800 & 64500 & 64300 & 56200 & 49400 \\
\hline 23 & 45400 & 42200 & 37400 & 35100 & 32600 & 29500 & 32300 & 46900 & 64500 & 64400 & 56000 & 50400 \\
\hline 24 & 45400 & 42000 & 37200 & 35100 & 32300 & 29400 & 32700 & 48200 & 64600 & 64500 & 55700 & 50300 \\
\hline 25 & 45300 & 41700 & 36700 & 34900 & 32200 & 29300 & 33200 & 49600 & 64500 & 64500 & 55500 & 50200 \\
\hline 26 & 45200 & 41300 & 36400 & 34800 & 32000 & 29300 & 34000 & 51200 & 64400 & 64500 & 55300 & 50100 \\
\hline 27 & 45100 & 41000 & 36000 & 34600 & 31700 & 29200 & 34500 & 52700 & 64200 & 64400 & 55000 & 50000 \\
\hline 28 & 45000 & 40700 & 35700 & 34300 & 31700 & 29200 & 34800 & 54000 & 63900 & 64000 & 54800 & 49900 \\
\hline 29 & 44900 & 40500 & 35400 & 34100 & --- & 29100 & 35000 & 55100 & 63700 & 63700 & 54600 & 49800 \\
\hline 30 & 44800 & 41500 & 35000 & 33800 & --- & 29200 & 35200 & 56100 & 63700 & 63300 & 54300 & 49700 \\
\hline 31 & 44700 & --- & 34700 & 33600 & --- & 29300 & --- & 57100 & --- & 63000 & 54100 & --- \\
\hline MAX & 48900 & 44600 & 42300 & 35100 & 34100 & 32700 & 35200 & 57100 & 64600 & 64500 & 62700 & 53800 \\
\hline MIN & 44700 & 40500 & 34700 & 30900 & 31700 & 29100 & 28900 & 35600 & 58100 & 61600 & 54100 & 49400 \\
\hline$a$ & 5532.66 & 5528.10 & 5517.84 & 5516.16 & 5513.14 & 5509.27 & 5518.53 & 5549.61 & 5557.81 & 5556.99 & 5545.44 & 5539.53 \\
\hline $\mathrm{b}$ & -4300 & -3200 & -6800 & -1100 & -1900 & -2400 & +5900 & +21900 & +6600 & -700 & -8900 & -4400 \\
\hline
\end{tabular}

CAL YR 1998 MAX 69300 MIN $27600 \quad b+1300$

WTR YR 1999 MAX 64600 MIN 28900 b +700

a Elevation, in feet, at end of month.

b Change in contents, in acre-feet. 


\section{BOWMAN-SPAULDING CANAL`ĀT JORDAN CREEK SIPHON VENTURI, NEAR EMIGRANT GAP, CA}

LOCATION.-Lat 39²0’32", long 120³8'26", in SW 1/4 NW 1/4 sec.16, T.17 N., R.12 E., Nevada County, Hydrologic Unit 18020125, at outlet of Jordan Creek Siphon, 0.6 mi downstream from Fuller Lake, and 3.5 mi northeast of Emigrant Gap.

PERIOD OF RECORD.-October 1964 to current year.

GAGE.-Water-stage recorder and Venturi section. Elevation of gage is 5,340 ft above sea level, from topographic map.

REMARKS.-Records show water diverted from Bowman Lake (station 11415500) plus numerous small tributaries before it enters Lake Spaulding (station 11414140). Most of the water at this gage flows downstream through Spaulding No. 3 Powerplant (station 11416200). See schematic diagrams of Yuba and Bear River Basins.

COOPERATION.-Records were collected by Pacific Gas \& Electric Co., under general supervision of the U.S. Geological Survey, in connection with a Federal Energy Regulatory Commission project.

EXTREMES FOR PERIOD OF RECORD.-Maximum daily discharge, $335 \mathrm{ft}^{3} / \mathrm{s}$, Dec. 25, 1983; no flow at times in most years.

DISCHARGE, CUBIC FEET PER SECOND, WATER YEAR OCTOBER 1998 TO SEPTEMBER 1999

DAILY MEAN VALUES

\begin{tabular}{|c|c|c|c|c|c|c|c|c|c|c|c|c|}
\hline DAY & OCT & NOV & $\mathrm{DEC}$ & JAN & FEB & MAR & APR & MAY & JUN & JUL & AUG & SEP \\
\hline 1 & 298 & 298 & 248 & 223 & 234 & 302 & 292 & 306 & 288 & 306 & 296 & 301 \\
\hline 2 & 298 & 293 & 230 & 222 & 231 & 311 & 286 & 319 & 312 & 309 & 299 & 301 \\
\hline 3 & 298 & 294 & 261 & 221 & 230 & 316 & 292 & 318 & 317 & 309 & 299 & 301 \\
\hline 4 & 298 & 290 & 256 & 220 & 230 & 305 & 288 & 297 & 306 & 307 & 305 & 304 \\
\hline 5 & 296 & 295 & 232 & 219 & 229 & 277 & 293 & 296 & 290 & 306 & 307 & 307 \\
\hline 6 & 302 & 305 & 229 & 218 & 220 & 261 & 296 & 305 & 283 & 305 & 300 & 303 \\
\hline 7 & 302 & 306 & 229 & 176 & 218 & 252 & 303 & 315 & 288 & 303 & 299 & 302 \\
\hline 8 & 301 & 269 & 228 & 83 & 254 & 249 & 307 & 306 & 302 & 301 & 298 & 300 \\
\hline 9 & 305 & 272 & 217 & 99 & 296 & 255 & 308 & 285 & 303 & 296 & 299 & 295 \\
\hline 10 & 306 & 282 & 213 & 99 & 247 & 253 & 305 & 278 & 301 & 245 & 299 & 295 \\
\hline 11 & 306 & 283 & 216 & 91 & 262 & 241 & 302 & 296 & 304 & 57 & 297 & 291 \\
\hline 12 & 304 & 282 & 228 & 153 & 263 & 238 & 302 & 307 & 310 & .00 & 296 & 289 \\
\hline 13 & 304 & 281 & 229 & 239 & 256 & 236 & 299 & 317 & 312 & .00 & 295 & 268 \\
\hline 14 & 304 & 271 & 229 & 230 & 250 & 236 & 309 & 309 & 313 & .00 & 294 & 246 \\
\hline 15 & 305 & 270 & 231 & 235 & 243 & 235 & 319 & 292 & 316 & .00 & 293 & 253 \\
\hline 16 & 308 & 279 & 235 & 256 & 243 & 235 & 318 & 280 & 313 & .00 & 289 & 188 \\
\hline 17 & 307 & 283 & 239 & 263 & 274 & 236 & 309 & 282 & 312 & .00 & 296 & 262 \\
\hline 18 & 308 & 284 & 237 & 308 & 285 & 239 & 309 & 301 & 306 & .00 & 296 & 284 \\
\hline 19 & 308 & 262 & 235 & 286 & 278 & 243 & 314 & 312 & 299 & .00 & 295 & 287 \\
\hline 20 & 308 & 211 & 235 & 309 & 260 & 246 & 320 & 319 & 292 & .00 & 297 & 287 \\
\hline 21 & 261 & 205 & 231 & 234 & 257 & 243 & 308 & 319 & 289 & .00 & 300 & 289 \\
\hline 22 & 267 & 215 & 230 & 217 & 248 & 239 & 289 & 318 & 299 & .00 & 299 & 289 \\
\hline 23 & 300 & 223 & 225 & 273 & 241 & 238 & 277 & 320 & 302 & .00 & 299 & 289 \\
\hline 24 & 307 & 242 & 220 & 289 & 237 & 238 & 297 & 321 & 302 & .00 & 300 & 289 \\
\hline 25 & 307 & 218 & 222 & 271 & 241 & 228 & 307 & 320 & 301 & .00 & 300 & 287 \\
\hline 26 & 312 & 206 & 218 & 262 & 237 & 270 & 320 & 319 & 299 & 17 & 300 & 286 \\
\hline 27 & 309 & 209 & 216 & 251 & 235 & 301 & 302 & 317 & 299 & 119 & 300 & 284 \\
\hline 28 & 303 & 212 & 221 & 246 & 244 & 307 & 287 & 313 & 299 & 244 & 300 & 276 \\
\hline 29 & 300 & 212 & 225 & 240 & --- & 306 & 272 & 308 & 299 & 284 & 300 & 286 \\
\hline 30 & 298 & 256 & 224 & 238 & --- & 307 & 285 & 295 & 300 & 294 & 300 & 291 \\
\hline 31 & 297 & --- & 224 & 236 & --- & 306 & --- & 285 & --- & 298 & 301 & --- \\
\hline TOTAL & 9327 & 7808 & 7113 & 6907 & 6943 & 8149 & 9015 & 9475 & 9056 & 4300.00 & 9248 & 8530 \\
\hline MEAN & 301 & 260 & 229 & 223 & 248 & 263 & 300 & 306 & 302 & 139 & 298 & 284 \\
\hline MAX & 312 & 306 & 261 & 309 & 296 & 316 & 320 & 321 & 317 & 309 & 307 & 307 \\
\hline MIN & 261 & 205 & 213 & 83 & 218 & 228 & 272 & 278 & 283 & .00 & 289 & 188 \\
\hline $\mathrm{AC}-\mathrm{FT}$ & 18500 & 15490 & 14110 & 13700 & 13770 & 16160 & 17880 & 18790 & 17960 & 8530 & 18340 & 16920 \\
\hline a & 19130 & 16000 & 14600 & 13860 & 14240 & 15850 & 18130 & 18840 & 18170 & 8010 & 18610 & 16190 \\
\hline
\end{tabular}

STATISTICS OF MONTHLY MEAN DATA FOR WATER YEARS 1965 - 1999, BY WATER YEAR (WY)

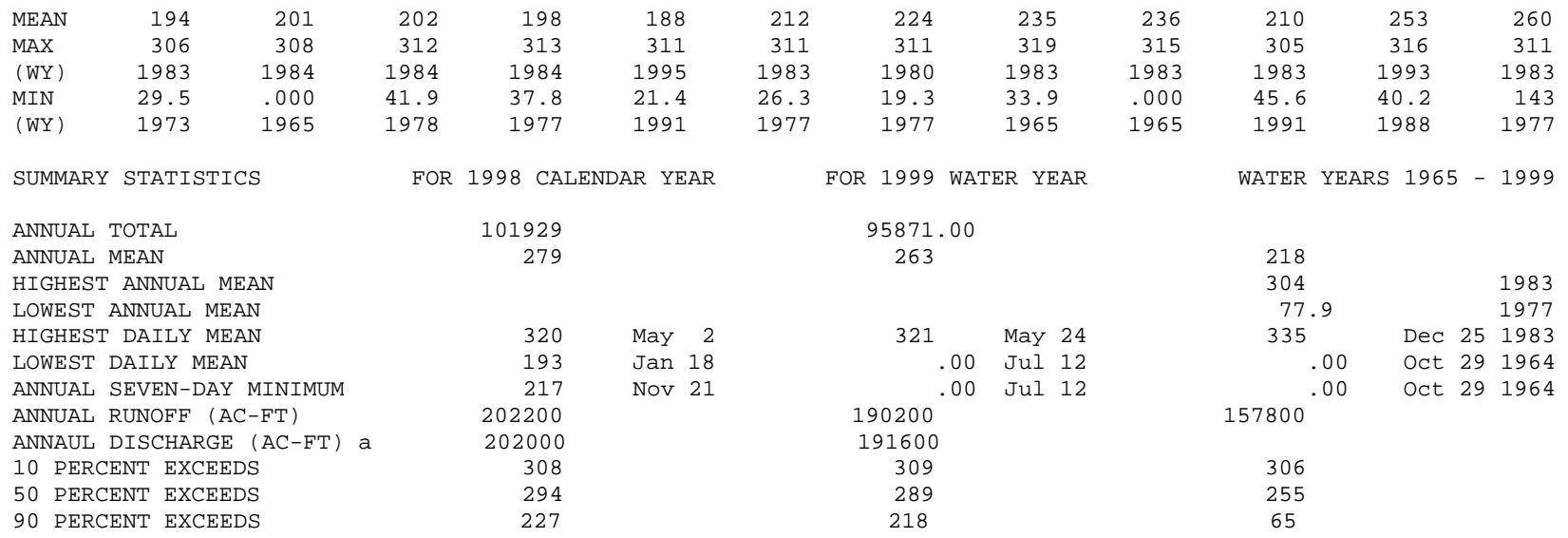

a Discharge, in acre-feet, through Spaulding No. 3 Powerplant, provided by Pacific Gas \& Electric Co. 


\section{CANYON CREEK BELOW BOWMAN LAKE, CA}

LOCATION.-Lat 39²6’23", long 120³9’37", in NE 1/4 SE 1/4 sec.7, T.18 N., R.12 E., Nevada County, Hydrologic Unit 18020125, on left bank 1 mi downstream from Bowman Dam, 3.5 mi upstream from Texas Creek, and 8.8 mi south of Sierra City.

DRAINAGE AREA.- $-28.3 \mathrm{mi}^{2}$.

PERIOD OF RECORD.- - January 1927 to current year.

REVISED RECORDS.-WSP 1315-A: 1930(M). WSP 1931: Drainage area.

GAGE.-Water-stage recorder. Elevation of gage is 5,300 ft above sea level, from topographic map.

REMARKS.-Flow regulated by Bowman Lake (station 11415500), several smaller reservoirs, and diversion into Bowman-Spaulding Canal (station 11416000). See schematic diagram of Yuba River Basin.

COOPERATION.-Records were collected by Nevada Irrigation District, under general supervision of the U.S. Geological Survey, in connection with a Federal Energy Regulatory Commission project.

EXTREMES FOR PERIOD OF RECORD.-Maximum discharge, about 5,500 ft 3 /s, Jan. 2, 1997, gage height, $13.01 \mathrm{ft}$, from floodmarks (backwater from debris), from rating curve extended above $1,500 \mathrm{ft}^{3} / \mathrm{s}$, on basis of computation of flow over Bowman Dam; no flow at times in some years.

DISCHARGE, CUBIC FEET PER SECOND, WATER YEAR OCTOBER 1998 TO SEPTEMBER 1999 DAILY MEAN VALUES

\begin{tabular}{|c|c|c|c|c|c|c|c|c|c|c|c|c|}
\hline DAY & $\mathrm{OCT}$ & NOV & DEC & JAN & FEB & MAR & APR & MAY & JUN & JUL & AUG & SEP \\
\hline 1 & 4.9 & 5.0 & 7.8 & 5.3 & 5.3 & 13 & 50 & 121 & 173 & 4.2 & 5.6 & 5.7 \\
\hline 2 & 4.8 & 5.0 & 7.9 & 5.3 & 5.3 & 7.9 & 58 & 124 & 174 & 4.0 & 5.6 & 5.6 \\
\hline 3 & 4.9 & 5.0 & 16 & 5.3 & 5.4 & 12 & 58 & 144 & 169 & 4.1 & 5.6 & 5.5 \\
\hline 4 & 4.9 & 5.0 & 7.2 & 5.3 & 5.5 & 7.2 & 58 & 150 & 161 & 4.1 & 5.7 & 5.4 \\
\hline 5 & 4.9 & 5.1 & 6.2 & 5.2 & 5.4 & 6.3 & 58 & 142 & 142 & 4.1 & 5.5 & 5.4 \\
\hline 6 & 4.8 & 5.2 & 5.8 & 5.2 & 5.4 & 6.0 & 49 & 141 & 142 & 4.1 & 5.5 & 5.3 \\
\hline 7 & 4.9 & 5.8 & 5.5 & 5.0 & 16 & 5.8 & 43 & 151 & 120 & 4.1 & 5.6 & 5.2 \\
\hline 8 & 4.8 & 5.5 & 5.5 & 4.9 & 11 & 5.8 & 43 & 185 & 103 & 4.1 & 5.5 & 5.3 \\
\hline 9 & 4.8 & 5.2 & 5.4 & 4.8 & 19 & 6.2 & 43 & 190 & 104 & 4.1 & 5.5 & 5.3 \\
\hline 10 & 4.8 & 5.1 & 5.4 & 4.8 & 7.2 & 5.6 & 43 & 124 & 105 & 3.7 & 5.6 & 5.5 \\
\hline 11 & 4.7 & 5.2 & 5.4 & 4.8 & 6.5 & 50 & 43 & 20 & 103 & 5.8 & 5.5 & 5.3 \\
\hline 12 & 4.7 & 5.1 & 5.5 & 5.1 & 6.1 & 87 & 44 & 20 & 94 & 7.2 & 5.5 & 4.8 \\
\hline 13 & 4.7 & 5.1 & 5.7 & 5.3 & 5.8 & 89 & 43 & 18 & 93 & 7.3 & 5.4 & 4.8 \\
\hline 14 & 4.7 & 5.1 & 5.8 & 5.3 & 5.8 & 90 & 53 & 16 & 93 & 7.2 & 5.4 & 5.4 \\
\hline 15 & 4.7 & 5.1 & 5.9 & 6.2 & 5.6 & 92 & 64 & 16 & 44 & 8.4 & 5.4 & 5.0 \\
\hline 16 & 4.6 & 5.1 & 6.6 & 7.9 & 7.3 & 93 & 93 & 16 & 10 & 10 & 5.4 & 5.0 \\
\hline 17 & 4.6 & 6.2 & 6.4 & 8.7 & 14 & 95 & 117 & 19 & 5.4 & 7.6 & 5.4 & 5.2 \\
\hline 18 & 4.6 & 5.6 & 5.9 & 15 & 8.6 & 97 & 124 & 24 & 4.3 & 6.9 & 5.4 & 5.2 \\
\hline 19 & 4.7 & 5.2 & 5.7 & 17 & 7.3 & 99 & 126 & 27 & 4.4 & 7.8 & 5.4 & 5.0 \\
\hline 20 & 4.8 & 5.0 & 5.7 & 21 & 6.4 & 101 & 138 & 27 & 4.4 & 7.3 & 5.3 & 4.8 \\
\hline 21 & 11 & 5.6 & 5.5 & 8.2 & 6.5 & 102 & 169 & 84 & 4.4 & 6.4 & 5.3 & 4.7 \\
\hline 22 & 4.9 & 8.3 & 5.4 & 7.0 & 5.9 & 103 & 169 & 169 & 4.4 & 6.0 & 5.4 & 4.5 \\
\hline 23 & 4.8 & 13 & 5.3 & 12 & 5.8 & 105 & 146 & 169 & 4.4 & 10 & 5.3 & 4.3 \\
\hline 24 & 5.0 & 8.0 & 5.2 & 6.9 & 5.8 & 108 & 130 & 185 & 4.3 & 7.3 & 5.3 & 4.4 \\
\hline 25 & 4.7 & 6.0 & 5.2 & 6.2 & 5.9 & 72 & 131 & 200 & 4.3 & 5.3 & 5.4 & 4.4 \\
\hline 26 & 4.6 & 5.5 & 5.2 & 5.9 & 5.7 & 51 & 168 & 195 & 4.3 & 5.4 & 5.4 & 4.4 \\
\hline 27 & 4.5 & 6.7 & 5.3 & 5.7 & 5.8 & 52 & 199 & 193 & 4.3 & 5.5 & 5.5 & 4.3 \\
\hline 28 & 4.6 & 6.8 & 5.3 & 5.6 & 12 & 52 & 176 & 188 & 4.3 & 5.8 & 5.5 & 4.4 \\
\hline 29 & 4.9 & 8.4 & 5.3 & 5.5 & --- & 52 & 157 & 179 & 4.2 & 5.7 & 5.5 & 4.5 \\
\hline 30 & 4.8 & 19 & 5.3 & 5.4 & --- & 53 & 129 & 175 & 4.2 & 5.7 & 5.5 & 4.5 \\
\hline 31 & 4.8 & --- & 5.4 & 5.4 & --- & 54 & --- & 173 & --- & 5.6 & 5.6 & --- \\
\hline TOTAL & 153.9 & 191.9 & 189.7 & 221.2 & 212.3 & 1772.8 & 2922 & 3585 & 1891.6 & 184.8 & 169.5 & 149.1 \\
\hline MEAN & 4.96 & 6.40 & 6.12 & 7.14 & 7.58 & 57.2 & 97.4 & 116 & 63.1 & 5.96 & 5.47 & 4.97 \\
\hline MAX & 11 & 19 & 16 & 21 & 19 & 108 & 199 & 200 & 174 & 10 & 5.7 & 5.7 \\
\hline MIN & 4.5 & 5.0 & 5.2 & 4.8 & 5.3 & 5.6 & 43 & 16 & 4.2 & 3.7 & 5.3 & 4.3 \\
\hline $\mathrm{AC}-\mathrm{FT}$ & 305 & 381 & 376 & 439 & 421 & 3520 & 5800 & 7110 & 3750 & 367 & 336 & 296 \\
\hline a & 17410 & 14670 & 12830 & 10680 & 10120 & 12870 & 13360 & 11680 & 13470 & 8280 & 17950 & 16490 \\
\hline
\end{tabular}

a Diversion, in acre-feet, to Bowman-Spaulding Canal, provided by Nevada Irrigation District. 
11416500 CANYON CREEK BELOW BOWMAN LAKE, CA—Continued

STATISTICS OF MONTHLY MEAN DATA FOR WATER YEARS 1927 - 1999, BY WATER YEAR (WY)

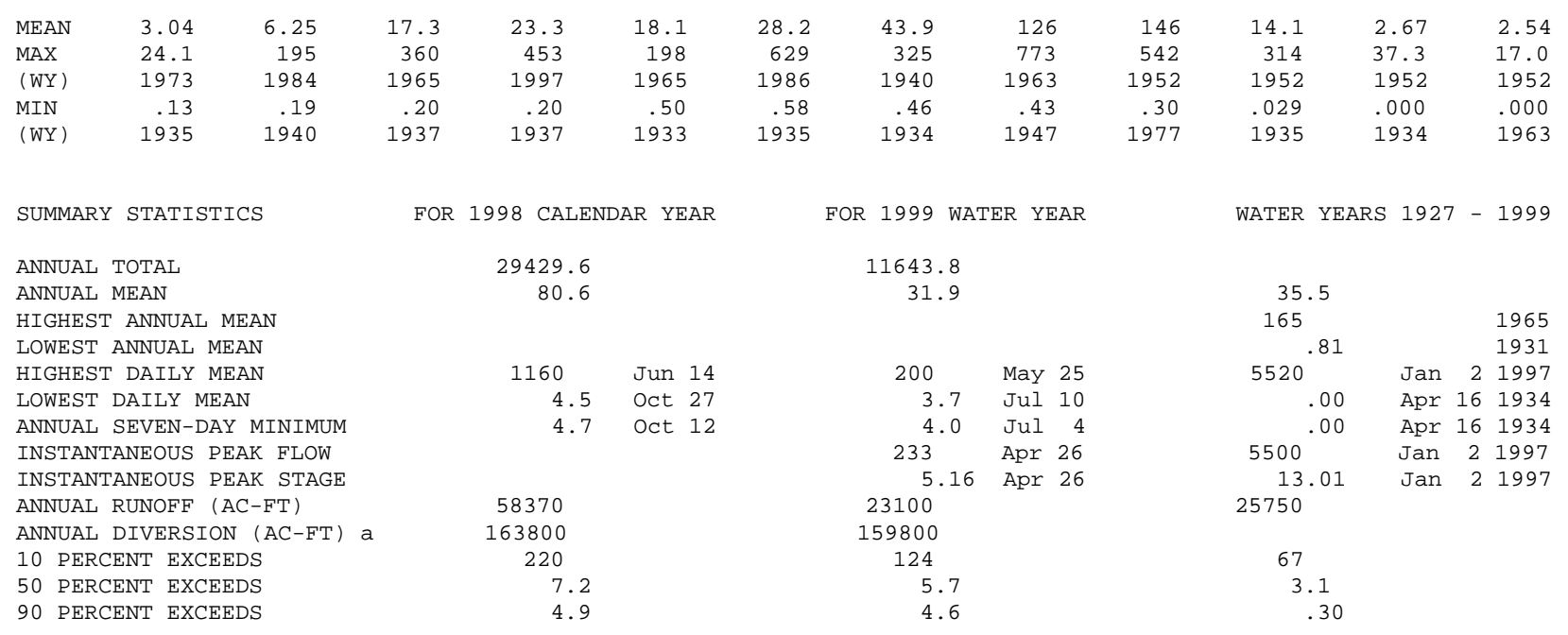

a Diversion, in acre-feet, to Bowman-Spaulding Canal, provided by Nevada Irrigation District. 


\section{TEXAS CREEK BELOW LOWER ROCK LAKE, NEAR GRANITEVILLE, CA}

LOCATION.-Lat 39 $25^{\prime} 42^{\prime \prime}$, long 120³7'19", in SW 1/4 NW 1/4 sec.15, T.18 N., R.12 E., Nevada County, Hydrologic Unit 18020125, Tahoe National Forest, on right bank $200 \mathrm{ft}$ downstream from outlet structure on Lower Rock Lake Dam and 6.4 mi east of Graniteville.

DRAINAGE AREA.- $-0.36 \mathrm{mi}^{2}$.

PERIOD OF RECORD.-October 1995 to current year (low-flow records only). Unpublished records for water years 1974 and 1979-95 available in files of the U.S. Geological Survey.

GAGE.-Water-stage recorder and Parshall flume. Elevation of gage is 6,615 ft above sea level, from topographic map. August 1965 to August 1995 , nonrecording gage at same site and datum.

REMARKS.-Records not computed for winter months or above $1.2 \mathrm{ft}^{3} / \mathrm{s}$. Flow regulated by Lower Rock Lake. See schematic diagram of Yuba River Basin.

COOPERATION.--Records were collected by Pacific Gas \& Electric Co., under general supervision of the U.S. Geological Survey, in connection with a Federal Energy Regulatory Commission project.

DISCHARGE, CUBIC FEET PER SECOND, WATER YEAR OCTOBER 1998 TO SEPTEMBER 1999 DAILY MEAN VALUES

\begin{tabular}{|c|c|c|c|c|c|c|c|c|c|c|c|c|}
\hline DAY & OCT & NOV & $\mathrm{DEC}$ & JAN & FEB & MAR & APR & MAY & JUN & JUL & AUG & SEP \\
\hline 1 & --- & --- & --- & --- & --- & --- & --- & --- & --- & .55 & .37 & .34 \\
\hline 2 & --- & --- & --- & --- & --- & --- & --- & --- & --- & .50 & .38 & .32 \\
\hline 3 & --- & --- & --- & --- & --- & --- & --- & --- & --- & .50 & .41 & .48 \\
\hline 4 & --- & --- & --- & --- & --- & --- & --- & --- & --- & .51 & .41 & .52 \\
\hline 5 & --- & --- & --- & --- & --- & --- & --- & --- & --- & .52 & .48 & .57 \\
\hline 6 & --- & --- & --- & --- & --- & --- & --- & --- & --- & .30 & .48 & .80 \\
\hline 7 & --- & --- & --- & --- & --- & --- & --- & --- & --- & .30 & .60 & .82 \\
\hline 8 & --- & --- & --- & --- & --- & --- & --- & --- & --- & .68 & .79 & --- \\
\hline 9 & --- & --- & --- & --- & --- & --- & --- & --- & --- & .69 & .61 & .92 \\
\hline 10 & --- & --- & --- & --- & --- & --- & --- & --- & --- & .81 & .64 & .86 \\
\hline 11 & --- & --- & --- & --- & --- & --- & --- & --- & --- & .87 & .54 & .84 \\
\hline 12 & --- & --- & --- & --- & --- & --- & --- & --- & --- & .61 & .51 & .82 \\
\hline 13 & --- & --- & --- & --- & --- & --- & --- & --- & --- & .64 & .45 & .81 \\
\hline 14 & --- & --- & --- & --- & --- & --- & --- & --- & --- & .53 & .41 & --- \\
\hline 15 & --- & --- & --- & --- & --- & --- & --- & --- & --- & .22 & .39 & --- \\
\hline 16 & --- & --- & --- & --- & --- & --- & --- & --- & --- & .13 & .39 & --- \\
\hline 17 & --- & --- & --- & --- & --- & --- & --- & --- & --- & .19 & .39 & --- \\
\hline 18 & --- & --- & --- & --- & --- & --- & --- & --- & --- & .27 & .39 & --- \\
\hline 19 & --- & --- & --- & --- & --- & --- & --- & --- & --- & .25 & .41 & --- \\
\hline 20 & --- & --- & --- & --- & --- & --- & --- & --- & --- & .23 & .41 & --- \\
\hline 21 & --- & --- & --- & --- & --- & --- & --- & --- & --- & .33 & .41 & --- \\
\hline 22 & --- & --- & --- & --- & --- & --- & --- & --- & --- & .45 & .41 & --- \\
\hline 23 & --- & --- & --- & --- & --- & --- & --- & --- & --- & .44 & .40 & --- \\
\hline 24 & --- & --- & --- & --- & --- & --- & --- & --- & --- & .43 & .38 & --- \\
\hline 25 & --- & --- & --- & --- & --- & --- & --- & --- & --- & .42 & .37 & --- \\
\hline 26 & --- & --- & --- & --- & --- & --- & --- & --- & 1.1 & .41 & .41 & --- \\
\hline 27 & --- & --- & --- & --- & --- & --- & --- & --- & 1.1 & .41 & .63 & --- \\
\hline 28 & --- & --- & --- & --- & --- & --- & --- & --- & .85 & .40 & .66 & --- \\
\hline 29 & --- & --- & --- & --- & --- & --- & --- & --- & .79 & .39 & .75 & --- \\
\hline 30 & --- & --- & --- & --- & --- & --- & --- & --- & .75 & .39 & .41 & --- \\
\hline 31 & --- & --- & --- & --- & --- & --- & --- & --- & --- & .37 & .37 & --- \\
\hline TOTAL & --- & --- & --- & --- & --- & --- & --- & --- & --- & 13.74 & 14.66 & --- \\
\hline MEAN & --- & --- & --- & --- & --- & --- & --- & --- & --- & .44 & .47 & --- \\
\hline MAX & --- & --- & --- & --- & --- & --- & --- & --- & --- & .87 & .79 & --- \\
\hline MIN & --- & --- & --- & --- & --- & --- & --- & --- & --- & .13 & .37 & --- \\
\hline $\mathrm{AC}-\mathrm{FT}$ & --- & --- & --- & --- & --- & --- & --- & --- & --- & 27 & 29 & --- \\
\hline
\end{tabular}


11416620 TEXAS CREEK TRIBUTARY BELOW CULBERTSON LAKE, NEAR GRANITEVILLE, CA

LOCATION.-Lat 39²5'17", long 120³7'21", in SW 1/4 SW 1/4 sec.15, T.18 N., R.12 E., Nevada County, Hydrologic Unit 18020125, Tahoe National Forest, on right bank $150 \mathrm{ft}$ downstream from outlet structure on Culbertson Lake Dam, 0.15 mi upstream from Texas Creek, and 6.4 mi east of Graniteville.

DRAINAGE AREA.-0.44 $\mathrm{mi}^{2}$.

PERIOD OF RECORD.- October 1988 to current year (low-flow records only). Unpublished records for water years 1965-88 available in files of the U.S. Geological Survey.

GAGE.-Water-stage recorder and V-notch sharp-crested weir. Elevation of gage is 6,420 ft above sea level. October 1965 to August 1988 , nonrecording gage at site $10 \mathrm{ft}$ downstream at different datum. August to September 1988, nonrecording gage at same site and datum.

REMARKS.- - Records not computed for winter months or above $1.2 \mathrm{ft}^{3} / \mathrm{s}$. Low and medium flow regulated by Culbertson Lake (capacity, 953 acre-ft). See schematic diagram of Yuba River Basin.

COOPERATION.—Records were collected by Pacific Gas \& Electric Co., under general supervision of the U.S. Geological Survey, in connection with a Federal Energy Regulatory Commission project.

DISCHARGE, CUBIC FEET PER SECOND, WATER YEAR OCTOBER 1998 TO SEPTEMBER 1999

DAILY MEAN VALUES

\begin{tabular}{|c|c|c|c|c|c|c|c|c|c|c|c|c|}
\hline DAY & OCT & NOV & $\mathrm{DEC}$ & JAN & $\mathrm{FEB}$ & MAR & $A P R$ & MAY & JUN & JUL & AUG & SEP \\
\hline 1 & --- & --- & --- & --- & --- & --- & --- & --- & --- & .90 & .79 & 1.1 \\
\hline 2 & --- & --- & --- & --- & --- & --- & --- & --- & --- & .89 & .87 & --- \\
\hline 3 & --- & .78 & --- & --- & --- & --- & --- & --- & --- & .85 & .85 & --- \\
\hline 4 & --- & --- & --- & --- & --- & --- & --- & --- & --- & .85 & .85 & --- \\
\hline 5 & --- & --- & --- & --- & --- & --- & --- & --- & --- & .75 & .83 & 1.1 \\
\hline 6 & --- & --- & --- & --- & --- & --- & --- & --- & --- & .59 & .81 & .66 \\
\hline 7 & --- & --- & --- & --- & --- & --- & --- & --- & --- & .73 & .79 & .64 \\
\hline 8 & --- & --- & --- & --- & --- & --- & --- & --- & --- & .96 & .79 & --- \\
\hline 9 & --- & --- & --- & --- & --- & --- & --- & --- & --- & .96 & .79 & --- \\
\hline 10 & --- & --- & --- & --- & --- & --- & --- & --- & --- & .95 & .79 & --- \\
\hline 11 & --- & --- & --- & --- & --- & --- & --- & --- & --- & .92 & .87 & --- \\
\hline 12 & --- & --- & --- & --- & --- & --- & --- & --- & --- & .92 & .92 & --- \\
\hline 13 & --- & --- & --- & --- & --- & --- & --- & --- & --- & .92 & .92 & --- \\
\hline 14 & --- & --- & --- & --- & --- & --- & --- & --- & --- & .91 & .91 & --- \\
\hline 15 & --- & --- & --- & --- & --- & --- & --- & --- & --- & .87 & .89 & --- \\
\hline 16 & --- & --- & --- & --- & --- & --- & --- & --- & --- & .85 & .89 & --- \\
\hline 17 & --- & --- & --- & --- & --- & --- & --- & --- & --- & .83 & .89 & --- \\
\hline 18 & --- & --- & --- & --- & --- & --- & --- & --- & --- & .83 & .89 & --- \\
\hline 19 & --- & --- & --- & --- & --- & --- & --- & --- & --- & .82 & .89 & --- \\
\hline 20 & --- & --- & --- & --- & --- & --- & --- & --- & --- & .81 & .86 & --- \\
\hline 21 & --- & --- & --- & --- & --- & --- & --- & --- & --- & .79 & .86 & --- \\
\hline 22 & --- & --- & --- & --- & --- & --- & --- & --- & --- & .78 & .89 & --- \\
\hline 23 & --- & --- & --- & --- & --- & --- & --- & --- & .76 & .79 & .89 & --- \\
\hline 24 & --- & --- & --- & --- & --- & --- & --- & --- & .71 & .80 & .86 & --- \\
\hline 25 & --- & --- & --- & --- & --- & --- & --- & --- & .72 & .81 & .82 & --- \\
\hline 26 & --- & --- & --- & --- & --- & --- & --- & --- & .72 & .79 & .82 & --- \\
\hline 27 & --- & --- & --- & --- & --- & --- & --- & --- & .70 & .78 & .82 & --- \\
\hline 28 & --- & --- & --- & --- & --- & --- & --- & --- & .68 & .76 & .82 & --- \\
\hline 29 & --- & --- & --- & --- & --- & --- & --- & --- & .67 & .76 & .83 & --- \\
\hline 30 & --- & --- & --- & --- & --- & --- & --- & --- & .80 & .75 & .82 & --- \\
\hline 31 & --- & --- & --- & --- & --- & --- & --- & --- & --- & .73 & .81 & --- \\
\hline TOTAL & --- & --- & --- & --- & --- & --- & --- & --- & --- & 25.65 & 26.33 & --- \\
\hline MEAN & --- & --- & --- & --- & --- & --- & --- & --- & --- & .83 & .85 & --- \\
\hline MAX & --- & --- & --- & --- & --- & --- & --- & --- & --- & .96 & .92 & --- \\
\hline MIN & --- & --- & --- & --- & --- & --- & --- & --- & --- & .59 & .79 & --- \\
\hline $\mathrm{AC}-\mathrm{FT}$ & --- & --- & --- & --- & --- & --- & --- & --- & --- & 51 & 52 & --- \\
\hline
\end{tabular}


LOCATION.-Lat 39²4'43", long 120³8'35", in NE 1/4 SE 1/4 sec.20, T.18 N., R.12 E., Nevada County, Hydrologic Unit 18020125, Tahoe National Forest, on left bank $10 \mathrm{ft}$ downstream from outlet structure on Lower Lindsey Lake Dam and 5.5 mi east of Graniteville.

DRAINAGE AREA.- $-0.91 \mathrm{mi}^{2}$.

PERIOD OF RECORD.-October 1988 to current year (low-flow records only). Unpublished records for water years $1965-88$ available in files of the U.S. Geological Survey.

GAGE.-Water-stage recorder and V-notch sharp-crested weir. Elevation of gage is 6,225 ft above sea level, from topographic map. October 1965 to July 1984, nonrecording gage at same site and different datum. July 1984 to August 1988, nonrecording gage at same site and different datum.

REMARKS.-Records not computed for winter months or above $1.2 \mathrm{ft}^{3} / \mathrm{s}$. Low and medium flow regulated by Lower Lindsey Lake, capacity, 293 acre-ft. Spillway flows bypass this station. See schematic diagram of Yuba River Basin.

COOPERATION.- Records were collected by Pacific Gas \& Electric Co., under general supervision of the U.S. Geological Survey, in connection with a Federal Energy Regulatory Commission project.

DISCHARGE, CUBIC FEET PER SECOND, WATER YEAR OCTOBER 1998 TO SEPTEMBER 1999 DAILY MEAN VALUES

\begin{tabular}{|c|c|c|c|c|c|c|c|c|c|c|c|c|}
\hline DAY & OCT & NOV & $\mathrm{DEC}$ & JAN & $\mathrm{FEB}$ & MAR & $\mathrm{APR}$ & MAY & JUN & JUL & AUG & SEP \\
\hline 1 & --- & --- & --- & --- & --- & --- & --- & --- & --- & .64 & .53 & .82 \\
\hline 2 & --- & --- & --- & --- & --- & --- & --- & --- & --- & .61 & .56 & .53 \\
\hline 3 & --- & --- & --- & --- & --- & --- & --- & --- & --- & .49 & .56 & .51 \\
\hline 4 & --- & --- & --- & --- & --- & --- & --- & --- & --- & .83 & .56 & .52 \\
\hline 5 & --- & --- & --- & --- & --- & --- & --- & --- & --- & --- & .53 & .51 \\
\hline 6 & --- & --- & --- & --- & --- & --- & --- & --- & --- & -- & .49 & .51 \\
\hline 7 & --- & --- & --- & --- & --- & --- & --- & --- & --- & .92 & .48 & .51 \\
\hline 8 & --- & --- & --- & --- & --- & --- & --- & --- & --- & .34 & .50 & .49 \\
\hline 9 & --- & --- & --- & --- & --- & --- & --- & --- & --- & .45 & .52 & .49 \\
\hline 10 & --- & --- & --- & --- & --- & --- & --- & --- & --- & .46 & .51 & .48 \\
\hline 11 & --- & --- & --- & --- & --- & --- & --- & --- & --- & .51 & .51 & .27 \\
\hline 12 & --- & --- & --- & --- & --- & --- & --- & --- & --- & .59 & .51 & .27 \\
\hline 13 & --- & --- & --- & --- & --- & --- & --- & --- & --- & .49 & .52 & .20 \\
\hline 14 & --- & --- & --- & --- & --- & --- & --- & --- & --- & .53 & .56 & .98 \\
\hline 15 & --- & --- & --- & --- & --- & --- & --- & --- & --- & .54 & .56 & --- \\
\hline 16 & --- & --- & --- & --- & --- & --- & --- & --- & --- & .50 & .56 & --- \\
\hline 17 & --- & --- & --- & --- & --- & --- & --- & --- & --- & .52 & .51 & --- \\
\hline 18 & --- & --- & --- & --- & --- & --- & --- & --- & --- & .57 & .48 & --- \\
\hline 19 & --- & --- & --- & --- & --- & --- & --- & --- & --- & .58 & .43 & --- \\
\hline 20 & --- & --- & --- & --- & --- & --- & --- & --- & --- & .56 & .56 & --- \\
\hline 21 & --- & --- & --- & --- & --- & --- & --- & --- & --- & .56 & .92 & --- \\
\hline 22 & --- & --- & --- & --- & --- & --- & --- & --- & .57 & .56 & .89 & --- \\
\hline 23 & --- & --- & --- & --- & --- & --- & --- & --- & .41 & .56 & .87 & --- \\
\hline 24 & --- & --- & --- & --- & --- & --- & --- & --- & .55 & .56 & .85 & --- \\
\hline 25 & --- & --- & --- & --- & --- & --- & --- & --- & .51 & .56 & .71 & --- \\
\hline 26 & --- & --- & --- & --- & --- & --- & --- & --- & .47 & .56 & .56 & --- \\
\hline 27 & --- & --- & --- & --- & --- & --- & --- & --- & .46 & .56 & .54 & --- \\
\hline 28 & --- & --- & --- & --- & --- & --- & --- & --- & .74 & .56 & .89 & --- \\
\hline 29 & --- & --- & --- & --- & --- & --- & --- & --- & .89 & .55 & 1.2 & --- \\
\hline 30 & e1.0 & --- & --- & --- & --- & --- & --- & --- & .67 & .55 & 1.1 & --- \\
\hline 31 & --- & --- & --- & --- & --- & --- & --- & --- & --- & .53 & 1.1 & --- \\
\hline TOTAL & --- & --- & --- & --- & --- & --- & --- & --- & --- & --- & 20.07 & --- \\
\hline MEAN & --- & --- & --- & --- & --- & --- & --- & --- & --- & --- & .65 & --- \\
\hline MAX & --- & --- & --- & --- & --- & --- & --- & --- & --- & --- & 1.2 & --- \\
\hline MIN & --- & --- & --- & --- & --- & --- & --- & --- & --- & --- & .43 & --- \\
\hline $\mathrm{AC}-\mathrm{FT}$ & --- & --- & --- & --- & --- & --- & --- & --- & --- & --- & 40 & --- \\
\hline
\end{tabular}




\section{SOUTH YUBA RIVER AT JONES BAR, NEAR GRASS VALLEY, CA}

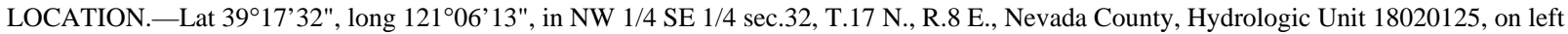
bank at Jones Bar, $100 \mathrm{ft}$ upstream from Rush Creek, 0.9 mi downstream from bridge on State Highway 49, and 5 mi northwest of Grass Valley. DRAINAGE AREA.-308 $\mathrm{mi}^{2}$.

PERIOD OF RECORD.-October 1940 to September 1948, April 1959 to current year. Published as South Fork Yuba River at Jones Bar 1940-48, and as South Yuba River at Jones Bar 1959-63. Yearly discharge for the 1947 water year published in WSP 1315-A. SEDIMENT DATA: Water years 1966-74.

WATER TEMPERATURE: Water years 1965-79 (daily records).

REVISED RECORDS.-WSP 1315-A: 1942-43(M), drainage area at former site. WSP 1931: Drainage area.

GAGE.-Water-stage recorder and crest-stage gage. Elevation of gage is 1,060 ft above sea level, from river-profile map. Oct. 1, 1940, to Sept. 30, 1948, at site $150 \mathrm{ft}$ upstream at datum $2.00 \mathrm{ft}$ higher.

REMARKS.- - Records fair. Flow regulated by Lake Spaulding, Fordyce Lake, and Bowman Lake (stations 11414140, 11414090, and 11415500) and many smaller reservoirs. Diversions into and out of basin for several powerplants and for irrigation. See schematic diagram of Yuba River Basin.

EXTREMES FOR PERIOD OF RECORD.-Maximum discharge, 53,600 ft ${ }^{3} / \mathrm{s}$, Dec. 22, 1964, gage height, $25.0 \mathrm{ft}$, from floodmarks, from rating curve extended above $23,000 \mathrm{ft}^{3} / \mathrm{s}$ on basis of slope-area measurement of peak flow; minimum daily, $1.0 \mathrm{ft}^{3} / \mathrm{s}, \mathrm{Sept}$. $10-13,1944$.

EXTREMES OUTSIDE PERIOD OF RECORD.-Flood of Dec. 23, 1955, reached a stage of $30.7 \mathrm{ft}$, from floodmarks, present datum, at site $100 \mathrm{ft}$ upstream.

DISCHARGE, CUBIC FEET PER SECOND, WATER YEAR OCTOBER 1998 TO SEPTEMBER 1999 DAILY MEAN VALUES

\begin{tabular}{|c|c|c|c|c|c|c|c|c|c|c|c|c|}
\hline DAY & $\mathrm{OCT}$ & NOV & $\mathrm{DEC}$ & JAN & FEB & MAR & $A P R$ & MAY & JUN & JUL & AUG & SEP \\
\hline 1 & 69 & 76 & 1270 & 133 & 397 & 2170 & 452 & 528 & 2150 & 96 & 63 & 55 \\
\hline 2 & 67 & 78 & 548 & 127 & 359 & 1450 & 406 & 554 & 1630 & 93 & 62 & 55 \\
\hline 3 & 67 & 70 & 1610 & 123 & 340 & 1890 & 418 & 705 & 1150 & 91 & 62 & 56 \\
\hline 4 & 67 & 68 & 981 & 120 & 330 & 1440 & 411 & 661 & 733 & 90 & 61 & 55 \\
\hline 5 & 66 & 69 & 531 & 118 & 318 & 1170 & 443 & 584 & 698 & 90 & 60 & 54 \\
\hline 6 & 64 & 71 & 408 & 116 & 411 & 1020 & 437 & 574 & 534 & 89 & 61 & 53 \\
\hline 7 & 63 & 130 & 320 & 115 & 2740 & 902 & 418 & 581 & 619 & 87 & 63 & 53 \\
\hline 8 & 62 & 162 & 295 & 112 & 2710 & 851 & 583 & 582 & 595 & 80 & 64 & 52 \\
\hline 9 & 62 & 98 & 278 & 107 & 6750 & 1010 & 527 & 577 & 597 & 72 & 63 & 52 \\
\hline 10 & 62 & 84 & 247 & 103 & 2690 & 810 & 476 & 557 & 597 & 71 & 64 & 52 \\
\hline 11 & 62 & 109 & 229 & 102 & 1670 & 734 & 581 & 514 & 602 & 76 & 68 & 52 \\
\hline 12 & 63 & 98 & 220 & 101 & 1260 & 797 & 604 & 530 & 670 & 83 & 64 & 52 \\
\hline 13 & 64 & 89 & 222 & 100 & 1030 & 766 & 613 & 561 & 923 & 103 & 61 & 50 \\
\hline 14 & 64 & 86 & 258 & 99 & 888 & 754 & 670 & 590 & 1220 & 104 & 60 & 50 \\
\hline 15 & 62 & 84 & 229 & 112 & 771 & 713 & 691 & 556 & 1580 & 99 & 59 & 50 \\
\hline 16 & 60 & 83 & 226 & 262 & 940 & 681 & 676 & 541 & 1550 & 96 & 59 & 50 \\
\hline 17 & 59 & 122 & 230 & 279 & 2560 & 663 & 709 & 535 & 1170 & 100 & 59 & 50 \\
\hline 18 & 59 & 160 & 215 & 1390 & 1690 & 647 & 719 & 531 & 857 & 94 & 58 & 50 \\
\hline 19 & 58 & 113 & 200 & 2100 & 1560 & 640 & 711 & 529 & 841 & 91 & 58 & 54 \\
\hline 20 & 57 & 100 & 195 & 4060 & 1270 & 653 & 710 & 534 & 802 & 89 & 57 & 58 \\
\hline 21 & 57 & 94 & 174 & 2080 & 1500 & 634 & 714 & 535 & 748 & 75 & 56 & 58 \\
\hline 22 & 66 & 252 & 165 & 1090 & 1130 & 603 & 677 & 568 & 751 & 73 & 56 & 57 \\
\hline 23 & 62 & 409 & 155 & 2710 & 1020 & 590 & 635 & 603 & 791 & 70 & 55 & 57 \\
\hline 24 & 67 & 902 & 153 & 1450 & 939 & 583 & 602 & 638 & 734 & 72 & 54 & 57 \\
\hline 25 & 104 & 293 & 149 & 975 & 1390 & 612 & 598 & 644 & 663 & 73 & 54 & 59 \\
\hline 26 & 78 & 204 & 148 & 775 & 1080 & 512 & 646 & 651 & 515 & 62 & 54 & 63 \\
\hline 27 & 69 & 277 & 144 & 625 & 948 & 506 & 719 & 791 & 246 & 61 & 57 & 57 \\
\hline 28 & 68 & 368 & 140 & 532 & 1090 & 486 & 624 & 1630 & 201 & 59 & 57 & 57 \\
\hline 29 & 105 & 307 & 137 & 469 & --- & 459 & 582 & 2140 & 147 & 58 & 55 & 55 \\
\hline 30 & 71 & 2070 & 133 & 423 & --- & 468 & 530 & 2190 & 102 & 57 & 54 & 55 \\
\hline 31 & 68 & --- & 134 & 439 & --- & 502 & --- & 2260 & --- & 58 & 54 & --- \\
\hline TOTAL & 2072 & 7126 & 10344 & 21347 & 39781 & 25716 & 17582 & 23974 & 24416 & 2512 & 1832 & 1628 \\
\hline MEAN & 66.8 & 238 & 334 & 689 & 1421 & 830 & 586 & 773 & 814 & 81.0 & 59.1 & 54.3 \\
\hline MAX & 105 & 2070 & 1610 & 4060 & 6750 & 2170 & 719 & 2260 & 2150 & 104 & 68 & 63 \\
\hline MIN & 57 & 68 & 133 & 99 & 318 & 459 & 406 & 514 & 102 & 57 & 54 & 50 \\
\hline $\mathrm{AC}-\mathrm{FT}$ & 4110 & 14130 & 20520 & 42340 & 78910 & 51010 & 34870 & 47550 & 48430 & 4980 & 3630 & 3230 \\
\hline
\end{tabular}




\section{SOUTH YUBA RIVER AT JONES BAR, NEAR GRASS VALLEY, CA—Continued}

STATISTICS OF MONTHLY MEAN DATA FOR WATER YEARS 1941 - 1999, BY WATER YEAR (WY)

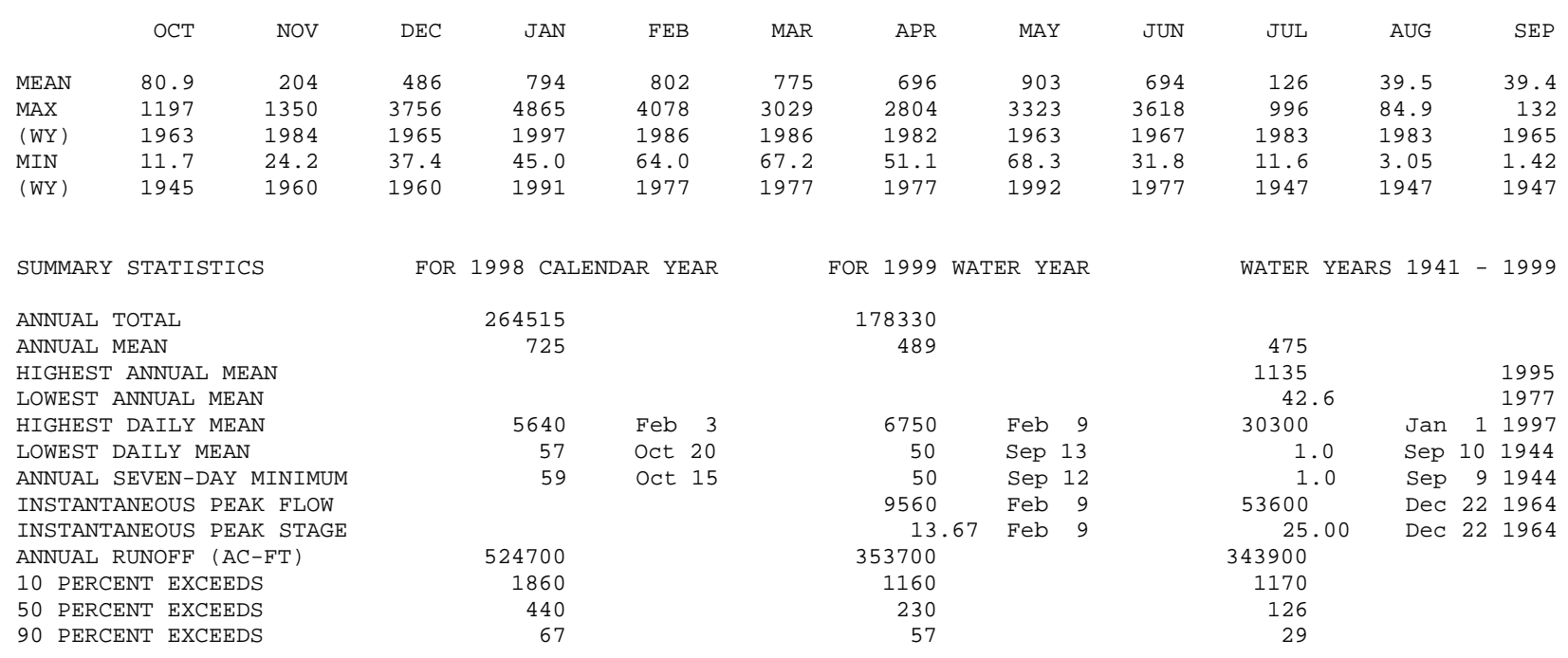


11418000 YUBA RIVER BELOW ENGLEBRIGHT DAM, NEAR SMARTVILLE, CA

LOCATION.-Lat $39^{\circ} 14^{\prime} 07^{\prime \prime}$, long $121^{\circ} 16^{\prime} 23^{\prime \prime}$, in NW 1/4 NW 1/4 sec.23, T.16 N., R.6 E., Yuba County, Hydrologic Unit 18020125, on right bank 2,000 ft downstream from Englebright Dam, $0.5 \mathrm{mi}$ upstream from Deer Creek, and $2.3 \mathrm{mi}$ northeast of Smartville.

DRAINAGE AREA.-1,108 $\mathrm{mi}^{2}$.

PERIOD OF RECORD.—October 1941 to current year. Prior to October 1953, published as "at Narrows Dam." October 1953 to September 1969 , published as "at Englebright Dam." If records for Deer Creek near Smartville (station 11418500) since 1941 are added to records at this station, records equivalent to those published from 1903 to 1941 as Yuba River at Smartville (station 11419000) can be obtained.

WATER TEMPERATURE: Water years 1973-78.

REVISED RECORDS.-WSP 1931: Drainage area.

GAGE.-Water-stage recorder and acoustic-velocity meters. Datum of gage is $278.68 \mathrm{ft}$ above sea level (levels by International Engineering Co.). Prior to Sept. 19, 1958, at site 2,000 ft upstream at datum $248.31 \mathrm{ft}$ higher, and Sept. 19, 1958, to Sept. 30, 1969, at datum $278.68 \mathrm{ft}$ lower. Supplementary gage 2,000 ft upstream since Oct. 1, 1969, at Englebright Dam at datum $248.31 \mathrm{ft}$ higher.

REMARKS.-Diversions up to $1,800 \mathrm{ft}^{3} / \mathrm{s}$ (see stations 11413250, 11414190, and 11414200) out of basin for power and irrigation upstream from station. Flow regulation by Lake Spaulding (station 11414140), Jackson Meadows and New Bullards Bar Reservoirs (stations 11407800 and 11413515), Englebright Reservoir beginning in 1941, capacity, 70,000 acre-ft, Bowman and Fordyce Lakes (stations 11415500 and 11414090), and many smaller reservoirs. Flow is determined by adding the discharges provided by Narrows Powerplant No. 1 (11417970), Narrows Powerplant No. 2 (11417980), and spill over Englebright Dam (11417950). See schematic diagram of Yuba River Basin.

COOPERATION.--Records were collected by Pacific Gas \& Electric Co., under general supervision of the U.S. Geological Survey, in connection with a Federal Energy Regulatory Commission project.

EXTREMES FOR PERIOD OF RECORD.-Maximum discharge, 171,000 ft $\mathrm{ft}^{3} / \mathrm{s}$, Dec. 22, 1964, gage height, $546.14 \mathrm{ft}$, site and datum then in use, from rating curve extended above $25,000 \mathrm{ft}^{3} / \mathrm{s}$ on basis of computation of peak flow over spillway of dam at gage heights 544.72 and $546.14 \mathrm{ft}$; no flow at times in 1942, 1949, 1956, 1958-61, 1968-69.

REVISIONS.-The maximum discharge for the water-year 1997 has been revised to 154,000 ft $3 / \mathrm{s}$, Jan. 2, 1997, gage height, $41.53 \mathrm{ft}$.

DISCHARGE, CUBIC FEET PER SECOND, WATER YEAR OCTOBER 1998 TO SEPTEMBER 1999 DAILY MEAN VALUES

\begin{tabular}{|c|c|c|c|c|c|c|c|c|c|c|c|c|}
\hline DAY & $\mathrm{OCT}$ & NOV & $\mathrm{DEC}$ & JAN & FEB & MAR & APR & MAY & JUN & JUL & AUG & SEP \\
\hline 1 & 1210 & 1370 & 4240 & 2650 & 3460 & 8920 & 3090 & 2970 & 3200 & 2280 & 2510 & 2080 \\
\hline 2 & 1210 & 1360 & 2680 & 2640 & 3560 & 10400 & 3090 & 2970 & 2950 & 2280 & 2660 & 1590 \\
\hline 3 & 1210 & 1360 & 2620 & 2610 & 3540 & 10800 & 3100 & 2970 & 2790 & 2270 & 2770 & 1520 \\
\hline 4 & 1210 & 1360 & 3200 & 2600 & 3540 & 10100 & 3110 & 3000 & 2790 & 2260 & 2770 & 1510 \\
\hline 5 & 1210 & 1370 & 2570 & 2570 & 3510 & 9490 & 3100 & 3020 & 2610 & 2260 & 2780 & 1510 \\
\hline 6 & 1210 & 1360 & 2550 & 2550 & 3490 & 8050 & 3120 & 2390 & 2480 & 2260 & 2770 & 1500 \\
\hline 7 & 1200 & 1360 & 2530 & 2510 & 4050 & 7000 & 3140 & 1640 & 2590 & 2260 & 2760 & 1260 \\
\hline 8 & 1200 & 1370 & 2510 & 2480 & 7210 & 5710 & 3140 & 2690 & 2680 & 2260 & 2770 & 1210 \\
\hline 9 & 1200 & 1380 & 2530 & 2460 & 18600 & 4630 & 3370 & 2740 & 2110 & 2260 & 2770 & 1210 \\
\hline 10 & 1210 & 1380 & 2530 & 2450 & 9440 & 4540 & 3730 & 2350 & 2510 & 2260 & 2770 & 1210 \\
\hline 11 & 1210 & 1660 & 2550 & 2430 & 6180 & 4400 & 3900 & 2400 & 2510 & 2260 & 2770 & 1210 \\
\hline 12 & 1210 & 1800 & 2560 & 2410 & 5220 & 4420 & 3920 & 2560 & 2520 & 2270 & 2770 & 1210 \\
\hline 13 & 1210 & 1820 & 2550 & 2420 & 4740 & 4420 & 3950 & 2580 & 2510 & 2270 & 2740 & 1210 \\
\hline 14 & 1220 & 1820 & 2540 & 2360 & 4530 & 4350 & 3860 & 2600 & 2500 & 1130 & 2770 & 1210 \\
\hline 15 & 1220 & 1810 & 2540 & 2320 & 4420 & 4350 & 3660 & 2610 & 2500 & 2510 & 2760 & 1180 \\
\hline 16 & 1210 & 1790 & 2550 & 2270 & 4430 & 4330 & 3680 & 2570 & 2420 & 2500 & 2760 & 1210 \\
\hline 17 & 1210 & 1800 & 2550 & 2230 & 8800 & 4330 & 3740 & 2540 & 2110 & 2490 & 2760 & 1210 \\
\hline 18 & 1210 & 1810 & 2550 & 2170 & 6120 & 4300 & 3850 & 2560 & 2520 & 2470 & 2750 & 1210 \\
\hline 19 & 1210 & 1750 & 2570 & 3420 & 6060 & 4110 & 3710 & 2560 & 2530 & 2390 & 2760 & 1210 \\
\hline 20 & 1210 & 1810 & 2580 & 9340 & 5250 & 4090 & 3490 & 2210 & 2510 & 2470 & 2780 & 1210 \\
\hline 21 & 1210 & 1810 & 2660 & 6170 & 6350 & 4080 & 3230 & 2380 & 2470 & 2480 & 2780 & 1170 \\
\hline 22 & 1210 & 1810 & 2730 & 4890 & 5180 & 4060 & 3230 & 2360 & 2480 & 2280 & 2770 & 1210 \\
\hline 23 & 1210 & 1810 & 2740 & 9300 & 4900 & 4020 & 2970 & 2360 & 2480 & 2500 & 2770 & 1150 \\
\hline 24 & 1210 & 1820 & 2740 & 5800 & 4760 & 4020 & 3000 & 1020 & 2480 & 2520 & 2770 & 1180 \\
\hline 25 & 1210 & 1820 & 2720 & 4120 & 5240 & 3800 & 2990 & 2380 & 2030 & 2490 & 2770 & 1180 \\
\hline 26 & 1310 & 1820 & 2710 & 3320 & 4990 & 4010 & 2990 & 2410 & 2110 & 2470 & 2780 & 1180 \\
\hline 27 & 1370 & 1830 & 2690 & 3300 & 4510 & 3910 & 2990 & 2120 & 1800 & 2480 & 2780 & 1180 \\
\hline 28 & 1370 & 1920 & 2680 & 3260 & 4720 & 3860 & 2990 & 3270 & 2260 & 2490 & 2780 & 1180 \\
\hline 29 & 1370 & 2320 & 2660 & 3280 & --- & 3860 & 2990 & 3140 & 2260 & 2480 & 2180 & 1180 \\
\hline 30 & 1370 & 2710 & 2660 & 3340 & --- & 3550 & 2970 & 3270 & 2270 & 2490 & 2090 & 1180 \\
\hline 31 & 1370 & --- & 2610 & 3270 & --- & 3150 & --- & 3210 & --- & 2500 & 2090 & --- \\
\hline TOTAL & 38400 & 51210 & 83100 & 106940 & 156800 & 165060 & 100100 & 79850 & 73980 & 72590 & 83510 & 38460 \\
\hline MEAN & 1239 & 1707 & 2681 & 3450 & 5600 & 5325 & 3337 & 2576 & 2466 & 2342 & 2694 & 1282 \\
\hline MAX & 1370 & 2710 & 4240 & 9340 & 18600 & 10800 & 3950 & 3270 & 3200 & 2520 & 2780 & 2080 \\
\hline MIN & 1200 & 1360 & 2510 & 2170 & 3460 & 3150 & 2970 & 1020 & 1800 & 1130 & 2090 & 1150 \\
\hline $\mathrm{AC}-\mathrm{FT}$ & 76170 & 101600 & 164800 & 212100 & 311000 & 327400 & 198500 & 158400 & 146700 & 144000 & 165600 & 76290 \\
\hline $\mathrm{a}$ & 19970 & 18450 & 12780 & 7620 & 195 & 234 & 12130 & 44940 & 45680 & 55010 & 46050 & 13920 \\
\hline
\end{tabular}

a Combined flow, in acre-feet, from Browns Valley Irrigation Ditch (11420750), Brophy South Canal (11420760) and Hallwood-Cordua Irrigation District Canal (11420770). 


\section{YUBA RIVER BELOW ENGLEBRIGHT DAM, NEAR SMARTVILLE, CA—Continued}

STATISTICS OF MONTHLY MEAN DATA FOR WATER YEARS 1942 - 1999, BY WATER YEAR (WY)

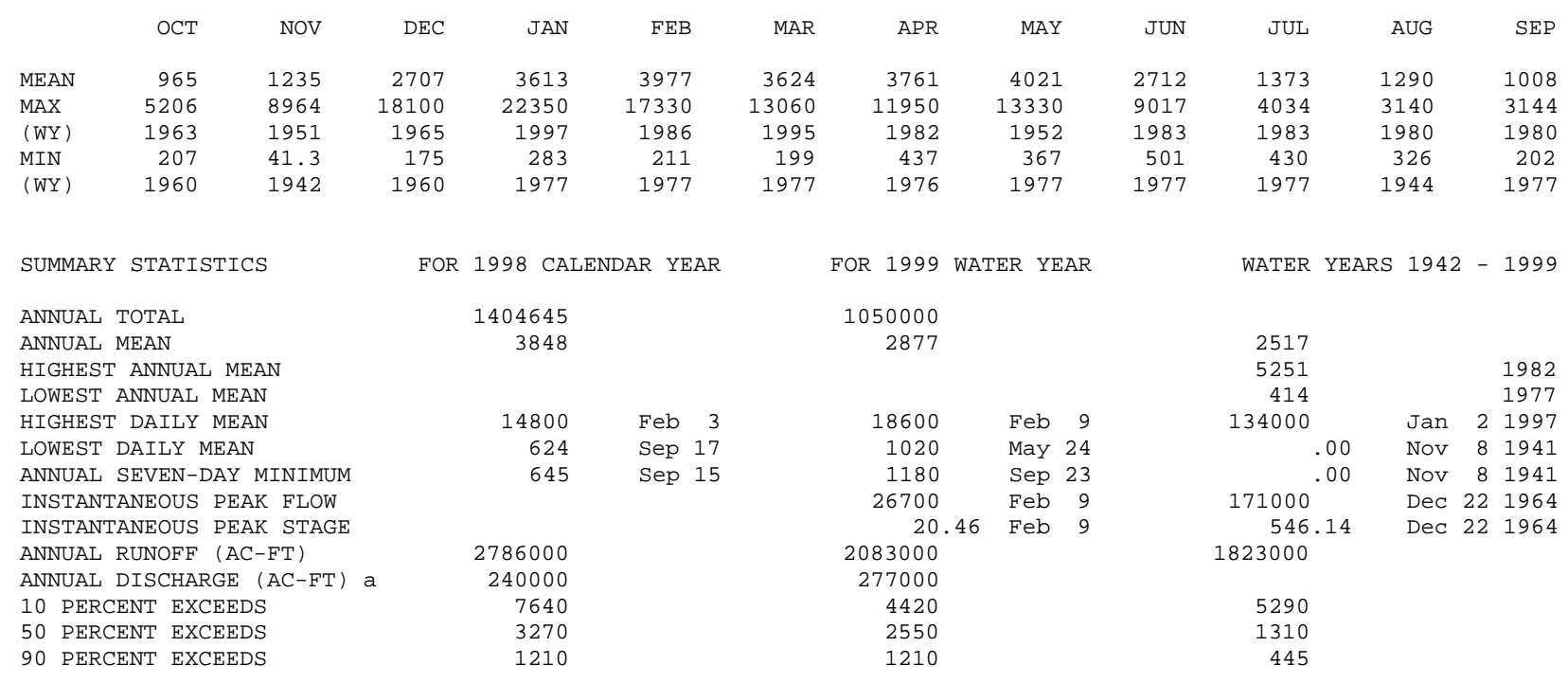

a Combined flow, in acre-feet, from Browns Valley Irrigation Ditch (11420750), Brophy South Canal (11420760) and Hallwood-Cordua Irrigation District Canal (11420770). 
LOCATION.-Lat $39^{\circ} 13^{\prime} 28^{\prime \prime}$, long $121^{\circ} 16^{\prime} 03 "$, in SW 1/4 SE 1/4 sec.23, T.16 N., R.6 E., Nevada County, Hydrologic Unit 18020125, on left bank $400 \mathrm{ft}$ upstream from county road bridge, $0.9 \mathrm{mi}$ upstream from mouth, and 2 mi northeast of Smartville.

DRAINAGE AREA.- $84.6 \mathrm{mi}^{2}$.

PERIOD OF RECORD.- June 1935 to current year.

WATER TEMPERATURE: Water years 1974-79.

SEDIMENT DATA: Water years 1974-79.

REVISED RECORDS.-WSP 1395: Drainage area.

GAGE.-Water-stage recorder. Elevation of gage is $630 \mathrm{ft}$ above sea level, from river-profile map. June 21, 1935, to Nov. 30, 1938, nonrecording gage at same site and datum.

REMARKS.- - Records good. Natural flow of stream is affected by Scotts Flat Reservoir beginning in 1949, usable capacity, 26,300 acre-ft, increased to 49,000 acre-ft in July 1964; Deer Creek Reservoir, capacity, 1,400 acre-ft beginning 1949; Lake Wildwood, capacity, 3,840 acre-ft beginning in 1970, power developments, and diversion for irrigation. At times water from South Yuba River is diverted to Deer Creek and water from Deer Creek is diverted to Bear River. See schematic diagram of Yuba River Basin.

EXTREMES FOR PERIOD OF RECORD.-Maximum discharge, 12,100 ft³/s, Feb. 17, 1986, gage height, $14.05 \mathrm{ft}$, from rating curve extended above $5,200 \mathrm{ft}^{3} / \mathrm{s}$; minimum daily, $0.06 \mathrm{ft}^{3} / \mathrm{s}$, Aug. 5,1977 .

EXTREMES OUTSIDE PERIOD OF RECORD.-Flood of March 1928 reached a stage of $14.5 \mathrm{ft}$ from floodmarks, discharge, $14,000 \mathrm{ft}{ }^{3} / \mathrm{s}$.

DISCHARGE, CUBIC FEET PER SECOND, WATER YEAR OCTOBER 1998 TO SEPTEMBER 1999 DAILY MEAN VALUES

\begin{tabular}{|c|c|c|c|c|c|c|c|c|c|c|c|c|}
\hline DAY & $\mathrm{OCT}$ & NOV & $\mathrm{DEC}$ & JAN & FEB & MAR & APR & MAY & JUN & JUL & AUG & SEP \\
\hline 1 & 12 & 16 & 349 & 28 & 280 & 677 & 175 & 46 & 10 & 7.9 & 6.2 & 4.5 \\
\hline 2 & 11 & 18 & 112 & 27 & 206 & 526 & 160 & 49 & 14 & 8.2 & 5.8 & 4.9 \\
\hline 3 & 9.9 & 17 & 1080 & 27 & 174 & 755 & 157 & 69 & 19 & 8.0 & 5.5 & 5.6 \\
\hline 4 & 9.5 & 16 & 334 & 25 & 172 & 539 & 151 & 117 & 20 & 7.8 & 5.4 & 7.7 \\
\hline 5 & 8.8 & 17 & 128 & 24 & 166 & 459 & 201 & 114 & 19 & 8.0 & 5.5 & 8.3 \\
\hline 6 & 6.5 & 14 & 116 & 24 & 251 & 413 & 225 & 90 & 15 & 6.3 & 5.1 & 6.9 \\
\hline 7 & 8.3 & 26 & 87 & 24 & 1740 & 370 & 181 & 52 & 12 & 6.6 & 6.5 & 4.8 \\
\hline 8 & 9.4 & 45 & 78 & 24 & 1540 & 359 & 287 & 69 & 12 & 8.2 & 7.3 & 4.3 \\
\hline 9 & 8.1 & 36 & 76 & 23 & 3880 & 529 & 268 & 87 & 9.8 & 7.7 & 6.3 & 4.3 \\
\hline 10 & 7.8 & 28 & 59 & 23 & 1340 & 362 & 201 & 66 & 9.8 & 7.5 & 7.0 & 4.3 \\
\hline 11 & 7.8 & 42 & 50 & 23 & 797 & 317 & 269 & 51 & 9.9 & 7.3 & 7.8 & 5.9 \\
\hline 12 & 8.2 & 34 & 46 & 22 & 592 & 291 & 201 & 43 & 11 & 5.8 & 8.1 & 6.7 \\
\hline 13 & 8.2 & 28 & 47 & 22 & 483 & 273 & 169 & 38 & 10 & 5.3 & 7.9 & 6.5 \\
\hline 14 & 8.3 & 24 & 78 & 22 & 431 & 261 & 139 & 33 & 9.7 & 5.2 & 8.8 & 5.6 \\
\hline 15 & 8.3 & 23 & 56 & 26 & 375 & 250 & 121 & 31 & 9.5 & 5.4 & 7.1 & 4.9 \\
\hline 16 & 7.4 & 19 & 48 & 71 & 711 & 237 & 120 & 29 & 9.5 & 5.4 & 5.6 & 5.0 \\
\hline 17 & 6.6 & 11 & 41 & 90 & 1720 & 226 & 111 & 27 & 10 & 5.6 & 5.8 & 4.9 \\
\hline 18 & 143 & 8.6 & 39 & 698 & 914 & 215 & 102 & 26 & 9.4 & 6.2 & 5.3 & 5.6 \\
\hline 19 & 301 & 7.2 & 38 & 1340 & 701 & 197 & 98 & 25 & 11 & 5.4 & 5.4 & 5.7 \\
\hline 20 & 279 & 6.5 & 37 & 1200 & 675 & 197 & 97 & 23 & 12 & 5.5 & 5.9 & 4.7 \\
\hline 21 & 252 & 6.5 & 35 & 350 & 1270 & 193 & 89 & 23 & 11 & 6.8 & 7.0 & 5.2 \\
\hline 22 & 215 & 21 & 35 & 262 & 587 & 189 & 78 & 20 & 12 & 7.5 & 7.6 & 5.2 \\
\hline 23 & 103 & 85 & 33 & 1460 & 469 & 186 & 62 & 17 & 11 & 7.5 & 6.0 & 4.8 \\
\hline 24 & 48 & 85 & 32 & 470 & 413 & 192 & 65 & 15 & 9.2 & 7.5 & 5.9 & 5.3 \\
\hline 25 & 42 & 19 & 32 & 442 & 663 & 218 & 56 & 19 & 7.8 & 8.0 & 5.9 & 5.8 \\
\hline 26 & 29 & 13 & 33 & 393 & 482 & 177 & 57 & 18 & 8.5 & 6.3 & 5.2 & 5.5 \\
\hline 27 & 22 & 83 & 32 & 342 & 412 & 165 & 56 & 13 & 7.5 & 8.4 & 5.4 & 3.9 \\
\hline 28 & 18 & 85 & 31 & 277 & 549 & 157 & 50 & 13 & 7.3 & 8.9 & 6.4 & 4.1 \\
\hline 29 & 15 & 69 & 29 & 240 & --- & 152 & 49 & 12 & 7.9 & 7.3 & 6.5 & 3.1 \\
\hline 30 & 14 & 462 & 29 & 214 & --- & 160 & 47 & 14 & 8.3 & 7.1 & 5.0 & 3.1 \\
\hline 31 & 13 & --- & 29 & 317 & --- & 223 & --- & 13 & --- & 7.2 & 5.0 & --- \\
\hline TOTAL & 1640.1 & 1364.8 & 3249 & 8530 & 21993 & 9465 & 4042 & 1262 & 333.1 & 215.8 & 194.2 & 157.1 \\
\hline MEAN & 52.9 & 45.5 & 105 & 275 & 785 & 305 & 135 & 40.7 & 11.1 & 6.96 & 6.26 & 5.24 \\
\hline MAX & 301 & 462 & 1080 & 1460 & 3880 & 755 & 287 & 117 & 20 & 8.9 & 8.8 & 8.3 \\
\hline MIN & 6.5 & 6.5 & 29 & 22 & 166 & 152 & 47 & 12 & 7.3 & 5.2 & 5.0 & 3.1 \\
\hline $\mathrm{AC}-\mathrm{FT}$ & 3250 & 2710 & 6440 & 16920 & 43620 & 18770 & 8020 & 2500 & 661 & 428 & 385 & 312 \\
\hline
\end{tabular}




\section{DEER CREEK NEAR SMARTVILLE, CA—Continued}

STATISTICS OF MONTHLY MEAN DATA FOR WATER YEARS 1936 - 1999, BY WATER YEAR (WY)

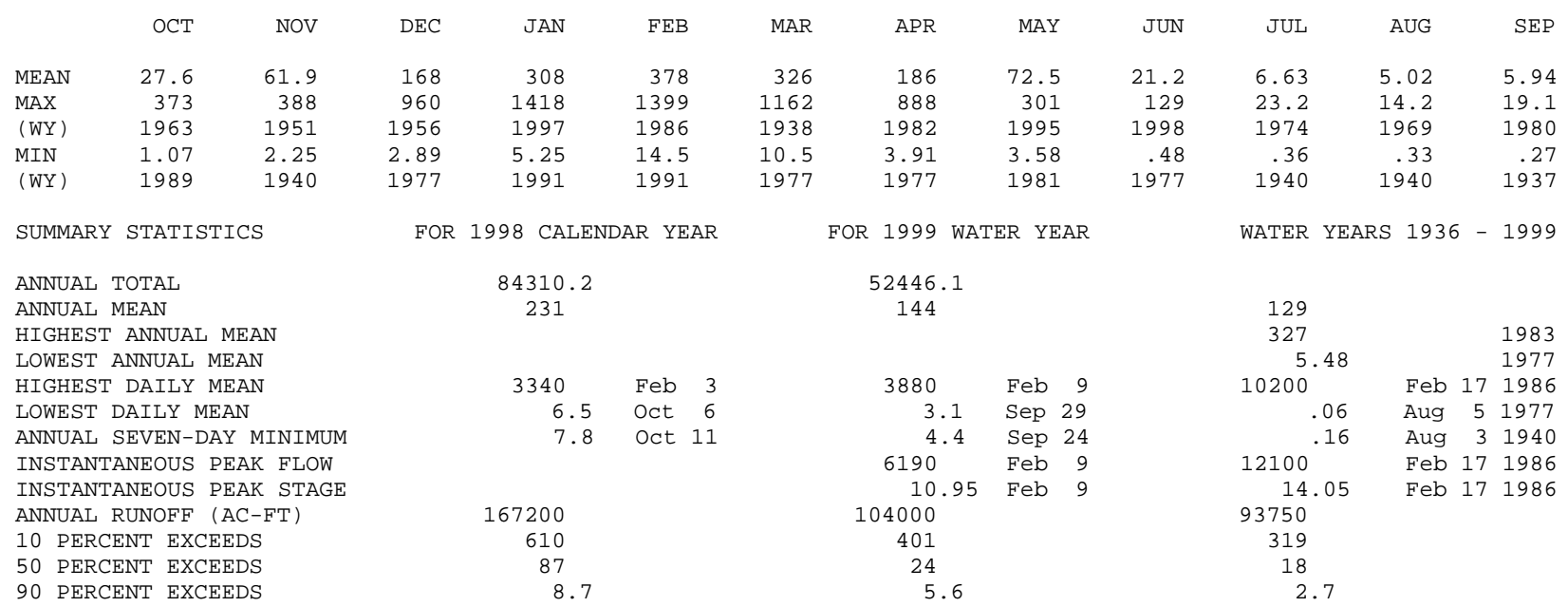




\section{YUBA RIVER NEAR MARYSVILLE, CA}

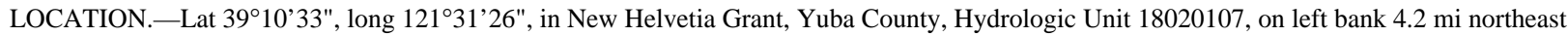
of Marysville and $5 \mathrm{mi}$ downstream from Dry Creek.

DRAINAGE AREA.-1,339 $\mathrm{mi}^{2}$.

\section{WATER-DISCHARGE RECORDS}

PERIOD OF RECORD.-October 1940 to current year (prior to October 1943, low-water periods only). Published as "at Marysville" October 1940 to September 1957. Separate records published for two sites August 1954 to September 1955. Yearly discharge for the 1945 water year published in WSP 1315-A.

REVISED RECORDS.-WSP 1715: 1956(M). WSP 1931: Drainage area.

GAGE.-Water-stage recorder and crest-stage gage. Datum of gage is $2.95 \mathrm{ft}$ below sea level. Prior to August 1954 and Oct. 1, 1956, to Sept. 30, 1957, at Simpson Lane Bridge in Marysville 4.2 mi downstream at same datum. Sept. 3, 1963, to Sept. 23, 1968, auxiliary water-stage recorder at Simpson Lane Bridge at same datum.

REMARKS. - Records good. Flow regulated by New Bullards Bar Reservoir since January 1969, and several other reservoirs. Many diversions upstream from station for power and for irrigation. See schematic diagrams of Yuba and lower Sacramento River Basins.

EXTREMES FOR PERIOD OF RECORD.-Maximum discharge (water years 1944, 1947-99), 180,000 ft³/s, Dec. 22, 1964, gage height, $90.15 \mathrm{ft}$, from floodmarks, from rating curve extended above $91,000 \mathrm{ft}^{3} / \mathrm{s}$ on basis of U.S. Army Corps of Engineers flood-routing study, maximum gage height $91.64 \mathrm{ft}$, from floodmarks, Jan. 2, 1997; minimum recorded, $10 \mathrm{ft}^{3} / \mathrm{s}$, July 2, 1959.

REVISIONS.-The maximum discharge for the water year 1997 has been revised to $161,000 \mathrm{ft}^{3} / \mathrm{s}$, Jan. 2, 1997, gage height, $91.64 \mathrm{ft}$.

DISCHARGE, CUBIC FEET PER SECOND, WATER YEAR OCTOBER 1998 TO SEPTEMBER 1999

DAILY MEAN VALUES

\begin{tabular}{|c|c|c|c|c|c|c|c|c|c|c|c|c|}
\hline DAY & OCT & NOV & $\mathrm{DEC}$ & JAN & $\mathrm{FEB}$ & MAR & APR & MAY & JUN & JUL & AUG & SEP \\
\hline 1 & 1080 & 1070 & 4730 & 2560 & 4230 & 10200 & 3600 & 2460 & 2750 & 1510 & 1820 & 1720 \\
\hline 2 & 1060 & 1050 & 2920 & 2530 & 4230 & 11800 & 3590 & 2480 & 2650 & 1520 & 1920 & 1410 \\
\hline 3 & 1050 & 1060 & 3640 & 2510 & 4130 & 12200 & 3580 & 2510 & 2400 & 1510 & 2080 & 1200 \\
\hline 4 & 1040 & 1060 & 3790 & 2490 & 4090 & 11400 & 3580 & 2530 & 2420 & 1510 & 2060 & 1190 \\
\hline 5 & 1030 & 1060 & 2730 & 2470 & 4060 & 10000 & 3640 & 2510 & 2290 & 1510 & 2080 & 1200 \\
\hline 6 & 1040 & 1080 & 2740 & 2430 & 4120 & 8860 & 3720 & 2400 & 2080 & 1510 & 2100 & 1230 \\
\hline 7 & 1030 & 1140 & 2630 & 2410 & 7170 & 7940 & 3690 & 2210 & 2060 & 1500 & 2110 & 1110 \\
\hline 8 & 961 & 1170 & 2570 & 2360 & 10300 & 6760 & 3840 & 2210 & 2030 & 1490 & 2120 & 1040 \\
\hline 9 & 910 & 1170 & 2570 & 2340 & e28000 & 5850 & 4320 & 2230 & 2040 & 1480 & 2120 & 1070 \\
\hline 10 & 932 & 1150 & 2580 & 2320 & e16400 & 5430 & 4480 & 2190 & 2020 & 1480 & 2130 & 1100 \\
\hline 11 & 950 & 1340 & 2550 & 2300 & 8190 & 5150 & 4720 & 1910 & 1940 & 1500 & 2150 & 1090 \\
\hline 12 & 956 & 1560 & 2530 & 2260 & 6760 & 5040 & 4660 & 1920 & 1880 & 1490 & 2170 & 1080 \\
\hline 13 & 958 & 1560 & 2530 & 2270 & 5930 & 5000 & 4580 & 1930 & 1890 & 1490 & 2190 & 1090 \\
\hline 14 & 978 & 1560 & 2560 & 2240 & 5560 & 4900 & 4470 & 1940 & 1880 & 1550 & 2190 & 1100 \\
\hline 15 & 983 & 1570 & 2530 & 2190 & 5340 & 4860 & 4170 & 2010 & 1860 & 1740 & 2200 & 1110 \\
\hline 16 & 974 & 1590 & 2520 & 2210 & 5550 & 4810 & 4100 & 2020 & 1860 & 1740 & 2220 & 1110 \\
\hline 17 & 938 & 1610 & 2500 & 2270 & 13800 & 4780 & 4120 & 2050 & 1860 & 1750 & 2240 & 1120 \\
\hline 18 & 956 & 1600 & 2500 & 3020 & 8230 & 4760 & 4210 & 2150 & 1870 & 1750 & 2250 & 1150 \\
\hline 19 & 1170 & 1580 & 2500 & 4530 & 8070 & 4590 & 4080 & 2170 & 1870 & 1760 & 2260 & 1180 \\
\hline 20 & 1170 & 1580 & 2500 & 12700 & 6550 & 4560 & 3860 & 2090 & 1870 & 1780 & 2260 & 1180 \\
\hline 21 & 1130 & 1590 & 2540 & 8040 & 9850 & 4530 & 3590 & 1980 & 1850 & 1790 & 2270 & 1170 \\
\hline 22 & 1100 & 1620 & 2620 & 5490 & 6910 & 4510 & 3260 & 1950 & 1840 & 1620 & 2280 & 1180 \\
\hline 23 & 1040 & 1680 & 2620 & 13100 & 5990 & 4470 & 3000 & 1960 & 1840 & 1790 & 2280 & 1170 \\
\hline 24 & 997 & 1810 & 2620 & 7760 & 5610 & 4470 & 2920 & 1990 & 1830 & 1810 & 2320 & 1150 \\
\hline 25 & 973 & 1650 & 2620 & 5440 & 6410 & 4540 & 2830 & 1980 & 1570 & 1800 & 2350 & 1130 \\
\hline 26 & 1010 & 1640 & 2620 & 4310 & 6210 & 4460 & 2800 & 1990 & 1580 & 1790 & 2350 & 1130 \\
\hline 27 & 1130 & 1720 & 2600 & 4180 & 5500 & 4400 & 2740 & 2180 & 1590 & 1790 & 2360 & 1130 \\
\hline 28 & 1130 & 1790 & 2590 & 4020 & 5520 & 4340 & 2600 & 2590 & 1580 & 1800 & 2390 & 1120 \\
\hline 29 & 1120 & 2150 & 2580 & 3930 & --- & 4310 & 2530 & 2620 & 1540 & 1790 & 1930 & 1100 \\
\hline 30 & 1100 & 2730 & 2570 & 3910 & --- & 4120 & 2490 & 2750 & 1500 & 1810 & 1670 & 1080 \\
\hline 31 & 1090 & --- & 2580 & 4040 & --- & 3730 & --- & 2750 & --- & 1830 & 1690 & --- \\
\hline TOTAL & 31986 & 44940 & 84680 & 124630 & 212710 & 186770 & 109770 & 68660 & 58240 & 51190 & 66560 & 34840 \\
\hline MEAN & 1032 & 1498 & 2732 & 4020 & 7597 & 6025 & 3659 & 2215 & 1941 & 1651 & 2147 & 1161 \\
\hline MAX & 1170 & 2730 & 4730 & 13100 & 28000 & 12200 & 4720 & 2750 & 2750 & 1830 & 2390 & 1720 \\
\hline MIN & 910 & 1050 & 2500 & 2190 & 4060 & 3730 & 2490 & 1910 & 1500 & 1480 & 1670 & 1040 \\
\hline $\mathrm{AC}-\mathrm{FT}$ & 63440 & 89140 & 168000 & 247200 & 421900 & 370500 & 217700 & 136200 & 115500 & 101500 & 132000 & 69110 \\
\hline
\end{tabular}


11421000 YUBA RIVER NEAR MARYSVILLE, CA—Continued

STATISTICS OF MONTHLY MEAN DATA FOR WATER YEARS 1944 - 1968, BY WATER YEAR (WY)

\begin{tabular}{|c|c|c|c|c|c|c|c|c|c|c|c|c|}
\hline & OCT & NOV & $\mathrm{DEC}$ & JAN & FEB & MAR & APR & MAY & JUN & JUL & AUG & SEP \\
\hline MEAN & 507 & 846 & 3323 & 3574 & 4555 & 3928 & 4965 & 5064 & 2610 & 514 & 218 & 240 \\
\hline MAX & 6222 & 8586 & 18650 & 13160 & 12470 & 7321 & 10400 & 13750 & 8712 & 2669 & 551 & 458 \\
\hline$(W Y)$ & 1963 & 1951 & 1965 & 1956 & 1958 & 1958 & 1952 & 1952 & 1952 & 1952 & 1967 & 1952 \\
\hline MIN & 50.5 & 116 & 157 & 573 & 965 & 1360 & 2139 & 1264 & 265 & 30.5 & 35.3 & 47.9 \\
\hline (WY) & 1962 & 1960 & 1960 & 1960 & 1948 & 1964 & 1961 & 1947 & 1959 & 1959 & 1959 & 1961 \\
\hline
\end{tabular}

SUMMARY STATISTICS

ANNUAL MEAN

HIGHEST ANNUAL MEAN

LOWEST ANNUAL MEAN

HIGHEST DAILY MEAN

LOWEST DAILY MEAN

ANNUAL SEVEN-DAY MINIMUM

INSTANTANEOUS PEAK FLOW

INSTANTANEOUS PEAK STAGE

ANNUAL RUNOFF (AC-FT)

10 PERCENT EXCEEDS

50 PERCENT EXCEEDS

90 PERCENT EXCEEDS
WATER YEARS $1944-1968$

$\begin{array}{rlll}2518 & & & \\ 5393 & & & 1952 \\ 882 & & & 1961 \\ 136000 & \text { Dec } 23 & 1955 \\ 15 & \text { Nov } 7 & 1959 \\ 15 & \text { Nov } 5 & 1959 \\ 180000 & \text { Dec } 22 & 1964 \\ 90.15 & \text { Dec } 22 & 1964 \\ 1824000 & & & \\ 6450 & & & \\ 822 & & & \\ 108 & & & \end{array}$

STATISTICS OF MONTHLY MEAN DATA FOR WATER YEARS 1970 - 1999, BY WATER YEAR (WY)

\begin{tabular}{|c|c|c|c|c|c|c|c|c|c|c|c|c|}
\hline & $\mathrm{OCT}$ & NOV & $\mathrm{DEC}$ & JAN & FEB & MAR & APR & MAY & JUN & JUL & AUG & SEP \\
\hline MEAN & 1142 & 1451 & 2531 & 4509 & 4650 & 4528 & 2992 & 2368 & 2005 & 1287 & 1477 & 1348 \\
\hline MAX & 2731 & 4475 & 11430 & 26180 & 20970 & 15100 & 14280 & 9721 & 8633 & 3735 & 2829 & 2900 \\
\hline (WY) & 1976 & 1984 & 1984 & 1997 & 1986 & 1983 & 1982 & 1995 & 1983 & 1983 & 1984 & 1980 \\
\hline MIN & 132 & 182 & 371 & 230 & 211 & 188 & 173 & 166 & 155 & 88.4 & 71.7 & 85.8 \\
\hline (WY) & 1970 & 1970 & 1977 & 1977 & 1977 & 1977 & 1977 & 1977 & 1977 & 1977 & 1977 & 1977 \\
\hline
\end{tabular}

SUMMARY STATISTICS

ANNUAL TOTAL

ANNUAL MEAN

HIGHEST ANNUAL MEAN

LOWEST ANNUAL MEAN

HIGHEST DAILY MEAN

LOWEST DAILY MEAN

ANNUAL SEVEN-DAY MINIMUM

INSTANTANEOUS PEAK FLOW

INSTANTANEOUS PEAK STAGE

ANNUAL RUNOFF (AC-FT)

10 PERCENT EXCEEDS

50 PERCENT EXCEEDS

90 PERCENT EXCEEDS
FOR 1998 CALENDAR YEAR

\begin{tabular}{|c|c|}
\hline $\begin{array}{r}1544129 \\
4230\end{array}$ & \\
\hline 21400 & Feb \\
\hline 494 & Sep 19 \\
\hline 511 & Sep 17 \\
\hline 3063000 & \\
\hline 8180 & \\
\hline 3180 & \\
\hline 1050 & \\
\hline
\end{tabular}

FOR 1999 WATER YEAR

$\begin{array}{rll}1074976 & & \\ 2945 & & \\ & & \\ 28000 & \text { Feb } & 9 \\ 910 & \text { Oct } & 9 \\ 949 & \text { Oct } & 8 \\ 35100 & \text { Feb } & 9 \\ 74.23 & \text { Feb } & 9 \\ 2132000 & & \\ 5430 & & \\ 2200 & & \\ 1100 & & \end{array}$

WATER YEARS 1970 - 1999

\begin{tabular}{|c|c|c|c|}
\hline 2514 & & & \\
\hline 5818 & & & 1982 \\
\hline 229 & & & 1977 \\
\hline 140000 & Jan & 2 & 1997 \\
\hline 62 & Jul 1 & 19 & 1977 \\
\hline 65 & Jul 3 & 31 & 1977 \\
\hline 161000 & Jan & 2 & 1997 \\
\hline 91.64 & Jan & 2 & 1997 \\
\hline 1821000 & & & \\
\hline 5230 & & & \\
\hline 1360 & & & \\
\hline 318 & & & \\
\hline
\end{tabular}




\section{WATER-QUALITY RECORDS}

PERIOD OF RECORD.-Water years 1951-52, 1973-80, 1990 to current year. Published as Yuba River at Marysville (station 11421500) during water years 1966, 1973-76.

CHEMICAL DATA: Water years 1951-52, 1973-80, 1996. Published as Yuba River at Marysville (station 11421500) water years 1966, 1973-76

WATER TEMPERATURE: Water years 1973-78, 1990 to current year.

SEDIMENT DATA: Water year 1996.

PERIOD OF DAILY RECORD.-

WATER TEMPERATURE: November 1972 to September 1978, October 1989 to current year.

INSTRUMENTATION.-Temperature recorder November 1972 to September 1978, October 1989 to current year.

REMARKS.-Water temperatures can be affected by releases from Englebright Reservoir located approximately 13 mi upstream from station.

EXTREMES FOR PERIOD OF DAILY RECORD.-

WATER TEMPERATURE: Maximum recorded, 28.5 C, July 16, 30, 1977, Aug. 11, 1992; minimum recorded, $4.5^{\circ} \mathrm{C}$, Dec. $22,23,29-31,1990$. EXTREMES FOR CURRENT YEAR.-

WATER TEMPERATURE: Maximum recorded, $19.5^{\circ} \mathrm{C}$, June 23, 25, 28-30; minimum recorded, 7.0 ${ }^{\circ} \mathrm{C}$, Dec. 21,24 .

TEMPERATURE, WATER (DEG. C), WATER YEAR OCTOBER 1998 TO SEPTEMBER 1999

\begin{tabular}{|c|c|c|c|c|c|c|c|c|c|c|c|c|}
\hline DAY & MAX & MIN & MAX & MIN & MAX & MIN & MAX & MIN & MAX & MIN & MAX & MIN \\
\hline & \multicolumn{2}{|c|}{ OCTOBER } & \multicolumn{2}{|c|}{ NOVEMBER } & \multicolumn{2}{|c|}{ DECEMBER } & \multicolumn{2}{|c|}{ JANUARY } & \multicolumn{2}{|c|}{ FEBRUARY } & \multicolumn{2}{|c|}{$\mathrm{MARCH}$} \\
\hline 1 & 17.0 & 13.5 & 13.0 & 11.5 & 11.5 & 10.5 & 9.0 & 7.5 & 9.0 & 7.5 & 10.0 & 8.5 \\
\hline 2 & 16.5 & 13.0 & 13.5 & 11.0 & 11.0 & 10.5 & 9.5 & 7.5 & 9.0 & 7.5 & 10.5 & 8.5 \\
\hline 3 & 16.5 & 13.0 & 13.5 & 11.5 & 11.0 & 10.0 & 8.5 & 8.0 & 9.5 & 7.5 & 10.0 & 9.0 \\
\hline 4 & 16.5 & 12.5 & 13.5 & 11.0 & 10.5 & 9.5 & 8.5 & 7.5 & 9.0 & 8.0 & 10.5 & 9.0 \\
\hline 5 & 16.5 & 12.5 & 13.5 & 11.5 & 10.0 & 9.0 & 8.5 & 7.5 & 9.0 & 7.5 & 10.0 & 8.5 \\
\hline 6 & 16.0 & 12.5 & 12.0 & 11.0 & 10.0 & 9.0 & 8.5 & 7.5 & 8.5 & 8.0 & 10.0 & 8.5 \\
\hline 7 & 16.5 & 12.5 & 12.0 & 11.0 & 10.0 & 8.5 & 8.0 & 8.0 & 9.5 & 8.5 & 10.0 & 8.5 \\
\hline 8 & 17.0 & 13.0 & 12.0 & 11.0 & 10.5 & 9.0 & 9.0 & 7.5 & 9.0 & 8.5 & 9.0 & 8.5 \\
\hline 9 & 16.5 & 13.0 & 13.0 & 10.5 & 10.0 & 8.5 & 8.0 & 7.5 & 9.0 & 8.5 & 10.0 & 8.0 \\
\hline 10 & 16.0 & 12.5 & 12.0 & 11.0 & 10.0 & 8.5 & 8.0 & 7.5 & 9.0 & 8.0 & 9.5 & 8.0 \\
\hline 11 & 16.0 & 12.0 & 12.5 & 10.5 & 10.0 & 8.5 & 8.5 & 7.5 & 9.0 & 8.0 & 10.0 & 8.0 \\
\hline 12 & 15.0 & 12.0 & 12.5 & 10.0 & 10.0 & 8.5 & 9.0 & 7.5 & 9.0 & 8.0 & 10.5 & 8.0 \\
\hline 13 & 16.0 & 13.0 & 12.5 & 10.5 & 10.0 & 8.5 & 9.5 & 7.5 & 9.0 & 7.5 & 10.5 & 8.0 \\
\hline 14 & 15.5 & 12.5 & 12.5 & 10.0 & 10.0 & 8.5 & 9.0 & 8.0 & 8.5 & 8.0 & 9.5 & 8.5 \\
\hline 15 & 15.0 & 12.0 & 12.5 & 10.5 & 10.0 & 8.5 & 9.0 & 8.5 & 9.0 & 7.5 & 10.5 & 8.5 \\
\hline 16 & 15.0 & 11.5 & 11.5 & 10.5 & 10.0 & 8.5 & 9.5 & 8.5 & 8.5 & 8.0 & 9.5 & 8.5 \\
\hline 17 & 15.0 & 11.5 & 12.5 & 10.5 & 10.0 & 8.5 & 9.5 & 8.5 & 9.0 & 8.5 & 10.5 & 8.5 \\
\hline 18 & 15.0 & 11.5 & 12.0 & 10.0 & 10.0 & 8.5 & 10.0 & 9.5 & 9.0 & 8.5 & 10.5 & 8.5 \\
\hline 19 & 15.5 & 11.5 & 12.0 & 10.0 & 9.5 & 8.0 & 9.5 & 9.0 & 10.0 & 8.0 & 10.5 & 8.5 \\
\hline 20 & 15.5 & 12.0 & 12.0 & 10.0 & 9.0 & 7.5 & 9.5 & 9.0 & 9.0 & 8.0 & 10.5 & 8.5 \\
\hline 21 & 15.5 & 12.0 & 11.5 & 10.5 & 9.0 & 7.0 & 10.0 & 9.0 & 9.5 & 8.5 & 10.5 & 8.5 \\
\hline 22 & 15.0 & 12.0 & 12.0 & 11.0 & 9.0 & 7.5 & 9.5 & 9.0 & 9.5 & 8.5 & 10.5 & 8.5 \\
\hline 23 & 15.0 & 12.0 & 11.5 & 11.0 & 8.5 & 7.5 & 9.5 & 8.5 & 10.0 & 8.5 & 10.5 & 8.5 \\
\hline 24 & 13.5 & 12.5 & 11.5 & 10.5 & 8.5 & 7.0 & 9.5 & 8.5 & 9.5 & 8.5 & 9.5 & 8.5 \\
\hline 25 & 15.0 & 12.0 & 12.0 & 10.0 & 9.0 & 7.5 & 9.5 & 8.5 & 9.5 & 8.5 & 11.5 & 9.0 \\
\hline 26 & 15.0 & 11.5 & 12.0 & 10.0 & 9.0 & 7.5 & 9.5 & 8.5 & 9.5 & 8.0 & 11.5 & 8.5 \\
\hline 27 & 14.5 & 11.5 & 11.0 & 10.5 & 9.0 & 7.5 & 9.5 & 8.0 & 10.0 & 8.5 & 11.0 & 8.5 \\
\hline 28 & 13.0 & 12.0 & 12.0 & 10.5 & 9.0 & 7.5 & 9.5 & 8.0 & 9.5 & 8.5 & 11.5 & 8.5 \\
\hline 29 & 14.0 & 11.5 & 10.5 & 10.0 & 9.0 & 7.5 & 9.5 & 7.5 & --- & --- & 11.0 & 8.5 \\
\hline 30 & 14.0 & 11.0 & 11.0 & 10.5 & 9.0 & 7.5 & 9.0 & 7.5 & --- & --- & 11.0 & 8.5 \\
\hline 31 & 13.0 & 10.5 & --- & --- & 9.5 & 8.0 & 8.5 & 8.0 & --- & --- & 11.0 & 8.0 \\
\hline MONTH & 17.0 & 10.5 & 13.5 & 10.0 & 11.5 & 7.0 & 10.0 & 7.5 & 10.0 & 7.5 & 11.5 & 8.0 \\
\hline
\end{tabular}


11421000 YUBA RIVER NEAR MARYSVILLE, CA—Continued

TEMPERATURE, WATER (DEG. C), WATER YEAR OCTOBER 1998 TO SEPTEMBER 1999

\begin{tabular}{|c|c|c|c|c|c|c|c|c|c|c|c|c|}
\hline DAY & MAX & MIN & MAX & MIN & MAX & MIN & MAX & MIN & MAX & MIN & MAX & MIN \\
\hline & \multicolumn{2}{|c|}{ APRIL } & \multicolumn{2}{|c|}{ MAY } & \multicolumn{2}{|c|}{ JUNE } & \multicolumn{2}{|c|}{ JULY } & \multicolumn{2}{|c|}{ AUGUST } & \multicolumn{2}{|c|}{ SEPTEMBER } \\
\hline 1 & 11.5 & 8.5 & 15.0 & 10.5 & 17.0 & 14.0 & 19.0 & 14.0 & 16.5 & 11.5 & 16.5 & 11.5 \\
\hline 2 & 11.5 & 8.5 & 12.5 & 10.0 & 16.5 & 14.0 & 18.5 & 13.5 & 16.5 & 12.0 & 17.0 & 12.0 \\
\hline 3 & 10.5 & 8.5 & 13.0 & 10.5 & 15.5 & 13.5 & 18.0 & 13.0 & 16.5 & 12.0 & 17.0 & 12.5 \\
\hline 4 & 11.5 & 8.0 & 14.5 & 10.0 & 16.5 & 13.0 & 18.0 & 12.5 & 17.0 & 12.0 & 17.5 & 12.5 \\
\hline 5 & 9.0 & 8.5 & 14.5 & 10.0 & 17.0 & 12.5 & 18.0 & 13.0 & 16.0 & 12.0 & 17.0 & 12.5 \\
\hline 6 & 11.0 & 8.5 & 15.5 & 10.5 & 17.0 & 12.5 & 18.5 & 13.0 & 15.5 & 12.0 & 17.5 & 12.5 \\
\hline 7 & 10.5 & 8.5 & 15.0 & 10.5 & 16.5 & 12.0 & 18.0 & 12.5 & 16.0 & 12.0 & 17.5 & 12.5 \\
\hline 8 & 10.0 & 8.5 & 15.0 & 10.5 & 16.5 & 11.5 & 18.5 & 13.0 & 16.5 & 12.0 & 18.0 & 13.0 \\
\hline 9 & 11.0 & 8.0 & 14.5 & 10.5 & 16.5 & 11.5 & 18.0 & 12.5 & 16.0 & 12.0 & 16.5 & 13.0 \\
\hline 10 & 10.0 & 8.5 & 14.5 & 10.5 & 16.5 & 11.5 & 18.0 & 12.5 & 15.0 & 12.0 & 17.5 & 13.0 \\
\hline 11 & 11.5 & 8.5 & 16.5 & 11.0 & 17.0 & 12.0 & 18.5 & 13.0 & 16.0 & 12.0 & 17.5 & 13.0 \\
\hline 12 & 12.0 & 8.5 & 16.0 & 11.5 & 17.0 & 12.0 & 18.5 & 13.0 & 16.5 & 11.5 & 17.5 & 13.0 \\
\hline 13 & 12.0 & 9.0 & 15.5 & 11.0 & 17.0 & 12.0 & 18.0 & 13.0 & 16.0 & 11.5 & 17.5 & 13.0 \\
\hline 14 & 12.5 & 9.0 & 15.5 & 11.0 & 18.0 & 12.5 & 18.0 & 13.0 & 16.5 & 11.5 & 17.5 & 13.0 \\
\hline 15 & 13.0 & 9.5 & 15.5 & 10.5 & 17.0 & 12.5 & 17.5 & 12.5 & 16.5 & 11.5 & 17.5 & 13.0 \\
\hline 16 & 13.0 & 9.5 & 16.0 & 11.0 & 18.0 & 13.0 & 17.5 & 12.0 & 16.5 & 12.0 & 17.5 & 13.0 \\
\hline 17 & 13.5 & 10.0 & 16.5 & 11.0 & 18.0 & 13.5 & 17.5 & 12.0 & 16.5 & 12.0 & 17.5 & 13.0 \\
\hline 18 & 13.5 & 10.0 & 16.0 & 11.5 & 17.5 & 13.5 & 17.5 & 12.0 & 16.0 & 12.0 & 16.0 & 13.0 \\
\hline 19 & 13.5 & 10.5 & 16.0 & 11.0 & 18.0 & 13.5 & 17.0 & 12.0 & 16.0 & 11.5 & 17.5 & 13.0 \\
\hline 20 & 13.5 & 10.5 & 16.0 & 11.0 & 18.0 & 13.5 & 17.0 & 12.0 & 16.0 & 11.5 & 17.5 & 13.0 \\
\hline 21 & 14.0 & 10.5 & 16.5 & 11.5 & 18.5 & 14.0 & 17.0 & 12.0 & 16.5 & 12.0 & 18.0 & 13.0 \\
\hline 22 & 14.0 & 10.5 & 17.0 & 11.5 & 19.0 & 14.0 & 18.5 & 12.0 & 16.5 & 12.0 & 18.0 & 13.5 \\
\hline 23 & 14.5 & 11.0 & 16.5 & 12.0 & 19.5 & 14.0 & 17.0 & 12.0 & 17.0 & 12.5 & 17.5 & 13.5 \\
\hline 24 & 14.5 & 10.5 & 17.0 & 12.0 & 18.5 & 14.0 & 16.5 & 11.5 & 16.5 & 12.0 & 17.5 & 13.5 \\
\hline 25 & 14.0 & 10.5 & 17.0 & 12.0 & 19.5 & 14.0 & 17.0 & 11.5 & 16.5 & 12.0 & 18.0 & 13.5 \\
\hline 26 & 13.5 & 10.5 & 17.5 & 12.5 & 19.0 & 14.0 & 17.0 & 12.0 & 15.0 & 12.0 & 17.5 & 13.0 \\
\hline 27 & 14.0 & 10.0 & 17.0 & 12.5 & 19.0 & 14.0 & 17.0 & 12.0 & 16.5 & 12.5 & 17.0 & 13.0 \\
\hline 28 & 14.0 & 10.0 & 17.0 & 12.5 & 19.5 & 14.0 & 17.0 & 12.0 & 16.0 & 12.0 & 17.0 & 12.5 \\
\hline 29 & 14.0 & 10.0 & 17.0 & 12.5 & 19.5 & 14.0 & 17.0 & 11.5 & 17.5 & 12.5 & 17.0 & 13.0 \\
\hline 30 & 14.5 & 10.5 & 17.5 & 13.5 & 19.5 & 14.0 & 17.0 & 12.0 & 16.5 & 12.5 & 17.5 & 13.0 \\
\hline 31 & --- & --- & 17.5 & 13.5 & --- & --- & 16.5 & 12.0 & 16.5 & 12.0 & --- & --- \\
\hline MONTH & 14.5 & 8.0 & 17.5 & 10.0 & 19.5 & 11.5 & 19.0 & 11.5 & 17.5 & 11.5 & 18.0 & 11.5 \\
\hline
\end{tabular}




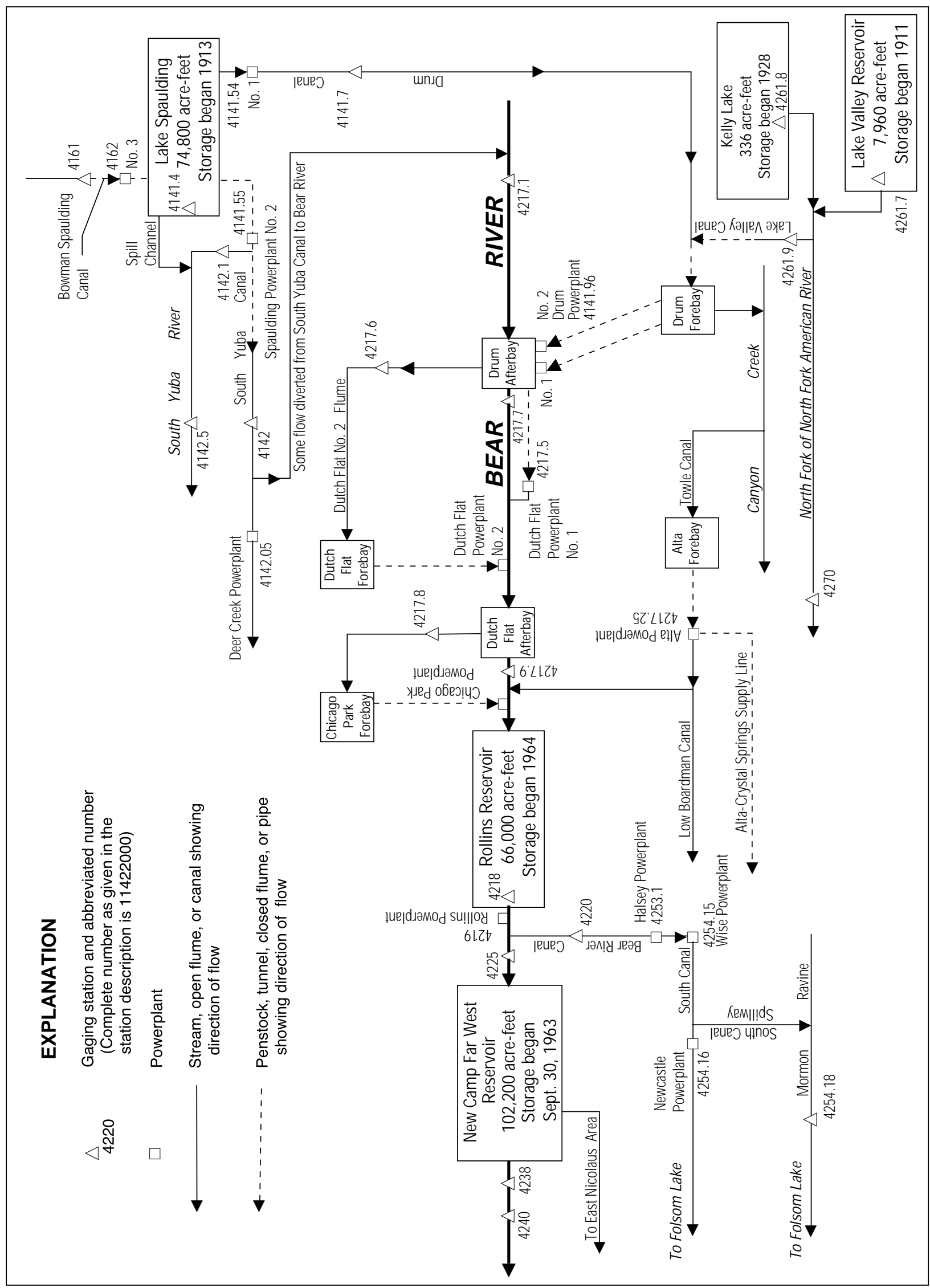




\section{BEAR RIVER NEAR EMIGRANT GAP, CA}

LOCATION.-Lat 39¹8'23", long 12040'41", in NW 1/4 SW 1/4 sec.30, T.17 N., R.12 E., Placer County, Hydrologic Unit 18020126, on left bank $20 \mathrm{ft}$ upstream from Highway 20 Bridge and 0.7 mi northwest of Emigrant Gap.

DRAINAGE AREA. $-0.76 \mathrm{mi}^{2}$.

PERIOD OF RECORD.-OCtober 1987 to current year (low-flow records only). Unpublished records for water years 1981-87 available in files of the U.S. Geological Survey.

GAGE.-Water-stage recorder and concrete culvert. Elevation of gage is 4,550 ft above sea level, from topographic map. Prior to October 1987, nonrecording gage at same site and datum.

REMARKS.—No records computed above $160 \mathrm{ft}^{3} / \mathrm{s}$. Some water is diverted into stream from South Yuba Canal (station 11414200). See schematic diagram of Bear River Basin.

COOPERATION.-Records were collected by Pacific Gas \& Electric Co., under general supervision of the U.S. Geological Survey, in connection with a Federal Energy Regulatory Commission project.

DISCHARGE, CUBIC FEET PER SECOND, WATER YEAR OCTOBER 1998 TO SEPTEMBER 1999

DAILY MEAN VALUES

\begin{tabular}{|c|c|c|c|c|c|c|c|c|c|c|c|c|}
\hline DAY & OCT & NOV & $\mathrm{DEC}$ & JAN & FEB & MAR & $A P R$ & MAY & JUN & JUL & AUG & SEP \\
\hline 1 & 5.4 & 6.5 & 18 & 6.7 & 9.6 & 127 & 149 & 151 & 129 & 54 & 6.3 & 6.7 \\
\hline 2 & 5.5 & 6.1 & 15 & 6.3 & 9.2 & 112 & 149 & 154 & --- & 94 & 6.2 & 6.9 \\
\hline 3 & 5.3 & 6.1 & 61 & 6.1 & 9.0 & 141 & 147 & --- & 104 & 75 & 6.1 & 6.7 \\
\hline 4 & 5.4 & 6.2 & 43 & 5.8 & 9.1 & 113 & 112 & --- & 90 & 64 & 6.3 & 6.5 \\
\hline 5 & 5.3 & 6.1 & 24 & 5.7 & 9.0 & --- & 58 & 153 & 88 & 61 & 6.5 & 6.9 \\
\hline 6 & 5.5 & 12 & 9.7 & 5.7 & 9.7 & 109 & 56 & 150 & 88 & 64 & 6.7 & 6.9 \\
\hline 7 & 5.3 & 8.1 & 8.3 & 5.4 & 69 & 105 & 55 & 150 & 88 & 63 & 5.9 & 7.1 \\
\hline 8 & 5.6 & 6.9 & 8.7 & 5.0 & 62 & 103 & 56 & 154 & 89 & 64 & 6.6 & 7.1 \\
\hline 9 & 5.6 & 6.4 & 8.7 & 5.6 & 97 & 103 & 44 & 151 & 89 & 64 & 6.4 & 6.9 \\
\hline 10 & 5.5 & 7.5 & 8.5 & 5.6 & 26 & 157 & 14 & 152 & 89 & 64 & 6.3 & 6.9 \\
\hline 11 & 6.1 & 6.8 & 8.2 & 5.7 & 19 & --- & 14 & 153 & 89 & 64 & 6.7 & 6.6 \\
\hline 12 & 5.7 & 6.4 & 8.2 & 5.7 & 17 & --- & 17 & 152 & 89 & 63 & 6.5 & 6.5 \\
\hline 13 & 5.4 & 6.1 & 8.7 & 5.4 & 15 & --- & 50 & 150 & 88 & 63 & 6.2 & 6.5 \\
\hline 14 & 5.5 & 6.1 & 8.7 & 6.0 & 15 & --- & 123 & 148 & 87 & 63 & 6.3 & 6.5 \\
\hline 15 & 5.7 & 6.1 & 9.4 & 8.9 & 14 & --- & 124 & 148 & 85 & 38 & 6.3 & 6.5 \\
\hline 16 & 6.1 & 9.5 & 10 & 12 & 52 & --- & 124 & 148 & 85 & 8.3 & 6.5 & 6.5 \\
\hline 17 & 6.3 & 9.2 & 9.7 & 15 & 142 & --- & 122 & 146 & 84 & 7.1 & 7.4 & 6.5 \\
\hline 18 & 5.9 & 6.9 & 8.8 & 43 & 133 & --- & 139 & 143 & 68 & 6.9 & 6.8 & 5.9 \\
\hline 19 & 6.0 & 6.5 & 8.3 & 57 & 116 & --- & --- & 141 & 49 & 6.7 & 6.4 & 6.8 \\
\hline 20 & 6.1 & 6.0 & 8.1 & 76 & 105 & --- & --- & 142 & 45 & 6.5 & 6.2 & 12 \\
\hline 21 & 6.1 & 11 & 7.9 & 29 & 104 & --- & --- & 142 & 44 & 6.6 & 6.4 & 13 \\
\hline 22 & 6.1 & 7.5 & 7.5 & 27 & 101 & 158 & --- & 142 & 43 & 6.5 & 6.3 & 13 \\
\hline 23 & 6.1 & 23 & 7.1 & 79 & 101 & 157 & --- & 141 & 43 & 6.6 & 6.1 & 13 \\
\hline 24 & 8.1 & 11 & 6.9 & 25 & 101 & 156 & --- & 141 & 42 & 6.9 & 6.1 & 13 \\
\hline 25 & 7.2 & 7.7 & 6.9 & 17 & 104 & 153 & --- & 141 & 43 & 7.1 & 6.3 & 13 \\
\hline 26 & 6.3 & 6.6 & 6.9 & 15 & 98 & 153 & --- & 130 & 43 & 7.0 & 6.1 & 13 \\
\hline 27 & 6.1 & 10 & 6.9 & 13 & 98 & 153 & --- & 137 & 43 & 6.7 & 6.6 & 13 \\
\hline 28 & 6.1 & 10 & 6.9 & 12 & 107 & 152 & --- & 133 & 24 & 6.3 & 6.7 & 13 \\
\hline 29 & 6.1 & 24 & 6.6 & 12 & --- & 151 & --- & 132 & 6.4 & 6.2 & 6.5 & 11 \\
\hline 30 & 6.0 & 38 & 6.5 & 11 & --- & 152 & 156 & 131 & 6.5 & 6.6 & 7.0 & 24 \\
\hline 31 & 5.7 & --- & 6.6 & 11 & --- & 151 & --- & 131 & --- & 6.6 & 6.9 & --- \\
\hline TOTAL & 183.1 & 290.3 & 369.7 & 543.6 & 1751.6 & --- & --- & --- & --- & 1066.6 & 199.6 & 277.9 \\
\hline MEAN & 5.91 & 9.68 & 11.9 & 17.5 & 62.6 & --- & --- & --- & --- & 34.4 & 6.44 & 9.26 \\
\hline MAX & 8.1 & 38 & 61 & 79 & 142 & --- & --- & --- & --- & 94 & 7.4 & 24 \\
\hline MIN & 5.3 & 6.0 & 6.5 & 5.0 & 9.0 & --- & --- & --- & --- & 6.2 & 5.9 & 5.9 \\
\hline $\mathrm{AC}-\mathrm{FT}$ & 363 & 576 & 733 & 1080 & 3470 & --- & --- & --- & --- & 2120 & 396 & 551 \\
\hline
\end{tabular}


LOCATION.-Lat 39¹5'16", long 12046'26", in SW 1/4 NW 1/4 sec.17, T.16 N., R.11 E., Placer County, Hydrologic Unit 18020126, on left bank $60 \mathrm{ft}$ downstream from Drum Afterbay Dam and 3.5 mi west of Blue Canyon.

DRAINAGE AREA.- $-12.3 \mathrm{mi}^{2}$.

PERIOD OF RECORD.—April 1966 to current year, low flows only April to September 1966 and since October 1998.

GAGE.-Water-stage recorder and 4-ft steel Cipolletti weir set in a concrete broad-crested weir. Elevation of gage is 3,300 ft above sea level, from topographic map. April 1966 to May 25, 1967, water-stage recorder at present site at different datum. May 26, 1967, to Feb. 11, 1968, waterstage recorder at site $1,000 \mathrm{ft}$ downstream at different datum.

REMARKS.-Records not computed above $13.5 \mathrm{ft}^{3} / \mathrm{sec}$. Water for Dutch Flat No. 1 Powerplant (station 11421750) and Dutch Flat No. 2 Flume (station 11421760) is diverted from Drum Afterbay just upstream from station. See schematic diagram of Bear River Basin.

COOPERATION.-Records were collected by Pacific Gas \& Electric Co., under general supervision of the U.S. Geological Survey, in connection with a Federal Energy Regulatory Commission project.

DISCHARGE, CUBIC FEET PER SECOND, WATER YEAR OCTOBER 1998 TO SEPTEMBER 1999 DAILY MEAN VALUES

\begin{tabular}{|c|c|c|c|c|c|c|c|c|c|c|c|c|}
\hline DAY & $\mathrm{OCT}$ & NOV & $\mathrm{DEC}$ & JAN & FEB & MAR & $\mathrm{APR}$ & MAY & JUN & JUL & AUG & SEP \\
\hline 1 & 5.7 & 6.2 & 6.3 & 6.5 & --- & --- & 12 & 12 & 12 & 12 & 11 & --- \\
\hline 2 & 6.0 & 6.3 & 6.2 & 6.4 & --- & 12 & 12 & 12 & 12 & 12 & --- & --- \\
\hline 3 & 5.7 & 5.8 & 6.7 & 6.5 & --- & 12 & 12 & 12 & 12 & 12 & --- & --- \\
\hline 4 & 5.7 & 5.9 & 6.2 & 6.2 & 6.4 & --- & 12 & 12 & 12 & 12 & --- & 11 \\
\hline 5 & 5.7 & 6.2 & 6.1 & 6.4 & --- & --- & 12 & 12 & 12 & 12 & --- & 11 \\
\hline 6 & 5.7 & 6.3 & 6.2 & 6.1 & --- & 12 & 12 & 12 & 12 & 12 & --- & 11 \\
\hline 7 & 5.7 & 6.4 & 6.1 & 6.2 & --- & 12 & 12 & 12 & 12 & 13 & 13 & --- \\
\hline 8 & 5.7 & 6.2 & 6.3 & 6.3 & --- & 12 & 12 & 12 & 12 & 12 & 11 & --- \\
\hline 9 & 5.7 & 6.0 & 6.4 & 6.1 & --- & 12 & 12 & 12 & 12 & 12 & --- & 11 \\
\hline 10 & 5.8 & 5.9 & 6.3 & 6.4 & --- & --- & 12 & 12 & 12 & 12 & --- & 11 \\
\hline 11 & 6.1 & 6.3 & 6.2 & 6.4 & 6.9 & --- & 12 & 12 & 12 & 12 & --- & 11 \\
\hline 12 & 6.2 & 6.0 & 6.3 & 6.6 & 6.5 & --- & 12 & --- & 12 & 12 & --- & 11 \\
\hline 13 & 5.8 & 6.5 & 6.3 & 6.3 & 6.1 & 12 & 12 & 12 & 12 & 12 & --- & 11 \\
\hline 14 & 6.0 & 6.4 & 5.9 & 6.0 & 6.4 & --- & --- & 12 & 12 & 12 & 11 & 11 \\
\hline 15 & 6.3 & 6.0 & 6.4 & 6.0 & 6.3 & 12 & --- & 12 & 12 & 12 & 10 & 11 \\
\hline 16 & 6.3 & 5.9 & 6.4 & 6.0 & 6.8 & 12 & 12 & 12 & 12 & 12 & --- & 11 \\
\hline 17 & 5.9 & 6.2 & 6.6 & 6.0 & 6.8 & 12 & 12 & 12 & 12 & 13 & --- & 11 \\
\hline 18 & 6.3 & 6.3 & 6.5 & 6.2 & 6.7 & --- & 12 & 12 & 12 & 12 & --- & 11 \\
\hline 19 & 6.2 & 6.0 & 6.2 & 6.1 & 6.2 & 12 & --- & 12 & 12 & 12 & --- & 11 \\
\hline 20 & 6.0 & 6.0 & 6.5 & 6.2 & 6.3 & 12 & 12 & 12 & 12 & 13 & --- & 11 \\
\hline 21 & 6.1 & 6.3 & 6.4 & 6.1 & 6.1 & --- & 12 & 12 & 12 & 11 & 11 & 11 \\
\hline 22 & 6.2 & 6.0 & 6.2 & 6.4 & --- & 12 & --- & 12 & 12 & 11 & 10 & 11 \\
\hline 23 & 6.0 & 6.5 & 6.1 & --- & --- & --- & 12 & --- & 12 & 11 & --- & 11 \\
\hline 24 & 6.2 & 6.1 & 6.0 & 6.0 & --- & 12 & --- & 12 & 12 & 11 & --- & 11 \\
\hline 25 & 6.2 & 6.4 & 6.4 & 6.6 & --- & 12 & 12 & 12 & 12 & 11 & --- & 11 \\
\hline 26 & 6.2 & 6.1 & 6.6 & 6.7 & --- & 12 & --- & 12 & 12 & 11 & --- & 11 \\
\hline 27 & 6.4 & 6.4 & 6.6 & 6.2 & 8.8 & 12 & 12 & 12 & 12 & 11 & --- & 11 \\
\hline 28 & 6.3 & 6.5 & 6.5 & 6.5 & 12 & 12 & 12 & 12 & 12 & 11 & 11 & 11 \\
\hline 29 & 6.0 & 6.7 & 6.6 & 6.6 & --- & 12 & 12 & 12 & 12 & 11 & 11 & --- \\
\hline 30 & 6.6 & 6.1 & 6.5 & 6.5 & --- & 12 & 12 & 12 & 12 & 11 & --- & --- \\
\hline 31 & 6.3 & --- & 6.6 & 6.6 & --- & 12 & --- & 12 & --- & 11 & --- & --- \\
\hline TOTAL & 187.0 & 185.9 & 196.6 & --- & --- & --- & --- & --- & 360 & 364 & --- & --- \\
\hline MEAN & 6.03 & 6.20 & 6.34 & --- & --- & --- & --- & --- & 12.0 & 11.7 & --- & --- \\
\hline MAX & 6.6 & 6.7 & 6.7 & --- & --- & --- & --- & --- & 12 & 13 & --- & --- \\
\hline MIN & 5.7 & 5.8 & 5.9 & --- & --- & --- & --- & --- & 12 & 11 & --- & --- \\
\hline $\mathrm{AC}-\mathrm{FT}$ & 371 & 369 & 390 & --- & --- & --- & --- & --- & 714 & 722 & --- & --- \\
\hline$a$ & 22050 & 19980 & 26440 & 24050 & 24590 & 31970 & 30470 & 32880 & 27450 & 22060 & 251 & 6180 \\
\hline $\mathrm{b}$ & 10330 & 10560 & 18920 & 17740 & 16530 & 27330 & 24370 & 25920 & 22640 & 23300 & 32500 & 13130 \\
\hline
\end{tabular}

CAL YR 1998 a 358400 b 185700

WTR YR 1999 a 268400 b 243300

a Diversion, in acre-feet, to Dutch Flat No. 2 Flume, provided by Nevada Irrigation District.

b Diversion, in acre-feet, to Dutch Flat No. 1 Powerplant, provided by Pacific Gas \& Electric Co. 
LOCATION.—Lat $39^{\circ} 12^{\prime} 55^{\prime \prime}$, long 12050'23", in NE 1/4 NW 1/4 sec.34, T.16 N., R.10 E., Placer County, Hydrologic Unit 18020126, at left bank downstream end of spillway on Dutch Flat Afterbay Dam, 0.6 mi north of Dutch Flat.

DRAINAGE AREA.-21.5 $\mathrm{mi}^{2}$.

PERIOD OF RECORD.-December 1965 to current year.

REVISED RECORDS.-WDR CA-82-4: 1978, 1979(M), 1980.

GAGE.-Water-stage recorder and concrete control. Elevation of gage is 2,600 ft above sea level, from topographic map.

REMARKS.-Water is imported from South Yuba River Basin via Drum Canal above forebay. Chicago Park Flume (station 11421780) diverts upstream from station to Chicago Park Powerplant. Records include spill over Dutch Flat Afterbay Dam. See schematic diagram of Bear River Basin.

COOPERATION.- Records were collected by Nevada Irrigation District, under general supervision of the U.S. Geological Survey, in connection with a Federal Energy Regulatory Commission project.

EXTREMES FOR PERIOD OF RECORD.-Maximum discharge, 4,240 ft $3 / \mathrm{s}$, Feb. 17, 1986; minimum daily, $0.08 \mathrm{ft}^{3} / \mathrm{s}$, Mar. 8-19, 1968.

DISCHARGE, CUBIC FEET PER SECOND, WATER YEAR OCTOBER 1998 TO SEPTEMBER 1999 DAILY MEAN VALUES

\begin{tabular}{|c|c|c|c|c|c|c|c|c|c|c|c|c|}
\hline DAY & $\mathrm{OCT}$ & NOV & $\mathrm{DEC}$ & JAN & FEB & MAR & $\mathrm{APR}$ & MAY & JUN & JUL & AUG & SEP \\
\hline 1 & 11 & 9.4 & 8.1 & 8.0 & 8.0 & 39 & 23 & 11 & 11 & 11 & 11 & 11 \\
\hline 2 & 11 & 8.2 & 8.2 & 8.0 & 7.9 & 129 & 55 & 11 & 11 & 11 & 11 & 11 \\
\hline 3 & 11 & 8.1 & 8.4 & 7.8 & 58 & 241 & 9.8 & 44 & 11 & 11 & 11 & 11 \\
\hline 4 & 11 & 8.0 & 8.1 & 7.8 & 7.9 & 79 & 30 & 54 & 11 & 11 & 11 & 11 \\
\hline 5 & 11 & 8.0 & 8.1 & 7.8 & 7.9 & 12 & 8.7 & 37 & 11 & 11 & 11 & 11 \\
\hline 6 & 11 & 8.1 & 8.1 & 7.8 & 8.0 & 50 & 8.4 & 14 & 11 & 11 & 11 & 11 \\
\hline 7 & 11 & 8.1 & 8.0 & 7.8 & 332 & 15 & 8.4 & 11 & 11 & 11 & 11 & 11 \\
\hline 8 & 11 & 8.1 & 8.0 & 7.9 & 249 & 10 & 8.5 & 11 & 11 & 11 & 11 & 11 \\
\hline 9 & 11 & 7.9 & 7.9 & 8.0 & 763 & 8.5 & 8.5 & 11 & 11 & 11 & 11 & 11 \\
\hline 10 & 11 & 8.1 & 8.0 & 8.0 & 14 & 8.3 & 8.4 & 11 & 11 & 11 & 11 & 11 \\
\hline 11 & 11 & 8.0 & 8.0 & 8.0 & 8.0 & 24 & 8.4 & 11 & 11 & 11 & 11 & 11 \\
\hline 12 & 11 & 8.0 & 8.1 & 8.0 & 8.1 & 61 & 8.4 & 11 & 11 & 11 & 11 & 11 \\
\hline 13 & 11 & 8.0 & 8.1 & 8.0 & 8.1 & 59 & 8.5 & 11 & 11 & 11 & 11 & 11 \\
\hline 14 & 11 & 8.0 & 8.0 & 8.0 & 8.0 & 71 & 8.5 & 11 & 11 & 11 & 11 & 11 \\
\hline 15 & 11 & 8.4 & 8.0 & 7.9 & 8.0 & 77 & 40 & 11 & 11 & 11 & 11 & 11 \\
\hline 16 & 11 & 8.1 & 8.0 & 8.0 & 8.1 & 59 & 12 & 11 & 11 & 11 & 11 & 11 \\
\hline 17 & 11 & 8.0 & 8.0 & 8.1 & 286 & 64 & 8.5 & 11 & 11 & 11 & 11 & 11 \\
\hline 18 & 11 & 8.1 & 8.1 & 8.2 & 101 & 28 & 8.5 & 11 & 11 & 11 & 11 & 11 \\
\hline 19 & 11 & 8.1 & 8.0 & 9.0 & 68 & 115 & 45 & 11 & 11 & 11 & 11 & 21 \\
\hline 20 & 11 & 8.0 & 8.0 & 339 & 35 & 39 & 120 & 11 & 11 & 11 & 11 & 40 \\
\hline 21 & 11 & 8.0 & 8.0 & 28 & 14 & 73 & 71 & 11 & 11 & 11 & 11 & 52 \\
\hline 22 & 11 & 8.0 & 7.9 & 7.9 & 8.0 & 93 & 125 & 11 & 11 & 11 & 11 & 39 \\
\hline 23 & 11 & 8.0 & 8.0 & 117 & 8.0 & 36 & 64 & 11 & 11 & 11 & 11 & 31 \\
\hline 24 & 11 & 8.0 & 8.0 & 13 & 8.0 & 113 & 44 & 11 & 11 & 11 & 11 & 31 \\
\hline 25 & 11 & 8.0 & 8.2 & 8.0 & 8.1 & 63 & 57 & 11 & 11 & 11 & 11 & 31 \\
\hline 26 & 11 & 8.0 & 8.3 & 8.0 & 8.1 & 33 & 86 & 11 & 11 & 11 & 11 & 30 \\
\hline 27 & 11 & 8.0 & 8.0 & 8.0 & 8.1 & 68 & 26 & 11 & 11 & 11 & 11 & 30 \\
\hline 28 & 11 & 8.2 & 8.0 & 7.8 & 8.1 & 59 & 49 & 11 & 11 & 11 & 11 & 17 \\
\hline 29 & 11 & 8.2 & 7.8 & 7.8 & --- & 46 & 50 & 11 & 11 & 11 & 11 & 12 \\
\hline 30 & 11 & 8.2 & 8.0 & 8.0 & --- & 36 & 14 & 11 & 11 & 11 & 11 & 12 \\
\hline 31 & 11 & --- & 8.0 & 8.0 & --- & 55 & --- & 11 & --- & 11 & 11 & --- \\
\hline TOTAL & 341 & 243.3 & 249.4 & 712.6 & 2064.4 & 1863.8 & 1022.5 & 446 & 330 & 341 & 341 & 544 \\
\hline MEAN & 11.0 & 8.11 & 8.05 & 23.0 & 73.7 & 60.1 & 34.1 & 14.4 & 11.0 & 11.0 & 11.0 & 18.1 \\
\hline MAX & 11 & 9.4 & 8.4 & 339 & 763 & 241 & 125 & 54 & 11 & 11 & 11 & 52 \\
\hline MIN & 11 & 7.9 & 7.8 & 7.8 & 7.9 & 8.3 & 8.4 & 11 & 11 & 11 & 11 & 11 \\
\hline $\mathrm{AC}-\mathrm{FT}$ & 676 & 483 & 495 & 1410 & 4090 & 3700 & 2030 & 885 & 655 & 676 & 676 & 1080 \\
\hline a & 33790 & 32510 & 48030 & 46170 & 53290 & 62340 & 57160 & 60290 & 52900 & 49710 & 42390 & 22870 \\
\hline
\end{tabular}

a Diversion, in acre-feet, to Chicago Park Flume. 
11421790 BEAR RIVER BELOW DUTCH FLAT AFTERBAY, NEAR DUTCH FLAT, CA—Continued

STATISTICS OF MONTHLY MEAN DATA FOR WATER YEARS 1966 - 1999, BY WATER YEAR (WY)

\begin{tabular}{|c|c|c|c|c|c|c|c|c|c|c|c|c|}
\hline MEAN & 18.9 & 11.9 & 43.9 & 53.1 & 58.3 & 63.5 & 61.3 & 25.1 & 12.9 & 10.9 & 10.6 & 14.3 \\
\hline MAX & 266 & 71.1 & 350 & 531 & 380 & 395 & 602 & 142 & 63.5 & 22.0 & 13.1 & 21.3 \\
\hline (WY) & 1968 & 1984 & 1997 & 1997 & 1986 & 1966 & 1969 & 1998 & 1998 & 1970 & 1969 & 1983 \\
\hline MIN & 4.81 & 2.65 & 2.42 & 4.94 & 4.10 & 4.26 & 3.94 & 5.30 & 5.13 & 5.00 & 5.00 & 5.00 \\
\hline (WY) & 1978 & 1968 & 1968 & 1975 & 1974 & 1973 & 1973 & 1977 & 1977 & 1977 & 1977 & 1977 \\
\hline
\end{tabular}

SUMMARY STATISTICS

ANNUAL TOTAL

ANNUAL MEAN

HIGHEST ANNUAL MEAN

LOWEST ANNUAL MEAN

HIGHEST DAILY MEAN

LOWEST DAILY MEAN

ANNUAL SEVEN-DAY MINIMUM

INSTANTANEOUS PEAK FLOW

ANNUAL RUNOFF (AC-FT)

ANNUAL DIVERSION (AC-FT) a

10 PERCENT EXCEEDS

50 PERCENT EXCEEDS

90 PERCENT EXCEEDS
FOR 1998 CALENDAR YEAR

$\begin{array}{rrr}13144.0 & & \\ 36.0 & & \\ & & \\ 441 & \text { Jun } 22 \\ 7.3 & \text { Apr } & 8 \\ 7.3 & \text { Apr } & 8 \\ & & \\ 26070 & & \\ 579500 & & \\ 127 & \\ 11 & & \\ 7.8 & \end{array}$

FOR 1999 WATER YEAR

$\begin{array}{rrr}8499.0 & & \\ 23.3 & & \\ & & \\ 763 & \text { Feb } & 9 \\ 7.8 & \text { Dec } & 29 \\ 7.8 & \text { Jan } & 2 \\ 1360 & \text { Feb } & 9 \\ 16860 & & \\ 561400 & & \\ 50 & & \\ 11 & & \\ 8.0 & & \end{array}$

WATER YEARS 1966 - 1999

\begin{tabular}{|c|c|c|}
\hline 28.8 & & \\
\hline 80.1 & & 1982 \\
\hline 5.53 & & 1977 \\
\hline 3400 & Feb 17 & 1986 \\
\hline .08 & Mar 8 & 1968 \\
\hline .08 & Mar & 1968 \\
\hline 4240 & Feb 17 & 1986 \\
\hline 20890 & & \\
\hline 29 & & \\
\hline 9.6 & & \\
\hline 5.0 & & \\
\hline
\end{tabular}

a Diversion, in acre-feet, to Chicago Park Flume. 


\section{ROLLINS RESERVOIR NEAR COLFAX, CA}

LOCATION.—Lat 3908'08", long 12056'57", in NE 1/4 SE 1/4 sec.22, T.15 N., R.9 E., Placer County, Hydrologic Unit 18020126, on left bank $300 \mathrm{ft}$ upstream from Rollins Dam on Bear River and 2.3 mi north of Colfax.

DRAINAGE AREA.-104 $\mathrm{mi}^{2}$.

PERIOD OF RECORD.-December 1964 to current year.

GAGE.-Water-stage recorder. Datum of gage is sea level (levels by Nevada Irrigation District).

REMARKS.- - Reservoir is formed by an earthfill dam. Storage began Dec. 15, 1964. Usable capacity, 66,000 acre-ft between elevations $1,970.0 \mathrm{ft}$, invert of outlet tunnel, and 2,171.0 ft, spillway crest. Dead storage, 270 acre-ft. Several diversions into and out of basin upstream for power development and irrigation. Water is normally released through Rollins Powerplant (station 11421900). Part of the water then is diverted to Bear River Canal (station 11422000) for power development. Water is later used for irrigation. See schematic diagram of Bear River Basin.

COOPERATION.-Records were collected by Nevada Irrigation District, under general supervision of the U.S. Geological Survey, in connection with a Federal Energy Regulatory Commission project.

EXTREMES FOR PERIOD OF RECORD.-Maximum contents, 71,700 acre-ft, Feb. 17, 1986, elevation, 2,177.7 ft; minimum since reservoir first filled, 4,250 acre-ft, Oct. 10, 1977, elevation, 2,022.5 ft.

EXTREMES FOR CURRENT YEAR.-Maximum contents, 68,200 acre-ft, Feb. 9, elevation, 2,173.58 ft; minimum, 39,900 acre-ft, Oct. 18, elevation, $2,133.38 \mathrm{ft}$.

Capacity table (elevation, in feet, and contents, in acre-feet)

(Based on table provided by Nevada Irrigation District in 1964)

\begin{tabular}{|c|c|c|c|c|c|c|c|}
\hline 2,020 & 3,920 & 2,050 & 8,940 & 2,100 & 23,900 & 2,160 & 57,300 \\
\hline 2,030 & 5,320 & 2,060 & 11,200 & 2,120 & 32,700 & 2,178 & 72,000 \\
\hline 2,040 & 6,990 & 2,080 & 16,800 & 2,140 & 43,800 & & \\
\hline
\end{tabular}

\section{RESERVOIR STORAGE (ACRE-FEET), WATER YEAR OCTOBER 1998 TO SEPTEMBER 1999} DAILY OBSERVATION AT 2400 HOURS

\begin{tabular}{|c|c|c|c|c|c|c|c|c|c|c|c|c|}
\hline DAY & OCT & NOV & $\mathrm{DEC}$ & JAN & $\mathrm{FEB}$ & MAR & APR & MAY & JUN & JUL & AUG & SEP \\
\hline 1 & 44700 & 42200 & 57500 & 63700 & 66500 & 67100 & 66800 & 66600 & 66600 & 66100 & 62400 & 65000 \\
\hline 2 & 44700 & 43100 & 57800 & 63900 & 66500 & 67000 & 66800 & 66600 & 66500 & 66400 & 62500 & 65000 \\
\hline 3 & 44700 & 44500 & 59600 & 64000 & 66000 & 67300 & 66800 & 66800 & 66500 & 66100 & 63000 & 65100 \\
\hline 4 & 44600 & 45500 & 60800 & 64000 & 66300 & 67100 & 66800 & 66700 & 66600 & 66300 & 63600 & 64900 \\
\hline 5 & 44600 & 46600 & 61100 & 63900 & 66200 & 67000 & 66800 & 66700 & 66500 & 66300 & 64100 & 64700 \\
\hline 6 & 44600 & 47400 & 61300 & 63900 & 66300 & 66900 & 66800 & 66600 & 66500 & 66300 & 64700 & 64300 \\
\hline 7 & 44600 & 47900 & 61200 & 63600 & 67900 & 66800 & 66700 & 66600 & 66600 & 66200 & 64800 & 64400 \\
\hline 8 & 44600 & 48500 & 61300 & 63200 & 68100 & 66900 & 66900 & 66600 & 66500 & 66400 & 64600 & 64400 \\
\hline 9 & 44500 & 48800 & 61600 & 62200 & 68200 & 66900 & 66800 & 66600 & 66500 & 66400 & 64700 & 64300 \\
\hline 10 & 43000 & 49100 & 61800 & 61200 & 67300 & 66800 & 66800 & 66600 & 66600 & 66300 & 64700 & 64300 \\
\hline 11 & 41900 & 49500 & 61900 & 61100 & 67100 & 66900 & 66800 & 66600 & 66500 & 66300 & 64800 & 64100 \\
\hline 12 & 41700 & 49900 & 62000 & 61400 & 67000 & 66900 & 66900 & 66600 & 66500 & 66300 & 64900 & 63700 \\
\hline 13 & 41700 & 50300 & 62400 & 61900 & 66900 & 66800 & 66800 & 66600 & 66500 & 66300 & 65100 & 63800 \\
\hline 14 & 41800 & 50500 & 62800 & 62300 & 66900 & 66800 & 66800 & 66500 & 66500 & 66200 & 64900 & 63900 \\
\hline 15 & 41600 & 50700 & 62800 & 62800 & 66800 & 66800 & 66900 & 66600 & 66500 & 66300 & 64800 & 63900 \\
\hline 16 & 41600 & 51500 & 62800 & 62900 & 67100 & 66800 & 66900 & 66600 & 66600 & 66300 & 65000 & 64000 \\
\hline 17 & 40400 & 51800 & 63000 & 63100 & 67400 & 66800 & 66800 & 66600 & 66500 & 65400 & 65000 & 64200 \\
\hline 18 & 39900 & 52400 & 63100 & 65700 & 67300 & 66800 & 66800 & 66600 & 66400 & 65400 & 65200 & 63700 \\
\hline 19 & 40200 & 53000 & 63300 & 67300 & 67100 & 66900 & 66800 & 66600 & 66500 & 65400 & 65300 & 62600 \\
\hline 20 & 40500 & 53000 & 63300 & 67800 & 67100 & 66800 & 66900 & 66600 & 66400 & 65400 & 65400 & 61600 \\
\hline 21 & 40900 & 52900 & e 63200 & 67000 & 67100 & 66800 & 66900 & 66600 & 66500 & 65300 & 65200 & 60600 \\
\hline 22 & 41100 & 53200 & e 63300 & 66900 & 67000 & 66800 & 66700 & 66600 & 66400 & 65100 & 65200 & 59500 \\
\hline 23 & 41500 & 53400 & 63500 & 67500 & 67000 & 66800 & 66700 & 66600 & 66400 & 65000 & 65200 & 58500 \\
\hline 24 & 41500 & 53600 & 63700 & 67000 & 66900 & 66900 & 66600 & 66600 & 66400 & 65000 & 65400 & 57400 \\
\hline 25 & 41400 & 53600 & 63600 & 66800 & 67000 & 66800 & 66700 & 66600 & 66300 & 64900 & 65500 & 56400 \\
\hline 26 & 41600 & 53400 & 63700 & 66700 & 66900 & 66800 & 66700 & 66600 & 66300 & 64800 & 65400 & 55300 \\
\hline 27 & 41600 & 53400 & 63700 & 66700 & 66900 & 66800 & 66600 & 66600 & 66300 & 65000 & 65400 & 54200 \\
\hline 28 & 41600 & 53400 & 63700 & 66600 & 67100 & 66800 & 66600 & 66600 & 66300 & 64600 & 65200 & 53100 \\
\hline 29 & 42000 & 53500 & 63900 & 66600 & -- & 66800 & 66600 & 66600 & 66300 & 64100 & 65000 & 52000 \\
\hline 30 & 42100 & 56200 & 63800 & 66600 & --- & 66800 & 66600 & 66600 & 66300 & 63200 & 64800 & 50600 \\
\hline 31 & 42200 & --- & 63700 & 66700 & --- & 66800 & --- & 66600 & --- & 62600 & 65000 & --- \\
\hline MAX & 44700 & 56200 & 63900 & 67800 & 68200 & 67300 & 66900 & 66800 & 66600 & 66400 & 65500 & 65100 \\
\hline MIN & 39900 & 42200 & 57500 & 61100 & 66000 & 66800 & 66600 & 66500 & 66300 & 62600 & 62400 & 50600 \\
\hline a & 2137.29 & 2158.48 & 2168.19 & 2171.78 & 2172.30 & 2172.01 & 2171.72 & 2171.73 & 2171.34 & 2166.83 & 2169.82 & 2150.55 \\
\hline $\mathrm{b}$ & -2500 & +14000 & +7500 & +3000 & +400 & -300 & -200 & 0 & -300 & -3700 & +2400 & -14400 \\
\hline C & 38300 & 25420 & 55060 & 55240 & 56920 & 63970 & 66380 & 64820 & 62400 & 63940 & 41320 & 39250 \\
\hline
\end{tabular}

CAL YR 1998 MAX 67800 MIN $39900 \quad b+18100 \quad$ C 606300

WTR YR 1999 MAX 68200 MIN 39900 b +5900 c 633000

e Estimated.

a Elevation, in feet, at end of month.

b Change in contents, in acre-feet.

c Discharge, in acre-feet, through Rollins Powerplant, provided by Pacific Gas \& Electric Co. 
11422000 BEAR RIVER CANAL INTAKE NEAR COLFAX, CA

LOCATION.-Lat 3907'58", long 12057'12", in SW 1/4 SE 1/4 sec.22, T.15 N., R.9 E., Placer County, Hydrologic Unit 18020126, on right bank $400 \mathrm{ft}$ downstream from canal inlet, $0.2 \mathrm{mi}$ downstream from Rollins Dam, and $2.2 \mathrm{mi}$ north of Colfax.

PERIOD OF RECORD.-January 1912 to September 1953, October 1964 to current year. Monthly discharge only for some periods published in WSP 1315-A. Prior to October 1912, published as Pacific Gas \& Electric Co.'s Canal near Colfax; October 1912 to September 1953, published as Bear River Canal near Colfax.

GAGE.-Water-stage recorder. Elevation of gage is 1,950 ft above sea level, from topographic map. Prior to Mar. 25, 1946, water-stage recorder at site $1.5 \mathrm{mi}$ downstream at different datum.

REMARKS.-Canal diverts from left bank of Bear River. Water is used to develop power at Halsey and Wise Powerplants (stations 11425310 and 11425415). Part of the water is distributed for irrigation, and the remainder is eventually spilled into North Fork American River. Capacity of canal is believed to have been increased in 1917 and 1931. See schematic diagram of Bear River Basin.

COOPERATION.--Records were collected by Pacific Gas \& Electric Co., under general supervision of the U.S. Geological Survey, in connection with a Federal Energy Regulatory Commission project.

EXTREMES FOR PERIOD OF RECORD.-Maximum daily discharge, $531 \mathrm{ft}^{3} / \mathrm{s}$, Oct. 5, 6, 1980; no flow at times in most years.

\section{DISCHARGE, CUBIC FEET PER SECOND, WATER YEAR OCTOBER 1998 TO SEPTEMBER 1999}

\section{DAILY MEAN VALUES}

\begin{tabular}{|c|c|c|c|c|c|c|c|c|c|c|c|c|}
\hline DAY & OCT & NOV & $\mathrm{DEC}$ & JAN & FEB & MAR & APR & MAY & JUN & JUL & AUG & SEP \\
\hline 1 & 442 & 181 & 340 & 429 & 442 & 431 & 444 & 433 & 430 & 422 & 423 & 432 \\
\hline 2 & 443 & 8.4 & 340 & 430 & 441 & 427 & 443 & 433 & 429 & 432 & 424 & 432 \\
\hline 3 & 444 & 6.9 & 340 & 430 & 441 & 395 & 443 & 430 & 428 & 441 & 423 & 432 \\
\hline 4 & 445 & 4.4 & 367 & 438 & 439 & 392 & 442 & 432 & 429 & 440 & 423 & 433 \\
\hline 5 & 446 & 4.4 & 383 & 442 & 427 & 391 & 424 & 432 & 428 & 437 & 423 & 433 \\
\hline 6 & 448 & 4.4 & 383 & 442 & 366 & 394 & 406 & 432 & 428 & 439 & 424 & 433 \\
\hline 7 & 449 & 3.0 & 383 & 440 & 338 & 396 & 406 & 433 & 428 & 439 & 424 & 433 \\
\hline 8 & 450 & .00 & 382 & 443 & 352 & 396 & 401 & 433 & 428 & 439 & 424 & 431 \\
\hline 9 & 451 & .00 & 382 & 444 & 338 & 395 & 406 & 433 & 425 & 439 & 425 & 433 \\
\hline 10 & 452 & .00 & 382 & 444 & 377 & 395 & 406 & 433 & 428 & 440 & 425 & 433 \\
\hline 11 & 454 & .00 & 382 & 443 & 435 & 394 & 406 & 433 & 428 & 439 & 425 & 434 \\
\hline 12 & 455 & .00 & 381 & 442 & 447 & 418 & 424 & 433 & 427 & 439 & 425 & 434 \\
\hline 13 & 456 & .00 & 381 & 441 & 444 & 422 & 432 & 432 & 427 & 439 & 426 & 434 \\
\hline 14 & 458 & .00 & 381 & 441 & 444 & 422 & 432 & 433 & 427 & 439 & 426 & 434 \\
\hline 15 & 459 & .00 & 379 & 431 & 442 & 423 & 432 & 433 & 428 & 439 & 426 & 435 \\
\hline 16 & 460 & 211 & 378 & 350 & 299 & 427 & 431 & 432 & 427 & 439 & 427 & 435 \\
\hline 17 & 434 & 332 & 378 & 368 & 299 & 427 & 431 & 432 & 427 & 432 & 427 & 434 \\
\hline 18 & 65 & 358 & 378 & 376 & 299 & 433 & 429 & 432 & 427 & 425 & 427 & 434 \\
\hline 19 & 62 & 289 & 377 & 374 & 358 & 428 & 428 & 432 & 426 & 425 & 427 & 434 \\
\hline 20 & 63 & 402 & 377 & 375 & 383 & 412 & 428 & 432 & 426 & 425 & 428 & 434 \\
\hline 21 & 64 & 402 & 377 & 375 & 339 & 424 & 428 & 431 & 426 & 424 & 428 & 434 \\
\hline 22 & 66 & 402 & 377 & 375 & 410 & 429 & 428 & 431 & 426 & 423 & 428 & 435 \\
\hline 23 & 67 & 390 & 376 & 375 & 429 & 438 & 429 & 430 & 426 & 423 & 429 & 435 \\
\hline 24 & 142 & 365 & 376 & 375 & 412 & 438 & 434 & 431 & 426 & 423 & 430 & 435 \\
\hline 25 & 173 & 385 & 377 & 376 & 415 & 437 & 430 & 431 & 425 & 423 & 430 & 435 \\
\hline 26 & 174 & 397 & 376 & 418 & 441 & 437 & 430 & 430 & 425 & 423 & 431 & 435 \\
\hline 27 & 174 & 397 & 376 & 445 & 445 & 436 & 430 & 430 & 424 & 423 & 431 & 435 \\
\hline 28 & 175 & 396 & 401 & 445 & 445 & 436 & 430 & 430 & 424 & 423 & 432 & 435 \\
\hline 29 & 177 & 396 & 427 & 445 & --- & 441 & 431 & 430 & 423 & 423 & 432 & 435 \\
\hline 30 & 178 & 360 & 428 & 435 & --- & 445 & 432 & 429 & 423 & 424 & 432 & 436 \\
\hline 31 & 179 & --- & 428 & 433 & --- & 444 & --- & 429 & --- & 424 & 433 & --- \\
\hline TOTAL & 9405 & 5694.50 & 11793 & 12920 & 11147 & 13023 & 12796 & 13380 & 12799 & 13365 & 13238 & 13017 \\
\hline MEAN & 303 & 190 & 380 & 417 & 398 & 420 & 427 & 432 & 427 & 431 & 427 & 434 \\
\hline MAX & 460 & 402 & 428 & 445 & 447 & 445 & 444 & 433 & 430 & 441 & 433 & 436 \\
\hline MIN & 62 & .00 & 340 & 350 & 299 & 391 & 401 & 429 & 423 & 422 & 423 & 431 \\
\hline $\mathrm{AC}-\mathrm{FT}$ & 18650 & 11300 & 23390 & 25630 & 22110 & 25830 & 25380 & 26540 & 25390 & 26510 & 26260 & 25820 \\
\hline $\mathrm{a}$ & 13850 & 9000 & 21000 & 23030 & 20380 & 23830 & 23440 & 23680 & 21870 & 22750 & 22710 & 22010 \\
\hline $\mathrm{b}$ & 12030 & 4960 & 17920 & 21230 & 19240 & 21250 & 19470 & 20200 & 21570 & 22880 & 21110 & 19830 \\
\hline
\end{tabular}

a Discharge, in acre-feet, to Halsey Powerplant, provided by Pacific Gas \& Electric Co.

b Discharge, in acre-feet, to Wise Powerplant, provided by Pacific Gas \& Electric Co. 
11422000 BEAR RIVER CANAL INTAKE NEAR COLFAX, CA-Continued

STATISTICS OF MONTHLY MEAN DATA FOR WATER YEARS 1918 - 1931, BY WATER YEAR (WY)

\begin{tabular}{|c|c|c|c|c|c|c|c|c|c|c|c|c|}
\hline MEAN & 184 & 158 & 156 & 124 & 139 & 154 & 200 & 253 & 253 & 250 & 251 & 235 \\
\hline MAX & 300 & 285 & 281 & 257 & 265 & 257 & 286 & 278 & 300 & 317 & 300 & 300 \\
\hline (WY) & 1929 & 1929 & 1925 & 1925 & 1925 & 1922 & 1925 & 1925 & 1927 & 1931 & 1926 & 1927 \\
\hline MIN & .000 & .000 & .000 & .000 & .000 & .000 & 53.2 & 158 & 190 & 162 & 167 & 93.7 \\
\hline (WY) & 1930 & 1930 & 1930 & 1930 & 1930 & 1930 & 1931 & 1931 & 1931 & 1918 & 1918 & 1924 \\
\hline
\end{tabular}

SUMMARY STATISTICS

ANNUAL MEAN

HIGHEST ANNUAL MEAN

LOWEST ANNUAL MEAN

HIGHEST DAILY MEAN

LOWEST DAILY MEAN

ANNUAL SEVEN-DAY MINIMUM

ANNUAL RUNOFF (AC-FT)

10 PERCENT EXCEEDS

50 PERCENT EXCEEDS

90 PERCENT EXCEEDS
WATER YEARS $1918-1931$

\begin{tabular}{|c|c|c|}
\hline \multicolumn{3}{|l|}{197} \\
\hline 245 & & 1929 \\
\hline 121 & & 1931 \\
\hline 345 & Aug 2 & 1931 \\
\hline .00 & Nov 12 & 1917 \\
\hline .00 & Mar 17 & 1918 \\
\hline$\$ 2400$ & & \\
\hline 300 & & \\
\hline 232 & & \\
\hline .00 & & \\
\hline
\end{tabular}

STATISTICS OF MONTHLY MEAN DATA FOR WATER YEARS 1932 - 1999, BY WATER YEAR (WY)

\begin{tabular}{|c|c|c|c|c|c|c|c|c|c|c|c|c|}
\hline MEAN & 334 & 313 & 372 & 357 & 347 & 321 & 312 & 389 & 402 & 410 & 410 & 396 \\
\hline MAX & 492 & 495 & 488 & 479 & 478 & 485 & 490 & 498 & 499 & 493 & 497 & 496 \\
\hline (WY) & 1968 & 1968 & 1976 & 1979 & 1980 & 1980 & 1978 & 1978 & 1978 & 1967 & 1967 & 1967 \\
\hline MIN & 69.8 & 27.9 & 52.7 & 8.65 & 27.8 & 18.5 & 18.4 & 106 & 139 & 143 & 136 & 114 \\
\hline (WY) & 1978 & 1978 & 1977 & 1946 & 1946 & 1977 & 1940 & 1977 & 1977 & 1977 & 1977 & 1977 \\
\hline
\end{tabular}

SUMMARY STATISTICS

ANNUAL TOTAL

ANNUAL MEAN

HIGHEST ANNUAL MEAN

LOWEST ANNUAL MEAN

HIGHEST DAILY MEAN

LOWEST DAILY MEAN

ANNUAL SEVEN-DAY MINIMUM

ANNUAL RUNOFF (AC-FT)

ANNUAL DISCHARGE (AC-FT) a

ANNUAL DISCHARGE (AC-FT) b

10 PERCENT EXCEEDS

50 PERCENT EXCEEDS

90 PERCENT EXCEEDS
FOR 1998 CALENDAR YEAR

\begin{tabular}{|c|c|c|}
\hline $\begin{array}{c}135734.50 \\
372\end{array}$ & & \\
\hline 460 & Jan & 2 \\
\hline .00 & Nov & 8 \\
\hline .00 & Nov & 8 \\
\hline 269200 & & \\
\hline 238800 & & \\
\hline 212700 & & \\
\hline 436 & & \\
\hline 400 & & \\
\hline 253 & & \\
\hline
\end{tabular}

FOR 1999 WATER YEAR

\begin{tabular}{|c|c|}
\hline $\begin{array}{c}142577.50 \\
391\end{array}$ & \\
\hline 460 & Oct 16 \\
\hline .00 & Nov 8 \\
\hline .00 & Nov \\
\hline 282800 & \\
\hline 247600 & \\
\hline 221700 & \\
\hline 442 & \\
\hline 428 & \\
\hline 338 & \\
\hline
\end{tabular}

WATER YEARS 1932 - 1999

\begin{tabular}{|c|c|c|}
\hline 364 & & \\
\hline 462 & & 1980 \\
\hline 118 & & 1977 \\
\hline 531 & Oct 5 & 1980 \\
\hline .00 & Mar 12 & 1932 \\
\hline .00 & Mar 12 & 1932 \\
\hline 263500 & & \\
\hline 475 & & \\
\hline 424 & & \\
\hline 138 & & \\
\hline
\end{tabular}

a Discharge, in acre-feet, to Halsey Powerplant, provided by Pacific Gas \& Electric Co.

b Discharge, in acre-feet, to Wise Powerplant, provided by Pacific Gas \& Electric Co. 
11422500 BEAR RIVER BELOW ROLLINS DAM, NEAR COLFAX, CA

LOCATION.-Lat 3907'53", long 12057'29", in SE 1/4 SW 1/4 sec.22, T.15 N., R.9 E., Nevada County, Hydrologic Unit 18020126, on right bank $20 \mathrm{ft}$ upstream from new highway bridge, $0.5 \mathrm{mi}$ downstream from Rollins Dam, and $2.2 \mathrm{mi}$ north of Colfax.

DRAINAGE AREA.- $105 \mathrm{mi}^{2}$.

PERIOD OF RECORD._January 1912 to September 1913, October 1913 to July 1915 (gage heights and discharge measurements only), August 1915 to June 1917, November 1949 to September 1953, August 1964 to current year. Monthly discharge only for some periods, published in WSP 1315-A. Prior to August 1964, published as Bear River near Colfax. Records for November and December 1911 include diversion to Bear River Canal and are not equivalent.

GAGE.-Water-stage recorder and concrete control. Datum of gage is $1,927.41 \mathrm{ft}$ above sea level. Prior to Aug. 8, 1915, nonrecording gages at several sites above diversion dam $0.3 \mathrm{mi}$ upstream at different datums. Aug. 8, 1915, to June 30, 1917, nonrecording gage 0.7 mi downstream at different datum. Nov. 1, 1949, to Sept. 30, 1953, at site 0.2 mi downstream at different datum. Aug. 17, 1964, to Feb. 4, 1986, at present site and datum. Feb. 5, 1986, to Mar. 19, 1987, at site $160 \mathrm{ft}$ downstream at datum $8.00 \mathrm{ft}$ lower.

REMARKS.-Flow regulated by Rollins Reservoir (station 11421800) beginning Dec. 15, 1964. Bear River Canal (station 11422000) diverts upstream from station. See schematic diagram of Bear River Basin.

COOPERATION.-Records were collected by Nevada Irrigation District, under general supervision of the U.S. Geological Survey, in connection with a Federal Energy Regulatory Commission project.

EXTREMES FOR PERIOD OF RECORD.-Maximum discharge (prior to construction of Rollins Dam in 1964), 9,620 ft $3 / \mathrm{s}$, Nov. 20, 1950, gage height, $21.40 \mathrm{ft}$, site and datum then in use, from rating curve extended above $3,600 \mathrm{ft}^{3} / \mathrm{s}$ on basis of slope-area measurement of peak flow; no flow at times in 1912, 1952. Maximum discharge since construction of Rollins Dam, 34,300 ft $3 / \mathrm{s}$, Jan. 2, 1997, gage height, $18.01 \mathrm{ft}$, maximum gage height, $20.62 \mathrm{ft}$, Feb. 17, 1986, site and datum then in use, from rating curve extended above 11,600 ft $\mathrm{ft}^{3} / \mathrm{s}$; minimum daily, $0.5 \mathrm{ft}^{3} / \mathrm{s}$, Nov. 17, 1964.

DISCHARGE, CUBIC FEET PER SECOND, WATER YEAR OCTOBER 1998 TO SEPTEMBER 1999 DAILY MEAN VALUES

\begin{tabular}{|c|c|c|c|c|c|c|c|c|c|c|c|c|}
\hline DAY & OCT & NOV & $\mathrm{DEC}$ & JAN & $\mathrm{FEB}$ & MAR & APR & MAY & JUN & JUL & AUG & SEP \\
\hline 1 & 303 & 369 & 412 & 394 & 592 & 1790 & 856 & 784 & 425 & 465 & 280 & 255 \\
\hline 2 & 303 & 77 & 412 & 393 & 485 & 1630 & 815 & 796 & 563 & 471 & 217 & 257 \\
\hline 3 & 303 & 29 & 425 & 395 & 488 & 1960 & 831 & 939 & 557 & 416 & 158 & 263 \\
\hline 4 & 303 & 29 & 395 & 388 & 381 & 1750 & 802 & 953 & 612 & 438 & 160 & 259 \\
\hline 5 & 304 & 29 & 380 & 384 & 407 & 1520 & 844 & 887 & 541 & 455 & 161 & 254 \\
\hline 6 & 304 & 114 & 380 & 383 & 471 & 1310 & 856 & 841 & 514 & 440 & 164 & 254 \\
\hline 7 & 306 & 384 & 379 & 383 & 2650 & 1280 & 766 & 809 & 624 & 429 & 162 & 252 \\
\hline 8 & 308 & 385 & 379 & 383 & 2780 & 1220 & 850 & 780 & 582 & 460 & 162 & 251 \\
\hline 9 & 308 & 386 & 380 & 382 & 7330 & 1290 & 921 & 773 & 547 & 466 & 168 & 248 \\
\hline 10 & 305 & 386 & 381 & 378 & 3190 & 1190 & 858 & 757 & 570 & 442 & 206 & 251 \\
\hline 11 & 300 & 387 & 380 & 190 & 1920 & 1040 & 853 & 757 & 581 & 439 & 205 & 251 \\
\hline 12 & 297 & 387 & 385 & 28 & 1480 & 1120 & 884 & 748 & 554 & 430 & 202 & 253 \\
\hline 13 & 299 & 389 & 382 & 30 & 1410 & 1100 & 821 & 747 & 554 & 422 & 201 & 257 \\
\hline 14 & 298 & 382 & 383 & 30 & 1140 & 1070 & 888 & 721 & 525 & 416 & 199 & 260 \\
\hline 15 & 295 & 369 & 411 & 34 & 793 & 1060 & 925 & 703 & 493 & 424 & 203 & 255 \\
\hline 16 & 296 & 85 & 430 & 46 & 1130 & 1020 & 940 & 706 & 554 & 425 & 203 & 255 \\
\hline 17 & 291 & 33 & 430 & 43 & 2780 & 986 & 915 & 692 & 520 & 416 & 203 & 170 \\
\hline 18 & 350 & 29 & 430 & 135 & 2080 & 937 & 882 & 682 & 487 & 422 & 205 & 128 \\
\hline 19 & 352 & 30 & 430 & 1010 & 1880 & 934 & 874 & 666 & 504 & 422 & 205 & 121 \\
\hline 20 & 353 & 27 & 431 & 3160 & 1640 & 969 & 914 & 642 & 471 & 422 & 204 & 125 \\
\hline 21 & 366 & 27 & 432 & 2400 & 1960 & 916 & 952 & 663 & 498 & 421 & 203 & 133 \\
\hline 22 & 370 & 27 & 434 & 1440 & 1610 & 952 & 1020 & 642 & 483 & 419 & 204 & 130 \\
\hline 23 & 372 & 167 & 431 & 2810 & 1450 & 885 & 921 & 667 & 461 & 420 & 201 & 127 \\
\hline 24 & 374 & 330 & 432 & 2030 & 1390 & 889 & 865 & 666 & 459 & 420 & 202 & 125 \\
\hline 25 & 369 & 344 & 435 & 1210 & 1530 & 921 & 860 & 625 & 458 & 419 & 250 & 123 \\
\hline 26 & 363 & 344 & 432 & 900 & 1370 & 853 & 892 & 614 & 457 & 419 & 262 & 126 \\
\hline 27 & 356 & 345 & 433 & 865 & 1250 & 824 & 885 & 625 & 442 & 421 & 259 & 128 \\
\hline 28 & 368 & 348 & 410 & 773 & 1270 & 850 & 833 & 669 & 453 & 421 & 271 & 127 \\
\hline 29 & 369 & 350 & 385 & 698 & --- & 832 & 833 & 609 & 485 & 420 & 271 & 124 \\
\hline 30 & 368 & 399 & 385 & 660 & --- & 829 & 808 & 615 & 479 & 421 & 267 & 231 \\
\hline 31 & 368 & --- & 387 & 704 & --- & 870 & --- & 608 & --- & 418 & 259 & --- \\
\hline TOTAL & 10221 & 6987 & 12611 & 23059 & 46857 & 34797 & 26164 & 22386 & 15453 & 13339 & 6517 & 5993 \\
\hline MEAN & 330 & 233 & 407 & 744 & 1673 & 1122 & 872 & 722 & 515 & 430 & 210 & 200 \\
\hline MAX & 374 & 399 & 435 & 3160 & 7330 & 1960 & 1020 & 953 & 624 & 471 & 280 & 263 \\
\hline MIN & 291 & 27 & 379 & 28 & 381 & 824 & 766 & 608 & 425 & 416 & 158 & 121 \\
\hline $\mathrm{AC}-\mathrm{FT}$ & 20270 & 13860 & 25010 & 45740 & 92940 & 69020 & 51900 & 44400 & 30650 & 26460 & 12930 & 11890 \\
\hline
\end{tabular}




\section{BEAR RIVER BELOW ROLLINS DAM, NEAR COLFAX, CA—Continued}

STATISTICS OF MONTHLY MEAN DATA FOR WATER YEARS 1912 - 1953, BY WATER YEAR (WY)

\begin{tabular}{|c|c|c|c|c|c|c|c|c|c|c|c|c|}
\hline MEAN & 46.0 & 300 & 474 & 804 & 778 & 635 & 586 & 314 & 133 & 46.2 & 36.3 & 47.0 \\
\hline MAX & 73.8 & 1016 & 1372 & 1103 & 1354 & 1110 & 1126 & 578 & 226 & 109 & 102 & 89.7 \\
\hline (WY) & 1951 & 1951 & 1951 & 1951 & 1916 & 1916 & 1952 & 1952 & 1953 & 1916 & 1916 & 1916 \\
\hline MIN & 12.7 & 19.8 & 58.4 & 287 & 201 & 127 & 151 & 165 & 35.1 & .000 & .000 & .000 \\
\hline (WY) & 1913 & 1953 & 1953 & 1913 & 1913 & 1913 & 1912 & 1916 & 1913 & 1913 & 1913 & 1913 \\
\hline
\end{tabular}

SUMMARY STATISTICS

ANNUAL MEAN

HIGHEST ANNUAL MEAN

LOWEST ANNUAL MEAN

HIGHEST DAILY MEAN

LOWEST DAILY MEAN

ANNUAL SEVEN-DAY MINIMUM

INSTANTANEOUS PEAK FLOW

INSTANTANEOUS PEAK STAGE

ANNUAL RUNOFF (AC-FT)

10 PERCENT EXCEEDS

50 PERCENT EXCEEDS

90 PERCENT EXCEEDS
WATER YEARS $1912-1953$

\begin{tabular}{|c|c|c|}
\hline 356 & & \\
\hline 534 & & 1951 \\
\hline 126 & & 1913 \\
\hline 5760 & Nov 20 & 1950 \\
\hline .00 & Jul 5 & 1912 \\
\hline .00 & Sep 11 & 1912 \\
\hline 9620 & Nov 20 & 1950 \\
\hline 21.40 & Nov 20 & 1950 \\
\hline 58000 & & \\
\hline 879 & & \\
\hline 138 & & \\
\hline 1.0 & & \\
\hline
\end{tabular}

STATISTICS OF MONTHLY MEAN DATA FOR WATER YEARS 1966 - 1999, BY WATER YEAR (WY)

\begin{tabular}{|c|c|c|c|c|c|c|c|c|c|c|c|c|}
\hline MEAN & 115 & 197 & 369 & 649 & 715 & 745 & 654 & 507 & 354 & 256 & 202 & 157 \\
\hline MAX & 330 & 1267 & 1957 & 2973 & 2889 & 2324 & 2516 & 1211 & 757 & 538 & 420 & 383 \\
\hline (WY) & 1999 & 1984 & 1997 & 1997 & 1986 & 1983 & 1982 & 1995 & 1998 & 1983 & 1995 & 1983 \\
\hline MIN & 21.3 & 10.3 & 6.53 & 6.67 & 5.14 & 4.56 & 16.6 & 21.8 & 15.2 & 22.8 & 34.3 & \\
\hline (WY) & 1978 & 1978 & 1978 & 1977 & 1977 & 1977 & 1976 & 1977 & 1977 & 1977 & 1977 & 197 \\
\hline
\end{tabular}

$\begin{array}{ccccccccccccc}\text { MIN } & 21.3 & 10.3 & 6.53 & 6.67 & 5.14 & 4.56 & 16.6 & 21.8 & 15.2 & 22.8 \\ \text { (WY } & 1978 & 1978 & 1978 & 1977 & 1977 & 1977 & 1976 & 1977 & 1977 & 1977\end{array}$

SUMMARY STATISTICS

ANNUAL TOTAL

ANNUAL MEAN

HIGHEST ANNUAL MEAN

LOWEST ANNUAL MEAN

HIGHEST DAILY MEAN

LOWEST DAILY MEAN

ANNUAL SEVEN-DAY MINIMUM

INSTANTANEOUS PEAK FLOW

INSTANTANEOUS PEAK STAGE

ANNUAL RUNOFF (AC-FT)

10 PERCENT EXCEEDS

50 PERCENT EXCEEDS

90 PERCENT EXCEEDS
FOR 1998 CALENDAR YEAR

260571

714

$3990 \quad$ Feb 3

$\begin{array}{lll}22 & \text { Jan } 1 \\ 24 & \text { Jan } & 1\end{array}$

516800

1270

561

303
FOR 1999 WATER YEAR

224384

615

7330 Feb 9

27 Nov 20

37 Nov 16

11300 Feb 9

10.48 Feb 9

445100

1130

429

163
WATER YEARS 1966 - 1999

\begin{tabular}{|c|c|c|}
\hline 409 & & \\
\hline 972 & & 1983 \\
\hline 19.0 & & 1977 \\
\hline 22800 & Jan 2 & 1997 \\
\hline 3.6 & Dec 26 & 1977 \\
\hline 4.4 & Mar 23 & 1977 \\
\hline 34300 & Jan 2 & 1997 \\
\hline 20.62 & Feb 17 & 1986 \\
\hline 296000 & & \\
\hline 972 & & \\
\hline 196 & & \\
\hline 22 & & \\
\hline
\end{tabular}


LOCATION.-Lat 3902’30", long 121¹9'52", in NE 1/4 NW 1/4 sec.29, T.14 N., R.6 E., Placer County, Hydrologic Unit 18020108, on left bank $5.4 \mathrm{mi}$ northeast of Wheatland and $1.2 \mathrm{mi}$ downstream from New Camp Far West Reservoir.

DRAINAGE AREA.-Not determined.

PERIOD OF RECORD.-October 1989 to current year.

GAGE.-Water-stage recorder. Elevation of gage is $120 \mathrm{ft}$ above sea level, from topographic map.

REMARKS.- The gage measures required fish-release flow and is entirely regulated by New Camp Far West Reservoir. See schematic diagrams of lower Sacramento River and Bear River Basins.

COOPERATION.-Records provided by South Sutter Water District, under general supervision of the U.S. Geological Survey, in connection with a Federal Energy Regulatory Commission project.

EXTREMES FOR PERIOD OF RECORD.-Maximum daily discharge, $43 \mathrm{ft}^{3} / \mathrm{s}$, Dec. 4, 1994; minimum daily, $8.0 \mathrm{ft}^{3} / \mathrm{s}, \mathrm{July} 2,1995$.

DISCHARGE, CUBIC FEET PER SECOND, WATER YEAR OCTOBER 1998 TO SEPTEMBER 1999 DAILY MEAN VALUES

\begin{tabular}{|c|c|c|c|c|c|c|c|c|c|c|c|c|}
\hline DAY & OCT & NOV & $\mathrm{DEC}$ & JAN & $\mathrm{FEB}$ & MAR & $A P R$ & MAY & JUN & JUL & AUG & SEP \\
\hline 1 & 12 & 13 & 13 & 11 & 13 & 14 & 13 & 28 & 27 & 15 & 11 & 11 \\
\hline 2 & 12 & 13 & 12 & 11 & 13 & 14 & 13 & 28 & 27 & 11 & 11 & 11 \\
\hline 3 & 12 & 13 & 12 & 11 & 13 & 15 & 13 & 28 & 27 & 11 & 11 & 11 \\
\hline 4 & 12 & 13 & 12 & 11 & 13 & 15 & 13 & 28 & 27 & 11 & 11 & 11 \\
\hline 5 & 12 & 13 & 12 & 11 & 13 & 15 & 13 & 28 & 27 & 11 & 12 & 11 \\
\hline 6 & 12 & 13 & 12 & 11 & 14 & 15 & 13 & 28 & 27 & 11 & 12 & 11 \\
\hline 7 & 12 & 14 & 12 & 11 & 14 & 14 & 13 & 27 & 27 & 12 & 12 & 11 \\
\hline 8 & 12 & 14 & 13 & 11 & 14 & 14 & 13 & 27 & 27 & 12 & 12 & 11 \\
\hline 9 & 12 & 14 & 13 & 11 & 14 & 15 & 20 & 27 & 27 & 11 & 12 & 11 \\
\hline 10 & 12 & 14 & 13 & 11 & 14 & 15 & 28 & 27 & 27 & 11 & 12 & 11 \\
\hline 11 & 12 & 14 & 13 & 11 & 15 & 14 & 28 & 27 & 26 & 11 & 12 & 11 \\
\hline 12 & 12 & 14 & 13 & 11 & 15 & 14 & 28 & 27 & 26 & 11 & 12 & 12 \\
\hline 13 & 12 & 14 & 13 & 11 & 15 & 14 & 28 & 27 & 26 & 11 & 12 & 12 \\
\hline 14 & 12 & 14 & 13 & 12 & 15 & 14 & 28 & 27 & 27 & 10 & 12 & 12 \\
\hline 15 & 12 & 14 & 13 & 12 & 15 & 14 & 28 & 27 & 28 & 11 & 12 & 12 \\
\hline 16 & 11 & 14 & 13 & 12 & 15 & 14 & 28 & 27 & 27 & 11 & 11 & 12 \\
\hline 17 & 11 & 14 & 13 & 12 & 15 & 14 & 28 & 27 & 28 & 11 & 11 & 12 \\
\hline 18 & 11 & 14 & 13 & 12 & 15 & 14 & 28 & 27 & 27 & 11 & 11 & 12 \\
\hline 19 & 13 & 14 & 13 & 12 & 15 & 14 & 28 & 27 & 27 & 11 & 11 & 12 \\
\hline 20 & 13 & 35 & 13 & 15 & 15 & 13 & 28 & 27 & 27 & 11 & 11 & 12 \\
\hline 21 & 13 & 35 & 13 & 15 & 14 & 13 & 28 & 27 & 26 & 11 & 11 & 12 \\
\hline 22 & 13 & 35 & 12 & 13 & 15 & 13 & 28 & 27 & 26 & 11 & 11 & 12 \\
\hline 23 & 13 & 35 & 12 & 13 & 14 & 13 & 28 & 27 & 27 & 11 & 11 & 12 \\
\hline 24 & 13 & 20 & 12 & 14 & 14 & 13 & 28 & 27 & 28 & 11 & 11 & 12 \\
\hline 25 & 13 & 13 & 12 & 12 & 14 & 14 & 28 & 27 & 28 & 11 & 11 & 12 \\
\hline 26 & 13 & 13 & 12 & 12 & 14 & 14 & 28 & 27 & 27 & 11 & 11 & 12 \\
\hline 27 & 13 & 13 & 12 & 13 & 14 & 13 & 28 & 27 & 27 & 11 & 11 & 14 \\
\hline 28 & 13 & 13 & 12 & 13 & 14 & 13 & 28 & 27 & 27 & 11 & 11 & 14 \\
\hline 29 & 13 & 13 & 12 & 13 & --- & 13 & 28 & 27 & 27 & 11 & 11 & 14 \\
\hline 30 & 13 & 13 & 11 & 13 & --- & 13 & 28 & 27 & 27 & 11 & 11 & 14 \\
\hline 31 & 13 & --- & 11 & 13 & --- & 13 & --- & 27 & --- & 11 & 11 & --- \\
\hline TOTAL & 382 & 498 & 385 & 374 & 398 & 430 & 712 & 843 & 809 & 346 & 352 & 357 \\
\hline MEAN & 12.3 & 16.6 & 12.4 & 12.1 & 14.2 & 13.9 & 23.7 & 27.2 & 27.0 & 11.2 & 11.4 & 11.9 \\
\hline MAX & 13 & 35 & 13 & 15 & 15 & 15 & 28 & 28 & 28 & 15 & 12 & 14 \\
\hline MIN & 11 & 13 & 11 & 11 & 13 & 13 & 13 & 27 & 26 & 10 & 11 & 11 \\
\hline $\mathrm{AC}-\mathrm{FT}$ & 758 & 988 & 764 & 742 & 789 & 853 & 1410 & 1670 & 1600 & 686 & 698 & 708 \\
\hline
\end{tabular}

STATISTICS OF MONTHLY MEAN DATA FOR WATER YEARS 1990 - 1999, BY WATER YEAR (WY)

\begin{tabular}{|c|c|c|c|c|c|c|c|c|c|c|c|c|}
\hline MEAN & 12.6 & 12.9 & 12.9 & 13.6 & 13.5 & 14.0 & 27.5 & 28.0 & 27.7 & 11.3 & 11.4 & 11.4 \\
\hline MAX & 14.5 & 18.0 & 16.4 & 21.7 & 18.7 & 21.7 & 32.0 & 30.5 & 30.1 & 12.9 & 13.0 & 13.0 \\
\hline (WY) & 1998 & 1996 & 1996 & 1995 & 1995 & 1995 & 1995 & 1995 & 1995 & 1995 & 1995 & 1995 \\
\hline MIN & 11.0 & 11.0 & 11.0 & 10.9 & 11.0 & 11.2 & 23.7 & 25.9 & 25.8 & 11.0 & 10.8 & 10.8 \\
\hline (WY) & 1991 & 1991 & 1991 & 1991 & 1991 & 1991 & 1999 & 1990 & 1900 & 1997 & 1990 & 1990 \\
\hline
\end{tabular}

SUMMARY STATISTICS

ANNUAL TOTAL

ANNUAL MEAN

HIGHEST ANNUAL MEAN

LOWEST ANNUAL MEAN

HIGHEST DAILY MEAN

LOWEST DAILY MEAN

ANNUAL SEVEN-DAY MINIMUM

ANNUAL RUNOFF (AC-FT)

10 PERCENT EXCEEDS

50 PERCENT EXCEEDS

90 PERCENT EXCEEDS
FOR 1998 CALENDAR YEAR

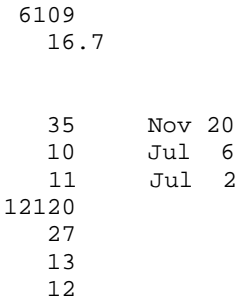

FOR 1999 WATER YEAR

5886

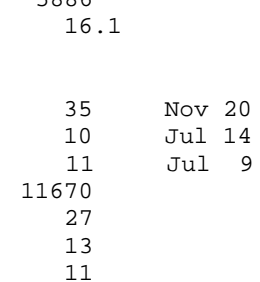

WATER YEARS 1990 - 1999

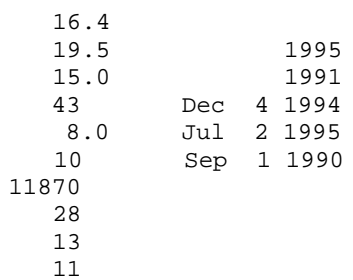




\section{BEAR RIVER NEAR WHEATLAND, CA}

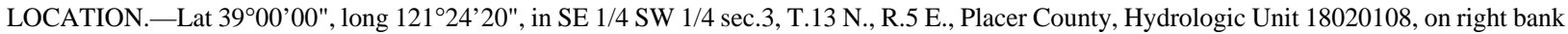
$200 \mathrm{ft}$ downstream from bridge on State Highway 65, 1 mi southeast of Wheatland, and 6.5 mi downstream from New Camp Far West Reservoir.

DRAINAGE AREA.-292 $\mathrm{mi}^{2}$.

\section{WATER-DISCHARGE RECORDS}

PERIOD OF RECORD.-October 1928 to current year.

REVISED RECORDS.-WSP 1931: Drainage area.

GAGE.-Water-stage recorder. Datum of gage is $71.92 \mathrm{ft}$ above sea level. See WSP 2131 for history of changes prior to May $28,1970$.

REMARKS.-Records good. Natural flow of stream affected by inflow from Yuba and American River Basins. Flow regulated by Lake Combie, usable capacity, 7,840 acre-ft, since 1928; Rollins Reservoir (station 11421800), since December 1964; and New Camp Far West Reservoir, usable capacity, 102,200 acre-ft, since October 1963. Many diversions for irrigation and power. See schematic diagrams of Bear River and lower Sacramento River Basins.

EXTREMES FOR PERIOD OF RECORD.-Maximum discharge, 48,000 ft 3 /s, Feb. 17, 1986, gage height, $21.60 \mathrm{ft}$, maximum gage height, $23.72 \mathrm{ft}$, Jan. 2, 1997 (backwater from Feather River); no flow at times.

DISCHARGE, CUBIC FEET PER SECOND, WATER YEAR OCTOBER 1998 TO SEPTEMBER 1999 DAILY MEAN VALUES

\begin{tabular}{|c|c|c|c|c|c|c|c|c|c|c|c|c|}
\hline DAY & OCT & NOV & $\mathrm{DEC}$ & JAN & FEB & MAR & $\mathrm{APR}$ & MAY & JUN & JUL & AUG & SEP \\
\hline 1 & 79 & 318 & 29 & 488 & 1260 & 2390 & 1100 & 490 & 98 & 30 & 19 & 43 \\
\hline 2 & 234 & 316 & 23 & 486 & 1120 & 2380 & 1080 & 420 & 131 & 16 & 19 & 48 \\
\hline 3 & 233 & 315 & 28 & 487 & e 960 & 2590 & 1020 & 376 & 148 & 18 & 17 & 16 \\
\hline 4 & 237 & 311 & 22 & 485 & e850 & 2680 & 984 & 459 & 149 & 16 & 19 & 16 \\
\hline 5 & 235 & 322 & 24 & 485 & e740 & 2240 & 995 & 482 & 151 & 16 & 22 & 19 \\
\hline 6 & 256 & 315 & 29 & 485 & 709 & 1980 & 1080 & 443 & 179 & 15 & 23 & 20 \\
\hline 7 & 274 & 322 & 34 & 482 & 2460 & 1780 & 863 & 390 & 173 & 15 & 21 & 17 \\
\hline 8 & 250 & 322 & 151 & 482 & 5370 & 1660 & 1200 & 342 & 161 & 16 & 25 & 22 \\
\hline 9 & 242 & 320 & 466 & 484 & 10400 & 1790 & 1270 & 310 & 138 & 14 & 25 & 20 \\
\hline 10 & 243 & 319 & 515 & 486 & 7520 & 1780 & 1230 & 286 & 113 & 15 & 25 & 17 \\
\hline 11 & 244 & 315 & 505 & 484 & 3740 & 1600 & 1180 & 238 & 78 & 17 & 22 & 17 \\
\hline 12 & 241 & 318 & 698 & 484 & 2760 & 1460 & 1180 & 281 & 50 & 16 & 22 & 19 \\
\hline 13 & 238 & 318 & 786 & 482 & 2280 & 1440 & 1110 & 269 & 42 & 13 & 21 & 17 \\
\hline 14 & 217 & 318 & 690 & 247 & 2060 & 1400 & 988 & 266 & 50 & 12 & 21 & 16 \\
\hline 15 & 207 & 319 & 680 & 21 & 1860 & 1350 & 1080 & 264 & 52 & 12 & 19 & 17 \\
\hline 16 & 259 & 319 & 675 & 21 & 1770 & 1240 & 1070 & 264 & 44 & 14 & 22 & 17 \\
\hline 17 & 295 & 319 & 672 & 20 & 5040 & 1310 & 1050 & 238 & 39 & 15 & 20 & 17 \\
\hline 18 & 297 & 312 & 670 & e25 & 3910 & 1240 & 1040 & 188 & 35 & 20 & 19 & 15 \\
\hline 19 & 295 & 316 & 672 & e1440 & 3450 & 1170 & 1170 & 154 & 36 & 17 & 22 & 16 \\
\hline 20 & 283 & 319 & 674 & e5540 & 2710 & 1150 & 1170 & 139 & 39 & 17 & 24 & 15 \\
\hline 21 & 296 & 320 & 672 & 4590 & 4120 & 1190 & 676 & 137 & 38 & 17 & 22 & 13 \\
\hline 22 & 296 & 321 & 517 & 2700 & 3250 & 1150 & 755 & 131 & 35 & 16 & 22 & 11 \\
\hline 23 & 292 & 327 & 308 & 4260 & 2490 & 1130 & 855 & 129 & 35 & 14 & 25 & 13 \\
\hline 24 & 301 & 317 & 384 & 4300 & 2150 & 1100 & 885 & 125 & 33 & e14 & 23 & 11 \\
\hline 25 & 301 & 316 & 486 & 2660 & 2200 & 1130 & 838 & 148 & 33 & e14 & 24 & 12 \\
\hline 26 & 299 & 317 & 487 & 1860 & 2150 & 1120 & 766 & 168 & 33 & e16 & 22 & 12 \\
\hline 27 & 300 & 320 & 486 & 1490 & 1940 & 1070 & 720 & 129 & 36 & 18 & 20 & 12 \\
\hline 28 & 277 & 319 & 486 & 1290 & 1820 & 1020 & 650 & 96 & 35 & 17 & 21 & 13 \\
\hline 29 & 306 & 323 & 481 & 1140 & --- & 1000 & 578 & 95 & 34 & 17 & 20 & 14 \\
\hline 30 & 304 & 213 & 480 & 1030 & --- & 896 & 516 & 95 & 34 & 18 & 20 & 12 \\
\hline 31 & 311 & --- & 484 & 1050 & --- & 1090 & --- & 93 & --- & 17 & 17 & --- \\
\hline TOTAL & 8142 & 9446 & 13314 & 39984 & 81089 & 46526 & 29099 & 7645 & 2252 & 502 & 663 & 527 \\
\hline MEAN & 263 & 315 & 429 & 1290 & 2896 & 1501 & 970 & 247 & 75.1 & 16.2 & 21.4 & 17.6 \\
\hline MAX & 311 & 327 & 786 & 5540 & 10400 & 2680 & 1270 & 490 & 179 & 30 & 25 & 48 \\
\hline MIN & 79 & 213 & 22 & 20 & 709 & 896 & 516 & 93 & 33 & 12 & 17 & 11 \\
\hline $\mathrm{AC}-\mathrm{FT}$ & 16150 & 18740 & 26410 & 79310 & 160800 & 92280 & 57720 & 15160 & 4470 & 996 & 1320 & 1050 \\
\hline
\end{tabular}


11424000 BEAR RIVER NEAR WHEATLAND, CA—Continued

STATISTICS OF MONTHLY MEAN DATA FOR WATER YEARS 1930 - 1963, BY WATER YEAR (WY)

\begin{tabular}{|c|c|c|c|c|c|c|c|c|c|c|c|c|}
\hline & OCT & NOV & $\mathrm{DEC}$ & JAN & $\mathrm{FEB}$ & MAR & $\mathrm{APR}$ & MAY & JUN & JUL & AUG & SEP \\
\hline MEAN & 92.8 & 184 & 565 & 826 & 1240 & 1033 & 770 & 306 & 79.0 & 12.6 & 16.7 & 18.4 \\
\hline MAX & 1348 & 1980 & 3501 & 3004 & 3360 & 2918 & 2553 & 939 & 245 & 55.4 & 148 & 215 \\
\hline (WY) & 1963 & 1951 & 1956 & 1956 & 1936 & 1938 & 1958 & 1942 & 1932 & 1952 & 1935 & 1935 \\
\hline MIN & 2.05 & 9.14 & 21.3 & 68.0 & 156 & 192 & 11.3 & .57 & .71 & .53 & .65 & .30 \\
\hline (WY) & 1961 & 1960 & 1960 & 1947 & 1933 & 1933 & 1959 & 1959 & 1959 & 1959 & 1939 & 1939 \\
\hline
\end{tabular}

SUMMARY STATISTICS

ANNUAL MEAN

HIGHEST ANNUAL MEAN

LOWEST ANNUAL MEAN

HIGHEST DAILY MEAN

LOWEST DAILY MEAN

ANNUAL SEVEN-DAY MINIMUM

INSTANTANEOUS PEAK FLOW

INSTANTANEOUS PEAK STAGE

ANNUAL RUNOFF (AC-FT)

10 PERCENT EXCEEDS

50 PERCENT EXCEEDS

90 PERCENT EXCEEDS
WATER YEARS $1930-1963$

\begin{tabular}{|c|c|c|}
\hline 424 & & \\
\hline 891 & & 1951 \\
\hline 70.0 & & 1933 \\
\hline 22100 & Dec 23 & 1955 \\
\hline .00 & Sep 18 & 1939 \\
\hline .00 & Sep 18 & 1939 \\
\hline 33000 & Dec 22 & 1955 \\
\hline 20.83 & Nov 21 & 1950 \\
\hline 307500 & & \\
\hline 1060 & & \\
\hline 77 & & \\
\hline 3.6 & & \\
\hline
\end{tabular}

STATISTICS OF MONTHLY MEAN DATA FOR WATER YEARS 1966 - 1999, BY WATER YEAR (WY)

\begin{tabular}{|c|c|c|c|c|c|c|c|c|c|c|c|c|}
\hline & $\mathrm{OCT}$ & NOV & $\mathrm{DEC}$ & JAN & FEB & MAR & APR & MAY & JUN & JUL & AUG & SEP \\
\hline MEAN & 23.1 & 147 & 469 & 1010 & 1257 & 1166 & 739 & 251 & 73.6 & 19.7 & 15.6 & 16.2 \\
\hline MAX & 263 & 1606 & 2668 & 3954 & 5201 & 3845 & 3796 & 1035 & 484 & 72.6 & 29.5 & 73.2 \\
\hline (WY) & 1999 & 1984 & 1984 & 1997 & 1986 & 1983 & 1982 & 1983 & 1998 & 1995 & 1967 & 1998 \\
\hline MIN & .002 & .056 & .000 & .14 & .62 & 1.07 & .60 & 4.05 & 3.17 & 2.95 & 4.72 & 1.31 \\
\hline (WY) & 1978 & 1977 & 1977 & 1977 & 1977 & 1977 & 1977 & 1977 & 1977 & 1977 & 1977 & 1977 \\
\hline
\end{tabular}

SUMMARY STATISTICS

ANNUAL TOTAL

ANNUAL MEAN

HIGHEST ANNUAL MEAN

LOWEST ANNUAL MEAN

HIGHEST DAILY MEAN

LOWEST DAILY MEAN

ANNUAL SEVEN-DAY MINIMUM

INSTANTANEOUS PEAK FLOW

INSTANTANEOUS PEAK STAGE

ANNUAL RUNOFF (AC-FT)

10 PERCENT EXCEEDS

50 PERCENT EXCEEDS

90 PERCENT EXCEEDS
FOR 1998 CALENDAR YEAR

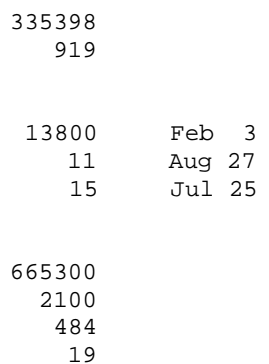

FOR 1999 WATER YEAR

$\begin{array}{rlr}239189 & & \\ 655 & & \\ & & \\ 10400 & \text { Feb } & 9 \\ 11 & \text { Sep } & 22 \\ 12 & \text { Sep } & 21 \\ 14400 & \text { Feb } & 9 \\ 15.42 & \text { Feb } & 9 \\ 474400 & & \\ 1780 & & \\ 299 & & \\ 17 & & \end{array}$

WATER YEARS 1966 - 1999

\begin{tabular}{|c|c|c|}
\hline 428 & & \\
\hline 1191 & & 1983 \\
\hline 3.42 & & 1977 \\
\hline 35900 & Feb 17 & 1986 \\
\hline .00 & Oct 14 & 1976 \\
\hline .00 & Oct 29 & 1976 \\
\hline 48000 & Feb 17 & 1986 \\
\hline 23.72 & $\operatorname{Jan} 2$ & 1997 \\
\hline 310200 & & \\
\hline 1240 & & \\
\hline 23 & & \\
\hline 8.0 & & \\
\hline
\end{tabular}


WATER-QUALITY RECORDS

PERIOD OF RECORD.-Water years 1953 to July 1980, June 1999 to September 1999. CHEMICAL DATA: Water years 1953 to July 1980, June 1999 to September 1999. SEDIMENT DATA: June 1999 to September 1999.

\section{WATER-QUALITY DATA, WATER YEAR OCTOBER 1998 TO SEPTEMBER 1999}

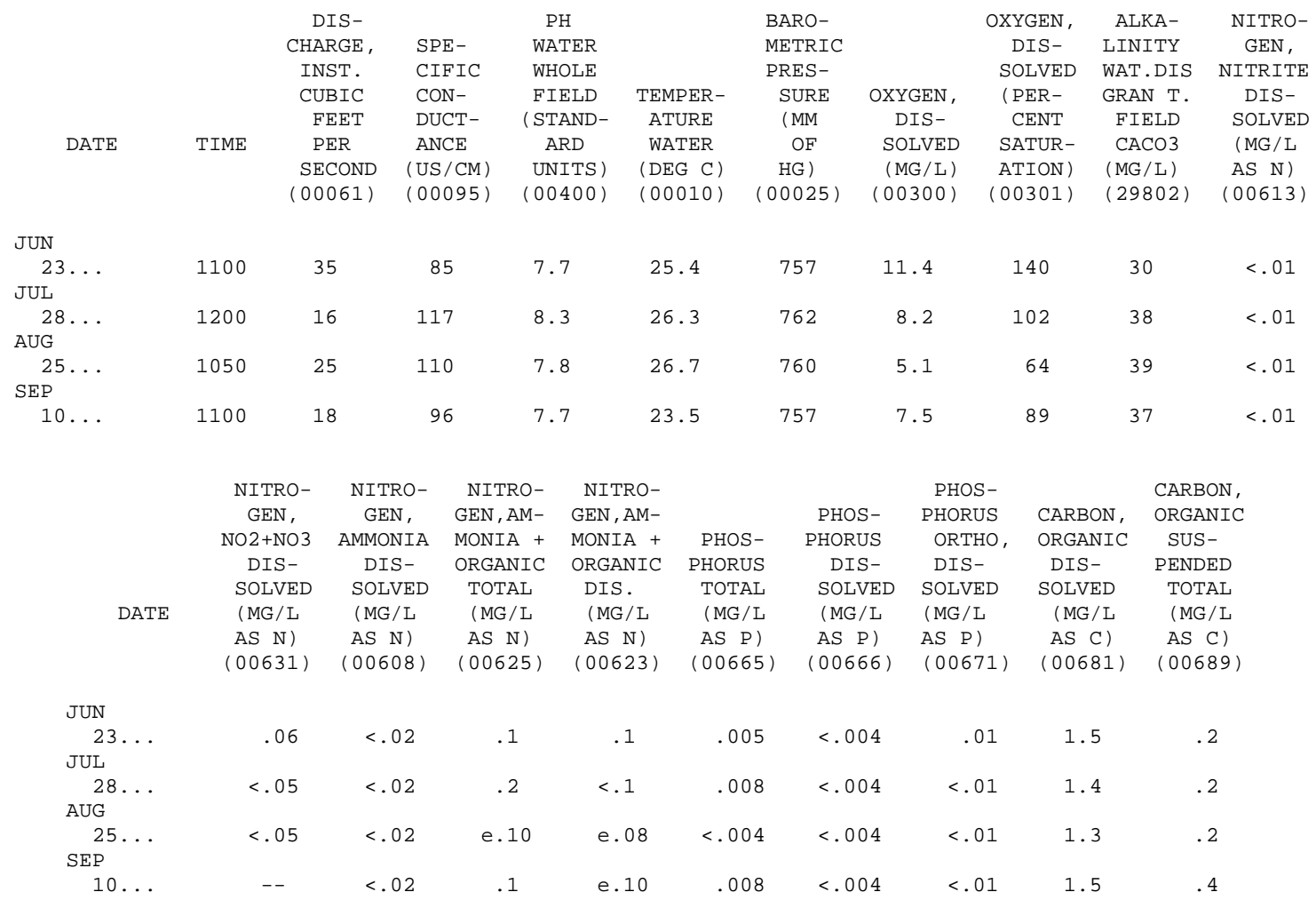

PARTICLE-SIZE DISTRIBUTION, WATER YEAR OCTOBER 1998 TO SEPTEMBER 1999

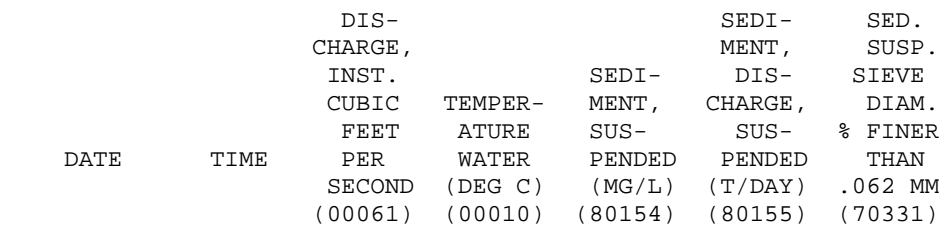

\begin{tabular}{|c|c|c|c|c|c|c|}
\hline \multicolumn{7}{|l|}{ JUN } \\
\hline $23 \ldots N$ & 1100 & 35 & 25.4 & 9 & .85 & 87 \\
\hline \multicolumn{7}{|l|}{ JUL } \\
\hline $28 \ldots N$ & 1200 & 16 & 26.3 & 4 & .17 & 92 \\
\hline \multicolumn{7}{|l|}{ AUG } \\
\hline $25 \ldots N$ & 1050 & 25 & 26.7 & 10 & .68 & 91 \\
\hline \multicolumn{7}{|l|}{ SEP } \\
\hline $10 \ldots N$ & 1100 & 18 & 23.5 & 6 & .29 & 60 \\
\hline
\end{tabular}

e Estimated.

$<$ Actual value is known to be less than the value shown.

N Suspended-sediment concentration value determined from a sample collected and processed according to National Water-Quality Assessment (NAWQA) protocol. 
11425418 MORMON RAVINE NEAR NEWCASTLE, CA

LOCATION.-Lat 3850'12", long 12105'36", in SE 1/4 NW 1/4 sec.4, T.11 N., R.8 E., Placer County, Hydrologic Unit 18020128, on right bank $200 \mathrm{ft}$ upstream from Folsom Lake, $700 \mathrm{ft}$ north of Newcastle Powerplant, and $3.3 \mathrm{mi}$ southeast of Newcastle.

DRAINAGE AREA.-3.84 $\mathrm{mi}^{2}$.

PERIOD OF RECORD.-October 1989 to current year (low-flow records only).

GAGE.-Water-stage recorder and V-notch sharp-crested weir. Elevation of gage is $500 \mathrm{ft}$ above sea level, from topographic map.

REMARKS.- Records not computed above $8.5 \mathrm{ft}^{3} / \mathrm{s}$. Low flow augmented by release from end of South Canal. Most of the water in South Canal is diverted to Newcastle Powerplant (station 11425416). See schematic diagram of Bear River Basin.

COOPERATION.--Records were collected by Pacific Gas \& Electric Co., under general supervision of the U.S. Geological Survey, in connection with a Federal Energy Regulatory Commission project.

DISCHARGE, CUBIC FEET PER SECOND, WATER YEAR OCTOBER 1998 TO SEPTEMBER 1999

DAILY MEAN VALUES

\begin{tabular}{|c|c|c|c|c|c|c|c|c|c|c|c|c|}
\hline DAY & OCT & NOV & $\mathrm{DEC}$ & JAN & $\mathrm{FEB}$ & MAR & $A P R$ & MAY & JUN & JUL & AUG & SEP \\
\hline 1 & --- & 2.7 & --- & 8.0 & --- & --- & --- & --- & 6.4 & 8.5 & --- & --- \\
\hline 2 & --- & 3.5 & --- & 8.0 & --- & --- & --- & --- & --- & 7.5 & --- & --- \\
\hline 3 & --- & 6.0 & --- & 8.0 & --- & --- & --- & --- & 8.5 & --- & --- & 7.3 \\
\hline 4 & --- & 5.4 & --- & 7.9 & --- & --- & --- & --- & --- & --- & --- & 7.0 \\
\hline 5 & --- & 5.3 & --- & 7.5 & --- & --- & --- & --- & 8.5 & --- & --- & 6.3 \\
\hline 6 & --- & 4.5 & --- & 7.6 & --- & --- & --- & --- & 7.7 & --- & --- & 5.8 \\
\hline 7 & --- & 8.0 & --- & 7.7 & --- & --- & --- & 8.0 & 7.3 & --- & --- & 5.6 \\
\hline 8 & --- & 7.8 & --- & 8.0 & --- & --- & --- & 7.7 & 6.8 & --- & --- & 5.8 \\
\hline 9 & --- & 5.7 & --- & 8.0 & --- & --- & --- & 7.6 & 6.3 & --- & --- & 6.1 \\
\hline 10 & --- & 4.1 & --- & 7.9 & --- & --- & --- & 7.6 & 6.3 & --- & --- & 6.9 \\
\hline 11 & --- & --- & --- & 8.0 & --- & --- & --- & 7.5 & 6.1 & --- & --- & 6.6 \\
\hline 12 & --- & --- & --- & 8.2 & --- & --- & --- & 7.4 & 6.4 & --- & --- & 6.6 \\
\hline 13 & --- & 6.9 & --- & 8.0 & --- & --- & --- & 7.7 & 6.2 & --- & --- & 6.7 \\
\hline 14 & 7.6 & 7.8 & --- & 7.7 & --- & --- & --- & 7.7 & 6.2 & --- & --- & 6.3 \\
\hline 15 & --- & 5.9 & --- & --- & --- & --- & --- & 7.9 & 7.1 & --- & --- & 6.8 \\
\hline 16 & --- & --- & --- & --- & --- & --- & --- & 7.6 & 7.2 & --- & --- & 6.4 \\
\hline 17 & --- & --- & 8.2 & --- & --- & --- & --- & 7.0 & 6.5 & --- & --- & 7.0 \\
\hline 18 & --- & --- & 7.8 & --- & --- & --- & --- & 6.4 & --- & --- & --- & 7.6 \\
\hline 19 & 2.2 & --- & 7.9 & --- & --- & --- & --- & 7.1 & --- & --- & --- & 7.6 \\
\hline 20 & .96 & --- & 7.9 & --- & --- & --- & --- & 7.2 & --- & --- & --- & 7.6 \\
\hline 21 & .87 & --- & 7.5 & --- & --- & --- & --- & 6.8 & --- & --- & --- & 7.3 \\
\hline 22 & 1.4 & --- & 7.5 & --- & --- & --- & --- & 6.8 & --- & --- & --- & 7.4 \\
\hline 23 & .86 & --- & 7.4 & --- & --- & --- & --- & 7.1 & --- & --- & --- & 7.0 \\
\hline 24 & 3.8 & --- & 8.2 & --- & --- & --- & --- & 7.7 & --- & --- & --- & 7.0 \\
\hline 25 & 3.0 & 8.4 & 8.2 & --- & --- & --- & --- & 7.3 & --- & --- & --- & 7.0 \\
\hline 26 & 6.3 & 7.9 & 8.5 & --- & --- & --- & --- & 6.8 & --- & --- & --- & 6.7 \\
\hline 27 & 5.5 & --- & 8.4 & --- & --- & --- & --- & 6.5 & --- & --- & --- & 6.7 \\
\hline 28 & 2.2 & --- & 8.2 & --- & --- & --- & --- & 6.6 & --- & --- & --- & 6.7 \\
\hline 29 & 2.2 & --- & --- & --- & --- & --- & --- & 7.5 & --- & --- & --- & 6.5 \\
\hline 30 & 1.5 & --- & 8.2 & --- & --- & --- & 7.8 & 7.1 & --- & --- & --- & 6.4 \\
\hline 31 & 1.6 & --- & 8.2 & --- & --- & --- & --- & 6.7 & --- & --- & --- & --- \\
\hline TOTAL & --- & --- & --- & --- & --- & --- & --- & --- & --- & --- & --- & --- \\
\hline MEAN & --- & --- & --- & --- & --- & --- & --- & --- & --- & --- & --- & --- \\
\hline MAX & --- & --- & --- & --- & --- & --- & --- & --- & --- & --- & --- & --- \\
\hline MIN & --- & --- & --- & --- & --- & --- & --- & --- & --- & --- & --- & --- \\
\hline $\mathrm{AC}-\mathrm{FT}$ & --- & --- & --- & --- & --- & --- & --- & --- & --- & --- & --- & --- \\
\hline $\mathrm{a}$ & 6440 & 5670 & 17290 & 17660 & 16220 & 17710 & 13580 & 6030 & 4500 & 0 & 0 & 9900 \\
\hline
\end{tabular}

CAL YR 1998 a 131800

WTR YR 1999 a 115000

a Diversion, in acre-feet, to Newcastle Powerplant, provided by Pacific Gas \& Electric Co. 


\section{SACRAMENTO RIVER AT VERONA, CA}

LOCATION.—Lat 3846’28", long 121³5'50", in SW 1/4 NW 1/4 sec.25, T.11 N., R.3 E., Sutter County, Hydrologic Unit 18020109, on left bank 1.3 mi southeast of Verona, 1.5 mi downstream from Feather River, 6.2 mi east of Knights Landing, and at mile 19.1 upstream from Sacramento.

DRAINAGE AREA.-21,251 $\mathrm{mi}^{2}$.

PERIOD OF RECORD.-May 1926 to September 1929 (low-water periods only), October 1929 to current year.

CHEMICAL DATA: Water years 1952, 1969-70, 1996-98.

SPECIFIC CONDUCTANCE: Water years 1995-98.

WATER TEMPERATURE: Water years 1980, 1995-98.

SEDIMENT: Water years 1980, 1996-98.

REVISED RECORDS.-WDR CA-77-4: Drainage area.

GAGE.-Water-stage recorder and crest-stage gage. Datum of gage is $3.00 \mathrm{ft}$ below sea level. May 1926 to Sept. 30, 1987, at site $0.5 \mathrm{mi}$ upstream at same datum.

REMARKS.- - Records good. Natural flow of stream affected by storage reservoirs, power developments, diversions for irrigation, return flow from irrigated areas, and bypassing for flood control. When discharge exceeds about 55,000 ft $\mathrm{ft}^{3} / \mathrm{s}$, flow begins over Fremont Weir, $3.5 \mathrm{mi}$ upstream on right bank, into Yolo Bypass (station 11453000). See schematic diagram of lower Sacramento River Basin.

EXTREMES FOR PERIOD OF RECORD.-Maximum discharge, 94,000 ft $\mathrm{ft}^{3} / \mathrm{s}$, Jan. 2, 1997, gage height, $42.09 \mathrm{ft}$; maximum gage height, Feb. 20, 1986, $42.11 \mathrm{ft}$, site then in use; minimum daily, $304 \mathrm{ft}^{3} / \mathrm{s}$, July 23, 24, 1931.

DISCHARGE, CUBIC FEET PER SECOND, WATER YEAR OCTOBER 1998 TO SEPTEMBER 1999 DAILY MEAN VALUES

\begin{tabular}{|c|c|c|c|c|c|c|c|c|c|c|c|c|}
\hline DAY & $\mathrm{OCT}$ & NOV & $\mathrm{DEC}$ & JAN & FEB & MAR & $A P R$ & MAY & JUN & JUL & AUG & SEP \\
\hline 1 & 18300 & 11300 & 33600 & 17200 & 37000 & 60100 & 28000 & 19800 & 15700 & 16900 & 19400 & 14700 \\
\hline 2 & 18000 & 11300 & 40900 & 16800 & 34200 & 61100 & 25700 & 19300 & 15300 & 17000 & 19600 & 14100 \\
\hline 3 & 17800 & 11300 & 46800 & 16600 & 30700 & 61600 & 24000 & 19200 & 15200 & 16800 & 19600 & 13900 \\
\hline 4 & 17200 & 11100 & 52000 & 16300 & 28200 & 62100 & 22800 & 19300 & 15800 & 16500 & 19200 & 13900 \\
\hline 5 & 17000 & 11000 & 55600 & 16200 & 27100 & 62100 & 22000 & 19000 & 16900 & 16300 & 18700 & 14000 \\
\hline 6 & 16200 & 11000 & 57500 & 16000 & 29500 & 62100 & 21700 & 18300 & 17200 & 16600 & 18300 & 13900 \\
\hline 7 & 15700 & 11200 & 58100 & 15900 & 37200 & 61800 & 21600 & 16700 & 16600 & 16600 & 17400 & 14300 \\
\hline 8 & 15300 & 11800 & 58000 & 15300 & 48000 & 61200 & 22200 & 16100 & 15600 & 16700 & 16800 & 14400 \\
\hline 9 & 14500 & 12600 & 57300 & 14800 & 57300 & 61000 & 22600 & 16500 & 14600 & 16900 & 16300 & 14200 \\
\hline 10 & 13800 & 13100 & 55800 & 14400 & 63700 & 60300 & 23200 & 16200 & 13700 & 17000 & 15500 & 14300 \\
\hline 11 & 13200 & 13000 & 54300 & 14200 & 63600 & 59700 & 24400 & 15500 & 13000 & 16900 & 14800 & 14600 \\
\hline 12 & 12900 & 13100 & 52400 & 14200 & 62700 & 58800 & 26100 & 14600 & 12500 & 17200 & 14700 & 14600 \\
\hline 13 & 12500 & 13800 & 50600 & 14200 & 61100 & 58000 & 30100 & 14000 & 12100 & 17500 & 14700 & 14300 \\
\hline 14 & 12000 & 13900 & 49200 & 14100 & 59900 & 57100 & 30700 & 13800 & 12100 & 17700 & 14500 & 13900 \\
\hline 15 & 11700 & 14200 & 48100 & 13800 & 58800 & 55600 & 29800 & 13500 & 11800 & 18200 & 14400 & 13800 \\
\hline 16 & 11500 & 14700 & 46600 & 14000 & 58100 & 52600 & 29300 & 12800 & 11600 & 17800 & 14300 & 13700 \\
\hline 17 & 11400 & 15300 & 43300 & 15000 & 59600 & 49000 & 29000 & 12300 & 11600 & 18300 & 14000 & 13600 \\
\hline 18 & 11100 & 15600 & 39200 & 16500 & 62300 & 45300 & 29000 & 12300 & 11800 & 18600 & 13400 & 13600 \\
\hline 19 & 11000 & 17000 & 36500 & 20800 & 63000 & 42000 & 28900 & 12200 & 11500 & 18900 & 12700 & 13700 \\
\hline 20 & 11000 & 19400 & 34600 & 31600 & 63000 & 39400 & 28300 & 12400 & 11400 & 19500 & 12500 & 13600 \\
\hline 21 & 10700 & 20100 & 32700 & 41400 & 63300 & 36600 & 27600 & 12400 & 11700 & 19800 & 12800 & 13100 \\
\hline 22 & 10600 & 20300 & 30600 & 44600 & 63600 & 34100 & 27000 & 12700 & 11700 & 19800 & 13300 & 12900 \\
\hline 23 & 10400 & 21600 & 28200 & 47400 & 62800 & 31600 & 25700 & 13500 & 11800 & 19600 & 13700 & 13000 \\
\hline 24 & 10300 & 24800 & 25600 & 53500 & 61300 & 29100 & 24100 & 13800 & 11600 & 19500 & 13900 & 12500 \\
\hline 25 & 10700 & 29000 & 23200 & 55300 & 60300 & 27800 & 22800 & 13800 & 11500 & 19500 & 14000 & 12200 \\
\hline 26 & 11400 & 30400 & 21400 & 54400 & 59900 & 32100 & 22000 & 13600 & 11600 & 19800 & 14100 & 12000 \\
\hline 27 & 12100 & 28900 & 20400 & 52700 & 59500 & 36500 & 21400 & 13300 & 11700 & 20100 & 14400 & 11700 \\
\hline 28 & 12500 & 29100 & 19900 & 49900 & 59300 & 37600 & 20900 & 13700 & 13300 & 20100 & 15200 & 12200 \\
\hline 29 & 12200 & 30700 & 19600 & 46700 & --- & 37000 & 20500 & 14400 & 15300 & 20000 & 15600 & 12900 \\
\hline 30 & 11800 & 30200 & 19000 & 43300 & --- & 34800 & 20300 & 14900 & 16500 & 19900 & 15400 & 12800 \\
\hline 31 & 11500 & --- & 18000 & 39800 & --- & 31400 & --- & 15600 & --- & 19400 & 15300 & --- \\
\hline TOTAL & 406300 & 530800 & 1229000 & 856900 & 1495000 & 1499500 & 751700 & 465500 & 402700 & 565400 & 478500 & 406400 \\
\hline MEAN & 13110 & 17690 & 39650 & 27640 & 53390 & 48370 & 25060 & 15020 & 13420 & 18240 & 15440 & 13550 \\
\hline MAX & 18300 & 30700 & 58100 & 55300 & 63700 & 62100 & 30700 & 19800 & 17200 & 20100 & 19600 & 14700 \\
\hline MIN & 10300 & 11000 & 18000 & 13800 & 27100 & 27800 & 20300 & 12200 & 11400 & 16300 & 12500 & 11700 \\
\hline $\mathrm{AC}-\mathrm{FT}$ & 805900 & 1053000 & 2438000 & 1700000 & 2965000 & 2974000 & 1491000 & 923300 & 798800 & 1121000 & 949100 & 806100 \\
\hline
\end{tabular}


11425500 SACRAMENTO RIVER AT VERONA, CA—Continued

STATISTICS OF MONTHLY MEAN DATA FOR WATER YEARS 1930 - 1943, BY WATER YEAR (WY)

\begin{tabular}{|c|c|c|c|c|c|c|c|c|c|c|c|c|}
\hline & $\mathrm{OCT}$ & NOV & $\mathrm{DEC}$ & JAN & FEB & MAR & APR & MAY & JUN & JUL & AUG & SEP \\
\hline MEAN & 5623 & 8493 & 17140 & 28130 & 33500 & 35320 & 34370 & 24600 & 12750 & 3943 & 2603 & 4242 \\
\hline MAX & 7816 & 23510 & 41690 & 56930 & 57860 & 57700 & 55330 & 53730 & 33480 & 9176 & 5036 & 5895 \\
\hline (WY) & 1939 & 1938 & 1938 & 1941 & 1942 & 1938 & 1938 & 1938 & 1938 & 1938 & 1938 & 1938 \\
\hline MIN & 3462 & 3923 & 5968 & 7819 & 11730 & 13860 & 5932 & 3103 & 1872 & 497 & 846 & 2960 \\
\hline (WY) & 1933 & 1933 & 1937 & 1937 & 1933 & 1931 & 1931 & 1931 & 1931 & 1931 & 1931 & 1934 \\
\hline
\end{tabular}

SUMMARY STATISTICS

ANNUAL MEAN

HIGHEST ANNUAL MEAN

LOWEST ANNUAL MEAN

HIGHEST DAILY MEAN

LOWEST DAILY MEAN

ANNUAL SEVEN-DAY MINIMUM

INSTANTANEOUS PEAK FLOW

INSTANTANEOUS PEAK STAGE

ANNUAL RUNOFF (AC-FT)

10 PERCENT EXCEEDS

50 PERCENT EXCEEDS

90 PERCENT EXCEEDS
WATER YEARS 1930 - 1943

$\begin{array}{rlrr}17470 & & & \\ 31300 & & & 1938 \\ 6286 & & & 1931 \\ 76900 & \text { Feb } 8 & 1942 \\ 304 & \text { Jul } 23 & 1931 \\ 313 & \text { Jul } 18 & 1931 \\ 79200 & \text { Mar } 1 & 1940 \\ 41.20 & \text { Mar } & 1 & 1940 \\ 12650000 & & & \\ 50700 & & & \\ 8620 & & & \\ 2680 & & & \end{array}$

STATISTICS OF MONTHLY MEAN DATA FOR WATER YEARS 1946 - 1999, BY WATER YEAR (WY)

\begin{tabular}{|c|c|c|c|c|c|c|c|c|c|c|c|c|}
\hline & $\mathrm{OCT}$ & NOV & $\mathrm{DEC}$ & JAN & FEB & MAR & APR & MAY & JUN & JUL & AUG & SEP \\
\hline MEAN & 10640 & 14000 & 22870 & 30110 & 34930 & 32210 & 25190 & 20280 & 14610 & 11700 & 12110 & 12810 \\
\hline MAX & 24920 & 43300 & 64470 & 71040 & 70030 & 71340 & 62140 & 51600 & 45560 & 24550 & 21400 & 22110 \\
\hline (WY) & 1963 & 1974 & 1984 & 1997 & 1998 & 1983 & 1982 & 1952 & 1998 & 1983 & 1983 & 1971 \\
\hline MIN & 4725 & 5987 & 6586 & 8561 & 7591 & 6731 & 6188 & 5118 & 4858 & 4848 & 5385 & 6300 \\
\hline (WY) & 1978 & 1993 & 1960 & 1991 & 1991 & 1977 & 1977 & 1992 & 1992 & 1947 & 1947 & 197 \\
\hline
\end{tabular}

SUMMARY STATISTICS

ANNUAL TOTAL

ANNUAL MEAN

HIGHEST ANNUAL MEAN

LOWEST ANNUAL MEAN

HIGHEST DAILY MEAN

LOWEST DAILY MEAN

ANNUAL SEVEN-DAY MINIMUM

INSTANTANEOUS PEAK FLOW

INSTANTANEOUS PEAK STAGE

ANNUAL RUNOFF (AC-FT)

10 PERCENT EXCEEDS

50 PERCENT EXCEEDS

90 PERCENT EXCEEDS
FOR 1998 CALENDAR YEAR

$\begin{array}{rlr}13078400 & & \\ 35830 & & \\ & & \\ 76300 & \text { Feb } 8 \\ 10300 & \text { Oct } 24 \\ 10700 & \text { Oct } 19 \\ & & \\ & & \\ & & \\ 640000 & & \\ 32700 & & \\ 13600 & \end{array}$

FOR 1999 WATER YEAR

\begin{tabular}{|c|c|}
\hline $\begin{array}{r}9087700 \\
24900\end{array}$ & \\
\hline 63700 & Feb 10 \\
\hline 10300 & Oct 24 \\
\hline 10700 & Oct 19 \\
\hline 64200 & Feb 10 \\
\hline 34.00 & Feb 22 \\
\hline 18030000 & \\
\hline 57300 & \\
\hline 17200 & \\
\hline 11900 & \\
\hline
\end{tabular}

WATER YEARS 1946 - 1999

$\begin{array}{cccc}20050 & & & \\ 39150 & & & 1983 \\ 7178 & & 1977 \\ 92300 & \text { Feb } 20 & 1986 \\ 3590 & \text { Jun } 24 & 1992 \\ 3960 & \text { Jun } 22 & 1992 \\ 94000 & \text { Jan } 2 & 1997 \\ 42.11 & \text { Feb } 20 & 1986 \\ 4520000 & & & \\ 47800 & & & \end{array}$




\section{SACRAMENTO WEIR SPILL TO YOLO BYPASS, NEAR SACRAMENTO, CA}

LOCATION.-Lat 38³6'25", long 121³3'15", unsurveyed, Sacramento County, Hydrologic Unit 18020109, on right bank $100 \mathrm{ft}$ upstream from weir, 3.2 mi upstream from American River, 4 mi northwest of Sacramento, and 4.2 mi upstream from Sacramento.

PERIOD OF RECORD.-October 1939 to current year. Monthly discharge only for water years 1940-51, published in WSP 1735. Published as Sacramento Weir near Sacramento 1939-61. Gage-height records collected at same site February 1926 to September 1934 and major flood flows only October 1934 to September 1939 are contained in reports of California Department of Water Resources.

GAGE.-Water-stage recorder and concrete weir crest. Datum of gage is $3.00 \mathrm{ft}$ below sea level. October 1939 to September 1942, October 1959 to September 1963, water-stage recorder or nonrecording gage at downstream end of weir. October 1942 to September 1959, water-stage recorder on left bank of Sacramento River opposite center of weir. February 1963 to September 1985, water-stage recorder on right bank of Sacramento River $100 \mathrm{ft}$ downstream from end of weir.

REMARKS.-Crest of weir is at gage height $20.2 \mathrm{ft}$ and top of movable gates at $28.0 \mathrm{ft}$. Weir consists of 48 gates each $38.1 \mathrm{ft}$ long. Flow over weir enters Yolo Bypass by way of Sacramento Bypass. Flow regulated by weir gates. February 1963 to September 1985, stage was obtained by averaging the stage obtained at sites on the Sacramento River above and below the weir. See schematic diagram of lower Sacramento River Basin.

COOPERATION.- - Records provided by California Department of Water Resources; not reviewed by the U.S. Geological Survey.

EXTREMES FOR PERIOD OF RECORD.—Maximum discharge, 128,000 ft 3 /s, Feb. 20, 1986, gage height, $30.84 \mathrm{ft}$; maximum gage height, $33.01 \mathrm{ft}$, Dec. 23, 1955; no flow all or most of each year.

EXTREMES FOR CURRENT YEAR.-No flow for 1999 water year.

STATISTICS OF MONTHLY MEAN DATA FOR WATER YEARS 1943 - 1999, BY WATER YEAR (WY)

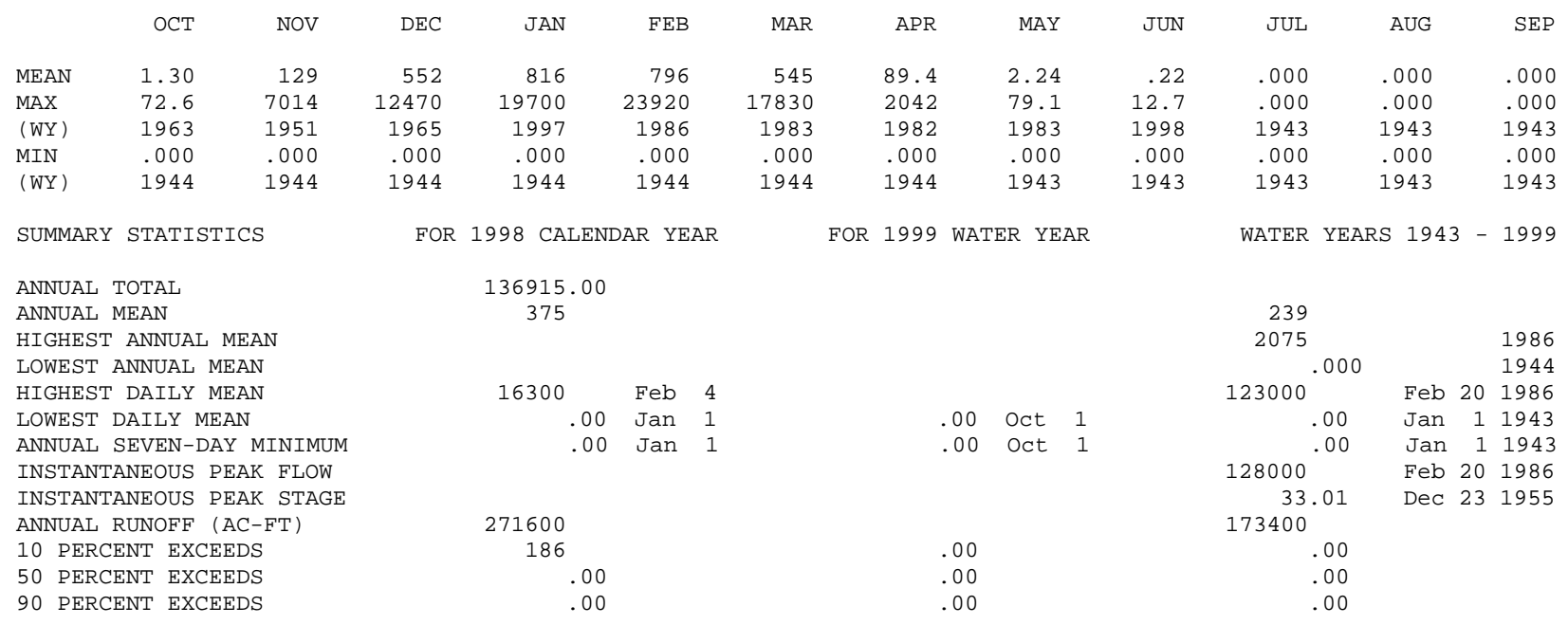


11426170 LAKE VALLEY RESERVOIR NEAR CISCO, CA

LOCATION.—Lat $39^{\circ} 18^{\prime} 01^{\prime \prime}$, long $120^{\circ} 35^{\prime} 46^{\prime \prime}$, in NE 1/4 NW 1/4 sec.35, T.17 N., R.12 E., Placer County, Hydrologic Unit 18020128, on dam near left abutment on North Fork of North Fork American River and 1.3 mi west of Cisco.

DRAINAGE AREA. $-4.54 \mathrm{mi}^{2}$.

PERIOD OF RECORD._-July 1987 to current year. Unpublished records for water years 1980-86 available in files of the U.S. Geological Survey.

GAGE.-Water-stage recorder. Datum of gage is 5,727.4 ft above sea level (levels by Pacific Gas \& Electric Co.). Prior to July 1987, nonrecording gage at same site and datum.

REMARKS.- - Lake is formed by an earthfill dam; storage began in 1911. Usable capacity, 7,960 acre-ft between gage heights $6.2 \mathrm{ft}$, natural rim of lake, and $57.5 \mathrm{ft}$, top of flashboards. Released water is diverted downstream to Lake Valley Canal (station 11426190) and then to several powerplants. Records, including extremes, represent usable contents at 2400 hours. See schematic diagrams of Bear and Yuba River Basins.

COOPERATION.-Records were collected by Pacific Gas \& Electric Co., under general supervision of the U.S. Geological Survey, in connection with a Federal Energy Regulatory Commission project.

EXTREMES FOR PERIOD OF RECORD.—Maximum contents, 8,225 acre-ft, Jan. 1, 1997, gage height, $58.35 \mathrm{ft}$; minimum, 1,153 acre-ft, Feb. 28, 1990, gage height, $25.01 \mathrm{ft}$.

EXTREMES FOR CURRENT YEAR.-Maximum contents, 7,943 acre-ft, May 22, June 2, gage height, $57.43 \mathrm{ft}$; minimum, about 2,119 acre-ft, Jan. 13, gage height, unknown.

Capacity table (gage height, in feet, and contents, in acre-feet)

(Based on survey by Pacific Gas \& Electric Co., dated June 18, 1965)

$\begin{array}{rrrrrr}8 & 41 & 17 & 476 & 40 & 3,455 \\ 10 & 102 & 20 & 693 & 50 & 5,810 \\ 12 & 189 & 25 & 1,152 & 59 & 8,411 \\ 14 & 304 & 30 & 1,830 & & \end{array}$

RESERVOIR STORAGE (ACRE-FEET), WATER YEAR OCTOBER 1998 TO SEPTEMBER 1999 DAILY OBSERVATION AT 2400 HOURS

\begin{tabular}{|c|c|c|c|c|c|c|c|c|c|c|c|c|}
\hline DAY & $\mathrm{OCT}$ & NOV & DEC & JAN & FEB & MAR & APR & MAY & JUN & JUL & AUG & SEP \\
\hline 1 & 5314 & 3973 & 3021 & 2397 & 3210 & 4037 & 4259 & 5727 & 7934 & 7836 & 7450 & 6507 \\
\hline 2 & 5269 & 3930 & 3012 & 2365 & 3192 & 4084 & 4238 & 5865 & 7943 & 7824 & 7438 & 6471 \\
\hline 3 & 5225 & 3884 & 3127 & 2331 & 3174 & 4166 & 4227 & 5975 & 7907 & 7815 & 7423 & 6438 \\
\hline 4 & 5181 & 3840 & 3130 & 2316 & 3159 & 4196 & 4200 & 6047 & 7871 & 7803 & 7408 & 6397 \\
\hline 5 & 5128 & 3799 & 3119 & e2294 & 3141 & 4223 & 4207 & 6121 & 7868 & 7794 & 7393 & 6364 \\
\hline 6 & 5085 & 3760 & 3105 & e2271 & 3150 & 4243 & 4187 & 6228 & 7880 & 7782 & 7381 & 6328 \\
\hline 7 & 5041 & 3746 & 3083 & e2249 & 3304 & 4257 & 4162 & 6355 & 7883 & 7773 & 7369 & 6297 \\
\hline 8 & 4997 & 3708 & 3064 & e2236 & 3432 & 4286 & 4173 & 6465 & 7883 & 7764 & 7354 & 6281 \\
\hline 9 & 4955 & 3659 & 3036 & e2214 & 3669 & 4309 & 4148 & 6567 & 7889 & 7752 & 7345 & 6261 \\
\hline 10 & 4908 & 3615 & 3012 & e2191 & 3701 & 4291 & 4125 & 6668 & 7901 & 7743 & 7331 & 6248 \\
\hline 11 & 4864 & 3567 & 2987 & e2169 & 3703 & 4270 & 4104 & 6786 & 7916 & 7731 & 7292 & 6228 \\
\hline 12 & 4818 & 3514 & 2963 & e2143 & 3705 & 4250 & 4081 & 6937 & 7919 & 7722 & 7253 & 6209 \\
\hline 13 & 4777 & 3462 & 2944 & e2119 & 3699 & 4229 & 4072 & 7086 & 7922 & 7713 & 7212 & 6193 \\
\hline 14 & 4732 & e3445 & 2921 & e2196 & 3690 & 4209 & 4072 & 7194 & 7922 & 7701 & 7173 & 6176 \\
\hline 15 & 4689 & e 3428 & 2897 & e2273 & 3673 & 4189 & 4107 & 7286 & 7916 & 7686 & 7137 & 6157 \\
\hline 16 & 4655 & e3411 & 2877 & e2350 & 3726 & 4169 & 4169 & 7375 & 7910 & 7671 & 7098 & 6138 \\
\hline 17 & 4594 & e3365 & 2864 & e2427 & 3831 & 4151 & 4254 & 7483 & 7901 & 7659 & 7059 & 6124 \\
\hline 18 & 4549 & e3319 & 2830 & e2504 & 3868 & 4144 & 4357 & 7528 & 7898 & 7644 & 7020 & 6108 \\
\hline 19 & 4502 & e3273 & 2802 & e2581 & 3870 & 4144 & 4472 & 7590 & 7889 & 7632 & 6981 & 6091 \\
\hline 20 & 4454 & e3227 & 2774 & e2655 & 3884 & 4153 & 4589 & 7716 & 7877 & 7617 & 6940 & 6078 \\
\hline 21 & 4407 & e3181 & 2737 & e2735 & 3898 & 4153 & 4695 & 7854 & 7866 & 7605 & 6905 & 6061 \\
\hline 22 & 4364 & e 3165 & 2703 & e2812 & 3881 & 4148 & 4791 & 7943 & 7866 & 7590 & 6868 & 6047 \\
\hline 23 & 4318 & e 3147 & 2674 & e2899 & 3865 & 4144 & 4869 & 7925 & 7874 & 7575 & 6825 & 6030 \\
\hline 24 & 4309 & e3129 & 2644 & e2966 & 3849 & 4139 & 4962 & 7916 & 7877 & 7563 & 6791 & 6016 \\
\hline 25 & 4266 & e3111 & 2615 & e 3043 & 3856 & 4137 & 5073 & 7913 & 7874 & 7549 & 6755 & 6000 \\
\hline 26 & 4227 & e3093 & 2585 & e3120 & 3835 & 4151 & 5229 & 7901 & 7866 & 7537 & 6721 & 5983 \\
\hline 27 & 4180 & e3075 & 2553 & e3197 & 3817 & 4173 & 5343 & 7892 & 7860 & 7522 & 6690 & 5972 \\
\hline 28 & 4137 & e3058 & 2522 & e3275 & 3893 & 4191 & 5430 & 7871 & 7854 & 7510 & 6657 & 5942 \\
\hline 29 & 4097 & e3040 & 2491 & e3259 & --- & 4209 & 5498 & 7842 & 7848 & 7495 & 6617 & 5925 \\
\hline 30 & 4051 & 3033 & 2459 & e 3243 & --- & 4245 & 5589 & 7851 & 7842 & 7480 & 6581 & 5911 \\
\hline 31 & 4005 & --- & 2430 & 3227 & --- & 4259 & --- & 7889 & --- & 7465 & 6543 & --- \\
\hline MAX & 5314 & 3973 & 3130 & 3275 & 3898 & 4309 & 5589 & 7943 & 7943 & 7836 & 7450 & 6507 \\
\hline MIN & 4005 & 3033 & 2430 & 2119 & 3141 & 4037 & 4072 & 5727 & 7842 & 7465 & 6543 & 5911 \\
\hline$a$ & 42.40 & 37.93 & 34.05 & 39.01 & 41.91 & 43.51 & 49.20 & 57.25 & 57.09 & 55.83 & 52.66 & 50.37 \\
\hline $\mathrm{b}$ & -1355 & -972 & -603 & +797 & +666 & +366 & +1330 & +2300 & -47 & -377 & -922 & -632 \\
\hline C & 1610 & 1540 & 2080 & 1520 & 1850 & 2340 & 2320 & 2430 & 1540 & 0 & 610 & 171 \\
\hline
\end{tabular}

CAL YR 1998 MAX 7925 MIN $2430 \quad$ b -768 C 19570 WTR YR 1999 MAX 7943 MIN 2119 b +551 c 18010

e Estimated.

a Gage height, in feet, at end of month.

b Change in contents, in acre-feet.

c Diversion, in acre-feet, to Lake Valley Canal, provided by Pacific Gas \& Electric Co. 
LOCATION.-Lat 39¹8'40", long 120³4'49", in SE 1/4 NW 1/4 sec.25, T.17 N., R.12 E., Placer County, Hydrologic Unit 18020128, Tahoe National Forest, on outlet structure on Kelly Lake Dam on unnamed tributary to North Fork of North Fork American River and 2.2 mi west of Cisco.

DRAINAGE AREA.- $-0.58 \mathrm{mi}^{2}$.

PERIOD OF RECORD.-October 1991 to current year. Unpublished records for water years 1965-91 available in files of the U.S. Geological Survey.

GAGE.-Water-stage recorder. Datum of gage is 5,888.9 ft above sea level (levels by Pacific Gas \& Electric Co.). Prior to October 1991, nonrecording gage at same site and datum.

REMARKS.-No records computed during the winter months. Reservoir is formed on natural lake by rock-fill dam completed in 1928. Usable capacity, 336 acre-ft between gage heights $0.0 \mathrm{ft}$, invert of outlet, and $17.1 \mathrm{ft}$, top of flashboards. Water is used for power development downstream. Records, including extremes, represent usable contents at 2400 hours. See schematic diagram of Bear River Basin.

COOPERATION.-Records were collected by Pacific Gas \& Electric Co., under general supervision of the U.S. Geological Survey, in connection with a Federal Energy Regulatory Commission project.

EXTREMES FOR PERIOD OF RECORD.-Maximum contents, 359 acre-ft, May 15, 1996, gage height, $17.96 \mathrm{ft}$; no storage many days in October 1991.

EXTREMES FOR CURRENT YEAR.-Maximum contents recorded, 346 acre-ft, May 6, gage height, $17.47 \mathrm{ft}$; minimum recorded, 80 acre-ft, Nov. 4, gage height, $5.15 \mathrm{ft}$.

Capacity table (gage height, in feet, and contents, in acre-feet)

(Based on survey by Pacific Gas \& Electric Co., dated December 1933)

$\begin{array}{rrrr}0 & 0 & 12 & 213 \\ 4 & 61 & 16 & 308 \\ 8 & 130 & 19 & 387\end{array}$

RESERVOIR STORAGE (ACRE-FEET), WATER YEAR OCTOBER 1998 TO SEPTEMBER 1999

DAILY OBSERVATION AT 2400 HOURS

\begin{tabular}{|c|c|c|c|c|c|c|c|c|c|c|c|c|}
\hline DAY & OCT & $\mathrm{NOV}$ & DEC & JAN & FEB & MAR & APR & MAY & JUN & JUL & AUG & SEP \\
\hline 1 & 268 & 81 & 164 & --- & --- & --- & --- & --- & 339 & 332 & 296 & 282 \\
\hline 2 & 265 & 80 & 169 & --- & --- & --- & --- & --- & 339 & 331 & 296 & 282 \\
\hline 3 & 261 & 80 & 197 & --- & --- & --- & --- & --- & 337 & 330 & 296 & 282 \\
\hline 4 & 261 & 80 & 203 & --- & --- & --- & --- & --- & 339 & 328 & 297 & 280 \\
\hline 5 & 255 & 80 & 207 & --- & --- & --- & --- & 343 & 339 & 322 & 297 & 280 \\
\hline 6 & 244 & 80 & 210 & --- & --- & --- & --- & 346 & 339 & 318 & 297 & 279 \\
\hline 7 & 231 & 92 & 213 & --- & --- & --- & --- & 344 & 338 & 314 & 297 & 279 \\
\hline 8 & 220 & 92 & 215 & --- & --- & --- & --- & 342 & 336 & 309 & 298 & 278 \\
\hline 9 & 208 & 92 & 217 & --- & --- & --- & --- & 338 & 336 & 305 & 298 & 277 \\
\hline 10 & 198 & 92 & 218 & --- & --- & --- & --- & 337 & 337 & 313 & 298 & 277 \\
\hline 11 & 186 & 92 & 222 & --- & --- & --- & --- & 338 & 337 & 320 & 297 & 276 \\
\hline 12 & 175 & 92 & 224 & --- & --- & --- & --- & 335 & 337 & 317 & 298 & 276 \\
\hline 13 & 166 & 92 & 226 & --- & --- & --- & --- & 338 & 337 & 317 & 297 & 275 \\
\hline 14 & 151 & 91 & 229 & --- & --- & --- & --- & 334 & 338 & 316 & 296 & 274 \\
\hline 15 & 138 & 91 & 231 & --- & --- & --- & --- & 332 & 338 & 314 & 296 & 273 \\
\hline 16 & 126 & 91 & 235 & --- & --- & --- & --- & 331 & 338 & 313 & 296 & 271 \\
\hline 17 & 115 & 93 & --- & --- & --- & --- & --- & 334 & 338 & 312 & 295 & 271 \\
\hline 18 & 106 & 93 & --- & --- & --- & --- & --- & 336 & 338 & 311 & 294 & 272 \\
\hline 19 & 102 & 93 & --- & --- & --- & --- & --- & 337 & 338 & 311 & 294 & 272 \\
\hline 20 & 100 & 93 & --- & --- & --- & --- & --- & 338 & 338 & 311 & 293 & 270 \\
\hline 21 & 96 & 94 & --- & --- & --- & --- & --- & 339 & 337 & 311 & 293 & 271 \\
\hline 22 & 93 & 94 & --- & --- & --- & --- & --- & 339 & 337 & 308 & 292 & 271 \\
\hline 23 & 88 & 109 & --- & --- & --- & --- & --- & 327 & 336 & 308 & 290 & 272 \\
\hline 24 & 89 & 110 & --- & --- & --- & --- & --- & 332 & 336 & 308 & 289 & 271 \\
\hline 25 & 88 & 111 & --- & --- & --- & --- & --- & 337 & 335 & 307 & 288 & 272 \\
\hline 26 & 86 & 111 & --- & --- & --- & --- & --- & 337 & 335 & 307 & 287 & 270 \\
\hline 27 & 86 & 116 & --- & --- & --- & --- & --- & 336 & 334 & 305 & 286 & 270 \\
\hline 28 & 85 & 119 & --- & --- & --- & --- & --- & 337 & 334 & 303 & 284 & 268 \\
\hline 29 & 83 & 127 & --- & --- & --- & --- & --- & 337 & 333 & 302 & 284 & 269 \\
\hline 30 & 82 & 158 & --- & --- & --- & --- & --- & 337 & 333 & 299 & 284 & 269 \\
\hline 31 & 80 & --- & --- & --- & --- & --- & --- & 338 & --- & 296 & 283 & --- \\
\hline MAX & 268 & 158 & --- & --- & --- & --- & --- & --- & 339 & 332 & 298 & 282 \\
\hline MIN & 80 & 80 & --- & --- & --- & --- & --- & --- & 333 & 296 & 283 & 268 \\
\hline $\mathrm{a}$ & 5.20 & 9.41 & & & & & & 17.17 & 16.95 & 15.54 & 14.99 & 14.44 \\
\hline b & -191 & +78 & & & & & & & -5 & -37 & -13 & -14 \\
\hline
\end{tabular}

WTR YR 1999 b -2

a Gage height, in feet, at end of month.

b Change in contents, in acre-feet. 
LOCATION.-Lat 3856'10", long 12101'22", in SW 1/4 NW 1/4 sec.31, T.13 N., R.9 E., Placer County, Hydrologic Unit 18020128, on left bank $50 \mathrm{ft}$ upstream from crest of North Fork Dam, 2 mi upstream from Middle Fork, and 4 mi northeast of Auburn.

DRAINAGE AREA.-342 $\mathrm{mi}^{2}$.

PERIOD OF RECORD.-October 1941 to current year.

CHEMICAL DATA: Water years 1977-80.

WATER TEMPERATURE: Water years 1959-83.

SEDIMENT DATA: Water year 1980 (periodic record).

REVISED RECORDS.-WSP 1931: Drainage area.

GAGE.-Water-stage recorder and ogee section of concrete debris dam. Datum of gage is $715.0 \mathrm{ft}$ above sea level (levels by U.S. Army Corps of Engineers).

REMARKS.--Records good. Minor regulation by Lake Clementine, usable capacity, 12,800 acre-ft, formed by North Fork Dam. Storage in Big Reservoir and Lake Valley Reservoir (station 11426170), combined capacity, 10,300 acre-ft upstream from station. Lake Valley Canal (station 11426190) diverts from North Fork of North Fork American River into Bear River Basin for power development in powerplants of Pacific Gas \& Electric Co. Combined storage and diversion have small effect on natural flow. See schematic diagrams of Bear and lower Sacramento River Basins.

EXTREMES FOR PERIOD OF RECORD.-Maximum discharge, 65,400 ft 3 /s, Dec. 23, 1964, gage height, $11.87 \mathrm{ft}$, from rating curve extended above $24,000 \mathrm{ft}^{3} / \mathrm{s}$ on basis of computed flow over crest of dam at gage height $10.22 \mathrm{ft}$; no flow, Aug. 27-30, Sept. 2-11, 1944; Oct. 5, 6, 1963; Nov. 7-10, 1965, caused by operation of valve in North Fork Dam.

EXTREMES FOR CURRENT YEAR.-Peak discharges greater than base discharge of 4,300 $\mathrm{ft}^{3} / \mathrm{s}$, or maximum:

\begin{tabular}{lcccccc} 
& Discharge & Gage height & \multicolumn{2}{c}{$\begin{array}{c}\text { Discharge } \\
\left(\mathrm{ft}^{3} / \mathrm{s}\right)\end{array}$} \\
Date & Time & $\left(\mathrm{ft}^{3} / \mathrm{s}\right)$ & $(\mathrm{ft})$ & Date & Time & 6,390 \\
Dec. 3 & 1800 & 4,410 & 3.59 & Feb. 17 & 0830 & 4.13 \\
Jan. 20 & 0445 & 8,730 & 4.68 & Mar. 1 & 0830 & 4,680 \\
Feb. 9 & 1230 & 17,300 & 6.23 & & & 3.67
\end{tabular}

DISCHARGE, CUBIC FEET PER SECOND, WATER YEAR OCTOBER 1998 TO SEPTEMBER 1999

DAILY MEAN VALUES

\begin{tabular}{|c|c|c|c|c|c|c|c|c|c|c|c|c|}
\hline DAY & $\mathrm{OCT}$ & NOV & $\mathrm{DEC}$ & JAN & FEB & MAR & APR & MAY & JUN & JUL & AUG & SEP \\
\hline 1 & 95 & 99 & 2320 & 265 & 983 & 3870 & 979 & 1520 & 1870 & 463 & 108 & 71 \\
\hline 2 & 90 & 105 & 1060 & 255 & 888 & 3130 & 908 & 1870 & 1890 & 441 & 107 & 70 \\
\hline 3 & 87 & 97 & 2220 & 245 & 828 & 3750 & 883 & 1840 & 1520 & 422 & 104 & 70 \\
\hline 4 & 85 & 91 & 2010 & 236 & 809 & 3230 & 837 & 1570 & 1190 & 377 & 102 & 67 \\
\hline 5 & 85 & 91 & 1090 & 227 & 777 & 2570 & 868 & 1410 & 1060 & 336 & 100 & 66 \\
\hline 6 & 83 & 95 & 828 & 223 & 789 & 2200 & 879 & 1540 & 1300 & 302 & 98 & 64 \\
\hline 7 & 81 & 136 & 648 & 220 & 5430 & 1930 & 867 & 1940 & 1350 & 288 & 99 & 63 \\
\hline 8 & 80 & 211 & 565 & 215 & 5560 & 1740 & 1010 & 1970 & 1190 & 276 & 99 & 62 \\
\hline 9 & 80 & 153 & 528 & 214 & 12500 & 1800 & 1070 & 1770 & 1130 & 261 & 99 & 64 \\
\hline 10 & 80 & 123 & 465 & 199 & 6350 & 1610 & 996 & 1670 & 1080 & 248 & 100 & 65 \\
\hline 11 & 79 & 134 & 432 & 194 & 3690 & 1500 & 1080 & 1680 & 1110 & 240 & 100 & 65 \\
\hline 12 & 78 & 129 & 411 & 202 & 2780 & 1400 & 1180 & 2010 & 1220 & 240 & 104 & 64 \\
\hline 13 & 78 & 113 & 406 & 191 & 2250 & 1330 & 1280 & 2340 & 1230 & 232 & 103 & 62 \\
\hline 14 & 77 & 107 & 467 & 188 & 1930 & 1320 & 1500 & 1910 & 1250 & 222 & 95 & 62 \\
\hline 15 & 77 & 105 & 431 & 210 & 1680 & 1260 & 1620 & 1550 & 1270 & 215 & 93 & 61 \\
\hline 16 & 75 & 104 & 416 & 651 & 1660 & 1190 & 1640 & 1480 & 1230 & 198 & 90 & 61 \\
\hline 17 & 73 & 157 & 480 & 806 & 4880 & 1190 & 1840 & 1570 & 1150 & 182 & 89 & 60 \\
\hline 18 & 73 & 226 & 490 & 3280 & 3530 & 1220 & 2050 & 1850 & 1060 & 172 & 86 & 60 \\
\hline 19 & 80 & 166 & 448 & 3590 & 3310 & 1270 & 2190 & 1960 & 976 & 164 & 85 & 60 \\
\hline 20 & 77 & 140 & 412 & 8040 & 2620 & 1260 & 2280 & 2030 & 902 & 158 & 84 & 59 \\
\hline 21 & 73 & 131 & 363 & 4880 & 3360 & 1190 & 2210 & 2000 & 848 & 154 & 83 & 57 \\
\hline 22 & 70 & 224 & 339 & 2600 & 2660 & 1100 & 1940 & 2310 & 814 & 148 & 82 & 56 \\
\hline 23 & 70 & 353 & 319 & 5590 & 2220 & 1060 & 1690 & 2670 & 843 & 143 & 81 & 55 \\
\hline 24 & 80 & 1540 & 308 & 3530 & 1970 & 1040 & 1650 & 2920 & 803 & 139 & 79 & 56 \\
\hline 25 & 107 & 530 & 300 & 2350 & 2260 & 1040 & 1790 & 3220 & 723 & 135 & 77 & 56 \\
\hline 26 & 105 & 339 & 293 & 1840 & 2030 & 1090 & 2120 & 3100 & 596 & 132 & 77 & 56 \\
\hline 27 & 90 & 309 & 284 & 1490 & 1830 & 1240 & 2180 & 2880 & 518 & 129 & 77 & 54 \\
\hline 28 & 85 & 386 & 275 & 1260 & 1800 & 1190 & 1750 & 2620 & 482 & 126 & 80 & 53 \\
\hline 29 & 85 & 383 & 267 & 1100 & --- & 1090 & 1470 & 2350 & 467 & 120 & 76 & 52 \\
\hline 30 & 86 & 2490 & 262 & 991 & --- & 1050 & 1330 & 1850 & 467 & 114 & 73 & 52 \\
\hline 31 & 88 & --- & 262 & 1020 & --- & 1090 & --- & 1980 & --- & 111 & 70 & --- \\
\hline TOTAL & 2552 & 9267 & 19399 & 46302 & 81374 & 50950 & 44087 & 63380 & 31539 & 6888 & 2800 & 1823 \\
\hline MEAN & 82.3 & 309 & 626 & 1494 & 2906 & 1644 & 1470 & 2045 & 1051 & 222 & 90.3 & 60.8 \\
\hline MAX & 107 & 2490 & 2320 & 8040 & 12500 & 3870 & 2280 & 3220 & 1890 & 463 & 108 & 71 \\
\hline MIN & 70 & 91 & 262 & 188 & 777 & 1040 & 837 & 1410 & 467 & 111 & 70 & 52 \\
\hline $\mathrm{AC}-\mathrm{FT}$ & 5060 & 18380 & 38480 & 91840 & 161400 & 101100 & 87450 & 125700 & 62560 & 13660 & 5550 & 3620 \\
\hline
\end{tabular}




\section{NORTH FORK AMERICAN RIVER AT NORTH FORK DAM, CA—Continued}

STATISTICS OF MONTHLY MEAN DATA FOR WATER YEARS 1942 - 1999, BY WATER YEAR (WY)

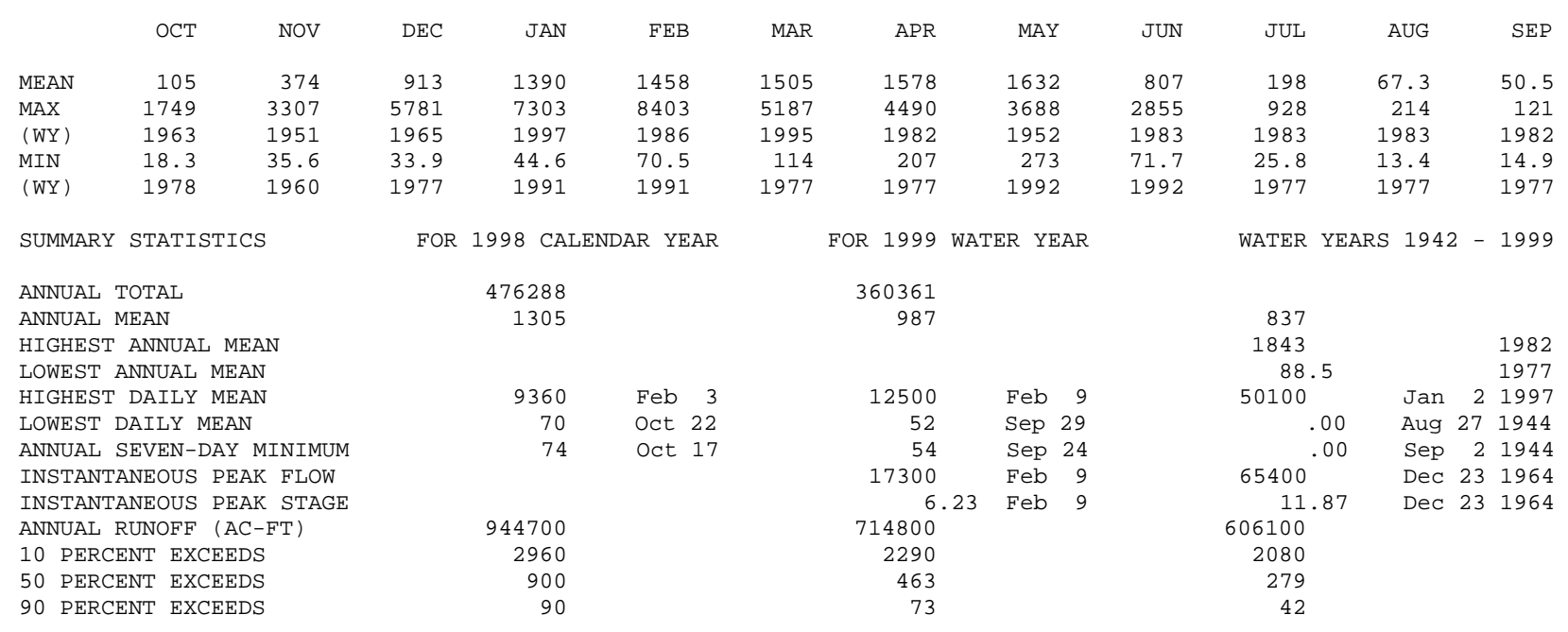




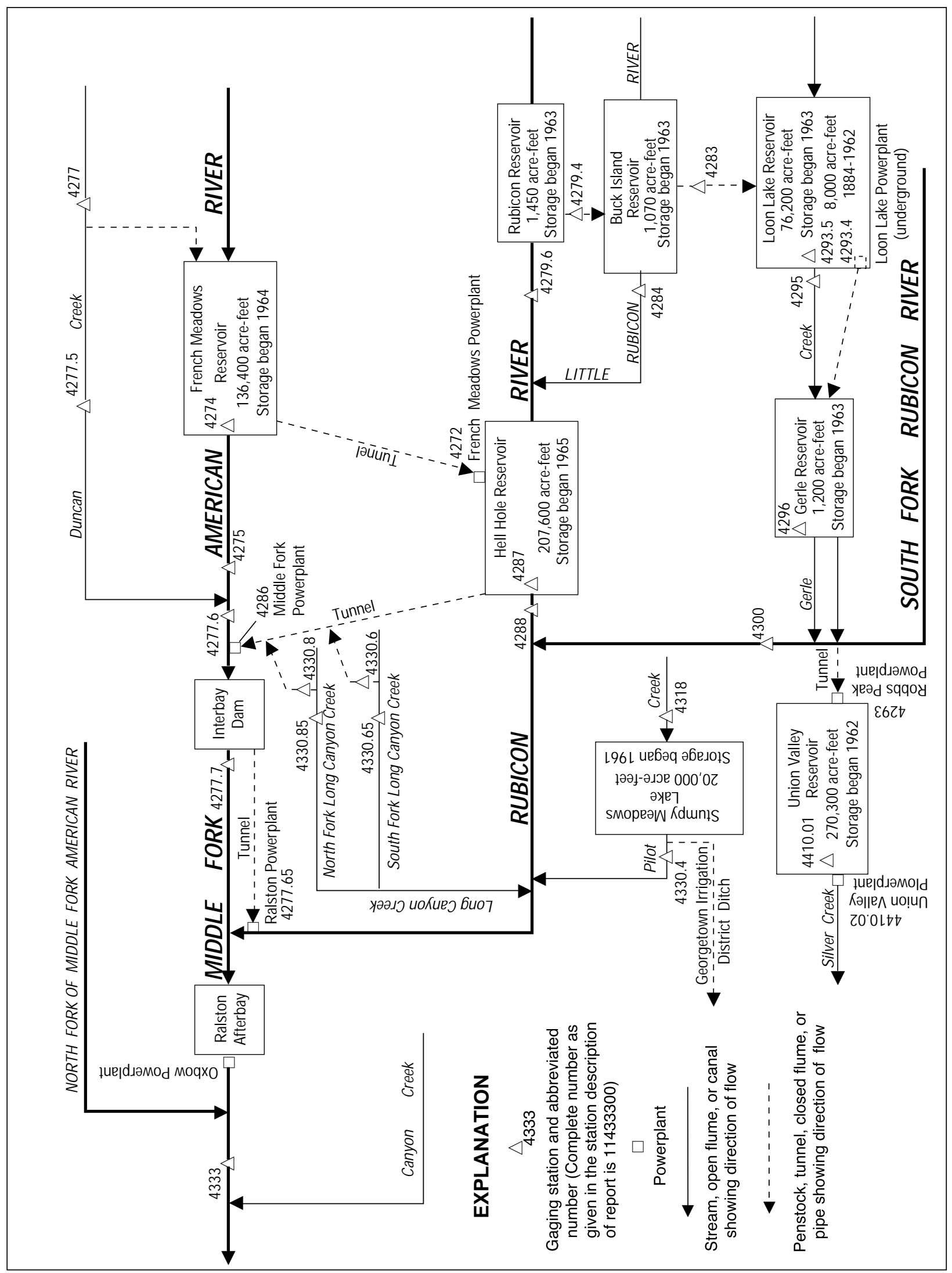

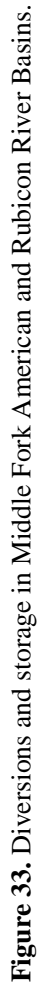


LOCATION.—Lat 3906'32", long 120²5'49", in SW 1/4 NE 1/4 sec.32, T.15 N., R.14 E., Placer County, Hydrologic Unit 18020128, Tahoe National Forest, on left bank 2.2 mi upstream from dam on Middle Fork American River, 6.9 mi upstream from Chipmunk Creek, and 21 mi northeast of Foresthill.

DRAINAGE AREA.- $-47.0 \mathrm{mi}^{2}$.

PERIOD OF RECORD.-December 1964 to current year.

GAGE.-Water-stage recorder. Datum of gage is sea level (levels by Placer County Water Agency).

REMARKS.- Reservoir is formed by rockfill dam with earth core. Storage began Dec. 21, 1964. Usable capacity, 125,601 acre-ft between elevations 5,125 ft, minimum operating level, and 5,263 ft, top of radial gates. Dead storage, 10,804 acre- $\mathrm{ft}$. Reservoir is used to store water for hydroelectric power. Up to $400 \mathrm{ft}^{3} / \mathrm{s}$ diverted from Duncan Creek through a tunnel to reservoir. Water is released through a tunnel to French Meadows Powerplant (station 11427200) at Hell Hole Reservoir (station 11428700) on the Rubicon River; releases began Dec. 13, 1965. See schematic diagram of Middle Fork American and Rubicon River Basins.

COOPERATION.-Records provided by Placer County Water Agency, under general supervision of the U.S. Geological Survey, in connection with a Federal Energy Regulatory Commission project.

EXTREMES FOR PERIOD OF RECORD.-Maximum contents, 137,700 acre-ft, May 19, 1966, elevation, 5,263.9 ft; minimum since reservoir first filled, 28,500 acre-ft, Oct. 21-24, 1991, elevation, 5,157.6 ft.

EXTREMES FOR CURRENT YEAR.-Maximum contents, 131,500 acre-ft, June 27, elevation, 5,259.5 ft; minimum, 62,100 acre-ft, Nov. 16, elevation, $5,199.6 \mathrm{ft}$

Capacity table (elevation, in feet, and contents, in acre-feet)

(Based on a survey by Placer County Water Agency in 1965)

$\begin{array}{rrrr}5,125 & 10,800 & 5,200 & 62,400 \\ 5,130 & 13,100 & 5,230 & 94,100 \\ 5,150 & 23,700 & 5,270 & 146,500\end{array}$

RESERVOIR STORAGE (ACRE-FEET), WATER YEAR OCTOBER 1998 TO SEPTEMBER 1999 DAILY OBSERVATION AT 2400 HOURS

\begin{tabular}{|c|c|c|c|c|c|c|c|c|c|c|c|c|}
\hline DAY & $\mathrm{OCT}$ & NOV & $\mathrm{DEC}$ & JAN & FEB & MAR & APR & MAY & JUN & JUL & AUG & SEP \\
\hline 1 & 88000 & 70400 & 65200 & 70500 & 84800 & 100400 & 91900 & 93700 & 118200 & 130800 & 119900 & 105400 \\
\hline 2 & 87500 & 69700 & 65400 & 70600 & 84900 & 100900 & 91400 & 94200 & 119100 & 130500 & 119300 & 104900 \\
\hline 3 & 86900 & 69200 & 66500 & 70600 & 85100 & 101100 & 91000 & 94400 & 119300 & 130400 & 118800 & 104500 \\
\hline 4 & 86400 & 68400 & 66900 & 70700 & 85400 & 101100 & 90500 & 94200 & 119500 & 130400 & 118300 & 104200 \\
\hline 5 & 85800 & 67600 & 67100 & 70800 & 85600 & 100900 & 90200 & 94100 & 119700 & 130200 & 117900 & 103900 \\
\hline 6 & 85400 & 66800 & 67300 & 70800 & 85900 & 100700 & 89700 & 94400 & 120000 & 129800 & 117400 & 103600 \\
\hline 7 & 84800 & 66200 & 67500 & 70900 & 87100 & 100300 & 89200 & 95000 & 120300 & 129400 & 117000 & 103100 \\
\hline 8 & 84400 & 65900 & 67600 & 70900 & 88400 & 100000 & 88700 & 95500 & 120800 & 129100 & 116600 & 102700 \\
\hline 9 & 83900 & 65300 & 67700 & 71000 & 90400 & 99600 & 88100 & 95700 & 121200 & 128700 & 116200 & 102200 \\
\hline 10 & 83300 & 64700 & 67800 & 71000 & 91200 & 99200 & 87700 & 96100 & 121600 & 128300 & 115800 & 101700 \\
\hline 11 & 82700 & 64200 & 67900 & 71100 & 91800 & 98800 & 87100 & 96500 & 122100 & 128200 & 115300 & 101500 \\
\hline 12 & 82200 & 63600 & 68000 & 71100 & 92200 & 98300 & 86600 & 97300 & 123000 & 127800 & 114800 & 101100 \\
\hline 13 & 81500 & 63000 & 68100 & 71200 & 92700 & 97800 & 86300 & 98200 & 124100 & 127400 & 114300 & 100800 \\
\hline 14 & 81100 & 62700 & 68300 & 71300 & 93000 & 97300 & 85900 & 98500 & 124900 & 127000 & 113800 & 100300 \\
\hline 15 & 80600 & 62400 & 68400 & 71600 & 93400 & 96900 & 85800 & 98800 & 126000 & 126700 & 113400 & 99800 \\
\hline 16 & 80000 & 62100 & 68600 & 72000 & 94000 & 96400 & 85700 & 99000 & 127100 & 126300 & 112900 & 99400 \\
\hline 17 & 79500 & 62200 & 68800 & 72600 & 94900 & 96100 & 85900 & 99400 & 127800 & 126000 & 112500 & 98900 \\
\hline 18 & 79000 & 62200 & 69000 & 74800 & 95600 & 95700 & 86400 & 100000 & 128500 & 125700 & 112000 & 98600 \\
\hline 19 & 78300 & 62300 & 69200 & 76400 & 95900 & 95400 & 86900 & 100500 & 129300 & 125300 & 111400 & 98300 \\
\hline 20 & 77700 & 62300 & 69400 & 78800 & 96400 & 95000 & 87600 & 101600 & 130000 & 125100 & 110800 & 97900 \\
\hline 21 & 77200 & 62300 & 69500 & 79800 & 96800 & 94700 & 88300 & 103000 & 130400 & 124500 & 110600 & 97300 \\
\hline 22 & 76500 & 62400 & 69600 & 80600 & 97100 & 94300 & 88700 & 104700 & 130700 & 124100 & 110200 & 97000 \\
\hline 23 & 76100 & 62900 & 69700 & 81800 & 97300 & 93800 & 88900 & 106600 & 130900 & 123700 & 109700 & 96400 \\
\hline 24 & 75400 & 63100 & 69800 & 82400 & 97600 & 93400 & 89300 & 108700 & 131200 & 123300 & 109200 & 95900 \\
\hline 25 & 75000 & 63200 & 69900 & 82800 & 97900 & 93000 & 90000 & 111000 & 131200 & 123000 & 108700 & 95500 \\
\hline 26 & 74100 & 63200 & 70000 & 83300 & 98200 & 92800 & 90900 & 113100 & 131400 & 122500 & 108300 & 95000 \\
\hline 27 & 73300 & 63300 & 70100 & 83500 & 98500 & 92700 & 91500 & 115000 & 131400 & 122000 & 107700 & 94500 \\
\hline 28 & 72500 & 63400 & 70100 & 83800 & 99000 & 92500 & 92200 & 116300 & 131200 & 121600 & 107200 & 94100 \\
\hline 29 & 71900 & 63700 & 70200 & 84000 & --- & 92200 & 93200 & 116900 & 131200 & 121000 & 106900 & 93600 \\
\hline 30 & 71100 & 64800 & 70300 & 84300 & --- & 92200 & 93500 & 117200 & 131100 & 120500 & 106400 & 93000 \\
\hline 31 & 70700 & --- & 70300 & 84600 & --- & 92100 & --- & 117500 & --- & 120300 & 105800 & --- \\
\hline MAX & 88000 & 70400 & 70300 & 84600 & 99000 & 101100 & 93500 & 117500 & 131400 & 130800 & 119900 & 105400 \\
\hline MIN & 70700 & 62100 & 65200 & 70500 & 84800 & 92100 & 85700 & 93700 & 118200 & 120300 & 105800 & 93000 \\
\hline$a$ & 5208.4 & 5202.5 & 5208.0 & 5221.6 & 5234.2 & 5228.3 & 5229.5 & 5249.1 & 5259.2 & 5251.2 & 5239.8 & 5229.1 \\
\hline b & -17800 & -5900 & +5500 & +14300 & +14400 & -6900 & +1400 & +24000 & +13600 & -10800 & -14500 & -12800 \\
\hline
\end{tabular}

CAL YR 1998 b +19700

WTR YR 1999 b +4500 
LOCATION.-Lat 3906'35", long 120²8'49", in SW 1/4 NW 1/4 sec.36, T.15 N., R.13 E., Placer County, Hydrologic Unit 18020128, Tahoe National Forest, on left bank 0.6 mi downstream from French Meadows Dam, 4.1 mi upstream from Chipmunk Creek, and 14 mi south of Cisco.

DRAINAGE AREA.-47.9 $\mathrm{mi}^{2}$.

PERIOD OF RECORD.-October 1951 to current year.

REVISED RECORDS.-WSP 1445: 1953-54. WSP 1931: Drainage area.

GAGE.-Water-stage recorder. Elevation of gage is 4,920 ft above sea level, from topographic map. Prior to Oct. 1, 1962 , at site 0.8 mi upstream at different datum.

REMARKS.-Considerable regulation by French Meadows Reservoir (station 11427400) 0.6 mi upstream beginning December 1964. Water diverted into basin from Duncan Creek to French Meadows Reservoir since December 1964. Water diverted out of basin from French Meadows Reservoir through French Meadows Powerplant (station 11427200) to Hell Hole Reservoir (station 11428700) since December 1965. See schematic diagram of Middle Fork American and Rubicon River Basins.

COOPERATION.-Records provided by Placer County Water Agency, under general supervision of the U.S. Geological Survey, in connection with a Federal Energy Regulatory Commission project.

EXTREMES FOR PERIOD OF RECORD.-Maximum discharge, 21,500 ft $\mathrm{ft}^{3} / \mathrm{s}$, Jan. 31, 1963, gage height, $14.20 \mathrm{ft}$, from rating curve extended above $1,100 \mathrm{ft}^{3} / \mathrm{s}$ on basis of peak flow at former site; minimum, $0.3 \mathrm{ft}^{3} / \mathrm{s}$, Oct. 4, 5, 21-25, 1960, Oct. 5, 6, 1961. Maximum discharge since construction of French Meadows Dam in 1964, 6,050 ft 3 /s, May 16, 1996, gage height, $11.61 \mathrm{ft}$, from flow over spillway of French Meadows Reservoir; minimum daily, $0.8 \mathrm{ft}^{3} / \mathrm{s}$, Oct. $22-25,1964$

DISCHARGE, CUBIC FEET PER SECOND, WATER YEAR OCTOBER 1998 TO SEPTEMBER 1999 DAILY MEAN VALUES

\begin{tabular}{|c|c|c|c|c|c|c|c|c|c|c|c|c|}
\hline DAY & $\mathrm{OCT}$ & NOV & $\mathrm{DEC}$ & JAN & FEB & MAR & $\mathrm{APR}$ & MAY & JUN & JUL & AUG & SEP \\
\hline 1 & 9.9 & 9.8 & 12 & 10 & 13 & 26 & 14 & 512 & 142 & 10 & 10 & 10 \\
\hline 2 & 9.9 & 9.5 & 12 & 10 & 12 & 19 & 14 & 513 & 11 & 10 & 10 & 10 \\
\hline 3 & 9.9 & 9.5 & 17 & 10 & 12 & 27 & 14 & 513 & 11 & 9.9 & 10 & 10 \\
\hline 4 & 9.9 & 9.5 & 13 & 10 & 12 & 21 & 13 & 513 & 11 & 9.9 & 10 & 10 \\
\hline 5 & 9.9 & 9.5 & 11 & 10 & 12 & 18 & 13 & 513 & 11 & 9.9 & 10 & 10 \\
\hline 6 & 9.9 & 9.5 & 11 & 10 & 12 & 17 & 13 & 513 & 11 & 9.9 & 10 & 10 \\
\hline 7 & 9.9 & 10 & 11 & 10 & 40 & 16 & 13 & 513 & 11 & 9.8 & 10 & 10 \\
\hline 8 & 9.9 & 9.7 & 11 & 10 & 32 & 15 & 13 & 513 & 11 & 9.8 & 10 & 10 \\
\hline 9 & 9.9 & 9.5 & 10 & 10 & 59 & 15 & 13 & 513 & 10 & 9.8 & 10 & 10 \\
\hline 10 & 9.9 & 9.6 & 10 & 10 & 21 & 14 & 13 & 513 & 10 & 9.7 & 10 & 10 \\
\hline 11 & 9.9 & 9.6 & 10 & 10 & 18 & 14 & 13 & 513 & 10 & 9.7 & 10 & 10 \\
\hline 12 & 9.9 & 9.5 & 10 & 10 & 16 & 14 & 14 & 514 & 10 & 9.8 & 10 & 10 \\
\hline 13 & 9.9 & 9.5 & 10 & 10 & 16 & 14 & 14 & 514 & 10 & 9.9 & 10 & 10 \\
\hline 14 & 9.9 & 9.5 & 10 & 10 & 15 & 14 & 15 & 515 & 10 & 10 & 10 & 10 \\
\hline 15 & 9.9 & 9.3 & 10 & 11 & 15 & 14 & 16 & 514 & 10 & 9.9 & 10 & 10 \\
\hline 16 & 9.8 & 9.3 & 11 & 12 & 17 & 14 & 16 & 514 & 10 & 10 & 10 & 10 \\
\hline 17 & 9.7 & 9.8 & 11 & 13 & 32 & 14 & 17 & 515 & 10 & 10 & 10 & 9.9 \\
\hline 18 & 9.7 & 9.5 & 11 & 23 & 20 & 14 & 17 & 515 & 11 & 10 & 10 & 9.9 \\
\hline 19 & 9.7 & 9.4 & 11 & 34 & 18 & 15 & 18 & 515 & 10 & 10 & 10 & 9.9 \\
\hline 20 & 9.7 & 9.3 & 11 & 51 & 16 & 15 & 18 & 395 & 10 & 10 & 10 & 9.9 \\
\hline 21 & 9.7 & 9.4 & 10 & 23 & 16 & 15 & 18 & 324 & 10 & 10 & 10 & 9.8 \\
\hline 22 & 9.7 & 9.9 & 10 & 18 & 15 & 14 & 17 & 325 & 10 & 10 & 10 & 9.8 \\
\hline 23 & 9.6 & 11 & 10 & 30 & 15 & 15 & 16 & 326 & 10 & 10 & 10 & 9.9 \\
\hline 24 & 9.9 & 11 & 10 & 18 & 14 & 15 & 16 & 307 & 10 & 10 & 10 & 9.8 \\
\hline 25 & 9.6 & 10 & 10 & 16 & 14 & 15 & 16 & 333 & 10 & 10 & 10 & 9.8 \\
\hline 26 & 9.5 & 9.8 & 10 & 15 & 14 & 15 & 17 & 335 & 10 & 10 & 10 & 9.8 \\
\hline 27 & 9.5 & 9.8 & 10 & 14 & 14 & 15 & 16 & 336 & 10 & 10 & 10 & 9.8 \\
\hline 28 & 9.5 & 10 & 10 & 13 & 17 & 15 & 14 & 337 & 11 & 10 & 10 & 9.7 \\
\hline 29 & 9.6 & 12 & 10 & 13 & --- & 15 & 13 & 337 & 10 & 10 & 10 & 9.7 \\
\hline 30 & 9.5 & 16 & 10 & 13 & --- & 15 & 302 & 338 & 11 & 10 & 10 & 9.7 \\
\hline 31 & 9.5 & --- & 10 & 13 & --- & 14 & --- & 337 & --- & 10 & 10 & --- \\
\hline TOTAL & 302.7 & 299.7 & 333 & 470 & 527 & 493 & 736 & 13788 & 442 & 308.0 & 310 & 297.4 \\
\hline MEAN & 9.76 & 9.99 & 10.7 & 15.2 & 18.8 & 15.9 & 24.5 & 445 & 14.7 & 9.94 & 10.0 & 9.91 \\
\hline MAX & 9.9 & 16 & 17 & 51 & 59 & 27 & 302 & 515 & 142 & 10 & 10 & 10 \\
\hline MIN & 9.5 & 9.3 & 10 & 10 & 12 & 14 & 13 & 307 & 10 & 9.7 & 10 & 9.7 \\
\hline $\mathrm{AC}-\mathrm{FT}$ & 600 & 594 & 661 & 932 & 1050 & 978 & 1460 & 27350 & 877 & 611 & 615 & 590 \\
\hline $\mathrm{a}$ & 17070 & 8720 & .00 & .00 & .00 & 21920 & 20860 & 2630 & 12060 & 14190 & 14580 & 12150 \\
\hline
\end{tabular}

a Diversion, in acre-feet, from French Meadows Reservoir to Hell Hole Reservoir through French Meadows Powerplant, provided by Placer County Water Agency. 
11427500 MIDDLE FORK AMERICAN RIVER AT FRENCH MEADOWS, CA—Continued

STATISTICS OF MONTHLY MEAN DATA FOR WATER YEARS 1952 - 1964, BY WATER YEAR (WY)

\begin{tabular}{|c|c|c|c|c|c|c|c|c|c|c|c|c|}
\hline & OCT & NOV & $\mathrm{DEC}$ & JAN & FEB & MAR & APR & MAY & JUN & JUL & AUG & SEP \\
\hline MEAN & 19.8 & 20.3 & 101 & 92.5 & 143 & 151 & 356 & 550 & 297 & 52.4 & 6.04 & 2.10 \\
\hline MAX & 222 & 106 & 882 & 377 & 561 & 367 & 537 & 1110 & 775 & 232 & 25.3 & 5.06 \\
\hline (WY) & 1963 & 1964 & 1956 & 1956 & 1963 & 1960 & 1962 & 1958 & 1952 & 1952 & 1952 & 1952 \\
\hline MIN & .40 & 1.60 & 1.76 & 5.57 & 40.1 & 55.2 & 187 & 210 & 69.7 & 6.22 & 1.57 & .64 \\
\hline (WY) & 1961 & 1960 & 1960 & 1960 & 1955 & 1962 & 1955 & 1959 & 1959 & 1959 & 1959 & 1961 \\
\hline
\end{tabular}

SUMMARY STATISTICS

ANNUAL MEAN

HIGHEST ANNUAL MEAN

LOWEST ANNUAL MEAN

HIGHEST DAILY MEAN

LOWEST DAILY MEAN

ANNUAL SEVEN-DAY MINIMUM

INSTANTANEOUS PEAK FLOW

INSTANTANEOUS PEAK STAGE

ANNUAL RUNOFF (AC-FT)

10 PERCENT EXCEEDS

50 PERCENT EXCEEDS

90 PERCENT EXCEEDS
WATER YEARS 1952 - 1964

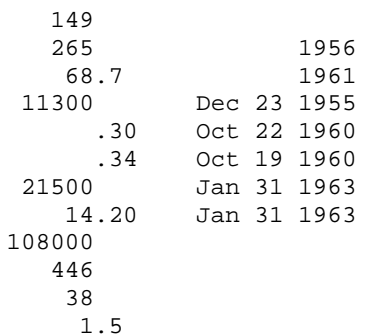

STATISTICS OF MONTHLY MEAN DATA FOR WATER YEARS 1965 - 1999, BY WATER YEAR (WY)

\begin{tabular}{|c|c|c|c|c|c|c|c|c|c|c|c|c|}
\hline & $\mathrm{OCT}$ & NOV & $\mathrm{DEC}$ & JAN & FEB & MAR & $\mathrm{APR}$ & MAY & JUN & JUL & AUG & SEP \\
\hline MEAN & 15.2 & 10.3 & 12.9 & 20.0 & 18.4 & 22.9 & 23.9 & 61.8 & 43.9 & 16.5 & 8.53 & 11.8 \\
\hline MAX & 266 & 42.7 & 83.3 & 249 & 200 & 375 & 248 & 518 & 272 & 136 & 15.0 & 136 \\
\hline (WY) & 1966 & 1966 & 1965 & 1997 & 1982 & 1986 & 1965 & 1965 & 1995 & 1983 & 1965 & 1965 \\
\hline MIN & 1.67 & 3.16 & 3.91 & 4.37 & 4.53 & 4.40 & 4.47 & 3.95 & 3.68 & 2.98 & 2.76 & 2.70 \\
\hline (WY) & 1965 & 1978 & 1977 & 1977 & 1977 & 1977 & 1977 & 1976 & 1977 & 1977 & 1977 & 1977 \\
\hline
\end{tabular}

SUMMARY STATISTICS

ANNUAL TOTAL

ANNUAL MEAN

HIGHEST ANNUAL MEAN

LOWEST ANNUAL MEAN

HIGHEST DAILY MEAN

LOWEST DAILY MEAN

ANNUAL SEVEN-DAY MINIMUM

INSTANTANEOUS PEAK FLOW

INSTANTANEOUS PEAK STAGE

ANNUAL RUNOFF (AC-FT)

TOTAL DIVERSION (AC-FT) a

10 PERCENT EXCEEDS

50 PERCENT EXCEEDS

90 PERCENT EXCEEDS
FOR 1998 CALENDAR YEAR

$$
\begin{array}{r}
9283.8 \\
25.4
\end{array}
$$

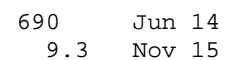

9.3 Nov 15

18410

170700

20
11
9

\begin{tabular}{|c|c|}
\hline $\begin{array}{r}18306.8 \\
50.2\end{array}$ & \\
\hline 515 & May 14 \\
\hline 9.3 & Nov 15 \\
\hline 9.4 & Nov 15 \\
\hline 672 & Apr 30 \\
\hline 8.42 & Apr 30 \\
\hline $\begin{array}{c}36310 \\
124200\end{array}$ & \\
\hline 36 & \\
\hline 10 & \\
\hline
\end{tabular}

FOR 1999 WATER YEAR

\begin{tabular}{|c|c|c|}
\hline \multicolumn{3}{|l|}{22.2} \\
\hline 97.3 & & 1965 \\
\hline 3.90 & & 1977 \\
\hline 3430 & May 16 & 1996 \\
\hline .80 & oct 22 & 1964 \\
\hline .84 & Oct 21 & 1964 \\
\hline 6050 & May 16 & 1996 \\
\hline 11.61 & May 16 & 1996 \\
\hline 16090 & & \\
\hline $\begin{array}{r}16 \\
9.6 \\
5.7\end{array}$ & & \\
\hline
\end{tabular}

WATER YEARS 1965 - 1999

a Diversion, in acre-feet, from French Meadows Reservoir to Hell Hole Reservoir through French Meadows Powerplant, provided by Placer County Water Agency. 
LOCATION.-Lat 3908'09", long 120²8'39", in NE 1/4 NW 1/4 sec.24, T.15 N., R.13 E., Placer County, Hydrologic Unit 18020128, Tahoe National Forest, on left bank 0.2 mi upstream from diversion dam, $0.5 \mathrm{mi}$ downstream from Little Duncan Creek, 2 mi northwest of French Meadows, and 20 mi northeast of Foresthill.

DRAINAGE AREA.-9.94 $\mathrm{mi}^{2}$.

PERIOD OF RECORD.-August 1960 to current year.

GAGE.-Water-stage recorder. Elevation of gage is 5,270 ft above sea level, from topographic map. Prior to Sept. 3, 1965, at site $150 \mathrm{ft}$ upstream at datum $9.56 \mathrm{ft}$ higher.

REMARKS.-No regulation or diversion upstream from station. See schematic diagram of Middle Fork American and Rubicon River Basins.

COOPERATION.--Records provided by Placer County Water Agency, under general supervision of the U.S. Geological Survey, in connection with a Federal Energy Regulatory Commission project.

EXTREMES FOR PERIOD OF RECORD.-Maximum discharge, 3,650 ft 3 /s, Dec. 22, 1964, gage height, $10.6 \mathrm{ft}$, from floodmarks, from rating curve extended above $400 \mathrm{ft}^{3} / \mathrm{s}$ on basis of computation of flow over diversion dam; maximum gage height, 10.95, Jan. 1, 1997 (backwater from debris dam); minimum daily, $0.10 \mathrm{ft}^{3} / \mathrm{s}$, several days during July and August 1977.

EXTREMES FOR CURRENT YEAR.-Peak discharges greater than base discharge of $250 \mathrm{ft}^{3} / \mathrm{s}$, or maximum:

$\begin{array}{lcccccrc}\text { Date } & \text { Time } & \begin{array}{c}\text { Discharge } \\ \left(\mathrm{ft}^{3} / \mathrm{s}\right)\end{array} & \begin{array}{c}\text { Gage height } \\ (\mathrm{ft})\end{array} & \text { Date } & \text { Time } & \begin{array}{c}\text { Discharge } \\ \left(\mathrm{ft}^{3} / \mathrm{s}\right)\end{array} & \begin{array}{c}\text { Gage height } \\ (\mathrm{ft})\end{array} \\ \text { Jan. 18 } & 0715 & 485 & 7.64 & \text { May } 24 & 1730 & 590 & 7.80\end{array}$

DISCHARGE, CUBIC FEET PER SECOND, WATER YEAR OCTOBER 1998 TO SEPTEMBER 1999 DAILY MEAN VALUES

\begin{tabular}{|c|c|c|c|c|c|c|c|c|c|c|c|c|}
\hline DAY & $\mathrm{OCT}$ & NOV & $\mathrm{DEC}$ & JAN & $\mathrm{FEB}$ & MAR & APR & MAY & JUN & JUL & AUG & SEP \\
\hline 1 & 2.3 & 5.3 & 39 & 13 & 25 & 147 & 32 & 130 & 218 & 14 & 2.5 & 1.2 \\
\hline 2 & 2.2 & 3.1 & 32 & 13 & 24 & 93 & 30 & 137 & 201 & 14 & 2.4 & 1.2 \\
\hline 3 & 2.1 & 2.5 & 140 & 13 & 24 & 90 & 29 & 108 & 154 & 13 & 2.4 & 1.2 \\
\hline 4 & 2.1 & 2.3 & 54 & 12 & 24 & 73 & 27 & 90 & 121 & 12 & 2.3 & 1.2 \\
\hline 5 & 2.0 & 2.3 & 35 & 12 & 22 & 60 & e26 & 94 & 123 & 11 & 2.3 & 1.1 \\
\hline 6 & 1.8 & 2.5 & 27 & 12 & 24 & 52 & e24 & 120 & 129 & 9.7 & 2.4 & 1.1 \\
\hline 7 & 1.8 & 6.0 & 22 & 11 & 103 & 45 & 24 & 149 & 119 & 9.0 & 2.5 & 1.1 \\
\hline 8 & 1.7 & 5.1 & 20 & 11 & 66 & 41 & 25 & 153 & 107 & 8.3 & 2.4 & 1.0 \\
\hline 9 & 1.7 & 3.6 & 18 & 11 & 142 & 38 & 23 & 145 & 99 & 7.7 & 2.4 & 1.0 \\
\hline 10 & 1.7 & 3.5 & 17 & 11 & 81 & 34 & 21 & 141 & 94 & 7.1 & 2.8 & 1.0 \\
\hline 11 & 1.7 & 4.1 & 16 & 11 & 61 & 31 & 22 & 161 & 98 & 6.6 & 2.6 & .96 \\
\hline 12 & 1.7 & 4.0 & 16 & 11 & 52 & 30 & 26 & 199 & 101 & 6.1 & 2.3 & .93 \\
\hline 13 & 1.7 & 4.5 & 17 & 11 & 45 & 31 & 35 & 198 & 102 & 6.1 & 2.2 & .93 \\
\hline 14 & 1.7 & 4.9 & 16 & 11 & 40 & 31 & 45 & 166 & 101 & 5.8 & 2.1 & .91 \\
\hline 15 & 1.6 & 4.7 & 16 & 22 & 35 & 29 & 49 & 143 & 97 & 5.3 & 2.0 & .88 \\
\hline 16 & 1.6 & 4.5 & 21 & 51 & 36 & 30 & 60 & 141 & 89 & 4.9 & 1.9 & .86 \\
\hline 17 & 1.6 & 7.8 & 23 & 69 & 72 & 33 & 86 & 157 & 77 & 4.7 & 1.8 & .86 \\
\hline 18 & 1.6 & 5.4 & 22 & 296 & 54 & 37 & 110 & 171 & 68 & 4.5 & 1.7 & .92 \\
\hline 19 & 1.5 & 5.0 & 20 & 182 & 43 & 38 & 134 & 188 & 59 & 4.3 & 1.7 & 1.1 \\
\hline 20 & 1.5 & 4.9 & 22 & 266 & 38 & 37 & 149 & 202 & 52 & 4.2 & 1.5 & 1.0 \\
\hline 21 & 1.5 & 4.9 & 23 & 143 & 34 & 32 & 147 & 224 & 46 & 4.0 & 1.5 & 1.1 \\
\hline 22 & 1.5 & 12 & 21 & 100 & 31 & 31 & 131 & 268 & 41 & 3.9 & 1.5 & .96 \\
\hline 23 & 1.4 & 61 & 23 & 111 & 29 & 32 & 119 & 305 & 37 & 3.7 & 1.7 & .95 \\
\hline 24 & 3.3 & 27 & 19 & 71 & 28 & 32 & 123 & 375 & 33 & 3.5 & 1.4 & 1.0 \\
\hline 25 & 3.5 & 14 & 15 & 56 & 27 & 34 & 142 & 408 & 28 & 3.4 & 1.3 & .93 \\
\hline 26 & 2.9 & 11 & 14 & 46 & 24 & 44 & 165 & 392 & 24 & 3.2 & 1.4 & .90 \\
\hline 27 & 2.3 & 11 & 13 & 39 & 25 & 48 & 161 & 371 & 21 & 3.1 & 1.7 & .83 \\
\hline 28 & 2.1 & 10 & 13 & 34 & 49 & 45 & 132 & 333 & 19 & 2.9 & 1.4 & .81 \\
\hline 29 & 2.3 & 28 & 13 & 31 & --- & 42 & 107 & 269 & 17 & 2.8 & 1.2 & .79 \\
\hline 30 & 2.3 & 132 & 13 & 29 & --- & 39 & 109 & 241 & 16 & 2.7 & 1.2 & .78 \\
\hline 31 & 2.0 & --- & 13 & 27 & --- & 35 & --- & 230 & --- & 2.6 & 1.3 & --- \\
\hline TOTAL & 60.7 & 396.9 & 773 & 1736 & 1258 & 1414 & 2313 & 6409 & 2491 & 194.1 & 59.8 & 29.50 \\
\hline MEAN & 1.96 & 13.2 & 24.9 & 56.0 & 44.9 & 45.6 & 77.1 & 207 & 83.0 & 6.26 & 1.93 & .98 \\
\hline MAX & 3.5 & 132 & 140 & 296 & 142 & 147 & 165 & 408 & 218 & 14 & 2.8 & 1.2 \\
\hline MIN & 1.4 & 2.3 & 13 & 11 & 22 & 29 & 21 & 90 & 16 & 2.6 & 1.2 & .78 \\
\hline $\mathrm{AC}-\mathrm{FT}$ & 120 & 787 & 1530 & 3440 & 2500 & 2800 & 4590 & 12710 & 4940 & 385 & 119 & 59 \\
\hline
\end{tabular}




\section{DUNCAN CREEK NEAR FRENCH MEADOWS, CA—Continued}

STATISTICS OF MONTHLY MEAN DATA FOR WATER YEARS 1960 - 1999, BY WATER YEAR (WY)

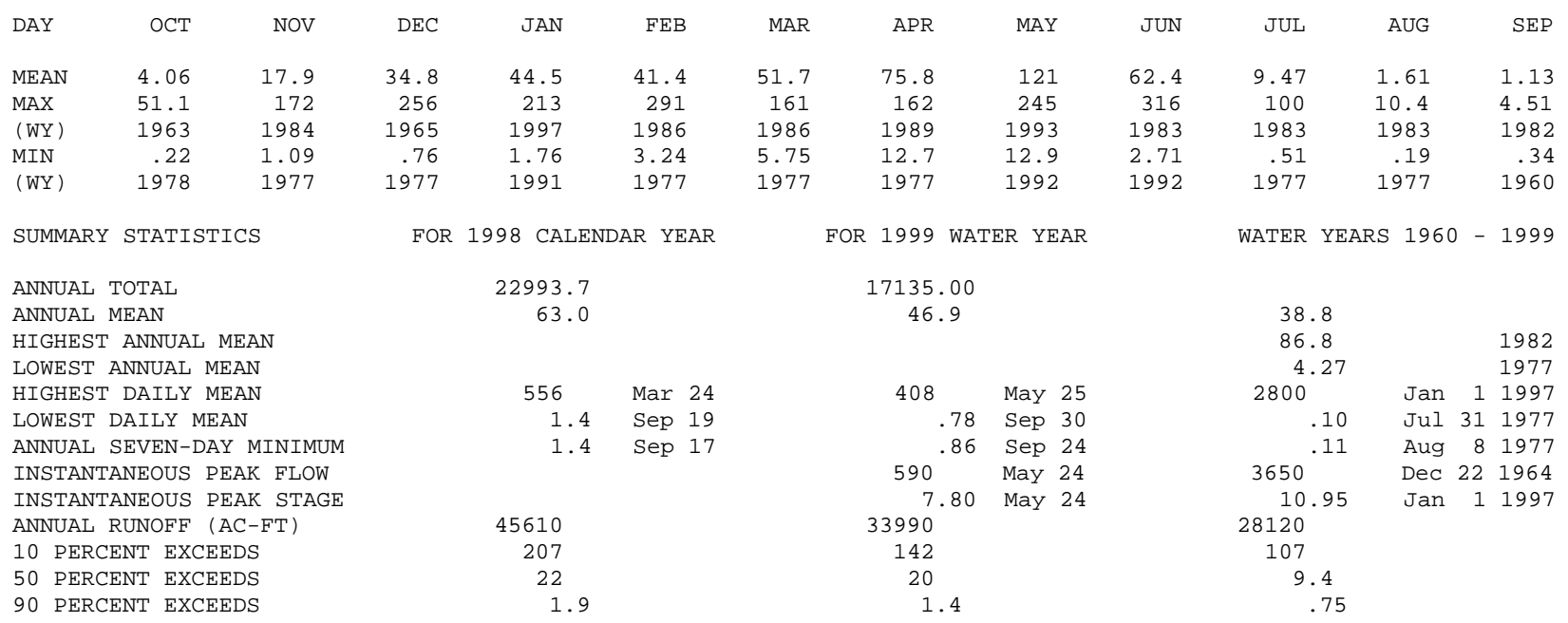


LOCATION.-Lat 3907'59", long 120²8'58", in NE 1/4 SE 1/4 sec.23, T.15 N., R.13 E., Placer County, Hydrologic Unit 18020128, Tahoe National Forest, on right bank $800 \mathrm{ft}$ downstream from unnamed right bank tributary, 1,000 ft downstream from Duncan Creek Diversion Dam, and 20 mi northeast of Foresthill.

DRAINAGE AREA.- $-10.5 \mathrm{mi}^{2}$.

PERIOD OF RECORD.-October 1964 to current year.

GAGE.-Water-stage recorder. Elevation of gage is 5,210 ft above sea level, from topographic map.

REMARKS.- Natural flow affected by transmountain diversion through Duncan Creek Diversion Tunnel to French Meadows Reservoir (station 11427400). Maximum design flow of tunnel is $400 \mathrm{ft}^{3} / \mathrm{s}$. See schematic diagram of Middle Fork American and Rubicon River Basins.

COOPERATION.-Records provided by Placer County Water Agency, under general supervision of the U.S. Geological Survey, in connection with a Federal Energy Regulatory Commission project.

EXTREMES FOR PERIOD OF RECORD.-Maximum discharge, 3,640 ft $3 / \mathrm{s}$, Dec. 22, 1964, gage height, $8.74 \mathrm{ft}$, in gage well, $10.0 \mathrm{ft}$, from floodmarks, from rating curve extended above $400 \mathrm{ft}^{3} / \mathrm{s}$ on basis of computation of peak flow over diversion dam; no flow at times in $1965-66$.

DISCHARGE, CUBIC FEET PER SECOND, WATER YEAR OCTOBER 1998 TO SEPTEMBER 1999

\begin{tabular}{|c|c|c|c|c|c|c|c|c|c|c|c|c|}
\hline DAY & $\mathrm{OCT}$ & NOV & $\mathrm{DEC}$ & JAN & FEB & MAR & APR & MAY & JUN & JUL & AUG & SEP \\
\hline 1 & 2.4 & 5.3 & 15 & 12 & 13 & 23 & 15 & 20 & 13 & 10 & 2.5 & 1.4 \\
\hline 2 & 2.3 & 3.3 & 14 & 12 & 13 & 20 & 15 & 20 & 13 & 10 & 2.4 & 1.3 \\
\hline 3 & 2.2 & 2.5 & 26 & 12 & 13 & 21 & 14 & 18 & 13 & 10 & 2.4 & 1.3 \\
\hline 4 & 2.1 & 2.3 & 17 & 12 & 13 & 19 & 14 & 17 & 13 & 9.9 & 2.3 & 1.2 \\
\hline 5 & 2.0 & 2.3 & 15 & 12 & 13 & 17 & 14 & 17 & 13 & 9.9 & 2.3 & 1.2 \\
\hline 6 & 1.9 & 2.5 & 14 & 12 & 13 & 16 & 14 & 19 & 13 & 9.8 & 2.3 & 1.1 \\
\hline 7 & 1.8 & 5.8 & 13 & 12 & e20 & 15 & 14 & 21 & 13 & 8.8 & 2.4 & 1.1 \\
\hline 8 & 1.8 & 5.6 & 13 & 12 & 19 & 15 & e13 & 20 & 12 & 8.2 & 2.3 & 1.1 \\
\hline 9 & 1.8 & 3.8 & 13 & 11 & 27 & e15 & 13 & 19 & 12 & 7.7 & 2.4 & 1.0 \\
\hline 10 & 1.8 & 3.6 & 13 & 11 & 20 & 14 & 13 & 18 & 12 & 7.2 & 2.7 & 1.0 \\
\hline 11 & 1.8 & 4.2 & 13 & 11 & 17 & 14 & 13 & 18 & 12 & 6.7 & 2.6 & 1.0 \\
\hline 12 & 1.7 & 4.1 & 13 & 11 & 16 & 14 & 13 & 19 & 12 & 6.2 & 2.4 & .95 \\
\hline 13 & 1.7 & 4.5 & 13 & 11 & 15 & 14 & 14 & 18 & 12 & 6.0 & 2.1 & .95 \\
\hline 14 & 1.7 & 4.9 & 13 & 11 & 15 & 14 & 16 & 17 & 12 & 6.1 & 2.0 & .95 \\
\hline 15 & 1.7 & 4.9 & 13 & 12 & 14 & 14 & 17 & 16 & 11 & 5.4 & 1.9 & .93 \\
\hline 16 & 1.7 & 4.6 & 14 & 14 & 15 & 14 & 19 & 16 & 11 & 5.0 & 1.9 & .88 \\
\hline 17 & 1.7 & 7.8 & 14 & 16 & 20 & 15 & 22 & 16 & 11 & 4.8 & 1.8 & .88 \\
\hline 18 & 1.7 & 5.7 & 14 & 70 & 17 & 16 & 25 & 15 & 11 & 4.6 & 1.7 & .91 \\
\hline 19 & 1.6 & 5.2 & 14 & 26 & 16 & 17 & 26 & 15 & 11 & 4.4 & 1.7 & 1.1 \\
\hline 20 & 1.5 & 5.1 & 13 & 37 & 15 & 17 & 26 & 15 & 11 & 4.2 & 1.6 & 1.0 \\
\hline 21 & 1.5 & 4.8 & e13 & 23 & 15 & 16 & 24 & 15 & 11 & 4.0 & 1.5 & 1.2 \\
\hline 22 & 1.5 & 12 & 13 & 19 & 14 & 15 & 22 & 16 & 11 & 3.9 & 1.5 & 1.0 \\
\hline 23 & 1.5 & 35 & e13 & 22 & 14 & 15 & 21 & 28 & 11 & 3.7 & 1.7 & .95 \\
\hline 24 & 3.2 & 26 & 13 & 18 & 14 & 15 & 21 & 76 & 11 & 3.6 & 1.6 & 1.0 \\
\hline 25 & 3.7 & 12 & 12 & 16 & 13 & 15 & 23 & 108 & 11 & 3.4 & 1.4 & .96 \\
\hline 26 & 3.0 & 11 & 12 & 15 & 13 & 16 & 24 & 98 & 10 & 3.3 & 1.5 & .93 \\
\hline 27 & 2.3 & 10 & 12 & 14 & 13 & 17 & 22 & 83 & 10 & 3.1 & 1.9 & .87 \\
\hline 28 & 2.2 & 10 & 12 & 14 & 15 & 17 & 20 & 55 & 10 & 2.9 & 1.6 & .83 \\
\hline 29 & 2.3 & 12 & 12 & 14 & --- & 17 & 18 & 19 & 10 & 2.8 & 1.4 & .81 \\
\hline 30 & 2.3 & 24 & 12 & 14 & --- & 16 & 18 & 14 & 10 & 2.7 & 1.4 & .81 \\
\hline 31 & 2.1 & --- & 12 & 13 & --- & 16 & --- & 13 & --- & 2.6 & 1.4 & --- \\
\hline TOTAL & 62.5 & 244.8 & 423 & 519 & 435 & 499 & 543 & 879 & 346 & 180.9 & 60.6 & 30.61 \\
\hline MEAN & 2.02 & 8.16 & 13.6 & 16.7 & 15.5 & 16.1 & 18.1 & 28.4 & 11.5 & 5.84 & 1.95 & 1.02 \\
\hline MAX & 3.7 & 35 & 26 & 70 & 27 & 23 & 26 & 108 & 13 & 10 & 2.7 & 1.4 \\
\hline MIN & 1.5 & 2.3 & 12 & 11 & 13 & 14 & 13 & 13 & 10 & 2.6 & 1.4 & .81 \\
\hline $\mathrm{AC}-\mathrm{ET}$ & 124 & 486 & 839 & 1030 & 863 & 990 & 1080 & 1740 & 686 & 359 & 120 & 61 \\
\hline
\end{tabular}

STATISTICS OF MONTHLY MEAN DATA FOR WATER YEARS 1965 - 1999, BY WATER YEAR (WY)

\begin{tabular}{|c|c|c|c|c|c|c|c|c|c|c|c|c|}
\hline MEAN & 2.15 & 8.68 & 22.2 & 31.0 & 21.9 & 19.3 & 15.7 & 29.1 & 13.8 & 4.04 & 1.44 & 1.09 \\
\hline MAX & 17.3 & 76.1 & 244 & 225 & 237 & 80.3 & 91.7 & 149 & 107 & 21.9 & 5.87 & 3.61 \\
\hline$(W Y)$ & 1983 & 1982 & 1965 & 1997 & 1986 & 1986 & 1982 & 1967 & 1998 & 1983 & 1983 & 1983 \\
\hline MIN & .061 & 1.15 & .76 & 1.69 & 2.02 & 2.63 & 4.80 & 3.88 & 2.15 & .44 & .28 & .090 \\
\hline (WY) & 1966 & 1991 & 1977 & 1991 & 1974 & 1965 & 1974 & 1976 & 1965 & 1965 & 1977 & 1965 \\
\hline
\end{tabular}

\section{SUMMARY STATISTICS}

ANNUAL TOTAL

ANNUAL MEAN

HIGHEST ANNUAL MEAN

LOWEST ANNUAL MEAN

HIGHEST DAILY MEAN

LOWEST DAILY MEAN

ANNUAL SEVEN-DAY MINIMUM

INSTANTANEOUS PEAK FLOW

INSTANTANEOUS PEAK STAGE

ANNUAL RUNOFF (AC-FT)

10 PERCENT EXCEEDS

50 PERCENT EXCEEDS

90 PERCENT EXCEEDS

e Estimated.
FOR 1998 CALENDAR YEAR

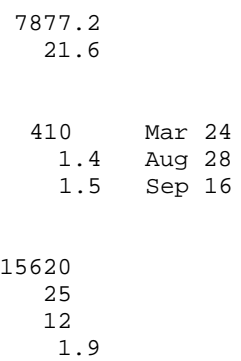

FOR 1999 WATER YEAR

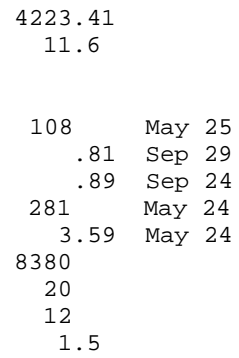

WATER YEARS 1965 - 1999

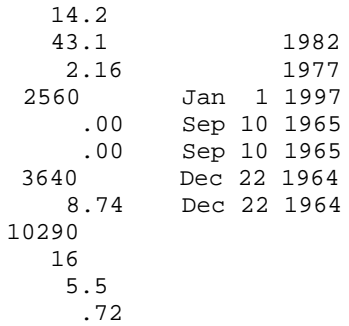


11427760 MIDDLE FORK AMERICAN RIVER ABOVE MIDDLE FORK POWERPLANT, NEAR FORESTHILL, CA

LOCATION.-Lat 3901'31", long 120³5'40", in NW 1/4 NW 1/4 sec.36, T.14 N., R.12 E., Placer County, Hydrologic Unit 18020128, Tahoe National Forest, on right bank $300 \mathrm{ft}$ upstream from Middle Fork Powerplant, 3.7 mi upstream from Big Mosquito Creek, and 11 mi east of Foresthill.

DRAINAGE AREA. $-87.8 \mathrm{mi}^{2}$.

PERIOD OF RECORD.-_August 1965 to current year.

GAGE.-Water-stage recorder. Elevation of gage is 2,540 ft above sea level, from topographic map. Prior to May 15,1980 , at datum $5.00 \mathrm{ft}$ higher. May 15, 1980, to Oct. 11, 1984, at datum $4.00 \mathrm{ft}$ higher.

REMARKS.-Considerable regulation by French Meadows Reservoir (station 11427400) 11 mi upstream. Transbasin diversions from French Meadows Reservoir to Hell Hole Reservoir (station 11428700) through French Meadows Powerplant (station 11427200). See schematic diagram of Middle Fork American and Rubicon River Basins.

COOPERATION.-Records provided by Placer County Water Agency, under general supervision of the U.S. Geological Survey, in connection with a Federal Energy Regulatory Commission project.

EXTREMES FOR PERIOD OF RECORD.-Maximum discharge, 13,900 ft 3 /s, Jan. 2, 1997, gage height, $14.6 \mathrm{ft}$, from floodmark, from rating curve extended above 4,200 $\mathrm{ft}^{3} / \mathrm{s}$; minimum daily, $5.3 \mathrm{ft}^{3} / \mathrm{s}$, Sept. $11,1977$.

DISCHARGE, CUBIC FEET PER SECOND, WATER YEAR OCTOBER 1998 TO SEPTEMBER 1999 DAILY MEAN VALUES

\begin{tabular}{|c|c|c|c|c|c|c|c|c|c|c|c|c|}
\hline DAY & $\mathrm{OCT}$ & NOV & $\mathrm{DEC}$ & JAN & FEB & MAR & $\mathrm{APR}$ & MAY & JUN & JUL & AUG & SEP \\
\hline 1 & 23 & 26 & 116 & 48 & 123 & 417 & 149 & 637 & 248 & 45 & 24 & 18 \\
\hline 2 & 22 & 25 & 85 & 48 & 117 & 345 & 143 & 646 & 90 & 44 & 24 & 18 \\
\hline 3 & 22 & 22 & 188 & 47 & 113 & 442 & 141 & 662 & 88 & 43 & 24 & 18 \\
\hline 4 & 21 & 21 & 129 & 46 & 114 & 384 & 134 & 645 & 83 & 43 & 24 & 18 \\
\hline 5 & 21 & 22 & 92 & 46 & 111 & 329 & 140 & 642 & 81 & 43 & 24 & 18 \\
\hline 6 & 21 & 22 & 80 & 45 & 117 & 294 & 134 & 650 & 77 & 41 & 24 & 18 \\
\hline 7 & 21 & 37 & 70 & 45 & 481 & 264 & 128 & 656 & 74 & 39 & 23 & 18 \\
\hline 8 & 21 & 35 & 70 & 44 & 552 & 245 & 136 & 651 & 72 & 38 & 23 & 18 \\
\hline 9 & 21 & 25 & 65 & 43 & 963 & 235 & 126 & 638 & 70 & 37 & 23 & 18 \\
\hline 10 & 21 & 24 & 61 & 42 & 529 & 210 & 122 & 627 & 68 & 36 & 24 & 19 \\
\hline 11 & 21 & 29 & 60 & 42 & 386 & 197 & 132 & 625 & 67 & 35 & 23 & 19 \\
\hline 12 & 20 & 25 & 59 & 42 & 322 & 186 & 140 & 628 & 65 & 33 & 22 & 19 \\
\hline 13 & 20 & 24 & 61 & 41 & 276 & 180 & 154 & 623 & 63 & 33 & 22 & 18 \\
\hline 14 & 21 & 24 & 63 & 41 & 247 & 176 & 181 & 615 & 62 & 33 & 21 & 18 \\
\hline 15 & 21 & 24 & 61 & 51 & 221 & 169 & 195 & 608 & 60 & 32 & 21 & 18 \\
\hline 16 & 20 & 24 & 64 & 89 & 246 & 166 & 209 & 598 & 59 & 31 & 21 & 18 \\
\hline 17 & 20 & 35 & 67 & 102 & 535 & 167 & 233 & 594 & 57 & 31 & 21 & 18 \\
\hline 18 & 20 & 30 & 66 & 305 & 401 & 169 & 249 & 593 & 56 & 30 & 20 & 18 \\
\hline 19 & 20 & 26 & 63 & 384 & 350 & 173 & 260 & 590 & 55 & 29 & 19 & 18 \\
\hline 20 & 20 & 25 & 63 & 753 & 312 & 177 & 264 & 480 & 54 & 29 & 19 & 18 \\
\hline 21 & 20 & 24 & 57 & 447 & 289 & 172 & 258 & 376 & 53 & 28 & 19 & 19 \\
\hline 22 & 20 & 44 & 57 & 290 & 257 & 165 & 245 & 376 & 52 & 28 & 19 & 18 \\
\hline 23 & 20 & 66 & 54 & 445 & 240 & 163 & 228 & 377 & 51 & 27 & 19 & 18 \\
\hline 24 & 24 & 107 & 55 & 308 & 225 & 161 & 221 & 390 & 50 & 27 & 19 & 18 \\
\hline 25 & 27 & 50 & 54 & 248 & 239 & 159 & 219 & 447 & 49 & 27 & 18 & 18 \\
\hline 26 & 23 & 43 & 53 & 212 & 214 & 164 & 231 & 435 & 48 & 27 & 18 & 18 \\
\hline 27 & 22 & 43 & 52 & 182 & 207 & 169 & 224 & 422 & 47 & 26 & 20 & 18 \\
\hline 28 & 21 & 46 & 51 & 163 & 231 & 165 & 206 & 400 & 47 & 25 & 19 & 18 \\
\hline 29 & 22 & 68 & 50 & 148 & --- & 160 & 190 & 376 & 46 & 25 & 19 & 17 \\
\hline 30 & 22 & 177 & 50 & 138 & --- & 160 & 388 & 362 & 45 & 24 & 18 & 17 \\
\hline 31 & 21 & --- & 50 & 134 & --- & 161 & --- & 358 & --- & 23 & 19 & --- \\
\hline TOTAL & 659 & 1193 & 2166 & 5019 & 8418 & 6724 & 5780 & 16727 & 2037 & 1012 & 653 & 542 \\
\hline MEAN & 21.3 & 39.8 & 69.9 & 162 & 301 & 217 & 193 & 540 & 67.9 & 32.6 & 21.1 & 18.1 \\
\hline MAX & 27 & 177 & 188 & 753 & 963 & 442 & 388 & 662 & 248 & 45 & 24 & 19 \\
\hline MIN & 20 & 21 & 50 & 41 & 111 & 159 & 122 & 358 & 45 & 23 & 18 & 17 \\
\hline $\mathrm{AC}-\mathrm{FT}$ & 1310 & 2370 & 4300 & 9960 & 16700 & 13340 & 11460 & 33180 & 4040 & 2010 & 1300 & 1080 \\
\hline
\end{tabular}


11427760 MIDDLE FORK AMERICAN RIVER ABOVE MIDDLE FORK POWERPLANT, NEAR FORESTHILL, CA—Continued

STATISTICS OF MONTHLY MEAN DATA FOR WATER YEARS 1966 - 1999, BY WATER YEAR (WY)

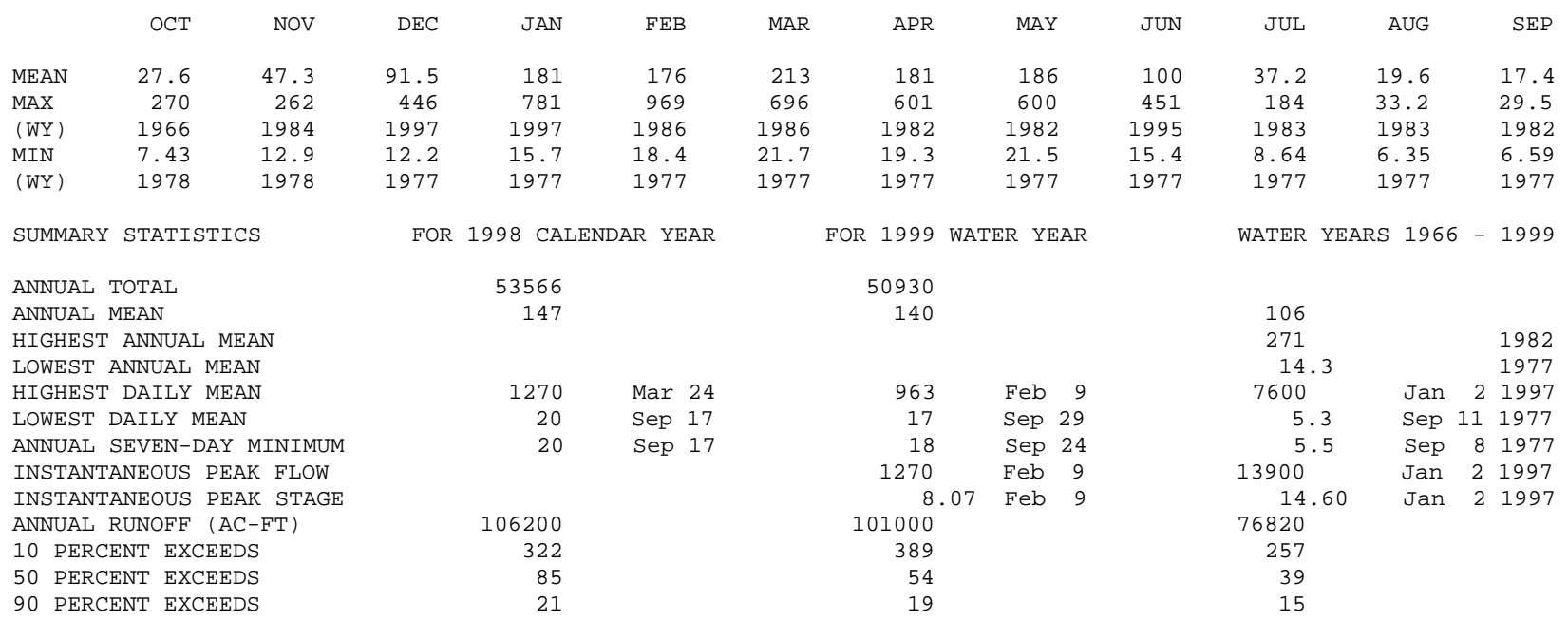


LOCATION.—Lat 3901'35", long 120³6'09", in SW 1/4 SE 1/4 sec.26, T.14 N., R.12 E., Placer County, Hydrologic Unit 18020128, Tahoe National Forest, on left bank at Interbay Dam, 3.3 mi upstream from Big Mosquito Creek, and 10.6 mi east of Foresthill.

DRAINAGE AREA.- $-89.1 \mathrm{mi}^{2}$.

PERIOD OF RECORD.-October 1965 to current year (since October 1985, operated as low-flow station only).

GAGE.-Acoustic-velocity meter system. Elevation of gage is 2,470 ft above sea level, from topographic map. Prior to February 1986, water-stage recorder at same site. March 1986 to September 1987, nonrecording gage and V-notch sharp-crested weir at same site and datum as previous gage.

REMARKS.-Flow regulated by French Meadows Reservoir (station 11427400) and after Aug. 22, 1966, by Interbay Reservoir (usable capacity, 130 acre-ft between normal operating limits) $500 \mathrm{ft}$ upstream. Water is diverted out of the basin from French Meadows Reservoir to Hell Hole Reservoir (station 11428700) and from Interbay Reservoir to Ralston Powerplant (station 11427765). Water is diverted into the basin from Hell Hole Reservoir to Middle Fork Powerplant (station 11428600) and through South Fork and Middle Fork Long Canyon Creek Diversion Tunnels (stations 11433060 and 11433080). See schematic diagram of Middle Fork American and Rubicon River Basins. Beginning October 1985 , only flows less than $35 \mathrm{ft}^{3} / \mathrm{s}$ are computed.

COOPERATION.-Records provided by Placer County Water Agency, under general supervision of the U.S. Geological Survey, in connection with a Federal Energy Regulatory Commission project.

EXTREMES FOR PERIOD OF RECORD.-Maximum discharge (water years 1966-85), 9,900 ft ${ }^{3} / \mathrm{s}$, Jan. 13, 1980, gage height, $7.95 \mathrm{ft}$; minimum daily, $1.0 \mathrm{ft}^{3} / \mathrm{s}$, Oct. 25-30, 1966, Jan. 19, 1967.

DISCHARGE, CUBIC FEET PER SECOND, WATER YEAR OCTOBER 1998 TO SEPTEMBER 1999 DAILY MEAN VALUES

\begin{tabular}{|c|c|c|c|c|c|c|c|c|c|c|c|c|}
\hline DAY & $\mathrm{OCT}$ & NOV & $\mathrm{DEC}$ & JAN & FEB & MAR & APR & MAY & JUN & JUL & AUG & SEP \\
\hline 1 & e25 & e23 & 23 & 23 & 23 & --- & 23 & 23 & 23 & 23 & 23 & 23 \\
\hline 2 & e25 & e23 & 23 & 23 & 23 & --- & 23 & 23 & 23 & 23 & 23 & 23 \\
\hline 3 & e25 & e23 & 23 & 23 & 23 & --- & 23 & 23 & 23 & 23 & 23 & 23 \\
\hline 4 & e25 & e23 & 23 & 23 & 23 & --- & 23 & 23 & 23 & 23 & 23 & 23 \\
\hline 5 & e25 & 23 & 23 & 23 & 23 & --- & 23 & 23 & 23 & 23 & 23 & 23 \\
\hline 6 & e25 & 23 & 23 & 23 & 23 & --- & 23 & 23 & 23 & 23 & 23 & 23 \\
\hline 7 & e25 & 23 & 23 & 23 & 23 & --- & 23 & 23 & 23 & 23 & 23 & 23 \\
\hline 8 & e25 & 23 & 23 & 23 & --- & --- & 23 & 23 & 23 & 23 & 23 & 23 \\
\hline 9 & e24 & 23 & 23 & 23 & --- & --- & 23 & 23 & 23 & 23 & 23 & 23 \\
\hline 10 & e24 & 23 & 23 & 23 & --- & --- & 23 & 23 & 23 & 23 & 23 & 23 \\
\hline 11 & e24 & 23 & 23 & 23 & 23 & --- & 23 & 23 & 23 & 23 & 23 & 23 \\
\hline 12 & e24 & 23 & 23 & 23 & 23 & --- & 23 & --- & 23 & 23 & 23 & 23 \\
\hline 13 & e24 & 23 & 23 & 23 & 23 & --- & 23 & 23 & 23 & 23 & 23 & 23 \\
\hline 14 & e24 & 23 & 23 & 23 & 23 & --- & 23 & 23 & 23 & 23 & 23 & 23 \\
\hline 15 & e25 & 23 & 23 & 23 & 23 & --- & --- & --- & 23 & 23 & 23 & 23 \\
\hline 16 & e24 & 23 & 23 & 23 & 23 & --- & --- & 23 & 23 & 23 & 23 & 23 \\
\hline 17 & e24 & 23 & 23 & 23 & 23 & --- & 23 & 23 & 23 & 23 & 23 & 23 \\
\hline 18 & e24 & 23 & 23 & 23 & 23 & --- & 23 & 23 & 23 & 23 & 23 & 23 \\
\hline 19 & e23 & 23 & 23 & --- & 23 & --- & --- & 23 & 23 & 23 & 23 & 22 \\
\hline 20 & e23 & 23 & 23 & --- & 23 & --- & --- & 23 & 23 & 23 & 23 & 20 \\
\hline 21 & e22 & 23 & 23 & 23 & 23 & --- & 23 & 23 & 23 & 23 & 23 & 22 \\
\hline 22 & e22 & 23 & 23 & 23 & 23 & --- & 23 & 23 & 23 & 23 & 23 & 21 \\
\hline 23 & e22 & 23 & 23 & 23 & 23 & --- & 23 & 23 & 23 & 23 & 23 & 20 \\
\hline 24 & e23 & 23 & 23 & 23 & --- & --- & 23 & 23 & 23 & 23 & 23 & 20 \\
\hline 25 & e23 & 23 & 23 & 23 & --- & --- & 23 & --- & 23 & 23 & 23 & 20 \\
\hline 26 & e23 & 23 & 23 & 23 & --- & 23 & --- & 23 & 23 & 23 & 23 & 20 \\
\hline 27 & e23 & 23 & 23 & 23 & --- & 23 & 23 & 23 & 23 & 23 & 23 & 20 \\
\hline 28 & e23 & 23 & 23 & 23 & 23 & 23 & 23 & 23 & 23 & 23 & 23 & 20 \\
\hline 29 & e23 & 23 & 23 & 23 & --- & 23 & 23 & 23 & 23 & 23 & 23 & 20 \\
\hline 30 & e23 & 23 & 23 & 23 & --- & 23 & 23 & 23 & 23 & 23 & 23 & 20 \\
\hline 31 & e23 & --- & 23 & 23 & --- & 23 & --- & 23 & --- & 23 & 23 & --- \\
\hline TOTAL & 737 & 690 & 713 & --- & --- & --- & --- & --- & 690 & 713 & 713 & 659 \\
\hline MEAN & 23.8 & 23.0 & 23.0 & --- & --- & --- & --- & --- & 23.0 & 23.0 & 23.0 & 22.0 \\
\hline MAX & 25 & 23 & 23 & --- & --- & --- & --- & --- & 23 & 23 & 23 & 23 \\
\hline MIN & 22 & 23 & 23 & --- & --- & --- & --- & --- & 23 & 23 & 23 & 20 \\
\hline $\mathrm{AC}-\mathrm{FT}$ & 1460 & 1370 & 1410 & --- & --- & --- & --- & --- & 1370 & 1410 & 1410 & 1310 \\
\hline a & 3100 & 29220 & 40390 & 34630 & 43280 & 52780 & 47450 & 51650 & 46410 & 41380 & 42210 & 25360 \\
\hline
\end{tabular}

e Estimated.

a Diversion, in acre-feet, through Ralston Powerplant, provided by Placer County Water Agency. 
11427770 MIDDLE FORK AMERICAN RIVER BELOW INTERBAY DAM, NEAR FORESTHILL, CA—Continued STATISTICS OF MONTHLY MEAN DATA FOR WATER YEARS 1966 - 1985, BY WATER YEAR (WY)

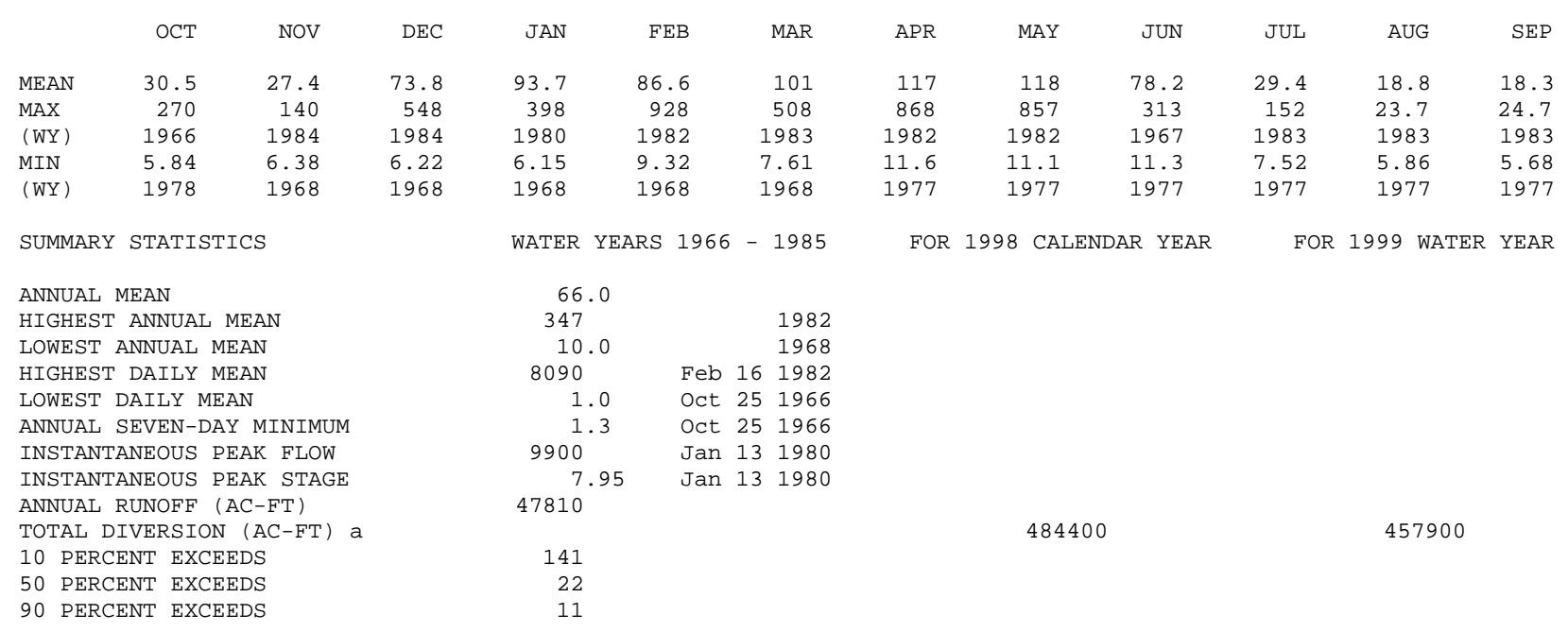

a Diversion, in acre-feet, through Ralston Powerplant, provided by Placer County Water Agency. 


\section{RUBICON-ROCKBOUND TUNNEL NEAR MEEKS BAY, CA}

LOCATION.-Lat 3859'16", long 120¹3’29", in NE 1/4 SE 1/4 sec.8, T.13 N., R.16 E., El Dorado County, Hydrologic Unit 18020128, Eldorado National Forest, on right bank at tunnel intake $100 \mathrm{ft}$ upstream from diversion dam on Rubicon River, 3.5 mi upstream from Rubicon Springs, and 6.4 mi southwest of Meeks Bay.

PERIOD OF RECORD.-December 1963 to current year.

GAGE.-Water-stage recorder. Datum of gage is 6,533.23 ft above sea level (levels by Sacramento Municipal Utility District). Auxiliary waterstage recorder since Aug. 26, 1966, $220 \mathrm{ft}$ downstream from tunnel outlet at different datum.

REMARKS.-Tunnel diverts water from Rubicon River to Rockbound Lake which flows into Buck Island Lake. Water is then diverted via BuckLoon Tunnel (station 11428300) to Loon Lake (station 11429350) for power development. See schematic diagram of Middle Fork American and Rubicon River Basins.

COOPERATION.-Records were collected by Sacramento Municipal Utility District, under general supervision of the U.S. Geological Survey, in connection with a Federal Energy Regulatory Commission project.

\section{DISCHARGE, CUBIC FEET PER SECOND, WATER YEAR OCTOBER 1998 TO SEPTEMBER 1999}

\section{DAILY MEAN VALUES}

\begin{tabular}{|c|c|c|c|c|c|c|c|c|c|c|c|c|}
\hline DAY & $\mathrm{OCT}$ & NOV & $\mathrm{DEC}$ & JAN & FEB & MAR & $\mathrm{APR}$ & MAY & JUN & JUL & AUG & SEP \\
\hline 1 & 14 & 29 & 191 & 18 & 22 & 39 & 36 & 157 & 646 & 392 & 20 & .03 \\
\hline 2 & 9.9 & 35 & 103 & 17 & 21 & 52 & 31 & 241 & 597 & 412 & 18 & .00 \\
\hline 3 & 7.4 & 19 & 125 & 16 & 22 & 43 & 29 & 173 & 323 & 379 & 16 & .00 \\
\hline 4 & 5.6 & 12 & 116 & 16 & 25 & 38 & 28 & 121 & 191 & 290 & 14 & .00 \\
\hline 5 & 4.1 & 8.5 & 76 & 15 & 25 & 29 & 25 & 122 & 184 & 230 & 12 & .00 \\
\hline 6 & 2.5 & 6.9 & 55 & 15 & 23 & 24 & 27 & 206 & 342 & 212 & 11 & .00 \\
\hline 7 & 1.2 & 6.7 & 43 & 15 & 36 & 21 & 25 & 331 & 406 & 218 & 11 & .00 \\
\hline 8 & .24 & 8.1 & 39 & 15 & 53 & 19 & 24 & 342 & 342 & 205 & 8.8 & .00 \\
\hline 9 & .00 & 9.7 & 34 & 14 & 48 & 20 & 27 & 284 & 328 & 185 & 9.6 & .00 \\
\hline 10 & .00 & 9.7 & 31 & 13 & 49 & 24 & 25 & 253 & 349 & 170 & 27 & .00 \\
\hline 11 & .00 & 9.8 & 31 & 13 & 45 & 18 & 22 & 313 & 435 & 178 & 40 & .00 \\
\hline 12 & .00 & 10 & 31 & 13 & 38 & 17 & 29 & 455 & 530 & 191 & 23 & .00 \\
\hline 13 & .00 & 11 & 34 & 13 & 27 & 19 & 52 & 519 & 619 & 182 & 15 & .00 \\
\hline 14 & .00 & 15 & 33 & 14 & 24 & 26 & 98 & 336 & 686 & 171 & 11 & .00 \\
\hline 15 & .00 & 19 & 30 & 23 & 22 & 25 & 114 & 233 & 767 & 159 & 8.4 & .00 \\
\hline 16 & .00 & 19 & 30 & 61 & 21 & 23 & 124 & 244 & 761 & 136 & 6.6 & .00 \\
\hline 17 & .00 & 21 & 38 & 63 & 42 & 29 & 156 & 326 & 688 & 108 & 5.4 & .00 \\
\hline 18 & .00 & 21 & 40 & 127 & 40 & 40 & 190 & 445 & 664 & 90 & 4.5 & .00 \\
\hline 19 & .00 & 19 & 35 & 104 & 29 & 53 & 236 & 447 & 623 & 76 & 4.2 & .00 \\
\hline 20 & .00 & 16 & 29 & 77 & 24 & 46 & 264 & 493 & 543 & 67 & 3.9 & .00 \\
\hline 21 & .00 & 14 & 27 & 60 & 24 & 36 & 264 & 508 & 553 & 31 & 3.3 & .00 \\
\hline 22 & .00 & 17 & 25 & 50 & 24 & 30 & 210 & 651 & 574 & .23 & 3.1 & .00 \\
\hline 23 & .00 & 75 & 23 & 48 & 21 & 31 & 148 & 775 & 655 & .08 & 6.9 & .00 \\
\hline 24 & .00 & 127 & 21 & 40 & 20 & 36 & 120 & 808 & 677 & 7.4 & 6.6 & .00 \\
\hline 25 & .00 & 72 & 19 & 39 & 21 & 41 & 170 & 848 & 554 & 26 & 4.7 & .00 \\
\hline 26 & .00 & 42 & 19 & 35 & 22 & 66 & 258 & 845 & 370 & 33 & 3.2 & .00 \\
\hline 27 & .00 & 30 & 18 & 29 & 20 & 93 & 235 & 826 & 299 & 35 & 2.5 & .00 \\
\hline 28 & .00 & 26 & 17 & 26 & 25 & 80 & 177 & 800 & 294 & 32 & 2.0 & .00 \\
\hline 29 & .11 & 28 & 17 & 24 & --- & 63 & 124 & 706 & 329 & 29 & 1.6 & .00 \\
\hline 30 & 4.5 & 272 & 17 & 23 & --- & 50 & 106 & 566 & 356 & 25 & .77 & .00 \\
\hline 31 & 11 & --- & 17 & 23 & --- & 42 & --- & 718 & --- & 22 & .33 & --- \\
\hline TOTAL & 60.55 & 1008.4 & 1364 & 1059 & 813 & 1173 & 3374 & 14092 & 14685 & 4291.71 & 304.40 & 0.03 \\
\hline MEAN & 1.95 & 33.6 & 44.0 & 34.2 & 29.0 & 37.8 & 112 & 455 & 490 & 138 & 9.82 & .001 \\
\hline MAX & 14 & 272 & 191 & 127 & 53 & 93 & 264 & 848 & 767 & 412 & 40 & .03 \\
\hline MIN & .00 & 6.7 & 17 & 13 & 20 & 17 & 22 & 121 & 184 & .08 & .33 & .00 \\
\hline $\mathrm{AC}-\mathrm{FT}$ & 120 & 2000 & 2710 & 2100 & 1610 & 2330 & 6690 & 27950 & 29130 & 8510 & 604 & .06 \\
\hline
\end{tabular}

STATISTICS OF MONTHLY MEAN DATA FOR WATER YEARS 1964 - 1999, BY WATER YEAR (WY)

\begin{tabular}{|c|c|c|c|c|c|c|c|c|c|c|c|c|}
\hline MEAN & 16.1 & 49.1 & 46.2 & 50.1 & 43.4 & 67.1 & 154 & 359 & 327 & 118 & 18.9 & 10.6 \\
\hline MAX & 149 & 277 & 204 & 222 & 187 & 196 & 295 & 655 & 789 & 519 & 168 & 91.0 \\
\hline (WY) & 1983 & 1984 & 1965 & 1970 & 1986 & 1986 & 1989 & 1969 & 1983 & 1983 & 1983 & 1982 \\
\hline MIN & .000 & .000 & .000 & .000 & 3.44 & 13.5 & 24.6 & 110 & 33.8 & .77 & .000 & .000 \\
\hline (WY) & 1964 & 1964 & 1977 & 1977 & 1991 & 1977 & 1975 & 1977 & 1976 & 1976 & 1964 & 1964 \\
\hline
\end{tabular}

SUMMARY STATISTICS

ANNUAL TOTAL

ANNUAL MEAN

HIGHEST ANNUAL MEAN

LOWEST ANNUAL MEAN

HIGHEST DAILY MEAN

LOWEST DAILY MEAN

ANNUAL SEVEN-DAY MINIMUM

ANNUAL RUNOFF (AC-FT)

10 PERCENT EXCEEDS

50 PERCENT EXCEEDS

90 PERCENT EXCEEDS
FOR 1998 CALENDAR YEAR

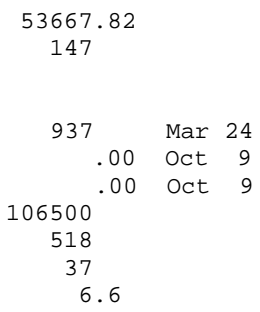

FOR 1999 WATER YEAR

42225.09

116

$848 \quad$ May 25

$\begin{array}{lll}.00 & \text { Oct } 9 \\ .00 & \text { Oct } 9\end{array}$

83750

384

27.00
WATER YEARS 1964 - 1999

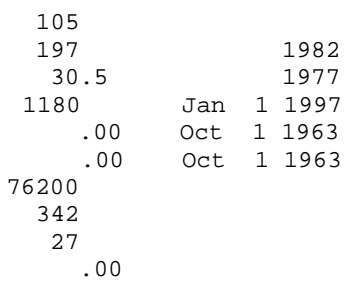


LOCATION._Lat 3859'20", long 120¹3'20", in NW 1/4 SW 1/4 sec.9, T.13 N., R.16 E., El Dorado County, Hydrologic Unit 18020128, Eldorado National Forest, at outlet structure on diverson dam on Rubicon River, 3.3 mi upstream from Rubicon Springs, and 6.2 mi southwest of Meeks Bay.

PERIOD OF RECORD.-October 1991 to current year (low-flow records only). Unpublished records for water years 1964-91 available in files of the U.S. Geological Survey.

GAGE.-Differential-pressure gage and orifice control in outlet pipes. Auxiliary nonrecording gage 1,300 ft downstream at different datum. Datum of gage is $6,520 \mathrm{ft}$ above sea level from topographic map. Prior to Sept. 4, 1991, nonrecording gage at site 1,300 $\mathrm{ft}$ downstream at different datum.

REMARKS.--Records not computed above $10 \mathrm{ft}^{3} / \mathrm{s}$. Flow regulated by Rubicon Reservoir. Flow over the spillway bypasses this station. Most of the water is diverted through Rubicon-Rockbound Tunnel (station 11427940) to Rockbound Lake, which flows into Buck Island Lake. Water is then diverted via Buck-Loon Tunnel (station 11428300) to Loon Lake (station 11429350) for power development. See schematic diagram of Middle Fork American and Rubicon River Basins.

COOPERATION.-_Records were collected by Sacramento Municipal Utility District, under general supervision of the U.S. Geological Survey, in connection with a Federal Energy Regulatory Commission project.

DISCHARGE, CUBIC FEET PER SECOND, WATER YEAR OCTOBER 1998 TO SEPTEMBER 1999 DAILY MEAN VALUES

\begin{tabular}{|c|c|c|c|c|c|c|c|c|c|c|c|c|}
\hline DAY & OCT & NOV & $\mathrm{DEC}$ & JAN & $\mathrm{FEB}$ & MAR & $A P R$ & MAY & JUN & JUL & AUG & SEP \\
\hline 1 & 6.9 & 6.2 & 6.8 & 6.6 & 6.6 & 6.8 & 6.7 & 7.5 & e8. 6 & e7.0 & e7. 3 & 6.7 \\
\hline 2 & 6.7 & 6.2 & 6.5 & 6.6 & 6.6 & 6.8 & 6.7 & 7.8 & e8. 5 & e7.0 & e7. 3 & 6.7 \\
\hline 3 & 6.7 & 6.1 & 6.5 & 6.6 & 6.6 & 6.8 & 6.4 & 7.6 & e7.9 & e7.0 & e7. 3 & 6.7 \\
\hline 4 & 6.6 & 6.0 & 6.5 & 6.6 & 6.6 & 6.8 & 6.6 & 7.4 & e7. 5 & e 6.8 & e7. 3 & 6.7 \\
\hline 5 & e 6.6 & 6.0 & 6.3 & 6.6 & 6.6 & 6.6 & 6.6 & 7.4 & e7. 4 & e 6.6 & e7. 3 & 6.6 \\
\hline 6 & e 6.6 & 6.0 & 6.3 & 6.6 & 6.6 & 6.6 & 6.6 & 7.7 & e7.9 & e6. 5 & e7.3 & 6.6 \\
\hline 7 & 6.6 & 6.0 & 6.2 & 6.5 & 6.7 & 6.6 & 6.6 & 8.1 & e8.1 & e 6.6 & e7. 3 & 5.4 \\
\hline 8 & 6.5 & 6.0 & 6.2 & 6.5 & 6.8 & 6.5 & 6.6 & 8.1 & e 8.0 & e 6.5 & e7. 3 & 3.3 \\
\hline 9 & 6.5 & 6.0 & 6.2 & 6.5 & 6.8 & 6.5 & 6.6 & 8.0 & e7.9 & e 6.4 & e7. 3 & 3.3 \\
\hline 10 & 6.5 & 6.0 & 6.4 & 6.5 & 6.8 & 6.5 & 6.6 & 7.9 & e 6.8 & e 6.1 & e7. 3 & 3.3 \\
\hline 11 & 6.5 & 6.0 & 6.7 & 6.5 & 6.8 & 6.5 & 6.6 & 8.0 & e 6.8 & e 6.4 & e7. 3 & 3.4 \\
\hline 12 & 6.4 & 6.0 & 6.7 & 6.6 & 6.7 & 6.5 & 6.6 & 8.3 & e7. 3 & e6. 5 & e7. 3 & 3.4 \\
\hline 13 & 5.4 & 6.0 & 6.7 & 6.6 & 6.6 & 6.5 & 6.7 & 8.5 & e 7.4 & e 6.4 & e7.3 & 3.4 \\
\hline 14 & 4.0 & 6.0 & 6.7 & 6.6 & 6.7 & 6.6 & 6.9 & 8.2 & e7. 6 & e6. 3 & e7. 3 & 3.3 \\
\hline 15 & 3.9 & 6.0 & 6.7 & 6.6 & 6.6 & 6.6 & 7.2 & 7.9 & e7. 6 & e6.3 & e7. 3 & 3.3 \\
\hline 16 & 4.0 & 6.0 & 6.7 & 6.8 & 6.6 & 6.6 & 7.4 & 7.9 & e7. 6 & e 6.2 & e7. 3 & 3.3 \\
\hline 17 & 3.9 & 6.1 & 6.7 & 6.8 & 6.7 & 6.7 & 7.5 & 8.1 & e 7.6 & e 6.1 & e7. 3 & 4.7 \\
\hline 18 & 3.9 & 6.1 & 6.7 & 7.1 & 6.7 & 6.7 & 7.6 & 8.4 & e7. 5 & e6. 2 & e7. 3 & 6.5 \\
\hline 19 & 3.9 & 6.1 & 6.7 & 7.0 & 6.6 & 6.8 & 7.8 & 8.4 & e 7.4 & e 6.4 & 7.2 & 6.5 \\
\hline 20 & 3.9 & 6.0 & 6.7 & 6.9 & 6.6 & 6.8 & 7.8 & 8.4 & e7. 3 & e 6.4 & 6.8 & 6.5 \\
\hline 21 & 3.9 & 6.0 & 6.7 & 6.8 & 6.6 & 6.7 & 7.9 & 8.5 & e 6.4 & e 6.4 & 6.8 & 5.6 \\
\hline 22 & 3.9 & 6.0 & 6.6 & 6.8 & 6.6 & 6.7 & 7.7 & 8.8 & e7. 3 & e 6.7 & 6.8 & 4.0 \\
\hline 23 & 3.8 & 6.3 & 6.6 & 6.8 & 6.7 & 6.7 & 7.5 & e8.9 & e7. 5 & e7.0 & 6.8 & 4.0 \\
\hline 24 & 3.8 & 6.6 & 6.6 & 6.7 & 6.6 & 6.7 & 7.4 & e8. 9 & e7. 5 & e7. 3 & 6.8 & 4.1 \\
\hline 25 & 3.9 & 6.4 & 6.6 & 6.7 & 6.6 & 6.7 & 7.6 & e8. 7 & e7. 3 & e7. 4 & 6.8 & 4.0 \\
\hline 26 & 3.9 & 6.3 & 6.6 & 6.7 & 6.6 & 6.8 & 7.9 & e8.7 & e7.0 & e7. 4 & 6.8 & 4.0 \\
\hline 27 & 4.0 & 6.2 & 6.6 & 6.7 & 6.7 & 7.0 & 7.8 & e8.7 & e 6.8 & e7. 4 & 6.8 & 4.0 \\
\hline 28 & 4.0 & 6.2 & 6.6 & 6.6 & 6.7 & 6.9 & 7.6 & e8. 8 & e 6.8 & e 7.4 & 6.8 & 4.0 \\
\hline 29 & 4.0 & 6.2 & 6.6 & 6.6 & --- & 6.8 & 7.4 & e8.7 & e 6.9 & e7. 4 & 6.8 & 4.0 \\
\hline 30 & 4.1 & 7.0 & 6.6 & 6.6 & --- & 6.8 & 7.3 & e8. 5 & e 6.9 & e7. 3 & 6.7 & 4.0 \\
\hline 31 & 5.4 & --- & 6.6 & 6.6 & --- & 6.7 & --- & e8.7 & --- & 7.3 & 6.7 & --- \\
\hline TOTAL & 156.7 & 184.0 & 203.6 & 206.7 & 186.4 & 207.3 & 214.2 & 255.5 & 223.1 & 208.7 & 220.0 & 141.3 \\
\hline MEAN & 5.05 & 6.13 & 6.57 & 6.67 & 6.66 & 6.69 & 7.14 & 8.24 & 7.44 & 6.73 & 7.10 & 4.71 \\
\hline MAX & 6.9 & 7.0 & 6.8 & 7.1 & 6.8 & 7.0 & 7.9 & 8.9 & 8.6 & 7.4 & 7.3 & 6.7 \\
\hline MIN & 3.8 & 6.0 & 6.2 & 6.5 & 6.6 & 6.5 & 6.4 & 7.4 & 6.4 & 6.1 & 6.7 & 3.3 \\
\hline $\mathrm{AC}-\mathrm{FT}$ & 311 & 365 & 404 & 410 & 370 & 411 & 425 & 507 & 443 & 414 & 436 & 280 \\
\hline
\end{tabular}

CAL YR 1998 TOTAL 2496.5 MEAN 6.84 MAX 8.4 MIN 3.8 AC-FT 4950 WTR YR 1999 TOTAL 2407.5 MEAN 6.60 MAX 8.9 MIN 3.3 AC-FT 4780 


\section{BUCK-LOON TUNNEL NEAR MEEKS BAY, CA}

LOCATION.—Lat 3900'17", long 120¹5’21", in SE 1/4 NW 1/4 sec.6, T.13 N., R.16 E., El Dorado County, Hydrologic Unit 18020128, Eldorado National Forest, on right bank at tunnel intake near left abutment of diversion dam and 7.4 mi southwest of Meeks Bay.

PERIOD OF RECORD.--November 1963 to current year.

GAGE.-Water-stage recorder. Datum of gage is 6,425.0 ft above sea level (levels by Sacramento Municipal Utility District).

REMARKS.-Tunnel diverts water from Buck Island Lake and discharges into Loon Lake (station 11429350). Buck Island Lake receives water from Rubicon River via Rubicon-Rockbound Tunnel (station 11427940). Gates are closed at the tunnel entrance during the summer to raise the level of Buck Island Lake for recreational purposes. See schematic diagram of Middle Fork American and Rubicon River Basins.

COOPERATION.- - Records were collected by Sacramento Municipal Utility District, under general supervision of the U.S. Geological Survey, in connection with a Federal Energy Regulatory Commission project.

DISCHARGE, CUBIC FEET PER SECOND, WATER YEAR OCTOBER 1998 TO SEPTEMBER 1999 DAILY MEAN VALUES

\begin{tabular}{|c|c|c|c|c|c|c|c|c|c|c|c|c|}
\hline DAY & OCT & NOV & $\mathrm{DEC}$ & JAN & FEB & MAR & $\mathrm{APR}$ & MAY & JUN & JUL & AUG & SEP \\
\hline 1 & 8.7 & 12 & 375 & 19 & 29 & 45 & 48 & 172 & 877 & 511 & 27 & 1.1 \\
\hline 2 & 129 & 46 & 191 & 19 & 26 & 61 & 39 & 314 & 838 & 540 & 24 & 1.0 \\
\hline 3 & 63 & 37 & 165 & 18 & 26 & 64 & 36 & 289 & 582 & 540 & 21 & 1.0 \\
\hline 4 & 11 & 24 & 188 & 18 & 28 & 55 & 34 & 193 & 338 & 443 & 18 & 1.0 \\
\hline 5 & 5.6 & 15 & 125 & 17 & 30 & 43 & 34 & 151 & 247 & 350 & 15 & 1.0 \\
\hline 6 & 2.8 & 11 & 84 & 16 & 31 & 35 & 34 & 218 & 408 & 298 & 14 & 1.0 \\
\hline 7 & 1.8 & 12 & 61 & 16 & 50 & 29 & 31 & 405 & 560 & 292 & 13 & 1.0 \\
\hline 8 & .76 & 13 & 52 & 16 & 73 & 26 & 31 & 478 & 514 & 289 & 12 & 1.0 \\
\hline 9 & .24 & 11 & 46 & 15 & 84 & 29 & 31 & 423 & 478 & 264 & 12 & 1.0 \\
\hline 10 & .04 & 11 & 40 & 14 & 69 & 25 & 28 & 363 & 479 & 237 & 21 & .99 \\
\hline 11 & .00 & 12 & 38 & 14 & 55 & 23 & 26 & 395 & 555 & 232 & 35 & .99 \\
\hline 12 & .00 & 12 & 38 & 13 & 49 & 21 & 28 & 549 & 667 & 255 & 38 & .98 \\
\hline 13 & .00 & 12 & 38 & 13 & 40 & 22 & 41 & 704 & 751 & 254 & 29 & .98 \\
\hline 14 & .00 & 13 & 41 & 14 & 34 & 26 & 80 & 540 & 845 & 234 & 19 & .97 \\
\hline 15 & .00 & 17 & 38 & 19 & 29 & 30 & 129 & 379 & 924 & 213 & 14 & .97 \\
\hline 16 & .00 & 22 & 37 & 56 & 28 & 29 & 147 & 329 & 944 & 185 & 11 & .96 \\
\hline 17 & .00 & 29 & 40 & 77 & 44 & 30 & 183 & 402 & 895 & 150 & 8.2 & .96 \\
\hline 18 & .00 & 30 & 45 & 160 & 53 & 38 & 240 & 557 & 842 & 120 & 6.1 & .96 \\
\hline 19 & .00 & 26 & 45 & 190 & 45 & 51 & 302 & 598 & 812 & 96 & 5.0 & .95 \\
\hline 20 & .00 & 22 & 43 & 142 & 35 & 57 & 354 & 639 & 721 & 80 & 4.2 & .95 \\
\hline 21 & .00 & 18 & 37 & 100 & 37 & 48 & 368 & 645 & 695 & 45 & 3.7 & .94 \\
\hline 22 & .00 & 21 & 33 & 69 & 31 & 40 & 321 & 782 & 707 & .76 & 3.4 & .94 \\
\hline 23 & .00 & 55 & 29 & 73 & 28 & 37 & 232 & 941 & 784 & .80 & 3.8 & .93 \\
\hline 24 & .00 & 186 & 26 & 60 & 26 & 38 & 164 & 974 & 827 & .82 & 4.1 & .93 \\
\hline 25 & .00 & 133 & 24 & 48 & 29 & 43 & 194 & 982 & 774 & .85 & 4.4 & .92 \\
\hline 26 & .00 & 75 & 22 & 45 & 27 & 59 & 319 & 985 & 577 & .91 & 4.2 & .92 \\
\hline 27 & .00 & 50 & 21 & 40 & 25 & 94 & 361 & 975 & 447 & 1.0 & 3.6 & .91 \\
\hline 28 & .00 & 41 & 20 & 35 & 28 & 101 & 275 & 964 & 407 & 16 & 2.9 & .91 \\
\hline 29 & .00 & 39 & 19 & 32 & --- & 82 & 190 & 927 & 439 & 33 & 2.4 & .90 \\
\hline 30 & .00 & 260 & 19 & 30 & --- & 67 & 139 & 758 & 472 & 33 & 1.8 & .90 \\
\hline 31 & .00 & --- & 19 & 29 & --- & 60 & --- & 868 & --- & 30 & 1.4 & --- \\
\hline TOTAL & 222.94 & 1265 & 1999 & 1427 & 1089 & 1408 & 4439 & 17899 & 19406 & 5745.14 & 382.2 & 28.96 \\
\hline MEAN & 7.19 & 42.2 & 64.5 & 46.0 & 38.9 & 45.4 & 148 & 577 & 647 & 185 & 12.3 & .97 \\
\hline MAX & 129 & 260 & 375 & 190 & 84 & 101 & 368 & 985 & 944 & 540 & 38 & 1.1 \\
\hline MIN & .00 & 11 & 19 & 13 & 25 & 21 & 26 & 151 & 247 & .76 & 1.4 & .90 \\
\hline $\mathrm{AC}-\mathrm{FT}$ & 442 & 2510 & 3970 & 2830 & 2160 & 2790 & 8800 & 35500 & 38490 & 11400 & 758 & 57 \\
\hline
\end{tabular}

STATISTICS OF MONTHLY MEAN DATA FOR WATER YEARS 1964 - 1999, BY WATER YEAR (WY)

$\begin{array}{lrrrrrrrrr}\text { MEAN } & 21.0 & 66.6 & 63.2 & 68.5 & 58.1 & 87.9 & 197 & 459 & 409 \\ \text { MAX } & 182 & 405 & 264 & 297 & 254 & 239 & 356 & 861 & 994 \\ \text { (WY) } & 1983 & 1984 & 1965 & 1970 & 1986 & 1989 & 1989 & 1969 & 1983 \\ \text { MIN } & .000 & .000 & .000 & .25 & 5.46 & 19.1 & 36.8 & 145 & 31.8 \\ \text { (WY) } & 1964 & 1964 & 1977 & 1991 & 1991 & 1977 & 1967 & 1977 & 1976\end{array}$

$\begin{array}{rrr}141 & 20.7 & 13.1 \\ 643 & 197 & 116 \\ 1995 & 1983 & 1982 \\ .97 & .000 & .000 \\ 1987 & 1964 & 1964\end{array}$

SUMMARY STATISTICS

ANNUAL TOTAL

ANNUAL MEAN

HIGHEST ANNUAL MEAN

LOWEST ANNUAL MEAN

HIGHEST DAILY MEAN

LOWEST DAILY MEAN

ANNUAL SEVEN-DAY MINIMUM

ANNUAL RUNOFF (AC-FT)

10 PERCENT EXCEEDS

50 PERCENT EXCEEDS

90 PERCENT EXCEEDS
FOR 1998 CALENDAR YEAR

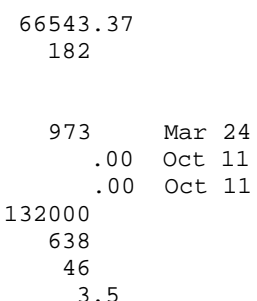

FOR 1999 WATER YEAR

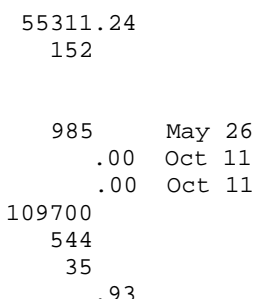

WATER YEARS 1964 - 1999

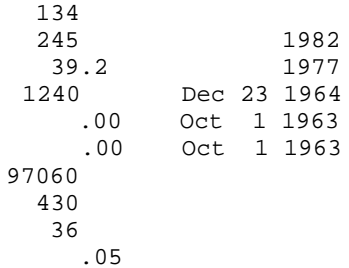


11428400 LITTLE RUBICON RIVER BELOW BUCK ISLAND DAM, NEAR MEEKS BAY, CA

LOCATION.-Lat 3900'18", long 120¹5'19", in SW 1/4 NW 1/4 sec.6, T.13 N., R.16 E., El Dorado County, Hydrologic Unit 18020128, Eldorado National Forest, at outlet structure on Buck Island Diversion Dam, 7.4 mi southwest of Meeks Bay.

DRAINAGE AREA.- $6.00 \mathrm{mi}^{2}$.

PERIOD OF RECORD._-October 1990 to current year (low-flow records only). Unpublished records for water years $1964-90$ available in files of the U.S. Geological Survey.

GAGE.-Water-stage recorder and V-notch sharp-crested weir. Elevation of gage is 6,420 ft above sea level, from topographic map. Aug. 14, 1964, to Oct. 4, 1973, nonrecording gage at site $60 \mathrm{ft}$ downstream at different datum. Nonrecording gage at present site Oct. 4, 1973, to Aug. 26, 1986, at different datum and Aug. 27, 1986, to Sept. 30, 1990, at same datum.

REMARKS.-No records computed above $2 \mathrm{ft}^{3} / \mathrm{s}$. Flow regulated by Buck Island Reservoir. Flow over the spillway bypasses this station. Most of the water is diverted at Buck Island Reservoir via Buck-Loon Tunnel (station 11428300) to Loon Lake (station 11429350). Buck Island Lake receives water from Rubicon River via Rubicon-Rockbound Tunnel (station 11427940). See schematic diagram of Middle Fork American and Rubicon River Basins.

COOPERATION.-Records were collected by Sacramento Municipal Utility District, under general supervision of the U.S. Geological Survey, in connection with a Federal Energy Regulatory Commission project.

DISCHARGE, CUBIC FEET PER SECOND, WATER YEAR OCTOBER 1998 TO SEPTEMBER 1999 DAILY MEAN VALUES

\begin{tabular}{|c|c|c|c|c|c|c|c|c|c|c|c|c|}
\hline DAY & $\mathrm{OCT}$ & NOV & $\mathrm{DEC}$ & JAN & FEB & MAR & APR & MAY & JUN & JUL & AUG & SEP \\
\hline 1 & 1.2 & 1.2 & 1.5 & 1.2 & 1.3 & 1.4 & 1.2 & 1.3 & 1.6 & 1.5 & 1.2 & 1.1 \\
\hline 2 & 1.3 & 1.2 & 1.3 & 1.2 & 1.3 & 1.4 & 1.2 & 1.4 & 1.6 & 1.5 & 1.2 & 1.1 \\
\hline 3 & 1.2 & 1.2 & 1.3 & 1.2 & 1.3 & 1.4 & 1.2 & 1.4 & 1.5 & 1.5 & 1.2 & 1.1 \\
\hline 4 & 1.2 & 1.2 & 1.3 & 1.2 & 1.3 & 1.3 & 1.2 & 1.3 & 1.4 & 1.4 & 1.2 & 1.1 \\
\hline 5 & e1.2 & 1.2 & 1.3 & 1.2 & 1.3 & 1.3 & 1.2 & 1.3 & 1.3 & 1.4 & 1.2 & 1.1 \\
\hline 6 & e1.2 & 1.2 & 1.3 & 1.2 & 1.3 & 1.3 & 1.2 & 1.3 & 1.5 & 1.3 & 1.2 & 1.1 \\
\hline 7 & 1.2 & 1.2 & 1.3 & 1.2 & 1.3 & 1.3 & 1.2 & 1.4 & 1.5 & 1.3 & 1.2 & 1.0 \\
\hline 8 & 1.2 & 1.2 & 1.3 & 1.2 & 1.3 & 1.3 & 1.2 & 1.5 & 1.5 & 1.3 & 1.2 & 1.1 \\
\hline 9 & 1.2 & 1.2 & 1.3 & 1.2 & 1.3 & 1.3 & 1.2 & 1.4 & 1.5 & 1.3 & 1.2 & 1.1 \\
\hline 10 & 1.2 & 1.2 & 1.2 & 1.2 & 1.3 & 1.3 & 1.2 & 1.4 & 1.5 & 1.3 & 1.2 & 1.1 \\
\hline 11 & 1.2 & 1.2 & 1.2 & 1.2 & 1.3 & 1.3 & 1.2 & 1.4 & 1.5 & 1.3 & 1.3 & 1.1 \\
\hline 12 & 1.2 & 1.2 & 1.2 & 1.2 & 1.4 & 1.3 & 1.2 & 1.5 & 1.6 & 1.3 & 1.3 & 1.1 \\
\hline 13 & 1.2 & 1.2 & 1.2 & 1.2 & 1.4 & 1.3 & 1.2 & 1.6 & 1.6 & 1.3 & 1.3 & 1.1 \\
\hline 14 & 1.2 & 1.2 & 1.2 & 1.2 & 1.3 & 1.3 & 1.2 & 1.5 & 1.6 & 1.3 & 1.4 & 1.1 \\
\hline 15 & 1.2 & 1.2 & 1.2 & 1.2 & 1.4 & 1.3 & 1.3 & 1.4 & --- & 1.3 & 1.4 & 1.1 \\
\hline 16 & 1.2 & 1.2 & 1.2 & 1.3 & 1.4 & 1.3 & 1.3 & 1.4 & --- & 1.3 & 1.5 & 1.1 \\
\hline 17 & 1.1 & 1.2 & 1.2 & 1.3 & 1.4 & 1.3 & 1.3 & 1.4 & --- & 1.2 & 1.6 & 1.1 \\
\hline 18 & 1.1 & 1.2 & 1.2 & 1.3 & 1.4 & 1.3 & 1.3 & 1.5 & 1.6 & 1.2 & 1.6 & 1.1 \\
\hline 19 & 1.1 & 1.2 & 1.2 & 1.3 & 1.4 & 1.2 & 1.4 & 1.5 & 1.6 & 1.2 & 1.6 & 1.1 \\
\hline 20 & 1.1 & 1.2 & 1.2 & 1.3 & 1.4 & 1.2 & 1.4 & 1.5 & 1.6 & 1.2 & 1.3 & 1.1 \\
\hline 21 & 1.1 & 1.2 & 1.2 & 1.3 & 1.4 & 1.2 & 1.4 & 1.5 & 1.6 & 1.2 & 1.2 & 1.1 \\
\hline 22 & 1.1 & 1.2 & 1.2 & 1.3 & 1.4 & 1.2 & 1.4 & 1.5 & 1.6 & 1.2 & 1.2 & 1.1 \\
\hline 23 & 1.1 & 1.3 & 1.2 & 1.3 & 1.4 & 1.2 & 1.3 & --- & 1.6 & 1.2 & 1.2 & 1.1 \\
\hline 24 & 1.1 & 1.3 & 1.3 & 1.3 & 1.4 & 1.2 & 1.3 & --- & 1.6 & 1.2 & 1.1 & 1.1 \\
\hline 25 & 1.1 & 1.3 & 1.3 & 1.3 & 1.4 & 1.2 & 1.3 & --- & 1.6 & 1.2 & 1.2 & 1.1 \\
\hline 26 & 1.1 & 1.3 & 1.3 & 1.3 & 1.4 & 1.2 & 1.4 & --- & 1.5 & 1.3 & 1.2 & 1.1 \\
\hline 27 & 1.1 & 1.3 & 1.3 & 1.3 & 1.4 & 1.3 & 1.4 & --- & 1.5 & 1.2 & 1.2 & 1.1 \\
\hline 28 & 1.1 & 1.2 & 1.3 & 1.3 & 1.5 & 1.3 & 1.3 & --- & 1.4 & 1.1 & 1.2 & 1.1 \\
\hline 29 & 1.1 & 1.2 & 1.3 & 1.3 & --- & 1.3 & 1.3 & --- & 1.4 & 1.2 & 1.2 & 1.2 \\
\hline 30 & 1.1 & 1.4 & 1.3 & 1.3 & --- & 1.2 & 1.3 & --- & 1.5 & 1.2 & 1.2 & 1.3 \\
\hline 31 & 1.1 & --- & 1.3 & 1.3 & --- & 1.2 & --- & --- & --- & 1.2 & 1.1 & --- \\
\hline TOTAL & 35.8 & 36.7 & 39.1 & 38.8 & 38.1 & 39.6 & 38.2 & --- & --- & 39.6 & 39.3 & 33.2 \\
\hline MEAN & 1.15 & 1.22 & 1.26 & 1.25 & 1.36 & 1.28 & 1.27 & --- & --- & 1.28 & 1.27 & 1.11 \\
\hline MAX & 1.3 & 1.4 & 1.5 & 1.3 & 1.5 & 1.4 & 1.4 & --- & --- & 1.5 & 1.6 & 1.3 \\
\hline MIN & 1.1 & 1.2 & 1.2 & 1.2 & 1.3 & 1.2 & 1.2 & --- & --- & 1.1 & 1.1 & 1.0 \\
\hline $\mathrm{AC}-\mathrm{FT}$ & 71 & 73 & 78 & 77 & 76 & 79 & 76 & --- & --- & 79 & 78 & 66 \\
\hline
\end{tabular}


LOCATION.-Lat $39^{\circ} 03^{\prime} 54^{\prime \prime}$, long 120²4'50", in SE 1/4 NW 1/4 sec.16, T.14 N., R.14 E., Placer County, Hydrologic Unit 18020128, Eldorado National Forest, on right bank 0.3 mi upstream from Hell Hole Dam on Rubicon River and 15.6 mi west of Meeks Bay.

DRAINAGE AREA.-114 $\mathrm{mi}^{2}$.

PERIOD OF RECORD.-December 1965 to current year.

GAGE.-Water-stage recorder. Datum of gage is sea level (levels by Placer County Water Agency).

REMARKS.--Reservoir is formed by rockfill dam with earth core. Storage began Dec. 6, 1965. Usable capacity, 207,342 acre-ft between elevations $4,287.65 \mathrm{ft}$, invert of river outlet, and 4,630.0 ft, crest of ogee spillway. Dead storage 248 acre- $\mathrm{ft}$. Reservoir is used to store water for hydroelectric power. Water is diverted into reservoir from French Meadows Reservoir (11427400) on the Middle Fork American River through French Meadows Powerplant (station 11427200). Water is diverted out of reservoir to the Middle Fork American River though Middle Fork Powerplant. See schematic diagram of Middle Fork American and Rubicon River Basins.

COOPERATION.-Records provided by Placer County Water Agency, under general supervision of the U.S. Geological Survey, in connection with a Federal Energy Regulatory Commission project.

EXTREMES FOR PERIOD OF RECORD.-Maximum contents, 217,400 acre-ft, Jan. 2, 1997, elevation, 4,637.7 ft; minimum since reservoir first filled, 37,499 acre-ft, Mar. 23, 1973, elevation, 4,428.28 ft.

EXTREMES FOR CURRENT YEAR.-Maximum contents, 204,600 acre-ft, June 18, elevation, 4,627.6 ft; minimum, 98,200 acre-ft, Apr. 12, elevation, $4,520.0 \mathrm{ft}$.

Capacity table (elevation, in feet, and contents, in acre-feet)

(Based on survey by Placer County Water Agency in 1966)

$\begin{array}{rrrrrr}4,340 & 5,220 & 4,400 & 24,200 & 4,550 & 122,700 \\ 4,360 & 9,840 & 4,450 & 49,600 & 4,600 & 171,900 \\ 4,380 & 16,200 & 4,500 & 83,000 & 4,650 & 233,400\end{array}$

RESERVOIR STORAGE (ACRE-FEET), WATER YEAR OCTOBER 1998 TO SEPTEMBER 1999 DAILY OBSERVATION AT 2400 HOURS

\begin{tabular}{|c|c|c|c|c|c|c|c|c|c|c|c|c|}
\hline DAY & $\mathrm{OCT}$ & NOV & $\mathrm{DEC}$ & JAN & FEB & MAR & APR & MAY & JUN & JUL & AUG & SEP \\
\hline 1 & 143100 & 155600 & 144200 & 114400 & 108700 & 101800 & 99900 & 118700 & 188800 & 200400 & 178100 & 149300 \\
\hline 2 & 143500 & 155400 & 143800 & 113200 & 108300 & 101800 & 99700 & 120200 & 191500 & 199900 & 177200 & 148200 \\
\hline 3 & 144100 & 155100 & 144200 & 112200 & 107800 & 102900 & 99700 & 121200 & 193000 & 198900 & 176400 & 146700 \\
\hline 4 & 144600 & 155100 & 144000 & 111200 & 107500 & 103300 & 99700 & 122000 & 193900 & 198400 & 175800 & 145600 \\
\hline 5 & 145000 & 155000 & 143800 & 110100 & 107100 & 103300 & 99500 & 122700 & 195100 & 197800 & 175300 & 144700 \\
\hline 6 & 145400 & 154900 & 143500 & 109100 & 106500 & 103400 & 99500 & 124300 & 196800 & 196800 & 174500 & 143800 \\
\hline 7 & 145800 & 154800 & 143100 & 108000 & 108100 & 103600 & 99400 & 126300 & 198400 & 196000 & 173700 & 142700 \\
\hline 8 & 146200 & 154500 & 142600 & 107000 & 109200 & 103300 & 99300 & 128000 & 199400 & 195100 & 172900 & 141500 \\
\hline 9 & 146700 & 154200 & 142100 & 106100 & 111300 & 102900 & 99100 & 129700 & 200500 & 194300 & 172100 & 140700 \\
\hline 10 & 147100 & 153900 & 141500 & 105500 & 111800 & 102600 & 98900 & 131000 & 201400 & 193600 & 171200 & 139600 \\
\hline 11 & 147700 & 153700 & 141000 & 104600 & 111400 & 102200 & 98500 & 132900 & 202400 & 192600 & 170200 & 138300 \\
\hline 12 & 148200 & 153400 & 140000 & 103800 & 111000 & 101700 & 98200 & 135300 & 202700 & 191600 & 169300 & 137700 \\
\hline 13 & 148800 & 153200 & 139200 & 103100 & 110500 & 101300 & 98400 & 137400 & 203100 & 191000 & 168500 & 136500 \\
\hline 14 & 149200 & 152700 & 138000 & 102500 & 110000 & 101000 & 98700 & 138900 & 203700 & 190400 & 168000 & 135400 \\
\hline 15 & 149600 & 152100 & 136800 & 101900 & 109300 & 100600 & 99100 & 139900 & 204000 & 189700 & 167200 & 134400 \\
\hline 16 & 150200 & 151600 & 135700 & 101700 & 108600 & 100200 & 99600 & 141100 & 204200 & 189000 & 166300 & 133300 \\
\hline 17 & 150500 & 150900 & 134700 & 101900 & 109400 & 100000 & 100600 & 142900 & 204500 & 188400 & 165400 & 132300 \\
\hline 18 & 151000 & 150100 & 133400 & 103900 & 109200 & 99900 & 102000 & 144800 & 204600 & 188100 & 164300 & 131400 \\
\hline 19 & 151600 & 149300 & 132300 & 105400 & 108600 & 99900 & 103400 & 146900 & 204300 & 187400 & 163300 & 131300 \\
\hline 20 & 152100 & 148400 & 130700 & 108000 & 108000 & 99800 & 105000 & 149000 & 204100 & 186900 & 162200 & 131600 \\
\hline 21 & 152600 & 147600 & 129100 & 108900 & 107600 & 99900 & 106900 & 151200 & 204000 & 186100 & 161300 & 132000 \\
\hline 22 & 153000 & 146900 & 127400 & 109100 & 106700 & 99600 & 107500 & 154000 & 204100 & 185500 & 160200 & 132400 \\
\hline 23 & 153500 & 147000 & 125700 & 110300 & 105800 & 99500 & 109200 & 157200 & 204100 & 184900 & 159200 & 133000 \\
\hline 24 & 154300 & 146600 & 124100 & 110700 & 104700 & 99200 & 110300 & 160900 & 204100 & 184300 & 158200 & 133200 \\
\hline 25 & 154800 & 145900 & 122900 & 110900 & 104000 & 99000 & 111800 & 164400 & 203700 & 183800 & 157100 & 133700 \\
\hline 26 & 155400 & 145100 & 121900 & 111000 & 102700 & 99200 & 113900 & 169000 & 203200 & 183000 & 156000 & 134100 \\
\hline 27 & 155500 & 144300 & 120800 & 110800 & 102000 & 99700 & 115500 & 173000 & 202600 & 182500 & 155000 & 134500 \\
\hline 28 & 156300 & 143500 & 119600 & 110400 & 101600 & 100200 & 116300 & 176900 & 202000 & 181600 & 153800 & 134900 \\
\hline 29 & 156500 & 143200 & 118300 & 110000 & --- & 100400 & 116700 & 180100 & 201400 & 180700 & 152800 & 135300 \\
\hline 30 & 156400 & 144400 & 116700 & 109500 & --- & 100200 & 117500 & 183000 & 200800 & 179700 & 151700 & 135600 \\
\hline 31 & 155900 & --- & 115200 & 109100 & --- & 100000 & --- & 186100 & --- & 178900 & 150500 & -- \\
\hline MAX & 156500 & 155600 & 144200 & 114400 & 111800 & 103600 & 117500 & 186100 & 204600 & 200400 & 178100 & 149300 \\
\hline MIN & 143100 & 143200 & 115200 & 101700 & 101600 & 99000 & 98200 & 118700 & 188800 & 178900 & 150500 & 131300 \\
\hline$a$ & 4585.3 & 4573.9 & 4541.1 & 4533.7 & 4524.3 & 4522.3 & 4543.8 & 4612.3 & 4624.5 & 4606.2 & 4580.0 & 4564.6 \\
\hline $\mathrm{b}$ & +13200 & -11500 & -29200 & -6100 & -7500 & -1600 & +17500 & +68600 & +14700 & -21900 & -28400 & -14900 \\
\hline
\end{tabular}

CAL YR 1998 b +60700

WTY YR 1999 b $\quad-7100$

a Elevation, in feet, at end of month.

b Change in contents, in acre-feet. 
LOCATION.-Lat $39^{\circ} 03^{\prime} 24^{\prime \prime}$, long $120^{\circ} 24^{\prime} 25^{\prime \prime}$, in NE 1/4 NE 1/4 sec.21, T.14 N., R.14 E., Placer County, Hydrologic Unit 18020128, Eldorado National Forest, on right bank $600 \mathrm{ft}$ downstream from outlet of dam, and $15.3 \mathrm{mi}$ west of Meeks Bay.

DRAINAGE AREA.-114 $\mathrm{mi}^{2}$.

PERIOD OF RECORD.-November 1965 to current year.

GAGE.-Water-stage recorder and V-notch sharp-crested weir. Datum of gage is 4,231.52 ft above sea level (levels by Placer County Water Agency).

REMARKS.-Flow completely regulated by Hell Hole Reservoir (station 11428700) $600 \mathrm{ft}$ upstream from station. During years when Hell Hole Dam spills, records include flow which bypasses the station. Transbasin diversions upstream from station through Buck-Loon Tunnel (station 11428300) to Loon Lake Reservoir (station 11429350); from Middle Fork American River Basin through tunnel from French Meadows Reservoir (station 11427400) to Hell Hole Reservoir; from Hell Hole Reservoir through tunnel to Middle Fork Powerplant (station 11428600). Diversion began Sept. 8, 1966. See schematic diagram of Middle Fork American and Rubicon River Basins.

COOPERATION.-Records provided by Placer County Water Agency, under general supervision of the U.S. Geological Survey, in connection with a Federal Energy Regulatory Commission project.

EXTREMES FOR PERIOD OF RECORD.-Maximum discharge, 28,800 ft³/s, Jan. 2, 1997, including flow over spillway; no flow Aug. 25 to Sept. 11, 1966.

DISCHARGE, CUBIC FEET PER SECOND, WATER YEAR OCTOBER 1998 TO SEPTEMBER 1999 DAILY MEAN VALUES

\begin{tabular}{|c|c|c|c|c|c|c|c|c|c|c|c|c|}
\hline DAY & $\mathrm{OCT}$ & NOV & $\mathrm{DEC}$ & JAN & FEB & MAR & $\mathrm{APR}$ & MAY & JUN & JUL & AUG & SEP \\
\hline 1 & 34 & 21 & 25 & 11 & 12 & 20 & 19 & 12 & 23 & 23 & 23 & 23 \\
\hline 2 & 34 & 21 & 24 & 12 & 12 & 15 & 13 & 13 & 23 & 23 & 23 & 23 \\
\hline 3 & 34 & 22 & 26 & 12 & 12 & 19 & 13 & 13 & 22 & 23 & 23 & 23 \\
\hline 4 & 34 & 23 & 25 & 12 & 12 & 16 & 13 & 13 & 23 & 23 & 23 & 23 \\
\hline 5 & 34 & 23 & 23 & 12 & 12 & 14 & 13 & 12 & 23 & 23 & 23 & 23 \\
\hline 6 & 34 & 23 & 22 & 12 & 12 & 14 & 13 & 13 & 23 & 23 & 23 & 23 \\
\hline 7 & 34 & 23 & 22 & 12 & 29 & 13 & 13 & 13 & 23 & 23 & 23 & 23 \\
\hline 8 & 34 & 23 & 22 & 12 & 25 & 13 & 13 & 13 & 22 & 23 & 23 & 23 \\
\hline 9 & 34 & 23 & 22 & 12 & 30 & 13 & 13 & 13 & 23 & 23 & 23 & 23 \\
\hline 10 & 34 & 23 & 22 & 12 & 16 & 13 & 13 & 12 & 23 & 23 & 23 & 23 \\
\hline 11 & 34 & 23 & 22 & 12 & 15 & 13 & 13 & 13 & 23 & 23 & 23 & 23 \\
\hline 12 & 29 & 23 & 22 & 12 & 15 & 13 & 15 & 14 & 23 & 23 & 23 & 23 \\
\hline 13 & 21 & 23 & 22 & 12 & 14 & 13 & 15 & 14 & 23 & 23 & 23 & 23 \\
\hline 14 & 21 & 23 & 22 & 12 & 14 & 13 & 16 & 17 & 23 & 23 & 23 & 23 \\
\hline 15 & 21 & 23 & 16 & 12 & 13 & 13 & 15 & 26 & 23 & 23 & 23 & 23 \\
\hline 16 & 21 & 23 & 13 & 13 & 16 & 12 & 15 & 26 & 23 & 23 & 23 & 22 \\
\hline 17 & 21 & 23 & 12 & 13 & 24 & 12 & 14 & 26 & 23 & 23 & 23 & 29 \\
\hline 18 & 21 & 23 & 11 & 20 & 16 & 13 & 15 & 26 & 23 & 23 & 23 & 31 \\
\hline 19 & 21 & 23 & 12 & 23 & 15 & 13 & 17 & 24 & 23 & 23 & 23 & 31 \\
\hline 20 & 21 & 23 & 12 & 27 & 14 & 13 & 17 & 23 & 23 & 23 & 23 & 29 \\
\hline 21 & 21 & 23 & 12 & 17 & 14 & 13 & 16 & 24 & 23 & 23 & 23 & 23 \\
\hline 22 & 21 & 24 & 12 & 15 & 13 & 13 & 15 & 24 & 23 & 23 & 23 & 23 \\
\hline 23 & 21 & 25 & 12 & 21 & 14 & 13 & 14 & 24 & 23 & 23 & 23 & 23 \\
\hline 24 & 21 & 25 & 12 & 15 & 14 & 12 & 14 & 23 & 23 & 23 & 23 & 23 \\
\hline 25 & 21 & 24 & 12 & 15 & 14 & 12 & 14 & 23 & 23 & 23 & 23 & 23 \\
\hline 26 & 21 & 24 & 12 & 14 & 13 & 12 & 15 & 23 & 23 & 23 & 23 & 23 \\
\hline 27 & 21 & 24 & 12 & 14 & 13 & 13 & 14 & 25 & 23 & 23 & 23 & 23 \\
\hline 28 & 21 & 23 & 12 & 13 & 16 & 12 & 13 & 22 & 23 & 23 & 23 & 23 \\
\hline 29 & 21 & 25 & 11 & 13 & --- & 12 & 12 & 21 & 23 & 23 & 23 & 23 \\
\hline 30 & 21 & 27 & 11 & 13 & --- & 18 & 12 & 23 & 23 & 23 & 23 & 23 \\
\hline 31 & 21 & --- & 11 & 12 & --- & 21 & --- & 23 & --- & 23 & 23 & --- \\
\hline TOTAL & 802 & 699 & 526 & 437 & 439 & 429 & 427 & 591 & 688 & 713 & 713 & 717 \\
\hline MEAN & 25.9 & 23.3 & 17.0 & 14.1 & 15.7 & 13.8 & 14.2 & 19.1 & 22.9 & 23.0 & 23.0 & 23.9 \\
\hline MAX & 34 & 27 & 26 & 27 & 30 & 21 & 19 & 26 & 23 & 23 & 23 & 31 \\
\hline MIN & 21 & 21 & 11 & 11 & 12 & 12 & 12 & 12 & 22 & 23 & 23 & 22 \\
\hline $\mathrm{AC}-\mathrm{FT}$ & 1590 & 1390 & 1040 & 867 & 871 & 851 & 847 & 1170 & 1360 & 1410 & 1410 & 1420 \\
\hline$a$ & 3170 & 28270 & 37940 & 24910 & 29950 & 45410 & 38610 & 19000 & 45450 & 41930 & 42860 & 25480 \\
\hline
\end{tabular}

a Diversion, in acre-feet, from Hell Hole Reservoir through Middle Fork Powerplant, provided by Placer County Water Agency. 


\section{RUBICON RIVER BELOW HELL HOLE DAM, NEAR MEEKS BAY, CA—Continued}

STATISTICS OF MONTHLY MEAN DATA FOR WATER YEARS 1966 - 1999, BY WATER YEAR (WY)

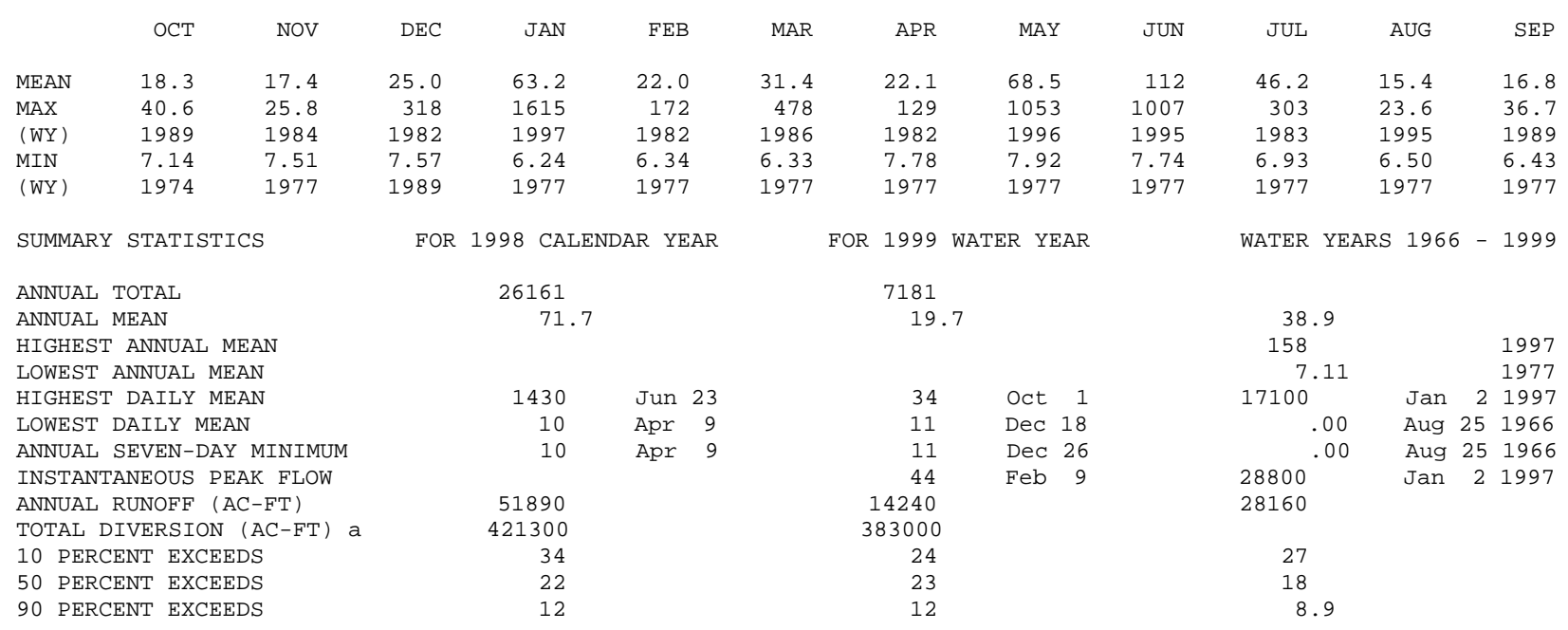

a Diversion, in acre-feet, from Hell Hole Reservoir through Middle Fork Powerplant, provided by Placer County Water Agency. 
LOCATION.-Lat 3858'59", long 120¹9'22", in SE 1/4 SW 1/4 sec.8, T.13 N., R.15 E., El Dorado County, Hydrologic Unit 18020128, Eldorado National Forest, in powerplant intake structure, $1.6 \mathrm{mi}$ southwest of right bank end of Loon Lake Dam on Gerle Creek, and 10 mi southwest of Meeks Bay.

DRAINAGE AREA.-7.96 $\mathrm{mi}^{2}$.

PERIOD OF RECORD.-December 1963 to current year.

CHEMICAL ANALYSES: June to September 1996.

REVISED RECORDS.-WDR CA-76-4: Drainage area.

GAGE.-Water-stage recorder. Datum of gage is sea level (levels by Sacramento Municipal Utility District). Prior to Sept. 23, 1975, at site 1.6 mi northeast on right bank end of Loon Lake Dam at same datum.

REMARKS.--Reservoir is formed by an earthfill dam completed Dec. 27, 1963; storage began Dec. 5, 1963. Prior to September 1962, reservoir was formed by granite-block dam built in 1884, capacity, 8,000 acre-ft. Usable capacity, 73,868 acre-ft, between elevations $6,325 \mathrm{ft}$, invert of fishwater release valve, and 6,410 ft, crest of spillway. Dead storage, 2,300 acre-ft. Lake receives water from Rubicon River via RubiconRockbound Tunnel to Buck Island Lake and from Buck Island Lake to Loon Lake via Buck-Loon Tunnel (stations 11427940, 11428300). Records, including extremes, represent total contents. See schematic diagram of Middle Fork American and Rubicon River Basins.

COOPERATION.-Records were collected by Sacramento Municipal Utility District, under general supervision of the U.S. Geological Survey, in connection with a Federal Energy Regulatory Commission project. Contents not rounded to U.S. Geological Survey standards.

EXTREMES FOR PERIOD OF RECORD.-Maximum contents, 77,700 acre-ft, June 6, 1969, elevation, 6,411.1 ft; minimum since reservoir first filled, 3,262 acre-ft, Nov. 8, 9, 1988, elevation, 6,328.70 ft.

EXTREMES FOR CURRENT YEAR.-Maximum contents, 75,288 acre-ft, July 11, elevation, 6,409.37 ft; minimum, 14,016 acre-ft, Apr. 1, elevation, $6352.40 \mathrm{ft}$

Capacity table (elevation, in feet, and contents, in acre-feet)

(Based on table provided by Sacramento Municipal Utility District recomputed October 1991)

$\begin{array}{rrrr}6,330 & 3,478 & 6,370 & 28,323 \\ 6,340 & 7,116 & 6,390 & 50,058 \\ 6,350 & 12,469 & 6,412 & 78,983\end{array}$

RESERVOIR STORAGE (ACRE-FEET), WATER YEAR OCTOBER 1998 TO SEPTEMBER 1999

DAILY OBSERVATION AT 2400 HOURS

\begin{tabular}{|c|c|c|c|c|c|c|c|c|c|c|c|c|}
\hline DAY & $\mathrm{OCT}$ & NOV & $\mathrm{DEC}$ & JAN & FEB & MAR & APR & MAY & JUN & JUL & AUG & SEP \\
\hline 1 & 46274 & 45236 & 48200 & 40249 & 39784 & 17096 & 14016 & 19729 & 47476 & 69587 & 70171 & 63280 \\
\hline 2 & 46497 & 45282 & 48366 & 40016 & 39497 & 17052 & 14116 & 19993 & 49311 & 70647 & 69696 & 63031 \\
\hline 3 & 46591 & 45305 & 48736 & 39739 & 39222 & 17111 & 14284 & 19937 & 50408 & 71685 & 69262 & 62652 \\
\hline 4 & 46591 & 45329 & 48832 & 39475 & 38817 & 16912 & 14337 & 20146 & 50990 & 72521 & 69181 & 62378 \\
\hline 5 & 46532 & 45340 & 48736 & 39123 & 37957 & 16670 & 14540 & 19737 & 51550 & 73113 & 69168 & 62013 \\
\hline 6 & 46485 & 45329 & 48390 & 38795 & 37471 & 16509 & 14663 & 19713 & 52530 & 73665 & 69154 & 61557 \\
\hline 7 & 46450 & 45398 & 48200 & 38467 & 37180 & 16321 & 14731 & 20536 & 53664 & 74219 & 69141 & 61129 \\
\hline 8 & 46403 & 45410 & 47855 & 38055 & 37213 & 16126 & 14909 & 21127 & 54521 & 74690 & 69127 & 60780 \\
\hline 9 & 46368 & 45410 & 47523 & 37968 & 37535 & 15990 & 14992 & 21810 & 55182 & 75163 & 69168 & 60418 \\
\hline 10 & 46345 & 45201 & 47298 & 37697 & 36966 & 15649 & 15068 & 22504 & 55671 & 75219 & 69181 & 60018 \\
\hline 11 & 46298 & 45224 & 47050 & 37492 & 36071 & 15417 & 15138 & 22993 & 56501 & 75288 & 69181 & 59967 \\
\hline 12 & 46263 & 45178 & 46556 & 37320 & 34902 & 15242 & 15228 & 23934 & 57945 & 74843 & 69181 & 59542 \\
\hline 13 & 46227 & 45189 & 46579 & 37191 & 34028 & 15047 & 15417 & 25099 & 59542 & 74885 & 69195 & 59132 \\
\hline 14 & 46204 & 45108 & 46216 & 36891 & 32919 & 14951 & 15727 & 25760 & 61155 & 75219 & 69181 & 58722 \\
\hline 15 & 46169 & 45015 & 45982 & 36955 & 32018 & 14930 & 16119 & 25842 & 62900 & 75247 & 69154 & 58327 \\
\hline 16 & 46017 & 44992 & 45573 & 37213 & 30829 & 14820 & 16444 & 26162 & 64399 & 75080 & 68533 & 57919 \\
\hline 17 & 45900 & 45131 & 45247 & 37579 & 30238 & 14752 & 16853 & 26605 & 65590 & 74885 & 68344 & 57411 \\
\hline 18 & 45876 & 45154 & 44922 & 38598 & 29428 & 14670 & 17529 & 27378 & 66575 & 74663 & 68277 & 57386 \\
\hline 19 & 45806 & 45178 & 44679 & 39354 & 28028 & 14711 & 18235 & 28437 & 67217 & 74788 & 67404 & 57183 \\
\hline 20 & 45690 & 45201 & 44414 & 40016 & 26902 & 14861 & 18962 & 29448 & 67833 & 74565 & 67150 & 56804 \\
\hline 21 & 45655 & 45189 & 43769 & 40360 & 25860 & 14889 & 19689 & 30356 & 68533 & 74233 & 66735 & 56476 \\
\hline 22 & 45620 & 45236 & 42969 & 40626 & 24598 & 14786 & 20114 & 31987 & 68722 & 73734 & 66308 & 55721 \\
\hline 23 & 45503 & 45596 & 42730 & 40961 & 23714 & 14670 & 20195 & 33729 & 69289 & 73251 & 65935 & 55345 \\
\hline 24 & 45515 & 45935 & 42469 & 41051 & 22573 & 14520 & 20114 & 35447 & 70334 & 72645 & 65391 & 55282 \\
\hline 25 & 45468 & 46157 & 42311 & 40604 & 21267 & 14445 & 20381 & 37180 & 70429 & 71986 & 65033 & 54908 \\
\hline 26 & 45433 & 46309 & 41690 & 40649 & 19921 & 14324 & 20797 & 38882 & 70293 & 71548 & 64768 & 54671 \\
\hline 27 & 45340 & 46403 & 41454 & 40582 & 18458 & 14561 & 21102 & 40404 & 69913 & 71110 & 64649 & 54633 \\
\hline 28 & 45271 & 46497 & 41353 & 40382 & 17439 & 14642 & 21143 & 42254 & 69370 & 71097 & 64399 & 54297 \\
\hline 29 & 45294 & 46791 & 40961 & 40182 & --- & 14351 & 20683 & 43655 & 68857 & 71110 & 64082 & 53838 \\
\hline 30 & 45201 & 47819 & 40682 & 40149 & --- & 14324 & 20090 & 44691 & 68735 & 70551 & 63569 & 53256 \\
\hline 31 & 45154 & --- & 40582 & 40226 & --- & 14043 & --- & 46028 & --- & 70565 & 63333 & --- \\
\hline MAX & 46591 & 47819 & 48832 & 41051 & 39784 & 17111 & 21143 & 46028 & 70429 & 75288 & 70171 & 63280 \\
\hline MIN & 45154 & 44992 & 40582 & 36891 & 17439 & 14043 & 14016 & 19713 & 47476 & 69587 & 63333 & 53256 \\
\hline $\mathrm{a}$ & 6385.86 & 6388.13 & 6381.84 & 6381.52 & 6357.24 & 6352.44 & 6360.65 & 6386.61 & 6404.59 & 6405.94 & 6400.53 & 6392.62 \\
\hline $\mathrm{b}$ & -1144 & +2665 & -7237 & -356 & -22787 & -3396 & +6047 & +25938 & +22707 & +1830 & -7232 & -10077 \\
\hline
\end{tabular}

CAL YR 1998 MAX 75707 MIN 17267 b +13977

WTR YR 1999 MAX 75288 MIN 14016 b $\quad+6958$

a Elevation, in feet, at end of month.

b Change in contents, in acre-feet. 
LOCATION.-Lat 3900'20", long 120¹8'52", in NE 1/4 NE 1/4 sec.5, T.13 N., R.15 E., El Dorado County, Hydrologic Unit 18020128, Eldorado National Forest, on right bank $0.3 \mathrm{mi}$ downstream from Loon Lake Dam, and $11 \mathrm{mi}$ southwest of Meeks Bay.

DRAINAGE AREA.- $-8.01 \mathrm{mi}^{2}$.

PERIOD OF RECORD._-July 1910 to April 1914 (fragmentary), August 1962 to current year. Prior to August 1962, published as "near Rubicon Springs."

GAGE.-Water-stage recorder and V-notch sharp-crested weir. Elevation of gage is 6,250 ft above sea level, from topographic map. Prior to August 1962 , nonrecording gage at site $1,400 \mathrm{ft}$ upstream at different datum.

REMARKS.-Records good. Beginning in 1884, flow regulated by Loon Lake (station 11429350). Original dam was dismantled during September and October 1962 to permit construction of a new earthfill dam, which was completed Dec. 27, 1963. Loon Lake receives water from Rubicon River via Buck-Loon Tunnel (station 11428300). Since August 1971, most of the water is diverted past the station via Loon Lake Powerplant (station 11429340) and returns to Gerle Creek at Gerle Creek Dam. See schematic diagram of Middle Fork American and Rubicon River Basins.

COOPERATION.-Records were collected by Sacramento Municipal Utility District, under general supervision of the U.S. Geological Survey, in connection with a Federal Energy Regulatory Commission project.

EXTREMES FOR PERIOD OF RECORD.-Maximum discharge, 3,240 ft 3 /s, unregulated, Feb. 1, 1963, gage height, $12.65 \mathrm{ft}$, from rating curve extended above $970 \mathrm{ft}^{3} / \mathrm{s}$ on basis of slope-area measurement of peak flow; no flow Oct. 15, 1913. Maximum discharge since construction of Loon Lake Dam in 1963, 1,050 ft³/s, June 5, 1969, gage height, $9.03 \mathrm{ft}$; minimum daily, $3.6 \mathrm{ft}^{3} / \mathrm{s}$, Sept. 27, 28, Nov. 3, 1977.

DISCHARGE, CUBIC FEET PER SECOND, WATER YEAR OCTOBER 1998 TO SEPTEMBER 1999 DAILY MEAN VALUES

\begin{tabular}{|c|c|c|c|c|c|c|c|c|c|c|c|c|}
\hline DAY & $\mathrm{OCT}$ & NOV & $\mathrm{DEC}$ & JAN & FEB & MAR & APR & MAY & JUN & JUL & AUG & SEP \\
\hline 1 & 12 & e12 & 12 & 12 & 12 & 14 & 11 & 14 & 15 & 12 & 12 & 12 \\
\hline 2 & 12 & 12 & 12 & 12 & 12 & 11 & 11 & 14 & 16 & 12 & 12 & 12 \\
\hline 3 & 12 & 11 & 13 & 12 & 12 & 11 & 11 & 13 & 15 & 12 & 12 & 12 \\
\hline 4 & 12 & 11 & 12 & 12 & 12 & 11 & 11 & 13 & 15 & 12 & 12 & 12 \\
\hline 5 & 12 & 11 & 12 & 12 & 12 & 11 & 11 & 14 & 15 & 12 & 12 & 12 \\
\hline 6 & 12 & 11 & 12 & 12 & 12 & 11 & 11 & 15 & 15 & 12 & 12 & 12 \\
\hline 7 & 12 & 11 & 11 & 12 & 13 & 11 & 11 & 15 & 15 & 12 & 12 & 12 \\
\hline 8 & 12 & 11 & 11 & 12 & 12 & 11 & 11 & 15 & 15 & 12 & 12 & 12 \\
\hline 9 & 12 & 11 & 11 & 12 & 14 & 11 & 11 & 14 & 15 & 12 & 12 & 12 \\
\hline 10 & 12 & 11 & 11 & 12 & 12 & 11 & 11 & 15 & 15 & 12 & 12 & 12 \\
\hline 11 & 12 & 11 & 11 & 12 & 12 & 12 & 11 & 15 & 15 & 12 & 12 & 12 \\
\hline 12 & 12 & 11 & 11 & 12 & 12 & 11 & 12 & 16 & 15 & 12 & 12 & 12 \\
\hline 13 & 12 & 11 & 11 & 12 & 12 & 11 & 12 & 15 & 15 & 12 & 12 & 12 \\
\hline 14 & 12 & 11 & 11 & 12 & 11 & 12 & 12 & 14 & 15 & 12 & 12 & 12 \\
\hline 15 & 12 & 11 & 11 & e12 & 11 & 11 & 12 & 14 & 15 & 12 & 12 & 12 \\
\hline 16 & 12 & 11 & 11 & e12 & 11 & 12 & 13 & 15 & 16 & 13 & 12 & 12 \\
\hline 17 & 12 & 11 & 11 & e12 & 12 & 12 & 13 & 15 & 14 & 12 & 12 & 12 \\
\hline 18 & 12 & 11 & 11 & e12 & 11 & 12 & 14 & 15 & 11 & 12 & 12 & 12 \\
\hline 19 & 12 & 11 & 11 & e12 & 11 & 12 & 14 & 15 & 11 & 12 & 12 & 12 \\
\hline 20 & 12 & 11 & 11 & e12 & 11 & 12 & 14 & 15 & 11 & 12 & 12 & 12 \\
\hline 21 & 12 & 11 & 11 & 12 & 11 & 12 & 14 & 15 & 12 & 12 & 12 & 12 \\
\hline 22 & 12 & 12 & 11 & 12 & 11 & 11 & 13 & 16 & 11 & 12 & 12 & 12 \\
\hline 23 & 12 & e12 & 11 & 12 & 12 & 12 & 13 & 16 & 11 & 12 & 12 & 12 \\
\hline 24 & e12 & 11 & 11 & 12 & 12 & 12 & 14 & 16 & 12 & 12 & 12 & 12 \\
\hline 25 & 12 & 11 & 12 & 12 & 12 & 12 & 15 & 16 & 12 & 12 & 12 & 12 \\
\hline 26 & 12 & 11 & 12 & 12 & 11 & 13 & 15 & 16 & 12 & 12 & 12 & 12 \\
\hline 27 & 12 & 11 & 12 & 12 & 11 & 12 & 14 & 16 & 12 & 12 & 12 & 12 \\
\hline 28 & 12 & 11 & 12 & 12 & 12 & 12 & 13 & 15 & 12 & 12 & 12 & 12 \\
\hline 29 & 12 & e12 & 12 & 12 & --- & 12 & 13 & 15 & 12 & 12 & 12 & 12 \\
\hline 30 & 12 & e12 & 12 & 12 & --- & 12 & 14 & 15 & 12 & 12 & 12 & 11 \\
\hline 31 & 12 & --- & 12 & 12 & --- & 11 & --- & 15 & --- & 12 & 12 & --- \\
\hline TOTAL & 372 & 336 & 355 & 372 & 329 & 361 & 375 & 462 & 407 & 373 & 372 & 359 \\
\hline MEAN & 12.0 & 11.2 & 11.5 & 12.0 & 11.8 & 11.6 & 12.5 & 14.9 & 13.6 & 12.0 & 12.0 & 12.0 \\
\hline MAX & 12 & 12 & 13 & 12 & 14 & 14 & 15 & 16 & 16 & 13 & 12 & 12 \\
\hline MIN & 12 & 11 & 11 & 12 & 11 & 11 & 11 & 13 & 11 & 12 & 12 & 11 \\
\hline $\mathrm{AC}-\mathrm{FT}$ & 738 & 666 & 704 & 738 & 653 & 716 & 744 & 916 & 807 & 740 & 738 & 712 \\
\hline a & 449 & 1040 & 11370 & 5370 & 24690 & 7500 & 7060 & 19620 & 17700 & 8220 & 6680 & 8580 \\
\hline
\end{tabular}

e Estimated.

a Diversion, in acre-feet, to Loon Lake Powerplant, provided by Sacramento Municipal Utility District. 
11429500 GERLE CREEK BELOW LOON LAKE DAM, NEAR MEEKS BAY, CA—Continued

STATISTICS OF MONTHLY MEAN DATA FOR WATER YEARS 1965 - 1970, BY WATER YEAR (WY)

\begin{tabular}{|c|c|c|c|c|c|c|c|c|c|c|c|c|}
\hline MEAN & 112 & 132 & 165 & 74.7 & 103 & 192 & 133 & 63.0 & 390 & 341 & 232 & 115 \\
\hline MAX & 190 & 356 & 343 & 134 & 261 & 347 & 244 & 209 & 721 & 493 & 351 & 338 \\
\hline (WY) & 1970 & 1966 & 1966 & 1968 & 1970 & 1970 & 1967 & 1969 & 1969 & 1967 & 1969 & 1967 \\
\hline MIN & 7.53 & 7.93 & 8.95 & 8.41 & 9.13 & 9.57 & 8.75 & 10.5 & 185 & 196 & 50.8 & 8.20 \\
\hline (WY) & 1965 & 1968 & 1969 & 1965 & 1968 & 1968 & 1965 & 1968 & 1966 & 1965 & 1965 & 1970 \\
\hline
\end{tabular}

SUMMARY STATISTICS

ANNUAL MEAN

HIGHEST ANNUAL MEAN

LOWEST ANNUAL MEAN

HIGHEST DAILY MEAN

LOWEST DAILY MEAN

ANNUAL SEVEN-DAY MINIMUM

INSTANTANEOUS PEAK FLOW

INSTANTANEOUS PEAK STAGE

ANNUAL RUNOFF (AC-FT)

10 PERCENT EXCEEDS

50 PERCENT EXCEEDS

90 PERCENT EXCEEDS
WATER YEARS $1965-1970$

\begin{tabular}{|c|c|c|c|}
\hline 171 & & & \\
\hline 217 & & & 1970 \\
\hline 127 & & & 1965 \\
\hline 1030 & Jun & 5 & 1969 \\
\hline 6.0 & Dec & 2 & 1969 \\
\hline 6.4 & Dec & 10 & 1969 \\
\hline 1050 & Jun & 5 & 1969 \\
\hline 9.03 & Jun & 5 & 1969 \\
\hline 124100 & & & \\
\hline 394 & & & \\
\hline 28 & & & \\
\hline 8.1 & & & \\
\hline
\end{tabular}

STATISTICS OF MONTHLY MEAN DATA FOR WATER YEARS 1972 - 1999, BY WATER YEAR (WY)

\begin{tabular}{|c|c|c|c|c|c|c|c|c|c|c|c|c|}
\hline MEAN & 8.97 & 8.81 & 9.45 & 9.19 & 9.11 & 9.18 & 9.11 & 11.0 & 9.17 & 9.11 & 8.73 & 8.75 \\
\hline MAX & 13.3 & 11.2 & 23.9 & 13.0 & 12.8 & 11.6 & 12.5 & 48.7 & 13.6 & 15.7 & 12.0 & 12.0 \\
\hline (WY) & 1993 & 1999 & 1984 & 1997 & 1996 & 1996 & 1999 & 1996 & 1999 & 1995 & 1999 & 1998 \\
\hline MIN & 3.93 & 4.00 & 4.45 & 4.61 & 5.12 & 4.67 & 4.27 & 4.64 & 4.13 & 4.30 & 4.09 & 3.99 \\
\hline (WY) & 1978 & 1978 & 1978 & 1978 & 1978 & 1977 & 1977 & 1977 & 1977 & 1977 & 1977 & 1977 \\
\hline
\end{tabular}

SUMMARY STATISTICS

ANNUAL TOTAL

ANNUAL MEAN

HIGHEST ANNUAL MEAN

LOWEST ANNUAL MEAN

HIGHEST DAILY MEAN

LOWEST DAILY MEAN

ANNUAL SEVEN-DAY MINIMUM

INSTANTANEOUS PEAK FLOW

INSTANTANEOUS PEAK STAGE

ANNUAL RUNOFF (AC-FT)

ANNUAL DIVERSION (AC-FT) a

10 PERCENT EXCEEDS

50 PERCENT EXCEEDS

90 PERCENT EXCEEDS
FOR 1998 CALENDAR YEAR

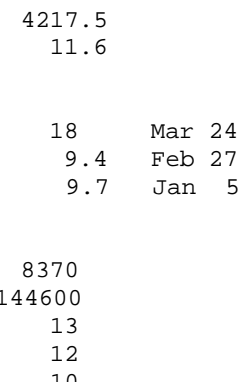

FOR 1999 WATER YEAR

$$
4473
$$$$
12.3
$$

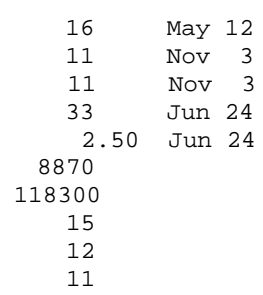

WATER YEARS 1972 - 1999

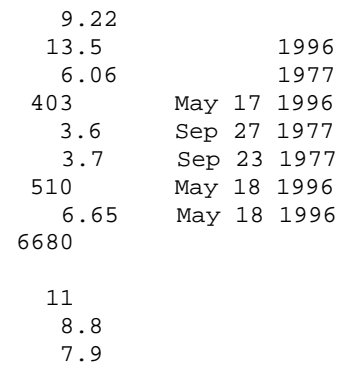

a Diversion, in acre-feet, to Loon Lake Powerplant, provided by Sacramento Municipal Utility District. 
LOCATION.-Lat 3857'59", long 120²3'33", in SE 1/4 SW 1/4 sec.15, T.13 N., R.14 E., El Dorado County, Hydrologic Unit 18020128, Eldorado National Forest, on left bank side of upstream face of dam on Gerle Creek, 0.2 mi downstream from Angel Creek, and $15.2 \mathrm{mi}$ southwest of Meeks Bay.

DRAINAGE AREA.-28.7 $\mathrm{mi}^{2}$.

PERIOD OF RECORD.—October 1993 to current year. Unpublished records for water years 1980-93 available in files of the U.S. Geological Survey.

GAGE.-Water-stage recorder. Datum of gage is sea level (levels by Sacramento Municipal Utility District). Prior to June 9, 1988, nonrecording gage at same site and datum.

REMARKS.- Reservoir is formed by concrete dam completed in 1970. Storage began in 1970. Usable capacity, 1,200 acre-ft, below elevation $5,230.9 \mathrm{ft}$, crest of spillway. Most of the water is diverted at this reservoir to Robbs Peak Powerplant (station 11429300). Records, including extremes, represent total contents at 2400 hours. See schematic diagram of Middle Fork American and Rubicon River Basins.

COOPERATION.-Records were collected by Sacramento Municipal Utility District, under general supervision of the U.S. Geological Survey, in connection with a Federal Energy Regulatory Commission project. Contents not rounded to U.S. Geological Survey standards.

EXTREMES FOR PERIOD OF RECORD.—Maximum contents, 1,469 acre-ft, Jan. 1, 1997, elevation, 5,235.39 ft; minimum, 845 acre-ft, Dec. 15, 1994, elevation, $5,222.15 \mathrm{ft}$.

EXTREMES FOR CURRENT YEAR.-Maximum contents, 1,174 acre-ft, May 21, elevation, 5,229.60 ft; minimum, 873 acre-ft, Nov. 7, elevation, $5,222.84 \mathrm{ft}$.

Capacity table (elevation, in feet, and contents, in acre-feet)

(Based on table provided by Sacramento Municipal Utility District, recomputed October 1991)

$\begin{array}{rrrr}5,200 & 203 & 5,220 & 761 \\ 5,205 & 304 & 5,225 & 964 \\ 5,210 & 431 & 5,230 & 1,193 \\ 5,215 & 583 & 5,235 & 1,448\end{array}$

RESERVOIR STORAGE (ACRE-FEET), WATER YEAR OCTOBER 1998 TO SEPTEMBER 1999 DAILY OBSERVATION AT 2400 HOURS

\begin{tabular}{|c|c|c|c|c|c|c|c|c|c|c|c|c|}
\hline DAY & OCT & NOV & DEC & JAN & $\mathrm{FEB}$ & MAR & APR & MAY & JUN & JUL & AUG & SEP \\
\hline 1 & 986 & 925 & 1099 & 981 & 991 & 1046 & 944 & 1120 & 1058 & 994 & 1040 & 1088 \\
\hline 2 & 949 & 969 & 942 & 1002 & 954 & 1076 & 940 & 1101 & 1094 & 1076 & 1062 & 1040 \\
\hline 3 & 904 & 922 & 1053 & 987 & 967 & 1006 & 963 & 1089 & 1041 & 1142 & 1054 & 1066 \\
\hline 4 & 911 & 929 & 959 & 1009 & 1004 & 1023 & 966 & 997 & 1042 & 986 & 1027 & 1079 \\
\hline 5 & e916 & 946 & 937 & 1038 & 1100 & 967 & 938 & 1115 & 1053 & 1083 & 1032 & 1124 \\
\hline 6 & 921 & 948 & 974 & 1047 & 989 & 987 & 911 & 1127 & 1041 & 1128 & 1037 & 1111 \\
\hline 7 & 925 & 873 & 939 & 968 & 1063 & 963 & 946 & 1123 & 1052 & 951 & 1043 & 1142 \\
\hline 8 & 930 & 903 & 994 & 1043 & 1089 & 965 & 898 & 1143 & 1074 & 1012 & 1049 & 1065 \\
\hline 9 & 934 & 927 & 987 & 966 & 1096 & 1010 & 900 & 1074 & 1063 & 1052 & 1055 & 1077 \\
\hline 10 & 937 & 983 & 945 & 1021 & 1084 & 977 & 898 & 1054 & 1080 & 1108 & 1112 & 1055 \\
\hline 11 & 940 & 967 & 951 & 1002 & 1129 & 966 & 914 & 1103 & 1055 & 1021 & 1099 & 1023 \\
\hline 12 & 943 & 882 & 944 & 972 & 1072 & 980 & 892 & 1141 & 1054 & 1082 & 1127 & 1090 \\
\hline 13 & 946 & 906 & 939 & 989 & 1106 & 987 & 966 & 1090 & 1048 & 1049 & 1065 & 1041 \\
\hline 14 & 948 & 975 & 994 & 977 & 1148 & 976 & 987 & 1117 & 1068 & 970 & 1067 & 1112 \\
\hline 15 & 893 & 930 & 945 & 982 & 1039 & 967 & 982 & 1082 & 1055 & 1031 & 1089 & 1121 \\
\hline 16 & 996 & 913 & 989 & 961 & 1139 & 987 & 1032 & 1097 & 1070 & 1112 & 1105 & 1094 \\
\hline 17 & 995 & 951 & 1017 & 988 & 1106 & 1006 & 1038 & 1132 & 1146 & 1062 & 1026 & 1066 \\
\hline 18 & 996 & 992 & 948 & 1021 & 1094 & 1002 & 1050 & 1120 & 1130 & 1043 & 977 & 1068 \\
\hline 19 & 947 & 924 & 948 & 1018 & 1076 & 987 & 1060 & 1096 & 1084 & 1062 & 1094 & 1064 \\
\hline 20 & 962 & 949 & 1046 & 1033 & 1103 & 994 & 1060 & 1100 & 1146 & 1073 & 1055 & 1154 \\
\hline 21 & 964 & 930 & 989 & 949 & 1120 & 984 & 1048 & 1174 & 1165 & 1039 & 1047 & 1118 \\
\hline 22 & 967 & 908 & 1022 & 961 & 1120 & 987 & 1072 & 1120 & 1157 & 1070 & 1070 & 1127 \\
\hline 23 & 964 & 947 & 958 & 999 & 1050 & 997 & 1065 & 1151 & 1062 & 1061 & 1046 & 1049 \\
\hline 24 & 913 & 923 & 961 & 987 & 1105 & 975 & 1127 & 1135 & 1148 & 1080 & 1120 & 1067 \\
\hline 25 & 897 & 946 & 968 & 992 & 1139 & 1001 & 1074 & 1104 & 1168 & 1119 & 1086 & 1102 \\
\hline 26 & 922 & 931 & 1093 & 940 & 1128 & 1002 & 1124 & 1114 & 1162 & 1024 & 1104 & 1114 \\
\hline 27 & 961 & 985 & 981 & 979 & 1152 & 998 & 1121 & 1137 & 1162 & 1089 & 987 & 1096 \\
\hline 28 & 961 & 942 & 973 & 958 & 1104 & 983 & 1078 & 1042 & 1126 & 942 & 1044 & 1077 \\
\hline 29 & 969 & 912 & 991 & 983 & --- & 1119 & 1132 & 1051 & 1140 & 954 & 1018 & 991 \\
\hline 30 & 984 & 984 & 952 & 940 & --- & 988 & 1157 & 1068 & 1107 & 1048 & e1053 & 1011 \\
\hline 31 & 991 & --- & 966 & 935 & --- & 1109 & --- & 1068 & --- & 1003 & 1088 & --- \\
\hline MAX & 996 & 992 & 1099 & 1047 & 1152 & 1119 & 1157 & 1174 & 1168 & 1142 & 1127 & 1154 \\
\hline MIN & 893 & 873 & 937 & 935 & 954 & 963 & 892 & 997 & 1041 & 942 & 977 & 991 \\
\hline$a$ & 5225.60 & 5225.44 & 5225.03 & 5224.31 & 5228.11 & 5228.23 & 5229.24 & 5227.32 & 5228.17 & 5225.88 & 5227.76 & 5225.07 \\
\hline $\mathrm{b}$ & +11 & -7 & -18 & -31 & +169 & +5 & +48 & -89 & +39 & -104 & +85 & -77 \\
\hline
\end{tabular}

CAL YR 1998 MAX 1291 MIN 873 b +66

WTR YR 1999 MAX 1174 MIN 873 b +31

e Estimated.

a Elevation, in feet, at end of month.

b Change in contents, in acre-feet. 
11430000 SOUTH FORK RUBICON RIVER BELOW GERLE CREEK, NEAR GEORGETOWN, CA

LOCATION._Lat 3857' 17", long $120^{\circ} 24^{\prime} 02^{\prime \prime}$, in SW 1/4 SW 1/4 sec.22, T.13 N., R.14 E., El Dorado County, Hydrologic Unit 18020128, Eldorado National Forest, on left bank $600 \mathrm{ft}$ downstream from Gerle Creek, 1.2 mi downstream from South Fork Rubicon River Diversion Dam, and 18 mi east of Georgetown.

DRAINAGE AREA.-47.6 $\mathrm{mi}^{2}$.

PERIOD OF RECORD.-February 1910 to June 1914 (published as Little South Fork Rubicon River below Gerle Creek near Quintette), August 1961 to current year.

REVISED RECORDS.-WSP 1931: Drainage area.

GAGE.-Water-stage recorder. Elevation of gage is 4,970 ft above sea level, from topographic map. Feb. 1, 1910, to June 21, 1914, nonrecording gage at site about $700 \mathrm{ft}$ downstream at different datum.

REMARKS.-Beginning in 1884, flow regulated by Loon Lake (station 11429350). Original dam was dismantled during September and October 1962 to permit construction of a new earthfill dam completed Dec. 27, 1963. Loon Lake receives water from Rubicon River via RubiconRockbound Tunnel to Buck Island Lake and from Buck Island Lake to Loon Lake via Buck-Loon Tunnel (stations 11427940 and 11428300 ). Prior to Dec. 3, 1961, water was diverted out of the basin in Georgetown Divide Ditch. Water is diverted 1.2 mi upstream at South Fork Rubicon River Diversion Dam to Robbs Peak Powerplant (station 11429300). Diversion of up to 1,440 $\mathrm{ft}^{3} / \mathrm{s}$ to Silver Creek Basin began in October 1962. See schematic diagram of Middle Fork American and Rubicon River Basins.

COOPERATION.-Records were collected by Sacramento Municipal Utility District, under general supervision of the U.S. Geological Survey, in connection with a Federal Energy Regulatory Commission project.

EXTREMES FOR PERIOD OF RECORD.-Maximum discharge, 12,600 ft 3 /s, Jan. 1, 1997, gage height, $12.65 \mathrm{ft}$, from rating curve extended above $2,500 \mathrm{ft}^{3} / \mathrm{s}$ on basis of slope-area measurement of peak flow; minimum, $0.8 \mathrm{ft}^{3} / \mathrm{s}$, Sept. $21,1962$.

DISCHARGE, CUBIC FEET PER SECOND, WATER YEAR OCTOBER 1998 TO SEPTEMBER 1999 DAILY MEAN VALUES

\begin{tabular}{|c|c|c|c|c|c|c|c|c|c|c|c|c|}
\hline DAY & OCT & NOV & $\mathrm{DEC}$ & JAN & FEB & MAR & $\mathrm{APR}$ & MAY & JUN & JUL & AUG & SEP \\
\hline 1 & 12 & 12 & 11 & 7.3 & 9.4 & 20 & 8.4 & 10 & 11 & 10 & 11 & 12 \\
\hline 2 & 12 & 11 & 10 & 7.5 & 9.5 & 15 & 7.9 & 10 & 12 & 11 & 11 & 11 \\
\hline 3 & 12 & 9.4 & 13 & 7.4 & 9.2 & 23 & 7.8 & 12 & 12 & 12 & 11 & 11 \\
\hline 4 & 12 & 7.6 & 11 & 7.3 & 9.3 & 16 & 7.8 & 11 & 12 & 12 & 11 & 11 \\
\hline 5 & 11 & 6.2 & 9.6 & 7.2 & 9.4 & 12 & 8.3 & 11 & 11 & 11 & 11 & 11 \\
\hline 6 & 11 & 6.4 & 9.2 & 7.1 & 10 & 11 & 7.7 & 12 & 11 & 12 & 11 & 12 \\
\hline 7 & 11 & 9.0 & 8.3 & 7.3 & 36 & 10 & 7.4 & 12 & 11 & 12 & 11 & 12 \\
\hline 8 & 11 & 6.9 & 8.2 & 7.1 & 34 & 9.7 & 7.6 & 12 & 11 & 11 & 11 & 12 \\
\hline 9 & 11 & 6.2 & 8.0 & 7.3 & 84 & 9.7 & 7.0 & 12 & 11 & 11 & 11 & 12 \\
\hline 10 & 11 & 6.3 & 7.8 & 7.3 & 19 & 9.2 & 6.7 & 11 & 11 & 12 & 11 & 12 \\
\hline 11 & 11 & 7.0 & 7.6 & 7.0 & 15 & 8.9 & 7.3 & 11 & 11 & 12 & 11 & 11 \\
\hline 12 & 11 & 6.6 & 7.8 & 7.0 & 14 & 8.7 & 8.5 & 11 & 11 & 12 & 12 & 11 \\
\hline 13 & 11 & 6.1 & 7.7 & 6.7 & 13 & 8.9 & 9.3 & 11 & 11 & 12 & 12 & 12 \\
\hline 14 & 11 & 6.1 & 7.7 & 7.1 & 12 & 9.1 & 10 & 11 & 11 & 11 & 12 & 11 \\
\hline 15 & 11 & 6.3 & 7.8 & e 7.1 & 12 & 9.1 & 11 & 11 & 11 & 11 & 12 & 11 \\
\hline 16 & 11 & 6.1 & 8.4 & e7. 5 & 14 & 9.3 & 11 & 11 & 11 & 11 & 12 & 11 \\
\hline 17 & 12 & 7.6 & 8.3 & e7. 5 & 27 & 9.9 & 11 & 10 & 11 & 11 & 12 & 12 \\
\hline 18 & 12 & 6.9 & 8.2 & e8.0 & 17 & 10 & 11 & 11 & 11 & 11 & 11 & 11 \\
\hline 19 & 12 & 6.9 & 7.8 & e15 & 15 & 10 & 11 & 11 & 11 & 11 & 11 & 12 \\
\hline 20 & 12 & 6.3 & 7.8 & e20 & 14 & 10 & 11 & 11 & 11 & 11 & 11 & 11 \\
\hline 21 & 12 & 6.4 & 8.1 & 18 & 14 & 9.4 & 10 & 14 & 11 & 11 & 11 & 11 \\
\hline 22 & 12 & 7.9 & 8.5 & 14 & 13 & 9.1 & 9.8 & 16 & 11 & 11 & 11 & 11 \\
\hline 23 & 12 & 11 & 8.5 & 30 & 12 & 9.3 & 9.3 & 10 & 11 & 11 & 11 & 12 \\
\hline 24 & 13 & 10 & 8.5 & 15 & 12 & 9.3 & 9.0 & 11 & 10 & 11 & 11 & 11 \\
\hline 25 & 12 & 7.8 & 8.3 & 13 & 12 & 9.3 & 9.0 & 11 & 11 & 11 & 11 & 11 \\
\hline 26 & 11 & 7.4 & 7.7 & 12 & 12 & 9.9 & 9.2 & 10 & 11 & 11 & 12 & 11 \\
\hline 27 & 11 & 7.2 & 7.3 & 11 & 12 & 9.7 & 9.0 & 10 & 11 & 11 & 12 & 11 \\
\hline 28 & 12 & 7.2 & 7.0 & 11 & 14 & 9.1 & 8.9 & 10 & 11 & 11 & 11 & 11 \\
\hline 29 & 12 & 9.0 & 7.3 & 10 & --- & 8.6 & 10 & 10 & 11 & 10 & 11 & 11 \\
\hline 30 & 12 & 14 & 7.5 & 9.7 & --- & 8.9 & 10 & 10 & 11 & 11 & 11 & 10 \\
\hline 31 & 12 & --- & 7.3 & 9.5 & --- & 8.8 & --- & 10 & --- & 11 & 11 & --- \\
\hline TOTAL & 359 & 234.8 & 261.2 & 318.9 & 483.8 & 330.9 & 271.9 & 344 & 332 & 347 & 349 & 339 \\
\hline MEAN & 11.6 & 7.83 & 8.43 & 10.3 & 17.3 & 10.7 & 9.06 & 11.1 & 11.1 & 11.2 & 11.3 & 11.3 \\
\hline MAX & 13 & 14 & 13 & 30 & 84 & 23 & 11 & 16 & 12 & 12 & 12 & 12 \\
\hline MIN & 11 & 6.1 & 7.0 & 6.7 & 9.2 & 8.6 & 6.7 & 10 & 10 & 10 & 11 & 10 \\
\hline $\mathrm{AC}-\mathrm{FT}$ & 712 & 466 & 518 & 633 & 960 & 656 & 539 & 682 & 659 & 688 & 692 & 672 \\
\hline $\mathrm{a}$ & 674 & 3430 & 16040 & 16390 & 36760 & 21120 & 26110 & 48470 & 27560 & 9560 & 6700 & 8680 \\
\hline
\end{tabular}

e Estimated.

a Diversion, in acre-feet, to Robbs Peak Powerplant, provided by Sacramento Municipal Utility District. 


\section{SOUTH FORK RUBICON RIVER BELOW GERLE CREEK, NEAR GEORGETOWN, CA—Continued}

STATISTICS OF MONTHLY MEAN DATA FOR WATER YEARS 1963 - 1999, BY WATER YEAR (WY)

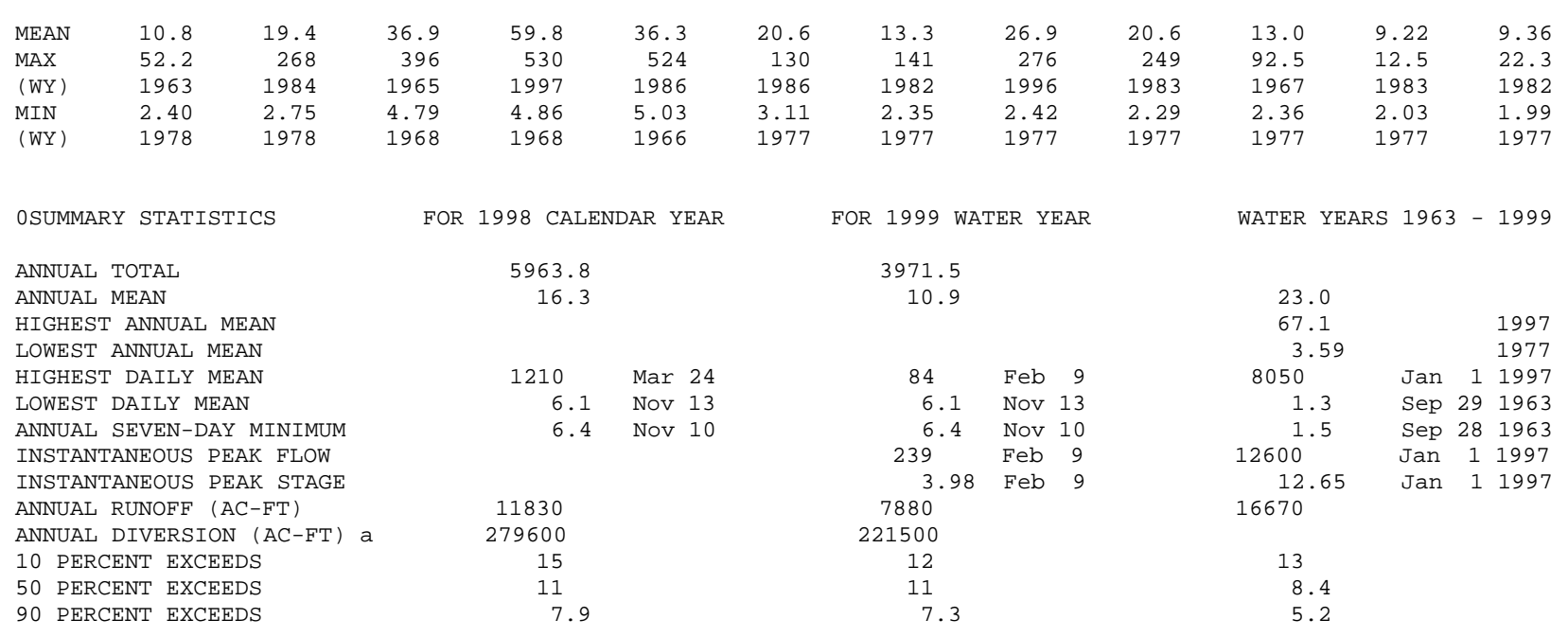

a Diversion, in acre-feet, to Robbs Peak Powerplant, provided by Sacramento Municipal Utility District. 
LOCATION.-Lat 3853'41", long 120³4'02", in NE 1/4 NW 1/4 sec.18, T.12 N., R.13 E., El Dorado County, Hydrologic Unit 18020128, on right bank 2.1 mi upstream from Stumpy Meadows Dam and 12.5 mi east of Georgetown.

DRAINAGE AREA.- $-11.7 \mathrm{mi}^{2}$.

PERIOD OF RECORD.-October 1960 to current year. Prior to October 1971, published as "above Stumpy Meadows Reservoir."

GAGE.-Water-stage recorder. Elevation of gage is 4,280 ft above sea level, from topographic map.

REMARKS.--Records good except estimated daily discharges, which are fair. No regulation or diversion upstream from station. See schematic diagram of Middle Fork American and Rubicon River Basins.

EXTREMES FOR PERIOD OF RECORD.-Maximum discharge, 3,510 ft $3 / \mathrm{s}$, Feb. 17, 1986, gage height, $7.15 \mathrm{ft}$, from rating curve extended above $540 \mathrm{ft}^{3} / \mathrm{s}$ on basis of slope-area measurement at gage height $6.31 \mathrm{ft}$; maximum gage height, $8.05 \mathrm{ft}$, Jan. 31,1963 ; minimum daily, $0.14 \mathrm{ft}^{3} / \mathrm{s}$, Aug. 16, 1977.

EXTREMES FOR CURRENT YEAR.-Peak discharges greater than base discharge of $140 \mathrm{ft}^{3} / \mathrm{s}$, or maximum:

$\begin{array}{lccccccc}\text { Date } & \text { Time } & \begin{array}{c}\text { Discharge } \\ \left(\mathrm{ft}^{3} / \mathrm{s}\right)\end{array} & \begin{array}{c}\text { Gage height } \\ (\mathrm{ft})\end{array} & \text { Date } & \text { Time } & \begin{array}{c}\text { Discharge } \\ \left(\mathrm{ft}^{3} / \mathrm{s}\right)\end{array} & \begin{array}{c}\text { Gage height } \\ (\mathrm{ft})\end{array} \\ \text { Jan. } 20 & 0532 & 382 & 3.32 & \text { Mar. 3 } & 0930 & 177 & 2.48 \\ \text { Feb. } 9 & 0747 & 762 & 4.22 & & & \end{array}$

DISCHARGE, CUBIC FEET PER SECOND, WATER YEAR OCTOBER 1998 TO SEPTEMBER 1999 DAILY MEAN VALUES

\begin{tabular}{|c|c|c|c|c|c|c|c|c|c|c|c|c|}
\hline DAY & $\mathrm{OCT}$ & NOV & $\mathrm{DEC}$ & JAN & FEB & MAR & APR & MAY & JUN & JUL & AUG & SEP \\
\hline 1 & 9.0 & 11 & 26 & 11 & 28 & 118 & 40 & 44 & 19 & 12 & 8.7 & 6.0 \\
\hline 2 & 8.8 & 8.9 & 21 & 11 & 27 & 98 & 37 & 45 & 21 & 12 & 8.5 & 5.9 \\
\hline 3 & 8.7 & 8.5 & 36 & 11 & 26 & 143 & 38 & 51 & 20 & 12 & 7.6 & 5.9 \\
\hline 4 & 8.7 & 8.2 & 26 & 11 & 25 & 122 & 38 & 46 & 20 & 12 & 7.2 & 5.7 \\
\hline 5 & 8.3 & 8.5 & 20 & 10 & 24 & 104 & 40 & 43 & 19 & 11 & 7.3 & 5.6 \\
\hline 6 & 8.2 & 8.8 & 18 & 10 & 26 & 92 & 38 & 41 & 19 & 11 & 7.6 & 5.5 \\
\hline 7 & 8.1 & 16 & 16 & 10 & 252 & 83 & 36 & 40 & 18 & 11 & 7.8 & 5.4 \\
\hline 8 & 8.2 & 14 & 15 & 10 & 320 & 75 & 38 & 38 & 18 & 10 & 7.7 & 5.4 \\
\hline 9 & 8.5 & 11 & 14 & 10 & 511 & 71 & 36 & 36 & 17 & 10 & 7.7 & 5.3 \\
\hline 10 & 8.6 & 10 & 14 & 10 & 206 & 61 & 35 & 35 & 17 & 9.9 & 7.8 & 5.4 \\
\hline 11 & 8.4 & 11 & 13 & 10 & 141 & 56 & 37 & 34 & 17 & 9.5 & 7.6 & 5.3 \\
\hline 12 & 8.1 & 10 & 13 & 10 & 112 & 50 & 39 & 33 & 17 & 9.2 & 7.3 & 5.4 \\
\hline 13 & 7.9 & 9.5 & 13 & 9.9 & 92 & 48 & 43 & 32 & 16 & 9.0 & 7.1 & 5.5 \\
\hline 14 & 8.0 & 9.3 & 13 & 9.8 & 79 & 49 & 49 & 31 & 16 & 9.0 & 7.0 & 5.4 \\
\hline 15 & 8.1 & 9.1 & 13 & 13 & 69 & 47 & 56 & 31 & 15 & 9.4 & 7.0 & 5.4 \\
\hline 16 & 7.9 & 8.6 & 14 & 21 & 77 & 46 & 63 & 30 & 15 & 9.6 & 7.0 & 5.3 \\
\hline 17 & 7.8 & 12 & 14 & 21 & 161 & 46 & 75 & 28 & 15 & 9.5 & 6.8 & 5.4 \\
\hline 18 & 7.8 & 11 & e13 & 76 & 127 & 46 & 79 & 28 & 15 & 9.5 & 7.0 & 5.6 \\
\hline 19 & 7.7 & 9.3 & e13 & 138 & 111 & 46 & 79 & 27 & 14 & 9.4 & 7.0 & 5.9 \\
\hline 20 & 7.6 & 8.8 & e13 & 304 & 100 & 47 & 78 & 26 & 14 & 9.4 & 6.9 & 5.6 \\
\hline 21 & 7.5 & 8.6 & e13 & 141 & 92 & 45 & 74 & 25 & 14 & 9.3 & 6.8 & 5.4 \\
\hline 22 & 7.4 & 13 & e13 & 83 & 81 & 44 & 70 & 24 & 13 & 9.6 & 6.8 & 5.4 \\
\hline 23 & 7.4 & 20 & e13 & 156 & 73 & 45 & 64 & 24 & 13 & 9.5 & 6.9 & 5.5 \\
\hline 24 & 10 & 26 & e12 & 102 & 67 & 44 & 62 & 23 & 13 & 9.4 & 6.2 & 5.5 \\
\hline 25 & 10 & 15 & e12 & 76 & 73 & 45 & 58 & 23 & 13 & 9.3 & 5.9 & 5.4 \\
\hline 26 & 8.8 & 12 & e12 & 60 & 64 & 45 & 60 & 22 & 13 & 9.3 & 5.9 & 5.3 \\
\hline 27 & 8.2 & 12 & e12 & 47 & 61 & 45 & 55 & 21 & 13 & 9.0 & 6.5 & 5.2 \\
\hline 28 & 8.2 & 12 & e11 & 40 & 67 & 43 & 50 & 20 & 13 & 8.8 & 6.0 & 5.1 \\
\hline 29 & 8.3 & 17 & e11 & 35 & --- & 42 & 47 & 20 & 13 & 9.1 & 5.8 & 5.1 \\
\hline 30 & 8.3 & 35 & 12 & 32 & --- & 42 & 45 & 20 & 12 & 8.8 & 5.9 & 5.1 \\
\hline 31 & 8.1 & --- & 12 & 30 & --- & 42 & --- & 20 & --- & 8.6 & 6.0 & --- \\
\hline TOTAL & 256.6 & 374.1 & 471 & 1518.7 & 3092 & 1930 & 1559 & 961 & 472 & 305.1 & 217.3 & 163.9 \\
\hline MEAN & 8.28 & 12.5 & 15.2 & 49.0 & 110 & 62.3 & 52.0 & 31.0 & 15.7 & 9.84 & 7.01 & 5.46 \\
\hline MAX & 10 & 35 & 36 & 304 & 511 & 143 & 79 & 51 & 21 & 12 & 8.7 & 6.0 \\
\hline MIN & 7.4 & 8.2 & 11 & 9.8 & 24 & 42 & 35 & 20 & 12 & 8.6 & 5.8 & 5.1 \\
\hline $\mathrm{AC}-\mathrm{FT}$ & 509 & 742 & 934 & 3010 & 6130 & 3830 & 3090 & 1910 & 936 & 605 & 431 & 325 \\
\hline
\end{tabular}




\section{PILOT CREEK ABOVE STUMPY MEADOWS LAKE, CA—Continued}

STATISTICS OF MONTHLY MEAN DATA FOR WATER YEARS 1961 - 1999, BY WATER YEAR (WY)

\begin{tabular}{|c|c|c|c|c|c|c|c|c|c|c|c|c|}
\hline MEAN & 6.55 & 12.8 & 26.3 & 48.4 & 50.0 & 54.4 & 47.9 & 36.6 & 15.6 & 8.51 & 5.44 & 4.86 \\
\hline MAX & 24.8 & 74.1 & 159 & 268 & 373 & 195 & 139 & 118 & 50.4 & 17.8 & 16.2 & 16.3 \\
\hline (WY) & 1963 & 1984 & 1965 & 1997 & 1986 & 1983 & 1982 & 1967 & 1967 & 1998 & 1961 & 1961 \\
\hline MIN & .87 & 2.79 & 3.35 & 4.55 & 4.64 & 4.82 & 3.38 & 4.06 & 1.93 & .64 & .18 & .50 \\
\hline (WY) & 1978 & 1977 & 1977 & 1991 & 1977 & 1977 & 1977 & 1977 & 1977 & 1977 & 1977 & 1977 \\
\hline
\end{tabular}

SUMMARY STATISTICS

ANNUAL TOTAL

ANNUAL MEAN

HIGHEST ANNUAL MEAN

LOWEST ANNUAL MEAN

HIGHEST DAILY MEAN

LOWEST DAILY MEAN

ANNUAL SEVEN-DAY MINIMUM

INSTANTANEOUS PEAK FLOW

INSTANTANEOUS PEAK STAGE

ANNUAL RUNOFF (AC-FT)

10 PERCENT EXCEEDS

50 PERCENT EXCEEDS

90 PERCENT EXCEEDS
FOR 1998 CALENDAR YEAR

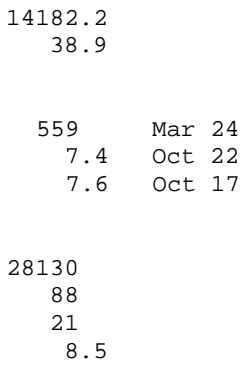

FOR 1999 WATER YEAR

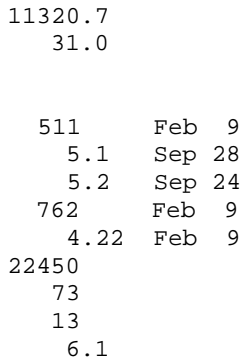

WATER YEARS 1961 - 1999

\begin{tabular}{|c|c|c|}
\hline 26.3 & & \\
\hline 64.8 & & 1983 \\
\hline 2.96 & & 1977 \\
\hline 2840 & Feb 17 & 1986 \\
\hline .14 & Aug 16 & 1977 \\
\hline .15 & Aug 12 & 1977 \\
\hline 3510 & Feb 17 & 1986 \\
\hline 8.05 & Jan 31 & 1963 \\
\hline 19080 & & \\
\hline 60 & & \\
\hline 10 & & \\
\hline 3.4 & & \\
\hline
\end{tabular}


LOCATION.-Lat 3855'25", long 120³8'27", in NE 1/4 NW 1/4 sec.4, T.12 N., R.12 E., El Dorado County, Hydrologic Unit 18020128, Eldorado National Forest, on left bank $450 \mathrm{ft}$ downstream from Mutton Canyon, $500 \mathrm{ft}$ downstream from Georgetown Divide Diversion Dam, 2.5 mi downstream from Stumpy Meadows Dam, and 10 mi east of Georgetown.

DRAINAGE AREA.-21.1 $\mathrm{mi}^{2}$.

PERIOD OF RECORD.- - June 1961 to current year.

GAGE.-Water-stage recorder. Elevation of gage is 3,760 ft above sea level, from topographic map.

REMARKS.--Records good. Flow regulated by Stumpy Meadows Lake 2.5 mi upstream, usable capacity, 17,500 acre-ft, completed in November 1961. Georgetown Irrigation District Ditch, capacity, about $60 \mathrm{ft}^{3} / \mathrm{s}$, diverts water out of Pilot Creek, $500 \mathrm{ft}$ upstream from station. See schematic diagram of Middle Fork American and Rubicon River Basins.

EXTREMES FOR PERIOD OF RECORD.-Maximum discharge, 7,830 ft $3 / \mathrm{s}, \mathrm{Jan} .2,1997$, gage height, $10.95 \mathrm{ft}$, from rating curve extended above $970 \mathrm{ft}^{3} / \mathrm{s}$ on basis of slope-area measurement at gage height $10.06 \mathrm{ft}$; minimum daily, $0.20 \mathrm{ft}^{3} / \mathrm{s}$, Sept. 24, Nov. 1-5, 1966.

DISCHARGE, CUBIC FEET PER SECOND, WATER YEAR OCTOBER 1998 TO SEPTEMBER 1999 DAILY MEAN VALUES

\begin{tabular}{|c|c|c|c|c|c|c|c|c|c|c|c|c|}
\hline DAY & $\mathrm{OCT}$ & NOV & $\mathrm{DEC}$ & JAN & FEB & MAR & APR & MAY & JUN & JUL & AUG & SEP \\
\hline 1 & 4.5 & 4.7 & 16 & 4.7 & 58 & 221 & 63 & 57 & 14 & 4.8 & 4.4 & 4.3 \\
\hline 2 & 4.5 & 4.5 & 7.8 & 4.7 & 51 & 195 & 59 & 59 & 19 & 4.8 & 4.4 & 4.6 \\
\hline 3 & 4.5 & 4.5 & 19 & 4.5 & 46 & 270 & 58 & 91 & 23 & 4.8 & 4.4 & 4.9 \\
\hline 4 & 4.5 & 4.3 & 12 & 4.5 & 44 & 232 & 55 & 75 & 19 & 4.8 & 4.5 & 4.7 \\
\hline 5 & 4.3 & 4.3 & 8.1 & 4.5 & 43 & 188 & 66 & 63 & 17 & 4.8 & 4.6 & 4.6 \\
\hline 6 & 4.6 & 4.5 & 7.2 & 4.5 & 54 & 165 & 67 & 57 & 15 & 4.7 & 4.5 & 4.5 \\
\hline 7 & 4.9 & 7.0 & 6.3 & 4.4 & 458 & 145 & 56 & 53 & 13 & 4.6 & 4.6 & 4.5 \\
\hline 8 & 4.8 & 5.8 & 6.2 & 4.3 & 597 & 131 & 67 & 49 & 12 & 4.6 & 4.6 & 4.5 \\
\hline 9 & 4.7 & 5.1 & 5.9 & 4.3 & 1360 & 144 & 59 & 47 & 11 & 4.6 & 5.2 & 4.5 \\
\hline 10 & 4.7 & 4.8 & 5.6 & 4.3 & 632 & 114 & 52 & 45 & 9.2 & 4.6 & 5.2 & 4.5 \\
\hline 11 & 4.7 & 5.7 & 5.5 & 4.3 & 365 & 103 & 57 & 43 & 8.9 & 4.5 & 4.6 & 4.5 \\
\hline 12 & 4.7 & 5.0 & 5.5 & 4.3 & 262 & 95 & 57 & 42 & 8.6 & 4.6 & 4.5 & 4.7 \\
\hline 13 & 4.7 & 4.7 & 5.6 & 4.2 & 206 & 89 & 60 & 40 & 7.8 & 4.9 & 4.5 & 4.7 \\
\hline 14 & 4.7 & 4.5 & 5.8 & 4.2 & 173 & 87 & 67 & 36 & 6.8 & 5.0 & 4.4 & 4.7 \\
\hline 15 & 4.7 & 4.5 & 5.8 & 5.3 & 145 & 83 & 75 & 36 & 6.3 & 5.1 & 4.3 & 5.0 \\
\hline 16 & 4.9 & 4.3 & 5.7 & 11 & 163 & 79 & 82 & 34 & 5.4 & 5.1 & 4.3 & 5.1 \\
\hline 17 & 4.7 & 6.0 & 5.6 & 9.8 & 345 & 77 & 93 & 32 & 5.1 & 5.1 & 4.4 & 5.1 \\
\hline 18 & 4.7 & 5.3 & 5.5 & 68 & 279 & 75 & 105 & 29 & 5.1 & 5.0 & 4.5 & 5.1 \\
\hline 19 & 4.7 & 4.8 & 5.5 & 230 & 248 & 72 & 108 & 27 & 5.1 & 5.0 & 4.5 & 5.1 \\
\hline 20 & 4.7 & 4.5 & 5.5 & 611 & 217 & 76 & 105 & 26 & 5.0 & 5.0 & 4.5 & 5.1 \\
\hline 21 & 4.7 & 4.5 & 5.3 & 363 & 229 & 76 & 100 & 25 & 5.0 & 5.0 & 4.5 & 5.1 \\
\hline 22 & 4.7 & 6.6 & 5.3 & 194 & 173 & 71 & 95 & 24 & 4.9 & 4.9 & 4.5 & 4.9 \\
\hline 23 & 4.6 & 8.7 & 5.2 & 346 & 153 & 70 & 91 & 23 & 4.8 & 4.9 & 4.5 & 5.0 \\
\hline 24 & 5.3 & 8.4 & 5.1 & 232 & 140 & 69 & 79 & 25 & 4.8 & 4.9 & 4.5 & 5.0 \\
\hline 25 & 5.1 & 5.6 & 5.1 & 164 & 174 & 68 & 76 & 23 & 4.7 & 4.8 & 4.5 & 4.9 \\
\hline 26 & 4.7 & 5.1 & 5.1 & 133 & 138 & 66 & 76 & 20 & 4.7 & 4.6 & 4.5 & 4.9 \\
\hline 27 & 4.5 & 5.1 & 5.1 & 102 & 128 & 65 & 74 & 18 & 4.7 & 4.6 & 4.5 & 4.9 \\
\hline 28 & 4.3 & 5.5 & 5.1 & 85 & 140 & 63 & 69 & 17 & 4.5 & 4.5 & 4.5 & 4.9 \\
\hline 29 & 4.3 & 8.7 & 5.0 & 72 & --- & 61 & 65 & 15 & 4.7 & 4.5 & 4.4 & 4.9 \\
\hline 30 & 4.3 & 23 & 4.9 & 64 & --- & 62 & 60 & 15 & 4.8 & 4.5 & 4.3 & 4.9 \\
\hline 31 & 4.3 & --- & 4.7 & 68 & --- & 74 & --- & 15 & --- & 4.5 & 4.3 & --- \\
\hline TOTAL & 144.0 & 180.0 & 206.0 & 2819.8 & 7021 & 3386 & 2196 & 1161 & 263.9 & 148.1 & 139.9 & 144.1 \\
\hline MEAN & 4.65 & 6.00 & 6.65 & 91.0 & 251 & 109 & 73.2 & 37.5 & 8.80 & 4.78 & 4.51 & 4.80 \\
\hline MAX & 5.3 & 23 & 19 & 611 & 1360 & 270 & 108 & 91 & 23 & 5.1 & 5.2 & 5.1 \\
\hline MIN & 4.3 & 4.3 & 4.7 & 4.2 & 43 & 61 & 52 & 15 & 4.5 & 4.5 & 4.3 & 4.3 \\
\hline $\mathrm{AC}-\mathrm{FT}$ & 286 & 357 & 409 & 5590 & 13930 & 6720 & 4360 & 2300 & 523 & 294 & 277 & 286 \\
\hline
\end{tabular}

STATISTICS OF MONTHLY MEAN DATA FOR WATER YEARS 1961 - 1999, BY WATER YEAR (WY)

\begin{tabular}{|c|c|c|c|c|c|c|c|c|c|c|c|c|}
\hline MEAN & 2.87 & 5.78 & 30.5 & 64.8 & 80.9 & 78.0 & 68.9 & 39.2 & 9.87 & 4.29 & 3.32 & 2.87 \\
\hline MAX & 7.19 & 28.6 & 340 & 621 & 585 & 370 & 289 & 171 & 54.4 & 15.6 & 13.4 & 8.54 \\
\hline (WY) & 1963 & 1984 & 1965 & 1997 & 1986 & 1983 & 1982 & 1995 & 1967 & 1983 & 1983 & 1983 \\
\hline MIN & .46 & .46 & .54 & .53 & .89 & 1.21 & .98 & 1.12 & .66 & .45 & .38 & .37 \\
\hline (WY) & 1962 & 1962 & 1962 & 1962 & 1991 & 1977 & 1977 & 1977 & 1977 & 1977 & 1977 & 1977 \\
\hline
\end{tabular}

SUMMARY STATISTICS

ANNUAL TOTAL

ANNUAL MEAN

HIGHEST ANNUAL MEAN

LOWEST ANNUAL MEAN

HIGHEST DAILY MEAN

LOWEST DAILY MEAN

ANNUAL SEVEN-DAY MINIMUM

INSTANTANEOUS PEAK FLOW

INSTANTANEOUS PEAK STAGE

ANNUAL RUNOFF (AC-FT)

10 PERCENT EXCEEDS

50 PERCENT EXCEEDS

90 PERCENT EXCEEDS
FOR 1998 CALENDAR YEAR

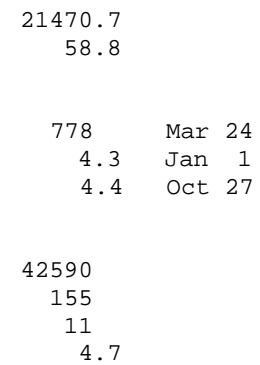

FOR 1999 WATER YEAR

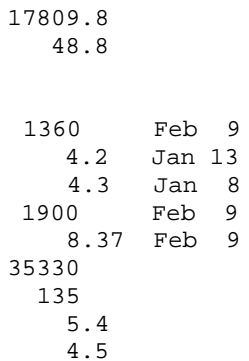

WATER YEARS 1961 - 1999

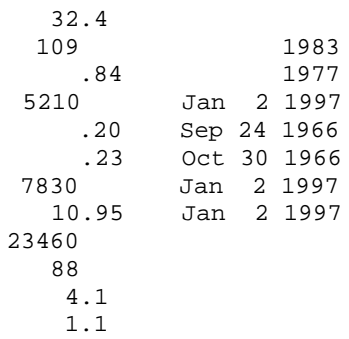


LOCATION.-Lat $39^{\circ} 03^{\prime} 04^{\prime \prime}$, long 120²8'14", in SW 1/4 NE 1/4 sec.24, T.14 N., R.13 E., Placer County, Hydrologic Unit 18020128, Eldorado National Forest, on right bank at diversion dam, 3.3 mi upstream from confluence with North and South Forks Long Canyon Creek, and 17.2 mi east of Volcanoville.

PERIOD OF RECORD.-October 1965 to current year.

GAGE.-Water-stage recorder and sharp-crested weir. Elevation of gage is 4,630 ft above sea level, from topographic map.

REMARKS.--Tunnel completed in September 1965; diversion began in February 1966. Flow is diverted from South Fork Long Canyon Creek to a tunnel from Hell Hole Reservoir to Middle Fork Powerplant on the Middle Fork American River. See schematic diagram of Middle Fork American and Rubicon River Basins.

COOPERATION.-Records provided by Placer County Water Agency, under general supervision of the U.S. Geological Survey, in connection with a Federal Energy Regulatory Commission project.

EXTREMES FOR PERIOD OF RECORD.-Maximum daily discharge, $251 \mathrm{ft}^{3} / \mathrm{s}$, Nov. 12, 1973; no flow for part of each year.

DISCHARGE, CUBIC FEET PER SECOND, WATER YEAR OCTOBER 1998 TO SEPTEMBER 1999

\begin{tabular}{|c|c|c|c|c|c|c|c|c|c|c|c|c|}
\hline DAY & OCT & NOV & $\mathrm{DEC}$ & JAN & FEB & MAR & APR & MAY & JUN & JUL & AUG & SEP \\
\hline 1 & .00 & .00 & .00 & .00 & 13 & 104 & 26 & 52 & 33 & .00 & .00 & .00 \\
\hline 2 & .00 & .00 & .00 & .00 & 13 & 71 & 23 & 54 & 34 & .00 & .00 & .00 \\
\hline 3 & .00 & .00 & .00 & .00 & 14 & 94 & 23 & 51 & 30 & .00 & .00 & .00 \\
\hline 4 & .00 & .00 & .00 & .00 & 16 & 67 & 22 & 49 & 26 & .00 & .00 & .00 \\
\hline 5 & .00 & .00 & .00 & .00 & 14 & 52 & 21 & 52 & 22 & .00 & .00 & .00 \\
\hline 6 & .00 & .00 & .00 & .00 & 15 & 44 & 21 & 58 & 20 & .00 & .00 & .00 \\
\hline 7 & .00 & .00 & .00 & .00 & 95 & 38 & 20 & 59 & 19 & .00 & .00 & .00 \\
\hline 8 & .00 & .00 & .00 & .00 & 102 & 35 & 19 & 56 & 18 & .00 & .00 & .00 \\
\hline 9 & .00 & .00 & .00 & .00 & 166 & 32 & 18 & 53 & 17 & .00 & .00 & .00 \\
\hline 10 & .00 & .00 & .00 & .00 & 80 & 30 & 17 & 52 & 15 & .00 & .00 & .00 \\
\hline 11 & .00 & .00 & .00 & .00 & 54 & 28 & 19 & 54 & 13 & .00 & .00 & .00 \\
\hline 12 & .00 & .00 & .00 & .00 & 44 & 26 & 22 & 60 & 13 & .00 & .00 & .00 \\
\hline 13 & .00 & .00 & .00 & .00 & 38 & 27 & 30 & 58 & 12 & .00 & .00 & .00 \\
\hline 14 & .00 & .00 & .00 & .00 & 35 & 28 & 40 & 53 & 11 & .00 & .00 & .00 \\
\hline 15 & .00 & .00 & .00 & .00 & 31 & 27 & 45 & 49 & 11 & .00 & .00 & .00 \\
\hline 16 & .00 & .00 & .00 & .00 & 39 & 28 & 50 & 47 & 9.4 & .00 & .00 & .00 \\
\hline 17 & .00 & .00 & .00 & 12 & 115 & 31 & 57 & 48 & 5.3 & .00 & .00 & .00 \\
\hline 18 & .00 & .00 & .00 & 56 & 67 & 34 & 61 & 49 & .00 & .00 & .00 & .00 \\
\hline 19 & .00 & .00 & .00 & 75 & 48 & 35 & 65 & 49 & .00 & .00 & .00 & .00 \\
\hline 20 & .00 & .00 & .00 & 137 & 41 & 34 & 66 & 49 & .00 & .00 & .00 & .00 \\
\hline 21 & .00 & .00 & .00 & 67 & 36 & 31 & 63 & 50 & .00 & .00 & .00 & .00 \\
\hline 22 & .00 & .00 & .00 & 44 & 33 & 28 & 59 & 53 & .00 & .00 & .00 & .00 \\
\hline 23 & .00 & .00 & .00 & 82 & 31 & 29 & 56 & 56 & .00 & .00 & .00 & .00 \\
\hline 24 & .00 & .00 & .00 & 45 & 30 & 30 & 57 & 60 & .00 & .00 & .00 & .00 \\
\hline 25 & .00 & .00 & .00 & 34 & 30 & 30 & 62 & 62 & .00 & .00 & .00 & .00 \\
\hline 26 & .00 & .00 & .00 & 27 & 27 & 36 & 74 & 57 & .00 & .00 & .00 & .00 \\
\hline 27 & .00 & .00 & .00 & 22 & 27 & 36 & 69 & 53 & .00 & .00 & .00 & .00 \\
\hline 28 & .00 & .00 & .00 & 19 & 42 & 34 & 58 & 49 & .00 & .00 & .00 & .00 \\
\hline 29 & .00 & .00 & .00 & 18 & --- & 31 & 50 & 43 & .00 & .00 & .00 & .00 \\
\hline 30 & .00 & .00 & .00 & 16 & --- & 30 & 50 & 42 & .00 & .00 & .00 & .00 \\
\hline 31 & .00 & --- & .00 & 15 & --- & 27 & --- & 35 & --- & .00 & .00 & --- \\
\hline TOTAL & 0.00 & 0.00 & 0.00 & 669.00 & 1296 & 1207 & 1263 & 1612 & 308.70 & 0.00 & 0.00 & 0.00 \\
\hline MEAN & .000 & .000 & .000 & 21.6 & 46.3 & 38.9 & 42.1 & 52.0 & 10.3 & .000 & .000 & .000 \\
\hline MAX & .00 & .00 & .00 & 137 & 166 & 104 & 74 & 62 & 34 & .00 & .00 & .00 \\
\hline MIN & .00 & .00 & .00 & .00 & 13 & 26 & 17 & 35 & .00 & .00 & .00 & .00 \\
\hline $\mathrm{AC}-\mathrm{FT}$ & .00 & .00 & .00 & 1330 & 2570 & 2390 & 2510 & 3200 & 612 & .00 & .00 & .00 \\
\hline
\end{tabular}

STATISTICS OF MONTHLY MEAN DATA FOR WATER YEARS 1966 - 1999, BY WATER YEAR (WY)

\begin{tabular}{|c|c|c|c|c|c|c|c|c|c|c|c|c|}
\hline MEAN & .002 & 3.33 & 5.47 & 10.7 & 13.5 & 21.9 & 27.7 & 25.5 & 8.89 & .33 & .002 & .000 \\
\hline MAX & .034 & 37.2 & 38.6 & 42.1 & 77.3 & 77.7 & 67.8 & 80.6 & 54.0 & 4.54 & .067 & .001 \\
\hline (WY) & 1980 & 1974 & 1984 & 1974 & 1996 & 1989 & 1980 & 1975 & 1998 & 1983 & 1983 & 1972 \\
\hline MIN & .000 & .000 & .000 & .000 & .000 & .000 & .000 & .000 & .000 & .000 & .000 & .000 \\
\hline (WY) & 1966 & 1966 & 1966 & 1966 & 1991 & 1974 & 1974 & 1974 & 1966 & 1966 & 1966 & 1966 \\
\hline
\end{tabular}

SUMMARY STATISTICS

ANNUAL TOTAL

ANNUAL MEAN

HIGHEST ANNUAL MEAN

LOWEST ANNUAL MEAN

HIGHEST DAILY MEAN

LOWEST DAILY MEAN

ANNUAL SEVEN-DAY MINIMUM

ANNUAL RUNOFF (AC-FT)

10 PERCENT EXCEEDS

50 PERCENT EXCEEDS

90 PERCENT EXCEEDS
FOR 1998 CALENDAR YEAR

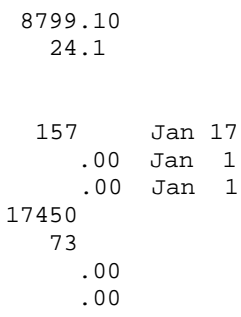

FOR 1999 WATER YEAR

$$
\begin{array}{rrrr}
6355.70 & & \\
17.4 & & \\
166 & & \text { Feb } & 9 \\
.00 & \text { Oct } & 1 \\
.00 & \text { Oct } & 1 \\
12610 & & & \\
55 & & & \\
.00 & & \\
.00 & &
\end{array}
$$

WATER YEARS 1966 - 1999

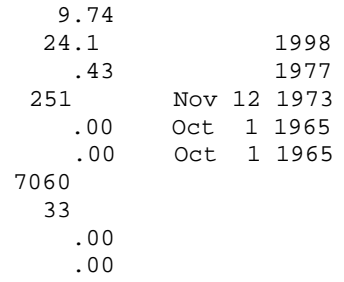


LOCATION.-Lat 3903'04", long 120²8'14", in SW 1/4 NE 1/4 sec.24, T.14 N., R.13 E., Placer County, Hydrologic Unit 18020128, Eldorado National Forest, on right bank $21 \mathrm{ft}$ below diversion dam, $3.3 \mathrm{mi}$ upstream from confluence of North and South Forks Long Canyon Creek, and 17.2 mi east of Volcanoville.

PERIOD OF RECORD.-October 1988 to current year.

GAGE.-Water-stage recorder. Elevation of gage is 4,630 ft above sea level, from topographic map.

REMARKS.-Discharge is computed only during periods of operation of South Fork Long Canyon Creek Diversion Tunnel (station 11433060). See schematic diagram of Middle Fork American and Rubicon River Basins.

COOPERATION.--Records provided by Placer County Water Agency, under general supervision of the U.S. Geological Survey, in connection with a Federal Energy Regulatory Commission project.

DISCHARGE, CUBIC FEET PER SECOND, WATER YEAR OCTOBER 1998 TO SEPTEMBER 1999 DAILY MEAN VALUES

\begin{tabular}{|c|c|c|c|c|c|c|c|c|c|c|c|c|}
\hline DAY & $\mathrm{OCT}$ & NOV & $\mathrm{DEC}$ & JAN & FEB & MAR & APR & MAY & JUN & JUL & AUG & SEP \\
\hline 1 & --- & --- & --- & --- & 6.2 & 7.0 & 6.0 & 6.5 & 6.3 & --- & --- & --- \\
\hline 2 & --- & --- & --- & --- & 6.0 & 6.8 & 6.0 & 6.6 & 6.3 & --- & --- & --- \\
\hline 3 & --- & --- & --- & --- & 6.2 & 6.9 & 6.0 & 6.6 & 6.3 & --- & --- & --- \\
\hline 4 & --- & --- & --- & --- & 6.2 & 6.8 & 6.0 & 6.5 & 6.3 & --- & --- & --- \\
\hline 5 & --- & --- & --- & --- & 6.3 & 6.6 & 6.0 & 6.5 & 6.2 & --- & --- & --- \\
\hline 6 & --- & --- & --- & --- & 6.3 & 6.6 & 6.0 & 6.5 & 6.2 & --- & --- & --- \\
\hline 7 & --- & --- & --- & --- & 7.0 & 6.5 & 6.0 & 6.5 & 6.2 & --- & --- & --- \\
\hline 8 & --- & --- & --- & --- & 7.1 & 6.5 & 6.0 & 6.5 & 6.0 & --- & --- & --- \\
\hline 9 & --- & --- & --- & --- & 10 & 6.5 & 6.0 & 6.5 & 6.0 & --- & --- & --- \\
\hline 10 & --- & --- & --- & --- & 7.3 & 6.4 & 6.0 & 6.5 & 6.0 & --- & --- & --- \\
\hline 11 & --- & --- & --- & --- & 6.8 & 6.4 & 6.2 & 6.5 & 6.0 & --- & --- & --- \\
\hline 12 & --- & --- & --- & --- & 6.6 & 6.3 & 6.2 & 6.5 & 6.0 & --- & --- & --- \\
\hline 13 & --- & --- & --- & --- & 6.6 & 6.3 & 6.3 & 6.5 & 6.0 & --- & --- & --- \\
\hline 14 & --- & --- & --- & --- & 6.5 & 6.3 & 6.3 & 6.5 & 6.0 & --- & --- & --- \\
\hline 15 & --- & --- & --- & --- & 6.5 & 6.3 & 6.4 & 6.4 & 6.0 & --- & --- & --- \\
\hline 16 & --- & --- & --- & --- & 6.6 & 6.3 & 6.4 & 6.4 & 6.0 & --- & --- & --- \\
\hline 17 & --- & --- & --- & 6.8 & 7.3 & 6.4 & 6.5 & 6.4 & 6.7 & --- & --- & --- \\
\hline 18 & --- & --- & --- & 6.3 & 6.8 & 6.4 & 6.5 & 6.4 & --- & --- & --- & --- \\
\hline 19 & --- & --- & --- & 6.3 & 6.5 & 6.4 & 6.5 & 6.4 & --- & --- & --- & --- \\
\hline 20 & --- & --- & --- & 6.5 & 6.5 & 6.4 & 6.5 & 6.5 & --- & --- & --- & --- \\
\hline 21 & --- & --- & --- & 5.9 & 6.4 & 6.4 & 6.5 & 6.5 & --- & --- & --- & --- \\
\hline 22 & --- & --- & --- & 6.2 & 6.4 & 6.4 & 6.4 & 6.5 & --- & --- & --- & --- \\
\hline 23 & --- & --- & --- & 6.9 & 6.4 & 6.4 & 6.4 & 6.5 & --- & --- & --- & --- \\
\hline 24 & --- & --- & --- & 6.5 & 6.4 & 6.4 & 6.4 & 6.5 & --- & --- & --- & --- \\
\hline 25 & --- & --- & --- & 6.4 & 6.4 & 6.4 & 6.4 & 6.5 & --- & --- & --- & --- \\
\hline 26 & --- & --- & --- & 6.3 & 6.3 & 6.4 & 6.5 & 6.5 & --- & --- & --- & --- \\
\hline 27 & --- & --- & --- & 6.2 & 6.3 & 6.4 & 6.5 & 6.5 & --- & --- & --- & --- \\
\hline 28 & --- & --- & --- & 6.2 & 6.5 & 6.3 & 6.5 & 6.5 & --- & --- & --- & --- \\
\hline 29 & --- & --- & --- & 6.2 & --- & 6.2 & 6.5 & 6.5 & --- & --- & --- & --- \\
\hline 30 & --- & --- & --- & 6.2 & --- & 6.2 & 6.5 & 6.5 & --- & --- & --- & --- \\
\hline 31 & --- & --- & --- & 6.2 & --- & 6.2 & --- & 6.4 & --- & --- & --- & --- \\
\hline TOTAL & --- & --- & --- & --- & 186.4 & 199.8 & 188.4 & 201.1 & --- & --- & --- & --- \\
\hline MEAN & --- & --- & --- & --- & 6.66 & 6.45 & 6.28 & 6.49 & --- & --- & --- & --- \\
\hline MAX & --- & --- & --- & --- & 10 & 7.0 & 6.5 & 6.6 & --- & --- & --- & --- \\
\hline MIN & --- & --- & --- & --- & 6.0 & 6.2 & 6.0 & 6.4 & --- & --- & --- & --- \\
\hline $\mathrm{AC}-\mathrm{FT}$ & --- & --- & --- & --- & 370 & 396 & 374 & 399 & --- & --- & --- & --- \\
\hline
\end{tabular}


LOCATION.-Lat $39^{\circ} 02^{\prime} 57^{\prime \prime}$, long $120^{\circ} 28^{\prime} 56^{\prime \prime}$, in SW 1/4 NW 1/4 sec.24, T.14 N., R.13 E., Placer County, Hydrologic Unit 18020128, Eldorado National Forest, on left bank at diversion dam, $3.2 \mathrm{mi}$ upstream from confluence of North and South Forks Long Canyon Creek, and $16.9 \mathrm{mi}$ east of Volcanoville.

PERIOD OF RECORD.-October 1965 to current year.

GAGE.-Water-stage recorder and Parshall flume. Elevation of gage is 4,700 ft above sea level, from topographic map.

REMARKS.--Tunnel completed in September 1965 and diversions began in February 1966. Flow is diverted from North Fork Long Canyon Creek to a tunnel from Hell Hole Reservoir to Middle Fork Powerplant (stations 11428700 and 11428600) on the Middle Fork American River. See schematic diagram of Middle Fork American and Rubicon River Basins.

COOPERATION.-Records provided by Placer County Water Agency, under general supervision of the U.S. Geological Survey, in connection with a Federal Energy Regulatory Commission project.

EXTREMES FOR PERIOD OF RECORD.-Maximum daily discharge, $100 \mathrm{ft}^{3} / \mathrm{s}$, Jan. 15, 1998; no flow for part of each year.

DISCHARGE, CUBIC FEET PER SECOND, WATER YEAR OCTOBER 1998 TO SEPTEMBER 1999

\begin{tabular}{|c|c|c|c|c|c|c|c|c|c|c|c|c|}
\hline DAY & $\mathrm{OCT}$ & NOV & DEC & JAN & FEB & MAR & $\mathrm{APR}$ & MAY & JUN & JUL & AUG & SEP \\
\hline 1 & .00 & .00 & .00 & .00 & 5.9 & 68 & 12 & 30 & 6.7 & .00 & .00 & .00 \\
\hline 2 & .00 & .00 & .00 & .00 & 5.9 & 39 & 11 & 29 & 7.1 & .00 & .00 & .00 \\
\hline 3 & .00 & .00 & .00 & .00 & 7.1 & 56 & 10 & 25 & 6.9 & .00 & .00 & .00 \\
\hline 4 & .00 & .00 & .00 & .00 & 7.9 & 39 & 9.2 & 25 & 6.3 & .00 & .00 & .00 \\
\hline 5 & .00 & .00 & .00 & .00 & 7.1 & 29 & 9.5 & 32 & 5.1 & .00 & .00 & .00 \\
\hline 6 & .00 & .00 & .00 & .00 & 6.7 & 24 & 8.6 & 36 & 4.3 & .00 & .00 & .00 \\
\hline 7 & .00 & .00 & .00 & .00 & 64 & 21 & 7.5 & 36 & 3.5 & .00 & .00 & .00 \\
\hline 8 & .00 & .00 & .00 & .00 & 61 & 18 & 6.9 & 32 & 2.9 & .00 & .00 & .00 \\
\hline 9 & .00 & .00 & .00 & .00 & 89 & 17 & 6.5 & 29 & .73 & .00 & .00 & .00 \\
\hline 10 & .00 & .00 & .00 & .00 & 46 & 14 & 5.9 & 28 & .00 & .00 & .00 & .00 \\
\hline 11 & .00 & .00 & .00 & .00 & 31 & 13 & 6.7 & 30 & .00 & .00 & .00 & .00 \\
\hline 12 & .00 & .00 & .00 & .00 & 25 & 13 & 12 & 33 & .00 & .00 & .00 & .00 \\
\hline 13 & .00 & .00 & .00 & .00 & 21 & 14 & 20 & 29 & .00 & .00 & .00 & .00 \\
\hline 14 & .00 & .00 & .00 & .00 & 19 & 14 & 26 & 25 & .00 & .00 & .00 & .00 \\
\hline 15 & .00 & .00 & .00 & .00 & 16 & 14 & 28 & 23 & .00 & .00 & .00 & .00 \\
\hline 16 & .00 & .00 & .00 & .00 & 19 & 15 & 33 & 22 & .00 & .00 & .00 & .00 \\
\hline 17 & .00 & .00 & .00 & 21 & 69 & 18 & 38 & 22 & .00 & .00 & .00 & .00 \\
\hline 18 & .00 & .00 & .00 & 57 & 41 & 20 & 41 & 22 & .00 & .00 & .00 & .00 \\
\hline 19 & .00 & .00 & .00 & 63 & 26 & 19 & 43 & 22 & .00 & .00 & .00 & .00 \\
\hline 20 & .00 & .00 & .00 & 92 & 21 & 18 & 43 & 21 & .00 & .00 & .00 & .00 \\
\hline 21 & .00 & .00 & .00 & 42 & 18 & 15 & 38 & 21 & .00 & .00 & .00 & .00 \\
\hline 22 & .00 & .00 & .00 & 25 & 16 & 14 & 34 & 21 & .00 & .00 & .00 & .00 \\
\hline 23 & .00 & .00 & .00 & 44 & 14 & 14 & 32 & 21 & .00 & .00 & .00 & .00 \\
\hline 24 & .00 & .00 & .00 & 24 & 14 & 14 & 33 & 23 & .00 & .00 & .00 & .00 \\
\hline 25 & .00 & .00 & .00 & 18 & 13 & 16 & 37 & 21 & .00 & .00 & .00 & .00 \\
\hline 26 & .00 & .00 & .00 & 14 & 11 & 21 & 41 & 18 & .00 & .00 & .00 & .00 \\
\hline 27 & .00 & .00 & .00 & 11 & 12 & 21 & 36 & 14 & .00 & .00 & .00 & .00 \\
\hline 28 & .00 & .00 & .00 & 9.5 & 28 & 18 & 29 & 12 & .00 & .00 & .00 & .00 \\
\hline 29 & .00 & .00 & .00 & 8.3 & --- & 16 & 26 & 10 & .00 & .00 & .00 & .00 \\
\hline 30 & .00 & .00 & .00 & 7.5 & --- & 15 & 27 & 9.0 & .00 & .00 & .00 & .00 \\
\hline 31 & .00 & --- & .00 & 6.7 & --- & 13 & --- & 7.7 & --- & .00 & .00 & --- \\
\hline TOTAL & 0.00 & 0.00 & 0.00 & 443.00 & 714.6 & 660 & 710.8 & 728.7 & 43.53 & 0.00 & 0.00 & 0.00 \\
\hline MEAN & .000 & .000 & .000 & 14.3 & 25.5 & 21.3 & 23.7 & 23.5 & 1.45 & .000 & .000 & .000 \\
\hline MAX & .00 & .00 & .00 & 92 & 89 & 68 & 43 & 36 & 7.1 & .00 & .00 & .00 \\
\hline MIN & .00 & .00 & .00 & .00 & 5.9 & 13 & 5.9 & 7.7 & .00 & .00 & .00 & .00 \\
\hline $\mathrm{AC}-\mathrm{FT}$ & .00 & .00 & .00 & 879 & 1420 & 1310 & 1410 & 1450 & 86 & .00 & .00 & .00 \\
\hline
\end{tabular}

STATISTICS OF MONTHLY MEAN DATA FOR WATER YEARS 1966 - 1999, BY WATER YEAR (WY)

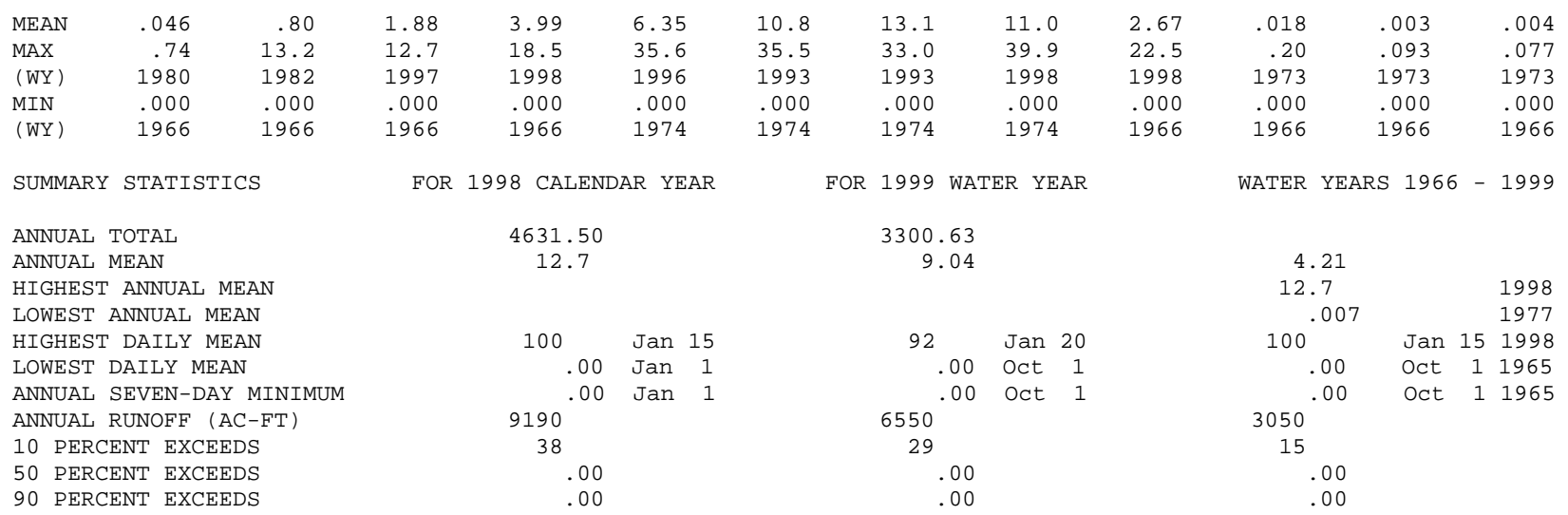


LOCATION.-Lat 3902'57", long 120²8'56", in SW 1/4 NW 1/4 sec.24, T.14 N., R.13 E., Placer County, Hydrologic Unit 18020128, Eldorado National Forest, on right bank $26 \mathrm{ft}$ below diversion dam, $3.2 \mathrm{mi}$ upstream from confluence of North and South Forks Long Canyon Creek, and 16.9 mi east of Volcanoville.

PERIOD OF RECORD.-October 1988 to current year.

GAGE.-Water-stage recorder. Elevation of gage is 4,700 ft above sea level, from topographic map.

REMARKS.-Discharge is computed only during periods of operation of North Fork Long Canyon Creek Diversion Tunnel (station 11433080). See schematic diagram of Middle Fork American and Rubicon River Basins.

COOPERATION.--Records provided by Placer County Water Agency, under general supervision of the U.S. Geological Survey, in connection with a Federal Energy Regulatory Commission project.

DISCHARGE, CUBIC FEET PER SECOND, WATER YEAR OCTOBER 1998 TO SEPTEMBER 1999 DAILY MEAN VALUES

\begin{tabular}{|c|c|c|c|c|c|c|c|c|c|c|c|c|}
\hline DAY & $\mathrm{OCT}$ & NOV & $\mathrm{DEC}$ & JAN & FEB & MAR & APR & MAY & JUN & JUL & AUG & SEP \\
\hline 1 & --- & --- & --- & --- & 3.9 & 4.8 & 3.7 & 4.1 & 3.3 & --- & --- & --- \\
\hline 2 & --- & --- & --- & --- & 3.5 & 4.1 & 3.6 & 4.0 & 3.4 & --- & --- & --- \\
\hline 3 & --- & --- & --- & --- & 3.4 & 4.5 & 3.6 & 3.9 & 3.4 & --- & --- & --- \\
\hline 4 & --- & --- & --- & --- & 3.5 & 4.2 & 3.6 & 3.9 & 3.3 & --- & --- & --- \\
\hline 5 & --- & --- & --- & --- & 3.5 & 3.9 & 3.6 & 4.1 & 3.3 & --- & --- & --- \\
\hline 6 & --- & --- & --- & --- & 3.5 & 3.8 & 3.6 & 4.2 & 3.3 & --- & --- & --- \\
\hline 7 & --- & --- & --- & --- & 5.4 & 3.7 & 3.5 & 4.2 & 3.2 & --- & --- & --- \\
\hline 8 & --- & --- & --- & --- & 5.1 & 3.6 & 3.5 & 4.1 & 3.1 & --- & --- & --- \\
\hline 9 & --- & --- & --- & --- & 7.7 & 3.6 & 3.5 & 4.1 & 3.8 & --- & --- & --- \\
\hline 10 & --- & --- & --- & --- & 6.2 & 3.5 & 3.4 & 4.1 & --- & --- & --- & --- \\
\hline 11 & --- & --- & --- & --- & 5.6 & 3.5 & 3.4 & 4.2 & --- & --- & --- & --- \\
\hline 12 & --- & --- & --- & --- & 5.3 & 3.4 & 3.6 & 4.2 & --- & --- & --- & --- \\
\hline 13 & --- & --- & --- & --- & 5.1 & 3.4 & 3.8 & 4.1 & --- & --- & --- & --- \\
\hline 14 & --- & --- & --- & --- & 4.1 & 3.4 & 4.0 & 4.0 & --- & --- & --- & --- \\
\hline 15 & --- & --- & --- & --- & 3.8 & 3.4 & 4.1 & 4.0 & --- & --- & --- & --- \\
\hline 16 & --- & --- & --- & --- & 3.9 & 3.5 & 4.2 & 4.0 & --- & --- & --- & --- \\
\hline 17 & --- & --- & --- & 4.7 & 5.1 & 3.5 & 4.4 & 3.9 & --- & --- & --- & --- \\
\hline 18 & --- & --- & --- & 4.8 & 4.2 & 3.7 & 4.4 & 3.9 & --- & --- & --- & --- \\
\hline 19 & --- & --- & --- & 4.8 & 4.0 & 3.8 & 4.5 & 3.8 & --- & --- & --- & --- \\
\hline 20 & --- & --- & --- & 7.4 & 3.9 & 3.7 & 4.5 & 3.7 & --- & --- & --- & --- \\
\hline 21 & --- & --- & --- & 5.7 & 3.8 & 3.7 & 4.4 & 3.7 & --- & --- & --- & --- \\
\hline 22 & --- & --- & --- & 5.0 & 3.8 & 3.6 & 4.2 & 3.7 & --- & --- & --- & --- \\
\hline 23 & --- & --- & --- & 5.5 & 3.8 & 3.6 & 4.2 & 3.8 & --- & --- & --- & --- \\
\hline 24 & --- & --- & --- & 4.8 & 3.7 & 3.6 & 4.2 & 3.8 & --- & --- & --- & --- \\
\hline 25 & --- & --- & --- & 4.5 & 3.7 & 3.7 & 4.4 & 3.8 & --- & --- & --- & --- \\
\hline 26 & --- & --- & --- & 4.4 & 3.7 & 3.8 & 4.5 & 3.7 & --- & --- & --- & --- \\
\hline 27 & --- & --- & --- & 4.1 & 3.6 & 3.9 & 4.4 & 3.6 & --- & --- & --- & --- \\
\hline 28 & --- & --- & --- & 4.0 & 3.9 & 3.8 & 4.1 & 3.6 & --- & --- & --- & --- \\
\hline 29 & --- & --- & --- & 4.0 & --- & 3.8 & 4.0 & 3.5 & --- & --- & --- & --- \\
\hline 30 & --- & --- & --- & 4.0 & --- & 3.8 & 4.0 & 3.4 & --- & --- & --- & --- \\
\hline 31 & --- & --- & --- & 3.9 & --- & 3.7 & --- & 3.4 & --- & --- & --- & --- \\
\hline TOTAL & --- & --- & --- & --- & 120.7 & 116.0 & 118.9 & 120.5 & --- & --- & --- & --- \\
\hline MEAN & --- & --- & --- & --- & 4.31 & 3.74 & 3.96 & 3.89 & --- & --- & --- & --- \\
\hline MAX & --- & --- & --- & --- & 7.7 & 4.8 & 4.5 & 4.2 & --- & --- & --- & --- \\
\hline MIN & --- & --- & --- & --- & 3.4 & 3.4 & 3.4 & 3.4 & --- & --- & --- & --- \\
\hline $\mathrm{AC}-\mathrm{FT}$ & --- & --- & --- & --- & 239 & 230 & 236 & 239 & --- & --- & --- & --- \\
\hline
\end{tabular}




\section{MIDDLE FORK AMERICAN RIVER NEAR FORESTHILL, CA}

LOCATION.-Lat 3900'22", long 12045'35", in NW 1/4 NW 1/4 sec.4, T.13 N., R.11 E., Placer County, Hydrologic Unit 18020128, Tahoe National Forest, on right bank $1.6 \mathrm{mi}$ downstream from Oxbow Powerplant and $3.3 \mathrm{mi}$ east of Foresthill.

DRAINAGE AREA.- $524 \mathrm{mi}^{2}$.

PERIOD OF RECORD.—October 1958 to current year.

CHEMICAL DATA: Water year 1979.

BIOLOGICAL DATA: Water year 1979.

GAGE.-Water-stage recorder. Elevation of gage is 1,070 ft above sea level, from topographic map. Prior to Oct. 22, 1965, at site $3.2 \mathrm{mi}$ downstream at different datum. Oct. 22, 1965, to Aug. 28, 1985, at site $400 \mathrm{ft}$ downstream at different datum.

REMARKS.-Flow regulated by French Meadows Reservoir, Hell Hole Reservoir, Loon Lake (stations 11427400, 11428700, and 11429350), Stumpy Meadows Lake, usable capacity, 17,500 acre-ft, and several smaller reservoirs. Robbs Peak Powerplant (station 11429300) and Georgetown Divide Ditch, capacity about $60 \mathrm{ft}^{3} / \mathrm{s}$, divert water out of basin upstream from station. See schematic diagrams of lower Sacramento River Basin and Middle Fork American and Rubicon River Basins.

COOPERATION.--Records provided by Placer County Water Agency, under general supervision of the U.S. Geological Survey, in connection with a Federal Energy Regulatory Commission project.

EXTREMES FOR PERIOD OF RECORD.-Maximum discharge, 310,000 ft 3 /s, Dec. 23, 1964, gage height, $69.0 \mathrm{ft}$ from floodmarks, site and datum then in use, caused by overtopping of the partly constructed Hell Hole Dam on the Rubicon River, from rating curve extended above $28,000 \mathrm{ft}^{3} / \mathrm{s}$ on basis of slope-area measurement at gage height $38.0 \mathrm{ft}$ and slope-conveyance study at gage height $69.0 \mathrm{ft}$, at site and datum then in use; next highest peak, $123,000 \mathrm{ft}^{3} / \mathrm{s}$, Jan. 2, 1997, gage height, $29.56 \mathrm{ft}$, from rating curve extended above $37,000 \mathrm{ft}^{3} / \mathrm{s} ;$ minimum, $35 \mathrm{ft}^{3} / \mathrm{s}$, Oct. $10-20,1961$.

DISCHARGE, CUBIC FEET PER SECOND, WATER YEAR OCTOBER 1998 TO SEPTEMBER 1999 DAILY MEAN VALUES

\begin{tabular}{|c|c|c|c|c|c|c|c|c|c|c|c|c|}
\hline DAY & OCT & NOV & $\mathrm{DEC}$ & JAN & FEB & MAR & APR & MAY & JUN & JUL & AUG & SEP \\
\hline 1 & 182 & 641 & 1760 & 860 & 1110 & 4440 & 1640 & 1890 & 1040 & 1120 & 731 & 919 \\
\hline 2 & 171 & 643 & 1290 & 816 & 1210 & 3670 & 1720 & 1870 & 846 & 1120 & 889 & 909 \\
\hline 3 & 171 & 623 & 1730 & 821 & 1200 & 4290 & 1620 & 2130 & 1070 & 1170 & 809 & 1070 \\
\hline 4 & 171 & 593 & 1650 & 815 & 1110 & 3910 & 1530 & 2020 & 1050 & 876 & 713 & 882 \\
\hline 5 & 166 & 624 & 999 & 852 & 1140 & 3340 & 1720 & 1920 & 974 & 926 & 616 & 824 \\
\hline 6 & 162 & 626 & 878 & 909 & 1310 & 2940 & 1670 & 1870 & 926 & 1120 & 853 & 735 \\
\hline 7 & 159 & 697 & 817 & 821 & 5430 & 2640 & 1590 & 1880 & 872 & 1060 & 710 & 766 \\
\hline 8 & 160 & 760 & 817 & 782 & 6240 & 2700 & 1770 & 1830 & 922 & 1070 & 734 & 900 \\
\hline 9 & 159 & 663 & 737 & 716 & 11900 & 2700 & 1750 & 1720 & 827 & 1050 & 863 & 814 \\
\hline 10 & 159 & 641 & 770 & 617 & 5790 & 2450 & 1640 & 1760 & 992 & 922 & 844 & 831 \\
\hline 11 & 160 & 667 & 745 & 726 & 4080 & 2320 & 1840 & 1780 & 1010 & 941 & 955 & 733 \\
\hline 12 & 160 & 657 & 912 & 662 & 3280 & 2250 & 2020 & 1890 & 1150 & 945 & 858 & 765 \\
\hline 13 & 160 & 629 & 910 & 656 & 2850 & 2240 & 2010 & 1830 & 1200 & 820 & 772 & 866 \\
\hline 14 & 153 & 637 & 1020 & 608 & 2590 & 2340 & 2280 & 1730 & 1210 & 869 & 721 & 878 \\
\hline 15 & 147 & 631 & 1040 & 679 & 2380 & 1920 & 2410 & 1710 & 1210 & 797 & 725 & 868 \\
\hline 16 & 146 & 613 & 1100 & 938 & 2530 & 2190 & 2360 & 1660 & 1260 & 770 & 779 & 867 \\
\hline 17 & 146 & 700 & 1110 & 994 & 5170 & 2040 & 2500 & 1600 & 1270 & 699 & 861 & 849 \\
\hline 18 & 144 & 711 & 1200 & 2510 & 4070 & 1880 & 2430 & 1600 & 1250 & 588 & 921 & 814 \\
\hline 19 & 142 & 667 & 1060 & 3570 & 3730 & 2040 & 2470 & 1620 & 1260 & 709 & 920 & 500 \\
\hline 20 & 140 & 642 & 1280 & 7250 & 3320 & 2030 & 2440 & 1540 & 1220 & 595 & 968 & 164 \\
\hline 21 & 138 & 637 & 1270 & 4440 & 3140 & 1870 & 2200 & 1430 & 1210 & 872 & 780 & 173 \\
\hline 22 & 131 & 752 & 1260 & 2750 & 2930 & 1930 & 2150 & 1460 & 1220 & 723 & 792 & 169 \\
\hline 23 & 115 & 811 & 1210 & 5170 & 2750 & 1900 & 2080 & 1450 & 1220 & 770 & 873 & 175 \\
\hline 24 & 114 & 1300 & 1260 & 3240 & 2760 & 1900 & 2010 & 1480 & 1190 & 733 & 923 & 179 \\
\hline 25 & 129 & 820 & 1060 & 2340 & 2910 & 1900 & 1990 & 1650 & 1210 & 554 & 909 & 170 \\
\hline 26 & 110 & 737 & 1100 & 1970 & 2780 & 1850 & 2080 & 1540 & 1200 & 746 & 852 & 159 \\
\hline 27 & 107 & 722 & 767 & 1810 & 2440 & 1770 & 2040 & 1460 & 1160 & 689 & 983 & 142 \\
\hline 28 & 114 & 740 & 807 & 1610 & 2530 & 1690 & 1900 & 1390 & 1160 & 872 & 993 & 116 \\
\hline 29 & 303 & 794 & 970 & 1540 & --- & 1670 & 1580 & 1210 & 1180 & 829 & 836 & 109 \\
\hline 30 & 618 & 1980 & 1170 & 1490 & --- & 1760 & 1610 & 1110 & 1180 & 863 & 940 & 111 \\
\hline 31 & 613 & --- & 1100 & 1440 & --- & 1780 & --- & 1120 & --- & 760 & 986 & --- \\
\hline TOTAL & 5650 & 22358 & 33799 & 54402 & 92680 & 74350 & 59050 & 51150 & 33489 & 26578 & 26109 & 17457 \\
\hline MEAN & 182 & 745 & 1090 & 1755 & 3310 & 2398 & 1968 & 1650 & 1116 & 857 & 842 & 582 \\
\hline MAX & 618 & 1980 & 1760 & 7250 & 11900 & 4440 & 2500 & 2130 & 1270 & 1170 & 993 & 1070 \\
\hline MIN & 107 & 593 & 737 & 608 & 1110 & 1670 & 1530 & 1110 & 827 & 554 & 616 & 109 \\
\hline $\mathrm{AC}-\mathrm{FT}$ & 11210 & 44350 & 67040 & 107900 & 183800 & 147500 & 117100 & 101500 & 66430 & 52720 & 51790 & 34630 \\
\hline
\end{tabular}


11433300 MIDDLE FORK AMERICAN RIVER NEAR FORESTHILL, CA—Continued

STATISTICS OF MONTHLY MEAN DATA FOR WATER YEARS 1959 - 1999, BY WATER YEAR (WY)

\begin{tabular}{|c|c|c|c|c|c|c|c|c|c|c|c|c|}
\hline & $\mathrm{OCT}$ & NOV & $\mathrm{DEC}$ & JAN & FEB & MAR & APR & MAY & JUN & JUL & AUG & SEP \\
\hline MEAN & 449 & 656 & 1176 & 1684 & 1889 & 1869 & 1784 & 1577 & 1035 & 670 & 625 & 523 \\
\hline MAX & 1634 & 2952 & 7172 & 8778 & 8815 & 5076 & 5572 & 4642 & 3300 & 1836 & 1142 & 1084 \\
\hline (WY) & 1963 & 1984 & 1965 & 1997 & 1986 & 1983 & 1982 & 1963 & 1983 & 1983 & 1983 & 1983 \\
\hline MIN & 54.3 & 47.1 & 64.8 & 85.2 & 111 & 240 & 110 & 120 & 124 & 99.2 & 47.2 & 42.8 \\
\hline (WY) & 1961 & 1960 & 1960 & 1991 & 1991 & 1977 & 1977 & 1977 & 1977 & 1966 & 1959 & 1962 \\
\hline
\end{tabular}

SUMMARY STATISTICS

FOR 1998 CALENDAR YEAR

FOR 1999 WATER YEAR

587965

1611

ANNUAL MEAN

HIGHEST ANNUAL MEAN

LOWEST ANNUAL MEAN

HIGHEST DAILY MEAN

LOWEST DAILY MEAN

ANNUAL SEVEN-DAY MINIMUM

INSTANTANEOUS PEAK FLOW

INSTANTANEOUS PEAK STAGE

ANNUAL RUNOFF (AC-FT)

10 PERCENT EXCEEDS

50 PERCENT EXCEEDS

90 PERCENT EXCEEDS

9790

$9790 \quad$ Mar 24

117 Oct 27

1166000

2770

1290
WATER YEARS 1959 - 1999

1158
2723
179
65000
35
38
310000
69.00
838600
2430
763
97

$\begin{array}{lll} & & 1982 \\ & 1977 \\ \text { Dec } & 23 & 1964 \\ \text { Oct } & 19 & 1961 \\ \text { Oct } & 14 & 1961 \\ \text { Dec } & 23 & 1964 \\ \text { Dec } & 23 & 1964\end{array}$

1982
Feb 9

Det 27

20.06 Feb 9

985900

2500

174 $\begin{array}{lll}\text { Dec } & 23 & 1964 \\ \text { Dec } & 23 & 1964\end{array}$ 


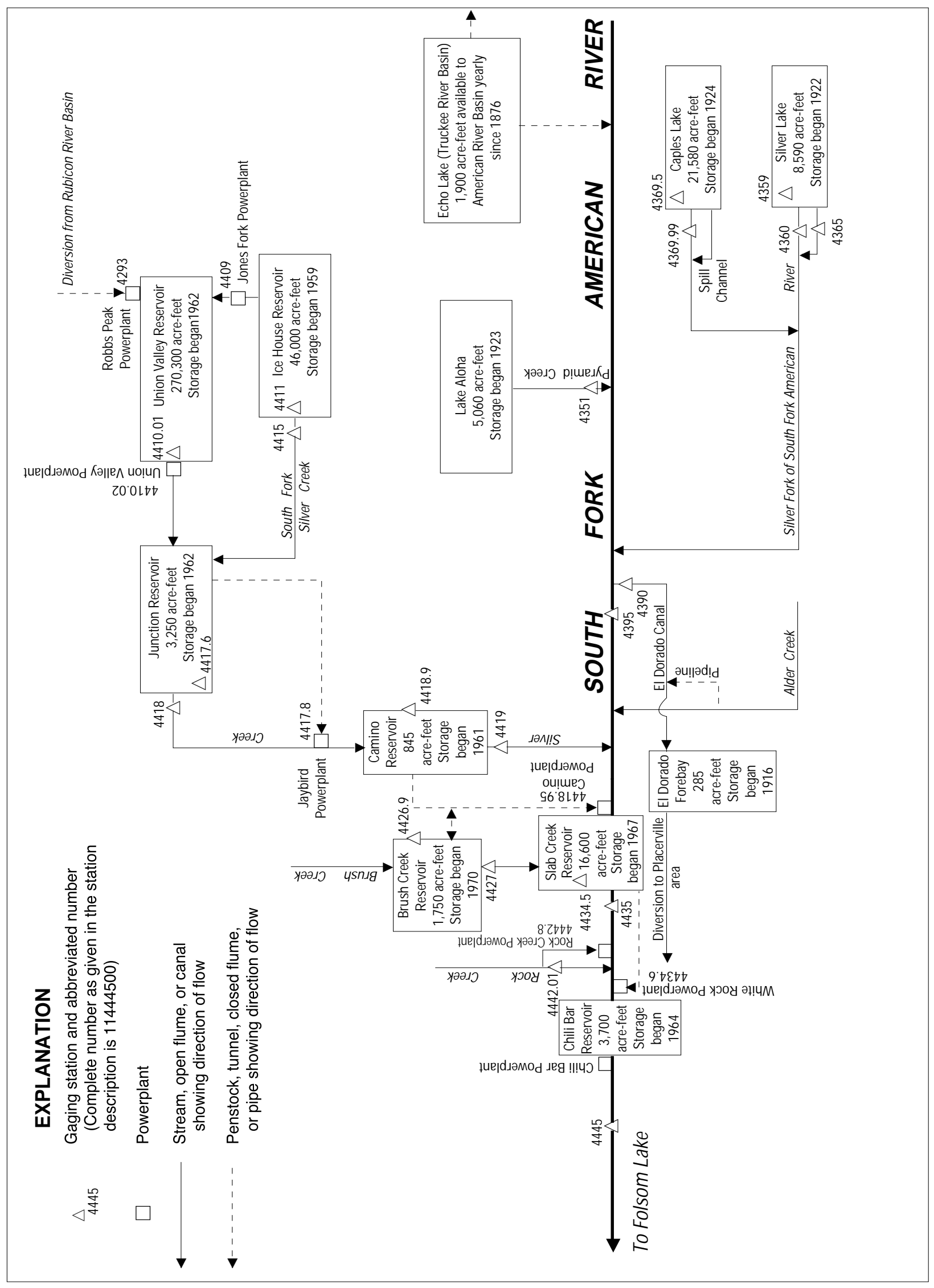


11433790 NORTH FORK AMERICAN RIVER AT AUBURN DAM SITE, NEAR AUBURN, CA

LOCATION.-Lat 39 $51^{\prime} 06^{\prime \prime}$, long 121 03 '26", in SW 1/4 NW 1/4 sec.23, T.12 N., R.8 E., Placer County, Hydrologic Unit 18020128, on right bank upstream side of the Auburn Dam Site diversion tunnel, 0.7 mi upstream from Knickerbocker Creek, and 1.3 mi southeast of Auburn. DRAINAGE AREA. - $972 \mathrm{mi}^{2}$.

PERIOD OF RECORD.-June to September 1999.

WATER TEMPERATURE.- - June to September 1999.

PERIOD OF DAILY RECORD.-June to September 1999.

WATER TEMPERATURE.-June to September 1999.

INSTRUMENTATION.-Water-temperature recorder since June 4, 1999.

REMARKS.-Water temperture can be affected by upstream releases. Interruption in record was due to the malfunction of the recording instrument. EXTREMES FOR PERIOD OF DAILY RECORD.-

WATER TEMPERATURE: Maximum recorded, $20.5^{\circ} \mathrm{C}$, July 12, 1999; minimum recorded, $12.5^{\circ} \mathrm{C}$, Sept. 2, 1999.

EXTREMES FOR CURRENT YEAR.-

WATER TEMPERATURE: Maximum recorded, $20.5^{\circ} \mathrm{C}$, July 12; minimum recorded, $12.5^{\circ} \mathrm{C}$, Sept. 2.

TEMPERATURE, WATER (DEG. C), WATER YEAR OCTOBER 1998 TO SEPTEMBER 1999

\begin{tabular}{|c|c|c|c|c|c|c|c|c|c|c|c|c|}
\hline \multirow[t]{2}{*}{ DAY } & MAX & MIN & MAX & MIN & MAX & MIN & MAX & MIN & MAX & MIN & MAX & MIN \\
\hline & \multicolumn{2}{|c|}{ APRIL } & \multicolumn{2}{|c|}{ MAY } & \multicolumn{2}{|c|}{ JUNE } & \multicolumn{2}{|c|}{ JULY } & \multicolumn{2}{|c|}{ AUGUST } & \multicolumn{2}{|c|}{ SEPTEMBER } \\
\hline 1 & --- & --- & --- & --- & --- & --- & 19.5 & 17.5 & --- & --- & --- & --- \\
\hline 2 & --- & --- & --- & --- & --- & --- & 19.5 & 17.5 & --- & --- & 15.5 & 12.5 \\
\hline 3 & --- & --- & --- & --- & --- & --- & 18.5 & 17.0 & --- & --- & -- & 13.0 \\
\hline 4 & --- & --- & --- & --- & --- & --- & 19.5 & 16.0 & --- & --- & 16.0 & 13.0 \\
\hline 5 & --- & --- & --- & --- & 15.0 & 13.0 & 20.0 & 17.0 & --- & --- & 17.5 & 14.0 \\
\hline 6 & --- & --- & --- & --- & 15.5 & 14.0 & 18.5 & 17.0 & --- & --- & 17.5 & 14.0 \\
\hline 7 & --- & --- & --- & --- & 16.0 & 14.5 & 18.5 & 16.5 & --- & --- & 18.0 & 14.5 \\
\hline 8 & --- & --- & --- & --- & 16.0 & 15.0 & 18.5 & 16.5 & --- & --- & 17.0 & 14.5 \\
\hline 9 & --- & --- & --- & --- & 16.0 & 14.5 & 18.5 & 16.5 & --- & --- & 16.5 & 14.5 \\
\hline 10 & --- & --- & --- & --- & 16.5 & 14.5 & --- & --- & --- & --- & 17.5 & 14.0 \\
\hline 11 & --- & --- & --- & --- & 16.5 & 15.0 & 20.0 & 17.5 & --- & --- & 17.5 & 14.5 \\
\hline 12 & --- & --- & --- & --- & 16.5 & 15.0 & 20.5 & 18.0 & 18.5 & 17.0 & 18.0 & 15.0 \\
\hline 13 & --- & --- & --- & --- & 17.0 & 15.5 & -- & -- & -- & -- & 18.0 & 15.0 \\
\hline 14 & --- & --- & --- & --- & 17.0 & 15.5 & --- & --- & --- & --- & 17.5 & 14.5 \\
\hline 15 & --- & --- & --- & --- & 17.0 & 16.0 & --- & --- & --- & --- & 17.0 & 14.5 \\
\hline 16 & --- & --- & --- & --- & 17.0 & 15.5 & --- & --- & --- & --- & 17.0 & 14.5 \\
\hline 17 & --- & --- & --- & --- & 17.5 & 15.5 & --- & --- & --- & --- & 17.0 & 14.0 \\
\hline 18 & --- & --- & --- & --- & 17.0 & 16.0 & --- & --- & --- & --- & 15.5 & 14.5 \\
\hline 19 & --- & --- & --- & --- & 17.5 & 15.5 & --- & --- & --- & --- & 17.0 & 14.0 \\
\hline 20 & --- & --- & --- & --- & 17.5 & 15.5 & --- & --- & --- & --- & --- & --- \\
\hline 21 & --- & --- & --- & --- & 18.0 & 16.0 & --- & --- & --- & --- & --- & --- \\
\hline 22 & --- & --- & --- & --- & 18.5 & 16.5 & --- & --- & --- & --- & --- & --- \\
\hline 23 & --- & --- & --- & --- & 19.0 & 17.0 & --- & --- & --- & --- & --- & --- \\
\hline 24 & --- & --- & --- & --- & 19.0 & 17.5 & --- & --- & --- & --- & --- & --- \\
\hline 25 & --- & --- & --- & --- & 19.0 & 17.5 & --- & --- & --- & --- & --- & --- \\
\hline 26 & --- & --- & --- & --- & 18.5 & 16.5 & --- & --- & --- & --- & --- & --- \\
\hline 27 & --- & --- & --- & --- & 18.5 & 16.5 & --- & --- & --- & --- & --- & --- \\
\hline 28 & --- & --- & --- & --- & 18.5 & 16.5 & --- & --- & --- & --- & --- & --- \\
\hline 29 & --- & --- & --- & --- & 18.5 & 17.0 & --- & --- & --- & --- & --- & --- \\
\hline 30 & --- & --- & --- & --- & 19.0 & 17.0 & --- & --- & --- & --- & --- & --- \\
\hline 31 & --- & --- & --- & --- & -- & -- & --- & --- & --- & --- & --- & --- \\
\hline MONTH & --- & --- & --- & --- & --- & --- & --- & --- & --- & --- & --- & --- \\
\hline
\end{tabular}




\section{PYRAMID CREEK AT TWIN BRIDGES, CA}

LOCATION.-Lat $38^{\circ} 48^{\prime} 57^{\prime \prime}$, long $120^{\circ} 06^{\prime} 58^{\prime \prime}$, in NW 1/4 SW 1/4 sec.9, T.11 N., R.17 E., El Dorado County, Hydrologic Unit 18020129 ,

Eldorado National Forest, on right bank 0.5 mi northeast of Twin Bridges, $2.2 \mathrm{mi}$ west of Phillips, and 3.6 mi downstream from Lake Aloha.

DRAINAGE AREA. $-8.76 \mathrm{mi}^{2}$.

PERIOD OF RECORD.-October 1970 to current year.

GAGE.-Water-stage recorder. Elevation of gage is 6,320 ft above sea level, from topographic map. Prior to October 1987, at datum 1.00 ft higher.

REMARKS.-Flow regulated by Lake Aloha, capacity, 5,060 acre-ft. Lake of the Woods, Ropi Lake, and Toem Lake (unknown capacities) also regulate at times. See schematic diagram of South Fork American River Basin.

COOPERATION.-Records were collected by the Pacific Gas \& Electric Co., under general supervision of the U.S. Geological Survey, in connection with a Federal Energy Regulatory Commission project.

EXTREMES FOR PERIOD OF RECORD.-Maximum discharge, 2,920 ft $\mathrm{ft}^{3} / \mathrm{s}$, Jan. 2, 1997, gage height, $7.22 \mathrm{ft}$, from rating curve extended above $300 \mathrm{ft}^{3} / \mathrm{s}$; minimum daily, $0.03 \mathrm{ft}^{3} / \mathrm{s}$, Oct. 26-28, 1992.

DISCHARGE, CUBIC FEET PER SECOND, WATER YEAR OCTOBER 1998 TO SEPTEMBER 1999

DAILY MEAN VALUES

\begin{tabular}{|c|c|c|c|c|c|c|c|c|c|c|c|c|}
\hline DAY & OCT & NOV & $\mathrm{DEC}$ & JAN & FEB & MAR & APR & MAY & JUN & JUL & AUG & SEP \\
\hline 1 & 48 & 18 & 34 & 10 & 12 & 19 & 15 & 50 & 164 & 114 & 27 & 50 \\
\hline 2 & 46 & 15 & 21 & 10 & 11 & 18 & 14 & 63 & 161 & 116 & 26 & 50 \\
\hline 3 & 45 & 10 & 43 & 9.9 & 12 & 19 & 13 & 43 & 122 & 108 & 26 & 49 \\
\hline 4 & 43 & 8.1 & 30 & 9.7 & 13 & 17 & 13 & 34 & 106 & 96 & 25 & 48 \\
\hline 5 & 41 & 7.1 & 24 & 9.7 & 11 & 16 & 13 & 42 & 111 & 88 & 25 & 48 \\
\hline 6 & 37 & 6.8 & 22 & 9.6 & 11 & 15 & 14 & 97 & 147 & 84 & 25 & 47 \\
\hline 7 & 33 & 9.4 & 21 & 9.1 & 16 & 14 & 13 & 126 & 142 & 83 & 25 & 46 \\
\hline 8 & 13 & 10 & 20 & 8.8 & 23 & 14 & 14 & 105 & 128 & 82 & 24 & 43 \\
\hline 9 & 7.7 & 9.4 & 19 & 8.6 & 26 & 15 & 20 & 91 & 128 & 79 & 24 & 15 \\
\hline 10 & 6.8 & 8.0 & 18 & 8.6 & 24 & 15 & 18 & 90 & 134 & 77 & 24 & 9.5 \\
\hline 11 & 6.5 & 9.0 & 16 & 8.8 & 20 & 14 & 16 & 112 & 149 & 76 & 24 & 8.9 \\
\hline 12 & 6.2 & 8.4 & 15 & 8.7 & 17 & 14 & 14 & 139 & 159 & 76 & 25 & 8.7 \\
\hline 13 & 6.2 & 9.4 & 14 & 8.7 & 16 & 15 & 19 & 127 & 173 & 76 & 40 & 8.8 \\
\hline 14 & 6.2 & 11 & 14 & 8.6 & 15 & 15 & 28 & 98 & 177 & 75 & 46 & 8.8 \\
\hline 15 & 6.1 & 11 & 13 & 14 & 15 & 14 & 31 & 87 & 192 & 73 & 49 & 8.8 \\
\hline 16 & 6.0 & 10 & 15 & 19 & 15 & 14 & 38 & 96 & 185 & 69 & 50 & 8.7 \\
\hline 17 & 5.7 & 11 & 16 & 26 & 22 & 17 & 52 & 118 & 174 & 65 & 50 & 8.7 \\
\hline 18 & 5.5 & 11 & 16 & 32 & 17 & 21 & 65 & 130 & 173 & 63 & 54 & 8.6 \\
\hline 19 & 5.5 & 9.3 & 13 & 28 & 17 & 21 & 76 & 132 & 164 & 60 & 55 & 8.6 \\
\hline 20 & 5.3 & 8.3 & 15 & 27 & 15 & 18 & 74 & 135 & 160 & 57 & 55 & 8.6 \\
\hline 21 & 5.2 & 8.6 & 20 & 22 & 17 & 16 & 63 & 146 & 163 & 38 & 55 & 9.1 \\
\hline 22 & 5.2 & 12 & 20 & 17 & 16 & 15 & 45 & 162 & 150 & 33 & 55 & 51 \\
\hline 23 & 5.1 & 17 & 20 & 16 & 15 & 15 & 33 & 176 & 155 & 32 & 56 & 60 \\
\hline 24 & 5.8 & 19 & 20 & 21 & 14 & 16 & 33 & 175 & 157 & 31 & 55 & 60 \\
\hline 25 & 7.3 & 14 & 20 & 19 & 15 & 18 & 53 & 196 & 132 & 31 & 54 & 59 \\
\hline 26 & 9.2 & 12 & 20 & 19 & 16 & 26 & 67 & 193 & 113 & 30 & 54 & 58 \\
\hline 27 & 11 & 11 & 20 & 18 & 15 & 26 & 53 & 188 & 104 & 30 & 53 & 57 \\
\hline 28 & 9.7 & 10 & 19 & 17 & 16 & 22 & 43 & 182 & 103 & 30 & 52 & 55 \\
\hline 29 & 9.2 & 14 & 15 & 16 & --- & 19 & 32 & 161 & 108 & 29 & 52 & 54 \\
\hline 30 & 11 & 52 & 11 & 15 & --- & 17 & 32 & 156 & 111 & 28 & 51 & 53 \\
\hline 31 & 10 & --- & 11 & 13 & --- & 16 & --- & 168 & --- & 28 & 51 & --- \\
\hline TOTAL & 468.4 & 369.8 & 595 & 467.8 & 452 & 531 & 1014 & 3818 & 4345 & 1957 & 1287 & 1008.8 \\
\hline MEAN & 15.1 & 12.3 & 19.2 & 15.1 & 16.1 & 17.1 & 33.8 & 123 & 145 & 63.1 & 41.5 & 33.6 \\
\hline MAX & 48 & 52 & 43 & 32 & 26 & 26 & 76 & 196 & 192 & 116 & 56 & 60 \\
\hline MIN & 5.1 & 6.8 & 11 & 8.6 & 11 & 14 & 13 & 34 & 103 & 28 & 24 & 8.6 \\
\hline$A C-F T$ & 929 & 733 & 1180 & 928 & 897 & 1050 & 2010 & 7570 & 8620 & 3880 & 2550 & 2000 \\
\hline
\end{tabular}

STATISTICS OF MONTHLY MEAN DATA FOR WATER YEARS 1971 - 1999, BY WATER YEAR (WY)

\begin{tabular}{|c|c|c|c|c|c|c|c|c|c|c|c|c|}
\hline MEAN & 12.1 & 18.2 & 16.6 & 20.5 & 18.1 & 24.8 & 40.5 & 96.1 & 104 & 71.3 & 44.9 & 18.4 \\
\hline MAX & 35.8 & 57.1 & 53.2 & 133 & 55.6 & 63.2 & 70.2 & 160 & 249 & 198 & 90.2 & 77.4 \\
\hline (WY) & 1996 & 1997 & 1997 & 1997 & 1982 & 1982 & 1997 & 1974 & 1998 & 1995 & 1974 & 1983 \\
\hline MIN & .18 & .74 & 1.93 & 2.25 & 3.54 & 7.13 & 14.7 & 29.5 & 18.4 & 32.3 & 2.52 & .28 \\
\hline (WY) & 1991 & 1991 & 1991 & 1991 & 1991 & 1977 & 1975 & 1977 & 1987 & 1991 & 1981 & 1981 \\
\hline
\end{tabular}

SUMMARY STATISTICS

ANNUAL TOTAL

ANNUAL MEAN

HIGHEST ANNUAL MEAN

LOWEST ANNUAL MEAN

HIGHEST DAILY MEAN

LOWEST DAILY MEAN

ANNUAL SEVEN-DAY MINIMUM

INSTANTANEOUS PEAK FLOW

INSTANTANEOUS PEAK STAGE

ANNUAL RUNOFF (AC-FT)

10 PERCENT EXCEEDS

50 PERCENT EXCEEDS

90 PERCENT EXCEEDS
FOR 1998 CALENDAR YEAR

$$
\begin{aligned}
& 21982.2 \\
& 60.2 \\
& 350 \text { Jun } 25 \\
& 5.1 \text { Oct } 23 \\
& 43600 \\
& 167 \\
& \begin{array}{l}
30 \\
10
\end{array}
\end{aligned}
$$

FOR 1999 WATER YEAR

$$
\begin{array}{ccc}
16313.8 & & \\
44.7 & & \\
& & \\
196 & \text { May } 25 \\
5.1 & \text { Oct } 23 \\
5.4 & \text { Oct } 17 \\
237 & \text { May } 25 \\
3.93 & \text { May } 25 \\
32360 & & \\
128 & & \\
21 & & \\
8.8 & &
\end{array}
$$

WATER YEARS 1971 - 1999

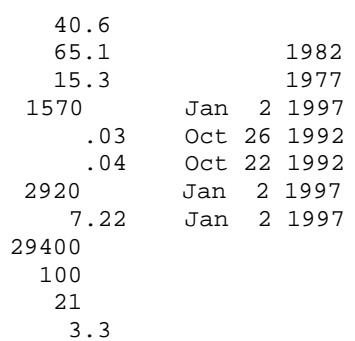


LOCATION.—Lat $38^{\circ} 40^{\prime} 07^{\prime \prime}$, long 12007' 14", in NW 1/4 SE 1/4 sec.32, T.10 N., R.17 E., Amador County, Hydrologic Unit 18020129, Eldorado National Forest, on outlet structure, 3.5 mi southwest of Kirkwood.

DRAINAGE AREA.- $-15.2 \mathrm{mi}^{2}$.

PERIOD OF RECORD.-October 1985 to current year. Unpublished records for water years 1981-85 available in files of U.S. Geological Survey.

GAGE.-Water-stage recorder. Datum of gage is 7,184.3 ft above sea level (levels by Pacific Gas \& Electric Co.). October 1985 to Mar. 5 , 1991, nonrecording gage at same site and datum.

REMARKS. - Lake is formed by earthfill and rock masonry dam initially constructed in 1876 and enlarged in 1929. Capacity, 8,590 acre-ft between gage heights $0.0 \mathrm{ft}$, invert of outlet, and $22.7 \mathrm{ft}$, top of radial gates and flashboards. Released water is used for power development on South Fork American River. See schematic diagram of South Fork American River Basin.

COOPERATION.-Records were collected by Pacific Gas \& Electric Co., under general supervision of the U.S. Geological Survey, in connection with a Federal Energy Regulatory Commission project. Contents not rounded to U.S. Geological Survey standards.

EXTREMES FOR PERIOD OF RECORD.-Maximum contents, 8,791 acre-ft, June 3, 1996, gage height, $23.10 \mathrm{ft}$; minimum, 0 acre-ft, Feb. 13, 15, 20, 22, 27, 1991, gage height, $0 \mathrm{ft}$.

EXTREMES FOR CURRENT YEAR.-Maximum contents, 8,746 acre-ft, June 29, gage height, $23.01 \mathrm{ft}$; minimum, 977 acre-ft, Jan. 10-14, gage height, $3.51 \mathrm{ft}$.

Capacity table (gage height, in feet, and contents, in acre-feet)

(Based on table provided by Pacific Gas \& Electric Co., recomputed Oct. 1, 1989)

$\begin{array}{rrrr}0.0 & 0 & 12.0 & 3,840 \\ 2.0 & 540 & 15.0 & 5,010 \\ 4.0 & 1,120 & 18.0 & 6,350 \\ 6.0 & 1,720 & 21.0 & 7,740 \\ 9.0 & 2,730 & 24.0 & 9,241\end{array}$

RESERVOIR STORAGE (ACRE-FEET), WATER YEAR OCTOBER 1998 TO SEPTEMBER 1999 DAILY OBSERVATION AT 2400 HOURS

\begin{tabular}{|c|c|c|c|c|c|c|c|c|c|c|c|c|}
\hline DAY & OCT & NOV & $\mathrm{DEC}$ & JAN & FEB & MAR & $A P R$ & MAY & JUN & JUL & AUG & SEP \\
\hline 1 & 5263 & 2547 & 1357 & 1038 & 1156 & 1111 & 1278 & 3289 & 6000 & 8665 & 7712 & 6547 \\
\hline 2 & 5151 & 2460 & 1357 & 1024 & 1147 & 1114 & 1266 & 3419 & 6170 & 8670 & 7669 & 6515 \\
\hline 3 & 5057 & 2377 & 1387 & 1018 & 1138 & 1141 & 1263 & 3456 & 6262 & 8670 & 7627 & 6483 \\
\hline 4 & 4958 & 2291 & 1378 & 1015 & 1138 & 1129 & 1236 & 3453 & 6290 & 8650 & 7584 & 6451 \\
\hline 5 & 4861 & 2206 & 1351 & 1006 & 1135 & 1123 & 1254 & 3513 & 6405 & 8635 & 7537 & 6414 \\
\hline 6 & 4813 & 2124 & 1348 & 1001 & 1171 & 1117 & 1248 & 3802 & 6657 & 8640 & 7499 & 6382 \\
\hline 7 & 4793 & 2070 & 1309 & 992 & 1213 & 1105 & 1242 & 4140 & 6869 & 8650 & 7457 & 6350 \\
\hline 8 & 4777 & 1995 & 1275 & 986 & 1245 & 1123 & 1260 & 4274 & 7039 & 8660 & 7415 & 6318 \\
\hline 9 & 4757 & 1921 & 1248 & 983 & 1275 & 1135 & 1242 & 4290 & 7256 & 8645 & 7387 & 6253 \\
\hline 10 & 4745 & 1847 & 1236 & 977 & 1248 & 1120 & 1228 & 4294 & 7547 & 8620 & 7368 & 6170 \\
\hline 11 & 4725 & 1784 & 1219 & 977 & 1228 & 1108 & 1210 & 4403 & 7899 & 8605 & 7335 & 6087 \\
\hline 12 & 4650 & 1720 & 1213 & 977 & 1213 & 1108 & 1192 & 4551 & 8285 & 8585 & 7298 & 5995 \\
\hline 13 & 4528 & 1651 & 1198 & 977 & 1201 & 1108 & 1204 & 4528 & 8575 & 8565 & 7256 & 5913 \\
\hline 14 & 4407 & 1591 & 1180 & 977 & 1180 & 1103 & 1245 & 4407 & 8575 & 8540 & 7214 & 5826 \\
\hline 15 & 4282 & 1531 & 1186 & 1012 & 1165 & 1091 & 1296 & 4329 & 8505 & 8500 & 7177 & 5745 \\
\hline 16 & 4166 & 1474 & 1177 & 1021 & 1186 & 1091 & 1372 & 4313 & 8395 & 8455 & 7141 & 5668 \\
\hline 17 & 4045 & 1438 & 1183 & 1038 & 1174 & 1100 & 1489 & 4434 & 8445 & 8420 & 7099 & 5592 \\
\hline 18 & 3934 & 1381 & 1195 & 1117 & 1174 & 1108 & 1654 & 4489 & 8585 & 8375 & 7058 & 5511 \\
\hline 19 & 3809 & 1336 & 1195 & 1186 & 1153 & 1123 & 1883 & 4547 & 8635 & 8330 & 7021 & 5440 \\
\hline 20 & 3687 & 1284 & 1186 & 1210 & 1171 & 1135 & 2124 & 4586 & 8630 & 8275 & 6984 & 5375 \\
\hline 21 & 3585 & 1245 & 1180 & 1207 & 1168 & 1141 & 2329 & 4690 & 8610 & 8210 & 6943 & 5280 \\
\hline 22 & 3471 & 1231 & 1171 & 1210 & 1156 & 1144 & 2449 & 4869 & 8645 & 8164 & 6906 & 5155 \\
\hline 23 & 3363 & 1287 & 1156 & 1242 & 1138 & 1150 & 2491 & 4958 & 8660 & 8109 & 6869 & 5040 \\
\hline 24 & 3278 & 1260 & 1138 & 1222 & 1126 & 1159 & 2540 & 4930 & 8620 & 8069 & 6833 & 4922 \\
\hline 25 & 3167 & 1242 & 1123 & 1207 & 1138 & 1165 & 2667 & 4946 & 8555 & 8029 & 6801 & 4809 \\
\hline 26 & 3090 & 1216 & 1108 & 1201 & 1123 & 1186 & 2859 & 5203 & 8575 & 7979 & 6773 & 4698 \\
\hline 27 & 2992 & 1192 & 1094 & 1192 & 1114 & 1213 & 3025 & 5538 & 8640 & 7934 & 6741 & 4575 \\
\hline 28 & 2902 & 1171 & 1082 & 1183 & 1117 & 1233 & 3119 & 5795 & 8731 & 7889 & 6708 & 4465 \\
\hline 29 & 2830 & 1192 & 1070 & 1165 & --- & 1245 & 3138 & 5817 & 8746 & 7844 & 6667 & 4356 \\
\hline 30 & 2719 & 1327 & 1059 & 1165 & --- & 1260 & 3171 & 5836 & 8696 & 7800 & 6621 & 4247 \\
\hline 31 & 2621 & --- & 1056 & 1165 & --- & 1281 & --- & 5881 & --- & 7755 & 6584 & --- \\
\hline MAX & 5263 & 2547 & 1387 & 1242 & 1275 & 1281 & 3171 & 5881 & 8746 & 8670 & 7712 & 6547 \\
\hline MIN & 2621 & 1171 & 1056 & 977 & 1114 & 1091 & 1192 & 3289 & 6000 & 7755 & 6584 & 4247 \\
\hline$a$ & 8.69 & 4.69 & 3.78 & 4.15 & 3.99 & 4.54 & 10.22 & 16.98 & 22.91 & 21.03 & 18.51 & 13.07 \\
\hline $\mathrm{b}$ & -2737 & -1294 & -271 & +109 & -48 & +164 & +1890 & +2710 & +2815 & -941 & -1171 & -2337 \\
\hline
\end{tabular}

CAL YR 1998 MAX 8625 MIN 1056 b $\quad-744$ WTR YR 1999 MAX 8746 MIN 977 b -1111 
LOCATION.-Lat 3840'18", long 12007'19", in NE 1/4 SW 1/4 sec.32, T.10 N., R.17 E., El Dorado County, Hydrologic Unit 18020129, Eldorado National Forest, on right bank 1,000 ft downstream from Silver Lake Dam and 3.5 mi southwest of Kirkwood.

DRAINAGE AREA.- $-15.2 \mathrm{mi}^{2}$.

PERIOD OF RECORD.-September 1922 to current year. Records for water year 1923 incomplete, yearly estimate published in WSP 1315-A.

REVISED RECORDS.—WDR CA-75-4: 1927(M), 1929(M), 1932(M), 1937-38(M), 1940-45(M), 1950-53(M), 1955-58(M), 1963(M), 1965(M), 1967(M), 1969-70(M), 1973(M).

GAGE.-Water-stage recorder. Concrete control since Sept. 8, 1986. Datum of gage is 7,198.0 ft above sea level (levels by Pacific Gas \& Electric Co).

REMARKS.-Low and medium flow regulated by Silver Lake (station 11435900) 1,000 ft upstream. Some water, in addition to that released through dam and over spillway, escapes from Silver Lake through porous rock formation and is measured at staff gage (station 11436500) $0.25 \mathrm{mi}$ east of station. For leakage from Silver Lake, refer to monthly figures below. See schematic diagram of South Fork American River Basin.

COOPERATION.--Records were collected by Pacific Gas \& Electric Co., under general supervision of the U.S. Geological Survey, in connection with a Federal Energy Regulatory Commission project.

EXTREMES FOR PERIOD OF RECORD.-Maximum discharge, 2,170 ft $\mathrm{ft}^{3} / \mathrm{s}$, Jan. 2, 1997, gage height, $7.79 \mathrm{ft}$, from rating curve extended above $430 \mathrm{ft}^{3} / \mathrm{s}$; no flow many days in February and March 1948, Jan. 13, 14, 1954, Nov. 3, 1959, to Feb. 5, 1960.

DISCHARGE, CUBIC FEET PER SECOND, WATER YEAR OCTOBER 1998 TO SEPTEMBER 1999 DAILY MEAN VALUES

\begin{tabular}{|c|c|c|c|c|c|c|c|c|c|c|c|c|}
\hline DAY & $\mathrm{OCT}$ & NOV & $\mathrm{DEC}$ & JAN & FEB & MAR & $A P R$ & MAY & JUN & JUL & AUG & SEP \\
\hline 1 & 56 & 45 & 23 & 13 & 14 & 12 & 21 & 51 & 205 & 78 & 5.9 & 5.8 \\
\hline 2 & 54 & 44 & 23 & 12 & 13 & 12 & 21 & 52 & 119 & 52 & 6.0 & 5.8 \\
\hline 3 & 54 & 43 & 24 & 12 & 13 & 13 & 20 & 52 & 66 & 31 & 6.2 & 5.7 \\
\hline 4 & 53 & 42 & 24 & 11 & 13 & 13 & 20 & 53 & 66 & 30 & 6.3 & 5.7 \\
\hline 5 & 52 & 41 & 24 & 11 & 13 & 13 & 19 & 53 & 66 & 25 & 6.3 & 5.6 \\
\hline 6 & 24 & 40 & 25 & 10 & 13 & 13 & 20 & 54 & 61 & 13 & 6.2 & 5.7 \\
\hline 7 & 4.8 & 39 & 25 & 10 & 16 & 13 & 20 & 67 & 58 & 6.5 & 6.1 & 5.6 \\
\hline 8 & 4.7 & 38 & 24 & 9.9 & 17 & 13 & 20 & 129 & 58 & 6.4 & 6.0 & 5.5 \\
\hline 9 & 4.7 & 37 & 22 & 9.3 & 19 & 14 & 19 & 155 & 31 & 6.3 & 6.0 & 18 \\
\hline 10 & 4.7 & 36 & 21 & 9.1 & 19 & 13 & 19 & 156 & 16 & 6.4 & 6.0 & 30 \\
\hline 11 & 4.7 & 36 & 19 & 8.8 & 18 & 13 & 18 & 174 & 16 & 6.5 & 6.1 & 30 \\
\hline 12 & 36 & 35 & 19 & 8.7 & 17 & 13 & 17 & 229 & 20 & 6.5 & 6.1 & 29 \\
\hline 13 & 59 & 34 & 19 & 8.6 & 16 & 12 & 17 & 249 & 76 & 6.5 & 5.9 & 29 \\
\hline 14 & 58 & 33 & 18 & 8.3 & 16 & 12 & 18 & 235 & 244 & 6.5 & 5.8 & 29 \\
\hline 15 & 58 & 32 & 18 & 8.5 & 15 & 12 & 20 & 187 & 289 & 6.6 & 5.8 & 29 \\
\hline 16 & 57 & 31 & 17 & 9.0 & 15 & 12 & 22 & 161 & 278 & 6.7 & 5.8 & 29 \\
\hline 17 & 56 & 30 & 17 & 9.3 & 15 & 12 & 25 & 167 & 194 & 6.7 & 5.8 & 28 \\
\hline 18 & 55 & 28 & 18 & 10 & 15 & 12 & 27 & 213 & 109 & 6.7 & 5.9 & 28 \\
\hline 19 & 55 & 26 & 18 & 13 & 15 & 13 & 35 & 222 & 147 & 6.7 & 5.9 & 28 \\
\hline 20 & 54 & 25 & 18 & 15 & 14 & 14 & 38 & 256 & 161 & 6.6 & 5.8 & 28 \\
\hline 21 & 53 & 24 & 18 & 16 & 15 & 14 & 41 & 282 & 159 & 6.6 & 5.8 & 40 \\
\hline 22 & 53 & 21 & 17 & 16 & 14 & 14 & 42 & 348 & 159 & 6.5 & 5.8 & 57 \\
\hline 23 & 52 & 22 & 17 & 17 & 14 & 15 & 43 & 409 & 163 & 6.4 & 5.8 & 56 \\
\hline 24 & 52 & 22 & 16 & 18 & 13 & 15 & 44 & 408 & 163 & 6.3 & 5.8 & 56 \\
\hline 25 & 51 & 21 & 16 & 17 & 14 & 15 & 45 & 416 & 123 & 6.3 & 5.8 & 55 \\
\hline 26 & 51 & 20 & 15 & 16 & 13 & 16 & 47 & 345 & 59 & 6.3 & 5.9 & 55 \\
\hline 27 & 50 & 19 & 15 & 16 & 13 & 18 & 48 & 289 & 30 & 6.2 & 5.8 & 54 \\
\hline 28 & 49 & 19 & 14 & 15 & 12 & 19 & 50 & 279 & 21 & 6.0 & 5.8 & 54 \\
\hline 29 & 48 & 19 & 14 & 15 & --- & 19 & 51 & 292 & 59 & 6.0 & 5.8 & 53 \\
\hline 30 & 47 & 21 & 13 & 14 & --- & 20 & 51 & 293 & 92 & 5.9 & 5.8 & 53 \\
\hline 31 & 46 & --- & 13 & 14 & --- & 21 & --- & 269 & --- & 5.9 & 5.8 & --- \\
\hline TOTAL & 1356.6 & 923 & 584 & 380.5 & 414 & 440 & 898 & 6545 & 3308 & 389.0 & 183.8 & 913.4 \\
\hline MEAN & 43.8 & 30.8 & 18.8 & 12.3 & 14.8 & 14.2 & 29.9 & 211 & 110 & 12.5 & 5.93 & 30.4 \\
\hline MAX & 59 & 45 & 25 & 18 & 19 & 21 & 51 & 416 & 289 & 78 & 6.3 & 57 \\
\hline MIN & 4.7 & 19 & 13 & 8.3 & 12 & 12 & 17 & 51 & 16 & 5.9 & 5.8 & 5.5 \\
\hline $\mathrm{AC}-\mathrm{FT}$ & 2690 & 1830 & 1160 & 755 & 821 & 873 & 1780 & 12980 & 6560 & 772 & 365 & 1810 \\
\hline$a$ & 22 & 0 & 0 & 0 & 0 & 0 & 0 & 29 & 515 & 717 & 390 & 79 \\
\hline
\end{tabular}

a Leakage, in acre-feet, from Silver Lake, provided by Pacific Gas \& Electric Co. 
11436000 SILVER LAKE OUTLET NEAR KIRKWOOD, CA—Continued

STATISTICS OF MONTHLY MEAN DATA FOR WATER YEARS 1923 - 1999, BY WATER YEAR (WY)

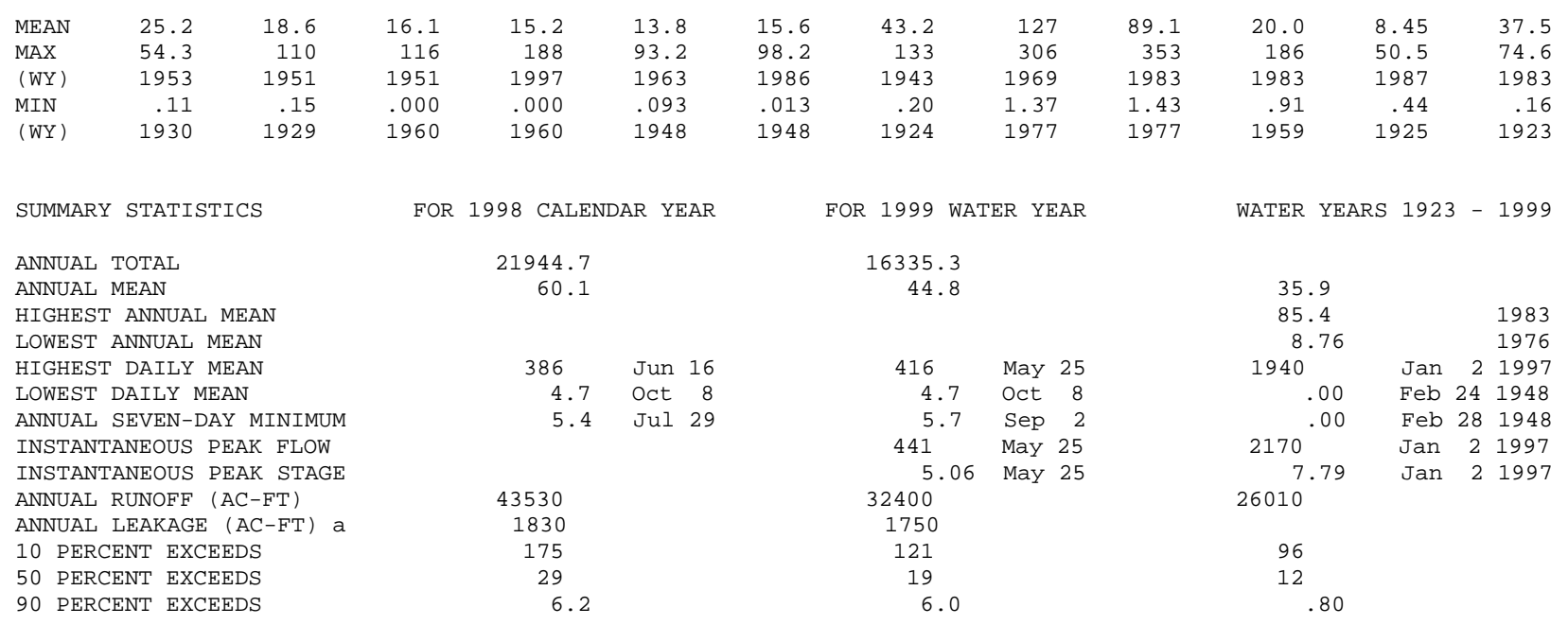

a Leakage, in acre-feet, from Silver Lake, provided by Pacific Gas \& Electric Co. 


\section{CAPLES LAKE NEAR KIRKWOOD, CA}

LOCATION.-Lat $38^{\circ} 42^{\prime} 27^{\prime \prime}$, long $120^{\circ} 02^{\prime}$ '55", in SW 1/4 SW 1/4 sec.18, T.10 N., R.18 E., Alpine County, Hydrologic Unit 18020129, Eldorado National Forest, on Caples Lake Dam near the center of the earthfill portion and 1.3 mi east of Kirkwood.

DRAINAGE AREA.- $-13.5 \mathrm{mi}^{2}$.

PERIOD OF RECORD.-OCtober 1985 to current year. Unpublished records for water years 1981-85 available in files of the U.S. Geological Survey.

GAGE.-Water-stage recorder since Oct. 1, 1991. Datum of gage is 7,894.0 ft above sea level (levels by Pacific Gas \& Electric Co.). Prior to Oct. 1, 1991, nonrecording gage read periodically except for the periods Oct. 16, 1986, to Sept. 30, 1987, Dec. 18, 1990, to May 26, 1991, and July 30 to Sept. 16, 1991, when there was a water-stage recorder at same site and datum.

REMARKS.-Lake is formed by one earthfill and one concrete dam at spillway; dam was completed and storage began in 1924. Capacity, 21,581 acre-ft, between gage heights 6.0 and $62.0 \mathrm{ft}$, top of $3 \mathrm{ft}$ of flashboards; capacity, 19,751 acre- $\mathrm{ft}$ at spillway level. Released water is measured at Caples Creek Release (station 11436999). When gage height is above spillway crest of $59.0 \mathrm{ft}$, there is leakage or spill which is not measured. Released water is used for power development on South Fork American River. See schematic diagram of South Fork American River Basin.

COOPERATION.-Records were collected by Pacific Gas \& Electric Co., under general supervision of the U.S. Geological Survey, in connection with a Federal Energy Regulatory Commission project. Contents not rounded to U.S. Geological Survey standards.

EXTREMES FOR PERIOD OF RECORD.—Maximum contents, 21,696 acre-ft, July 9, 10, 1997, gage height, $62.19 \mathrm{ft}$; minimum, 2,427 acre-ft, Mar. 30, 31, 1987, gage height, $20.7 \mathrm{ft}$.

EXTREMES FOR CURRENT YEAR.—Maximum contents, 21,519 acre-ft, July 8, gage height, $61.90 \mathrm{ft}$; minimum, 16,754 acre-ft, Apr. 14, gage height $53.89 \mathrm{ft}$.

Capacity table (gage height, in feet, and contents, in acre-feet)

(Based on survey by Pacific Gas \& Electric Co., dated Mar. 24, 1934)

$\begin{array}{llll}15.0 & 1,061 & 45.0 & 12,037 \\ 20.0 & 2,238 & 50.0 & 14,609 \\ 25.0 & 3,703 & 55.0 & 17,390 \\ 30.0 & 5,442 & 60.0 & 20,356 \\ 35.0 & 7,432 & 63.0 & 22,201 \\ 40.0 & 9,648 & & \end{array}$

RESERVOIR STORAGE (ACRE-FEET), WATER YEAR OCTOBER 1998 TO SEPTEMBER 1999 DAILY OBSERVATION AT 2400 HOURS

\begin{tabular}{|c|c|c|c|c|c|c|c|c|c|c|c|c|}
\hline DAY & OCT & NOV & $\mathrm{DEC}$ & JAN & FEB & MAR & $A P R$ & MAY & JUN & JUL & AUG & SEP \\
\hline 1 & 19271 & 17923 & 17540 & e 17200 & 17252 & 17598 & e17083 & 16874 & 19229 & 21433 & 21383 & 21003 \\
\hline 2 & 19212 & 17923 & 17557 & e17188 & 17252 & 17569 & e17056 & 16880 & 19050 & 21439 & 21383 & 20972 \\
\hline 3 & 19170 & 17894 & 17592 & e17177 & 17229 & 17609 & e17029 & 16874 & 18712 & 21420 & 21383 & 20935 \\
\hline 4 & 19098 & 17877 & 17592 & e 17160 & 17217 & 17609 & e17002 & 16817 & 18310 & 21427 & 21377 & 20935 \\
\hline 5 & 19033 & 17877 & 17586 & e 17143 & 17194 & 17575 & e16975 & 16800 & 17947 & 21396 & 21340 & 20899 \\
\hline 6 & 18997 & 17877 & 17586 & e17114 & 17229 & 17575 & e16948 & 16874 & 17766 & 21451 & 21340 & 20868 \\
\hline 7 & 18961 & 17906 & 17569 & e17102 & 17361 & 17540 & e16921 & 16999 & 17708 & 21494 & 21328 & 20837 \\
\hline 8 & 18919 & 17894 & 17569 & e17091 & 17494 & 17569 & e16895 & 17120 & 17656 & 21519 & 21316 & 20794 \\
\hline 9 & 18854 & 17871 & 17569 & e17080 & 17563 & 17592 & e16868 & 17211 & 17696 & 21482 & 21309 & 20745 \\
\hline 10 & 18842 & 17865 & 17563 & e17068 & 17580 & 17563 & e16842 & 17309 & 17935 & 21482 & 21365 & 20684 \\
\hline 11 & 18771 & 17865 & 17552 & e17057 & 17580 & 17563 & e16815 & 17488 & 18281 & 21451 & 21365 & 20605 \\
\hline 12 & 18712 & 17842 & 17540 & e17039 & 17580 & 17563 & e16791 & 17760 & 18664 & 21451 & 21390 & 20544 \\
\hline 13 & 18652 & 17842 & 17528 & e17028 & 17557 & 17540 & e16765 & 17888 & 19140 & 21494 & 21365 & 20477 \\
\hline 14 & 18623 & 17842 & 17517 & e17017 & 17563 & 17511 & 16754 & 17853 & 19558 & 21513 & 21340 & 20380 \\
\hline 15 & 18588 & 17801 & 17511 & e17028 & 17534 & 17505 & e16784 & 17766 & 19883 & 21513 & 21340 & 20313 \\
\hline 16 & 18493 & 17783 & 17505 & e17034 & 17575 & 17482 & e16814 & 17725 & 20137 & 21488 & 21316 & 20240 \\
\hline 17 & 18457 & 17801 & e17476 & e17034 & 17580 & 17476 & e16844 & 17696 & 20368 & 21470 & 21279 & 20174 \\
\hline 18 & 18393 & 17778 & e17453 & e17091 & 17604 & 17465 & e16874 & 17749 & 20575 & 21439 & 21285 & 20137 \\
\hline 19 & 18339 & 17778 & e17430 & e 17183 & 17604 & 17448 & e16904 & 17795 & 20733 & 21439 & 21242 & 20059 \\
\hline 20 & 18281 & 17731 & e17407 & e 17240 & 17609 & 17453 & e16934 & 17906 & 20941 & 21396 & 21242 & 19980 \\
\hline 21 & 18222 & 17679 & e17384 & 17240 & 17650 & 17436 & 16965 & 18069 & 21187 & 21408 & 21199 & 19914 \\
\hline 22 & 18187 & 17650 & e17367 & 17257 & 17650 & 17413 & 17017 & 18316 & 21383 & 21408 & 21199 & 19835 \\
\hline 23 & 18151 & 17720 & e17344 & 17332 & 17621 & 17413 & 16977 & 18670 & 21457 & 21390 & 21174 & 19715 \\
\hline 24 & 18104 & 17667 & e17327 & 17344 & 17598 & 17373 & 16942 & 18931 & 21439 & 21396 & 21174 & 19570 \\
\hline 25 & 18075 & 17621 & e17309 & 17338 & 17633 & 17361 & 16914 & 19128 & 21340 & 21396 & 21144 & 19415 \\
\hline 26 & 18023 & 17546 & e17292 & 17332 & 17615 & 17303 & 16925 & 19289 & 21346 & 21402 & 21131 & 19271 \\
\hline 27 & 17988 & 17517 & e17275 & 17321 & 17615 & 17269 & 16931 & 19438 & 21396 & 21402 & 21131 & 19116 \\
\hline 28 & 17988 & 17471 & e17257 & 17303 & 17598 & 17234 & 16948 & 19558 & 21427 & 21402 & 21131 & 18955 \\
\hline 29 & 17982 & 17500 & e 17240 & 17303 & -- & 17177 & 16948 & 19528 & 21451 & 21402 & 21101 & 18812 \\
\hline 30 & 17958 & 17569 & e17223 & 17263 & --- & 17137 & 16908 & 19433 & 21433 & 21402 & 21033 & 18664 \\
\hline 31 & 17941 & -- & e17211 & 17269 & --- & e17110 & -- & 19361 & -- & 21402 & 21003 & --- \\
\hline MAX & 19271 & 17923 & 17592 & 17344 & 17650 & 17609 & 17083 & 19558 & 21457 & 21519 & 21390 & 21003 \\
\hline MIN & 17941 & 17471 & 17211 & 17017 & 17194 & 17110 & 16754 & 16800 & 17656 & 21390 & 21003 & 18664 \\
\hline a & 55.95 & 55.31 & & 54.79 & 55.36 & & 54.16 & 58.35 & 61.76 & 61.71 & 61.06 & 57.18 \\
\hline $\mathrm{b}$ & -1384 & -372 & -358 & $\begin{array}{l}+58 \\
\end{array}$ & +329 & -488 & -202 & +2453 & +2072 & -31 & -399 & -2339 \\
\hline
\end{tabular}

CAL YR 1998 MAX 21494 MIN 15520 b -765 WTR YR 1999 MAX 21519 MIN 16754 b -661

e Estimated.

a Gage height, in feet, at end of month.

b Change in contents, in acre-feet. 
LOCATION.-Lat $38^{\circ} 42^{\prime} 31^{\prime \prime}$, long $120^{\circ} 03^{\prime} 02^{\prime \prime}$, in NW 1/4 SW 1/4 sec.18, T.10 N., R.18 E., Alpine County, Hydrologic Unit 18020129, Eldorado National Forest, on right bank $500 \mathrm{ft}$ downstream from main dam and outlet gate of Caples Lake and 1.3 mi east of Kirkwood.

DRAINAGE AREA.- $-13.5 \mathrm{mi}^{2}$.

PERIOD OF RECORD.-October 1992 to current year. Records for September 1922 to September 1992 were published as station 11437000 , Caples Lake Outlet. This record combined the spillway discharge. Records for water year 1945 incomplete, yearly estimate published in WSP 1315-A. Prior to October 1969, published as Twin Lakes Outlet near Kirkwood.

REVISED RECORDS.-WSP 1931: Drainage area.

GAGE.-Water-stage recorder and concrete control. Elevation of gage is 7,730 ft above sea level, from topographic map.

REMARKS. - Flow regulated by Caples Lake (station 11436950) $500 \mathrm{ft}$ upstream. Flow over Caples Lake Spillway bypasses this gage. No diversion upstream from station. See schematic diagram of South Fork American River Basin.

COOPERATION.--Records were collected by Pacific Gas \& Electric Co., under general supervision of the U.S. Geological Survey, in connection with a Federal Energy Regulatory Commission project.

EXTREMES FOR PERIOD OF RECORD.—Maximum discharge, $292 \mathrm{ft}^{3} / \mathrm{s}$, May 28, 1999, gage height, $3.21 \mathrm{ft}$; minimum daily, $5.5 \mathrm{ft}^{3} / \mathrm{s}$, Sept. 10 , 1996.

DISCHARGE, CUBIC FEET PER SECOND, WATER YEAR OCTOBER 1998 TO SEPTEMBER 1999 DAILY MEAN VALUES

\begin{tabular}{|c|c|c|c|c|c|c|c|c|c|c|c|c|}
\hline DAY & $\mathrm{OCT}$ & NOV & $\mathrm{DEC}$ & JAN & FEB & MAR & APR & MAY & JUN & JUL & AUG & SEP \\
\hline 1 & 31 & 9.2 & 20 & 13 & 13 & 13 & 31 & 53 & 289 & 71 & 10 & 10 \\
\hline 2 & 30 & 9.2 & 12 & 13 & 13 & 13 & 31 & 53 & 289 & 71 & 11 & 10 \\
\hline 3 & 30 & 9.2 & 12 & 13 & 13 & 13 & 31 & 53 & 289 & 71 & 10 & 10 \\
\hline 4 & 30 & 9.2 & 12 & 13 & 13 & 13 & 31 & 53 & 288 & 71 & 10 & 10 \\
\hline 5 & 31 & 9.3 & 12 & 13 & 13 & 13 & 31 & 53 & 286 & 64 & 10 & 10 \\
\hline 6 & 22 & 9.4 & 12 & 13 & 13 & 13 & 31 & 53 & 232 & 49 & 10 & 10 \\
\hline 7 & 11 & 9.4 & 12 & 13 & 13 & 13 & 31 & 54 & 176 & 49 & 10 & 10 \\
\hline 8 & 17 & 9.4 & 12 & 13 & 13 & 13 & 31 & 54 & 158 & 49 & 10 & 14 \\
\hline 9 & 23 & 9.4 & 12 & 13 & 13 & 13 & 31 & 54 & 95 & 49 & 10 & 29 \\
\hline 10 & 23 & 9.4 & 13 & 13 & 13 & 13 & 31 & 54 & 44 & 49 & 11 & 33 \\
\hline 11 & 23 & 9.4 & 13 & 13 & 13 & 13 & 31 & 54 & 36 & 49 & 11 & 33 \\
\hline 12 & 23 & 9.4 & 13 & 13 & 13 & 13 & 31 & 55 & 36 & 49 & 11 & 33 \\
\hline 13 & 23 & 9.4 & 12 & 13 & 13 & 13 & 31 & 97 & 36 & 49 & 10 & 33 \\
\hline 14 & 23 & 9.4 & 13 & 13 & 13 & 13 & 32 & 130 & 86 & 49 & 10 & 33 \\
\hline 15 & 23 & 9.4 & 13 & 13 & 13 & 13 & 32 & 130 & 153 & 49 & 10 & 33 \\
\hline 16 & 23 & 9.4 & 13 & 13 & 13 & 13 & 32 & 130 & 154 & 49 & 10 & 33 \\
\hline 17 & 23 & 9.4 & 13 & 13 & 13 & 13 & 32 & 130 & 154 & 48 & 10 & 33 \\
\hline 18 & 23 & 9.4 & 13 & 13 & 13 & 13 & 32 & 130 & 155 & 40 & 10 & 33 \\
\hline 19 & 23 & 9.4 & 13 & 13 & 13 & 13 & 32 & 130 & 155 & 32 & 10 & 33 \\
\hline 20 & 23 & 13 & 13 & 13 & 13 & 13 & 32 & 130 & 130 & 25 & 10 & 33 \\
\hline 21 & 23 & 30 & 13 & 13 & 13 & 13 & 32 & 130 & 113 & 21 & 10 & 33 \\
\hline 22 & 23 & 30 & 13 & 13 & 13 & 13 & 45 & 130 & 113 & 18 & 10 & 56 \\
\hline 23 & 23 & 30 & 13 & 13 & 13 & 13 & 55 & 131 & 113 & 13 & 10 & 73 \\
\hline 24 & 23 & 30 & 13 & 13 & 13 & 23 & 55 & 172 & 113 & 13 & 10 & 73 \\
\hline 25 & 23 & 30 & 13 & 13 & 13 & 31 & 55 & 239 & 113 & 12 & 10 & 73 \\
\hline 26 & 23 & 30 & 13 & 13 & 13 & 31 & 55 & 262 & 99 & 11 & 10 & 73 \\
\hline 27 & 16 & 30 & 13 & 13 & 13 & 31 & 55 & 277 & 71 & 11 & 10 & 73 \\
\hline 28 & 10 & 30 & 13 & 13 & 13 & 31 & 30 & 290 & 71 & 10 & 10 & 73 \\
\hline 29 & 8.4 & 30 & 13 & 13 & --- & 31 & 35 & 290 & 71 & 10 & 10 & 73 \\
\hline 30 & 8.9 & 30 & 13 & 13 & --- & 31 & 53 & 290 & 71 & 10 & 10 & 73 \\
\hline 31 & 9.3 & --- & 13 & 13 & --- & 31 & --- & 289 & --- & 10 & 10 & --- \\
\hline TOTAL & 668.6 & 490.7 & 401 & 403 & 364 & 539 & 1097 & 4150 & 4189 & 1171 & 314 & 1149 \\
\hline MEAN & 21.6 & 16.4 & 12.9 & 13.0 & 13.0 & 17.4 & 36.6 & 134 & 140 & 37.8 & 10.1 & 38.3 \\
\hline MAX & 31 & 30 & 20 & 13 & 13 & 31 & 55 & 290 & 289 & 71 & 11 & 73 \\
\hline MIN & 8.4 & 9.2 & 12 & 13 & 13 & 13 & 30 & 53 & 36 & 10 & 10 & 10 \\
\hline $\mathrm{AC}-\mathrm{FT}$ & 1330 & 973 & 795 & 799 & 722 & 1070 & 2180 & 8230 & 8310 & 2320 & 623 & 2280 \\
\hline
\end{tabular}




\section{CAPLES CREEK RELEASE BELOW CAPLES DAM, NEAR KIRKWOOD, CA—Continued}

STATISTICS OF MONTHLY MEAN DATA FOR WATER YEARS 1993 - 1999, BY WATER YEAR (WY)

\begin{tabular}{|c|c|c|c|c|c|c|c|c|c|c|c|c|}
\hline MEAN & 25.5 & 18.0 & 16.9 & 34.6 & 31.0 & 23.3 & 34.4 & 65.8 & 119 & 72.1 & 27.6 & 32.8 \\
\hline MAX & 54.5 & 33.1 & 29.8 & 116 & 92.4 & 40.0 & 83.5 & 134 & 203 & 183 & 64.5 & 55.3 \\
\hline (WY) & 1996 & 1996 & 1996 & 1997 & 1997 & 1997 & 1995 & 1999 & 1995 & 1995 & 1995 & 1995 \\
\hline MIN & 6.72 & 6.75 & 6.60 & 13.0 & 9.54 & 9.87 & 9.37 & 8.63 & 9.34 & 11.6 & 10.1 & 17.0 \\
\hline (WY) & 1998 & 1998 & 1998 & 1999 & 1996 & 1996 & 1994 & 1994 & 1994 & 1994 & 1999 & 1994 \\
\hline
\end{tabular}

SUMMARY STATISTICS

ANNUAL TOTAL

ANNUAL MEAN

HIGHEST ANNUAL MEAN

LOWEST ANNUAL MEAN

HIGHEST DAILY MEAN

LOWEST DAILY MEAN

ANNUAL SEVEN-DAY MINIMUM

INSTANTANEOUS PEAK FLOW

INSTANTANEOUS PEAK STAGE

ANNUAL RUNOFF (AC-FT)

10 PERCENT EXCEEDS

50 PERCENT EXCEEDS

90 PERCENT EXCEEDS
FOR 1998 CALENDAR YEAR

$\begin{array}{rlr}18879.4 & & \\ 51.7 & & \\ & & \\ 260 & \text { Jun } 27 \\ 6.4 & \text { Jan } & 1 \\ 6.5 & \text { Jan } & 1 \\ & & \\ 37450 & & \\ 126 & & \\ 30 & & \\ 9.3 & & \end{array}$

FOR 1999 WATER YEAR

\begin{tabular}{|c|c|}
\hline $\begin{array}{r}14936.3 \\
40.9\end{array}$ & \\
\hline 290 & May 28 \\
\hline 8.4 & Oct 29 \\
\hline 9.1 & Oct 29 \\
\hline 292 & May 28 \\
\hline 3.21 & May 28 \\
\hline 29630 & \\
\hline 113 & \\
\hline 13 & \\
\hline 10 & \\
\hline
\end{tabular}

WATER YEARS 1993 - 1999

\begin{tabular}{|c|c|c|}
\hline \multicolumn{3}{|l|}{41.7} \\
\hline 63.1 & & 1995 \\
\hline 20.8 & & 1994 \\
\hline 290 & May 28 & 1999 \\
\hline 5.5 & Sep 10 & 1996 \\
\hline 6.4 & Oct 5 & 1997 \\
\hline 292 & May 28 & 1999 \\
\hline 3.21 & May 28 & 1999 \\
\hline \multicolumn{3}{|l|}{30210} \\
\hline 98 & & \\
\hline 23 & & \\
\hline 8.6 & & \\
\hline
\end{tabular}


LOCATION.-Lat 3845'49", long 120¹9’39", in SW 1/4 SW 1/4 sec.29, T.11 N., R.15 E., El Dorado County, Hydrologic Unit 18020129, Eldorado National Forest, on right bank 0.8 mi downstream from Silver Fork American River, and 1.9 mi southwest of Kyburz.

DRAINAGE AREA.-193 $\mathrm{mi}^{2}$.

PERIOD OF RECORD.-August to December 1907, October 1922 to current year. Prior to October 1956, records for river and El Dorado Canal published separately; combined flow only, October 1956 to September 1960.

CHEMICAL DATA: Water years 1979, 1980.

BIOLOGICAL DATA: Water years 1979, 1980

SUSPENDED SEDIMENT: Water year 1980.

WATER TEMPERATURE: Water years 1966-79.

REVISED RECORDS.-WSP 1445: 1923(M), 1925(M), 1927(M), 1928 (river only), 1935-37(M). WSP 1515: 1928 (combined). WSP 1931: Drainage area.

GAGE.-Water-stage recorder on river; water-stage recorder for canal diversion (station 11439000). Elevation of gage is 3,840 ft above sea level, from topographic map. Prior to Oct. 1, 1962, at datum $1.00 \mathrm{ft}$ higher.

REMARKS.-Low and medium flows regulated by Echo Lake, Silver Lake, Caples Lake (stations 10336608, 11435900, and 11436950), and Lake Aloha, total capacity, 37,100 acre-ft. Some water is diverted out of river 0.6 mi upstream at diversion dam to El Dorado Canal (station 11439000). Part of this water is used for irrigation and domestic use and the remainder is returned to river at El Dorado Powerplant. See schematic diagram of South Fork American River Basin.

COOPERATION.-Records were collected by Pacific Gas \& Electric Co., under general supervision of the U.S. Geological Survey, in connection with a Federal Energy Regulatory Commission project.

EXTREMES FOR PERIOD OF RECORD.-River only: Maximum discharge, 25,000 ft 3 /s, Jan. 2, 1997, gage height, $14.26 \mathrm{ft}$ (from floodmarks), from rating curve extended above $6,300 \mathrm{ft}^{3} / \mathrm{s}$ on basis of contracted-opening measurement at gage height $10.40 \mathrm{ft}$; minimum daily, $0.13 \mathrm{ft}^{3} / \mathrm{s}$, Nov. 26, 1977.

Combined flow: Maximum discharge, 25,000 ft³/s, Jan. 2, 1997; minimum daily, $10 \mathrm{ft}^{3} / \mathrm{s}$, Oct. 17, 19, 1929.

DISCHARGE, CUBIC FEET PER SECOND, WATER YEAR OCTOBER 1998 TO SEPTEMBER 1999

DAILY MEAN VALUES

\begin{tabular}{|c|c|c|c|c|c|c|c|c|c|c|c|c|}
\hline DAY & OCT & NOV & $\mathrm{DEC}$ & JAN & FEB & MAR & APR & MAY & JUN & JUL & AUG & SEP \\
\hline 1 & 190 & 100 & 324 & 122 & 193 & 410 & 419 & 1050 & 2240 & 872 & 120 & 79 \\
\hline 2 & 178 & 111 & 207 & 118 & 189 & 430 & 399 & 1200 & 2040 & 818 & 118 & 78 \\
\hline 3 & 171 & 95 & 265 & 117 & 195 & 584 & 390 & 985 & 1600 & 724 & 116 & 76 \\
\hline 4 & 163 & 90 & 220 & 114 & 210 & 552 & 371 & 866 & 1400 & 617 & 114 & 75 \\
\hline 5 & 160 & 87 & 179 & 114 & 203 & 449 & 376 & 932 & 1430 & 546 & 113 & 73 \\
\hline 6 & 151 & 87 & 176 & 113 & 201 & 401 & 369 & 1280 & 1610 & 455 & 114 & 71 \\
\hline 7 & 97 & 98 & 164 & 112 & 541 & 370 & 351 & 1570 & 1510 & 415 & 117 & 71 \\
\hline 8 & 76 & 101 & 174 & 108 & 649 & 343 & 336 & 1580 & 1380 & 394 & 112 & 70 \\
\hline 9 & 59 & 90 & 153 & 107 & 996 & 336 & 346 & 1500 & 1310 & 408 & 111 & 59 \\
\hline 10 & 53 & 90 & 149 & 106 & 584 & 318 & 329 & 1430 & 1220 & 364 & 128 & 82 \\
\hline 11 & 52 & 93 & 150 & 108 & 448 & 301 & 335 & 1630 & 1300 & 348 & 159 & 81 \\
\hline 12 & 54 & 88 & 149 & 107 & 398 & 291 & 359 & 1980 & 1370 & 331 & 104 & 80 \\
\hline 13 & 111 & 89 & 157 & 106 & 363 & 294 & 434 & 1990 & 1510 & 304 & 99 & 78 \\
\hline 14 & 119 & 93 & 155 & 106 & 337 & 314 & 594 & 1750 & 1690 & 293 & 101 & 86 \\
\hline 15 & 117 & 94 & 148 & 131 & 311 & 302 & 683 & 1560 & 1910 & 279 & 97 & 90 \\
\hline 16 & 115 & 90 & 167 & 223 & 327 & 301 & 786 & 1550 & 1820 & 258 & 94 & 90 \\
\hline 17 & 112 & 100 & 200 & 234 & 593 & 339 & 970 & 1710 & 1730 & 230 & 89 & 89 \\
\hline 18 & 109 & 91 & 188 & 496 & 484 & 379 & 1120 & 1880 & 1570 & 214 & 92 & 90 \\
\hline 19 & 106 & 85 & 172 & 614 & 400 & 420 & 1250 & 1870 & 1510 & 193 & 93 & 91 \\
\hline 20 & 103 & 86 & 138 & 690 & 369 & 412 & 1310 & 2010 & 1450 & 182 & 91 & 117 \\
\hline 21 & 104 & 103 & 136 & 453 & 358 & 368 & 1290 & 2160 & 1400 & 157 & 89 & 125 \\
\hline 22 & 103 & 132 & 150 & 344 & 338 & 350 & 1090 & 2510 & 1380 & 141 & 90 & 176 \\
\hline 23 & 101 & 183 & 142 & 368 & 325 & 365 & 964 & 2770 & 1450 & 130 & 96 & 235 \\
\hline 24 & 108 & 237 & 158 & 307 & 316 & 373 & 932 & 2800 & 1460 & 122 & 87 & 236 \\
\hline 25 & 112 & 152 & 148 & 285 & 332 & 409 & 1080 & 3030 & 1280 & 118 & 84 & 232 \\
\hline 26 & 113 & 135 & 134 & 260 & 308 & 496 & 1300 & 3070 & 988 & 111 & 86 & 229 \\
\hline 27 & 126 & 124 & 126 & 232 & 308 & 552 & 1200 & 2910 & 819 & 104 & 97 & 226 \\
\hline 28 & 106 & 122 & 123 & 219 & 336 & 511 & 1110 & 2790 & 788 & 100 & 87 & 222 \\
\hline 29 & 98 & 129 & 121 & 210 & --- & 487 & 869 & 2490 & 828 & 96 & 83 & 226 \\
\hline 30 & 96 & 478 & 122 & 209 & --- & 465 & 862 & 2370 & 890 & 97 & 80 & 206 \\
\hline 31 & 93 & --- & 127 & 205 & --- & 456 & --- & 2360 & --- & 122 & 80 & --- \\
\hline TOTAL & 3456 & 3653 & 5122 & 7038 & 10612 & 12378 & 22224 & 59583 & 42883 & 9543 & 3141 & 3739 \\
\hline MEAN & 111 & 122 & 165 & 227 & 379 & 399 & 741 & 1922 & 1429 & 308 & 101 & 125 \\
\hline MAX & 190 & 478 & 324 & 690 & 996 & 584 & 1310 & 3070 & 2240 & 872 & 159 & 236 \\
\hline MIN & 52 & 85 & 121 & 106 & 189 & 291 & 329 & 866 & 788 & 96 & 80 & 59 \\
\hline $\mathrm{AC}-\mathrm{FT}$ & 6850 & 7250 & 10160 & 13960 & 21050 & 24550 & 44080 & 118200 & 85060 & 18930 & 6230 & 7420 \\
\hline
\end{tabular}




\section{SOUTH FORK AMERICAN RIVER NEAR KYBURZ, CA—Continued}

STATISTICS OF MONTHLY MEAN DATA FOR WATER YEARS 1923 - 1999, BY WATER YEAR (WY)

\begin{tabular}{|c|c|c|c|c|c|c|c|c|c|c|c|c|}
\hline MEAN & 35.8 & 78.4 & 131 & 156 & 175 & 276 & 636 & 1207 & 856 & 188 & 26.7 & 25.4 \\
\hline MAX & 223 & 1283 & 1587 & 1964 & 1333 & 1252 & 1497 & 2765 & 3551 & 1628 & 343 & 417 \\
\hline (WY) & 1984 & 1951 & 1951 & 1997 & 1986 & 1986 & 1982 & 1969 & 1983 & 1995 & 1983 & 1983 \\
\hline MIN & .77 & .49 & .69 & .57 & .76 & 2.42 & 38.9 & 56.8 & .76 & .62 & .58 & .54 \\
\hline (WY) & 1929 & 1929 & 1931 & 1929 & 1931 & 1933 & 1977 & 1977 & 1924 & 1924 & 1926 & 1924 \\
\hline
\end{tabular}

SUMMARY STATISTICS

ANNUAL TOTAL

ANNUAL MEAN

HIGHEST ANNUAL MEAN

LOWEST ANNUAL MEAN

HIGHEST DAILY MEAN

LOWEST DAILY MEAN

ANNUAL SEVEN-DAY MINIMUM

INSTANTANEOUS PEAK FLOW

INSTANTANEOUS PEAK STAGE

ANNUAL RUNOFF (AC-FT)

10 PERCENT EXCEEDS

50 PERCENT EXCEEDS

90 PERCENT EXCEEDS
FOR 1998 CALENDAR YEAR

\begin{tabular}{|c|c|}
\hline $\begin{array}{r}249548 \\
684\end{array}$ & \\
\hline 3750 & Mar 24 \\
\hline 52 & Oct 11 \\
\hline 72 & Oct 7 \\
\hline 495000 & \\
\hline 2010 & \\
\hline 246 & \\
\hline 100 & \\
\hline
\end{tabular}

FOR 1999 WATER YEAR

$\begin{array}{rll}183372 & & \\ 502 & & \\ & & \\ 3070 & \text { May 26 } \\ 52 & \text { Oct } 11 \\ 71 & \text { Sep } 3 \\ 3890 & \text { May 25 } \\ 6.67 & \text { May 25 } \\ 363700 & & \\ 1480 & & \\ 223 & & \\ 90 & & \end{array}$

WATER YEARS 1923 - 1999

\begin{tabular}{|c|c|c|c|}
\hline 316 & & & \\
\hline 907 & & & 1983 \\
\hline 19.4 & & & 1977 \\
\hline 18000 & Jan & 2 & 1997 \\
\hline .13 & Nov 2 & 26 & 1977 \\
\hline .36 & Nov & 5 & 1928 \\
\hline 25000 & Jan & 2 & 1997 \\
\hline 14.26 & Jan & 2 & 1997 \\
\hline 228900 & & & \\
\hline 1040 & & & \\
\hline 52 & & & \\
\hline 2.8 & & & \\
\hline
\end{tabular}


11439501 SOUTH FORK AMERICAN RIVER NEAR KYBURZ, CA—Continued

SOUTH FORK AMERICAN RIVER AND EL DORADO CANAL NEAR KYBURZ, CA

DISCHARGE, CUBIC FEET PER SECOND, WATER YEAR OCTOBER 1998 TO SEPTEMBER 1999

DAILY MEAN VALUES

\begin{tabular}{|c|c|c|c|c|c|c|c|c|c|c|c|c|}
\hline DAY & $\mathrm{OCT}$ & NOV & $\mathrm{DEC}$ & JAN & FEB & MAR & $\mathrm{APR}$ & MAY & JUN & JUL & AUG & SEP \\
\hline 1 & 207 & 118 & 334 & 122 & 193 & 410 & 419 & 1080 & 2260 & 905 & 120 & 112 \\
\hline 2 & 195 & 129 & 217 & 118 & 189 & 430 & 399 & 1230 & 2070 & 851 & 118 & 111 \\
\hline 3 & 189 & 113 & 275 & 117 & 195 & 584 & 390 & 1020 & 1620 & 757 & 116 & 110 \\
\hline 4 & 181 & 108 & 230 & 114 & 210 & 552 & 371 & 899 & 1420 & 649 & 114 & 109 \\
\hline 5 & 177 & 105 & 185 & 114 & 203 & 449 & 376 & 965 & 1450 & 578 & 113 & 107 \\
\hline 6 & 171 & 105 & 176 & 113 & 201 & 401 & 369 & 1310 & 1630 & 487 & 114 & 105 \\
\hline 7 & 119 & 116 & 164 & 112 & 541 & 370 & 351 & 1600 & 1530 & 448 & 117 & 104 \\
\hline 8 & 101 & 119 & 174 & 108 & 649 & 343 & 336 & 1610 & 1410 & 426 & 112 & 102 \\
\hline 9 & 89 & 108 & 153 & 107 & 996 & 336 & 346 & 1530 & 1340 & 441 & 111 & 91 \\
\hline 10 & 85 & 108 & 149 & 106 & 584 & 318 & 329 & 1430 & 1250 & 397 & 128 & 115 \\
\hline 11 & 84 & 111 & 150 & 108 & 448 & 301 & 335 & 1630 & 1330 & 381 & 165 & 114 \\
\hline 12 & 85 & 106 & 149 & 107 & 398 & 291 & 359 & 1980 & 1400 & 363 & 131 & 113 \\
\hline 13 & 141 & 107 & 157 & 106 & 363 & 294 & 434 & 1990 & 1540 & 337 & 132 & 111 \\
\hline 14 & 149 & 111 & 155 & 106 & 337 & 314 & 594 & 1750 & 1720 & 326 & 134 & 119 \\
\hline 15 & 147 & 112 & 148 & 131 & 311 & 302 & 683 & 1560 & 1940 & 312 & 130 & 123 \\
\hline 16 & 145 & 108 & 167 & 223 & 327 & 301 & 786 & 1550 & 1850 & 291 & 127 & 123 \\
\hline 17 & 142 & 118 & 200 & 234 & 593 & 339 & 970 & 1710 & 1760 & 263 & 122 & 122 \\
\hline 18 & 139 & 109 & 188 & 496 & 484 & 379 & 1120 & 1880 & 1600 & 247 & 125 & 123 \\
\hline 19 & 136 & 103 & 172 & 614 & 400 & 420 & 1250 & 1870 & 1540 & 226 & 126 & 124 \\
\hline 20 & 133 & 99 & 138 & 690 & 369 & 412 & 1310 & 2010 & 1480 & 215 & 124 & 126 \\
\hline 21 & 134 & 103 & 136 & 453 & 358 & 368 & 1290 & 2170 & 1430 & 190 & 122 & 125 \\
\hline 22 & 133 & 134 & 150 & 344 & 338 & 350 & 1110 & 2530 & 1410 & 174 & 123 & 176 \\
\hline 23 & 131 & 190 & 142 & 368 & 325 & 365 & 997 & 2790 & 1480 & 163 & 130 & 235 \\
\hline 24 & 138 & 247 & 158 & 307 & 316 & 373 & 965 & 2820 & 1490 & 155 & 121 & 236 \\
\hline 25 & 143 & 162 & 148 & 285 & 332 & 409 & 1110 & 3060 & 1310 & 151 & 117 & 232 \\
\hline 26 & 138 & 145 & 134 & 260 & 308 & 496 & 1330 & 3100 & 1020 & 144 & 119 & 229 \\
\hline 27 & 138 & 134 & 126 & 232 & 308 & 552 & 1230 & 2940 & 849 & 137 & 131 & 226 \\
\hline 28 & 124 & 132 & 123 & 219 & 336 & 511 & 1140 & 2820 & 819 & 134 & 121 & 222 \\
\hline 29 & 116 & 139 & 121 & 210 & --- & 487 & 901 & 2510 & 859 & 130 & 117 & 226 \\
\hline 30 & 114 & 489 & 122 & 209 & --- & 465 & 895 & 2390 & 923 & 126 & 113 & 220 \\
\hline 31 & 111 & --- & 127 & 205 & --- & 456 & --- & 2380 & --- & 122 & 113 & --- \\
\hline TOTAL & 4235 & 4088 & 5168 & 7038 & 10612 & 12378 & 22495 & 60114 & 43730 & 10526 & 3806 & 4391 \\
\hline MEAN & 137 & 136 & 167 & 227 & 379 & 399 & 750 & 1939 & 1458 & 340 & 123 & 146 \\
\hline MAX & 207 & 489 & 334 & 690 & 996 & 584 & 1330 & 3100 & 2260 & 905 & 165 & 236 \\
\hline MIN & 84 & 99 & 121 & 106 & 189 & 291 & 329 & 899 & 819 & 122 & 111 & 91 \\
\hline $\mathrm{AC}-\mathrm{FT}$ & 8400 & 8110 & 10250 & 13960 & 21050 & 24550 & 44620 & 119200 & 86740 & 20880 & 7550 & 8710 \\
\hline a & 1550 & 864 & 90 & 0 & 0 & 0 & 551 & 1090 & 1700 & 1950 & 1320 & 1290 \\
\hline
\end{tabular}

STATISTICS OF MONTHLY MEAN DATA FOR WATER YEARS 1923 - 1999, BY WATER YEAR (WY)

\begin{tabular}{|c|c|c|c|c|c|c|c|c|c|c|c|c|}
\hline MEAN & 110 & 161 & 221 & 243 & 272 & 381 & 741 & 1329 & 986 & 313 & 149 & 134 \\
\hline MAX & 365 & 1301 & 1698 & 1964 & 1412 & 1344 & 1533 & 2905 & 3561 & 1637 & 357 & 424 \\
\hline (WY) & 1983 & 1951 & 1951 & 1997 & 1986 & 1986 & 1982 & 1969 & 1983 & 1995 & 1983 & 1983 \\
\hline MIN & 20.8 & 25.1 & 44.2 & 35.9 & 38.4 & 53.7 & 178 & 207 & 99.7 & 75.0 & 73.0 & 46.4 \\
\hline (WY) & 1978 & 1930 & 1960 & 1929 & 1977 & 1977 & 1977 & 1977 & 1924 & 1931 & 1994 & 1987 \\
\hline
\end{tabular}

SUMMARY STATISTICS

ANNUAL TOTAL

ANNUAL MEAN

HIGHEST ANNUAL MEAN

LOWEST ANNUAL MEAN

HIGHEST DAILY MEAN

LOWEST DAILY MEAN

ANNUAL SEVEN-DAY MINIMUM

INSTANTANEOUS PEAK FLOW

ANNUAL RUNOFF (AC-FT)

ANNUAL DIVERSION (AC-FT) a

10 PERCENT EXCEEDS

50 PERCENT EXCEEDS

90 PERCENT EXCEEDS
FOR 1998 CALENDAR YEAR

FOR 1999 WATER YEAR

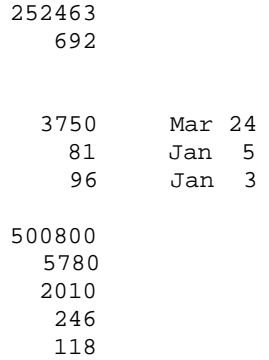

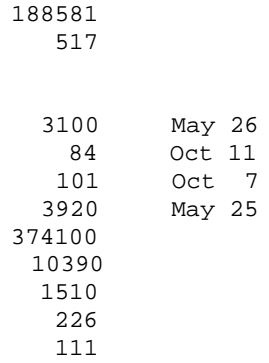

WATER YEARS 1923 - 1999

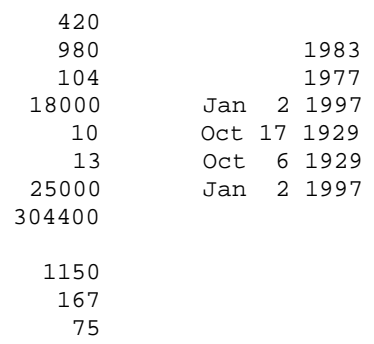

a Diversion, in acre-feet, to El Dorado Canal, provided by Pacific Gas \& Electric Co. 


\section{UNION VALLEY RESERVOIR NEAR RIVERTON, CA}

LOCATION.-Lat 3851'33", long 120²6'13", in NW 1/4 NW 1/4 sec.29, T.12 N., R.14 E., El Dorado County, Hydrologic Unit 18020129, Eldorado National Forest, in valve control house near left bank at Union Valley Dam on Silver Creek, 0.7 mi upstream from Little Silver Creek, and 6.6 mi north of Riverton.

DRAINAGE AREA.- $83.7 \mathrm{mi}^{2}$.

PERIOD OF RECORD.-October 1962 to current year.

CHEMICAL ANALYSES.-June to September 1996.

GAGE.-Water-stage recorder. Datum of gage is sea level (levels by Sacramento Municipal Utility District).

REMARKS.- Reservoir is formed by earthfill dam completed in December 1962; storage began May 1962. Usable capacity, 269,514 acre-ft between elevations 4,645.0 ft, minimum operating level, and 4,870.0 ft, top of radial spillway gates. Dead storage, 7,921 acre-ft. Reservoir receives water from the South Fork Rubicon River via Robbs Peak Powerplant (station 11429300) and from South Fork Silver Creek, since April 1985, via Jones Fork Powerplant (station 11440900). Water is used for power development in the South Fork American River Basin. Discharge to Union Valley Powerplant (station 11441002) is shown as a line item below this table. Records, including extremes, represent total contents. See schematic diagrams of Middle Fork American and Rubicon River Basins and South Fork American River Basin.

COOPERATION.-Records were collected by Sacramento Municipal Utility District, under general supervision of the U.S. Geological Survey, in connection with a Federal Energy Regulatory Commission project. Contents not rounded to U.S. Geological Survey standards.

EXTREMES FOR PERIOD OF RECORD.-Maximum contents, 279,100 acre-ft, July 9, 1974, elevation, 4,870.6 ft; minimum since reservoir first filled, 18,300 acre-ft, Jan. 13, 1977, elevation, 4,683.3 ft.

EXTREMES FOR CURRENT YEAR.-Maximum contents, 272,159 acre-ft, July 4, elevation, 4,868.23 ft; minimum, 147,049 acre-ft, Dec. 25, elevation, $4,816.13 \mathrm{ft}$.

Capacity table (elevation, in feet, and contents, in acre-feet)

(Based on table provided by Sacramento Municipal Utility District, recomputed October 1991)

$\begin{array}{rrrr}4,680 & 17,675 & 4,780 & 89,926 \\ 4,700 & 25,160 & 4,800 & 118,894 \\ 4,720 & 35,266 & 4,820 & 154,489 \\ 4,740 & 48,883 & 4,840 & 197,460 \\ 4,760 & 66,841 & 4,870 & 277,435\end{array}$

RESERVOIR STORAGE (ACRE-FEET), WATER YEAR OCTOBER 1998 TO SEPTEMBER 1999 DAILY OBSERVATION AT 2400 HOURS

\begin{tabular}{|c|c|c|c|c|c|c|c|c|c|c|c|c|}
\hline DAY & OCT & NOV & $\mathrm{DEC}$ & JAN & FEB & MAR & APR & MAY & JUN & JUL & AUG & SEP \\
\hline 1 & 164962 & 154665 & 151168 & 148831 & 175973 & 210745 & 187990 & 186051 & 247467 & 270534 & 242284 & 183438 \\
\hline 2 & 164983 & 153746 & 152211 & 149289 & 175306 & 211139 & 186523 & 187448 & 248519 & 271036 & 241278 & 182462 \\
\hline 3 & 164142 & 152909 & 153961 & 149403 & 174640 & 212373 & 184976 & 187741 & 248657 & 271479 & 239384 & 181115 \\
\hline 4 & 164080 & 152017 & 154881 & 149308 & 174576 & 212571 & 184061 & 187832 & 248990 & 272159 & 237179 & 180236 \\
\hline 5 & 163365 & 151361 & 154979 & 149212 & 174833 & 212596 & 182351 & 189511 & 249852 & 271390 & 234936 & 178727 \\
\hline 6 & 163365 & 150802 & 155195 & 149308 & 175715 & 212348 & 181115 & 191636 & 251470 & 270741 & 232206 & 178073 \\
\hline 7 & 163039 & 151457 & 154704 & 150015 & 180038 & 211805 & 179885 & 193340 & 252029 & 269828 & 230626 & 176794 \\
\hline 8 & 162713 & 151631 & 154920 & 149766 & 184016 & 211237 & 178836 & 194288 & 252197 & 268330 & 228272 & 175607 \\
\hline 9 & 162855 & 150648 & 154900 & 150207 & 189329 & 209567 & 177965 & 194985 & 252646 & 267833 & 225908 & 173934 \\
\hline 10 & 163079 & 150360 & 154371 & 150284 & 192004 & 209297 & 176686 & 195287 & 253123 & 266431 & 223640 & 173059 \\
\hline 11 & 162509 & 149785 & 153844 & 150015 & 193872 & 208028 & 175370 & 196360 & 253432 & 266081 & 221669 & 171299 \\
\hline 12 & 162448 & 149422 & 153376 & 150418 & 195893 & 206521 & 174084 & 197812 & 254587 & 265034 & 219052 & 170328 \\
\hline 13 & 162042 & 148565 & 153961 & 150860 & 196523 & 205191 & 172868 & 199721 & 255434 & 264222 & 216057 & 169425 \\
\hline 14 & 162327 & 147730 & 153473 & 151380 & 197694 & 204757 & 172549 & 201072 & 256085 & 263152 & 213836 & 168317 \\
\hline 15 & 162042 & 148071 & 152463 & 152114 & 198847 & 203412 & 172379 & 202383 & 256879 & 262027 & 211040 & 167193 \\
\hline 16 & 161556 & 147635 & 152521 & 153298 & 200076 & 201572 & 172379 & 202741 & 256794 & 260475 & 209297 & 166013 \\
\hline 17 & 161880 & 147181 & 152288 & 154626 & 204540 & 201025 & 173806 & 204108 & 257305 & 259844 & 207127 & 165209 \\
\hline 18 & 161091 & 147087 & 151592 & 158162 & 206763 & 200289 & 174898 & 205988 & 258387 & 259387 & 204637 & 163773 \\
\hline 19 & 160748 & 147332 & 151303 & 161657 & 207711 & 199083 & 176275 & 208028 & 259701 & 258701 & 202550 & 162733 \\
\hline 20 & 160567 & 147389 & 150744 & 166178 & 208320 & 198188 & 177617 & 210548 & 260704 & 257618 & 200787 & 161536 \\
\hline 21 & 160104 & 147597 & 150207 & 168213 & 208686 & 197014 & 178705 & 212942 & 1825 & 256993 & 199532 & 160406 \\
\hline 22 & 159823 & 147068 & 149862 & 169677 & 208028 & 196360 & 179578 & 216282 & 263556 & 255632 & 197929 & 159261 \\
\hline 23 & 158901 & 147162 & 148546 & 172188 & 208149 & 194822 & 179797 & 220523 & 264976 & 254502 & 191246 & 158282 \\
\hline 24 & 159261 & 147540 & 147332 & 173528 & 207687 & 193918 & 179753 & 224669 & 265586 & 253854 & 193409 & 157566 \\
\hline 25 & 158063 & 147484 & 147049 & 174790 & 208442 & 192694 & 181137 & 228141 & 266547 & 252871 & 192233 & 156515 \\
\hline 26 & 157685 & 147465 & 147768 & 175306 & 208540 & 192510 & 182484 & 231231 & 267540 & 251694 & 191063 & 155431 \\
\hline 27 & 156377 & 147654 & 148090 & 176189 & 208637 & 191636 & 183638 & 234882 & 268213 & 249880 & 189556 & 154019 \\
\hline 28 & 155844 & 147805 & 147578 & 176383 & 209273 & 190880 & 184484 & 237447 & 268946 & 248768 & 188466 & 152851 \\
\hline 29 & 155372 & 148546 & 147749 & 176405 & --- & 189624 & 184641 & 239816 & 269857 & 246777 & 187402 & 151979 \\
\hline 30 & 154783 & 150264 & 148318 & 176102 & --- & 189033 & 184752 & 242392 & 270652 & 244934 & 186546 & 150226 \\
\hline 31 & 154371 & --- & 148223 & 175715 & --- & 188058 & --- & 245236 & --- & 243620 & 184976 & --- \\
\hline MAX & 164983 & 154665 & 155195 & 176405 & 209273 & 212596 & 187990 & 245236 & 270652 & 272159 & 242284 & 183438 \\
\hline MIN & 154371 & 147068 & 147049 & 148831 & 174576 & 188058 & 172379 & 186051 & 247467 & 243620 & 184976 & 150226 \\
\hline $\mathrm{a}$ & 4819.94 & 4817.82 & 4816.75 & 4830.33 & 4844.93 & 4835.92 & 4834.45 & 4858.79 & 4867.72 & 4858.20 & 4834.55 & 4817.80 \\
\hline $\mathrm{b}$ & -10632 & -4107 & -2041 & +27492 & +33558 & -21215 & -3306 & +60484 & +25416 & -27032 & -58644 & -34750 \\
\hline C & 16380 & 15680 & 30720 & 9060 & 37640 & 72070 & 66110 & 54280 & 40600 & 40820 & 68100 & 48390 \\
\hline
\end{tabular}

CAL YR 1998 MAX 276027 MIN 131720 b +10996 c 583600 WTR YR 1999 MAX 272159 MIN 147049 b -14777 c 499900

a Elevation, in feet, at end of month.

b Change in contents, in acre-feet.

c Diversion, in acre-feet, to Union Valley Powerplant, provided by Sacramento Municipal Utility District. 
LOCATION.-Lat 3849'51", long 120²1'35", in SE 1/4 NW 1/4 sec.1, T.11 N., R.14 E., El Dorado County, Hydrologic Unit 18020129, Eldorado National Forest, in powerplant intake structure near right bank, $0.5 \mathrm{mi}$ north of Ice House Dam on South Fork Silver Creek, and $5.2 \mathrm{mi}$ northwest of Kyburz.

DRAINAGE AREA.- $-27.2 \mathrm{mi}^{2}$.

PERIOD OF RECORD.-October 1959 to current year.

CHEMICAL ANALYSES: June to September 1996.

REVISED RECORDS.-WSP 1931: 1960.

GAGE.-Water-stage recorder. Datum of gage is sea level (levels by Sacramento Municipal Utility District). Prior to July 15, 1985, at site 0.5 mi downstream at Ice House Dam at same datum.

REMARKS.--Reservoir is formed by an earthfill dam; storage began Dec. 15, 1959. Usable capacity, 45,839 acre-ft between elevations 5,327.5 ft, centerline of fishwater outlet, and 5,450.0 ft, top of spillway gates. Dead storage, 160 acre-ft. Reservoir is used to store water for power development. Reservoir is also forebay for Jones Fork Powerplant (station 11440900), which diverts up to $350 \mathrm{ft}^{3} / \mathrm{s}$ to powerplant completed in April 1985, then to Union Valley Reservoir (station 11441001). Records, including extremes, represent total contents. See schematic diagram of South Fork American River Basin.

COOPERATION.—Records were collected by Sacramento Municipal Utility District, under general supervision of the U.S. Geological Survey, in connection with a Federal Energy Regulatory Commission project. Contents not rounded to U.S. Geological Survey standards.

EXTREMES FOR PERIOD OF RECORD.-Maximum contents, 46,400 acre-ft, June 27, 1971, elevation, 5,450.6 ft; minimum since reservoir first filled, 1,450 acre-ft, Dec. 8, 1983, elevation, 5,347.9 ft.

EXTREMES FOR CURRENT YEAR.-Maximum contents, 43,549 acre-ft, July 10, elevation, 5,446.61 ft; minimum, 13,547 acre-ft, Apr. 16, elevation, $5,390.32 \mathrm{ft}$.

Capacity table (elevation, in feet, and contents in acre-feet)

(Based on table provided by Sacramento Municipal Utility District, recomputed in October 1991)

$\begin{array}{llll}5,345 & 1,080 & 5,400 & 17,665 \\ 5,350 & 1,801 & 5,420 & 27,406 \\ 5,360 & 3,751 & 5,440 & 39,167 \\ 5,380 & 9,663 & 5,451 & 46,721\end{array}$

RESERVOIR STORAGE (ACRE-FEET), WATER YEAR OCTOBER 1998 TO SEPTEMBER 1999 DAILY OBSERVATION AT 2400 HOURS

\begin{tabular}{|c|c|c|c|c|c|c|c|c|c|c|c|c|}
\hline DAY & OCT & NOV & DEC & JAN & $\mathrm{FEB}$ & MAR & APR & MAY & JUN & JUL & AUG & SEP \\
\hline 1 & 32337 & 27390 & 26318 & 23783 & 24381 & 19732 & 15372 & 16249 & 34445 & 42319 & 43086 & 39310 \\
\hline 2 & 32325 & 27315 & 26334 & 23818 & 24245 & 19478 & 15262 & 16538 & 35043 & 42551 & 43031 & 39024 \\
\hline 3 & 32308 & 27246 & 26449 & 23838 & 24220 & 19345 & 15166 & 16703 & 35433 & 42742 & 42962 & 38837 \\
\hline 4 & 32291 & 27166 & 26470 & 23858 & 24190 & 19093 & 15141 & 16659 & 35672 & 42887 & 42866 & 38708 \\
\hline 5 & 32262 & 27098 & 26476 & 23888 & 24044 & 18837 & 14919 & 16616 & 36090 & 43017 & 42845 & 38560 \\
\hline 6 & 32244 & 27045 & 26465 & 23918 & 23873 & 18570 & 14633 & 16807 & 36661 & 43134 & 42818 & 38367 \\
\hline 7 & 31967 & 27008 & 26339 & 23898 & 23878 & 18484 & 14406 & 17241 & 37036 & 43300 & 42797 & 38175 \\
\hline 8 & 31840 & 26939 & 26204 & 23848 & 23974 & e18344 & 14287 & 17616 & 37369 & 43404 & 42845 & 37920 \\
\hline 9 & 31651 & 26865 & 26151 & 23798 & 24039 & e18204 & 14049 & 17945 & 37666 & 43487 & 42749 & 37717 \\
\hline 10 & 31410 & 26802 & 26136 & 23748 & 23888 & e18064 & 13940 & 18259 & 37978 & 43549 & 42749 & 37521 \\
\hline 11 & 31388 & 26744 & 26105 & 23554 & 23718 & e17924 & 13838 & 18665 & 38380 & 43459 & 42763 & 37470 \\
\hline 12 & 31188 & 26670 & 26047 & 23514 & 23435 & e17784 & 13802 & 19216 & 38785 & 43169 & 42763 & 37256 \\
\hline 13 & 30978 & 26591 & 26016 & 23460 & 23350 & e17644 & 13757 & 19802 & 39303 & 43203 & 42749 & 37055 \\
\hline 14 & 30746 & 26528 & 25964 & 23420 & 23073 & e17504 & 13705 & 20155 & 39806 & 43265 & 42736 & 36836 \\
\hline 15 & 30554 & 26465 & 25886 & 23460 & 22764 & e17364 & 13628 & 20474 & 40353 & 43328 & 42708 & 36624 \\
\hline 16 & 30397 & 26413 & 25819 & 23484 & 22563 & e17244 & 13547 & 20917 & 40777 & 43376 & 42462 & 36362 \\
\hline 17 & 30156 & 26402 & 25808 & 23534 & 22378 & e17084 & 13600 & 21403 & 41144 & 43404 & 42319 & 36139 \\
\hline 18 & 30061 & 26355 & 25669 & 23798 & 22188 & e16944 & 13761 & 21960 & 41399 & 43425 & 42298 & 36115 \\
\hline 19 & 29672 & 26298 & 25426 & 24054 & 21936 & e16804 & 14017 & 22509 & 41594 & 43425 & 42122 & 35905 \\
\hline 20 & 29412 & 26245 & 25083 & 24265 & 21724 & e16664 & 14340 & 23054 & 41769 & 43418 & 41993 & 35684 \\
\hline 21 & 29126 & 26214 & 24863 & 24341 & 21527 & e16524 & 14480 & 23778 & 41850 & 43418 & 41776 & 35427 \\
\hline 22 & 28852 & 26178 & 24721 & 24401 & 21436 & 16382 & 14657 & 24950 & 41972 & 43231 & 41466 & 35171 \\
\hline 23 & 28590 & 26235 & 24568 & 24503 & 21159 & 16305 & 14790 & 26110 & 42183 & 43231 & 41231 & 34946 \\
\hline 24 & 28411 & 26157 & 24230 & 24533 & 20884 & 16236 & 14894 & 27076 & 42373 & 43231 & 40910 & 34849 \\
\hline 25 & 28195 & 26115 & 24180 & 24533 & 20662 & 16111 & 15078 & 28178 & 42421 & 43217 & 40684 & 34602 \\
\hline 26 & 27979 & 26079 & 24114 & 24533 & 20375 & 15885 & 15393 & 29357 & 42332 & 43217 & 40564 & 34271 \\
\hline 27 & 27904 & 26016 & 23984 & 24503 & 20132 & 15855 & 15651 & 30442 & 42292 & 43190 & 40346 & 34253 \\
\hline 28 & 27818 & 25990 & 23928 & 24437 & 19941 & 15791 & 15893 & 31433 & 42346 & 43183 & 40234 & 34008 \\
\hline 29 & 27609 & 26026 & 23883 & 24417 & --- & 15740 & 15979 & 32250 & 42169 & 43162 & 39957 & 33770 \\
\hline 30 & 27539 & 26240 & 23833 & 24386 & --- & 15689 & 16064 & 32949 & 42081 & 43127 & 39629 & 33450 \\
\hline 31 & 27459 & -- & 23803 & 24396 & --- & 15630 & -- & 33734 & -- & 43107 & 39342 & -- \\
\hline MAX & 32337 & 27390 & 26476 & 24533 & 24381 & 19732 & 16064 & 33734 & 42421 & 43549 & 43086 & 39310 \\
\hline MIN & 27459 & 25990 & 23803 & 23420 & 19941 & 15630 & 13547 & 16249 & 34445 & 42319 & 39342 & 33450 \\
\hline$a$ & 5420.10 & 5417.79 & 5413.02 & 5414.20 & 5405.01 & 5395.36 & 5396.38 & 5429.98 & 5444.55 & 5445.95 & 5440.33 & 5430.80 \\
\hline $\mathrm{b}$ & -4890 & -1219 & -2437 & $\begin{array}{r}+593 \\
+\end{array}$ & -4455 & -4311 & $\begin{array}{r}+434 \\
\end{array}$ & +17670 & $\begin{array}{r}+8347\end{array}$ & $\begin{array}{r}+1026 \\
\end{array}$ & -3765 & -5892 \\
\hline
\end{tabular}

1998 MAX 45942 MIN 13854 b -166

WTR YR 1999 MAX 43549 MIN 13547 b +1101

e Estimated.

a Elevation, in feet, at end of month

b Change in contents, in acre-feet. 


\section{SOUTH FORK SILVER CREEK NEAR ICE HOUSE, CA}

LOCATION.—Lat 3849’08", long 120²1'51", in NW 1/4 NW 1/4 sec.12, T.11 N., R.14 E., El Dorado County, Hydrologic Unit 18020129, Eldorado National Forest, on right bank $300 \mathrm{ft}$ upstream from Peavine Creek, $0.4 \mathrm{mi}$ downstream from Ice House Dam, and 4.8 mi northwest of Kyburz.

DRAINAGE AREA.-27.5 $\mathrm{mi}^{2}$.

PERIOD OF RECORD.-October 1924 to current year.

REVISED RECORDS.-WSP 1395: 1928, 1938. WSP 1635: Drainage area at former site.

GAGE.-Water-stage recorder and concrete control. Elevation of gage is 5,290 ft above sea level, from topographic map. Prior to Oct. 1, 1959, at site 0.3 mi upstream at different datum.

REMARKS.-Flow regulated by Ice House Reservoir (station 11441100) beginning in December 1959. Diversion to Jones Fork Powerplant (station 11440900) starting April 1985 bypasses station and returns to Silver Creek at Union Valley Reservoir (station 11441001). See schematic diagram of South Fork American River Basin.

COOPERATION.—Records were collected by Sacramento Municipal Utility District, under general supervision of the U.S. Geological Survey, in connection with a Federal Energy Regulatory Commission project.

EXTREMES FOR PERIOD OF RECORD.-Maximum discharge prior to construction of Ice House Dam in 1959, 3,940 ft $3 / \mathrm{s}$, Dec. 23, 1955, gage height, $6.71 \mathrm{ft}$, site and datum then in use, from rating curve extended above $540 \mathrm{ft}^{3} / \mathrm{s}$ on basis of slope-area measurement at gage height $6.69 \mathrm{ft}$; no flow Oct. 31 to Nov. 9, 1958. Maximum discharge since construction of the dam, 7,530 ft $3 / \mathrm{s}$, May 16, 1996, gage height, $7.64 \mathrm{ft}$, from rating curve extended above $730 \mathrm{ft}^{3} / \mathrm{s}$ on basis of computation of flow over dam at gage height $5.66 \mathrm{ft}$; minimum daily, $1.2 \mathrm{ft}^{3} / \mathrm{s}$, Mar. 17-19, 1960.

DISCHARGE, CUBIC FEET PER SECOND, WATER YEAR OCTOBER 1998 TO SEPTEMBER 1999

\section{DAILY MEAN VALUES}

\begin{tabular}{|c|c|c|c|c|c|c|c|c|c|c|c|c|}
\hline DAY & $\mathrm{OCT}$ & NOV & $\mathrm{DEC}$ & JAN & FEB & MAR & $A P R$ & MAY & JUN & JUL & AUG & SEP \\
\hline 1 & 15 & 13 & 6.2 & 5.2 & 4.5 & 7.2 & 4.9 & 12 & 12 & 15 & 16 & 16 \\
\hline 2 & 14 & 11 & 6.0 & 5.1 & 4.5 & 6.1 & 5.0 & 12 & 12 & 15 & 16 & 16 \\
\hline 3 & 14 & 11 & 6.7 & 5.1 & 4.6 & 8.1 & 5.1 & 12 & 12 & 15 & 16 & 16 \\
\hline 4 & 14 & 10 & 6.0 & 5.1 & 4.6 & 6.0 & 4.7 & 12 & 12 & 15 & 16 & 16 \\
\hline 5 & 14 & 10 & 5.9 & 4.5 & 4.7 & 5.4 & 4.6 & 11 & 12 & 15 & 16 & 16 \\
\hline 6 & 14 & 10 & 5.9 & 3.9 & 4.8 & 5.2 & 4.6 & 11 & 12 & 15 & 16 & 16 \\
\hline 7 & 14 & 11 & 5.9 & 5.2 & e 5.0 & 5.1 & 4.6 & 11 & 12 & 15 & 16 & 16 \\
\hline 8 & 14 & 11 & 6.0 & 5.3 & e5. 6 & 5.1 & 4.3 & 11 & 12 & 16 & 16 & 16 \\
\hline 9 & 13 & 10 & 6.2 & 5.4 & e 5.5 & 5.0 & 4.3 & 11 & 12 & 16 & 16 & 16 \\
\hline 10 & 13 & 11 & 6.5 & 5.4 & e 5.4 & 4.9 & 4.3 & 11 & 12 & 16 & 16 & 16 \\
\hline 11 & 13 & 11 & 6.5 & 4.6 & 5.4 & 4.9 & 4.4 & 11 & 12 & 16 & 16 & 16 \\
\hline 12 & 13 & 11 & 6.6 & 4.0 & 5.1 & 5.0 & 4.8 & 11 & 12 & 16 & 16 & 16 \\
\hline 13 & 13 & 12 & 6.3 & 4.0 & 5.1 & 5.3 & 5.2 & 11 & 12 & 16 & 16 & 16 \\
\hline 14 & 13 & 12 & 6.2 & 4.0 & 5.1 & 5.4 & 5.3 & 12 & 12 & 16 & 16 & 16 \\
\hline 15 & 13 & 12 & 6.1 & 4.4 & 4.9 & 5.3 & 5.2 & 11 & 12 & 16 & 16 & 16 \\
\hline 16 & 13 & 9.4 & 5.9 & 4.9 & 6.5 & 5.0 & 5.2 & 11 & 12 & 16 & 16 & 15 \\
\hline 17 & 13 & 5.6 & 6.0 & 4.8 & 8.9 & 5.0 & 5.0 & 11 & 13 & 16 & 16 & 15 \\
\hline 18 & 14 & 5.4 & 5.8 & e 5.0 & 6.3 & 5.0 & 4.9 & 11 & 13 & 16 & 16 & 15 \\
\hline 19 & 14 & 5.4 & 5.6 & e 5.2 & 5.8 & 5.0 & 4.8 & 11 & 13 & 16 & 16 & 15 \\
\hline 20 & 14 & 5.4 & 5.6 & e 5.2 & 5.5 & 5.1 & 4.7 & 11 & 12 & 16 & 16 & 16 \\
\hline 21 & 14 & 5.4 & e 5.6 & e 5.2 & 5.5 & 4.9 & 4.6 & 11 & 12 & 16 & 16 & 15 \\
\hline 22 & 13 & 5.8 & e 5.6 & 5.2 & 5.5 & 4.9 & 4.6 & 11 & 12 & 16 & 16 & 15 \\
\hline 23 & 13 & 6.1 & 5.4 & e 5.2 & 5.4 & 4.9 & 4.5 & 11 & 13 & 16 & 16 & 16 \\
\hline 24 & 13 & 5.8 & 5.4 & 5.1 & 5.4 & 4.9 & 4.4 & 11 & 12 & 16 & 15 & 16 \\
\hline 25 & 13 & 5.4 & 5.4 & 4.9 & 5.4 & 4.9 & 4.4 & 11 & 12 & 16 & 15 & 16 \\
\hline 26 & 13 & 5.4 & 5.4 & 4.9 & 5.4 & 5.0 & 4.4 & 11 & 12 & 16 & 16 & 16 \\
\hline 27 & 12 & 5.4 & 5.7 & 4.6 & 5.5 & 5.0 & 4.3 & 11 & 12 & 16 & 16 & 16 \\
\hline 28 & 13 & 5.4 & 6.3 & 4.6 & 6.2 & 4.7 & 4.4 & 11 & 12 & 16 & 16 & 16 \\
\hline 29 & 13 & 6.5 & 5.6 & 4.6 & --- & 4.6 & 8.8 & 12 & 14 & 16 & 15 & 16 \\
\hline 30 & 12 & 6.7 & 5.4 & 4.6 & --- & 4.8 & 12 & 12 & 15 & 16 & 15 & 16 \\
\hline 31 & 13 & --- & 5.4 & 4.6 & --- & 4.9 & --- & 12 & --- & 16 & 15 & --- \\
\hline TOTAL & 414 & 255.1 & 183.1 & 149.8 & 152.1 & 162.6 & 152.3 & 349 & 369 & 489 & 491 & 474 \\
\hline MEAN & 13.4 & 8.50 & 5.91 & 4.83 & 5.43 & 5.25 & 5.08 & 11.3 & 12.3 & 15.8 & 15.8 & 15.8 \\
\hline MAX & 15 & 13 & 6.7 & 5.4 & 8.9 & 8.1 & 12 & 12 & 15 & 16 & 16 & 16 \\
\hline MIN & 12 & 5.4 & 5.4 & 3.9 & 4.5 & 4.6 & 4.3 & 11 & 12 & 15 & 15 & 15 \\
\hline $\mathrm{AC}-\mathrm{ET}$ & 821 & 506 & 363 & 297 & 302 & 323 & 302 & 692 & 732 & 970 & 974 & 940 \\
\hline a & 4500 & 2240 & 4350 & 2320 & 8430 & 8610 & 7560 & 7260 & 6580 & 839 & 3140 & 4980 \\
\hline
\end{tabular}

e Estimated.

a Diversion, in acre-feet, to Jones Fork Powerplant, provided by Sacramento Municipal Utility District. 
11441500 SOUTH FORK SILVER CREEK NEAR ICE HOUSE, CA—Continued

STATISTICS OF MONTHLY MEAN DATA FOR WATER YEARS 1925 - 1959, BY WATER YEAR (WY)

\begin{tabular}{|c|c|c|c|c|c|c|c|c|c|c|c|c|}
\hline MEAN & 4.98 & 24.1 & 36.6 & 31.3 & 35.8 & 61.6 & 155 & 296 & 197 & 42.7 & 5.82 & 2.03 \\
\hline MAX & 28.0 & 326 & 305 & 163 & 91.7 & 191 & 280 & 531 & 418 & 132 & 22.8 & 7.62 \\
\hline (WY) & 1948 & 1951 & 1951 & 1956 & 1925 & 1928 & 1943 & 1952 & 1952 & 1952 & 1952 & 1952 \\
\hline MIN & .65 & .64 & 2.34 & 3.00 & 3.00 & 6.92 & 54.9 & 66.2 & 35.0 & 2.92 & .22 & .18 \\
\hline (WY) & 1933 & 1930 & 1933 & 1933 & 1933 & 1933 & 1944 & 1934 & 1931 & 1934 & 1931 & 1931 \\
\hline
\end{tabular}

SUMMARY STATISTICS

ANNUAL MEAN

HIGHEST ANNUAL MEAN

LOWEST ANNUAL MEAN

HIGHEST DAILY MEAN

LOWEST DAILY MEAN

ANNUAL SEVEN-DAY MINIMUM

INSTANTANEOUS PEAK FLOW

INSTANTANEOUS PEAK STAGE

ANNUAL RUNOFF (AC-FT)

10 PERCENT EXCEEDS

50 PERCENT EXCEEDS

90 PERCENT EXCEEDS
WATER YEARS $1925-1959$

\begin{tabular}{|c|c|c|}
\hline \multicolumn{3}{|l|}{74.5} \\
\hline 123 & & 1956 \\
\hline 25.3 & & 1931 \\
\hline 2780 & Dec 23 & 1955 \\
\hline .00 & Oct 31 & 1958 \\
\hline .00 & Oct 31 & 1958 \\
\hline 3940 & Dec 23 & 1955 \\
\hline 6.71 & Dec 23 & 1955 \\
\hline 53970 & & \\
\hline 237 & & \\
\hline 20 & & \\
\hline 1.4 & & \\
\hline
\end{tabular}

STATISTICS OF MONTHLY MEAN DATA FOR WATER YEARS 1961 - 1984, BY WATER YEAR (WY)

\begin{tabular}{|c|c|c|c|c|c|c|c|c|c|c|c|c|}
\hline MEAN & 112 & 87.6 & 49.4 & 57.1 & 71.2 & 43.6 & 56.0 & 125 & 157 & 78.1 & 80.9 & 90.1 \\
\hline MAX & 330 & 332 & 171 & 216 & 316 & 199 & 348 & 449 & 382 & 363 & 378 & 360 \\
\hline (WY) & 1970 & 1966 & 1980 & 1982 & 1971 & 1969 & 1983 & 1982 & 1983 & 1983 & 1983 & 1983 \\
\hline MIN & 5.64 & 5.05 & 5.21 & 4.76 & 5.48 & 3.67 & 2.94 & 4.17 & 3.80 & 4.02 & 3.79 & 3.97 \\
\hline (WY) & 1965 & 1963 & 1963 & 1967 & 1973 & 1984 & 1977 & 1977 & 1977 & 1977 & 1977 & 1977 \\
\hline
\end{tabular}

SUMMARY STATISTICS

ANNUAL MEAN

HIGHEST ANNUAL MEAN

LOWEST ANNUAL MEAN

HIGHEST DAILY MEAN

LOWEST DAILY MEAN

ANNUAL SEVEN-DAY MINIMUM

INSTANTANEOUS PEAK FLOW

INSTANTANEOUS PEAK STAGE

ANNUAL RUNOFF (AC-FT)

10 PERCENT EXCEEDS

50 PERCENT EXCEEDS

90 PERCENT EXCEEDS
WATER YEARS 1961 - 1984

\begin{tabular}{|c|c|c|}
\hline \multicolumn{3}{|l|}{84.0} \\
\hline 226 & & 1983 \\
\hline 24.8 & & 1977 \\
\hline 1560 & Jan 22 & 1970 \\
\hline 1. 3 & $\operatorname{Jan} 26$ & 1984 \\
\hline 1.4 & $\operatorname{Jan} 24$ & 1984 \\
\hline 1930 & May 26 & 1982 \\
\hline 5.74 & May 26 & 1982 \\
\hline 60830 & & \\
\hline 256 & & \\
\hline 12 & & \\
\hline 5.3 & & \\
\hline
\end{tabular}

STATISTICS OF MONTHLY MEAN DATA FOR WATER YEARS 1986 - 1999, BY WATER YEAR (WY)

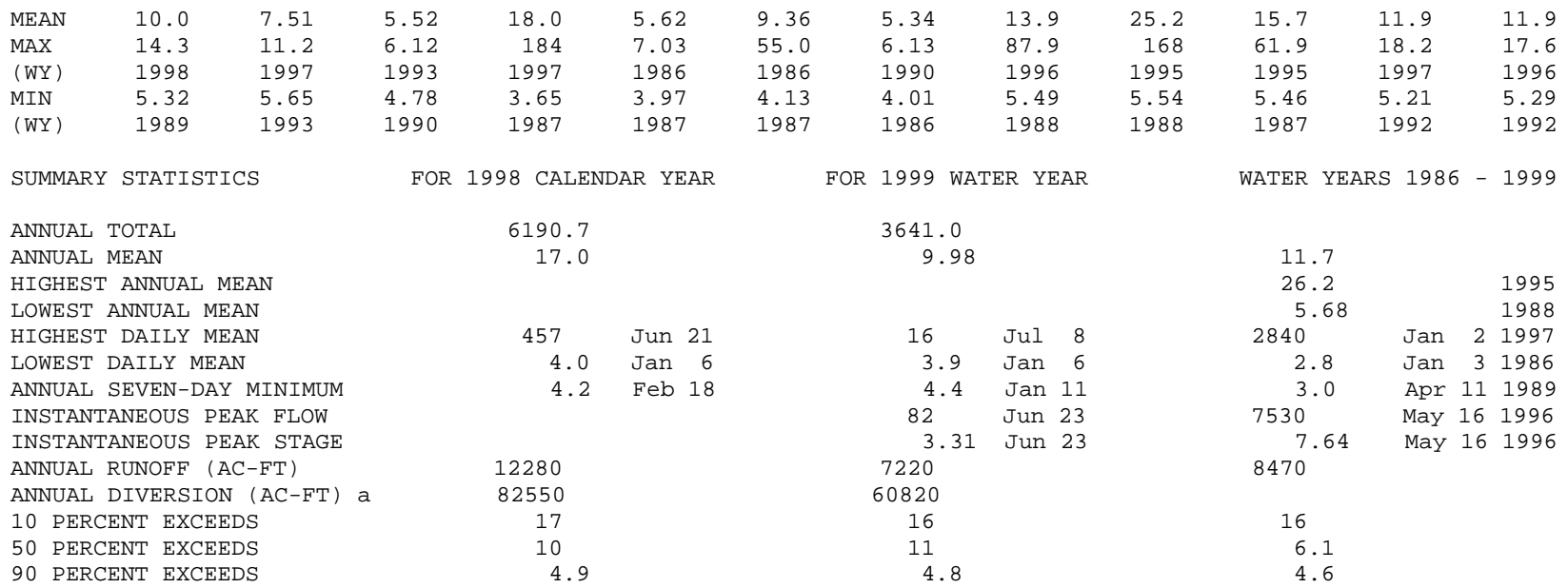

a Diversion, in acre-feet, to Jones Fork Powerplant, provided by Sacramento Municipal Utility District. 


\section{JUNCTION RESERVOIR NEAR POLLOCK PINES, CA}

LOCATION.—Lat 38 51'07", long 120²7’22", in SW 1/4 SW 1/4 sec.30, T.12 N., R.14 E., El Dorado County, Hydrologic Unit 18020129, in outlet structure to Jaybird Powerplant $100 \mathrm{ft}$ upstream from left abutment of Junction Diversion Dam, 0.3 mi downstream from South Fork Silver Creek, and 9.0 mi northeast of Pollock Pines.

DRAINAGE AREA.-147 $\mathrm{mi}^{2}$.

PERIOD OF RECORD.-October 1991 to current year. Unpublished records for water years 1980-91 available in files of the U.S. Geological Survey.

GAGE.-Water-stage recorder. Datum of gage is sea level (levels by Sacramento Municipal Utility District). Prior to Apr. 13, 1987, nonrecording gage at same site and datum.

REMARKS.--Reservoir is formed by concrete arch dam completed in 1962. Storage began in 1962. Usable capacity, 2,368 acre-ft, between elevations 4,397 ft, maximum drawdown level, and 4,450 ft, crest of spillway. Dead storage, 862 acre-ft. Most of the flow is diverted at this reservoir to Jaybird Powerplant (station 11441780). Records, including extremes, represent total contents at 2400 hours. See schematic diagram of South Fork American River Basin.

COOPERATION.-Records were collected by Sacramento Municipal Utility District, under general supervision of the U.S. Geological Survey, in connection with a Federal Energy Regulatory Commission project. Contents not rounded to U.S. Geological Survey standards.

EXTREMES FOR PERIOD OF RECORD.-Maximum contents, 3,737 acre-ft, Jan. 2, 1997, elevation, 4,459.10 ft; minimum, 875 acre-ft, Oct. 3 , 1991, elevation, 4,397.47 ft.

EXTREMES FOR CURRENT YEAR.-Maximum contents, 3,360 acre-ft, Apr. 6, elevation, 4,452.34 ft; minimum, 1,555 acre-ft, Nov. 20 , elevation, $4,416.58 \mathrm{ft}$.

Capacity table (elevation, in feet, and contents, in acre-feet)

(Based on table provided by Sacramento Municipal Utility District, recomputed October 1991)

$\begin{array}{rrrr}4,390 & 692 & 4,420 & 1,703 \\ 4,400 & 949 & 4,440 & 2,687 \\ 4,410 & 1,290 & 4,460 & 3,788\end{array}$

RESERVOIR STORAGE (ACRE-FEET), WATER YEAR OCTOBER 1998 TO SEPTEMBER 1999 DAILY OBSERVATION AT 2400 HOURS

\begin{tabular}{|c|c|c|c|c|c|c|c|c|c|c|c|c|}
\hline DAY & OCT & NOV & $\mathrm{DEC}$ & JAN & $\mathrm{FEB}$ & MAR & APR & MAY & JUN & JUL & AUG & SEP \\
\hline 1 & 2762 & 2693 & 3050 & 2806 & 2338 & 2560 & 2582 & 2403 & 2686 & 3038 & 3061 & 2993 \\
\hline 2 & 2541 & 3132 & 2818 & 2550 & 2375 & 2567 & 2559 & 2342 & 2848 & 2717 & 2644 & 3024 \\
\hline 3 & 3096 & 3122 & 2790 & 2672 & 2408 & 2725 & 2317 & 2639 & 2972 & 2769 & 2798 & 2795 \\
\hline 4 & 2877 & 3056 & 2723 & 2724 & 2475 & 2792 & 2065 & 2910 & 2958 & 2821 & 2883 & 2682 \\
\hline 5 & e2932 & 3186 & 3068 & 2935 & 2301 & 2798 & 2908 & 3044 & 2780 & 3085 & 2752 & 2751 \\
\hline 6 & 2990 & 2827 & 2870 & 2932 & 1989 & 2819 & 3360 & 2588 & 2891 & 2694 & 3077 & 2891 \\
\hline 7 & 3085 & 2647 & 2910 & 2473 & 2218 & 2466 & 3351 & 2415 & 2988 & 2885 & 2701 & 2780 \\
\hline 8 & 3135 & 2442 & 3111 & 3001 & 2598 & 2108 & 3323 & 2577 & 3054 & 3039 & 2724 & 2743 \\
\hline 9 & 2991 & 2832 & 2883 & 2804 & 3007 & 2604 & 3183 & 2549 & 2874 & 2886 & 2779 & 2741 \\
\hline 10 & 2778 & 2930 & 2979 & 2804 & 3062 & 2474 & 2842 & 2488 & 2977 & 2697 & 2951 & 3092 \\
\hline 11 & 3066 & 3061 & 2733 & 3049 & 2843 & 2308 & 2512 & 2575 & 2886 & 2874 & 2733 & 3116 \\
\hline 12 & 2927 & 2970 & 2842 & 3051 & 2677 & 2413 & 2669 & 2712 & 2820 & 2630 & 2567 & 3055 \\
\hline 13 & 2895 & 2953 & 2583 & 2794 & 2921 & 2675 & 2758 & 2548 & 2864 & 2682 & 2915 & 3021 \\
\hline 14 & 2822 & 3110 & 2675 & 2789 & 2756 & 2272 & 2765 & 2665 & 2827 & 2631 & 2546 & 2968 \\
\hline 15 & 3050 & 3142 & 2839 & 2858 & 2553 & 2205 & 2823 & 2523 & 2786 & 2781 & 2733 & 2845 \\
\hline 16 & 3129 & 3010 & 2527 & 3004 & 2384 & 2428 & 2711 & 2285 & 2839 & 2759 & 2792 & 2916 \\
\hline 17 & 2794 & 2991 & 2221 & 3036 & 2825 & 2538 & 2688 & 2492 & 2758 & 3021 & 2730 & 2914 \\
\hline 18 & 2862 & 2521 & 2711 & 2979 & 2655 & 2367 & 2566 & 2376 & 2647 & 2974 & 2590 & 2866 \\
\hline 19 & 3102 & 1747 & 2903 & 2603 & 2513 & 2241 & 2460 & 2433 & 2724 & 2747 & 3017 & 2927 \\
\hline 20 & 2981 & 1555 & 2895 & 2912 & 2488 & 2203 & 2520 & 2396 & 2977 & 2809 & 2823 & 2919 \\
\hline 21 & 3139 & 1618 & 2955 & 2532 & 2401 & 2190 & 2433 & 2646 & 2846 & 2600 & 2654 & 3027 \\
\hline 22 & 2941 & 2341 & 2783 & 2300 & 2758 & 2296 & 2409 & 2574 & 3088 & 2809 & 2792 & 3154 \\
\hline 23 & 3138 & 2820 & 2899 & 2465 & 2640 & 2243 & 2580 & 2713 & 2916 & 2910 & 2685 & 2966 \\
\hline 24 & 2695 & 2748 & 2832 & 2287 & 2605 & 2125 & 2405 & 2542 & 2906 & 2700 & 3123 & 2598 \\
\hline 25 & 2976 & 2736 & 2744 & 2501 & 2603 & 2254 & 2619 & 2549 & 3091 & 2765 & 3102 & 2562 \\
\hline 26 & 3091 & 2894 & 2574 & 2587 & 2585 & 2464 & 2636 & 2218 & 3091 & 2947 & 2823 & 2699 \\
\hline 27 & 3018 & 2606 & 2834 & 2390 & 2516 & 2190 & 2606 & 2411 & 3159 & 2752 & 3144 & 2859 \\
\hline 28 & 3155 & 2517 & 2795 & 2494 & 2343 & 2233 & 2583 & 2462 & 3201 & 2847 & 2965 & 2862 \\
\hline 29 & 2862 & 2399 & 2992 & 2294 & --- & 2604 & 2545 & 2403 & 3175 & 2705 & 2850 & 2882 \\
\hline 30 & 3052 & 2732 & 2671 & 2251 & --- & 2579 & 2238 & 2287 & 3158 & 2976 & e2965 & 2848 \\
\hline 31 & 2967 & --- & 3031 & 2262 & --- & 2654 & --- & 2466 & --- & 2788 & 3081 & --- \\
\hline MAX & 3155 & 3186 & 3111 & 3051 & 3062 & 2819 & 3360 & 3044 & 3201 & 3085 & 3144 & 3154 \\
\hline MIN & 2541 & 1555 & 2221 & 2251 & 1989 & 2108 & 2065 & 2218 & 2647 & 2600 & 2546 & 2562 \\
\hline$a$ & 4445.20 & 4440.84 & 4446.38 & 4431.78 & 4433.37 & 4439.37 & 4431.30 & 4435.77 & 4448.69 & 4441.89 & 4447.29 & 4443.00 \\
\hline $\mathrm{b}$ & -59 & -235 & +299 & -769 & +81 & +311 & -416 & +228 & +692 & -370 & +293 & -233 \\
\hline
\end{tabular}

CAL YR 1998 MAX 3412 MIN 1555 b +445 WTR YR 1999 MAX 3360 MIN 1555 b -178

e Estimated.

a Elevation, in feet, at end of month.

b Change in contents, in acre-feet. 
LOCATION.-Lat 3851'08", long 120²7'22", in SW 1/4 SW 1/4 sec.30, T.12 N., R.14 E., El Dorado County, Hydrologic Unit 18020129, Eldorado National Forest, at outlet structure on Junction Dam and 9 mi northeast of Pollock Pines.

DRAINAGE AREA.-147 $\mathrm{mi}^{2}$.

PERIOD OF RECORD.-October 1987 to current year (low-flow records only). Unpublished records for water years 1965-87 available in files of the U.S. Geological Survey.

GAGE.-Differential-pressure gage and orifice control in outlet pipe. Auxiliary nonrecording gage $550 \mathrm{ft}$ downstream at different datum. Elevation of gage is 4,280 ft above sea level, from topographic map. August 1964 to December 1986, nonrecording gage at site $500 \mathrm{ft}$ downstream at different datum. December 1986 to September 1987, nonrecording gage at site $550 \mathrm{ft}$ downstream.

REMARKS.-Records not computed above $30 \mathrm{ft}^{3} / \mathrm{s}$. Flow completely regulated by Junction Dam. Flow over the spillway bypasses this station. Diversion through Jaybird Powerplant (station 11441780) since 1962 bypasses this station. See schematic diagram of South Fork American River Basin.

COOPERATION.--Records were collected by Sacramento Municipal Utility District, under general supervision of the U.S. Geological Survey, in connection with a Federal Energy Regulatory Commission project.

DISCHARGE, CUBIC FEET PER SECOND, WATER YEAR OCTOBER 1998 TO SEPTEMBER 1999 DAILY MEAN VALUES

\begin{tabular}{|c|c|c|c|c|c|c|c|c|c|c|c|c|}
\hline DAY & OCT & NOV & $\mathrm{DEC}$ & JAN & FEB & MAR & APR & MAY & JUN & JUL & AUG & SEP \\
\hline 1 & 21 & 21 & 11 & 11 & 11 & 11 & 11 & 21 & 21 & 23 & 23 & 23 \\
\hline 2 & 21 & 14 & 11 & 11 & 11 & 11 & 11 & 21 & 21 & 22 & 22 & 23 \\
\hline 3 & 21 & 11 & 11 & 10 & 11 & 11 & 11 & 21 & 21 & 22 & 22 & 22 \\
\hline 4 & 21 & 11 & 11 & 11 & 11 & 11 & 11 & 21 & 21 & 22 & 23 & 22 \\
\hline 5 & 21 & 11 & 11 & 11 & 11 & 11 & 11 & 21 & 21 & 23 & 22 & 22 \\
\hline 6 & 21 & 11 & 11 & 11 & 11 & 11 & --- & 21 & 21 & 22 & 23 & 23 \\
\hline 7 & 21 & 11 & 11 & 11 & 11 & 11 & --- & 21 & 21 & 22 & 22 & 22 \\
\hline 8 & 21 & 11 & 11 & 11 & 11 & 11 & --- & 21 & 21 & 23 & 22 & 22 \\
\hline 9 & 21 & 11 & 11 & 10 & 11 & 11 & --- & 21 & 21 & 23 & 22 & 22 \\
\hline 10 & 21 & 11 & 11 & 11 & 11 & 11 & 11 & 21 & 21 & 22 & 23 & 23 \\
\hline 11 & 21 & 10 & 10 & 11 & 11 & 11 & 11 & 21 & 21 & 22 & 22 & 23 \\
\hline 12 & 21 & 11 & 11 & 11 & 11 & 11 & 11 & 21 & 21 & 22 & 22 & 23 \\
\hline 13 & 21 & 11 & 11 & 11 & 11 & 11 & 11 & 21 & 21 & 22 & 22 & 21 \\
\hline 14 & 21 & 11 & 11 & 11 & 11 & 11 & 11 & 21 & 21 & 22 & 22 & 21 \\
\hline 15 & 21 & 11 & 11 & 11 & 10 & 11 & 11 & 21 & 20 & 22 & 22 & 21 \\
\hline 16 & 21 & 11 & 11 & 11 & 11 & 11 & 11 & 21 & 21 & 22 & 22 & 21 \\
\hline 17 & 21 & 11 & 11 & 11 & 11 & 11 & 11 & 21 & 21 & 23 & 22 & 21 \\
\hline 18 & 21 & 11 & 11 & 11 & 11 & 11 & 11 & 21 & 21 & 23 & 22 & 21 \\
\hline 19 & 21 & 11 & 11 & 10 & 11 & 11 & 11 & 21 & 21 & 23 & 23 & 21 \\
\hline 20 & 21 & 11 & 11 & 11 & 11 & 11 & 11 & 21 & 21 & 22 & 22 & 21 \\
\hline 21 & 21 & 11 & 11 & 11 & 11 & 11 & 11 & 21 & 21 & 22 & 22 & 21 \\
\hline 22 & 21 & 11 & 11 & 11 & 11 & 11 & 11 & 21 & 22 & 22 & 22 & 21 \\
\hline 23 & 21 & 11 & 11 & 11 & 11 & 11 & 11 & 21 & 23 & 22 & 22 & 21 \\
\hline 24 & 21 & 10 & 11 & 10 & 11 & 11 & 11 & 21 & 22 & 22 & 23 & 21 \\
\hline 25 & 21 & 11 & 11 & 11 & 11 & 11 & 11 & 21 & 22 & 22 & 23 & 21 \\
\hline 26 & 21 & 11 & 11 & 11 & 11 & 11 & 11 & 21 & 23 & 23 & 23 & 21 \\
\hline 27 & 21 & 11 & 11 & 11 & 11 & 11 & 11 & 21 & 23 & 22 & 23 & 21 \\
\hline 28 & 21 & 11 & 10 & 11 & 11 & 11 & 11 & 21 & 23 & 22 & 23 & 21 \\
\hline 29 & 21 & 11 & 11 & 11 & --- & 11 & 11 & 21 & 23 & 22 & 22 & 21 \\
\hline 30 & 21 & 11 & 11 & 11 & --- & 11 & 17 & 21 & 23 & 22 & 23 & 21 \\
\hline 31 & 21 & --- & 11 & 11 & --- & 11 & --- & 21 & --- & 22 & 23 & --- \\
\hline TOTAL & 651 & 341 & 339 & 337 & 307 & 341 & --- & 651 & 644 & 690 & 694 & 648 \\
\hline MEAN & 21.0 & 11.4 & 10.9 & 10.9 & 11.0 & 11.0 & --- & 21.0 & 21.5 & 22.3 & 22.4 & 21.6 \\
\hline MAX & 21 & 21 & 11 & 11 & 11 & 11 & --- & 21 & 23 & 23 & 23 & 23 \\
\hline MIN & 21 & 10 & 10 & 10 & 10 & 11 & --- & 21 & 20 & 22 & 22 & 21 \\
\hline$A C-F T$ & 1290 & 676 & 672 & 668 & 609 & 676 & --- & 1290 & 1280 & 1370 & 1380 & 1290 \\
\hline a & 17190 & 17860 & 33420 & 19490 & 53720 & 84000 & 71690 & 60490 & 42390 & 42590 & 68980 & 49190 \\
\hline
\end{tabular}

CAL YR 1998 a 653700 WTR YR 1999 a 561000

a Diversion, in acre-feet, to Jaybird Powerplant, provided by Sacramento Municipal Utility District. 
LOCATION.-Lat 3849'44", long 120³2'09", in NW 1/4 NW 1/4 sec.4, T.11 N., R.13 E., El Dorado County, Hydrologic Unit 18020129, Eldorado National Forest, in outlet tower to Camino Powerplant $100 \mathrm{ft}$ upstream from right abutment of Camino Diversion Dam, $0.3 \mathrm{mi}$ upstream from Round Tent Canyon, and 5.3 mi northwest of Pollock Pines.

DRAINAGE AREA.-160 $\mathrm{mi}^{2}$.

PERIOD OF RECORD.-October 1991 to current year. Unpublished records for water years 1980-91 available in files of the U.S. Geological Survey.

GAGE.-Water-stage recorder. Datum of gage is sea level (levels by Sacramento Municipal Utility District). Prior to Apr. 8, 1987, nonrecording gage at same site and datum.

REMARKS.-Reservoir is formed by concrete-arch dam completed in 1961. Storage began in 1961. Usable capacity, 763 acre-ft, between elevations 2,840 ft, centerline of outlet valve, and 2,915 ft, maximum water surface level. Dead storage, 50 acre-ft. Most of the water is diverted at this reservoir to Camino Powerplant (station 11441895). Records, including extremes, represent total contents at 2400 hours. See schematic diagram of South Fork American River Basin.

COOPERATION.-Records were collected by Sacramento Municipal Utility District, under general supervision of the U.S. Geological Survey, in connection with a Federal Energy Regulatory Commission project.

EXTREMES FOR PERIOD OF RECORD.-Maximum contents, 819 acre-ft, Jan. 21, 1993, elevation, 2,915.29 ft; minimum, 208 acre-ft, Oct. 28 , 1997, elevation, 2,868.19 ft.

EXTREMES FOR CURRENT YEAR.-Maximum contents, 731 acre-ft, May 4, elevation, 2,910.44 ft; minimum, 276 acre-ft, Nov. 20, elevation, $2,876.00 \mathrm{ft}$.

Capacity table (elevation, in feet, and contents, in acre-feet)

(Based on table provided by Sacramento Municipal Utility District, recomputed October 1991)

$\begin{array}{llll}2,860 & 149 & 2,900 & 564 \\ 2,870 & 223 & 2,910 & 724 \\ 2,880 & 315 & 2,920 & 910 \\ 2,890 & 428 & & \end{array}$

RESERVOIR STORAGE (ACRE-FEET), WATER YEAR OCTOBER 1998 TO SEPTEMBER 1999 DAILY OBSERVATION AT 2400 HOURS

\begin{tabular}{|c|c|c|c|c|c|c|c|c|c|c|c|c|}
\hline DAY & $\mathrm{OCT}$ & NOV & $\mathrm{DEC}$ & JAN & FEB & MAR & APR & MAY & JUN & JUL & AUG & SEP \\
\hline 1 & 630 & 644 & 658 & 565 & 611 & 704 & 605 & 532 & 615 & 586 & 719 & 690 \\
\hline 2 & 635 & 602 & 644 & 627 & 670 & 715 & 579 & 622 & 644 & 579 & 630 & 681 \\
\hline 3 & 674 & 668 & 671 & 598 & 613 & 679 & 661 & 723 & 687 & 640 & 682 & 624 \\
\hline 4 & 682 & 643 & 623 & 609 & 628 & 626 & 592 & 731 & 671 & 694 & 650 & 655 \\
\hline 5 & e 665 & 612 & 642 & 631 & 644 & 693 & 663 & 644 & 664 & 574 & 661 & 644 \\
\hline 6 & 648 & 660 & 670 & 586 & 629 & 614 & 678 & 568 & 624 & 619 & 587 & 568 \\
\hline 7 & 619 & 633 & 648 & 612 & 591 & 601 & 634 & 654 & 644 & 661 & 652 & 651 \\
\hline 8 & 637 & 612 & 660 & 603 & 649 & 636 & 622 & 659 & 651 & 696 & 639 & 585 \\
\hline 9 & 638 & 653 & 652 & 575 & 572 & 590 & 655 & 589 & 659 & 665 & 653 & 693 \\
\hline 10 & 629 & 644 & 674 & 546 & 675 & 557 & 571 & 573 & 652 & 632 & 655 & 658 \\
\hline 11 & 623 & 661 & 570 & 639 & 612 & 579 & 587 & 591 & 685 & 657 & 623 & 687 \\
\hline 12 & 641 & 623 & 587 & 571 & 556 & 618 & 694 & 576 & 650 & 589 & 683 & 711 \\
\hline 13 & 684 & 600 & 583 & 633 & 650 & 676 & 653 & 580 & 636 & 720 & 592 & 594 \\
\hline 14 & 584 & 685 & 604 & 647 & 625 & 576 & 689 & 548 & 650 & 582 & 671 & 565 \\
\hline 15 & 580 & 680 & 651 & 649 & 639 & 584 & 689 & 568 & 661 & 555 & 648 & 621 \\
\hline 16 & 686 & 618 & 608 & 565 & 664 & 610 & 710 & 591 & 668 & 641 & 643 & 657 \\
\hline 17 & 624 & 557 & 573 & 609 & 666 & 677 & 698 & 577 & 711 & 708 & 611 & 617 \\
\hline 18 & 596 & 593 & 631 & 521 & 664 & 594 & 682 & 637 & 636 & 674 & 673 & 632 \\
\hline 19 & 637 & 602 & 609 & 540 & 638 & 519 & 655 & 648 & 645 & 694 & 557 & 631 \\
\hline 20 & 662 & 276 & 629 & 690 & 648 & 639 & 624 & 696 & 663 & 669 & 573 & 653 \\
\hline 21 & 644 & e 362 & 702 & 651 & 660 & 630 & 658 & 629 & 653 & 716 & 630 & 675 \\
\hline 22 & 546 & 447 & 698 & 672 & 653 & 660 & 634 & 610 & 517 & 708 & 724 & 622 \\
\hline 23 & 678 & 591 & 630 & 649 & 627 & 601 & 636 & 567 & 568 & 679 & 589 & 614 \\
\hline 24 & 649 & 599 & 605 & 664 & 655 & 622 & 661 & 546 & 544 & 701 & 683 & 572 \\
\hline 25 & 631 & 607 & 674 & 628 & 682 & 636 & 604 & 600 & 512 & 579 & 630 & 571 \\
\hline 26 & 624 & 599 & 718 & 614 & 691 & 590 & 646 & 612 & 544 & 629 & 632 & 575 \\
\hline 27 & 604 & 556 & 609 & 597 & 680 & 570 & 566 & 536 & 521 & 614 & 593 & 606 \\
\hline 28 & 600 & 612 & 619 & 603 & 655 & 583 & 611 & 548 & 535 & 648 & 649 & 679 \\
\hline 29 & 614 & 602 & 562 & 607 & --- & 641 & 572 & 569 & 532 & 569 & 625 & 646 \\
\hline 30 & 647 & 629 & 650 & 596 & --- & 579 & 577 & 581 & 604 & 695 & e 670 & 694 \\
\hline 31 & 667 & --- & 561 & 601 & --- & 640 & --- & 560 & --- & e707 & 716 & --- \\
\hline MAX & 686 & 685 & 718 & 690 & 691 & 715 & 710 & 731 & 711 & 720 & 724 & 711 \\
\hline MIN & 546 & 276 & 561 & 521 & 556 & 519 & 566 & 532 & 512 & 555 & 557 & 565 \\
\hline$a$ & 2906.59 & 2904.23 & 2899.76 & 2902.46 & 2905.86 & 2904.97 & 2900.90 & 2899.72 & 2902.67 & & 2909.52 & 2908.26 \\
\hline $\mathrm{b}$ & +48 & -38 & -68 & +40 & +54 & -15 & -63 & -17 & +44 & +103 & +9 & -22 \\
\hline
\end{tabular}

CAL YR 1998 MAX 724 MIN 276 b -89

WTR YR 1999 MAX 731 MIN $276 \quad$ b +75

e Estimated.

a Elevation, in feet, at end of month.

b Change in contents, in acre-feet. 
LOCATION._Lat 3849’26", long 120³2'18", on line between secs.4 and 5, T.11 N., R.13 E., El Dorado County, Hydrologic Unit 18020129, Eldorado National Forest, on right bank $300 \mathrm{ft}$ downstream from Round Tent Canyon, 0.4 mi downstream from diversion dam, and 5 mi northeast of Pollock Pines.

DRAINAGE AREA.-171 $\mathrm{mi}^{2}$.

PERIOD OF RECORD.-October 1960 to current year.

GAGE.-Water-stage recorder. Datum of gage is 2,754.06 ft above sea level (Sacramento Municipal Utility District benchmark).

REMARKS.-Flow is regulated by Ice House Reservoir (station 11441100) since 1959, Union Valley Reservoir (station 11441001) since 1962, and Junction and Camino Reservoirs (stations 11441760 and 11441890). Diversion to Camino Powerplant (station 11441895) since 1961 bypasses this station. See schematic diagram of South Fork American River Basin.

COOPERATION.-Records were collected by Sacramento Municipal Utility District, under general supervision of the U.S. Geological Survey, in connection with a Federal Energy Regulatory Commission project.

EXTREMES FOR PERIOD OF RECORD.-Maximum discharge, about 47,700 ft ${ }^{3} / \mathrm{s}$, Jan. 2, 1997, gage height, $15.72 \mathrm{ft}$, backwater from log jam, from rating curve extended above $4,700 \mathrm{ft}^{3} / \mathrm{s}$ on basis of slope-area measurement at gage height $11.28 \mathrm{ft}$; minimum daily, $1.0 \mathrm{ft}^{3} / \mathrm{s}$, Nov. 1 , 1980.

DISCHARGE, CUBIC FEET PER SECOND, WATER YEAR OCTOBER 1998 TO SEPTEMBER 1999 DAILY MEAN VALUES

\begin{tabular}{|c|c|c|c|c|c|c|c|c|c|c|c|c|}
\hline DAY & $\mathrm{OCT}$ & NOV & $\mathrm{DEC}$ & JAN & $\mathrm{FEB}$ & MAR & $A P R$ & MAY & JUN & JUL & AUG & SEP \\
\hline 1 & 28 & 28 & 19 & 20 & 20 & 832 & 48 & 44 & 28 & 28 & 28 & 28 \\
\hline 2 & 28 & 22 & 20 & 20 & 20 & 832 & 121 & 47 & 30 & 28 & 27 & 27 \\
\hline 3 & 28 & 20 & 25 & 20 & 19 & 922 & 70 & 57 & 30 & 27 & 26 & 27 \\
\hline 4 & 28 & 20 & 19 & 20 & 19 & 894 & 143 & 56 & 30 & 28 & 28 & 27 \\
\hline 5 & 28 & 20 & 20 & 20 & 19 & 678 & 50 & 54 & 29 & 28 & 28 & 27 \\
\hline 6 & 28 & 20 & 20 & 19 & 20 & 663 & 76 & 52 & 29 & 28 & 27 & 27 \\
\hline 7 & 28 & 20 & 20 & 19 & 648 & 748 & 47 & 50 & 29 & 28 & 27 & 27 \\
\hline 8 & 28 & 21 & 20 & 20 & 497 & 650 & 100 & 48 & 29 & 27 & 27 & 27 \\
\hline 9 & 28 & 22 & 20 & 19 & 1850 & 565 & 45 & 45 & 29 & 27 & 27 & 27 \\
\hline 10 & 28 & 21 & 20 & 20 & 193 & 168 & 45 & 43 & 28 & 26 & 27 & 27 \\
\hline 11 & 28 & 20 & 20 & 20 & 141 & 119 & 47 & 36 & 28 & 27 & 28 & 27 \\
\hline 12 & 28 & 20 & 20 & 19 & 118 & 114 & 55 & 34 & 28 & 27 & 28 & 28 \\
\hline 13 & 28 & 20 & 20 & 20 & 100 & 87 & 45 & 33 & 28 & 26 & 27 & 28 \\
\hline 14 & 28 & 20 & 20 & 20 & 94 & 198 & 25 & 33 & 27 & 27 & 28 & 26 \\
\hline 15 & 28 & 20 & 21 & 20 & 89 & 263 & 29 & 33 & 27 & 27 & 27 & 26 \\
\hline 16 & 27 & 20 & 21 & 19 & 85 & 109 & 32 & 32 & 28 & 26 & 27 & 28 \\
\hline 17 & 27 & 20 & 20 & 20 & 147 & 173 & 35 & 32 & 28 & 27 & 27 & 33 \\
\hline 18 & 27 & 20 & 20 & 70 & 457 & 238 & 31 & 33 & 28 & 27 & 28 & 32 \\
\hline 19 & 27 & 20 & 20 & 114 & 900 & 195 & 26 & 34 & 27 & 27 & 26 & 33 \\
\hline 20 & 27 & 28 & 20 & 179 & 791 & 98 & 23 & 32 & 27 & 27 & 26 & 32 \\
\hline 21 & 27 & 44 & 20 & 107 & 793 & 164 & 23 & 30 & 27 & 27 & 27 & 31 \\
\hline 22 & 27 & 77 & 21 & 75 & 748 & 97 & 25 & 29 & 27 & 27 & 28 & 31 \\
\hline 23 & 28 & 68 & 20 & 105 & 722 & 145 & 42 & 29 & 28 & 27 & 27 & 30 \\
\hline 24 & 27 & 19 & 20 & 79 & 658 & 99 & 60 & 28 & 29 & 27 & 28 & 29 \\
\hline 25 & 28 & 20 & 21 & 91 & 680 & 281 & 57 & 28 & 29 & 26 & 28 & 28 \\
\hline 26 & 27 & 20 & 20 & 37 & 645 & 141 & 55 & 28 & 29 & 27 & 27 & 29 \\
\hline 27 & 28 & 20 & 20 & 25 & 669 & 83 & 52 & 28 & 29 & 27 & 26 & 30 \\
\hline 28 & 28 & 20 & 20 & 21 & 709 & 62 & 51 & 27 & 29 & 27 & 25 & 29 \\
\hline 29 & 28 & 20 & 20 & 19 & --- & 47 & 49 & 27 & 29 & 27 & 26 & 30 \\
\hline 30 & 28 & 24 & 20 & 19 & --- & 220 & 47 & 28 & 24 & 26 & 26 & 30 \\
\hline 31 & 28 & --- & 20 & 20 & --- & 48 & --- & 28 & --- & 28 & 27 & --- \\
\hline TOTAL & 859 & 754 & 627 & 1296 & 11851 & 9933 & 1554 & 1138 & 847 & 839 & 839 & 861 \\
\hline MEAN & 27.7 & 25.1 & 20.2 & 41.8 & 423 & 320 & 51.8 & 36.7 & 28.2 & 27.1 & 27.1 & 28.7 \\
\hline MAX & 28 & 77 & 25 & 179 & 1850 & 922 & 143 & 57 & 30 & 28 & 28 & 33 \\
\hline MIN & 27 & 19 & 19 & 19 & 19 & 47 & 23 & 27 & 24 & 26 & 25 & 26 \\
\hline $\mathrm{AC}-\mathrm{FT}$ & 1700 & 1500 & 1240 & 2570 & 23510 & 19700 & 3080 & 2260 & 1680 & 1660 & 1660 & 1710 \\
\hline a & 18360 & 19690 & 36540 & 28210 & 55180 & 81360 & 84020 & 64860 & 45740 & 44820 & 71150 & 50760 \\
\hline
\end{tabular}

a Diversion, in acre-feet, to Camino Powerplant, provided by Sacramento Municipal Utility District. 


\section{SILVER CREEK BELOW CAMINO DIVERSION DAM, CA—Continued}

STATISTICS OF MONTHLY MEAN DATA FOR WATER YEARS 1961 - 1999, BY WATER YEAR (WY)

\begin{tabular}{|c|c|c|c|c|c|c|c|c|c|c|c|c|}
\hline MEAN & 28.0 & 45.1 & 73.1 & 205 & 130 & 118 & 118 & 191 & 148 & 66.7 & 32.6 & 26.4 \\
\hline MAX & 138 & 1088 & 856 & 4122 & 1168 & 1207 & 956 & 1505 & 1019 & 503 & 364 & 188 \\
\hline (WY) & 1995 & 1984 & 1965 & 1997 & 1986 & 1986 & 1962 & 1995 & 1995 & 1995 & 1962 & 1962 \\
\hline MIN & 3.12 & 3.44 & 5.39 & 5.21 & 5.45 & 3.56 & 3.14 & 3.30 & 3.29 & 2.98 & 3.11 & 3.18 \\
\hline (WY) & 1978 & 1978 & 1977 & 1977 & 1977 & 1977 & 1977 & 1977 & 1977 & 1977 & 1977 & 1977 \\
\hline
\end{tabular}

SUMMARY STATISTICS

ANNUAL TOTAL

ANNUAL MEAN

HIGHEST ANNUAL MEAN

LOWEST ANNUAL MEAN

HIGHEST DAILY MEAN

LOWEST DAILY MEAN

ANNUAL SEVEN-DAY MINIMUM

INSTANTANEOUS PEAK FLOW

INSTANTANEOUS PEAK STAGE

ANNUAL RUNOFF (AC-FT)

ANNUAL DIVERSION (AC-FT) a

10 PERCENT EXCEEDS

50 PERCENT EXCEEDS

90 PERCENT EXCEEDS
FOR 1998 CALENDAR YEAR

$\begin{array}{rrr}44719 & & \\ 123 & & \\ & & \\ 2380 & \text { Jun } 17 \\ 11 & \text { Jan } 1 \\ 11 & \text { Jan } 1 \\ & & \\ 88700 & & \\ 740800 & & \\ 117 & \\ 26 & & \\ 19 & & \end{array}$

FOR 1999 WATER YEAR

$\begin{array}{rrr}31398 & & \\ 86.0 & & \\ & & \\ 1850 & \text { Feb } & 9 \\ 19 & \text { Nov } & 24 \\ 19 & \text { Jan } & 6 \\ 3460 & \text { Feb } & 9 \\ 7.60 & \text { Feb } & 9 \\ 62280 & & \\ 600700 & & \\ 144 & & \\ 28 & & \\ 20 & & \end{array}$

WATER YEARS 1961 - 1999

\begin{tabular}{|c|c|c|c|}
\hline 98.4 & & & \\
\hline 461 & & & 1997 \\
\hline 4.16 & & & 1977 \\
\hline 32900 & Jan & 2 & 1997 \\
\hline 1.0 & Nov & 1 & 1980 \\
\hline 2.7 & Mar & 2 & 1977 \\
\hline 47700 & Jan & 2 & 1997 \\
\hline 15.72 & Jan & 2 & 1997 \\
\hline 71270 & & & \\
\hline 145 & & & \\
\hline $\begin{array}{r}19 \\
6.9\end{array}$ & & & \\
\hline
\end{tabular}

a Diversion, in acre-feet, to Camino Powerplant, provided by Sacramento Municipal Utility District. 
11442690 BRUSH CREEK RESERVOIR NEAR POLLOCK PINES, CA

LOCATION.-Lat 3848'42", long 120³7'14", in NW 1/4 SE 1/4 sec.10, T.11 N., R.12 E., El Dorado County, Hydrologic Unit 18020129, Eldorado National Forest, in outlet tower to Camino Powerplant $200 \mathrm{ft}$ upstream from left abutment of Brush Creek Diversion Dam and $4.0 \mathrm{mi}$ northwest of Pollock Pines.

DRAINAGE AREA.-7.99 $\mathrm{mi}^{2}$

PERIOD OF RECORD.-October 1991 to current year. Unpublished records for water years 1980-91 available in files of the U.S. Geological Survey.

GAGE.-Water-stage recorder. Datum of gage is sea level (levels by Sacramento Municipal Utility District). Prior to Apr. 7, 1987, nonrecording gage at same site and datum.

REMARKS.--Reservoir is formed by concrete-arch dam completed in 1970. Storage began in 1970. Usable capacity, 1,273 acre-ft, between elevations 2,825 ft, invert of tunnel, and 2,915 ft, crest of spillway. Dead storage, 259 acre- $\mathrm{ft}$. Most of the water is diverted at this reservoir to Camino Powerplant (station 11441895). Records, including extremes, represent total contents at 2400 hours. See schematic diagram of South Fork American River Basin.

COOPERATION.-Records were collected by Sacramento Municipal Utility District, under general supervision of the U.S. Geological Survey, in connection with a Federal Energy Regulatory Commission project. Contents not rounded to U.S. Geological Survey standards.

EXTREMES FOR PERIOD OF RECORD.-Maximum contents, 1,546 acre-ft, Jan. 25, 1997, elevation, 2,915.72 ft; minimum, 541 acre-ft, June 29, 1995, elevation, 2,853.64 ft.

EXTREMES FOR CURRENT YEAR.—Maximum contents, 1,505 acre-ft, Feb. 8, elevation, 2,913.72 ft; minimum, 902 acre-ft, Apr. 13, elevation, $2,880.14 \mathrm{ft}$.

Capacity table (elevation, in feet, and contents, in acre-feet)

(Based on table provided by Sacramento Municipal Utility District, recomputed October 1991)

$\begin{array}{rrrr}2,820 & 220 & 2,870 & 753 \\ 2,830 & 300 & 2,880 & 900 \\ 2,840 & 393 & 2,890 & 1,062 \\ 2,850 & 499 & 2,900 & 1,239 \\ 2,860 & 619 & 2,915 & 1,532\end{array}$

RESERVOIR STORAGE (ACRE-FEET), WATER YEAR OCTOBER 1998 TO SEPTEMBER 1999 DAILY OBSERVATION AT 2400 HOURS

\begin{tabular}{|c|c|c|c|c|c|c|c|c|c|c|c|c|}
\hline DAY & $\mathrm{OCT}$ & NOV & DEC & JAN & FEB & MAR & APR & MAY & JUN & JUL & AUG & SEP \\
\hline 1 & 1301 & 1407 & 1299 & 1221 & 1251 & 1444 & 1444 & 1064 & 1406 & 1289 & 1322 & 1263 \\
\hline 2 & 1306 & 1406 & 1310 & 1221 & 1259 & 1429 & 1195 & 1086 & 1343 & 1352 & 1215 & 1269 \\
\hline 3 & 1311 & 1404 & 1343 & 1223 & 1268 & 1416 & 1171 & 1123 & 1356 & 1356 & 1071 & 1267 \\
\hline 4 & 1316 & 1401 & 1363 & 1224 & 1279 & 1440 & 1166 & 1204 & 1374 & 1367 & 1219 & 1268 \\
\hline 5 & e1321 & 1399 & 1373 & 1224 & 1289 & 1469 & 1187 & 1314 & 1391 & 1254 & 1214 & 1270 \\
\hline 6 & 1325 & 1398 & 1379 & 1224 & 1305 & 1411 & 1209 & 1169 & 1407 & 1240 & 1215 & 1272 \\
\hline 7 & 1328 & 1404 & 1333 & 1224 & 1439 & 1288 & 1068 & 1225 & 1422 & 1282 & 1221 & 1273 \\
\hline 8 & 1332 & 1406 & 1347 & 1225 & 1505 & 1276 & 995 & 1227 & 1437 & 1288 & 1225 & 1275 \\
\hline 9 & 1336 & 1406 & 1298 & 1225 & 1376 & 1279 & 956 & 1229 & 1453 & 1295 & 1229 & 1276 \\
\hline 10 & 1340 & 1406 & 1266 & 1225 & 1408 & 1289 & 944 & 1233 & 1345 & 1302 & 1234 & 1277 \\
\hline 11 & 1344 & 1407 & 1247 & 1225 & 1390 & 1308 & 961 & 1195 & 1343 & 1309 & 1238 & 1279 \\
\hline 12 & 1347 & 1407 & 1240 & 1225 & 1365 & 1333 & 907 & 1184 & 1357 & 1101 & 1242 & 1281 \\
\hline 13 & 1351 & 1407 & 1243 & 1224 & 1346 & 1365 & 902 & 1195 & 1370 & 1282 & 1246 & 1282 \\
\hline 14 & 1355 & 1406 & 1247 & 1339 & 1345 & 1397 & 943 & 1212 & 1384 & 1360 & 1249 & 1283 \\
\hline 15 & 1358 & 1406 & 1249 & 1338 & 1358 & 1432 & 957 & 1228 & 1398 & 1349 & 1253 & 1284 \\
\hline 16 & 1362 & 1405 & 1252 & 1356 & 1405 & 1467 & 971 & 1243 & 1411 & 1351 & 1257 & 1286 \\
\hline 17 & 1365 & 1407 & 1244 & 1366 & 1434 & 1370 & 999 & 1258 & 1227 & 1354 & 1261 & 1288 \\
\hline 18 & 1369 & 1408 & 1231 & 1436 & 1405 & 1295 & 1033 & 1273 & 1355 & 1425 & 1265 & 1289 \\
\hline 19 & 1371 & 1408 & 1208 & 1442 & 1418 & 1243 & 1061 & 1289 & 1362 & 1426 & 1269 & 1291 \\
\hline 20 & 1374 & 1407 & 1192 & 1413 & 1438 & 1205 & 1086 & 1304 & 1228 & 1430 & 1273 & 1293 \\
\hline 21 & 1377 & 1405 & 1182 & 1428 & 1399 & 1206 & 1108 & 1319 & 1219 & 1436 & 1276 & 1294 \\
\hline 22 & 1379 & 1411 & 1230 & 1389 & 1375 & 1224 & 1127 & 1332 & 1031 & 1340 & 1316 & 1296 \\
\hline 23 & 1382 & 1425 & 1270 & 1421 & 1366 & 1249 & 1128 & 1345 & 1231 & 1333 & 1304 & 1297 \\
\hline 24 & 1388 & 1435 & 1215 & 1437 & 1372 & 1273 & 1147 & 1359 & 1322 & 1338 & 1295 & 1298 \\
\hline 25 & 1393 & 1437 & 1215 & 1455 & 1419 & 1297 & 1170 & 1372 & 989 & 1343 & 1295 & 1300 \\
\hline 26 & 1397 & 1438 & 1212 & 1300 & 1406 & 1321 & 1088 & 1386 & 1019 & 1347 & 1297 & 1301 \\
\hline 27 & 1400 & 1225 & 1216 & 1290 & 1375 & 1343 & 1095 & 1398 & 1196 & 1352 & 1299 & 1302 \\
\hline 28 & 1404 & 1219 & 1215 & 1265 & 1373 & 1365 & 1039 & 1410 & 1113 & 1356 & 1301 & 1303 \\
\hline 29 & 1408 & 1228 & 1217 & 1247 & --- & 1387 & 1031 & 1422 & 1210 & 1344 & 1303 & 1301 \\
\hline 30 & 1409 & 1265 & 1218 & 1236 & --- & 1410 & 1044 & 1434 & 1269 & 1256 & 1304 & 1297 \\
\hline 31 & 1407 & --- & 1220 & 1239 & --- & 1434 & --- & 1446 & --- & 1252 & 1306 & --- \\
\hline MAX & 1409 & 1438 & 1379 & 1455 & 1505 & 1469 & 1444 & 1446 & 1453 & 1436 & 1322 & 1303 \\
\hline MIN & 1301 & 1219 & 1182 & 1221 & 1251 & 1205 & 902 & 1064 & 989 & 1101 & 1071 & 1263 \\
\hline$a$ & 2908.82 & 2901.43 & 2898.96 & 2900.01 & 2907.12 & 2910.19 & 2888.92 & 2910.79 & 2901.66 & 2900.70 & 2903.60 & 2903.11 \\
\hline $\mathrm{b}$ & +112 & -142 & -45 & +19 & +134 & +61 & -390 & +402 & -177 & -17 & +54 & -9 \\
\hline CAL Y & YR 1998 & $\operatorname{MAX} 1523$ & MIN 854 & $b+21$ & & & & & & & & \\
\hline WTR Y & YR 1999 & MAX 1505 & MIN 902 & $\mathrm{~b}+2$ & & & & & & & & \\
\hline
\end{tabular}

e Estimated.

a Elevation, in feet, at end of month.

b Change in contents, in acre-feet. 
LOCATION.-Lat 3848’41", long 120³7’20", in NW 1/4 SE 1/4 sec.10, T.11 N., R.12 E., El Dorado County, Hydrologic Unit 18020129, Eldorado National Forest, at outlet structure on Brush Creek Dam and 4.0 mi northwest of Pollock Pines.

DRAINAGE AREA.-7.99 $\mathrm{mi}^{2}$.

PERIOD OF RECORD.-October 1987 to current year. Unpublished records for water years 1971-87 available in files of the U.S. Geological Survey.

GAGE.-Differential-pressure gage and orifice control in outlet pipe. Auxiliary water-stage recorder $200 \mathrm{ft}$ downstream at different datum. Elevation of gage is $2,700 \mathrm{ft}$ above sea level, from topographic map. Prior to October 1987, nonrecording gage $400 \mathrm{ft}$ downstream at different datum.

REMARKS.-Flow completely regulated by Brush Creek Reservoir (station 11442690). See schematic diagram of South Fork American River Basin.

COOPERATION.- Records were collected by Sacramento Municipal Utility District, under general supervision of the U.S. Geological Survey, in connection with a Federal Energy Regulatory Commission project.

EXTREMES FOR PERIOD OF RECORD.-Maximum daily discharge, $620 \mathrm{ft}^{3} / \mathrm{s}$, Jan. 2, 1997; minimum daily, $2.1 \mathrm{ft}^{3} / \mathrm{s}$, many days in 1988 .

DISCHARGE, CUBIC FEET PER SECOND, WATER YEAR OCTOBER 1998 TO SEPTEMBER 1999 DAILY MEAN VALUES

\begin{tabular}{|c|c|c|c|c|c|c|c|c|c|c|c|c|}
\hline DAY & $\mathrm{OCT}$ & NOV & $\mathrm{DEC}$ & JAN & FEB & MAR & APR & MAY & JUN & JUL & AUG & SEP \\
\hline 1 & 3.6 & 7.1 & 6.3 & 6.6 & 6.6 & 6.8 & 6.7 & 6.6 & 4.4 & 3.2 & 3.2 & 3.2 \\
\hline 2 & 3.6 & 7.2 & 6.3 & 6.7 & 6.6 & 6.8 & 6.6 & 6.7 & 3.1 & 3.2 & 3.1 & 3.1 \\
\hline 3 & 3.7 & 7.2 & 6.4 & 6.6 & 6.7 & 6.7 & 6.7 & 6.7 & 3.1 & 3.1 & 3.1 & 3.1 \\
\hline 4 & 3.7 & 7.2 & 6.3 & 6.7 & 6.7 & 6.7 & 6.7 & 6.7 & 3.1 & 3.1 & 3.2 & 3.1 \\
\hline 5 & e3.7 & 7.2 & 6.3 & 6.7 & 6.6 & 6.8 & 6.7 & 6.6 & 3.1 & 3.1 & 3.1 & 3.1 \\
\hline 6 & e3.7 & 7.2 & 6.4 & 6.7 & 6.7 & 6.7 & 6.8 & 6.7 & 3.1 & 3.0 & 3.1 & 3.1 \\
\hline 7 & 3.7 & 7.0 & 6.3 & 6.6 & 7.0 & 6.7 & 6.7 & 6.7 & 3.1 & 3.1 & 3.1 & 3.1 \\
\hline 8 & 3.7 & 7.0 & 6.3 & 6.7 & 10 & 6.7 & 6.8 & 6.6 & 3.2 & 3.1 & 3.1 & 3.1 \\
\hline 9 & 3.7 & 6.6 & 6.3 & 6.7 & 8.0 & 6.7 & 6.8 & 6.7 & 3.2 & 3.1 & 3.1 & 3.1 \\
\hline 10 & 3.7 & 6.2 & 6.3 & 6.7 & 6.8 & 6.7 & 6.9 & 6.6 & 3.2 & 3.1 & 3.1 & 3.0 \\
\hline 11 & 3.7 & 6.2 & 6.4 & 6.7 & 6.7 & 6.7 & 6.9 & 6.6 & e 3.2 & 3.1 & 3.1 & 3.1 \\
\hline 12 & 3.7 & 6.2 & 6.4 & 6.6 & 6.7 & 6.7 & 6.8 & 6.6 & 3.2 & 3.1 & 3.2 & 3.1 \\
\hline 13 & 3.7 & 6.2 & 6.4 & 6.7 & 6.7 & 6.7 & 6.8 & 6.7 & 3.2 & 3.1 & 3.2 & 3.1 \\
\hline 14 & 3.7 & 6.2 & 6.4 & 6.6 & 6.7 & 6.6 & 6.9 & 6.7 & 3.2 & 3.1 & 3.2 & 3.0 \\
\hline 15 & 3.7 & 6.2 & 6.5 & 6.7 & 6.7 & 6.5 & 6.9 & 6.7 & 3.2 & 3.6 & 3.2 & 3.0 \\
\hline 16 & 3.7 & 6.2 & 6.5 & 6.7 & 6.8 & 6.6 & 6.9 & 6.7 & 3.2 & 3.1 & 3.2 & 3.0 \\
\hline 17 & 3.7 & 6.2 & 6.5 & 6.7 & 6.9 & 6.5 & 6.8 & 6.7 & 3.2 & 3.1 & 3.2 & 3.0 \\
\hline 18 & 3.7 & 6.2 & 6.5 & 6.8 & 6.7 & 6.5 & 6.8 & 6.7 & 3.3 & 3.1 & 3.2 & 3.0 \\
\hline 19 & 3.7 & 6.2 & 6.5 & 6.9 & 6.7 & 6.5 & 6.9 & 6.7 & 3.2 & 3.1 & 3.2 & 3.0 \\
\hline 20 & 3.7 & 6.2 & 6.5 & 6.8 & 6.8 & 6.5 & 6.9 & 6.7 & 3.2 & 3.1 & 3.2 & 3.0 \\
\hline 21 & 3.7 & 6.2 & 6.4 & 6.7 & 6.8 & 6.5 & 6.9 & 6.6 & 3.2 & 3.1 & 3.2 & 3.0 \\
\hline 22 & 3.7 & 6.2 & 6.4 & 6.7 & 6.7 & 6.5 & 6.8 & 6.6 & 3.2 & 3.1 & 3.1 & 3.0 \\
\hline 23 & 3.7 & 6.3 & 6.4 & 6.7 & 6.7 & 6.5 & 6.8 & 6.6 & 3.3 & 3.1 & 3.1 & 3.0 \\
\hline 24 & 3.7 & 6.2 & 6.4 & 6.7 & 6.7 & 6.6 & 6.7 & 6.6 & 3.2 & 3.1 & 3.2 & 3.0 \\
\hline 25 & 3.7 & 6.2 & 6.5 & 6.7 & 6.7 & 6.6 & 6.7 & 6.6 & 3.2 & 3.1 & 3.1 & 3.0 \\
\hline 26 & 3.7 & 6.2 & 6.5 & 6.6 & 6.8 & 6.6 & 6.7 & 6.7 & 3.2 & 3.1 & 3.1 & 3.0 \\
\hline 27 & 3.7 & 6.2 & 6.5 & 6.5 & 6.7 & 6.6 & 6.7 & 6.7 & 3.2 & 3.1 & 3.0 & 3.0 \\
\hline 28 & 3.7 & 6.2 & 6.5 & 6.5 & 6.7 & 6.6 & 6.6 & 6.7 & 3.2 & 3.1 & 3.0 & 3.0 \\
\hline 29 & 3.7 & 6.2 & 6.5 & 6.7 & --- & 6.6 & 6.6 & 6.7 & 3.2 & 3.1 & 3.1 & 3.0 \\
\hline 30 & 5.0 & 6.3 & 6.5 & 6.6 & --- & 6.7 & 6.6 & 6.6 & 3.2 & 3.2 & 3.1 & 3.0 \\
\hline 31 & 7.0 & --- & 6.5 & 6.6 & --- & 6.7 & --- & 6.6 & --- & 3.2 & 3.1 & --- \\
\hline TOTAL & 119.1 & 194.1 & 198.9 & 206.9 & 192.9 & 205.6 & 203.1 & 206.4 & 96.8 & 96.9 & 97.2 & 91.3 \\
\hline MEAN & 3.84 & 6.47 & 6.42 & 6.67 & 6.89 & 6.63 & 6.77 & 6.66 & 3.23 & 3.13 & 3.14 & 3.04 \\
\hline MAX & 7.0 & 7.2 & 6.5 & 6.9 & 10 & 6.8 & 6.9 & 6.7 & 4.4 & 3.6 & 3.2 & 3.2 \\
\hline MIN & 3.6 & 6.2 & 6.3 & 6.5 & 6.6 & 6.5 & 6.6 & 6.6 & 3.1 & 3.0 & 3.0 & 3.0 \\
\hline$A C-F T$ & 236 & 385 & 395 & 410 & 383 & 408 & 403 & 409 & 192 & 192 & 193 & 181 \\
\hline
\end{tabular}


11442700 BRUSH CREEK BELOW BRUSH CREEK DAM, NEAR POLLOCK PINES, CA—Continued

STATISTICS OF MONTHLY MEAN DATA FOR WATER YEARS 1988 - 1999, BY WATER YEAR (WY)

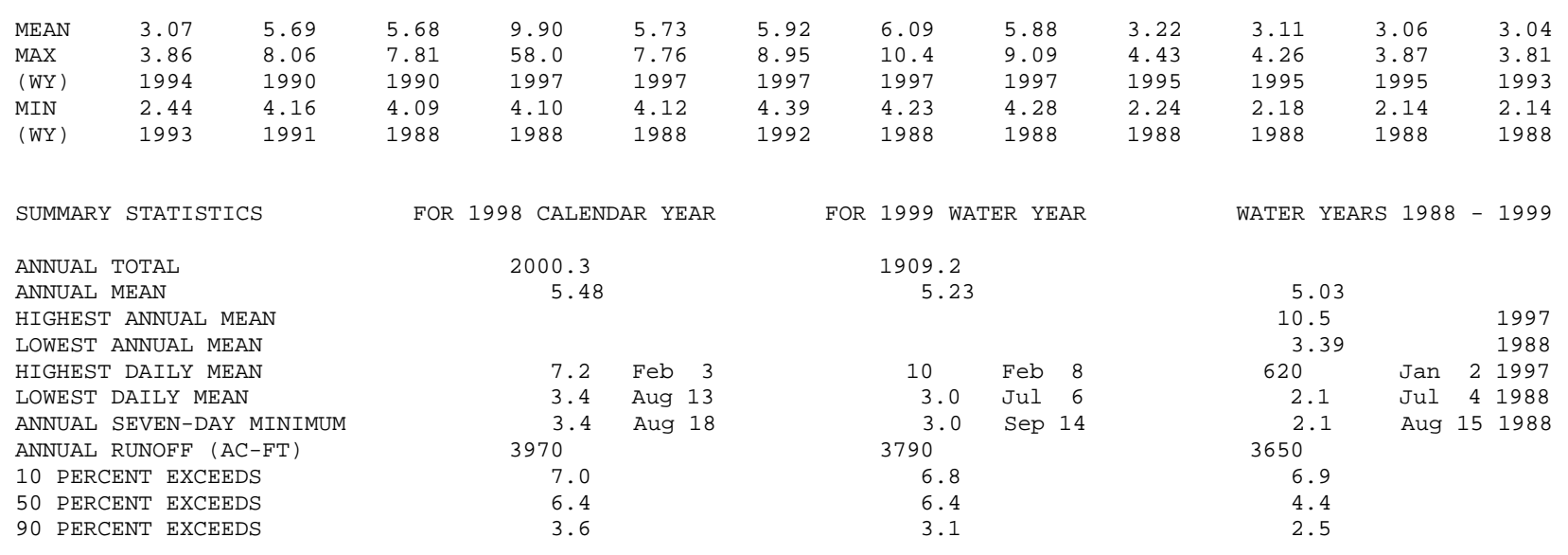




\section{SLAB CREEK RESERVOIR NEAR CAMINO, CA}

LOCATION.-Lat 3846’21", long 12041'58", in SW 1/4 NE 1/4 sec.25, T.11 N., R.11 E., El Dorado County, Hydrologic Unit 18020129, Eldorado National Forest, on left bank $100 \mathrm{ft}$ upstream from dam on South Fork American River, 1,600 ft upstream from Iowa Canyon, and 2.7 mi northwest of Camino.

DRAINAGE AREA.-493 $\mathrm{mi}^{2}$.

PERIOD OF RECORD.-May 1987 to current year. Unpublished records for water years 1969-86 available in files of the U.S. Geological Survey.

GAGE.-Water-stage recorder. Datum of gage is sea level (levels by Sacramento Municipal Utility District). Prior to May 26, 1987, nonrecording gage at same site and datum. September 1980 to October 1993, supplementary water-stage recorder at left abutment of dam operated by U.S. Geological Survey during periods of spill.

REMARKS.- Reservoir is formed by concrete-arch dam completed in 1967. Storage began in October 1967. Usable capacity, 16,567 acre-ft, between elevations 1,670 ft, invert of tunnel, and 1,850 ft, crest of spillway. Dead storage, 600 acre-ft. Reservoir receives water from South Fork American River and Silver Creek via El Dorado and Camino Powerplants (station 11441895) 10 mi upstream. Nearly the entire flow is diverted at this reservoir to White Rock Powerplant (station 11443460). See South Fork American River near Camino (station 11443500) for additional information on diversions and releases from Slab Creek Reservoir. Records, including extremes, represent usable contents. See schematic diagram of South Fork American River Basin.

COOPERATION.—Records were collected by Sacramento Municipal Utility District, under general supervision of the U.S. Geological Survey, in connection with a Federal Energy Regulatory Commission project. Contents not rounded to U.S. Geological Survey standards.

EXTREMES FOR PERIOD OF RECORD.—Maximum contents, 18,637 acre-ft, Jan. 1, 1997, elevation, 1,859.70 ft; minimum, 3,917 acre-ft, Oct. 27, 1991, elevation, unknown.

EXTREMES FOR CURRENT YEAR.-Maximum contents, 17,124 acre-ft, Feb. 8, elevation, 1,852.68 ft, minimum, 9,360 acre-ft, May 18 , elevation, $1,808.84$.

Capacity table (elevation, in feet, and contents, in acre-feet)

(Based on survey by Sacramento Municipal Utility District recomputed October 1991)

$\begin{array}{rrrr}1,730 & 1,688 & 1,800 & 8,124 \\ 1,740 & 2,276 & 1,820 & 11,073 \\ 1,750 & 2,966 & 1,840 & 14,587 \\ 1,760 & 3,763 & 1,850 & 16,567 \\ 1,780 & 5,700 & 1,855 & 17,615\end{array}$

RESERVOIR STORAGE (ACRE-FEET), WATER YEAR OCTOBER 1998 TO SEPTEMBER 1999 DAILY OBSERVATION AT 2400 HOURS

\begin{tabular}{|c|c|c|c|c|c|c|c|c|c|c|c|c|}
\hline DAY & $\mathrm{OCT}$ & NOV & $\mathrm{DEC}$ & JAN & FEB & MAR & APR & MAY & JUN & JUL & AUG & SEP \\
\hline 1 & 15836 & 15641 & 16614 & 14393 & 13052 & 16801 & 11413 & 11801 & 14308 & 14009 & 14628 & 14749 \\
\hline 2 & 16086 & 15461 & 15198 & 14261 & 12471 & 16780 & 11783 & 12224 & 15917 & 14059 & 15172 & 13970 \\
\hline 3 & 16049 & 15390 & 14303 & 14306 & 13134 & 16867 & 12549 & 11556 & 16035 & 14476 & 15019 & 14678 \\
\hline 4 & 15973 & 15274 & 13324 & 13968 & 13091 & 16809 & 12613 & 11566 & 14955 & 14471 & 14739 & 14171 \\
\hline 5 & e15779 & 15227 & 12552 & 13624 & 12365 & 16751 & 11336 & 11326 & 14711 & 14372 & 14579 & 14028 \\
\hline 6 & 15585 & 15575 & 12701 & 13558 & 11387 & 16757 & 10876 & 11887 & 15170 & 14732 & 13299 & 13400 \\
\hline 7 & 15780 & 14340 & 12743 & 13617 & 14880 & 16691 & 11232 & 12266 & 14912 & 15215 & 14306 & 13885 \\
\hline 8 & 15554 & 14070 & 12302 & 13426 & 17124 & 16011 & 12260 & 11556 & 14342 & 15382 & 15572 & 14473 \\
\hline 9 & 15514 & 14431 & 12629 & 13520 & 17080 & 14408 & 12338 & 12192 & 12811 & 14592 & 15465 & 15270 \\
\hline 10 & 15530 & 14239 & 12570 & 13776 & 16838 & 12158 & 12269 & 11964 & 10815 & 15510 & 15731 & 15274 \\
\hline 11 & 15188 & 14164 & 13418 & 13944 & 16763 & 10813 & 12141 & 12291 & 10445 & 15327 & 15593 & 15433 \\
\hline 12 & 15210 & 14318 & 13529 & 14151 & 16722 & 11428 & 12168 & 12042 & 10494 & 14272 & 14435 & 15534 \\
\hline 13 & 15471 & 15126 & 13659 & 14380 & 16687 & 11411 & 12192 & 12632 & 10100 & 14254 & 14547 & 15591 \\
\hline 14 & 14905 & 16031 & 13837 & 14033 & 16658 & 11074 & 11596 & 11078 & 10863 & 14684 & 14359 & 15544 \\
\hline 15 & 14843 & 15133 & 14242 & 13712 & 16627 & 12343 & 12098 & 11316 & 10794 & 15015 & 13688 & 15842 \\
\hline 16 & 14914 & 14799 & 14024 & 14070 & 16728 & 11905 & 11568 & 11184 & 10470 & 14812 & 14416 & 16108 \\
\hline 17 & 15040 & 15597 & 14303 & 14581 & 16813 & 12850 & 12141 & 10580 & 12024 & 15122 & 14372 & 16336 \\
\hline 18 & 14916 & 15901 & 14556 & 15262 & 16913 & 12643 & 12618 & 9360 & 12391 & 15163 & 14070 & 16003 \\
\hline 19 & 14909 & 16195 & 14211 & 16836 & 16838 & 11846 & 12778 & 11176 & 14849 & 15052 & 14423 & 15868 \\
\hline 20 & 14573 & 16468 & 14239 & 16911 & 16848 & 11333 & 11736 & 13407 & 15605 & 14874 & 14082 & 15627 \\
\hline 21 & 14640 & 15766 & 13431 & 16674 & 16825 & 11241 & 11885 & 16096 & 14726 & 15475 & 14444 & 15581 \\
\hline 22 & 14860 & 15339 & 14000 & 16256 & 16751 & 11289 & 12481 & 16944 & 14154 & 14444 & 14465 & 15272 \\
\hline 23 & 14810 & 15192 & 13617 & 16720 & 16730 & 11485 & 11515 & 16963 & 14393 & 14714 & 14872 & 15473 \\
\hline 24 & 14533 & 14895 & 13866 & 16454 & 16720 & 11184 & 11568 & 16950 & 14241 & 14320 & 15079 & 15917 \\
\hline 25 & 14554 & 14897 & 13507 & 15597 & 16749 & 10946 & 12778 & 17076 & 14188 & 14310 & 14613 & 15473 \\
\hline 26 & 14847 & 14938 & 13522 & 14878 & 16724 & 11505 & 12984 & 16990 & 14310 & 14333 & 15132 & 15736 \\
\hline 27 & 14828 & 15011 & 13206 & 13791 & 16722 & 11867 & 12533 & 17026 & 14930 & 14512 & 14932 & 15258 \\
\hline 28 & 15067 & 14745 & 13741 & 13145 & 16774 & 11123 & 12339 & 17009 & 14166 & 14336 & 15429 & 15211 \\
\hline 29 & 15633 & 14235 & 13607 & 12701 & --- & 10636 & 11031 & 16898 & 14214 & 15149 & 15923 & 15532 \\
\hline 30 & 15671 & 15750 & 13888 & 12903 & --- & 11456 & 10793 & 16542 & 13831 & 14440 & e15293 & 14503 \\
\hline 31 & 15838 & --- & 14220 & 13156 & --- & 11146 & --- & 15298 & --- & 14613 & 14663 & --- \\
\hline MAX & 16086 & 16468 & 16614 & 16911 & 17124 & 16867 & 12984 & 17076 & 16035 & 15510 & 15923 & 16336 \\
\hline MIN & 14533 & 14070 & 12302 & 12701 & 11387 & 10636 & 10793 & 9360 & 10100 & 14009 & 13299 & 13400 \\
\hline$a$ & 1846.41 & 1845.97 & 1838.06 & 1832.25 & 1851.00 & 1820.45 & 1818.25 & 1843.68 & 1835.97 & 1840.14 & 1840.40 & 1839.56 \\
\hline $\mathrm{b}$ & +263 & -88 & -1530 & -1064 & +3618 & -5628 & -353 & +4505 & -1467 & +782 & +50 & -160 \\
\hline
\end{tabular}

CAL YR 1998 MAX 17204 MIN 9576.0 b $\quad-871$

WTR YR 1999 MAX 17124 MIN 9360.0 b -1072

e Estimated.

a Elevation, in feet, at end of month

b Change in contents, in acre-feet. 
LOCATION.-Lat 38 46'23", long 120²'02", in SW 1/4 NE 1/4 sec.25, T.11 N., R.11 E., El Dorado County, Hydrologic Unit 18020129, on right bank $500 \mathrm{ft}$ upstream from Iowa Canyon Creek and 2.8 mi northwest of Camino.

DRAINAGE AREA.-493 $\mathrm{mi}^{2}$.

PERIOD OF RECORD.-October 1922 to current year. Monthly discharge only for October 1922, WSP 1315-A. Records for river and American River Flume, published separately October 1922 to September 1956, October 1962 to December 1964 when flume was destroyed. Records of river and flume combined October 1956 to September 1962.

REVISED RECORDS.-WSP 931: 1928, 1938, 1940(M). WSP 1931: Drainage area at former site.

GAGE.-Acoustic-velocity meter. Elevation of gage is 1,625 ft above sea level, from topographic map. Prior to May 26, 1987, water-stage recorder at different datum at site 1,000 ft downstream. Auxiliary water-stage recorder on Slab Creek Dam records spill discharges which are combined with release discharges. See WSP 2131 for history of changes prior to Oct. 12, 1966.

REMARKS.-Flow regulated by several reservoirs. Since 1967 diversion from Slab Creek Dam to White Rock Powerplant (station 11443460) bypasses this station. Echo Lake Conduit (station 11434500) imports up to 1,900 acre-ft each year from Truckee River Basin. Variable amounts of El Dorado Canal water, up to $40 \mathrm{ft}^{3} / \mathrm{s}$ May to October, and about $7 \mathrm{ft}^{3} / \mathrm{s}$ remainder of the year, diverted for irrigation and domestic use between Pollock Pines and Placerville. Water from Jenkinson Lake in North Fork Cosumnes River Basin diverted to Camino and substituted for flow from El Dorado Canal in some years. Since October 1962, water is imported from the Upper Rubicon River Basin by way of Robbs Peak Powerplant (station 11429300). See schematic diagram of South Fork American River Basin.

COOPERATION.- - Records were collected by Sacramento Municipal Utility District, under general supervision of the U.S. Geological Survey, in connection with a Federal Energy Regulatory Commission project.

EXTREMES FOR PERIOD OF RECORD.-Maximum discharge, $62,300 \mathrm{ft}^{3} / \mathrm{s}$, Jan. 2, 1997, from rating curve extended above $24,000 \mathrm{ft}^{3} / \mathrm{s}$ on basis of computation of peak flow over dam; minimum daily, $1.3 \mathrm{ft}^{3} / \mathrm{s}$, Aug. 24, 1931.

DISCHARGE, CUBIC FEET PER SECOND, WATER YEAR OCTOBER 1998 TO SEPTEMBER 1999

DAILY MEAN VALUES

\begin{tabular}{|c|c|c|c|c|c|c|c|c|c|c|c|c|}
\hline DAY & $\mathrm{OCT}$ & NOV & $\mathrm{DEC}$ & JAN & FEB & MAR & APR & MAY & JUN & JUL & AUG & SEP \\
\hline 1 & 41 & 41 & 314 & 40 & 39 & 1300 & 38 & 38 & 39 & 40 & 40 & 37 \\
\hline 2 & 41 & 40 & 48 & 40 & 39 & 1180 & 38 & 39 & 39 & 40 & 41 & 37 \\
\hline 3 & 41 & 40 & 40 & 40 & 39 & 1540 & 39 & 38 & 40 & 40 & 41 & 37 \\
\hline 4 & 41 & 40 & 39 & 40 & 39 & 1620 & 39 & 38 & 39 & 40 & 41 & 37 \\
\hline 5 & 41 & 40 & 39 & 40 & 39 & 1120 & 38 & 38 & 39 & 40 & 41 & 37 \\
\hline 6 & 41 & 40 & 38 & 39 & 38 & 898 & 38 & 38 & 39 & 41 & 40 & 37 \\
\hline 7 & 41 & 40 & 39 & 39 & 38 & 944 & 38 & 38 & 39 & 41 & 40 & 37 \\
\hline 8 & 41 & 40 & 38 & 39 & 851 & 606 & 38 & 38 & 39 & 41 & 41 & 37 \\
\hline 9 & 40 & 40 & 38 & 39 & 5260 & 40 & 39 & 38 & 38 & 41 & 41 & 37 \\
\hline 10 & 40 & 40 & 39 & 39 & 1990 & 39 & 39 & 38 & 37 & 41 & 41 & 38 \\
\hline 11 & 40 & 40 & 38 & 39 & 964 & 38 & 39 & 39 & 36 & 41 & 41 & 38 \\
\hline 12 & 40 & 40 & 39 & 40 & 634 & 38 & 39 & 38 & 36 & 41 & 41 & 38 \\
\hline 13 & 40 & 40 & 39 & 40 & 396 & 38 & 39 & 39 & 36 & 40 & 41 & 38 \\
\hline 14 & 40 & 40 & 39 & 40 & 243 & 38 & 38 & 38 & 38 & 40 & 40 & 38 \\
\hline 15 & 40 & 40 & 39 & 39 & 184 & 38 & 38 & 38 & 38 & 41 & 40 & 38 \\
\hline 16 & 40 & 40 & 40 & 39 & 148 & 39 & 39 & 37 & 37 & 41 & 40 & 38 \\
\hline 17 & 40 & 40 & 40 & 40 & 1330 & 39 & 38 & 37 & 38 & 41 & 40 & 38 \\
\hline 18 & 40 & 40 & 40 & 40 & 1040 & 39 & 39 & 36 & 39 & 41 & 40 & 38 \\
\hline 19 & 40 & 41 & 40 & 376 & 1450 & 39 & 39 & 40 & 40 & 41 & 40 & 38 \\
\hline 20 & 40 & 41 & 40 & 2260 & 1110 & 38 & 38 & 40 & 41 & 41 & 40 & 38 \\
\hline 21 & 40 & 41 & 39 & 102 & 1210 & 38 & 38 & 39 & 41 & 41 & 40 & 38 \\
\hline 22 & 40 & 40 & 39 & 116 & 1000 & 38 & 39 & 1070 & 40 & 41 & 40 & 38 \\
\hline 23 & 40 & 40 & 39 & 269 & 880 & 38 & 39 & 1560 & 40 & 40 & 41 & 38 \\
\hline 24 & 40 & 40 & 39 & 141 & 758 & 38 & 38 & 1820 & 40 & 41 & 41 & 38 \\
\hline 25 & 40 & 40 & 39 & 41 & 936 & 38 & 39 & 2100 & 40 & 40 & 41 & 38 \\
\hline 26 & 40 & 40 & 39 & 40 & 796 & 38 & 39 & 2500 & 41 & 40 & 41 & 38 \\
\hline 27 & 40 & 40 & 39 & 40 & 748 & 38 & 39 & 2430 & 41 & 40 & 39 & 38 \\
\hline 28 & 40 & 40 & 39 & 39 & 758 & 38 & 39 & 2380 & 41 & 40 & 38 & 38 \\
\hline 29 & 40 & 40 & 39 & 39 & --- & 37 & 38 & 2040 & 40 & 40 & 38 & 38 \\
\hline 30 & 40 & 40 & 39 & 39 & --- & 38 & 37 & 1430 & 40 & 41 & 38 & 38 \\
\hline 31 & 41 & --- & 40 & 39 & --- & 38 & --- & 40 & --- & 40 & 38 & --- \\
\hline TOTAL & 1249 & 1204 & 1496 & 4253 & 22957 & 10088 & 1155 & 18172 & 1171 & 1257 & 1245 & 1131 \\
\hline MEAN & 40.3 & 40.1 & 48.3 & 137 & 820 & 325 & 38.5 & 586 & 39.0 & 40.5 & 40.2 & 37.7 \\
\hline MAX & 41 & 41 & 314 & 2260 & 5260 & 1620 & 39 & 2500 & 41 & 41 & 41 & 38 \\
\hline MIN & 40 & 40 & 38 & 39 & 38 & 37 & 37 & 36 & 36 & 40 & 38 & 37 \\
\hline $\mathrm{AC}-\mathrm{FT}$ & 2480 & 2390 & 2970 & 8440 & 45540 & 20010 & 2290 & 36040 & 2320 & 2490 & 2470 & 2240 \\
\hline a & 27750 & 30920 & 54040 & 56920 & 95270 & 139000 & 150500 & 159500 & 136800 & 64840 & 78290 & 58740 \\
\hline
\end{tabular}

a Diversion, in acre-feet, to White Rock Powerplant, provided by Sacramento Municipal Utility District. 
11443500 SOUTH FORK AMERICAN RIVER NEAR CAMINO, CA—Continued

STATISTICS OF MONTHLY MEAN DATA FOR WATER YEARS 1923 - 1957, BY WATER YEAR (WY)

\begin{tabular}{|c|c|c|c|c|c|c|c|c|c|c|c|c|}
\hline MEAN & 54.8 & 254 & 569 & 601 & 855 & 1171 & 2069 & 2681 & 1557 & 285 & 39.7 & 31.1 \\
\hline MAX & 221 & 3951 & 4780 & 3422 & 2125 & 3367 & 4015 & 6382 & 4031 & 1310 & 168 & 150 \\
\hline (WY) & 1952 & 1951 & 1951 & 1956 & 1927 & 1943 & 1952 & 1952 & 1952 & 1952 & 1951 & 1951 \\
\hline MIN & 4.43 & 5.46 & 12.9 & 43.0 & 116 & 146 & 620 & 418 & 13.8 & 1.97 & 2.01 & 6.97 \\
\hline (WY) & 1930 & 1930 & 1950 & 1929 & 1929 & 1924 & 1924 & 1934 & 1924 & 1931 & 1931 & 1955 \\
\hline
\end{tabular}

SUMMARY STATISTICS

WATER YEARS 1923 - 1957

ANNUAL MEAN

HIGHEST ANNUAL MEAN

LOWEST ANNUAL MEAN

HIGHEST DAILY MEAN

LOWEST DAILY MEAN

ANNUAL SEVEN-DAY MINIMUM

INSTANTANEOUS PEAK FLOW

INSTANTANEOUS PEAK STAGE

ANNUAL RUNOFF (AC-FT)

10 PERCENT EXCEEDS

50 PERCENT EXCEEDS

90 PERCENT EXCEEDS

\begin{tabular}{|c|c|c|}
\hline 846 & & \\
\hline 1760 & & 1951 \\
\hline 161 & & 1924 \\
\hline 40000 & Dec 23 & 1955 \\
\hline 1.3 & Aug 24 & 1931 \\
\hline 1.5 & Jul 29 & 1931 \\
\hline 49800 & Dec 23 & 1955 \\
\hline 32.6 & Dec 23 & 1955 \\
\hline \multicolumn{3}{|l|}{612700} \\
\hline \multicolumn{3}{|l|}{2520} \\
\hline \multicolumn{3}{|l|}{230} \\
\hline 13 & & \\
\hline
\end{tabular}

STATISTICS OF MONTHLY MEAN DATA FOR WATER YEARS 1923 - 1957, COMBINED RIVER PLUS FLUME, BY WATER YEAR (WY)

\begin{tabular}{|c|c|c|c|c|c|c|c|c|c|c|c|c|}
\hline MEAN & 167 & 364 & 684 & 713 & 959 & 1259 & 2176 & 2815 & 1695 & 413 & 154 & 142 \\
\hline MAX & 288 & 4051 & 4780 & 3422 & 2229 & 3490 & 4181 & 6552 & 4201 & 1474 & 324 & 227 \\
\hline (WY) & 1948 & 1951 & 1951 & 1956 & 1927 & 1943 & 1952 & 1952 & 1952 & 1952 & 1952 & 1952 \\
\hline MIN & 44.1 & 49.8 & 134 & 141 & 212 & 252 & 727 & 533 & 97.3 & 50.2 & 35.5 & 53.4 \\
\hline (WY) & 1930 & 1930 & 1924 & 1929 & 1933 & 1924 & 1924 & 1934 & 1924 & 1931 & 1931 & 1924 \\
\hline
\end{tabular}

SUMMARY STATISTICS

ANNUAL MEAN

HIGHEST ANNUAL MEAN

LOWEST ANNUAL MEAN

HIGHEST DAILY MEAN

LOWEST DAILY MEAN

ANNUAL SEVEN-DAY MINIMUM

ANNUAL RUNOFF (AC-FT)

10 PERCENT EXCEEDS

50 PERCENT EXCEEDS

90 PERCENT EXCEEDS
WATER YEARS 1923 - 1957

$\begin{array}{rlll}960 & & & \\ 1860 & & & 1952 \\ 249 & & & 1924 \\ 40000 & \text { Dec 23 } & 1955 \\ 20 & \text { Aug 24 } 1931 \\ 30 & \text { Aug } 19 & 1931 \\ 695700 & & & \\ 2660 & & & \\ 350 & & & \\ 120 & & & \end{array}$

STATISTICS OF MONTHLY MEAN DATA FOR WATER YEARS 1968 - 1999, BY WATER YEAR (WY)

\begin{tabular}{|c|c|c|c|c|c|c|c|c|c|c|c|c|}
\hline MEAN & 47.8 & 87.8 & 135 & 348 & 232 & 130 & 129 & 343 & 305 & 83.5 & 34.8 & 34.7 \\
\hline MAX & 453 & 1093 & 1112 & 4836 & 2709 & 1090 & 1402 & 2434 & 2619 & 936 & 45.1 & 48.2 \\
\hline (WY) & 1968 & 1968 & 1984 & 1997 & 1986 & 1986 & 1971 & 1995 & 1995 & 1995 & 1980 & 1980 \\
\hline MIN & 9.97 & 10.2 & 10.0 & 10.0 & 5.62 & 10.9 & 10.0 & 9.73 & 9.98 & 9.93 & 10.4 & 10.1 \\
\hline (WY) & 1978 & 1978 & 1988 & 1988 & 1970 & 1992 & 1988 & 1977 & 1977 & 1977 & 1977 & 1977 \\
\hline
\end{tabular}

SUMMARY STATISTICS

ANNUAL TOTAL

ANNUAL MEAN

HIGHEST ANNUAL MEAN

LOWEST ANNUAL MEAN

HIGHEST DAILY MEAN

LOWEST DAILY MEAN

ANNUAL SEVEN-DAY MINIMUM

INSTANTANEOUS PEAK FLOW

ANNUAL RUNOFF (AC-FT)

ANNUAL RUNOFF (AC-FT) a

10 PERCENT EXCEEDS

50 PERCENT EXCEEDS

90 PERCENT EXCEEDS
FOR 1998 CALENDAR YEAR

$\begin{array}{rr}83324 & \\ 228 & \\ & \\ 4260 & \text { Mar } 25 \\ 36 & \text { Jan } 25 \\ 37 & \text { Jan } 25 \\ 165300 & \\ 1389000 & \\ 459 & \\ 40 & \\ 38 & \end{array}$

FOR 1999 WATER YEAR

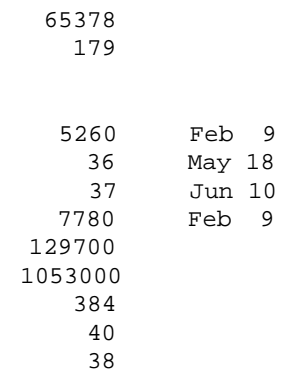

WATER YEARS 1968 - 1999

\begin{tabular}{|c|c|c|c|}
\hline \multicolumn{4}{|l|}{159} \\
\hline 608 & & & 1995 \\
\hline 13.3 & & & 1977 \\
\hline 48900 & Jan & 2 & 1997 \\
\hline 2.4 & Feb & 12 & 1970 \\
\hline 2.6 & Feb & 9 & 1970 \\
\hline 62300 & Jan & 2 & 1997 \\
\hline 115100 & & & \\
\hline 74 & & & \\
\hline 36 & & & \\
\hline
\end{tabular}

a Diversion, in acre-feet, to White Rock Powerplant, provided by Sacramento Municipal Utility District. 
LOCATION.—Lat 3847’39", long 12046'28", in NE 1/4 NW 1/4 sec.20, T.11 N., R.11 E., El Dorado County, Hydrologic Unit 18020129, on left bank $500 \mathrm{ft}$ downstream from Rock Creek Road and 4.0 mi north of Placerville.

DRAINAGE AREA.-73.0 $\mathrm{mi}^{2}$.

PERIOD OF RECORD.-October 1986 to current year.

GAGE.-Water-stage recorder and broad-crested weir; water-stage recorder and sharp-crested weir. Elevation of gages is 1,305 ft above sea level, from topographic map.

REMARKS.-Flow at this station has two components, which are combined for publication: flow over a broad-crested weir (station 11444200) and flow over a sharp-crested weir (station 11444260). Water is diverted upstream from weirs through a tunnel to Rock Creek Powerplant (station 11444280), returning to Rock Creek at its confluence with the South Fork American River. Extremes also represent combined flows. See schematic diagram of South Fork American River Basin.

COOPERATION.-Records provided by Sithe Energies, Inc., under general supervision of the U.S. Geological Survey, in connection with a Federal Energy Regulatory Commission project.

EXTREMES FOR PERIOD OF RECORD.-Maximum discharge, 6,690 ft³/s, Jan. 2, 1997; no flow Sept. 29 to Oct. 3, 1987.

DISCHARGE, CUBIC FEET PER SECOND, WATER YEAR OCTOBER 1998 TO SEPTEMBER 1999 DAILY MEAN VALUES

\begin{tabular}{|c|c|c|c|c|c|c|c|c|c|c|c|c|}
\hline DAY & OCT & NOV & $\mathrm{DEC}$ & JAN & $\mathrm{FEB}$ & MAR & $\mathrm{APR}$ & MAY & JUN & JUL & AUG & $\mathrm{SEP}$ \\
\hline 1 & 25 & 30 & 142 & 30 & 22 & 235 & 22 & 25 & 22 & 41 & 28 & 25 \\
\hline 2 & 25 & 27 & 22 & 30 & 22 & 130 & 22 & 26 & 22 & 41 & 39 & 25 \\
\hline 3 & 26 & 29 & 128 & 29 & 22 & 378 & 22 & 69 & 21 & 41 & 44 & 23 \\
\hline 4 & 25 & 26 & 90 & 29 & 22 & 199 & 22 & 24 & 22 & 41 & 52 & 23 \\
\hline 5 & 25 & 25 & 22 & 29 & 22 & 136 & 22 & 23 & 22 & 41 & 52 & 23 \\
\hline 6 & 24 & 28 & 22 & 28 & 22 & 92 & 23 & 22 & 22 & 40 & 54 & 22 \\
\hline 7 & 24 & 36 & 22 & 28 & 1350 & 68 & 23 & 26 & 22 & 40 & 55 & 22 \\
\hline 8 & 23 & 35 & 21 & 28 & 876 & 44 & 65 & 28 & 22 & 40 & 53 & 22 \\
\hline 9 & 24 & 28 & 22 & 28 & 1790 & 67 & 55 & 26 & 22 & 39 & 52 & 22 \\
\hline 10 & 24 & 27 & 22 & 28 & 693 & 36 & 27 & 24 & 22 & 39 & 54 & 23 \\
\hline 11 & 24 & 37 & 21 & 28 & 296 & 24 & 65 & 23 & 19 & 39 & 52 & 23 \\
\hline 12 & 23 & 28 & 23 & 28 & 109 & 22 & 40 & 22 & 22 & 38 & 53 & 22 \\
\hline 13 & 23 & 25 & 32 & 27 & 62 & 22 & 29 & 22 & 22 & 37 & 52 & 22 \\
\hline 14 & 24 & 24 & 36 & 27 & 33 & 21 & 27 & 22 & 22 & 35 & 51 & 22 \\
\hline 15 & 24 & 23 & 21 & 32 & 22 & 22 & 27 & 22 & 21 & 35 & 51 & 21 \\
\hline 16 & 23 & 23 & 21 & 34 & 72 & 25 & 21 & 22 & 21 & 36 & 50 & 21 \\
\hline 17 & 23 & 39 & 38 & 26 & 990 & 23 & 22 & 22 & 21 & 35 & 50 & 21 \\
\hline 18 & 23 & 33 & 37 & 267 & 335 & 23 & 22 & 22 & 21 & 34 & 50 & 22 \\
\hline 19 & 26 & 29 & 36 & 419 & 302 & 23 & 22 & 23 & 21 & 33 & 49 & 22 \\
\hline 20 & 26 & 27 & 36 & 1200 & 219 & 23 & 22 & 24 & 21 & 32 & 34 & 21 \\
\hline 21 & 25 & 26 & 36 & 392 & 500 & 23 & 22 & 22 & 21 & 32 & 24 & 21 \\
\hline 22 & 24 & 55 & 35 & 54 & 295 & 23 & 22 & 25 & 21 & 32 & 24 & 22 \\
\hline 23 & 23 & 39 & 35 & 662 & 205 & 22 & 22 & 21 & 25 & 32 & 23 & 21 \\
\hline 24 & 31 & 62 & 35 & 253 & 172 & 22 & 22 & 30 & 27 & 31 & 19 & 21 \\
\hline 25 & 31 & 42 & 35 & 46 & 203 & 22 & 22 & 42 & 29 & 31 & 19 & 22 \\
\hline 26 & 26 & 34 & 32 & 48 & 110 & 21 & 22 & 41 & 27 & 30 & 19 & 22 \\
\hline 27 & 26 & 37 & 32 & 28 & 70 & 21 & 22 & 43 & 44 & 30 & 22 & 22 \\
\hline 28 & 23 & 42 & 31 & 22 & 68 & 22 & 23 & 36 & 30 & 30 & 21 & 22 \\
\hline 29 & 25 & 26 & 31 & 22 & --- & 21 & 22 & 22 & 28 & 30 & 21 & 22 \\
\hline 30 & 25 & 156 & 30 & 22 & --- & 21 & 22 & 22 & 33 & 29 & 23 & 21 \\
\hline 31 & 26 & --- & 30 & 26 & --- & 22 & --- & 22 & --- & 28 & 25 & --- \\
\hline TOTAL & 769 & 1098 & 1176 & 3950 & 8904 & 1853 & 821 & 843 & 715 & 1092 & 1215 & 663 \\
\hline MEAN & 24.8 & 36.6 & 37.9 & 127 & 318 & 59.8 & 27.4 & 27.2 & 23.8 & 35.2 & 39.2 & 22.1 \\
\hline MAX & 31 & 156 & 142 & 1200 & 1790 & 378 & 65 & 69 & 44 & 41 & 55 & 25 \\
\hline MIN & 23 & 23 & 21 & 22 & 22 & 21 & 21 & 21 & 19 & 28 & 19 & 21 \\
\hline $\mathrm{AC}-\mathrm{FT}$ & 1530 & 2180 & 2330 & 7830 & 17660 & 3680 & 1630 & 1670 & 1420 & 2170 & 2410 & 1320 \\
\hline $\mathrm{a}$ & .00 & 30 & 875 & 2380 & 5530 & 7910 & 6840 & 3410 & 1760 & .00 & .00 & .00 \\
\hline
\end{tabular}

a Discharge, in acre-feet, through Rock Creek Powerplant near Placerville, provided by Sithe Energies U.S.A., Inc. 


\section{ROCK CREEK NEAR PLACERVILLE, CA—Continued}

STATISTICS OF MONTHLY MEAN DATA FOR WATER YEARS 1987 - 1999, BY WATER YEAR (WY)

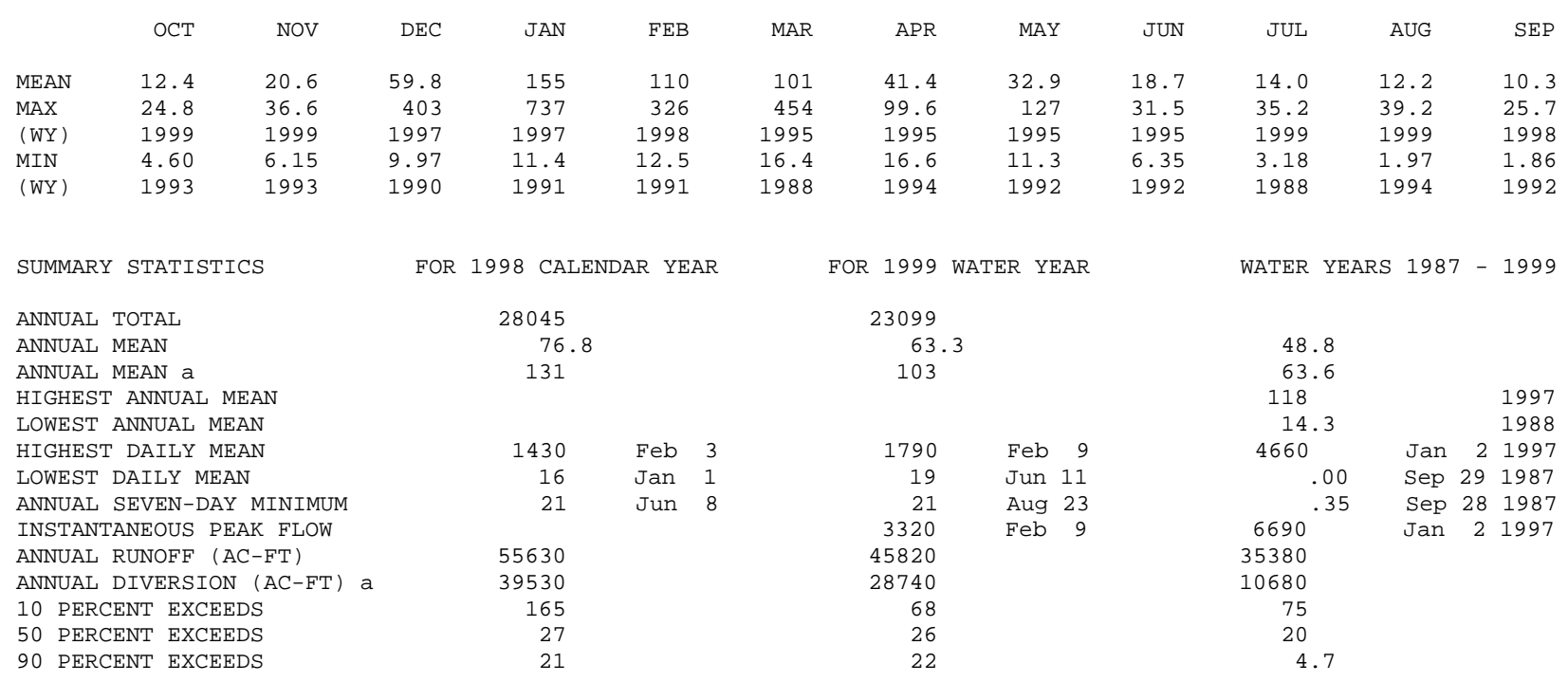

a Discharge, in acre-feet, through Rock Creek Powerplant near Placerville, provided by Sithe Energies U.S.A., Inc. 
11444500 SOUTH FORK AMERICAN RIVER NEAR PLACERVILLE, CA

LOCATION.-Lat 3846'16", long 12048'55", in NE 1/4 SW 1/4 sec.25, T.11 N., R.10 E., El Dorado County, Hydrologic Unit 18020129, on right bank $700 \mathrm{ft}$ downstream from Chili Bar Dam, $0.5 \mathrm{mi}$ upstream from Big Canyon, and 2.5 mi north of Placerville.

DRAINAGE AREA. $-598 \mathrm{mi}^{2}$.

PERIOD OF RECORD._-August 1911 to July 1920 (monthly discharge only for some periods, published in WSP 1315-A), July 1964 to current year.

REVISED RECORDS.-WSP 1931: Drainage area.

GAGE.-Water-stage recorder. Elevation of gage is $925 \mathrm{ft}$ above sea level, from topographic map. Aug. 11, 1911, to July 31, 1920, nonrecording gage $0.6 \mathrm{mi}$ downstream at different datum.

REMARKS.-Flow regulated by Chili Bar Reservoir, capacity, 3,700 acre-ft, Chili Bar Powerplant, and other storage and powerplants (see station 11443500). See schematic diagrams of South Fork American River and lower Sacramento River Basins.

COOPERATION.--Records provided by Pacific Gas \& Electric Co., under general supervision of the U.S. Geological Survey, in connection with a Federal Energy Regulatory Commission project.

EXTREMES FOR PERIOD OF RECORD.-Maximum discharge, 71,000 ft $3 / \mathrm{s}$, Jan. 2, 1997, gage height, unknown, on basis of computations of flow over dam, maximum gage height, $17.4 \mathrm{ft}$, from floodmarks, datum then in use, Dec. 23, 1964; minimum daily, $0.2 \mathrm{ft}^{3} / \mathrm{s}, \mathrm{Nov} .12,1964$.

DISCHARGE, CUBIC FEET PER SECOND, WATER YEAR OCTOBER 1998 TO SEPTEMBER 1999

DAILY MEAN VALUES

\begin{tabular}{|c|c|c|c|c|c|c|c|c|c|c|c|c|}
\hline DAY & $\mathrm{OCT}$ & NOV & $\mathrm{DEC}$ & JAN & FEB & MAR & APR & MAY & JUN & JUL & AUG & SEP \\
\hline 1 & 154 & 357 & 267 & 250 & 1160 & 3830 & 2490 & 2500 & 3920 & 1840 & 1220 & 1350 \\
\hline 2 & 308 & 738 & e1550 & 484 & 2000 & 3620 & 2330 & 3070 & 2670 & 1190 & 1280 & 1570 \\
\hline 3 & 705 & 808 & e2020 & 428 & 1300 & 4260 & 1980 & 3620 & 2910 & 862 & 1480 & 820 \\
\hline 4 & 557 & 1230 & e2100 & 670 & 1490 & 4180 & 2510 & 2800 & 3280 & 829 & 1490 & 1280 \\
\hline 5 & 870 & 452 & e1580 & 825 & 1890 & 3610 & 2730 & 2740 & 2340 & 1200 & 1810 & 1300 \\
\hline 6 & 397 & 858 & e1170 & 520 & 2000 & 3250 & 2480 & 2380 & 2090 & 1180 & 2200 & 1180 \\
\hline 7 & 229 & 1360 & e1220 & 554 & 4000 & 3320 & 2350 & 3140 & 2750 & 1010 & 1280 & 949 \\
\hline 8 & 706 & 535 & e1220 & 441 & 4040 & 3380 & 2170 & 3820 & 2990 & 1390 & 867 & 875 \\
\hline 9 & 303 & 363 & e971 & 439 & 8510 & 4070 & 2640 & 3240 & 3520 & 1620 & 1400 & 725 \\
\hline 10 & 424 & 833 & e1170 & 261 & 5070 & 4070 & 2640 & 3640 & 3560 & 1000 & 1290 & 766 \\
\hline 11 & 539 & 504 & e1210 & 504 & 3580 & 3480 & 2740 & 3420 & 2980 & 1230 & 2000 & 1100 \\
\hline 12 & 321 & 861 & e905 & 271 & 3070 & 2410 & 2700 & 4100 & 2340 & 2410 & 2380 & 1190 \\
\hline 13 & 400 & 216 & e532 & 204 & 2740 & 2720 & 2700 & 3770 & 2450 & 1570 & 1730 & 1150 \\
\hline 14 & 676 & 218 & e983 & 448 & 2530 & 2850 & 3220 & 4080 & 2410 & 1250 & 1760 & 1110 \\
\hline 15 & 637 & 554 & 896 & 495 & 2430 & 1960 & 2820 & 3230 & 3110 & 921 & 2150 & 1140 \\
\hline 16 & 234 & 1210 & 1350 & 296 & 2380 & 2750 & 3330 & 3430 & 3190 & 1380 & 1570 & 950 \\
\hline 17 & 584 & 302 & 1030 & 379 & 4760 & 2090 & 3020 & 3560 & 2370 & 981 & 1620 & 711 \\
\hline 18 & 280 & 342 & 1130 & 975 & 3710 & 2790 & 3110 & 4060 & 2670 & 950 & 1810 & 1240 \\
\hline 19 & 994 & 649 & 1070 & 1890 & 4080 & 3160 & 3390 & 2560 & 1250 & 960 & 1570 & 1180 \\
\hline 20 & 593 & 525 & 1450 & 3400 & 3580 & 2870 & 4110 & 2180 & 1810 & 1250 & 1870 & 1440 \\
\hline 21 & 573 & 563 & 1690 & 3130 & 4170 & 2710 & 3470 & 2130 & 2660 & 1280 & 1350 & 1120 \\
\hline 22 & 728 & 838 & 706 & 2530 & 3600 & 2620 & 2990 & 3030 & 2700 & 1280 & 1190 & 1560 \\
\hline 23 & 632 & 757 & 1510 & 2840 & 3340 & 2410 & 3550 & 3890 & 2150 & 1050 & 1360 & 1360 \\
\hline 24 & 872 & 955 & 1130 & 2750 & 3150 & 2660 & 3060 & 4230 & 2440 & 1130 & 1300 & 853 \\
\hline 25 & 732 & 722 & 804 & 2340 & 3410 & 2740 & 2450 & 4490 & 2530 & 1380 & 1820 & 1340 \\
\hline 26 & 678 & 389 & 681 & 2210 & 3200 & 2330 & 3310 & 4790 & 1860 & 1150 & 1020 & 1030 \\
\hline 27 & 749 & 572 & 1070 & 2150 & 3070 & 2530 & 3490 & 4460 & 1520 & 1180 & 1290 & 1140 \\
\hline 28 & 733 & 481 & 389 & 1980 & 3060 & 2980 & 3340 & 4430 & 2060 & 1410 & 746 & 1490 \\
\hline 29 & 362 & 1200 & 836 & 1730 & --- & 2920 & 3510 & 4120 & 1690 & 1010 & 989 & 1210 \\
\hline 30 & 642 & 327 & 339 & 1730 & --- & 2070 & 3020 & 3990 & 1860 & 1390 & 1280 & 1810 \\
\hline 31 & 575 & --- & 236 & 1340 & --- & 2750 & --- & 4080 & --- & 1240 & 1460 & --- \\
\hline TOTAL & 17187 & 19719 & 33215 & 38464 & 91320 & 93390 & 87650 & 108980 & 76080 & 38523 & 46582 & 34939 \\
\hline MEAN & 554 & 657 & 1071 & 1241 & 3261 & 3013 & 2922 & 3515 & 2536 & 1243 & 1503 & 1165 \\
\hline MAX & 994 & 1360 & 2100 & 3400 & 8510 & 4260 & 4110 & 4790 & 3920 & 2410 & 2380 & 1810 \\
\hline MIN & 154 & 216 & 236 & 204 & 1160 & 1960 & 1980 & 2130 & 1250 & 829 & 746 & 711 \\
\hline$A C-F T$ & 34090 & 39110 & 65880 & 76290 & 181100 & 185200 & 173900 & 216200 & 150900 & 76410 & 92400 & 69300 \\
\hline
\end{tabular}

e Estimated. 


\section{SOUTH FORK AMERICAN RIVER NEAR PLACERVILLE, CA—Continued}

STATISTICS OF MONTHLY MEAN DATA FOR WATER YEARS 1965 - 1999, BY WATER YEAR (WY)

\begin{tabular}{|c|c|c|c|c|c|c|c|c|c|c|c|c|}
\hline & OCT & NOV & $\mathrm{DEC}$ & JAN & $\mathrm{FEB}$ & MAR & $\mathrm{APR}$ & MAY & JUN & JUL & AUG & SEP \\
\hline MEAN & 517 & 761 & 1297 & 1873 & 1837 & 1974 & 2083 & 2556 & 2049 & 1209 & 970 & 842 \\
\hline MAX & 935 & 3806 & 5386 & 9673 & 6613 & 5561 & 5382 & 6159 & 6496 & 3648 & 1719 & 1401 \\
\hline (WY) & 1984 & 1984 & 1965 & 1997 & 1986 & 1983 & 1982 & 1995 & 1983 & 1983 & 1998 & 1995 \\
\hline MIN & 204 & 106 & 320 & 188 & 125 & 124 & 255 & 295 & 228 & 88.2 & 142 & 244 \\
\hline (WY) & 1988 & 1978 & 1977 & 1977 & 1977 & 1977 & 1977 & 1977 & 1977 & 1977 & 1977 & 1977 \\
\hline
\end{tabular}

SUMMARY STATISTICS

FOR 1998 CALENDAR YEAR

FOR 1999 WATER YEAR

WATER YEARS 1965 - 1999

ANNUAL TOTAL

ANNUAL MEAN

TST ANNUAL MEAN

LOWEST ANNUAL MEAN

HIGHEST DAILY MEAN

LOWEST DAILY MEAN

ANNUAL SEVEN-DAY MINIMUM

INSTANTANEOUS PEAK FLOW

INSTANTANEOUS PEAK STAGE

ANNUAL RUNOFF (AC-FT)

10 PERCENT EXCEEDS

50 PERCENT EXCEEDS

90 PERCENT EXCEEDS
886873

2430

686049

1880

$7260 \quad$ Mar 25

154 Oct 1

417 Oct 6

1759000

4430

2040

574

1361000

3570

1520

491
1496

$3275 \quad 1983$

$224 \quad 1977$

57100 Jan 21997

$\begin{array}{rrrccrr}8510 & \text { Feb } & 9 & 57100 & \text { Jan } & 2 & 1997 \\ 154 & \text { Oct } & 1 & 20 & \text { Nov } & 12 & 1964 \\ 354 & \text { Jan } 10 & 20 & \text { Feb } & 11 & 1977\end{array}$

$11700 \quad$ Feb 9071000 Jan 21997

11.51 Feb $9 \quad 17.40 \quad$ Dec 231964
1084000

3370

1010 


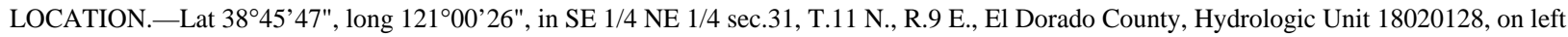
bank 0.1 mi downstream from Weber Creek and 5.0 mi south of Pilot Hill.

DRAINAGE AREA.- $801 \mathrm{mi}^{2}$.

PERIOD OF RECORD.—August to September 1999.

WATER TEMPERATURE: August to September 1999.

PERIOD OF DAILY RECORD.-August to September 1999.

WATER TEMPERATURE: August to September 1999.

INSTRUMENTATION.-Water-temperature recorder since Aug. 4, 1999.

REMARKS.-Water temperature can be affected by upstream powerplant releases.

EXTREMES FOR PERIOD OF DAILY RECORD.-

WATER TEMPERATURE: Maximum recorded, $17.5^{\circ} \mathrm{C}$, several days in August and September 1999; minimum recorded, $11.5^{\circ} \mathrm{C}$, Aug. 14, 15, 19, 21, 1999.

EXTREMES FOR CURRENT YEAR.-

WATER TEMPERATURE: Maximum recorded, $17.5^{\circ} \mathrm{C}$, several days in August and September; minimum recorded, $11.5^{\circ} \mathrm{C}$, Aug. 14, 15, 19, 21.

TEMPERATURE, WATER (DEG. C), WATER YEAR OCTOBER 1998 TO SEPTEMBER 1999

\begin{tabular}{|c|c|c|c|c|c|c|c|c|c|c|c|c|}
\hline \multirow[t]{2}{*}{ DAY } & $\operatorname{MAX}$ & MIN & $\operatorname{MAX}$ & MIN & MAX & MIN & MAX & MIN & MAX & MIN & MAX & MIN \\
\hline & \multicolumn{2}{|c|}{ APRIL } & \multicolumn{2}{|c|}{ MAY } & \multicolumn{2}{|c|}{ JUNE } & \multicolumn{2}{|c|}{ JULY } & \multicolumn{2}{|c|}{ AUGUST } & \multicolumn{2}{|c|}{ SEPTEMBER } \\
\hline 1 & --- & --- & --- & --- & --- & --- & --- & --- & --- & --- & 15.0 & 12.0 \\
\hline 2 & --- & --- & --- & --- & --- & --- & --- & --- & --- & --- & 15.0 & 12.0 \\
\hline 3 & --- & --- & --- & --- & --- & --- & --- & --- & --- & --- & 15.5 & 12.0 \\
\hline 4 & --- & --- & --- & --- & --- & --- & --- & --- & --- & --- & 16.5 & 14.0 \\
\hline 5 & --- & --- & --- & --- & --- & --- & --- & --- & 17.0 & 13.5 & 16.0 & 12.5 \\
\hline 6 & --- & --- & --- & --- & --- & --- & --- & --- & 16.5 & 13.5 & 16.5 & 13.0 \\
\hline 7 & --- & --- & --- & --- & --- & --- & --- & --- & 16.5 & 12.5 & 16.5 & 13.0 \\
\hline 8 & --- & --- & --- & --- & --- & --- & --- & --- & 17.0 & 14.5 & 17.0 & 13.5 \\
\hline 9 & --- & --- & --- & --- & --- & --- & --- & --- & 17.5 & 15.0 & 16.0 & 14.5 \\
\hline 10 & --- & --- & --- & --- & --- & --- & --- & --- & 16.5 & 13.0 & 17.5 & 14.5 \\
\hline 11 & --- & --- & --- & --- & --- & --- & --- & --- & 16.5 & 12.5 & 17.0 & 14.5 \\
\hline 12 & --- & --- & --- & --- & --- & --- & --- & --- & 15.5 & 12.0 & 17.0 & 13.5 \\
\hline 13 & --- & --- & --- & --- & --- & --- & --- & --- & 16.0 & 12.0 & 16.5 & 13.5 \\
\hline 14 & --- & --- & --- & --- & --- & --- & --- & --- & 15.5 & 11.5 & 16.5 & 13.5 \\
\hline 15 & --- & --- & --- & --- & --- & --- & --- & --- & 15.5 & 11.5 & 16.5 & 14.5 \\
\hline 16 & --- & --- & --- & --- & --- & --- & --- & --- & 16.0 & 12.0 & 17.0 & 14.0 \\
\hline 17 & --- & --- & --- & --- & --- & --- & --- & --- & 16.0 & 12.0 & 17.0 & 14.0 \\
\hline 18 & --- & --- & --- & --- & --- & --- & --- & --- & 16.0 & 12.0 & 16.5 & 15.0 \\
\hline 19 & --- & --- & --- & --- & --- & --- & --- & --- & 15.0 & 11.5 & 17.0 & 14.0 \\
\hline 20 & --- & --- & --- & --- & --- & --- & --- & --- & 15.5 & 12.0 & 16.5 & 13.5 \\
\hline 21 & --- & --- & --- & --- & --- & --- & --- & --- & 15.5 & 11.5 & 17.0 & 14.0 \\
\hline 22 & --- & --- & --- & --- & --- & --- & --- & --- & 17.0 & 13.5 & 17.0 & 14.0 \\
\hline 23 & --- & --- & --- & --- & --- & --- & --- & --- & 16.5 & 13.0 & 17.5 & 14.0 \\
\hline 24 & --- & --- & --- & --- & --- & --- & --- & --- & 16.5 & 12.5 & 17.5 & 14.5 \\
\hline 25 & --- & --- & --- & --- & --- & --- & --- & --- & 16.0 & 12.5 & 17.5 & 14.5 \\
\hline 26 & --- & --- & --- & --- & --- & --- & --- & --- & 15.0 & 12.5 & 17.0 & 14.0 \\
\hline 27 & --- & --- & --- & --- & --- & --- & --- & --- & 17.5 & 14.0 & 17.0 & 14.0 \\
\hline 28 & --- & --- & --- & --- & --- & --- & --- & --- & 16.5 & 13.0 & 17.0 & 13.5 \\
\hline 29 & --- & --- & --- & --- & --- & --- & --- & --- & 17.0 & 15.5 & 17.0 & 14.0 \\
\hline 30 & --- & --- & --- & --- & --- & --- & --- & --- & 17.0 & 15.0 & 17.0 & 14.0 \\
\hline 31 & --- & --- & --- & --- & --- & --- & --- & --- & 15.5 & 12.5 & -- & -- \\
\hline MONTH & --- & --- & --- & --- & --- & --- & --- & --- & --- & --- & 17.5 & 12.0 \\
\hline
\end{tabular}




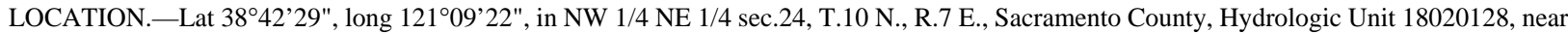
center of dam on American River, 0.7 mi downstream from South Fork American River, and 2.3 mi northeast of Folsom.

DRAINAGE AREA.-1,861 $\mathrm{mi}^{2}$.

PERIOD OF RECORD.—February 1955 to current year. Prior to October 1959, published as Folsom Reservoir near Folsom.

REVISED RECORDS.-WSP 1931: Drainage area.

GAGE.-Water-stage recorder. Datum of gage is sea level (levels by U.S. Bureau of Reclamation).

REMARKS.- Reservoir is formed by concrete gravity-type dam with rolled-earth-wing dams, auxiliary dams, and dikes, completed May 14, 1956; storage began Feb. 25, 1955. Total capacity, 1,010,300 acre-ft between elevations $205.5 \mathrm{ft}$, invert of lower tier of river outlets, and $466.0 \mathrm{ft}$ gross pool elevation, all of which are available for release. Spillway design flood pool elevation, $475.4 \mathrm{ft}$, capacity, 1,120,200 acre-ft. Records, including extremes, represent usable contents at 2400 hours. See schematic diagram of lower Sacramento River Basin.

COOPERATION.-Records provided by U.S. Bureau of Reclamation.

EXTREMES FOR PERIOD OF RECORD.-Maximum contents, 1,024,400 acre-ft, June 15, 1963, elevation, $467.23 \mathrm{ft}$; minimum since storage pool first filled, 140,600 acre-ft, Nov. 20, 21, 1977, elevation, $347.57 \mathrm{ft}$.

EXTREMES FOR CURRENT YEAR.—Maximum contents, 950,200 acre-ft, June 25, elevation, 463.59 ft; minimum, 495,600 acre-ft, Feb. 28 , elevation, $415.97 \mathrm{ft}$.

\begin{tabular}{|c|c|c|c|c|c|}
\hline \multicolumn{6}{|c|}{$\begin{array}{l}\text { Capacity table (elevation, in feet, and contents, in acre-feet) } \\
\text { (Based on survey by U.S. Bureau of Reclamation in 1992) }\end{array}$} \\
\hline 345 & 123,600 & 380 & 258,600 & 440 & 703,800 \\
\hline 350 & 137,900 & 390 & 314,100 & 460 & 908,400 \\
\hline 360 & 170,600 & 400 & 376,900 & 479 & $1,125,000$ \\
\hline 370 & 210,500 & 420 & 525,500 & & \\
\hline
\end{tabular}

RESERVOIR STORAGE (ACRE-FEET), WATER YEAR OCTOBER 1998 TO SEPTEMBER 1999 DAILY OBSERVATION AT 2400 HOURS

\begin{tabular}{|c|c|c|c|c|c|c|c|c|c|c|c|c|}
\hline DAY & $\mathrm{OCT}$ & NOV & $\mathrm{DEC}$ & JAN & FEB & MAR & APR & MAY & JUN & JUL & AUG & SEP \\
\hline 1 & 705700 & 611500 & 562000 & 561300 & 532900 & 503300 & 618500 & 757300 & 915200 & 932600 & 783100 & 751800 \\
\hline 2 & 699400 & 609600 & 564800 & 559200 & 533300 & 505600 & 620800 & 760900 & 916200 & 928800 & 779000 & 751400 \\
\hline 3 & 694800 & 607900 & 570500 & 558100 & 533900 & 513200 & 622200 & 766900 & 918100 & 925300 & 776100 & 751400 \\
\hline 4 & 691000 & 606500 & 578100 & 556500 & 533300 & 519000 & 624100 & 771200 & 919300 & 920400 & 774500 & 750200 \\
\hline 5 & 687900 & 605200 & 580400 & 554400 & 531400 & 520100 & 627200 & 774300 & 919400 & 915600 & 774200 & 749500 \\
\hline 6 & 684100 & 603300 & 581300 & 554300 & 526300 & 519000 & 630400 & 776300 & 919200 & 911600 & 772100 & 748300 \\
\hline 7 & 679800 & 602800 & 580700 & 552500 & 537300 & 519700 & 633400 & 780600 & 919400 & 907600 & 772000 & 746900 \\
\hline 8 & 676300 & 602300 & 580100 & 550700 & 551000 & 522300 & 636700 & 786400 & 919600 & 903600 & 769700 & 746000 \\
\hline 9 & 674700 & 600800 & 579100 & 549500 & 612900 & 529600 & 641000 & 790500 & 922000 & 899200 & 767100 & 745100 \\
\hline 10 & 672100 & 599200 & 577600 & 547000 & 611500 & 536700 & 645600 & 795800 & 925200 & 894100 & 765900 & 743800 \\
\hline 11 & 669300 & 595700 & 576400 & 544500 & 592700 & 540700 & 650100 & 800100 & 928100 & 888400 & 765500 & 742900 \\
\hline 12 & 666500 & 593100 & 576000 & 543000 & 577400 & 541800 & 655500 & 806700 & 929600 & 884600 & 766200 & 741900 \\
\hline 13 & 663400 & 588800 & 574800 & 541400 & 564000 & 543900 & 659300 & 813600 & 931000 & 880000 & 766000 & 742800 \\
\hline 14 & 661200 & 584400 & 573200 & 539000 & 554500 & 547700 & 665500 & 819900 & 932600 & 874900 & 765400 & 744500 \\
\hline 15 & 658100 & 580300 & 572900 & 537500 & 547600 & 550700 & 671800 & 824300 & 936000 & 870000 & 764600 & 745500 \\
\hline 16 & 655300 & 576300 & 571800 & 536700 & 541600 & 554600 & 676800 & 827700 & 939300 & 863100 & 763500 & 746600 \\
\hline 17 & 652800 & 574100 & 571800 & 537300 & 542900 & 559500 & 683400 & 832100 & 941800 & 857200 & 762600 & 747200 \\
\hline 18 & 649300 & 570500 & 572100 & 544200 & 516800 & 563600 & 689900 & 837100 & 945000 & 850800 & 762400 & 746300 \\
\hline 19 & 646400 & 567500 & 571600 & 565000 & 503100 & 569300 & 697000 & 841000 & 945200 & 845200 & 763300 & 745100 \\
\hline 20 & 644100 & 564400 & 571500 & 595200 & 495700 & 574100 & 706000 & 842800 & 944900 & 838800 & 762400 & 743500 \\
\hline 21 & 641100 & 560900 & 572100 & 589800 & 500800 & 578800 & 712600 & 844500 & 945600 & 833900 & 762700 & 742200 \\
\hline 22 & 637100 & 559600 & 572400 & 568700 & 499200 & 582900 & 718400 & 847400 & 947500 & 829000 & 761400 & 740100 \\
\hline 23 & 634400 & 557200 & 572200 & 567100 & 497800 & 586100 & 724200 & 853600 & 948500 & 824600 & 759900 & 737800 \\
\hline 24 & 633100 & 559100 & 571500 & 562600 & 497200 & 590300 & 728800 & 861200 & 949500 & 819400 & 759000 & 735100 \\
\hline 25 & 631100 & 559200 & 571300 & 555600 & 498800 & 594500 & 731500 & 870600 & 950200 & 814000 & 759500 & 732400 \\
\hline 26 & 628200 & 556900 & 569700 & 548900 & 499200 & 598100 & 736800 & 879900 & 948800 & 810100 & 759300 & 731000 \\
\hline 27 & 625300 & 554800 & 567800 & 542900 & 497600 & 601300 & 743300 & 888200 & 944600 & 804300 & 757900 & 728600 \\
\hline 28 & 622500 & 552700 & 565800 & 538600 & 495600 & 605500 & 748100 & 895700 & 940800 & 799700 & 756800 & 726200 \\
\hline 29 & 618200 & 550700 & 564100 & 535600 & --- & 609100 & 752100 & 901400 & 937800 & 795200 & 755200 & 723600 \\
\hline 30 & 615800 & 555300 & 563800 & 533700 & --- & 611400 & 755600 & 905300 & 934800 & 791300 & 753400 & 721600 \\
\hline 31 & 613800 & --- & 563000 & 533200 & --- & 615400 & --- & 910300 & --- & 787300 & 752500 & --- \\
\hline MAX & 705700 & 611500 & 581300 & 595200 & 612900 & 615400 & 755600 & 910300 & 950200 & 932600 & 783100 & 751800 \\
\hline MIN & 613800 & 550700 & 562000 & 533200 & 495600 & 503300 & 618500 & 757300 & 915200 & 787300 & 752500 & 721600 \\
\hline$a$ & 430.02 & 423.28 & 424.19 & 420.63 & 415.97 & 430.20 & 445.04 & 459.94 & 462.19 & 448.21 & 444.73 & 441.58 \\
\hline b & -99100 & -58500 & +7700 & -29800 & -37600 & +119800 & +140200 & +154700 & +24500 & -147500 & -34800 & -30900 \\
\hline C & 2699 & 882 & 964 & 563 & 814 & 1615 & 3507 & 5445 & 6826 & 7321 & 5919 & 4611 \\
\hline
\end{tabular}

CAL YR 1998 b +141800

WTR YR 1999 b +8700

a Elevation, in feet, at end of month.

b Change in contents, in acre-feet.

c Total evaporation, in acre-feet, provided by U..S. Bureau of Reclamation; not reviewed by U.S. Geological Survey. 


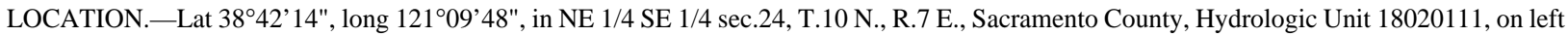
bank 0.3 mi downstream from Folsom Dam and 1.5 mi north of Folsom.

DRAINAGE AREA.- $1,863 \mathrm{mi}^{2}$.

PERIOD OF RECORD.-October 1998 to September 1999.

WATER TEMPERATURE.-October 1998 to September 1999.

PERIOD OF DAILY RECORD.-October 1998 to September 1999.

WATER TEMPERATURE.-October 1998 to September 1999.

INSTRUMENTATION.-Water-temperature recorder since Oct. 23, 1998.

REMARKS.-Water temperature is affected by upstream releases from Folsom Dam. Interruption in record was due to malfunction of the recording instrument.

EXTREMES FOR PERIOD OF DAILY RECORD.-

WATER TEMPERATURE: Maximum recorded, $17.5^{\circ} \mathrm{C}$, Aug. 11, 15, 16, 1999; minimum recorded, 7.0 ${ }^{\circ}$ C, Feb. 6, 15-17, 1999.

EXTREMES FOR CURRENT YEAR.-

WATER TEMPERATURE: Maximum recorded, $17.5^{\circ} \mathrm{C}$, Aug. $11,15,16$; minimum recorded, $7.0^{\circ} \mathrm{C}$, Feb. 6, 15-17.

TEMPERATURE, WATER (DEG. C), WATER YEAR OCTOBER 1998 TO SEPTEMBER 1999

\begin{tabular}{|c|c|c|c|c|c|c|c|c|c|c|c|c|}
\hline \multirow[t]{2}{*}{ DAY } & MAX & MIN & MAX & MIN & MAX & MIN & $\operatorname{MAX}$ & MIN & MAX & MIN & MAX & MIN \\
\hline & \multicolumn{2}{|c|}{ OCTOBER } & \multicolumn{2}{|c|}{ NOVEMBER } & \multicolumn{2}{|c|}{ DECEMBER } & \multicolumn{2}{|c|}{ JANUARY } & \multicolumn{2}{|c|}{ FEBRUARY } & \multicolumn{2}{|c|}{ MARCH } \\
\hline 1 & --- & --- & 16.0 & 15.0 & 13.5 & 13.0 & 8.5 & 8.0 & 8.5 & 8.0 & 8.5 & 8.0 \\
\hline 2 & --- & --- & 16.0 & 15.0 & 13.5 & 13.0 & 9.0 & 8.0 & 8.5 & 8.0 & 8.5 & 8.0 \\
\hline 3 & --- & --- & 15.5 & 15.0 & 13.0 & 12.5 & 8.5 & 8.0 & 8.5 & 8.0 & 8.5 & 8.0 \\
\hline 4 & --- & --- & 15.5 & 15.0 & 13.0 & 12.0 & 8.5 & 8.0 & 8.5 & 8.0 & 9.0 & 8.0 \\
\hline 5 & --- & --- & 15.5 & 15.0 & 12.5 & 11.5 & 8.5 & 7.5 & 8.0 & 7.5 & 9.0 & 8.5 \\
\hline 6 & --- & --- & 15.5 & 14.5 & 12.5 & 11.5 & 8.5 & 8.0 & 8.0 & 7.0 & 9.0 & 8.5 \\
\hline 7 & --- & --- & 15.5 & 14.5 & 12.5 & 11.5 & 8.5 & 7.5 & 8.0 & 7.5 & 9.0 & 8.5 \\
\hline 8 & --- & --- & 15.5 & 15.0 & 12.0 & 11.5 & 8.5 & 7.5 & 8.0 & 7.5 & 9.0 & 8.0 \\
\hline 9 & --- & --- & 15.5 & 14.5 & 12.0 & 11.0 & 8.5 & 7.5 & 8.5 & 7.5 & 9.0 & 8.5 \\
\hline 10 & --- & --- & 15.0 & 14.5 & 12.5 & 11.0 & 8.5 & 7.5 & 8.5 & 8.0 & 9.0 & 8.5 \\
\hline 11 & --- & --- & 15.0 & 14.5 & 12.5 & 11.0 & 8.5 & 7.5 & 9.0 & 8.0 & 9.0 & 8.5 \\
\hline 12 & --- & --- & 15.0 & 14.5 & 11.0 & 10.5 & 8.5 & 7.5 & 8.5 & 8.0 & 9.0 & 8.5 \\
\hline 13 & --- & --- & 15.0 & 14.5 & 11.0 & 10.0 & 8.0 & 7.5 & 8.5 & 7.5 & 9.0 & 8.5 \\
\hline 14 & --- & --- & 15.0 & 14.5 & 12.0 & 10.0 & 9.0 & 7.5 & 8.0 & 7.5 & 9.0 & 8.5 \\
\hline 15 & --- & --- & 15.0 & 14.5 & --- & 10.0 & 8.5 & 8.0 & 7.5 & 7.0 & 9.0 & 8.0 \\
\hline 16 & --- & --- & 15.0 & 14.0 & 11.0 & --- & 8.5 & 8.0 & 7.5 & 7.0 & 8.5 & 8.0 \\
\hline 17 & --- & --- & 14.5 & 14.0 & 10.5 & 10.0 & 8.5 & 8.0 & 8.0 & 7.0 & 9.0 & 8.5 \\
\hline 18 & --- & --- & 14.5 & 13.5 & 10.5 & 10.0 & 8.5 & 8.0 & 8.0 & 7.5 & 9.0 & 8.0 \\
\hline 19 & --- & --- & 14.5 & 13.5 & 10.5 & 10.0 & 9.0 & 8.0 & 8.0 & 7.5 & 9.0 & 8.0 \\
\hline 20 & --- & --- & 14.5 & 13.5 & 10.5 & 10.0 & 8.5 & 7.5 & 8.0 & 7.5 & 9.0 & 8.0 \\
\hline 21 & --- & --- & 14.5 & 13.5 & 10.5 & 9.5 & 8.5 & 8.0 & 8.0 & 7.5 & 9.0 & 8.5 \\
\hline 22 & --- & --- & 14.5 & 13.5 & 10.0 & 9.5 & 9.0 & 8.0 & 8.0 & 7.5 & 9.0 & 8.5 \\
\hline 23 & --- & --- & 14.0 & 13.5 & 10.0 & 9.5 & 9.0 & 8.5 & 8.5 & 8.0 & 9.0 & 8.5 \\
\hline 24 & 15.5 & 15.0 & 14.0 & 13.0 & 10.0 & 9.0 & 9.0 & 8.5 & 8.5 & 8.0 & 9.0 & 8.0 \\
\hline 25 & 15.5 & 15.0 & 14.0 & 13.5 & 9.5 & 9.0 & 9.0 & 8.5 & 8.0 & 7.5 & 9.5 & 8.5 \\
\hline 26 & --- & --- & 14.0 & 13.5 & 9.5 & 8.5 & 9.0 & 8.5 & 8.5 & 8.0 & 9.0 & 8.5 \\
\hline 27 & 15.5 & 15.0 & 14.0 & 13.5 & 9.0 & 8.5 & 9.0 & 8.5 & 8.5 & 8.0 & 9.0 & 8.5 \\
\hline 28 & 15.5 & 15.0 & 13.5 & 13.0 & 9.0 & 8.5 & 8.5 & 8.5 & 8.5 & 8.0 & 9.5 & 8.5 \\
\hline 29 & 15.5 & 15.0 & 13.5 & 13.0 & 9.0 & 8.0 & 8.5 & 8.5 & --- & --- & 9.5 & 8.5 \\
\hline 30 & 16.0 & 15.0 & --- & --- & 9.0 & 8.0 & 8.5 & 8.0 & --- & --- & 9.0 & 8.5 \\
\hline 31 & 16.0 & 15.0 & --- & --- & 8.5 & 8.0 & 8.5 & 8.0 & --- & --- & 9.5 & 8.5 \\
\hline MONTH & --- & --- & --- & --- & --- & --- & 9.0 & 7.5 & 9.0 & 7.0 & 9.5 & 8.0 \\
\hline
\end{tabular}




\section{AMERICAN RIVER BELOW FOLSOM DAM, NEAR FOLSOM, CA—Continued}

TEMPERATURE, WATER (DEG. C), WATER YEAR OCTOBER 1998 TO SEPTEMBER 1999

\begin{tabular}{|c|c|c|c|c|c|c|c|c|c|c|c|c|}
\hline \multirow[t]{2}{*}{ DAY } & MAX & MIN & MAX & MIN & MAX & MIN & MAX & MIN & MAX & MIN & MAX & MIN \\
\hline & \multicolumn{2}{|c|}{ APRIL } & \multicolumn{2}{|c|}{ MAY } & \multicolumn{2}{|c|}{ JUNE } & \multicolumn{2}{|c|}{ JULY } & \multicolumn{2}{|c|}{ AUGUST } & \multicolumn{2}{|c|}{ SEPTEMBER } \\
\hline 1 & 10.0 & 9.5 & 11.5 & 10.5 & 12.0 & 11.5 & 14.0 & 13.0 & 17.0 & 12.0 & 16.5 & 13.5 \\
\hline 2 & 10.0 & 9.0 & 11.5 & 10.5 & 12.0 & 11.5 & 14.0 & 13.5 & 17.0 & 16.0 & 16.5 & 13.5 \\
\hline 3 & 10.5 & 9.0 & 11.5 & 10.5 & 13.5 & 10.5 & 14.0 & 13.5 & 17.0 & 16.5 & 16.5 & 13.0 \\
\hline 4 & 10.0 & 9.0 & 12.0 & 11.0 & 12.5 & 11.5 & 14.5 & 13.5 & 17.0 & 16.5 & 16.5 & 12.5 \\
\hline 5 & 9.5 & 9.0 & 12.0 & 11.0 & 12.5 & 11.5 & 14.0 & 13.5 & 17.0 & 12.5 & 16.5 & 13.5 \\
\hline 6 & 10.0 & 9.5 & 12.0 & 11.0 & 12.5 & 12.0 & 14.0 & 13.5 & 17.0 & 16.0 & 16.5 & 13.0 \\
\hline 7 & 10.0 & 9.0 & 12.0 & 11.0 & 12.5 & 12.0 & 14.5 & 14.0 & 17.0 & 16.0 & 16.5 & 13.5 \\
\hline 8 & 9.5 & 9.0 & 12.0 & 11.0 & 13.0 & 12.0 & 14.5 & 13.5 & 17.0 & 13.5 & 15.5 & 13.5 \\
\hline 9 & 10.0 & 9.0 & 12.0 & 11.0 & 12.5 & 12.0 & 14.5 & 14.0 & 17.0 & 12.5 & 15.5 & 13.5 \\
\hline 10 & 10.0 & 9.0 & 12.0 & 11.0 & 12.5 & 12.0 & 14.5 & 14.0 & 17.0 & 12.5 & 15.5 & 13.0 \\
\hline 11 & 10.0 & 9.0 & 12.0 & 10.0 & 12.5 & 12.0 & 14.5 & 14.0 & 17.5 & 12.0 & 15.5 & 13.5 \\
\hline 12 & 10.0 & 9.5 & 12.0 & 10.5 & 12.5 & 12.0 & 14.5 & 14.0 & 17.0 & 12.5 & 15.5 & 14.0 \\
\hline 13 & 10.5 & 9.5 & 12.0 & 11.0 & 13.0 & 12.0 & 14.5 & 14.0 & 17.0 & 12.5 & 15.5 & 13.5 \\
\hline 14 & 11.0 & 10.0 & 11.5 & 11.0 & 12.5 & 12.0 & 15.0 & 14.0 & 17.0 & 12.5 & 15.5 & 14.0 \\
\hline 15 & 10.5 & 10.0 & 12.5 & 11.0 & 13.0 & 12.0 & 15.0 & 12.0 & 17.5 & 13.0 & 16.0 & 14.5 \\
\hline 16 & 11.0 & 10.0 & 12.0 & 11.0 & 13.0 & 12.5 & 15.0 & 14.0 & 17.5 & 12.5 & 16.0 & 15.0 \\
\hline 17 & 11.0 & 10.0 & 12.0 & 11.0 & 13.0 & 12.5 & 15.0 & 14.5 & 16.5 & 12.0 & 16.0 & 14.0 \\
\hline 18 & 11.0 & 10.0 & 11.5 & 11.0 & 13.0 & 12.5 & 15.5 & 14.5 & 15.5 & 13.0 & 16.0 & 14.5 \\
\hline 19 & 11.0 & 10.0 & 12.0 & 11.0 & 13.0 & 12.5 & 15.5 & 12.0 & 14.5 & 13.0 & 16.0 & 15.5 \\
\hline 20 & 11.0 & 10.0 & 12.0 & 11.0 & 13.0 & 12.5 & 15.5 & 15.0 & 16.0 & 12.5 & 16.0 & 15.5 \\
\hline 21 & 11.0 & 10.0 & 12.0 & 11.0 & 13.5 & 12.5 & 16.0 & 15.0 & 16.5 & 13.5 & 16.0 & 15.0 \\
\hline 22 & 13.0 & 10.0 & 12.0 & 11.0 & 13.0 & 12.5 & 16.0 & 15.0 & 16.0 & 13.0 & 16.0 & 15.5 \\
\hline 23 & 12.5 & 11.0 & 12.0 & 11.0 & 13.0 & 12.5 & 15.5 & 15.0 & 16.5 & 13.0 & 16.5 & 15.5 \\
\hline 24 & 11.5 & 10.5 & 12.0 & 11.5 & 13.5 & 12.5 & 16.0 & 15.0 & 16.5 & 13.0 & 16.5 & 16.0 \\
\hline 25 & 11.5 & 10.5 & 12.0 & 11.0 & 13.5 & 12.5 & 16.0 & 15.0 & 16.0 & 13.0 & 16.5 & 16.0 \\
\hline 26 & 11.5 & 10.5 & 12.0 & 11.0 & 13.0 & 12.5 & 16.0 & 12.0 & 16.0 & 14.0 & 16.5 & 16.0 \\
\hline 27 & 11.5 & 10.5 & 12.0 & 11.5 & 13.5 & 13.0 & 16.5 & 15.0 & 17.0 & 14.0 & 16.5 & 16.0 \\
\hline 28 & 12.0 & 10.5 & 12.5 & 11.5 & 13.5 & 13.0 & 16.5 & 15.0 & 17.0 & 14.5 & 16.5 & 16.0 \\
\hline 29 & 12.5 & 11.5 & 12.0 & 11.5 & 13.5 & 13.0 & 16.5 & 15.5 & 16.0 & 14.5 & 16.5 & 16.0 \\
\hline 30 & 12.0 & 11.0 & 12.0 & 11.5 & 14.0 & 13.0 & 16.5 & 16.0 & 16.5 & 14.5 & 16.5 & 16.0 \\
\hline 31 & --- & --- & 12.0 & 11.5 & --- & --- & 16.5 & 15.5 & 16.5 & 14.5 & --- & --- \\
\hline MONTH & 13.0 & 9.0 & 12.5 & 10.0 & 14.0 & 10.5 & 16.5 & 12.0 & 17.5 & 12.0 & 16.5 & 12.5 \\
\hline
\end{tabular}


11446500 AMERICAN RIVER AT FAIR OAKS, CA

LOCATION.-Lat 38³8'08", long 121¹3’36", in SE 1/4 NE 1/4 sec.17, T.9 N., R.7 E., Sacramento County, Hydrologic Unit 18020111, on right bank 2,100 ft downstream from Nimbus Dam, 2.4 mi east of Fair Oaks, 8.1 mi downstream from South Fork, and at mile 22.2.

DRAINAGE AREA.- $1,888 \mathrm{mi}^{2}$.

\section{WATER-DISCHARGE RECORDS}

PERIOD OF RECORD.-November 1904 to current year. Monthly discharge only for some periods, published in WSP 1315-A.

REVISED RECORDS._WSP 1181: 1928(M). WSP 1515: 1907(M), 1910, 1931(M), 1943(M). WSP 1931: Drainage area.

GAGE.-Water-stage recorder. Datum of gage is $71.53 \mathrm{ft}$ above sea level. See WSP 2131 for history of changes prior to July 15 , 1970.

REMARKS.-Records good. Flow regulated by Folsom Lake beginning Feb. 25, 1955 (station 11446200). Some minor regulation of high flows by temporary pondage during period of construction January 1953 to February 1955. Diurnal fluctuations from Folsom Powerplant re-regulated by Nimbus Reservoir, capacity, 2,800 acre-ft between normal operating elevations 118.5 and $125.0 \mathrm{ft}$ and by Nimbus Powerplant. Many diversions upstream from station for irrigation, municipal, and domestic water supply. Diversions for San Juan Suburban Water District, city of Folsom, city of Roseville, and State of California are made at Folsom Dam. Diversion to Folsom South Canal from Nimbus Reservoir started in June 1973. Some inflow from Bear and Yuba River Basins. See schematic diagram of lower Sacramento River Basin.

EXTREMES FOR PERIOD OF RECORD.-Maximum discharge, 180,000 $\mathrm{ft}^{3} / \mathrm{s}$, Nov. 21, 1950, gage height, $31.85 \mathrm{ft}$, site and datum then in use; minimum, $3.6 \mathrm{ft}^{3} / \mathrm{s}$, Aug. 16, 1924. Maximum discharge since regulation by Folsom Lake in 1955, 134,000 ft $3 / \mathrm{s}, \mathrm{Feb} .19,1986$, gage height, $27.96 \mathrm{ft}$; minimum daily, $160 \mathrm{ft}^{3} / \mathrm{s}$, Apr. $17,1955$.

\section{DISCHARGE, CUBIC FEET PER SECOND, WATER YEAR OCTOBER 1998 TO SEPTEMBER 1999} DAILY MEAN VALUES

\begin{tabular}{|c|c|c|c|c|c|c|c|c|c|c|c|c|}
\hline DAY & $\mathrm{OCT}$ & NOV & $\mathrm{DEC}$ & JAN & FEB & MAR & APR & MAY & JUN & JUL & AUG & SEP \\
\hline 1 & 4140 & 2160 & 3080 & 2570 & 4610 & e9300 & 4090 & 4520 & 4340 & 4530 & 3640 & 2280 \\
\hline 2 & 3640 & 2060 & 3110 & 2560 & 4560 & e10400 & 4090 & 4550 & 4340 & 4160 & 3640 & 2300 \\
\hline 3 & 3140 & 1980 & 3100 & 2560 & 4180 & e10400 & 4090 & 4570 & 4340 & 4040 & 3170 & 2300 \\
\hline 4 & 2760 & 1980 & 3120 & 2530 & 4560 & e10400 & 4100 & 4560 & 4340 & 4040 & 2620 & 2300 \\
\hline 5 & 2590 & 1990 & 3120 & 2530 & 4950 & e10400 & 4080 & 4570 & 4350 & 4030 & 2540 & 2260 \\
\hline 6 & 2460 & 1990 & 3110 & 2530 & 6770 & 10200 & 4090 & 4580 & 4340 & 4050 & 2510 & 2210 \\
\hline 7 & 2430 & 1990 & 3100 & 2520 & 13000 & 8840 & 4100 & 4590 & 4350 & 4070 & 2510 & 2190 \\
\hline 8 & 2100 & 2010 & 3080 & 2510 & 13900 & 7480 & 4110 & 4370 & 3950 & 4340 & 2510 & 2010 \\
\hline 9 & 1950 & 2050 & 3100 & 2500 & 15500 & 6670 & 4120 & 4340 & 3840 & 4600 & 2500 & 2020 \\
\hline 10 & 1970 & 2050 & 3120 & 2500 & 23900 & 6140 & 4120 & 4340 & 3580 & 4620 & 2520 & 2020 \\
\hline 11 & 1980 & 2930 & 3170 & 2480 & 22400 & 6140 & 4130 & 4350 & 3640 & 4650 & 2510 & 2030 \\
\hline 12 & 2010 & 3040 & 3210 & 2510 & 18200 & 6110 & 4160 & 4370 & 3650 & 4700 & 2510 & 2010 \\
\hline 13 & 2010 & 3090 & 3170 & 2520 & 15200 & 5690 & 4160 & 4360 & 3660 & 4640 & 2520 & e1150 \\
\hline 14 & 2030 & 3070 & 3180 & 2550 & 12700 & 5240 & 4150 & 4370 & 3590 & 4620 & 2500 & e910 \\
\hline 15 & 2010 & 3080 & 3120 & 2520 & 11000 & 4800 & 4140 & 4380 & 3530 & 4530 & 2500 & e1440 \\
\hline 16 & 2030 & 3070 & 3110 & 2590 & 10700 & 4500 & 4680 & 4370 & 3520 & 4620 & 2470 & e 980 \\
\hline 17 & 2070 & 3100 & 3130 & 2660 & 16800 & 4130 & 4630 & 4370 & 3510 & 4680 & 2280 & e1270 \\
\hline 18 & 2050 & 3140 & 3180 & 2560 & 21800 & 4030 & 4590 & 4380 & 3490 & 4630 & 2230 & e2300 \\
\hline 19 & 2050 & 3060 & 3110 & 3590 & 20000 & 4040 & 4600 & 4380 & 3480 & 4550 & 2250 & e2290 \\
\hline 20 & 2050 & 3060 & 3080 & 11900 & 15300 & 4060 & 4610 & 4360 & 3510 & 4430 & 2260 & e2300 \\
\hline 21 & 2030 & 3070 & 3270 & 19600 & 13900 & 4040 & 4560 & 4360 & 3510 & 4090 & 2250 & e2300 \\
\hline 22 & 1990 & 3120 & 3170 & 19100 & 12700 & 4050 & 4650 & 4380 & 3530 & 4120 & 2240 & e2300 \\
\hline 23 & 2000 & 3110 & 2990 & 17100 & 10800 & 4050 & 4740 & 4380 & 3530 & 4040 & 2250 & 2360 \\
\hline 24 & 2050 & 3120 & 3000 & 14800 & 9950 & 4050 & 4500 & 4370 & 3510 & 4090 & 2250 & 2330 \\
\hline 25 & 2200 & 3110 & 3010 & 12000 & 9650 & 4070 & 4440 & 4370 & 3630 & 4110 & 2270 & 2360 \\
\hline 26 & 2110 & 3140 & 3000 & 10500 & 9520 & 4080 & 4480 & 4370 & 4430 & 4140 & 2250 & 2370 \\
\hline 27 & 2100 & 3150 & 3000 & 9160 & 9500 & 4080 & e 4560 & 4410 & 4780 & 4150 & 2320 & 2420 \\
\hline 28 & 2130 & 3120 & 3010 & 7760 & 9550 & 4070 & e 4530 & 4410 & 4830 & 4170 & 2320 & 2320 \\
\hline 29 & 2040 & 3080 & 2580 & 6500 & --- & 4080 & e 4530 & 4380 & 4830 & 4170 & 2250 & 2190 \\
\hline 30 & 2020 & 3080 & 2520 & 5700 & --- & 4080 & 4510 & 4360 & 4790 & 3750 & 2280 & 2400 \\
\hline 31 & 2250 & --- & 2560 & 5200 & --- & 4080 & --- & 4340 & --- & 3730 & 2290 & --- \\
\hline TOTAL & 70390 & 82000 & 94610 & 188610 & 345600 & 183700 & 130340 & 136810 & 118720 & 133090 & 77160 & 61920 \\
\hline MEAN & 2271 & 2733 & 3052 & 6084 & 12340 & 5926 & 4345 & 4413 & 3957 & 4293 & 2489 & 2064 \\
\hline MAX & 4140 & 3150 & 3270 & 19600 & 23900 & 10400 & 4740 & 4590 & 4830 & 4700 & 3640 & 2420 \\
\hline MIN & 1950 & 1980 & 2520 & 2480 & 4180 & 4030 & 4080 & 4340 & 3480 & 3730 & 2230 & 910 \\
\hline$A C-F T$ & 139600 & 162600 & 187700 & 374100 & 685500 & 364400 & 258500 & 271400 & 235500 & 264000 & 153000 & 122800 \\
\hline
\end{tabular}


11446500 AMERICAN RIVER AT FAIR OAKS, CA—Continued

STATISTICS OF MONTHLY MEAN DATA FOR WATER YEARS 1905 - 1954, BY WATER YEAR (WY)

\begin{tabular}{|c|c|c|c|c|c|c|c|c|c|c|c|c|}
\hline & OCT & NOV & $\mathrm{DEC}$ & JAN & $\mathrm{FEB}$ & MAR & $\mathrm{APR}$ & MAY & JUN & JUL & AUG & SEP \\
\hline MEAN & 455 & 1327 & 2504 & 4483 & 5831 & 6647 & 8258 & 8656 & 5149 & 1293 & 342 & 269 \\
\hline MAX & 1430 & 16450 & 17360 & 24290 & 15540 & 24710 & 15640 & 18200 & 17720 & 6336 & 1497 & 813 \\
\hline (WY) & 1905 & 1951 & 1951 & 1909 & 1909 & 1907 & 1907 & 1952 & 1911 & 1906 & 1907 & 1907 \\
\hline MIN & 100 & 85.0 & 254 & 284 & 650 & 879 & 1998 & 1488 & 206 & 26.8 & 15.8 & 24.4 \\
\hline (WY) & 1930 & 1930 & 1906 & 1918 & 1920 & 1924 & 1924 & 1924 & 1924 & 1924 & 1924 & 1924 \\
\hline
\end{tabular}

SUMMARY STATISTICS

ANNUAL MEAN

HIGHEST ANNUAL MEAN

LOWEST ANNUAL MEAN

HIGHEST DAILY MEAN

LOWEST DAILY MEAN

ANNUAL SEVEN-DAY MINIMUM

INSTANTANEOUS PEAK FLOW

INSTANTANEOUS PEAK STAGE

ANNUAL RUNOFF (AC-FT)

10 PERCENT EXCEEDS

50 PERCENT EXCEEDS

90 PERCENT EXCEEDS
WATER YEARS 1905 - 1954

$\begin{array}{cccc}3752 & & & \\ 7896 & & & 1907 \\ 731 & & & 1924 \\ 132000 & \text { Nov } 21 & 1950 \\ 4.6 & \text { Jul } 29 & 1924 \\ 4.8 & \text { Jul } 29 & 1924 \\ 180000 & \text { Nov } 21 & 1950 \\ 31.85 & \text { Nov } 21 & 1950 \\ 2718000 & & & \\ 9980 & & & \\ 1420 & & & \\ 216 & & & \end{array}$

STATISTICS OF MONTHLY MEAN DATA FOR WATER YEARS 1956 - 1999, BY WATER YEAR (WY)

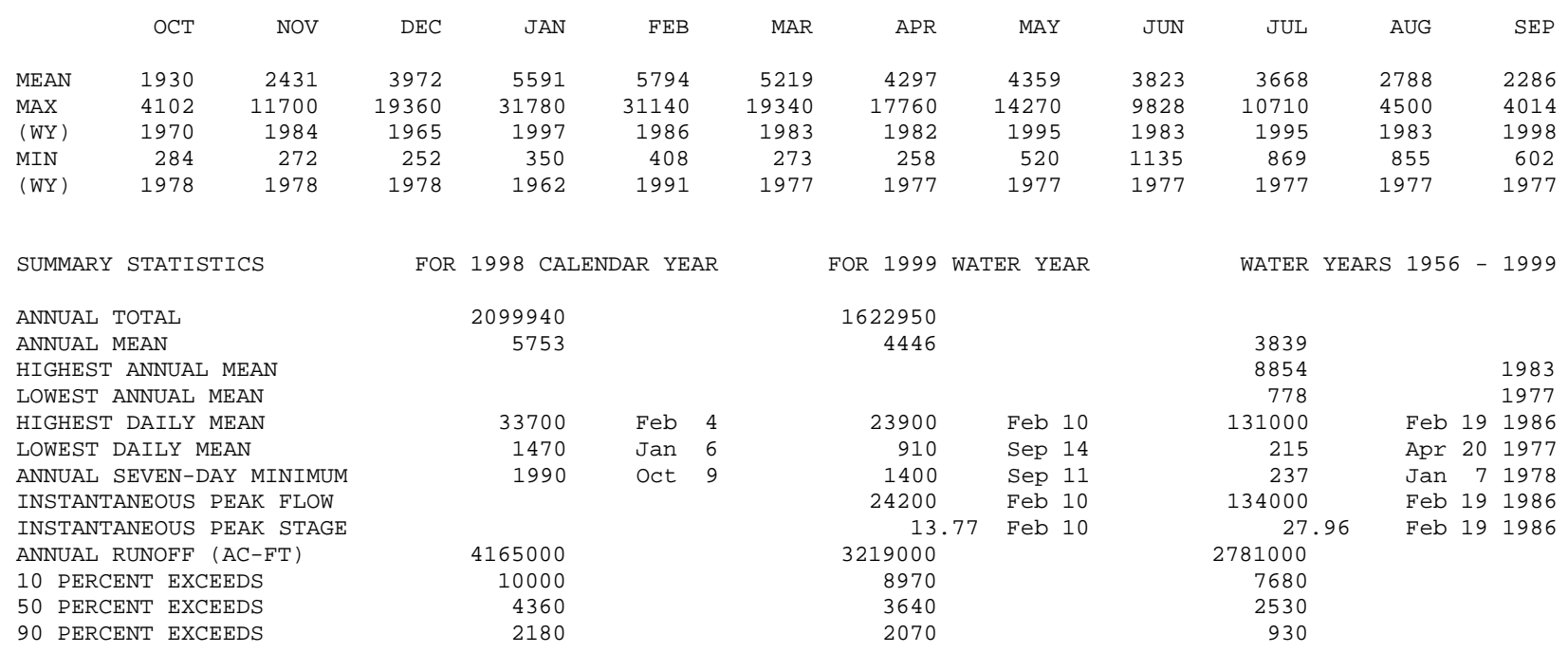




\section{WATER-QUALITY RECORDS}

PERIOD OF RECORD.-Water years 1960-65, October 1998 to September 1999.

WATER TEMPERATURE: Water years 1961-65, October 1998 to September 1999.

CHEMICAL DATA: Water years 1960-62.

PERIOD OF DAILY RECORD.-Water years 1961-65, October 1998 to September 1999.

WATER TEMPERATURE: Water years 1961-65, October 1998 to September 1999.

INSTRUMENTATION.-Water-temperature recorder since Oct. 29, 1998.

REMARKS.-Water temperature is affected by upstream releases from Nimbus Dam. Interruption in record was due to malfunction of the recording instrument.

EXTREMES FOR PERIOD OF DAILY RECORD.-

WATER TEMPERATURE: Maximum recorded, $18.5^{\circ} \mathrm{C}$, on several days during August and September 1999 ; minimum recorded, $7.5^{\circ} \mathrm{C}$, Jan. 10, 1999.

EXTREMES FOR CURRENT YEAR.-

WATER TEMPERATURE: Maximum recorded, $18.5^{\circ} \mathrm{C}$, on several days during August and September; minimum recorded, $7.5^{\circ} \mathrm{C}$, Jan. 10 .

TEMPERATURE, WATER (DEG. C), WATER YEAR OCTOBER 1998 TO SEPTEMBER 1999

\begin{tabular}{|c|c|c|c|c|c|c|c|c|c|c|c|c|}
\hline DAY & MAX & MIN & MAX & MIN & MAX & MIN & MAX & MIN & MAX & MIN & MAX & MIN \\
\hline & \multicolumn{2}{|c|}{ OCTOBER } & \multicolumn{2}{|c|}{ NOVEMBER } & \multicolumn{2}{|c|}{ DECEMBER } & \multicolumn{2}{|c|}{ JANUARY } & \multicolumn{2}{|c|}{ FEBRUARY } & \multicolumn{2}{|c|}{ MARCH } \\
\hline 1 & --- & --- & 16.0 & 15.5 & 13.5 & 13.0 & 9.5 & 8.5 & 9.0 & 8.5 & 9.5 & 9.0 \\
\hline 2 & --- & --- & 16.5 & 15.5 & 13.5 & 13.0 & 9.5 & 8.5 & 9.0 & 8.5 & 9.5 & 8.5 \\
\hline 3 & --- & --- & 16.5 & 15.5 & 13.5 & 13.0 & 9.0 & 8.5 & 9.5 & 9.0 & 9.5 & 9.0 \\
\hline 4 & --- & --- & 16.5 & 15.5 & 13.5 & 12.5 & 9.0 & 8.5 & 9.5 & 8.5 & 9.5 & 9.0 \\
\hline 5 & --- & --- & 16.5 & 15.5 & 12.5 & 12.0 & 9.0 & 8.5 & 9.0 & 8.5 & 10.0 & 9.0 \\
\hline 6 & --- & --- & 16.0 & 15.0 & 12.5 & 12.0 & 8.5 & 8.0 & 9.0 & 8.0 & 9.5 & 9.0 \\
\hline 7 & --- & --- & 15.5 & 15.0 & 12.5 & 11.5 & 8.5 & 8.0 & 8.5 & 8.0 & 10.0 & 9.0 \\
\hline 8 & --- & --- & 15.5 & 14.5 & 12.0 & 11.5 & 9.0 & 8.0 & 9.0 & 8.5 & 9.5 & 9.0 \\
\hline 9 & --- & --- & 15.5 & 14.5 & 12.0 & 11.5 & 8.5 & 8.0 & 9.0 & 8.0 & 9.5 & 8.5 \\
\hline 10 & --- & --- & 15.5 & 14.5 & 12.0 & 11.5 & 8.5 & 7.5 & 9.0 & 8.0 & 10.0 & 9.0 \\
\hline 11 & --- & --- & 15.0 & 14.5 & 12.0 & 11.5 & 8.5 & 8.0 & 9.0 & 8.5 & 10.0 & 9.5 \\
\hline 12 & --- & --- & 15.0 & 14.5 & 12.0 & 11.0 & 8.5 & 8.0 & 9.0 & 8.5 & 10.0 & 9.5 \\
\hline 13 & --- & --- & 15.5 & 14.5 & 11.5 & 11.0 & 9.0 & 8.0 & 9.0 & 8.5 & 10.0 & 9.5 \\
\hline 14 & --- & --- & 15.5 & 14.5 & 11.5 & 11.0 & 8.5 & 8.0 & 8.5 & 8.0 & 10.0 & 9.5 \\
\hline 15 & --- & --- & 15.0 & 14.5 & 11.5 & 11.0 & 9.0 & 8.0 & 8.5 & 8.0 & 10.0 & 9.5 \\
\hline 16 & --- & --- & 15.0 & 14.5 & 11.5 & 11.0 & 9.0 & 8.5 & 8.5 & 8.0 & 9.5 & 9.0 \\
\hline 17 & --- & --- & 15.0 & 14.5 & 11.5 & 10.5 & 9.0 & 8.5 & 8.5 & 8.0 & 10.0 & 9.0 \\
\hline 18 & --- & --- & 15.0 & 14.5 & 11.0 & 10.5 & 9.0 & 8.5 & 9.0 & 8.5 & 10.5 & 9.5 \\
\hline 19 & --- & --- & 15.0 & 14.0 & 11.0 & 10.5 & 9.5 & 9.0 & 9.0 & 8.5 & 10.0 & 9.5 \\
\hline 20 & --- & --- & 14.5 & 14.0 & 10.5 & 9.5 & 9.5 & 8.5 & 9.0 & 8.5 & 10.5 & 9.5 \\
\hline 21 & --- & --- & 14.5 & 14.0 & 10.0 & 9.5 & 9.0 & 8.0 & 9.0 & 8.0 & 10.0 & 9.0 \\
\hline 22 & --- & --- & 14.5 & 14.0 & 10.0 & 9.5 & 9.0 & 8.5 & 9.0 & 8.5 & 10.0 & 9.5 \\
\hline 23 & --- & --- & 14.5 & 14.0 & 10.0 & 9.5 & 9.0 & 8.5 & 9.0 & 8.5 & 10.5 & 9.5 \\
\hline 24 & --- & --- & 14.5 & 14.0 & 10.0 & 9.5 & 9.0 & 8.5 & 9.0 & 8.5 & 10.0 & 9.5 \\
\hline 25 & --- & --- & 14.0 & 13.5 & 10.0 & 9.5 & 9.0 & 8.5 & 9.0 & 8.5 & 10.5 & 9.5 \\
\hline 26 & --- & --- & 14.5 & 13.5 & 10.0 & 9.5 & 9.0 & 8.5 & 9.0 & 8.5 & 11.0 & 9.5 \\
\hline 27 & --- & --- & 14.0 & 13.5 & 10.0 & 9.5 & 9.0 & 8.5 & 9.5 & 8.5 & 10.5 & 9.5 \\
\hline 28 & --- & --- & 14.0 & 13.5 & 10.0 & 9.0 & 9.0 & 8.5 & 9.5 & 8.5 & 10.5 & 9.5 \\
\hline 29 & --- & --- & 14.0 & 13.0 & 10.0 & 9.0 & 9.0 & 8.5 & --- & --- & 10.5 & 9.5 \\
\hline 30 & 16.5 & 15.5 & 13.5 & 13.0 & 9.5 & 9.0 & 9.0 & 8.0 & --- & --- & 10.5 & 9.5 \\
\hline 31 & 16.0 & 15.5 & --- & --- & 9.5 & 8.5 & 9.0 & 8.5 & --- & --- & 10.5 & 9.5 \\
\hline MONTH & --- & --- & 16.5 & 13.0 & 13.5 & 8.5 & 9.5 & 7.5 & 9.5 & 8.0 & 11.0 & 8.5 \\
\hline
\end{tabular}


11446500 AMERICAN RIVER AT FAIR OAKS, CA—Continued

\section{TEMPERATURE, WATER (DEG. C), WATER YEAR OCTOBER 1998 TO SEPTEMBER 1999}

\begin{tabular}{|c|c|c|c|c|c|c|c|c|c|c|c|c|}
\hline DAY & MAX & MIN & MAX & MIN & MAX & MIN & MAX & MIN & MAX & MIN & MAX & MIN \\
\hline & \multicolumn{2}{|c|}{ APRIL } & \multicolumn{2}{|c|}{ MAY } & \multicolumn{2}{|c|}{ JUNE } & \multicolumn{2}{|c|}{ JULY } & \multicolumn{2}{|c|}{ AUGUST } & \multicolumn{2}{|c|}{ SEPTEMBER } \\
\hline 1 & 11.0 & 9.0 & 13.0 & 12.0 & 13.5 & 12.5 & 15.5 & 14.0 & 18.0 & 16.5 & 17.0 & 16.0 \\
\hline 2 & 11.0 & 10.0 & 12.5 & 11.0 & 13.5 & 12.5 & 15.5 & 14.5 & 18.0 & 17.0 & 17.5 & 16.0 \\
\hline 3 & 11.0 & 10.0 & 12.0 & 11.0 & 13.0 & 12.0 & 15.5 & 14.5 & 18.5 & 17.0 & 17.5 & 16.0 \\
\hline 4 & 10.5 & 9.5 & 12.0 & 11.0 & 13.5 & 12.5 & 15.5 & 14.5 & 18.5 & 17.0 & 17.5 & 16.5 \\
\hline 5 & 10.5 & 9.5 & 13.0 & 11.5 & 14.0 & 13.0 & 15.5 & 14.5 & 18.5 & 17.0 & 17.5 & 16.5 \\
\hline 6 & 10.0 & 9.0 & 13.0 & 12.0 & 14.0 & 13.0 & 15.5 & 14.5 & 18.5 & 17.0 & 17.5 & 16.5 \\
\hline 7 & 10.5 & 9.5 & 13.0 & 12.0 & 14.0 & 13.0 & 16.0 & 14.5 & 18.5 & 17.0 & 18.0 & 16.5 \\
\hline 8 & 10.5 & 9.5 & 13.0 & 12.0 & 14.0 & 12.5 & 16.0 & 15.0 & 18.5 & 17.0 & 18.0 & 16.5 \\
\hline 9 & 10.5 & 9.5 & 13.0 & 12.0 & 14.0 & 13.0 & 16.0 & 15.0 & 18.5 & 17.5 & 17.5 & 16.5 \\
\hline 10 & 10.5 & 9.5 & 13.0 & 12.0 & 14.5 & 13.0 & 16.0 & 15.0 & 18.5 & 17.5 & 17.5 & 16.5 \\
\hline 11 & 11.0 & 10.0 & 13.0 & 12.0 & 14.5 & 13.0 & 16.0 & 15.0 & 18.5 & 17.5 & 17.5 & 16.5 \\
\hline 12 & 11.0 & 10.0 & 13.5 & 12.0 & 14.5 & 13.0 & 16.5 & 15.5 & 18.5 & 17.5 & 17.5 & 16.5 \\
\hline 13 & 11.5 & 10.5 & 13.5 & 12.5 & 14.5 & 13.0 & 16.5 & 15.5 & 18.5 & 17.5 & 18.0 & 16.5 \\
\hline 14 & 11.5 & 10.5 & 13.0 & 12.0 & 14.5 & 13.0 & 16.5 & 15.5 & 18.5 & 17.0 & --- & --- \\
\hline 15 & 12.0 & 11.0 & 13.0 & 12.0 & 14.5 & 13.0 & 16.5 & 15.5 & 18.5 & 17.0 & 18.0 & 16.5 \\
\hline 16 & 12.0 & 11.0 & 13.0 & 12.0 & 14.5 & 13.5 & 16.0 & 15.5 & 18.0 & 17.0 & --- & 16.5 \\
\hline 17 & 12.0 & 11.0 & 13.5 & 12.0 & 15.0 & 13.5 & 16.5 & 15.5 & 18.0 & 16.5 & 18.5 & 16.5 \\
\hline 18 & 12.0 & 11.0 & 13.0 & 12.0 & 14.5 & 13.5 & 16.5 & 15.5 & 17.5 & 16.5 & 17.0 & 16.5 \\
\hline 19 & 12.0 & 11.0 & 13.0 & 12.0 & 15.0 & 13.5 & 17.0 & 16.0 & 18.0 & 16.5 & 17.5 & 16.5 \\
\hline 20 & 12.0 & 11.0 & 13.0 & 12.0 & 15.0 & 13.5 & 16.5 & 16.0 & 17.0 & 15.5 & 17.5 & 16.5 \\
\hline 21 & 12.0 & 11.0 & 13.0 & 12.0 & 15.0 & 13.5 & 17.0 & 16.0 & 17.0 & 15.5 & 17.5 & 16.5 \\
\hline 22 & 12.0 & 11.0 & 13.5 & 12.5 & 15.5 & 14.0 & 17.0 & 16.0 & 18.0 & 16.5 & 17.5 & 16.5 \\
\hline 23 & 13.0 & 11.0 & 13.5 & 12.0 & 15.5 & 14.0 & 17.5 & 16.0 & 17.5 & 16.5 & 17.5 & 16.5 \\
\hline 24 & 13.0 & 12.0 & 13.5 & 12.5 & 15.5 & 14.0 & 17.0 & 16.0 & 18.0 & 16.5 & 17.5 & 16.5 \\
\hline 25 & 12.5 & 11.5 & 13.5 & 12.5 & 15.5 & 14.0 & 17.5 & 16.5 & 17.5 & 16.5 & 17.5 & 16.5 \\
\hline 26 & 12.0 & 11.5 & 13.5 & 12.5 & 15.5 & 14.0 & 18.0 & 16.5 & 17.5 & 16.5 & 17.5 & 16.5 \\
\hline 27 & 12.0 & 11.0 & 13.5 & 12.5 & 15.0 & 14.0 & 17.5 & 16.5 & 17.5 & 16.0 & 17.5 & 16.5 \\
\hline 28 & 12.5 & 11.5 & 13.5 & 12.5 & 15.0 & 14.0 & 17.5 & 16.5 & 17.5 & 16.0 & 17.5 & 16.5 \\
\hline 29 & 12.5 & 11.5 & 13.5 & 12.5 & 15.0 & 14.0 & 17.5 & 16.5 & 17.5 & 16.0 & 17.5 & 16.5 \\
\hline 30 & 13.0 & 12.0 & 13.5 & 12.5 & 15.5 & 14.5 & 18.0 & 16.5 & 17.5 & 16.0 & 17.5 & 16.5 \\
\hline 31 & --- & --- & 13.5 & 12.5 & --- & --- & 18.0 & 17.0 & 17.5 & 16.0 & --- & --- \\
\hline MONTH & 13.0 & 9.0 & 13.5 & 11.0 & 15.5 & 12.0 & 18.0 & 14.0 & 18.5 & 15.5 & --- & --- \\
\hline
\end{tabular}


LOCATION.—Lat $38^{\circ} 34^{\prime} 32^{\prime \prime}$, long 121 $23^{\prime} 14^{\prime \prime}$, in SE 1/4 NW 1/4 sec.12, T.8 N., R.5 E., Sacramento County, Hydrologic Unit 18020111, on right bank 15 mi downstream from Folsom Dam and 5 mi southwest of Carmichael.

DRAINAGE AREA.- $1,938 \mathrm{mi}^{2}$.

PERIOD OF RECORD.-November 1998 to September 1999.

WATER TEMPERATURE.-November 1998 to September 1999.

PERIOD OF DAILY RECORD.--November 1998 to September 1999.

WATER TEMPERATURE.-November 1998 to September 1999.

INSTRUMENTATION.-Water-temperature recorder since Nov. 13, 1998.

REMARKS.-Water temperature can be affected by releases from Folsom and Nimbus Dams.

EXTREMES FOR PERIOD OF DAILY RECORD.-

WATER TEMPERATURE: Maximum recorded, $21.5^{\circ} \mathrm{C}$, Sept. 14, 1999; minimum recorded, $7.5^{\circ} \mathrm{C}$, several days in January and February 1999. EXTREMES FOR CURRENT YEAR.-

WATER TEMPERATURE: Maximum recorded, $21.5^{\circ} \mathrm{C}$, Sept. 14 ; minimum recorded, $7.5^{\circ} \mathrm{C}$, several days in January and February.

TEMPERATURE, WATER (DEG. C), WATER YEAR OCTOBER 1998 TO SEPTEMBER 1999

\begin{tabular}{|c|c|c|c|c|c|c|c|c|c|c|c|c|}
\hline \multirow[t]{2}{*}{ DAY } & MAX & MIN & MAX & MIN & MAX & MIN & MAX & MIN & MAX & MIN & MAX & MIN \\
\hline & \multicolumn{2}{|c|}{ OCTOBER } & \multicolumn{2}{|c|}{ NOVEMBER } & \multicolumn{2}{|c|}{ DECEMBER } & \multicolumn{2}{|c|}{ JANUARY } & \multicolumn{2}{|c|}{ FEBRUARY } & \multicolumn{2}{|c|}{ MARCH } \\
\hline 1 & --- & --- & --- & --- & 14.0 & 13.0 & 9.5 & 8.5 & 9.5 & 8.0 & 10.5 & 9.0 \\
\hline 2 & --- & --- & --- & --- & 14.0 & 13.0 & 10.0 & 8.5 & 9.5 & 8.0 & 10.0 & 8.5 \\
\hline 3 & --- & --- & --- & --- & 13.5 & 13.0 & 9.0 & 8.5 & 10.0 & 8.0 & 10.0 & 9.0 \\
\hline 4 & --- & --- & --- & --- & 13.0 & 12.0 & 9.5 & 9.0 & 9.5 & 8.5 & 9.5 & 8.5 \\
\hline 5 & --- & --- & --- & --- & 12.5 & 11.5 & 9.0 & 8.0 & 9.5 & 7.5 & 10.5 & 8.5 \\
\hline 6 & --- & --- & --- & --- & 12.5 & 11.5 & 8.5 & 8.0 & 8.5 & 8.0 & 10.5 & 9.5 \\
\hline 7 & --- & --- & --- & --- & 12.0 & 11.0 & 8.5 & 8.0 & 8.0 & 8.0 & 11.0 & 9.5 \\
\hline 8 & --- & --- & --- & --- & 12.0 & 11.5 & 9.0 & 8.0 & 8.5 & 8.0 & 10.0 & 9.0 \\
\hline 9 & --- & --- & --- & --- & 12.0 & 10.5 & 8.5 & 8.0 & 8.5 & 8.0 & 10.5 & 9.0 \\
\hline 10 & --- & --- & --- & --- & 12.0 & 11.0 & 8.5 & 8.0 & 9.0 & 7.5 & 10.5 & 9.0 \\
\hline 11 & --- & --- & --- & --- & 12.0 & 11.0 & 8.5 & 7.5 & 9.0 & 8.0 & 11.0 & 9.5 \\
\hline 12 & --- & --- & --- & --- & 12.0 & 11.0 & 8.5 & 7.5 & 8.5 & 8.0 & 11.5 & 9.5 \\
\hline 13 & --- & --- & 15.5 & 14.0 & 11.5 & 11.0 & 9.0 & 7.5 & 9.0 & 8.0 & 11.5 & 9.5 \\
\hline 14 & --- & --- & 15.5 & 14.0 & 12.0 & 10.5 & 9.0 & 7.5 & 8.5 & 7.5 & 10.5 & 9.5 \\
\hline 15 & --- & --- & 15.5 & 14.0 & 11.5 & 10.5 & 9.5 & 8.5 & 8.5 & 7.5 & 11.5 & 9.5 \\
\hline 16 & --- & --- & 14.5 & 14.0 & 12.0 & 10.5 & 9.5 & 9.0 & 8.0 & 7.5 & 10.5 & 9.5 \\
\hline 17 & --- & --- & 15.0 & 14.0 & 12.0 & 10.5 & 10.0 & 9.0 & 8.0 & 7.5 & 11.5 & 9.0 \\
\hline 18 & --- & --- & 15.0 & 13.5 & 11.5 & 10.5 & 10.0 & 9.5 & 8.0 & 8.0 & 11.5 & 9.5 \\
\hline 19 & --- & --- & 14.5 & 13.0 & 11.0 & 10.0 & 10.0 & 9.5 & 8.5 & 7.5 & 11.5 & 9.5 \\
\hline 20 & --- & --- & 15.0 & 13.5 & 10.5 & 9.5 & 10.0 & 8.5 & 8.5 & 8.0 & 11.5 & 9.5 \\
\hline 21 & --- & --- & 15.0 & 13.5 & 10.0 & 8.5 & 9.0 & 8.5 & 8.5 & 8.0 & 12.0 & 9.5 \\
\hline 22 & --- & --- & 15.0 & 14.0 & 10.0 & 8.5 & 9.0 & 8.5 & 8.5 & 8.0 & 11.0 & 9.5 \\
\hline 23 & --- & --- & 14.5 & 14.0 & 10.0 & 9.0 & 9.0 & 8.5 & 9.0 & 8.0 & 11.5 & 9.5 \\
\hline 24 & --- & --- & 14.5 & 13.5 & 9.5 & 8.5 & 9.5 & 8.5 & 8.5 & 8.0 & 11.0 & 9.5 \\
\hline 25 & --- & --- & 14.5 & 13.0 & 10.0 & 8.5 & 9.0 & 8.5 & 9.0 & 8.0 & 12.0 & 9.5 \\
\hline 26 & --- & --- & 14.5 & 13.0 & 10.0 & 9.0 & 9.0 & 8.5 & 9.0 & 7.5 & 12.5 & 9.5 \\
\hline 27 & --- & --- & 14.0 & 14.0 & 10.0 & 9.0 & 9.5 & 8.0 & 10.0 & 8.0 & 12.0 & 9.5 \\
\hline 28 & --- & --- & 14.5 & 13.5 & 10.0 & 9.0 & 9.5 & 8.5 & 9.5 & 9.0 & 12.0 & 9.5 \\
\hline 29 & --- & --- & 13.5 & 13.0 & 10.0 & 9.0 & 9.5 & 8.5 & --- & --- & 12.0 & 9.0 \\
\hline 30 & --- & --- & 13.5 & 13.0 & 10.0 & 8.5 & 9.0 & 8.0 & --- & --- & 12.0 & 9.5 \\
\hline 31 & --- & --- & --- & --- & 10.0 & 9.0 & 9.0 & 8.5 & --- & --- & 11.5 & 9.0 \\
\hline MONTH & --- & --- & --- & --- & 14.0 & 8.5 & 10.0 & 7.5 & 10.0 & 7.5 & 12.5 & 8.5 \\
\hline
\end{tabular}


11446980 AMERICAN RIVER BELOW WATT AVENUE BRIDGE, NEAR CARMICHAEL, CA—Continued

\section{TEMPERATURE, WATER (DEG. C), WATER YEAR OCTOBER 1998 TO SEPTEMBER 1999}

\begin{tabular}{|c|c|c|c|c|c|c|c|c|c|c|c|c|}
\hline \multirow[t]{2}{*}{ DAY } & MAX & MIN & MAX & MIN & MAX & MIN & MAX & MIN & MAX & MIN & MAX & MIN \\
\hline & \multicolumn{2}{|c|}{ APRIL } & \multicolumn{2}{|c|}{ MAY } & \multicolumn{2}{|c|}{ JUNE } & \multicolumn{2}{|c|}{ JULY } & \multicolumn{2}{|c|}{ AUGUST } & \multicolumn{2}{|c|}{ SEPTEMBER } \\
\hline 1 & 12.0 & 9.0 & 15.5 & 12.5 & 16.0 & 13.0 & 18.5 & 15.0 & 20.0 & 16.5 & 18.5 & 15.5 \\
\hline 2 & 12.5 & 9.5 & 13.0 & 12.0 & 15.5 & 13.0 & 18.5 & 14.5 & 20.5 & 17.0 & 19.0 & 15.5 \\
\hline 3 & 12.0 & 10.0 & 13.0 & 11.0 & 15.0 & 12.5 & 18.0 & 14.5 & 21.0 & 17.0 & 19.0 & 16.0 \\
\hline 4 & 12.0 & 9.0 & 14.0 & 11.0 & 16.0 & 12.5 & 18.5 & 14.5 & 21.0 & 17.5 & 19.5 & 16.5 \\
\hline 5 & 11.0 & 9.5 & 15.0 & 11.5 & 17.0 & 13.0 & 18.5 & 15.0 & 20.0 & 17.0 & 19.5 & 16.0 \\
\hline 6 & 11.0 & 9.0 & 15.5 & 12.5 & 17.0 & 13.5 & 18.5 & 14.5 & 20.0 & 17.5 & 19.5 & 16.0 \\
\hline 7 & 11.0 & 9.5 & 15.5 & 12.5 & 16.5 & 13.0 & 18.5 & 14.5 & 20.5 & 17.0 & 19.5 & 16.5 \\
\hline 8 & 11.0 & 9.5 & 15.0 & 12.0 & 17.0 & 13.0 & 18.5 & 15.0 & 20.5 & 17.0 & 20.0 & 16.5 \\
\hline 9 & 12.0 & 9.0 & 15.0 & 12.0 & 17.0 & 13.0 & 19.0 & 15.0 & 20.5 & 17.5 & 19.5 & 17.0 \\
\hline 10 & 11.5 & 9.5 & 15.0 & 12.0 & 17.5 & 13.5 & 18.5 & 15.0 & 20.5 & 17.5 & 19.5 & 16.5 \\
\hline 11 & 12.0 & 10.0 & 16.0 & 12.5 & 17.5 & 13.5 & 19.0 & 15.0 & 21.0 & 17.5 & 19.0 & 16.5 \\
\hline 12 & 13.0 & 9.5 & 16.0 & 13.0 & 17.5 & 13.5 & 19.0 & 15.5 & 21.0 & 17.5 & 19.5 & 16.5 \\
\hline 13 & 13.5 & 10.5 & 15.5 & 12.5 & 18.0 & 13.5 & 19.0 & 15.5 & 21.0 & 17.0 & 20.5 & 16.5 \\
\hline 14 & 14.0 & 10.5 & 15.0 & 12.0 & 18.0 & 14.0 & 19.0 & 15.5 & 21.0 & 17.5 & 21.5 & 18.0 \\
\hline 15 & 14.0 & 11.0 & 15.5 & 12.0 & 17.5 & 13.5 & 19.0 & 15.5 & 21.0 & 17.0 & 19.5 & 18.0 \\
\hline 16 & 14.0 & 11.0 & 15.5 & 12.0 & 18.0 & 14.0 & 18.5 & 15.5 & 21.0 & 17.0 & 21.0 & 17.0 \\
\hline 17 & 14.5 & 11.5 & 16.0 & 12.0 & 18.0 & 14.0 & 18.5 & 15.0 & 20.5 & 17.0 & 21.0 & 18.0 \\
\hline 18 & 14.0 & 11.0 & 15.5 & 12.5 & 18.0 & 14.0 & 19.0 & 15.0 & 20.0 & 16.5 & 19.5 & 16.5 \\
\hline 19 & 14.0 & 11.0 & 15.5 & 12.0 & 18.5 & 14.0 & 19.0 & 15.5 & 20.0 & 16.0 & 19.0 & 16.5 \\
\hline 20 & 13.5 & 11.0 & 15.5 & 12.0 & 18.0 & 14.0 & 19.0 & 15.5 & 19.5 & 16.5 & 19.0 & 16.0 \\
\hline 21 & 14.0 & 11.0 & 16.0 & 12.0 & 18.0 & 14.0 & 19.5 & 15.5 & 19.5 & 15.5 & 19.5 & 16.5 \\
\hline 22 & 14.0 & 11.0 & 16.5 & 12.5 & 18.5 & 14.5 & 19.5 & 15.5 & 20.5 & 16.5 & 19.5 & 17.0 \\
\hline 23 & 14.5 & 10.5 & 16.5 & 13.0 & 18.5 & 14.5 & 19.5 & 16.0 & 20.0 & 17.0 & 19.5 & 17.0 \\
\hline 24 & 15.5 & 12.5 & 16.0 & 12.5 & 19.0 & 14.0 & 19.5 & 16.0 & 20.5 & 16.5 & 19.5 & 16.5 \\
\hline 25 & 14.0 & 12.0 & 16.5 & 12.5 & 18.5 & 14.5 & 20.0 & 16.0 & 20.0 & 17.0 & 19.5 & 16.5 \\
\hline 26 & 13.0 & 11.5 & 16.5 & 13.0 & 18.5 & 14.0 & 20.0 & 16.5 & 19.5 & 17.0 & 19.5 & 17.0 \\
\hline 27 & 14.0 & 11.0 & 16.5 & 13.0 & 18.0 & 14.5 & 20.0 & 16.5 & 20.5 & 16.5 & 19.0 & 17.0 \\
\hline 28 & 14.0 & 11.0 & 16.5 & 12.5 & 18.0 & 14.5 & 19.5 & 16.0 & 20.0 & 16.5 & 19.0 & 16.5 \\
\hline 29 & 14.0 & 11.0 & 16.5 & 12.5 & 18.0 & 14.5 & 20.0 & 16.0 & 20.0 & 16.5 & 19.5 & 16.5 \\
\hline 30 & 15.0 & 12.0 & 16.5 & 13.0 & 18.5 & 14.5 & 20.0 & 16.5 & 19.5 & 16.0 & 19.5 & 16.5 \\
\hline 31 & --- & --- & 16.5 & 13.0 & --- & --- & 20.5 & 16.5 & 19.0 & 16.0 & --- & --- \\
\hline MONTH & 15.5 & 9.0 & 16.5 & 11.0 & 19.0 & 12.5 & 20.5 & 14.5 & 21.0 & 15.5 & 21.5 & 15.5 \\
\hline
\end{tabular}


11447293 DRY CREEK AT VERNON STREET BRIDGE, AT ROSEVILLE, CA

LOCATION.-Lat $38^{\circ} 44^{\prime} 04^{\prime \prime}$, long 121¹7'55", NW 1/4 SW 1/4 sec.11, T.10 N, R6 E, in Placer County, Hydrologic Unit 18021111, on right bank upstream side of bridge and 0.5 mi below confluence of Cirby Creek, at Roseville, Ca.

DRAINAGE AREA.- $-80.08 \mathrm{mi}^{2}$.

PERIOD OF RECORD.-October 1996 to current year.

GAGE.-Water-stage recorder. Datum of gage is $99.86 \mathrm{ft}$ above sea level (levels by City of Roseville).

REMARKS.-Records good. Records computed only for gage heights above the bottom of the stilling well (11.55 $\mathrm{ft}$ and above), as the well sits above the intakes. Low summer flow sustained by ground-water seepage and residential and industrial waste water.

EXTREMES FOR PERIOD OF RECORD.-Maximum discharge, 7,950 ft³/s, Jan. 22, 1997, gage height, $24.39 \mathrm{ft}$.

EXTREMES FOR CURRENT YEAR.-Peak discharges greater than a base discharge of $1,500 \mathrm{ft}^{3} / \mathrm{s}$, or maximum:

$\begin{array}{ccccccc} & \text { Discharge } & \text { Gage height } & \text { Discharge } & \text { Gage height } \\ \text { Date } & \text { Time } & \left(\mathrm{ft}^{3} / \mathrm{s}\right) & (\mathrm{ft}) & \text { Date } & \text { Time } & (\mathrm{ft})\end{array}$

Feb. 9

1,990

15.60

DISCHARGE, CUBIC FEET PER SECOND, WATER YEAR OCTOBER 1998 TO SEPTEMBER 1999

DAILY MEAN VALUES

\begin{tabular}{|c|c|c|c|c|c|c|c|c|c|c|c|c|}
\hline DAY & $\mathrm{OCT}$ & NOV & $\mathrm{DEC}$ & JAN & FEB & MAR & APR & MAY & JUN & JUL & AUG & SEP \\
\hline 1 & --- & --- & --- & --- & --- & --- & --- & --- & --- & --- & --- & --- \\
\hline 2 & --- & --- & --- & --- & --- & --- & --- & --- & --- & --- & --- & --- \\
\hline 3 & --- & --- & --- & --- & --- & e 372 & --- & --- & --- & --- & --- & --- \\
\hline 4 & --- & --- & --- & --- & --- & --- & --- & --- & --- & --- & --- & --- \\
\hline 5 & --- & --- & --- & --- & --- & --- & --- & --- & --- & --- & --- & --- \\
\hline 6 & --- & --- & --- & --- & --- & --- & --- & --- & --- & --- & --- & --- \\
\hline 7 & --- & --- & --- & --- & e 776 & --- & --- & --- & --- & --- & --- & --- \\
\hline 8 & --- & --- & --- & --- & 410 & --- & --- & --- & --- & --- & --- & --- \\
\hline 9 & --- & --- & --- & --- & 1290 & --- & --- & --- & --- & --- & --- & --- \\
\hline 10 & --- & --- & --- & --- & 366 & --- & --- & --- & --- & --- & --- & --- \\
\hline 11 & --- & --- & --- & --- & --- & --- & --- & --- & --- & --- & --- & --- \\
\hline 12 & --- & --- & --- & --- & --- & --- & --- & --- & --- & --- & --- & --- \\
\hline 13 & --- & --- & --- & --- & --- & --- & --- & --- & --- & --- & --- & --- \\
\hline 14 & --- & --- & --- & --- & --- & --- & --- & --- & --- & --- & --- & --- \\
\hline 15 & --- & --- & --- & --- & --- & --- & --- & --- & --- & --- & --- & --- \\
\hline 16 & --- & --- & --- & --- & --- & --- & --- & --- & --- & --- & --- & --- \\
\hline 17 & --- & --- & --- & --- & 838 & --- & --- & --- & --- & --- & --- & --- \\
\hline 18 & --- & --- & --- & --- & e404 & --- & --- & --- & --- & --- & --- & --- \\
\hline 19 & --- & --- & --- & 382 & --- & --- & --- & --- & --- & --- & --- & --- \\
\hline 20 & --- & --- & --- & 547 & --- & --- & --- & --- & --- & --- & --- & --- \\
\hline 21 & --- & --- & --- & --- & 842 & --- & --- & --- & --- & --- & --- & --- \\
\hline 22 & --- & --- & --- & --- & --- & --- & --- & --- & --- & --- & --- & --- \\
\hline 23 & --- & --- & --- & e374 & --- & --- & --- & --- & --- & --- & --- & --- \\
\hline 24 & --- & --- & --- & --- & --- & --- & --- & --- & --- & --- & --- & --- \\
\hline 25 & --- & --- & --- & --- & --- & --- & --- & --- & --- & --- & --- & --- \\
\hline 26 & --- & --- & --- & --- & --- & --- & --- & --- & --- & --- & --- & --- \\
\hline 27 & --- & --- & --- & --- & --- & --- & --- & --- & --- & --- & --- & --- \\
\hline 28 & --- & --- & --- & --- & --- & --- & --- & --- & --- & --- & --- & --- \\
\hline 29 & --- & --- & --- & --- & --- & --- & --- & --- & --- & --- & --- & --- \\
\hline 30 & --- & --- & --- & --- & --- & --- & --- & --- & --- & --- & --- & --- \\
\hline 31 & --- & --- & --- & e409 & --- & --- & --- & --- & --- & --- & --- & --- \\
\hline TOTAL & --- & --- & --- & --- & --- & --- & --- & --- & --- & --- & --- & --- \\
\hline MEAN & --- & --- & --- & --- & --- & --- & --- & --- & --- & --- & --- & --- \\
\hline MAX & --- & --- & --- & --- & --- & --- & --- & --- & --- & --- & --- & --- \\
\hline MIN & --- & --- & --- & --- & --- & --- & --- & --- & --- & --- & --- & --- \\
\hline $\mathrm{AC}-\mathrm{FT}$ & --- & --- & --- & --- & --- & --- & --- & --- & --- & --- & --- & \\
\hline
\end{tabular}

e Estimated. 


\section{ARCADE CREEK NEAR DEL PASO HEIGHTS, CA}

LOCATION.-Lat 38³8’01", long 121²2' 54", in Del Paso Grant, Sacramento County, Hydrologic Unit 18021111, on right bank $500 \mathrm{ft}$ upstream from bridge on Watt Avenue at intersection with Longview Drive and 1.3 mi east of Del Paso Heights.

DRAINAGE AREA.- $-31.5 \mathrm{mi}^{2}$.

PERIOD OF RECORD.—July 1963 to June 1978, December 1995 to current year.

CHEMICAL DATA: Water years 1996-98.

SPECIFIC CONDUCTANCE: Water years 1997-98.

WATER TEMPERATURE: Water years 1997-98.

SEDIMENT DATA: Water years 1996-98.

GAGE.-Water-stage recorder. Elevation of gage is $50 \mathrm{ft}$ above sea level, from topographic map. Prior to December 1995, at site 0.3 mi upstream at different datum.

REMARKS.- Records good except for discharges below $1 \mathrm{ft}^{3} / \mathrm{s}$ which are poor. Low summer flow sustained by residential and industrial waste water.

EXTREMES FOR PERIOD OF RECORD.-Maximum discharge, 3,320 ft 3 s, Feb. 3, 1998, gage height, 15.63; no flow for many days in most years. See schematic diagram of lower Sacramento River Basin.

EXTREMES FOR CURRENT YEAR.-Peak discharges greater than a base discharge of $500 \mathrm{ft}^{3} / \mathrm{s}$, or maximum:

$\begin{array}{lccclcrc}\text { Date } & \text { Time } & \begin{array}{c}\text { Discharge } \\ \left(\mathrm{ft}^{3} / \mathrm{s}\right)\end{array} & \begin{array}{c}\text { Gage height } \\ (\mathrm{ft})\end{array} & \text { Date } & \text { Time } & \begin{array}{c}\text { Discharge } \\ \left(\mathrm{ft}^{3} / \mathrm{s}\right)\end{array} & \begin{array}{c}\text { Gage height } \\ (\mathrm{ft})\end{array} \\ \text { Oct. 24 } & 1515 & 572 & 9.58 & \text { Jan. 31 } & 1030 & 687 & 10.28 \\ \text { Nov. 30 } & 1700 & 602 & 9.77 & \text { Feb. } 9 & 1000 & 1,040 & 11.69 \\ \text { Jan. 20 } & 0245 & 613 & 9.84 & \text { Feb. 21 } & 0100 & 710 & 10.39\end{array}$

DISCHARGE, CUBIC FEET PER SECOND, WATER YEAR OCTOBER 1998 TO SEPTEMBER 1999 DAILY MEAN VALUES

\begin{tabular}{|c|c|c|c|c|c|c|c|c|c|c|c|c|}
\hline DAY & $\mathrm{OCT}$ & NOV & $\mathrm{DEC}$ & JAN & FEB & MAR & APR & MAY & JUN & JUL & AUG & SEP \\
\hline 1 & 1.4 & 12 & 30 & .55 & 12 & 67 & 4.9 & 1.9 & 2.5 & 2.2 & 2.3 & 1.1 \\
\hline 2 & 1.8 & 2.4 & 4.3 & .43 & 3.1 & 6.1 & 2.8 & 2.9 & 2.1 & 2.1 & 2.4 & 1.3 \\
\hline 3 & 1.2 & 1.2 & 128 & .35 & 1.9 & 57 & 2.3 & 4.9 & 16 & 2.1 & 2.1 & 1.3 \\
\hline 4 & 1.2 & 1.2 & 10 & .26 & 1.4 & 6.3 & 1.9 & 2.0 & 3.1 & 1.9 & 2.2 & 1.4 \\
\hline 5 & 1.1 & .50 & 8.3 & .24 & 1.1 & 4.4 & 67 & 1.4 & 2.6 & 2.0 & 2.0 & 1.5 \\
\hline 6 & 1.0 & 7.0 & 33 & .11 & 22 & 3.8 & 23 & 1.5 & 2.5 & 2.2 & 2.0 & 1.4 \\
\hline 7 & 1.2 & 77 & 2.8 & .33 & 440 & 3.5 & 4.3 & 1.5 & 2.4 & 2.2 & 2.1 & 1.4 \\
\hline 8 & 1.2 & 12 & 2.0 & .56 & 83 & 31 & 28 & 1.5 & 2.2 & 2.1 & 2.1 & 1.3 \\
\hline 9 & .99 & 3.1 & 1.6 & .27 & 527 & 94 & 7.0 & 1.6 & 2.3 & 2.1 & 2.0 & 1.0 \\
\hline 10 & 1.1 & 1.7 & 1.1 & .21 & 21 & 5.7 & 4.1 & 1.5 & 2.7 & 2.1 & 2.0 & 1.0 \\
\hline 11 & 1.3 & 46 & 1.0 & .20 & 6.6 & 4.0 & 4.1 & 1.7 & 2.2 & 2.2 & 2.0 & 1.1 \\
\hline 12 & 1.1 & 3.9 & .91 & .19 & 3.9 & 3.3 & 3.5 & 1.8 & 2.9 & 2.2 & 1.9 & 1.2 \\
\hline 13 & 1.1 & 1.4 & .64 & .23 & 5.4 & 3.3 & 2.8 & 1.9 & 2.9 & 2.2 & 1.9 & 1.4 \\
\hline 14 & 1.1 & .92 & 3.5 & .21 & 21 & 2.9 & 2.2 & 1.7 & 2.8 & 2.1 & 1.9 & 1.2 \\
\hline 15 & .99 & .64 & 1.2 & 9.9 & 3.1 & 2.7 & 2.1 & 1.8 & 2.2 & 2.0 & 1.9 & 1.0 \\
\hline 16 & .87 & .43 & .64 & 58 & 65 & 2.7 & 2.2 & 1.9 & 2.2 & 2.1 & 1.7 & .97 \\
\hline 17 & .72 & 26 & .55 & 25 & 200 & 2.7 & 2.5 & 1.9 & 2.4 & 2.2 & 1.7 & .99 \\
\hline 18 & .95 & 2.6 & .66 & 148 & 43 & 2.5 & 2.5 & 1.9 & 2.3 & 2.1 & 1.7 & .93 \\
\hline 19 & 1.6 & .95 & .40 & 80 & 17 & 2.4 & 2.5 & 2.0 & 2.5 & 2.3 & 1.8 & 1.1 \\
\hline 20 & 1.0 & .39 & .29 & 205 & 119 & 11 & 2.1 & 2.1 & 2.3 & 2.4 & 1.7 & 1.1 \\
\hline 21 & 1.2 & .55 & .08 & 9.5 & 224 & 3.4 & 1.9 & 1.9 & 2.5 & 2.5 & 1.6 & .95 \\
\hline 22 & 1.3 & 57 & .25 & 3.1 & 10 & 2.3 & 1.9 & 2.1 & 2.5 & 2.5 & 1.6 & .92 \\
\hline 23 & 1.3 & 60 & .93 & 83 & 6.2 & 3.9 & 1.8 & 2.0 & 2.3 & 2.7 & 1.7 & .82 \\
\hline 24 & 182 & 41 & 1.2 & 8.1 & 5.1 & 4.9 & 1.7 & 1.9 & 2.3 & 2.8 & 1.7 & .76 \\
\hline 25 & 15 & 3.1 & 1.0 & 2.5 & 28 & 14 & 2.2 & 1.9 & 2.3 & 2.9 & 1.7 & .85 \\
\hline 26 & 2.9 & 1.4 & .90 & 31 & 5.4 & 3.5 & 2.0 & 2.2 & 2.3 & 2.9 & 1.6 & .81 \\
\hline 27 & 2.0 & 90 & .75 & 3.4 & 4.1 & 2.4 & 2.1 & 2.3 & 2.2 & 2.5 & 1.9 & .70 \\
\hline 28 & 1.4 & 112 & .62 & 1.4 & 42 & 2.1 & 1.7 & 2.4 & 2.3 & 2.5 & 1.8 & .48 \\
\hline 29 & 1.6 & 30 & .58 & .98 & --- & 2.1 & 1.7 & 2.3 & 2.2 & 2.2 & 1.7 & .47 \\
\hline 30 & 1.2 & 217 & .55 & .86 & --- & 1.9 & 1.5 & 2.1 & 2.3 & 2.3 & 1.5 & .43 \\
\hline 31 & .93 & --- & .51 & 243 & --- & 9.5 & --- & 2.2 & --- & 2.4 & 1.3 & --- \\
\hline TOTAL & 233.75 & 813.38 & 238.26 & 916.88 & 1921.3 & 366.3 & 190.3 & 62.7 & 86.3 & 71.0 & 57.5 & 30.88 \\
\hline MEAN & 7.54 & 27.1 & 7.69 & 29.6 & 68.6 & 11.8 & 6.34 & 2.02 & 2.88 & 2.29 & 1.85 & 1.03 \\
\hline MAX & 182 & 217 & 128 & 243 & 527 & 94 & 67 & 4.9 & 16 & 2.9 & 2.4 & 1.5 \\
\hline MIN & .72 & .39 & .08 & .11 & 1.1 & 1.9 & 1.5 & 1.4 & 2.1 & 1.9 & 1.3 & .43 \\
\hline $\mathrm{AC}-\mathrm{ET}$ & 464 & 1610 & 473 & 1820 & 3810 & 727 & 377 & 124 & 171 & 141 & 114 & 61 \\
\hline
\end{tabular}


11447360 ARCADE CREEK NEAR DEL PASO HEIGHTS, CA—Continued

STATISTICS OF MONTHLY MEAN DATA FOR WATER YEARS 1963 - 1999, BY WATER YEAR (WY)

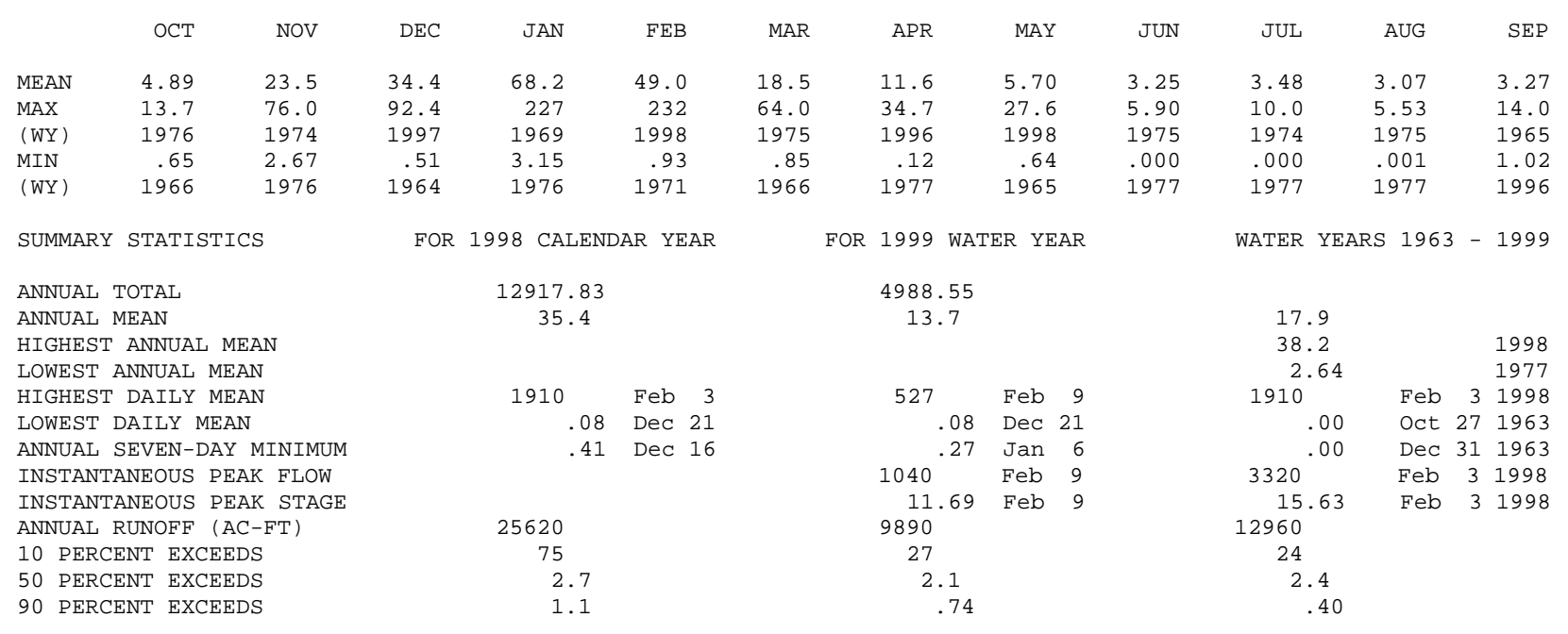




\section{SACRAMENTO RIVER AT FREEPORT, CA}

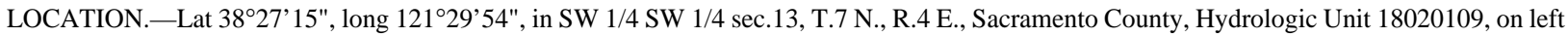
bank $630 \mathrm{ft}$ downstream from drawbridge at Freeport and $11 \mathrm{mi}$ south of Sacramento.

DRAINAGE AREA.--Indeterminate.

\section{WATER-DISCHARGE RECORDS}

PERIOD OF RECORD._January 1904 to July 1905 (gage heights only), June to November 1921, October 1948 to current year. Prior to October 1979, published as Sacramento River at Sacramento (station 11447500).

REVISED RECORD.-WDR CA-96-4: 1994-1995 (P).

GAGE.-Water-stage recorder and acoustic-velocity system. Datum of gage is sea level.

REMARKS.- - Records good. Natural flow of stream affected by storage reservoirs, power development, diversions for irrigation, return flow from irrigated areas, and tide. Floodflows bypass station through Sacramento Weir Spill to Yolo Bypass (stations 11426000 and 11453000). See schematic diagram of lower Sacramento River Basin.

EXTREMES FOR PERIOD OF RECORD (since 1949).—Maximum discharge, 117,000 ft 3 /s, Feb. 19, 1986, elevation, $25.00 \mathrm{ft}$; minimum daily, $3,970 \mathrm{ft}^{3} / \mathrm{s}$, Oct. $15,1977$.

EXTREMES OUTSIDE PERIOD OF RECORD.—Maximum discharge known prior to Nov. 21, 1950, 103,000 ft $3 / \mathrm{s}$, Jan. 17, 1909, elevation, $29.6 \mathrm{ft}$, site then in use at present datum, from reports of California Department of Water Resources.

DISCHARGE, CUBIC FEET PER SECOND, WATER YEAR OCTOBER 1998 TO SEPTEMBER 1999 DAILY MEAN VALUES

\begin{tabular}{|c|c|c|c|c|c|c|c|c|c|c|c|c|}
\hline DAY & $\mathrm{OCT}$ & NOV & $\mathrm{DEC}$ & JAN & FEB & MAR & APR & MAY & JUN & JUL & AUG & SEP \\
\hline 1 & 22900 & e13900 & 37200 & 21600 & 44900 & 71800 & 34300 & 24900 & 19800 & 20900 & 22900 & 17300 \\
\hline 2 & 22200 & e14300 & 43300 & 21000 & 41600 & 73400 & 31400 & 24800 & 19400 & 21000 & 23300 & 16600 \\
\hline 3 & 21500 & e14100 & 49400 & 20900 & 37900 & 74000 & 29800 & 24400 & 20000 & 20600 & 23200 & 16500 \\
\hline 4 & 20300 & 13200 & 54700 & 20600 & 34800 & 74700 & 28600 & 24800 & 20200 & 20800 & 22400 & 16500 \\
\hline 5 & 20000 & 13200 & 58300 & 20500 & 33900 & 74700 & 27700 & 24400 & 21200 & 20400 & 21500 & 16600 \\
\hline 6 & 19000 & 13200 & 60700 & 20200 & 35300 & 74600 & 27700 & 23800 & 21800 & 20500 & 21400 & 16300 \\
\hline 7 & 18200 & 13200 & 61900 & 20100 & 48400 & 72900 & 27300 & 22500 & 21500 & 20800 & 20600 & 16400 \\
\hline 8 & 18000 & 14500 & 62300 & 19700 & 60300 & 71300 & 27500 & 21300 & 20400 & 20700 & 19700 & 16400 \\
\hline 9 & 16800 & 15100 & 61900 & 19200 & 69200 & 70600 & 28200 & 21400 & 18700 & 21200 & 18900 & 16200 \\
\hline 10 & 16300 & 15800 & 60700 & 18400 & 83100 & 69300 & 28300 & 21200 & 17600 & 21400 & 17800 & 16200 \\
\hline 11 & 15800 & 16600 & 59400 & 18000 & 85400 & 68200 & 29400 & 20500 & 16400 & 21300 & 17400 & 16500 \\
\hline 12 & 15500 & 16800 & 57600 & 17900 & 82500 & 67700 & 30700 & 19300 & 16200 & 21400 & 17100 & 16900 \\
\hline 13 & 15100 & 17100 & 55700 & 17900 & 78700 & 66600 & 34400 & 18700 & 15600 & 21500 & 17200 & 16200 \\
\hline 14 & 14400 & 17200 & 54200 & 17800 & 75200 & 65600 & 36500 & 18100 & 15600 & 21500 & 17100 & 15100 \\
\hline 15 & 14100 & 17400 & 53100 & 17400 & 72400 & 63900 & 35700 & 18200 & 15000 & 22100 & 17100 & 15300 \\
\hline 16 & 13900 & 18000 & 51900 & 17600 & 71200 & 61000 & 34900 & 17500 & 15200 & 22200 & 17000 & 15400 \\
\hline 17 & 13500 & 18600 & 49300 & 18300 & 74800 & 57200 & 34600 & 16900 & 15100 & 22500 & 16600 & 15000 \\
\hline 18 & 13300 & 19200 & 45300 & 20100 & 86700 & 53000 & 34600 & 16600 & 15200 & 22900 & 15600 & 16000 \\
\hline 19 & 13100 & 19800 & 42100 & 23600 & 85600 & 49200 & 34700 & 16700 & 15200 & 23100 & 15500 & 16300 \\
\hline 20 & 13100 & 22400 & 40000 & 36400 & 81300 & 46600 & 34200 & 17100 & 14900 & 23900 & 14800 & 16700 \\
\hline 21 & 12900 & 23500 & 38400 & 56800 & 80000 & 43500 & 33600 & 17100 & 15000 & 23900 & 15100 & 15900 \\
\hline 22 & 12700 & 24200 & 36300 & 61700 & 79400 & 40900 & 33200 & 16800 & 14900 & 23800 & 15300 & 15300 \\
\hline 23 & 12700 & 24400 & 34000 & 63100 & 76600 & 38300 & 31800 & 17400 & 14700 & 23200 & 15600 & 15400 \\
\hline 24 & 13000 & 28500 & 31500 & 66700 & 74000 & 35800 & 29900 & 18400 & 14600 & 23300 & 16100 & 15000 \\
\hline 25 & 13400 & 31900 & 29000 & 67500 & 72600 & 34000 & 28600 & 18400 & 14900 & 23200 & 16100 & 14800 \\
\hline 26 & 13700 & 34600 & 26900 & 65700 & 71900 & 36000 & 27500 & 17900 & 15400 & 23400 & 16300 & 14600 \\
\hline 27 & 14200 & 33900 & 25600 & 63700 & 71300 & 41400 & 26900 & 17700 & 15700 & 23600 & 16500 & 14400 \\
\hline 28 & 15400 & 33400 & 24900 & 60200 & 71100 & 43200 & 26700 & 17700 & 17100 & 23700 & 17400 & 14500 \\
\hline 29 & e14400 & 34800 & 24200 & 56200 & --- & 43100 & 26100 & 18600 & 19100 & 23900 & 17700 & 15200 \\
\hline 30 & e14700 & 34800 & 23400 & 52000 & --- & 41300 & 25500 & 18900 & 20700 & 23700 & 17900 & 15400 \\
\hline 31 & e14400 & --- & 22400 & 48800 & --- & 38200 & --- & 19800 & --- & 23100 & 17800 & --- \\
\hline TOTAL & 488500 & 627600 & 1375600 & 1069600 & 1880100 & 1762000 & 920300 & 611800 & 517100 & 689500 & 558900 & 474900 \\
\hline MEAN & 15760 & 20920 & 44370 & 34500 & 67150 & 56840 & 30680 & 19740 & 17240 & 22240 & 18030 & 15830 \\
\hline MAX & 22900 & 34800 & 62300 & 67500 & 86700 & 74700 & 36500 & 24900 & 21800 & 23900 & 23300 & 17300 \\
\hline MIN & 12700 & 13200 & 22400 & 17400 & 33900 & 34000 & 25500 & 16600 & 14600 & 20400 & 14800 & 14400 \\
\hline $\mathrm{AC}-\mathrm{FT}$ & 968900 & 1245000 & 2729000 & 2122000 & 3729000 & 3495000 & 1825000 & 1214000 & 1026000 & 1368000 & 1109000 & 942000 \\
\hline
\end{tabular}


11447650 SACRAMENTO RIVER AT FREEPORT, CA—Continued

STATISTICS OF MONTHLY MEAN DATA FOR WATER YEARS 1949 - 1999, BY WATER YEAR (WY)

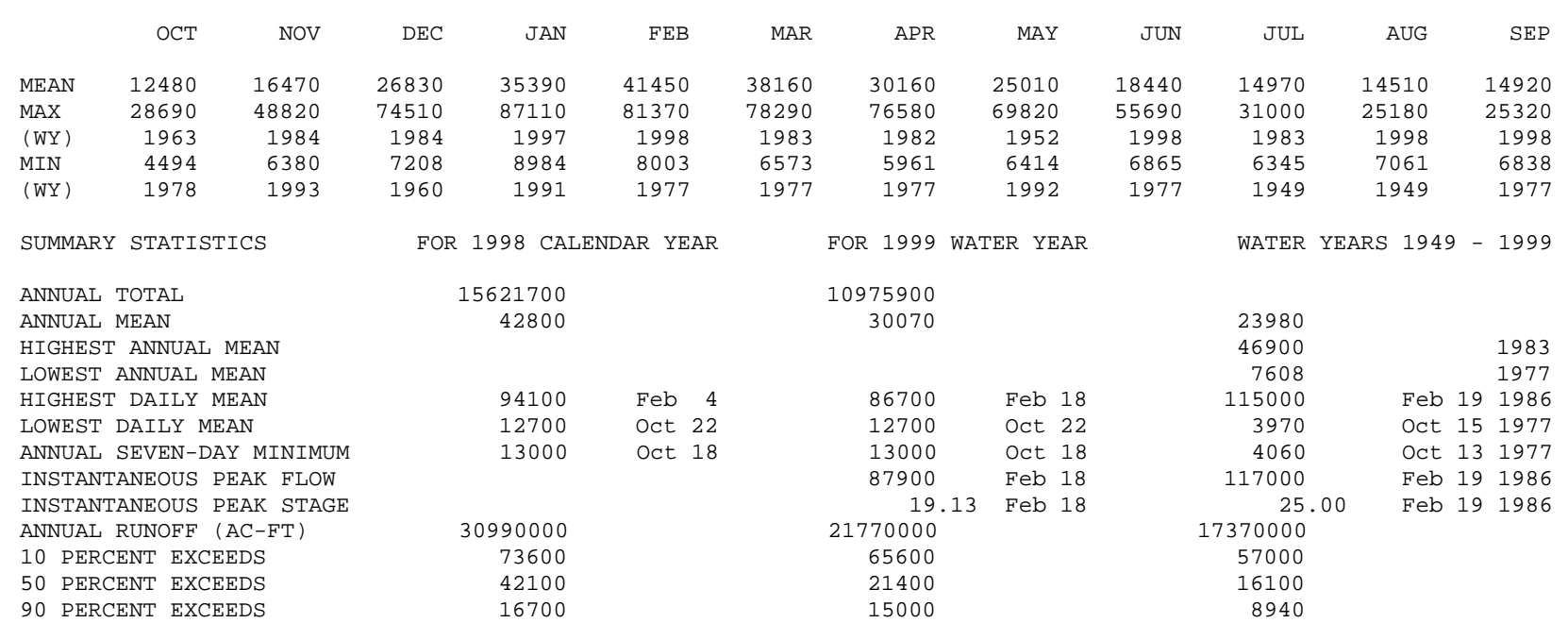




\section{WATER-QUALITY RECORDS}

PERIOD OF RECORD.-Water year 1957 to current year. CHEMICAL DATA: Water years 1959 to current year.

BIOLOGICAL DATA: Water years 1974-81.

SPECIFIC CONDUCTANCE: Water years 1974-75, 1989-98.

WATER TEMPERATURE: Water year 1960 to current year.

SEDIMENT DATA: Water year 1957 to current year (prior to water year 1980, published as 11447500 Sacramento River at Sacramento).

PERIOD OF DAILY RECORD.-October 1956 to current year.

CHEMICAL DATA: June 1960 to June 1963.

SPECIFIC CONDUCTANCE: Water years 1974-75, 1989-94, 1996-98.

WATER TEMPERATURE: June 1960 to current year.

SUSPENDED SEDIMENT: October 1956 to current year.

INSTRUMENTATION.-Temperature recorder June 1960 to November 1988. Water-quality monitor since November 1988.

REMARKS.-Records of sediment discharge from 1957 to 1979 were obtained at Sacramento and are considered equivalent. Additional specificconductance and monthly chemical and trace-element data are available in files of the U.S. Geological Survey. Interruption in record was due to malfunction of recording instrument.

EXTREMES FOR PERIOD OF DAILY RECORD.-

SPECIFIC CONDUCTANCE: Maximum recorded, 318 microsiemens, Nov. 22, 1974; minimum recorded, 32 microsiemens, Apr. 6, 1974.

WATER TEMPERATURE: Maximum recorded, $27.0^{\circ} \mathrm{C}$, Sept. 8, 1977; minimum recorded, 3.0 ${ }^{\circ} \mathrm{C}$, Dec. $25-27,1990$.

SEDIMENT CONCENTRATION: Maximum daily mean, 1,960 mg/L, Dec. 24, 1964; minimum daily, 2 mg/L, Jan. 27,31 , and Nov. 21, 1991.

SEDIMENT LOAD: Maximum daily, 525,000 tons, Dec. 24, 1964; minimum daily, 35 tons, Jan. 31, 1991.

EXTREMES FOR CURRENT YEAR.-

WATER TEMPERATURE: Maximum recorded, $22.5^{\circ} \mathrm{C}$, several days in June, July, and August; minimum recorded, $6.5^{\circ} \mathrm{C}$, Dec. $23-26$.

SEDIMENT CONCENTRATION: Maximum daily mean, $204 \mathrm{mg} / \mathrm{L}$, Dec. 3, minimum daily mean, $10 \mathrm{mg} / \mathrm{L}, \mathrm{Nov} .4,5$.

SEDIMENT LOAD: Maximum daily, 36,500 tons, Feb. 10; minimum daily, 362 tons, Nov. 5.

WATER-QUALITY DATA, WATER YEAR OCTOBER 1998 TO SEPTEMBER 1999

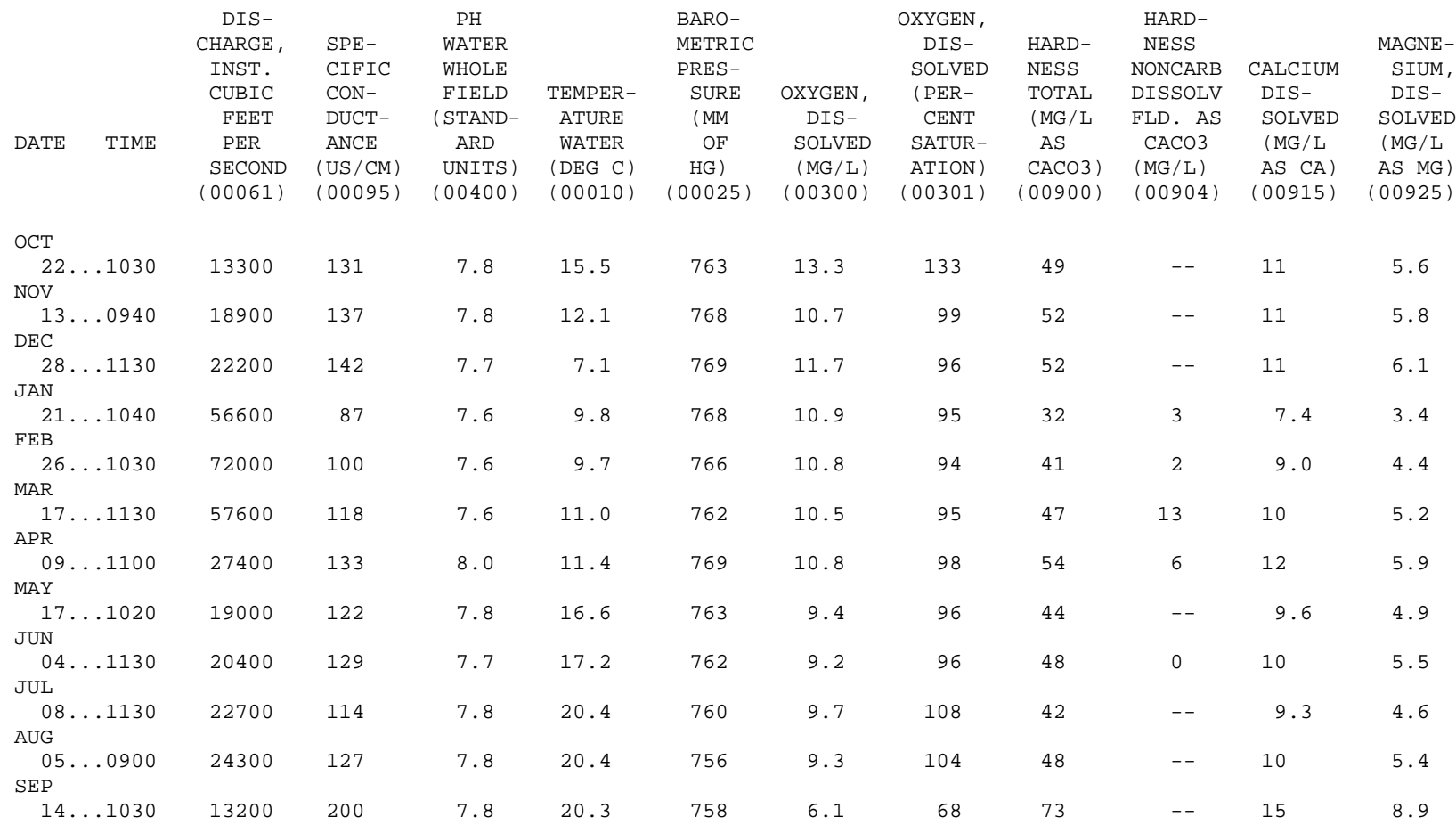


11447650 SACRAMENTO RIVER AT FREEPORT, CA—Continued

\section{WATER-QUALITY DATA, WATER YEAR OCTOBER 1998 TO SEPTEMBER 1999}

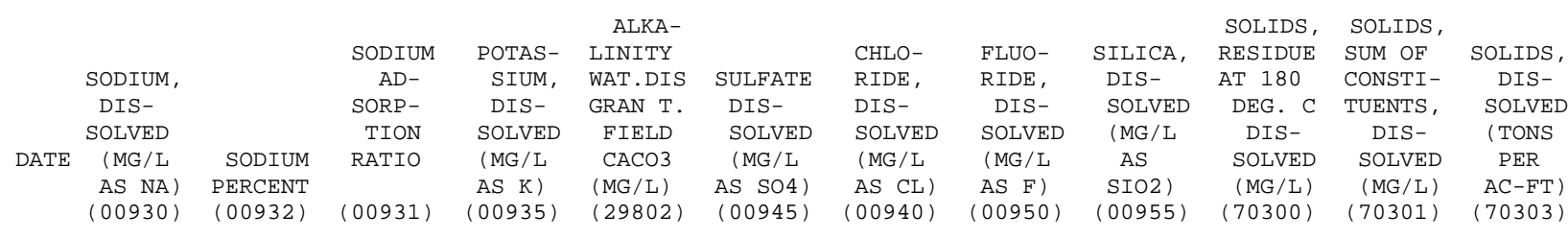

\begin{tabular}{|c|c|c|c|c|c|c|c|c|c|c|c|c|}
\hline \multicolumn{13}{|l|}{$\mathrm{OCT}$} \\
\hline 22 & $\ldots 6.3$ & 21 & .4 & 1 & 51 & 4.4 & 3.8 & $<.1$ & 16 & 90 & 82 & .12 \\
\hline \multicolumn{13}{|c|}{ NOV } \\
\hline 13 & ... 7.9 & 24 & .5 & 1.2 & 53 & 6.3 & 5.5 & $<.1$ & 17 & 98 & 91 & .13 \\
\hline \multicolumn{13}{|c|}{ DEC } \\
\hline 28 & ... 7.4 & 23 & .4 & 1.1 & 53 & 6.4 & 5.6 & $<.1$ & 18 & 93 & 92 & .13 \\
\hline \multicolumn{13}{|c|}{ JAN } \\
\hline 21 & $\ldots 3.8$ & 20 & .3 & .9 & 29 & 3.8 & 2.9 & $<.1$ & 13 & 64 & 58 & .09 \\
\hline \multicolumn{13}{|c|}{ FEB } \\
\hline 26 & ... 4.9 & 20 & .3 & 1.0 & 39 & 4.3 & 2.9 & $<.1$ & 16 & 75 & 71 & .10 \\
\hline \multicolumn{13}{|c|}{ MAR } \\
\hline 17 & $\ldots 6.0$ & 21 & .4 & 1.1 & 34 & 4.3 & 3.2 & $<.1$ & 18 & 80 & 69 & .11 \\
\hline \multicolumn{13}{|c|}{ APR } \\
\hline 09 & $\ldots 6.5$ & 20 & .4 & 1 & 48 & 5.5 & 4.3 & $<.1$ & 17 & 89 & 82 & .12 \\
\hline \multicolumn{13}{|c|}{ MAY } \\
\hline & $\ldots 6.3$ & 23 & .4 & .9 & 45 & 5.4 & 5.1 & $<.1$ & 16 & 79 & 75 & .11 \\
\hline \multicolumn{13}{|c|}{ JUN } \\
\hline 04 & .. 7.9 & 26 & .5 & .9 & 48 & 5.6 & 5.2 & $<.1$ & 16 & 101 & 80 & .14 \\
\hline \multicolumn{13}{|c|}{ JUL } \\
\hline 08 & $\ldots 6.6$ & 25 & .4 & .8 & 46 & 21 & 9.1 & $<.1$ & 16 & 114 & 95 & .16 \\
\hline \multicolumn{13}{|c|}{ AUG } \\
\hline 05 & $\ldots 7.5$ & 25 & .5 & .9 & 52 & 5.1 & 1.7 & $<.1$ & 16 & 90 & 78 & .12 \\
\hline \multicolumn{13}{|c|}{ SEP } \\
\hline \multirow[t]{4}{*}{14.} & .. 13 & 28 & .7 & 1.2 & 82 & 8.5 & 9.0 & $<.1$ & 19 & 124 & 124 & .17 \\
\hline & $\begin{array}{c}\text { NITRO- } \\
\text { GEN, } \\
\text { NITRITE }\end{array}$ & $\begin{array}{c}\text { NITRO- } \\
\text { GEN, } \\
\text { NO2+NO3 }\end{array}$ & $\begin{array}{l}\text { NITRO- } \\
\text { GEN, } \\
\text { AMMONIA }\end{array}$ & $\begin{array}{r}\text { NITRO- } \\
\text { GEN, AM- } \\
\text { MONIA + }\end{array}$ & $\begin{array}{l}\text { NITRO- } \\
\text { GEN, AM- } \\
\text { MONIA + }\end{array}$ & & $\begin{array}{l}\text { PHOS- } \\
\text { PHORUS }\end{array}$ & $\begin{array}{r}\text { PHOS- } \\
\text { PHORUS }\end{array}$ & & $\begin{array}{l}\text { MANGA- } \\
\text { NFSF }\end{array}$ & CARBON, & $\begin{array}{l}\text { CARBON, } \\
\text { ORGANIC }\end{array}$ \\
\hline & DIS- & DIS- & DIS- & ORGANIC & ORGANIC & PHORUS & DIS- & DIS- & $\begin{array}{l}\text { IRUN, } \\
\text { DIS- }\end{array}$ & DIS- & DIS- & PENDED \\
\hline & SOLVED & SOLVED & SOLVED & TOTAL & DIS. & TOTAL & SOLVED & SOLVED & SOLVED & SOLVED & SOLVED & TOTAL \\
\hline \multirow[t]{3}{*}{ DATE } & (MG/L & (MG / L & (MG/L & (MG / L & (MG / L & (MG/L & (MG / L & (MG/L & (UG/L & (UG/L & (MG / L & (MG / L \\
\hline & AS N) & AS N) & AS N) & AS N) & AS N) & AS P) & AS P) & AS P) & AS FE) & AS MN) & AS C) & AS C) \\
\hline & $(00613)$ & $(00631)$ & $(00608)$ & $(00625)$ & $(00623)$ & $(00665)$ & $(00666)$ & $(00671)$ & $(01046)$ & $(01056)$ & $(00681)$ & $(00689)$ \\
\hline
\end{tabular}

OCT

\begin{tabular}{|c|c|c|c|c|c|c|c|c|c|c|c|}
\hline \multicolumn{12}{|l|}{ OCT } \\
\hline $22 \ldots<.01$ & .16 & $<.02$ & .1 & $<.1$ & $<.05$ & .03 & .01 & $<10$ & $<3$ & 1.4 & .2 \\
\hline \multicolumn{12}{|l|}{ NOV } \\
\hline $13 \ldots .01$ & .21 & .03 & .2 & .2 & .05 & .03 & .04 & 12 & $<3$ & 2.1 & .3 \\
\hline \multicolumn{12}{|l|}{$\mathrm{DEC}$} \\
\hline $28 \ldots .02$ & .20 & $<.02$ & .2 & $<.1$ & .06 & .02 & .02 & 14 & 3 & 1.4 & .4 \\
\hline \multicolumn{12}{|l|}{ JAN } \\
\hline $21 \ldots<.01$ & .12 & .05 & .3 & .1 & .13 & $<.01$ & .01 & 11 & $<3$ & 2.8 & 1 \\
\hline \multicolumn{12}{|l|}{ FEB } \\
\hline $26 \ldots<.01$ & .14 & .02 & .3 & $<.1$ & .07 & .02 & .01 & 21 & 4 & 1.9 & .4 \\
\hline \multicolumn{12}{|l|}{ MAR } \\
\hline $17 \ldots<.01$ & .11 & $<.02$ & .2 & $<.1$ & .06 & .02 & .02 & 12 & 3 & 1.4 & -- \\
\hline \multicolumn{12}{|l|}{ APR } \\
\hline $09 \ldots<.01$ & .16 & $<.02$ & .2 & $<.1$ & .05 & .02 & .01 & $<10$ & $<3$ & 1.2 & .5 \\
\hline \multicolumn{12}{|l|}{ MAY } \\
\hline $17 \ldots<.01$ & .08 & $<.02$ & .1 & $<.1$ & .05 & .02 & .02 & $<10$ & 4 & 1.5 & .3 \\
\hline \multicolumn{12}{|l|}{ JUN } \\
\hline $04 \ldots<.01$ & $<.05$ & .02 & .2 & .1 & .06 & .03 & $<.01$ & 11 & $<3$ & 1.4 & .2 \\
\hline \multicolumn{12}{|l|}{ JUL } \\
\hline $08 \ldots<.01$ & .08 & $<.02$ & .2 & .1 & .05 & .02 & .03 & $<10$ & $<3$ & 1.4 & 1.0 \\
\hline \multicolumn{12}{|l|}{ AUG } \\
\hline $05 \ldots<.01$ & .06 & $<.02$ & .2 & $<.1$ & .05 & .03 & .03 & $<10$ & $<3$ & 1.5 & .3 \\
\hline \multicolumn{12}{|l|}{ SEP } \\
\hline $14 \ldots<.01$ & .07 & $<.02$ & .2 & .1 & .06 & .04 & .03 & 11 & e2 & 2.2 & .7 \\
\hline
\end{tabular}

$<$ Actual value known to be less than value shown. 


\section{SACRAMENTO RIVER AT FREEPORT, CA—Continued}

\section{WATER-QUALITY DATA, WATER YEAR OCTOBER 1998 TO SEPTEMBER 1999}

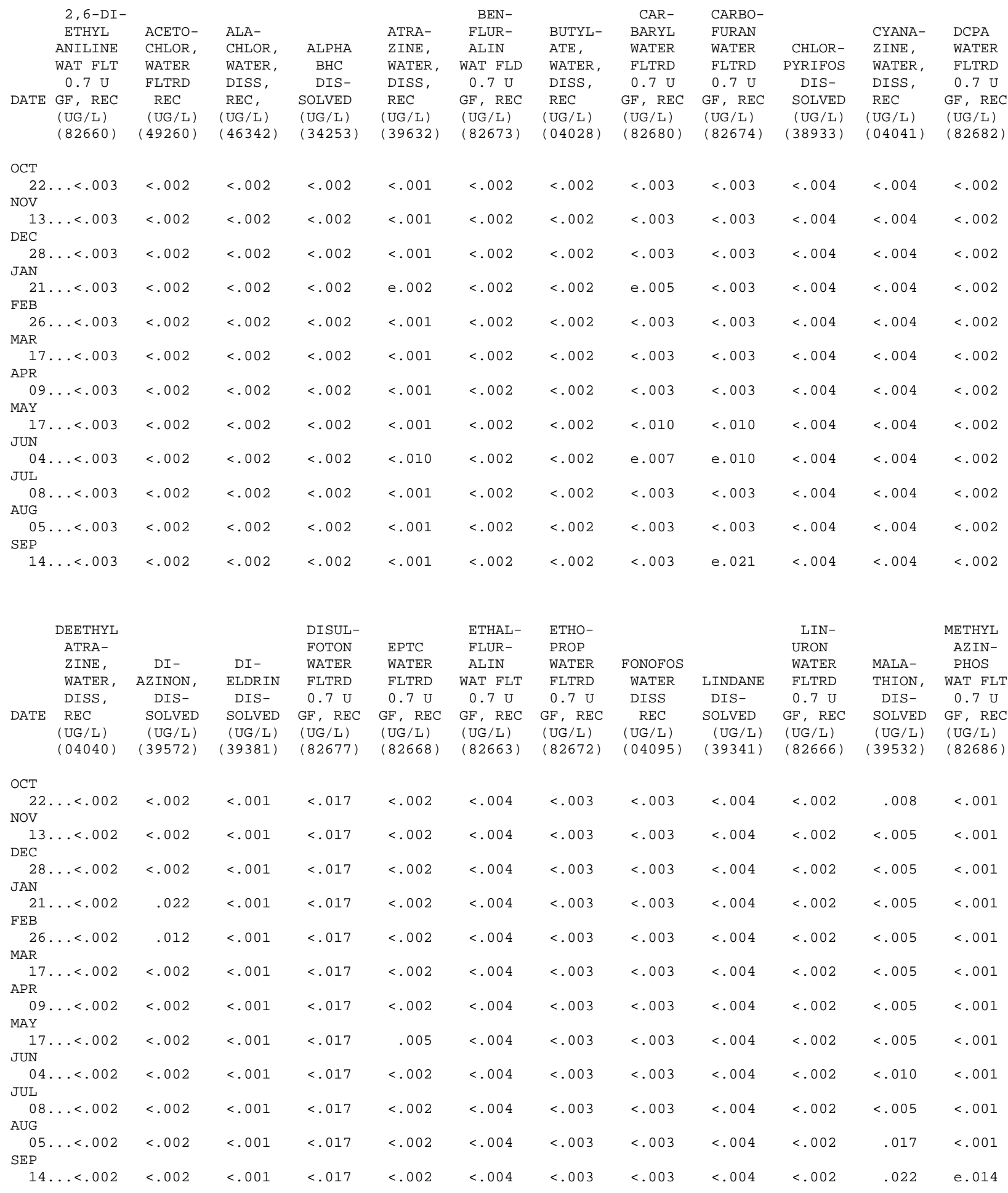

e Estimated.

$<$ Actual value known to be less than value shown. 
11447650 SACRAMENTO RIVER AT FREEPORT, CA—Continued

\section{WATER-QUALITY DATA, WATER YEAR OCTOBER 1998 TO SEPTEMBER 1999}

\begin{tabular}{|c|c|c|c|c|c|c|c|c|c|c|c|c|}
\hline & $\begin{array}{c}\text { METHYL } \\
\text { PARA- } \\
\text { THION }\end{array}$ & METO- & $\begin{array}{l}\text { METRI- } \\
\text { BUZIN }\end{array}$ & $\begin{array}{l}\text { MOL- } \\
\text { INATE } \\
\text { WATER }\end{array}$ & $\begin{array}{c}\text { NAPROP- } \\
\text { AMIDE } \\
\text { WATER }\end{array}$ & & PARA- & $\begin{array}{r}\text { PEB- } \\
\text { ULATE } \\
\text { WATER }\end{array}$ & $\begin{array}{l}\text { PENDI- } \\
\text { METH- } \\
\text { ALIN }\end{array}$ & $\begin{array}{c}\text { PER- } \\
\text { METHRIN } \\
\text { CIS }\end{array}$ & $\begin{array}{c}\text { PHORATE } \\
\text { WATER }\end{array}$ & $\begin{array}{l}\text { PRO- } \\
\text { METON, }\end{array}$ \\
\hline & WAT FLT & LACHLOR & SENCOR & FLTRD & FLTRD & $\mathrm{P}, \mathrm{P}^{\prime}$ & THION, & FILTRD & WAT FLT & WAT FLT & FLTRD & WATER, \\
\hline & $0.7 \mathrm{U}$ & WATER & WATER & $0.7 \mathrm{U}$ & $0.7 \mathrm{U}$ & DDE & DIS- & $0.7 \mathrm{U}$ & $0.7 \mathrm{U}$ & $0.7 \mathrm{U}$ & $0.7 \mathrm{U}$ & DISS, \\
\hline DATE & $\begin{array}{l}\text { GF, REC } \\
(\mathrm{UG} / \mathrm{L}) \\
(82667)\end{array}$ & $\begin{array}{l}\text { DISSOLV } \\
\text { (UG/L) } \\
(39415)\end{array}$ & $\begin{array}{l}\text { DISSOLV } \\
\text { (UG/L) } \\
(82630)\end{array}$ & $\begin{array}{l}\text { GF, REC } \\
(\mathrm{UG} / \mathrm{L}) \\
(82671)\end{array}$ & $\begin{array}{l}\text { GF, REC } \\
\text { (UG/L) } \\
(82684)\end{array}$ & $\begin{array}{l}\text { DISSOLV } \\
\text { (UG/L) } \\
(34653)\end{array}$ & $\begin{array}{r}\text { SOLVED } \\
(\text { UG/L) } \\
(39542)\end{array}$ & $\begin{array}{l}\text { GF, REC } \\
\text { (UG/L) } \\
(82669)\end{array}$ & $\begin{array}{l}\text { GE, REC } \\
\text { (UG/L) } \\
(82683)\end{array}$ & $\begin{array}{l}\text { GF, REC } \\
\text { (UG/L) } \\
(82687)\end{array}$ & $\begin{array}{l}\text { GF, REC } \\
\text { (UG/L) } \\
(82664)\end{array}$ & $\begin{array}{l}\text { REC } \\
(\mathrm{UG} / \mathrm{L}) \\
(04037)\end{array}$ \\
\hline \multicolumn{13}{|l|}{ OCT } \\
\hline 22 & $\ldots<.006$ & $<.002$ & $<.004$ & $<.004$ & $<.003$ & $<.006$ & $<.004$ & $<.004$ & $<.004$ & $<.005$ & $<.002$ & $<.018$ \\
\hline \multicolumn{13}{|c|}{ NOV } \\
\hline 13 & $\ldots<.006$ & $<.002$ & $<.004$ & .016 & $<.003$ & $<.006$ & $<.004$ & $<.004$ & $<.004$ & $<.005$ & $<.002$ & $<.018$ \\
\hline \multicolumn{13}{|c|}{$\mathrm{DEC}$} \\
\hline $\begin{array}{l}28 \\
\text { JAN }\end{array}$ & $\ldots<.006$ & $<.002$ & $<.004$ & $<.010$ & $<.003$ & $<.006$ & $<.004$ & $<.004$ & $<.004$ & $<.005$ & $<.002$ & $<.018$ \\
\hline 21 & $\ldots<.006$ & $<.002$ & $<.004$ & $<.004$ & $<.003$ & $<.006$ & $<.004$ & $<.004$ & $<.004$ & $<.005$ & $<.002$ & $<.018$ \\
\hline \multicolumn{13}{|c|}{ FEB } \\
\hline $\begin{array}{r}26 \\
\text { MAR }\end{array}$ & $\ldots<.006$ & $<.002$ & $<.004$ & $<.004$ & $<.003$ & $<.006$ & $<.004$ & $<.004$ & $<.004$ & $<.005$ & $<.002$ & $<.018$ \\
\hline 17 & $\ldots<.006$ & $<.002$ & $<.004$ & $<.004$ & $<.003$ & $<.006$ & $<.004$ & $<.004$ & $<.004$ & $<.005$ & $<.002$ & $<.018$ \\
\hline \multicolumn{13}{|c|}{$\mathrm{APR}$} \\
\hline 09 & $\ldots<.006$ & $<.002$ & $<.004$ & $<.004$ & $<.003$ & $<.006$ & $<.004$ & $<.004$ & $<.004$ & $<.005$ & $<.002$ & $<.018$ \\
\hline \multicolumn{13}{|c|}{ MAY } \\
\hline $\begin{array}{c}17 \\
\text { JUN }\end{array}$ & \multicolumn{12}{|c|}{ JUN } \\
\hline $\begin{array}{l}04 \\
\text { JUL }\end{array}$ & $\ldots<.006$ & .052 & $<.004$ & 1.34 & $<.003$ & $<.006$ & $<.004$ & $<.004$ & $<.004$ & $<.005$ & $<.002$ & $<.018$ \\
\hline 08 & $\ldots<.006$ & .005 & $<.004$ & .055 & $<.003$ & $<.006$ & $<.004$ & $<.004$ & $<.004$ & $<.005$ & $<.002$ & $<.018$ \\
\hline \multicolumn{13}{|c|}{ AUG } \\
\hline 05 & $\ldots<.006$ & .007 & $<.004$ & .023 & $<.003$ & $<.006$ & $<.004$ & $<.004$ & $<.004$ & $<.005$ & $<.002$ & $<.018$ \\
\hline \multicolumn{13}{|l|}{ SEP } \\
\hline 14 & $\ldots<.006$ & .005 & $<.004$ & .057 & $<.003$ & $<.006$ & $<.004$ & $<.004$ & $<.004$ & $<.005$ & $<.002$ & $<.018$ \\
\hline
\end{tabular}

\begin{tabular}{|c|c|c|c|c|c|c|c|c|c|c|c|}
\hline & $\begin{array}{l}\text { PRON- } \\
\text { AMIDE }\end{array}$ & $\begin{array}{r}\text { PRO- } \\
\text { PANIL }\end{array}$ & $\begin{array}{c}\text { PRO- } \\
\text { PARGITE }\end{array}$ & PROP- & SI- & $\begin{array}{l}\text { TEBU- } \\
\text { THIURON }\end{array}$ & $\begin{array}{r}\text { TER- } \\
\text { BACIL }\end{array}$ & $\begin{array}{r}\text { TER- } \\
\text { BUFOS }\end{array}$ & $\begin{array}{c}\text { THIO- } \\
\text { BENCARB }\end{array}$ & $\begin{array}{l}\text { TRIAL- } \\
\text { LATE }\end{array}$ & $\begin{array}{r}\text { TRI- } \\
\text { FLUR- }\end{array}$ \\
\hline & WATER & WATER & WATER & CHLOR, & MAZINE, & WATER & WATER & WATER & WATER & WATER & ALIN \\
\hline & FLTRD & FLTRD & FLTRD & WATER, & WATER, & FLTRD & FLTRD & FLTRD & FLTRD & FLTRD & WAT FLT \\
\hline & $0.7 \mathrm{U}$ & $0.7 \mathrm{U}$ & $0.7 \mathrm{U}$ & DISS, & DISS, & $0.7 \mathrm{U}$ & $0.7 \mathrm{U}$ & $0.7 \mathrm{U}$ & $0.7 \mathrm{U}$ & $0.7 \mathrm{U}$ & $0.7 \mathrm{U}$ \\
\hline DATE & $\begin{array}{l}\text { GF, REC } \\
(\mathrm{UG} / \mathrm{L}) \\
(82676)\end{array}$ & $\begin{array}{l}\text { GF, REC } \\
\text { (UG/L) } \\
(82679)\end{array}$ & $\begin{array}{l}\text { GF， REC } \\
\text { (UG/L) } \\
(82685)\end{array}$ & $\begin{array}{l}\text { REC } \\
(\mathrm{UG} / \mathrm{L}) \\
(04024)\end{array}$ & $\begin{array}{l}\text { REC } \\
(\mathrm{UG} / \mathrm{L}) \\
(04035)\end{array}$ & $\begin{array}{l}\text { GF, REC } \\
(\mathrm{UG} / \mathrm{L}) \\
(82670)\end{array}$ & $\begin{array}{l}\text { GF， REC } \\
\text { (UG/L) } \\
(82665)\end{array}$ & $\begin{array}{l}\text { GF， REC } \\
\text { (UG/L) } \\
(82675)\end{array}$ & $\begin{array}{l}\text { GF, REC } \\
(\mathrm{UG} / \mathrm{L}) \\
(82681)\end{array}$ & $\begin{array}{l}\text { GF， REC } \\
(\mathrm{UG} / \mathrm{L}) \\
(82678)\end{array}$ & $\begin{array}{l}\text { GF, REC } \\
\text { (UG/L) } \\
(82661)\end{array}$ \\
\hline \multicolumn{12}{|l|}{ OCT } \\
\hline $22 \ldots$ & $<.003$ & $<.004$ & $<.013$ & $<.007$ & .005 & $<.010$ & $<.007$ & $<.013$ & $<.002$ & $<.001$ & $<.002$ \\
\hline \multicolumn{12}{|l|}{ NOV } \\
\hline${ }_{\mathrm{DEC}}^{13 \ldots}$ & \multicolumn{10}{|c|}{$\mathrm{DEC}$} & $<.002$ \\
\hline$\underset{\text { JAN }}{28 \ldots}$ & $<.003$ & $<.004$ & $<.013$ & $<.007$ & $<.005$ & $<.010$ & $<.007$ & $<.013$ & $<.002$ & $<.001$ & $<.002$ \\
\hline$\underset{F E B}{21 \ldots}$ & $<.003$ & $<.004$ & $<.013$ & $<.007$ & .015 & $<.010$ & $<.007$ & $<.013$ & $<.002$ & $<.001$ & $<.002$ \\
\hline$\underset{\text { MAR }}{26 \ldots}$ & $<.003$ & $<.004$ & $<.013$ & $<.007$ & .012 & $<.010$ & $<.007$ & $<.013$ & $<.002$ & $<.001$ & $<.002$ \\
\hline $17 \ldots$ & $<.003$ & $<.004$ & $<.013$ & $<.007$ & $<.005$ & $<.010$ & $<.007$ & $<.013$ & $<.002$ & $<.001$ & $<.002$ \\
\hline \multicolumn{12}{|l|}{$\mathrm{APR}$} \\
\hline$\underset{M A Y}{0 .}$ & $<.003$ & $<.004$ & $<.013$ & $<.007$ & $<.005$ & $<.010$ & $<.007$ & $<.013$ & $<.002$ & $<.001$ & $<.002$ \\
\hline $\begin{array}{l}\text { MAY } \\
\quad 17 \ldots\end{array}$ & $<.003$ & $<.004$ & $<.013$ & $<.007$ & $<.005$ & $<.010$ & $<.007$ & $<.013$ & .071 & $<.001$ & $<.002$ \\
\hline \multicolumn{12}{|l|}{ JUN } \\
\hline $\begin{array}{l}04 \ldots \\
\text { JUL }\end{array}$ & \multicolumn{11}{|c|}{ JUL } \\
\hline $08 \ldots$ & $<.003$ & $<.004$ & $<.013$ & $<.007$ & $<.005$ & $<.010$ & $<.007$ & $<.013$ & .017 & $<.001$ & $<.002$ \\
\hline \multicolumn{12}{|l|}{ AUG } \\
\hline $05 \ldots$ & $<.003$ & .087 & $<.013$ & $<.007$ & $<.005$ & $<.010$ & $<.007$ & $<.013$ & .009 & $<.001$ & $<.002$ \\
\hline \multicolumn{12}{|l|}{ SEP } \\
\hline $14 \ldots$ & $<.003$ & $<.004$ & $<.013$ & $<.007$ & .016 & $<.010$ & $<.007$ & $<.013$ & .020 & $<.001$ & $<.002$ \\
\hline
\end{tabular}

$<$ Actual value known to be less than value shown. 
11447650 SACRAMENTO RIVER AT FREEPORT, CA—Continued

PARTICLE-SIZE DISTRIBUTION OF SUSPENDED SEDIMENT, WATER YEAR OCTOBER 1998 TO SEPTEMBER 1999

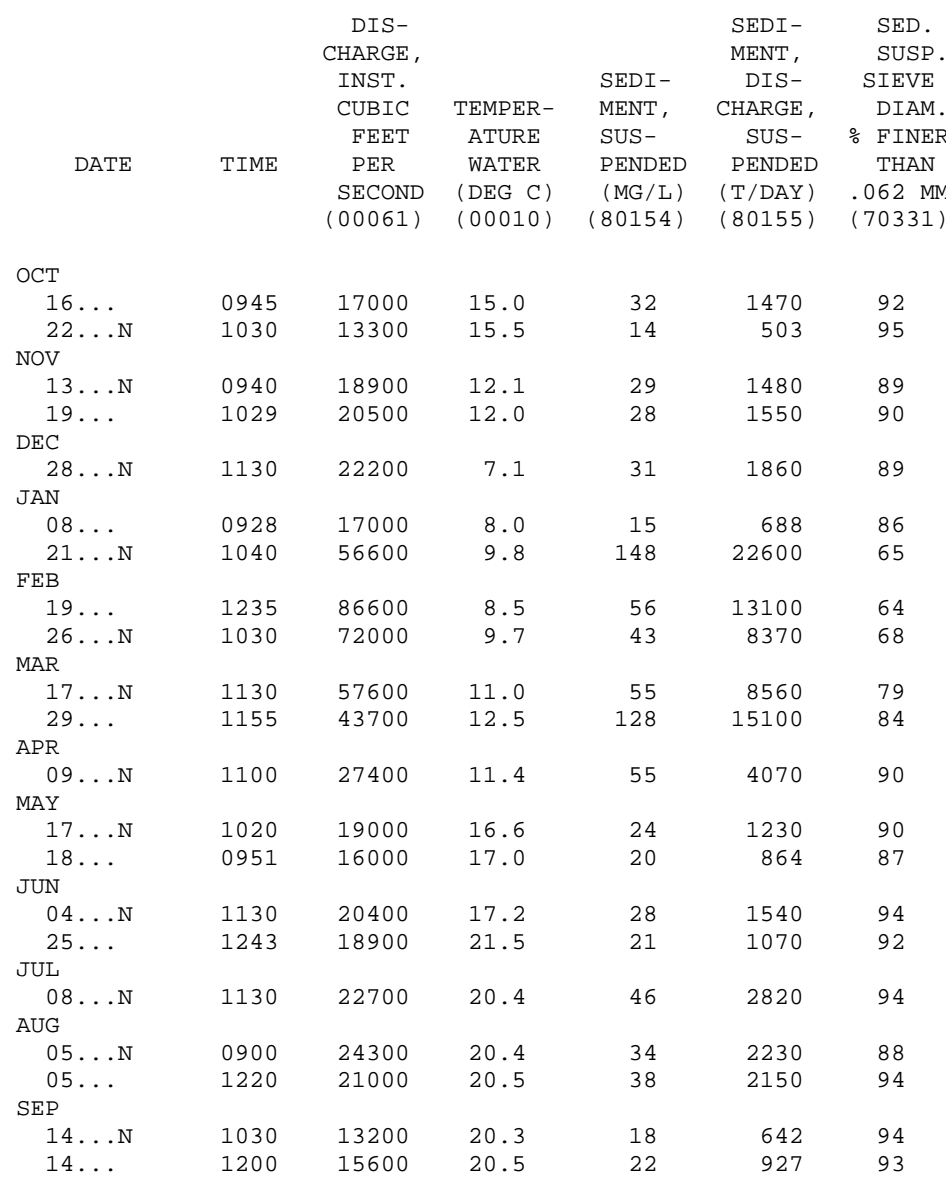

N Suspended-sediment concentration value determined from a sample collected and processed according to National Water-Quality Assessment (NAWQA) protocol. 
11447650 SACRAMENTO RIVER AT FREEPORT, CA—Continued

PARTICLE-SIZE DISTRIBUTION OF SURFACE BED MATERIAL, WATER YEAR OCTOBER 1998 TO SEPTEMBER 1999

\begin{tabular}{|c|c|c|c|c|c|c|c|}
\hline & & & DIS- & & BED & BED & BED \\
\hline & & NUMBER & CHARGE, & & MAT. & MAT. & MAT. \\
\hline & & $\mathrm{OF}$ & INST. & & SIEVE & SIEVE & SIEVE \\
\hline & & SAM- & CUBIC & TEMPER- & DIAM. & DIAM. & DIAM. \\
\hline & & PLING & FEET & ATURE & $\because$ FINER & $\because$ FINER & $\because$ FINER \\
\hline DATE & TIME & POINTS & PER & WATER & THAN & THAN & THAN \\
\hline & & (COUNT) & SECOND & $(\mathrm{DEG} C \mathrm{C})$ & $.062 \mathrm{MM}$ & $.125 \mathrm{MM}$ & $.250 \mathrm{MM}$ \\
\hline & & $(00063)$ & $(00061)$ & $(00010)$ & $(80164)$ & $(80165)$ & $(80166)$ \\
\hline FEB & & & & & & & \\
\hline $19 \ldots$ & 1146 & 1 & 86800 & 8.5 & -- & -- & 10 \\
\hline $19 \ldots$ & 1150 & 1 & 86800 & 8.5 & -- & -- & 2 \\
\hline $19 \ldots$ & 1156 & 1 & 86800 & 8.5 & -- & -- & 12 \\
\hline $19 \ldots$ & 1200 & 1 & 86800 & 8.5 & -- & -- & 7 \\
\hline $19 \ldots$ & 1204 & 1 & 86800 & 8.5 & -- & 1 & 46 \\
\hline AUG & & & & & & & \\
\hline $05 \ldots$ & 1255 & 1 & 20100 & 20.5 & 1 & 12 & 24 \\
\hline $05 \ldots$ & 1300 & 1 & 20100 & 20.5 & -- & -- & 1 \\
\hline $05 \ldots$ & 1305 & 1 & 20100 & 20.5 & 1 & 4 & 26 \\
\hline $05 \ldots$ & 1310 & 1 & 20100 & 20.5 & 1 & 4 & 18 \\
\hline $05 \ldots$ & 1315 & 1 & 20100 & 20.5 & -- & 1 & 20 \\
\hline & & $\mathrm{BED}$ & BED & $\mathrm{BED}$ & $\mathrm{BED}$ & $\mathrm{BED}$ & BED \\
\hline & & MAT. & MAT. & MAT. & MAT. & MAT. & MAT. \\
\hline & & SIEVE & SIEVE & SIEVE & SIEVE & SIEVE & SIEVE \\
\hline & & DIAM. & DIAM. & DIAM. & DIAM. & DIAM. & DIAM. \\
\hline & & $\because$ FINER & $\because$ FINER & $\because$ FINER & \% FINER & \% FINER & \% FINER \\
\hline DATE & TIME & THAN & THAN & THAN & THAN & THAN & THAN \\
\hline & & $.500 \mathrm{MM}$ & $1.00 \mathrm{MM}$ & $2.00 \mathrm{MM}$ & $4.00 \mathrm{MM}$ & $8.00 \mathrm{MM}$ & $16.0 \mathrm{MM}$ \\
\hline & & $(80167)$ & $(80168)$ & $(80169)$ & $(80170)$ & $(80171)$ & $(80172)$ \\
\hline $\mathrm{FEB}$ & & & & & & & \\
\hline $19 \ldots$ & 1146 & 78 & 96 & 99 & 100 & -- & -- \\
\hline $19 \ldots$ & 1150 & 50 & 91 & 98 & 100 & -- & -- \\
\hline $19 \ldots$ & 1156 & 73 & 98 & 100 & -- & -- & -- \\
\hline $19 \ldots$ & 1200 & 59 & 83 & 94 & 99 & 100 & -- \\
\hline $19 \ldots$ & 1204 & 100 & -- & -- & -- & -- & -- \\
\hline AUG & & & & & & & \\
\hline $05 \ldots$ & 1255 & 53 & 95 & 99 & 100 & -- & -- \\
\hline $05 \ldots$ & 1300 & 50 & 96 & 100 & -- & -- & -- \\
\hline $05 \ldots$ & 1305 & 70 & 91 & 94 & 96 & 99 & 100 \\
\hline $05 \ldots$ & 1310 & 87 & 99 & 100 & -- & -- & -- \\
\hline $05 \ldots$ & 1315 & 93 & 100 & -- & -- & -- & -- \\
\hline
\end{tabular}


11447650 SACRAMENTO RIVER AT FREEPORT, CA—Continued

\section{TEMPERATURE, WATER (DEG. C), WATER YEAR OCTOBER 1998 TO SEPTEMBER 1999}

\begin{tabular}{|c|c|c|c|c|c|c|c|c|c|c|c|c|}
\hline DAY & MAX & MIN & MAX & MIN & MAX & MIN & MAX & MIN & MAX & MIN & MAX & MIN \\
\hline & \multicolumn{2}{|c|}{ OCTOBER } & \multicolumn{2}{|c|}{ NOVEMBER } & \multicolumn{2}{|c|}{ DECEMBER } & \multicolumn{2}{|c|}{ JANUARY } & \multicolumn{2}{|c|}{ FEBRUARY } & \multicolumn{2}{|c|}{ MARCH } \\
\hline 1 & 17.0 & 17.0 & 14.0 & 13.5 & 11.5 & 11.0 & 9.0 & 8.5 & 9.0 & 9.0 & 10.5 & 10.5 \\
\hline 2 & 17.0 & 16.5 & 14.0 & 13.5 & 11.0 & 11.0 & 9.0 & 8.5 & 9.0 & 8.5 & 11.0 & 10.5 \\
\hline 3 & 17.0 & 16.5 & 14.0 & 13.5 & 11.0 & 11.0 & 9.0 & 9.0 & 9.0 & 9.0 & 11.0 & 11.0 \\
\hline 4 & 16.5 & 16.0 & 14.0 & 13.5 & 11.0 & 10.5 & 9.0 & 8.5 & 9.5 & 9.0 & 11.0 & 11.0 \\
\hline 5 & 16.5 & 16.0 & 14.0 & 13.5 & 10.5 & 10.0 & 8.5 & 8.5 & 9.5 & 9.5 & 11.0 & 10.5 \\
\hline 6 & 16.5 & 16.0 & 13.5 & 13.0 & 10.0 & 9.0 & 8.5 & 8.5 & 9.5 & 9.0 & 10.5 & 10.5 \\
\hline 7 & 16.5 & 16.0 & 13.0 & 12.5 & 9.0 & 8.5 & 8.5 & 8.0 & 9.5 & 9.0 & 10.5 & 10.5 \\
\hline 8 & 16.5 & 16.0 & 12.5 & 12.5 & 9.0 & 8.5 & 8.0 & 8.0 & 9.5 & 9.5 & 10.5 & 10.0 \\
\hline 9 & 16.5 & 16.0 & 12.5 & 12.0 & 9.0 & 9.0 & 8.0 & 8.0 & 10.0 & 9.5 & 10.0 & 9.5 \\
\hline 10 & 16.5 & 16.0 & 12.0 & 11.5 & 9.0 & 9.0 & 8.0 & 7.5 & 9.5 & 9.0 & 9.5 & 9.5 \\
\hline 11 & 16.0 & 16.0 & 12.0 & 11.5 & 9.0 & 9.0 & 8.0 & 7.5 & 9.5 & 9.0 & 9.5 & 9.5 \\
\hline 12 & 16.0 & 16.0 & 12.0 & 11.5 & 9.5 & 9.0 & 7.5 & 7.5 & 9.5 & 9.0 & 10.0 & 9.5 \\
\hline 13 & 16.0 & 15.5 & 12.0 & 11.5 & 10.0 & 9.5 & 8.0 & 7.5 & 9.0 & 9.0 & 10.5 & 10.0 \\
\hline 14 & 16.0 & 15.5 & 12.0 & 11.5 & 10.0 & 10.0 & 8.5 & 8.0 & 9.0 & 9.0 & 10.5 & 10.5 \\
\hline 15 & 16.0 & 15.5 & 12.0 & 12.0 & 10.5 & 10.0 & 9.0 & 8.5 & 9.0 & 9.0 & 10.5 & 10.5 \\
\hline 16 & 15.5 & 14.5 & 12.0 & 12.0 & 10.5 & 10.5 & 10.0 & 9.0 & 9.0 & 9.0 & 11.0 & 10.5 \\
\hline 17 & 14.5 & 14.5 & 12.0 & 12.0 & 10.5 & 10.5 & 10.5 & 10.0 & 9.5 & 9.0 & 11.0 & 11.0 \\
\hline 18 & 14.5 & 14.0 & 12.0 & 12.0 & 10.5 & 10.5 & 11.0 & 10.5 & 9.5 & 9.5 & 11.5 & 11.0 \\
\hline 19 & 14.5 & 14.5 & 12.0 & 12.0 & 10.5 & 10.0 & 11.5 & 11.0 & 9.5 & 9.5 & 12.0 & 11.0 \\
\hline 20 & 15.0 & 14.5 & 12.0 & 11.5 & 10.0 & 9.5 & 11.5 & 11.0 & 10.0 & 9.5 & 12.0 & 11.5 \\
\hline 21 & 15.0 & 14.5 & 12.0 & 12.0 & 9.5 & 8.0 & 11.0 & 10.5 & 9.5 & 9.5 & 12.0 & 11.5 \\
\hline 22 & 15.5 & 15.0 & 12.5 & 12.0 & 8.0 & 7.5 & 10.5 & 10.5 & 9.5 & 9.5 & 12.0 & 11.5 \\
\hline 23 & 15.5 & 15.0 & 12.5 & 12.5 & 7.5 & 6.5 & 10.5 & 10.0 & 10.0 & 9.5 & 12.0 & 11.5 \\
\hline 24 & 15.5 & 15.0 & 12.5 & 12.0 & 6.5 & 6.5 & 10.0 & 9.5 & 10.5 & 10.0 & 12.0 & 11.5 \\
\hline 25 & 15.0 & 14.5 & 12.5 & 12.0 & 6.5 & 6.5 & 9.5 & 9.5 & 10.5 & 10.5 & 11.5 & 11.5 \\
\hline 26 & 15.0 & 14.5 & 12.0 & 11.5 & 7.0 & 6.5 & 9.5 & 9.0 & 10.5 & 10.0 & 12.0 & 11.5 \\
\hline 27 & 15.0 & 15.0 & 12.0 & 11.5 & 7.5 & 7.0 & 9.0 & 8.5 & 10.5 & 10.0 & 12.0 & 11.5 \\
\hline 28 & 15.0 & 14.5 & 12.0 & 11.5 & 8.0 & 7.5 & 9.0 & 8.5 & 10.5 & 10.5 & 12.0 & 11.5 \\
\hline 29 & 15.0 & 14.5 & 12.0 & 11.5 & 8.5 & 7.5 & 9.0 & 8.5 & --- & --- & 12.5 & 12.0 \\
\hline 30 & 14.5 & 14.5 & 11.5 & 11.5 & 8.5 & 8.0 & 9.0 & 8.5 & --- & --- & 12.0 & 11.5 \\
\hline 31 & 14.5 & 14.0 & --- & --- & 9.0 & 8.5 & 9.0 & 8.5 & --- & --- & 12.0 & 11.5 \\
\hline MONTH & 17.0 & 14.0 & 14.0 & 11.5 & 11.5 & 6.5 & 11.5 & 7.5 & 10.5 & 8.5 & 12.5 & 9.5 \\
\hline \multirow[t]{2}{*}{ DAY } & MAX & MIN & MAX & MIN & MAX & MIN & MAX & MIN & MAX & MIN & MAX & MIN \\
\hline & \multicolumn{2}{|c|}{ APRIL } & \multicolumn{2}{|c|}{ MAY } & & & & & & & SEI & MBER \\
\hline 1 & 11.5 & 11.5 & 16.0 & 15.0 & 19.5 & 19.0 & 22.5 & 21.5 & 20.5 & 19.0 & 20.5 & 20.0 \\
\hline 2 & 11.5 & 11.5 & 15.5 & 15.0 & 19.0 & 18.0 & 22.0 & 21.0 & 20.5 & 19.5 & 20.0 & 19.5 \\
\hline 3 & 11.5 & 11.0 & 15.0 & 14.5 & 18.0 & 17.5 & 21.0 & 20.0 & 21.0 & 19.5 & 19.5 & 19.5 \\
\hline 4 & 11.5 & 11.0 & 15.0 & 14.0 & 18.0 & 17.0 & 20.5 & 19.5 & 21.0 & 20.0 & 20.0 & 19.5 \\
\hline 5 & 11.0 & 11.0 & 16.0 & 15.0 & 18.0 & 17.0 & 20.0 & 19.5 & 21.0 & 20.0 & 20.0 & 19.5 \\
\hline 6 & 11.0 & 10.5 & 16.5 & 15.5 & 18.0 & 17.0 & 20.5 & 19.5 & 21.0 & 20.0 & 20.5 & 20.0 \\
\hline 7 & 11.0 & 10.5 & 17.0 & 16.0 & 18.0 & 17.5 & 20.5 & 19.5 & 20.5 & 19.5 & 20.5 & 20.0 \\
\hline 8 & 11.5 & 11.0 & 17.0 & 16.0 & 18.5 & 18.0 & 20.5 & 19.5 & 20.0 & 19.5 & 20.5 & 20.5 \\
\hline 9 & 11.5 & 11.0 & 16.5 & 16.0 & 19.0 & 18.0 & 20.5 & 19.5 & 20.0 & 19.5 & 21.0 & 20.5 \\
\hline 10 & 12.0 & 11.0 & 16.5 & 15.5 & 19.5 & 18.5 & 21.0 & 20.0 & 20.0 & 19.5 & 21.0 & 20.5 \\
\hline 11 & 11.5 & 11.0 & 16.5 & 15.5 & 20.0 & 19.0 & 21.0 & 20.5 & 20.0 & 19.5 & 20.5 & 20.5 \\
\hline 12 & 12.0 & 11.5 & 17.5 & 16.5 & 20.0 & 19.5 & 21.5 & 21.0 & 20.0 & 19.5 & 20.5 & 20.5 \\
\hline 13 & 13.0 & 12.0 & 18.0 & 17.0 & 20.0 & 19.5 & 22.0 & 21.0 & 20.0 & 20.0 & 20.5 & 20.5 \\
\hline 14 & 13.5 & 12.5 & 18.0 & 17.0 & 20.5 & 20.0 & 21.5 & 21.0 & 20.5 & 20.0 & 20.5 & 20.5 \\
\hline 15 & 14.5 & 13.0 & 17.5 & 17.0 & 20.5 & 20.0 & 21.5 & 20.5 & 21.0 & 20.0 & 20.5 & 20.5 \\
\hline 16 & 15.0 & 14.5 & 17.5 & 17.0 & 20.5 & 20.0 & 21.0 & 20.0 & 21.0 & 20.5 & 21.0 & 20.5 \\
\hline 17 & 15.5 & 15.0 & 17.5 & 16.5 & 21.0 & 20.5 & 20.0 & 19.5 & 21.5 & 21.0 & 21.0 & 20.5 \\
\hline 18 & 16.0 & 15.5 & 17.5 & 17.0 & 21.5 & 21.0 & 20.0 & 19.0 & 21.5 & 21.0 & 20.5 & 20.0 \\
\hline 19 & 16.0 & 15.5 & 18.0 & 17.0 & 21.5 & 21.0 & 19.5 & 19.0 & 21.5 & 21.0 & 20.0 & 19.5 \\
\hline 20 & 16.0 & 16.0 & 18.0 & 17.5 & 21.5 & 21.0 & 19.5 & 19.0 & 21.5 & 21.0 & 19.5 & 19.5 \\
\hline 21 & 16.0 & 15.5 & 18.5 & 17.5 & 21.5 & 21.0 & 19.5 & 18.5 & 21.5 & 21.5 & 20.0 & 19.5 \\
\hline 22 & 16.0 & 16.0 & 19.0 & 18.0 & 22.0 & 21.0 & 20.0 & 18.5 & 22.0 & 21.5 & --- & --- \\
\hline 23 & 16.0 & 15.5 & 19.5 & 18.5 & 22.0 & 21.5 & 20.0 & 19.0 & 22.5 & 22.0 & --- & --- \\
\hline 24 & 16.0 & 15.5 & 19.5 & 19.0 & 22.0 & 21.5 & 20.0 & 19.0 & 22.5 & 22.0 & --- & --- \\
\hline 25 & 16.0 & 15.5 & 19.5 & 19.0 & 22.0 & 21.0 & 20.0 & 19.0 & 22.5 & 22.0 & --- & --- \\
\hline 26 & 15.5 & 15.0 & 20.0 & 19.0 & 22.0 & 21.0 & 20.5 & 19.0 & 22.5 & 22.0 & --- & --- \\
\hline 27 & 15.5 & 15.0 & 20.0 & 19.5 & 22.0 & 21.0 & 20.5 & 19.5 & 22.0 & 21.5 & --- & --- \\
\hline 28 & 15.5 & 15.0 & 20.0 & 19.5 & 22.0 & 21.0 & 20.0 & 19.0 & 21.5 & 21.0 & --- & --- \\
\hline 29 & 15.0 & 14.5 & 20.0 & 19.5 & 22.0 & 21.5 & 20.0 & 19.0 & 21.5 & 21.0 & 19.5 & 19.0 \\
\hline 30 & 15.5 & 14.5 & 20.0 & 19.0 & 22.5 & 21.5 & 20.0 & 19.0 & 21.0 & 20.5 & 19.0 & 18.5 \\
\hline 31 & --- & --- & 20.0 & 19.0 & --- & --- & 20.0 & 19.0 & 21.0 & 20.5 & --- & -- \\
\hline MONTH & 16.0 & 10.5 & 20.0 & 14.0 & 22.5 & 17.0 & 22.5 & 18.5 & 22.5 & 19.0 & --- & --- \\
\hline
\end{tabular}


11447650 SACRAMENTO RIVER AT FREEPORT, CA—Continued

\section{SEDIMENT DISCHARGE, SUSPENDED (TONS/DAY), WATER YEAR OCTOBER 1998 TO SEPTEMBER 1999}

\begin{tabular}{|c|c|c|c|c|c|c|c|c|c|}
\hline \multirow{3}{*}{ DAY } & \multicolumn{3}{|c|}{ MEAN } & \multicolumn{3}{|c|}{ MEAN } & \multicolumn{3}{|c|}{ MEAN } \\
\hline & $\begin{array}{c}\text { MEAN } \\
\text { DISCHARGE } \\
\text { (CFS) }\end{array}$ & $\begin{array}{l}\text { CONCEN- } \\
\text { TRATION } \\
(\mathrm{MG} / \mathrm{L})\end{array}$ & $\begin{array}{l}\text { SEDIMENT } \\
\text { DISCHARGE } \\
\text { (TONS/DAY) }\end{array}$ & $\begin{array}{c}\text { MEAN } \\
\text { DISCHARGE } \\
(\mathrm{CFS})\end{array}$ & $\begin{array}{l}\text { CONCEN- } \\
\text { TRATION } \\
(\mathrm{MG} / \mathrm{L})\end{array}$ & $\begin{array}{l}\text { SEDIMENT } \\
\text { DISCHARGE } \\
\text { (TONS/DAY) }\end{array}$ & $\begin{array}{c}\text { MEAN } \\
\text { DISCHARGE } \\
\text { (CFS) }\end{array}$ & $\begin{array}{l}\text { CONCEN- } \\
\text { TRATION } \\
(M G / L)\end{array}$ & $\begin{array}{l}\text { SEDIMENT } \\
\text { DISCHARGE } \\
\text { (TONS / DAY) }\end{array}$ \\
\hline & \multicolumn{3}{|c|}{ OCTOBER } & \multicolumn{3}{|c|}{ NOVEMBER } & \multicolumn{3}{|c|}{ DECEMBER } \\
\hline 1 & 22900 & 29 & 1780 & e13900 & 11 & 426 & 37200 & 105 & 10600 \\
\hline 2 & 22200 & 27 & 1590 & e14300 & 11 & 425 & 43300 & 199 & 23300 \\
\hline 3 & 21500 & 27 & 1560 & e14100 & 11 & 407 & 49400 & 204 & 27200 \\
\hline 4 & 20300 & 28 & 1530 & 13200 & 10 & 369 & 54700 & 190 & 28000 \\
\hline 5 & 20000 & 29 & 1570 & 13200 & 10 & 362 & 58300 & 147 & 23100 \\
\hline 6 & 19000 & 29 & 1470 & 13200 & 12 & 413 & 60700 & 150 & 24500 \\
\hline 7 & 18200 & 23 & 1150 & 13200 & 14 & 492 & 61900 & 122 & 20300 \\
\hline 8 & 18000 & 20 & 953 & 14500 & 16 & 643 & 62300 & 106 & 17900 \\
\hline 9 & 16800 & 20 & 929 & 15100 & 19 & 791 & 61900 & 102 & 17000 \\
\hline 10 & 16300 & 22 & 966 & 15800 & 21 & 901 & 60700 & 83 & 13600 \\
\hline 11 & 15800 & 24 & 1000 & 16600 & 22 & 1010 & 59400 & 69 & 11100 \\
\hline 12 & 15500 & 25 & 1060 & 16800 & 24 & 1080 & 57600 & 63 & 9740 \\
\hline 13 & 15100 & 27 & 1100 & 17100 & 25 & 1180 & 55700 & 60 & 9080 \\
\hline 14 & 14400 & 29 & 1130 & 17200 & 27 & 1250 & 54200 & 59 & 8590 \\
\hline 15 & 14100 & 31 & 1180 & 17400 & 27 & 1290 & 53100 & 57 & 8140 \\
\hline 16 & 13900 & 32 & 1190 & 18000 & 28 & 1350 & 51900 & 53 & 7480 \\
\hline 17 & 13500 & 29 & 1070 & 18600 & 28 & 1420 & 49300 & 50 & 6680 \\
\hline 18 & 13300 & 27 & 969 & 19200 & 29 & 1480 & 45300 & 54 & 6600 \\
\hline 19 & 13100 & 25 & 880 & 19800 & 28 & 1500 & 42100 & 57 & 6500 \\
\hline 20 & 13100 & 23 & 811 & 22400 & 33 & 1990 & 40000 & 60 & 6470 \\
\hline 21 & 12900 & 21 & 736 & 23500 & 42 & 2640 & 38400 & 61 & 6310 \\
\hline 22 & 12700 & 20 & 669 & 24200 & 53 & 3440 & 36300 & 48 & 4710 \\
\hline 23 & 12700 & 18 & 616 & 24400 & 66 & 4380 & 34000 & 43 & 3910 \\
\hline 24 & 13000 & 17 & 582 & 28500 & 82 & 6320 & 31500 & 40 & 3370 \\
\hline 25 & 13400 & 15 & 553 & 31900 & 86 & 7400 & 29000 & 37 & 2900 \\
\hline 26 & 13700 & 14 & 521 & 34600 & 87 & 8120 & 26900 & 35 & 2510 \\
\hline 27 & 14200 & 13 & 501 & 33900 & 88 & 8050 & 25600 & 32 & 2200 \\
\hline 28 & 15400 & 13 & 527 & 33400 & 89 & 8040 & 24900 & 29 & 1950 \\
\hline 29 & e14400 & 12 & 481 & 34800 & 91 & 8590 & 24200 & 26 & 1700 \\
\hline 30 & e14700 & 12 & 478 & 34800 & 94 & 8840 & 23400 & 24 & 1500 \\
\hline 31 & e14400 & 12 & 455 & --- & --- & --- & 22400 & 21 & 1260 \\
\hline TOTAL & 488500 & --- & 30007 & 627600 & --- & 84599 & 1375600 & --- & 318200 \\
\hline
\end{tabular}

JANUARY

FEBRUARY

MARCH

\begin{tabular}{|c|c|}
\hline 1 & 21600 \\
\hline 2 & 21000 \\
\hline 3 & 20900 \\
\hline 4 & 20600 \\
\hline 5 & 20500 \\
\hline 6 & 20200 \\
\hline 7 & 20100 \\
\hline 8 & 19700 \\
\hline 9 & 19200 \\
\hline 10 & 18400 \\
\hline 11 & 18000 \\
\hline 12 & 17900 \\
\hline 13 & 17900 \\
\hline 14 & 17800 \\
\hline 15 & 17400 \\
\hline 16 & 17600 \\
\hline 17 & 18300 \\
\hline 18 & 20100 \\
\hline 19 & 23600 \\
\hline 20 & 36400 \\
\hline 21 & 56800 \\
\hline 22 & 61700 \\
\hline 23 & 63100 \\
\hline 24 & 66700 \\
\hline 25 & 67500 \\
\hline 26 & 65700 \\
\hline 27 & 63700 \\
\hline 28 & 60200 \\
\hline 29 & 56200 \\
\hline 30 & 52000 \\
\hline 31 & 48800 \\
\hline TOTAL & 1069600 \\
\hline
\end{tabular}

\begin{tabular}{|c|c|}
\hline 21 & 1240 \\
\hline 23 & 1300 \\
\hline 25 & 1400 \\
\hline 26 & 1470 \\
\hline 25 & 1410 \\
\hline 25 & 1350 \\
\hline 22 & 1180 \\
\hline 18 & 949 \\
\hline 20 & 1040 \\
\hline 20 & 994 \\
\hline 19 & 946 \\
\hline 14 & 683 \\
\hline 11 & 552 \\
\hline 11 & 550 \\
\hline 12 & 560 \\
\hline 12 & 582 \\
\hline 17 & 829 \\
\hline 25 & 1330 \\
\hline 42 & 2650 \\
\hline 99 & 9690 \\
\hline 121 & 18500 \\
\hline 135 & 22500 \\
\hline 143 & 24400 \\
\hline 116 & 20800 \\
\hline 94 & 17100 \\
\hline 100 & 17800 \\
\hline 80 & 13800 \\
\hline 61 & 9850 \\
\hline 55 & 8290 \\
\hline 54 & 7620 \\
\hline 55 & 7200 \\
\hline--- & 198565 \\
\hline
\end{tabular}

44900
41600
37900
34800
33900
35300
48400
60300
69200
83100

85400
82500
78700
75200
72400
71200
74800
86700
85600
81300
80000
79400
76600
74000
72600
71900
71300
71100
---
---
---
1880100

\begin{tabular}{|c|c|}
\hline 55 & 6620 \\
\hline 50 & 5610 \\
\hline 43 & 4390 \\
\hline 44 & 4160 \\
\hline 49 & 4490 \\
\hline 53 & 5060 \\
\hline 104 & 13600 \\
\hline 120 & 19600 \\
\hline 169 & 31600 \\
\hline 163 & 36500 \\
\hline 105 & 24200 \\
\hline 96 & 21400 \\
\hline 88 & 18600 \\
\hline 79 & 16000 \\
\hline 71 & 13900 \\
\hline 64 & 12300 \\
\hline 58 & 11700 \\
\hline 59 & 13700 \\
\hline 60 & 13800 \\
\hline 60 & 13100 \\
\hline 56 & 12000 \\
\hline 52 & 11100 \\
\hline 49 & 10100 \\
\hline 49 & 9770 \\
\hline 49 & 9580 \\
\hline 47 & 9200 \\
\hline 47 & 8990 \\
\hline 46 & 8910 \\
\hline--- & --- \\
\hline--- & --- \\
\hline--- & --- \\
\hline--- & 369980 \\
\hline
\end{tabular}

71800
73400
74000
74700
74700
74600
72900
71300
70600
69300
68200
67700
66600
65600
63900
61000
57200
53000
49200
46600
43500
40900
38300
35800
34000
36000
41400
43200
43100
41300
38200
1762000

\begin{tabular}{rr}
46 & 8870 \\
43 & 8520 \\
52 & 10400 \\
56 & 11300 \\
51 & 10200 \\
45 & 8990 \\
39 & 7760 \\
36 & 6910 \\
47 & 8960 \\
35 & 6610 \\
& \\
40 & 7420 \\
46 & 8390 \\
47 & 8460 \\
48 & 8500 \\
49 & 8440 \\
50 & 8220 \\
51 & 7900 \\
56 & 8000 \\
60 & 7930 \\
63 & 7940 \\
& \\
67 & 7830 \\
68 & 7510 \\
51 & 5290 \\
46 & 4460 \\
40 & 3640 \\
77 & 7450 \\
122 & 13600 \\
121 & 14100 \\
120 & 14000 \\
86 & 9540 \\
74 & 7600 \\
& \\
--- & 264740 \\
& \\
\hline 7
\end{tabular}

e Estimated. 
11447650 SACRAMENTO RIVER AT FREEPORT, CA—Continued

\section{SEDIMENT DISCHARGE, SUSPENDED (TONS/DAY), WATER YEAR OCTOBER 1998 TO SEPTEMBER 1999}

\begin{tabular}{|c|c|c|c|c|c|c|c|c|c|}
\hline \multirow[b]{2}{*}{ DAY } & \multicolumn{3}{|c|}{ MEAN } & \multicolumn{3}{|c|}{ MEAN } & \multicolumn{3}{|c|}{ MEAN } \\
\hline & $\begin{array}{c}\text { MEAN } \\
\text { DISCHARGE } \\
\text { (CFS) }\end{array}$ & $\begin{array}{l}\text { CONCEN- } \\
\text { TRATION } \\
\text { (MG/L) }\end{array}$ & $\begin{array}{l}\text { SEDIMENT } \\
\text { DISCHARGE } \\
\text { (TONS/DAY) }\end{array}$ & $\begin{array}{c}\text { MEAN } \\
\text { DISCHARGE } \\
\text { (CFS) }\end{array}$ & $\begin{array}{l}\text { CONCEN- } \\
\text { TRATION } \\
\text { (MG/L) }\end{array}$ & $\begin{array}{l}\text { SEDIMENT } \\
\text { DISCHARGE } \\
\text { (TONS/DAY) }\end{array}$ & $\begin{array}{c}\text { MEAN } \\
\text { DISCHARGE } \\
\text { (CFS) }\end{array}$ & $\begin{array}{l}\text { CONCEN- } \\
\text { TRATION } \\
\text { (MG/L) }\end{array}$ & $\begin{array}{l}\text { SEDIMENT } \\
\text { DISCHARGE } \\
\text { (TONS/DAY) }\end{array}$ \\
\hline & & APRI & & & MAY & & & JUNE & \\
\hline 1 & 34300 & 69 & 6380 & 24900 & 45 & 3040 & 19800 & 40 & 2140 \\
\hline 2 & 31400 & 64 & 5470 & 24800 & 47 & 3160 & 19400 & 40 & 2100 \\
\hline 3 & 29800 & 60 & 4860 & 24400 & 49 & 3260 & 20000 & 40 & 2160 \\
\hline 4 & 28600 & 57 & 4360 & 24800 & 50 & 3370 & 20200 & 40 & 2180 \\
\hline 5 & 27700 & 53 & 3960 & 24400 & 41 & 2720 & 21200 & 40 & 2290 \\
\hline 6 & 27700 & 50 & 3700 & 23800 & 42 & 2700 & 21800 & 40 & 2350 \\
\hline 7 & 27300 & 46 & 3400 & 22500 & 43 & 2600 & 21500 & 40 & 2320 \\
\hline 8 & 27500 & 44 & 3270 & 21300 & 43 & 2500 & 20400 & 40 & 2200 \\
\hline 9 & 28200 & 47 & 3570 & 21400 & 44 & 2550 & 18700 & 39 & 1980 \\
\hline 10 & 28300 & 51 & 3860 & 21200 & 45 & 2550 & 17600 & 38 & 1810 \\
\hline 11 & 29400 & 53 & 4180 & 20500 & 42 & 2300 & 16400 & 37 & 1640 \\
\hline 12 & 30700 & 54 & 4500 & 19300 & 38 & 1970 & 16200 & 36 & 1570 \\
\hline 13 & 34400 & 79 & 7320 & 18700 & 35 & 1760 & 15600 & 35 & 1470 \\
\hline 14 & 36500 & 105 & 10400 & 18100 & 33 & 1620 & 15600 & 34 & 1430 \\
\hline 15 & 35700 & 123 & 11900 & 18200 & 32 & 1560 & 15000 & 33 & 1340 \\
\hline 16 & 34900 & 86 & 8140 & 17500 & 30 & 1430 & 15200 & 32 & 1320 \\
\hline 17 & 34600 & 77 & 7220 & 16900 & 27 & 1240 & 15100 & 30 & 1220 \\
\hline 18 & 34600 & 72 & 6750 & 16600 & 22 & 982 & 15200 & 28 & 1140 \\
\hline 19 & 34700 & 68 & 6330 & 16700 & 27 & 1230 & 15200 & 26 & 1050 \\
\hline 20 & 34200 & 63 & 5800 & 17100 & 27 & 1230 & 14900 & 24 & 950 \\
\hline 21 & 33600 & 60 & 5480 & 17100 & 29 & 1330 & 15000 & 22 & 883 \\
\hline 22 & 33200 & 65 & 5810 & 16800 & 31 & 1430 & 14900 & 20 & 817 \\
\hline 23 & 31800 & 66 & 5670 & 17400 & 34 & 1610 & 14700 & 20 & 806 \\
\hline 24 & 29900 & 66 & 5330 & 18400 & 37 & 1850 & 14600 & 21 & 814 \\
\hline 25 & 28600 & 66 & 5100 & 18400 & 37 & 1810 & 14900 & 22 & 870 \\
\hline 26 & 27500 & 62 & 4620 & 17900 & 38 & 1820 & 15400 & 23 & 973 \\
\hline 27 & 26900 & 47 & 3390 & 17700 & 40 & 1890 & 15700 & 25 & 1060 \\
\hline 28 & 26700 & 42 & 3020 & 17700 & 40 & 1910 & 17100 & 27 & 1230 \\
\hline 29 & 26100 & 41 & 2920 & 18600 & 40 & 2010 & 19100 & 28 & 1460 \\
\hline 30 & 25500 & 43 & 2980 & 18900 & 40 & 2040 & 20700 & 30 & 1670 \\
\hline 31 & --- & --- & --- & 19800 & 40 & 2140 & --- & --- & --- \\
\hline TOTAL & 920300 & --- & 159690 & 611800 & --- & 63612 & 517100 & --- & 45243 \\
\hline
\end{tabular}

JULY

AUGUST

SEPTEMBER

\begin{tabular}{|c|c|}
\hline 1 & 20900 \\
\hline 2 & 21000 \\
\hline 3 & 20600 \\
\hline 4 & 20800 \\
\hline 5 & 20400 \\
\hline 6 & 20500 \\
\hline 7 & 20800 \\
\hline 8 & 20700 \\
\hline 9 & 21200 \\
\hline 10 & 21400 \\
\hline 11 & 21300 \\
\hline 12 & 21400 \\
\hline 13 & 21500 \\
\hline 14 & 21500 \\
\hline 15 & 22100 \\
\hline 16 & 22200 \\
\hline 17 & 22500 \\
\hline 18 & 22900 \\
\hline 19 & 23100 \\
\hline 20 & 23900 \\
\hline 21 & 23900 \\
\hline 22 & 23800 \\
\hline 23 & 23200 \\
\hline 24 & 23300 \\
\hline 25 & 23200 \\
\hline 26 & 23400 \\
\hline 27 & 23600 \\
\hline 28 & 23700 \\
\hline 29 & 23900 \\
\hline 30 & 23700 \\
\hline 31 & 23100 \\
\hline TOTAL & 689500 \\
\hline
\end{tabular}

$\begin{array}{ll}30 & 1710 \\ 31 & 1740 \\ 31 & 1730 \\ 31 & 1770 \\ 32 & 1750 \\ 32 & 1780 \\ 33 & 1830 \\ 33 & 1850 \\ 35 & 1990\end{array}$

22900
23300
23200
22400
21500
21400
20600
19700
18900
17800

$\begin{array}{ll}40 & 2480 \\ 40 & 2540 \\ 41 & 2540 \\ 41 & 2470 \\ 38 & 2200 \\ 35 & 2000 \\ 34 & 1900 \\ 34 & 1790 \\ 33 & 1690 \\ 33 & 1570\end{array}$

17300
16600
16500
16500
16600
16300
16400
16400
16200
16200

17400

17100

17200

17100

17100

17000
16600

15600

15500

1520

1470

1460

1430
1410

1390

1340

1240

1210

16500

16900

16200

15100

15300

15400

15000

16000
16300

16700

15100

15300

15600
16100

16100
16100

16300

16500

17400

17900

17800

1150

1150

1160
1180

1180
1160

1160

1160

1210

1210

1190

15900

15300

15400

15000
14800

14600

14400

14500

15200

2550

558900

47730

474900

1140
1080
1060
1050
1050
1010
1010
997
974
962

969
981
929
896
936
928
891
936
940
948
890
843
836
803
780
758
737
731
755
754
---
27574

YEAR 10975900 
LOCATION.-Lat 3855'39", long 12250'33", in SE 1/4 SE 1/4 sec.34, T.13 N., R.9 W., Lake County, Hydrologic Unit 18020116, on left bank 1.6 mi downstream from Widow Creek and $3.5 \mathrm{mi}$ south of Kelseyville.

DRAINAGE AREA.- $36.6 \mathrm{mi}^{2}$.

PERIOD OF RECORD.-October 1946 to current year.

REVISED RECORDS.-WSP 1285: 1947-48(M), 1950-52(P). WSP 1931: Drainage area. WDR CA-96-4: 1956-93(P).

GAGE.-Water-stage recorder. Datum of gage is 1,475.44 ft above sea level. Prior to July 16, 1955, at site $600 \mathrm{ft}$ upstream at different datum. REMARKS.-Records good. Some minor diversions upstream from station. See schematic diagram of lower Sacramento River Basin.

EXTREMES FOR PERIOD OF RECORD.-Maximum discharge, 8,600 $\mathrm{ft}^{3} / \mathrm{s}$, Mar. 9, 1995, gage height, $13.80 \mathrm{ft}$; minimum daily, $0.13 \mathrm{ft}^{3} / \mathrm{s}$, Sept. 6-11, 1992.

EXTREMES FOR CURRENT YEAR.-Peak discharges greater than base discharge of 2,400 $\mathrm{ft}^{3} / \mathrm{s}$, or maximum:

$\begin{array}{lccccccc}\text { Date } & \text { Time } & \begin{array}{c}\text { Discharge } \\ \left(\mathrm{ft}^{3} / \mathrm{s}\right)\end{array} & \begin{array}{c}\text { Gage height } \\ (\mathrm{ft})\end{array} & \text { Date } & \text { Time } & \begin{array}{c}\text { Discharge } \\ \left(\mathrm{ft}^{3} / \mathrm{s}\right)\end{array} & \begin{array}{c}\text { Gage height } \\ (\mathrm{ft})\end{array} \\ \text { Nov. 30 } & 0915 & 2,520 & 9.26 & \text { Feb. } 9 & 0415 & 5,100 & 11.62\end{array}$

DISCHARGE, CUBIC FEET PER SECOND, WATER YEAR OCTOBER 1998 TO SEPTEMBER 1999 DAILY MEAN VALUES

\begin{tabular}{|c|c|c|c|c|c|c|c|c|c|c|c|c|}
\hline DAY & $\mathrm{OCT}$ & NOV & $\mathrm{DEC}$ & JAN & FEB & MAR & APR & MAY & JUN & JUL & AUG & SEP \\
\hline 1 & 8.2 & 9.7 & 294 & 19 & 60 & 264 & 120 & 49 & 21 & 9.3 & 6.7 & 5.8 \\
\hline 2 & 8.3 & 9.7 & 144 & 18 & 52 & 206 & 106 & 48 & 22 & 9.2 & 6.8 & 5.9 \\
\hline 3 & 8.3 & 9.9 & 382 & 18 & 48 & 234 & 95 & 51 & 23 & 9.2 & 6.8 & 5.9 \\
\hline 4 & 8.1 & 9.8 & 157 & 17 & 45 & 173 & 88 & 48 & 22 & 9.6 & 6.7 & 5.8 \\
\hline 5 & 8.0 & 9.5 & 110 & 17 & 41 & 148 & 98 & 45 & 21 & 9.4 & 6.8 & 5.5 \\
\hline 6 & 7.9 & 9.6 & 106 & 17 & 521 & 130 & 91 & 43 & 20 & 9.2 & 7.1 & 5.5 \\
\hline 7 & 7.8 & 23 & 71 & 17 & 1110 & 115 & 80 & 42 & 19 & 9.0 & 7.3 & 5.3 \\
\hline 8 & 8.1 & 17 & 65 & 16 & 703 & 297 & 168 & 40 & 19 & 8.9 & 7.2 & 5.3 \\
\hline 9 & 8.3 & 13 & 56 & 16 & 2120 & 368 & 127 & 40 & 18 & 8.5 & 7.1 & 5.3 \\
\hline 10 & 8.3 & 11 & 50 & 16 & 489 & 234 & 116 & 39 & 17 & 8.2 & 7.0 & 5.4 \\
\hline 11 & 8.4 & 11 & 45 & 15 & 285 & 183 & 666 & 38 & 17 & 7.9 & 7.2 & 5.2 \\
\hline 12 & 8.4 & 11 & 41 & 15 & 205 & 150 & 302 & 37 & 17 & 7.6 & 7.3 & 5.2 \\
\hline 13 & 8.4 & 10 & 42 & 15 & 161 & 130 & 203 & 36 & 16 & 7.4 & 7.0 & 5.2 \\
\hline 14 & 8.5 & 10 & 47 & 15 & 135 & 180 & 158 & 35 & 16 & 7.3 & 6.8 & 5.2 \\
\hline 15 & 8.6 & 11 & 39 & 16 & 110 & 183 & 132 & 34 & 16 & 7.6 & 6.7 & 5.1 \\
\hline 16 & 8.5 & 10 & 36 & 20 & 359 & 143 & 116 & 33 & 16 & 7.8 & 6.6 & 5.0 \\
\hline 17 & 8.3 & 15 & 33 & 21 & 441 & 124 & 104 & 32 & 15 & 7.8 & 6.4 & 5.1 \\
\hline 18 & 8.2 & 13 & 32 & 123 & 330 & 111 & 95 & 31 & 14 & 7.6 & 6.2 & 5.1 \\
\hline 19 & 8.3 & 11 & 30 & 250 & 244 & 103 & 86 & 31 & 14 & 7.6 & 6.4 & 5.1 \\
\hline 20 & 8.3 & 11 & 29 & 336 & 307 & 106 & 79 & 30 & 13 & 7.8 & 6.2 & 5.1 \\
\hline 21 & 8.2 & 12 & 27 & 197 & 433 & 93 & 75 & 30 & 13 & 7.9 & 6.1 & 5.1 \\
\hline 22 & 8.2 & 17 & 26 & 254 & 287 & 86 & 72 & 28 & 13 & 7.7 & 5.9 & 5.3 \\
\hline 23 & 8.4 & 462 & 24 & 434 & 220 & 105 & 68 & 27 & 12 & 7.6 & 5.8 & 5.2 \\
\hline 24 & 15 & 156 & 23 & 185 & 236 & 406 & 65 & 25 & 11 & 7.6 & 5.8 & 5.2 \\
\hline 25 & 13 & 50 & 23 & 125 & 486 & 638 & 62 & 25 & 12 & 7.4 & 5.7 & 5.0 \\
\hline 26 & 10 & 45 & 23 & 92 & 261 & 304 & 59 & 24 & 11 & 7.2 & 5.6 & 5.0 \\
\hline 27 & 9.6 & 48 & 22 & 72 & 206 & 217 & 57 & 23 & 11 & 7.2 & 5.7 & 4.9 \\
\hline 28 & 9.5 & 33 & 21 & 61 & 305 & 174 & 55 & 22 & 11 & 7.1 & 5.6 & 4.9 \\
\hline 29 & 9.5 & 63 & 20 & 55 & --- & 148 & 54 & 22 & 9.8 & 7.2 & 5.5 & 4.8 \\
\hline 30 & 9.4 & 1360 & 20 & 50 & --- & 146 & 52 & 22 & 9.6 & 7.1 & 5.5 & 4.9 \\
\hline 31 & 9.3 & --- & 20 & 86 & --- & 150 & --- & 21 & --- & 6.9 & 5.7 & --- \\
\hline TOTAL & 275.3 & 2481.2 & 2058 & 2608 & 10200 & 6049 & 3649 & 1051 & 469.4 & 247.8 & 199.2 & 157.3 \\
\hline MEAN & 8.88 & 82.7 & 66.4 & 84.1 & 364 & 195 & 122 & 33.9 & 15.6 & 7.99 & 6.43 & 5.24 \\
\hline MAX & 15 & 1360 & 382 & 434 & 2120 & 638 & 666 & 51 & 23 & 9.6 & 7.3 & 5.9 \\
\hline MIN & 7.8 & 9.5 & 20 & 15 & 41 & 86 & 52 & 21 & 9.6 & 6.9 & 5.5 & 4.8 \\
\hline $\mathrm{AC}-\mathrm{FT}$ & 546 & 4920 & 4080 & 5170 & 20230 & 12000 & 7240 & 2080 & 931 & 492 & 395 & 312 \\
\hline
\end{tabular}




\section{KELSEY CREEK NEAR KELSEYVILLE, CA—Continued}

STATISTICS OF MONTHLY MEAN DATA FOR WATER YEARS 1947 - 1999, BY WATER YEAR (WY)

$\begin{array}{lrrrrrrrrrrrr} & \text { OCT } & \text { NOV } & \text { DEC } & \text { JAN } & \text { FEB } & \text { MAR } & \text { APR } & \text { MAY } & \text { JUN } & \text { JUL } & \text { AUG } & \text { SEP } \\ \text { MEAN } & 11.3 & 46.5 & 127 & 209 & 213 & 152 & 78.7 & 32.0 & 13.4 & 5.81 & 3.63 & 3.80 \\ \text { MAX } & 154 & 334 & 688 & 929 & 919 & 640 & 429 & 163 & 64.1 & 19.2 & 9.40 & 16.3 \\ \text { (WY) } & 1963 & 1974 & 1956 & 1995 & 1986 & 1983 & 1982 & 1983 & 1998 & 1998 & 1998 & 1957 \\ \text { MIN } & 1.22 & 3.55 & 4.19 & 4.83 & 8.97 & 11.4 & 5.67 & 6.12 & 1.98 & .46 & .20 & .16 \\ \text { (WY) } & 1992 & 1991 & 1991 & 1991 & 1977 & 1977 & 1977 & 1977 & 1977 & 1977 & 1977 & 1992\end{array}$

SUMMARY STATISTICS

FOR 1998 CALENDAR YEAR

FOR 1999 WATER YEAR

WATER YEARS 1947 - 1999

ANNUAL TOTAL

ANNUAL MEAN

52824.4

145

TEST ANNUAL MEAN

LOWEST ANNUAL MEAN

HIGHEST DAILY MEAN

LOWEST DAILY MEAN

ANNUAL SEVEN-DAY MINIMUM

INSTANTANEOUS PEAK FLOW

INSTANTANEOUS PEAK STAGE

ANNUAL RUNOFF (AC-FT)

10 PERCENT EXCEEDS

50 PERCENT EXCEEDS

90 PERCENT EXCEEDS

104800

406

43
8.2
29445.2

80.7

2120 Feb 9

4.8 Sep 29

5.0 Sep 24

$5100 \quad$ Feb 9

11.62 Feb 9
58400

218

19
5.8

\begin{tabular}{|c|c|c|c|}
\hline 74.1 & & & \\
\hline 206 & & & 1983 \\
\hline 4.78 & & & 1977 \\
\hline 6020 & Feb & 17 & 1986 \\
\hline .13 & Sep & 6 & 1992 \\
\hline .13 & Sep & 5 & 1992 \\
\hline 8600 & Mar & 9 & 1995 \\
\hline 13.80 & Mar & 9 & 1995 \\
\hline 3700 & & & \\
\hline 156 & & & \\
\hline 13 & & & \\
\hline 2.6 & & & \\
\hline
\end{tabular}


11450000 CLEAR LAKE AT LAKEPORT, CA

LOCATION.-Lat 3902’21", long 122 54'44", in NE 1/4 NE 1/4 sec.25, T.14 N., R.10 W., Lake County, Hydrologic Unit 18020116, on pier behind 410 Esplanade Street in Lakeport.

DRAINAGE AREA. $-528 \mathrm{mi}^{2}$.

PERIOD OF RECORD.-1874-1900 (incomplete), January 1913 to April 1982, October 1984 to current year.

GAGE.-Water-stage recorder. Datum of gage is 1,318.26 ft above sea level (California State Land Commission Benchmark). Prior to July 8, 1947, nonrecording gage, and July 8, 1947, to Mar. 17, 1949, at municipal wharf at foot of Third Street in Lakeport at datum $0.33 \mathrm{ft}$ higher. Mar. 18 , 1949, to Sept. 30, 1967, at private pier at foot of Fourth Street at datum $0.33 \mathrm{ft}$ higher. Gage relocated at same datum, Apr. 20, 1982, and published as "at Clearlake" for 1982-84.

REMARKS. - This natural lake is regulated by gates on a dam at outlet, completed in 1915 . Capacity between gage heights 0.00 and $7.56 \mathrm{ft}$, limits stipulated by court decree of 1920 , about 319,000 acre-ft. Water is released down natural channel of Cache Creek (station 11451000 ), from which it is diverted for irrigation. See schematic diagram of lower Sacramento River Basin.

EXTREMES FOR PERIOD OF RECORD.-Maximum gage height, 11.44 ft, Feb. 24, 1998, minimum observed, -3.50 ft, Sept. $24-27,1920$.

EXTREMES OUTSIDE PERIOD OF RECORD.-Flood of Mar. 4, 1983, reached a stage of $11.24 \mathrm{ft}$, present datum, from floodmarks.

GAGE HEIGHT, FEET, WATER YEAR OCTOBER 1998 TO SEPTEMBER 1999 DAILY MEAN VALUES

\begin{tabular}{|c|c|c|c|c|c|c|c|c|c|c|c|c|}
\hline DAY & $\mathrm{OCT}$ & NOV & $\mathrm{DEC}$ & JAN & FEB & MAR & $\mathrm{APR}$ & MAY & JUN & JUL & AUG & SEP \\
\hline 1 & 3.12 & 2.79 & 3.36 & 3.82 & 4.72 & 7.49 & 7.45 & 7.57 & 6.40 & 5.26 & 3.77 & 2.47 \\
\hline 2 & 3.08 & 2.78 & 3.43 & 3.83 & 4.74 & 7.46 & 7.46 & 7.53 & 6.32 & 5.19 & 3.72 & 2.45 \\
\hline 3 & 3.04 & 2.78 & 3.48 & 3.83 & 4.75 & 7.41 & 7.41 & 7.50 & 6.34 & 5.09 & 3.68 & 2.42 \\
\hline 4 & 3.04 & 2.77 & 3.56 & 3.83 & 4.75 & 7.36 & 7.45 & 7.49 & 6.30 & 5.07 & 3.63 & 2.40 \\
\hline 5 & 3.02 & 2.73 & 3.57 & 3.83 & 4.76 & 7.33 & 7.49 & 7.48 & 6.25 & 5.03 & 3.57 & 2.39 \\
\hline 6 & 3.00 & 2.75 & 3.60 & 3.83 & 4.83 & 7.32 & 7.53 & 7.44 & 6.20 & 4.98 & 3.53 & 2.37 \\
\hline 7 & 2.97 & 2.76 & 3.63 & 3.84 & 5.07 & 7.31 & 7.52 & 7.39 & 6.15 & 4.94 & 3.50 & 2.35 \\
\hline 8 & 2.94 & 2.78 & 3.67 & 3.84 & 5.32 & 7.36 & 7.53 & 7.35 & 6.13 & 4.91 & 3.45 & 2.32 \\
\hline 9 & 2.90 & 2.79 & 3.68 & 3.84 & 5.72 & 7.48 & 7.55 & 7.32 & 6.10 & 4.87 & 3.42 & 2.30 \\
\hline 10 & 2.91 & 2.79 & 3.69 & 3.84 & 6.07 & 7.49 & 7.60 & 7.28 & 6.06 & 4.83 & 3.38 & 2.27 \\
\hline 11 & 2.90 & 2.79 & 3.70 & 3.84 & 6.23 & 7.45 & 7.71 & 7.26 & 6.01 & 4.79 & 3.34 & 2.25 \\
\hline 12 & 2.89 & 2.79 & 3.71 & 3.84 & 6.32 & 7.46 & 7.68 & 7.21 & 5.97 & 4.76 & 3.30 & 2.22 \\
\hline 13 & 2.87 & 2.79 & 3.71 & 3.85 & 6.35 & 7.50 & 7.63 & 7.16 & 5.94 & 4.72 & 3.25 & 2.20 \\
\hline 14 & 2.86 & 2.78 & 3.75 & 3.85 & 6.41 & 7.57 & 7.58 & 7.09 & 5.92 & 4.67 & 3.21 & 2.18 \\
\hline 15 & 2.85 & 2.79 & 3.76 & 3.87 & 6.44 & 7.63 & 7.59 & 7.09 & 5.87 & 4.63 & 3.18 & 2.15 \\
\hline 16 & 2.85 & 2.78 & 3.78 & 3.90 & 6.61 & 7.63 & 7.61 & 7.04 & 5.84 & 4.57 & 3.15 & 2.12 \\
\hline 17 & 2.84 & 2.77 & 3.79 & 3.95 & 6.92 & 7.58 & 7.62 & 7.02 & 5.81 & 4.52 & 3.12 & 2.09 \\
\hline 18 & 2.83 & 2.79 & 3.79 & 3.97 & 7.05 & 7.50 & 7.62 & 6.97 & 5.76 & 4.48 & 3.07 & 2.06 \\
\hline 19 & 2.83 & 2.78 & 3.77 & 4.03 & 7.14 & 7.44 & 7.61 & 6.95 & 5.72 & 4.43 & 3.03 & 2.03 \\
\hline 20 & 2.81 & 2.77 & 3.75 & 4.14 & 7.17 & 7.45 & 7.59 & 6.91 & 5.68 & 4.37 & 2.99 & 2.00 \\
\hline 21 & 2.81 & 2.77 & 3.78 & 4.22 & 7.27 & 7.45 & 7.58 & 6.89 & 5.65 & 4.33 & 2.95 & 1.99 \\
\hline 22 & 2.79 & 2.79 & 3.78 & 4.29 & 7.33 & 7.47 & 7.61 & 6.86 & 5.63 & 4.28 & 2.92 & 1.96 \\
\hline 23 & 2.78 & 2.86 & 3.78 & 4.40 & 7.34 & 7.50 & 7.62 & 6.81 & 5.59 & 4.22 & 2.88 & 1.93 \\
\hline 24 & 2.82 & 2.95 & 3.78 & 4.51 & 7.34 & 7.61 & 7.62 & 6.78 & 5.52 & 4.16 & 2.85 & 1.91 \\
\hline 25 & 2.83 & 2.97 & 3.79 & 4.54 & 7.43 & 7.77 & 7.63 & 6.76 & 5.48 & 4.12 & 2.81 & 1.89 \\
\hline 26 & 2.84 & 2.99 & 3.79 & 4.60 & 7.47 & 7.79 & 7.61 & 6.72 & 5.44 & 4.07 & 2.78 & 1.86 \\
\hline 27 & 2.82 & 3.00 & 3.80 & 4.62 & 7.46 & 7.76 & 7.56 & 6.67 & 5.40 & 4.03 & 2.75 & 1.84 \\
\hline 28 & 2.82 & 2.99 & 3.81 & 4.64 & 7.47 & 7.71 & 7.58 & 6.61 & 5.37 & 3.98 & 2.70 & 1.80 \\
\hline 29 & 2.80 & 3.06 & 3.81 & 4.67 & --- & 7.62 & 7.60 & 6.55 & 5.34 & 3.92 & 2.65 & 1.77 \\
\hline 30 & 2.80 & 3.20 & 3.82 & 4.69 & --- & 7.54 & 7.60 & 6.50 & 5.31 & 3.88 & 2.54 & 1.74 \\
\hline 31 & 2.79 & --- & 3.82 & 4.70 & --- & 7.47 & --- & 6.46 & --- & 3.82 & 2.51 & --- \\
\hline MEAN & 2.89 & 2.84 & 3.71 & 4.11 & 6.30 & 7.51 & 7.57 & 7.05 & 5.85 & 4.55 & 3.15 & 2.12 \\
\hline MAX & 3.12 & 3.20 & 3.82 & 4.70 & 7.47 & 7.79 & 7.71 & 7.57 & 6.40 & 5.26 & 3.77 & 2.47 \\
\hline MIN & 2.78 & 2.73 & 3.36 & 3.82 & 4.72 & 7.31 & 7.41 & 6.46 & 5.31 & 3.82 & 2.51 & 1.74 \\
\hline
\end{tabular}


11451000 CACHE CREEK NEAR LOWER LAKE, CA

LOCATION.-Lat 3855'27", long 122³3'53", in sec.6, T.12 N., R.6 W., Lake County, Hydrologic Unit 18020116 , on left bank 500 ft downstream from Clear Lake Dam, 1.9 mi downstream from Copsey Creek, and 2.5 mi northeast of Lower Lake.

DRAINAGE AREA. $-528 \mathrm{mi}^{2}$.

PERIOD OF RECORD.-May 1944 to current year.

GAGE.-Water-stage recorder and rain gage (station 385525122335501). Datum of gage is 1,279.64 ft above sea level. Prior to Oct. 2, 1987, at datum $1.00 \mathrm{ft}$ higher.

REMARKS.-Records fair. Flow completely regulated by Clear Lake (station 11450000) $500 \mathrm{ft}$ upstream. See schematic diagram of lower Sacramento River Basin.

EXTREMES FOR PERIOD OF RECORD.-Maximum discharge, 10,200 ft ${ }^{3} / \mathrm{s}$, Feb. 17, 1998, gage height, $11.01 \mathrm{ft}$, present datum; no flow, Nov. 8-20, 1977, Apr. 5, 6, 1987.

DISCHARGE, CUBIC FEET PER SECOND, WATER YEAR OCTOBER 1998 TO SEPTEMBER 1999 DAILY MEAN VALUES

\begin{tabular}{|c|c|c|c|c|c|c|c|c|c|c|c|c|}
\hline DAY & $\mathrm{OCT}$ & NOV & $\mathrm{DEC}$ & JAN & FEB & MAR & APR & MAY & JUN & JUL & AUG & SEP \\
\hline 1 & 220 & 6.2 & 5.2 & 5.5 & 7.0 & 2530 & 883 & 435 & 694 & 553 & 553 & 300 \\
\hline 2 & 200 & 6.3 & 5.2 & 5.5 & 7.1 & 2510 & 419 & 681 & 525 & 569 & 544 & 277 \\
\hline 3 & 201 & 6.4 & 5.5 & 5.5 & 7.3 & 2550 & 276 & 676 & 445 & 559 & 565 & 237 \\
\hline 4 & 196 & 5.1 & 5.5 & 5.5 & 7.5 & 2400 & 211 & 669 & 398 & 555 & 583 & 223 \\
\hline 5 & 197 & 5.3 & 5.6 & 6.0 & 7.5 & 1620 & 210 & 670 & 361 & 557 & 567 & 232 \\
\hline 6 & 207 & 5.3 & 5.7 & 7.8 & 7.6 & 1000 & 459 & 698 & 342 & 551 & 538 & 260 \\
\hline 7 & 210 & 5.4 & 6.0 & 6.9 & 8.1 & 620 & 469 & 737 & 402 & 541 & 522 & 274 \\
\hline 8 & 213 & 5.5 & 5.9 & 6.5 & 8.3 & 405 & 471 & 718 & 507 & 538 & 466 & 300 \\
\hline 9 & 213 & 5.6 & 6.2 & 6.3 & 1040 & 1680 & 340 & 702 & 569 & 549 & 418 & 323 \\
\hline 10 & 123 & 5.5 & 6.5 & 6.0 & 95 & 2330 & 557 & 726 & 625 & 551 & 389 & 294 \\
\hline 11 & 10 & 5.6 & 6.5 & 6.1 & 9.4 & 1350 & 2340 & 730 & 566 & 544 & 458 & 271 \\
\hline 12 & 9.7 & 5.7 & 6.5 & 6.1 & 9.5 & 556 & 2660 & 742 & 478 & 541 & 475 & 275 \\
\hline 13 & 9.5 & 5.6 & 6.3 & 6.2 & 9.7 & 277 & 2650 & 744 & 441 & 541 & 530 & 281 \\
\hline 14 & 9.3 & 5.7 & 7.1 & 6.3 & 9.8 & 448 & 1190 & 737 & 483 & 510 & 519 & 280 \\
\hline 15 & 9.0 & 5.5 & 6.8 & 6.3 & 10 & 1250 & 412 & 729 & 542 & 547 & 477 & 276 \\
\hline 16 & 8.5 & 5.5 & 6.6 & 6.3 & 10 & 2070 & 397 & 731 & 573 & 559 & 458 & 273 \\
\hline 17 & 8.0 & 5.6 & 6.9 & 6.3 & 756 & 2560 & 529 & 724 & 584 & 578 & 466 & 271 \\
\hline 18 & 7.7 & 5.5 & 7.1 & 6.5 & 2070 & 2510 & 526 & 747 & 598 & 591 & 517 & 273 \\
\hline 19 & 7.3 & 5.6 & 7.5 & 6.5 & 2230 & 1210 & 530 & 717 & 594 & 574 & 554 & 274 \\
\hline 20 & 7.0 & 5.5 & 7.8 & 6.5 & 2290 & 496 & 284 & 678 & 586 & 487 & 569 & 272 \\
\hline 21 & 6.8 & 5.6 & 7.0 & 6.5 & 2440 & 480 & 140 & 692 & 527 & 522 & 532 & 270 \\
\hline 22 & 6.8 & 5.4 & 5.8 & 6.5 & 2460 & 290 & 46 & 698 & 525 & 611 & 509 & 312 \\
\hline 23 & 6.9 & 5.5 & 5.8 & 6.5 & 2450 & 337 & 20 & 696 & 599 & 701 & 532 & 331 \\
\hline 24 & 7.0 & 5.2 & 5.8 & 6.6 & 2430 & 1970 & 20 & 713 & 629 & 742 & 574 & 337 \\
\hline 25 & 6.8 & 5.0 & 5.8 & 6.5 & 2630 & 2870 & 19 & 749 & 578 & 761 & 589 & 340 \\
\hline 26 & 6.6 & 5.0 & 5.7 & 6.6 & 2550 & 2680 & 255 & 801 & 527 & 700 & 562 & 350 \\
\hline 27 & 6.6 & 5.0 & 5.8 & 6.8 & 2540 & 2680 & 261 & 823 & 521 & 599 & 528 & 340 \\
\hline 28 & 6.6 & 5.0 & 5.8 & 6.8 & 2520 & 2660 & 23 & 833 & 487 & 582 & 517 & 334 \\
\hline 29 & 6.7 & 4.9 & 5.8 & 6.8 & --- & 2540 & 28 & 823 & 475 & 596 & 516 & 334 \\
\hline 30 & 6.5 & 5.2 & 5.8 & 6.8 & --- & 2580 & 20 & 793 & 484 & 601 & 503 & 343 \\
\hline 31 & 6.3 & --- & 5.6 & 7.0 & --- & 2190 & --- & 788 & --- & 579 & 391 & --- \\
\hline TOTAL & 2139.6 & 164.2 & 191.1 & 198.0 & 28619.8 & 51649 & 16645 & 22400 & 15665 & 17989 & 15921 & 8757 \\
\hline MEAN & 69.0 & 5.47 & 6.16 & 6.39 & 1022 & 1666 & 555 & 723 & 522 & 580 & 514 & 292 \\
\hline MAX & 220 & 6.4 & 7.8 & 7.8 & 2630 & 2870 & 2660 & 833 & 694 & 761 & 589 & 350 \\
\hline MIN & 6.3 & 4.9 & 5.2 & 5.5 & 7.0 & 277 & 19 & 435 & 342 & 487 & 389 & 223 \\
\hline $\mathrm{AC}-\mathrm{FT}$ & 4240 & 326 & 379 & 393 & 56770 & 102400 & 33020 & 44430 & 31070 & 35680 & 31580 & 17370 \\
\hline a & 0.90 & 6.45 & 0.97 & 2.90 & 8.81 & 4.38 & 2.14 & 0.16 & 0.24 & 0.01 & 0.00 & 0.00 \\
\hline
\end{tabular}

STATISTICS OF MONTHLY MEAN DATA FOR WATER YEARS 1945 - 1999, BY WATER YEAR (WY)

\begin{tabular}{|c|c|c|c|c|c|c|c|c|c|c|c|c|}
\hline & $\mathrm{OCT}$ & NOV & DEC & JAN & $\mathrm{FEB}$ & MAR & APR & MAY & JUN & JUL & AUG & SEP \\
\hline MEAN & 30.7 & 16.2 & 113 & 635 & 868 & 840 & 551 & 327 & 376 & 400 & 318 & 167 \\
\hline MAX & 191 & 683 & 2584 & 3047 & 4988 & 4919 & 3538 & 951 & 684 & 651 & 514 & 325 \\
\hline$(W Y)$ & 1996 & 1984 & 1984 & 1997 & 1998 & 1983 & 1958 & 1983 & 1998 & 1998 & 1999 & 1995 \\
\hline MIN & .40 & .17 & .14 & .18 & .17 & .32 & .42 & .40 & .29 & .41 & .71 & .55 \\
\hline (WY) & 1978 & 1978 & 1991 & 1991 & 1991 & 1955 & 1990 & 1990 & 1991 & 1977 & 1977 & 1977 \\
\hline
\end{tabular}

SUMMARY STATISTICS

ANNUAL TOTAL

ANNUAL MEAN

HIGHEST ANNUAL MEAN

LOWEST ANNUAL MEAN

HIGHEST DAILY MEAN

LOWEST DAILY MEAN

ANNUAL SEVEN-DAY MINIMUM

INSTANTANEOUS PEAK FLOW

INSTANTANEOUS PEAK STAGE

ANNUAL RUNOFF (AC-FT)

10 PERCENT EXCEEDS

50 PERCENT EXCEEDS

90 PERCENT EXCEEDS
FOR 1998 CALENDAR YEAR

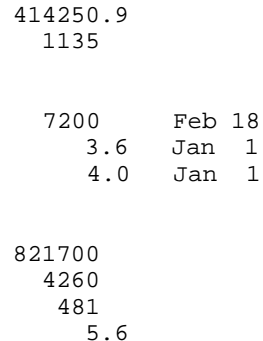

FOR 1999 WATER YEAR

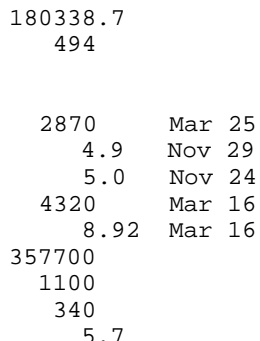

WATER YEARS 1945 - 1999

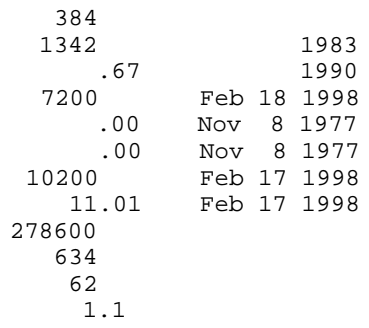

a Precipitation, in inches. 
LOCATION.-Lat 3909'56", long 122³7’08", in SE 1/4 NW 1/4 sec.10, T.15 N., R.7 W., Lake County, Hydrologic Unit 18020116, on right bank $0.5 \mathrm{mi}$ upstream from Spanish Creek, 0.9 mi upstream from Hough Springs, and 10 mi northeast of Clearlake Oaks.

DRAINAGE AREA.- $-60.2 \mathrm{mi}^{2}$.

PERIOD OF RECORD.-October 1971 to current year.

GAGE.-Water-stage recorder and crest-stage gage. Elevation of gage is 1,534.13 ft above sea level. Prior to Jan. 13, 1980 , at datum $2.0 \mathrm{ft}$ higher. Recording rain gage (station 391056122420801 ) 4.7 mi northwest of gage. Elevation of rain gage is 2,050 ft above sea level, from topographic map.

REMARKS.-Records good. No regulation or diversion upstream from station. See schematic diagram of lower Sacramento River Basin.

EXTREMES FOR PERIOD OF RECORD.-Maximum discharge, 13,200 ft ${ }^{3} / \mathrm{s}$, Jan. 1, 1997, gage height, $14.14 \mathrm{ft}$, from rating curve extended above $3,900 \mathrm{ft}^{3} / \mathrm{s}$ on basis of slope-area measurement at gage height $11.23 \mathrm{ft}$; no flow at times in 1972, 1976-77, 1987-88, 1990-92, 1994.

EXTREMES FOR CURRENT YEAR.-Peak discharges greater than base discharge of 1,500 $\mathrm{ft}^{3} / \mathrm{s}$, or maximum.

\begin{tabular}{cccccccc} 
& \multicolumn{3}{c}{ Discharge } & Gage height & \multicolumn{2}{c}{$\begin{array}{c}\text { Discharge } \\
\left(\mathrm{ft}^{3} / \mathrm{s}\right)\end{array}$} \\
Date & Time & $\left(\mathrm{ft}^{3} / \mathrm{s}\right)$ & $(\mathrm{ft})$ & Date & Time & 1,620 & 7.61
\end{tabular}

DISCHARGE, CUBIC FEET PER SECOND, WATER YEAR OCTOBER 1998 TO SEPTEMBER 1999 DAILY MEAN VALUES

\begin{tabular}{|c|c|c|c|c|c|c|c|c|c|c|c|c|}
\hline DAY & OCT & NOV & $\mathrm{DEC}$ & JAN & $\mathrm{FEB}$ & MAR & APR & MAY & JUN & JUL & AUG & SEP \\
\hline 1 & 6.7 & 9.6 & 434 & 47 & 106 & 712 & 226 & 74 & 31 & 11 & 4.9 & 3.3 \\
\hline 2 & 6.6 & 9.2 & 310 & 45 & 97 & 565 & 205 & 73 & 31 & 11 & 5.0 & 3.3 \\
\hline 3 & 6.9 & 9.2 & 641 & 44 & 93 & 531 & 189 & 76 & 33 & 10 & 5.0 & 3.2 \\
\hline 4 & 6.9 & 9.2 & 338 & 43 & 89 & 432 & 174 & 70 & 31 & 10 & 4.9 & 3.2 \\
\hline 5 & 6.4 & 9.2 & 236 & 42 & 83 & 357 & 176 & 66 & 29 & 10 & 4.6 & 3.1 \\
\hline 6 & 6.4 & 9.6 & 188 & 41 & 465 & 305 & 162 & 63 & 27 & 9.5 & 5.6 & 2.9 \\
\hline 7 & 6.3 & 42 & 146 & 41 & 1440 & 261 & 151 & 60 & 27 & 9.2 & 5.8 & 2.9 \\
\hline 8 & 6.5 & 26 & 173 & 39 & 1000 & 316 & 178 & 58 & 26 & 8.9 & 5.4 & 2.7 \\
\hline 9 & 6.8 & 16 & 140 & 38 & 2520 & 399 & 161 & 57 & 25 & 8.7 & 5.2 & 2.7 \\
\hline 10 & 6.8 & 15 & 121 & 38 & 1020 & 323 & 177 & 55 & 25 & 8.4 & 5.2 & 2.7 \\
\hline 11 & 6.9 & 16 & 109 & 38 & 592 & 286 & 446 & 54 & 24 & 7.8 & 5.4 & 2.7 \\
\hline 12 & 6.7 & 14 & 102 & 37 & 464 & 261 & 378 & 52 & 22 & 7.5 & 5.2 & 2.6 \\
\hline 13 & 6.9 & 13 & 105 & 36 & 394 & 253 & 343 & 51 & 22 & 7.2 & 5.0 & 2.6 \\
\hline 14 & 6.9 & 12 & 112 & 36 & 303 & 376 & 301 & 49 & 21 & 7.0 & 4.8 & 2.7 \\
\hline 15 & 7.0 & 12 & 99 & 39 & 242 & 431 & 259 & 48 & 21 & 6.8 & 4.6 & 2.5 \\
\hline 16 & 6.7 & 13 & 91 & 59 & 626 & 356 & 225 & 47 & 20 & 6.7 & 4.4 & 2.5 \\
\hline 17 & 6.5 & 24 & 86 & 64 & 916 & 310 & 198 & 45 & 19 & 6.7 & 4.2 & 2.5 \\
\hline 18 & 6.5 & 21 & 82 & 290 & 694 & 274 & 177 & 44 & 18 & 6.7 & 4.1 & 2.4 \\
\hline 19 & 6.4 & 16 & 77 & 207 & 599 & 245 & 158 & 43 & 18 & 6.5 & 4.1 & 2.4 \\
\hline 20 & 6.0 & 14 & 74 & 413 & 613 & 252 & 142 & 43 & 17 & 6.5 & 4.1 & 2.3 \\
\hline 21 & 6.0 & 15 & 66 & 408 & 743 & 223 & 132 & 43 & 16 & 6.6 & 4.0 & 2.4 \\
\hline 22 & 6.1 & 25 & 63 & 388 & 632 & 207 & 122 & 40 & 16 & 6.5 & 3.9 & 2.3 \\
\hline 23 & 6.2 & 541 & 61 & 727 & 590 & 223 & 111 & 39 & 15 & 6.3 & 3.7 & 2.3 \\
\hline 24 & 15 & 248 & 58 & 394 & 589 & 684 & 105 & 38 & 14 & 6.0 & 3.3 & 2.3 \\
\hline 25 & 12 & 96 & 56 & 286 & 884 & 1080 & 98 & 38 & 14 & 5.9 & 3.2 & 2.2 \\
\hline 26 & 9.1 & 89 & 55 & 219 & 632 & 623 & 93 & 36 & 14 & 5.7 & 3.2 & 2.2 \\
\hline 27 & 8.8 & 91 & 54 & 172 & 552 & 479 & 88 & 35 & 14 & 5.5 & 3.3 & 2.1 \\
\hline 28 & 8.6 & 66 & 52 & 142 & 736 & 377 & 85 & 34 & 13 & 5.3 & 3.2 & 2.0 \\
\hline 29 & 8.8 & 214 & 51 & 125 & --- & 312 & 81 & 34 & 12 & 5.2 & 3.1 & 2.0 \\
\hline 30 & 8.5 & 818 & 49 & 114 & --- & 283 & 76 & 33 & 12 & 5.2 & 3.0 & 2.1 \\
\hline 31 & 8.6 & --- & 49 & 130 & --- & 257 & --- & 31 & --- & 5.0 & 3.2 & --- \\
\hline TOTAL & 230.5 & 2513.0 & 4278 & 4742 & 17714 & 11993 & 5417 & 1529 & 627 & 229.3 & 134.6 & 77.1 \\
\hline MEAN & 7.44 & 83.8 & 138 & 153 & 633 & 387 & 181 & 49.3 & 20.9 & 7.40 & 4.34 & 2.57 \\
\hline MAX & 15 & 818 & 641 & 727 & 2520 & 1080 & 446 & 76 & 33 & 11 & 5.8 & 3.3 \\
\hline MIN & 6.0 & 9.2 & 49 & 36 & 83 & 207 & 76 & 31 & 12 & 5.0 & 3.0 & 2.0 \\
\hline $\mathrm{AC}-\mathrm{FT}$ & 457 & 4980 & 8490 & 9410 & 35140 & 23790 & 10740 & 3030 & 1240 & 455 & 267 & 153 \\
\hline $\mathrm{a}$ & 1.61 & 12.49 & 1.98 & 5.41 & 14.72 & 6.18 & 2.61 & 0.10 & 0.08 & 0.00 & 0.00 & 0.00 \\
\hline
\end{tabular}

a Precipitation, in inches. 
11451100 NORTH FORK CACHE CREEK AT HOUGH SPRINGS, NEAR CLEARLAKE OAKS, CA—Continued

STATISTICS OF MONTHLY MEAN DATA FOR WATER YEARS 1972 - 1999, BY WATER YEAR (WY)

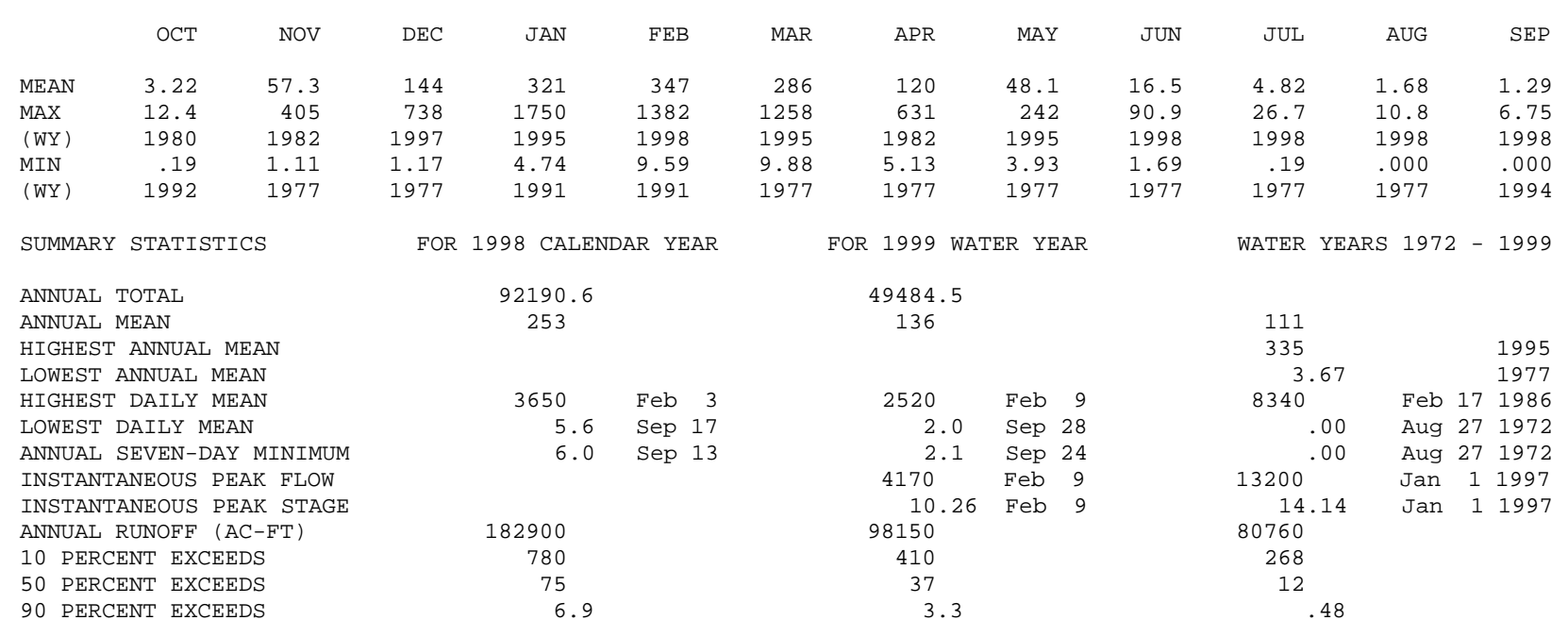


11451300 NORTH FORK CACHE CREEK NEAR CLEARLAKE OAKS, CA

LOCATION.-Lat 3904'50", long 122³2’07", in SE 1/4 SW 1/4 sec.4, T.14 N., R.6 W., Lake County, Hydrologic Unit 18020116, on right bank 2,500 ft downstream from Indian Valley Dam and 8 mi northeast of Clearlake Oaks.

DRAINAGE AREA.-121 $\mathrm{mi}^{2}$.

PERIOD OF RECORD.-October 1983 to September 1985 (operated as a low-flow station only), October 1985 to current year.

GAGE.-Water-stage recorder and crest-stage gage. Elevation of gage is 1,320 ft above sea level, from topographic map. Recording rain gage (station 390500122321601) located on top of Indian Valley Dam.

REMARKS.- Records good except for estimated daily discharges, which are fair. Flow completely regulated by Indian Valley Reservoir, capacity 300,000 acre-ft. See schematic diagram of lower Sacramento River Basin.

EXTREMES FOR PERIOD OF RECORD.-Maximum discharge, 7,950 ft ${ }^{3} / \mathrm{s}$, Feb. 11, 1998, gage height 10.61; maximum gage height, 10.62 ft, Jan. 2, 1997; minimum daily, $0.37 \mathrm{ft}^{3} / \mathrm{s}$, Oct.15, 1994.

EXTREMES OUTSIDE PERIOD OF RECORD.-Flood of Jan. 26, 1983, reached a stage of $12.74 \mathrm{ft}$, present datum, discharge about 9,500 ft ${ }^{3} / \mathrm{s}$.

DISCHARGE, CUBIC FEET PER SECOND, WATER YEAR OCTOBER 1998 TO SEPTEMBER 1999

DAILY MEAN VALUES

\begin{tabular}{|c|c|c|c|c|c|c|c|c|c|c|c|c|}
\hline DAY & $\mathrm{OCT}$ & NOV & $\mathrm{DEC}$ & JAN & FEB & MAR & $\mathrm{APR}$ & MAY & JUN & JUL & AUG & SEP \\
\hline 1 & 77 & 20 & 15 & 15 & 265 & 990 & 14 & 164 & 177 & 370 & 139 & 249 \\
\hline 2 & 102 & 20 & 15 & 15 & 118 & 970 & 13 & 12 & 254 & 355 & 138 & 249 \\
\hline 3 & 101 & 18 & 15 & 15 & 13 & 690 & 13 & 12 & 254 & 356 & 138 & 246 \\
\hline 4 & 101 & 13 & 291 & 15 & 13 & 374 & 13 & 12 & 253 & 355 & 138 & 201 \\
\hline 5 & 100 & 13 & 431 & 15 & 13 & 271 & 13 & 12 & 254 & 355 & 138 & 155 \\
\hline 6 & 57 & 14 & 453 & 18 & 13 & 473 & 13 & 12 & 254 & 355 & 138 & 133 \\
\hline 7 & 13 & 14 & 457 & 14 & 124 & 440 & 13 & 12 & 178 & 358 & 138 & 119 \\
\hline 8 & 13 & 14 & 457 & 15 & 997 & 271 & 13 & 12 & 130 & 367 & 138 & 161 \\
\hline 9 & 13 & 14 & 439 & 15 & 2260 & 524 & 13 & 12 & 127 & 366 & 163 & 186 \\
\hline 10 & 119 & 14 & 439 & 15 & 2380 & 661 & 13 & 12 & 135 & 366 & 153 & 186 \\
\hline 11 & 188 & 14 & 439 & 15 & 2340 & 565 & 13 & 12 & 214 & 366 & 56 & 167 \\
\hline 12 & 192 & 14 & 438 & 15 & 2300 & 370 & 13 & 12 & 258 & 367 & 11 & 128 \\
\hline 13 & 212 & 14 & 438 & 15 & 1870 & 271 & 13 & 12 & 257 & 367 & 11 & 113 \\
\hline 14 & 212 & 14 & 437 & 15 & 1630 & 333 & 13 & 12 & 259 & 368 & 11 & 113 \\
\hline 15 & 212 & 15 & 334 & 15 & 722 & 489 & 13 & 12 & 261 & 312 & 11 & 113 \\
\hline 16 & 211 & 14 & 171 & 15 & 276 & 455 & 13 & 12 & 260 & 274 & 11 & e111 \\
\hline 17 & 124 & 14 & 133 & 15 & 970 & 393 & 13 & 12 & 260 & 275 & 11 & e113 \\
\hline 18 & 22 & 14 & 86 & 15 & 1670 & 267 & 13 & 12 & 260 & 275 & 11 & e113 \\
\hline 19 & 22 & 14 & 56 & 16 & 1920 & 267 & 20 & 12 & 261 & 275 & 10 & e111 \\
\hline 20 & 22 & 14 & 55 & 16 & 1230 & 267 & 162 & 12 & 261 & 275 & 15 & e113 \\
\hline 21 & 23 & 14 & 35 & 15 & 790 & 191 & 257 & 12 & 260 & 184 & 11 & e115 \\
\hline 22 & 23 & 14 & 15 & 183 & 791 & 14 & 316 & 12 & 260 & 138 & 11 & e112 \\
\hline 23 & 23 & 14 & 15 & 265 & 912 & 14 & 344 & 12 & 261 & 138 & 10 & e 51.0 \\
\hline 24 & 23 & 14 & 15 & 265 & 1230 & 14 & 344 & 12 & 263 & 137 & 11 & 5.4 \\
\hline 25 & 23 & 14 & 15 & 265 & 1470 & 413 & 344 & 12 & 334 & 137 & 10 & 5.2 \\
\hline 26 & 23 & 14 & 15 & 265 & 1090 & 775 & 154 & 12 & 405 & 226 & 10 & 5.2 \\
\hline 27 & 23 & 14 & 15 & 265 & 786 & 777 & 199 & 12 & 405 & 274 & 10 & 5.2 \\
\hline 28 & 23 & 14 & 15 & 265 & 829 & 774 & 401 & 12 & 404 & 184 & 10 & 5.5 \\
\hline 29 & 23 & 14 & 15 & 265 & --- & 450 & 427 & 12 & 404 & 139 & 11 & 5.5 \\
\hline 30 & 27 & 15 & 15 & 265 & --- & 277 & 492 & 12 & 404 & 139 & 10 & 5.9 \\
\hline 31 & 31 & --- & 15 & 265 & --- & 122 & --- & 12 & --- & 139 & 180 & --- \\
\hline TOTAL & 2378 & 436 & 5784 & 2887 & 29022 & 13162 & 3695 & 524 & 7967 & 8592 & 1863 & 3395.9 \\
\hline MEAN & 76.7 & 14.5 & 187 & 93.1 & 1036 & 425 & 123 & 16.9 & 266 & 277 & 60.1 & 113 \\
\hline MAX & 212 & 20 & 457 & 265 & 2380 & 990 & 492 & 164 & 405 & 370 & 180 & 249 \\
\hline MIN & 13 & 13 & 15 & 14 & 13 & 14 & 13 & 12 & 127 & 137 & 10 & 5.2 \\
\hline $\mathrm{AC}-\mathrm{FT}$ & 4720 & 865 & 11470 & 5730 & 57570 & 26110 & 7330 & 1040 & 15800 & 17040 & 3700 & 6740 \\
\hline $\mathrm{a}$ & 0.92 & 3.92 & 1.04 & 2.59 & 6.90 & 3.31 & 1.26 & 0.04 & 0.08 & 0.00 & 0.00 & 0.00 \\
\hline
\end{tabular}

e Estimated.

a Precipitation, in inches. 


\section{NORTH FORK CACHE CREEK NEAR CLEARLAKE OAKS, CA—Continued}

STATISTICS OF MONTHLY MEAN DATA FOR WATER YEARS 1986 - 1999, BY WATER YEAR (WY)

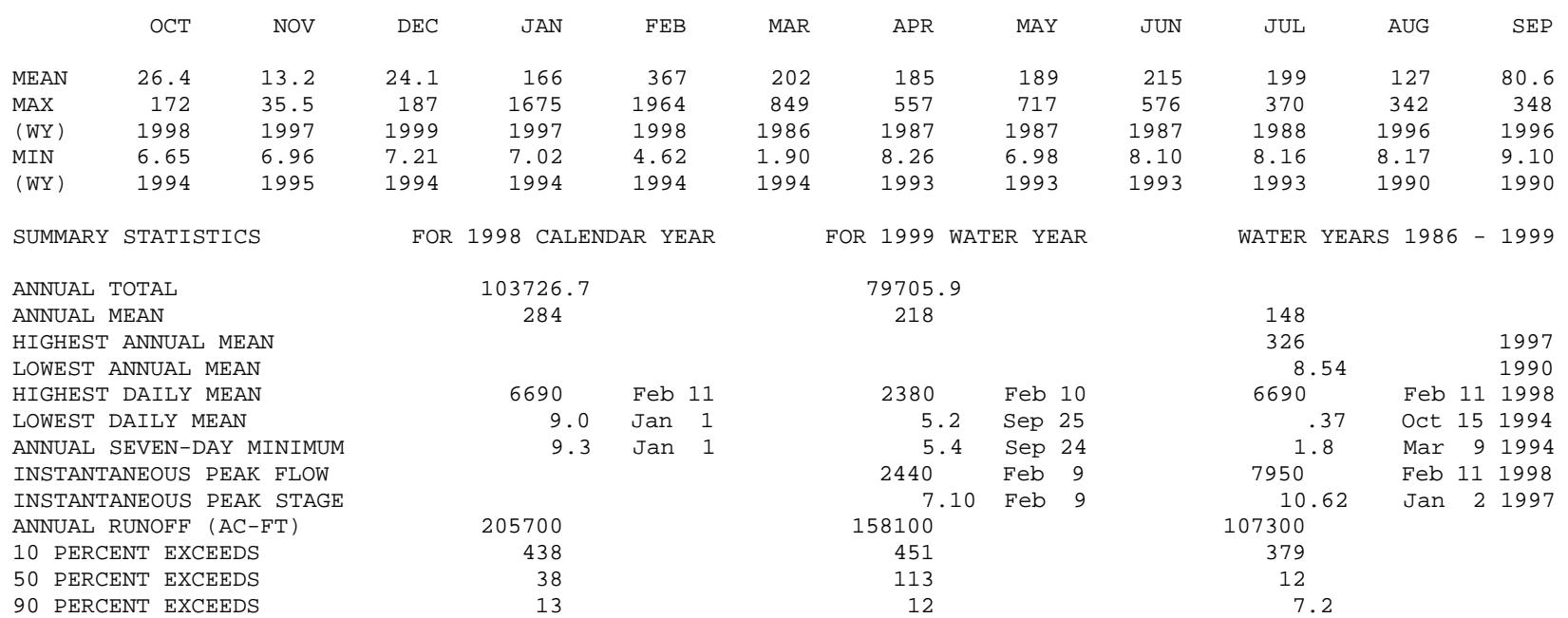


11451715 BEAR CREEK ABOVE HOLSTEN CHIMNEY CANYON, NEAR RUMSEY, CA

LOCATION.-Lat 3857’28", long 122²0’30", in NW 1/4 SE 1/4 sec.19, T.13 N., R.4 W., Colusa County, Hydrologic Unit 18020116, on left bank, downstream side Highway 16 bridge, 2.9 mi upstream from confluence with Cache Creek, 7.4 mi northwest of Rumsey.

DRAINAGE AREA.-94.90 $\mathrm{mi}^{2}$.

PERIOD OF RECORD.-November 1997 to current year.

GAGE.-Water-stage recorder and crest-stage gage. Elevation of gage is $920 \mathrm{ft}$ above sea level, from topographic map.

REMARKS.-Records fair except for estimated daily discharges, which are poor. Some minor diversions upstream from station. See schematic diagram of lower Sacramento River Basin.

EXTREMES FOR PERIOD OF RECORD.-Maximum discharge, 8,510 $\mathrm{ft}^{3} / \mathrm{s}$, Feb. 2, 1998, gage height, $13.57 \mathrm{ft}$ from rating curve extended above $3,000 \mathrm{ft}^{3} / \mathrm{s}$; minimum daily, $1.7 \mathrm{ft}^{3} / \mathrm{s}$, Sept. 28, 1999.

EXTREMES OUTSIDE PERIOD OF RECORD.-Maximum discharge 9,200 ft 3 /s, Jan. 5, 1965.

EXTREMES FOR CURRENT YEAR.-Peak discharges greater than base discharge of 2,000 $\mathrm{ft}^{3} / \mathrm{s}$, or maximum.

$\begin{array}{ccccccc}\text { Date } & \text { Time } & \begin{array}{c}\text { Discharge } \\ \left(\mathrm{ft}^{3} / \mathrm{s}\right)\end{array} & \begin{array}{c}\text { Gage height } \\ (\mathrm{ft})\end{array} & \text { Date } & \text { Time } & \begin{array}{c}\text { Discharge } \\ \left(\mathrm{ft}^{3} / \mathrm{s}\right)\end{array} \\ \text { Feb. } 9 & 0645 & 4,120 & 11.34 & & \text { Gage height } \\ (\mathrm{ft}) & \end{array}$

DISCHARGE, CUBIC FEET PER SECOND, WATER YEAR OCTOBER 1998 TO SEPTEMBER 1999 DAILY MEAN VALUES

\begin{tabular}{|c|c|c|c|c|c|c|c|c|c|c|c|c|}
\hline DAY & $\mathrm{OCT}$ & NOV & $\mathrm{DEC}$ & JAN & FEB & MAR & APR & MAY & JUN & JUL & AUG & SEP \\
\hline 1 & e4.8 & 6.1 & 143 & 7.4 & 28 & 87 & 68 & 28 & 10 & e5.4 & 3.1 & 2.7 \\
\hline 2 & e 4.7 & 6.1 & 38 & 7.4 & 20 & 73 & 58 & 28 & 11 & e5.3 & 3.2 & 3.0 \\
\hline 3 & 4.7 & 6.1 & 34 & 7.6 & 18 & 65 & 53 & 29 & 14 & e 5.2 & 3.2 & 2.8 \\
\hline 4 & 4.5 & 6.1 & 31 & 7.4 & 17 & 58 & 49 & 28 & 13 & e 5.1 & 3.1 & 2.7 \\
\hline 5 & 4.3 & 6.1 & 21 & 7.4 & 16 & 56 & 52 & e27 & 12 & e 5.0 & 3.2 & 2.7 \\
\hline 6 & 4.3 & 5.9 & 21 & 7.7 & 26 & 55 & 55 & e26 & 11 & e 4.9 & 3.6 & 2.7 \\
\hline 7 & 4.6 & 7.3 & 17 & 7.8 & 499 & 51 & 49 & e24 & 10 & e 4.8 & 3.8 & 2.6 \\
\hline 8 & 5.3 & 6.9 & 16 & 7.5 & 143 & 54 & 85 & e23 & 10 & e 4.7 & 3.7 & 2.5 \\
\hline 9 & 5.1 & 6.0 & 15 & 7.2 & 1410 & 175 & 59 & e22 & 10 & e 4.6 & 3.5 & 2.5 \\
\hline 10 & 5.2 & 5.8 & 13 & 7.3 & 183 & 85 & 51 & e21 & 10 & 4.5 & 3.7 & 2.3 \\
\hline 11 & 5.2 & 6.3 & 12 & 7.5 & 86 & 62 & 213 & e20 & 9.6 & 4.4 & 4.0 & 2.5 \\
\hline 12 & 5.1 & 5.8 & 12 & 7.6 & 63 & 52 & 135 & e19 & 9.3 & 4.4 & 3.8 & 2.2 \\
\hline 13 & 5.4 & 5.5 & 11 & 7.7 & 52 & 48 & 76 & e18 & 9.1 & 4.2 & 3.4 & 2.1 \\
\hline 14 & 5.4 & 5.4 & 12 & 7.5 & 49 & 55 & 62 & e17 & 8.9 & 4.0 & 3.5 & 1.9 \\
\hline 15 & 5.7 & 5.5 & 10 & 8.2 & 38 & 169 & 56 & e17 & 8.8 & 3.9 & 3.4 & 2.0 \\
\hline 16 & 5.2 & 5.5 & 9.6 & 10 & 385 & 76 & 52 & e16 & 8.7 & 3.9 & 3.2 & 1.9 \\
\hline 17 & 5.0 & 6.0 & 9.3 & 9.4 & 421 & 61 & 49 & e16 & 8.6 & 3.8 & 3.0 & 2.0 \\
\hline 18 & 5.1 & 5.8 & 9.3 & 9.4 & 143 & 55 & 47 & 15 & 8.2 & 3.8 & 2.9 & 1.9 \\
\hline 19 & 5.3 & 5.2 & 9.1 & 13 & 117 & 53 & 44 & e15 & 8.0 & 3.6 & 2.9 & 1.9 \\
\hline 20 & 5.1 & 5.5 & 8.8 & 32 & 112 & 61 & 44 & e14 & 7.8 & 3.5 & 3.0 & 2.1 \\
\hline 21 & 5.1 & 6.2 & 8.3 & 30 & 365 & 63 & 43 & e14 & 7.5 & 3.5 & 3.2 & 2.2 \\
\hline 22 & 5.4 & 7.2 & 8.0 & 21 & 125 & 55 & 41 & e14 & 7.3 & 3.4 & 3.0 & 2.2 \\
\hline 23 & 5.5 & 15 & 7.9 & 33 & 81 & 52 & 38 & e14 & 6.8 & 3.5 & 3.1 & 2.2 \\
\hline 24 & 7.9 & 28 & 7.9 & 28 & 67 & 142 & 37 & e14 & 6.4 & 3.3 & 3.1 & 2.3 \\
\hline 25 & 9.1 & 17 & 7.7 & 19 & 157 & 612 & 36 & e13 & 6.2 & 3.5 & 3.0 & 2.2 \\
\hline 26 & 6.5 & 11 & 7.9 & 17 & 82 & 171 & 34 & e13 & 6.3 & 3.6 & 3.0 & 2.1 \\
\hline 27 & 6.0 & 10 & 8.1 & 15 & 65 & 107 & 33 & e13 & 6.2 & 3.7 & 3.1 & 1.8 \\
\hline 28 & 5.9 & 9.4 & 7.8 & 13 & 67 & 85 & 33 & 12 & 6.0 & 3.3 & 3.1 & 1.7 \\
\hline 29 & 6.4 & 17 & 7.7 & 13 & --- & 75 & 31 & 11 & 5.8 & 3.3 & 2.8 & 1.8 \\
\hline 30 & 6.1 & 179 & 7.7 & 13 & --- & 70 & 30 & 11 & 5.5 & 3.2 & 2.6 & 1.8 \\
\hline 31 & 5.9 & --- & 7.7 & 34 & --- & 79 & --- & 11 & --- & 3.2 & 2.5 & --- \\
\hline TOTAL & 169.8 & 418.7 & 538.8 & 423.0 & 4835 & 2962 & 1713 & 563 & 262.0 & 126.5 & 99.7 & 67.3 \\
\hline MEAN & 5.48 & 14.0 & 17.4 & 13.6 & 173 & 95.5 & 57.1 & 18.2 & 8.73 & 4.08 & 3.22 & 2.24 \\
\hline MAX & 9.1 & 179 & 143 & 34 & 1410 & 612 & 213 & 29 & 14 & 5.4 & 4.0 & 3.0 \\
\hline MIN & 4.3 & 5.2 & 7.7 & 7.2 & 16 & 48 & 30 & 11 & 5.5 & 3.2 & 2.5 & 1.7 \\
\hline $\mathrm{AC}-\mathrm{FT}$ & 337 & 830 & 1070 & 839 & 9590 & 5880 & 3400 & 1120 & 520 & 251 & 198 & 133 \\
\hline
\end{tabular}

e Estimated. 
11451715 BEAR CREEK ABOVE HOLSTEN CHIMNEY CANYON, NEAR RUMSEY, CA—Continued

STATISTICS OF MONTHLY MEAN DATA FOR WATER YEARS 1998 - 1999, BY WATER YEAR (WY)

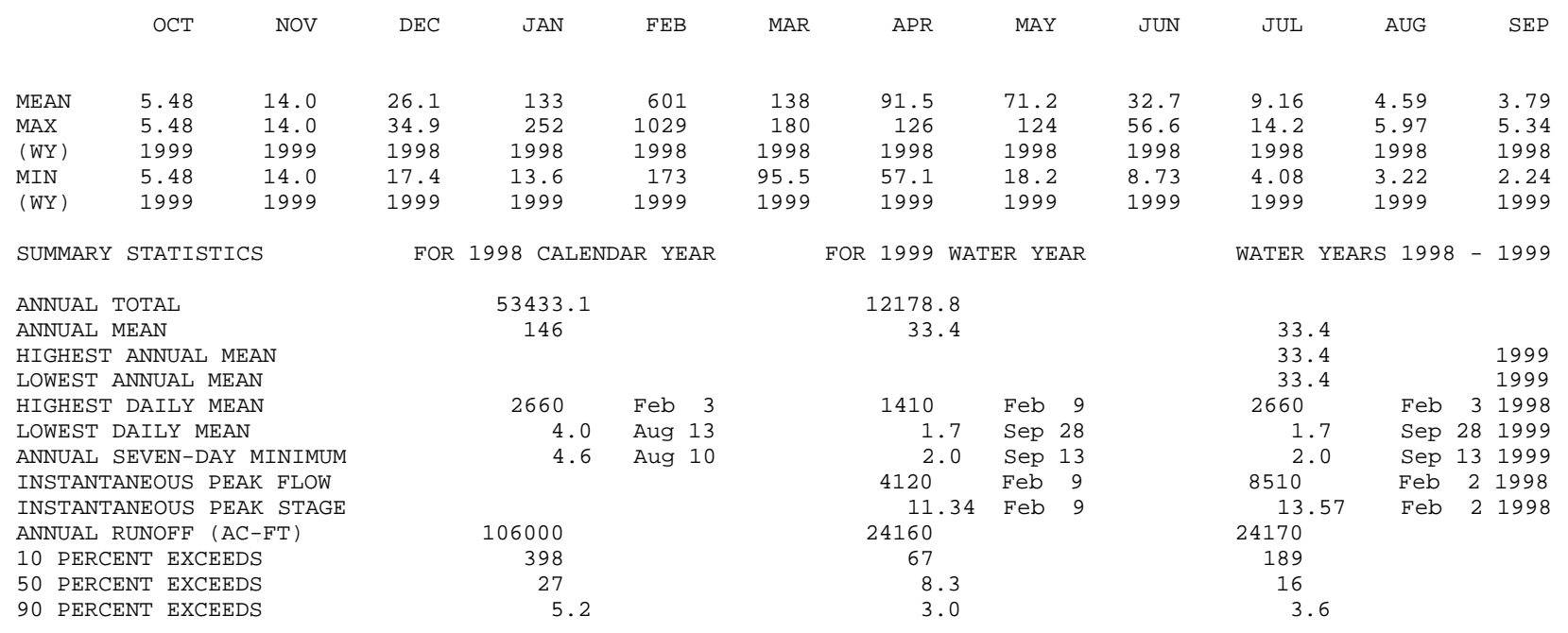




\section{CACHE CREEK AT RUMSEY, CA}

LOCATION.-Lat 3853'26", long 122 '14'14", in Canada de Capay Grant, Yolo County, Hydrologic Unit 18020110, midstream on Arbuckle Bridge at Rumsey.

DRAINAGE AREA.- $-963.55 \mathrm{mi}^{2}$.

PERIOD OF RECORD.-Water years 1976, 1996 to current year.

CHEMICAL DATA: February 1996 to current year.

SEDIMENT DATA: December 1975 to September 1976, February 1996 to current year.

REMARKS.- Records of sediment discharge from December 1975 to September 1976 were obtained from the California Department of Water Resources. DWR has provided discharge data from December 1975 to current year. This station replaced former station 11451760 (Cache Creek above Rumsey) in September 1976 and was reestablished February 1996 for NAWQA water-quality and sediment sampling purposes.

WATER-QUALITY DATA, WATER YEAR OCTOBER 1998 TO SEPTEMBER 1999

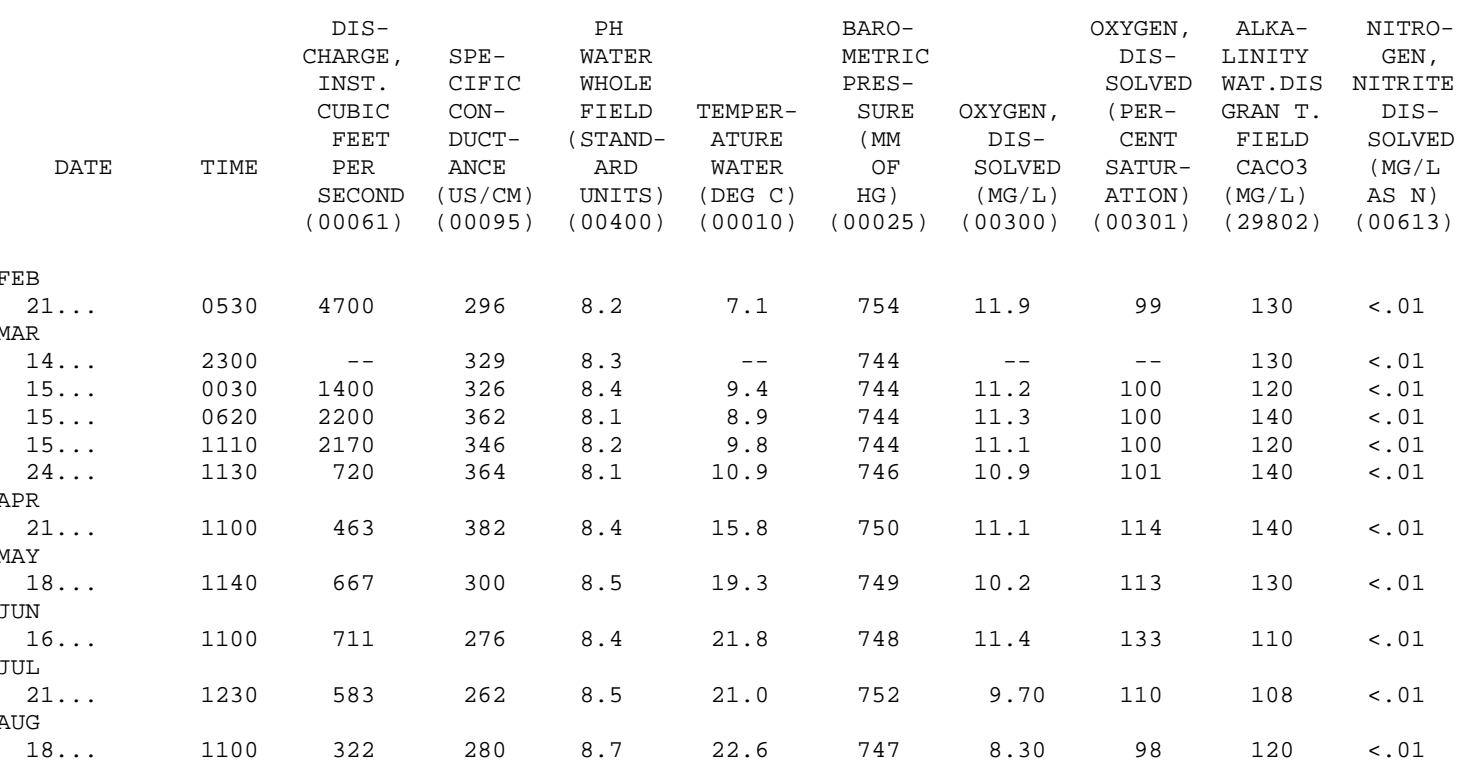

\begin{tabular}{|c|c|c|c|c|c|c|c|c|c|}
\hline \multirow{7}{*}{ DATE } & NITRO- & NITRO- & NITRO- & NITRO- & & \multicolumn{3}{|c|}{ PHOS- } & CARBON, \\
\hline & $\begin{array}{c}\text { GEN, } \\
\text { NO2 }+ \text { NO3 }\end{array}$ & $\begin{array}{c}\text { GEN, } \\
\text { AMMONIA }\end{array}$ & $\begin{array}{l}\text { GEN, AM- } \\
\text { MONIA + }\end{array}$ & $\begin{array}{l}\text { GEN, AM- } \\
\text { MONIA + }\end{array}$ & PHOS- & $\begin{array}{l}\text { PHOS- } \\
\text { PHORUS }\end{array}$ & $\begin{array}{c}\text { PHORUS } \\
\text { ORTHO, }\end{array}$ & $\begin{array}{l}\text { CARBON, } \\
\text { ORGANIC }\end{array}$ & $\begin{array}{l}\text { ORGANIC } \\
\text { SUS- }\end{array}$ \\
\hline & DIS- & DIS- & ORGANIC & ORGANIC & PHORUS & DIS- & DIS- & DIS- & PENDED \\
\hline & SOLVED & SOLVED & TOTAL & DIS . & TOTAL & SOLVED & SOLVED & SOLVED & TOTAL \\
\hline & (MG/L & (MG / L & (MG / L & (MG / L & (MG / L & (MG / L & (MG / L & (MG/L & (MG/L \\
\hline & $\begin{array}{l}\text { AS N) } \\
(00631)\end{array}$ & $\begin{array}{c}\text { AS N) } \\
(00608)\end{array}$ & $\begin{array}{c}\text { AS N) } \\
(00625)\end{array}$ & $\begin{array}{c}\text { AS N) } \\
(00623)\end{array}$ & $\begin{array}{c}\text { AS P ) } \\
(00665)\end{array}$ & $\begin{array}{c}\text { AS P) } \\
(00666)\end{array}$ & $\begin{array}{l}\text { AS P ) } \\
(00671)\end{array}$ & $\begin{array}{c}\text { AS C) } \\
(00681)\end{array}$ & $\begin{array}{c}\text { AS C) } \\
(00689)\end{array}$ \\
\hline & & & & & & & & & \\
\hline
\end{tabular}

\begin{tabular}{|c|c|c|c|c|c|c|c|c|c|}
\hline \multicolumn{10}{|l|}{$\mathrm{FEB}$} \\
\hline $21 \ldots$ & .08 & $<.02$ & .8 & .2 & .53 & .02 & .02 & 3.0 & 3.2 \\
\hline \multicolumn{10}{|l|}{ MAR } \\
\hline $14 \ldots$ & .10 & $<.02$ & .2 & e. 08 & .091 & .009 & $<.01$ & 1.9 & .7 \\
\hline $15 \ldots$ & .11 & $<.02$ & .3 & .1 & .11 & .008 & .01 & 2.1 & .8 \\
\hline $15 \ldots$ & .10 & $<.02$ & .6 & .1 & .26 & .012 & .01 & 2.4 & 1.8 \\
\hline $15 \ldots$ & .08 & .02 & .4 & .1 & .14 & .012 & .01 & 2.6 & .7 \\
\hline $24 \ldots$ & .09 & $<.02$ & .3 & .2 & .051 & .008 & $<.01$ & 2.3 & .4 \\
\hline \multicolumn{10}{|l|}{ APR } \\
\hline $21 \ldots$ & .10 & $<.02$ & .1 & e. 08 & .007 & .006 & $<.01$ & 2.0 & .3 \\
\hline \multicolumn{10}{|l|}{ MAY } \\
\hline $18 \ldots$ & $<.05$ & $<.02$ & .3 & .2 & .065 & .023 & .02 & 4.0 & .7 \\
\hline \multicolumn{10}{|l|}{ JUN } \\
\hline $16 \ldots$ & .06 & $<.02$ & .4 & .3 & $<.004$ & .01 & $<.01$ & 2.8 & 1.0 \\
\hline \multicolumn{10}{|l|}{ JUL } \\
\hline $21 \ldots$ & $<.05$ & $<.02$ & .4 & .3 & .036 & .013 & $<.01$ & 2.9 & .4 \\
\hline \multicolumn{10}{|l|}{ AUG } \\
\hline $18 \ldots$ & $<.05$ & $<.02$ & 1.5 & .4 & .20 & .018 & $<.01$ & 4.2 & -- \\
\hline
\end{tabular}

e Estimated.

$<$ Actual value is known to be less than the value shown. 
11451800 CACHE CREEK AT RUMSEY, CA—Continued

\section{PARTICLE-SIZE DISTRIBUTION, WATER YEAR OCTOBER 1998 TO SEPTEMBER 1999}

\begin{tabular}{|c|c|c|c|c|c|c|}
\hline & & $\begin{array}{c}\text { DIS- } \\
\text { CHARGE, }\end{array}$ & & & $\begin{array}{l}\text { SEDI- } \\
\text { MENT, }\end{array}$ & $\begin{array}{l}\text { SED. } \\
\text { SUSP }\end{array}$ \\
\hline & & INST. & & SEDI- & DIS- & SIEVE \\
\hline & & CUBIC & TEMPER- & MENT, & CHARGE, & DIAM. \\
\hline & & FEET & ATURE & SUS- & SUS- & $\%$ FINER \\
\hline DATE & TIME & PER & WATER & PENDED & PENDED & THAN \\
\hline & & SECOND & $(\mathrm{DEG} \quad \mathrm{C})$ & (MG / L ) & $(\mathrm{T} / \mathrm{DAY})$ & $.062 \mathrm{MN}$ \\
\hline & & $(00061)$ & $(00010)$ & $(80154)$ & $(80155)$ & $(70331)$ \\
\hline $\mathrm{FEB}$ & & & & & & \\
\hline $21 \ldots N$ & 0530 & 4700 & 7.1 & 607 & 7700 & 91 \\
\hline MAR & & & & & & \\
\hline $14 \ldots \mathrm{N}$ & 2300 & -- & -- & 90 & -- & 79 \\
\hline $15 \ldots N$ & 0030 & 1400 & 9.4 & 113 & 427 & 86 \\
\hline $15 \ldots \mathrm{N}$ & 0620 & 2200 & 8.9 & 313 & 1860 & 90 \\
\hline $15 \ldots \mathrm{N}$ & 1110 & 2170 & 9.8 & 158 & 926 & 91 \\
\hline $24 \ldots N$ & 1130 & 720 & 10.9 & 31 & 60 & 82 \\
\hline APR & & & & & & \\
\hline $21 \ldots N$ & 1100 & 463 & 15.8 & 9 & 11 & 88 \\
\hline MAY & & & & & & \\
\hline $18 \ldots \mathrm{N}$ & 1140 & 667 & 19.3 & 27 & 49 & 86 \\
\hline JUN & & & & & & \\
\hline $15 \ldots N$ & 1100 & 711 & 21.8 & 32 & 61 & 84 \\
\hline JUL & & & & & & \\
\hline $21 \ldots N$ & 1230 & 583 & 21.0 & 12 & 19 & 91 \\
\hline AUG & & & & & & \\
\hline $18 \ldots N$ & 1100 & 322 & 22.6 & 39 & 34 & 94 \\
\hline
\end{tabular}

N Suspended-sediment concentration value determined from a sample collected and processed according to National Water-Quality Assessment (NAWQA) protocol. 


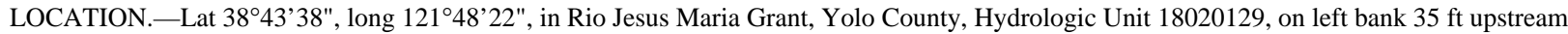
from Interstate 5 highway bridge, $0.5 \mathrm{mi}$ south of Yolo, and $7.3 \mathrm{mi}$ downstream from Moore Dam.

DRAINAGE AREA.-1,139 $\mathrm{mi}^{2}$.

PERIOD OF RECORD.—January 1903 to current year. Records for water year 1903 incomplete; yearly estimate published in WSP 1315-A.

WATER TEMPERATURE: Water years 1959-65, November 1966 to February 1967.

SEDIMENT DATA: Water years 1959-65, November 1966 to February 1967 (daily record), 1986 (periodic record).

REVISED RECORDS.-WSP 1315-A: 1914(M). WSP 1345: 1906. WSP 1445: 1955. WSP 1931: Drainage area.

GAGE.-Water-stage recorder. Datum of gage is sea level. See WSP 2131 for history of changes prior to Apr. 25, 1969. Apr. 25, 1969, to July 1976, at site $765 \mathrm{ft}$ upstream at same datum.

REMARKS.--Records good. Some regulation by Clear Lake (station 11450000) beginning in 1915 and Indian Valley Reservoir beginning in 1974, capacity, 300,000 acre-ft. Diversions for irrigation of about 30,000 acres between Capay and Yolo, from data furnished by Clear Lake Water Co. See schematic diagram of lower Sacramento River Basin.

EXTREMES FOR PERIOD OF RECORD.-Maximum discharge, 41,400 ft ${ }^{3} / \mathrm{s}$, Feb. 25, 1958, gage height, $85.35 \mathrm{ft}$, present datum; maximum stage observed, $86.4 \mathrm{ft}$ (corrected), present datum, Mar. 10, 1904; no flow at times in most years.

DISCHARGE, CUBIC FEET PER SECOND, WATER YEAR OCTOBER 1998 TO SEPTEMBER 1999 DAILY MEAN VALUES

\begin{tabular}{|c|c|c|c|c|c|c|c|c|c|c|c|c|}
\hline DAY & $\mathrm{OCT}$ & NOV & $\mathrm{DEC}$ & JAN & FEB & MAR & $\mathrm{APR}$ & MAY & JUN & JUL & AUG & SEP \\
\hline 1 & 108 & 24 & 633 & 66 & 441 & 4660 & 2870 & 61 & 25 & 8.4 & 43 & 14 \\
\hline 2 & 110 & 48 & 308 & 66 & 369 & 4600 & 1400 & 58 & 36 & 18 & 59 & 23 \\
\hline 3 & 117 & 51 & 180 & 64 & 311 & 4540 & 1020 & 123 & 66 & 38 & 63 & 37 \\
\hline 4 & 118 & 53 & 271 & 61 & 156 & 4280 & 805 & 91 & 48 & 47 & 53 & 29 \\
\hline 5 & 116 & 51 & 314 & 58 & 122 & 3650 & 683 & 93 & 55 & 52 & 45 & 31 \\
\hline 6 & 109 & 52 & 482 & 58 & 120 & 2350 & 685 & 82 & 54 & 52 & 48 & 17 \\
\hline 7 & 118 & 59 & 502 & 57 & 752 & 2240 & 905 & 71 & 40 & 47 & 49 & 5.6 \\
\hline 8 & 92 & 57 & 497 & 57 & 1630 & 1500 & 953 & 77 & 32 & 42 & 48 & 9.1 \\
\hline 9 & 71 & 56 & 500 & 58 & 5320 & 1600 & 1130 & 74 & 40 & 29 & 51 & 12 \\
\hline 10 & 68 & 56 & 476 & 56 & 5060 & 4120 & 791 & 56 & 39 & 31 & 48 & 21 \\
\hline 11 & 70 & 56 & 472 & 55 & 3150 & 3880 & 1410 & 51 & 28 & 40 & 59 & 29 \\
\hline 12 & 68 & 54 & 470 & 54 & 2770 & 2270 & 4310 & 55 & 26 & 38 & 63 & 35 \\
\hline 13 & 62 & 54 & 466 & 54 & 2610 & 1420 & 3890 & 64 & 29 & 20 & 46 & 43 \\
\hline 14 & 69 & 55 & 466 & 53 & 2040 & 1150 & 3580 & 69 & 13 & 19 & 29 & 34 \\
\hline 15 & 74 & 53 & 461 & 54 & 1860 & 1870 & 1340 & 65 & 14 & 26 & 51 & 24 \\
\hline 16 & 75 & 52 & 403 & 56 & 1020 & 2770 & 805 & 54 & 35 & 46 & 49 & 25 \\
\hline 17 & 75 & 52 & 306 & 59 & 2840 & 3810 & 607 & 58 & 34 & 44 & 30 & 23 \\
\hline 18 & 79 & 53 & 201 & 57 & 3960 & 3960 & 668 & 51 & 36 & 44 & 15 & 22 \\
\hline 19 & 68 & 53 & 192 & 60 & 4870 & 3660 & 627 & 46 & 25 & 52 & 13 & 22 \\
\hline 20 & 30 & 53 & 143 & 99 & 4950 & 1690 & 563 & 54 & 26 & 53 & 25 & 33 \\
\hline 21 & 15 & 53 & 124 & 237 & 5070 & 1380 & 362 & 50 & 38 & 46 & 50 & 38 \\
\hline 22 & 13 & 56 & 117 & 198 & 4790 & 1210 & 325 & 31 & 44 & 52 & 48 & 35 \\
\hline 23 & 11 & 60 & 107 & 233 & 4570 & 771 & 250 & 47 & 20 & 35 & 45 & 43 \\
\hline 24 & 15 & 68 & 89 & 554 & 4550 & 811 & 205 & 47 & 18 & 17 & 33 & 30 \\
\hline 25 & 13 & 103 & 82 & 435 & 5110 & 4770 & 156 & 51 & 37 & 24 & 30 & 18 \\
\hline 26 & 13 & 100 & 78 & 389 & 5140 & 5090 & 106 & 47 & 37 & 37 & 31 & 30 \\
\hline 27 & 13 & 82 & 75 & 364 & 4610 & 4810 & 118 & 57 & 55 & 46 & 37 & 58 \\
\hline 28 & 14 & 75 & 72 & 345 & 4450 & 4660 & 145 & 70 & 62 & 63 & 38 & 67 \\
\hline 29 & 13 & 75 & 71 & 335 & --- & 4540 & 106 & 62 & 53 & 61 & 41 & 63 \\
\hline 30 & 12 & 91 & 69 & 333 & --- & 4140 & 78 & 56 & 24 & 31 & 38 & 61 \\
\hline 31 & 13 & --- & 68 & 355 & --- & 4100 & --- & 31 & --- & 21 & 30 & --- \\
\hline TOTAL & 1842 & 1805 & 8695 & 4980 & 82641 & 96302 & 30893 & 1902 & 1089 & 1179.4 & 1308 & 931.7 \\
\hline MEAN & 59.4 & 60.2 & 280 & 161 & 2951 & 3107 & 1030 & 61.4 & 36.3 & 38.0 & 42.2 & 31.1 \\
\hline MAX & 118 & 103 & 633 & 554 & 5320 & 5090 & 4310 & 123 & 66 & 63 & 63 & 67 \\
\hline MIN & 11 & 24 & 68 & 53 & 120 & 771 & 78 & 31 & 13 & 8.4 & 13 & 5.6 \\
\hline $\mathrm{AC}-\mathrm{FT}$ & 3650 & 3580 & 17250 & 9880 & 163900 & 191000 & 61280 & 3770 & 2160 & 2340 & 2590 & 1850 \\
\hline
\end{tabular}


11452500 CACHE CREEK AT YOLO, CA—Continued

STATISTICS OF MONTHLY MEAN DATA FOR WATER YEARS 1903 - 1999, BY WATER YEAR (WY)

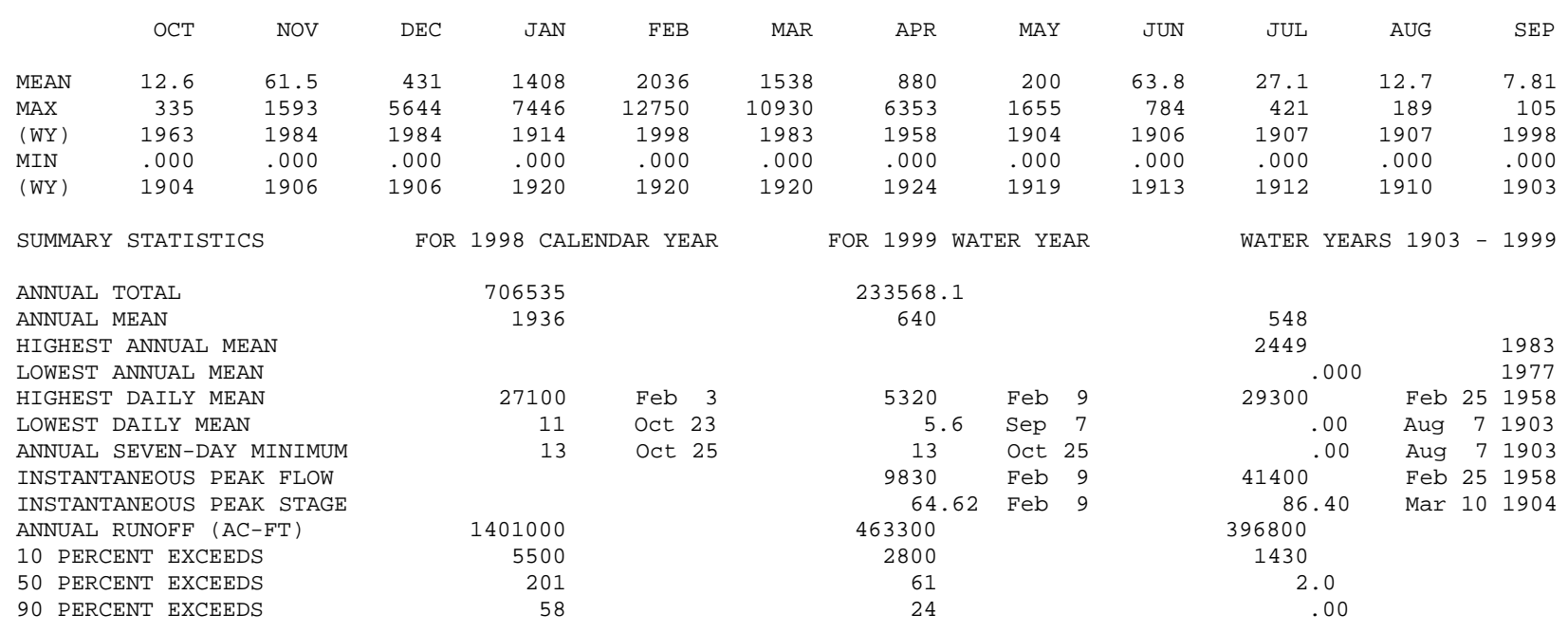


11453000 YOLO BYPASS NEAR WOODLAND, CA

LOCATION.-Lat 3840'40", long 12138'35", unsurveyed, Yolo County, Hydrologic Unit 18020109, on left bank $300 \mathrm{ft}$ upstream from Sacramento and Woodland Railroad Bridge, 6 mi upstream from Sacramento Bypass, 6 mi downstream from Fremont Weir, and 7 mi east of Woodland.

PERIOD OF RECORD.-October 1939 to current year (since October 1977, high-flow records only). Monthly discharge only for some periods, published in WSP 1315-A.

SEDIMENT DATA: Water years 1957-61, 1980.

REVISED RECORDS.-WDR CA-96-4: 1995(M).

GAGE.-Water-stage recorder. Datum of gage is $3.41 \mathrm{ft}$ below sea level. Prior to Dec. 17, 1941, nonrecording gage, and Dec. 18-31, 1941, waterstage recorder, at datum $0.73 \mathrm{ft}$ higher. Prior to Sept. 30, 1977, a supplementary water-stage recorder 6 mi downstream at different datum recorded low flow.

REMARKS.-Flow is from Cache Creek and Knights Landing Ridge Cut plus floodwater passing over Fremont Weir. Beginning October 1977, only flows above $1,000 \mathrm{ft}^{3} / \mathrm{s}$ are computed. See schematic diagram of lower Sacramento River Basin.

EXTREMES FOR PERIOD OF RECORD.-Maximum discharge, 374,000 ft $3 / \mathrm{s}$, Feb. 20, 1986, gage height, $34.87 \mathrm{ft}$; no flow at times in several years.

EXTREMES FOR CURRENT YEAR.-Maximum discharge, 33,900 ft³/s, Feb. 10, gage height, $26.47 \mathrm{ft}$.

DISCHARGE, CUBIC FEET PER SECOND, WATER YEAR OCTOBER 1998 TO SEPTEMBER 1999

\begin{tabular}{|c|c|c|c|c|c|c|c|c|c|c|c|c|}
\hline DAY & $\mathrm{OCT}$ & NOV & $\mathrm{DEC}$ & JAN & FEB & MAR & APR & MAY & JUN & JUL & AUG & SEP \\
\hline 1 & --- & --- & 1280 & --- & --- & 12700 & 4080 & --- & --- & --- & --- & --- \\
\hline 2 & --- & --- & 1880 & --- & --- & 16300 & 3240 & --- & --- & --- & --- & --- \\
\hline 3 & --- & --- & 2110 & --- & --- & 18400 & 2450 & --- & --- & --- & --- & --- \\
\hline 4 & --- & --- & 1960 & --- & --- & 20000 & 1940 & --- & --- & --- & --- & --- \\
\hline 5 & --- & --- & 1760 & --- & --- & 19700 & 1540 & --- & --- & --- & --- & --- \\
\hline 6 & --- & --- & 1550 & --- & --- & 19100 & 1710 & --- & --- & --- & --- & --- \\
\hline 7 & --- & --- & 1460 & --- & --- & 17600 & 1450 & --- & --- & --- & --- & --- \\
\hline 8 & --- & --- & 1660 & --- & --- & 15700 & 1450 & --- & --- & --- & --- & --- \\
\hline 9 & --- & --- & 1620 & --- & 1380 & 14200 & 1620 & --- & --- & --- & --- & --- \\
\hline 10 & --- & --- & 1160 & --- & 15400 & 12500 & 1620 & --- & --- & --- & --- & --- \\
\hline 11 & --- & --- & --- & --- & 31100 & 10200 & 1600 & --- & --- & --- & --- & --1 \\
\hline 12 & --- & --- & --- & --- & 27700 & 7460 & 2790 & --- & --- & --- & --- & --- \\
\hline 13 & --- & --- & --- & --- & 21200 & 5090 & 3960 & --- & --- & --- & --- & --- \\
\hline 14 & --- & --- & --- & --- & 15500 & 3620 & 4070 & --- & --- & --- & --- & --- \\
\hline 15 & --- & --- & --- & --- & 10700 & 2520 & 3450 & --- & --- & --- & --- & --- \\
\hline 16 & --- & --- & --- & --- & 6900 & 2890 & 2350 & --- & --- & --- & --- & --- \\
\hline 17 & --- & --- & --- & --- & 6440 & 3510 & 1770 & --- & --- & --- & --- & --- \\
\hline 18 & --- & --- & --- & --- & 20800 & 3950 & 1410 & --- & --- & --- & --- & --- \\
\hline 19 & --- & --- & --- & --- & 29200 & 3970 & 1200 & --- & --- & --- & --- & --- \\
\hline 20 & --- & --- & --- & --- & 31300 & 3550 & 1150 & --- & --- & --- & --- & --- \\
\hline 21 & --- & --- & --- & --- & 31500 & 2520 & 1180 & --- & --- & --- & --- & --- \\
\hline 22 & --- & --- & --- & --- & 33100 & 2150 & 1130 & --- & --- & --- & --- & --- \\
\hline 23 & --- & --- & --- & --- & 30300 & 1870 & --- & --- & --- & --- & --- & --- \\
\hline 24 & --- & --- & --- & --- & 23800 & 1550 & --- & --- & --- & --- & --- & --- \\
\hline 25 & --- & 1120 & --- & --- & 18700 & 2430 & --- & --- & --- & --- & --- & --- \\
\hline 26 & --- & 1110 & --- & --- & 16400 & 4770 & --- & --- & --- & --- & --- & --- \\
\hline 27 & --- & 1090 & --- & --- & 14400 & 5150 & --- & --- & --- & --- & --- & --- \\
\hline 28 & --- & 1060 & --- & --- & 12500 & 5000 & --- & --- & --- & --- & --- & --- \\
\hline 29 & --- & --- & --- & --- & --- & 4740 & --- & --- & --- & --- & --- & --- \\
\hline 30 & --- & --- & --- & --- & --- & 4490 & --- & --- & --- & --- & --- & --- \\
\hline 31 & --- & --- & --- & --- & --- & 4290 & --- & --- & --- & --- & --- & --- \\
\hline TOTAL & --- & --- & --- & --- & --- & 251920 & --- & --- & --- & --- & --- & -- \\
\hline MEAN & --- & --- & --- & --- & --- & 8126 & --- & --- & --- & --- & --- & --- \\
\hline MAX & --- & --- & --- & --- & --- & 20000 & --- & --- & --- & --- & --- & --- \\
\hline MIN & --- & --- & --- & --- & --- & 1550 & --- & --- & --- & --- & --- & --- \\
\hline $\mathrm{AC}-\mathrm{FT}$ & --- & --- & --- & --- & --- & 499700 & --- & --- & --- & --- & --- & --- \\
\hline
\end{tabular}

STATISTICS OF MONTHLY MEAN DATA FOR WATER YEARS 1946 - 1977, BY WATER YEAR (WY)

\begin{tabular}{|c|c|c|c|c|c|c|c|c|c|c|c|c|}
\hline MEAN & 441 & 738 & 5638 & 13230 & 11240 & 3398 & 3849 & 430 & 144 & 20.7 & 26.1 & 51.0 \\
\hline MAX & 13420 & 10890 & 48790 & 86470 & 92890 & 27910 & 37310 & 4546 & 1420 & 107 & 84.9 & 155 \\
\hline (WY) & 1963 & 1951 & 1956 & 1970 & 1958 & 1958 & 1958 & 1952 & 1967 & 1958 & 1958 & 1954 \\
\hline MIN & 1.01 & 2.19 & .92 & 2.43 & .88 & 3.55 & .083 & .55 & .53 & .000 & .000 & .63 \\
\hline (WY) & 1977 & 1960 & 1977 & 1977 & 1977 & 1977 & 1976 & 1977 & 1977 & 1966 & 1966 & 1977 \\
\hline
\end{tabular}

SUMMARY STATISTICS

WATER YEARS 1946 - 1977

ANNUAL MEAN

HIGHEST ANNUAL MEAN

LOWEST ANNUAL MEAN

HIGHEST DAILY MEAN

LOWEST DAILY MEAN

ANNUAL SEVEN-DAY MINIMUM

INSTANTANEOUS PEAK FLOW

INSTANTANEOUS PEAK STAGE

ANNUAL RUNOFF (AC-FT)

10 PERCENT EXCEEDS

50 PERCENT EXCEEDS

90 PERCENT EXCEEDS

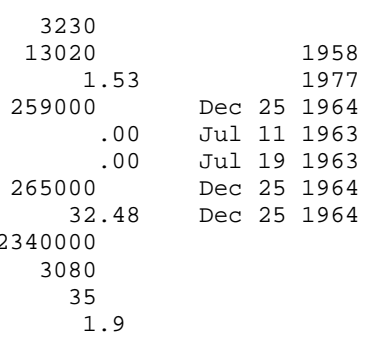




\section{$11453120^{`}$ YOLO BYPASS AT INTERSTATE HIGHWAY 80, NEAR WEST SACRAMENTO, CA}

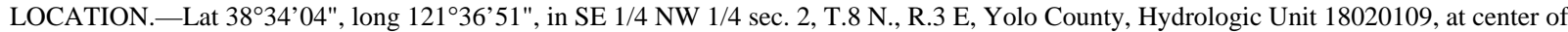
bikepath bridge on Interstate Highway 80, 1.9 mi west of West Capitol Avenue, approximately 2.8 mi west of West Sacramento.

DRAINAGE AREA.-Indeterminate.

PERIOD OF RECORD.—January 1997 to current year.

CHEMICAL DATA: January 1997 to current year.

SEDIMENT DATA: January 1997 to current year.

INSTRUMENTATION.-None.

REMARKS.-Discharge values were determined from USGS station 11453000, Yolo Bypass Near Woodland, and California Department of Water Resourses discharge data.

WATER-QUALITY DATA, WATER YEAR OCTOBER 1998 TO SEPTEMBER 1999

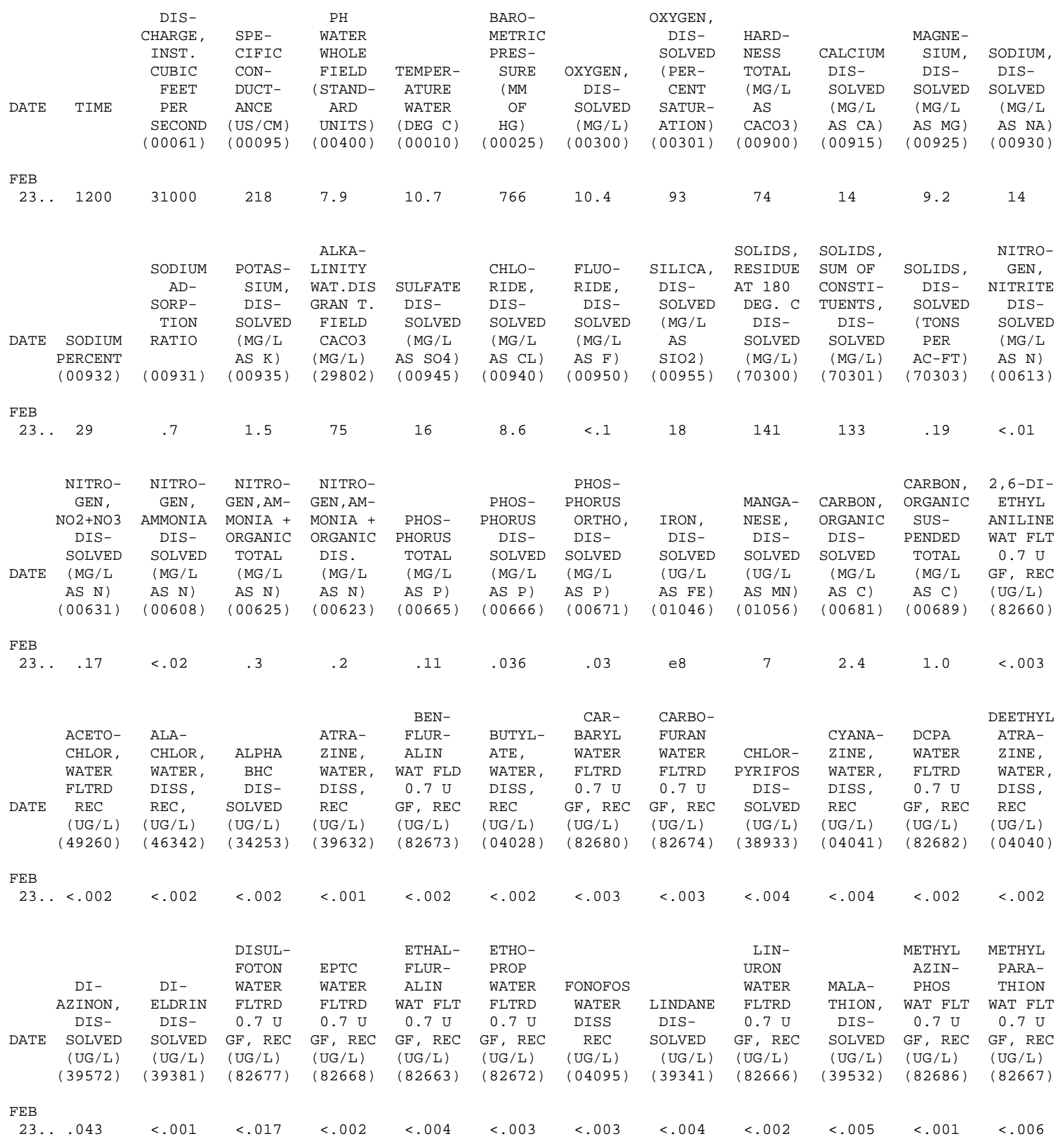

e Estimated.

$<$ Actual value is known to be less than value shown. 
11453120 YOLO BYPASS AT INTERSTATE HIGHWAY 80, NEAR WEST SACRAMENTO, CA—Continued

\section{WATER-QUALITY DATA, WATER YEAR OCTOBER 1998 TO SEPTEMBER 1999}

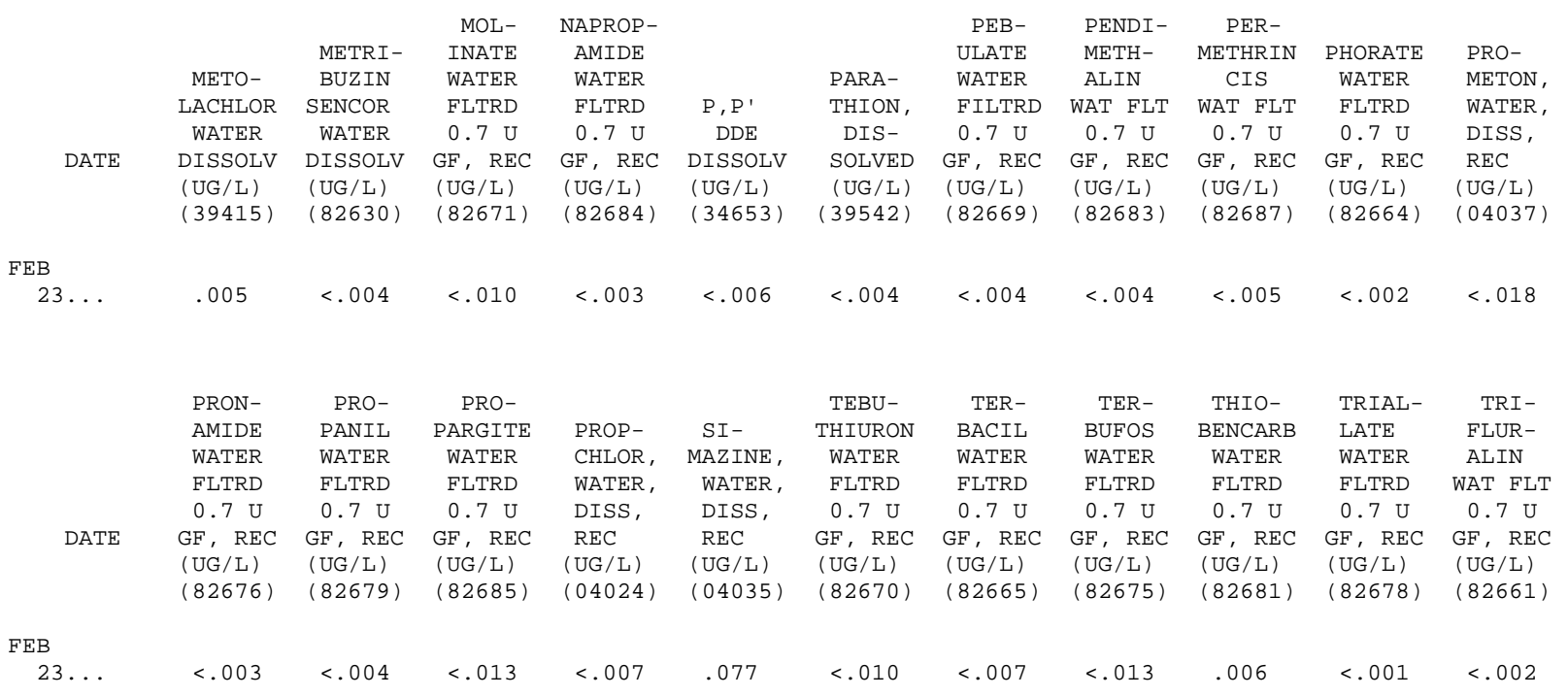

PARTICLE-SIZE DISTRIBUTION, WATER YEAR OCTOBER 1998 TO SEPTEMBER 1999

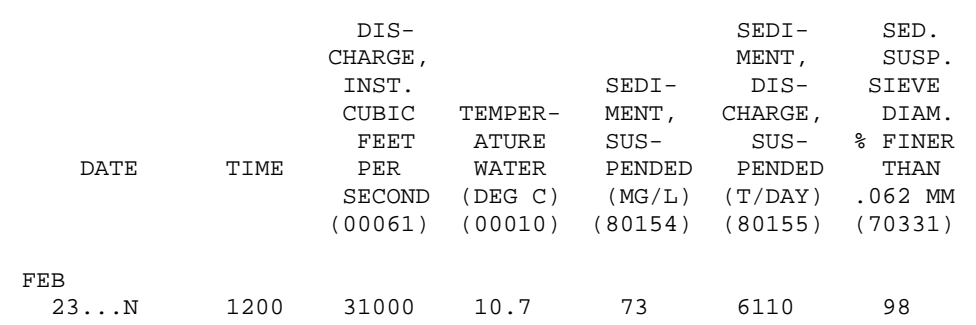

$<$ Actual value is known to be less than value shown.

N Suspended-sediment concentration value determined from a sample collected and processed according to National Water-Quality Assessment (NAWQA) protocol. 


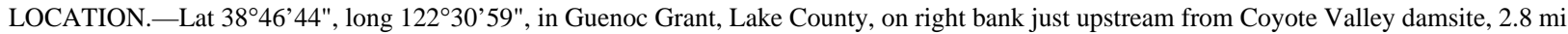
upstream from Soda Creek and $3.2 \mathrm{mi}$ downstream from highway bridge at Guenoc.

DRAINAGE AREA.-113 $\mathrm{mi}^{2}$.

PERIOD OF RECORD.-February 1904 to September 1906, July 1930 to September 1976, and April 1998 to current year. Monthly discharge only for some periods, published in WSP 1315-A.

REVISED RECORDS.--WSP 1285: 1937(M), 1938, 1940, 1943(M), 1951(M).

GAGE.-Water-stage recorder. Datum of gage is $911.18 \mathrm{ft}$ above sea level. February 1904 to September 1906, nonrecording gage 0.2 mi upstream at different datum, July 1930 to September 1976, at datum $3.00 \mathrm{ft}$ higher.

REMARKS.--Records good. Some regulation by Hartmann Dam on Coyote Creek since 1969, capacity, 3,000 acre-ft; diversions and ground-water withdrawals for irrigation of about 1,600 acres above station. See schematic diagram of lower Sacramento River Basin.

EXTREMES FOR PERIOD OF RECORD.-Maximum discharge, 32,000 ft $3 / \mathrm{s}$, Dec. 11, 1937, gage height, $22.7 \mathrm{ft}$, from rating curve extended above $13,000 \mathrm{ft}^{3} / \mathrm{s}$; no flow many days in 1964, 1970, 1974-76.

EXTREMES FOR CURRENT YEAR.-Peak discharges greater than base discharge of $5,000 \mathrm{ft}^{3} / \mathrm{s}$, or maximum:

$\begin{array}{cccccccc}\text { Date } & \text { Time } & \begin{array}{c}\text { Discharge } \\ \left(\mathrm{ft}^{3} / \mathrm{s}\right)\end{array} & \begin{array}{c}\text { Gage height } \\ (\mathrm{ft})\end{array} & \text { Date } & \text { Time } & \begin{array}{c}\text { Discharge } \\ \left(\mathrm{ft}^{3} / \mathrm{s}\right)\end{array} & \begin{array}{c}\text { Gage height } \\ (\mathrm{ft})\end{array} \\ \text { Feb. } 9 & 0500 & 16,300 & 19.61 & & & \end{array}$

DISCHARGE, CUBIC FEET PER SECOND, WATER YEAR OCTOBER 1998 TO SEPTEMBER 1999

DAILY MEAN VALUES

\begin{tabular}{|c|c|c|c|c|c|c|c|c|c|c|c|c|}
\hline DAY & OCT & NOV & $\mathrm{DEC}$ & JAN & FEB & MAR & APR & MAY & JUN & JUL & AUG & SEP \\
\hline 1 & 9.2 & 9.4 & 946 & 50 & 282 & 906 & 359 & 117 & 41 & 12 & 3.3 & 2.2 \\
\hline 2 & 9.7 & 9.2 & 538 & 47 & 215 & 642 & 316 & 113 & 42 & 11 & 3.0 & 2.3 \\
\hline 3 & 9.7 & 9.2 & 1360 & 46 & 188 & 711 & 283 & 117 & 44 & 11 & 2.8 & 1.9 \\
\hline 4 & 9.4 & 9.9 & 544 & 45 & 170 & 543 & 258 & 111 & 42 & 11 & 3.3 & 2.0 \\
\hline 5 & 8.5 & 11 & 333 & 44 & 154 & 464 & 298 & 104 & 39 & 13 & 3.1 & 2.0 \\
\hline 6 & 7.9 & 12 & 355 & 43 & 1710 & 410 & 308 & 97 & 37 & 12 & 3.4 & 1.8 \\
\hline 7 & 7.7 & 28 & 233 & 42 & 4110 & 364 & 261 & 91 & 35 & 11 & 4.1 & .99 \\
\hline 8 & 7.2 & 36 & 205 & 41 & 1930 & 651 & 502 & 88 & 35 & 10 & 5.3 & .63 \\
\hline 9 & 5.3 & 24 & 172 & 40 & 6780 & 1180 & 423 & 86 & 34 & 9.7 & 4.8 & 1.3 \\
\hline 10 & 5.5 & 21 & 145 & 39 & 1660 & 692 & 356 & 82 & 33 & 10 & 4.2 & 2.0 \\
\hline 11 & 5.6 & 21 & 127 & 39 & 1000 & 541 & 1550 & 79 & 31 & 9.6 & 4.2 & 1.1 \\
\hline 12 & 6.4 & 19 & 116 & 38 & 724 & 453 & 838 & 74 & 29 & 8.7 & 3.8 & 1.8 \\
\hline 13 & 7.4 & 17 & 110 & 37 & 569 & 399 & 585 & 72 & 27 & 8.5 & 2.8 & 1.6 \\
\hline 14 & 7.1 & 17 & 120 & 37 & 496 & 461 & 470 & 71 & 26 & 8.1 & 2.7 & 1.2 \\
\hline 15 & 7.2 & 17 & 103 & 37 & 405 & 541 & 397 & 69 & 25 & 5.7 & 2.4 & .50 \\
\hline 16 & 5.0 & 17 & 95 & 44 & 1020 & 402 & 346 & 67 & 24 & 6.2 & 2.9 & 1.8 \\
\hline 17 & 5.9 & 20 & 89 & 46 & 1560 & 346 & 305 & 66 & 24 & 6.7 & 3.6 & 1.8 \\
\hline 18 & 5.7 & 20 & 84 & 516 & 1050 & 310 & 272 & 65 & 22 & 6.2 & 3.3 & 1.9 \\
\hline 19 & 5.7 & 19 & 79 & 856 & 860 & 290 & 245 & 63 & 22 & 5.8 & 3.1 & 1.9 \\
\hline 20 & 5.4 & 18 & 75 & 1470 & 904 & 312 & 223 & 61 & 21 & 6.2 & 3.0 & 1.5 \\
\hline 21 & 5.7 & 19 & 71 & 738 & 1540 & 278 & 206 & 59 & 20 & 6.8 & 3.0 & 1.3 \\
\hline 22 & 5.4 & 29 & 67 & 557 & 1050 & 258 & 193 & 55 & 20 & 7.2 & 2.9 & 1.5 \\
\hline 23 & 5.5 & 1390 & 65 & 1490 & 780 & 309 & 179 & 53 & 18 & 7.2 & 2.8 & 2.0 \\
\hline 24 & 11 & 520 & 63 & 624 & 649 & 1030 & 168 & 52 & 17 & 7.3 & 1.4 & 2.0 \\
\hline 25 & 13 & 169 & 60 & 415 & 1390 & 1970 & 158 & 51 & 17 & 6.7 & 1.7 & 1.8 \\
\hline 26 & 10 & 125 & 59 & 319 & 806 & 906 & 150 & 48 & 17 & 5.0 & 1.3 & 2.2 \\
\hline 27 & 8.7 & 145 & 58 & 253 & 637 & 642 & 139 & 46 & 17 & 4.3 & 1.4 & 2.4 \\
\hline 28 & 9.0 & 101 & 56 & 208 & 834 & 514 & 134 & 44 & 16 & 5.2 & 3.0 & 2.0 \\
\hline 29 & 10 & 209 & 54 & 181 & --- & 439 & 129 & 43 & 15 & 4.5 & 3.0 & .97 \\
\hline 30 & 8.0 & 2570 & 53 & 164 & --- & 412 & 123 & 43 & 14 & 4.9 & 2.6 & .44 \\
\hline 31 & 8.4 & --- & 51 & 464 & --- & 450 & --- & 42 & --- & 4.8 & 2.3 & --- \\
\hline TOTAL & 236.2 & 5631.7 & 6486 & 8970 & 33473 & 17826 & 10174 & 2229 & 804 & 246.3 & 94.5 & 48.83 \\
\hline MEAN & 7.62 & 188 & 209 & 289 & 1195 & 575 & 339 & 71.9 & 26.8 & 7.95 & 3.05 & 1.63 \\
\hline MAX & 13 & 2570 & 1360 & 1490 & 6780 & 1970 & 1550 & 117 & 44 & 13 & 5.3 & 2.4 \\
\hline MIN & 5.0 & 9.2 & 51 & 37 & 154 & 258 & 123 & 42 & 14 & 4.3 & 1.3 & .44 \\
\hline $\mathrm{AC}-\mathrm{FT}$ & 469 & 11170 & 12860 & 17790 & 66390 & 35360 & 20180 & 4420 & 1590 & 489 & 187 & 97 \\
\hline
\end{tabular}


11453500 PUTAH CREEK NEAR GUENOC, CA—Continued

STATISTICS OF MONTHLY MEAN DATA FOR WATER YEARS 1905 - 1999, BY WATER YEAR (WY)

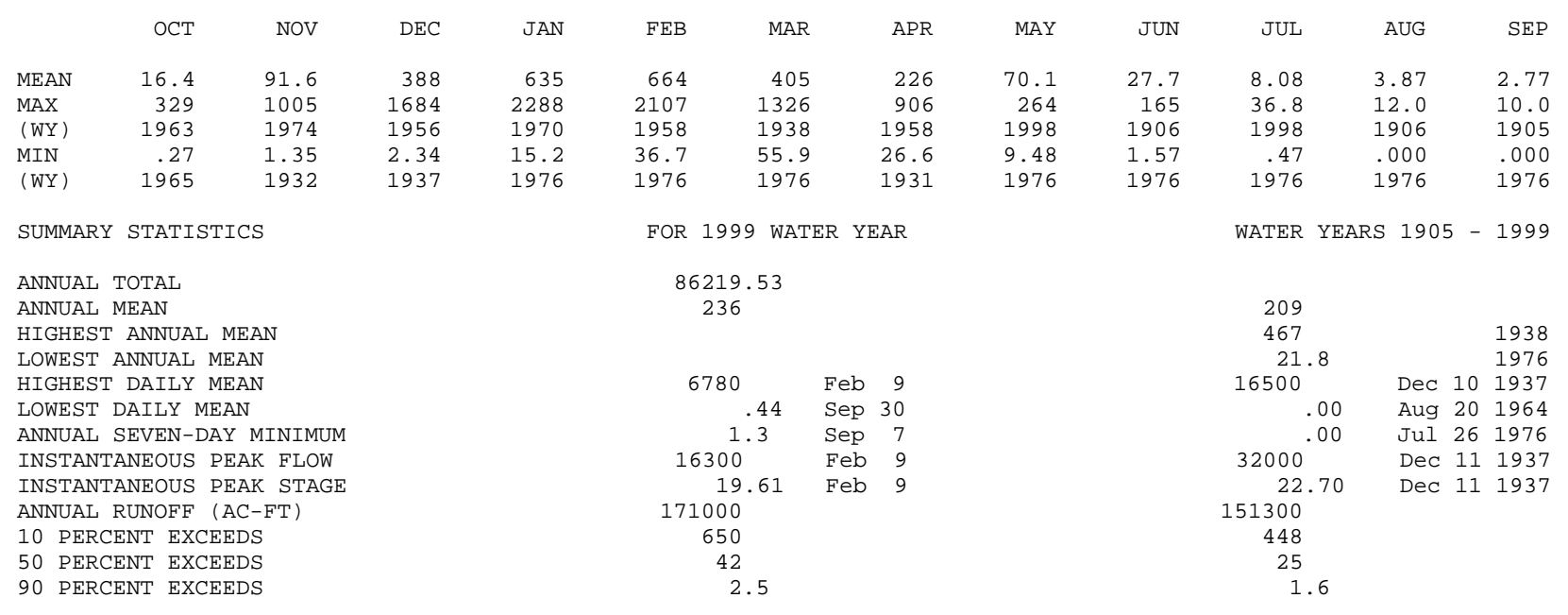




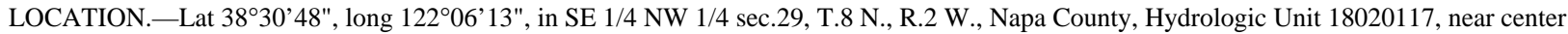
of Monticello Dam on Putah Creek and 7.4 mi west of Winters.

DRAINAGE AREA.-566 $\mathrm{mi}^{2}$.

PERIOD OF RECORD.- -January 1957 to current year.

REVISED RECORDS.-WSP 1735: 1958-60. WSP 1931: Drainage area.

GAGE.-Water-stage recorder. Datum of gage is sea level (levels by U.S. Bureau of Reclamation).

REMARKS.--Reservoir is formed by concrete arch-gravity dam completed November 1956. Usable capacity, 1,592,000 acre-ft between elevations $253.25 \mathrm{ft}$, invert of outlet valves, and $440 \mathrm{ft}$ crest of glory-hole spillway. Dead storage, 10,340 acre-ft. Water is released down Putah Creek and is diverted into Putah South Canal for irrigation of about 46,000 acres in the lower Sacramento Valley. Total diverted during current year was 204,735 acre-ft. Releases for irrigation began in May 1959. Records, including extremes, show total contents at 2400 hours. See schematic diagram of lower Sacramento River Basin.

COOPERATION.-Records provided by U.S. Bureau of Reclamation.

EXTREMES FOR PERIOD OF RECORD.-Maximum contents, 1,733,500 acre-ft, Mar. 2, 1983, elevation, $446.67 \mathrm{ft}$; minimum since irrigation pool first filled, 422,130 acre-ft, Dec. 1, 1992, elevation, $361.73 \mathrm{ft}$.

EXTREMES FOR CURRENT YEAR.-Maximum contents, 1,621,400 acre-ft, Mar. 26, elevation, 440.99 ft; minimum, 1,392,300 acre-ft, Sept 30, elevation, $428.80 \mathrm{ft}$.

\begin{tabular}{lllrrr}
\multicolumn{7}{c}{ Capacity table (elevation, in feet, and contents, in acre-feet) } \\
(Based on survey by U.S. Bureau of Reclamation in 1956) \\
360 & 404,550 & 390 & 765,730 & 420 & $1,236,000$ \\
370 & 511,760 & 400 & 911,200 & 430 & $1,414,200$ \\
380 & 632,360 & 410 & $1,068,100$ & 450 & $1,799,900$
\end{tabular}

RESERVOIR STORAGE (ACRE-FEET), WATER YEAR OCTOBER 1998 TO SEPTEMBER 1999 DAILY OBSERVATION AT 2400 HOURS

\begin{tabular}{|c|c|c|c|c|c|c|c|c|c|c|c|c|}
\hline DAY & $\mathrm{OCT}$ & NOV & $\mathrm{DEC}$ & JAN & FEB & MAR & APR & MAY & JUN & JUL & AUG & SEP \\
\hline 1 & 1455700 & 1437300 & 1457500 & 1462700 & 1483200 & 1612100 & 1616400 & 1599800 & 1564300 & 1520400 & 1467200 & 1422400 \\
\hline 2 & 1454700 & 1436800 & 1458100 & 1462700 & 1484000 & 1612100 & 1615000 & 1599400 & 1563100 & 1518700 & 1465900 & 1421200 \\
\hline 3 & 1454000 & 1436800 & 1461000 & 1463300 & 1484000 & 1612500 & 1614100 & 1598400 & 1562400 & 1517000 & 1464400 & 1420000 \\
\hline 4 & 1454000 & 1436800 & 1463100 & 1463800 & 1484700 & 1612300 & 1613300 & 1597100 & 1561000 & 1515100 & 1463100 & 1418600 \\
\hline 5 & 1453600 & 1436200 & 1464900 & 1463300 & 1485300 & 1611800 & 1612500 & 1596100 & 1559500 & 1513800 & 1461000 & 1417700 \\
\hline 6 & 1452100 & 1435900 & 465700 & 1462200 & 1500600 & 1611200 & 1612100 & 1595500 & 1558600 & 1512100 & 1459400 & 1415100 \\
\hline 7 & 1451800 & 1437000 & 465900 & 1461200 & 1518500 & 1610600 & 1611000 & 1594800 & 1557400 & 1510200 & 1458100 & 1415500 \\
\hline 8 & 1451200 & 1437000 & 1466400 & 1461000 & 1544800 & 1611600 & 1612100 & 1593600 & 1556100 & 1508500 & 1456000 & 1413800 \\
\hline 9 & 1450500 & 1436800 & 1467500 & 1460500 & 1566400 & 1614100 & 1611900 & 1592500 & 1554900 & 1506800 & 1454700 & 1412700 \\
\hline 10 & 1450100 & 1436800 & 1468100 & 1460100 & 1571400 & 1614800 & 1611600 & 1591500 & 1553400 & 1505100 & 1453800 & 1411600 \\
\hline 11 & 448600 & 1435900 & 1469000 & 1461000 & 1573800 & 1614800 & 1616800 & 1590500 & 1552300 & 1503300 & 2700 & 1410500 \\
\hline 12 & 1447700 & 1435900 & 1469600 & 1461400 & 1574800 & 1614300 & 1617900 & 1589600 & 1550000 & 1501600 & 51000 & 09800 \\
\hline 13 & 1447700 & 1435300 & 1471500 & 1460900 & 1575200 & 1613500 & 1617800 & 1588600 & 1548600 & 1500100 & 1449700 & 1408500 \\
\hline 14 & 1447300 & 1435300 & 1472200 & 1460300 & 1575400 & 1613700 & 1617200 & 1587100 & 1547500 & 1498200 & 1448200 & 1407400 \\
\hline 15 & 1447000 & 1435100 & 1472900 & 1460300 & 1575400 & 1613700 & 1616600 & 1585900 & 1546400 & 1496300 & 1446600 & 1406500 \\
\hline 16 & 446000 & $\$ 100$ & 200 & 9900 & 9200 & & & & & & & 5400 \\
\hline 17 & 1444700 & 1434900 & 1463600 & 1459900 & 1581500 & 1612300 & 1614700 & 3600 & 15439 & 1492900 & 4200 & 4300 \\
\hline 18 & 1443800 & 1434800 & 1463600 & 1460300 & 1586500 & 1611600 & 1614100 & 1582500 & 1542600 & 1491400 & 1442500 & 3200 \\
\hline 19 & 1443100 & 1434800 & 1462900 & 1461400 & 1588000 & 1611200 & 1612900 & 31100 & 1539700 & 1489800 & 0900 & 2100 \\
\hline 20 & 1442900 & 1434600 & 1462300 & 1467000 & 1591700 & 1610600 & 1611900 & 1580200 & 1538200 & 1488200 & 1439400 & 1400800 \\
\hline 21 & & 500 & 200 & 800 & 500 & 200 & 000 & & & 5800 & 100 & 100 \\
\hline 22 & 1441600 & 1434600 & 1462200 & 1472000 & 1600400 & 1609600 & 1610200 & 1577300 & 35700 & 1484900 & 800 & 9200 \\
\hline 23 & 1440900 & 1436800 & 1462300 & 5200 & 3100 & 0200 & 8500 & 500 & 4600 & 3200 & 500 & 8300 \\
\hline 24 & 1440700 & 1437000 & 1462300 & 1476700 & 1605400 & 1613100 & 1607100 & 75000 & 1532700 & 1481700 & 34000 & 1397500 \\
\hline 25 & 1440700 & 1437000 & 1462200 & 1477600 & 1608500 & 1621200 & 1606000 & 1573700 & 1531400 & 1480400 & 1432900 & 1396600 \\
\hline 26 & & & & & & & & & & & & 95700 \\
\hline 27 & 1439600 & 1437500 & 1462300 & 1479500 & 1610000 & 1620700 & 1604200 & 1571500 & 1527600 & 1477000 & 1430000 & 1394600 \\
\hline 28 & 1439000 & 1437500 & 1462700 & 1479700 & 1610600 & 1620100 & 1603100 & 1570200 & 1525900 & 1474800 & 1428700 & 1393700 \\
\hline 29 & 1438500 & 1440500 & 1463300 & 1479900 & --- & 1618900 & 1601300 & 1568700 & 1524400 & 1472000 & 27000 & 1393000 \\
\hline 30 & 1437900 & 1454700 & 1462300 & 1480800 & --- & 8300 & 1600700 & 1567100 & 1522100 & 1470100 & 25400 & 1392300 \\
\hline 31 & 1437500 & --- & 1462300 & 1481900 & --- & 1617200 & --- & 1565600 & --- & 1468500 & 1423900 & --- \\
\hline MAX & & & 1472900 & & 1610600 & & 1617900 & & & & 57200 & 22400 \\
\hline MIN & 1437500 & 1434600 & 1457500 & 1459900 & 1483200 & 1609600 & 1600700 & 1565600 & 1522100 & 1468500 & 1423900 & 1392300 \\
\hline $\mathrm{a}$ & 431.27 & 432.20 & 432.61 & 433.66 & 440.43 & 440.77 & 439.92 & 438.09 & 435.80 & 432.94 & 430.53 & 428.80 \\
\hline $\mathrm{b}$ & -18900 & +17200 & +7600 & +19600 & +128700 & +6600 & -16500 & -35100 & -43500 & -53600 & -44600 & -31600 \\
\hline C & 4687 & 1198 & 1157 & 1216 & 1271 & 3132 & 6050 & 8657 & 9346 & 10054 & 8694 & 7165 \\
\hline
\end{tabular}

CAL YR 1998 b +74000

WTY YR 1999 b -64100

a Elevation, in feet, at end of month.

b Change in contents, in acre-feet.

c Total evaporation, in acre-feet, provided by U.S. Bureau of Reclamation, not reviewed by U.S. Geological Survey. 


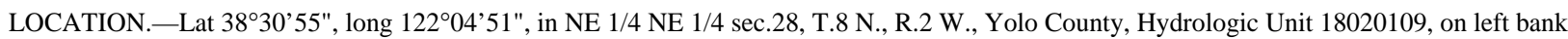
1 mi downstream from Cold Canyon, 1.3 mi downstream from Monticello Dam, and 6 mi west of Winters.

DRAINAGE AREA.- $574 \mathrm{mi}^{2}$.

PERIOD OF RECORD.—July 1930 to current year.

CHEMICAL DATA: Water years 1951-66, 1973-81.

WATER TEMPERATURE: Water years 1966-81.

REVISED RECORDS._WSP 901: 1937-38(M). WSP 1285: 1932(M), 1935-36(M), 1940(M), 1942-43(M), 1951, $1952(M)$. WSP $1565: 1957$. WSP 1931: Drainage area.

GAGE.-Water-stage recorder. Datum of gage is $160.75 \mathrm{ft}$ above sea level (river-profile survey). June 28, 1930, to Feb. 29, 1940, at datum about $1 \mathrm{ft}$ higher.

REMARKS.-Records good. Flow completely regulated by Lake Berryessa (station 11453900) beginning January 1957. See schematic diagram of lower Sacramento River Basin.

EXTREMES FOR PERIOD OF RECORD.-Maximum discharge, $81,000 \mathrm{ft}^{3} / \mathrm{s}$, Feb. 27, 1940, gage height, $30.5 \mathrm{ft}$, present datum, from rating curve extended above 30,000 ft 3 /s; no flow, Sept. 6-15, 1950, July 26 to Sept. 1, Sept. 6-9, 1955. Since completion of Monticello Dam in 1957, maximum discharge, 18,700 ft $33 / \mathrm{s}$, Mar. 2, 1983, gage height, $19.55 \mathrm{ft}$; minimum daily, $6.1 \mathrm{ft}^{3} / \mathrm{s}$, Dec. $19,1967$.

EXTREMES OUTSIDE PERIOD OF RECORD.-Maximum stage known since at least 1905, that of Feb. 27, 1940, on basis of records for station at Winters.

DISCHARGE, CUBIC FEET PER SECOND, WATER YEAR OCTOBER 1998 TO SEPTEMBER 1999 DAILY MEAN VALUES

\begin{tabular}{|c|c|c|c|c|c|c|c|c|c|c|c|c|}
\hline DAY & $\mathrm{OCT}$ & NOV & $\mathrm{DEC}$ & JAN & FEB & MAR & $\mathrm{APR}$ & MAY & JUN & JUL & AUG & SEP \\
\hline 1 & 271 & 96 & 72 & 86 & 72 & 1060 & 1490 & 709 & 715 & 826 & 598 & 518 \\
\hline 2 & 266 & 75 & 88 & 86 & 72 & 1090 & 1420 & 711 & 684 & 822 & 598 & 484 \\
\hline 3 & 256 & 75 & 102 & 86 & 72 & 1120 & 1350 & 712 & 642 & 743 & 617 & 486 \\
\hline 4 & 280 & 99 & 93 & 85 & 72 & 1110 & 1280 & 666 & 600 & 682 & 622 & 454 \\
\hline 5 & 298 & 140 & 86 & 85 & 72 & 1080 & 1230 & 603 & 545 & 684 & 603 & 422 \\
\hline 6 & 325 & 136 & 86 & 85 & 79 & 1050 & 1200 & 550 & 543 & 711 & 595 & 456 \\
\hline 7 & 325 & 107 & 86 & 85 & 136 & 1020 & 1150 & 555 & 560 & 783 & 564 & 483 \\
\hline 8 & 313 & 98 & 86 & 85 & 80 & 990 & 1180 & 586 & 597 & 740 & 510 & 483 \\
\hline 9 & 294 & 98 & 99 & 85 & 455 & 1190 & 1180 & 600 & 627 & 788 & 525 & 483 \\
\hline 10 & 271 & 98 & 110 & 85 & 676 & 1280 & 1150 & 625 & 642 & 836 & 562 & 472 \\
\hline 11 & 256 & 98 & 104 & 82 & 694 & 1280 & 1280 & 681 & 679 & 795 & 567 & 426 \\
\hline 12 & 264 & 83 & 99 & 81 & 635 & 1250 & 1470 & 721 & 660 & 784 & 564 & 424 \\
\hline 13 & 266 & 74 & 99 & 81 & 688 & 1210 & 1480 & 651 & 663 & 784 & 593 & 419 \\
\hline 14 & 260 & 82 & 99 & 80 & 688 & 1170 & 1470 & 572 & 651 & 776 & 601 & 398 \\
\hline 15 & 246 & 86 & 82 & 82 & 688 & 1210 & 1420 & 567 & 644 & 764 & 587 & 396 \\
\hline 16 & 247 & 110 & 84 & 85 & 688 & 1170 & 1380 & 619 & 681 & 724 & 563 & 396 \\
\hline 17 & 240 & 131 & 99 & 85 & 695 & 1140 & 1330 & 693 & 665 & 649 & 556 & 394 \\
\hline 18 & 235 & 111 & 99 & 85 & 693 & 1090 & 1280 & 727 & 593 & 581 & 589 & 374 \\
\hline 19 & 235 & 111 & 95 & 85 & 691 & 1050 & 1220 & 717 & 570 & 617 & 652 & 362 \\
\hline 20 & 262 & 111 & 90 & 86 & 692 & 1030 & 1160 & 689 & 601 & 656 & 613 & 385 \\
\hline 21 & 282 & 96 & 79 & 86 & 702 & 920 & 1120 & 655 & 630 & 687 & 601 & 418 \\
\hline 22 & 302 & 83 & 70 & 86 & 692 & 663 & 1060 & 654 & 643 & 707 & 611 & 397 \\
\hline 23 & 285 & 79 & 142 & 86 & 692 & 374 & 980 & 642 & 738 & 730 & 559 & 353 \\
\hline 24 & 248 & 82 & 86 & 86 & 708 & 538 & 914 & 630 & 796 & 787 & 572 & 324 \\
\hline 25 & 212 & 105 & 86 & 86 & 807 & 1640 & 866 & 636 & 741 & 770 & 600 & 308 \\
\hline 26 & 193 & 110 & 86 & 86 & 901 & 1780 & 811 & 654 & 717 & 720 & 616 & 304 \\
\hline 27 & 185 & 94 & 86 & 86 & 940 & 1770 & 782 & 657 & 717 & 688 & 593 & 289 \\
\hline 28 & 174 & 87 & 86 & 86 & 976 & 1720 & 751 & 664 & 760 & 660 & 550 & 295 \\
\hline 29 & 141 & 87 & 86 & 84 & --- & 1670 & 731 & 685 & 790 & 635 & 534 & 329 \\
\hline 30 & 123 & 82 & 86 & 79 & --- & 1590 & 711 & 704 & 812 & 668 & 534 & 317 \\
\hline 31 & 123 & --- & 86 & 72 & --- & 1550 & --- & 731 & --- & 637 & 534 & --- \\
\hline TOTAL & 7678 & 2924 & 2837 & 2608 & 15056 & 36805 & 34846 & 20266 & 19906 & 22434 & 17983 & 12049 \\
\hline MEAN & 248 & 97.5 & 91.5 & 84.1 & 538 & 1187 & 1162 & 654 & 664 & 724 & 580 & 402 \\
\hline MAX & 325 & 140 & 142 & 86 & 976 & 1780 & 1490 & 731 & 812 & 836 & 652 & 518 \\
\hline MIN & 123 & 74 & 70 & 72 & 72 & 374 & 711 & 550 & 543 & 581 & 510 & 289 \\
\hline $\mathrm{AC}-\mathrm{FT}$ & 15230 & 5800 & 5630 & 5170 & 29860 & 73000 & 69120 & 40200 & 39480 & 44500 & 35670 & 23900 \\
\hline
\end{tabular}




\section{PUTAH CREEK NEAR WINTERS, CA—Continued}

STATISTICS OF MONTHLY MEAN DATA FOR WATER YEARS 1931 - 1956, BY WATER YEAR (WY)

\begin{tabular}{|c|c|c|c|c|c|c|c|c|c|c|c|c|}
\hline & OCT & NOV & $\mathrm{DEC}$ & JAN & $\mathrm{FEB}$ & MAR & $\mathrm{APR}$ & MAY & JUN & JUL & AUG & SEP \\
\hline MEAN & 8.62 & 96.0 & 993 & 1284 & 1716 & 976 & 514 & 137 & 42.1 & 12.5 & 6.94 & 5.84 \\
\hline MAX & 45.4 & 807 & 5110 & 3957 & 6468 & 3506 & 2729 & 452 & 156 & 63.7 & 31.7 & 20.8 \\
\hline (WY) & 1951 & 1951 & 1956 & 1952 & 1938 & 1938 & 1941 & 1941 & 1942 & 1941 & 1941 & 1941 \\
\hline MIN & .89 & 3.17 & 7.16 & 44.6 & 66.7 & 118 & 40.8 & 12.3 & 6.72 & 2.39 & .000 & 1.47 \\
\hline$(W Y)$ & 1956 & 1956 & 1931 & 1947 & 1948 & 1932 & 1931 & 1931 & 1931 & 1955 & 1955 & 1931 \\
\hline
\end{tabular}

SUMMARY STATISTICS

ANNUAL MEAN

HIGHEST ANNUAL MEAN

LOWEST ANNUAL MEAN

HIGHEST DAILY MEAN

LOWEST DAILY MEAN

ANNUAL SEVEN-DAY MINIMUM

INSTANTANEOUS PEAK FLOW

INSTANTANEOUS PEAK STAGE

ANNUAL RUNOFF (AC-FT)

10 PERCENT EXCEEDS

50 PERCENT EXCEEDS

90 PERCENT EXCEEDS
WATER YEARS 1931 - 1956

\begin{tabular}{|c|c|c|}
\hline 477 & & \\
\hline 1387 & & 1941 \\
\hline 48.1 & & 1931 \\
\hline 54500 & Feb 27 & 1940 \\
\hline .00 & Sep 6 & 1950 \\
\hline .00 & Sep 6 & 1950 \\
\hline 81000 & Feb 27 & 1940 \\
\hline 30.5 & Feb 27 & 1940 \\
\hline 345500 & & \\
\hline 924 & & \\
\hline 38 & & \\
\hline 3.0 & & \\
\hline
\end{tabular}

STATISTICS OF MONTHLY MEAN DATA FOR WATER YEARS 1960 - 1999, BY WATER YEAR (WY)

\begin{tabular}{|c|c|c|c|c|c|c|c|c|c|c|c|c|}
\hline & $\mathrm{OCT}$ & NOV & $\mathrm{DEC}$ & JAN & FEB & MAR & APR & MAY & JUN & JUL & AUG & SEP \\
\hline MEAN & 225 & 89.5 & 110 & 506 & 713 & 776 & 655 & 547 & 596 & 627 & 547 & \\
\hline MAX & 476 & 263 & 1625 & 4406 & 6271 & 7791 & 5023 & 1018 & 773 & 802 & 681 & \\
\hline$(W Y)$ & 1972 & 1987 & 1984 & 1970 & 1998 & 1983 & 1982 & 1983 & 1981 & 1984 & 1975 & \\
\hline MIN & 13.3 & 14.9 & 11.6 & 11.6 & 21.6 & 40.9 & 110 & 155 & 328 & 338 & 298 & \\
\hline (WY) & 1960 & 1963 & 1961 & 1960 & 1960 & 1962 & 1960 & 1960 & 1960 & 1960 & 1960 & \\
\hline
\end{tabular}

SUMMARY STATISTICS

ANNUAL TOTAL

ANNUAL MEAN

HIGHEST ANNUAL MEAN

LOWEST ANNUAL MEAN

HIGHEST DAILY MEAN

LOWEST DAILY MEAN

ANNUAL SEVEN-DAY MINIMUM

INSTANTANEOUS PEAK FLOW

INSTANTANEOUS PEAK STAGE

ANNUAL RUNOFF (AC-FT)

10 PERCENT EXCEEDS

50 PERCENT EXCEEDS

90 PERCENT EXCEEDS
FOR 1998 CALENDAR YEAR

372755

1021

$10900 \quad$ Feb 8

64 Jan 11

73 Jan 22

739400

1370

566

83
FOR 1999 WATER YEAR

$\begin{aligned} 195392 & \\ 535 & \\ & \\ 1780 & \text { Mar 26 } \\ 70 & \text { Dec 22 } \\ 73 & \text { Jan 30 } \\ 1830 & \text { Mar 26 } \\ 10.34 & \text { Mar 26 } \\ 387600 & \\ 1130 & \\ 567 & \\ 85 & \end{aligned}$

WATER YEARS 1960 - 1999

\begin{tabular}{|c|c|c|c|}
\hline 481 & & & \\
\hline 1580 & & & 1983 \\
\hline 132 & & & 1960 \\
\hline 17700 & Mar & 2 & 1983 \\
\hline 6.1 & Dec & 19 & 1967 \\
\hline 8.3 & Nov & 7 & 1963 \\
\hline 18700 & Mar & 2 & 1983 \\
\hline 19.55 & Mar & 2 & 1983 \\
\hline 348700 & & & \\
\hline 723 & & & \\
\hline 357 & & & \\
\hline 52 & & & \\
\hline
\end{tabular}


11454210 PUTAH SOUTH CANAL NEAR WINTERS, CA

LOCATION.-Lat 38²9’534, long 12200’07", in Rio De Los Putos Grant, T.8 N., R.1 W., Solano County, Hydrologic Unit 18020109, on left bank, $500 \mathrm{ft}$ downstream from diversion headgate structure on Lake Solano, and $2.7 \mathrm{mi}$ southwest of Winters.

PERIOD OF RECORD.-October 1994 to September 1997, October 1998 to September 1999. Monthly and yearly totals were published during water years 1972-93.

GAGE.-Water-stage recorder. Elevation of gage is $160 \mathrm{ft}$ above sea level, from topographic map.

REMARKS.-Water from canal is diverted for irrigation, municipal, and industrial use. See schematic diagram of lower Sacramento River Basin. COOPERATION.-Records provided by U.S. Bureau of Reclamation.

EXTREMES FOR PERIOD OF RECORD.-Maximum daily discharge, $725 \mathrm{ft}^{3}$ /s, July 1, 1999; no flow on some days during most years.

DISCHARGE, CUBIC FEET PER SECOND, WATER YEAR OCTOBER 1998 TO SEPTEMBER 1999 DAILY MEAN VALUES

\begin{tabular}{|c|c|c|c|c|c|c|c|c|c|c|c|c|}
\hline DAY & $\mathrm{OCT}$ & NOV & $\mathrm{DEC}$ & JAN & FEB & MAR & APR & MAY & JUN & JUL & AUG & SEP \\
\hline 1 & 223 & 74 & 75 & 50 & 40 & 47 & 109 & 393 & 647 & 725 & 555 & 501 \\
\hline 2 & 220 & 20 & 75 & 43 & 40 & 50 & 107 & 419 & 621 & 705 & 558 & 476 \\
\hline 3 & 220 & 30 & 63 & 40 & 40 & 50 & 105 & 432 & 593 & 672 & 558 & 455 \\
\hline 4 & 227 & 92 & 50 & 45 & 40 & 50 & 105 & 461 & 561 & 622 & 571 & 427 \\
\hline 5 & 237 & 100 & 55 & 50 & 40 & 50 & 105 & 499 & 503 & 615 & 565 & 408 \\
\hline 6 & 265 & 86 & 60 & 50 & 40 & 50 & 105 & 518 & 495 & 620 & 561 & 434 \\
\hline 7 & 271 & 73 & 60 & 50 & 34 & 50 & 105 & 543 & 519 & 670 & 525 & 461 \\
\hline 8 & 270 & 63 & 60 & 55 & 30 & 50 & 98 & 538 & 557 & 653 & 475 & 468 \\
\hline 9 & 249 & 58 & 60 & 60 & 30 & 40 & 95 & 560 & 565 & 669 & 495 & 446 \\
\hline 10 & 226 & 55 & 60 & 48 & 37 & 50 & 97 & 582 & 587 & 708 & 515 & 436 \\
\hline 11 & 221 & 51 & 60 & 37 & 50 & 45 & 100 & 610 & 614 & 694 & 537 & 411 \\
\hline 12 & 215 & 53 & 60 & 35 & 50 & 40 & 100 & 629 & 609 & 671 & 531 & 396 \\
\hline 13 & 215 & 58 & 60 & 43 & 43 & 47 & 104 & 596 & 615 & 665 & 554 & 383 \\
\hline 14 & 218 & 60 & 45 & 50 & 40 & 50 & 103 & 552 & 605 & 675 & 546 & 369 \\
\hline 15 & 208 & 38 & 40 & 52 & 50 & 55 & 100 & 549 & 589 & 665 & 523 & 361 \\
\hline 16 & 203 & 87 & 60 & 55 & 52 & 73 & 107 & 583 & 609 & 645 & 509 & 367 \\
\hline 17 & 190 & 85 & 55 & 55 & 46 & 74 & 127 & 627 & 605 & 596 & 516 & 370 \\
\hline 18 & 189 & 61 & 50 & 55 & 40 & 70 & 142 & 651 & 562 & 550 & 549 & 355 \\
\hline 19 & 186 & 73 & 57 & 55 & 40 & 77 & 159 & 649 & 552 & 560 & 597 & 343 \\
\hline 20 & 210 & 48 & 45 & 52 & 45 & 80 & 170 & 617 & 570 & 595 & 582 & 366 \\
\hline 21 & 227 & 50 & 45 & 50 & 50 & 80 & 183 & 611 & 567 & 620 & 565 & 385 \\
\hline 22 & 240 & 50 & 45 & 50 & 50 & 80 & 208 & 606 & 606 & 632 & 558 & 379 \\
\hline 23 & 248 & 50 & 84 & 47 & 50 & 93 & 215 & 598 & 643 & 655 & 528 & 335 \\
\hline 24 & 209 & 63 & 50 & 45 & 44 & 37 & 240 & 590 & 674 & 675 & 545 & 301 \\
\hline 25 & 169 & 75 & 50 & 45 & 40 & 88 & 273 & 598 & 666 & 669 & 559 & 283 \\
\hline 26 & 154 & 50 & 50 & 45 & 40 & 100 & 334 & 607 & 635 & 640 & 571 & 261 \\
\hline 27 & 150 & 40 & 50 & 45 & 40 & 100 & 350 & 608 & 630 & 611 & 555 & 258 \\
\hline 28 & 132 & 49 & 50 & 45 & 40 & 87 & 342 & 610 & 664 & 594 & 511 & 281 \\
\hline 29 & 104 & 50 & 47 & 45 & --- & 80 & 318 & 632 & 690 & 581 & 487 & 290 \\
\hline 30 & 92 & 67 & 50 & 42 & --- & 75 & 366 & 640 & 706 & 606 & 508 & 280 \\
\hline 31 & 86 & --- & 50 & 40 & --- & 85 & --- & 662 & --- & 591 & 507 & --- \\
\hline TOTAL & 6274 & 1809 & 1721 & 1479 & 1181 & 2003 & 5072 & 17770 & 18059 & 19849 & 16716 & 11286 \\
\hline MEAN & 202 & 60.3 & 55.5 & 47.7 & 42.2 & 64.6 & 169 & 573 & 602 & 640 & 539 & 376 \\
\hline MAX & 271 & 100 & 84 & 60 & 52 & 100 & 366 & 662 & 706 & 725 & 597 & 501 \\
\hline MIN & 86 & 20 & 40 & 35 & 30 & 37 & 95 & 393 & 495 & 550 & 475 & 258 \\
\hline $\mathrm{AC}-\mathrm{FT}$ & 12440 & 3590 & 3410 & 2930 & 2340 & 3970 & 10060 & 35250 & 35820 & 39370 & 33160 & 22390 \\
\hline
\end{tabular}

STATISTICS OF MONTHLY MEAN DATA FOR WATER YEARS 1995 - 1999, BY WATER YEAR (WY)

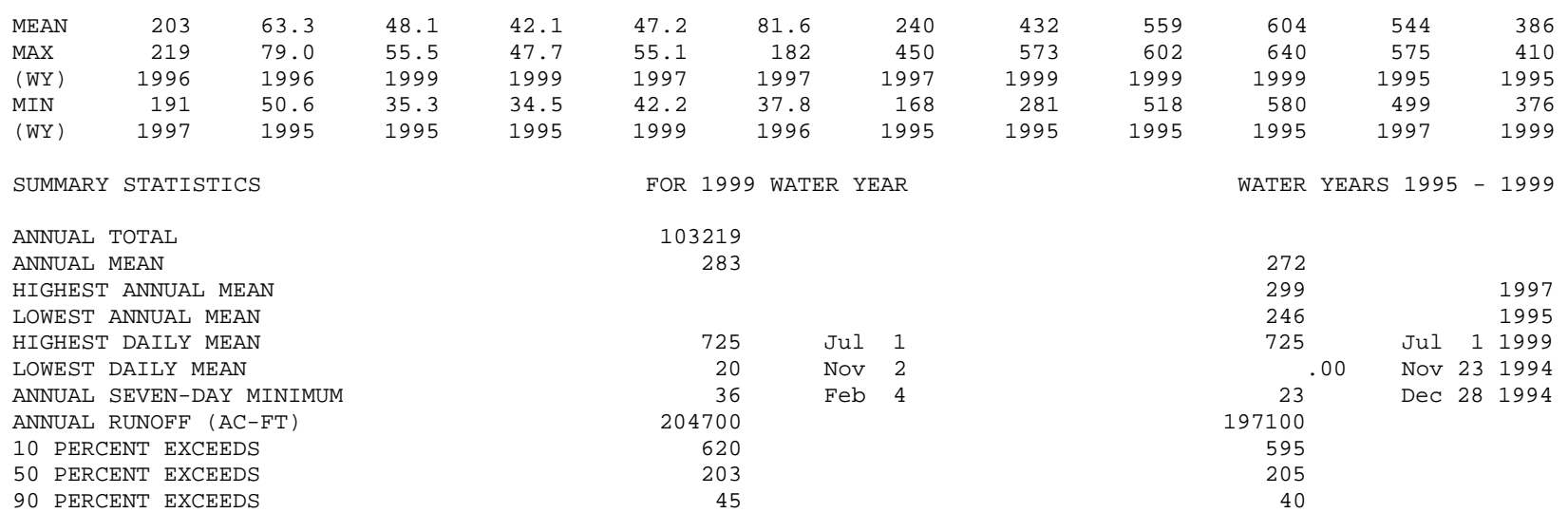




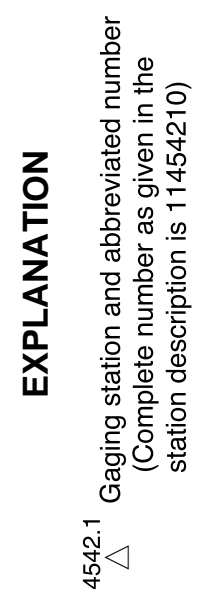


As the number of streams on which streamflow information is likely to be desired far exceeds the number of stream-gaging stations feasible to operate at one time, the U.S. Geological Survey collects limited streamflow data at sites other than stream-gaging stations. When limited streamflow data are collected on a systematic basis over a period of years for use in hydrologic analyses, the site at which the data are collected is called a partial-record station. Data collected at these partial-record stations are usable in low- or flood-flow analyses, depending on the type of data collected.

Discharge measurements made at miscellaneous sites during water year 1999

\begin{tabular}{|c|c|c|c|c|c|c|}
\hline \multirow[b]{2}{*}{ Station no. } & \multirow[b]{2}{*}{ Station name } & \multirow[b]{2}{*}{ Location } & \multirow{2}{*}{$\begin{array}{l}\text { Drainage } \\
\text { area } \\
\left(\mathrm{mi}^{2}\right)\end{array}$} & \multirow{2}{*}{$\begin{array}{l}\text { Period of } \\
\text { record }\end{array}$} & \multicolumn{2}{|c|}{ Measurements } \\
\hline & & & & & Date & $\begin{array}{l}\text { Discharge } \\
\left(\mathrm{ft}^{3} / \mathrm{s}\right)\end{array}$ \\
\hline \multicolumn{7}{|c|}{ SACRAMENTO RIVER BASIN } \\
\hline 11341900 & $\begin{array}{l}\text { Dog Creek at Delta, } \\
\text { CA }\end{array}$ & $\begin{array}{l}\text { Lat } 40^{\circ} 56^{\prime} 17^{\prime}, \text {, long } 122^{\circ} 25^{\prime} 13^{\prime} \text {, in SE } 1 / 4 \\
\text { NE } 1 / 4 \text { sec. } 34 \text {, T.36 N., R.5 W., Shasta } \\
\text { County, Hydrologic Unit } 18020005,0.1 \mathrm{mi} \\
\text { upstream from mouth, } 0.5 \text { mi southwest of } \\
\text { Delta, and } 25 \text { mi north of Redding. }\end{array}$ & 17.3 & $\begin{array}{r}\text { a1975, } \\
1976-84 \\
1986-99\end{array}$ & $\begin{array}{l}11-02-98 \\
02-10-99 \\
03-03-99 \\
09-10-99\end{array}$ & $\begin{array}{l}\text { b9.17 } \\
241 \\
264 \\
\text { b5.72 }\end{array}$ \\
\hline $\begin{array}{l}38352512143 \\
\quad 4601\end{array}$ & $\begin{array}{l}\text { Willow Slough } \\
\text { Bypass near Davis, } \\
\text { CA }\end{array}$ & $\begin{array}{l}\text { Lat } 38^{\circ} 35^{\prime} 25^{\prime}, \text {, long } 121^{\circ} 43^{\prime} 46^{\prime \prime}, \text { in SE } 1 / 4 \\
\text { SE } 1 / 4 \text { sec. } 27 \text {, T.9 N., R.2 E., Yolo County, } \\
\text { Hydrologic Unit } 18020109 \text {, at County Road } \\
102,3.5 \text { mi northeast of Davis. }\end{array}$ & - & - & $\begin{array}{l}08-18-99 \\
08-27-99 \\
09-03-99 \\
09-10-99 \\
09-17-99 \\
09-24-99\end{array}$ & \\
\hline $\begin{array}{l}38374912143 \\
3701\end{array}$ & $\begin{array}{l}\text { Willow Slough near } \\
\text { Woodland, CA }\end{array}$ & $\begin{array}{l}\text { Lat } 38^{\circ} 37^{\prime} 49^{\prime}, \text {, long } 121^{\circ} 43^{\prime} 37^{\prime \prime}, \text { in NW } 1 / 4 \\
\text { NW } 1 / 4 \text { sec.14, T.9 N., R.2 E., Yolo County, } \\
\text { Hydrologic Unit } 18020109,1,000 \mathrm{ft} \\
\text { downstream of County Road } 102 \text {, and } 3.8 \\
\text { miles southeast of Woodland. }\end{array}$ & - & - & $\begin{array}{l}08-18-99 \\
08-27-99 \\
09-03-99 \\
09-10-99 \\
09-17-99 \\
09-24-99\end{array}$ & \\
\hline
\end{tabular}

a Published as a miscellaneous measurement.

b Base flow. 
